A RESPONSIBLE INDUSTRY PROMOTING ENVIRONMENTAL PROTECTION, BIODIVERSITY AND OCCUPATIONAL HEALTH THROUGH SUSTAINABLE AGRICULTURAL TECHNOLOGY

\section{Mutiso}

Three years of concerted effort between industry, government and development agencies have led to measurable improvements in compliance with the FAO Code of Conduct on the Distribution and Use of Pesticides in the pilot countries of the GIFAP Safe Use Project - Guatemala, Kenya and Thailand. Apart from the field achievements in the training of farmers, retailers and medical professionals and the creation of awareness among pre-school leavers, the Project has generated several data bases which will help chart the direction for its next three years and provide a reference point for expanding the Project into other countries.

One significant conclusion of the Project's work to date is that Prior Informed Consent is an inadequate alternative to the establishment of proper national pesticide registration schemes. There has been a close correlation between developing countries which have strong national agrochemical industry associations and those which have national pesticide registration schemes. Clearly industry must continue to take a leading role.

Integrated Pest Management will be the key to pest management strategies in the next quarter century if we are to provide double the present world population with safe and affordable food and fibre while halting and reversing the encroachment of agriculture on our diminishing natural heritage.

A detailed exposition of the methodology development by the Project in the pilot countries of Africa, Asia and Latin America will demonstrate that the Safe Use Project Modules are an ideal vehicle for rapid and cost effective technology transfer into other third world countries.

Dr. Clive Mutiso, Village Plaza, Ngara Road, P O Box 60723, Kenya,

tel $+254 / 2742006$
THE CONSUMER, THE CASH-DESK, CATERPILLARS AND CHEMICALS

\section{Calvert}

The paper addresses the question of 'Affordable Food' through consideration of the real cost of different food production systems to the farmer, to the consumer and to the environment. The author is in a unique position, as the head of a very large consumer-owned farming business, to comment on the external factors likely to influence the development of agricultural production techniques. The paper is based on work done and experience obtained within the U.K. but many of the principles developed apply across a wide range of different environments.

The cost to the farmer of different farming systems may be measured through the relationship between varying levels of input use and the quality, yield and end product price achieved.

The cost to the consumer may be direct through the price paid for goods purchased, indirect through the taxation required to support subsidised agricultural systems or subjective through the effects on choice.

The cost to the environment may be positive, negative or neutral depending on the techniques of food production employed.

The paper compares the impact of conventional high input systems with organic farming methods and integrated crop management techniques, drawing on original research of international significance.

An attempt is made to balance the financial and ethical costs of the different systems and potentially controversial conclusions are drawn about the likely future direction of food production techniques.

Dr. Mike Calvert, CWS Agriculture, The White House, Stoughton-Leicester, OE2 2FL Great Britain, tel +44533714278, fax +44533720640

THE SAFEST, LOWEST-COST AND MOST SUSTAINABLE FARMING IN HISTORY

\title{
D.T. Avery
}

The high-yield modern agriculture which increasingly dominates the world food system:

1) provides the safest food ever available on the planet;

2) provides the most affordable food in history, and will produce food at even lower real costs in the years ahead - despite the huge expected increase in food and protein demand; and

3 ) is the most sustainable food production system devised by man in the 10,000 -year history of agriculture.

High-yield agriculture minimizes the contamination of grains and oilseeds by fungal toxins, both through the use of fungicides and through the suppression of insect and rodent damage which give fungi access to crop seeds. An even more important food safety benefit is the production of ample supplies of attractively-priced fruits and vegetables - which can cut human cancer risks in half.

The risks to humans from the pesticides and fertilizers used in these bigh-yield farming systems are nearly zero. In fact, tests show that there is $\mathbf{1 0 , 0 0 0}$ times more cancer risks in the natural chemicals found in our food that in the residues of modern pesticides. The so-called 'contamination' of our groundwater by nitrate and pesticide traces is even more trivial as a health threat than the residues on our food. Neither is as dangerous as eating mustard or pickles.

High-yield agriculture has been lowering the real cost of food for more than 50 years, during a doubling of the world's population and a major upgrading of consumer diets. It does this by raising productivity - more crop from each acre of land, more crop from each hour of labor expended, ańd more crop from each dollar invested in production. There is no reason to doubt that science and technology wil continue to raise agricultural productivity, so long as we will continue to make adequate investments in research and infrastructure.

Soil erosion is the true long-term threat to sustainable food production on this planet, and high-yield farming today has achieved the ability to virtually stop soil erosion in its tracks. When we double the yields on the best and safest acres, we cut soil erosion by more than half (for a given level of output). Even better, herbicides have now given the modern farmer the opportunity to substitute conservation tillage systems for the old 'bare earth' farming techniques still used by both organic and traditional farmers. Using herbicides for weed control, modern farmers should now be able to build topsoil on the good farmlands - in that midst of high-yield crop production! 
HERBICIDE-RESISTANT WEEDS: EVOLUTION, MECHANISMS AND POSSTBLE MANAGEMENT

B. Rubin

The rapid increase in the number of worldwide reports of weeds resistant to virtually all classes of herbicides, indicates the importance of the phenomenon. Herbicide resistance closely associated with high selection pressure imposed by an intensive agriculture, e.g., monoculture, mono-herbicide and minimum tillage. Under these circumstances, repeated application of high rates of the same or similar residual herbicide usually practiced. However, herbicide resistance has appeared also when non-residual herbicides commonly used. The harmful effect of herbicide-resistant weed populations endangers the usefulness of chemical weed control, and poses a real threat to the environment. Altered herbicide target site, enhanced detoxification and sequestration of the herbicide away from its target site are the major reported resistance mechanisms. In addition, weed populations exhibiting cross-resistance and multiple-resistance to more than one chemical, were also reported. To improve the combat against herbicideresistant weeds, there is an urgent need for rapid and reliable identification methods. Management practices to prevent or delay herbicide resistance evolution will be discussed, including crop and herbicide rotations, use of herbicide mixtures and combinations with synergists and safeners.

Department of Field Crops, Vegetables and Genetics, Faculty of Agricuiture, The Hebrew University of Jerusalem, Rehovot 76100 , Israel.
INSECTICIDE RESISTANCE ACTION COMMITEE (IRAC) : INDUSTRY'S RESPONSE TO RESISTANCE

\section{H. Buholzer , S. Iving}

The Intemational Organisation of Plant Protection Agenis Manufacturers (GIFAP) initiated the fomation of the Insecticide Resistance Action committee (IRAC) to act as a consultative body on all scientific and technical matters relating to insecticide/acaricide resistance.

IRAC acts to coordinate efforts of industy to combat resistance and thus prolong the life of insect/mite control agents. Such actions are in the best interests of industry, the grower and environment.

The existence of IRAC shows that industry is concemed about resistance and wishes to develop strategies to combot resistance. IRAC is also dedicated to article 3.10 of the FAO-Codex, which emphasises the joint efforts of authorities, industry, national and intemational organisations and public research institutes.

IRAC is involved in (1) global surveys to assess the status of resistance on a crop/country/insect or mite bcsis, (2) developing management strategies based on good agricultural practice. IRAC accomplishes this through its crop working groups ot an intemotional level, (3) sponsoring research in cructal areas of resistance monitoring/resistonce strategies in order to increcse our understanding.

IRAC is responding to the increased need for resistance management by contributing to local research and implementation programs and by conmbuting to local research and implementation programs and by communicating its findings
involved in crop protection.

These efforts are leading to increased cooperation between companies. scientific institutes and authorities to improve our understanding and implementation of use management strategies to benefit sustaingble agnculture and the long term use of IPM.

H. Buholzer, Ciba, Crop Protection Division, $\mathrm{CH}-4002$, Bosel, Switzeriand
7

BACILLUS THURINGIENSIS RESISTANCE IN INSECTS: MECHANISMS AND MANAGEMENT

\section{M.E. Whalon, M. Bush, U. Rahardja and D. Norris}

Eight species of arthropods have evolved resistance to Bacilhus thuringiensis (B.t.) in the laboratory and field. Although B.t. endotoxins offer an environmentally-sufe alternative to chemical insecticides increasing numbers of pest manageneat workers are becoming concerned that the increased use of conventionally applies B.t. and biotechnologically engineered plants with $B . . t$ toxin genes will result in broad spread resistance.

Several workers have suggested the use of these toxins in various deployment strategies including mixtures, rotations and sequences to delay the onset of resistance. Others have suggested manipulation of pest refugia, low-dosages or even altematjve promoter strategies in transgenic plants.

We report the suspected mechanjsms and proposed management of B.t. resistance in Colorado potato beetle, Leptinotarsa decemlineata, including: 1) current levels of laboratory and field resistance, 2) monitoring tools and tactics and 3) results of mechanism studies and field maragement strategies, tactics and tools.

B-11 Pesticide Research Center

Michigan State University

East Lansing, Michigan 48824-1311
8

INSECTICIDE RESISTANCE MANAGEMENT OF Plutella xylostella L. ON CRUCIFERS IN SOUTHEAST ASIA. ASFECTS OF IMFLEMENTATYON

S. Uk

\section{Abstract}

The Diamondback moth (DBM), Plutella xylostella L., is one of the most serious pests of cruciferous crops in sourheast Asia. Farmers have developed the habit of spraying their crops once or twice a week starting two weeks after transplanting, regardless of infestation level. This has resulted in widespread resistance of this pest to almost all insecticides on the market before 1990 .

Recently, new types of products such as avermectin, diafenthiuron, fipronil, tebufenozide and $\mathrm{AC} 303,630$ have been introduced in close succession in some parts of Southeast Asia for use on vegetables. These products have new modes of action quite distinct from each other. They ald have good activity on DBM and appear not to suffer from crossresistance to existing organophosphates, carbamates, pyrethroids, or acylureas. This gives a good opportunity to avoid or delay the resistance development to these new compounds if farmers do not solely use a single product during the course of a growing season.

In a case study in Malaysia and Thailand involving selection experiments in field-cages over a period of three years, no obvious resistance development by DBM to diafenthjuron was observed. Nevertheless, the ability of the DBM to develop resistance cannot be excluded. Diafenthiuron should never be used alone throughout the season year after year. It should be alternated with any of the products listed above. An effective B.t. product should also be included in the alternation regime.

The active participation of pesticide dealers and farmers is the key to success in the management of DBM resistance in southeast Asia. Aspects of practical implementation of insecticide resistance management strategy (IRM) involving closer co-operation between pesticice producers, users, advisers and regulators are suggested.

Ciba-Geigy Ltd, Crop Protection Division, R-1004.810, $\mathrm{CH}-4002$, Basie, Switzerland. 
MANAGEMENT OF RESISTANCE TO STEROL BIOSYNTHESIS INHIBITING FUNGICIDES

\section{D.W. Hollomon}

Fungicides that inhibit the biosynthesis and function of sterols are important components of plant disease control world-wide. At least 30 different active ingredients provide opportunities to control most diseases, except those caused by Phycomycete fungi. The larger group (DMIs) inhibit primarily the sterol 14a demethylase step in the pathway, whilst "morpholinen fungicides inhibit two other steps. Morpholines are especially useful against powdery mildew diseases. Monitoring baseline sensitivities has revealed extensive variation within target pathogen populations. involvement of many genetic factors in controlling sensitivity. Changes in sensitivity are often exratic and difficult to relate to performance difficulties and use. After 25 years there are still no confirmed cases of practical resistance to morpholines, and this durability has played a major part in many anti-resistance strategies. Resistance to DMIs has emerged as a practical problem in a few diseases, and only for some of the older products. Restricting use of DMIs to once or twice a season, or reducing dose rate, may reduce selection pressures, but often with conflicting results. Mixtures with fungicides with different modes of action offer the main approaches to combating DMI resistance, but evidence of success of this strategy is fragmentary. Nevertheless, the mixture option bas expanded recently through introduction of new chemistry. Differences in resistance factors between different DMIs has created scope to control resistance through DMI mixtures. Exploitation of novel modes of action will see several new fungicides being registered for use in the near future, providing a significant boost to anti-resistance strategies in general. Integration of these new fungicides ixto disease control strategies will provide a challenge which, if successful,

IACR-Long Ashton Research Station, Department of Agricultural Sciences, University of Bristol, Long Ashton, Bristol, BS18 9AF, UK. Some of this variation is undoubtedly experimental, but the remainder reflects should maintain the usefulness of DMIs for many years to come.

COUNTERMEASLRE OF INSECT RESIGTANCE LO INSECTICIDES CONSULT FHOM PAST EXPERLMENTAL DATA

T. Saito, T. Mi yata, N. Sinchaisri, O. Sarnthoy, A. Vattanatangum, W. Rushtapakronchai and P.Keiameesuke

In spite of the probelms of insect resistance to insecticides have long history,died away of excellent insecticides by insecticide resistance is very regretable matter because development of new insecticide is need large quantity money and time. resistance is distress not only for company also nation and ublic organization. Because they cannot establish insect control program and cannot recommend effective insecticides to their farmers. We have many reports on the insecticide resistance from farmers. Ve have many reports on the insecticide resistance from past experimental data following suggestions could

countermeasure of insect resistance to insecticide. IRAC has been proposed insecticide/acaricide susceptibility tests IRAC has been proposed insecticide/acaricide susceptibility tests
but some improvements stil1 needed such as set up standard insectbut some improvements still needed, such as set up standard insect-
icide and insect strain, physical condition (temperature, humidity light,etc., control, bioassay methods afapt to IGR and B.t., etc. Regarding on development network for resistance monitoring, colla: Doration of federal, state, university, industry and farmer are necessary. After detected developed resistance in the insecticide ivoid nore high dosage application of the insecticide because it will produce more high level resistance, more broad cross resistance and more stable resistance.

2 . Insecticide rotation use of no cross resistance insecticides. The origine of insect resistance to insecticide is biological adaptation so that as possible as avoid continue use the same insecticide. However choice of no cross resistance insecticide is hard work. As much as many insect pest collect from field where usual high population area and select with candidate insecticide in the laboratory on successive generations. After resistant ratio gave 50 or more, then we can use it for cross resistance test to other insecticides. If no development resistance, its experiment is falure then agin collect the insect from the field because collected strain was no included resistance factor (gene). However wo must mind for some difference between laboratory breed strain anc field resistant strain.

3. The tactics for delying of insect resistance to insecticides. The tactics of integrated pest management (IPM) is contribut to the delaying of insect resistance to insecticide, however more attentions to the countermeasure of resistance is pesticide manazement (PM) such as judicious and efficent use of insecticide and ipplication of synergist.

we are working international Joint Research Project between Japan and Thailand on the Insect Toxicological Studies on Resistance to our results and discuss how approach to countermeasure of insect resistance problems.

School of Agricultural Science, Nagoya University, Furo, Chikusa, 464-01, Japan

12

PESTICIDE RESISTANCE OF Aphid gOSSYPIi AND ITS MANAGEMENT IN PROVINCE SHANXI, PR CHINA

X. Qiago, J. Zhu, J. Ean, C. Zhang, J. Li

Cotton aphid (Aphid gossypii Glover) damage was one of the factors that delimited the yields and benefits of the cotton production. in province Shanxi, PR China. It became more and more serious in recent years. Failed in a effective technical consulting system and a suitable management strategy, fast all the possible insecticides were used to control the cotton aphid. As a result, resistance of cotton aphid to some of the insecticides was developed, so that some pesticides lost their control effects and economical usefulness. In order to explain the practical situation of insecti cide resistance of cotton aphid and to find out a way to managing the problem in the cotton production, we have made field trials to observe the efficacies of most frequently used insecticides fenvalerate, omethoate, monocrotophos, and carbofuran, bioassay to determine the resistance level of this pesticide, and management of resistance of cotton aphid with alternative insecticides and pesticide mixtures.

Fenvalerate resistance was reached. the very high level (16.3 to 4730 times higher of LD50 than that of sensitive strain). With a longer than 12 years application history the fenvalerate has lost its usefulness in the practice (relative efficacy $<50$ a 1 day after spraying). The resistance of cotton aphid to omethoate were between 1-10 times higher than that of sensitive strain and to monocrotophos were 1-2.5 times higher than that of sensitive strain. Both the organophosphorous insecticides were the main varieties in practice, but the application dosages were more and more larger. The resistance to carbofuran was 1-16 times higher than that of sensitive strain. Notable was that although carbaryl was used in cotton aphid control in shanxi only 2-3 years, the tolerance of cotton aphid increased to 3.7-8.1 times higher than that of sensitive strain. The resistance level relied on the history and intensity of pesticide application.

As a practice realizable measure we took alternative insecticides and insecticide mixtures to overcome resistance problems. The results shown that the mixture of monocrotophos and carbaryl was a synergistic and rentable combination, while mixture of fervalerate and omethoate or malathion was worthless. Use of alternative agent with other structure and act mechanism would be a good strategy for resistance management. We have applied imidacloprid, a pyridine-like insecticide, to control cotton aphid. The relative efficacy was hold in $>90 \%$ in 3 weeks, as other conventional agents acted on 7-10 days. A alternative use of different agents would be recommended for reducina the risk of resistance developmeint.

Institute of Plant Protection, Shanxi Academy of Agriculturai Sciences, 64 North Nongke Road. Taiyuan 030031, FR China
FLUDIOXONIL AND IPRODIONE RESISTANCE IN BOTRYTIS CINEREA

\section{Steel, C.C.}

Fungicides registered for the control of Botrytis cinerea on grapes in Australia include the non-specific inhibitor, chlorothalonil and the site specific fungicides, the benzimidazoles (benomyl and carbendazim) and the dicarboximides (iprodione, vinclozolin and procymidone). Resistance to the benzimidazoles and dicarboximides is a common occurrence worldwide. Relatively recently, the phenylpyrroles have been developed which are a novel fungicide group with a unique mode of action (inhibition of transport associated phosphorylation of glucose). Fludioxonil is a phenylpyrrole developed for the control of $B$. cinerea which isn't yet registered in the Australasian region. Pheny!pyrroles show some similarities to the dicarboximide fungicides and consequently there may be a risk of cross resistance between these two fungicide groups. If so, then this would limit the usefulness of fludioxonil. The initial aim of this study was to obtain baseline sensitivity data for fludioxonil in $B$. cinerea isolates from grapes, strawberry, cucumber and tomato grown in Australia and to determine if cross-resistance exists to the cicarboximides. Fludioxonil proved to be a potent inhibitor of mycelial growth of both dicarboximide-sensitive and resistant isolates of growth of both dicarboximide-sensitive and resistan
$B$. cinerea with $L D_{50}$ values in the range $0.032-0.1 \mu \mathrm{M}$. Resistance to the dicarboximide fungicide group is thought to be mediated by enhanced activities of free radical detoxifying enzymes (e.g. catalase, superoxide dismutase, glutathione reductase), and their continued use is dependent upon adequate field monitoring, traditionally carried out using growth bioassays on a range of fungicide concentrations. This is necessary as it is possible to identify isolates with a range of dicarboximide sensitivities from field populations. An enzyme assay for catalase based on the release of oxygen from hydrogen peroxide was therefore evaluated as a marker for iprodione-resistance. Catalase activity was found to be positively correlated with iprodione resistance $(r=0.77, P<0.001)$ in field isolates of $B$. cinerea confirming that dicarboximide resistance is at least partially mediated by enhanced oxidative protective mechanisms.

Steel, C.C. Cooperative Research Centre for Viticulture, NSW Agriculture, Biological and Chemical Research Institute, PMB No. 10, Rydalmere, NSW 2116 Australia. 
SYSTEMIC ACQUIRED RESISTANCE SIGNALING IN PLANTS

John Ryals 1, Leslie Friedrich 1 , Kay Lawton 1, Helmut Kessmann ${ }^{2}$.

Many plants respond to a pathogen infection by inducing a broadspectrum, systemic resistant state that is effective against many pathogens for several weeks to months. This systemic acquired resistance (SAR) is an important defense response in plants and it could serve as the basis for future crop protection strategies by providing either engineered plants with increased pathogen tolerance or novel mode-of-action chemicals that stimulate the plants inherent defense mechanisms. SAR is also a facile, reliable system to study signal transduction in plants. We have identified a number of genes (SAR genes) whose expression correlates well with the onset of SAR. Plants expressing these CDNA's provide significant levels of pathogen tolerance. The expression of these genes increases in healthy plants from very low levels to levels approaching $1 \%$ of the total mRNA. Thus, the system provides for a dynamic range of gene expression. We have studied the SAR signaling pathway using Arabidopsis genetics. Several mutants have been isolated that constitutively express SAR. Also, one mutant has been isolated that can no longer be induced by INA, SA or other chemical induces. The progress of understanding these mutants will be discussed

${ }^{1}$ Ciba Agricultural Biotechnology Research Unit, Research Triangle Park, NC. ${ }^{2}$ Ciba Crop Protection Division, Basel , Switzerland.

15

FEASIBILITY OF ENGINEERING BACTERIAL DISEASE RESISTANCE INTO CROPS

\section{Florack, S. Allefs, D. Bosch and W. Stiekema}

Bacterial diseases can cause a severe decrease of yield in many crops. Breeding for bacterial disease resistance therefore is of utmost importance. A novel approach in producing resistance to plant parasites, is the application of genetic engineering technology. For engineering bacterial disease resistance into crops, several possibilities can be thought of. A general overview will be presented.

Some methods have been examined in more detail for feasibility. One of these methods is the introduction and expression of genes coding for antibacterial peptides in plants. We have studied the expression of three such peptides, namely the hordothionins from barley (Hordeum vulgare), cecropin B from the giant silkmoth (Hyalophora cecropia) and tachyplesin 1 from the Southeast Asian horseshoe crab (Tachypleus tridentatus), in plants. Expression, processing of precursot peptides, accumulation of biologically active peptide and targeting were studied either in tobacco, tomato and/or potato. Biologically active hordothionins are accumulated intracellularly up to high levels in transgenic tobacco, tomato and potato plants (see Plant Molecular Biology 24 (1994) 83-96). Cecropin B could never be detected in transgenic plants, most likely as the result of degradation by endogenous proteases (Transgenic Research 4 (1995) 132-141) and tachyplesin I was expressed at low levels in transgenic plants (submitted for publication). Results will be presented. Finally, numerous transgenic plants were evaluated for resistance to diverse bacterial pathogens to estimate the feasibility of this approach for engineering disease resistance. Especially the expression of tachyplesin I in potato looks promising with respect to engineering Enwinia soft rot resistance. Experiments to further evaluate the use of these peptides in plants ate in progress. Results from growth chamber, greenhouse and field trials with transgenic plants expressing one of these peptides will be presented.

DLO Centre for Plant Breeding and Reproduction Research (CPRODLO), Department of Molecular Biology, P.O. Box 16, NL 6700 AA WAGENINGEN, The Netherlands. Telefax: + 31837018094.
DEVELOPMENT OF A TWO-COMPONENT SYSTEM TO ENGINEER NEMATODE RESISTANCE

PC Sijmons, F vd Lee, $\rfloor$ Klap, J Spiegeler, R Krutwagen and $\underline{S} \underline{O}$ hl

The formation of nematode feeding structures (NFS) in plant roots after infection with cyst or root-knot nematodes is a complex, highly regulated process involving a prolonged interaction between nematode and the affected root tissue. By analyzing promoter-gusA fusions in Arabidopsis after infection with Heterodera schachtii or Meloidogyne incognita a number of well known promoters, such as CaMV $35 S$ and RolD, were found to be down-regulated in NFS. Using a gene-tagging strategy, plant promoters were identified which confer fairly specific expression in the NFS. These regulatory sequences were isolated, recloned in GUS cassettes and transformed back to Arabidopsis to confirm their specificity in nematode infection assays.

Both types of regulatory sequences were used in a twocomponent system aimed at the disruption of developing NFS. In this system, the NFS-active promoter confers the local expression of the ribonuclease barnase derived from Bacillus amyloliquefaciens. Leaky expression of barnase in other parts of the plant is neutralized by the concomitant expression of the second component barstar, a highly specific barnase-inhibitor. The promoter driving barstar expression is down-regulated inside the NFS. Both induction of the RNase and down-regulation of its inhibitor are triggered by the nematode shortly after seiecting its feeding cell. Here we present data indicating that the system confers increased resistance in Arabidopsis against the beet cyst nematode Heterodera schachtii.

MOGEN International nv, Einsteinweg 97, $2333 \mathrm{CB}$ Leiden, The Netherlands

16 COMMERCIALIZATION STRATEGIES FOR INSECT RESISTANT COTTON

David M. Stalker, Kevin E. McBride, John E. Kiser, Catherine M. Houck

Cotton is one of the most intensely managed field crops with respect to natural pest problems, with the most damaging crop loss resulting from insect pressure. A biotechnological and environmentally preferred solution is the introduction into and expression of Bacillus thuringiensis $\mathrm{Bt}$ ) toxin genes in cotton. We have generated cotton lines expressing the crylA(c) Bt gene toxin gene linked genetically to a gene $(b x n)$ conferring resistance to BUCTRIL ${ }^{(3)}$ herbicide. These $\mathrm{BXN}^{\mathrm{MM}} / \mathrm{Bt}$ cotton lines now exhibit various degrees of resistance to the cotton insect pests $H$, virescens, $H$, zea and $S$. exigua. Generation, screening and field trial development of these potential new transgenic cotton varieties will be detailed during this presentation as well as a novel method for the high-level expression of $\mathrm{Bt}$ toxin genes in plants.

Calgene Inc. 1920 Fifth St., Davis, CA. 95616, USA 
Dlsease resistance conferred by expression of a hydrogen peroxldegenerating enzyme In transgenle potato

Gusul Wu, Barry J. Shortt, Ellen B. Lawrence, Elaine B. Levine and Dilip M. Shah. Agricultural Group, Monsanto Company, 700 Chesterfield Village Parkway, St. Louis, MO. 63198, USA.

Plent defense responses to pathogen infection involve the production of active oxygen species (AOS) including hydrogen peroxide $\left(\mathrm{H}_{2} \mathrm{O}_{2}\right) . \mathrm{H}_{2} \mathrm{O}_{2}$ Inhibits the growth of bacterial and fungal pathogens in vitro and aiso plays an important role in triggering a battery of blochemical processes associated with plent disease resistance. We have obtained transgenic potato plants expressing a $\mathrm{H}_{2} \mathrm{O}_{2} \cdot g$ enerating glucose oxidase from Aspergillus niger. Tubers from transgenlc lines exhibited strong reslstance to a bacterial soft rot disease. Resistance to soft rot disease was mediated by $\mathrm{H}_{2} \mathrm{O}_{2}$. Transgenic potato lines with elevated levels of $\mathrm{H}_{2} \mathrm{O}_{2}$ also exhibited enhanced resistance to late blight and Verticillium wilt diseases.
WEED CONTROL. IN GLUFOSINATE RESISTANT CROPS.

\section{Ed Pieters.}

Glufosinate is a contact, non-selective herbicide that provides effective postemergence control of many broadleaf and grassy weods. It is highly biodegradable, has no residual activity, and has very low toxicity for humans and wild fauna. Genetically engineered glufosinate resistant crops will provide a selective use for glufosinate, and a valuable new weed management tool for agricultural producers.

Genetically engineered glufosinate resistant plants showed excellent tolerance to post emergence applications of glufosinate up to $1500 \mathrm{~g}$ ai ha. Broadleaf and grassy weeds in a vegetative growth stage were effectively controlled with post emergence applications of glufosinate at $400-600 \mathrm{~g}$ ai ha. Residual control and broadening of weed spectrum was provided by tank mixing glufosinate with other post emergence herbicides registered for use on crops. A second application of glufosinate or timely cultivations helped to control weeds that had not emerged at the time of the first application of glufosinate.

Genetically engineered crops did not differ from non-transtormed crops in regard to agronomic characteristics, pest susceptibility and sensitivity to other herbicides.

AgrEvo USA Company

Little Falis Centre one

2711 Centerville Road

Wimington, DE 19808

\section{CGA 245704: MODE OF ACTION OF A NEW PLANT ACTIVATOR}

H, Kessmann, J. Ryals", T. Staub, M. Oostendorp, P. Ahl Goy, C. Hołmann, L. Friedrich", T. Delaney", K. Lawton", K. Weymann", H. Ligon", B. Vemooij", E. Ward", S. Uknes"

Ciba - Geigy AG, Division Crop Protection, CH-4002 Basle; "Ciba - Geigy Corp., P.O. Box 12257 Research Triangle Park. NC 27709. USA

CGA 245704 represents a new class of chemical plant activators which has been discovered and developed by Ciba - Geigy Ltd. for use on various mono- and dicol crops.

CGA 245704 is able to mimic the biological induction of systemic acquired resistance (SAR). In vitro studies where the compound did not exhibit significant direct activity against phytopathogenic fungi and bacteria indicated that disease control activity was not due to antinicrobial etfects. Furthemore, CGA 245704 protected tobacso, cucumber, and Arabicopsis thatians against the same spectrum of diseases as observed after induction of SAR by a localized infection with a necrotizing pathogen. For example, tobacco is protected by a local infection with TMV or foliar spray of CGA 245704 against fungal (Peronospora tabacina, Cercospora nicotianae, Phytophthora parasitica), bacterial (Pseudornonas tabaci) and viral (TMV) diseases. Neither of the treatments protected tobacco against Botrytis cinerea.

Detailed studies in several dicot species showed that the compound induced the same biochemical and molecular changes as observed in systemic tissue following a local infection with a biological inducer. In all dicot species studied so far SAR correlates woll with the accumulation of pathogenesis - related (PR) - proteins (e.g. chitinases or $\beta$-1,3-glucanases). Current studies also focus on SAF in wheat and rice. As in other monocot species, induction of PR - proteins is tar less pronounced compared to dicots and involvernent of other, yet unidentified SAR - mechanisms is likely. For rice, there is good evidence that lipoxygenase is involved in SAR since it is systernically induced after a local infection with Pseudomonas syringae pv. syningae or treatment with CGA 245704

Mutants of Arabidopsis thaliana were isolated which are unable to exhibit SAR after biological induction or treatment with either salicylic acid or CGA 245704. This is an additional indication that these plant activators act only through the plant mimicking the biological induction of SAR. Transgenic tobacco and Arabidopsis thaliana expressing the bacterial enzyme salicylate hyoroxylase did not accumulate salicylic acid and did not exhibit SAR. CGA 245704 was still active in these plants which indicates that its target is the salicyite acid binding protein or a step downstream. The status of our studies on identification of the target of CGA 245704 witi be presented.

Our results suppon the hypothesis that plant activators like CGA 245704 act as functiona analogues of salicylic acid in the pathway leading to SAA.
Expression in plants of a synthetic gene encoding for a putative proctolin precursor as a strategy for crop protection.

R. Rao, P. Petrilli, E. Filippone, F. Pennacchio, C. Malva, A. Bavoso E. Tremblay and L. Monti

The expression in plants of foreign genes conferring pest resistance evidenced the existence of three major constraints: a) the use of genes encoding for Bactlus thuringtensts endotoxins induces a rapld selection of insects resistant strains (Van Rie et al, Science 247:72-74, 1990); b) the overexpression of naturally occuring plant defense proteins should be avolded since they can be toxic for mammalian (Kessler et al Science 256:1747, 1992): cl a single molecule can not protect plants agalnst all 256:1747, identfication of new genes that could overcome these problems should be an important step towards a more effectlve strategy of integrated be an important step towards a more effectlve strategy of integrated pest management. An innovative approach is the use of peptides able to blological activity of proctolin, a neuropeptide flve amminoacid long, on Helicoverpa armigera larvae. Haemocoelic injections of proctolin (0.1$10 \mu \mathrm{g}$ ) into last instar Helicoverpa armigera larvae induced a catatonic syndrome of short duration and totally reversible. This biological effect was also observed after oral injection of $10 \mu \mathrm{g}$ of proctolin. Proctolin gut absorption was futher assessed by oral injection of th. Proctolin gut peptide and monitoring its subsequent occurrence in the haeolymph. The recorded absorption rate of undegraded proctolin was $0.5 \%$. On the basis of this observation, new blocontrol strategles such as expression of neurohormone in crop plants ean be more reasonably attempted and proctolin was used as model system to define a new biotechnological approach to crop protection against insect pests. A putative proctolin precursor was synthetised and used for genetic trasformation of tobacco plants. The obtained plants showed stable insertion and expression of the synthetic gene. To reduce the degradation rate of tngested proctolin, enhanclng. then, the effleiency of the proposed strategy, a
new analogue has been designed, which proved to be more stable when exposed in vitro to gut proteases of $H$. armigera.

Department of Agronomy and Plant Genetics, via Universita'100, 80055 Portic1, Italy 
DEVELOPMENT OF GLIOCLADIUM VIRENS FOR BIOLOGICAL CONTROL OF PYTHIUM AND RHIZOCTONIA DAMPING-OFF DISEASES OF SEEDLINGS

\section{R. D Lumsden}

Gliocladium virens (=Trichoderma virens) effectively suppresses damping-off diseases caused by Pythium ultimum and Rhizoctonia solomi. The effective strain (GL-21), along with several other factors attractive to private industry, facilitated a technology transfer arrangement with W. R. Grace \& $\mathrm{Co}$., which resulted in registration of $G$. virens with the U.S. Environmental Protection Agency. Early phases of marketing for use of the product against dampingoff diseases of vegetable and omamental seedlings in glasshouse production sites are initiated. A major component of the $G$. virens biocontrol mechanism, the antibiotic metabolite gliotoxin, has been identified and isolated. Both Pythium and Rhizoctonia are sensitive to the action of this metabolite

Biocontrol of Plant Diseases Laboratory, USDA/ARS, Beltsville, MD 20705

\section{COMMERCIAL, DEVELOPMENT OP BIOFUNGICIDES}

B. Fridiendec, R. Hofstein, A. Bercovitz, H. Katz, D. Beit-Din and M. Keren-Zur

Ecogen Israel Partherahip (EIP), a fully owned subsidiayy of Bcogen Inc, in Langhome, PA has been dedicated, for the lat few years to the research, development and manufacturing of biofungicides. Based on proprietary microorganisms, EIP' $B$ first two protucts have been regittered by the United States Environmental Protection Agency (EPA). The product designatod as AQ $10^{\mathrm{Tm}}$ la the first biofungicido registered by the EPA to protect crops from powdery mildew. The product's active ingredient is a unique isolate of the fungus Ampelomyces quisgualis identified in 1980. Anpelomyces qutsqualis behaves in nature as a hyperparasize of the powdery mildew fungun. This hyperperastitem efiect hes been used as an advanuge for the contsol of powdery mildew disease. At fint the company will focus on the applieation of AQ 10 for the control of powdery mildew on

\section{Brapes.}

The recond product developed by EIP, Aspirerix was registered by tho EPA in February of 1995 as the first blofunglelde for the control of portharvent rot in fuits and vegetables. Asplre's actlve ingredient is the raturally existing yeast Cartida oleophila. The capability of thi microorganism to outgrow sorse of the fungal pachogene cuung postharven rot has servod as the main meahanism involved in the blocontrol effect of Aopire. This product will Inicially be commerclalized for applicetion againx rot control in citrus fruit.

Both of these products are poritoned as part of Integrated Pest Management (IPM) regimes. In this manner, the ardounts of chemical pesticides used can be considerably decreased. These two products have been formulated as Water Dispersibje Granules (WDG) which are applied by using existing conventional spraying equipment. Both products have demonstrated considerable shelf stability when norod packaged at ambient conditions.

Extentiye fleld studles done with $A Q 10$ on grapes in Israel, France, Italy and California have demanserad the efifcecy of this product in the control of powdery mildew. Its application in IPM rogimes can replace reveral of the chemical sprays presently used for the control of the disease. The efficacy of Aspire has been extencively studied on citrus fruit at EIP's pilot packaging line. The combination of Aspire with 5 to $10 \%$ chernical fungiciden presently ured for the control of posthervest rot consirtently provides similar protection to that obtsined with full chemisal commercial treatments.
STABILITY AND COST EFFECTIVENESS OF GRANULAR FORMULATION OF ENTOMOPATHOGENIC NEMATODES

\section{R. Georgis}

Stability at various temperatures and ease of use are the major obstacles in expanding the commercial potential of steinernematid and heterorhabditid nematode-based products. Lipid is a major energy reserve for infective juveniles and initial lipid level appears to have a direct impact on shelf-life. The rate of utilization of stored energy depends on many factors such as temperature, environmental stress and nematode activity and behavior. A breakthrough in nematode formulation was achieved with the development of water dispersible granular (WDG) formulation that allows the nematodes to enter into an anhydrobiotic state preserving nematode survival and pathogenicity for up to 6 months at $4-25^{\circ} \mathrm{C}$. The formulation is suited for a wide variety of consumer, agricultural and horticultural application.

biosys, 1057 East Meadow Circle, Palo Alto, CA 94303

24 FORMULATION OF MYCOINSECTICIDES WITH SPECIAL
REFERENCE TO THE BIOLOGICAL CONTROL OF ACRIDID PESTS

R.P. Bateman, \& C. Prior

\section{Abstract}

The potential of insecticides based on entomopathogenic fungi has been recognised for over a century. However their widespread adoption may have been constrained by a perceived need to adapt to conventional application systems; these have included dusts, baits and spraying with hydraulic atomisers.

UItra low volume (ULV) application using oil-based formulations is the Ultra low volume (ULV) application using oil-based formulations
conventional means of pesticide application for locust control. conventional means of pesticide application for locust control.
LUBILOSA ${ }^{*}$ and ol her projects have been able to benefit from an enhanced activity of Deuteromycete fungus conidia conferred by formulation in vegetable and mineral oils.

ULV suspension (SU) formulations of Metarhizium and Beouveria can be prepared easily and cheaply (<US\$10 per ha.) from aerial conidia, that have been separated from solid culture media. It is easier to stare and have been separated from solid culture media. It is easier to store and
provide dry conidia or oil miscible concentrates than to prepare $S U$ provide dry conidia or oil miscible concentrates than to prepare SU
formulations that are ready to spray. Liquid fermentation processes probably will be employed in commercial mycoinsecticides and various techniques have been developed to convert hydrophilic matter into products that can be sprayed with oil. Two important concepts in mycoinsecticide product development are that:
1. Mass production, formulation, Jogistics of formulation supply, and application are related and essential issues that cannot be treated independently of one another.

2. Although "intermediate technology" production and formulation systems have been reported, reliable applications require rigourou. quality control at these stages.

*: The "LUtte BIologique contre les LOcustes et SAuteriaux" is a collaborative research programme being executed by the International Institute of Biological Control (IIBC), The International Institute of Tropical Agriculture, Cotonou, Benin and The AGRHYMET Centre, Niamey, Niger.

The authors work at: IIBC, Ascot, Berkshire, SL5 7TA, UK. 


\section{M.P. Grezves, I. Potyka and J. Lawrie}

The introduction of the first commercial microbial herbicides, Devine and Collego, in the USA in the carly 1980's led to a marked increase in research interest in weed biocontrol. Since then many potential microbial herbicides have been identified and characterised. It is significant that only a few of these have been registered and none have appeared on the market. It is widely accepted that this is due, primarily, to unreliable performance over a range of climatic conditions. In particular, these microbials are heavily dependent on the presence of exogenous water for periods sufficient to allow spore germination and infection to occur. Such conditions are not guaranteed to occur regularly during the early part of the cropping season, when microbial herbicides must be used. Consequently, some attention has been paid to formulation of microbial herbicides to provide the necessary water and to proiect them from other environmental constraints, such as UV irradiation.

The range of basic formulations that have been considered is wide, encompassing granular and liquid preparations. Granules based on grains, such as cracked wheat or rice, grain components, like gluten and starch, and on polysaccharides, principally alginates, have all been assessed. Liquid formulations range from simple aqueous suspensions of spores or vegetative proparules to those fortified with a range of adjuvants including surfactants, humectants, stickers and even clay minerals. More recently attention has been focused on oil-based formulations, either as invert (water-in-oil) or standard (oil-in-water) emulsions. All these developments will be briefly reviewed with particular attention being paid to the latest research into oil-in-water emulsions, formulations now being used increasingly for delivery of chemical herbicides. Some results showing a possible mode of action involving oil penetration into the target weed's leaf tissue and conversion of the emulsion to an invert emulsion form during spraying will be presented. The potential for such formulations to improve commercialisation of microbial herbicides will be discussed.

IACR-Long Ashton Research Station, Department of Agricultural Sciences, University of Bristol, Long Ashton, Bristol, BS18 9AF, UK

DEVELOPMENT AND COMMRRCIALIZATION OF BACILLUS SUBTILIS (GB03) AS A RHIZOSPHERE INOCULANT

Paul A. Backman
Biological Control Institute and Department of Flant Pathology Auburn University, AL 36849 U.S.A.

Bacillus subtilis GB03 was used to inoculate more than 2 million hectares of crops in 1994. This paper will trace the development of $\mathrm{GB} 03$, from the discovery of its parent isolate, A-13, through its present state of commercial development. First isolated from a sclerotium from Australian soi by Pricilla Broadbent in the late 1960's, it was later classified as a spore-forming isolate of Bacillus subtilis (A-13) and reported to control several seed and seedling diseases. Typical application methods were as drenches of mixtures of vegetative cells and spores. Results in controlled environments were good. but were much more erratic when the technology was brought to the field By the mid $1970 \%$ after several publications and continued variability of field results. the further development of this A-13 was abandoned.

Epaluation of A-13 began in the United States in 1980, when a preparation of formulated endospones was applied to peanut seed (Arachis hypogaea $7.0 \times 10^{7} /$ seed) for control of seed and soedling diseases. Although there was a significant increase in emergence, the most unex-pected results were earlier emergence, and vigor lomprovements that were apparent through midseason. These results were not dissimilax from the plant growth promotion noted two years earlier by Kloepper and Schroth following root olonization by Pseudomonads. When these trials were repested it was found that colonization of peanut was always sucoessful, but that vigor differenoen and emergence improve-ments were variable. By 1982, a key observation was made: It wss found that mining $B$. subtilis with traditional sed treatment chemicals ansured good emergence without compromizing colonization. Yield improvements were recond-ed in most trials, often without signs of either emergence or vigor differences. Evaluation of treated peanut root aystems indicated that the root mass was increased, as were the number of Bradyrhizobium nodules and levels of geveral plant nutrients. Damage by Rhizortonis solani AG-4 was frequently suppressed, but was not required for yield enhancement. Variability was much reduced if treatments were limited to seed planted to nonrotated fields, andor plant-ed into cool (<22C) soils. A-13 was registered in 1985 for use on peanut.. Acceptance was slow because the cost per hectare was high, primarily because of a high seeding rate.

Cotton research with A-13 began in the late 1980's with early results again showing erratic responses. Here, again, treatments were always made in combination with seed treatment pesticides and after the first year a second key improvement was made, the A-13 isolate was adapted to cotton by multiple host-passages (the strain name was then changed to GBO3). When data were developed that indicated that root mass was increased, Phizoctonia AG-4 seed and seedling disesses were reduced, plus that there was suppression of Fusarium wilt caused by Fusarium oxysporum f.sp. vesinfectum, commercial seed vendors were quick to incorporate the GBO3 atrain into their blend of treatment chemicals. Since seding rates are $1 / 7$ of the levels of peanut, costs were redurod by a similar factor. A third factor facilitating sales of this biological control system was that the companies were not promoting the increase in emergence, nor the vigor differences as the expected product of treatment. Rather, they promoted the product. for its improvements in season-long root bealth and for the increases in yield. For the 1995 season, more than $80 \%$ of the cotton planted in the U.S.A. is expected to be treated with GBO3. More than 50\% of the cotton will be treated in cornbination with a second Bacillus subtilis (strain GB07). Data points to the second strain reducing Pythium damage while slso possessing Rhizoctonia and Fusarium activity

Strain GBO3 of B. subtjils is a promiscuous colonizer of both monocots and dicots. Althought it has detailed registrations on 7 crops in the USA, it can be used on all crops. Research date on soybeans, corn and small grains shows a great. potential for continued commercial develop-ment. In addition it promotes vegetable growth whether incorporated into the media of trans-plants, drenched in with transplant water, or applied by the seed delivery system. The success of GBO3 and that of $\mathrm{GBO}$ t as well comes from the fact that it produces endospores, colonizes most plants, is environmentally insensitive, and is extremely tolerant of other agricultural chemicals. It is sold worldwide by the various subdivisions of Uniroyal Agricultural Cbemicals.

\section{8}

FORMULATION AND COMMERCIAL ASPECTS IN DEVELOPING A NEW BtPRODUCT

\section{N.C.J. Schmidt and D. Hobbs}

The market for biological products has grown with a considerably rate over the last decade, but still the biologicals only account for less than $1 \%$ of the total agrochemical market. The only real commercial success has been the use of Bacillus thuringiensis Berliner.

In developing a new Bt-product the steps and milestones taken are very different from a normal agrochemical product development. Chemists as well as marketing people face a number of problems originating from the fact that the active ingredients in a Bt-product ere derived from a living organism. The market needs for a new Btproduct can differ from region to region, but generally issues like cost-effectiveness, reliability, easy to use, as well as safety are the main issues. This makes the formulation of the Bt-product so critical in obtaining a commercial success.

This paper will review work done on Bt-formulations in relation to shelf life, particle size, Bt-persistence in the environment (UV-protectants) and formulation adjuvants. Furthermore some of the commercial aspects in product development will be discussed, with emphasis on issues critical for a successful market penetration.

Novo Nordisk AS, Plant Protection Division, Novo Alle, DK-2880 Bagsvaerd Denmark. 
WHAT'S THE DIFFERENCE OF FOOD WEB IH THE STRUCTURE AND FUNCTION BETWEEN INTENSIVE AND SUSTAINABLE RICE PHODUCTION IN JAPAN ?

\section{Razunasa HIDARA}

Food web structure of agroecosystem is closely related to several ecological functions (pest population regulation, nutrient cycling, bio-diversity etc.) Some comparative examinations for structure and functions of food web in conventionally intensive rice production and organic faraing production which a fe farmers tried to cultivate ithout pesticides in Japan. Organic farning can be divided tro types as traditional type and low-input ones in Japan (Hidaka. 1994). For example. Masanobu Fukuoka's practice (Fukuoka, 1976) is typical for the later. that do not tillage, chenicals treatment and intensive weeding. In intensive rice production. food web was the simplest one awong these systems and species diversity decreased because of strong disturbance. For example, so effective parasite of rice planthopper, Agamernis unka (Neastoda) becose very rare in paddy fields farmed under intensive rice production using pesticides excessivly (Hidaka 1994). This newatoda was comon species before 1950's in the southern part of Japan, as can be seen in some classical papers (Kaburaki and lmamura. 1932: Esaki and Hashimoto. 1937). However. in alnost of older (nore than $5 \sim 10$ years) traditional farming paddies, ve could find out easily these this residental natural eneny. In old traditional rice paddies. 1 . unka density and parasitise could sustain in the much higher than conventional rice paddies. Furthermore. found out some unkown organisus included benificial organisas for sustainable rice production. Tro species of Colleabola eat hyphae of fungus including $r$ ice blast desease, and are important prey for nymphe of spiders and Microvellia. However tadpole shrimp which effective biological control agent of meeds, become disappear in older rice paddies farmed under traditional practice. In traditional farming padies is not practical, because of too higt input of humane labor, lower productivity and frequent injury of food eb structure in LISA based on reduced tillage and legume living aulching similating Fukuoka's was quite different from traditional and intensive one, the most diversified food meb. Especially polyphaugous predators. lycosidids were dominant to be able to surpress colonization of mojor pest. WBPH and BPH. But some residental minor pest species occurred in this disturbance-reduced systems. In conclusion, this new systems is thougt to be best choice to design functional food web for sustanbale rice production in the southern part of Japan.

Adress: Department of Bio-environnental Conservation. College of Agriculture, Ehiwe University, Tarumi, Matsuyawa, 790, Japan.

Quantifying the Impact of Insecticides on Food Web Structure of Rice Arthropod Populations

K. G. Schoenly and Heong, K.L.

Louisiana Tech University, Ruston, USA and

International Rice Research Institute, Los Baños, Philippines

The impact of insecticide sprays on the food web structure of ricearthropod populations was studied in control and sprayed fields in farmers' irrigated fields in Central Luzon, Philippines. Arthropod populations were vacuum-sampled weekly from transplantation of seedlings to harvest on a total of 14 sampling dates. To mimic field practices of local farmers, foliar sprays of deltamethrin, a high-toxicity pyrethroid, were applied to the sprayed field on 28 , 38 , and 49 days after transplanting (DT). Time-specific webs for control and sprayed fields were constructed from time-series of species abundance and a 546-taxa cumulative Philippines-wide web. Numbers of sampled taxa and individuals, over all sampling dates, were higher in the sprayed field ( 83 taxa and 29,638 individuals) than the control field ( 80 taxa and 15,849 individuals) Pests increased nearly 4 -fold in the sprayed field over the control field $(11,454$ and 2,994 , respectively). To assess variation between treatments and sampling dates, six measures of food web structure were used: the percentage abundances of herbivore $(\% \mathrm{H})$, natural enemy $(\% \mathrm{E})$, and other $(\% \mathrm{O}$, detritivorous and planktonic) taxa; the direction of differences in $\mathrm{H}$ and $\mathrm{E}$ abundances between control and sprayed fields; and mean food chain length $(\mu)$. Deltamethrin sprays caused a dramatic rise in $\% \mathrm{H}_{r}$ a reduction in $\mu$, a mixed response in \%E, and a negligible change in $\% \mathrm{O}$ in the sprayed field relative to the control field. The estimated recovery time from sprays, based on analysis of trophic groups and $\mu$, varied from 22 to 30 days. Temporal trends in $\% \mathrm{H}, \% \mathrm{E}$ and $\mu$ and comparisons between control and sprayed fields are translated into ecological indicators (increased herbivores, decreased natural enemies) that may be used for pest management decisions.
AGROCHEMICAL EFFECTS ON THE ROLE OF AQUATIC INVERTEBRATES IN THE DECOMPOSITION OF RICE CROP RESIDUES

\section{IC Simpson and I F Grant.}

The role of aquatic invertebrates in the decomposition of rice crop residues under different agrochemical management was investigated in intensively cropped fields at the International Rice Research Institute (IRRI) Philippines. Decomposition of rice roots and straw was quantified using nylon bags of different mesh sizes. Bags containing the crop residues were placed in four treatments of a field experiment; 1) $\mathrm{N}$ fertilizer + carbofuran 2) $\mathrm{N}$ fertilizer - carbofuran,

3) no $\mathrm{N}$ fertilizer + carbofuran,4) no $\mathrm{N}$ fertiliser no carbofuran (control). At transplanting, bags were positioned in the plots in pairs, one horizontally at the soilfloodwater interface and one vertically in the soil. Crop residues remaining in the bags were recovered throughout the crop season, processed, weighed and analyzed for $\mathrm{C}$ and $\mathrm{N}$. Invertebrates found in the bags were enumerated.

Crop residue decomposition was exponential and significantly different between bag positions, mesh sizesand agrochemical treatments. At 77 days after transplanting, mass losses of rice roots and straw were typically in the ranges 35 to $50 \%$ and 70 to $90 \%$, respectively. Carbofuran applicationssignificantly retarded rice straw decomposition. Nitrogen fertilizer additions stimulated the decomposition of rice rootsand straw. During the crop season, $\mathrm{C}$ content of the crop residues was relatively constant, $\mathrm{N}$ content increased and therefore the $C: N$ ratio decreased. Changes in crop residue composition were influenced by bag position, mesh size and $\mathrm{N}$ fertilizer. $\mathrm{N}$ content and $\mathrm{C}: \mathrm{N}$ ratio of the crop residues were highly correlated with mass loss.

Invertebrate populations in the bags positioned in the soil were dominated by oligochaetes. Organisms in the bags at the soil-floodwater interface were more diverse and numerous. The most abundant organisms were snails, chironomid larvae, ostracods and oligochaetes. Invertebrate community structures changed over the crop season.

Results indicate that the overall role of aquatic invertebrates in the decomposition of rice crop residues is relatively small, but that the agrochemical regimes significantly alter decomposition patterns. Differences found in decomposition patterns must influence the timing and quantity of nutrient release from crop residues. These differences, although small, could be important relative to crop mutrient demands.

Natural Resources Institute, Central Avenue, Chatham Maritime, Chatham, Kent, ME4 4TB, UK. 
EFFECTS OF THE PYRETHROID INSECTICIDE LAMBDA-CYHALOTHRIN ON NATURAL. ENEMIES IN THE RICE AGROECOSYSTEM

ED Pilling and $F J$ Lewis

Due to the high level of awareness of IPM issues regarding the control of pests in $S E$ Asian rice farming, extensive research programmes are now well established to define the ideal IPM approach. Current IPM technologies for rice however, will only be adopted by farmers when they are less complex and offer better improvements in yield and profits. Responsible agrochemical companies should thus promote the safe and sensible use of existing pesticides combined with biological and physical manipulation techniques to control rice pests.

In collaboration with the Philippines Rice Research institute (PhilRice), Zeneca Agrochernicals conducted a large scale field trial in the Philippines during the wet season of 1994 to provide more information regarding the environmental effects of the pyrethroid insecticide lambda-cyhalothrin (Karate) in the rice agroecosystem. More specifically, the trial was designed to assess the magnitude and duration of effects on natural enemies of insect pests in rice, and monitor subsequent re-invasion after treatment. The substantial information generated from the trial will be used to assess the potential for a sensible and optimum use pattern for Karate within an IPM programme.

Karate was applied under different spray regimes at application rates ranging from low to high input. Throughout the season, pest and predator population numbers were assessed using suction sampling and sweep netting methods, pest damage were assessed using suction sampling and sweep netting methods, pest damage
estimated by visual counting, and stem borer egg parasitoid numbers assessed by percentage emergence from field exposed eggs. Estimations of final yield were made to determine if any impact of treatments on predators and pest resurgence translated into yield reductions.

During the trial, over 50 different species of beneficial arthropods were identified and these were fivided into tcur main groups which appeared to be important in terms of abundance; spiders, damselflies, ladybeetles and parasitoid wasps. Initial results indicate that as expected, predator population numbers were reduced immediately after treatment but substantially recover within 15 days. More mobile groups of important beneficials, for example damselflies and parasitoid wasps, reinvade the crop rapidly atter treatment reaching control levels within to days. Karate was also found to provide good control of pest populations with no resurgence problems.

Dr E D Pilling

Ecology and Soil Science Section

Zeneca Agrochemicals

Jealort's Hill Research Station

Bracknell

Berkshire RG12 6EY

United Kingdom

35

AN INTEGRATED APPROACH TO INVESTIGATE THE IMPACT OF REPEATED APPLICATIONS OF FUNGICIDES ON SOIL ORGANISMS UNDER FIELD CONDITIONS.

F J LEWIS, J M COULSON AND J F H COLE

Repeated application of pesticides to broad-acre crops can result in the prolonged exposure of soil dwelling organisms to low levels of pesticide residues. Considering the importance of soil soil dwelling organisms to low levels of pesticide residues. Considering the importance of soil flora and fauna to the continued fertility of the sol
on the function or activity of these organisms.

An integrated field trial was conducted over a period of 10 years to examine the possible impact on this important group of organisms from long term exposure to residues of two triazole fungicides, hexaconazole and flutriatol. In this study three major groups of organisms were studied; soil microarthropods, earthworms and soil microflora.

Replicated plots were established in continuous pasture at Jealott's Hill Research Station in the south of England in the 1980s. Each fungicide was applied at rates up to $2.5 \mathrm{~kg} \mathrm{ai} \mathrm{ha}^{-1}$ (10x greater than the maximum label rates). Populations were sampled at regular intervals for earthworms and after more prolonged exposure for soil microarthropods and eantworms. Comparisons were made with unsprayed control plots to examine the effects of exposure to the fungicide on (a) the community structure and diversity of earthworm and microarthropod populations, and on (b) the activity of soil microflora in respect to their ability for mineral tumover and nutrient cycling.

The effects seen for both chemicals on all groups of organisms studied were minimal and it was concluded that probonged exposure to soil residues resulting from the repeated annual use of either of these products would not affect either the populations of the organisms studied or the important soil fertility processes in which they are involved.

ZENECA Agrochemicals,

Jealott's Hill Research Station,

Bracknell,

Berkshire, RG12 6EY

UK
EFFECTS OF PESTICIDES ON MICROBIAL DETRITIVORRE INTERACTIONS DURING TROPICAL LEAF LITTER DECOMPOSITION.

\section{K Vink 1) and NM Van Straalen 2)}

The effect of the pesticides benomyl and diazinon in the interaction between the fungal population of litter and isopods as representatives of the saprotrophic soil invertebrates were evaluated using microcosm experiments.

The litter used in the experiments was a mixture of Bauhinia sp, Ceiba pentandra, Hevea brasilienis and Swietenia mahagoni. The pesticides were seiected on their expected interference with the two functional groups. With regular time intervals the carbondioxide production and nitrogen mineralization were measured as parameters for the decomposition processes.

Microcosms containing the litter mixture were incubated for two weeks before isopods (Porcellionides pruinosus) were added to part of the microcosms. The effect of the pesticides were tested separately and as a mixture.

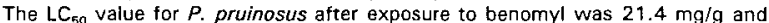
for diazinon $110 \mathrm{\mu g} / \mathrm{g}$. Judged by tha toxic unit approach, benomyl and diazinon acted synergistically in their toxicity to isopods with a toxic strength of the mixture of 0.17 units.

The results of the carbondioxide measurements lead to the conclusion that the microbial community changed as a result of the pesticide treatment. This was also supported by the results of the measurements of the mineral nitrogen concentrations. The effect of the isopods on the mineralization was clear especially for the nitrification part of the process.

The conclusions of the study were: 1 . Microcosm experiments provide important additional information about the pesticide effects compared to classical soil toxicity test. 2 Microcosm experiments usefull tools to evaluate of the effect on sensitive processes such as nitrification.

VU- Amsterdam, Dept. Ecology \& Ecotoxicology, de Boelelaan 1087, 1081 HV Amsterdam, The Netherlands.

Current address: LU-Wageningen, Dept. Toxicology, Post Box 8000, 6700 BX Wageningen, The Netherlands.

36

\section{BIODEGRADATION OF CARBOFURAN IN THE UNSATURATED ZONE OF SOIL}

\author{
John P.E. Anderson
}

The purpose of the investigation was to provide a data base for modelling pesticide degradation in the unsaturated zone of soils. To this end, fluxes in temperature, moisture and soil air composition were followed for 4 years at 6 levels in an $8 \mathrm{~m}$ profile of a silty loam soil Using 2 different sets of cores taken from the profile, the spatial distribution and diversity of the microflora, and the degradation of ${ }^{14} \mathrm{C}$-Carbofuran were investigated. Cores were collected to a depth of $8 \mathrm{~m}$; the 2 sites chosen for collection were $25 \mathrm{~m}$ apart.

The physical characteristics of the cores from the 2 sites were similar, however, the $\mathrm{pH}$ of the cores differed substantially. The numbers and distribution of aerobic and anaerobic bacteria determined by dilution plate counting, and fungi, determined by a soil-wash technique, were similar at mined by dilution plate counting, and fungi, determined by a soil-wash technique, were similar at
both sites. The microbial biomass, determined by the substrate induced respiration technique, decreased at both sites to a depth of $2.4 \mathrm{~m}$. Between 3.5 and $7 \mathrm{~m}$, it again increased and reached about one tenth of that found in the upper $25 \mathrm{~cm}$ of the soils.

Using ${ }^{14} \mathrm{C}$-carbonyl Carbofuran as a model pesticide, degradation was followed in sterilized and microbiologically active samples from 12 depths of both $8 \mathrm{~m}$ profifes. Studies with different concentrations of Carbofuran, incubated at different temperatures and soil moisture contents, showed that the rates of degradation were dependent on soil pH, the quantities of soil microbial biomass, the availability of Carbofuran to the biomass, and the activity levels of the biomass. A predictive model, which is being expanded to describe the degradation of other pesticides in subsoils, was developed.

The research was generously supported by the Environment program adopted by the European Council of Ministers.

Bayer AG, PF-E/OE, 51368 Leverkusen, Germany 
A METHODOLOCY TO DEVELOP PROTOTYPES OF INTECRATED AND ECOLOCICAL ARABLE FARMING SYSTEMS IN EUROPE, ILLUSTRATED BY EXPERIENCES IN THE NETHERLANDS

\section{E.G. Wijnands}

To alleviate the consequences of the ongoing agricultural crisis in the European Union, new farming systems need to be developed. The current complex of problems however, cannot be solved on an ad hoc basis or $a$ one-by-one approach. They have to be treated as integral and fundamental. Such intogral approsches are at present mainly represented in two major visions: integrated and ecological farming system (I/BES). Over the last 15 years these I/BFS systems are being developed on experimental farrs all over Western Europe. In the last 5 years substantial experiences were also gained with developing these proto-
type systems in cooperation with comerclal farms: Innovative pilot type syst

The methodology of this type of research however is still yourg, dyra The wethodolosy of this type of research bowever is still yourg, dyra mic and developing. It is subject of a 4 year EU concerted action program. Stepwlse the methodology comprises to establish a hierachy of
objectives, quantifying these objectives in a sultable set of paraobjectives, quantifying these objectives in a sultable set of paraweters, Identifying sultable farming methods snd techniques to suffi of a theoretical prototype linking methods and parameters to objectives, the design of a multi-Functional crop rotation, the lay out of ves, the design of a multi-functional crop rotation, the lay out of agro-ecological territorial unity, operating the methods and testing agro-ecological territorial unity, operating the methods and testing vel), improviag and optimizing the methods till finally the objectives are achleved snd the syster is resdy for disseminarion.

In the Netherlands prototypes of I/EFS systems are developed on three regional oxperimental farms. The IFs systems were tested oll performan ce and feasibility with a group of 38 comercial farms in a 4 year profect (1990-1993). The 1nnovation of EFS systems is at present sutb ject of a longer term pilot farm project. In this project 10 farmers in one region participate. The results of IFS clearly show that pest cide inputs can be substituted to the greater part by integrated crop protection and that the integrated nutrient management reduces the $N / P$ mineral fertilizer input, waintains the soll fertility at the wanted leval and reduces the $\mathrm{N}$-leaching drastically. The economic evalustions of IFS show comparable results to conventional or reference farms. IFS systers elearly are the succeeders of the current systems alleviating fastly and substantially the environmental risks. However on a longer terw the only comprehensive solution might be advanced ecological farwing systems principally resting on a strong domestic marker with quality lables and premium prices to ensure sufficient managenent achlevements and econowle marglns.

Research Station for Arable Farming and Field Production of Vegetables, P.0. Box 430,8200 AK Lelystad, The Netherlands

39

THE FUTURE OF COMPREHENSIYE CROP MONITORING IN THE DEVELOPMENT OF CROP PROTECTION SYSTEMS EOR SUS'TAINABLE PRODUCTION. THE COIRE PROIECT IN SCOTLAND, A CASE STUDY.

N. McRoberts, D,H.K. Davies, K.A. Evans, G.N. Foster \& S.J. Wale

One of the key problems facing intensive agriculture is how to get from its current position to one of sustainable production. Verejjken (1992) has pointed out the problem is one which requires the involvenent of society as a whole, but this notwithstanding it is clear that changes in crop protection must play an important role in the transition from intensive to sustainable production. Vereijken suggests that localisation of markets and production production. Vereijken suggests that localisation of markets and prod production systems. Adopting Vereijken's proposition, it seems reasonable to suggest, that, if overall levels of pesticide use are to be reduced, more efficient use must be made of those pesticides which are retained in efficient use wust be made of those pesticides which are retained in
sustainable systems. Since it is known that both production constraints sustainable systems. Since it is known that both production constraints (pests, weeds and diseases) and farmers practices vary between regions
(Zadoks, 1989; Savary et al, 1994), it is adyisable to base crop protection (Zadoks, 1989; Savary et al, 1994), it is advisable to base crop prote

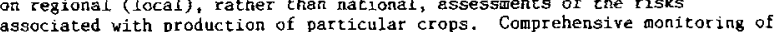
associated with production of particular crops. Comprehensive monitoring of field and crop characteristics, and weather. Such monitoring of crops now, in intensive production, will provide the following:

1. An index of current production constraints. This index will allow us to assess whether broad changes in husbandry have an effect on pest, weed and disease populations. The data can also be used to verify or cest predictive models for crop loss or the occurrence of pest, weed or disease problems. An additional use for this type of information be as an index for assessment of the effects of climate change.

2. An assessment of the particular combinations of production constraints which pose a risk on a regional basis.

3. Identification of farmers' practices. This information could be used, for example, by extension workers to identify regions in which new practices are adopted slowly.

4. Identification of sites thich exemplify regional characteristics, or which have particularly high or los risk of attack. These sites would form the basis of regionally based monitoring programmes in sustainable production systems.

Continued monitoring of indicator sites as sustainable production systems are adopted on a wider scale will allow assessment of the impact of changes in husbandry.

The COIRE (Crop Optimisation by Integrated Risk Evaluation) project is a comprehensive monitoring programe in autumn-sown wheat and oilseed rape in Scotland, which will provide the information listed above. During the thr year period of the project a database of curxent practeristics will be husbandry practices, weather data, and variety choracteristick multivariat methods, Analysis of this dacabase as ilow to characterise regional field types on the basis of their geographic, biological and hustandry characteristics. This paper this type of research to crop protection in sustainable production.

N. McRoberts, Plant Science Department, SAC Auchincruive, Ayr, KAG SHW, U.K.
INTEX - AN INTERDISCIPLINARY PROJECT COMBINING FARMING SYSTEM DEVELOPMENT WITH APPLIED RESEARCH

B. Gerowitt

Three topics are inffuencing agriculfural production in Europe: Environmental effects recieve a growing public attention, numerous detailed information is offered io tarmers to make production more efficient, poitical decisions concentrate on reducing surplus production. How arable production could develop guided by these decisions concentrate on reducing surplus production. How arable prod investigated in an experimental farming system approach.

factors is investigated in an expenmental farming system approach.
Since 1989 large scaled fietd experiments are running on threa locations in the south eastem part of Since 1989 lerge scaled field experiments are running on Ihrea locations in the south eastern part of Niedersacnsen (lower saxony). Including three farms (fertile fluvial soil in a flat landscape, heavy clay soil in
a hilly landscape, sandy soil) and crop rotations which are dominated by winter sown cereals and oil seed

a hilly landscape, sandy soil) and crop rotations which are dominated by winter sown cer
rape, four arable taming systems with descending intensity are grown simutaneously:
Conventional: three course rotation, ploughing, production due to "good farming practice";

Conventional: three course rotation, ploughing, production due to "good farming practice",
integrated: four course rotation, reduced tillage, reduced fertilization, reduced use of pesticides, $3 \mathrm{~m}$ fallow strips around all fields;

Reduced: like Conventional, but only $50 \%$ of the $\mathrm{N}$-fertilization and no insecticides; Extensiv: like integrated, but no use of mineral fertilizers and pesticides.

Single fields without replications have a size of 1.3 to 4.1 ha - the complete experimental area covers 94 ha. Conventional is the reference system, which represents a moderate local agriculture. In Integrated agncultural goals like profitability and long term handling of the system drive the instruments as well as ecological goals. The latter are formulated qualitatively, e.g. 'to stabilize or increase biodiversity in arable areas' or "to guarantee groundwater quality'. All actions are to assess, whether they add to ecological goals and whether they are not completely unreasonable for the tarmer. Compared to Conventional in Reduced two main instruments are restricted, while in Extensiv all agro-chemicals are withdrawn, but little agronomic adaptions were taken to compensate. The Extensiv system is artificial, since in reality stronger adaptions will be taken under these restrictions ( 500 Organic agriculture).

Various working groups (Agronomy, Botany, Economy, Entomology, Plant Nutrition, Plant Pathology, Soil Science, Weed Science, Zoologie) cooperate to accompany and inf uence the development of the systems: Furthermere all have opporturities to follow detailed questions in specfic experimental approaches within the farming systems. 'Applied' refers not only to farmers requieries, but also to those stated in the beginning:
After five years project time some general conclusions of the system development can be drawn: Yields de; crease in the sequence Conventional, Integrated, Reduced, Extensi. The distances between the systems are strongly depending on the site. Gross margins of the nat arabie area (without faliow sitrips) are equal for Conventional and integrated under fertile conditions, in poorer situations up to 182 DM/ha was lost in Integrated. Mean gross margins in Reduced went down to 72 - $82 \%$ of Conventional. Extensiv is even less profitable, moreover weed problems, which could not be managed, increase very rapidly.

Diversity of plants increased in the sequence Conventional, Integrated, Extensiv, while diversity of arthropodes (including beneficials) was highest in Integrated. The fallow strips are valuable for wild lite - an agricult tural value seems reasonable, but cannot be assessed quantitavely yet.

The field of crop protection in the less intensive faming systems must be clearly divided into either weeds, diseases and pests: while disease and pest (cereal aohids) management is effective with preventive methods like resistant varieties, variety mixtures, moderate N-tertilization, support of beneficiais and occasionally pesticide use, weed management offers problems which cannot be solved without a rather frequent use of herbicides.

Detailed appited experments tocus on key factors of the system development. Reduced soll cultivation fno ploughing) is an important factor for several disciplines in either a negative or positive way: to prevent erosion, to support soil siructure, to support soil fauna, to prevent nitrate mobilization are to mention as positive effects, while the interference with crop establishment and increasing weed populations, which induce more effects, while the interference with cop
herbicide use, are negative effects.

Some difficulties rise of the bivalent approach of system development and applied research, but synergistic Some difficulties nise of the bivalent approach of system development and applied research, but synergistic
effecis are predominating. Experiments like INTEX will never replace fundamental research, but can help to effects are predominating. Experiments like INTEX will never replace fundamental research, but can heip to
evaluate and integrate various components of sustainabls agriculure and to make the ideas more concrete evaluate and integrate various components of sus

Research Center Agriculture and the Environment, University of Göttingen, Am Vogelsang 6 D.37075 Götingen, Germany

40

THE DEVELOPMENT OF ECOLOGICAL AGRIGULTURE IN LATIN AMERICA

Miguel A. Altere

Many trends have influenced the development of ecological agricul-

ture in Latin Amer1ca. First of ali the regton is characterfzed

by an ecologicaliy heterogeneous agriculture and by a great diversity of ethnic groups that manage agricultural systems in complex and varied ways. Ethnoecological descriptions of such systems have provided agroecologists with much inputs on how to design sustainable agroecosystems. The urgency of dealing with rural poverty and environmental degradation has also inspired professionals masty from ing, with innovations to manage agroecosys tems with low-1nputs and resource conserving techniques to provide tems with low-1nputs and resource conserving techniques to provide foctor of agriculeture some farmers have tinder their own 1nitlative converted thefr systems to organic management. In some cases, researchers and consultant 3 have collaborated with Earmers during the transition phase towards agroecological management, deriving important sclentiflc principles for the conversion of high. Input systems to low-1nput management. Some environmental economists are using natural resource accounting techniques to evaluate benefits of agroeselogical systems. This paper provides concrete examples of the systems and trends described above.

Division of Bfological Control, University of Calfornia, Berkeley 1050 San Eabio Ave., Albany, Califormia 94706, USA and Consorcio
Lat1noAmericano sobre Agroecologla y Desarrollo (CLADES); Santiago, LatinoA
Chile. 


\section{COMMUNITY APPROACHES TO IPM PROGRAM DEVELOPMENT}

Kevin D. Gallagher, Peter E. Kenmore, Douglas Russell Dilts, John Pontius, Peter. A.C. Ooi, Alma Linda Morales, Mario Corado, and Nugroho Wienarto

Experiences in farmer learning programs over the past 14 years in .16 member countries of the FAO Inter-Country Programme have led to the conclusion that when IPM is used by farmers it must be highly local. IPM is best accomplished through community action programs that are supported by national and local government agencies and/or non-govemment organizations.

National government support to IPM is through extension staff training on facilitation methods, adult education, and IPM experiential leaming activities. National agencies also support IPM through policy changes that reduce pesticide supports like subsidies or extension staff selling pesticides, and provide staff assignments oriented towards IPM. Local government support is often through investment of operational funds to support farmer training, and assignment of IPM facilitators to work with farmer groups. At the community level, local farmer groups are organized by local extension trainers. These "Farmer Field Schools" (FFS) join or become core community groups that begin with a season-long IPM field training. The graduates of the FFS can then help others leam about IPM in their community, often with the technical support of IPM facilitators. FFS take many directions to follow up the first season, often embarking on field training activities for the larger community, or studying other agricultural issues like soil fertility, nutrient management, crop physiological compensation from damage or stress, rice fish culture, or IPM in rotation crops like vegetables or cotton. FFS graduates become well-informed research cooperators carrying out studies in the local fields. This "Field Lab" approach places farmers and researchers in the field, solving problems together.

Currently FFS are developing towards integration of crop production issues through a plant protection gateway. "Grow a healthy crop" (the first principle of IPM in the FFS) embraces integrated plant nutrient systems, conservation and management of water and genetic diversity, and tillage. IPM community groups are exploring the direct impact these agronomic practices have on the compensatory abilities of plants, plant defense mechanisms, factor productivity and profitability under their local conditions.

Examples and data are given in the main paper on community approaches to IPM development within national programs in Asia.

FAO Inter-Country Programme for Rice IPM in South and SouthEast Asia P.O. Box 3700 MCPO, 1200 Makati, Metro Manila, Philippines

\section{3}

UNITED STATES AGENCY FOR INTERNATIONAL DEVELOPMENT (USAID); STRATEGY FOR SUSTAINABLE CROP PROTECTION - AN OVERVIEN

\section{A. . R. Bennett} Sustainable crop protection has much to contribute to economic
development, food security, and to the preservation of the environment in less developed countries. In order to accelerate sustainable agricultural development, the united states Agency for International Development (USAID), office of Agriculture and Food Security, utilizes international partnerships to generate new techrologles through research in sustainable crop protection methodologles. Key linkages encompass U.S. universities, the CGIAR, other multilateral initiatives, and national agricultural CGIAR, other multilateral initiatives, and national agricultural
research systems (NARS). USAID's Agricultural Biotechnology for research systems (NARS). USAID's Agricultural Biotechnology for Sustainable productivity project utilizes biotechnology research to develop environmentally-compatible, insect and disease resistant also includes research oriented projects with strong crop
protection components. The sustainable Agricultural systems cRsp protection components. The Sustainable Agricultural systems CRSP
applies systems-based research to identify and develop sustainable applies systems-based research to identify and develop sustainable which results in improved environmental quality in developing countries. The Integrated Pest Management (IPM) CRSP is designed to develop and implement economically and environmentally sound crop protection methods through collaborative IPM research. The IPM CRSP contributes to the resolution of difficult, persistent constraints to the adoption of IPM, particularly within the context of horticultural export crops and transitional agricultural system in all areas of the world. The Sorghum and Millet CRSP (INTSORMIL) increases protection of sorghum and millet crops in developing countries where these are principal food crops, through the development of improved varieties with insect and disease resistance, drought tolerance, striga resistance, and bird resistance. These programs and other USAID linkages to the intersational research compunity increase agricultural productivity economic growth and enhance food security in developing nations.

U.S. Agency for International Development, office of Agriculture and Food security, SA-2, Room 311-H, Washington, DC 20523-0214 USA.
IPM FOR THE SMALI FARMER IN ASIA - HOW TO MOVE ON FROM RICE TO COTTON

$$
\text { K.G. Eveleens and J. Mangan }
$$

Cotton is a major small farmers' crop in South and East Asia and usually beset by serious insect pests, which has led to heavy and ever-increasing dependency on chemical control practices. It is argued that this trend could be effectively countered by implementing IMP strategies which are based on a matching of:

implementing IMp strategies which are based on a matching of restoration of the potential of naturally occurring parasites and predators as biotic mortality agents, and

(2), farmers' training in IPM according to the successful participatory field school model, developed within the region for rice growing.

Department of Theoretical Production Ecology Wageningen Agricultural University P.0. Box 430

$6700 \mathrm{AK}$ Wageningen
44

\section{IPM-IN AUSTRALIAN COTTON: USING ECOLOGY AND HOST.PLANT RESISTANCE TO MANAGE INSECT PESTS \\ GARY P. FITT}

While Australian cotton production is highly efficient and maintains the world's highest average yields, pest management is a significant and increasing problem. As elsewhere, the crop is attacked by a range of insect pests, the most significant being two species of Helicoverpa, sucking pests (mirids, aphids) and mites (T.urticae). Current pest management includes several IPM concepts: regular and objective crop checking, action thresholds, disease resistant and insect tolerant varieties, destriction of crop residues, and the use of "soft" chemicals where possible to maintain some beneficial insects. However, despite a broad commitment to some kind of IPM, commercial pest management still relies heavily on pesticides and could not be
accurately described as IPM (Fitt 1994, Ann. Rev. Ent. 39: 543-62). With increasing pesticide accurately described as IPM (Fitt 1994, Ann. Rev. Ent. 39: 543-62). With increasing pes
resistance and environmental concerns less disruptive options are now being considered.

The population ecology of the key pests, Helicoverpa and mites, is now well understood. Models of Helicoverpa populations have been developed which take account of local and migratory movements and indicate the linkages between cotton, other crops and other non-crop habitats. The importance of overwintering dynamics of $H$. armigera in the maintenance of SP resistance is now established and underpins efforts to destroy overwintering pupae by crop
residue destruction. There is now renewed interest in the rich fauna of beneficials and residue destruction. There is now renewed interest in the rich fauna of beneficials and
techniques to maintain them in the crop environment or to enhance their activity. True techniques to maintain them in the crop environment or to enhance their activity. True
integration of beneficials as an explicit part of decision making may well occur in the next 5-10 years, co-incident with another major change with the introduction of $B t$ transgenic cottons.

The development of cotton varieties with natural or genesically engineered resistance to pests is another obvious avenue for improved pest management. Insect pest resistance is being sought by modifying plant morphology, chemistry, phenology and fruiting pattem. In Australia major advances have been with morphological characters; okra leaf, glabrousness, frego bract and nectariless, which alone or in combination modify the suitability of the crop for Helicoverpa spp. and mites. All but nectariless have been incorporated into high yielding commercial
varieties, with okra leaf cottons now accounting for $40-50 \%$ of Australian production. With varieties, with okra leaf cottons now accounting for $40-50 \%$ of Australian production. With
respect to Helicoverpa none of these characters provides substantial resistance. Gossypium respect to Helicoverpa none of these characters provides substantial resistance. Gossypium
spp., both cultivated and wild, are rich in terpenoid compounds and condensed tannins, but progress with these biochemical resistance factors has been mixed, partly because these mechanisms often seem associated with reduced yield potential.

The ability of cotton to tolerate insect damage via compensatory fruiting is widely recognised and compensation has been utilised as an IPM tactic, particularly in low yielding situations. However, in high inpur, intensive production systems compensation is less well understood at the physiological level and consequently less well utilised. Similarly the potential role of
induced defensive responses as part of the compensation process have not been fully explored. induced defensive responses as part of the compensation
These areas offer scope for improved pest management.

Genetic enginoering of cotton to achieve pest resistance through the introduction of insecticidal protein genes from Bacillus thuringiensis is well advanced. There is also considerable effort to identify management requirements, environmental impacts and in particular resistance management strategies to be implemented before Bt cottons are commercialised. Resistance management based on the concepts of refugia for susceptible insects and high expression of $\mathrm{Bt}$ proteins will draw heavily on the ecological understanding built up over the last 20 years.

CSIRO Cotton Research Unit, Australian Cotton Research Institute, P.O. Box 59, Narrabri, NSW, 2390, Australia. 
THE IMPLENENTATION OF IPM BY FARMERS GROWIMG
GROUNDNUT ALONG THE EAST COAST OF INDIA

\section{J A Wightean and $G \vee$ Ranga Rao}

Groundnut is often sown in the postrainy season after a rice crop in the $50-100 \mathrm{~km}$ wide strip of light soil a long the east coast of India. This tract is about $1000 \mathrm{~km}$ long indicating that a considerable amount of groundnut can be grown here. As yields exceed 2,5 times the national rainy season average of $0.7 \mathrm{t} \mathrm{ha} \mathrm{h}^{-1}$ in this production zone, it contributes as much as $25 \%$ of India's total groundnut production of $8-\theta \mathrm{mt} \mathrm{pa}$. Farmers growing such a valuable crop are risk averse. They apply insecticides up to seven times in a 100 day season - and suffer excessive defoliation caused by Spodoptera litura. This is a pest induced by the destruction of the natural control process (mainly predators) and the failure, through resistance, of insecticides to kill it. This conclusion was the result of 7 years research at nearby ICRISAT Asia trap monitoring, the relationships between caterpillar trap monitoring, the relationships between caterpillar density/leaf damage and yield, natural control and mechanisms of host plant resistance, Farmers were delighted by the outcome of following advice not to apply insecticide, but to apply fungicide according to need. The former saved them Rs foo0-2000 ha in input posts, the latter increased the yield by about 0.5 tha There was virtually no defoliation caused by caterpillars or late leaf spot. Farmer support included a trained scout who monitored crops and pheromone traps. Farmers quickly came to understand the role of birds and other predators in the natural control process. An NGO supported Farm Support Group took over the extension process and, in consultation with the scientists, reinforced the basic procedure with indigenous IPM components, such as growing castor and sunflower as trap crops. The area 'under IPM' has increased from about 20 ha in 1882-3 to over 1000 ha in 1984-5. Further clusters of villages will be contacted in the next season. The scientists continue to learn about insect and disease managenent, but they also have a unique opportunity to learn about and become involved this a range of technology exchange procedures. Thus, this paper reports the conpletion of a research project

INTERMATIONAL CROPS RESEARCH INSTITUTE FOR IHE SEMI-ARIO TROPICS Patancheru, Andhra Pradesh 502324 , India

INTBGRATRD PEST MANAGEMRNT OP COPFEE FOR SMALI, SCALR PARMERS IN EAST AMRICA: NRRDS AND IIMUTTATIONS

B.T. Nyambo! D.M. Masaba, and G.J. Hakiza

Coffee in East Africa (Kenya, Janzania and Uganda) is an important cash and export crop for small scale farmers. The crop suffers heavy yield losses due to damage caused by a wide range of indigenous pests (insects, diseases, nematodes and weed). Current recommended pest control measures include a combination of cultural, resistant/tolerant cultivars and the use of broad spectrum chemical pesticides. Chemical pesticides are far more popular at the farm level than any of the other recommended pest control measures. coffee pest control strategies are often aimed at individual pests with little consideration of the implications for the total coffee pest complex and its agro-system. This unifateral approach has resulted in increased pest pressure on coffee and some of itg companion crops, outbreak of new pests of coffee, development of pest strains resistant to the cheap and commonly available chemical pesticides, increased environmental problems, increased health risks to man and livestock and an overall increase in costs of coffee production, thus forcing many farmers to neglect their coffee plantations. Measures to elleviate the above problems, particularly the high production costs, are needed to inpzove coffee production and increase the cash-return to the small scale farmer. Integrated pest management (IFM) offers the best prospects for solving the above problems. however, lack of national IPM policies, poor extension systems, inefficient research/extension/farmer Iinkage and the lack of holistic approseh will delay the development and implementation of appropriate, acceptable and sustainable IPM practices.

1. International Institue of Biological Control, Kenya Station, P.O. Box 76520, Nairobi, KENYA

2. Coffee Research Foundation, P.o. Box 4, Ruiru, KENYA

3. Kawanda Agricultural Research Station, P.0. Box 7066 , Kampala, UGANDA

\section{IPM IN APPLE ORCHARDS IN EUROPE.}

\section{H. M. Blommers}

In Europe, opportunities increase to reduce chemical control of arthropod pests in apple orchards. Natural control of phytophagous mites is now realized in most countries. Similarly, other man-made pests, like leafminers and woolly aphid, are becoming less troublesome. The progress is mainly due to the availability of a few selective insecticides and control methods for omnipresent pests like codling moth, some leafrollers and aphids.

Where these selective means are used, the orchard fauna is seen to recover. Natural enemies of yarious pests return, but some previously scarce pests like apple sawfly, blossom weevil, some shoot and tree borers are seen to recover as well. After decades of neglect, improved management of these, often still local, pests draws attention, too.

The implementation of IPM is seen to create new research targets; i.e. some less known pests and many more promising beneficial arthropods. A major challenge will be to develop, and commercialize, new practicable control tactics for the former that have no detrimental effects on the latter.

Experimental Orchard De Schuilenburg, Schuilenburg 3, 4041 BK Kesteren, The Netherlands.

48

COMPATIBILITY OF PARTIAL RESISTANCE IN COTTON WTTH BIOLOGICAL CONTROL FOR BAMGERENT OF Hellicoverps araigers

\section{S. UTHAMASAMY}

Cotton is an important fibre crop infested by mony insects pests. Increasing public concern shout the use of toxic chemicals and their impact on environmenthas necessitated the search for alternative methods. Among the olternatives, development of insect resjstant cultjvars assumes much signjficance in view of tis sustajnable nature. A number of insect resistant genotypes with characters such as glabrousness, frego bract and nectarjless have been identifjed. These characters reduce the attractiveness of cotton plant to Helicoverpa specjes. The larvae hatching from eggs rematn longer on frego bract cotton types and are more exposed to predators than Jarvae on susceptible Deltapine smooth leaf variety LK 861 , a genotype with 10 trichomes per $\mathrm{cm}^{2}$ on leaves was favourable for intra plant dispersal of the predator. Chrysopa scelestes resulting in increased predation of eggs and neonate iorvae of H.armigers compared with JK.276-4 and $\mathrm{TCH} 1002$ with 126 and 107 trjchomes per $\mathrm{cm}^{2}$ respectively. The latter two genotypes interfered with movement of predatary grubs and adults. Parasitization by Trjehogrammo chilonis was maximum on genotypes with lesser number of trichomes. There appears to be an inverse relationship between trichome density on cotton Jeaves and parasitism by the eqg parasitold. T.chilonis. Compatiblitty of partial resistance with bjologicel control may result in synergism. The morphological characters such os frego bract, trichomes on abaxiol ond adaxjal surfaces of leaves mey provide a favourable envisonment for increased predation and parasitism. It is also relatively easier to olter the corpholegical characters of cotion plant by traditional breeding approeches than to manipulate the secondary/ allelochemicals. Such genotypes with moderste/partisl reststance would strategies. Department of Entomology. Tamil Nodu Agricultural University, Colmbetore, 


\section{N. S. Talekar and W. J. Hu}

Diamondback moth (DBM), Plutella xylostella L., is the most widespread pest of crucifers in the world. The insect larvae feed on all aboveground parts of the plant. which reduces yield and quality of the produce. Cost of control of this pest is about US\$ 1 billion per year worldwide. Continuous avaliability of host-plants, absence of effective natural enemies. high fecundity and quick turnover of generations and the ability to develop resistance to insecticides quickly have all contributed to this European insect becoming the most destructive pest of all economically important cruciferous vegetables in tropical to subtropical economically important cruciferous vegetables in tropical to subtropical Asia. At present, farmers use only insecticides to combat this and most
other vegetable insect pests. As a result of intensive insecticide use, this insect has become resistant to practically all insecticides throughout Asia. A collaborative IPM research, funded by the Asian Development Bank, was initiated in 1989 between the Asian Vegetable Research and Development Center (AVRDC) and national programs of Thailand, Malaysia, Indonesia, and the Philippines to combat this pest. This program has since been extended to Paktstan, India, Nepal, Bhutan, Bangladesh. Sri Lanka, Cambodia, Laos, and Vietnam. The major component of this IPM project is the introduction of exotic parasites, mainly of European origin. This is supplemented by innudative releases of existing natural enemies, trap cropping, and where it is a must, use of Bacillus thuringiensis Berliner or a limited amount of selective insecticides. At the start of the project, scientists from each country were trained at AVRDC in mass-rearing of parasites and other techniques. Based mainly on their tolerance to temperatures, Diadegma semiclausum (Hellen) and Diadromus collaris (Gravenhorst) are being introduced in cooler highland areas, and Cotesia plutellae (Kurdjumov) and Oomyzus sokolowskii (Kurdjumov) in warmer lowland areas of Asia. Diadegma semiclausum and C. plutellae are now established in respective ecological zones in major crucifer production areas of Asia resulting in 60 to $80 \%$ savings in the use of insecticides. The incidence of hyperparasites is being monitored and efforts are under way to introduce supplemental parasites to obtain sustainable control of DBM as well as other crucifer insect pests. This IPM is being extended to control other important insect pests of crucifers.

Asian Vegetable Research and Development Center, P. O. Box 42 , Shanhua. Tainan 741, Taiwan, ROC

\section{SOYBEAN INTEGRATED PEST MANAGEMENT IN BRAZIL}

\section{E.MOSCARDI}

A pilot soybean IPH programe was imp lemented during 1974 and 1975 in Southern Brazil. It consisted of weekly scouting of insect populations, assessment of defoliation and plant growth, act ion thresholds based on pest density, damage and plant growth, and use of selected insecticides when the threshold was and plant growth, and use of se lected insecticides when the threshold was reached. Paired fields of 10 to 30 ha were compared in each of nine farms;
one followed the IPM scheme and the other was managed according to farmers' established methods. The number of insecticide applications in IPM fields was reduced by $78 \%$ in relation to farner-treated fields, with no differential effect on yields found. Following the pilot programe, concentrated efforts were directed towards effective IPM implementation, through specific tactics of technology transfer to extension services and farmers, which included: demonstration fields for farmers, set up joint ly by federal and state research organtzations, extension services, and farmer cooperatives; theoretical and pract ical courses for extension workers; publications for extension ists and farmers; intensive use of mass media a imed at soybean growers; implementat ion of an a lert system; and provision of supporting materials to extension. As a result, a dramatic decrease in insect icide. usage occurred in Parana State. Among assisted farmers, the average number of applications fell form 5.8 in the $1976 / 77$ season to 0.74 in the $1979 / 80$ season. In the same period, the average number of applications fell to ca. 2.0 among non-assisted farmers. Similar strategies were employed in other soybean - producing regions. Significant research advances were gradually incorporated in the programme, such as: a) the use of a nuclear polyhedros is virus of Anticarsia gemmatalis in ca 1.0 million ha annualy; b) the use of an egg parasitoid, Irissolcus basalis, against the stinkbug complex; and c) the release of a variety
resistant to stinkbugs, among others. Most important constraints to the soybean IPH programe in Brazil are: 1) size of farms (too large) in some regions; 2) low participation (comitment) of farmer cooperatives; 3) inadequate structure of research and extension in some regions; 4) insuf icient government funds for needed IPM activities; 5) lack of government policies towards IPM initiatives; 6) changing policies at state level with changes in the government; 7) inadequate curriculum at Universities with reference to IPM, among others. In spite of the constraints, after about 20 years the programe is adopted in ca. 4.0 million ha (about $35 \%$ of the soybean area), avoiding the application of many million of litres of chemicals in the environment. Economic figures point from conservative returns of US\$ 550 million to more opt imistic estimates of up to USs 3.5 billions in the same period.

EMBRAPA-Nat ional Centre for Soybean Research, C.P.1061,86001-970, Londrina, PR, Brazi1. Ph (55)43) 320.4166, Fax (55)(43)320.4186.

\section{INTEGRATED PEST MANAGEMI." OF SAN JOAQUIN VALLEY CITRUS USING AUGM: SIOLOGICAI CONTROL}

R. E. Luck ${ }^{1}$, L. D. Forster', 3 rse', H. Griffth ${ }^{2}$, and J. Richardson ${ }^{3}$.

${ }^{1}$ Department of Entomology, University of California, Riverside, CA, 92521;

${ }^{2}$ Entomological Services Integrated, $5101 / 2$ W. Chase Dr., Corona, CA, 91720; ${ }^{3} 27845$ Avenue 138 , Porterville, CA 93257

An integrated pest management program is under development for citrus in the San Joaquin Valley of central California. Almost $60 \%$ of the citrus grown in California is grown in this Valley. Traditionally, the pests in this region are suppressed with four to five annual treatments of broad-spectrum pesticides. However, increasing pesticide resistance in several key pests and growing public opposition lo pesticide use is forcing changes in pest management practices. In contrast to the Valley, southem Califomia citrus is treated infrequently with pesticides. The pests in this rcgion ane suppressed biologically.

Beginning in 1986, a team of entomologists, pest control advisors, and growers began modifying the traditional pest control practices experimentally in 45 hectares of commercial citns. They sought to eliminate the use of broad spectrum pesticides and to rely, instead, on blological control and selective pesticides. Elimination of the broadspectrum chemicals was essential if the augmentative biological control agent and the resident natural enemies were to survive in the grove. Selective pesticides, such as Bacillus thurengiensis, botanticals, and highly refined oils, replacod the broad-spectrum chemicals. The entomologists developed intervention thresholds and alternative control thetics for each of the pests. A key element in the program involved the release of Aphytis melinus, an armored scale parasitoid, to suppress Aonidiella citrina and $A$. aunantii. Amored sale suppression depended on releasing this parasitoid, beginning in mid-winter (about 15 February). Releases were made every two weeks until late Autumn at a rate of 10,500 parasitoids per hectare per Jelease (ca. 200,000 wasps per hectare per year). The wasps were (and are) available commercially. Currently, research seeks to docunent the role of natural enemies in the suppression of armored scales and the other pests, and to develop tochniques of implementing the program more widely.

The IPM program has reduced pesticide treatments in several Valley groves to less than one a year. It has produced fruit of equal or better quality to those produced under the traditional pest control program and at a cost equal to or less than that incurred with the traditional program. Current refinements to the program are improving its reliability and roducing its costs.

\author{
INTERNATIONAL PLANT PROTECTION CONGRESS \\ THE HAGUE - THE NETHERLANDS - 2-7 July 1995
}

ENVIRONMENTAL RISK ASSESSMENTS:
ARE WE ASKING THE RIGHT OUESTIONS ?

RISK ASSESSMENT (GERMANY vis-a-vis UNITED STATES)

\section{A.W. Klein, W.Klein}

Different national procedures and criteria for testing, evaluation and registration of plant protection agents as well as with concern to the risk and benefit analysis and control measures can lead to non-tarriff trade barriers and distortion of normal trading conditions. Nevertheless, the decisions about registration and control of plant protection products are taken under administrative, political and socio-economic conditions, which may yary widely from country to country. Under economic, administrative and trade aspects, therefore, all those involved, i.e. competent authorities, industries, and academia, see the urgent need for the development of internationally compatible control strategies.

The essential prerequisite of these efforts is the availability of scientifically valid findings, test citeria and assessment procedures as a basis for the evaluation of plant profection agents with concern to their effects on man and the environment. The internationally hamonized decision principles to be developed must be based on good science and include concrete instructions for acting which enable the mutual acceptance of risk assessments and registrations. Harmonization must preserve a high safety standard in order to prevent harmful effects on man and the environment. Harmonization assessment practices, data collection and methods development can improve the scientific basis of decisions and save administrative reseurces in all countries.

Risk assessment and risk management are discussed under different national legal specifications (Germany vis-a-vis United States) and possible approaches of tasks sharings are presented taking into account other international activities (OECD, EU, IUPAC, SCOPE). Special concem is focused on the worldwide interest to keep the wording of the label as part of both the regulation and the registration process concise, legible, easy to understand and focused on the major issues. Since most plant protection products are being produced and marketed internationally, not only a severe interference with the environmental policy of other countries but also high public pressure may result from non-harmonized statements.

Therefore, an urgent recessity is recognized for harmonization of risk management, too, taking into account only enforceable measures that have direct relevance to the use of the compound and the protection of human health and the environment.

Unweltbundesamt, Federal Environmental Agency

Mauerstraße 45-52, D-10117 Berlin, Germany 
MANAGING RISK WITH PRODUCT STEWARDSHIP

Jonathan E. Bryant

Much of the creative efforts of industry, government and academia have been directed into pinpointing the areas and magnifude of risk. The regulatory community creates and entorces rules that manage that risk by quantifying exposures and assessing the risk for specific compounds and uses. Traditionally, industry action on managing risk has been lead by these shifting regulations; only after regulations changed did industry alfer its use pattems to conform to new risk standard's, particutanty whete markets might suffer

Gurrent philosophy is changing, both in industry and in many govemment and academic circles, such that the paradigm of product stewardship becomes a leading force in shaping the research and use of pesticides. In contrast to much o tradition, advances in the manner of a pesticide product's use that reduces risk may well happen both outside of the regulatory framework, and before regulations requiring change appesr.

For full industry involvement, the driving forces for the product stewardship effort must address commercial issues: competitive advantage as well as product profitability. They must address the carrot of continued profitability, not the traditional stick of regulatory action. Such encoutagement can come through efforts to reduce infustry's regulatory turden, where acoropriate, or where data to assess risk can be replaced by product slewardship to manage risk.

Encouragement may also come, in the short term, from greater market acceptance if the stewardship effort can help consumers (growers) overcome their nisk or regulatory problems. Benefits can also be provided to industry members whose stewardship effort provide new compounds that pose low risk by rapid regulatory approvai. Short term costs for adoption can be outweighed by increased longevity of a product in the market and regulatory arena.

Recent industry stewardship efforts have taken many avenues: the adoption of new packing concepts for reduced applicator risk (such as bulk packaging. returnable containers and water soluble packages); acoption of novel applicatlon lechnologies to reduce environmental exposure hooded sprayers or early cut-o devices for sub-soll granular applicators), efforts to develop label language that provides users information to minimize nisk (run-off avoidance, resistance buildup, ar-larget spray movement, mixemoaderlapplicator exposure), education (such as where and exclusive to proft and have load to improved risk assessment for our industry.

SANDOZ AGRO, Inc., 1300 E. Toughy Ave., Des Plaines, IL 60018, USA

ROLE OF RISK ASSESSMENT IN INTEGRATED PEST MANAGEMENT

\section{$\underline{\text { S, Miyai }}$}

Protecting the environment is a serious issue in developed countries and is also gaining importance in developing countries, so there is increasing demand for a reduction in the use of chemical pesticides, particularly the traditional broad spectrum pesticides. As a best possible management practice to reduce negative environmental impacts from these pesticides, integrated pest management (IPM) has attracted special attention recently. Although there are still many pests for which IPM implementation is rather difficult and current control practices are generally pesticide based, IPM programs have been successfully developed for many crops in many countries. The decision rule for undertaking management actions adopted in IPM comprises of the economic injury level (EIL) and economic threshold (ET). Although this conventional EIL does not evaluate environmental costs, its appropriate use has succeeded in reducing pesticide use by decreasing the frequency of application. Currently, IPM is desired to place emphasis on the use of environmentally benign control tactics and to develop decision rules which explicitly consider environmental factors. The attempt has just started to develop environmental EILs into which environmental costs as well as economical costs and benefits are incorporated. Environmental risk assessment can and should play a critical role to develop them.

Tohoku National Agricultural Experiment Station, Shimo-kuriyagawa, Morioka, Iwate 020-01, Japan

\section{DEVELOPING COUNTRY PERSPECTIVES ON RISK ASSESSMENT: THE CENTRAL AMERICAN EXAMPLE}

M.F. Lowe

Until recently, relatively little attention has been paid to the risk assessment/risk management needs of developing countries. In part due to growing international trade, increasing interdependency and global communication networks, this is changing, as evidenced by enactment of many laws in developing countries and initiation of new international activities. Available models from industrialized countries with sophisticated regulatory regimes may not be workable for most developing countries, however, especially given limited scientific and enforcement resources. While industrialized countries are recoguizing the need to work toward more international harmonization in risk assessment, developing countries often have specific needs for data that are not necessarily generated in connection with regulatory decisions by industrialized countries or international bodies, particularly with respect to exposure. Enhanced data assessment capabilities will also be required to enable developing countries to make sound risk assessroent and management decisions. This presentation examines some of the specific issues facing developing countries, focusing on the experience of Central American countries in working toward safer pesticide management policies and practices through international, regional and bilateral cooperative programs.

c/o US-EPA, Environmental Fate and Effects Division, 7507 G, 401 M-street S.W., Washington DC 20460, USA.

56

\section{RISK MITIGATION}

W. Klein, A W. Klein

There exists a number of tools for environmental risk mitigation which have a different agree of practicability as well as effectivity. Tools to be used in risk mitigation after the development of an a.i. for use in agriculture are especially the type of formulation and application (e.g. seed dressing), rate and time of application and buffer zones to protect. ecotones and aquatic ecosystems. The application of these tools requires multivariate consideration aways keeping in mind the degree of risk mitigation versus impact on the agricultural use of the pesticide. Especially experience with the risk mitigation based an the use of buffer zones for spraydrift and run-off will be presented.

Preferable to the a posterior risk mitigation is the selection of the optimal a.t. upon new pesticide development. Frequently, there are several candidates to be developed: The inclusion of information on differences of these candidates as regards degradability. degradation pathways, accumulation, sorption properties as well as ecological effects potential in the developmental optimization procedure avoids problems at later stages. in this process, a.i-group specific as well as general QSAR's play a role to reduce developmental costs.

Fraunhofer-Institut für Umweltchemie und Ókotoxikologie

P.O. Box $1260,0-57377$ Schmallenberg, Germany 
CHARACTERIZING RISKS FROM PESTICLDES IN AQUATIC ECOSYSTEMS.

\section{K.R. Solomon, P. Takacs, R. Robinson and J. Bestari.}

A characterization process was developed for assessing levels of concern, risks to aquatic ecosystems, and needs for mitigation of the effects of pesticides that present unacceptable risks to aquatic organisms. This process was based on a probabilistic characterization of susceptibility (toxicity) in aquatic organisms based on the estimation of low level effects (LC5 for acute exposures and MATCs from chronic exposures) derived from the results of laboratory tests and a comparison of this to a distribution of concentrations measured or expected to be found in the aquatic ecosystem. This procedure makes better use of all the data developed for regisuration and allows for flexible integration with risk mitigation and management options. The suggested process is tiered to reduce unnecessary evaiuation of compounds that do not pose a threat to the aquatic environment. Early stages of the tiers can be implemented on the basis of current registration requirements, while later tiers will require more toxicological information and computer modelling than is currently required. This procedure was validated using the insecticide azinphos methyl in freshwater mesocosms. Acute and chronic toxicity data for azinphos methyl were obtained from the literature and the registrant and manufacturer of the product. The LC5 concentrations and the chronic NOECs were estimated by graphic extrapolation. These values were plotted in a probabilistic distribution diagram and a series of concentrations chosen to span the range of toxic responses.

Mesocosms were allowed to allow to stabilize after biological communities were established During this period, water from the irrigation pond was allowed to circulate to ensure uniformity of the planktonic flora and fauna. Sediment trays were placed on half of the bottom area of the mesocosms and aquatic macrophytes (Myriophyllum spp.) plants were rooted in pots and placed in the mesocosms. Mature fathead minnows (Pimephales promelas) were added to the mesocosms.

Mesocosms were treated with azinphos methyl. Initially, enough azinphos methyl was added to ottain the intended concentration. Thereafter, half the amount was added every two days to compensate for hydrolytic and other losses. The half life of azinphos methy! is 2 days under these conditions. Treatment was continued for a further 20 days.

Sampling was carried out on days 2 and 4 post-treatment and weekly thereafter, with a total post-treatment sampling period of 6 weeks. Samples to measure general water quality, chemical residues, phytoplankton and periphyton biomass, benthos, zooplankton and nekton, and fish mortality and reproduction were taken.

This presentation will address the triggers use for chronic testing, determination of levels of concern and the use of the probabilistic approach to assessment, both in theoretical terms and by the use of data from field studies and observations in the validation study with azinphos methyl.

Cenure for Toxicology, L'niversity of Guelph, 620 Gordon St., Guelph ON Canada:
Models for the Assessment of the Translocation In Soll

Dr. Helniv! Schäter

For estimating the potential of a pesticide to reach :ne ground water miodels have first been developed in the U.S. (i.e PRZM, 1984); development of models in Europe soon followed. In the Netherlands and in Germany the models PESTLA and PELMO are now used within the regulation procedure foliowing a standard protocol

baching models consist generally of two parts, one which describes the soil hydrolngy and the second which simulates the pesticide behaviour. The hydrology part is in most cases restricted to the unsaturated zone and modelled either by the Richards equation or by the tipping bucket approach. For modelling the pesticide, relevant processes such as adsorption, degradation, volatilisation, interception. and plant uplake have to be considered Not all of these processes are yet well understood crop growth infomation and etfects of agrlcultural messurec should also be implemented in the modcl.

The assessment should be done in a tiered approach. On a first level foxed standard environmental scenarios and standard pesticide information should be used. This has the environmental scenarios and standard pesticide information should be used. This has the advantege of a fast assessment. It diso makes it more simple to compare modelting stu
conducted by authorities and by industry. On the other hand this approach may not be representative for a specific compound. Therefore on a second level environmental scenartos should be used which reflect in detall the proposed use of tha pesticide. Finally on a third level probabilistic modelling snould be conducted.

While a lot of information is available concerning the pesticide, there is a lack of representative environmental data sets, which are essential for level two and three modelling. In Europe only for par of the U.K. a data base with soil and climate information

is availatle.
The resulting concentrations in the soil water in a predefined soil depth are averaged over the resulting concentrations in the soll water in a predefined soil depth are averaged over time or space and compared inth fixed or ecotoxicologically based threshold values. Based on this compart

Bayer AG

Gebäude 6600

Q-51371 Loverkusen, Bayenwerk

Germony

THE 'NEW ASSOCIATION HYPOTHESIS' IN THBORY AND PRACTICE

B. M. T. Hokkanen

Biological control on $t+$ is based on an ecological association between the $\quad \because$ organism and the biocontrol agent employed. This ation may be for example predatory. parasitic, pata.., ic, or antagonistic, depending on the situation. The capacity to provide effective biological control is dependent on the severity of the interaction in the exploiter-victin system: thus symbiotic and mutualistic interactions will not normally be useful, whereas highly virulent or aggressive ones may provide adequate pest suppression. The 'new association hypothesis' was introduced in the 1980's to stress the importance of evolution in exploiter-victim systems to the success rate of biological control. Coevolved systems ('old associations') tend to approach interspecific homeostasis, where the interaction is not so severe as before the coevolution has taken place: i.e. evolution brings the system towards mutualistic interaction. Therefore it was proposed that blological control might be more effective and more successful if 'new associations' were used, i.e. control organlsms which have not had a possibility to coevolve with the target host. Several individual studies on interscting parasite-host or pathogen-host systems indicate loss of control or increase in resistance as a result of coevolution. Analyses of past biocontrol success rates association agents indeed appear to have provided about twice as many successes relative to attempts, as old association agents. In some systems, however, no differences could be demonstrated. The actual utilisation of the new association hypothesis in practical biological control has met with conceptual, organisatory, practical, scientific, and regulatory constraints, even exceeding those normally confronted in biological control. Despite these the growing number - possibly also a growing share - of biocontrol successes utilising new association agents shows, however that biocontrol still has a lot of underutilised potential in controlling exotic as well as native pests. This should be exploited much more thoroughly as a means of environmentally friendly. scientifically sound, and economically attractive means of plant protection.

Department of Applied Zoology, Box 27, Viikki C, FIN-00014 University of Helsinki, Finland 
MICROBIAL ANTAGONISTS FROM HETEROLOGOUS HOSTS: PROBLEMS AND POTENTIAL

C. Prior

Two important and perhaps irreconcilable attributes which are of ten demanded of a microbia pest control agent are high virulence across a taxonomically arbitrary tange of target organisms combined with a host range limited to those targets, or which at least does not encompass specified, also arbitrary, non-target organisms (NTOs). Homologous (HOM) and heterologous (HET) hosts may be the source of isolates and in addition, saprophytes with no definable host have been chosen for plant disease control. Choice is influenced by the desire for increased virulence which may result from the "new encounter" effect of a HET isolate, countered by a perception of risks from using a HET agent with a host range that may encompass an unknown number of non-related NTOs as well as the targets.

The very narrow host range of some microbial agents (eg most insect viruses and plant rust fungi) limits choice to HOM isolates. Others have no definable host (eg Trichoderma spp. and fuorescent pseudomonad bacteria for soil diseases). Controversy over selection of HOM versus HET isolates therefore arises with organisms where both HOM and HET isolates are virulent to the target. This occurs particularly in the fungal deuteromycete genera Metarhizium, Beauveria, Verticillium and Paecilomyces favoured for mycopesticide development and also in Bacillus thuringiensis (BT), where the host sources and host range may be delimited at a supra-specific taxonomic level.

Where both target pest and antagonist isolate are indigenous, the augmentative use of both HOM and HET isolates is perceived to pose two risks: an alteration in host range due to genetic instability and a change in the range and proportion of NTOs affected, both agravated by increased inoculum levels. HET isolates are perceived to offer a greater risk of the latter due to their inherently wider host range.

Where the target pest is exotic to the outbreak area, HOM isolates will not be available from that area, but may be found in the pest's centre of origin. In this case, in the outbreak area there is the option to use indigenous HET isolates, or exotic HOM or HET isolates. For the former, augmentative use poses a similar threat to the use of HET isolates against indigenous pests. For the latter, an additional risk with exotic HOM or HET agents from any source outside the outbreak area is an unpredictable effect on indigenous NTOs not previously challenged by these agents. This issue of the limit to host range has caused controversy in amily as the indigenous target, but from a region exotic to the target area.

Factors to consider in assessing these risks are: 1 . the sub-and supra-specific taxonomic levels at which agents and hosts are defined; 2 . the geographic range of HOM or HET genotypes; 3. the quantitative rather than qualitative nature of non-host resistance; 4. the ecological limitations on host and NTO infection and post-application spread of infection; 5 . the effects of formulation and application techniques on host range. The greatest perceived risk is from the use of HET isolates which are exotic to the range of the target host and whose host range in the new environment cannot be predicted or determined easily. However, both the biological properies of the organisms concerred and the availabie data from actual field releases suggest that there are not likely to be any deleterious consequences from the use of HET rather than HOM isolates. The final choice, except in the case of exotic HET isolates, is likely to be made pragmatically on the basis of virulence, NTO effects and production costs.

Intemational Institute of Biological Control, Silwood Park, Ascot SL5 7TA, UK

63

TNTERESTS . AND CONTRAINTS OF MICROBIAL ASSOCIATIONS NN BIOCONTROL OF PLANT PATHOGENS

\section{ALABOUVEUE and P. LEMANCEAU}

Despite progress made during the two last decades, biological control of plant diseases remains of exceptional usage in comparison to chemical control or even to biccontrol of insects. Many papers have reviewed the reasons for the failure of commercial applications of biocontrol, although succes has often been reported in the scientific literature. One of the most common reproach made to biocontrol is its inconsistency under field conditions. This inconsistency is partly due to the fuct that biological control is oflen based on a single agent, or a single mechanism although biological control systems operating in rature are always complex and multifactorial. Therefore, one wzy to improve consistency of biocontrol of plant diseases would be the use of mixtures of antagonists having different modes of action and different coological requirements.

At least four broad categories of mochanisms are responsible for the control of plant diseases by microbial antagonists : competition for nutrients and infection sites, antagonism by antibiosis parasitism or predarion, and induction of hos resistance. All these modes of action can be partly responsible for the reduction of disease severity and in many cases, several of them contribute to disease control. Indeed it has been demonstrated that most of the well known biocontrol agent (Trichoderma spp., fluorescent Pseudomonas spp., Agrobacterium radiobacter, Gliocladium spp. (Trichaderm spp., fluorescent Pseudomonas spp., Agrobacterium radiobacter, Gliocladium spp. have several modes of action, some being predonnangt in certain circumstances, others in other conditions. For exsmple, competition for iron has been showed to play a major role in biocontrol of several pathogens by fluorescent pseudomonsds, but production of antibiotics seens to be the major factor in controlling other pathogens. Therefore it bas been proposod to use an association of several strains of fluorescent pseudomonads having different modes of action to improve the efficacy of biological control. It is also possible to associate fluorescent pseudomonads with nonpathogenic Fusarium oxysporum to improve not onty the efficacy but also the consistency of biocontrol of fusarium wilts. In that case, siderophore mediated competition for iron due to fluorescent pseudomonads enhances the intensity of competition for carbon between pathogenic and nonpathogenic F.oxysporum. But other mechanisms not yet fully understood might also account for the improved efficacy of the mixture

Association of different antagonists may also belp to solve a problem linked to the specificity of biocontrol. Most of the antagonists being target specific, control of different pathogens that can affort a same srop requires application of fungicides compatible with biocontrol. In such conditions it would be better to apply mixtures of biocontrol agents as it has been suggested to control Fythium root rot and Fusarium wilts with an association of fluorescent Pseudomonas spp. with nonpathogenic F.oxysporum.

However it will be difficult to solve the problens linked to mass production, formulation and application of mixtures of biocontrol agents.

Registration will also be more difficult to obtain for a mixture than for a single antagonist, but we Registration will also be more difficult to obtain for a mixture than for a single antagonist, but we
have to remember that only complex interactions between pathogen, antagonist and the saprophytic have to remember that only complex interactions betwe
microflora lead to biological control operating in nature.

I.N.R.A., Center de Microbiologie du Sol et de l'Eaviroanement

Laboratoire de ragherches sur la Flore Pathogene du Sol

BV $1540-521034$ Dijon cedex
HOST SPECIFICITY PATTERNS AND DETERMINANTS IN HYMENOPTEROUS PARASITOWS: CONSTRAINT TO THE USE OF NEW ASSOCIATIONS?

\section{E. F. Legner}

Expanding restrictions are being placed on the selection of hymenopterous parasitoids for biological control. Such restrictions are developed largely by paraprofessional bureaucrats without significant input by governmental regulatory authorities, industry and researchers. The scope of restrictions being broad include many kinds of organisms such as vertebrates, invertebrates, and microbial pathogens, when the requirements for introduction differ greatly among them. With over 100 years of biological control practice by the professional community, there has never been any evidence for even negligible negative effects of professionally selected agents on the environment. The guidelines for judgment are straight forward and would never allow for the devastating accidental invasions as have occurred with organisms such as Dutch Elm Disease, chestnut blight, Bermuda cedar scale, the Asian mongoose, Norwegian rat, giant toad, etc. Particularly impeding to the selection of hymenopterous parasitoids is the requirement of host specificity, when such is so rare in nature. At a time when the earth beseeches respites from environmental contaminants, the deployment of parasitoids in the biological control of native and introduced pest insects is a viable altemative. Host specificiry in this case impedes rather than improves the chances for significant nonpolluting results. The "new host association" approach in insect biological control provides an arsenal of infinite benefits to mankind without elevating the risk factor. This has been proven by numerous successful biological control projects in which both highly host specific and non host specific organisms were used. Restricting imported organisms to operating on specific habitats eliminates most of the envisioned threats to nontarget organisms.

Department of Entomology, University of California, Riverside, CA. 92521

64

SOME OBSERVATIONS ON THE CONSTRAINTS TO THE USE OF ENTOMOPATHOGENIC NEMATODES WORLDWIDE

\section{N G M Hague}

Steinemematids can be produced in fermenters and formulated satisfactorily, but their commercial use has been restricted to crops such as ornamentals, cranberries, mushrooms, citrus and turf grass because of the high cost of the nematode product. Expansion into wider markets is essential if the unit cost is to be competitive with insecticides. The single, most important constraint on the development of these biological products is legislation. Although nematodes are exempt from safety regulations in countries where they are presently sold, the use of non-indigenous species is prohibited in most countries, and in some countries legislation insists on the use of indigenous species/isolates only.

It is clearly environmentally undesirable for non-indigenous nematodes to be introduced where they may affect non-target organisms, but nematodes are unlikely to be as damaging to the environment as the widespread use of certain insecticides have been. The recent discovery of a Steinemenatid in Texas, USA, $S$ riobravis, with a high temperature profile, highlights the potential problem. This nematode is effective against armyworms/bollworms of the genera Helicoverpa and Spodoptera and could be used on a wide variety of tropical crops against those damaging insects which are high on the list as targets by agrochemical companies for insecticides. It has now become clear that the future commercial development of entomopathogenic nematodes will only occur if suitable agricultural markets can be found. If these markets are to be worldwide, nematode products will only be produced and formulated by companies if legislation is realistic - it is not commercially feasible to produce individual nematodes for specialised crop systems.

Department of Agricuiture, The University of Reading, Earley Gate, PO Box 236, Reading, Berkshire, RG6 2AT, UK. 


$$
\text { D. Rosen }
$$

The ongoing population explosion has faced mankind with imminent starvation, depletion of energy sources and worldwide environmental pollution. Homo sapiens may fast become an endangered species, and it is imperative that Solutions for one probiem do not aggravate the othars. Efrective pest management is an absolute necessity in modern pesticides may involve grave problems of prohibitive cost, development of resistance, and toxicity to non-target organisms, and has become, a major source of environmental organisms, and has become a major source of environmenta pollution. Classical biological control, i.e. importation and establishment of exotic natural enemies, has been by far the most successful, and is the most promising, alternative to the wholesale use of pesticides. When it works, as it often does, ciassical biological control is permanent, inexpensive, and essentially free of environmental hazards. Unfortunately, some petty environnental concerns about possible detrimental effects of imported natural enemies on native ecosystems may lead to undesirable legislative are discussed, and a plea is made for reversal of this al arming trend.

Department of Entomology, Faculty of Agriculture, The Hebrew University of Jerusalem, P.0. Box 12, Rehovot 76100, Israel.

67

\section{DISCUSSION POINTS}

\section{H.M.T. Hokkanen}

How small of a genetic change is ecologically relevant?

What ecological features are critical in terms of environmental impact?

How do changes in critical features affect the ecological competency of an organism?

Does genetic engineering always - or ever - result in 'new associations' in the release environment?

Does an engineered 'old association' biocontrol agent become a 'new association' agent?

Are exotic control agents in a new environment intrinsically safer than engineered control agents based on species which already occur in the target ecosystem? ... or ... Should engineered agents be treated as exotic agents?
N.J. Fokkema, M. Gerlagh, J. Köhl

In this presentation three different strategies in biological control of diseases of above-ground plant parts will be compared with respect to their potential efficacy in disease control as well as to their possible constraints to implementation. 1. Microbial suppression of infection. The leaf surface is colonized by saprophytic bacteria, yeasts and hyphal fungi. In germinate and penetrate the leaf in order to cause disease. When not being disturbed by broad-spectrum fungicides, naturally occurring yeast populations in the phyllosphere may create unfavourable nutritional conditions for leaf infection by necrotunfavourable nutritional conditions for leaf infection by necrotrophic pathogens. Spraying biological control agents (BCA's) onto healthy leaves, however, is generally not a very effective means of disease control because the pathogen may escape from antagonism by rapid penetration of the leaf. The always existing problem of introducing the antagonist to the proper place at the proper time, however, is simplified in the microbial protection of man-made wounds on e.g. fruits. In this area successful commercialization of BCA's is to be expected. Interesting BCA's can be selected from the natural microtlora of above-ground plant parts. Among the possible mechanisms of interaction, competition for nutrients is to be preferred above antibiotic production. 2. Mcrobial suppression of spore production of the pathogen. effective against diseases with several infection cycles per season. This approach allows a longer period of interaction between the antagonist and the pathogen than microbial suppression of infection, and this increases the reliability of biological control. This strategy aims at delaying the progress of epidemics. Research on the use of inycoparasites for the control of powdery mildew and rust is well known in this respect. Biocontrol products against powdery mildew based on specific mycoparasites are appearing on the market. Promising, and relatively new, is the suppression of spore production of necrotrophic pathogens sporulating on dead plant material. Several saprophytic fungi naturally colonizing plant debris are resistant to variations in micro-climatic conditions and able to compete successfully with e.g. Botrytis cinerea in dead plant tissue resulting in a strongly reduced sporulation of the pathogen. Further exploration of this approach may generate new broad-spectrum biocontrol products.

3. Microbial suppression of pathogen survival. Mycoparasites may also interfere with the formation and vitality of sclexotia in infected above-ground plant tissue and crop remains. Depending on the relative importance of initial inoculum in a hostpathosystem, treatment of infected plant material may reduce the severity of the disease in following seasons. Treatment of crops infected by Sclerotinia sclerotiorum with the mycoparasite coniochyrium minitans at the end of the season reduces soilborne inoculum by $90 \%$.

DLO Research Institute for Plant Protection (IPO-DLO).

P.O.Box 9060 , NL-6700 GW Wageningen, The Netherlands

68

\section{SAPONIN DETOXIFICATION BY PLANT PATHOGENIC FUNGI}

Anne Osbourn, P. Bowyer, G. Bryan, P. Lunnessa, B. Clarke and M. Daniels

Saponins occur in many plant species, and because of their antifungal properties they have been implicated as pre-formed determinants of resistance to fungal attack. Some fungi produce enzymes which remove sugars from saponins, to give molecules which are less fungitoxic. Mutants of the cerealinfecting fungus Gaeumannomyces graminis var. avenae which do not produce the saponin glucosyl hydrolase avenacinase can no longer infect the saponincontaining host oats (but are still fully pathogenic to wheat, which does not contain saponins) (see accompanying poster by Bowyer et al.). Southern blots using avenacinase cDNA as a probe revealed cross-hybridising DNA sequences in a number of other phytopathogenic fungi, suggesting that enzymes related to avenacinase may be widespread. We have demonstrated for one of these fungi (the tomato pathogen, Septoria lycopersici), that the cross-hydridising DNA in this fungus also encodes a saponin detoxyfying enzyme (in this case tomatinase). While avenacinase and tomatinase are clearly related, the relative activities of these enzymes towards avenacin and tomatine reflect the host specificity of the fungi from which they originate. Structure/function analysis of these two highly conserved saponin glucenyl hydrolases should allow us to identify the regions of the enzymes which are important for activity and for substrate specificity, and to design inhibitors of enxyme action which may have significance for crop protection strategies. The occurrence of DNA sequences which hybridise to avenacinase CDNA in genomic DNA or other phytopathogenic fungi suggest that saponin-saponinase combinations may be more important in determining host range than has previously been appreciated. 
INTERACTION BETWEEN CLADOSPORIUM FULVUM AVIRULENCE GENES AVI AND AVI 4 WITH THEIR COMPLEMENTARY TOMATO RESISTANCE GENES C 9 AND $C F / 4$

Pierre J.G.M. de Wit, M. Kooman-Gersmann, R. VogeIsang,

The interaction between $C$. fulvum and tomato has a gene-forgene basis. Two race-specific elicitors encoded bij the 作 mature AVk hypersensitive response (HR) in Cf9 tomato genotypes. By in same, higher or lower HR-inducing activities than the wild-typ same, higher or lower HR-inducing activities than the wild-type
AVR9 peptide. 225 -AVR9 peptide binds to membranes of different comato genotypes.

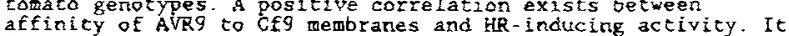
is yer unclict is yet unclear whether the cloned Crg resiscance gene encodes the receptor for the AVR elicitor. The macure Avid elicitor is a 106 - andino acid-peptide. All strains avirulent on Cru genotypes concaln an identical Avr4 gene, while virulent coring contain. alleles wich single basepair changes in the coding region, or a frameshift mutation. Peptides encoded by

Department of Phytopathology, Wageningen Agricultural University, P. O, Box 8025,6700 EE Wageningen, The Netherlands

71

MAPPING AND MOLECULAR ORGANIZATION OF LEUCINE-RJCH-REPEAT (LRR) ELEMENTS AROUND THE CF4/CG LOCUS IN TOMATO

Guusje Bonnema ${ }^{1}$, Jan Hontelez ${ }^{1}$, Ruud Verkerk 1 , Yong Qing $2 \mathrm{hang}^{2}$, Neline van der Hout ${ }^{1}$, Ab van Kammen ${ }^{1}$ and Pim Zabell

1) Department of Molecular Biology. Agricultural University. Dreyenlaan 3, 6703HA Wageningen, The Netherlands.

fax +318370 83584; e-mail Guusje.Bonnema@mac.mb.wau.nl

2) present address: Department of Virology, Institute of Microbiology, The Chinese Academy of Sciences, Bejjing 100080 , China.

With the final goal of isolating the tomato $\mathrm{Cf} 4$ gene that confers resistance to Cladosporium fulvum by means of a map based cloning approach, we are currently studying the genetic and physical organization of the $\mathrm{Cf} 4 \mathrm{l}$ locus on chromosome 1. A detailed map of this chromosomal region will be presented, showing genetic recombination units in relation to physical disiances.

Vinually all resislance $(R)$ genes recently isolated do contain a vanable number of leucinerich repeat motifs (LRRs). The finding that the $C$ ' gene (isolated by ihe group of J Jones) contains 29 LRRs and is a member of a gene family, with all the members mapping at or near the CF loxus, prompted us to study the distribution of these LRRs at the comato Cf4/Cf9 locus.

Two tomato YAC libraries (genotypes MMCf4 and VFNT Cherry), with average insert sizes of $250 \mathrm{~kb}$, have been constructed in our lab. These YAC libraries were screened using primers, designed to amplify a $540 \mathrm{bp}$ fragment containing part of the Cf9 gene LRR's. Nine YACs were identified, containing each one or more LRR sequences. End clones, isolated by plasmid rescue and inverse $\mathrm{PCR}$, and physical maps of the selected $\mathrm{YACs}$, were informative for the building of $Y A C$ contigs. The extremities of the $Y A C$ contigs were mapped relative to the $\mathrm{C} 4 \mathrm{4}$ gene using a set of plants with recombination events around the $\mathrm{Cf} 4 \mathrm{gene}$. The organization and genetic localization of several YACs containing LRRs will be presented.
GENE SLENCING AND VIRUS RESISTANCE IN TRANSGENIC PLANTS

Baulcombe DC, Mueller E, English $\mathbf{J}$ and Davenport $\mathrm{G}$.

It is now well established that plants may be genetically engineered to resist virus infection by transformation with segments of virus genomes. There are at least four types of mechanism that underlie this pathogen derived resistance. If the virus transgene encodes a protein or functional RNA motif there may be resistance due to an imbalance in the levels of components of the infection cycle. Alternatively if the transgene specifies a dysfunctional protein or RNA the resistance may be due to a dominant negative mutant interference effect. Recently a third type of mechanism has been discovered in which the resistance is due to activation of a host mechanism by the tansgene. This mechanism is the same as the mechanism giving rise to transgene silencing and is based on accelerated degradation of the viral RNA in the cytoplasm of resistant cells.

The relationship of this resistance mechanism with gene silencing was first suggested by analyses demonstrating a correlation of virus resistance with low level accumulation of the virus transgene RNA. This work was carried out with plants carrying a transgene based on the coat protein genes of either poty- or tospoviruses. Subsequently we have shown with plants expressing the RNA polymerase gene of a potexvirus that the same correlation applies. We have also shown by genetical analysis that a transgene that confers resistance is also able to suppress RNA accumulation of an homologous transgene.

This resistance mechanism is referred to as homology dependent resistanve because the mechanism is related to a mechanism of homology dependent gene silencing. For the resistance mechanism to be effective it is only necessary that there is sequence homology of the inoculated virus with a transgene with gene silencing potential. Although the mecharism of this resistance is only beginaing to be understood it is bikely that many previously reported examples of transgenic virus resistance involve the same mechanism. An advantage of homology dependent resistance over other forms of transgenic virus resistance is the extreme degree of resistance: in some instances the plants are effectively immune to the target virus of the resistance mechanism. This type of resistance is therefore likely to have practical utility

The Sainsbury Laboratory, John Innes Centre, Norwich Research Park, Colney, Norwich, NR4 7UH, U.K.

72

PRODUCTION OF JRANSGENIC TOMATO AND TOBACCO PLANTS RESISTANT TO TOMATO SPOTTED WILT YIRUS [TSWV]

M.Prins, C. Anker, M van Veller, T. Ultzen', J.Gielen', P. de Hzan', M. van Grinsveri', and R. Goldhach

Dept. of Virolagy, Wageningen Agricult. University, P.O. box 8045, 6700 EM Wageringen, The Netherlands

' S \& G Seeds B.Y.. P.O. 60x 26, 1600 AA Enkhuizen, The Netherlands

High levels of resistance to tomato spotted wilt virus (TSWV), type species of the tospoviruses, were obtained by transforming host plants, e.g. tobacco and tomato, with the viral nucleoprotein (N) gene. This resistance was also operational when transoenic plants were challenged with virus (N) gene. This resistance was also operational when transoenic plants were challenged with virus
in a natural way, i.e. using viruliferous thrips, and under greenhouse and field conditions.
Further analyses revealed that similar levels of resistance could be obtained using untranslatable Further analyses revealed that similar lovels of resistance could be obtained using untranslatable forms of the transoens, indicating that the antiviral state in the plant was induced by the transegnically expressed viral RNA sequences rather than the viral protein. By transforming various genes and sequences, togather representing $70 \%$ of tha virai genome, it was determined that not all viral RNA sequancBs. but only thase derived from the $N$ or NSm gene, provided high level resistance when tranzosnically expressed, indicating the

replication and intertolutar movement respectively, in plants. The RNA mediated wasislanco obrained was virus-specife, but broad spoctrum rosistanco opainst Aimultanoous 
Cloning and Characterization of the Tomato Pto Gene Family

Gregory Martin, Department of Agronomy, Purdue University, West Lafayette, IN 47907-1150, USA. gmartin@dept.agry.purdue.edu

Genes that confer resistance to pathogenic microbes occur in all economically important plant species. In tomato, resistance to bacterial speck disease is governed by a gene-for-gene interaction in which a single resistance locus (Pto) in the plant responds to the expression of a specific avirulence gene (avrPto) in the bacterial pathogen, Pseudomonas syringae pv. tomato. Disease susceptibility results if either Pto or avrPto are lacking from the corresponding organisms. Leaves of tomato cultivars that contain the Pto locus also exhibit a hypersensitive-like resistance response upon exposure to an organophosphorous insecticide, fenthion. The Pto gene was isolated by a map-based cloning approach and was found to be a member of a clustered multigene family with similarity to various serine/threonine protein kinases. Another member of this family, termed Fen, was found to confer sensitivity to fenthion. Current research is directed at three primary areas: 1) elucidation of the structural and functional differences among the Pto/Fen family members; 2) identification of other proteins that may be involved in the Pto/Fen signal transduction pathway; and 3) assessment of Pto function in other plant species.

Thilmony, R. T., Z. Chen, R. A. Bressan, G. B. Martin (1995). The tomato Pto disease resistance gene functions in tobacto. (submitted)

Loh, Y.-T., G. B. Martin (1995). The disease resistance gene $P$ to and the fenthion sensitivity gene Fen are closely related, functional protein kinases. Proc. Natl. Acad. Sci. USA (in press).

Martin, G. B., A. Frary, T. Wu, S. Brommonschenkel, J. Chunwongse, E. D. Earle, S. D. Tanksley (1994). A member of the Pto gene family confers sensitivity to fenthion resulting in rapid cell (1994). A member of the Pto gene
death. Plant Cell 6:1543-1552

Martir G. B., S. H. Brommonscherkel, J. Chunwongse, A. Frary, M. W. Ganal, R. Spivey, T. Wu E. D. Earle, S. D. Tanksley (1993). Map-based cloning of a protein kinase gene conferring disease resistance in tomato. Science 262:1432-1436.
75

ROOT-KNOT NEMATODE RESISTANCE IN TOMATO

\section{M. Williamson.}

Root-knot nematodes (Meloidogyne spp.) are endoparasitic roundworms that infect tomato (Lycopersicon esculentum) and many other crops. These nematodes cause the formation of root galls, and produce stunting, wilting, and poor yield in the host. Some tomato varieties carry a genetic locus, Mi. that confers effective resistance to these pests. Mi is a dominant, or semidominant, gene that confers resistance to the three major root-knot nematode species that infect tomato. Resistance is determined within the first 24 hours of infection and is characterized by localized necrosis of host cells near the head of the infecting pathogen. In order to understand the resistance process, we are investigating changes in gene expression in root tips that occur early after infection. Several genes that are induced by 12 houxs after infection have been identified by differential cDNA screening and theit role in resistance is under investigation.

A particularly valuable tool for understanding $\mathrm{Mi}_{i}$ mediated resistance would be a clone of the Mi gene itself. A collaborative effort is underway to identify Mi by a mapbased cloning approach. DNA clones that span the region containing $M i$ and fine mapping are being used to localize the gene. We recently found that there is a gene, Meu-1, that confers resistance to the potato aphid, Macrosiphum euphorbiae, tightly linked to $M i$. Meu-1 appears to have been introgressed into tomato along with $M i$ from the wild tomato species L. peruvianum. So far we have not been able to separate Mi and Meu-I genetically so it is possible that they are the same gene. Alternatively, they may be closely linked and, perhaps, related genes. Although very different pests. there are interesting parallels in the Eeeding modes of aphids and root-knot nematodes. We are currently investigating the mechanism of aphid resistance mediated by Meu-1.

$M_{i}$ does not confer resistance at temperatures above 32 ${ }^{\prime} C$, and nematode variants that can infect plants with $M i$ do occur. Additional genes conferring resistance at $32^{\circ} \mathrm{C}$ and resistance to $M i$-infecting nematode variants have been identified in $i$. peruvianum. one of these genes, Mis, has recently been mapped to chromosome 12 , and tightiy linked oNA markers have been identified. Transfer of Mi3 to tomato would enhance the genetic basis of nematode resistance and perhaps increase durability of resistance.

Department of Nematology, University of California, Davis, CA 95616 , USA

\section{GENES REQUIRED FOR FUNCTION OF RESISTANCE GENES IN THE BARLEY-POWDERY MILDEW INTERACTION}

\section{Paul Schulze-Lefert, Christoph Peterhänsel and Andreas Freialdenhoven}

Erysiphe graminis $\mathrm{f}$ sp hordei is an obligate biotrophic fungus which exclusively attacks epidermal leaf tissue of its host, barley. Resistance to the fungus is controlled by a multidude of either race-specifically acting resistance genes or by the non race-specifically acting mlo resistance locus. A mutational approach has been used to identify host genes required for function of resistance genes $M l a_{12}$ and $m l o$. Rar-l and Rar-2 have been identified which are required for function of race-specific resistance controlled by $M_{1 / 2}$. Ror -1 and Ror-2 define two loci, which are required for function of all tested $m l o$ resistance alleles. Gene interaction studies suggest that the Rar genes are not required for mlo function and, vice versa, that the Ror genes are not required for race-specific resistance specified by the Mla locus. This finding suggests that genetically separable components act in race-specific and non race-specific resistance reactions to a single pathogen.

RWTH Aachen, Biologie I, Worringer Weg I, 52074 Aachen, Germany

76

\section{INVOLVEMENT OF WOMEN IN CROP PROTECTION}

IN THE REPUBLIC OF BENIN

\section{o. Adjadi}

As farmers, women contribute greatly to food crops, vegetables and cash crop production in the Republic of Benin. These crops need care for their survival from the competition of weeds and attack of insect pests and diseases. Alt the farmers know that they must protect their crops in order to ensure a gaod yield but most of them do not have the necessary resources for controlling food crops, insect pests and diseases.

Unlike, their male counterparts, women have limited access to pesticides on cotton and vegetables though the ones on cotton are sold on credit. Very few of them are trained in agricultural techniques recommended by the extension services. In 1993, $9 \%$ of 4238 farmers trained on different aspects of crop protection were women. Considerable efforts made by government services and special projects to deliver technical messages related to crop protection to farmers have very little impact on women farmers.

As a consequence, those who can afford pesticides do not respect technical recommendations in terms of type, dosage and precautions, so they constitute serious hazard for themselves and their environment. Thus there is a need to undertake appropriate action with the participation of female farmers who are concerned for the improvement of this situation.

CARDER Atlantique, BP 648, Cotonou, Benin 
THE ROLE OF RURAL WOMEN IN THE IMPLEMENTATION OF THE INTEGRATED PEST MANAGEMENT PROGRAM IN INDONESIA

Delima H. Darmawan

Policy interest in rural women began in the early 1970 s when widespread disillusionment with the effects of development policies on the agrarian sectors of less developed countries was being felt. These effects included stagnating levels of food production, uutritional decline and the destruction of rural communities fueling massive rural-to-urban migration (Kandiyoti, 1985). These effects imply that, in countries such as Indonesia, where a large portion of the population lives in rural areas, the relationship between rural women and agricultural development is important to examine. This paper will focus on the relationship between rural Indonesian women and development issues especially in relation of the implementation of the nationwide Integrated Pest Management (IPM) Program. Rural households discussed will be restricted to a particular crop. The intent of the paper is to provide a geueral overview of some of the major issues for consideration for development researcbers, planners and funding agents within a desirable context for development. Desirable development is a process in which ecological, cultural, social, economic institutional and political facets are understood and interrelated. It should also be in harmony with the environment and be committed to the eradication of poverty. Before addressing the pertinent questions surrounding this issue, it is important to describe the women involved; their roles and differing positions in society and farm activities. The question to be asked is whether women are neglected in the Integrated Pest Management Program, which is answered by a review of literature. The results of the review lead into the next question of how the IPM program excludes wonen and why. Finally, the basis for women's inclusion in the IPM program is established.

Office of the Coordinating Minister for Economy, Finance and Development Supervision, Gedung Utama Departemen Keneangan, Lantai V, Jalan Capangan Banteng Timur No. 2-4, Jakarta 10710, Indonesia.

79

GENDER ASPECTS OF A VILLAGE-BASED IPM PROGRAMME FOR CITRUS IN BHUTAN

\section{F. H. J. van Schoubroeck, Choeki Wangmo}

In eastern Bhutan an integrated research and extension programme is being implemented in which practical IPM is being developed for citrus (mandarins) emphasizing participation. In households, both men and women are active in citrus cultivation and both sexes are involved in decision making. Thus, both genders should be involved in an IPM citrus programme. The Bhutan government extension practice does not specifically discriminate between members of bouseholds. In two "pilot villages" and six other villages of the IPM programme, the extension staff and community members have been interacting to develop a village-specific programme, and a way of working that is "community sensitive". Staff promote imterest in IPM by community sensitive methods: farmers are addressed within their villages, staff takes part in local social functions and the local language is used. Feed-back on support by the community and adoption of cultivation practices and participation in pest control activities has been constantly looked for. Female farmers have been participating at all levels of the extension programme. However, female participation often declines when speaking skills and literacy are required, as in committees, because, traditionally, men have been in a better position to acquire such stills. In poorer communities female farmers cutmumber male farmers and subsequently female participation in the programme is relatively bigher than in richer villages. For communicating with women, it is important that extension staff respect social codes and speak the local language. Gender participation rates are not influenced by the sex of the extension agent in extension activities in eastem Bhutan.

IPMD Project, P.O. Box 639, Thimphu, Bhutan, Fax +975-2-23940
GENDERED PLANNING AND IMPLEMENTATION OF A COWPEA IPM PROGRAMME IN GHANA

\section{B.J. Vander Mey}

The Clemson/Ghana IPM on Cowpea Project is a multidisciplinary project. It seeks to create more pest resistant varieties of cowpea, to hone the knowledge base regarding the extent of and purposes for growing cowpea in Ghana, and to implement farmer-driven IPM technologies on cowpea.

Assumptions guiding the planning and implementation include: farmers' indigenous knowledge is vital to IPM; projects are successful when farmers are partner participants; women's vital roles - and inequality - in the farm family and the farming economy must be fully considered; women farmers' knowledge, needs and practices should inform any farming project; Extension Service Agents' attitudes toward and service delivery to women farmers must be included in all stages of research and project implementation. In sum, to be successful, both planning and implementation must be gendered.

The Clemson/Ghana IPM on Cowpea Team is taking a multi-method, multi-site approach to its socioeconomic knowledge base. In depth, qualitative interviews with farmers and key informants have been conducted. Nationwide surveys of farms and Extension Agents are being conducted. On-farm test pilots will be tailored to the gendered realities of farmers in different areas, sensitive also to environmental and economic realities.

The traditional gendered division of labor generalized to Ghanaian farm families was that men tilled and women did the planting, harvesting, and carrying to market. Our research shows complex deviations from this traditional picture. A special challenge for this project is addressing the serious leisure gap between men and women farms in Ghana. The gap is deeply rooted in gendered traditions. Finally, in the past, farming innovations tended to be directed toward men farmers by researchers and Extension Agents alike. The current project enjoys a supportive role from the Extension Service.

In this paper, we provide a background and rationale for each guiding assumption. We then present findings from the in-depth interviews and nationwide surveys. The paper concludes with an overview of gendered challenges to IPM as a crop protection measure.

Department of Sociology, Clemson University, Box 341513, 132 Brackett Hall, Clemson, SC, U.S.A., 29634-1513.
80

HOW CAN WE IMPROVE THE PARTICIPATION OF WOMEN IN IPM TRAIMING? A PILOT STUDY OF IPM TRAINING WITH INOIGENOUS AND MESTIZO WOMEN IN HONDURAS

C. Meir

Zamorano, the Panamerican School of Agricul ture based in Monduras, has offerad innovative training in Natural Control to Central American snallholder farmars since 1991. The overall goal of the programe is to improve the standard of living of smallholder farmers by complamenting the farmers' knowledge of pest management, in order help thew round out the tr concept of the system they manage and thus better ensble
outside help is not available.

The training is offered to farmers, irrespective of their sex. However, the dichotomy between the roles of rural men and women is typically atrong in Honduras and many more men attend than women. In order to ses how wh can best increase the participation of women in our training prooramses we conducted increase the participation of women in our training propramses we conducted
pllot training aimed exclus ively at momen in one mastizo community and one pllot training aimed exclusively at women in one mestizo community and one
indigenous Lencan community in Honduras. The mestizo community was typical in that the farming was mostly subsistence crops, the women tended not to be in that the farming was mostly subsistence crops, the women tended not to be indigenous community, on the other hand, farmed both subsistence maizo and beans and cash cropped potatoes. The women farmed a small area cooperatively, and helped the men out in their own fields when other activities allowed. We found that both groups of women loved the training. The women were interested and enthusiastic, proud to be learning more about their world, and particularly appreciative (despite having very lifilted time ayaliable), because such training is raraly offered to wonen. At the end of both courses the women had gained good grasp of the new ideas presented and wers ablo to use thig hach magement tochnologies. Subsequent to the training, in the mestizo comannity there was little transfer of inforation to the untrained men, in part dus to the women's lack of creditirity with the men. Thus, although the mestizo women remained convinced of the need to protect natural anemies, and management on the largely ornanental plants imadiacely around their housea, they had little opportunity to out their knowledge into wider practice. Communication between man and women was better in the indigenous comnunity, but the wonen still had iinited credibility with the men. The nas conferonce was that the ind genous womon ware able to impleme sone of they gained directly, and so become surer of their now tools.

Aithough this study is still continuing, we have made some initial conclusions. Women are interosted in learning more about pest management and can absorb and apply new concepts. However, IPA training for women needs to be specially designed: it needs to fit in with their time constraints and to concentrate on providing an opportunity for the women to try out their new skills for themseives. Longer term training in the community of fered through local extensionists and concentrating on small vegetablo plots farmed by the women seems to offer potential. If both men and women are trained and both have the opportunity to put thair new ideas into practice. together they may reinforce what they have learnt and create a stronger learning envirarment and
more self-sufficient rura? families.

Department of Crop Protection. Panamerican School of Agriculture, PO Box 93, Tegucigalpa, Honduras C.A., and Imperial College Centre for Environmenta Technology, 48 Prince's Gardens, London, SW7 $2 \mathrm{PE}$, United Kingdom. 
USE OF PESTICIDES BY WOMEN IN THE MANTARO VALLEY OF PERU

\section{Scurrah, M. Fernandez and N. Canales}

Women have traditionally played an important role in Andean Agriculture. Important changes in peasant agriculture are taking place as migration takes more men away from the land and women slowly take on wider responsibilities. At the same time, modern agriculturat achoology has encroached on traditional farming practices.

As a result of emphasis on increased yields in western agriculture a host of agrochemicals have been developed which support a system in which a single high yielding variety is planted as often as possible on the same land and sold for cash. In contrast subsistence agriculture relies on a different range of technologies involving genetic diversity, careful and long rotations, and the spread of seeding dates and plots to offset the risks of frosts, droughts, pests and diseases. These
siffent two systems are often juxtaposed, while government policy lends its support mainly to change from subsistence to a market orientation and encourages the use of modern varieties and agrochemicals.

This paper is a small study of the use of pesticides in agriculture in the Mantaro Valley in the Central Andes of Peru. 52 people participated in this study of which 20 were men. The women were categorized by age and status. The main finding of this study is the high level of acceptability of the technology involving pesticides, especially by young women. They learn about pesticides when they travel to the coast where they are hired as seasonal workers and where they are first when they travel to the coast where they are
introduced to chemical control of insect pests.

The pesticides they mentioned they used most frequently were: ALDRN, PARATHION, CURATER, AND FOLDOL. The majority could identify the different insect pests that affected their crops, especially the potato crop. They seemed more knowledgeable than men about the pests affecting the crops and the chemicals to use. These pesticides have been banned in the developed world, some for over a decade, their persistence in the soil and food chain and their high toxicity. However, they are available in open markets and agricultural supply stores all over rural Peru.

None of the women wore any protective clothing or gloves. Pesticides were mixed and often used in higher than recommended dosages. The women appreciated the new lighter back-packs available which made it possible for them to perform a task which previously only men were able to do. They said that they were not aware of any deleterious effects of the chemicals on the soil or their health, although they mentioned that they would not spray if pregnant. Pesticide residues are left in unlabelled used containers around the house and are an occasional cause of death when taken accidentally.

It is ironic that while agriculnure in the first world is trying to decrease their chemical input of toxic persistent insecticides, the poorest sector in the Andes is just being introduced to the agrochemical agriculture without the benefit of a regulatory service which would provide information to farmers on dosage and effects on health and the environment

Grupo Yanapai

Casilla 264

Huancayo, Perí

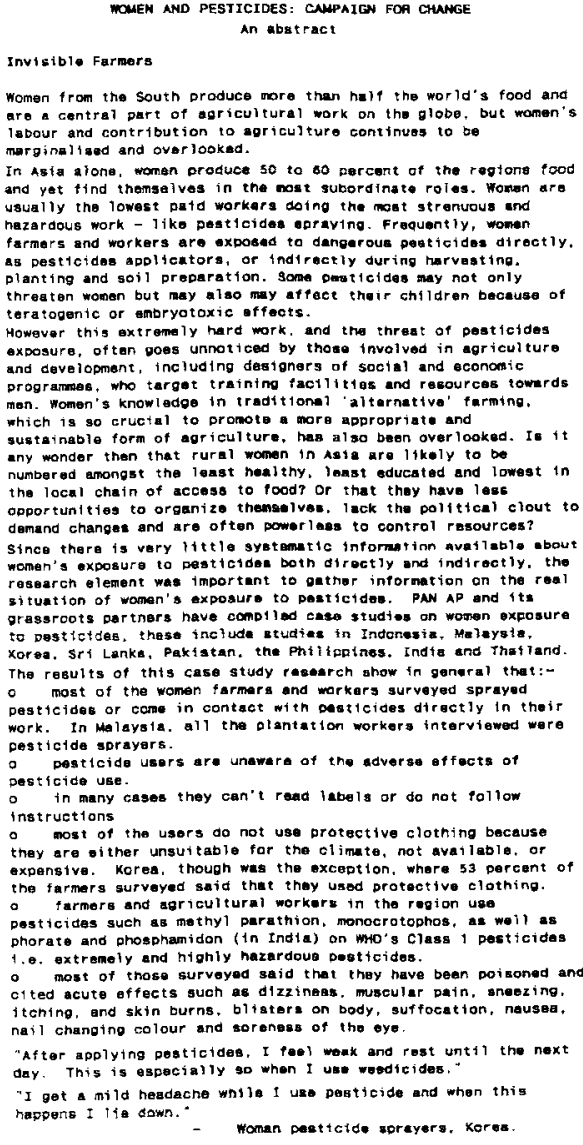

84

USE OF MOLECULAR MARKERS TO IMPROVE MALE FOR DISEASE RESISTANCE: APPLICATION TO PLANT BREEDING PROGRAMS

\section{G. GAY, M. RAGOT, M. MEGHI, U. WEBER and L. CASTOR.}

Within the last five years, molecular markers have been used to localise quantitative trait loci involved in disease resistance in a large number of species. In several cases, molecular markers were also used to select for favourable alleles at these quantitative trait loci in segregating populations. Despite this outstanding new technical potential, there seems to be a relatively modest use of those technologies made in the seed industry. Some of the problems and limitations that we, as a seed company, faced when using these methodologies for disease resistance are thustrated through a model example.

A project aimed at localising and manipulating loci involved in Northem Corn Leaf Blight resistance is described. In addition to already known mechanisms of resistance to Northern Com Leaf Blight $\left(\mathrm{Ht}, \mathrm{H} \mathbf{H}_{2}\right.$ and $\left.\mathrm{Ht} 3\right)$, HiN was identified in 1976 in the maize cultivar Pepetilla. While $\mathrm{Ht}, \mathrm{Ht} 2$ and $\mathrm{Ht}^{3}$ confer resistance through the production of chlorotic lesions, HtN results in delay of lesion development. Hypothesis have been formulated that several genes, including a number of modifiers, might be involved in the HtN resistance. To better understand the genetics of HiN, an RFLP study was conducted, based on a population developed from a cross B37HtN (resistant line) with CG148 a susceptible experimental inbred line

After identification of the major QTLs involved in the HtN resistance mechanism, those were introgressed into CG468 another susceptible experimental inbred line using RFLP markers. A marker assisted introgression of two chromosome segments involved in Northern Corn Leaf Blight resistance was conducted for four generations using molecular markers to both recover the donor alleles at the QTL loci and the recurrent parent allele for the rest of the genome. Isogenic lines were obtained and field tested. The isogenic lines showed a clear improvement for Northern Corn Leaf Blight resistance.

This program has been a success. However, such a strategy is limited to relatively specific cases. Strategies that allow a more general use of molecular markers for disease resistance in a commercial context will be presented

Gilles GAY

Ciba Seeds

R-1096-2-16

CH-4002 BASEL

Tel: 41616973550

Fax: 41616974256

E-mail:GAYG@ABRU.CG.COM
International Institute for Agricine, Farmer-to-Farmer Program, Winrock Moscow, Russia 125040. 
BIOLOGY AND MOLECULAR-GENETIC ANALYSIS OF CEREAL CYST NEMATODE RESISTANCE GENES FROM THE DIPLOID D GENOME OF WHEAT

\section{R.E Eastrood, E.S. Lagudah and R. Appels}

Resistance to cereal cyst nematode is measured as a reduction in the number of white female nematodes per plant, compared with susceptible plants. Two genes $C^{3}{ }^{3}$ and $C r e 4$ (synonyms $C C n D 1$ and $C \mathrm{CnD} 2$ ) for resistance to the Australian pathotype (Hal3) of cereal cyst nematode have been identified in Triticum tauschii, the $\mathrm{D}$ genome progenitor of wheat, and transferred to hexaploid wheat. Cre 3 is more highly expressed than Cre4 to the Hal3 pathotype of the nematode, but against the European pathotypes $\mathrm{Hall}$ and $\mathrm{Hal2}$, Cre 4 is highly effective while Cre3 is ineffective. The Cre3 gene is inherited as a single dominant locus in bread wheat.

The effect of the Cre3 and Cre4 genes on the development of females can first be observed at around 20 days after invasion. This is similar to the effect of the Crel gene present in bread wheat. The number of male nematodes produced is reduced on cultivars possessing the Crel, Cre3 and Cre4 genes compared with the number produced on the susceptible cultivar Egret. In addition the length of males produced on cultivars possessing the $\mathrm{Cr}$ l 1 and $\mathrm{Cre3}^{3}$ genes is reduced.

Using a combination of bulked segregant analysis and enrichment for low copy DNA sequences, RAPD markers were identified with potential linkage to the Cre3 nematode resistance gene. One of these (CSE20-2) was cloned and mapped to the distal end of the long arm of chromosome 2D. csE20-2 was used to assay an RFLP locus and complete cosegregation was obtained with the Cre 3 locus from $178 \mathrm{~F}_{2}$ progeny in a segregating cross. This marker is being developed as a PCR-based assay for the Cre3 resistance gene. Joint segregation analysis from a genome-wide mapping of RFLP markers and the Cret gene showed it to be located in the proximal region of chromosome 2D.

The proximal location of Cre4 on chromosome 2D suzgests a homocologous association with the $\mathrm{Crel}$ and $\mathrm{Ha} 2$ resistance genes of wheat and barley which are located in the proximal regions of chromosomes $2 \mathrm{~B}$ and $2 \mathrm{H}$ respectively. These also share a similar reaction to Cre4 against the $\mathrm{Hal3}, \mathrm{Hall}$ and $\mathrm{Hal2}$ pathotypes. This suggests and evolutionary link between the $\mathrm{Cre4}, \mathrm{Crel}$ and $\mathrm{Ha} 2$ genes and possibly a similar mechanism of resistance, whereas Cre3 has a separate location and different pathotype reaction.

Victorian Institute for Dryland Agriculture, Private Bag 260 Horsham, Victoria 3400 Australiz.

MOLECULAR MARKERS FOR LEAF RUST DISEASE RESISTANCE GENES IN WHEAT

B.Keller G. Schachermayr, M. Messmer, H. Winzeler, M. Winzeler and C. Feuillet

Leaf rust is one of the most important fungal diseases of wheat. To achieve a more durable resistance against this disease we are breeding for combinations of several leaf rust (Lr) disease resistance genes. This strategy of pyramiding resistance genes is only efficient if molecular markers for the different genes are available. Using near isogenic lines we have delined markers for the genes $L r l, L r 9$ and $L r 24$. These genes are still highly effective in Switzerland. The detection of the markers for the three resistance genes is based on PCR. In addition, we have found an isozyme marker for the $\operatorname{Lr} 19$ resistance gene. These four markers should allow an efficient marker-assisted selection for the desired combinations of resistance genes in our breeding program.

Dept. of Plant Breeding, Swiss Federal Research Station for Agronomy, Reckenholzstrasse 191, CH-8046 Zürich, Switzerland
MARKER ASSISTED SELECTION FOR MILDEW RESISTANCE IN CULTIVATED Triticinae

A. Jahoor, M. Schönfeld, G. Backes, L. Hartl and V. Mohler

Powdery mildew (Erysiphe graminis f. sp. hordei) is one of the most important diseases of cultivated Triticinae. The most effective way to control this disease is the introduction of resistance genes in present cultivars. Several genes conferring powdery mildew have been identified in cultivars, land varieties or wild relatives of cultivated crops. With the application of gene-for-gene model, the presence of a specific gene for mildew resistance can be ascertained by a inoculation with the mildew culture that lacks the corresponding gene for virulence. With the number of resistance genes which can be combined in a cultivar increasing, the use of the genefor-gene model for the identification of these resistance genes will become difficult, since the number of isolates needed for analysis will becone probibitive.

Restriction fragment length polymorphism (RFLP) marker bas been developed to determine genetic differentiations at the DNA level in order to improve the diagnosis of resistance genes. The Mia locus of barley possesses a complex polymorphism, up to 31 different alleles or several closely linked loci have been confirmed for this locus. Seven near-isogenic lines containing $M \mathrm{Ha}$ alleles of different geographic origin and one line (MLa2O) derived from Hordeum spontaneum were used to detect intralocus recombination. Two susceptible recombinants were isolated from the crosses involving Mlal, Mal2, Mal3 and Ma20. To confirm intralocus recombination at the $M a$ locus, very closely linked molecular markers were applied. Furtbermore, different powdery mildew isolates were employed to detect the specificity of mildew resistance at this locus. These experiments were in agreement with the fact that the specificity of recombinant plants differs from the one of the parental lines. Thereafter the origin of new alleles at these complex loci has been discussed.

Near-isogenic lines in the background of $\mathrm{cv}$. 'Chancellor' polymorphic RFLP probes for the genes $P m 1, P m 2, P m 3 a, P m 3 b$ and $P m 3 c$. The probes WHS178 and WHS295 tagged the genes $P m l$ and $P m 2$ and were assigned to the corresponding chromosomes $7 \mathbf{A}$ and $5 D$ with null-tetrasomic lines and with a mapping population. Both probes were located at a distance of $3 \mathrm{cM}$ to the resistance genes. These markers can be used to identify the presence of the correspouding genes in commercial cultivars. Probe WHSI79 was able to detected polymorphisms not only between the near-isogenic lines and the recurrent parent but also between the alleies $P m 3 a, P m 3 b$ and $P m 3 c$ at this locus. With nulli-tetrasomic lines the probe was assigned to the homologous group 1 and located at a distance of $3 \mathrm{cM}$ to this locus.

Furthermore, quantitative mildew resistance genes has been investigated on the DH populations from the cross between Igri and Danilo. For the chromosomal localization of this resistance, RLFP markers were employed. These (three) loci were detected on the chromosomes $5 \mathrm{H}, 6 \mathrm{H}$ and $7 \mathrm{H}$ and explain $20 \%$ genetic variation.

A relationship between powdery resistance loci in wheat, barley and rye has been demonstrated. This observation indicates the presence of synteny among different mildew resistance genes.

88 
MOLECULAR MARKERS FOR MARKER-ASSISTED SELECTION OF DISEASE RESISTANCE IN POTATO

C. Gebhardt, J. Niewöhner, D. Leister, A. Ballvora, P. Oberhagemann, F. Salamini

A number of qualitative and quantitative disease resistance genes have been mapped on the genetic map of potato using RFLP markers. The use of RFLPs for marker-assisted selection schemes in potato breeding is hampered by the fact that RFLP technology requires good laboratory facilities, technical skills and high financial input. Marker technology based on the polymerase chain reaction (PCR) would facilitate the application of marker assisted selection. We have developed, therefore, PCR-based assays for RFLP markers that are closely linked to various resistance alleles. One of those alleles, $\mathrm{HI}$, confers resistance to the root cyst nematode Globodera rostochiensis. This resistance allele can now be selected in diploid and tetraploid potatoes using an allele specific PCR assay.

Max-Planck-Institut für Züchtungsforschung, Carl-von-Linné-Weg 10, D-50829 Köln, Germany

91

\section{MAPPING OF WHITEFLY RESISTANCE IN TOMATO}

C.A. Maliepaard, N. Bas, A.W. van Heusden, J. Kos, G. Pet, R. Verkerk, M. Vrielink, P. Zabel \& P. Lindhout

An $F_{2}$ of an interspecific cross between cultivated tomato ( Lycopersicon esculentum cv Moneymaker) and $L$ hirsutum f. glabratum was used to generate an RFLP linkage map. In order to identify quantitative trait loci (QTLs) for greenhouse whitefly (Trialeurodes vaporariorum ) resistance from L. hisutum f. glabratum, life history components of the greenhouse whitefly were evaluated in this population. The $F_{2}$ population was also evaluated for the segregation of type IV and type VI glandular trichome densities. These glandular trichomes and their exudates have been associated with resistance to a number of insects. Two QTLs affecting whitefly oviposition rate, two QTLs affecting trichome type IV density and one QTL affecting trichome type VI density were identified and mapped on the linkage map. On the basis of the $\mathrm{F}_{2}$ RFLP data, $F_{3}$ lines were selected which were homozygous for either the L. esculentum allele or the L. hirsutum f. glabratum allele at one or both resistance loci. These $F_{3}$ lines were again evaluated for whitefly oviposition rate. The results of the $\mathrm{F}_{3}$ experiment confirmed the presence of the two QTLs. The results do not support the hypothesis that the density of type IV trichomes is involved in whitefly resistance. The involvement of the QTL for type VI trichome density in whitefly resistance could not be excluded.
MAPPING PEST AND DISEASE RESISTANCE GENES IN TOMATO

\section{Pim Lindhout}

The cultivated tomato (Lycopersicon esculentum) is susceptible to a large number of pests and diseases. Wild Lycopersicon relatives harbour a wealth of resistance genes. Many of these have been introduced into the cultivated tomato and protect the crop against pests and diseases. Genetic studies with populations segregating for resistance genes and for molecular markers with known map positions have enabled the mapping of resistance genes the genus LYcopersicon, more than 1000 RFLP markers are available that have been proven to mapping resistance mapping resistance
genes. Already 18 resistance genes, against 11 pests and diseases, have been mapped by using RFLP and RAPD markers.

Different strategies have been followed: Firstiy, one can take advantage of the lack of polymorphism in the

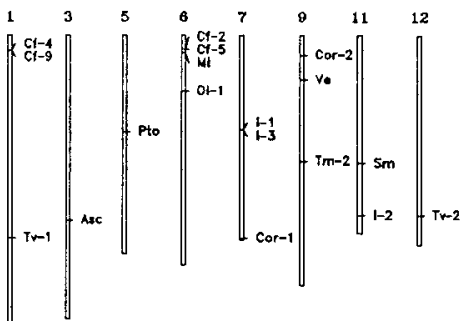
cultivated tomato relative to the The position of some dipesse resistance genes abundance in wild on the tomato genetic linkage map. relatives that harbour

resistance genes. Due to linkage drag resistant cultivars carry a disease resistance gene on a chromosome fragment that is derived from the original resistant donor species. Any RFLP marker on this fragment can be used as marker for the presence of this disease resistance gene. Knowledge of the map position of these markers also enables the mapping of the linked resistance gene. secondly, one can generate an interspecific $F_{2}$ population by crossing a susceptible tomato line with a resistant wild relative. By "Bulked Segregant Analysis" an RAPD marker is detected that is genetically linked to the resistance gene from the wild relative. This RAPD marker is mapped in a mapping population with known segregating data of RFLP markers, establishing also the map position of the resistance gene. Thirdly, QTL mapping procedures are executed for mapping genes involved in quantitative resistance, like bacterial canker or white fly resistance. To this end larger segregating populations are generated that are analysed for resistance and for marker alleles. By using an interval mapping method, genes are mapped that explain part of the genetic variation of the trait. Examples will be presented of strategies, that have been applied to map genes involved in resistance to cladosporium fulvum, oidium $l_{\text {ycopersicum and the greenhouse whitefly. }}$

Department of plant Breeding, Wageningen Agricultural University, PO Box 386, NL-6700AJ Wageningen, The Netherlands.

92

PHEROMONE BASED MASS TRAPPING OF Oryctes monoceros AND Rhynchophorus phoenicis (L) IN NIGERIA

\section{A.I. Oehlschlager* and C.I. Aisagbonhi**}

Three groups of 5 bucket traps containing Oryctes monoceros pheromone, enhanced with insecticide impregnated organic matter were set up on NIFOR date palm field at Dutse. A control set of 5 traps containing organic material alone, without pheromone, was also set up. Traps were placed at 6 palms $(52.8 \mathrm{~m})$ away from one another and examined once a week from April to May 1994. Pheromone traps enhanced with bits of sugar cane and cured cow dung or bits of palm fronds caught Oryctes while the traps without pheromone caught none. The difference was highly significant $(P<0.05)$. These traps lay out with little variation, and containing Rhynchophorus phoenicis pheromone were similarly set up on a replanted oil palm field in NIFOR, Benin city. Recoveries collated from February to May 1994 indicated that the Rynchophoporus pheromone was highly efficient $(P<0.05)$ in catching the adult insects. Pheromone based mass trapping will help immensely in protecting living palms from extensive destruction by these insects in Nigeria.

* Department of chemistry, Simon Fraser University, Burnaby, British Columbia V5A 1S6, Canada

** Entomology Division, Nigerian Institute for Oil Palm Research (NIFOR), P.M.B. 1030, Benin city, Edo state, Nigeria
Department of Vegetable and Fruit Crops

Centre for Plant Breeding and Reproduction Research (CPRO-DLO)

P.O. Box 16

6700 AA Wageningen

The Netherlands 
MANAGEMENT OF WHITEGRUBS IN AUSTRALIAN SUGARCANE: FUTURE DIRECITIONS

P.G. AlIsogo, L.N. Rohertson, K.J. Chandler, F.W. WaIker

Nineteen species of scarab larvae, collectively known as canegrubs, attack the root system of sugarcane in Australia. Thousands of hectares of sugarcane were damaged before the introduction of organochlorine insecticides in 2948. Current control is reliant on a controlled-release formulation of chlorpyrifos (suscon Blue) and non-residual formulations of ethoprophog (Mocap) and cadusafos (Rugby). Recent failureg of both euSCon and Mocap in some fields have intersified the search for alcernative controls. Management options co minimise the impact of canegrubs are, and must, move from a reliance on symthetic insecticides to a system integrating a range of cultural, biological and insecticidal controls. This change is being underpinned by a wide range of research projects aimed at improving the efficiency of current control strategies, devising substitute atrategies, and in changing the sugarcane system to minimise the impact of the pegts. A summary of these projects is presented; where they are at and where they are going, and how they relate to improving and integrating canegrub control strategies.

Bureau of Sugar Experiment Stations, P.O. Box 651, Bundaberg 4670, Australia

95

CULTURAL MANAGEMENT-BASED PEST MANAGEMENT SYSTEM FOR LOWLAND RICE IPM IN THE PHITIPPINES

\section{I. Calderon and J. S. Soriano, Jr.}

In the Philippines, populations of chronic pests of irrigated rice often reach levels that can cause yield loss. These pests include stemborers, leaffolders, whorl maggots, and some defoliators.

Yield loss due to damage caused by these pests has been the major constraint in most rice production programs.

A pest management concept which simultaneously addresses the Filipino farmers' needs for a rational, cost-effective, and environmentally sound pest control method for irrigated lowland rice has been developed.

Results of 16 field trials conducted in major rice producing areas in the Philippines showed that a low dose pesticide application adjusted in relation to the pest's peak population density at the crop's growth stages, effectively controlled peits. No adverse effects oD beneficials was observed. Furthermore, higher marginal benefit cost ratios and lower variability was obtained in farms where the tactic was applied.

A furcher development of the strategy incorporates the use of a simple, "farmer-friendly" monitoring cool and a field validated computer model of a multi-pest threshold to technically support and simplify the concept.

A full discussion of the concept will be presented in this paper.

JOSE I. CALDERON

Ciba-Geigy (Phils.), Inc.

Agricultural Rivision

3rd Floor. Asiar Reinsurance Building

Salcedo Conner Gamboa Streets

Legaspi Village, Makati

Metro Manila 1229

PHILIPPINES
THE SYSTEM OF INTEGRATED PEST AND DISEASE MANAGEMENT ON CABBAGE UNDER THE CONDITIONS OF THE PROGRESSIVE TECHNOLOGY OF ITS CULTIVATION.

\section{B.P.Asyakin, O.V.Ivanova}

The uniformity of size and shape of crop plants and their productive organs together with plant resistance to pests and diseases is of great practical importance under the conditions of commercial cultivation of vegetables.

The comparison studjes of generally used and new technology of cabbage cultivation were carned out in Leningrad district in 1990-93. The growing of seedlings in conic misi-blocks which fulfils all the plant nutritional and environmental requiremedes is one the main distinctive feantres of the new technology. As a tesult of the study the lesser injury of seedlings by blscketing was observed under the conditions of new technology as compared with the generally used one $(0.5-1.2 \%$ and $15-32 \%$, respectively). The survival of seedlings in field constituted to $92 \%$. Thus method of seedling production provides as a whole a nomal plant metabolisin resulting in favourable influence at all subsequent stages of plant growth and development. The greater uniformity of plants, which were cultivated according to the new technology, ensured the reduction of yield losses due to cabbage root flies (Delia brassicae Boushe \& D.floralis Fall.), diamondback moth (Plutella xylostella L.) and slime (Erwinia carotovora).

The enhancement of plant resistance to pests and diseases is an important component of cabbage IPM. The providing of plants with all the necessary fertilizer elements at the stage of head forming intensified the action of cultural and chemical control measures resulting in enhancement of the plant compensatory ability and increase of amount of standard crop production. The best results were obtained when the leaf spray fertilization with agricultural salt (contains potassium, sodium and chlorine) and calumagpesia (potassium, magnesium and sulphur) was used. Thus the effectiveness of complex control of cabbage pests and diseases depends on technology of crop cultivation. The best results were received when the insecticides were used in combination with immunizators and best methods of cultivgtion. The additional yield sternuing from control measures constituted to 87 ceaters per hectare as compared with generally used technology of cabbage cultivation.

All-Russian Institute for Plant Protection, Podbelsky sh. 3, St.-Petersburg-Pushkin, 189620, Russia

96

IHTEGRATED PLANT DISEASE MANAGEYEHT (IDH) IN SIBERIA

Chuiktra Y.A. Hovosibirsk State Agricultural University

There are more then 160 diseases on crops in Siberia. They can be distributed into four epiphytotheological groups according to the main nechanisa of the pathogen transmission: 1)soilborne or root diseases; 2)air-droplet or foliar ones; 3)seedborne or natrixdaughter diseases; 4)insect transmissive ones.

IDH is developed 13 for every epiphytotheological group of infection in order to break off (to weaken) the rochanisa of the pathogen transwisston, and 2)on tho calendar-phenological bag for every crop or the group of orops in order to realize general potentinl productivity of the variety and to operate the elents of yleid.

633128 Russia, Noyosibirsk region, Rrasnoobsk, a/y 18. 
SUCCESSFUL USE OF MATING DISRUPTION AND GRANULOSIS VIRUS SPRAYS TO CONTROL CODLING MOTH IN ORGANIC APPLE ORCHARDS IN NEW ZEALAND

\section{I.R.CLEARWATER}

Four New zealand apple orchards have used mating disruption pheromone dispensers as the key input to control codling moth. the most important pest of organic apple orchards. All orchards showed a large improvement in control. These orchards cover the range of apple management systems in NZ. The use of dispensers in a low input gate-sales orchard allowed the ratio of dried apple slices (from codling damaged apples) to Eresh fruit change in favoux of fresh fruit. An intensively managed dwarf apple produced the first N2 export crop in 1992 with the help of dispensers. Two large tree orchards in an apple growing district surrounded by conventional. orchards have been able to maintain supplies to a large supermarket. In the last environment sprays of codling moth granulosis virus assisted in retaining control in the face of very large initial populations and maintaining consistent control allows a conficent recomendation to Nz apple consistent
growers.

Hort+Research, Mount Albert Research Centre, Private Bag 92169 Auckland New Zealand.

\section{RECENT ADVANCES IN THE USE OF PETROLEUM SPRAY OILS IN CITRUS}

\section{G.A.C. Beattie, G.A. Herron, Z.M. Liu and A.D. Clift}

Petroleum spray oils play an essential role in citrus IPM programs in Australia. Current recommendations are based on narrow-range oils that are less phytotoxic than their predecessors. We therefore believe that currently prescribed rates are no longer appropriate. To resolve this issue we have conducted laboratory bioassays to determine deposits required for the control of a range of pests and field experiments to determine dose-response relationships for Chinese wax scale, pink wax scale and red scale. We also rediscovered and validated the 1910-40 recommendations for the use of petroleum products against citrus leafminer.

Our bioassays were based on the novel use of a Potter spray tower. Although there was significant heterogeneity in most regressions we consider it well suited for establishing relative susceptibilities of a wide range of pests.

The field experiments established efficacies against scales versus volumes of spray and oil applied and deposits. These results indicated that applying slightly higher volumes of $1 \%$ sprays than $2 \%$ sprays can give equivalent mortalities at significantly lower deposits. This outcome re-emphasised the importance of wetting trees to form effective films of oil. This can only be achieved by run-off throughout canopies after the emulsion breaks, leaving a film of oil behind.

The citrus leafminer results with $0.5 \%$ sprays should provide a unique opportunity to implement sustainable IPM programs in citrus - worldwide following its recent dramatic spread. This view is supported by recent studies in China. The only limit is the amount of oil that can be applied annually without risk of phytotoxicity. However, this timit, equivalent to a single 2-3\% spray, is based on broad-range oils used in the $1920 \mathrm{~s}-30$ s and could be revised upwards for narrow-range oils.

Biological and Chemical Research institute, PMB 10, Rydalmere, New South Wales, 2116 Australia

Powdery mildew, caused by Sphaerothecs fuligines, is one of the most important diseases in greenhouse-grown cucumbers in The Netherlands. Research on alternative control methods is done, in order to reduce the input of chemical pesticides against this pathogen. The effect of several factors, such as cultivar choice, climate settings, composition of the mutrient solution and biological control is investigated separately and in combination.

The influence of relative humidity on powdery mildew epidemics is not very strong. Partial resistant cultivars slow down the epidemic. Adding extra calcium to the nutrient solution has no effect on powdery mildew, but silicon in the nutrient solution, in the form of potassium silicate, reduces the amount of disease. The effect of silicon only becomes apparent when silicon is added to the nutrient solution in a concentration of $0.75 \mathrm{mmol} / \mathrm{l}$ or higher. Application of plant extracts from Reynoutria sachalinensis strongly reduces powdery mildew, especially on a partial resistant cultivar. Several biological control agents have been tested and have been found to be effective. The effect of biological control agents is stronger on a partial resistant cultivar than on a susceptible cultivar. A combination of several factors offers perspectives for a future with limited use of fungicides. 


\section{N.G.M. Dulmans}

Nursery stock production in the Netherlands is a relatively small industry. An immensely vast range of hardy woody plants is grown in containers or in the open soil. As expor is very imporant, quality standards are high so relatively high amounts of fertilizers, soil sterilants and pesticides are used.

In 1991 a research project was started to develop integrated production systems to reduce the input of chemicals. The project consists of two prototype integrated nurseries for field production; one for ornamental shrubs and conifers and the other for seedlings of forest trees and rose rootstocks. On a third prototype nursery ornamental plants are grown in container. Besides, supportive research is done to investigate alternatives in detail. Economic evaluation on nursery-level must give insight in the feasibility of integrated methods. The main research themes are: crop rotation, mechanical in the feasibility of integrated methods. The main research themes are: crop rotation, mechanical weed control, biological and integrated control, soil fertility and organic matter, recirculation of
water, $\mathrm{N}$-mineralisation and nutrient balances. Some of these themes will be presented more in detail in other papers at the conference. This paper will give a general view on the set-up of the project and first results will be presented.

Integrated production of nursery stock has very good prospects. It appears that the input of fertilizers and pesticides can be reduced drastically, when knowledge is available to make better decisions.

As integrated production is involving the whole nursery management, certain aspects are now tried As integrated production is involving the whole nursery management, certain aspects are now tried
in practice. Although monitoring and discussions are taking up more time, most of the growers are positive about the results and want to continu.

Research Station for Nursery Stock,

P.O.Box 118, 2770 AC Boskoop, Netherlands

EFFECT OF SOIL SOLARIZATION ON THE INCIDENCE OF WHITE ROT DISEASE OF ONION UNDER FLOOD IRRIGATION IN EGYPT .

FAHHW, M.M. ; M. M. SATOUR ; A. E. OSMAN and S. A. EL- HADDAD .

Soil solarization by the mean of covering the well prepared wet soil with transparent polyettylene sheets, $100 \mathrm{u}$ in thickness, during July - August 1987 at Beni Sweef Govemorate, successfully controlled the white rot disease of onion incited by Scterotium ceptvorum. Disease incidence In the experimental ste in the three successive seasons ( $87 / 88,88 / 89$ and $89 / 90$ ) was 50 , 100 and $100 \%$ for the control (untreated) while it was $0.0,0.0$ and $0.7 \%$ in the treated plots, respectively. However, the infection of $0.7 \%$ was mainly restricted to the borders of the treated plots and may be due to recontamination from the adjacent infested soil . In addition plant growth was improved by solarization , plant height was increased by ( $65 \%$ ) number of leaves per plant by $(40 \%)$, bulb weight by ( $137 \%$ ), bulb diameter by $(42 \%)$, neck diameter by ( $51 \%$ ) and bulbing rate (bulb diameter / neck diameter) by ( $6 \%$ ). Bulb yield per feddan $(4200 \mathrm{~m}$ ) was in favoure of soil solarization being 19.14, 21.97 and 20.35 ton / feddan compared with 3.15, 0.0 and 0.0 ton I feddan in nonsolarized soll in the three successive years of experimentation.

Plant Pathology Research insttute ARC, GIza , EGYPT ( 12019 ).

\section{A.H, EL-HENEIDY and B.M. SERAMATTE}

cotton is a major crop in Uganda. Through the IFAD/WORLD BANK pilot project, 'Smallholder Cotton Rehabilitation Project' 1993-96, IPM has been introduced in the cotton agro-ecosystem in Uganda for the first time. IPM experiments were conducted in five districts, represent most of the cotton growing zones in the country. Preliminary results of the season $1993 / 94$ led to a serlous testing of IPM system in the season 1994/95. The key elements used were : (1) collection of field data of cotton plants, pests and natural enemies, (2) surveying indigenous natural enemies, cotton pests, (4) screening insecticides (biological cotton pests, (4) screening insecticides (biological and conventional), (5) using pheromone traps and scouting techniques for monitoring pests population, (6) developing adaptive field and laboratory research in research instiand extension agents in the principles of IPM. Data abtained showed:(1) cotton plant pattern, trends of major obtained showed: (1) cotton plant pattern, trends of major bugs, bollworms and stainers were the major pests, aphida were ocassionally important, (2) more than 20 species of parasitoids, malnly on aphids and bollworms plus g groups of predators, (3) preliminary economic threshold levels for the major pests; lygus and bollworms; (4) that numbers of sprays can be minimised depending on monitoring techniques of pests populations, timming and effiency of chemical application. Besides, several training courses and workshops were carried out to qualify IPM staff.

NAMULONGE AGRIC, AND ANIMAL PRODUCTION RESEARCH INSTITUTE (NAARI) -NATIONAI AGRIC. RESEARCH ORGANIZATION (NARO) P.O. BOX 5763, RAMPALA - UGANDA

104

TOWARDS SUSTAINABLE PRODUCTION OF DECIDUOUS FRUIT IN SOUTH AFRICA

J.H. Giliomee

South Africa exports large quantities of deciduous pome and stone fruit. The fruit is produced in extensive monocultures of apples, pears, peaches and plums, using intensive production methods which normally require heavy applications of fertilizer, irrigation water and pesticides. For various reasons this is probably not sustainable. As a result, a paradigm shift to Integrated Fruit Production (IFP) is advocated in which the emphasis is on longterm profitability, environmental conservation, and human health and welfare. Infegrated Pest Management (IPM) forms an important aspect of this. IPM requires the use of regular monitoring by well-trained orchard monitors, often using sophisticated methods such as pheromone traps. A variety of non-chemical methods are employed to protect the fruit from damage, e.g. orchard sanitation, stem barriers for fruit weevil and biological control for woolly apple aphid and phytophagous mites. Pesticides are still required for certain pests and these are classified into the following categories: permitted, permitted with restrictions, not permitted with exceptions and not permitted. A scheme has been devised whereby the producers are given penalty points for the application of non-preferred pesticides or pesticides outside the recommended period. If a certain number are accumulated, the producer will not be allowed to use a label indicating that the fruit was produced using environment-friendly methods.

Department of Entomology and Nematology, University of Stellenbosch, Stellenbosch 7600 , South Africa. 
NITROGEN FERTILIZATION AND CHEMICAL PROTECTION OF WINTER WHEAT AGAINST DISEASES IN IPM EXPERIMENTS IN POL.AND

\section{GLAZEK, S. PRUSZYŃSKI}

Since 1991-1992 a long - term research program of integrated pest management (IPM) has been undertaken in the Institute of Plant Protection, Poznan, Poland. The program was developed in the years 1993 and 1994. The results presented comprise a part of research work performed in that period of time. In the Branch of the Institute of Plant Protection located in Sosnicowice (south - western Poland) 2-year experiment with winter wheat cv. Rosa was performed to evaluate the effect of chemical control of diseases on their occurrence and grain yield, at various conditions of $\mathrm{N}$ fertilization. The economical profit (profitability) obtained in experimental variants is defined as a value of wheat grain minus costs of production. This profitability was analysed in Terms of its relation to a standard application of fungicides during the vegetative period, in comparison to non - treated variants. The occurrence of diseases in the years 1993 and 1994 was very low because of unfavourable conditions for their development. Under these circumstances the amount of grain yield was mainly related to the application of increased nitrogen rates up to 1.5 optimal standard rate of recommended fertilization. A mean net economical profit of increasing $\mathrm{N}$ fertilization from 0.5 to 1.5 optimal rate was highest and was equal to 2.6 and 4.6 million zl in the years 1993 and 1994 , respectively. A mean economical profit for fungicide application was very low in 1993 (58.000 $\mathrm{zt} / \mathrm{ha}$ ) and in 1994 this value approached $0 \mathrm{z} / \mathrm{ha}$. It was concluded that at low infection levels of wheat with pathogenic fungi standard application of chemical disease control during the vegetative period may not be economically justified. In such conditions other suitable agricultural practices including appropriate for a given type of soil nitrogen fertilization may be more suitable for obtaining high grain yield of winter wheat.

\section{PLANT PROTECTION INSTITUTE}

IN POZNAN

SOŚNICOWICE BRANCH

Gliwicka St. 29

44-153 SOSNICOWICE

POLAND

107

A YIABLE INTEGRATED PEST MANAGEMENT (IPM) STRATEGY FOR VEGETABLE PRODUCTION IN NORTHERV AUSTRAIIA.

\section{MARDEN \& J. HALL.}

An IPM strategy for vegetables has been developed in response to increases in pest pressure, a longer cropping season, pesticide resistance, the cost of chemical control and the environmental effects of pesticides.

The program developed for trellised tomatoes (Lycopersicon sp.) illustrates the essential components of successful I.P.M. All control methods are integrated into a program The major pests include Phythorimaea operculella (potato tuber moth), Helicoverpa sp Tetranychus urticas (two spotted mite), aphids, fruit flies, thrips, Alternaria sp. bacterial spot, sclerotinia, botrytis, bacterial and fusarium wilt, virus diseases, nematodes and grass and broad leafed weeds.

Pre-planting - soil preparation includes the addition of high rates of organic material for nematode suppression and deep ploughing of crop residue for insect, pathogen and weed control. These and crop location in relation to neighbouring crops and prevailing winds are all cultural controls. Plastic mulch is used over the crop row to provide physical control of weeds and methyl bromide applied under the mulch for control of Sclemtium, Pythium and Ritizoctonio and suppression of nematodes. Two spotted mites are monitored in the area and predatory mites (Phytoseitulus persimilis) released if required, (biological control), prior to crop establishment. Crop varieties are carefully selected where possible for pest resistance to Fusarium sp. races I, II, and III, bacterial wilt and nematodes. Fusarium race III is not widespread and host resistance and quarantine are used to control this pest. Equipment is disinfested and weed hosts of viruses controlled - quarantine and hygiene.

Post Planting - pest free seedlings are produced using clean media and protected using quarantine, physical and chemical control strategies. Seedlings are planted into the disinfected soil protected by the plastic mulch. All pests and beneficial organisms are monitored regularly with chemical and biological control recommendations based on thresholds established by applied research.

Interventions used include release of predatory mites and Trichogramo spp. and application of Bacillts thuringiensis (B.t) and chemical pesticides which are carefully selected and applied to minimise disruption of biological control.

Results include reductions in: pesticide use, losses to pests and cost of chemical control; increases in: use of biological control, pest monitoring and professional advice; and adoption of a sustainable IPM system for vegetable production. Professional plant protection advice, written and verbal, is essential to the success of this IPM program. Current research includes methods for targeting selected pesticides, use of inter-row vegetation/organic mulch to conserve and enhance beneficials and use of IPM in other solanaceous, cucurbit and brassica crops.

Plant Protection,

University of Queensland, Gatton College, Gatton. Australia 4343
108

EFFECTS OF STRIGA HERMONTHICA INFESTATION, WATER STRESS AND NITROGEN ON WATER USE EFFICIENCY AND ROOT AND SHOOT GROWTH OF PEARL MILLET.

\section{Boukar, D.E. Hess, and W.A. Payne}

The effects of nitrogen fertilization, water regime, and infestation by the root parasite Striga hermonthica (Del.) Benth., on water use efficiency (WUE) and root and shoot growth of pearl millet [Pennisetum glaucum (L.) R. Br.], was studied in $36 \mathrm{~L}$ pots in a glasshouse. Prior to sowing, the upper $5 \mathrm{~cm}$ of the very sandy soil was infested with 0 , $\{8,000$ or 71,000 viable Striga seeds. Half of the plants received $3.26 \mathrm{~g}$ of urea (equivalent to $60 \mathrm{~kg} \mathrm{~N} \mathrm{ha}^{-1}$ ) in two equal applications (at sowing and at 21 DAS). Pots were maintained for 45 days at one of two water regimes: $7 \%$ and $16 \%$ of volumetric water content (MWC). The soll in each pot was entirely enclosed within a polyethylene bag so that water loss was through transpiration only. After $\mathbf{4 5}$ days plants were harvested and roots recovered. Counts were made of haustoria attached to millet roots in the upper $10 \mathrm{~cm}$ of soil and root length density (RLD) and total root length (TRL) were determined in addition to host shoot and total root biomass. The number of haustoria per unit length of root was calculated.

Striga infestation decreased WUE of pearl millet independently of Striga seed number in the soil, whereas low water regime and nitrogen fertilization both increased WUE. With no nitrogen, host growth was limited and reduction $\{42 \%$ ) in shoot biomass was seen only at high infestation. With nitrogen, host growth was improved 5-6 fold and Striga infestation reduced shoot biomass by $21 \%$ and $36 \%$ at low and high infestation levels, respectively. On the contrary, host root biomass was unaffected by Striga infestation at $\mathrm{O} N$ but increased $26 \%$ with Striga infestation at high $\mathbf{N} .52 \%$ more Striga haustoria were present in pots infested with 71,000 seed than in pots infested with $: 18,000$ seed but Striga emergence was enhanced only in the presence of $\mathbf{N}$. With $\mathbf{N}$, drought favored haustoria ( $82 \%$ increase) and Striga emergence $(162 \%$ increase). At low infestation RLD and TRL were similar to the uninfested control but at high infestation an $18 \%$ increase in both was observed. The number of haustoria per unit length of host root was increased by water stress, $\mathbf{N}$ stress and increased Striga infestation. The increased number of haustoria per root length of the host elucidates a physiological mechanism by which Striga infestation further weakens plants already subject to environmental stresses such as nutrient and water stress.

Striga infestation decreased WUE of pearl millet, consistent with previousty reported gas exchange measurements. Host aerial development was reduced by Striga infestation, whereas root growth was stimulated. Although the level of $\mathrm{N}$ employed in this experiment $\left(60 \mathrm{~kg} \mathrm{ha}^{-1}\right)$ reduced the number of haustoria per unit length of host root it stimulated emergence of attached Striga. In addition host root and shoct development were enhanced by N. $16 \%$ WW reduced Striga and enhanced host growth, but only in the presence of $\mathrm{N}$.

ICRISAT Sahelian Center, B.P. 12404, Niamey, Niger 


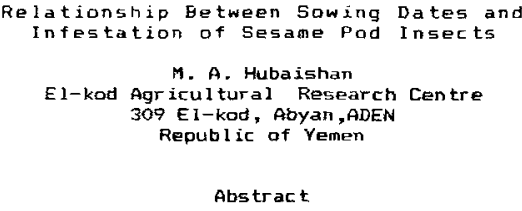

Abstract

At El-kad Agricultural Research Centre, two field trials were carried out to study the relationship between sesame sowing dates and infection with the sesame gallmidge catalaunalis Doponche, in two successive growing seasons.

The sowing dates were; 18 August, a Septenber, ist Oc tober, 20 Oc tober, 19 November, 10 December. Early sowing dates gave significantly higher yields and less infestation by both insects. Production was reduced at the rate of 5.9 $K g$ for $1 \%$ pod infestation, according to the equation
$Y=504.6+(-5.9 x)$.

Infestation by sesame pod worm was low in both seasons while infestation by sesame gall midge was high and the insect is responsible of $97 \%$ of total infestation. Results showed that only one sampling is required 50 days after sowing and will be enough to estimate expected losses at the end of the season, according to the above equation.

111

INTEGRATED PEST MANAGEMENT STUDIES IN NEWLY ESTABLISHED CITRUS ORCHARD IN THE ÇUKUROVA REGION OF TURKEY

\section{Ismail KARACA Nedim UYGUN M. Rifat ULUSOY Naime Z. TEKELL}

Integrated Pest Management is based on the principle of keeping all pests in the ecosystem under the economic threshold by inteorating all control methods and techniques instead of destroying them. Today, although this method has been developped to be used aginst cirrus pests in Turkey successfully, unfortunately many cirrus growers fail to use it

IPM of citrus pests was studied in a newly established citrus orchard since 1977. The orchard was about $100 \mathrm{ha}$ in size and consisted of lemons (20\%), sweet oranges (20\%), grapefruits (30\%), and mandarins (30\%). IPM was continously applied from 1977 on. In the presented paper we reported on the results in 1991-1994.

Aonidiella aurantil (Maskell) brought with infected seedlings during the establishment of the orchard, was primary pest in 1977 and contunied its imponance until today. Good pruning and other cultural practices and monitoring natural enemies and single application of mineral oil once in winter or summer gave successful control of $A$. aurantii.

Phyllocoptruta oleivora (Ashmead), Aceria sheldoni Ewing, Chrysomphalus dictiyosperm Morgan, Ceroplastes floridensis Const. Coccus pseudomagnoliarum Kuwana, Icerya purchasi Maskell, Planococcus cirri Risso, Parabemisia myricae (Kuwana), Dialeurodes cirri (Ashmead), Aphis cirricola v.d.Goot, were excellent control by imported and native naturat enemies or by single applications of selective insecticides and acaricides. The leafhoppers Asymmetrasca decedens (Paoli) and Empoasca decipiens Paoli suck on maturing fruits in autumn, resulting in yellowish or brownish patches. This damage does not affect yield but the quality of fruits. No control measures are used against these pests since the leafhopper damage does not influence the markability of citrus.

Most imporant problems arise with the recently introduced pest Polyphagotarsonemus latus (Banks) and Phyllocnistis citrella Stainton. The latter species developed to the most serjous citrus pests in the Eastem Mediterranean region. Although some native parasitoids of $P$. cirtella were determined, they did not show any impact on this pest. We are presently involved in importing and mass rearing of specific parasitoids of $P$. citrella to include these into the citrus IPM program.

\section{INTEGRATED PEST MANAGEMENT IN NICARAGUAN COTTON PRODUCTION}

\section{H.-G. Jansen, A.-E. Jansen}

Soon after its introduction as a cash crop in 1950, Cotton became a major source for rural employment and national exchange earnings in Nicaragua. Due to high production costs, driven up mainly by inafficient pest control, and relatively low yields, cotton has become unprofitable in Nicaragua at the beginning of the present decade. A system with up to 30 sprayings of chemical pesticides per season lover $50 \%$ of production with up to 30 sprayings 80 's could not survive the stricter market conditions with low cotton prices and high cost for chemical pest control.

Beginning in 1991, a new initiative for an integrated cotton production system in Nicaragua joined efforts of growers and the new government, assisted by international donor agencies. Cotton was declared priority crop. The aim was to put known methods of IPM, supplemented by agronomic measures into practice at large scale and thereby increase the crop's profitability and to lower the negative impact on the environment of pest control practices.

The boll weevil Anthonomus grandis Boheman is the key pest in Nicaragua's cotton production. Because of lack of alternative control methods, it determines the beginning and the number of chemical insecticide applications. The integrated system is based on the reduction of the weevil population outside the growing period of the commercial crop By early destruction of cotton stalks after harvest, use of trap crops and the Boll Weevil Attract and Control Tube (BWACT ${ }^{\mathrm{P}}$ ) a major breakthrough in boll weevil control was achieved. In 1993 less than $40 \%$ of the use of insecticides is made against that pest, while it was close to $100 \%$ only 4 years before. Boll wevil management is comple mented by improving sol prearation including subsoling, use of short season crop


varieties, that diminish the periad the crop needs protection, biological control of lepiequipment, and an intensive training program for farmers and technicians.

Within 5 years, the national average of the number of chemical applications in cotton production went from 26 to 10 , the production cost fell from 1.720 US\$ ha to 930 US\$ha, increasing the yieid level. Cotton can be groduced without massive insecticide usage and the production has achieved profitability again.

Proyecto Ampliación del Servicio de Protección Vegetal MAG-GTZ Apartado Postal 489. Managua, Nicaragua Tel./Fax: (505)-2-780224

112

EPIDEMIOLOGY AND SUPERVISED CONTROL OF POWDERY MILDEW IN ROSE (SPHAEROTHECA PANNOSA) GROWN UNDER GLASS.

A. Kerssies, H.D. Frinking, M.M.J. Pieters.

The epidemiology of powdery mildew on rose (Sphaerotheca pannosa) grown under glass was studied in a glasshouse of $300 \mathrm{~m}^{2}$ from 1991 to 1994

Colonies of $S$. pannosa on rose leaves were counted in the glasshouse. The numbers of $S$. pannosa colonies increased rapidly over time. $S$. pannosa is able to sporulate, germinate and penetrate at the temperatures $\left(17-30^{\circ} \mathrm{C}\right)$ and the relative humidities $(40-90 \%)$ in the glasshouse during most of the months of the year. In 1991 three epidemics and in 1992 seven epidemics of $S$ pannosa were observed. When all the rose plants were infected with $S$ pannosa, the crop was treated with a fungicide. After three fungicide treatments $S$. pannosa was completely controlled. The first new colonies were observed 8 to 10 days after the last treatment. From March untill November within 5 weeks most of the rose plants were infected with $S$. pannosa. In winter this increase in colony numbers was slower than in the other seasons, but still within 12-14 weeks $100 \%$ of the plants showed one or more colonies per plant.

The horizontal distribution of $S$. pannosa was fairly uniform. Only in winter, when air movements in the glasshouse were low, most of the colonies of $S$. pannosa were limited to a few locations.

With the results obtained from the epidemiological studies an economic action threshold for powdery mildew on rose was calculated as $5 \mathrm{colonies} / \mathrm{m}^{2}$ crop. This threshold was used for supervised control of powdery mildew in the less susceptible rose cultivar 'Kiss' and in the susceptible cultivar 'Sonia', from 1993 to 1994. Supervised control of powdery mildew in rose with the calculated action threshold significantly reduced the number of fungicide sprays against powdery mildew in 'Kiss', but not in 'Sonia'.

Research Station for Floriculture, Linnaeuslagn 2a, 1431 JV Aalsmeer, The Netherlands. 
A. LAGNAOUI and $H$. BEN SALAH. Potato tuber moth (PTM), Phthorimaea opercullela (Zeller),
is the most devastating potato pest in North Africa and the Middle East. Potato growers rely heavily on toxic chemical insecticides to control this economically important pest. insecticides to control this economically important pest. Trequent insecticide applications is a routine operat The present problems with insecticide resistance and residues in the environment have encouraged pest management management. Several control components have been identified over the years. At the International potato Center, we attempted to formulate an integrated pest management approach based on cultural practices (hilling irrigation and harvest date) and a spray of biopesticides (Granulosis virus and Bacillus thuriengiensis). In this paper, we will virus and Bacilius thuriengiensis). In this paper, we wIII integrated pest management strategy adopted in the region. integrated pest management strategy adopted in the region. pesticide use in the fields and a replacement of toxic chemical pesticides (Parathion, Fenitrithion) in storage by more effective and lesser toxic ones (Pyrethroids and Biologicals).

Abdelaziz LAGNAOUI, International Potato Center (CIP), 11 Rue des Orangers, 2080 Ariana - Tunisia.

DEVELOPING AN INTEGRATED PEST MANAGEMENT SYSTEM FOR FRENCH BEAN PRODUCTION NN KENYA.

B. Löhr, Susanne Michalik

Kenya is Africas langest exporter of French Beans, the main market is the European Union. Raised concem about possible pesticide residues in the consumer countries and new stringent MRL regulations introduced by the European Union necessitated the iritiation of a programme to produce high quality export beans using less pesticide.

In a planning meeting with all parties concerned in production and export, the pest problems of the crop were prioritised, the known information compiled and a research action plan developed. Bean Flies, Bean Flower Thrips, spider mites and flower and podfeeding caterpillars were identified as the most important insect pests and Bean Rust as the only disease of high priority. A strategy to avoid foliar pesticide applications as long as possible was developed using seed dressing and furrow application materials. In trials with those, the crop could be kept free of aphids and Bean Fly from planting to early flowering stage. The development of thrips and mite populations as well as their natural enemies in fields with conventional foliar applications as compared to seed treatments is currently being investigated and intervention thresholds for both pests are being developed. The integration of IGRs in the IPM system for the control of late-season pests like Maruca testulalis and Helicoverpo armigera are also investigated.

KARI/GTZ IPM Horticulture Project, P.O. Box 41607, Nairobi, Kenya

\section{M.Levitin. V.Ivashchenko, N.Shipilova, T.Gagkaeva, L.Nazarovskaja}

Fusarium head blight is a widely occuring disease on cereals in Russia. The disease is characterized by two forms. In Southern districts the disease has visual form; in Central Russia, Northern West and Siberia the disease has latent form. The loss only of wheat crop reached $25-50 \%$ and the contamination of cereal grain by mycotoxins increased more than 25 times. The use of fungicides for the plant protection was not effective. At present Russia hasn't cereals cultivars resistant to disease. The most perspective strategy for the protection is the use integrated control. For realization of this strategy the following researches were carried out. 1. The Fusarium species, causing head blight of cereals, and population structure one of the most widespread species - F. graminearum have been studied. 2. Biological pecularities of some species were researched. Role of infection sources and ascogenous stage in appearance of the disease were established. 3. The complex of agrotechnical measures allowing to decrease the damage of plant from this disease in two times was worked out. 4. The microbiological agents perspective for the control of Fusarium head blight were revealed. 5.Using the infectional phones we estimated the resistance to disease of many samples and cultivars of cereals. Among cultivars of wheat the forms with middle resistance were isolated. They could be succesfully used in IPM. 6. The conception of integrated control of Fusarium head blight was proposed.

All-Russian Institute of Plant Protection Podbelsky sh.,3, St.-Petersburg-Pushkin, Russia
116

INTEGRATED CONTROL OF CACAO WITCHES' BROOM IN BAHIA, BRAZIL BASED ON EPIDEMIOLOGICAL INFORMATIONS.

E.D.M.N. LUZ, J.L. BEZERRA, L.C.C. ALMEIDA and E.C.F. DOREA

Witches' broom disease of cacao caused by Crinipellis

perniciosa (Stahel) Singer, was first reported in Bahia, Brazil, perniciosa (Stahel) Singer, was first reported in Bania, Brazil plantations. An erradication attempt was made without success plantations. An erradication attempt was made without sucos because the pathogen was well established ln other areas detected by and today more than 708 of the cacao growing area of Bahia is contamlnated with the pathogen. In lggh, losses of 20 to 408 of the production were observed in some locations of the region where the disease has been more severe. Cultural and chemical minimize losses, using informations from epidemiological and phenological local studies. At least, three removals of all diseased tissues from the trees are needed during, the months of February/March, July/August and November/December. Five sprays with copper fungicides are recommended during flowering, protect the pods from infections by $C$. perniciosa and

phy tophthora spp. These integrated control measures have been proved effective. However, because the low prices of cacao growers need financial support from the government to cope with addicional costs of control.

CEPLAC/CEPEC/Fitopatologia, Km 22 rodovia Ilhéus/Itabuna, CX. postal 07, 45600-000 Itabuna-Bahia, Brazil. 


\section{Leo, Panebi Kong, LiogXiao}

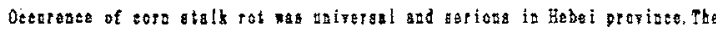
genctal disease pateg mere 8-a0r and could be oref 60 at wost. The plants of digease corn were prematere decay and the seeds nere not foll. Inus with dominast of plantigg reaigtant pariatieg againat coro stalk pot were carted ont frow 1986 to 1994 to costrol the disease. Aceording to the

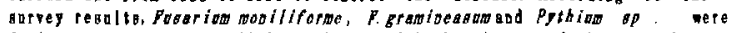
domlant pathogess. So soil inoculatiag with the mirtere of these three pathogens were done at seediog time of 6-8 leaves atage to ideotlfy the



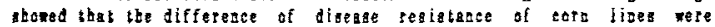

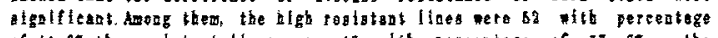

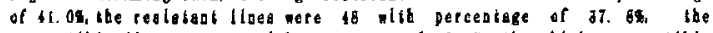
anacatible lloes were 28 with percentsge of 18 . 1\%. the bigh ouceptible

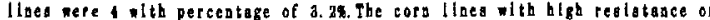
reatatence to alf of the three dominas patbogens contd be exterded is

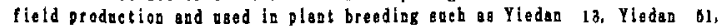
Yleden 52, Danje 16, Shendan 7, 478, 4017,5008 and 80 on. The diaesse

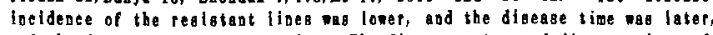
and the devoloping speed was alower. The diseare rateg and diacage bime of

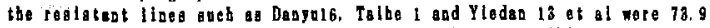
-92. 25 lawer and 6-10 days later thas that of the aysecptible ltaes anch as Jidan 17 and yedan 12. These cors liged were aot oals lowat digesse rate and later disaseg time, but also atconger adaptation. 2hoogdan 2 and Yledan 2, for exapmple, the gietda of apriog geediag, tatepplanting and ground corering with plastic membera were 11, 5-30.1\% and 4.1-18. 2\% higher than sumer geedlog reapectively. Sutable late platiog of apriag seeding cora lisea had good effects of corn atalk rot control. The diaese pate of aeading at the ead of April and the early of Mgg was 01. Ba lower than that of aeediag at the middle of April. The relative coustol effect of potatiog - ith fice coold be orer 82. 10. Up to oon the IBy of coin stalk cot bs

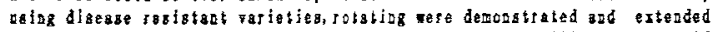

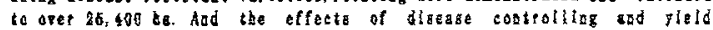
inescasiog rerc aigolficant.

lastitute of Plant Protection

Bebel Acedemg of Agricultural

73 Bast Pasa Strect

Qaoding Hebei 071000

P. R. China and Poreatry Sciences

\section{TO REDUCE FUNGICIDE DOSE IN CONTROLLLING APPLE SCAB}

\section{W. E. MacHardy, and D. K. Sutton}

Apple scab, caused by Venturia inaequalis (Cke.) Wint., is the major pathogen of apple in most regions worldwide where apples are grown commercially. It is controlled by the repeated application of fungicides that typically begin soon after bud break and end in late summer. In most regions, the primary inoculum consists only of ascospores produced in pseudothecia on overwintered scabbed leaves in the leaf litter; therefore. several early-season applications of fungicide are aimed at preventing infections by ascospores. Secondary inoculum consists of conidia produced in foliar and fruit lesions, and when primary scab is not controlled sufficiently, fungicide applications to control scab are continued to late summer rather then ending soon after petal-fall. Thus, the key to successful scab control is the prevention of primary infections. Because apple scab has secondary cycles that can result in significant economic crop loss, it has generally been considered that sanitation practices aimed at reducing the production, development, or availability of the primary (ascosporic) inoculum have limited utility in scab management. However, it has been shown in several years of field studies in commercial orchards in New Hampshire and other northeastern states in the US that a $25-75 \%$ reduction in yearly fungicide dose can be expected without increased risk to the crop in orchards that receive sanitation treatments to reduce the potential ascospore dose (PAD), i.e., the estimated production of ascospores per meter square of orchard floor, below a 'PAD' action threshold. Several sanitation treatments have been shown to reduce the production of ascosporic inoculum significantly. In one study with the following treatments, (i) leaf-shredding in autumn, (ii) leaf-shredding in early spring, (iii) leaf-shredding in autumn and spring, and (iv) ground treatment of $5 \%$ urea in spring, the mean total ascospores rapped in treatment plots (i) to (iv) during rain events in spring was reduced 91,73 89 , and $65 \%$, respectively, compared with the mean total ascospores trapped in untreated plots. A program to integrate sanitation practices with fungicide sprays to reduce the fungicide dose is presented. The key to this strategy is a 'Sanitation' action threshold based on an estimate of potential ascospore dose. The use of this threshold in relation to a 'PAD' action threshold for initiating a fungicide schedule in a truly integrated scab management program is presented.

Department of Plant Biology, 325 Nesmith Hall, University of New Hampshire, Durham, New Hampshire 03824, USA

\section{0}

CHALLENGES IN MMPLEMENTING IPM FOR LOW ECONOMIC THRESHOLD SOIL INSECT PESTS

\section{L.N. Matthiessen}

In parts of Australia soil-dwelling insects are major pests of potatoes where production is rotated with pasture. The main species are the accidental introductions African black beetle, Heteronychus arator (Fabricius ! and whitefringed weevil, Graphognathus leucoloma (Boheman). Both have a capacity to be highly damaging in low abundance. On average, a single insect of either species attacks from two to seven tubers, rendering them unsuitable for the market, and African black beetle adults attack stems causing yield reduction. As few as one or two insects per metre of crop row causes substantial economic loss in the potato production system which has a low tolerance for losses because of the high cost of production and the high value of the product.

Damage is primarily caused by insects resident in land to be cropped, imposing a need for pre-cropping control measures. The patchy distribution, low abundance, small size of many stages and concealment and protection by soil makes both scouting for prediction of the threat and management difficult. These factors have given rise to reliance on soilincorporated prophylactic control measures, typically with the most potent agents available, in order to minimise the risk of damage occurring. A recent trend has been the use of expensive soil fumigants as a control agent. The iong-term sustainability of such an approach is questionable.

This poster presents an overview of research within the CRC Resource Protection programme, the results of which will contribute to the development of pest management strategies that are effective, economic and environmentally safe.

CRC for Temperate Hardwood Forestry

Locked Bag No. 2

Post Office Sandy Bay

TASMANLA 7005 Australia
The challenge for researchers seeking to devise alternative, integrated-style managentent strategies for such soil insects with extremely low economic thresholds is to ensure a very high degree of efficacy while minimising undesirable effects. Sound biological knowledge, meaningful not just in the context of the potato cropping phase which is transient relative to the insects, is seen as essential for devising soundly-based management methods that may be unconventional, and helping to convince producers of their worth. A significant challenge is to establish a mind-set in which reducing soil insect populations can be viewed as a longer-term commitment, not solely an immediate component of crop husbandry.

For such pests, few totally environmentally benign control techniques such as classical biological control are, in isolation, likely to be effective in sufficiently reducing populations. They could only be conceived of as a component of an integrated management strategy with a focus on the longer period of the insects' life-cycle that typically occurs in the pasture phase of the rotation. Strategic use of relatively 'soft' insecticides, applied perhaps some time before the potato crop is planted and carried out without undesirable soil-incorporation, offers one such means of population reduction through targenting of prebreeding populations. A strategy seen as particularly worthy of attention is the use of rotation crops that do not favour the insects, or the use of break crops speciffcally selected to have biotoxic properties against them. 
Study on Integrated Pest Management for Durian

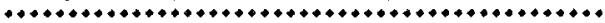

Su-apa Disthaporn (1)

Sirichai Limrateepong (1)

Vitsanu Uttayopas (1)

Sukkawat Chantarapannik (2)

Chaiwat Kraturoek (2)

Vit Namruangsri (2)

Somchin Palakul (3)

Integrated Pest Management for durian was carried out in Chantaburi, Prachinburi and Rayong provinces in eastem region of Thailand in the vear 19911994. It was found that IPM by using biological control and cultural practises as main components could control 5 major species of durian pests e.g. Allocaridara malcvensis, Euetranuchus africanus, Orgyia postica, Conogethes punctiferalis and Phytophthora palmrora effectively.

With the reduction of $70-90 \%$ of pesticide application in the PM plots. the dominant natural enemies e.g. chrysopids, coccinelids, parasitoids and spiders could be enhanced 4 times higher than in the conventional plots. Durian fuits were in special grade by average $70 \%$ without pesticide residue, which resulted in higher net return of about $200-400$ US $\$$ per rai. IPM in durian as it is practised nowadavs by durian growers gives higher economical net returns and is a ecologically sound crop production system

Zamorano, besides being an excellent agricultural school, has become an institution engaged in outreach programs that complement its teaching mandate. One of the programs has been IPM technology generation, validation and transfer. Small farmers have been our main target, but we also got involved in export crops (melons, onions) grown by highly resourceful and successful farmers. An IPM program for melon in Honduras and Nicaragua has developed a menu of alternatives for most pest problems. Most alternatives are based on non-chemical use. A successful program to manage bean slug (Sarasinula plebeia) was developed, validated and adopted in most dry bean producing areas in Central America. The program includes prevention measures as well as chemical control. The IPM program for corn and dry bean has created a new approach in technology generation. Farmers's knowledge is being complemented with education in basic pest biology and farmers are generating new ways to manage their pests. Farmers have generated and adapted many ideas to manage their pest problems, With this new approach, the technology is generated by the final user; these technologies generated have the advantages of being cheaper, usually are not chemical users, are viable options, focus on a real problems faced by the farmers and use farm available resources.

Zamorano, Department of Crop Protection, P.O. Box 93, Tegucigalpa, Honduras. Telephone (504) 76-6140, Fax (504) 76-6242, E-mail apitty@huracan.cr

INCIDENCE OF WEBWORM AND GALL-MIDGE AND THEIR FUTURE RESEARCH DIRECTION IN UGANDA

N. Ogwangit and J. ocan

ABSTRACT:

In two study seasons, viz. 1st rains (March - June) and 2 nd rains (Aupust - october) 1993 at serere Apricultural and Animal production Research Institute (SAARI), Uganda, studies were conducted to determine the incidence of Webworm (Antigastria catalaunalis Dup.) and Gall-midge (Asphondylia sesami FeIt.) in relation to sesame crop development. In Tst rains, A. catalaunalis infested the crop 17 days after emergence $(\mathrm{d}$.⿳亠丷厂巾.e. $)$ and its population increased progressively from 0.22 to a peak of 0.43 larvae/plant which coincided with peak leaf growth ( 35 d.a.e). In 2 nd rains the pest attacked the crop 20 d.a.e and its density increased from 0.18 to 0.35 larvae/plant when leaf growth was at its peak. A. sesami infested the crop at the onset of flowering (42, $\mathrm{d}, \mathrm{a}, \mathrm{e}$ ) and reached peak infestation at peak flower formation $(63 \mathrm{~d}$ a a $e$ ) The infestation ranged from 0.31 to 1.0 larvae/plant in 1st was recorded with maximum yield loss of $31.4 \%$. The forecasting the pest incidence, methods to save and use natural enemles, development of resistant sources, optinum plant population and optimum planting date.

Serere Agricultural and Animal production Research Institute, SOROTI, UGAIDA.
124

INTEGRATED CONTROL OF THE STRAWBERRY BLOSSOM WEEVIL ANTHONOMUS RUBI HERBST IN STRAWBERRY: THEORY AND PRACTICE

\section{S.Ya.Popov}

My concept of strawberry protection from the strawberry blossom weevil (SBW) is founded on the 15 years research programme which included the construction of reproductive and mortality life tables, the registration of insect phenology and plant damages, the estimation of energy budget and host plants, the development of an action threshold. Spring monitoring for SBW was based on: a) registration of food holes in the young leaves and floral parts, b) counting the number of aduits on one linear meter of row. It was found that counting the number of clipped buds as a method of monitoring was not suitable for Central Russia, where the period of damage was shorter than in USA and Canada. Phenological observations showed that pheromone monitoring could be incorporated into the SBW management. Parasitoids (Bracon intercessor Nees, Pteromalus (H.) grandis Walker, Trichomalus sp. and Lestodiplosis $s p$.) were not able to regulate pest populations effectively, that was why the standart control practice for SBW remained the use of pyrethroids. We concluded that the strategic management based on nonchemical basis was a reduction of reproductive success. The latter can be achieved by: a) impacts disturbing a stereotype of behaviour in females during their oviposition, b) selection of varieties with antibiotic, morphological or phenological resistance, c) infestation of adults by parasites or diseases affecting the reproductive system.

Entomology Department, Moscow Agricultural Academy, Timiryazevskaya str. 49, 127550 Moscow, Russia 
INTEGRATED MANAGEMENT OF THE SWRETPOTATO WII TEPLY BemiaLO tabQCi IN VENEZURLA J. Salas, 0 . Mendoza

The sweetpotato whitefly Bemisia tabaci (Gennadius) (Homoptera: Aleyrodidae) has been reported in verezuela since five decades as a secondary insect pest. population outbreaks were observed in melon (Cucumis melo $x_{\text {. }}$ ) from late 1980 s in several production areas and causing important economic damage in tomato (Lyeopersicon ebculentim), tobacco (Niootiana tabaom), sesame (Seramun indeverai vitus diseases in tometo the nost important damage. A research program was rapidly started on the biology, ecology and integrated control. progran was rapidiy started on the blology, ecology and lintegrated control, obtaining so far, the following results: a) the life cycle on tomato under laboratory conditions was reached in 22.26 days under a mean temperature and relative hutioity allows that under tropical conditions, 10 to 16 generations per year may be reached. An mportant menber of host plants including crops have been recorded, being the mot hiportant hoste crops: melon, potato, comon bean, sesame, tomato. sweet pepper, squash, etc. The parasitolds Brocreic $8 p$ and Bretmoecrup have beeper, squent plants. Predators as Delphastud, Chryoperie and unknown syrphic larvae have been abserved preying on eggs and inmatures. Entompathogenes fungi as Vewtichlian lecanti and pabeylowhes op have been fecorded arfecting $B$. tabaci per lations and ass production and conercial usage have been started by growexs. Yellow sticky traps are being used as an adule monitoring and/or control device. Cultural practices as traps crops, host weeds elimination, avoiding continuos host cropping and crop rotation have yielded good results reducing $B$. tabaci populations. Use or neem extracts have been encouraged to small farmers. The following insecticides have controlled s. tabaci populations: blphentrin, endosuphan, cypermethrin, alfacypezmethrin, methamdophos and dimethoate used aione, and the mixtures cypermetinin + deltamethrin, rotenone + cypermethrin, chlorpyrifos + dimethoate, methylpyrifos + mineral oil, alfacypermethrin + methomidophos and buprofezin + lambdacyalothrin. Additional practices as: plant check from seedbed thry harvest to decide spraying, rotation of insecticides, use of appropiate sprayers, keeping good agronomic practices as fertilization and irrigation are encouraged. All the thru an IPM program obtaining a reduction of 40 of insecticide sprays.

FonalAp. Centro de I rvestigaciones agropecuarias del Estado Lara. Apartado postal 592. Barquisimeto. Venezuela. CURRENT INTEGRATED PEST MANAGEMENT RESEARCH BY THE PLANT
PROTECTION RESEARCH INSTTTTTE OF THE AGRICULTURAL RESEARCH PROTECTION RESEARCH IN
COUNCIL IN SOUTH AFRICA

\section{E.R.T.C. Sandmann}

Current integrated pest management (IPM) research projects in the Plant Protection Research Institute of the Agricultural Research Council in south Africa are described. In the light of the detrimental in south Africa are described. In the light of the special in these projects. The needs of small-scale farmers in the in these projects. The needs of small-scale farmers in the developing communities in South Africa are also addressed. The programme provides what is essential for biological control, namely, an insect quarantine service available to all organisations in South Africa and southern Africa. This unit not only handies natural enemies for use in classical biological control, but also imported mass-reared natural enemies for inundative release purposes. In support of developing communties who practise intensive lntercropping, research on the effect of different plant systems or companion plants on crop pests is currently under way. The development of cost-effective biopesticides from plant extracts or from microbial cultures for use investigated. Target pests for these projects are the African bollworm, diamondback moth, and stemborers of grain crops. The classical biological control of stemborers in grain crops and diamondback moth is also investigated. A successful project aimed at reducing the dependence on pesticides against lepidopterous pests of raisins and groundnuts in storage, by using massreleases of a laboratory-reared hymenopterous parasite is described.

Plant pxotection Research Institute

Agricultural Research Institute

Private bag $\times 134$

Pretoria, 0001
South africa
Assessment and characterization of pest and disease constraints of plantain in Ghana

\section{P. F. Schill, K. Afreh-Nuamah}

Plantain (Musa AAB) is a primary food crop in the densely populated, high rainfall zones in Ghana. It is a key component in sustainable agricultural systems. Serious yield decline of plantain has been observed in recent years and has been attributed to soil exhaustion, worsening banana weevil, nematode and leaf disease problems. Although the combined action of pests and diseases clearly affects plantain yields, losses have been neither quantified nor partitioned among constraints. A diagnostic survey on the distribution, incidence, and severity of pests and diseases was carried out during the 94 major rainy season in Ghana. Site selection was based on Geographical information Systems (GIS) data based on population density (= land pressure), soil fertility, and length of rainy season. Fifty-two representative plantain growing sites were selected across Ghana through a stratified random sampling procedure. Two farms, with plantain in the first cycle, were selected per site and on each farm ten recently flowered plants were scored for plant parasitic nematode damage and sigatoka incidence (Mycosphaerella fijiensis, $M$. musicola). Banana weevil (Cosmopolites sordid us) damage was evaluated through destructive sampling on recently harvested plants. The results suggest, that sigatoka and plant parasitic nematodes are the major biotic constraints in the plantain systems in Ghana. Sigatoka was spread throughout the planeain growing belt in Ghana and generally with high severity. The plantain root systems showed severe nematode damage in nearly all of the sites. The plant parasitic nematodes Helicotylenchus multicinctus, and Pratylenchus coffeae were identified as the most wide spread nematode species. Radopholus similis was also present, but its occurrence localized. Meloidogyne incognita was widespread, but in low densities. Banana weevil damage was low in all of the sites sampled, suggesting that the importance of the banana weevil at least in the first cycle of plantain is negigible.

International Institute of Tropical Agriculture, IITA-Ghana

B.P. 9698 K.I.A., Accra, Ghana. 


\section{V.K. SEHGAL}

Arongst 54 species of insect pests of Chickpea, Cicer arietinum L., the winter season food legume, the pod borer, grain yield to millions of mostly resource poor farmers in Asia this loss has been estimated at 423.7 n US $\$$ (Ryan 1994):

Cultivated Chickpeas, after origin in Turkey, have diversified in the mixed and sustainable farming systems of India where even now $775 \%$ of world production of 7 . I "II MT is grown. For past 3 decades chemical insecticides have helped entomologists to demonstrate high on-tarm yields of $>2 \mathrm{t} / \mathrm{ha}$ cf. average yield of $0.7 \mathrm{t} / \mathrm{ha}$ in India. However now, frequent crop failures due to insecticide resistance in Helicoverpa, loss of natural enemies, resurgence of secondary pests besides, environmental and human health hazards have made the cultivation of Chickpeas unsustainable despite the favourable price of $4: 1$ vis-a-vis the cereal wheat. The farmers often loose $>50 \%$ of the realisable on-farm yields.

IPM for Helicoverpa has potential to double the present production of Chickpeas and to confer sustainability in Cotton, Sunflower and Tomatoes where it is a major pest. Resources are needed for data based bioric stress mapping, monitoring and forewarning systems, workable ET's and EIL's. Sustainable IPM includes combined use of cilltural practices, pest avoidance, host-plant resistance, ecofriendly and bio-pesticides (NPV, Bt, Neem etc.), to conserve natural enemy populations and prevent insecticide resistance. Incernational cooperation for Helicoverpa management is suggested.

Professor,

Department of Entomology,

G.B. Pant Univ. of Agric. \& Tech.

Pantnagax-263 145 ,

Pantnagax-263 145 , A

India

\section{Sher Hassan, Albert Munting and Akhtar Ali}

Malakand Division in the North West Frontier Province of Pakistan is the only frost tree zone whers tomatoes are grown in winter. However, a number of economically important viral diseases of tomato are prevalent mainly due to the intensified and continuous cultivation of tomato with no rotation, the cultivation of other solanaceous, cucurbitaceous, Malvaceous and cruciferous vegetables mixed or adjacent to tomato, the use of virus infected/contaminated seed and lack of proper phytosanitary measures. The important viral diseases prevalent in the area include tomato mosaic virus (ToMV). Tomato yellow leai curl virus (TYLCV), tomato yellow top virus (TYTV), cucumber mosaic virus (CMV) and potato virus $Y$ (PV). The damages due to the viral diseases result in $50 \%$ loss in yield and detioration in fruit quality causing a significant economic loss. Various management strategies tested to control tomato viral diseases included (a) Chemical and heat trealment of seed. (b) Vector control with insecticides, and (c) Changes in cultural practices. Incidence of TOMV decreased from $28 \%$ to $9 \%$ and yield increased from $219 \mathrm{Kg}$ to $235 \mathrm{Kg}$ with chemical and heat treatments of seed. A significant reduction in disease severity was also observed in the seed treated plots. Vector population decreased by $10 \%$ with concomitant decrease of virus incidence from $27 \%$ to $17 \%$ causing a $14 \%$ increase in yield as a result of insecticidal sprays. Incidence of tomato viruses which was $68 \%$ in mixed cropping reduced to $30 \%$ in monoculture with proportionate decrease in disease severity resulting in significant improvement in yield. Wead aradication from the experimental plots resulted in no significant difference in virus incidence, disease severity and yield. Significant reduction in viral incidence accompanied with a $21 \%$ increase in yield was observed in directly seeded plots as compared to those sown through transplant. It is concluded that the strategies used in this study were effective in reducing the incidence of viruses and improving the yield and quality of fruits.

Department of Plant Pathology, NWFP Agricultural University, Peshawar, Pakistan.

132

INTRODUCTION OF INTEGRATED PEST MANAGMENT (IPM) IN NURSERY STOCK. IN THE NETHERLANDS.

\section{R.W.H.M. Van Tol and M.J. Van der Horst}

Nursery stock in the Netherlands consists for more than $90 \%$ of field grown plants. Ouly a very small part of the nurseries grow plants in greenhouses and tunnels. So IPM in nursery stock has at least two different control strategies for pests and diseases. In greenhouses IPM is based on biological control of pests. Chemicals are only used against fungal diseases and as a correction for pests. Because most greenhouses are non-heated, climatic conditions (temperature, relative humidity) are variable and not always ideal for the biological control. Regular monitoring and correction is therefore essential in these greenhouses to keep the biological control optimal. In the field IPM consists mainly of chemical control based on selective compounds, monitoring and decision-making tools like damage thresholds. control based on selective compounds, monitoring and decision-making tools like damage thresholds.
By regular observations and use of monitoring plants we were able to use chemicals against the By regular observations and use of monitoring plants we were able to use chemicals against the
various pests on the right time and place and keep the damage below economic thresholds. For many plant species in nursery stock the economic damage level is very low because the visual attractiveness of these plants is an important quality factor on the market. Successful introduction of biological control agents antside are the insect-parasitic nematodes against the biack vine weevil. Other introductions (lace-wings, ladybirds and predatory mites) are promising but still experimental. The use of chemicals non-hazardous or limited hazardous for other insects suppor the survival and built-up of natural populations of beneficial insects to such an extent that they control several apbid species without using chemicals. Some of the more general problems for a successful introduction of IPM in nursery stock are the great variety of plant species on each nursery, the sparse knowledge on damage nursery stock ate the great variety of plant species on each nursery, the sparse knowledge on damage
thresholds and possible control strategies for the different pest-plant combinations and the costs of thresholds

Research on IPM in nursery stock started in 1992 in non-heated greenhouses and at nurseries growing plants in pots outdoors. In 1993 this research was extended to a nursery growing conifers and shrubs in the field. In a cooperation between the Research Station, Plant Health Service and Advisory Services the introduction programme for IPM has extended in 1994 to 22 nurseries. In this presentation the results of three introductions of IPM in nursery stock will be presented. These are introduction in a non-heated greenhouse, container field outside and in the field outside.

In the non-heated greenhouse a reduction in use of pesticides by $75 \%$ in 1992 and $96 \%$ in 1993 was reached. In 1993 all the pests (thrips, aphids, whitefly and two spotted spider mites) were controlled biologically with only a single chemical correction. The costs of crop protection in plant production are estimated on $0.3 \%$ in the reference year 1991 and $1.4 \%$ in 1993

At the nursery with pothed plants, problems were slightly different. There were not so many different plant species and the problems with insect pests were limited in 1992 and 1993. Special problems in pot plants were the control of mosses and fungal diseases like Phytophthora root rot. On this nursery we reached a reduction in pesticide use of $50 \%$ in 1992 and 1993 . These reductions were obtained by we reached a reduction in pesticide use of $50 \%$ in 1992 and 1993 . These reductions
chemical control based on monitoring diseases and by cultural methods like hygicne.

At the nursery in the field more than 200 plant species are grown. We were able to get a reduction in pesticide use by $42 \%$ in 1993 and $61 \%$ in 1994. The reduction in use of chenicals supported the survival and built-up of natural populations of hoverflies, ladybirds, anthocorid predarory bugs, lacewings and parasitic wasps. This resulted in 1993 in a limited reduction of aphids but in 1994 in a strong reduction without using chemicals. In 1994 the savings on insecticides were $87 \%$ compared to 1992. The costs of crop protection in plant production were $1.5 \%$ in 1992 and $2.9 \%$ in 1994. 
M. Bifat ULUSOY Nedim UYGUN Ulich KERSTING Ismail KARACA Serdar SATAR

Dialeurodes citri (Ashmead) and Parabemisia myricae (Kuwana) have become the most serious citrus pests in Turkey since 1965 and 1982, respectively. Recently, two new citrus whiteflies, Aleurothrixus floccosus (?) (Maskell) and Paraleyrodes minei (?) laccarino were determined in the East Mediterranean region of Turkey in August 1994

$D$. cirri and $P$. myricae thrive best in dense citas stands with high humidity. They are atacked by a number of indigenous predators which have, however, only a minor impact on their population dynamics. Studies on biological control of $D$. citri has been carried by mass culturing and releasing the parasitoid Encarsia lahorensis Howard in Aegean region of Turkey. Another promising biological control agent, the coccinellid Serangium parcesetosum Siccard, is mass cultured and released in East Mediterranean region of Turkey. Desipte promising results, complete biological control of $D$. citri by using $E$. lahorensis and $S$ parcesetosum have not been achieved yet. $D$. citri is controlled by cultural methods (wide-space planting, thorough pruning, weed control etc.) and by mineral oil sprays 1 ir 2 times per year. These measures are not sufficient to suppress $P$. myricae, but biological control with the aphelinid wasp Eretmocerus debachi Rose and Rosen has proved to be a complete success.

Integreated control measurements are being developed for A. floccosiss and $P$. minei.

Department of Plant Protection, Faculty of Agriculture, University of Cukurova, 01330 Adana, TURKEY

135

NEW OPPORTUNITIES FOR VEGETATION MANAGEMENT WITH HERBICIDE RESISTANT COTTON

J. W. Wilcut

New technological advances will soon make it possible to use previously nonselective herbicides for vegetation management in cotton (Gossypium hirsutum). There currently are no registered herbicides for postemergence control of annual broadleaf weeds in U.S. cotton without the risk of maturity delays and/or quality and yield reductions. Bromoxynilresistant cotton cultivars could be commercially available in
the 1995 growing season. Bromoxynil is a nonresidual. postemergence herbicide with a short soil half-life that controls numerous annual broadleaf weed species. Bromoxynil controls Ipomoea species, Xanthium strumarium, Chenopodium albur, Cassia occidentalis, Sida spinosa, Anoda cristata, and numerous other species. Bromoxynil does not control Senna obtusifolia, annual or perennial grasses, or sedge species. Glyphosate will control the aforementioned species plus annual and peremial grasses, cyperus esculentus, and cyperus rotundus. Bromoxynil and glyphosate will provide valuable options for controlling arsenical-resistant xanthium strumarium, and ALS-resistant Xanthium strumarium. Both bromoxynil and glyphosate are economically very cost effective.

Registration of herbicide-resistant cotton cultivars plus the aforementioned herbicides vill allow producers to use an IPM approach to vegetation management based on economic

thresholds. An extensive data base for economic thresholds of numerous weed species has been developed for U.S. cotton. Resistant cultivars and the use of the respective herbicide will be integrated into existing management programs. These programs include other management options including tillage and cultivation, and cover crops for weed suppression. The likelihood of weed populations developing resistance to bromoxynil or glyphosate is small since both herbicides are nonresidual and will be used with herbicides with different modes-of-action in a systems approach cotton tolerance to these other herbicides is based on differential metabolism and/or differential placement. This technology will increase no- and minimum-tillage, and stale seedbed production. It is no- and minimum-tillage, and stale seedbed production. It herbicide be used in a growing season to further reciuce the potential for resistance development in weed populations.

Crop Science Department, Box 7620, North Carolina state Crop Science Department, Box 7620 , North
University, Raleigh, NC $27695-7620$, USA

\section{UTHAMASAHY}

During the last 25 years, pest management in cotton has undergane a revolution. Increase in production and productivity have been as a result of developing high yiejding genotypes (varjeties and hybrids), improved production techniques coupled with innovative technologies for management of pests. Chemical hased pest management technologies have brought in newer problems such as pest resurgence. replacement and resistance. Excenewer problems such as pest resurgence. replacement and resistance. Exce-
ssive reliance and indiscriminate use of chemical jnsecticides hove eljmissive reliance and indjecriminate use of chemical jnsecticides hove eljmi-
nated the natural enemy complex of pests of cotton. Thus, a sejf sustainated the natural enemy complex of pests of cotton. Thus, a sejf sustai-
ning cotton protection system has become fragile. Cotton growers have realized that a single component approach involving the use of insecticides realized that a single component approach involving the use of insecticides
is no longer sustainable. Integrated Pest Management (IPM) has been is ho longer sustainabie. Integrated Pest Monagement iPM has been accepted as a sustainable alternative to chemical bosed approach in pest management. The Tamil Nadu Agricultural University (TNAU) has a mandate to develop sustainable strategies for IPM on cotton. The strategies
include pest surveillance, adoption of Jow cost and indigenous methads include pest surveillance, adoption of Jow cost and indigenous methads
such as cultural, conservation and augmentation of natural enemies. Besuch as cultural, conservation and augmentation of natural enemies. Besides, development of insect resistance cotton varieties is in progress. Genotypes, resistant to leafhopper. Amrasca devastans, white fly. Bemisia tabacj and moderately resistant to bollworms are cultivated by farmers. Pest monitoring imvolving cotton farmers, agricultural extension worker and plant protect $i$ on specialists is cone at village jevel and it forewarns
the farmers of the impending fjeld problems. Tools such as light, pheromone and yellow sticky traps are used to monitor the activity of pests. Maize, a nonhost crop is grown as a barrier crop on the borders of cotton fields to check the immigrant population of such pests as leafhopper and white fiy. Castor as a trap crop attracts the moths of Spotopterd jitura
for egg laying. Hand plcking and destruction of eggs, flared squares, caterpillats of bollworms, rosette flowers are pracriced by farmers.

The TNAU has developed technigues for mass production of the egg parasitojo, Trichogramma chllonis, aphid Jion. Chrysoperla carnea and the nuclear

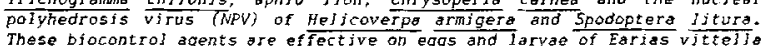
These biocontrol agents gre effectjve on eggs and larvae of Earias v vitiejla

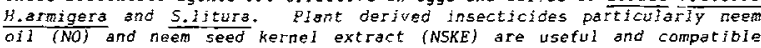
with biocontrol agents. Onfarm experjments in farmers fields indicate that in most cotton productjon zones, corservation and release of natural enemies during squaring and bolling stages could substantially reduce the need for chemical insecticides. These JPM technologies are environmentally sound and socially acceptable. It appears that IPM would go a long way in sustajnable crop protection in cotton in India in the coming years.

Department of Entomology. Tamil Nadu Agrjeultural University, Coimbatore, India 641003 .

136

EFFECTS OF CULTIVARS, PLANTING DATE, AND TILLAGE ON DISEASES OF SOYBEAN

J. A. Wrather, S. R. Kendig, T. L. Niblack, G. S. Smith, and A four year experiment, 1991-1994, was conducted in a field
where diseases had beer previously observed on soybean. The where diseases had been previously observed on soybean. The objective was to determine the effects of soybean cultivars, pymptoms of sudden death syndrome (SDS), brown spot, and downy mildew, and the soil population density of Macrophomina mildew, and the soil population density of Macrophomina phaseolina microsclerotia, and Heterodera glycines eggs. The soybean cultivars Essex, Forrest, Hartwig, and Rhodes were each planted in $75-c n$ wide rows in disc-till, ridge-till, and no-till
plots. The planting dates were inid-May, mid-June, and late-June plots. The planting dates were mid-May, mid-June, and late-Jun to early-July each year. The experimental design was a splitsplit piot with four repilications. The percentage of leaves with symptoms of SDS, brown spot, and downy mildew was estimated
at the R6 soybean growth stage. Soil samples were collected at at the R6 soybean growth stage. Soil samples were collected at
soybean planting each year and analyzed for colony forming units of $M$. phaseolina and $\mathrm{H}$. glycine eggs. The percentage of leaves with symptoms of SDs was higher in Essex, Forrest, and Rhodes than Hartwig, and the percentage was higher for no-till than either disc-till or ridge-till plots planted mid-May. The percentage of leaves with symptoms of brown spot was higher in mid-May than late-June to early-July plantings, and the percentage was higher in disc-till and ridge-till than no-till plots. The percentage of leaves with symptoms of downy mildew was higher in the last two planting dates than the first, and the percentage was similar among tillage treatments. The soil population of $M$. phaseolina was higher in ridge-tili than no-till or disc-till plots, was higher in mid-May than the later plantings, and was lowest where Rhodes was planted. Tillage, planting dates, and cultivars had no consistent effect on the population density of H. glycines.

University of Missouri-Columbia, P. O. Box 160, Portageville, Mo 63873 , USA. 


\section{NEGATIVE INFLUENCE OF SOME BIOFUNGICIDES ON}

POTATO SENSITINITY TO PHYTOPHTHORA INFESTANS (MONT.) D BY

\section{A.V. FILIPPOV., M.A. KUZNETZOVA}

Rhizoplan (strain of Pseudomonas putida) was used as a biological agent against Phytophthora infestans on potatoes in Russia. This agent inhibits germination sporangia and sporulation in vitro and on planta. Treatment of seed tubers before sowing delayes appearance of infection in the field for 8 days. However the losses of yield of potato were considerable. There were reports that the rhizoplan which had been applied as a seed spray to tubers influenced on immunite sistem of plants. Grown up plants from the treated tubers were less susceptible to the late blight than the plants from the untreated tubers until the development of $7-9$ leaves, when their susceptibility became egual. Plants from the treated tubers after the stage of flowering were beeing infected 1.8 times strongly then the plants from the untreated ubers. We expect that the Pseudomonas putida used as a commercial biological control agent alteres an immunologic status of the potato plants from positive to negative during the growth period.

143050, SCIENTIFIC RESEARCH INSTITUTE OF PHYTOPATHOLOGY, BOLSHIE VIAZEMI, MOSCOW REGION, RUSSIA

139

SAFETY OF PARASITIC PROTOZOANS NOSEMA SP. AND MATTESLA SP. FOR THE PREDATORY BEETLE TERETRIOSOMA NIGRESCENS USED IN BIOLOGICAL CONTROL OF PROSTEPHANUS TRUNCATUS (COLEOPTERA, BOSTRYCHIDAE)

Jerzy J. Lipa

The larger grain borer, Prostephanus truncalus Hom (Coleoptera, Bostrychidae) causes extremely serious damage to stored maixe (Zea mays) and cassava (Manuhot esculenta) in East and West Africa, after its accidental introduction in 1981 to Tanzania and in 1984 to Togo from Central and/or North America.

During an intensive survey for effective enemies occurring in natural populations of $P$. truncatus a oeogregarine Mantesia sp. (Apicomplexa, Neogregarinida), and a microsporidian Nosema sp. (Microsporida, Nosematidae) have been isolated and they have shown a good potential in biological control of its host.

However, the best potential in biological control of $P$. truncatus has a predatory beetle Teretriosoma nigrescens (Hom)(Coleoptera, Histeridae). This beetle naturally occurring in Meso-America is now being introduced to various regions in Africa.

While planning the introduction of $T$. nigrescess against $P$. truncalus in Africa it was necessary to evaluate various implications and to conduct various safety tests. The knowledge of safety of the above mentioned pathogens to $T$, nigrescens was needed in onder to avoid any failure which might take place if one or both pathogens would appear to be infectious to the predator and consequently diminish its role in biological control program against $P$. truncatus.

Due to small body size of larvae and aduits ( 1 to $3 \mathrm{~mm}$ ) of $T$. vigreacens it was not possible to microfed them with spore suspensions of Mattesia sp. and Nosema sp. The only feasible technique to expose $T$. rigrescens to infection was to offer to the predator as food the larvae, pupae and adults of $P$. truncatus heavily infected by both pathogens.

Short (21 days) and long term (8 months) exposure tests proved that Nosema 5p. and Mattesia sp. are not infectious to adults and larvae of $T$. nigrescens. Therefore it is concluded that the introduction of $T$ nigrescens to East and West Africa may be procured to healthy populations of $P$. truncatus or to its populations in which Nosema sp. and Mattesia sp. are present as all three biological control agents are compatible and can be used in an integrated manner.

Department of Biological Control and Quarantine, Institute of Plant Ptotection, Miczurina 20, 60-318 Poznan, Poland
BIOLOGICAL CONTROL VERSES HOST PLANT RESISTANCE? TRIPARTITE RELATIONSHIP PHTHORMAEA OPERCUNEULA ZELLER (LEPIDOPTERA: GELECHIIDAE), COPIDOSOMA KOEHLERIBLANCHARD (HYMENOPTERA: ENCYRTIDAE) AND SOLANACEAE.

\section{G. M. Gurr, L. R. Baggen, P. C. E. Bailey}

Results are presented which show that oviposition by the potato tuber moth, $P$. operculeila, is heavily influenced by the surface characteristics of host plant foliage and that this may be a behavioural adaptation to minimise egg mortality from egg parasitoids such as $C$. koehleri, a significant biological control agent. In choice tests the relatively glabrous leaves of Solanum pinnatisectum and S. chacoense were significantly less preferred than pubescent leaves of $S$.polytrichon and $S$. tuberosum $\mathrm{ssp}$. tuberosum $\mathrm{cv}$. Tarago. Lethal effects of glandular trichome exudates on $C$. koohleri were observed from laboratory exposure of adult parasitoids to leaves of tomato Lycopersicon esculentum, with dense glandular trichomes. Results will be presented from experiments aiming to establish the degree of sub-lethat influence of pubescence on the parasitoid via impaired host detection and subsequent rates of parasitism. Results are to be discussed in relation to breding of solanaceous crops for insect resistance based on glandular trichomes and the compatibility of host plant resistance with biological control for integrated pest management.

Orange Agriculturat College, the University of Sydney, Orange, NSW, 2800 .

140

Metabolic activity and ecological correlation between phytopathogen1c and nonpathogenic enterobacteria in different agricultural conditions.

Veronica P1shch1k, Irina Chernyaeva, Evgenia Lukyanenko

A comparative study of physlological, blochemical, morphological and cultural properties of 15 avirulent and 60 virulent strains of Erwinia carotovora was made by using numerical taxonomy. Avirulent bacteria differed in a number of properties: a lack of production of pectatliaze 1sozymes, in the activity of sugar, in the alcohol and glucoside fermentation rates, the tolerance to antibiotics, and sodium chloride and nitrate. He did not ind a difference between the character of colonization of tomato rhlzosphera by avirulent and virulent bacteria. But the zone with great quantity of tibrils was formed only around virulent bacteria. Virulent bacteria also were more tolerant (in comparison with avirulent bacteria) to the presence of nitrate. Nitrate quantity in potato tubers increased with increasing of dose of nitrogen fertilizer and in the condition of nitrogen nutrition disbalance. During this period a correlation between virulent and avirulent bacteria in the microbocenosis changed so, that the first prevalled. Also a quantity of rot tubers increased. The potato tubers were badly preserved. Th1s unfavorable situation for plants may be improved using the inoculation with avirulent strains of enterobacteria.

All - Russian Research Inst1tute for Agr1cultural microblology St - Petersburg, Pushkin, Podbelsky sh.3, 189620. Russ1a 
CULTIVATION OF BACULOVIRUS OF NUN MOTH (OCNERIA MONACHA L.) IN HETEROLOGOUS CELL LINES: BASIC RESULTS

\section{V.N.Bakhvalova. S.A.Bakhvalov}

Our positive experience of producing and applying the viral preparation based on baculovirus cultivated in vivo against the nun moth has encouraged our studies on cultivation of the virus in heterologous lines of celf culture of insects. We haveused 5 cell lines of Lepidoptera: Lymantria Dispar - JPLB-65z and SCLD-135); Antheraea Pernui - MCA p-1; Bombix Mori - SCB m-36; Euxoa Scendens - Es; and 2 cell lines of mosquito: Aedes Albopictus - Aa and Aedes Aegypti - L. The viral material hasbeen produced from infected larvae of the nun moth. Microscopic analysis has shown that in all the lines except Aa on the 3-d day after infecting pathologic changes occurred: hypertrophy of nuclei and their granulation; on the 7-th day polyhedron-like and highly polymorphous structures appeared. Only in a part of the nuclei of JPLB cell line typical polyhedrons for this baculovirus were detected. The larger part of polyhedron-like formations in the cells either did not contain virions or contained a small quantity of them. The structure of free virions in the nucleus and cytoplasm is very often non-typical: friability and considerable variability of size. The quantity of the polyhedron-like structures, polyhedrons andfree virions in the cells of all the lines was considerable less than in the nun moth cell in vivo.

Institute of Biology Siberian Branch of Russian Academy of Sciences, Frunze 11, Novosibirsk, Russia 630091

CONCEPTION OF REGIONAL SMALL-SCALE PRODUCTION OF BIOPESTICIDES.

L.W. Wolambet, $V_{0}$ E. Likhovidov

The regional principle of production of biopesticides (BP) implies timely supplying of the given agricultural region with the preparations in amounts and assortment determined from the analysis of phytosanitary, economical and ecological conditions. It the context of modern biotechnology the production of BP should be based on highly efficient, ecologically harmless technologies with the use of standard equipment. Among these, submerged cultivation of strains-producers seems to be he most production implies production implies this connecto ritions can be dropped. The latter should be substitited for liquid preparations and pastes, which are more profitable and -production lines, the major purposes of which are the developpreduction ines, the major purposes af which are the devel series of preparations for field trials;training of biotechseries of preparations for field trials;training of biotec holots. The pilot-production lines will serve a model is ruture entreprises. For realozation of this program,it is required to select biopreparations based on bacterial, fungous and actinonyctic strains-producers successfully grow by submerged cultivation. These biopreparations should provide strong protective effect against soil pests and phytopathogenes. 
Production methods and distribution strategy were crucial for the success of Invade ${ }^{\mathrm{R}}$, a microbial control of the New Zealand grass grub

Trevor A Jackson, Maureen O'Callaghan and Joan F. Pearson

Environmental Pest Management, AgResearch, PO Box 60, Lincoln, New Zealand.

Development of Invade $^{\mathrm{R}}$, the world's first microbial insecticide to be based on a bacterium from the enterobacteriaceae, has posed some unique problems. The active ingredient of Invade ${ }^{\mathbb{R}}$ is the nonsporeforming bacterium Serratia entomophila, the cause of amber disease in the grass grub (Costelytra zealandica, Coleoptera: Scarabaeidae), a pest of pastures in New Zealand. To successfully control the pest, live bacteria must be applied to the soil in sufficient quantities at a time when the larvae are feeding in initiate an epizootic of disease.

This posed problems of production, storage and distribution of the bacterium which had already proven efficacious in laboratory and small plot tests. The first problem to be overcome was bulk of product. The target quantity of $4 \times 10^{13}$ bacteria/ha was originally produced by fermentation in 20 litres of broth, but this was reduced by improved concentration within the fermenter. Bacterial survival remained a problem. Phage attack was overcome by use of phage resistant strains and lengevity extended by formulation and production methods.

The product lnvade ${ }^{\mathrm{R}}$ was safety tested and registered for commencial application in 1989 with characteristics of a 1 litre/ha product and a shelf life under refrigeration of $>3$ months. Distribution of a refrigerated product to the rural connunity still posed a problem. This was largely overcome by concentrating sales on agricultural contractors with cool stores and specialised equipment to apply the product.

Inyade is now firmly established as a commercial microbial pesticide and in the last 4 years has been applied to approximately 10,000 ha for the control of grass grut.

\section{S. Jigletsova, V.Shevtsov and E.Schyolokovo}

Determination of guarantee shelf-lifes for standard preparations of microbiological plant protection means (MPPM) and Inactivation forecasting of their manufactured formulations in different climatic zones are important problems. The applicability of "accelerated deterioration" method (ADM) (the storage at several raised temperatures) for MPPM guarantee shelf-ilfes prediction was investigated. For this purpose the inactivation kinetics of liquid concentrates and dry samples prepared by means of liophilisation and spray desiccation on the basis of different B.thuringlensis strains was studied at the temperature interval from +40 to $+70^{\circ} \mathrm{C}$. It was established that in most cases the $\operatorname{lnLC}_{50}$-time and in of survived spores number time relationships are represented the rectilinear functions With the correlation factors close by 1 . So, inactivation processes of MPРM preparations investigated had the $1^{\text {s }}$ kinetic oder. More complicated inactivation curves were observed for some liquid formulations. Thls facts served as a basis for deternination of inactivation mechanisms.

Values of inactivation constants $(K)$ at various temperatures were estimated from rectlilnear plots of Inactivation relationshlps. Functions $\mathrm{lnK}(I / T)$ were rectilinear with high correlation factors too. Extrapolation of straight 1 ines obtained affords to estimate the values of $K$ at every desireable temperature assuming that inactivation mechanisn does not change. The guarantee shelf-1ifes of MPPM at various temperatures were evaluated from data obtained assigning the acceptable level of inactivation. Predicted garantee shelf-11fes determined by ADM colnclded with experimental ones at the same temperature. So, ADM can be applied for prediction of activity preservation of biopreparations.

102, Lenin str., Serpukhov, Moscow Region 142283, Russia, $\operatorname{rax}(095) 1168980$.

148

A NEW POTENTIAL BIOLOGICAL CONTROL AGENT FOR SOYBEAN CYST NEMATODE AND ROOT-ROT DISEASES

Xing-Zhong Liu, Xin-De Zhang, Yun-Qin Xie, Guo-Sheng Shen, Man-Hong Sun \& Hui Liu

Soybean cyst nematode (Heterodera glycines) is a major pest in the Northeast, north and east region of China. More than $70 \%$ soybean planting area in China had been infested by this pest. Soybean root-rot has also became a serious disease in those regions. A new biological control agent as a seed-coating formulation containing antagonistic fungi, Paecilomyces lilacimus, organic materials, nematicidal plant broth and mineral fertilizers was developed for managing soybean cyst nematode. This agen was tested in green house, the cyst numbers on root and root rot soybean was reduced respectively $68.0 \%$ and $65.9 \%$ the growth of soybean seedlings was obviously enhanced. The agent was tested in field microplots and large-scale row plots in northeais:- China in 1993-1994. In 1993, six farms involved in 9 localities was conducted in the area of over 40 ha, the average cysts on root and root-rot plants were reduced respectively $66.4 \%$ and $32.2 \%$ and the average yield of $20.6 \%$ increase was obtained at an application rate of $5250 \mathrm{ml}$ agent/ha in 1994,52 localities involved in 38 farms and 14 counties were conducted in an area over 3600 ha the cysts on roo and root-rot plants were reduced $60.1 \%$ and $51.6 \%$ respectively and the average yield increase of $19.1 \%$ was obtained at an application rate $1500-2250 \mathrm{ml}$ agent/ha $(\mathrm{ca} .4 \$$ ha). The control efficacy and yields increase of this agent were comparable to the chemical treatments such as soybean chemical seed-coating formulation (product of Bayi Land-Reclamation \& Agricultural University) and chemical seed-coating formulation No. 3 and No. 23 (products of Beijing Agricultural University) but the cost of this agent per ha is much cheaper than that of chemical agents.

Institute of Biologica! Control, Chinese Academy of Agricultural Sciences, Beijing 100081 , China compound(s), giving in the presence of atmospheric oxygert and (or) daylght (or should reside separately from each other before the moment of dispersion) a thin surface film (membrane). The membrane must be permeable for oxygen, carbon dioxide and fungal hyphae and impermeable to water molecules. The film must adhere to the insect suface. The microcapsules should be about 30 microns in diameter each capsule bearing 5-10 spores. Film-forming compounds should be well-dispergable in the suspension and nontoxic to the humans, the fungus and non-target organisms. This approach allows to solve the problem of deficient air humidity, owing to the water supply in the described microcapsules Such approach may broaden application of EF in open ground (fields, orchards and forests). However, it should be noted the possibie problem of EF-hyphae orientation on insect surface

Institute of Chemical Kinetics and Combustion, Siberian Branch of the Russian Academy of Sciences, 630090 , Novosibirsk-90, Russia 
ASPOROGENIC BIOINSECTICIDES - NEXT STAGE OF ECOLOGICAI SAFEIY IN PEST CONTROL

V. Shevtsoy, E. Schyolokova and S. Jigletsova

Through chemical mutagenesis we have obtained the non-spore-forming strain Bacillus thuringiensis Sv, kurstaki (Btk) with entomocidal activity at the initial spore forming strain level. The electron-microscopic investigation of the mutant has shown the blocade of their spore-genesis process at the 3rd stage. Formation of toxic components is not violated and proceeds as it is with the parent strain.

On the basis of the asporogenic strain of Btk the ecologically safe biopreparation Asthur has been made. The comparative laboratory tests have shown that as regards biological activity relating to different test objects Asthur is on one level with analogs: Dipel, Thuricide, Lepidocide at al.

Small scale and field trials of Asthur against a wide range of lepidopterous insect pests have been carried out in Russia, Moldavia and Ckraina. Its biological activity is shown at the Lepidocide level against insect pests invading cabbage, leaf-chewing pests of fruit, forest and ornamental plants.

Within a three year period the preparation has been tested with six medicinal cultures and showed the same effectiveness as a concentrated Lepidocide, but surpassed the Dengrobacylline with beet webworm and Lucerne cutworm. The ecological aspects of application of Asthur have been investigated. Nu influence of the preparation upon the entomophages has been registrated.

The Asthur tests have also been carried out in seven states of India. For this and other countries which have a developed sericulture, the application of asporogenic plant protection preparation in the silk worm breeding areas is of considerable interest, as the absence of live spores in Asthur rules out the risk of epizootics in the populations of this economically important culture.

102, Lenin str., Serpukhov, Moscow Region 142283, Russia, Fax +7 (095) 1168980

151

DEVEIOANENT OF THE TECHNOLOGIY FOR SIJAEPGED OLLTIVATION OF FUNGOUS PREPARATIONS,

\section{Ri, Stepanova, V,E, LiKHOVidoV}

SUBMERGED AND SUBMERGED-SURFACE CULTIVATION WERE USED FOR PRODUCTION OF FUNGOUS PREPARATIONS ON THE BASE OF THE MOST ACTIVE ST-
RAINS OF P.P.BEAUVERIA, VERTICILLUM AND METHARRIZIUM, YEAST HYDRORAINS OF P,P.BEAUVER IA, ERT ICILLUM AND METHARRIZIUM, YEAST HYDRO-
YSATE WAS USED AS THE BASE OF THE NUTRIENT MEDIUM, OPTIMIZED WITH YSATE WAS USED AS THE BASE OF THE NUTRIENT MEDIUM, OPTIMIZED WITH
REGARD TO THE CONTENT OF CARBON, NITROGEN, MINERAL COMPOUNDS AND GXYGEN, TO THE CONTENT OF CARBON, NITROGEN, MINERAL COMPOUNDS AND

THE SUBMERGED CULTIVATION OF M, ANISOPLIAE, B, BASSIANA
$"$. LECANI I FOR $30-48$ HOURS YEILDED $2.5 \times 108,1.5 \times 10^{9}$ AND

$2.5 \times 10^{9}$ BLASTOSPORES PER $1 \mathrm{ML}$, RESPECTIVELY,

THE SUBMERGED-SURFACE TECHNOLOGY INYOLVES THE CULTIVATION OF FUNG I MYCELIUM FOR 120 HOURS UP TO THE FORMAIION OF CONIDIA SPORES (THE CULTIVATION DEVICE UKM-50 HAS BEEN CONSTRUCTED IN STATE Research Instituje of Phytopathology). The tItre of the final ProDUCT WAS $20 \times 10^{9}$ SPORES PER 1 ML.

ALL THE PREPARATIONS WERE TESTED WITH REGARD TO THEIR SHELFLIFE GAPABILITY AND INSECTICIDAL ACTIVITY

THE SPORES OF DRY BOYERINE AND VERTICEL WERE OBTAINED BY BOTH SUBMERGED AND SUBMERGED-SURFACE CILTIXAT ION. THE SHELF-LIFE OF THE FINAL PRODUCT WAS FEUND TO BE UP TQ $10-12$ MONTHS AT $4-100 \mathrm{C}$.

THE TESTING OF BOVERINE WITH COLORADO POTATO BEETLE IÁRVAS SHOWED NO CONSIDERABLE DIFFERENCES BETWEEN PRODUCTION TECHNOLOGIES USED.

THE EXPERIMENTS ON WHITE FLIES SHOWED THAT THE ACTIVITTY OF VERTICEL OBTAINED BY SUBMERGED CULTIVATION IS HIGHER THAN THAT OF THE PREPARATION OBTAINED BY SUBMERGED-SURFACE TECHNIQUE,

AT PRESENT, THE DEYELOPMENT OF LIQUID FUNGOUS PREPARATIONS, THE INSECTICIDAL ACTIVITY OF WHICH ARE CONSIDERABLY HIGHER THAN THAT OF DRY FUNGOUS PREPARATIONS, IS IN PROGRESS.
DEVELOPMENT OF CHAETOMIUM CUPREUM AS A SOLL MYCOFUNGICIDE FOR BIOLOGICAL CONTROL OF FUSARIUM WILT OF TOMATO.

Kasem Soytong, Faculty of Agricultural Technology, King Mongkut's Knstitute of Technology Ladkrabang, Bangkok 10520, Thailand.This research project was supported by IFS, Sweden and partly supported by IWAS, Italy.

Slide bi-culture test resulted to an antagonistic activity of Chaetomium cupreum KMIT 3003 agalnst Fusarium oxysporum f. sp. lycoperaic1. Withthis the conidia were lost of pathogenicity due to the broken cell and the protoplast was released from the fungal cells. It is implying as antiblosis. Soil mycofungicide was developed and performed inta 2-formulations as biopeliets whick formulated with sodium alginate and with mixed organic materials like ground rice straw, rice bran and pass through pellether. These biopellets were tested for survival which resulted to approximately $80-90 \%$ propagule survival more than 2 -years. In the field trial, applying biopellets one week before transplarting the tomato seedlings and then inoculated with the pathogen troculum resulted to reducer the disease incidence of tomato wilt as effectively as chemical treated one ( PCNB). Moreover, biological and chemical control had shown to be greater frlut width, fruit length, and yleld.

152

PRODUCTION OF ANTIFUNGAL ANTIBIOTIC FROM TRICHODERMA HARZIANUM ACTIVE AGAINST COLLETOTRICHUM GLOEDSPORIOIDES

Vijaya S. Kanapathipillad, Mawardi Rahmani and Eng Keng Joo

Irichoderma marzianum was studied as potentially active against Colletotrichum gloeosporioides, agent for anthracnose disease of tropical fruits such as mango, avocado, citrus etc. The Trichoderma species proved to be most efficient due to excretion of antibiotic molecules and fast growth over the pathogen. The antagonistic action shown through use of culture filtrates on mango fruits confirmed that $I$. harzianum can prevent or reduce infection by $\underline{c}$. gloeosporioides.

The crude antifungal ethyl acetate extract from cell free filtrates after 15 days of growth, was effective against c. gloeosporioides. By using silica gel absorption chromatography, an oily7gummy yellow product was obtained. The NMR and the mass spectrum indicated similarity to 1,2 , benzene dicarboxilic acid, bis ( 2 ethylhexyl) ester. Further work need to be carried out to confirm the structure of the active compound. Although crude ethy? acetate extract showed antagonistic effect on $c$. gloeosporiojides the partially purified compound (NX) did not show positive inhibitory effect on the growth or spore germination of $C$. gloeosporioides.

However, using HPLC to seperate another portion of the crude ethyl acetate extract, one of the seven fractions (EK5) obtained, significantly decreased the growth of the mycel ium and the spore gemination of $c$. gioeosporioides.

Biology Department, Faculty of Science and Environmental Studies, Universiti Pertanian Malaysia, 43400 UPM Serdang, Selangor, Malaysia. 
NATURALIS-L: A BIOLOGICAL CONTROL PRODUCT (BEAUVERIA BASSLANA) FOR CONTROL OF CROP AND GREENHOUSE INSECTS

\section{J.E. WRIGKTT}

Naturalis-Lis a natural microbial biological control product that has activity against major pests of cotton, vegetables and omamentals. The active ingredient, an insect specific fungus, is a patented strain of Beauveria bassiona, ATCC 74040. Naturalis-L has undergone extensive field evaluations in more than 52 countries and registration has occurred in many of these, ineluding the United States. Evaluations have determined that Naturalis-L is highly compatible with natural predators and parasites. Crop protection strategies, including resistant management, with the use of Naturalis-L has opened new alternatives for pest control in IPM (integrated pest management) and also OPM (organic pest management) programs. Its chemical and physical properties address current environmental issues and reflect a very favorable profile to mammals and terrestrial and aquatic organisms.

Troy Biasciences, Incorporation

2620 North 37th Drive

Pnoenix AZ 85009

['S.A
THE ANALYSIS OF RISKS IN THE CONVERSION TO SUSTAINABLE ARABLE FARMING: A DUTCH CASE OF WEED CONTROL IN SUGAR BEET

\section{A.J. de Buck ${ }^{1}$, H.B. Schoorlemmer ${ }^{2}$ and G.A.A. Wossink}

Arable farmers in the Netherlands have to integrate guality of the environment into their objectives. Despite stimulating research results in Integrated Arable Farming Systems, the conversion to IAFS only takes place slowly. One of the obstacles seems to be the perception of risk, associated with the new cropping techniques.

Under this setting a research project has started, focussing on normative aspects of risk and on risk perception by the farmer. This paper describes an approach of modelling normative aspects of risk. The model is based on multiple-year results of IAFS research on experimental farms, added by expert-knowledge. Risk is pictured by designing a decision tree. A decision tree is defined by various states, which can occur under the sequential stages of the decision process. Based on information, the choise for one of the pre-selected actions has to be made. Information about the current state as well as information about the possible outcomes with probabilities is important. Financial consequences as well as consequences for labour planning are considered. The model is tested by entering the strategies in existing farm situations of 38 intensively registrated IAFS farms.

Decisions are made about timing of weed control and about the technique to be used. Timing depends on crop stage, number of weed plants, workability and weather prospect. If the number of weed plants exceeds a certain treshold, weed control should be executed. Workability depends on current field and weather conditions, simulated from a set of relevant weather data. Chemical spraying, according to modern Low Dosage systems and two techniques without use of chemicals (harrowing and hoeing) are the choices in the decision for a techrique. Each technique demands a specific set of workability aspects. In case a technique is scarcely succesful, correction is possible with the use of a chemical product. With this information states under sequential stages are composed. The model chooses the optimal decision, based on the actual information (including joformation on forthcoming weather). The outcome of a decision is calculated under newly simulated weather. In the next stage it may appear that the wrong decision is taken. A large number of iterations gives insight in the distribution of the outcomes.

Lineair Programming with the use of Discrete Stochastic Programming is an appropriate technique to model this issue. Fixed strategies can be calculated by fixing a path through the decision tree. Hence, the consequences of a fixed strategy instead of an optimal strategy can be calculated. Comparison of optimized and (partially) fixed strategies pictures risk, associated with the outcomes.

1) Wageningen Agricultural University, Dept. of Farm Management, Hollandseweg 1, 6707 KN Wageningen, The Netherlands.

${ }^{2}$ ) Research Station for Arable Farming and Field Production of Vegetables, P.O. box 430, 8200 AK Lelystad, The Netherlands.
155

\section{AFRICAN FARMING HEADING TOWARDS SUSTAINABLE AGRICULTURE}

\section{Arif Gamal}

Wad Ramli's village lies in the rural areas of the Greater Khartoum State, about 60 $\mathrm{km}$ north of Khartoum. The population of WR which totals about 5,000 relies entirely on farming. Two thousand farmers form the core economical strength of the community producing tomatoes, potatoes and onions. The Central Market of Khartoum and the adjacent markets depend on North Khartoum farming communities for its regetables.

Heavy pesticide use and fertilizer use was the trend in WR till the beginning of the 1990's. Unfortunate and sad cases of acute poisoning resulting from ingestion of Aldicarb/Temik/Temick were current as weU as cases reported on endosulfan and DDT spray. The farmers applied up to about 6 sprays on tomatoes and potatoes to curb the white flies and cowpea aphids. Potatos were continuously sprayed with systemic insecticides to ward off root cutworms (Agrotis spp., Col.). Onions in its early stages were sprayed to control thrips.

With a modest grant for the campaign from the Dutch Small-scale Project Fund, NARP, a local implementing NGO, was able to elaborate a long-term involvement with rural farmers North of Khartoum. The Natural Resources Protection Group (NARP) was able to establish four years working with the farmers to curb irrational use of pesticide health hazards and to training the farmers on identification of major insect pests and their natural enemies.

The program on Integrated Pest Management (IPM) established in WR had many stages. The farmers were first trained to monitor their fields for presence of insect pests always relating it to the climatic conditions prevailing. In a second stage they were trained to identify the natural enemies, especially ladybirds, laceworms and yellow jackets. The application of pesticide was ouly to resume when the farmer assessed that the damage to the crop were excessive. The idea of spraying periodically according to a set agenda was totally abandoned. The results were overwhelming as most of the farmers gained more tirough not spending on pesticides and also most of the farmers who reduced their applications to only one-third according to need, found better and earlier yield, exceeding to about one and half time the barvest of the classically sprayed plots.

Nitrogen Fixing Bacteria (NFB) freely distributed by the National Council for Research was introduced on faba beans and alfalfa. The results of harvest were very successful. The amelioration of soils and crop growth were both an incentive for the farmer to adopt the technology. The results of the program which still continues is were overwhelming. About $50 \%$ of the farmers by 1993 turned to organic farming, while the rest of the farmers phased-out the use of their toxic chemicals to $60 \%$, i.e. only one-third of the habitual applications were maintained.
156

INTEGRATED CONTROL OF SUNFLOWER DISEASES IN THE FRAME OF THE MODERN CONCEPT OF SUSTAINABLE AGRICULTURE

\author{
H. I1lescu, A. Penescu
} The principles of disease integrated control have a very well
estabifshed place within the modern concept of sustainable estabilshed place within the modern concept of sustainable carried out over some years and which included both the technological means and the bid prevent and control diseases.

It is presented the luportance of soll works, chemlcalization, and crop rotation as well in preventing some pathogens attack that ometlus made uncertain the possibility to cultivate guntlower on certain areas. There are also lnciuded some preliminary results as regards the possibility to use superparasites or antagonists in

pathogens which produce sclerotia.

In the authors' opinion chemical control represented by seed dressing with funglcides with systemic or contact activity as well as by treatgents applied during vegetation period will be
another important means to decrease the attack.

In order to avold environmental pollution and at the same $t$ me to attain the concept of sustainable agriculture the economic threshold, the selectlvity of the chemicals which are used must be consldered and observed, treatments during vegetation
befing applied only at forecasting.

Research Institute for Plant Protection, B-dul Ion Ionescu de la Brad Nr.8, Sector I, Cod 71592, Bucharest, Romania 
ESCaPP is a regional project to develop, test, and adapt sustainable cassava plant protection technologies for the most important arthropods, pathogens and weeds found in West Africa. Multi-disciplinary teams of national plant protection experts join regionally with international experts to share expertise and pool efforts across agroecologies. The project is a collaborative effort between ITA, national plant protection staff, extension workers and farmers in Cameroon, Nigeria, Benin and Ghana, with a parallel component in Brazil involving CIAT and EMBRAPA. Project activities are divided into three interrelated and overlapping phases. The major cassava pests in targeted agroecologies are identified through diagnostic surveys in the first phase. This provides a baseline on the ecological, agronomic and socioeconomic concems as observed in the field and as recorded during group interviews with farmers. In the second phase, farmers participate in the development and testing of appropriate intervention technologies. Concurrently, in-service training of researchers, extension agents, and farmers in the principles and practices of sustainable crop production and protection is provided. Postgraduate training is also provided to selected women to strengthen their professional resource base in plant protection development activities. In the third phase, progress in achieving training objectives and technology implementation is evaluated. Unique features of the project include national teams of seconded multi-disciplinary scientists, shared local expertise on a regional basis, and $\mathrm{R} \& \mathrm{D}$ activities based on local diagnoses and priorities. The research, training, implementation and evaluation paradigm created in this project can serve as a model for developing appropriate plant protection technologies and pest management strategies for other cropping systems in the continent.

International Institute of Tropical Agriculture, Plant Health Management Division, B. P. 08-0932, Cotonou, Benin, West Africa

INTERACTIOIS IN SYSTEM YERTICILLIUM DAFLIEA - COTTON PIANT A. Kh. Akbarov, R.S.Sattarova, A.6. Khalmuradov.

Now the wide investigations on study fungal enzymes, which very perspective for using in national economic, are carrid-out. To number of such enzymes $\beta-1,3$-glucanases are related. They are used for biodegradation of wastes aiming oligosaccharines obtaining. The last may be used as inducers of defending reaction of phytoalecsins formation in tissues host-plant.

It has been established, that $Y$. dahliae synthetizes and reveals into medium two nolecular forms (I and II) of B-1,3-glucanase. To forms of $\beta-1,3$-glucanase in bomogenous state were isolated by methods of gel, ionexchange, adsorbtion chromatographies and praparative electrophoresis in polyacrylamide gel.

It.s possible, that phytoalexins synthesis may be induced by olygosaccharide fragnents of cell walls of $v$. dahliae. That,s why, wave extracted polysaccharides from cell wails of $\nabla$. dahiliae by method of alkali extraction, which main fraction by date of ini-spectroscopy 13 c was $\$-1,3$-glucan with molecular wight $80 \mathrm{DOO} \mathrm{D}$. Analysis of monosaccharide compasition showed, that isolated $\beta-1,3$-glucan consists frou glucose (7Dz), galactose (15z) and glucoseamine (15z).

We have studied physiologic action of enzyme hydrolysate of polysaccharide on cotton seedlings, beforehand determined the inducing ability of extracted polysaccharides on cotton defence reactions. It has been occured that $0,5 \mathrm{mg} / \mathrm{ml}$ polysaccharide concentration induces cotton seedlines defence reactions that was confirmed by absence of wilt at thein infection by Verticillim wilt. Then, polysaccharides were hydrolised by B-1,3-glucanase and action of enzyme hydrolysate of polysaccharides was investigated on seedlings. It has been establir. shed that enzyme hydrolyzate of polysaccharides from fungus cell walls begining from concentration $0,025 \mathrm{mg} / \mathrm{ml}$ includes cotton defence reactions.

Total DNA of $\mathrm{V}$. dahliae was obtained, restricted by EcoRI till $20 \mathrm{~kb}$ and ligation with cosmid vector plaFR 5 was carried out for determination of gene site and further cloning of B-1,3-glucanase.

Institute of microbiology of Academy of Sciences Republic of Uzbekistan, 700128, Tashkent, Kadiry st. $7^{\frac{1}{3}}$
TRANSGENIC TOBACCO PLANTS EXPRESSING THE PVX ORF2 PRODUCT ARE RESISTANT TO TMV AND Ob TOBAMOVIRUSES

\section{V.X.Ares, C.Hemenway, R.N.Beachy, A.Mentaberry}

The potato virus X (PVX) has a plus-sense RNA genome containing at least five open reading frames (ORFs). Products encoded by these ORFs comprise the viral replicase (ORF1), the proteins of 24,12 and $8 \mathrm{kDa}$ (ORFs 2-4) and the coat protein (ORF5). ORFs $2-4$ are partially overlapped and are commonly known as the "triple block". The triple block products, together with the coat protein, are involved in cell-to-cell viral movement by a mechanism not yet fully understood.

We obtained transgenic tobacco plants (Nicotiana tabacum Xanthi D8 NN) accumulating the ORF2 product (p24). Plants showing the highest levels of p24 accumulation had a stunted and chlorotic phenotype. These plants were able to complement the transport of a PVX infectious transcript harboring a p24 frameshift mutation, demonstrating that the transgenic protein is fully functional. While control plants developed the expected hypersensitive response upon challenge with tobacco mosaic virus (TMV), the size of necrotic local lesions on 24 plants was significantly smaller. Resistance to tobamoviruses was also evidenced when $\mathrm{p} 24$ plants were infected with $\mathrm{Ob}, \mathrm{a}$ virus able to overcome the resistance conferred by the $\mathrm{N}$ gene. In this case the plants showed no systemic symptoms and we could not detect viral accumulation in systemic leaves. On the other hand, symptoms obtained upon infection with PVX were more severe, although no differences were found on viral accumulation or timing of infection.

These results could be explained by assuming that both PVX and TMV transport mechanisms share common functions and that overexpression of p24 could inhibit TMV movement by competing for a putative host factor. Alternatively, another possible explanation is that p24 expression induces some general response impeding the establishment of virus infection. In particular, PVX infection would be allowed by some enhancing effect of the transgenic p24 on PVX cell-to-cell movement.

INGEBI-CONICET

Vuelta de Obligado $24902 \mathrm{p}$

(1428) Capital Federal

ARGENTINA 
PROPAGATION OF DIFFERENT POTATO VIRUS $X$ (PVX) ISOLATES IN PVX COAT PROTEIN EXPRESSING TOBACCO PLANTS AND PROTOPLASTS

\section{Marie-Lise Bouscaren, Lilian Järvekülg, Mart Saarma, Erkki Truve}

Several transgenic tobacco clones expressing coat protein (CP+ plants) or antisense RNA [CP $(-)$ plants] of the Russian isolate of $P V X$ were constructed. The infection analysis of seven PVX isolates showed that lines expressing detectable amounts of PVX CP were protected against Russian, HB, CP2, KD, KD4, S6111 and P551 isolates. However, the protection levels were different. Nearly complete resistance against strains KD and KD4 was achieved, whereas the two Finnish PVX isolates S6111 and P551 propagated in CP+ plants comparatively weII. In CP(-) clones we could not defect any inhibition of PVX isolates' propagation We have PCR-cloned and sequenced the coat protein genes of PVX isolates $\mathrm{cp} 2, \mathrm{~S} 6111$ and P551. Surprisingly, sequence comparisons revealed that isolates $S 6111$ and $P 551$ coat protein amino acid sequences were very similar to the Russian isolate's, whereas strains HB and $\mathrm{cp} 2$ were much more distantly related. Except for the amino acid change $A 88 \rightarrow V$, the other changes in S6111 and P551 coat protein sequence were conservative and should not change the structure compared to that of Russian isolate

We analyzed the replication of PVX isolates Russian, 56111 and P551 in protoplasts derived from $\mathrm{PVX} C \mathrm{CP}+$ transgenic tobaccos. In contrast to studies with intact plants, we were not able to detect any differences in replication rates between the three isolates. At low inoculum pressure the replication of all three isolates was inhibited in $\mathrm{CP}+$ cells, whereas the elevated inoculum concentrations abolished this effect.

The results of these experiments will be discussed in the light of the possible mechanisms of coat protein-mediated virus resistance in transgenic plants.
JOINT ACTION OP INTERSPECIFIC HXRRIDIZATION AND GENETIC TRANSFORMATION FOR TOMATO PLANT FROTECTION

Chesnokor Yu.V., Senyuk V.I., Bastrygtna A.S.

Introduction into Intact topato plante of exogenous plasmids which containing the NPIII gene by the procoss of pollination-fecundation realted.... in the production of kananycin resistant interspeoiflo iyoopersioon esoulentun (or. Fake1) M11. I Solanum pennellif Cor. hybride. All the resiotant genotypes aelected on selectire nedia wth kananyein ware trangferred to a field for further growth. Theae $F_{1}$ hybrida did not differ phenotypleally exoept for fruft aize and oolour. Fruitg were orange-red, yellow or yellow-green and their weight varled betweon 18 and $55 \mathrm{~g}$. Phe control $F_{1}$ genotypes had small (up to 14-16 g.) Ereen-yellow frutts. The presence of NPIII geno in zenome of regiatant $F_{1}$ hybrids, as rell as in their progeny, was shown by dot-hybridization and IPTII actirity analyala. The feat tissue of $F_{0}, F_{1}$ and $F_{2}$ plants atudied for their proteing with polyacrylamide gel electrophoresis and a comparatiro analyais of tometo transformante-hybrids was given on the basis of total protaln polymorphism. Ma aeleoted $R_{1}$ genotypen with different frutt colour and neight. Ptre populations rere eateblished ond atudied $\mathrm{from}{ }_{2}$ seeda of these hybrids. Segregstion for ehape and size of bush, sterilitj, aeod sotting and Iruit colour, shape, aize and weight, resiatance to somo diseaser and peats as well were obserted in the $p_{2}$ populations obteined.

The results auggert that utilization of exogenoug ors in interapeoffio hybridization can broaden the renge of genotypio rariability due to genetic transformation by meang of germinating pollen. This DNA transfer procedure reaulted in release of potential rariability, leading to ohenger in phenotyplo differences between genotyper and developing resistence to diaesas and perta.

Institute of Genet10a, Moldarian Aoadeny of Soienoes, Leanaya 20 Kishiner 277018, Moldore

164

THE BIOTIC ELICITOR OF DEFENSE RESPONSES IN ONION NDUCES RESISTANCE TO THE Peronospora destructor

A.P. Dmitriev, G.Y. Perkovskaya, L.L. Magomet*

Fungal pathogens are major agricultural pathogens world-wide. Currently, chemical control involves environmentally damaging and expensive fungicides. Breeding for resistance has been largely ineffective. Our long-term goal is to provide novel alternative plant resistance based upon stimulation of the plant's own defences against diseases by fungal elicitors. The elicitors, molecular signals activating defence responses in plants, fungal elicitors. The elicitors, molecular signals activating defence responses in plants,
are low molecular weight products reieased by the invading pathogen or the plant or both.

Using a drop diffusate technique we have shown that induced antibiotic substances are accumulated in the infection droplets of onion bulb scales. Non-specific pathogens to which onion is resistant (Fusarium solani, Botrytis cinerea) induce more fingitoxicity in which onion is resistant (Fusarium solani, Botrytis cinerea) induce more fingitoxicity in
the droplets, than the specific (Botrytis allin) or the non-pathogenic (Monilia fructigena) the droplets, than the specific (Botrytis allin) or the non-pathogenic (Monilia fructigena)
fungi. Isolated by TLC and HPLC, onion phytoalexins were further identified by UV, IR, NMR and chromato-mass spectra as 1,3-dion-5-octyl-cyclopentane (tsibulin ld) and 1,3-dion-5-hexyl-cyclopentane (tsibulin 2d). To elucidate the role of Tsibulin 1d and $2 \mathrm{~d}$ in onion resistance we have analysed the time-course of their accumulation in the in onion resistance we have analysed the time-course of their accumulation in the
infected tissues. The speed of phytoalexin accumulation in limited lestions (incompasble interaction) is much higher than in spreading lesions (compatible interactions). Analysis of bulb scales tissues by SDS-PAGE revealed that several PR-proteins accumulnted earlier in incompatible interactions than in compatible ones. Two of the accumulsting PR-proteins were purified by ion-exchange chromatography and used to raise polyclonal antibodies. One of the proteins $(27 \mathrm{kDa})$ showed chitinase activity, while the other (33

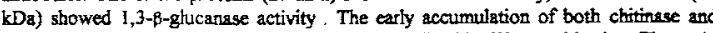
glucanase in incompatible interaction was also visualized by Westem blotting. The rapid accumulation of these enzymes, potentially capable of degrading the fungal byphat walls might play an important role as an active plant defence reaction.

At least two groups of elicitors were found in $F$, solcori mycelinm extract which differ in composition, molecular mass and inducing activity. The biotic glycoprotein elictor has been also isolated from mycelium of $B$. cinerea after removal of sohble cytosolic compounds. The elicitor activity depends on carbohydrate moiety of a glycoprotein as the activity is destroyed by periodate, but not by trypsin or pronase. Phytoalexin accumulation served as a physiological marker of resistance reactions increases upon application of very low concentrations of the elicitor $(<15 \mu \mathrm{g} / \mathrm{ml} \mathrm{glc.} \mathrm{eq.)}$

The results of 2-year field trials demonstrated that foliar spraying of onion plants with the elicitor from $F$, solmoni 2 days before artificial inoculation with $P$. destricior reduced significantly the incidence and extent of the disease. The fungal elicitors are not fungicides. They do not themselves kill the fungus but stimulate onion's own defenses against $P$. destructor and assures healthy plant growth

Institute of Cell Biology and Genetic Engineering Ukrainian Academy of Sciences,

- Skvira Agricultural Research Station, Ukrainian Agricultural Academy,

148 Zabolotnogo St., 252143 Kiev, Ukraine 
VARIAITION IN MITOCHONDRIAL GENOMIC DNA OF BAYISTIN AND IPRODIONE REBIBTANT MUTANTS OP RHIZOCTONYA SOLANI

A. Elavaxasan and D. Lalithakumari

Laboratory mutans of R.solani were produced by UV adaptation, EMS mutagenes is and adaptation using young mycelia, protoplast and sclerotia as source. Stable mutants with constant growth on high concentration of test fungicides for ten generations showing patrogenicity for ten generations showing pitochondria were chosen for the present investigation.

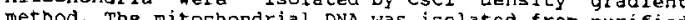
mithod. The mitochondrial DNA was isolated from purified Thioch for The R. solani mt DNA is GC rich unlike the other fungi $A$ t rich geromes. Digestion with hind 111 for $12 \mathrm{~h}$ at $37^{\circ} \mathrm{C}$ revealed a sertes of discrete bands when screened on agrose gel electrophoresis. The resulting restriction enzyme prodse was quite different from each other. The my sumationas of me DNA of each strain was calculated by sume molecular mass or htDNR of sensitlve strain was 38.5 and that of for , IaDp and rems respectively.

A.Elavarasan, Centre for Advanced studies in Botany, University of Madras, Guindy campus, Madras-
PRODUCTION OP PUBARIUM WILT RESIATANT TOMATOEB
BY IN VITRO CELI CULTURE METHOD

$$
\text { O. Ivanova }
$$

The objective of this study vas to obtain resist tomato plants to disease Fusarius oxysporum wilt. Beries of experiments vere done to: (i) choose the atralng of Pusarium and forms or theis action on plant celle; (ii)determine (iif) gearsh plast cell's cultivation and regeneration; choofing of cell selection cell phepar Iusarium acid biogyntbosis, pectinass, galacturodaso and callulase activity had beep phytotorical activity ought to be wpore than 50 follows. 2)


ought to be nigher tbeg cellulase ones; 3 ) fusarium acid biosynthesis ougte to be registrated from the beqinning of fungy oultivation. Culture filtrate of Fusarium and in some cases autoal ayed culture filtrate seem to be best suited es selective agents. Fethod of mathematical planning and result processing has been used succesfully for optimization of the culture media composition. Good balanced nutrient medium for cell morphogenesia and optimized medium for cell selection ware hormone-trea, that aimplify and reauce the price of culture medin preparation. The key for succes in obtaining or resistant plants is in optimar strategy of in vitro selection. Yore cetalle, sublothal dose of soleative agent has to be introduced in hormones-free culture media, then explants (hypocotylo fragments) plate and mutants select by (Ro) (Ro) resistance Bxamination carrying out in vivo by the seed posterity (R2) and to next step is to obtain resigtance on infoction and to make estimation of it latest step is clonal propagation of resigtant pethod. The technology improved for production of disease resistant tomato forms by in vitro culture methods makes the selstant process more raduco id price and gimplest. The tecbnolon had been approbated is Research instltute for vecetabies cultures (Moldova) and ngrfeulture Institute (It)hua) and may be recomended for creation of resistant forma of the vegetable cultures of ide spectrum.

Al1-Russian Researchinstitute for plant Protection, st. Petersburg, 189620, Russia

168

TNVASTIGATION OP SOME BIOCHEMTCAL ASPFCTS OP COMTON PATHOCENRASISTAHCE

Yuruskhanov Sh.Yu., Shapkin V:A., Aynabekov A.

The infection of different in resiatance to phytophthorosis and Verticilliun w1lt cotton varleties by corresponding infections was deternined to result in increasing protein blosgnthesis in leaves at the 1nitial perlod. The labeling in acid-soluble protein iraction including PR-proteins gufficiently increased in suaceptible to phytophthprosis Termez-16 varlety. The 3 hour exposition of leaf cuttings in an incubating medium with addition of Phytopithora вp. (P.op.) suspension also caueed the intensification of ${ }^{35} \mathrm{~s}$ - pethionnine incorporation in total protein (2,5 times) and ac1d- soluble protein fraction $(1,5$ times). The fluorescent in UV rays substances synthesized in cotton in responce to P.sp. Infection. These substances indices differ from onea produced when 71 t disease. Phenol compounds formation can indicate the occurence of spec1f1c mechanisto of cell protection agalnst P.sp.

Institute of Genetica, Acadeny of Sc1ences.

700000 Tashkent, Glavpochtamt, P.0.B.97, Uzbek1atan 
INHIBITORS OF $\alpha$-AMYLASES AND PROTEINASES AND THE RESISTANCE OF CEREALS AND POTATOES TO INSECTS

Al.V. Konarev, Fasulati S.R.

Hydrolase inhibitors is a part of the plant defence system. The differences between insect species in component compositions and relative activities of the main types of digestive amylases and proteinases as woll as the heterogeneity and intraspecific variability of plant inhibitors of pest enzymes must be taken into account in studies of protective role of the inhibitors. various types of the insect and microorganism amyiase and proteinase inhibitors were revealed among plant proteins with the use of isoelectric focusing in PAG, electrophoresis and thin. Iayer gel-filtration in combination with high sensitive and universal methods of amylase/starch/PAG and gelatine replicas. In the wheat group. the insect $d$-amylase inhibitors with Mr 12 kDa from endosperm of Triticum turgidum, T. timopheevil and other tetraploid species are especially of interest in relation with study of wheat resistance to storage pests. The varieties and wild forms of tetraploid wheats vary significantly in presence or absence of these inhibitors and in inhibitor activity. The varieties and forms of wheats differ largely in component composition and activity of endosperm trypsin inhibitors (TI). The differences among hard and bread wheat varieties in the $T I$ activity are correlated with the resistance to corn borer Rhyzopertha dominica $F$. in whose gut the trypsin-like proteinases prevail over others. The potatoes varieties are differed in capability to accumulate in leaves the trypsin, chymotrypsin. subtilisin and elastase inhibitors in response to injuries imitating the damage by Colorado bette larvae, with the reaction of resistant varieties being more expressive.

(Part of this work was supported by grant No. 93-04-21a90 from Russian Foundation for Pundamental Investigations).

Al1-Russian Institute for Plant Protection (VIZR), Podbelskogo

3, st.Petersburg-Pushkin, 189620 Russia
EXPRESSION OF INFECTION-RELATED TRANSCRIPTS DURING A COMPATIBLE INTERACTION BETWEEN MAIZE AND STRIGA HERMONTHICA.

M.J.Mayer, J. Steel, J.A. Hargreaves and J.A. Bailey.

Maize is completely susceptible to aftack by Striga hermonthica, a parasitic weed which causes major yield losses in many areas of Africa. Although extensive scteening programmes have discovered lines of maize with tolerance to parasitism, they have yet to identify an effective soutce of resistance. Knowledge of the effect of colonisation on gene expression at the infection site may yield information for use in the genetic engineering of novel resistance mechanisms. Young maize plants were cultured in vitro and inoculated with germinated $S$. hermonthica seedlings. Infected tissue was collected at 24,48 and 72 hours after inoculation (hai) The material was dissected into infection sites (consisting of c. $2 \mathrm{~mm}$ of infected maize root with the internal $S$. hermonthica tissue), the surrounding root tissue, and the external $S$. hermonshica seedling tissue. A PCR-generated cDNA library of c. $2.3 \times 10^{5}$ clones was constructed from lug total RNA isolated from the infection site at 72 hai. The library was then screened by differential bybridisation with analogous uninfected maize root tissue, to identify transcripts which were up-regulated or induced at the infection site. Seventy-four clones exhibiting differential expression were isolated in the first screen. Inserts from selected clones were amplified and used to probe Southern blots of maize and $S$. hermonthica genomic DNA in order to determine their origin. Seven clones bybridised to both maize and $\boldsymbol{S}$, hermonthica genomic DNA, one to only $S$. hermonthica DNA and one to only maize DNA. The temporal and spatial expression of the selected clone MM60, which hybridised to maize DNA and $S$. hermonthica genomic DNA, was examined in infected and uninfected tissue, using RNA from a range of infection stages and maize in vitro and pot-grown control tissue. Preliminary results indicate that this transcript is up-regulated in maize at the site of infection and in the surrounding tissue. The analysis of otber maize and $S$, hemonthica clones isolated from the differential screen is continuing. A number of clones of publisbed maize sequences bave been prepared by PCR from genomic DNA. These are being used to examine the expression of known pathogen-retated, stress-related and cell wall-related genes during the infection.

Institute of Arable Crops Research, Long Ashton Research Station, Department of Agricultural Sciences, University of Bristol, Long Ashton, Bristol, BS18 9AF, UK

172

POTATO BLOTECHNOLOGY FOR PRODUCING DISEASE RESISTANT VARIETIES

M. Usoltseya, N. Gusera and E. Aseera

The control of pests and diseases in agricuture today is hesvily dependent on the use of chemicals. Thus, even during a favourzble season, potato crops may be sprayed ten times for protection against late blight. The growing of resistant varieties is the most effective way to make agriculture ecologically friendly. Selection of resistant plants is a long process, but it can be accelerated by the use of biotechnological methods. Our research is concerned with obtaining potato forms that are resistant to late blight (Phytophthora infestans), black leg (Erwinia carotovora var. atroseptica) and insect pests by using sconaclongl rariation, cell selection and genetic engineering. While working with somaclonal variation phenomena among a collection of susceptihle potato varieties two forms with bigh morphogenic potential were selected. These were a Russian variety, "White Spring" with a very short (42 days) period of vegutation, and a German variety, "Adretta". We obtained a run of plant regeneranes, bech ria calli and straight on the explants of these varieties and chose forms with greater resistance than that of the original variety. One of the forms of the "Adretta" variety, similar to the initial form in morpholoy and yield, showed a considerable increase in resistance to the disease, both in the laboratory and the field. We identifled forms with resistance to both late blate and black leg. Cell selection is based on the fact that some pathogenic fungi (such as Phytophthora infestans) as well as many insects are unable to synthesize sterols independently. Therefore, for their development and reproduction they need to obtain sterol compounds from the host plants. The idea of using sterol deficiency in the host plants as a ner way of providing protection against pathogens suggested at the Deparment of Genetics of St. Petersburg State University. We worked out a scheme of selection of potato plants that were resistant to compounds which interrupt sterol biosytnthesis. For the initial material, we use stem and leaf explants where the heterogeneity of the tissues is enough to obtain forms with the desirable characteristics. Among plants resistant to selective agents, we have picked up some forms with resistance to late blight. Gene engineering approach required the production of transgenic plants that yield compounds that are toxic for insects and other parasites. We obtained a run of regenerants of "White Spring" whose explants were transformed by the agrobacterial vector strain pGV3850tt with the gene of endotoxin from Bacillus thuringiensis. (The strain as provided by Dr. L. Lutova, St. Petersburg State University). We obtained a run of regenerants that were resistant to kanamycin, which is a marker of transformation. The plants were tested in a laboratory model system called "potato-drosaphila", which was developed at the Dept. of Genetics, St. Petersburg State University. One form dramatically decreased the fecundity of insects and showed increased resistance to late blight and black leg. The latter can probably be explained by a spontaneous mutation caused by the integration of foreign genetic material in the plant genome.

137, Leninsky prospect, ff. 123, St. Petersburg, 198216, Russia 
IDENTIFICATION AND PURIFICATION OF A TOMATO CYSTEINE PROTEINASE INHIBITOR: POTENTIAL USE FOR CONTROL OF COLORADO POTATO BEETLE.

\section{S. Visal, S. Overney, S. Yelle}

Many defense responses in plants like activation of secondary metabolic pathway and production of defense related proteins are induced by signaling molecules in response to pathogen and insect attack. Plants, when treated with methyl jasmonate or its precursors are reported to induce the synthesis of defense proteins, among which are the proteinase inhibitor (PI) proteins. Bioengineering the plants with PIs is a promising method to control insect pests. Colorado Potato Bettle (CPB) is one of the most economically important pests of potato. Its effective control has not yet been achieved. Previous studies have shown that CPB has $80 \%$ of the digestive proteinases of cysteine type. Protein of $\approx 55 \mathrm{kDa}$ from gamma-linolenic acid treated tomato leaves shows cysteine proteinase inhibitory activity, characteristic to the cystatins. In vitro inhibition of larval proteases with tomato cystatin was compared with already available oryzacystatins (OCI and $\mathrm{OCII}$ ) and a human cystatin, Stefin A. In vitro inhibitory assays with individual PIs showed that the tomato cystatin inhibits $70 \%$ of proteinases which is 4 times more efficient than $O C I$ and 2 times more efficient than OCII and Stefin A. Also, when individual PIs- OCI, OCII and Stefin A were combined with tomato cystatin, no considerable additive increase in the inhibition of proteinases was seen as compared to the inhibition with the tomato cystatin alone. Further characterisation and application of this tomato cystatin in pest control will be discussed.

Horticultural Research Centre, Envirotron Building, Laval University, Quebec G1K 2P4, PQ, Canada .

SOMACLONAL VARIATION : BIOCHEMICAL CRTTERIA AND USE IN THE IN VTTRO SELECTION OF TOMATO PLANTS FOR RESISTANCE TO ALTERNARIA SOLANI

\section{A.O.Avrova, S.L.Tituterev, O.G.Kozyreva*, T.A.Evstigneeva}

Somactonal variation together with the genetical nonhomogenisity of plant cell and tissue populations can provide the material for the creation of disease resistant forms. The main problem of the in vitro selection of tomato plants resistant to the earty blight caused by Alternaria solani (Ell. et Mart.) as well as to the most other diseases is connected with the absence of the host- selective toxins among the metabolites of this fungus. Taking this into account we had created the principally new kind of selective background. A.solani have been cultivated on the fast-growing tomato calluses, which led to the essential increase in synthesis of phytotoxic polyketide pigments, especially altersolanol $A$ and $B$, followed by the considerable rise of vinulence of this pathogen. Introducing homogenate of mycelium with callus tissue residues including the complex of phytotoxic metabolites into the medium for regeneration before autoclaving we have drawn the cells of explants in the conditions near to those which occur in vivo during the pathogenesis. Specificity of action of this background on the tomato tissue have been checked on the explants of varieties with different sensitivity to this pathogen. Seed progeny of the plants regenerated from callus cultures initiated from cotyledons segments of tomato seedlings (variety Talalihin-186) were evaluated for the resistance to the complex of metabolites taking part in pathogenesis. Resistance to the described selective agent has been revealed in the first, the second and the third seed progenies of the regenerated plants. Most of the families resistant to the metabolites of the pathogen were also resistant to the fungus itself. Some of lines resistant in R1 generation were susceptible in generations R2 and R3. Among $R_{3} 4$ lines with high level of resistance were eliminated. Their resistance to A.solani 1,7-2,7 times exceeded the resistance of the initial variety and corresponded to the level of resistance of the well known variety Devon surprise. R4 of these lines is received. The studies of the origin and the nature of inheritance of this resistance go on

In the process of the studies of host-pathogen interaction we have created a biochemical test for evaluation of resistance of tomato forms to A.solani. It is based on the high level of induction of $\beta$-glucosidase activity in tomato tissues of susceptible varieties after their inoculation with this fungus. On the contrary in the tissues of resistant varieties practically no induction of activity of this enzyme was observed. This regularity remained also among the seed progeny of the regenerated plants.

All-Russia Research Institute of Plant Protection,

Podbelsky shosse 3, 189620, St.-Petersburg-Pushkin, Russia

* N.I.Vavilov Research Institute of Plant Industry,

B.Morskaya st. 42, 190000, St.-Petersburg, Russia

\section{WHEAT GENOME FUNCTION IN RESISTANCE TO PHYTOPATHOGENES}

\section{AYALALEEV A.YALALEEVA}

A correlation has been found out between wheat resistance to phytopathogenes and the genome composition of the Triticus genus. Wheat genomes differ essentially from each other in their immunologic reactions to phytopathogenes. The wheats with $A$ and $G$ genomes appear to be the most resistent. Hence, a search for resistance sources in selection should be focused at the forms with such genomes. Ferments and their protein inhibitors, lectins have been shown to be of great importance in forming wheat immunity to fungal diseases. The Bisol-2 immunostimulator has been advanched wheach reduces sick rates due to mobilization of the host-plant own mechanisms of resistance (potentialities). A specific effect of immunostimalators has been investigated upon both susceptible and resistent plant genotypes.

A.Yamaleev. Department of Biochemistry and Cytochemistry, Ufa Scientific Centre, Russian Academic of Sciences, Pr. Oktyabrya 69, Ufa 450054, Russia
176

CHARACTERIZATION OF A SYSTEM INHIBITOR-PROTEASE INVOLVED IN DEVELOPMENT OF A DEFENSE REACTION IN BUCKHHEAT SEEDS IN RESPONSE TO PATHOGEN ATTACR.

Y.E.Dunaevsky, G.A.Bellakova, E.B.Paviukova, H.A.Belozersky

Interrelations between higher plants and pathogentc fungi are complex and involve action of different chemical compounds directed both by fungi and planta tharoly tools used by the fung1 to attack plants and to damage their tissues. In turn. plants use specific inhibitors to defend themselves from these enzymes. We studied a protease inhibitor from the seeds of buckwhest Fagoprum escrientum and protease secreted by pathogenic fung1 fusarium oxysporum as molecular agents of pathogenic fungl Fusarium oxysporm as molecular agents of inhibitor was able to suppress the growth of mycelium of the inhibitor was able to suppress the growth of mycelium of the mentioned micromycete has been purified to homogeneity from
dry buckwheat seeds using affinity chromatography on
trypsin-sepharose and FPLC-technique on Mono 0 . The purified trypsin-sepharose and FPLC-technique on Mono Q. The purified
inhibitor is a protein of $7.6 \mathrm{kDa}$ and 51 . Its amino acid inhibitor is a protein of $7.6 \mathrm{kDa}$ and $\mathrm{pI} \mathrm{5.0.} \mathrm{Its} \mathrm{amino} \mathrm{acid}$ composition rovealed a high content of glutamic acid and valine and a low content of isoleucine, aromatic and sulfur containing anino acids. The inhibitor posessed high pH- and thermostability. It had no effect on endogenous buckwheat seed proteases but suppressed activity of a protease secreted by the lungi F.oxysporum. This enzyme was obtained highly purlfled and characterized, It is a serine protease with a molecular mass of $31 \mathrm{kDa}$. The data on the active centre of the enzyme and its action on various synthetic substrates indicate that secreted protease is a trypsin-like enzyme. Interaction between the studied rungi protease and the endogenous protease inhibitor plant defense against pathogenic microflora.

A.N.Belozersky Institute of Physico-Chemical Biology, Moscow State University, Moscow 119899 , Russta 
PROTEIN PROTEASE INIIBITORS AS MARKERS OF PROTECTIVE PROPERTTES OF AGRICULTURAL PLANTS BY THE ACTION OF NEGATTVE FACTORS OF ENVIRONMENT

1.A. Filonik

Institute of Biology Dnepropetrovkk State Univerity; Gagarin av., 72; GSP-10, Dnepropetrovsk, 320625, Ukraine

The exdension of chernicals application in agriculture more and mare increases the pollution of soils and plant products, that results in the decrease of their quality. Small doses of chronic irmadiation cansed by Chemobyl dieaster, influence negatively growth and development of agricultural planta, their phytoimmunity. It is the reason for investigation of the action of these negative factors of enviromment on the biochernical processee in high planis. It will allow to study the mechanism of their toxicological effect at the motecular level.

In high plants protein protease inhibitors except their perticipation in the activity regulation of own endogenous enxymes can execute protocting functions, an well suppreasing protease activity of ineects and phytopathogenice microorganima. Apart from protegre inhibitors can thow radioprotective properties. Therefore influence of perticide background and low dose radiation in rahural conditions on the activitice of protease inhibitors (trypein, hymotrypsin, subtilisine) in agricultural plants was atudied.

The decrease $(30-50 \%)$ of activitien of all three protease inhibitors at the earty The decrease $(30-50 \%)$ of activities of all three protease inhibitors at the earty
stage of nipening maize seeds and considerable increase of proteolytic activity in the milk-waxy ripen seeds on the pesticide beckground (atrazine, acenite) were found Distinctions in the levels of inhibitore and proteases activities in com disappeared

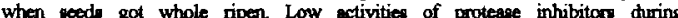
development of maize seeds testify to the decrease of protective properties of plants unders the perticide action.

The decrease (1.5 - 5 times) of protenes inhibitor activitiea in ripe soeds of cereals (wheat, rye, maixe) grown in Chernobyl zone in 1988 - 1993 was found. In two genetic forms of maize (W $64 \mathrm{~A}+1+, \mathrm{W} 64 \mathrm{~A}$. 22/02) the lower activitien of protease inhibitor: were discovered in ripening aeds during whole reproductive period under the action of low dose radiation. In model experiments the docrease of protease intither action inhibitor action of radiation $(2-10 \mathrm{R})$. The subtilisine indubitor was the most senvitive to the
pesticides and low dose radiation effect.

Distinction in the changes of protease inhibitos activitie in the of agricultural plants under the pesticides and natintion action can be cansed by different stability of the complex "enzyme-inhibitor" and reflect the decrease of plant immarity. The plants from Chernobyl zone were defeated by insects and diseases to more extent. The reduction of protease inhibitor activities in cersals is the evidence of lowering of protective properties of plant organisms by the action of negative factors of environment. This proteins can be suggested to biotesting of protective potential of agricultural plants at the different and complex pollution of environment.

\section{CHARACTERIZATION OF ELICITOR GLYCOPROTEIN FROM PHYTOPHTHORA INFESTANS BY USING MONOCLONAL ANTIBODIES}

\section{Naotgka Furvichi and Reiko Ikeda}

Plants respond to elicitor of a pathogen by rapid hypersensitive response. The glycoprotein produced in culture filtrate and byphal wall components (HWC) were reported as elicitors from

Phytophthora infestans (Pi).

We have isolated an intracellular glycoprotein from the hyphal homogenate of potato pathogen Pi by using HPLC, which is a potent elicitor of phytoalexin accumulation and defense gene activation in potato (Solanum tuberosum $\times \underline{\text { S }}$ demissum). We raised anti-HWC elicitor-monoclonal antibodies (Abs) to analyze the active site(s) in the elicitor. By pre-treatment of HWC elicitor with pronase, carboxypeptidase $Y$ (specific to L-amino acid), end-glycosidase $H$ (specific to $\beta-1,4$ linkage of $\mathrm{N}$-acetyl glucosamine) and sodium periodate, the binding activity of the elicitor to $2 \mathrm{Al1}$ Abs was greatly reduced. The results showed that HWC was glycoprotein, which contained $\mathrm{p}-1,4$ linked $\mathrm{N}$-acetyl glucosamine residues. In addition, these pre-treatment by a chemical and enzymes were effective upon elicitor activity of HWC, indicating that the active molecule of HWC might be glycoprotein. Binding capability of HWC elicitor to the $\mathrm{C} 8 \mathrm{Abs}$ was enhanced by pre-treatment of HWC with sodium periodate. This result indicates that the epitope of the $\mathrm{C} 8$ Abs may be protein covered with carbohydrate. The fact that potato lectin recognize HWC was confirmed by using ELISA assay with $2 \mathrm{All}$ anti-HWC Abs. The epitope of $2 \mathrm{All}$ Abs seems to be $200 \mathrm{kDa}$ in HPLC gel permeation analysis. Taking these results into consideration, we propose that both protein and carbohydrate moieties of HWC elicitor are important for expression of the elicitor activity.

Faculty of Agriculture, Niigata University , Niigata, 950-21, JAPAN
180

\section{dS RNA ISOTATION FROM F.OXYSPORUK AND V.DAHTIAF}

\section{O.S. Petrova, T. P. Bmakova, A. A. Abodukarimov}

The main goal of this investigation is to fsolate virus particles from natural 1solates of P.oxyyporum and V.dahliae. the fung1 wh1ch cause cotton wilt. Since genome of the most mycoriruses is represented by deRNA, its detection in mycellal tissue can serve as a basis for conrirmation of 71 rus particles presence. Double-stranded fNA has been determined in mycelia of romg1 by means of worr1s modification method which perm1ts to detect virus particles in a large number of 18olates. 23\% or V.dahllae 1solates and $25 \%$ of P.ozysporum ones of spectimens in the studied group contained deRNA. The electrophoret1c analgsis of nucleus acids 1solated from fungous mycelia showed that quantity and size of segments differ depending on a strain and vary from 1 to 4. The electron microscopy 1nvestigations showed the rod-like particles, $250 \mathrm{~nm}$ size, and sphertcal ones $12-15 \mathrm{~nm}$ size are present in F.oxgsporm 1solates. The rod-11ke particles, $450 \mathrm{~nm}$ size and spher1cal 33-35 $\mathrm{nm}$ s1ze, are present in v.dahliae 1solates. The study of protein composition of virus and non-virus strains of the fung1 has been fulfilled.

Institute of Genet1cs, Acadeny of Sc1ences of dzbek1stan 700000 Taghkent, Glavpochtamt, P.0.B. 97, Uzbektatan 
THE BIOCHEMICAL AND ULTRASTRUCTURAL ASPECTS OF A WINTER WHEAT RESISTANCE TO VIRUSES

I.T.Mighchenko, A.M.Silayeva, A.I.Boiko The winter wheat plants in several regions of Ukraine are af-
fected by viral diseages causing an appreciable damage to the agriculture. Our electron microscopical and biochemical agriculture. Our electron microscopical and biochemical investigations of disessed plants in field experiments show that the wlinter wheat is usually affected by the streaked mosaic virus of wheat (Sw, mosaic virus of winter wheat (MVWW), and yellow dwarfness virus of barley (YDVB). Greenhouse experiments were carried out with wheat plants systemicly infected by SMW. Some pathological changes in diseased plant leaf cells were obaerved. There are a dismption of cristae and a clarificetion of matrix in mitochondria. Phey enter into contacts with 1990 omes and become the centers of active hydrolytic procesese in plants. As to chloroplasts of diseased planta, there are a degtruction of the photosynthetio membrane complex, chlorophyll content is reduced by $60 \%$, the number and dimension of plestoglobuleg, are increased. At the same time oome chloroplasts pregerve their inner structure and photogynthetic function, forming numerous branched contacts wth mitochondria, peroxysomes, nucleus, endoplasmatio reticulum, etc. The cytoplasm becomeg more clear through a reduction of the ribosome content and s fucrease of $1 \mathrm{ysosome}$ and vecula numbers and sizes. There sppear various osmiophilic inclusions within the cytoplagm and vacuoles as well on the plasmaleme that may be connected whith forroation and accumulation of hydrolytic producta. The pathological changes in a cell atructure are accompanying with those in the biochemical composition of plants, in a yield and its quality. There $1 \mathrm{~s}$ a reduction of $\mathrm{za}, \mathrm{Cu}$, and $\mathrm{Fe}$ contents by $30 \%$, manganese, magnegium and calcium ones by $70 \%$ in diseaged plant leaves. An yleld falls by $20-60 \%$; a 1000 seed weight, protein and gluten contents are diminished too. An extra-root treatment of SWVW-disessed plants with Cu, B, Li, Mo, Mn and $\mathrm{Zn}$ galta has increased a yield capacity by 13-22\%, a 1000 seed weight by 10-14\% and has improved a protein-gluten content. Pre-sowing and extra-root treatnents using growth-regulatora with cytokinin activity have caused 32-104\% rlaing in the leaf chlorophyll content, $25 \%$ increasing in the dry plant mass, and twice decreasing in the quantity of diseased plants. Besides, some element in leaves has increased: zinc and iron by $50-60 \%$, calcium by $24 \%$, nagnesium by 15\%. At the oame tim potassium, sodium, manganese, cooper, and lithium contents were changed ingignificantly. Thua, uaing a pre-gowing aeed incruatation and an extra-root treatment of plants with certain oompounda, we are able to atabilize the blochemical, structural, and functional status of virug-diaeased plant that allowa

Tarea Shevchenko Kiyiv University, Departmeat of Biology,

Virusology Cha1r, 64 Volodymyraka St. Kiyiv, 252017, Ukraine.

183

THE ROLE OP CYTOKININS IN WHEAT RESISTANCE TO STEM AND BRONR RUSTS

\section{V.P.Muzykantov S.Yu. Larina}

It has been previously reported, that the cytokinin (CK) spectrum of wheat plante is closely connected with their resistance to the obl1gate pathogens (Vizarova, 1989; Larina et a1. 1993). The interrelationship between the CK spectrum of the hoet and the pathogen etill remaing unknown. He have invest1gated the interrelationship between $\mathrm{CK}$ apectrum of Pucoinia graminig triticl, P.recondita and wheat varieties with varioug degree of resiatance to these pathogenes. $\mathrm{CK}$ activity was detected using FPLC and Amaranthus bioassay. CK spectrums of healthy rust-susceptible wheat oultivara (CV) was found to contain more zeatin-glucoaid and zeatin-ribosid than those of resistant ones. The activities of zeatin and high RI. CK fraction were higher in mist-resistant wheat CV. Correlation coefficient $x$ was uged to determine the degree of similarity between CK apectrum of healthy (infected) wheat plants and rust. For healthy susceptible wheat CV Saratovekaja (S)29 and Grecum 114 (G 114) $r_{x y}$ was lees than 0,1 and for $\mathrm{CV}$ IIttle Club $r_{x y}$ was 0,50 . For healthy resistant wheat $\mathrm{CV}$ Khapli $r_{x y}$ was 0,80 and for CV $846 r_{x y}$ wa 0,52 . After inoculation of susceptible ov with stem or brown mate $r_{\text {fy }}$ was raised to 0,57 for CV 0114, to 0,86 for CV I. Club and 0,81 for CV 529 . After infection of:resistant $\mathrm{CV} \mathrm{I}_{\mathrm{XY}}$ was etrongly decreased to 0,21 and leas for CV KhapIi, to 0,39 for $\mathrm{CV}$ \$46. These invertigations have shown that CK spectrums of resiatant whest $\mathrm{CV}$ are close to those of rugts. These $\mathrm{CV}$ are able to maintain their CK status, rapidly metabolizing musta CK or weskly reating to thee. $r_{x y}$ can serve as a peculiar marker of wheat resigtance to stem and brown musts.
IMPLEMENTATION OF PROTOPLABT FUSANTS OF TRICHODERMA BPP. WITH EMHANCED BIOCONTROL EFFICACY TO CONTROL BHEATH BLIGHY ( $R$. SOLANI) OF RICE UNDER GREEM EOUSE CONDITIONS

C. Mrinalini and D. Lalithakumari

Protoplast fusion was carried out between two species of Trichoderma. T. harzianum and r.longibrachiatum, former with biocontrol efficacy and latter with fungicide tolerance. Fusants that resulted were more efflcient in the control of sheath blight (90) control) than the parent strains. Small scale preparations of the fusant Trichoderma were made into which $R$.solani was also inoculated and amended into the soil along with the paddy seeds. The paddy plants (30d) were transplanted in pots, and were incubated and observed. The taproved strain amended pots showed a profuse growth when compared to control. The susceptible IR 50 variety was taken for the present studies. Application of fusants resulted in increased height of the plants, length of the leaves and reduction in the number of lesions that appeared in the leaves and sheath caused by the pathogen. c. Mrinalini, Centre for Advanced Studies in Botany, university
of Madras, Guindy Campus, Madras 600025 , India.

184

\section{2'-5' OLIGOADENYLATE-BINDING PROTEIN(S) IN HIGHER PLANTS}

\section{Niqul، A. Aaspölitu, M. Saarma, E. Truve}

Pathogen-derived resistance approach for the construction of virustolerant transgenic plants have enabled to end up with plants protected (or even resistant) to one specific plant virus or in some cases to a group of closely related viruses. The airn to create plants displaying a broadspectrum virus resistance remains to be achieved.

We have demonstrated that plants expressing the mammalian $2^{\prime}-5^{\prime}$ oligoadenylate (2-5A) synthetase are protected against plant virus infections in greenhouse as well as undet the field conditions (Bio/Technology 11:1048, Arch. Virol. Suppl. 9:41). In order to understand better the mechanisms being responsible for this achieved protection, we have analyzed the influence of chemically synthesized 2-5A on plant cellfree translation and have shown that similarly to that in mammals, 2-5A are in vitro translation inhibiting agents also in plant systems. It is due to their ability to induce a rapid degradation of mRNA. We have also anatyzed the presence of 2-5A-binding protein(s) in plants using chemical cross-linking, ligand blotting and immunoaffinity techniques (using antibodies against mammalian 2-5A-dependent ribonuclease). We are currently screening plant CDNA and expression libraries for the presence of mammalian 2-5A-dependent ribonuclease analogs. At the same time we are also chracterizing the rat 2-5A-dependent ribonuclease in order to co-transform its cDNA into plants together with rat 2-5A synthetase. This coupled system should provide us with stronger protection against a braod range of plant RNA viruses than was achieved by expressing in planta 2-5A synthetase alone.

The results will be discussed in connection with other possibilities to confer braod spectrum virus resistance in plants as well as with the evolution of antiviral 2-5A pathway.

Institute of Chemical Physics and Biophysics, Akadeemia tee 23, EE0026 Tallinn, ESTONIA 
OCCURRANCE, PRODUCTION AND PROPERTIES OF LIGNINOCELLLLOLYTIC ENZYMES ON SUGARCANE BAGGASSE AND MANGO WOOD BY GANODERMA LUCIDUM

PERULAL x; TALAICHBLVAM P.T. Ganoderma lucidum is one of the basidiomycetous white rot plante. Some of the economically important plants affected by this disease are teak, coconut, palms. cocoa, mango, quercus and casuarina. As much as 55 \% of total infected plants have besuarina. As much as 55 , of total infected and $5-10$ ve been infected by G.luctdus in-and-around Madras and $5-10$ vere seve appeared regularly on winter season. In culture G.lucidus appeared regularly on winter season. In culture G.lucidue produces lignin peroxidase, laccase, cellulases and xylanases. On Tamarlndus trees infected by G.lucldum showed tissues towards the inner barke of th from the outer fungal were produced on the natke of the tree. These anzymes sugarcare baggasse, wango wood, eucalyptus wood, as well ast, in different gynthetic wood, eucalyptus wood, as well as glucose and maltose tavouredia. Among the carbon sources peroxidase and laccase extract also timulated higher production and yeast peroxidase and lacoases. If production were $33-37 \cdot \mathrm{C}, 6.5-7.2$ respective and $\mathrm{pH}$ for wall polyeccharide used in the medium such as lignin, cellulose, xylan were Native PAGE patterns as individualy and in combinations: different isozymes. Lip 1sozymes varied from 1-5 depending on the sources of the substrates. Two laccases 1-5 depending on $P \lambda G B$ res of the substrates. Two laccasas were observed polyacrylamide gels are in progress.

CENTRE FOR ADVANCED STUDIES IN BOTANY, UNIVERSTTY OF MADRAS, GUINDY CAMPUS, MADRAS - 600025 , 6. INDTA.
RUST FUNGI NUCLEAR DIVISION AND CONIROL OF THEIR ECTOPHYT IC DEVELOPMENT

\section{J.M. Plotnikova, V. V. Karpuk}

We studied $P$. graminis (f. sp. triticl and f.sp. secal is) infection structure differentiation in vivo and in vitro. Puccinia graminis appressoria were usually formed over host stomata and infection pegs penetrated into substomatal cavities. Some appressoria were aberrant, they produced outgrowths along the leaf surface and did not penetrate inside leaves. Deviation of heat shock temperature triggered the differentiation in vitro. Optimal heat shock temperature induced normal appressoria formation and further differentiation (type 1); higher temperature stopped differentiation at appressorial stage (type 2); lower temperature induced formation of smaller appressoria with lateral outgrowths (type 3). Appressoria and substomatal vesicles of type 1 contained 4 and 8 nuclei correspondingly, appressoria of type 2 had 4 nuclei, appressoria of type 3 contained 2 nuclei which moved later to lateral outgrowth. Thus, unfavourable conditions suppress mitosis, inhibit differentiation and fungal plant pathogen invasion. We suggested that triggering of nuclear division at appressorial stage by chemical and physical factors can control rust fungi invasion into leaf tissue of host plants.

Dep. Plant Protection, Main Botanical Garden, Russian Academy of Sciences, Botanicheskaya, 4, Noscow 127276, Russia

188

MACROAOLECULAR CHANGES OF CEREAL TRCTEITS ANI THEIR CCNECTICN WTH THE LEVELCERENT OF RESFCNSE REACTIC: WO FUNGAL INFECTION

Z.J.Serova, L.K.Ges, V.V.Karpuk, G.M.Fodchupharova

Listurbance of cooperative protein charecteristics (capacity for polymorphim, complementary ineraction, catelysis) in the pathogenesia course provide all the events, moving in contact of plant and fungous cella. The se events are shown through plural forms of winter rye reaction to the brown rust infection. They are: resistance, susceptibility, tolerance. The quentitative rediatribution of molecular forms in protein composition of diseased plants is detected. Content of slow- and middlemobile forms of protein increase on the first and second patogenesis stages (incubation period, etiolated spota) and decrease on the third one (sporulation). The level of slow-mobile proteins forma is connected with degree of protective reaction. In adition pathogenesis-related proteins (PR-proteins, so call resistance marker) in aumary protein preparation are show. The are several proteins with $\mathrm{MM} 25,28,40 \mathrm{kD}$ amang them. This proteins appear in 3 hours after infection then disappear in 15 hours and they are revealed in 19-24 hours later. The proteing spectrum changes again in 48 hours. PR-proteins functionate not only in rye cells, but inercellular liquid too. They regulate brown ruat hyphal development here. The marked changes in polymorphous function are accompanied by modificetion of complementary properties of interecting protein molecules of host and pathogen. According to the number of precipitation zones, the level of immunological affintty is low at the firat two diseage stages and on the complete stages is high. The deviation from norm in catalytic of protein fractions (oxidative-reductase, hydrolase, carboxylase, transferase and so on) changes ratio between the metabolic shunts. Thus host adaptive potentialties increase in the begining of pothogenesis and they decrease when it is over.

Institut of Experimental Botany Academy of Sciences, F Skorina at. , 27, Mingk 220072 Belarus 
EXTRACELIULAR ENZYMES OF NET BLOTH INCIMANT ANL THEIR ROIE IN BARLEY FATHOCENESIS

J.K.Ges. 2.J.Serova, G. R.Podchupharova

Enzymes wich excreted by Helmonthosporium teres Sacc. are major factor of pathogenecity. By means of them the fungus overcomes surface barriers of plant tissue, receive access to cell contenta and above all - Includes synthetic mechanisong of substances, reguired for nutrition. It was determined that intensity of enzym ecretion changes in dinanics of fungus development. At first pectolytic enzymesare isoleted. Their adtivity sherply increases in 2-3 dsya of H. teres growth then decresgeg and disappeara in 6 days, Excretion of proteolic enzymesby $H$, teres initiates in the 2-3 days too and continues duxing pathogenesis. The peaks of their activity appear in 4-5, 6-5, 11-12 and 17-19 days accordingly. Primery secretation of proteases and pectinases by fungas lead to hydrolygis of substances and as a consequence favour to pathogen intrugion into cells. Ag oistinct from pectinases wich are inactivated no sooner than cell cestruction happen. proteases functionate systematically during all the onthogenesiz of fungus. They correct content of constantly synthesized proteins wich can carry out the role of antigen factor as to pathogen. Secretion of nucleases into nutritetious environment occurs later on. The first peak of their activity comes in 5-6 days after what it disappear. If directlor of DNA-ase action doen't change in the following that RNA-gae activity incresse repestly. The aecond peak raises in $26-28$ says of sungus onthogenea1s. And this one is conslderably higher than the Pirst peek

Institut of Experimental Botany Academie of Science, F. Scortna street, 27. 220733, Minsk. Belarus

191 BIOELECTRUCAI PHENOMENA IN CHLOROPLAST AND CELL NUCLE] IN VIEW OF RESISTIVTY OF TRITICUM AESTIVUM TO PHYTOPATHOEENES

\section{A.Yamaleeva, R.Bashirova}

An effect of the Bisol immunostimulator has been studied upon the chloroplast and cell nuclei bioelectic activity among the wheats being either resistent or susceptible to phytopathogenic fungal infection - Tilletia caries, Fusarium gramenianum and Helminchosporium sativum. It has been found out that those wheats which display resistance to phytopathogenes are characterized by more intensive bioelectric processes. This fact is supported with higher and more stable values of redox-potential and electrophoretic mobility (EPM) of the chloroplasts and cell nuclei. The chloroplast EPM of the resistent wheaths ("Moskovskaya" and "Zarya" grades) is thigher than that of irresistent grades $\left(0.648\right.$ and $0.654 \cdot 10^{-8} \mathrm{~m}^{2} / \mathrm{Vs}$ correspondingly) The some applies to shoot nuclei of phytopathogene - resistant wheat. The Bisol introduction into the chloroplast suspension, as well preliminary treatment of wheat shoots reduce the chloroplast and cell nuclei EPM among the resistent wheats to a less degree than among the iresistent grades. In this case the higher level of bioelectrical processes is correlated to the intensity of biosynthetic processes: protein and nucleic acids syntheses.

Anna Yamoleeva, Frunse street 32, Ufa-74, 450074,Russia

\section{ANALYSIS OF MULTIPLE RESISTANCE IN THE PROTOPLAST FUSANTS OF VENTURIA INAEQUALIS}

\section{P.VIJAYAPALANI AND D. LALTHAKUMARI}

The present investigation was conducted to authenticate the development of multiple resistance in Venturia inaequalis by protoplast fusion technique. Stable in vitro mutants of $V$. inoequolis resistant to penconazole (ergostero! biosynthesis inhibitor) and carbendazim (benzimidazole) were developed. Protoplasts of these mutants were isolated and fused in the presence of polyethylene glycol (PEG). Progenies exhibited diverse phenotypes as parents, segregants and recombinants. Recombination frequency was $0.67 \%$. Recombinants exhibited multiple resistance and the stability of multiple resistance was evaluated. Stability of multiple resistance indicated the possibility of development of multiple resistance in in vivo. Biochemical aspects of resistance was analysed. Negative cross resistance was shown by multiple resistant strains for propiconazole, bitertanol and mancozeb.

\section{CENTRE FOR ADVANCED STUDIES IN BOTANY, UNIUERSITY OF MADRAS, GUNIDY CAMPUS, MADRAS - 600025 , TAMIL NADU, INDIA}

EFFECT OF POPULATION DENSITY ON SUSCEPTIBILITY OF GRASS MOTH Loxostege sticticais I (LEPIDOPTERA: PYRALIDAE) LARVAE TO INSECTICIDES

\section{O.V. Chankina, A.A.Alekseev, I.B.Knorr.}

Stress action of increased population density on physiological status of specimens is studied mainly on vertebrates. Such researches on insects are fragmentary and not numerous. All-round experiments to study of effect of density on population structures of meadow moth was carried out. In the course of these experiments the susceptibility of $3-4$ instar larvae to permethrin, $\alpha$-cypermethrin and methyl parathion has been evaluated. The meadow moth larvae were bred in two variants of densities: the low (I) and high density (II) (1 and 200 larvae/container respectively) that corresponds to optimum and extreme densities. The $\mathrm{LD}_{50}$ of all insecticides differed significantly and were: permethrin $-0.2 \pm$ 0.03 and $0.1 \pm 0.02$ ( $\pm \mathrm{Cl}, \mathrm{P}=0.05$ ); $\alpha$-cypermethrin $-0.034 \pm 0.004$ and $0.013 \pm 0.002$; methyl parathion $-4.5 \pm 0.6$ and $3.0 \pm 0.4 \mu \mathrm{g} / \mathrm{g}$ for I and II variants of densities respectively. Possibie mechanisms of these differences are discussed.

Institute of Chemical Kinetics and Combustion Russian Academy of Sciences, Institutskaya 3, Novosibirsk 630090, Russia. 
The Effect of Model Selection and Variability on the Evaluation of Pesticide Mixtures.

\section{Brutho d.A.}

Pesticide mixtures can be an important component of effective pest management programs and have been used to delay resistance development, to expand pest control spectrum and to combine desirable pest control atributes. The enhanced activity from synergy offers potential for reducing grower cos15, lowering use rates and enhancing commercial profitabilty and is thus a highly desirable trait to establish. Synergy associated with mixtures of fungicides with ditlerent modes of action has received considerable attention over the last decade. This report action has received considerable attention over the last decade. This report
examines the sensitivity of a particular measure of synergy, a synergy ratio, to examines the sensitivity of a particular measur
model selection and to experimental error.

model selection and to experimental error.
Two models, originally defined by Bliss (1939), have formed the basis for most evaluations of mixtures. The independent effects model is based on the assumption that the effect from each component of a mixture is expressed independently from the effects of the other mixture components. The similar action model is based on the assumption that the components of a mixture can substitute for each other at a constant rate. A synergy ratio based on ED90 estimates from the similar action model was proposed by Gisi, $\theta t$ al. (1985) as a quantitative measure of synergy and has been adopted in the plant pathology literature. A synergy ratio of 1.0 is expected for additive activity while values greater than 1.5 synergy ratio of 10 is expected tor additive activity while values greater than 1.5 show synergy and values less than 0.5 demonstrate antagonism. Two factors complicate the interpretation of this ratio. First, for mixtures of fungicides with differing modes of action, the method employs the similar action model when the independent effects model is the more likely null hypothesis. Second, synergy ratios have typically bean avaluated without an explicit consideration of experimental error.

Sensitivity analysis shows that the independent effects model and the similar action model can provide very different expectations for the efficacy of fungicide mixtures. This difference creates a bias in the estimate of synergy via the synergy ratio. Both the sign and magnitude of this bias is sensitive to the slope of the dosage response the sign and magnitude of this bias is sensitive to the slope of the dosage response
curve of the mixture components, the contribution of each component to mixture curve of the mixture components, the contribution of each component to mixture
efficacy and the level of pest control targeted for the mixture (e.g. ED90 vs ED50) efficacy and the level of pest control targeted for the mixture ( 8.9 . ED90 vs
Variability also atfects the estimation of synergy ratios. While any nile for interpreting synergy ratios is subject to the conditions of a particular experiment, it was found that synergy ratios based upon ED90 estimates from the similar action model must exceed at least $3.0-4.0$ before synergy is suggested. A less stringent rule applies when synergy is assessed with ED50 values. For mixtures containing fungicides with different modes of action an alternate approach, based on the independent effects model, for measuring the synergy of mixtures is proposed.

DuPont Agricutlural Products, Stine-Haskell Bldg 200, P.O. Box 30. Newark, DE, 19714, USA

THE POPULATION STRUCTURE CHANGE OF THE PERMETHRINRESISTANT STRAIN OF THE HOUSE FLY (MUSCa domestica L.) IN THE COURSE OF THE REVERSION OF RESISTANCE.

\section{T.Goriunova, A.Alekseev, O.Chankina}

The 1,3 and 5 generations of permethrin-resistant (R) strain of the house fly after the termination of permethrin $(\mathrm{Pm})$ selection were analyzed. $\mathrm{LD}_{50}$ of $\mathrm{Pm}$ were 25, 13, 4 and $2 \mu \mathrm{g} / \mathrm{g}$ for 3-4 day old fernales of the $1,3,5$ generations of $R$ and susceptible (S) strains respectively. The $R$ strain were selected from $S$ by permethrin with dose $L_{80}$ during 20 generations. The fate of topically applied [ $3 \mathrm{H}] \mathrm{Pm}$ (1.5 Ci/mmo) was analyzed. The high specific activity of [3H]permeth- rin makes it possible to analyze the individual flies. The quantity of radioactive material on a body surface, in excrement rinses and in an internal fractions of individual flies, as well as the excreted/penetrated radioactivity ratio ( $K_{\text {excr }}$ ) were evaluated in 1 and $8 \mathrm{hr}$ after application of $\left[{ }^{3} \mathrm{H}\right] \mathrm{Pm}$. The $\mathrm{K}_{\text {excr }}$ indirectly characterizes intensity of metabolism. The change of a population structure in the course of the reversion or resistance was studied using this parameter. The change of permethrin toxicokinetics was studied.

Institute of Chemical Kinetics \& Combustion Russian Academy of Sciences, Institutskaya 3, Novosibirsk 630090, Russia

\section{RESISTANCE OF LEAF ROLLERS TO INSECTICIDES AND WAYS OF THEIR OVERCOMING}

\section{L.A.Burkova}

Results of Jong-term experiments on studying sensitivity of Jeaf rollers complex to novel insecticides make it possible to conclude that long-term application of organophosphorous compounds to control codling moth and concomitant pests resulted in development of resistance to the most intensively used products. The level of resistance aquired vanes depending on the duration and intensity of the insecticide application and pest species-specificity. Among complex of leaf rollers, dominating in 3 regions of Russia, Pandemis heparava Den. and Schiff (18-72) is the most resistant to organophosphorous insecticides. Archips rosana L. and A.xylosteana L. (12-44) are less resistant. A.podana Sc. and Adoxophyes reticulana $\mathrm{Hb}$. (6-12) possess the lowest level of resistance to organophosphorous compounds. There exists a tendency of group and cross resistance development by leaf rollers, manifesting in dicrease of their sensitivity to novel compounds from organophosphorous and other chemical groups. To control resistant populations of leaf rollers and to prevent subsequent development of the resistance, schemes of insecticide altemations from different chemical groups have been developed and introduced into practice. The schemes are based on the following principles: - compounds are included in the scheme with the account of spectrum of their cross resistance and its supposed mechanism; compounds alternation during the season is determined not only by their toxicity to the controled pest bur by the level of their hazard for the beneficial arthropods; chemical treatment are conducted only at a economic damage threshold of major and concomitant pests. Weakening of organophosphorous toxicant dominating sole in alternation schemes and application of compounds possessing a quite different from organophosphorous compounds mode of action, favours the reversion of sensitivity of leaf roller resistant populations.

All-Russian Institute for Plant Ptotection, Shosse Podbelsky 3, St.Petersburg-Pushkin, 189620, Russia

196

THE TOXICINY OF SOME INSECTICIDES AGAINST KHAPRA BEBTLLE, TROGODERMA GRANARITM Everts.

B. J. Chekmenev, N.P.Kuzina, D. O. Andreev

In laboratory experiments carried out at $25 \pm 0,5^{\circ} \mathrm{C}$ and $90 \pm$ $50 \% \mathrm{RH}$ the toxicity of 10 ingecticides was determined sgainst 4-7 instar larvae of Trogodema granarium Everta. The organophosphorus Insecticides (Phoxim, Pirimiphos-metbyl, Malathion, Diazinon, Heptenophos) with 24-h LC50 values $0.00045,0.00072$, $0.0024,0.032$ and $0.030 \%$ respactively; the ICS5 values 0.0041 , $0.0024,0.0083,0.065$ and $0.18 \%$ respectively.The Pyrethrioid insecticides (Peovalerate, Lambda-Cyhalothrin, Del tamethrin. Permethrin and (ypermethrin) with 24-h LC50 values of 0.00096 , $0.0021,0.0089,0.023$ and $0.027 \%$ respectively; the LC95 values of $0.032,0.018,0.039,0.14$ and $0.33 \%$ respectively. The most toric insecticides were Organophosphorous Firimiphosmethyl and Phorlm and amongst Pyrethroids - Lambda-Cyhalothrin and Penvalerete.

117218, Moscon, Krahizanovaky str. 15 bld.2 Rusatan Academy of Agricultural seiences 
THE TOXICITY OF OROANOPHOSPHORUS AND PYRETHROID

INSECTICIDES AGAINST KHAPRA BEETLE, TROGODERMA GFANARIUM

\section{S.J.Chekmenev, N.P.Kusina, D.O.Andreev}

In laboratory experiments carried out at $25 \pm 0,5^{\circ} \mathrm{C}$ and $70 \pm 50 \%$ RH the toxicity of 10 insectlcldes was determined agelast $T \cdot V I$ Iatar larve of Trogoderna granarium Bverta. The Organophosphorus Insecticides (Phorim, Pirimiphoe-methyl, Malatbion, Diesinon, Meptenophos) with 24-h IC 50 values $0.00045,0,00072,0.0024,0.032$ and $0.030 \%$ respectively; the IC 95 values $0.0041,0.0024,0.0083,0.065$ and $0.18 \%$ respectively. The Pyrethroid Insecticides (Benvalerate, Lambda-Gyhalothrin, DeItamethrin, Permethrin and cypermethrin) wth 24-h LC 50 valuea of 0.00096 , $0.0021,0.0081,0.023$ and $0.027 \%$ respectively; the IC 95 values of $0.032,0.018,0.039,0.14$ and $0.33 \%$ respectively. Mongst the Organophoaphorus insecticides -Pirimiphos-methyl and Phoxim were the most toxic, amongat the Pyrethrolds - Fenvelerate and Lambde-Cyhalothrin weze the moat toxic.

117218, Moacow, kuss18, Krshtwanovaky otr. 15, bld.2 Russien Acedemy of Agricultural sclences SUSCEPTIBLE STRAIN OF TRIALEURODES VAPORARIORUM

\section{A. DE COCK \& D. DEGHEELE}

A resistant strain of the greenhouse whitefly, Trialeurodes vaporariorum, was collected in a commercial greenhouse after 24 treatments with buprofezin during four years. The susceptibility for buprofezin of the field collected and a susceptible laboratory strain was determined. The field collected strain had a resistance ratio of more than 159 in comparison with the laboratory strain ( $\mathrm{LC}_{50} 2.1 \mathrm{mg}$ (Al)/iter). Both strains were studied in relation to progeny formation. These assays indicated that rate of oviposition of the resistant strain, expressed as the number of eggs/female/day, much lower is than that of the susceptible strain $(0.33 \pm 0.04$ versus $3.61 \pm 0.42$, respectively).

The optimum conditions for trehalase and invertase, two key enzymes, were determined. For trehalase activity they involve a reaction mixture of $0.2 \mathrm{M}$ phosphate buffer (pH: 5.5) and $3 \%$ trehalose at $40^{\circ} \mathrm{C}$ for 60 minutes, for invertase activity: $0.2 \mathrm{M}$ phosphate buffer (pH:5.5) and $5 \%$ sucrose at $37^{\circ} \mathrm{C}$ for 50 minutes. At optimal conditions both invertase and trehalase activities were higher in the resistant strain.

The resistant strain exhibited a $16 \%$ lower level of tolal protein compared with the susceptible strain. This can be a possible explanation for the lower fecundity of this strain.

Laboratory of Agrozoology, Faculty of Agricultural and Applied Biological Sciences, University of Gent, Coupure Links 653, B-9000 Gent, Belgium
SURVEY OF AZINPHOSMETIL AND ESFENVALERATE RESISTANCE IN CODLING MOTH (LEPIDOPTERA: TORTRICIDAE) IN RIO NEGRO. ARGENTINA.

L. Cichón, D. Fernandez Azinphosmetil has been used for more than 20 years and
piretroides for more than 10 years to control codling moth in the Piretroides for more than 10 years to control codling

fruit region of Alto valle de Rio Negro $y$ Neuquen. It was speculate that the increase of damage during the past 5 years was produced because of resistance to any of these insectlcides. In 1994, an extenalve survey of 18 archards spreaded along the valiey was undertaken with a dscriminating concentration $75 \mu \mathrm{g}$ (AI) of azinphosmethy $I / g$ adhesive and 28.6 $\mu g$ (AI) of esfenvalerate/g adhesive in each one orchard. Moth mortality uas scorsd after $64 \mathrm{hs}$. at $20^{\circ} \mathrm{C}$ and above $85 \mathrm{~s} \mathrm{R} . \mathrm{H}$. These orchards had a severe control problems and most of the used 3 to 4 application of plretroldes and 1 to 4 applications of axinphosmethyl during several season. Fifteen of the 18 populations monitored had significant levels of resistance compared ith a susceptible laboratory strain based on nonoverlapping $958 \mathrm{CL}$. Only one orchard didn't show levels of resistance to Esfenvalerate. Bleven of the 18 orchards had corrected mortality below 40 and 6 orchards between 40 to 547 . Fifteen Qrchards had significant levels of resistance to the insecticides studied. These results demands an urgant change in the strategies of control looking for the alternatives to minimize the resistance problems.

INTA EEA Alto valle. CC 782, (8332) Gral. Roca. RIO NEGRO. ARGENTINA.

200

DIMETHOMORPH RESISTANT STRAINS OF PHYTOPHTHORA INPESTANS

An Jian Li, Bagirova S.F., Derevygina M., Dolgova A.V., Dyakov Yu.T.

Dimethomorph is a fungicide wich belongs to the morpholins group and characterised as being toxic to oomycetes, having slight systemic activity and an absence of cross-resistance to phenylamides which belongs to the morpholins group. Artemps to isolate resistant to dimethomorph clones from natural populations of P. cactorum and plasmopara viticola or to get resistant mutants were unsuccessful ( Blankenagel et al. 1990). Using the quantitative method (Dyakov et al.1988) we had obtained resistant clones of phytophthora infestans from potato eropps with fungiciales treated (frequency $2-5$ \%) and from laboratory collections of strains. The lethal dosage of dimethomorph for resistant strains reached $40 \mathrm{~m} k \mathrm{k} / \mathrm{ml}$ (for susceptible ones -i mkg' ml) However resistant strains had low fitness and died after several passages on agar medium. Resistant mutants of $P$.infestans were obtained with the help of $N$-nitrosomethylurea (frequancy $5-7$ $x 10^{7}$, in a dose which caused 10 survival. The lethal dosage of dimethomorph. For initial strain was $2 \mathrm{mkg} / \mathrm{ml}$, for mutants Dm1B and $\mathrm{Dm} 3 \mathrm{~B}-4 \mathrm{mkg} / \mathrm{ml}$. and $\mathrm{Dm} 2 \mathrm{~B}-6 \mathrm{mkg} / \mathrm{ml}$. Mutants DmlB and Dm3B serve as initial ones to obtain mutants which survived on a medium with $8 \mathrm{mkg} / \mathrm{ml}$ of dimethomorph. Both the speed of growth on an artificial medium and the pathogenicity to potato leaves of initial strain and mutants were similar. Bfficiency of cloning on tomato leaves for mutants was increased by about 3 times in dimethomorph and reduced by about 4 times in control (H2O) in comparison with the initial strain. So, resistant to dimethomorph. can change in result of step mutations and also nighresistant strains have low fitness. Hence dimethomorph belongs to the fungicides that have a moderate risk of resistance. The experiments were carried out with partial financial support from the fund of RAS, programan "Frontier of genetics".

Dep. Mycol, and Algol., Moscow University, Moscow 119899, Russia 
QUANTITATIVE METHOD OF MONITORING FOR FUNGICIDE REBISTANCE

Derevjagina M.K., Dolgova A.V., Dyakov Y.T.

The widely described failure of many systemic fungicides has led to the need for monitoring of resistance in pathogenic fungi. The purposes of monitoring: 1 .Assessing resistance risk during introduction of a new fungicide prediction of fungus population behavior after the wide spread of the product. $2 .^{\prime}$ Choosing the most effectivel fungicide, or a mixture of such, before the application. 3. Analysis of previous fungicide failures. 4. Assessing comparative efficlency of antiresistant strateegies. The most of present methods may be used for solving the $3-d$ and $4-$ th purposes but not good enough for the 1-st and 2-nd ones. The method having no disadvantages of standart techniques is described below. It may be used for any one of the above-stated purposes of monitoring.It is based on methodology of selective media, which is an important tool of microorganisms genetics. Potato leaves of variety without $\mathrm{R}$-genes with low horizontal resistance were placed on moist chambers and sprayed with water (control) or a fungicide.leaves with P. infestans sporulations are collected at random in the field and washed in a flask with delonised water. 8-10 hours after fungicide application the leaves in cameras were sprayed with cystospore suspension and covered with glass. In 2-4 days each leaf was searched for light-transparent spots and the number of lesions (without sporulation) countered. During the period of sporulation some lesion coalesce and in this case method can not be considered as a quantitative one. The ration of a number spots in control chanbera to number spores in inoculum shows on the main aggressiveness of population; the ration of a number spots in test chambera to number spots in control - on the percent of resistant clones. In the poster will be give the examples of uses this method for different purposes.

Dep.Mycol \& Algol. Moscow State Univ. Moscow,119899 Russia

203

\section{COMPARISON OF ESTERASE ACTIVITY IN VARIOUS KINDS OF THRIPS}

C. Y. Hsin

Thrips becocme an economic pest on many kinds of crops in Taiwan. Several organophosphate insecticides failed to control them. Changes in esterases was considered as a cause of insecticide resistance. Esterase activities identified by $\alpha$ naphthyl acetate, $\alpha$-naphthyl butyrate, $\beta$-naphthyl acetate and p-nitrophenyl acetate were different among grapevine thrips, flower thrips and cuban laurel thrips. The esterase activity with $\alpha$-naphthyl acetate as substrate was bighest in grapevine thrips, followed by flower thrips and lowest in cuban laurel thrips. Decreased levels of esterase activity appeared to be corrrelated with the decreased frequency of pesticide exposure of the thrips. Five and three molecular forms of carborylesterase were observed by using native polyacrylamide gel electrophoresis and isoelectric focusing electrophoresis respectively in grapevine thrips. The relationship of the most active one to the insecticide resistance in the grapevine thrips will be discussed.

Department of Plant Protection, National Pingtung Polytechnic Institute, Nei Pu, Pingtung, 91207, Taiwan, Republic of China

NEED OF FUNGICIDE RESISTANCE STUDY IN PLANT PATHOGENS FOR SUSTAINABI.E PESTICIDE INDUSTRY

L.V. Gangawane

Soil Microbiology and Pesticides Laboratory,

Department of Botany,

Dr. Babasaheb Ambedkar Marathwada University,

Dr, Babasaheb Ambedkar Mara
Aurangabad 431004 . MS, India

In recent years application of pesticides has plagued with several problems among which development of resistance in the pathogenic organisms is most important. Many examples of resistance of fungicide
in pathogens have been noted in developed and developing countries like USA, Japan, Australla, European and also south East Asian like USA, Japan, Australla, European and also south East Asian countries. In India fungicide resistance has been reported in some of the pathogens like Aspergillus flavus, Macrophomina phaseolina,

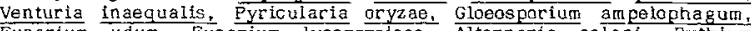
Fusarium udum. Fusarium lycopersicae, Alternaria solani, Pythium aphanidermatum enypophthora nicotianae, phytophtheradrechsleri f.sp. cajani, phytophthora infestans etc. against captafol, captan, carbendazin, carboxin, maneb, thiophanate methyl, kocide, metalaxyl ect. However, most of the reports are laboratory reports and resistant factor is based upon the base line sensitivity (MIC or $\mathrm{ED}_{50}$ ) of the wild sensitive isolate. Therefore, informtion regarding $1 .{ }^{50}$ pathogen life cycle, 2. survey from different fields, 3. development through mutagens or passage, 4. virulence of . resistant strain, 5 . cross resistance, 6 . fitness, 7 . compitition with other saprophytes, 8. management of resistance with other agrochemicals- alternate or in mixture gained in this laboratory against many of the pathogens suggested above will certainly be helpful in the immidiate implimentation policy for the management of resistant pathogens at the field if ineffectivenass of the fugicide is really reported. The data thus prepared in advance for the pathogen and fungicide will increase the sustainability of the product in the market and in the field.

Prof. L.V.Gangawane,Soil Microbiology and Pesticides Laboratory Department of Botany Dr, Babasaheb Ambedkar Marathwada University, Aurangabad 431004, MS, Indla.

204

IRSECTICIDE RESISTARCE IN DJAMONDBACR WOTH, Plntella yylostella (L.) ARD ITS MARAGBIBHT IN PUKJAB, INDIA

\section{B.S. Joia and R.P.Chaw $1 \mathrm{a}$}

Among the important insect pests of cole crops in punjab, India, diamondback moth (DBM) has become a major constraint for the production of quality vegetable heads due to emergence of insecticjde resistance. Monitoring of susceptibility status of the pest since 3970 s has established the development of resistance against all major groups of insectfcldes evolved along thejr use pattern. Currently as high as $70 x, 2880 x$ and $2700 x$ resistance has been recorded against commonly used insecticides - quinalphos, cypermethrin and fenvalerate, respectively jo Punjah. The emergence of resistance in the pest has escajated the treadmill of increased use and disuse of insecticldes at the cultivators' level. For want of susceptible straln, the understanding of the biochemical and genetic mechanisms of resistance is seriously handicapped.

Practical strategies for the insecticide resistance management against $D B M$ are being developed utilising the field observations that the insect is absent as pest during mid May to mid august, the sustained control of the pest achieveable with common insecticldes where natural enemy, Apanteles is still active and only low level of resistance against endosulfan, coupled with laboratory findings on cross-resistance spectrum that novel insectlcides - cartap, diafenthiuron and $\underline{B} \cdot \underline{t}$. show considerable promise agafnst DBM.

Department of Entomology, Punjab Agricultural University, Ludhyana-I4I 004 , India 


\section{BIOLOGICAL AND NEUROTOXIC JOINT ACTION OF} SEVERAL KINDS OF INSECTICIDE COMBINATIONS

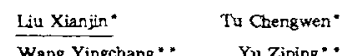

Activities of 13 kinds of two-way insecticide combinations on the Asian corborer (Ostrivia furmacalis), the rice brown planthopper (Nilaparuse bugens), and the rice stem borer (Chilo sappreodis) were tested in the laboratory and field experiments. Significant sinergistic joint action on the biological activties was found in 1 mixtures, summation joint action in 5 mixtures, and antagonistic in 4 . Combined analysis on the $\mathrm{co}-$ toxic coefficient values based on $L C_{50}$ and $L C_{s 0}$ was proved useful for the assessment of the biological joint action, the comparision of the $\mathrm{LD}-\mathrm{P}$ lines tested and theoretical would discribe the changes of the activity all - dosagely, and be urifying criteria of synergistic, summation and antagonistic joint action of the bio-assay and the field tests were disocussed.

The excitatory junction potentials (EJPs) of the drasophila larval neuromuscullar junction recorded by using enterceliular microelectrode showed the there are addition effects between the toxicological action of methothrin and deltamethrin during their interaction with the peripleral nervous system. Similar effects were also found in the neurotoxic joint action of endosulfan - deltamethrin of methamidophos-deltamethrin on the central nervous system of the American cocktoach (Periplenta creriona) by using spontaneous excitory postsynaptic potentials (SEPSPS) extracellular recording method, but antagonistic action in the methamidophos and imidacloprid joint treatment. These phenomers may suppose that neurotoxic joint action of the insecticide combinations is one of the most important mechanisms of the biological joint activity.

* : Inst. of Plant Protection, Jjangsu Acad. of Agric. Sci. Nanjing 210014, P. R. CHINA

* * Dept. of Plant Protection, Nanjing Agricultural University Nanjing 210095 , P. R. CHINA

207

THIRTY YEARS OF COLORADO POTATO BEETLE RESISTANCE MANAGEMENT IN CROATLA

\section{Maceliski}

The Colorado pocato beetle / $\mathrm{CPB} /$ is present in Croatia from 1946. We proved that the resistance to DDT and lindane started in the year 1965., to kelevan in 1972., to carbaryl about 1973, to OP and other OC insecticides in $1986-1987$, and to pyrethroids in 1988 . The resistance to chlorinated hydrocartons occured after 16 years, to kelevan after 4 years, to $O P$ insecticides after approx. 16 years and to pyretinroides after approx. 7 years of theis first larger use.

During the period 1986 - 1990., 42, and in the period $1992-1994$., 36 populations of different proveniences were tested. In the first period our adapted method very similar to IRAC method $\mathrm{N}^{\circ} 7$, and in the second period a simplified IRAC method $N^{0} 7$ was used.

At the end of the first mentioned period the resistance to $O P, O C$ and pyrethroids was developed on about $5 \%$ od potato fields in northern Croatia. At the end of the period 1992 - 1994., the resistance to OP and OC insecticides appeared on approx. $70 \%$ and to pyrethroids on $30 \%$ of potato fields in this region. The appearance of the resistance to $O P$ and $O C$ insecticides has a large blotch pattem, and to pyrethroids a small spot pattem.

It should be mentioned tiw no resistance was registered in southern Croatia Istria and Dalmatia/.

In regions where resistance appeared, the use of formulations based on bensultap, tiociklam, hexaflumuron, teflubenzuron and B.t.t., all approved in Croatia, are recomrnended. Comblnations of IGR-s and 8.t. with lower or sublethal dosages of $O P$ or pyrethroids were used in some trials in order to improve the initial action and the efficacy to adults.

Prof. dr. Milan Maceljski, Faculty of Agriculture, Institute for Agricultural Zoology, Svetoßimunska 25, 41.000 Zagreb, Croatia.
RESISTANCE OF ERYSIPHE GRAINIS F, SP. TRICITI TO TRIADIMEFON

Ma, Zhiqiaeg Liu, Guorong Zhang, Xiaofeeg

Minter whert is the second latgest ex op with sn sran of sbout it million ba. in China. Pondery tiden is one of the wain diacases on the crop. Triatimefon has been applyed since 1980 and still leep a good performace. By oo there is no other fungicide that can ingtead of it. Since the tesistance of B.grapiais to triazoles wa reported in Europe, it is necessary to make sura whether the resistance has occured in China. Four woditoring wethods have beea compased in greenhouse. Entire plant method is more seasitive than tast-tube and bait plast methods. But the detacked loaf-tip wothod can be used to test aumerious samples. Meanhile, the baseline sensitivity of the wild strain to triadimefon bas been established: $Y=-0.6309+2.2411 \times$. By teating of different methods in 1993, the existance of peaisteat-8traio in the mildew population was firatis obtriged in Fusban. Shadong propiace. 213 single pustules of milden samples, collected from Shandong prorince, were tested with detached leaf-tip wethod duriag 1992-1993. The resulta ghowed that the resiatance lerel of pathogen to triadimefor is lower. The MIC values azo ranged from 1 to 7 times of basalino. A mixture of triazole and worgholiae has been made. The field exporemeat showed that it bas a sieriat effect th thadimefor. Since the rather condition is very dry in racant geara (1991-1991), powdery wildew is lighter. Se we bare no chance to make bure the mixture' a action in fesictance management.

Institute of Plant Protettion

Hebei Academy of Agricultural 2 Forestes

Sciences

73 East Pass Street

Bading, Hebei 071000

P. R. Chins
208

EVALUATION OF BIOASSAY METHODS FOR DETECTING RESISTANCE IN COCOA MIRL, HELOPELTIS THEIVORA TO INSECTICIDES AND THEIR IMPLICATION IN RESISTANCE MANAGEMENT

Muhamad, R. and Dzolkhifli, O.

Three bioassay methods were compared to detect resistance in three populations of cocoa mirid, Helopeltis theivora (Waterhouse) (Hemiptera: Miridae) to $\gamma$ hexachlorocyclohexane $(\mathrm{HCH})$, cypermethrin and deltamethrin. The methods were time-response on treated filter paper, residual on filter paper and topical application. The populations were collected from Serdang, Kelang and Sungai Tekam within the Peninsular Malaysia and cultured in the laboratory. The first generation of fourth and fifth nymphal instar were used in all tests. The median knockdown time $\left(\mathrm{KT}_{50}\right)$, median lethal concentration $\left(\mathrm{LC}_{50}\right)$ and median lethal dose $\left(\mathrm{LD}_{50}\right)$ was obtained for time-response, residual and topical application bioassay respectively. The resistance factors (RF) were calculated by dividing $\mathrm{KT}_{50}, \mathrm{LC}_{50}$ and $\mathrm{LD}_{50}$ of the least with the most susceptible population. When the Serdang population was assumed as the susceptible population to $\gamma-\mathrm{HCH}$, the Sungai Tekam population showed a RF of 6.4 using topical application bioassay, while the time-response and residual bioassay showed RF of 1.7 and 1.9 respectively. For cypermethrin, assuming Kelang population was the susceptible population, the Serdang population showed a resistance factor of 7.0 by topical application in contrast to the time-response and residual bioassay that gave the RF of 2.8 and 2.5 respectively. Similar results were also obtained for deltamethrin when Sungai Tekam was assumed as the susceptible strain; the Serdang population gave a RF of 5.0 by topical application while the time-response and residual bioassay gave RF of 1.0 and 1.5 respectively. These results showed that the topical application method was more sensitive for detecting resistance in the cocoa mirid to $\gamma-\mathrm{HCH}$, cypermethrin and deltamethrin compared to the other methods. Nevertheless, the time-response method is the Food Agriculture Organisation (FAO) recommended method for measurement of the cocoa mirid resistance to insecticides. The FAO method is rapid and simple and it uses fewer insects. From these studies, the $R F$ obtained from the time-response method should be corrected by multiplying with a constant that varies according to the chemical used to provide a more accurate status of the cocoa mirid resistance to insecticides in the field.

Department of Plant Protection, Faculty of Agriculture

Universit: Pertanian Malaysia,

43400 Serdang, Selangor,

Malaysia 
EFFECT OF CONTINUOUS TREATMENT WITH BICARBONATE FORMULATIONS ON POWDERY MILDEW ON CUCUMBER LEAVES

\section{H. Murakami, Y. Homma and H.Negishi}

It is necessary for use of fungicides when we take the management for cultivation of horticultural plants. However, a decline of fungicidal effect for fungicide resistant pathogens is world-wide problem since 1970's. This time, bicarbonate formulations, Kaligreen ${ }^{80}$ (potassium bicarbonate formulation) and Harmomate (sodium bicarbonate formulation) which have inhibitory effect for various kinds of vegitable powdery mildows, cucumber, strawberry, eggplant and so on, was used for series of experiments. It was tested whether Sphaerotheca fuliginea get fungicide resistance by 10 times treatment of the bicarbonate formulations or not. $S$. fuliginea was inoculated on the leaf of cucumber true leaves (Variety : Sagamihanjiro 0.7 leaf stage) and bicarbonate formulations (each of 1000 times solution) were sprayed $(50 \mathrm{ml} / \mathrm{pot})$ onto cucumber leaves about seven days after inoculation. Three days after treatment, we counted collapsed lesions or not about all disease lesions. Several days after the count, $S$. fuliginea conidia regenerated at disease spot were inoculated onto true leaves of cucumber seedlings. This process is one time, the process was repeated for 10 times. As a result of these experiments, curative effect of bicarbonate formulation from first to tenth times treatment was always about $95 \%$. Therefore, we could not observe the bicarbonate resistant strains of $S$. fuliginea by our experiments.

Tokyo University of Agriculture,1-1-1,Sakuragaoka,Setagaya-ku,Tokyo, Japan 156

211

TOXICITY AMD RESISTAKCE RATIO OF CRRTAIN INSECTICIDRS TO DTAMONDBACK MOTH, Plutella xylostella (L.)

Raju,S.Y.S, and Choudhary, H.K.

Diamondback moth (DBH), Plutelia xylostella (L.), is the nost destructive norldride pest of crucifer vegetable crops. Extensive and indiecriningte use of insecticides and lack of pronisive alternate control nethods oreated a ghastly scenario of developnent of resistance to every synthetic insecticide used against this pest in the field across the world. Reeping these facts invien, pest resigtance studies were carriedout by exposing firat generation second instar DBH larvae, whose parental population were collected fron two different cabbage and cauliflower oxtensively growing areas of North-Eastern parts of India and nawed as strain-H and strain-S. The larvae were exposed to some conmonly available and used insecticides viz., endosulfan, quinalphos, cypermethrin, fenvalorate, cartap hydrochloride and neen oil extract. A naximum mortality of 95 and cent per cent was recorded after $72 \mathrm{~h}$ of exposure to reconmended field concentration of fenvalerate in strain-M and strain-S population, respectively. Cypernethrin and ondosulfan were observed to be maderately toxic to strain-H population. All these insecticides showed significant degreo of variation anong thenselves in regpect of toxicity to both the strains of DBH and together differed fron the per cent nortality recorded with nornal field dose of neem oil extract treatments, where on $1 y$ 45 and 50 per cont mortality was recorded aftar $72 \mathrm{~h}$ with strain-K and strain-s, respectivoly. Probit analysis of por oent nortality data obtained after $48 \mathrm{~h}$ of biossay tests clearly shown significantly high LC $50 / L_{50}$ values indicating degres of tolerance to all these insecticides by both the strains. Resistance ratios when conputed exhibited 42.68 and 25.10 fold resistance to cypermethrin and fonvalerate by strain-H, respectively. Nearly 20 fold resistance to these pyrethroids was also observed with strain-S population. High lovels of tolerance to cartap hydrochloride and guinalphos in strain-H and endosulfan in strain-s population were detected.

Department of Entonology \& Agril.zoology, Institute of Agricultural Soience, Banaras Hindu University, Varanasi221 005, INDIA.
ROLE OF ESTERASE AND GLUTATHIONE S-TRANSFERASE IN COTTON APHID (HOMOPTERA:APHIDIDAE) SUSCEPTIBILITY TO TWO INSECTICIDES

E. O. Owusu, C-S. Kim, M. Horiike, C. Hirano

Relationship between susceptibilities to dichlorvos (organophosphate) and permethrin (pyrethroid) insecticides, and activities of two detoxification enzymes (esterase and glutathione S-transferase) were assessed in vitro in clones of cotton aphid, Aphis gossypii.

Carboxylesterase activity (1-naphthyl acetate) was found to be positively correlated to dichlorvos susceptibility $\left(\mathrm{r}^{2}=0.97 ; P=0.001\right)$, but not to permethrin susceptibility. Cholinesterase (acetylthiocholinesterase and butyrylthiocholinesterase) activity was however found to be unrelated to susceptibility to both insecticides. Qualitative assessment on polyacrylamide gel further revealed that carboxylesterases E-7, E-9 and E-10 are the principal isozymes involved in dichlorvos resistance.

Furthermore, the existing differences in effects of temperature, $\mathrm{pH}$, and substrate (1-chloro-2,4-dinitrobenzene (CDNB)) concentration on activities of glutathione S-transferase from dichlorvos resistant E-D-R and susceptible E-S strains of cotton aphid were evaluated. Both strains had an optimum temperature of $30^{\circ} \mathrm{C}$. While activity of the $\mathrm{E}-\mathrm{S}$ strain peaked at 7,2, that of the E-D-R strain showed complete linear dependency up to $\mathrm{pH}$ 8.0. Both strains however, depicted a common Michaelis constant $\left(K_{m}\right)$ value of $0.69 \mathrm{mM}$. On the whole, the E-D-R strain exhibited enormous potential to produce higher enzyme activity in the presence of dichlorvos while the E-S strain did not. Conclusions drawn from the results indicate dichlorvos resistance in cotton aphid is due to production of altered forms of carboxylesterases E-7, E-9 and E10 , as well as emergency production of CDNB linked glutathione S-transferase as complimentary protective enzymes to confer resistance in close conjunction with other pre-existing mechanisms. On the contrary, permethrin resistance is likely to be expressed through other mechanisms rather than those outlined above. True resistance in this aphid is likely to be the product of multiple mechanisms rather than cross resistance from a single molecular mechanism. However, involvement of cytochrome P-450 is ruled out.

Kochi University, Faculty of Agriculture, B200 Monobe, Nankoku-shi, Kochi 783, Japan

212

COUNTER MEASURE TO CHECX BITERTANOL AND FENAPANIL RESIGTANCE IN VENTURTA TNAEQUALTS

R. Reyathi and D. Lalithakumari

Potent in vitro mutants of Venturia inaequalis resistant to bitertanol and fenapanil were developed by EMs mutagenesis and adaptation techniques. Bitertanol mutants showed very high resistance (nearly 500 folds) than fenapanil-mutants (nearly 6 folds) on fungicide amended potato dextrose yeast extract agar medium. To overcome this fungicide resistance, cross resistance work was carried out using twelve different funglcides of diverse groups. The $Q$ values indicated that fytolan, dithane $\mathrm{M}-45$ and penconazole were negatively correlated fungicides. These three fungicides were effective in controlling all the mutants. These chemicals can be used as alternate fungicides in the regular spray schedule for apple scab to control or delay the development of bitertanol and fenapanil resistance under field, conditions.

R. Revathi, Centre for Advanced Studies in Botany, University of Madras, Guindy Campus, Madras 600025 , India. 
RESISTANCE OF TETRANYCHUS URTICAE KOCH (TETRANYCHIDAE) TO BISCLOFENTEZIN

\section{T. Stamenkovi,c, S. Stamenkovj.ć, D. Marčić}

Investigations were carried out in the "Dedinje" glasshouse for flower growing (carnation, rose, etc.) in Belgrade.

Over the 1984-1989 period the population of Tetranychus urticae was high and it considerably endangered flower production in almost all glasshouses in Serbia, Over the above-mentioned period two treatments per year were applied (Bisclofentezin combined with (ihexatin), providing successful flower protection against acarinae. Bisclofentezin was therefore applied until 1992. However, since 1988 the effect of Bisclofentezin has been observed to be constderably lower, especially in the last few years. The objective of this paper was to determine the resistance degree of $T$. urticae after Bjsclofentezin has been applied for ten years.

The investigations were $\mathrm{c}$ arried out in $\mathrm{lab}$ conditions at $22^{\circ} \mathrm{C}$ and $\mathrm{RH}$ 55-658. Leaf discs of bean plant were used. Bisclofentezin (Apollo $50 \mathrm{EC}$ ) was applied in five concentrations $(0.0005 \%$, $0.0018,0.0058,0.0258,0.058$ a.s.).

Experiments were carried out in four repetitions, with 300 eggs per each repetition. The eggs were 24,48 and 72 hours old. Leaf dip method was used. The dipping of leaf discs lasted 10 seconds.

The results obtained with two concentrations $(0.00058$ and 0.05 t a.s.) indicate that mortalj.ty for 24 hour-old eggs ranged from 41.48 - 65.68, whereas the mortality range for 48 hour-old eggs was $37.58-58,38$. Mortality for 72 hour-old eggs ranged from $20.68-41,5 \%$.

The results obtained in this study show high degree of resistance in the studied population after lo years of permanent: treatments with Bisclofentezin. It is ncessary to.use some other acaricides instead of Bisclofentezin in the glasshouses where Bisclofentezin has been permanently applied for many years.

215

\section{ACTIVE OXYGEN AS THE MARKER OF INHERITABLE OR INDUCED RICE RESISTANCE TO BLAST}

\section{Aver'yanov A.A., Lapikova V.P., Kovalenko E.D.}

Active oxygen (AO) species (superoxide $\mathrm{O}_{2}^{-}$and hydroxyl $\cdot \mathrm{OH}$ free radicals, and hydrogen peroxide) are produced apparently in any aerobic cell. They are recognized now to mediate plant antiinfective responses. In this work we estimated the $\mathrm{AO}$ formation by rice leaves in relation to blast disease. Twenty two cultivars differing in their vertical resistance were tested. The drop diffusates collected from infected leaves during 3 days after inoculation inhibited spore germination of the causative fungus Magnaporthe grisea. The effect was significantly more pronounced with resistant cultivars than with susceptible ones. The addition of catalase, superoxide dismutase (SOD), or hydroxyl scavengers to diffusates diminished strongly the antifungal action, which, therefore, was $\mathrm{H}_{2} \mathrm{O}_{2}$ and $\mathrm{O}_{2}-$-dependent. The toxic property was rather unstable, possibly due to exhausting of $\mathrm{AO}$ precursors. The superoxide generation, more intense in resistant cultivars, was confirmed by chemical assay (SOD-sensitive epinephrine sxidation) in leaf diffusates. The similar AO-mediated high fungitoxicity of diffusates and chemically detected rise in the superoxide production were observed in plants of the susceptible cultivar with acquired disease resistance. This was found after of heating of plants or application of non-toxic fungicides (tricyclazole, fthalide, probenazole) or that of other blastcontrolling preparations (metaloorganic complexes and riboflavin derivatives). It is likely that the $A O$ formation on leaf surface being the anti-infectional factor is indicative of, at least, some kinds of disease resistance.

Research Institute of Phytopathology, p/o B.Vyazemy, Moscow region, 143050 Russia
THE PROBLEM OF COTTON PEST RESISTANCE TO PESTICIDES IN THE COMONWEALTH OF INDEPENDENT STATES (CIS)

Galina I. Sukhoruchenko

Pesticide resistance is a limiting factor for effective chemical control of cotton pests in the CIS (formerly USSR). In the past 30 years (1964-1994), the resistance to widespread chemical compounds was documented in populations of 7 arthropod species: spider mite Tethranychus urticae Koch., aphids Aphis gossypii Glov. and Acyrthosiphon gossypii Mordv., whiteflies Trialeurodes waporariorum Westw. and Bemisia tabaci Genn., plant bug Lygus pratensis $\mathrm{L}$., bollworm Heliothis armigera Hbn. The importance of resistance is closely connected with the pesticide situation in different regions of cotton cultivation. Thus, the resistance of mites to organophosphates was registered in the early sixties in Fadjikistan and Uzbekistan but only in the late eighties it was documented in Azerbaijan. The crossresistance between organophosphates and pyrethroids was formed in the population of whiteflies in Turkmenistan and in the population of plant bug in Tadjikistan. The development of multiresistance to cyclodiens, organophosphates and pyrethroids is observed in the populations of bollworms from Tadjikistan and Azerbaijan. For solving the problem, it is necessary to reduce the volumes of usage of organophosphates and pyrethroids dominating last years in the systems of control of cotton pests. The strategy of resistance management aimed at diminishing of pesticide load on fields is based on the monitoring of its levels in the populations of key pests, utilization of pesticides according the economic thresholds, seasonal rotation of pesticides with different modes of action including the insect growth regulators, use of microbiological products and activity of the natural and released beneficial arthropods.

All-Russian Institute of Plant Protection, 3 Podbelsky Shosse, St.Petersburg - Pushkin, 189620 Russia.

216

SIMULATION MODELING WITH INFORMATION USING OF BIOCHEMICAL PARAMETERS OF THE ALFALFA'S PROTECTION SYSTEM PRESENTED WITH PHENOL-CARBONIC ACIOS AND AMINOACIOS

Korol T.S., Smelyanets V.P.

Studies of the biochemical factors and their protective functions have shown that the immune system is multicomponent and consists of parameters with different functional applicance.

With the help of complex of the biochemical parameters (phenol-carbonic acids. aminoacids, sugars, saponins, proteins-inhibitors and other) it has been formed the simulative model of protective system to the pests on the basis of the images" theory.

The complicated terminal image of the protective system of resistant and The complicated terminal image of the protective system of resistant and
unresistant samples consists of parameters of three levels of singnificance nucleus situations, background components of the 1 st and 2 nd orders.

The nucleus situations are determined on the base of the computer experiments with functional simulative models of protective systems being different by the resistance degree of plants. They are an enumeration of casual-consequent hypothesises. It has been shown the mechanisms of the immunological reactions go in the presence of the background components of the 1st order that presented by the products of secondary metabolism and also on the background of the components of the 2 nd order being represented by nutriments.

Comparison of the imiges of the protective systems with different resistance level gives a possibility to determine that the samples have the analogical structures but are different in quantitative and qualitative composition of the components. It are different in quantitative and qualitat
determines the different defensive level.

Ukraine 252022 Kiev-22, Vasilkovskaya, 33, Plant Protection Institute 
BAM H1 RDSTRICTION FRAGMENTS OF POTATO CHLOROPLAST DNA AS POSS IBLE MOLECULAR MARKERS OF VIRUS RESISTANCE

\section{A.N.Palilova, V.r.Pyko}

Chloroplast DNA Bam H1, PVU1,, Hind111, EcoB1 restriction spectra of more than 30 potato samples differing in the type of virus resistance were studied. Among them were wild species - donorg of this character, species immuse to $x$ and $I$ viruses, resiatant and susceptible potato cultivated varietias and denfisotis (hybrias bearing s.demissum cytoplesin and $S . t u b e r o s u b$ genome). The most pronounced and stable distinctiong between resistant and susceptible gamples were sbserved under the action of Baml restrictase in 9-20 k.b.p. zone. Spocific features of thls zone of spectna for donors of immolty and extra-susceptibility were revealed. Resistant and ouscegtible cultivars of this zone differed in fragment number and molecular weight thus proving the idea of posaible use their as molecular markers of virus resistance.

Institute of Genetics and Cytology, Acadery of Saiences of Belarus, F.Skorina st.27, Hingk 220072, Republic of Belarus.

219

EFFECT OF HERBICIDES ON SOIL-BORNE ROOT DISEASES DF CEREALS

J. Aitman. Department of Plant Pathology and Weed Science, Colorado - State University, Fort Collins, Colorado 80523

Although pesticides are applied to plants and soils to control plant parasites and weeds, they also affect soil properties, microorganisms and hosts. Uneven herbicide distribution, coupled with the various effects of a herbicide on the metabolic activities and overall growth of a host and/or a pathogen, can account, in part, for an increase in soil-borne disease resulting in greater pathogen damage from Fusarium, Rhizoctonia, Gaemannomyces or Pyth ium as wel 1 as mematodes including the Cereal Cyst Nematode. Herbicides evaluated included Glean, a sulfonyl urea, Trifluralin, Dicamba and MCPA.

\section{EFFECTS OF INSECTICIDE AEROSOLS ON INSECTS OF GRASSLAND ECOSYSTEMS}

\section{A.A.Alekseev, E.I. Kirov, I.B.Knorr}

The effects of organophosphorous insecticide aerosols (methyl parathion) on the insects of herb layer was investigated. The experimental plots were taken in the parcel of virgin steppe of South-West Siberia (Novosibirsk region) adjacent with crop areas. The control plots were chosen in virgin parcel which has been never treated with pesticides. A single application of methyl parathion (200 g per hectare a.i.) was carried out at experimental plots that were located within treatment areas of $200-1000$ hectare. The sampling was carried out in ten-day intervals from June to August 1988 . The single treatments of experimental plots were made in June 1986, June 1987 and June 1988. Thus we could observe both acute insecticide stress and long-term effects of insecticide aerosol treatment. The analysis of seasonal abundances of main taxonomic groups and trophic groupings has been conducted. This analysis showed: 1) The Cicadoidea and phytophagous Hemiptera as bioindicator groups of insecticide stress can be used. 2) The numbers of all observed groups were decreased by acute insecticide stress. Returning to the control level after aerosol treatment within 20-30 days has been occurred. 3) We did not find the long-term effects of insecticide stress in a 1 and 2 years. Analysis of phytophagous and entomophagous insects numbers showed that theirs abundances returned to the control level in 20-30 days after acute stress, statistically significant long-term effects were not obser-ved. Thus, aerosols of optimum dispersion (droplet size $\sim 15$ microns) of methyl parathion affects mildly on insects of grassland ecosystems.

Institute of Chemical Kinetics and Combustion, Siberian Branch of Russian Academy of Sciences, Institutskaya 3, Novosibirsk, 630090, Russia,

220

CARBOFURAN IN WATER:

SUBACUTE AND SUBCHRONIC TOXIC EFFECTS ON RATS

Boškovie, D., Vitorovic, S.Lj., Karan, v. and Neł̌kovic, N.K.

Carbofuran (2,3-dihydro-2,2-dimetyl-7-benzofuranyl metyl-carbamate $)$ is a carbamate compound used as a systemic and stomach poison insecticidenematocide.

It is known that carbofuran is a potential contaminant of surface and ground water. Carbofuran has occasionally been detected in ground water in areas with sandy soll and water table aquifers and in well water samples, indicating that lesching does occur. It was also appesred in surface waters, presumably due to storm water runoff, soil drainaze and spills. It has also occasionally been detected in studies of drinking water quality.

The occurrence in ground and drinking water points to the importance of determining the margine of safety from the aspect of potential harmful effects.

In this study the biological effects of carbofurati administered to rats in drinking water $\{25,100,400$ pem $\}$ for period of 28 and 90 days i4 and 13 weeks) were studied. Water and food consumption, bady weight gain, organ weight, clinical, biochemical and hematological changes, such ss pathological changes in liver and kidneys were investigated.

The results obtained showed that carbofuran had no aignificant effect on water and food consumption nor on the body weight gain and orgen weight. However, atatistically significant changes on the plasma and erythrocite cholinestersse activity, as well as on the serum ensyme (AP, GOT/AST, GPT/ALT) activity in test animala (carbofuran concentration: 100 and $400 \mathrm{ppm}$ ) were found, in relation to control. Hematological data showed changes in bemoglobin concentration and total RBC count. The changes in glucose and urea content, and total bilirubin in serum were found.

The changes in all cases varied depending on sex, carbofuran concentration in water, exposure time, and parameter examined. 
E.D. 010 mé

\section{INTROPUCTION}

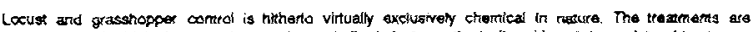

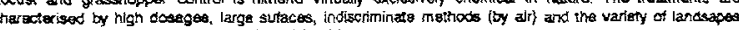
sprayed (natura arass as wall as ailfivated lands).

Concern by both local govemmints and the international (danor) community abous the omvironmemial hazard

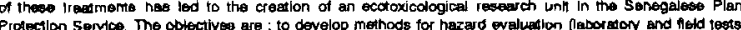

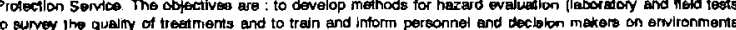
to sanvey the are

\section{IERAESTHIAL ECOTOXICQLOGY}

Since 1991 the Locustox Project carries out studies in Senegal to elucidate the side-effects of locust comtrol on non-target, benefficial, arthropods. Research has concentrzad specificaly on netural enemles of Sahelitan crop pests as well as on arthropods reaponsible for nutrtent cycling in the sol.

More then 100 experimertal plots were treated in different parts of the country (champs de mil, sevane semi-

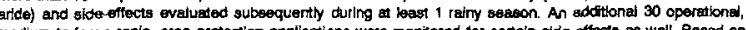

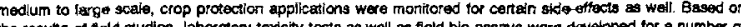
the resulte of held studiea laboratory toxicity tests as well as fleld blo-assays ware daveloped for a number of Important banefldal anthropods. They include the parasitold Bracon hebetor (Hyrm., Eraconidae), the predator Alesta striata (Col, Cave

\section{AgNATE ECOTOXICOLOGY}

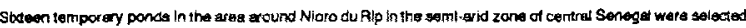

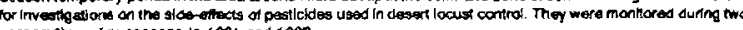

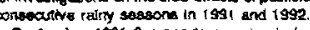

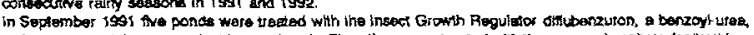

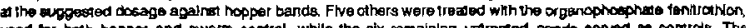

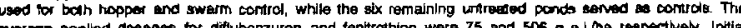

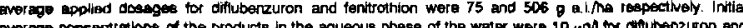

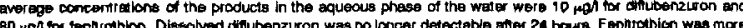

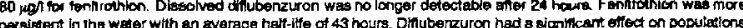

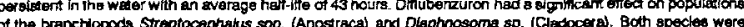
of the branchiopods streptocephalus Spp. (Anostraca) and Okpphnosoma sp. (Hedocera). Bath specleer were

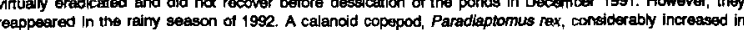
rember following the difluberzuron treatments, possibty due to the elimination of ine branchlopods. Other species of Cledocera, Copepoda, Ostracoda and different aquatic insects were not significantily affected.

\section{EMMRONMENTAL CHEMUSTRY}

Of a number of pesticldes used for locust control we studled behaviour and fast in the phitysical and biotic orvironment, The pesticldes (fenitrothion, diffubenzuron, bendiocarb, chlorpyritios anks deltamethrin) were elther applled expassimentraly or monitored after spray operations. We studlod degradation in soll on vegotaton (neature and cropos) and in cropped and stored products. We also described their behowiour in lakes (i.e. parttioning between water and suspended matter) and the subsequent degradation. Wo derived hat lives for various matrles and cllmatcl conditions. In combination with the toxcity deta, the results allow us to carry out rish assessmente tor the spectitic circumtances. The end products such as hazard ocealing of pesictedes wanting perlocis for consumer products, and buffer zones for sensitive areas, aro wet

Grop Profection Diteation. LOCUSTOX Ptoject, c/O FAO ER 3300. Dakar/SENEGR:

EPA WORKER PROTECTION STANDARD FOR AGRICULTURAL PESTICIDES: A HEALTH PERSPECTIVE

\section{w. K. Hock}

The EPA porker Protection Standard (WTPS) is designed to decrease pesticiderelated illness and injury for people who grow or maintzin plants in forests, greenhouses and nurseries, and on farms. The wPS covers aquicultural production workers and pesticide handlers. Agricultuxal workers may be engaged in harvesting, thinning, weeding, planting, pruning, roguing, and hand packing produce into containers in the field as well as certain other hand labor tasks. A pesticide handler includes persons who mix, load or apply peseicides; handle opened pesticide containers; clean, adjuse or repair application equipment; act as a flagger during derial applications; and dispose of pesticides or pesticide containers. An agriculeural emplayer must not allow any warker to enter or reduin in the treated area before the Restricted Entry Interval (REI) on the pestitide label has expired. An REI is the time immediately after a pesticide application when entry into the treated area is restricted. All agricultural pesticides covered by the WPS will have REIs listed on their labels. REIs will range from a minimum of 12 hours up to 48 hours and, in certain cases, up to 72 hours. Although early entry is allowed in a few specific circumstances, no entyy is permitted until at least four hours after the completion of a pesticide application. Employers are required to provide either oral or posted warnings, or both, for workers following all pesticide applications, unless employers can be assured that workers will not be exposed to pesticide residues during the REI. The WPS is very specific about the content, design, and size of the posting sign; only an EPA-approved sign is acceptable. workers and handlers must also be trained in health and safety issues before they can begin to work in areas where pesticides have been used. Handlers must be trained before they do any handling tasks and workers must be erained in a fanguage that they understand prior to entry into a treated area where a pesticide has been used within the past 30 days. An agricultural employer must provide and maintain personal protective equipment (PPE) for workers and handlers as specified on the product label, provide decontamination facilities for washing off pesticides and pesticide residues for up to 30 days after the expiration of the REX, and the employer must maxe prompt emergency assistance transport tion available to sny workers or handlers kelieved to have been poisonea or injured by pesticides. The wPS applies to all agricultural employers who have employees performing hand labor operations in fields, forests, nurseries, and greenhouses treated with pesticides, and/or employees handling pesticides in these locations. WPs provisions are complicated and will place an additional, but probably necessaxy, regulatory burden on agzicultural employers. The states will have the regulatory responsibility of enforcing the wPs.

113 Buckhout Lak

Penn State University

University Park, PA 16802

U.s.A.
M. Galiev

Several herbicide tank mixtuses were evaluated for their efficiency in controlling Chenopodium album L., Stellaria media (L.) Vill., Matricaria perforaa Merat, Capsella bursa-pastoris (L.) Medik., Spergula arvensis L., Erysimum cheiranthoides L., Senecio vulgaris L., Polygonum lapathifolium (L.) S.F. Gray. Field experiments were carried out at the Pushkin Research Station near St. Petersburg, Russia. The tank mixtures of Metribuzin with Prometryn (MP), Metribuzin with Fluorochloridone (MF), Metribuzin with Glufosinate-Ammonium (MGA) and Metrihuzin with Glyphosate (MG) were used before germination of potato tubers, Besides, the tank mixture of Metribuzin with Bentazone (MB) was used for weed control in potato after germination of tubers.

It was found that the MF tank mixture considerably increased the mortality and diminished relative dry weight of the weed Polygonum convolvalis L., as compared to MB mixture. The MB tank mixture caused the increase of damage and mortality of Cirsium arvense (L.) Scop., Sonchus arvensis $L$, Equisetum arvense $\mathbf{L}$. and Stachys palustris $\mathbf{L}$. However, the successive application of Metribuzin and Bentazone on potato before and after germination of tubers was higher than tank mixture of these herbicides. The MGA and MG tank mixtures had the highest efficiency for potato before germination of tubers. The use of such tank mixtures of herbicides allows to decrease their application rates, residues in soil as well as the damage to potato plants.

All-Russian Institute for Plant protection, Schosse Podbelskyi, 3, St petersburg-Pushkin, 189620 Russia

224

EFFECT OF SIX YEAR HERBICIDE USE DN POPULATION DYNAMICS OF FOUR SPECIES DF MIGRATORY PLANT PARASITIC NEMATODES

\section{S. Kornobis}

The 1987-1992 micraplot experiment was conducted in agricultural field conditions under routine crop, and herbicide rotation. Over the experimental time only the endoparasitic species, Pratylenchus neglectus revealed significant changes in the population fycramics. In a sugar beet the nematode population decreased in microplots treated with chloridazon ( $3.25 \mathrm{~kg} / \mathrm{ha}$ ) or cycloate $(4.44 \mathrm{~kg} / \mathrm{ha})$ but increased in the control. In a winter rape the population of $P$. neglectus increased in microplots treated with benazolin ( $0.45 \mathrm{~kg} / \mathrm{ha}$ ) but it decreased in the control. The observed trends in the dymamics of the nematode population persisted for one year only, and they were changed with the rotation of crops and herbicides. No differences between herbicide treated, and control microplots were observed when linuron + bentazone $(1.0 \mathrm{~kg} / \mathrm{ha}+1.5 \mathrm{~kg} / \mathrm{ha}$, respectively) was applied to pea culture, methabenzthiazuron $(2.8 \mathrm{~kg} / \mathrm{ha}$ ) was applied to a winter wheat, and MCPA as sodium salt + dicamba as sodium salt $(0.725 \mathrm{~kg} / \mathrm{ha}+0.08 \mathrm{~kg} / \mathrm{ha}$, respectively) was applied to a spring wheat. The examined crops and herbicides did nat markedly affect the population dynamics of ectoparasitic Criconemella curvata, Tylenchorhynchus dubius, afrd Helicotylenchus diganicus.

Instytut Ochrony Roslin, Ul. Miczurina 20, 60-318 Paznan, Poland. 
FATE OF CARBENDAZIM, METOLACHLOR AND 2,4-DCP IN THE ENVIRONMENT BASING ON LYSIMETER ASSAYS

\section{A. J. Lewandowska}

Field iysimeters $/ 100 \mathrm{~cm} 1 . \mathrm{d} ., 200 \mathrm{~cm}$ depth/ paoked with humus soil wero used to study disappearance dynamles of extraotable residues and formation of the so called bound residues of mentioned compounds in $15 \mathrm{~cm}$ layer as well as vertical displaoement in $30 \mathrm{~cm}$ soll profile and uptake from soll by plants. The investigations were carried out using ${ }^{14} \mathrm{C}$ labelled compounds. So11 and plant samples were Soxhlet extracted with methanol; the ${ }^{14} \mathrm{C}$ in the extracts and in dried extracted material was determined by combustion and Ilquid scintillation counting. It has been stated that the behaviour of the tested renobiotiog applied to the soll comprises two phases; the first one - disappearance of extractable and formation of bound residues occured simultaneously and the second phase - further degradation of both kinds of residues. The perlods of the first phase and dynazics were different for each compound; a.8. carbendazim incorporated into the soll / $/ 5,35 \mathrm{~kg}$ a, 1 , /ha/ lasts 12 weeks, for metolachlor $/ 1.9 \mathrm{~kg}$.t./ha/ and 2, 4-DCP /metabolite of 2,4-D herbletde/ at a dose $2.125 \mathrm{~kg}$ a.1./ha both gprayed on the soll surface were 4 and 2 weeks respootively. In the 30 em soll profiles sampled after vegetative season / seotioned $10 \times 3 \mathrm{~cm}$ for analyses/ both extracted and bound residues were determined, but most of them remained in the upper layers. Only traces of ${ }^{14} \mathrm{C}$ appeared in permeable water collected at the bottom of the lysimeter with metolachlor treated soil. In the test plants grown on treated solls both forms of ${ }^{14} \mathrm{C}$ residues were round in roots and green parts. The lowest residues levels were determined in seeds.

Plant Protection Institute, 20 Miczurina st., 60-318 POZNAN POLAND

227

\section{PESTICIDES AND FREE AMINO ACIDS IN MAIZE}

A.N. Vinnichenko, N.I.Stemenko.

Our previous investigation showd that some quantities of undestructured atrazine in maize grain grown on fields with overherbicidious concentrations was accompanied by rebuildings in amino acid content that depends on genotype of a plant. Especially significant rebuildings were found for maize lines in contrast to hybrids and concerned increase in free amino acids content. To follow these investigations a working hypothesis was developed: free amino acid accumulation is a protective mechanism of a plant cell to increase turgor and thus to prevent accumulation of xenobiotic; from the other hand, excess of amino acids switchs on some regulation mechanism of reduction of biosynthesis of stored proteins. Last consideration was at first discussed by Tsai C.(1983) and may be named a hypothesis of osmotically regulated biosynthesis of zeines. Some pesticides as harmony, acetal, primextra and other were applied in different dozes together with amino acids analysis of grain on some stages of ripening on the maize fields. In all experiments where we found decreased endosperm size ( reduced synthesis of stored proteins) the increased quantities of free amino acids was discovered too. The main osmolite was asparagine - a transport form of nitrogene in maize. Our experiments thus developed imagination about osmotically biosynthesis of stored proteins of plants and similarity of this regulation mechanism to the same in animal cells is discussed.

Institute of Biology of Dniepropetrovsk State University Gragarin Avenue 72, Dniepropetrovsk 320625, Ukraine

ECOLOGICAL ASPECTS OF THE INFLUENCE DF PESTICIDES ON AGRICULTURAL ENUIRONMENT

A.Sokolowski, W. Walkouski

The effoct of pesticides (insecticides, fungicides, herbicides) on dynamics of opigeal predecious insects and arachnide was studied. The invertigetione were carried out at vinnogora near Poznan in 1993-1994. Two flelds with different four-yoer crop rotation ware ootabliahed. In 1993 spring barley and malze were cultivated; 1994 - faba bean, pea; 1995 - winter wheat, winter rapeseed; 1996 - potato, sugar beet. Each of crop was divided into treated with pesticides part and untreated control. In control pielda no paticides since 1964 have been appliad. The epigeal pauna was collected by means of Barber trape every weak during the vegatation period. The most numerous arthropods were Carabidee (Calooptera), Staphylinidae (Coleoptera), Araneida and Oplliones. In all chemically protected pielda the total numbers of mentionad arthropods wes raduced. In spring barley the numbers of Carabidae was decreased by $23.1 \mathrm{x}$, Staphylinidae $95 . ? \times$, Rraneida $69.5 \times$, Opiliones $65.7 \times$; in maize - Carabidas 43.0 X, Staphylinida $18.5 \times$, Aranoida $58.3 \times$ and Opillones $64.1 \times$. In treated piold of Paba bean the reduction of numbers of Carabidas was $27.2 x$, Staphylinldas $60.7 \times$, Aranaida $2.4 \times$, Opiliones $23.4 x$; in pea - Carabida $39.3 \times$, Staphylinidae $39.9 \times$, Aranelda $33.9 \times$ and Oplliones $7.4 \times$. In some cases the trend to the aqualization of numbers of Carabidae in trated and untrated fields was observed.

Plant Protection Inatitute, 20 Miczurina st., 60-318 pOZNAN, POLAND 
SUSTAINABLE AGRICULTURE AND GENDER IN SUB-SAHARAN AFRICA: STUDY CASE OF ALLOBA EL SOUK, KORDOFAN STATE - WESTERN SUDAN

\section{Samia Abdel Majid, Hanan Mutwakil}

Alloba El Souk (APS) is a small village that lies in Sheikan province in north Kordofan state. It is allocated about $\mathbf{8 2} \mathrm{km}$ south of Kordofan's capital city El Obied town and $60 \mathrm{~km}$ west of E. Rahad town. This village is centered in a cluster of villages mith nearly the same agroecological characteristics. The nearest villages to Alloba being "lradibo" from the north, "Uum Soriba" from the east, "Alloba Alomda" from west and "Kortalla" in the south.

The main pests that are present in the field are piercing and sucking insects Dysedercus spp. (Hemiptera-Heteroptera) and Sesamia spp. (Lep.) on sorghum and millet. Seasonal outbreaks of locusts, Locusta migratoria and Schistocerca gregaria, as well as grasshoppers; Locustana spp. and Ailopus spp. are frequently highly damag ing pests. On farmers' vegetable crops the major pests are Heliothes spp. and Agrotis spp. (Lepidoptera). Other major losses occurring on field crops are the Quelea quelea birds' attacks. The post harvest damages are insect (Trogoderma spp.) and rodent related.

Natural Resources Protection Group (NARP) was established in 1990, as an environmental and uon-profit organization. $A$ meeting of citizen groups representing researchers, academies, students, farmers, labourers and women groups, NARP has been active in a wide range of issues spanning from sustainable development to child and mother health. The basic role of NARP is to improve management of natural resources, introduce reforms to uatural resources policies, empower rural/indigenous groups and seek social justice for its various constituencies. Through its mandate NARP elaborated on a program targeting at sustainable agriculture in AES, specifically pertaining to fond and security in rural areas.

The project supports the farmers in their endeavours to curb pesticide irrational use in their fields and improvement on the quality of seeds, soils and post harvest storage. The program in its second year targets at all farmers in the community and especially women as they form the bulk of the labour in the field. Through an IPM approach the program proceeds in training, education and information of the farmer on the recognition of the major insect pests in the field and storage facilities. Emphasis is laid on thresholds of pests and the periods before the damaging effect of the pests are anticipated to justify mobilization of pesticides/external inputs to control the pests. Also, the farmers are trained to identify the beneficial insects, Hymenoptera specifically, and monitor the development of these vital natural enemies in their fields.

NARP, P.O.Rox 10521, Khartoum, Sudan

WOMEN INVOLVEMENT IN IPM TRAINING AND IMPLEMENTATION IN INDONESIA

\section{Elske van de Fliert}

The importance of women's role in agriculture is widely acknowledged but seldom paid specific attention to in agricultural development programmes. IPM, as it is presently advocated in Indonesia, is a crop protection approach which emphasises the specific ecological and economic conditions of individual farms. and farmers' ability to take informed crop protection decisions based on routine observations of the crop and its environment and analysis of the farm conditions. Practices important for IPM implementation include choice of suitable and pest resistant varieties, good field preparation, adequate field sanitation, production of healthy seedling, appropriate water and fertiliser management, timely and adequate weeding and pest control measures, and timely harvesting. The key to success of IPM seems to lie in adequacy and timeliness of all management practices, so that a healthy crop and a healthy ecosystem are obtained which can resist pest and disease attack. In order to take adequate and timely decisions, farmers need to do routine monitoring and analysis of the crop ecosystem. Such an approach is knowledge-intensive and complex, and therefore requires training. Many of the IPM practices mentioned above deal with the farming responsibilies of women. The importance of the decision making process and possible savings on farm inputs in particular actually call for the involvement of women in IPM implementation and, thus, in IPM training.

Women involvement during the initial years of the IPM training programmes in Indonesia has been very low. During the first training cycles of the two major IPM programmes, participation of women was below $10 \%$, in many training groups even nil. The most important reason identified to explain this phenomenon was that no special effort was done to involve women. Automatically, as a result of the cultural habit to appoint men being the household heads to represent in formal activities, the majority of training participants were men (and predominantly men from the higher socio-economic layers in the community). Other constraints detected imply the little and unpredictable amount of spare time left by women farmers who also have many tasks as housewives, women's feelings of inferiority, and (in some areas) local customs not allowing wornen to mix with men other than their relatives. Several efforts of late, however, have shown that the se constraints are not impossibe to overcome. What is needed are deliberate efforts to increase women's involvement in programme activities. From the side of the programmes this should imply efforts to design selection procedures and training schedules that enable and encourage the active participation of women farmers. From the side of the communities this should imply efforts to analyse and resolve constraints resulting from cultural habits and customs.

JI. Bali 11, Widoro Baru, Ngropoh CC, Yogyakarta 55283, INDONESIA.
COMMUNTY DEVELOPMENT ORGANIZATIONS AS A POTENTIAL TOOL FOR INCREASING WOMEN'S PARTICIPATION IN CROP PROTECTION

\section{Bradshaw, L. A. Lawrence, J. L. Díaz Naranjo}

Community Development Associations and other comunurity groups provide a mechanism for neighborhoods to implement common projects at the local level outside of their municipal governments. Throughout Costa Rica these groups represent an important forum for popular participation, with women frequently active in them. These community groups exist as a potential medium for information transfer of crop protection methods. To examine this potential, with particular regard to women's roles in community groups and crop protection on their own farms, we surveyed and interviewed women from different neighborhoods in Atenas County who have farms within their immediate families. Atenas County, located in the west Central Valley of Costa Rica, is a largely agricultural region which is becoming more urbanized by the capital city, San Jose's, growing metropolitan area. Over one third of the women surveyed, and more than one half of their husbands are involved in community groups, with some women holding offices and/or being perceived as community leaders. Approximately two thirds of the women work on their farm in some capacity, including tending home gardens, harvesting, caring for animals, or general field labor. Although nearly one half of the women feel that pesticide use should be reduced, less than one quarter stated that they play any role in the decision making regarding pest management. No correlation exists between women's age and their participation in crop protection decision making. This low participation could be due to the fact that Costa Rica is a very "westernized" society where farming responsibilities are assumed predominantly by males. Main information sources for pest management identified were programs and Main information sources for pest management identified were programs and
courses from the local cooperative, neighbors or friends, and the Ministry of Agriculture extension. Men are usually the sole recipients of the information, although in some cases both women and men in the family receive information. We conclude that in order to increase women's involvement in crop protection decisions, information exchange can be channelled through community groups. Neighbors and friends and the Ministry of Agriculture are main sources of information which could be combined into community group meetings. The Costa Rican government recently mandated the Ministry of Agriculture to work with groups of producers rather than individual farmers, thus the incentive for this combination is already in place. If producer groups are formed from existing community groups in which women's participation is secured, then women could become more actively involved in crop protection decisions. We view such involvement as a positive change because women are the primary informal educators of future farmers, and as workers on farms, face the risks of whatever crop protection regime is implemented.

Centro de Estudios sobre Desarrollo Sostenible, School for Field Studies, Apdo. 150-4013 Atenas, Alajuela, Costa Rica

232

\section{GENDER IN FARMING SYSTEMS AND TECHNOLOGICAL CHANGE}

$$
\text { B.C.M. van Koppen }
$$

For effective design and implementation of crop protection measures and other agrarian technologies, insight is needed in farming strategies of technology users and the way in which technology can improve their livelihoods. Research and field experiments yielded a growing body of empirical insights. However, up till now few efforts have been made to systematize and conceptualize these experiences for a better understanding of women's and men's interests in agrarian technology and its specific characteristics. An exception is $C$. Safitiou. who also pointed out the importance of a more realistic concept of the farm household. She proposed to distinguish intra-household productive subsystems that are controled by either husband, wife and sometimes aduit children. Household members exchange resources, a process in which bargaining power of individual members, cultural values, etc play an important role. With this distinction of intra-household productive subsystems, overall farming systems can be classified into dual, male and female farming systems according to the gender of the main decision maker and controler of resources (1988).

Currently two fieid studies are carried out fram this perspective in Burkina Faso and Bangladesh. Both concern water management technology for improved rice cultivation. While the technology itself is gender neutral, differences between its adoption in women's and men's respective productive subsystems are studied. as well as the impact of the project approach.

Here it is noted that land and water management projects often define their target group according to the access to improved land and water. The other components of their extension packages, that may include HYV's, fertilizer, crop protection measures, credits, organisation etc. are exclusively directed at this user.

In South-west Burkina Faso rice cultivation in valleys is mainly, but not exclusively part of the female productive subsystem. Three categories of rice cultivators: men and younger and oider women of two neighbouring ethnies are compared. Differences in intra-household negotiations and cultural values on the mabilisation of labour, imputs and control over the harvest in the light of the overall dual farming system are studied

In Bangladesh pump irrigated rice cultivation in Bangladesh is a maie productive subsystem. However, privatization of purfip ownership, the development of a water market and the provision of loans for irrigation pumps by NGO's, make it even possible tor landless men and women to own and manage an irrigation pump and sell the water as entrepreneurs. Here intra-household involvement in female irrigation enterprises and the impact on women's status are studied. 
WOMEN IN PLANT PROTECTION: AS FARMERS, EXTENSION WORKERS AND RESEARCHERS

\section{A. A. Laurense and F, H. Ali}

Athough women constitute the majority of farmers in Africa their involvement in agriculturai extension and research activities is very restricted. This is due to limited educational opporturities for girls, social notions on appropriate jobs and family obligations as (unmarried) daughter or as mother and wife. Women who manage to reach a certain position against all odds are generally better educated and more motivated than their maje colieagues.

The limited involvement is very unfortunate since female extension workers, researchers and decision makers can be key role players in any attempt to improve the agricultural skills of the (fernale) African farmer. The female extension worker has hetter access to female farmers who feel less restricted and more equal in their contact than with her male counterpart. Male extension workers can, in several societies, only approach female farmers through their husbands or other male farmers.

The conventional pest control technology based on the use of pesticides restricts the involvement of both female farmer and female Plant Protection extension agent.

Fernale farmers are mostly involved in capital extensive subsistence farming with no or limited use of pesticides. Where used, application of pesticides is difficult for women due to the physical condition of the majority of fernale farmers (being either pregnant or lactating or generally in a poor health condition) and/or due to the application techniques and equipment.

Infegrated Pest Management (IPM) is an approach par excellence for female farmers. Cultural control methods such as planting, weeding and close monitoring of the crops, are activities which fall under the responsibility of wonen. Selection of seeds and planing material are also activities taken are of by wornen.

Where IPM involves food crops, not only the yield but also the opinion of the consumer on quality and properties of improved/introduced varieties is essential. Being a producer, a cook and consumer, the female farmer is in a key position to judge.

Stressing the need to involve female farmers in IPM development is not enough, nor is it to send (only) female extension and research staff to 'gender issue' meetings and workshops. Conditions allowing female staff to be trained, employed and promoted should be improved. Women should be given and use the opportunities in terms of education, jobs and promotion. While, at the same time, social obligations and expectations should be taken into account.

Plant Protection Division, Ministry of Agriculture, Livestock and Natural Resources, P.O. Box 1062, Zanzibar, TANZANIA

235

GENDER ANALYSIS AND SUCCESS OF INTEGRATED PEST MANAGEMENT STRATEGIES

Amina H. Mohammed

Even though women's position in decision making on plant protection activities was considered marginalized and non productive, the shift in status of crops (food vs cash) and the change in approach by the plant protection stafi, realize the values of women's low input protection practices influenced by their position.

The roles of men and women in protecting both food and cash crops are analyzed, its impact in relation to these changes is considered.

These issues are discussed in relation to the success of IPM strategies; a new approach in the country.

P.O. Box 2313, Zanzibar, Tanzania
THE ROLE OF WOMEN FARMERS IN RICE PEST MANAGEMENT

P. Menakanit

Farm women in Thailand play their role in all activities related to agricultural management. A number of research findings pointed out that they are involved in decision making in every step of farm activities. Recently, due to shortage of labourers, pest management became a responsibility of rice women farmers. Because of the important role in household chores, women farmers had very little chance to participate in formal training. Thus, the participatory training for rice women farmers was conducted in 1993 in Chainat province, Central Thailand in order to develop their knowledge of IPM concept. One year later after training this group of women farmers were investigated for sustaining IPM adoption. A group of thirty farmers who did not participate in IPM training were also assessed as the control. Results from the survey indicated that decision making capabilities of women farmers is improved. They were more skilled in insect pest-beneficial diagnosis and predator-prey relationships. Almost all women farmers admitted insecticide application does not contribute to yield, while less than half of the control group did not agree. Results also showed the trained women farmers had better attitude toward leaf-feeding insects than the control group. They agreed most leaf-feeding insects did not cause any yield loss and early spray was not necessary. Yield measurement indicated that without any insecticide applications, the trained women farmers obtained greater yield than the control group.

Plant Protection Service Division, Department of Agricultural Extension, Bangkok, Thailand

236

COTTON: TEN YEARS OF IPM IN THE STATE OF SAOO PAULO - BRAZIL

V. R. Cruz

From 1981 to 1991 were installed 831 IPM plots in cotton with a minimum area of 1 hectare each one on commercial crops. In these IPM plots were made sampling once a week, just right after the emergency till near the harvest; were examined 20 plants/ha noting the pests and natural enemies. Until 80 days after emergency we used selective pesticides (chlorfentezine, diflubenzuron, endosulfan and abamectin), less selective pestictdes as (dimethoate, triazophos, metfdathion and fenitrothion) and not seletive pesticfies (monocrotophos, betacyfluthrin, cypermethrin, deltamethrin, metyl parathion and fenvalerate) in selective doses or in restrict areas. In some plots were used phytoregulators (mepiquat chlorate and chlormequat) and unleafer (tidiazuron). In farmers plots control, they used their own technology of pests control. In IPM plots infested by Anthonomus grandis, were made in average 4.9 sprays while in the State were 7.6 sprays, with a decreasing of $35 \%$ in their number, without loss of productivity. The production of pit cotton was $2430 \mathrm{Kg} /$ ha in the IPM plots and $1860 \mathrm{Kg} / \mathrm{ha}$ in the State average, with an increase of $31 \%$. The cost of treatments was US\$27.96/ha in the IPM plots and US\$44.58/ha in the State ave; rage, with a decreasing of $37 \%$. In areas not infested by $A_{\text {. grandis, }}$ the results were nore meanlgful 1 fth 2.3 sprays in IPM plots and 6.9 in the state average, with a decreasing of $67 \%$. The production was $2454 \mathrm{Kg} / \mathrm{ha}$ in IPM plots and $1882 \mathrm{Kg} / \mathrm{ha}$ in the State average, with a increase of $30 \%$. The cost was US\$13.12/ha in IPM plots and US\$40.52/ha in the State average, with a decreasing of 68\%. These results showed that is efficient and economical the use of cotton IPM method by famers in the State of Săo PauTo.

CATI - Coordenadoria de Assistência Técnica Integral

Av. Brasil, 2340 - Caixa Postal 960 - FAX 00-55-192-418309

13073-001 - CAMPINAS - SP - BRAZIL 


\section{S. R. L. Dieh1}

In order to show to farmers the efficiency of soybean IPM on conmercial crops, were conducted 43 plots of 1 hectare each one, from 1991 to 1994. The IPM evaluations were done every week. The sprays were done only when the control levels to Anticarsia gemmatalis, Nezara viridula, Piezodorus guildinii and Euchistus heros reached. The pesticides used were: Baculovirus anticarsia, endosulfan, metamidophos, triflumuron, betacyfluthrin, monocrotophos and, in some sprays, were used salt ( $\mathrm{NaCl} 0.5 \%$ ) mixed with pesticides, reducing the pesticides doses. In checks, the farmers used their own technology of pests control. The medium number of sprays were 0.9 in the IPM plots, 1.5 in checks and 2.4 in the State usual average. The medium productivity were: $2501 \mathrm{~kg} / \mathrm{ha}$, $2435 \mathrm{Kg} / \mathrm{ha}$ and $2045 \mathrm{Kg} / \mathrm{ha}$ in the IPM plots, checks and State, respectivily. The average cost of sprays was: US\$4.10/ha in IPM plots, US\$7.40/ha in checks and US\$12.70/ha in the State. In conclusion, we can say that there was one reduction of $37.5 \%$ in the number of sprays and $32.3 \%$ in their cost, when we compare the data of IPM plots with the State average. The data showed too, that the productivity increase $18 \%$. These results confirm, in the field, the viablitity of soybean IPM under Săo Paulo State conditions.

CATI - Coordenadoria de Assistência Técnica Integral

Av. Brasil, 2340 - Caixa Postal 960 - FAX 00-55-192-418309

13073-001 - CAMPIKAS - SP - BRAZIL

\section{J. D. De Negri}

The IPM citrus programme developed by CATI, the State of São Paulo Extension Service, was begun in 1986 and has been carried out viewing the management of the two major important mites in our citriculture: citrus rust mite (CRM), Phyliocoptruta oleivora (Ashmead, 1879) Keffer, 1938 and leprosis mite (LM), Brevipalpus phoenicis (Geijskes, 1939) Sayed, 1946. For their control, the c1trus farmers spend $21 x$ of the yearly production cost only with miticides. Twenty six areas of 10 hectares each in commercial citrus orchards were followed up by sampling $1 \%$ of the plants every 10 days, inspecting 3 fruits on the canopy periphery for CRM and 3 inside frutis fo LM. Each fruit was considered infested when more than 20 (for fresh fruit) or 30 (for industry) mites of $\mathrm{CPM} / \mathrm{Cm}^{2}$ or at least 1 mite of $\mathrm{LM} /$ fruit were present. The control level used was $\geqslant 10 \%$ of infested fruits for CRM and $>5 \%$ of infested fruits for LM. The products used in the progranme were: azocyclothtn, bromopropylate, cyhexatin, dicofol, fenbutanthin oxide, fenpyroximate, hexityazox, propargite, sulfur and triazophos. The results of the $1993 / 94$ crop showed that there was a reduction of $35 \%$ in the number of sprays in the IPM plots when compared with the controls (IPM=2.92 spraying/year and controls $=4.52$ spraying/year), with a mite mortality efficiency higher than $80 \%$. There was also an increase of $31 \%$ in the praduction of the orchards with IPM, towards the controls (IPM=23.4 ton/ha and controls $=17.8 \mathrm{ton} / \mathrm{ha}$ ). Considering that the cost of one spraying is around $15 \$ 60.00 /$ hectare and that the São Paulo State has 800,000 hectares of citrus, we may estimate that, if this IPM technology would be adopted in all this area, we could save around US\$75 millions a year, only in the miticide item.

CAJI - Coordenadoria de Assistência Tëcnica Integral

Av. Brasi1, 2340 - Caixa Postal 960 - FAX 00-55-192-418309

13073-001 - CAMPINAS - SP - BRAZIL

\section{W. H. Merege}

In 1 hectare areas, on several commercial maize farms, a follow up management of the caterpillar Spodoptera frugiperda was carried out during 3 years, using the following products: triflumuron, betacyfluthrin, deltamethrin, cypermethrin, permethrin, diflubenzuron and lambdacyhalathrin. Plants were monitored looking for the "scratched leaves" phase, an indication that, at this moment, the caterpillars are 7 to $8 \mathrm{~mm}$ in lenght, being unable to perforate the leaves, only scratching them. When the crop has $20 \%$ of their leaves in that condition, product application shall start. The results obtained indicate that all products induced a mortality around $80 \%$ of the insect pest. In spite of only one spray being made there was an increase of productivity compared with the commercial crops of $15 \%$ for the pyrethroids and $18 \%$ for the physiological pesticides. Considering that the farmers in the conventional treatments apply up to 3 sprays for the management of the insect, a reduction in the pesticide use of about $66.7 \%$ was achieved by the use of IPM.

CATI - Coordenadoria de Assistência Tëcnica Integral

Av. Brasi], 2340 - Caixa Posta! $960-$ FAX 00-55-192-418309

13073-001 - CAMPINAS - SP - BRAZIL

240

\section{INTEGRATED NEMATODE MANAGEMENT ON BANANA IN CUBA.}

Femández, E., H Gandarilla, R Hernández, O. Acosta, R. Vázquez, M. Fernández, M. Paneque, M. Basterrechea, E. Duarte.

Banana crop is parasited in Cuba by different nematode speciea, but the most common are Radopholus similis and Meloidogyae incognita; Rotylenchulus reniformis and Heticotykenchus molticinctus are scarce.

Several Integrated Nematode Management (INM) systems were evaluated on different crops conditions. They inchuded preventive mesusures to avoid nematodes, cultural methods fike soil tillage and crop rotation, physical and chemical treatments of corms and plants and also the use of the fungus Paecilomyces hilacinus like biological control.

The best system of DNM requires the sampling of soil before nurseries and plantations, use of clean vitroplants inoculated before planting with P. liacinus a good goil banding with enough time to eliminate nematodes in crop residuca, establishment of nonsusceptible cropa before banana ininfeated areas, coms tratment with hot water or phenamiphos, chemical treatment with phenamiphos in young plantations and the aternative use of $\mathbf{P}$. lilacinus in areas with fight infestation kevels. This system sbowed good feasibility against the main nematode species.

Instituto de Imvestigaciones Sanidad Vegetal. Calle 110 \# $514 \%$ 5ta A y 5ta F. Zona Postal 13. Playa Ciudad Habana. Cuba. 
INTEGRATED RED MTE MANAGEMENT (Teranychus midus, Banks) IN BANANA AND PLANTAIN IN CUBA.

Almaguel Lendas Mayra Ramos, R Pérez A., R. Pérez S., J. Ovies, C. Murguido, Zuleika Martinez, Misleibia Mirquez, I. Cortifias and Maria Elens Márquez.

The integrated red mite management in banana and plantain was elaborated through a regearch develop during 1976-1994 and include the results in biology of the mite, population dynamic, prognocia, damage threshold, microbiological and chemical control methods, the natural enemy protection and inoculative release of mite predatore (Phytosetulus macropilis) according to the crop age. The biological effect was deternined in lab with "Macho 3/4" and "Victoria" clons by the survivor method and 30 replication by each case. The dynamic was made in growing atage and establishod plantation with those clones, the red mite populations was evaluated every week in $100 \mathrm{~cm}^{2}$ per leaf in 5 plants per plot. Temperature, relative humidity and rainfall were recorded. Intensity and distribution of this mite in differents places of Cuba in 9 banana clones, the losses over 200 plants in the more sensible clone and the most vulnerable crop stage were determined. Natural enemies was identified and its behavior and relation with $T$. tumichs. The optimal time and the threshold to use biological and chemical acaricides in nursery, growing stage and plantation was determined. Red mite affect mostly and growing stage, with highest damage in "Macho $3 / 4^{*}$, "Hembra $3 / 4^{"}$, "Robusta" and "Vietnatnita" clones. The highest population of red mite were with $21-25^{\circ} \mathrm{C}$ temperature and rain fall leas than $35 \mathrm{~mm}$. in 7 days; the effectivity of climatic and population prognosis methods had 87 and $70 \%$ respectively. Stethorus picipes, Scollotrips pallichus, Artronodix sp., Phytoserulus macropilis and Amblyseizus sp. were the natural enemies more abundant and relationed with red mitc. Differential threshold were extabished acconting to the relation pest-predator and emviromental favorable conditions; in nursery, with 1-2 red mite/plant attainment release of 1 predatos per 20 red mite; in growing stape of banam, with 20-30 red mite/predator in favorable conditions it's neceseary to apply Bacillus thuringiensis and with more than it, use acaricide; this later threshold is the same for 1 year plantation in favorable conditions to apply $B$. thuringiensis in oldest crop stages, only natural enemies protection. This integrated management was done in Lo Havana, Villa Clara and Cienfuegos provinces with satisfactory results.

Instituto de Investigaciones de Sanidad Vegetal. Calle 110 \# 514 e/ 5n A y $5^{\text {m }} \mathrm{F}$. Zona Postal 13. Ciudad Habana. Cuba.

\section{INTEGRATED WEED MANAGEMENT IN HORTICULTURAL CROPS}

Pérez E.; F. La O; E. Paredes; R. Labrada; R. Rodríguez; Pura Moreno; R. Gancia; Tamara Mateo and $A$. Sury.

During 10 years different researches were made in annuals crops, with the view to stablish a program of integrated Weed Management in Horticultural Crops (TWMHC). Technological package includes ammual prognosis of more impontant weeds and type of association and the method of ecological surveying of weed; cconomical and damage threshold of Cyperus rotundus L, Rotboellia conchinchinensis (Low) Clayton and Parthenium hysterophorus L. in potatoe, tomatoe, gartic, onion soyabean, bean corn and the critical period of competition of associated weed in those crops and cucumber, sweet potatoes and pepper, also it was inchuded the bioecological study the more distributed weeds in Red Ferralitic soil as important aspects of IWMHC.

As a result of the research about soil preparation in IWMHC it necesary to consider the type of implements, the season, the time between labours and the whole time of preparation; between the syatems evaluated the combiation of multiplough and tiller with interval of $10-12$ days during more than 45 days, highty reduced the incidence of $S$. halepense, $C$. rouunchus and $C$. dactylon In relathion to crop rotation and polycrop, sone aspects are distinguished which play importan role in IWMHC, those are the sequence of crop (order), the rotation system (open or close), the variety of clon the crop management the big influency of intercropping (raining season) in weed population, the allelopathic effects and the distance between plant in polycrops. There are good control of amual and perennial grasses and $C$. rotunctus with sweet potatoes as intercropping in rotation with potatoes or beans as main crop (dry season). On the other hand some result will be obteined with com an millet again broad leave weeds. Stizolobium deeringicanum Bort incorporste to the soil at finish cycle or corn hight population seediting and incorporate at $50 \mathrm{~cm}$. hight, both recticed herticides consumption in the next crop about 20 and $25 \%$ respectively. The phytotoxic threshold of principal herticide in three types of soil to differents horticulturals crops, permit the management of rotation crop taking into account soil residues, there also were stablished biologicals methods for residual detection. The practical role of this aspect in FWMHC were demostrated when trifluralin were detected at $0-20 \mathrm{~cm}$ soil deph at $0.034 \mathrm{mg} / \mathrm{kg}$ concentration 308 days after aptication and oxadiazon at $0.15 \mathrm{mg} / \mathrm{kg}$ concentration $270 \mathrm{dxys}$.

It was stablished the herbicide use with reduced dosis based of row aplication and optimal period of weed and different researches were doing in order to recomend the herbicides for each weed and crop.

As a part of IWMHC it was included a agricultural extension system in which the producer participated as a protagonist, with maximal exploitation of available resource.

Since 1991-93 the IWMHC were developed in Batabanó (south of Havana) in 1500 ha and later it has been developed in different regions of Cuba. The results in big areas were highly satisfactory with substantial population reduction of $S$. halepense, $C$. rotundus. $R$. cochinchinensis and other important weeds and yields increase

Instituto de Invertigaciones de Sanidad Vegetal, Calle 110 \# $514 \%$ 5ta A y 5ta F, Zona Postal \#13. Playa, C. Habana. Cuba. Fax 5-37-330535.

244

DIPTERAN LEAFMINERS ON HIGHLAND CROPS OF MALAYSIA AND AN INTEGRATED APPROACH TOWARDS IHEIR MANAGEMENI

\section{A. Sivapragasan, W.H. Loke, A.R. Syed and C.K. Heng}

Dipteran leafminers of the genus Lirionyza and Chromatomyia horticola (G.)

$900 \mathrm{~m})$ of Malaysia. Recently, the problem of leafminers has increased,

firstly, due to the $i$ ts expanding host range. In a recent survey, this

leafminer complex had been recorded on 29 vegetables, 8 ornamentals and

5 weed species, compared to only a few hosts in 1991 .

Secondly, these has been serious concern on the development of resistance

of leafminers against some of the commonly used insecticides. To reduce

the dependence on insecticides, a holistic approach towards the management

of leafminers was initiated focused on two crops as models, viz, sweetpeas,

Pisum sativun and Chrysanthemm morifolium, both of wich are of high

economic value. In this approach, emphasis was generally given towards

understanding and amplifying the role of natural mortality factors,

especially parasitoids, the use of other enviroment-friendly control

tactics such as neem, Azadirachta indica and the yellow sticky trap.

judicious use with selective insecticides, such as cyromazine and $\mathrm{L}^{-}$

cyhalothrin, was underscored. In sweetpeas treated with these insecticide
relatively high parasitoids to leafminer ratios were obtained. Further,

relatively high parasitoids to leafminer ratios were obtained.

insecticide sprays based on crop phenology and damage incidence

( $) 30 \%$ on young leaves) at weekly intervals before flowering but

effective compared to weekly sprays advocated by farmers. Three larval-

pupal parasitoids species vere recorded which include two eulophids,

Hemiptarsenus varicornis, and Chrysocharis sp and a bethylid, Goniozus sp.

Parasitism levels as high as $41 \%$ was obtained in an insecticide-free

sweetpess crop. Impact evaluation studies using exclusion cages also

showed that parasitoids could significantly affect survival rates of the

leafminers. Results are promising for use of neem whereby a water suspen-

sion of 3 neen kernel extract gave good control with concomitant higher

yield of sweetpeas than abamectin and non-insecticide control. Neem also

had some anti oviposition effect and favored parasitoids. Yellow sticky

traps are currently extensively used especially under protected

environments. About 5,000 leafminer adults could be caught per trap of

size 30 cr $\times 10$ cri during a trapping period of three days. In chrysanthemus

besides the utilisation of yellow sticky traps, insecticides stili remain

the major component of management. Fogging is not recomended due to

its detrimental nature to parasitoids. Chrysanthemm varieties showing low its detrimental (e.s. Crean Glance) could also be exploited to reduce leafminer infestations.

Basic Research Division, M.A.R.D.I., G.P.O. Box 12301, 50774 Kuala Lumpur MALAYSIA 
METABOLIC ACTIVITY OF TRICHODERMA ISOLATES PROMISSORY FOR BIOLOGICAL CONTROL, OF SOILBORNE PATHOGENS

Stefanova Marusia and Amada Leyva.

Among the fungi used for biological control of soiborne pathogens, various Trichoderma species have received mone attention. The effectivencess of Trichoderma lies on a combination of nutrients competition, mycoparasitism and antifungical metabolite production. The metabolic activity of four promisory Trichoderma isolates for the biological control of the economically important soilbome pathogens, Phytophthora nicoticnae, $P$. capsici, Rizoctonia solani and Pythium sp. was studied. The isolates A-53 (Trichoderma Sect), A-34 (T. harziamum) A-86 (T.viride) and TPR (Longibrachiatum Sect) produce non-volatile metabolites with antifungical activity, including tytic enzymea composed cellulose, chitinase and $\beta 1.3$ ghuconase. The isolate A-86 also yiclds a volatile lactose, probably G-pentyl alpha pyroese (6 PP). The growth of the tested organisans was intibited by the production of hoth difrusable and volatile metabolites and host cells exhibite vacuolation, granulation, cosgulasion, desintegrasion and bris.

Instituto de Investigaciones do Samidad Vegetal. Calle 110 \# $514 \%$ st A y sta F. Playa. C. Habars. Cuba.

247

ASSESSMENT OF SIDE-EFFECTS IN SOIL COMMUNITIES BASED ON ECOTOXICOLOGICAL RECOVEAY TIME

\section{N.M. van Straalen}

Vrije Universiteit, Department of Ecology and Ecotoxicology. De Boelelaan 1087, $1081 \mathrm{HV}$ Amsterdam. The Netherlands

Ecological side-effects of pesticides may be evaluated on the basis of two different apsects, the magnitude and the duration of the effect. Since every pesticide is expected to cause some unavoidable negative effect, particular attention must be payed to the potential for ecotoxicological recovery, after the action of the product has faded. This paper proposes a theoretical framework that joins the magnitude and the duration of the effect into a single criterion, which may be useful when classitying pesticides for regulatory purposes.

From laboratory experiments estimates may be obtained for the no-eftect concentrations of potential ecological receptors and for the degradation time
of the chemical. These data may be combined with intormation on the initial concentration after application. to estimate the time needed for achieving a "saf" concentration, the so-called ecotoxicological recovery time.

The newly developed methodology is applied to literature data for some pesticides applied to soil. Examples elaborated for carboturan, benomyl, lindane and parathion show that the joint assessment approach may lead tot a classification of pesticides that is different from the ones based on persistence or toxicity alone.

\section{WOMEN AND THE VIETNAM NATIONAL IPM PROGRAM}

Authors:

\section{Tran Thi Van Anth}

The Vietnam National IPM Program has developed rapidly since the first Farmer Field Schools were held in 1992. By the end of the current cropping season, about 100,000 rice farmers will have graduated from season-long IPM field schools. Data collected by the program, however, indicates that less than 15 percent of field school participants are women. This participation rate fails to reflect the large role played by women in rice farming. As a first step towards formulating policies to increase women's participation, the Center for Family and Women's Studies, in conjunction with active IPM trainers, carried out a survey on issues relating to women and IPM in three locations. The main objective of the survey was to examine the role of women in on-farm decision-making and to identify obstacles to increased participation of women in IPM field schools.

The study found substantial variation in the extent to which women assume direct responsibility for making important decisions on farm management. Nonetheless, women are generally indirectly involved in these decisions by virtue of their overall responsibility for the economic management of the household. In addition, women in all three locations account for a large share of household labour expended in rice cultivation. These findings provide support for the conclusion that the program should work to increase female participation in IPM field schools.

Various obstacles to increased female participation were identified. These include inadequate support from within the household, insuificient understanding and support from local officials and lack of direct contact and consultation between program administrators and existing institutions, such as the Wonen's Union, representing the interests in women at the local level. Time pressures on women, especially poor women, exacerbate these problems.

Although quotas in support of women's participation in IPM field schools could help improve the situation, quantitative targets must be combined with greater sensitivity among local leaders and program personnel. Improved consultation with women farmers at the village level, and direct involvement of existing institutions such as the Women's Union, would improve planning and hence make program activities more attractive to women. It is particularly important that Training of Trainers programs include workshops and modules on gender sensitivity and the role of women in agriculture.

Center for Family and Women's Studies, 6 Dinh Cong Tiang, Hanoi, Vietnam

248

\section{FORMULATION AND COMMERCIALISATION OF} INSECT PATHOGENIC VIRUSES

\author{
MICHEL GULLON
}

Natural Plant Protection, Boire Postale 80, Route d'Arix, 64150 Noguères, France

\section{SUMMARY}

Insect pathogenic viruses were discovered some decades ago.

Commercial development has been delayed for three main reasons

Industrial production to obtain cost effective product

Formulation improvement to improve shelf-life and prolonged activity of commercial

products

Education and training of farmers to ensure correct use of this living material

Integrated pest management is now developped world wide, and some viral insectiicides are now successfully commercialised.

The three main marketing arguments are that insect pathogenic viruses are

Environmentally safe,

Self replicating and also have some long term beneficial effects,

An alternative product in cross-resistance situations,

despite their inconvenients : specificity, fragility and application technicity 
CROP PROTECTION POLICY AS AN ESSENTIAL CONTRIBUTION TO SUSTAINABLE AGRICULTURE

\section{Degheele}

This half-day forum to discuss the importance and necessity of an adequate policy-framework concerning issues of plant protection and pesticide usage, as a prerequisite to sustainable development of agriculture and the environment, is organized by the Ministry of Agriculture, Nature Management and Fisheries, and the Ministry of Housing, Spatial Planning and the Environment of The Netherlands. Departments of these and other ministries as well as the agro-industry conduct an active crop protection policy, aiming at sustainable and socially acceptable forms of agriculture.

Six speakers will present the ways and means of and reasoning behind the current plant protection policy of their country or region. Each new situation requires an analysis of the internal and external factors in order to develop a national plant protection policy which complies with the national needs and internationsl obligations. The forum will end with a short summary and panel discussion.

The ways and means to achieve a sustainable agriculture can vary and consist of a blend of e.g. plant quarantine, integrated pest management (IPM) pesticide usage, genetically modified organisms and ecological or organic farming technology.

Four decades of research in Europe in biological and integrated pest control, co-ordinated by the IOBC/WPRS, have resulted in some crop protection and production systems with a significantly lower need for pesticide inputs (30-85\%, exceptionally $95 \%$ reduction). The economic and ecological feasibility of several systems has been proved and they can therefore be implemented on a much larger scale than at present. Constraints for implementing integrated crop protection are e.g. lack of knowledge about integrated protection and production (IPP) by farmers and policy makers, lack of well trained extension officers, insufficient economic incentives and research funding, unrealistic zero-tolerance, confusion about the standards for a labeled product and lack of confidence in IPP.

Several countries in the world declared already now IPM as a national policy. An overview will be given of the measures taken in Belgium to contribute to a sustainable agriculture

University of Ghent, Faculty of Agricultural and Applied Biological Sciences, Coupure Links 653, Gent B 9000, België.
SUSTANABLITY A NECESSARY CHANGE IN THE BANANA PLANTATION INDUSTRY

\section{Castillo}

Banana production in Costa Rica dates from the end of the XIX century and bananas have been one of the main agricultural export commodities for many decades. This has implied a strong governmental support in spite of indications of serious environmental and health problems linked to banana production.

Several pests and diseases affect banana plantations, two of the most important are the Black Sigatoka disease (Mycosphaerella fïiensis var. difformis) fungus which diminishes both yields and quality, and the nematode Radopholus similis, which damages the root system of the plant. The control of these organism relies on the intensive use of pesticides. It is estimated that more than a third of the pesticide volume imported in Costa Rica is used on banana plantations. Fungicides are applied up to 40 times a year by airplane, while highly toxic nematicides are applied 2-4 times a year directly to the soil. Weeds and insects are mostly controlled with chemical products. Mycosphaerella has developed pesticide resistance and the situation of the Sigatoka is currently almost out of control.

Banana plantation areas showed an almost fivefold risk for acute pesticide poisoning among agricultural workers. During the seventies more than thousand banana workers were sterilized because of chronic exposure to the nematicide DBCP. Severe soil contamination by copper compounds used in the banana plantations has damaged several hundreds of hectares of fertile soils. Contamination of surface and groundwater with pesticides has been demonstrated in banana plantation areas in Costa Rica. Banana exports to the United States, the principal market for Costa Rica, have been rejected several times due to violation of residue tolerance. The economic losses implied by these problems have not been quantified but are undoubtedly important.

Afthough sustainable development, environmental conditions and quality of human life are considered priorities by the Costa Rican government, there has been total government support towards the activities and expansion of the banan industry. However, there is a growing awareness both in the government and in the banana sector that changes must take place. A decree in 1990 establishes a tax to banana exports to promote research and environmental and occupational health programs. This together with the integration of the Environmental Committee of the banana sector are indications of new perspectives.

Studies of feasible changes towards sustainable agricultural practices, such as soll management practices and integrated pest management have been undertaken by scientist linked to academic institutions and to the banana industry, but more support for research towards an environment and health friendly production system is urgent.

Changes towards sustainability are imperative in the banana production system for economical, social, health and environmental ressons. These can only be achieved through the collaboration of government, industry and academic institutions.

Pesticide Program: Development, Health and Environment (PPUNA), Universidad Nacional, Heredia, Costa Rica
MATCHING POLICY ISSUES ON PLANT PROTECTION AND THE ENVTRONMENT AT GOVERNMENT AND PUBLIC LEVEL

T.J. Perfect

Concern over the potential environmental impacts of plant protection activities, both within the production systems in which they are undertaken and more generally in relation to human health and the sustainable use of natural resources, has led to a growing realisation that a better informed and more pro-active approach to formulating plant protection policies is required at the national and international level. Most of the well characterised problems that have led to inereased awareness derive from inappropriate use of chemical pesticides. They include direct effects on the sustainability of existing plant protection technologies, primariły pest resistance and resurgence, and indirect effects on the production base, producers and consumers.

There is widespread recognition that crop protection has a continuing and perhaps increasingly important role to play in meeting food security needs and optimising both sustained productivity and return on investment from agricultural land. Whilst the concept of Integrated Pest Management (IPM) is almost universally accepted as an optimal approach it remains broadly true that short-term gains in many systems are most easily secured through pesticide based technologies. This dilemma creates conflict between public and private sector interests that relate both to long-term sustainability of output for agricultural producers and of markets for the agrochemical industry. Consumers, often represented by NGOs, have an increasingly powerful voice in the debate.

Experience has shown that government policy has a profound infuence on adoption of approaches to crop protection that are less dependent on the use of pesticides. A number of countries have now moved to declare IPM as a national poliey following the example of Indonesia. Such a policy environment is considered by many to be a prerequisite of implementation on the scale required to achicve uptake and impact. It requires demonstrable proof that IPM can provide economic and environmental benefits within the major production systems of the country. Internationat initiatives are now in place to further promote the validation of IPM technologies and management systems and thus generate the basis for wide scale implementation.

The long-term surstainability of both public and private sector engagement in crop protection as a component of agricultural production systems that promote environmental quality will hinge on the success of such ventures.

Natural Resources Institute, UK Overseas Development Administration, Central Avenue, Chatham Maritime, Kent ME4 4TB, United Kingdom. 
THE DUTCH ATTEMPT TO SUSTAINABLE PLANT PROTECTION PRACTICE

E.A. Goewie

Agriculture in The Netherlands is very succesful. That favours export and employment. However it also causes unwanted effects leading to problems and threatening the crop production system in the long run. Therefore

disadvantages of intensive agricultural production should be considered.

Two disadvantages arose. Markets became satiated and consumers started to doubt the quality of agricultural products and of production circumstances. Agricultural policy of the Dutch government faces decreasing market prices and less social embedding of agriculture. Due to increasing knowledge about physiology and ecology, as well as emancipation of consumers, pesticides attracted attention of governments.

In order to stimulate a crop production less dependent on pesticides, the Dutch government launched a pesticide reduction plan. This so called "Multi-Year Crop Protection Plan" seems to be succesful. Farmers have become aware and accepted the necessary changes in order to safeguard their own future. The first steps have been taken. A signicant decrease of the use of some pesticides has been recorded. However there are doubts that crop protection really will become less dependent on pesticides at all. The plan strives ultimately for a kind of crop production without or at least with a very low input of pesticides. Such an approach should support sustainability in agricultural development in The Netherlands. Ideas for pesticide-poor crop production systems are awaited.

This paper launches ideas for the development of pesticide-poor crop protection systems. Those ideas are based on the preposition that agricultural production systems should selfregulate possible attacks by harmful organisms. Negative feedback as a kind of selfregulation in agricultural production systems may result in an agriculture which considers pesticides to be nothing more than pest population correcting substances. That means that future crop protection becomes less pest and more production system oriented. Important issues of that idea are predisposition of crops and animals on the one hand, and production systems on the other.

Wageningen Agricultural University, Department of Ecological Agriculture, Haanweg 333, $6709 \mathrm{RZ}$ Wageningen, The Netherlands.
PLANT PROTECTION AND PESTICIDE POLICY AT CENTRAL AND STATE GOVERNMENT LEVELS IN INDIA

S. Nagarajan

In the last thirty years population of India doubled and yet the per capita availability of rice and wheat increased by 14 and $100 \%$, respectively. Judicious use of inputs as fertilizer, water, pesticides backed by social and policy incentives brought about the much needed agricultural transformation. From 1954 onwards, the demand for pesticides quickly increased as the high yielding varieties were pest vulnerable and the government launched the national malaria control programme. From the beginning, India emphasised the adoption of Integrated Pest Management (IPM) strategy to minimise dependance on pesticides.

The quality, quantity and type of pesticide manufactured and marketed is covered by the Insecticides Act, 1968, Government of India and the Pesticide Acts of the various state governments. So far 143 biological and chemical pesticides have been either provisionally or regularly registered.

The Destructive Insect Pest Act of 1914 and the 1989 promulgation order govern various procedures and issues related to plant quarantine. The guidelines issued by the Department of Biotechnology on genetically transformed plants, related guidelines on environmental protection and the ongoing debate on plant variety protection (PVP) are all directed towards sustained agricultural growth.

Twenty years of IPM research by ICAR and the 26 State Agricultural Universities have been field tested through Operational Research Projects (ORP), adaptive villages and various other programmes. The Central Plant Protection Training Institute and the IPM centers in each state carry the research results to the end users. Withdrawal of pesticide subsidy by the Center and State governments promoted rational use of ecofriendly pesticides, better spray appliances, biological control and adoption of good agronomic practices. People's concern for cleaner environment, resource degradation and the anxiety to blend organic farming with modern agriculture is restrieting the pesticide usage and promoting IPM. Several companies are now marketing plant based pesticides, biocontrol agents and seeds of new varieties that are disense resistant. The ecohazardous old pesticides have been replaced by newer ones that are relatively safe. The outcome of all these effort is, that pesticide usage in agriculture has been down sized from 56700 tonnes of active ingredient in 1988 to 55000 tonnes in 1993 .

Indian Council of Agricultural Research, Directorate of Wheat Research, Postbox 158, Kunjpura Road, Karnal - 132001, India.

CONTRIBUTION OF INTERNATIONAL PREVENTION AND EXCLUSION POLICY TO SUSTAINABLE CROP PRODUCTION

I.M. Smith

Plant quarantine, traditionally, provides a way of preventing expensive and sometimes catastrophic losses from introduced plant pests by taking administrative and hegal exclusion measures which are relatively cheap for the administrative and legal exclusion measures which are relatively cheap for the been explicitly considered, although it is probably true that the countries which need the import trade least also apply the strictest measures. However, the relatively simple situation which existed in the past has been transformed by new developments. The establishment of "common markets" in Europe, North America and elsewhere forces importing countries to justify the measures they take, both in terms of their own economies and in reaction to challenges from exporters. The recent conclusion of the Uruguay round of GATT trade negotiations extends this concept of justifi-cation to all trade in agricultural products between GATT contracting parties.

A new approach, known as PRA (Pest Risk Analysis), is applied to phytosanitary measures. It aims to ensure that the measures taken are commensurate with the risk. This forces National Plant Protection Organizations to consider the exaet nature and magnitude of potential losses from introduced quarantine pests, and also the full trade consequences of phytosanitary measures. The impact of these ideas is only beginning to be felt, but it will inevitably strengthen in years to come. Potential losses have often been evaluated in cerms of the additional costs of control, since it is only the most dramatic quarantine pests which cause uncontrollable losses. The question now arises, in the contex of sustainable agriculture, what sort of control?

Various examples will be considered, leading to the conclusion that the protection of sustainable agriculture calls for stronger and more effective plant quarantine. On the other hand, producing material for export to meet strict quarantine standards may also call on control methods which are incompatible with sustainable agriculture. The types of measures which are acceptable to exporting countries may also change as a result. Finally, PRA invokes various social and economic conse-quences of pest introduction going beyond immediate losses or costs. Managing the agricultural environment as a whole depends on its stabi-lity; all new pest introductions are destabilizing agents, threatening integrated pest management systems which have been painstakingly built up.

European and Mediterranean Plant Protection Organization, 1, rue Le Nôtre, 75016, Paris, France. 


\section{B. A. Federici}

Insect pests and disease vectors are subject to diseases of their own caused by bacteria, viruses, fungi, protozoans, and nematodes. Many members of these groups have been evaluated as control agents, but few have proven operationally successful. Most attack larvae, and are most effective against this stage. The principal agents evaluated include nuclear polyhedrosis viruses (NPVs) and granulosis viruses (GVs), various subspecies of the bacterium, Bacillus thuringiensis (Bt), the imperfect fungi Metarhizium anisopliae and Beauveria bassiana, protozoa (microsporidia) of the genera Nosema and Vairimorpha, and steinernematid and (meterorhabditid nematodes. Of these, only three subspecies of $B$. thuringiensis have heterorhabditid nematodes. Of these, only three subspecies of $B$. thuringiensis have
proven an operational and economic success in highly industrialized countries, proven an operational and economic success in highly industrialized countries,
though the use of steinemematid nematodes and nuclear polyhedrosis viruses is increasing due to the decreasing availability and effectiveness of chemical insecticides. In countries with low labor costs, insect viruses and fungi as well as Bt are used to control many insect pests.

Bt's success is due to its cost-effectiveness and relative ease of use. These characteristics result from Bt's ability to be mass produced on relatively inexpensive media, and its suitability for formulation and application using conventional insecticide technology. Over 100 commercial formulations of Bt are now available. Insecticide technology. Over 100 commercial formulations of Bt are now available.
Most are based on B. $t$. subsp. kurstaki, used against caterpillar pests; $B$. $t$. subsp. Most are based on $B$. $t$. subsp. kurstaki, used against caterpillar pests; $B$. t. subsp.
israelensis, used against mosquito and blackfly larvae; and $B$. $t$. subsp. tenebrionis, used against certain coleopterous pests such as the Colorado potato beetle. The steinernematid nematode, Steinernema carpocapsae, has proven effective against caterpillar pests in moist or wet habitats and treo-boring beetle larvae. NPV use is also increasing, especially against forest pests such as the gypsy moth, and caterpillar pests of field, vegetable, and floricultural crops such as the beet armyworm, and the cotton bollworm/leafworm complex. Other important viruses include the NPV of the velvet bean caterpillar used to control this pest on soybeans include the NPV of the velvet bean caterpillar used to control this pest on soybeans
in Brazil, and GVs of cabbage worms used to control these pests in China and other in Brazil, and GVs of cabbage worms used to control these pests in China and other
countries in southeast Asia. Imperfect fungi have been used to control beetle, countries in southeast Asia. Imperfect fungi have been used to control beetle,
caterpillar, and homopteran pests on several crops in some countries, but poor costeffectiveness limits use in most others. More efficient methods of mass production for fungi are needed to expand use.

Looking ahead in this decade and into the next century, it is clear that genetic engineering will have an enormous impact on pest control. NPVs have already been improved by engineering them to produce insect-specific neurotoxins, which lead to quick paralysis of pests and cessation of feeding damage. Improved Bis have also quick paralysis of pests and cessation of feeding damage. Improved Bts have also been developed through engineering to increase efficacy and activity spectrum.
Perhaps most important is the use of recombinant DNA techniques to engineer pest resistant plants. Cotton, corn, potatoes, tomatoes, and rice have aiready been made resistant to several important insect pests and viral diseases. Though as with any new technology there are likely to be problems during early phases of implementation, it is probable that in 30-50 years, many, if not most, major insect pests and plant diseases will be controlled by engineering plants for resistance.

Department of Entomology, University of California, Riverside, California 92521 USA

SUCCESSFUL BIOLOGICAL CONTROL OF THE MANGO MEALYBUG BY TWO EXOTIC PARASITOIDS IN WEST AFRICA

\section{P. Neuenschwander, A. H. Bokonon-Ganta, C. Boavida, J. J. M. van Alphen}

Two specific endophagous parasitoids, Gyranusoidea tebygi and Anagyrus mangicola, of Indian origin, were mass-reared at the International Institute of Tropical Agriculture (IITA) in Cotonou and released against the mango mealybug, Rastrococcus invadens, in collaboration with national biological control programs and other organizations. G. tebygi was widely established in Benin, Gabon, Ghana, Nigeria, Sierra Leone, and Zaire, as well as in Togo where it had been released and studied in another project. Without previous release, it spread into Congo and Côte d'Ivoire.

Following $G$. tebygi's establishment, mealybug populations collapsed and were virtually wiped out in experimental orchards in Benin. This was attributed to the fact that $G$. tebygi had an intrinsic rate of increase $(\mathrm{rm})$ twice the one of its host. Several indigenous hyperparasitoids were numerous and showed a strong density dependent response to their host. Indigenous predators proved to be of little importance. G. tebygi's impact was confirmed by exclusion experiments with paired sleeves. On a tree where $R$. invadens populations persisted, $r_{m}$ was higher and escape lower than on a little infested, neighbouring tree. This difference was attributed to plant genotype.

Country-wide surveys confirmed a substantial reduction of the pest to below-damaging levels in most localities and a corresponding increase in mango yields. This observation was substantiated in farmers' interviews. The mango mealybug disappeared almost completely from most host plants that had been infested before biological control was established. In large towns, however, $R$. invadens populations remained a problem on mango.

On these infestations, $A$. mangicola was later released. In the beginning, releases of adult $A$. mangicola were not successful in the savanna zone; parasitized mealybugs had to be used instead. By the end of 1994, A. mangicola was established widely in Benin and locally in Ghana, Sierra Leone and, perhaps, Gabon. In Benin, within a year, $A$. mangicola became about equally abundant as $G$. tebygi, demonstrating that the two parasitoids coexist and are complementary. In fact, $G$. tebygi reproduced more efficiently on younger hosts that exhibited less defensive behaviour than large hosts, while the larger species, $A$. mangicola., did better on older hosts.

IITA, B.P. 08 0932, Cotonou, Benin (West Africa)
Bigler F.

Biological control of insect and mite pests by insect parasitoids and predators is discussed in the context of: 1 . Classical biological control by introduction of exotic natural enemies, 2. Augmentation by periodic release of mass reared natural enemies and 3. Conservation and enhancement of indigenous natural enemies. The overview of classical biological control is based mainly on data compiled by Greathead (1986, 1992).

Figures on numbers of worldwide introductions with percent successes and failures are presented, numbers of parasitoid and predator species introduced against the array of pest species are mentioned and the successful introductions are shown for orders of pests. Data on the principal agents used in biological control introductions and the countries with the highest numbers of introductions are given. Controversal aspects as to the superiority of parasitoids or predators, the success rate of introductions on islands and continents, the suitability of inoculations against native or exotic pests, the importance of size and number of releases and the impact of the crop environment on success are addressed. Augmentation by periodic release of mass reared predators and parasitoids is exemplified by an overview of biological control in greenhouses and the use of the egg parasitoids of the genus Trichogramma. Market values of commercialized natural enemies are presented and a table showing the number of producers and suppliers of these agents for the different world regions is given. All predators and parasitoids used in greenhouse biocontrol which are available on the market are listed. The total world area treated with Trichogramma in 1992 is shown and the target crops are mentioned. More detailed information on the yearly use of Trichogramma in corn in Western Europe complements this part of the study.

Conservation and enhancement of natural enemies are discussed in the light of habitat management, i.e. vegetational diversity within crops and adjacent areas. Polycultures and monocultures are compared with respect to the enhancement of predators and parasitoids and examples are given. Current hypotheses of enemy enhancement in polycultures are listed and applied to other examples of multispecies plant associations.

The future and applicability, constraints and promoting factors of the different biological control strategies are summarized.

Bigler F., Swiss Federal Research Station for Agronomy, Reckenholzstrasse 191/211, CH-8046 Zurich, Switzerland

412

BIOLOGICAL PEST AND DISEASE CONTROL IN PROTECTED CROPS A SCIENTIFIC AND APPLIED SUCCESS

J.C. van Lenteren

Biological control of pests and diseases is a reliable method and an economically profitable endeavour for growers of greenhouse crops The fast evaluation and introduction of a number of natural enemies in situations wly re chemical control was either insufficient, impossible or undesired, has taught policy makers, extensionists, growers and crop protection specialists that biological control is a powerful option in pest control.

The total world area covered by greenhouses is about 200,000 ha. Greenhouses offer an excellent opportunity to grow high quality products in large quantities on a small surface area, but demand great investments in construction and management. Production of crops in greenhouses requires well-trained, intelligent growers who will not afford to risk any damage from pests for ideological reasons. If chemical control works better, they will certainly use it. In tomatoes, for example, chemical pest control represents less than $2 \%$ of the total overall cost of production, so these low costs do not stimulate to use alternatives. Yet despite the serious constraint that chemical control is so easy and inexpensive, implementation of biological and integrated control has been remarkably quick in greenhouses.

The main reason to start research on biological pest control in the 1960 s was the occurrence of resistance to pesticides in several key pests in greenhouses. Small scale commercial application of biological control in greenhouses was started in 1968 with the use predatory mites. Interest in using whitefly parasites occurred at the start of the 1970 s when enormous outbreaks of this pest took place in Europe. Biological control of these two key pests, whitefly and spider mite, is applied nowadays in more than 20 out of a total of 35 countries having a greenhouse industry. Since 1970 some 25 natural enemy species have been identified and are commercially used against greenhouse pests. The number of companies that produce natural enemies for use in greenhouses has increased from one in 1968 to more than 50 in 1995. Most of the natural enemies are employed in IPM programmes, with differences in use of other control methods per crop and per country.

Biological control was limited mainly to the control of insects until a few years ago. In North-West Europe as well as in Mediterranean countries, disease problems are considerable, particularly in tomatoes, cucumbers and cut flowers. A number of fungicides integrates well with the use natural enemies, but problems with fungicide resistance are strongly increasing. Disease management is now evolving towards strategies relying on the use of resistant cultivars and manipulation of the environment. During the past decade several initiatives have led to research in non-chemical control, such as the effect of soil solarization on nematodes and fungi, the role of suppressive soils in reduction of fungi and the potential of antagonistic leaf fungi.

Scientifically the benefits of biological control research have been very rewarding as well. While developing practical biological control we have generated a set of criteria for efficient pre-introductory evaluation of natural enemies. These criteria prevent spending research money on useless natural enemies. Also, stochastic, explanatory simulation models have been designed of crop - pest - natural enemy systems, which can be used for strategic decisions about natural enemy applications. 


\section{BIOLOGICAL CONTROL OF DISEASES}

\section{LM. WHIPPS}

Commercial biological control agents for pests and weeds have been used for several years but those for diseases have only become more widely available telatively recently. Most of these products are for the control of soil-bome pathogens, particularly those causing danping-off, although a Trichoderma preparation for control of the foliar pathogen, Botrytis cinerea, causing grey mould of a tange of crops is now registered in several councries. These recent commercial products were a result of a synthesis of knowledge derived from advances made in several areas. For example, the aetiology of the pathogens was clearly defined; the ecological and physiological properties and mode of action of the control agents were identified; the antagonists were targeted at specific stages in the life cycle of the pathogens; the production, formulation and application techniques were simple and gave control that was reproducible and cost-effective. Use of two different biological control agents and integration with reduced pesticide applications have also proved feasible. In addition, numerous groups are considering the use of genetic modification to improve activity of biological control agents. This has been stimulated by the success of "Nogall", Agrobacterium radiobacter strain K1026, a transfer-deficient deletion mutant which is commercially available for the control of crown-gall caused by Agrobacterium tumefaciens of several woody species. Future developments in these areas will be discussed.

Department of Microbial Biotechnology,

Horticulture Research International.

Wellesbourne,

Warwick,

Warwick,
CV35 $9 \mathrm{EF}$.

UK.
BIOLOGICAL CONTROL OF FUSARIUM WLLT BY FLUORESCENT PSEUDOMONADS

P.A.H.M. Bakkex, B.J. Duijff, M. Leemar, J.M. Raa1jmakers, H.A. van Pelt, I. van der Sluis, B. Schippers, and L.C. van Loon

Department of Plant Ecology and Evolutionary Biology, Section of Plant Pathology, Utrecht University, 2.0. Box $800.84,3508$ TB Utrecht, The Netherlands. Specific straine of fluorescent Pseudomonas spp. were demonstrated to
control fusarium wilt in carnatton and $1 \mathrm{n}$ radish. The mechanisms involved control fusarium wilt in carnation and 1n radish, The mechanisms involved in this control were studied using specific mutants lacking the traits to subsequent competition for ferrfc fron between Fusarlum oxysporum f.sp. subsequent competition for ferric lron between Fusarlum oxysporum E.sp. dianthi respectively F.o. f.sp. conglutinans and speciftc fluorescent
Pseudomonas spp. stralns was demonstrated to be involved in the suppression of wilt. However, in some cases mutants defective in the production of of wit. However, in some cases mutants defective in the production of
their fluorescent siderophore stili significantly suppressed the disease, their fluorescent siderophore stili significantly suppressed the disease, suggesting another mechanism to be Involved. In a special experiwental setup, the pathogen was spatially separated from the applied pseudomonads and this spatial separation was confirmed to persist throughout the experiment. Some strains significantly reduced disease incidence in this system,
suggesting that they systemicaliy induce resistance. The possible fnvolvesuggesting that they systemicaliy induce resistance. The possible fnvolve
ment of the o-antigenic side chain of the 11popolysaccharides of some ment of the 0-antigenic side chain of the 11popolysaccharides of some
Pseudomonas strains in this phenomenon was studied using purified LPS and Pseudomonas strains in this phenomenon was studied using purlfied LPS and
mutants of the stratns, lacking the O-antigenic side chain. In these expemutants of the stratns, lacking the 0-antigenic side chain. In these expe
riments it was demonstrated that the LPS indeed is involved in induction riments it was demonstrated that the LPS Indeed is involved in induction
of systemic resistance. When Iron avaliability for the pseudomonads was of systeafc resistance. When Iron avaliabllity for the pseudomonads was
lowered In this experiment, suppression of disease was also observed for the mutants lacking the 0 -antigenic side chain. Thus, it appears that also iron-regulated metabolites of these bacterla have the ability to induce reststance. Currenty the involvement of salicylic actd and pyoverdine, both produced under low fron avaflability, is being studied.

In a long term fteld trial with radish a pseudomonas fluorescens strain suppressed fusartum wilt significantiy and increased marketable radishes with an average of $44 \%$. Based on these favourable results a comercial product was formulated by S\&G seeds and is sold now commercially in the Netherlands, under the name "Biocoat".

416

THE USE OF NEMATODES AS BIOLOGICAL CONTROL AGENTS.

\section{Fisher, C.M}

The demand for biological control agents has increased due to chemical pesticide effects on non target organisms, residues on food crops, ground water contanination and the development of insect resistance. Entomopathogenic nematodes of the genera Steinernema and Heterorhabditis have been used commercially as biological control agents in Europe and the USA for a number of years. These nematodes can be mass produced using conventional fermentation techniques. Research has shown that nematodes can control a wide range of pests, however they are only routinely used to control a few pests such as black vine weevil (Otiorhynchus sulcatus) and sciarid flies (Bradysia paupera and Lycoriella corripita). Recently attention has been focused on the use of the slug parasitic nematode Phasmarhabditis hermaphrodita which has been launched as a product in the UK.

This paper will discuss the use of nematodes as biological control agents with reference to their biology, ecology, production and formulation.

MicroBio Ltd, c/o Horticultural Research International, Worthing Road, Littlehampton, West Sussex, United Kingdom, BN17 5HE. 
NEW DEYELOPMENTS IN THE MODE OF ACTION OF PEROXIDIZING HERBICIDES

Peter BÖGER

This class of herbicides inhibits biosynthesis of chlorophyll and leads to accumulation of its precursor protoporphyrin IX. Light activation of this tetrapyrrole results in activated oxygen species which induces the breakdown of cell components. The talk will emphasize (1) enzymic modification of cyclic isoimides in the plant yielding products of higher phytotoxicity, (2) explain our fundings on safeners and (3) will present some data as to how excess protoporphyrin IX is produceed in the cell.

University of Konstanz, D-78434 Konstanz, Germany, Fax: $+49-7531-883042$

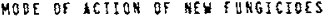
P. LEROUX

Many protectant fungicides, used since sovoral decades, are non spocific Inhibitors of nergy production. Systemic fungicioes, acting specificaliy on the respiratory chain have also baen found. The carboxaldes uhich are nainly effective touards basidonycotos, Inhibit the succinate-ubiquinone reductase; the most racant ones (og. flutolanil, trifluaztas) are highly toxic to Rhizoctonia sp. The stobilurine dorivativas (analogs of toxic entabolites producod by soveral agarics) aro promising broad spectrue fungicides which inhibit the ubiquinone cytochrome c roductase. The experinental anti-oanycotos dimefluazole (benzlaidazolo-sulfonanido) has also the sale target site.

Microtubulos are constituents of spindle and cellular cytaskeloton uhose inhibition loads to the arrest of mycolial grouth. The antimlarotubular effect of - ither benziaidazolos (eg. carbendazla) or phonylcarbanates (eg. diothofencarb) whlch exhiblt negative cross resistance, is probably due their oinding to tubulin, the min component of these nicrotubules. Zarilonido, a benzalide derivative a ainly offoctive tovards oonycotes, and poncycuron, a substituted uraa highly toxic to Rhizoctonia solant, are also inhibitors of icrotubule foration. rludioxonil and fonplicionil are synthetic phenylpyrcoles rolated to pyrrointerin, an antifungal compound produced by soveral bacteria. Thase phanylpyrroles have propertios siailar to those of dicarboxinides (og. iprodiona) or naromatic hydrocarbons" (og. quintozeno). In particular, all these compounds extitit positive cross resistance in laboratery nutants of eany fungal spoeies. The biochouical studios suggest that tho phenyipyrrolos intorfore vith transportassociated phospharylation of glucose.

The anillnopylaidinos (eg. cyprodinil, mepanipyrie, pyriecthanil) are new broad spectrua fungieldeg. They provent the secretion of bost-cell wall degrading enzyogs by fungt. Noditionnally they probably interfore with the biosynthesis of nethionine.

from this survey, it appoars that several nev types of highly active fungicides have been recently discovered. Sowe of then alj] be good alternatives or partners to the inhibitors of sterol biosynthese (SBIs), one of tho cain group of agricultural fungicides.

InRA. Unité de phytophermacie des Mádateurs Chimiques.

78026 versafles codex - Irance.

420

MODE OF ACTION OF THE NOVEL RICE BLAST FUNGICIDE KTU 3616(PROPOSED COMMON NAME : CARPROPAMID)

2-ARYLPYRROLES: A NEW CLASS OF INSECTICIDES STRUCTURE, ACTIVTYY AND MODE OF ACTION

David A. Hunt

The development of a new series of insecticidal pyrroles based on the natural product dioxapyrrolomycin and culminating in the current development candidate AC 303,630 is described.

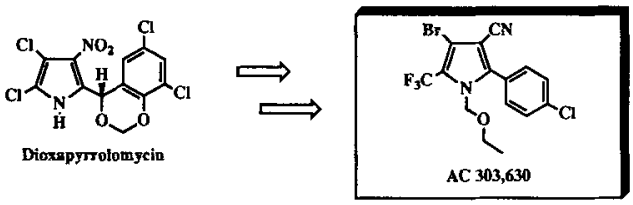
In particular, mode of action studies and structure activity comparisions are presented for an array of halogenated and triflucromethyl-substituted 2-arylpyrroles with the genezal formula 1 . This ne
class of insect control agents has been demonstrated to be active on a broad spectrum of insect pests.

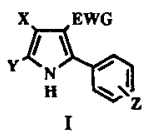

American Cyanamid Company, Agricultural Research Division, P.O. Box 400, Princeton, NJ 08543-0400, USA rice blast into cellophane membrane.
Y. KURAHASH, T. HATTOR

Nihon Bayer Agrochem K.K., Yuki Research Centre,

9511-4 Yuki, Yukj-shi, Ibaraki, 307 Japan

S. KAGABU

Gifu University, Faculty of Education, Gifu 501-11, Japan

\section{R. PONTZEN}

Bayer AG, Crop Protection Division, D-51368 Leverkusen, Germany

KTU 3616 (proposed common name : carpropamid) $\{(1 R, 3 S)-2,2-$ dichioro- $N-[(R)-1-$ (4-chlorophenyl) ethyl -1 -ethyl-3-

methylcyclopropanecarboxamide\} provides outstanding protective efficacy with systemic properties in the control of rice blast, and was jointly developed by Bayer AG and Nihon Bayer Agrochem K.K. In

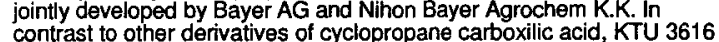
contrast to other derivatives of cyclo
shows a specific mode of action.

In culture tests of various plant pathogenic fungi and bacteria, KTU 3616 did not show any remarkable activity, but in several fungi the colour of the mycelium was changed. Spore germination and appressorium formation of Pyricularia oryzae was not influenced substantially but pigmentation of appressorium cells was strongly inhibited leading to pigmentation of appressorum cells was strongly inhibited leading to transparent appressoria. These results suggested KTU 3616 to be a
melanin biosynthesis inhibitor like tricyclazole, preventing the blast melanin biosynthesis inhibitor like tricyclazole, preventing the blast
fungus from penetration into the rice epidermal cell. In fact it was shown that KTU 3616 was an effective inhibitor of appressorial penetration of

in order to exactly characterize the target site within the melanin biosynthesis pathway a liquid culture of Pyricularia oryzae was treated

with KTU 3616 . Analysis of the culture filtrate by thin layer
chromatography demonstrated a strong accumulation of scytalone, an chromatography demonstrated a strong accumulation of scytalone, an
intermediate of fungal melanin biosynthesis. Further biochemical studies intermediate of fungal melanin biosynthesis. Further biochemical
with a crude enzyme extracted from blast mycelium showed that dehydration of scytalone to $1,3,8$-trihydroxynaphthalene was strongly inhibited by KTU 3616 . In culture tests using an albino blast strain the dehydration of vermelone to 1,8-dihydroxynaphthalene, a later step in the melanin blosynthesis pathway, was also blocked. It is concluded that the primary mode of action of KTU 3616 is the inhibition of melanin the primary mode of action of Kiosynthesis by blocking the dehydration of scytalone and vermelone. biosynthesis by blocking the dehydration of scytalone and vermelone. This is a novel mechan
biosynthesis inhibitors.

Nihon Bayer Agrochem K.K., Yuki Research Centre, 9511-4 Yuki, Yuki-shi, lbaraki, 307 Japan 


\section{THE CHALLENGE OF MICROENCAPSULATING SOLID ACTIVE INGREDIENTS}

\section{Hans-W. Hässlin ${ }^{(1)}$ and Rudolf Nastke ${ }^{(2)}$}

Microencapsulation of liquid pesticides mainly done by interfacial polycondensation is well established. Several products are on the market from different companies.

The microencapsulation of solid active ingredients by use of amino resins is a very challenging task as will be shown in this paper.

In the presentation the relevant scientific and technical questions of this process will be touched, which are:

- chemistry and interfacial activity of precursor polymer

- the process of wall-formation

- influence of reaction conditions on microcapsule properties

- multi-coating process

- a.j.-release properties from microcapsules produced by different approaches

- melt encapsulation

- influencing microcrystallinity of melt encapsulated active ingredients

(1) CIBA-GEIGY Limited, Crop Protection Division

CH-4333 Münchwilen, Switzerland

(2) Fraunhofergesellschaft (FhG), Institut for Applied Polymer Research, D-14504 Teltow, Germany
FORMULATION AND PACKAGING TRENDS IN TODAY'S INDUSTRIAL ENYIRONMENT

B. Frei, P. Nixon and P.A. Urech

The continuous evaluation and change in the crop protection business is characterized by several driving forces, 1. Pressure on use intensity of agrochemicals, 2. Stagnating markets, several drivo forces, 1 . Pressure on use 3. Safety to Decreasing margins, and 7. Legislation. This all means increasing economic pressure, and the crop protection therefore has to optimize all aspects of the business chain, from development over production to disposal.

Formulations and their packaging determine the delivery of products and their ultimate use at farm level. The social groups, which are active in the last part of the supply chain, are the custom formulators, packaging industry, distributors and farmers. In terms of finished goods, these groups have different, often conflicting, goals and needs.

\begin{tabular}{|c|c|c|}
\hline $\begin{array}{l}\text { Farmers } \\
\text {.Ease of use } \\
\text {.Fast and precise } \\
\text { measurement } \\
\text {.Safe to crops } \\
\text {.No waste, easy disposal } \\
\text { Safe use } \\
\text {.Safe to environment } \\
\text {.Cheap solutions } \\
\text {.IPM calls for fast } \\
\text { delivery }\end{array}$ & $\begin{array}{l}\text { Reguintion } \\
\text { Encourages packaging } \\
\text { waste reduction } \\
\text {. On farm regulation for } \\
\text { waste management } \\
\text {.Less solvents in the air } \\
\text { management } \\
\text { Industry to collect } \\
\text { containers } \\
\text {.Environment protection } \\
\text {.Safety in storage } \\
\text {.Safe for user }\end{array}$ & $\begin{array}{l}\text { Industry } \\
\text {.Clean environment } \\
\text {.Low cost of supply chatn } \\
\text {.Safe in storage and transport } \\
\text { Safe to crops, user, men } \\
\text {. Reduction of number af items } \\
\text {. No issues } \\
\text {.Differentiation from competitor } \\
\text {.JIT production and delivery } \\
\text {.Low stocks, few supply points } \\
\text {.Innovation } \\
\text {.Avoid overcapacity in } \\
\text { formulation and packaging } \\
\text {.Avoid non controlled } \\
\text { disposal of used containers }\end{array}$ \\
\hline
\end{tabular}

Conclusions. 1. Regulations are becoming more severe which forces industry and distribution to think about how these new needs can be met satisfactorily and efficiently. 2. Solvtions are actually being developed which integrate producers, distributors, and farmers. 3. Within industry new standards are being established. 4. Formulation and packagino their design ingredient is no longer the only success factor in the business chatr.

Formulations and packaging is an area where crop protection is changing fast. There are basically three areas in which this is confirmed. 1. Delivery (less products $>$ less risks, faster delivery $>$ refillables, less waste $>$ supply points). 2 . Design, innovation (safety improve ments (reduced worker exposure), user friendliness, WGs, water based formulations). 3 . Attitude (design is now more influenced by marketing, recognition that formulation and packaging is important and should be considered as a single entity, the user is in focus, new regulations are pushing innovation, learning from other industries).

In the paper, examples are presented showing innovative means and ways in order to cope with the new challenges and opportunities. In particular water dispersibje granules cope with the (WG) and water based formulations as well as the progress in new packaging concepts, such the fungicide TLLT as one of the examples.

Ciba-Geigy Limited, Crop Protection Division, CH-4002 Basel, Switzerland
423

\section{KTU 3616: A NOVEL, FUNGICIDE FOR RICE BLAST CONTROL}

\section{G. HANSSLER}

Bayer AG, Crop Protection Division, D-51368 Leverkusen, Germany

\section{T. HAT'TORI}

Nihon Bayer Agrochem K.K., Yuki Research Center, 9511-4 Yuki, Yuki-shi, Ibaraki, 307, Japan

\section{U. KRAATZ, J. KONZE}

Bayer AG, Crop Protection Division, D-51368 Leverkusen, Germany

\section{S. KAGABU}

Gifu University, Faculty of Education, Gifu 501-11, Japan

\section{ABSTRACI}

KTU 3616, a cyciopropanecarboxamide (proposed common aаme: carpropanid) is a novel fungicide for controlling rice blast caused by Pyricularia oryzae. It is characterized by a unique structure discovered, patented and developed jointly by Nihon Bayer Agrochem K.K. and Bayer AG. The compound has a good toxicological profile having a low toxicity to mammals and aquatic wildlife

KTU 3616 has been effective in field trials when applied either as a spray or dust to the foliage or as granules to the paddy water or to the rice seedling boxes prior to transplanting A dose of $100-200 \mathrm{~g} \mathrm{ai}$. / ha provided very high control of both leaf and panicle blast when either a SC-formulation or a DP-formulation was applied using the standard intervals. The systemic action and the long lasting effectiveness make KTU 3616 an outstanding product for the treatment of rice in seedling boxes before transplanting. Only one application $(2 \mathrm{~g} \mathrm{ai}$. $/$ box $=400 \mathrm{~g} \mathrm{ai}$. $/ \mathrm{ha})$ given at this time may control not only rice leaf blast but also panicle blast. KTU 3616 has shown excellent plant safety during all stages of the rice crop. The biochemical target of KTU 3616 seems to be the melanin biosynthesis pathway of Pyncularia oryzae.

As a single compound KTU 3616 will be avallable as SC and DP for foliar application and as GR for seedling box application.
424

\section{PLANT ACTIVATOR CGA 245704. A NEW TECHNOLOGY FOR DISEASE MANACEMENT}

W.Ruess . W.Kunz, Th. Staub. K.Müller, N.Poppinger, J.Speich, P.Ahl Goy Ciba-Geigy AG, Crop Protection Otulsion, CH-4002 Basle, Switzerland

CGA 245704 (Benzo [1,2,3]thiadiazole-7-carbothiolc acid S-methyl ester) in an chemical plant defense activalor representing a fundamentally new technology for disease management in agricultural crops. It has been discovered and developed by the Crop Protection Division of Ciba-Geigy.

The compound acts Indirectly by Inducing the self-defense mechanisms of plants. It thereby mimics a natural phenomenon known as Systemic Aquired Resistance (SAR). CGA 245704 is not a funglcide and shows no in vitro activity. In contrast to the naturally occuring SAR which is not predictable in time and intensity, the application of CGA 245704 can be timed such that it triggers the activation of plant defense mechanisms according to disease management requirements.

SAR provides protection against a broad range of fungi and bacteria. Level and spectrum of defense reaction triggered by CGA 245704 makes the compound particularly useful for pothosystems in wheat, rice, tobacco and vegetable crops. A single application of CGA 245704 at $30 \mathrm{~g}$ si/ha to wheat at the 2-node crop stege geve high level of protection apalnst Erysiphe araminis for 6-9 weeks up to ear emergence. If also provided some supression of Septoria spp and Puccinia spp. Histological studies with E.graminis on wheat indicate that the plant defense reaction interferes at multiple sites during the pathogen life cycle from penetration onwards.

Long lasting protectlon of 10-12 weeks is achieved agalnst rice leaf blast (Pyricularia oryzae) by a single application of $1 \mathrm{~g}$ ai to the seedling box just before trangplanting. At use rates of 10-50 $\mathrm{g}$ al/ha applied in 10-20 day intervals good protection was achieved agalnst blue mold (Peronosprora tabacina) on tobacco and downy mildew. leafspot and bacterial diseases in vegetable crops.

Because of a certaln lag-time required for activation of the plant defense mechanisms the applications of CGA 245704 have to be made protectively or early in the disease progress.

Since there is no direct effect of the compound on the pathogen, the development of fungal resistance is very unlikely. CGA 245704 is therefore a valuable tool to be included in anti-resistance strategies.

The plant activator CGA 245704 represents a breakthrough in disease management and is a suitable component for integrated crop protection and sustalnable agriculture.

Ciba-Geigy AG, R-1001A.218, CH-4002 Basle, Switzerland 
ECOLOGY OF GENETICALLY-MODIFIED BACTERIA AS A PRELUDE TO A FUNCTIONAL RELEASE FOR PEST CONTROL

\section{L.M.WHIPPS, F.A.A.M. de Leij, J.M. Lynch and M.J. Bailey*}

Through techniques of genetic engineering, microorganisms can now be modified to express novel traits. Thus, depending on the genes transferred or modified, numerous microorganisms can potentially be modified to act as biological control agents of pests and diseases. In terms of biological control, these genetically modified microorganisms (GMMs) should be able to establish and survive in the target ecological niche and provide long-term control. However, use of such ecologically robust GMMs is not without risk to the environment and, before widespread commercial use is possible, a thorough environmental risk assessment is required. A logical initial step in this process, would be to examine the ecology of a microorganism with potential for finctional modification but which is modified solely with marker genes, rather than a functional gene. Use of marker genes would enable monitoring of survival, establishment and dissemination of the GMM and gene exchange and, when combined with studies of the impact of the GMM on other microorganisms and processes, would provide a portfolio of baseline data of use for future development of functionally modified microorganisms. Over the past five years, HRI in collaboration with IVEM, Oxford has been carrying out such a study which has resulted in the first field releases of a doubly chromosomally-marked genetically-modified bacterium (Pseudomonas aureofaciens) onto wheat and sugar beet. Aspects of this wotk will be discussed.

Department of Microbial Biotechnology,

Horticulture Research International,

Wellesbourne,

Warwick,

CV35 9EF,

UK

* Institute of Virology and Environmental Microbiology, Mansfield Road, Oxford, OX1 3SR, UK

\section{SPECIFICITY OF BACILLUS THURINGIENSIS TOXINS.}

D. Bosch, B. Schipper, H. van der Klei, R. de Maagd and W.J. Stiekema.

To contribute to safeguard the longterm use of Bacillus thuringiensis $(\mathrm{Bt})$ in crop protection, we study the mode of action of Bt crystal proteins (Cry)

$\mathrm{CrylC}$ and $C$ ryiE are both toxic to lepidoptera, but each protein has its own specificity profile. For example, CrylC is much more active against Spodoptera exigua and Mamestra brassicae whereas both proteins are toxic to Manduca sexta. In order to identify regions in these proteins involved in insecticidal specificity, hybrids between crylC and crylE were generated by in vivo recombination. Analysis of the hybrid proteins showed that domain III of CryIC is involved in the toxicity towards 5 . exigua and $M$. brassicae which is lacking in CrylE. Transfer of this domain to CrylE resulted in mutant protein G27 with broader activity spectrum. Although the specificity of these proteins is apparently determined by domain III, heterologous competition experiments with 5 . exigua brush border membrane vesicles demonstrated that receptor binding followed domain II. $G 27$ and $C$ rylE recognized the same binding sites on midgut membranes and these sites differ from those recognized by $\mathrm{CrylC}$. Since $G 27$ and CrylC are equally active but bind to different receptors, G27 might be used in resistance management programs as an alternative for CrylC. For example, when S.exigua develops resistance towards $\mathrm{CrylC}$, by changing receptor binding sites, the insects could possibly still be controlled by $\mathrm{G} 27$

Preliminary experiments demonstrate that domain 11 of $\mathrm{CrylC}$ is, similarly as is the case with CrylE, capable to improve the toxicity of CrylAb towards S.exigua. These results show that domain ill of Cry proteins play an important role in the specificity and toxicity level to target insects, and that substitution of domain III may be a powerful tool to increase the repertoire of available toxins to control pest insects

Centre for Plant Breeding and Reproduction Research (CPRO-DLO), P.O. Box 16, 6700 AA Wageningen, The Netherlands.
APPLICATION OF GENETICALLY MODIFIED MICROORGANISMS IN CROP PROTECTION

L.D. van Elsas and L.S. van Overbeek

Microorganisms such as bacteria and fungi are increasingty being employed as biological alternatives to chemical agents in intensive agriculture. However, their application has met with varying degrees of success and consistency. Factors controlling the efficacy of biocontrol agents are mainly reizat to their ecology following release into a soil/plant system, in particular their survival and specific activities.

Genetic modification offers exciting possibilities for improvement of strains for biological control; strains with superior survival characteristics can be engineered so as to conditionally express a betreficial trait in a soil/plant system, waranting optimal persistence and conditional induction of this function. To this end, bacterial promoters which are differentially expressed under the influence of envirommental triggers bave been screened for by using promoter probe under the influence of envirommental triggers bave been screened for by using promoter probe
transposons with reponer genes. This way, reporter gene expression triggered by prevailing transposons with reponter genes. This way, reporter gene expression triggered by prevailing reponter gene activity has enabled in situ specific gene expression to be monitored. Thus, specific responses to both compound limitation (bulk soil) and the presence of specific compounds (rhizosphere soil) thave been identified. In addition, the response of bacterial populations in soil to nuerient scarcity has been sudied by following the physiological adaptations with regard to stress resistance inoculant cells undergo when confronted with soil conditions. Results with Pseudomonas fluorescens have shown that survivors in introduced populations quickly adapt to soil, leading to an overall enhanced cellular resistance to a variety of stresses. These findings bave a great bearing on the release of genetically modified bacteria into soil.

The application of $P$. fluorescens modified with differentially induced crystal protein or phenazine synthesis genes for the control of soil-borne insect larvae or fungal pathogens will be discussed as paradigms of genetically modified bacteria applied in crop protection.

Deparment of Soit Biotechwology, IPO-DLO, P.O.Box 9060 , 6700GW Wageningen, the Netherlands

428

\section{POTENTIAL OF GENETICALLY ENGINEERED BACILLUS THURINGIENSIS FOR INSECT CONTROL}

\author{
W. D. Gelernter and G. E. Schwab
}

The use of products based on naturally occuring isolates of Bacillus thuringiensis ( $B$ ) has gradually increased over the past io years due to development of insect resistance to conventional synthetic insecticides (and the corresponding lack of other insect control methods) and to growing legal and social requirements for safer pesticides. Despite this modest growth, products based on naturally occurring isolates of $B t$ are used primarity in specialized situations and have not enjoyed widespread adoption among growers. This is largely due to performance limitations such as brief residual activity, narrow host range and poor delivery to cryptic insects.

The tool of genetic engineering has now been avallable to Bt researchers for almost 15 years. In this paper, we will explere the extent to which the use of genetic engineering has corrected the limitations and expanded the markets for naturally occurring $B$ ts through a review of research, development and commercialization progress to date. In addition, we will discuss the potential for genetically engineered $B t$ products of the future.

\section{Mycogen Corporation, 4980 Carroll Canyon Road} San Dlego, CA 92121 USA 
ENGINEERING OF ENTOMOPATHOGENIC FUNGI AND ITS POTENTIAL FOR INSECT CONTROL

G. RIBA, Y. COUTEAUDDER, M. VLAUD, C. NEUVEGLISE, P. MAURER

The success of several fungal biopesticides prouved to be agronomically and economically comperitive offers a new chance in order to entarge the use of entomogenous Hyphomycetes.

Fungal molecular biology, just at its first steps, is yet very informative for a better understanding of mode of action, strain monitoring and strain characterization.

Cuticular penetration of fungi is the most important limiting factor of pathogenesis. It is characterized first by appressortum differentiation then by specific proteic excretions of hydrophobin-like protein and cuticle-degrading proteases $\left(\mathrm{PR}_{1}, \mathrm{PR}_{2}\right)$ and lipases. Penetration is also the key factor of tost specificity. Toxemic activity is an important process for host invasion since an over expression of entomotoxic proteins significantly reduces LT 50.

A better knowledge of genomic plasticity and chromosomic or genic recombinations through parasexual cycle and transposition will be highly useful to identify key functions of pathogenesis and to select strains with enhanced winulence. Repetitive DNA elements presenting the characteristics of active transposable elements have been identified in entomopathogenic fungi and stable hypervirulent recombinants can be obtained by forced parasexuality.

Complementarly, molecular biology provides tools for isolates characterization which is important for three applications : identify registrated or patented strains used in mycopesticides; establish risk assessment for agronomic use of natural or genetical manipulated fungal strains ; enable monitoring of environmental impact. Performant strategies, mainly based on PCR amplification are now allowing the further development of sensitive, fiable and specific detection methods that will effectively contribute to answer these ecological questions.

431

\section{GENETIC ENGINEERING OF BIOSAFE BACULOVIRUS INSECTICIDES Just M. Ylak}

Baculoviruses are insect pathogens that cause fatal disease in larvae of insects, mainly members of the Lepidoptera and Hymenoptera family. They have great potential as biological insect control agents, due to their safety for non-target organisms, their natural role in regulating the size of insect populations, their ease of production and the absence of noticeable resistance. A major limitation to a more wide-spread use of baculoviruses as insect control agent is their relatively siow speed of action as compared to chemical insecticides, in particular in crops with low damage thresholds. Upon infection insect stop feeding only after a few days, whereas immediate insecticidal effect is often required. In addition, baculovinuses have a restricted host range, which makes them less attractive commercially as a 'kills all' insecticide. In some cases they possess low virulence in particular against older instar larvae.

The current knowledge of baculovirus gene structure, function and regulation and the availability of suitable cell culture systems have allowed the modification of the viral genome by genetic engineering techniques. Strategies to improve the speed of action, for example, involved the introduction of genes coding for insect-specific toxins, hormones and enzymes into the baculovirus genome. Some of these modifications have resulted in baculovirus recombinants with enhanced speed of action and improvements are still being made. In one instance the deletion of a viral gene disturbed the interaction between virus and insect larva resulting in the premature death of the insect.

The combination of the regulated expression of a highly specific insecticidal toxin protein with the specificity of the baculovirus presents an attractive concept for the engineering of baculovirus recombinants with improved insecticidal properties. Although baculoviruses have a perfect safety record over many decades, the genetic modification calls for re-assessment of their safety in view of the potential risks associated with a large scale release in the environment. Engineering strategies to secure the biosafety of engineered baculoviruses, for example by reducing the ecological and environmental fitness of the virus without affecting their virulence, have been developed. The potential risks associated with relense of genetically modified baculoviruses in the environment as well as strategies to improve their biological safety will be discussed. The current thinking is that enhancement of the insecticidal activity of baculoviruses is an asset from the insect control perspective and at the same time a safety belt, since it reduces their ecological and environmental fitness.

This work was sponsored, in part, in the framework of BRDGE project Biot-CT910291 (Biosafety of genetically modified baculoviruses for insect control).

Department of Virology, Wageningen Agricultural University, Binnenhaven 11, 6709 PD Wageningen, The Netherlands (phone: $31-8370-8390$; FAX: 31-837084820; E-mail: just.vlak@medew.viro.wau.nl)
POTENTIAL OF ENGINEERING ENDOSYMBIONTS FOR CONTROL OF INSECTS

Richard Stouthamer

Recently we have come to the realisation that the majority of insect species carry symbiotic bacteria. The association between symbionts and host can range from the very specific such as the associations that are found between aphids and their Buchnera symbionts, to a more loose association as is the case for many insects and their Wobachia symbionts. The fact that many insects carry symbionts that somehow are essential for their host or influence the hosts reproduction opens up new possibilities of insect control through the manipulation of their symbionts. These manipulations can range from the very simple such as killing the symbionts by specific antibiotics to complex by genetically altering the symbionts to render their insect host less capable of causing injury to the crop. In this presentation I will give some examples of some of the manipulations of these symbionts to improve crop protection.

Department of Entomology, Wageningen Agricultural University, P.O. Box 8031,6700 EK Wageningen, The Nethertands
FIELD EFFICACY OF RECOMEINANT BACULOVIRUSES WXTH IMPROVED INSECTICIDAL PROPEATIES

$$
\text { J. S. Cory }
$$

In forests, or in other crops with high damage thresholds, insect specific baculoviruses have been used with success. However, in more sensitive systems, baculoviruses can act too slowly to prevent some crop damage occurring. Baculoviruses have thus become a prime target for genetic modification with the aim of increasing the1r speed of action to make them more competitive with synthetic chemical insecticides. This goal has primarily oeen addressed by synthe tic chemical lnsecticides. This goal has primarlyy oeen addressed by hormones into the baculovirus genome with the afm of producing a more rapid cessation ln feeding through paralysis or distuption of lnsect development. To date, a number of constructs have been created which have had varying success in terms of enhanced activity in laboratory assays. Some additional genes have falled to al ter baculovinys activity, whereas the most successful have produced a $20-40 \%$ increase in speed of kil. One of the most promising constructs is a nucleopolyhedrosis virus from the alfalfa looper, Autographa from the venom of the North Afrdcan scorpion Androctonus australis. The toxin is selectively toxic to certain species of insect and acts by irreversibly aitering sodium channel conductance of axons, leading to paralysis and eventual death. Laboratory assays using a sensitive host, the cabbage looper, Trichoplusia ni, showed that infection with the recombinant ACNPV expressing the scorpion toxin (ACST-3) resulted in a $25 \%$ reduction in time to death, compared to the wild-type parent virus. However, both pathogenicity and host compared to the wild-type parent virus. However, both pathogenicity and host range were unaltered. The virus was tested in the field in as the target host. The trial showed that AcST-3 kiIIed the using $r$, ni as the target host. The trial showed that AcST-3 kfiled the insects wore rapldy, resulting in a significant reduction in crop damage as compared to the wild-type virus. Early collections of insects from the fleld site (before any larvae had died from virus) showed that mortality levels from the viruses were not significantly different. However, the final sample from the ffeld showed that significantly more survivors were present in the recomcycling in the recombinant treated plots due to differences in virus yield and distribution after the epizootic.

It is also important to examine any environmental consequences which might result from the release of genetically modified baculoviruses. A key area of importance is their effect on susceptiole non-target hosts. Baculoviruses differ in the bredth of their host range, some viruses appearing monospecific whereas others will infect a range of species. These hosts are not equally susceptible. Laboratory tests indicate which species may be infected under ideal conditions, however, they tell us little about the invasive potential of baculoviruses in the field sitwation. Further field experiments designed to estimate virus transmission in permissive and semi-penissive hosts will be described as part of our framework for risk assessment of genetically modified baculovirus insecticides.

NERC Institute of Virology and Environmental Microbiology, Mansfield Road, OXFORD, OX1 3SR, UK 


\section{Marcel Dicke}

Plants can defend themselves against herbivores in 2 ways:

(i) directly through characteristics that negatively affect the herbivore such as trichomes and toxins;

(ii) indirectly through characteristics that enhance the effectiveness of carnivorous enemies of the herbivore, such as structures for shelter or the production of attractants.

In the past decade it was shown that plants can respond to herbivore damage by initiating the production of volatile chemicals that attract carnivorous arthropods: plants thus recruit bodyguards' after damage to their tissues occurs. This phenomenon has been reported for a range of plant species, including many important agricultural crops, and in response to a a range of plant species, including many important agricuitural crops, and in response to a range of herbivorous arthropods that include many important pest species. The natura
enemies that have been shown to utilize these 'SOS-signals' comprise predators and many enemies that have been show
species of parasitic wasps.

species of parasitic wasps. that are used in biological control. They have to find their herbivorous victims that have been under natural selection to reduce the emission of cues that may attract their enemies. Experiments show that carnivores usually hardly perceive any volatiles emitted by their victim. However, the plant betrays herbivore activities. The fact that the plant produces valatiles in reponse to herbivore than the herbivore itself: the 'SOS-signals' are produced by the infested leaf as well as by uninfested leaves of the infested plant.

This interesting phenomenon has received much attention from scientists in the field of biological control. However, since it has been shown that cultivars within a crop may differ in the degree to which they produce the 'SOS-signals' it may be of significant interest to plan breeders also. Through plant breeding programmes crop cultivars may be selected that contribute maximally to the success of a biological control programme. In coordinating the activities of plant breeders and biological control specialists, new and exciting options for environmentally friendly pest control are available.

I will present data to (i) illustrate the phenomenon of plants recruiting bodyguards, (ii) show the effects of different cultivars within a crop and (iit) how plant breeders may select cultivars that 'cry for help' rather than remain silent.

Department of Entomology, Wageningen Agricultural University, P.O. Box 8031, Wageningen, The Netherlands

\section{S. D. Eigenbrode}

Plant surface features can have large effects on the behaviors and efficiencies of entomophagous insects. Leaf form, leaf hairs (both glandular and nonglandular), and surface waxes can influence the third trophic level. In several cases the genetic control of these characteristics is understood, opening the way for deliberate manipulation of plant surface attributes to enhance biological control of pest herbivores (EB). The development of cucumbers with hair densities designed to maximize the efficiency of Encarsia formosa attacking Trialeurodes vaporariorum is a successful undertaking that. illustrates this potential. There is imminent potential in other systems as well. Surface lipids of Brassica can strongly influence the behaviors and effectiveness of generalist predatots and a hymenopteran parasitoid. Classical breeding and molecular technology now permits manipulation of leaf surface lipids of Brassica plants, with the potential to adjust these to maximize the efficacy of the third trophic level. Other crops with prominent surface lipid structures may be amenable to this approach as well. Features such as leaf shape, curling response to damage, and hair density also have the potential to be manipulated successfully to enhance biological control in several crops.

Development and deployment of host plant resistance (HPR) that depends in part on EB will be challenging. Screening trials incorporating organisms at three trophic levels are difficult and the results more variable. Therefore, elucidation of mechanisms is a requirement to permit screening for relevant plant attributes. Incorporation of BE and direct HPR can be difficult because of a requirement for multiple plant attributesq in breeding lines, and complicated because traits conferring BE and HPR can be antagonistic. Efficacy of this type of pest control in production will depend on adequate predator or parasitoid populations. Practices that enhance or augment these populations may be required. The potential for obtaining more stable effective pest management argue for development of $\mathrm{BE}$ in crops in spite of these difficulties.

Department of Entomology, 410 Forbes Building, University of Arizona, Tucson, AZ 85719 USA

436

PLANT RESISTANCE COMPLEMENTARY TO BIOCONTROL OF THE SOIL-BORNE PATHOGENIC FUNGUS RHIZOCTONIA SOLANI (Kühn)

\section{P.H.J.F. van den Boogert, A.J.G. Luttikholt and J. Keijer}

Rhizoctonia solani is a cosmopolitan soil-borne plant pathogen of almost unlimited host range. The pathogen significantly reduces the crop quality and quantity of several major crops (potatoes, sugar beet, rice) and specialist crops (flower bulbs, saffron and omamentais) of regional importance, and it contributes to poor seedling establishment in general. $R$. solani is currently controlled by chemical means but has the potential to be controlled by non-chemical methods including biological agents (e.g. the mycoparasitic fungus Verticillium biguttatum Gams) and integrated management techniques (Green Crop Harvesting) specific for individual crops and sites. In an attempt to trace natural variation in pathogenicity of $R$. solani, we discovered considerable differences in virulence and susceptibility among isolates and cultivars, respectively. In addition, variation in efficacy biocontrol by $V$. biguttatum occurs especially in heavily contaminated soils causing unpredictable levels of disease. Observations on yaration in resistance and antagonism lead to the supposition that (engineered) resistant cultivars and biocontrol are attractive strategies for non-chemical control.

We will discuss the usefullness of breeding for resistance and of biocontrol in Rhizoctonia-disease management, and will report on data of preliminary experiments in respect to non-chemical control of $R$. solani in diverse crop plants using partial resistant cultivars and the biocontrol agent $V$. biguttatum.

DLO Research Institute for Plant Protection, IPO-DLO, P.O. Box 9060, NL-6700 GW, Wageningen, The Netherlands 
INTEGRATION OF PLANT RESISTANCE INTO CROPPING SYSTEMS AND TRITROPHIC INTERACTION IMPLICATIONS

\section{S. S. Quisenberry and D. J. Schotzko}

Plant resistance to insects can play a major role in the management of insect pest populations. Interactions between pest management methods must be understood. The biology, distribution and population dynamics of the pest can also affect the plant resistance type used in a cropping system and influence other control methods. The use of plant resistance and natural enemies can be independent, synergistic or antagonistic because either method may enhance, reduce, or not affect the the other control. Thus, tritrophic relationships in a cropping system can be influenced by host plant-herbivore-natural enemy interactions

Department of Plant, Soil and Entomological Sciences, University of Idaho, Moscow, Idaho 83844-2339, U.S.A.

EVOLLTIONARY IHPACT OF SYNERGISII BETWEEN HOST PLANT RESISTANCE AND BIOLOGICAL CONTROL

11.T. Johnson, F. Gould, W.H. LU, G.G. Kennedy

It was at one time thought that the impact on pests of host plant resistance and of biological control agents were so distinct that the presence of one of these inimical agents would not influence the rate at which insect pests adapted to the other. We conducted a theoretical analysis of this tritrophic system using a single locus deterministic genetic model. Results of our modeling work indicated that the presence of natural enemies could increase, decrease or have no effect on the rate at which pests adapt to partial host plant resistance depending on the type of host plant resistance and the "foraging tactics" of the natura enemy. He selected two tritrophic systems in which there was synergism between host plant resistance and the effectiveness of a natural enemy where, based on theory, the natural enemy was expected to increase the rate of pest adaptation to the host plant resistance. We designed laboratory selection experiments in which the pest was selected for adaptation to the plant resistance mechanism either in the presence or absence of the natural enemy. We wi 11 present results of these selection experiments and discuss how they relate to our theoretical results.

North Carolina State University, Department of Entomology, Box 7634, Raleigh, Morth Carolina 27695-7634
BIOLOGICAL CONTROL AND PLANT RESISTANCE COMPATIBILITY: A COMPONENT FOR PLANT BREEDING PROGRAMS

\section{D.J. Boethel}

Over the past decade, the compatibility of biological control and plant resistance to insects has been examined through studies involving parasitoids, predators, and most recently, entomopathogens. A majority of this research has dealt with plant genotypes exhibiting antibiosis, to a lesser extent, antixenosis, and an even lesser extent, tolerance. The interactions of these two prominent pest management tactics have demonstrated antagonistic, synergistic, and additive outcomes, with the results dependent on the mechanism of resistance. Compatibility with biological control has not been the focus of most plant breeding programs and certainly would rank after desirable agronomic traits and insect resistance per se. Whether evaluations of the impact on natural enemies should become a routine component of cultivar development certainly warrants discussion. Adoption of this component may lead to refined pest management and more sustainable crop production.

Department of Entomology, Louisiana Agricultural Experiment Station, Louisiana State University Agricultural Center, Baton Rouge, Louisiana, USA 70808 
AN AGRONOMIST, VIEW OF ROTATIONAL SET-ASIDE

T. Doré, A. Dalbiès, J.M. Meynard

The set-aside introducec by the CAP reform has led to new uses of farmed land in Europe. As a result, set-aside has raised several guestions for agronomists. These include how accupation of this space will influence the productivity of cropping systems, how should set-aside fields be integrated into crop management, or what rationale can be used to into crop management, or what rationale can be used to manage a set-aside field, as it cannot be simply left agrononic questions raised by this problem differ from those associated with standard cultural practises, rather than reporting results on the effects of set-aside. These questions therefore require a certain reviaion of our approach to crop successions. Emphasis is thus placed on three aspects of land removal from cultivation, as it apree aspects of land removal from cultivation, as it administrative and technical conditions for applying setaside rules lead to a wide variation in the land set-aside. This is true both for the type of farm land from which this land is drawn, which reflects the diversity of their faming history more than in standard farming situations, and for the way farmers treat set-aside fields. This diversity of land management reflects the differences in the objectives given for the management of the set-aside, with respect to the land itself or the whole farm. Fe then analyze the new agronomic questions raised by set-aside. Rather than providing a complete list of questions, we aim to show that their emergence is clearly linked to the appearance of new physical-chenical and biological phenomena that must be analyeed and understood. We also focus on the particular importance of interactions between these phenomena within the set-aside area. Lastly, we discuss the conseguences of these relationships for the development of faming practices for managing these set-aside areas, with emphasis on the difficulties raised by their diversity. The conclusion proposes two ideas. One concerns the agronomic gignificance of a set-aside field, showing that it is not like any other form of fallow plot. The second points out the opportunities offered by set-aside to re-examine crop successions.

Laboratoire d'Agronomie INA-PG/INRA, 16 rue C. Bernard, 75231 Paris Cedex 05, France

CONSEQUENCES OF THE EXTENSION OF FALLOWNG FOR THE EVALUATION OF WEED POPULATIONS

D Atkinson, N M Fisher, D H K Davies, M A Ford

The EC requirement for set-aside as a component of arable crop production has reintroduced fallowing as a major practice within ley-arable farming. The compulsory period of set-aside or fallowing may either be a bureaucratic intervention to be endured, or a break period which may be used positively to aid the development of farming systems with reduced chemical inputs. Whether the period of fallowing is actively managed or not, it will have major implications for the development of the weed flora of a particular land area. It will also have major implications for the subsequent management of weed populations. The elassification of a particular element of vegetation as a weed will depend upon the subsequent use of the land. The breadth of issues involved in fallowing have resulted in this paper being focused upon the use of set-aside periods in arable systems destined to remain as part of long-term arable culture. Where farming systems traditionally depend upon crop rotations, i.e. a sequence of cropping including both arable and grass crops, then the rules imposed by the EC in relation to set-aside involve more of a change in the use of materials produced during the fallowing period and the management of the fallow cycle than a major change in farming practice. In situations where continuous arable cropping was practised, the introduction of a fallow period represents a more significan change. Within the above context, it is usefiul to review the effects of either one year or three to five year periods of fallowing and of systems where fallowing involves either a sown grass cover or a vegetative cover derived from natural regeneration. The implications for the development of weed populations in these four types of situations will be discussed and related to future developments in farming systems, especially reduced input systems and systems using transgenic cropping material. In experiments carried out on SAC farms in Scotland, where fallowing involved the sowing of a grass clover-based cover, this had a major effect upon the appearance of vegetation whether assessed after a period of one year or a longer period. In most situations, the sown species remained dominant. Where vegetation was allowed to develop as a consequence of natural regeneration, then the weed lora present differed with time. For both time periods, the species present were heavily influenced by the composition of the soil seed-bank. For both sown and unsown fallows, the development of the weed flora was affected by the fertility of the site and the size of the seed bank increased. The magnitude of the increase and the composition of the weed flora varied between types of fallow. Treatments, such as cutting and disturbance, had only minor effects. Effects of fallowing seemed to be governed by major generic principles, although unmanged factors, e.g. rabbit grazing could have a mejor impact. The husbandry employed during a fallowed period will influence the ability to manage low chemical input systems. The general principles from set-aside studies will influence the development of transgenic crop species (GMOs) as weeds and the flow of genes from residual crop plants in fallow vegetation into related weedy species. Despite recent emphasis upon herbicide resistance, other transgenic traits, c.g. pest resistance, may have a larger impact on the evolution of weed populations. These aspects will be discussed.

SAC, West Mains Road, Edinburgh EH9 $3 \mathrm{JG}$
FALLOWNG AND ITS IMPLICATIONS FOR CROP PROTECTION: EFFECTS ON PLANT PATHOGENS AND ESPECLALLY VIRUSES

\section{Lepoivse, J.B. Quiot, J.M. Thresh}

The incidence of pests, pathogens and weeds is greatly influenced by the type and sequence of periods of fallow when no vegetation is allowed to grow, or when natural recolonization occurs spontaneously, or when non-crop species are deliberately introduced. Such practices have long been used and for diverse reasons in agriculture and horticulture in tropical, subtropical and temperate regions. The various practices adopted include traditional "bare fallowing", "bush fallowing" and the current use of "set-aside" in Europe.

Examples will be given from the literature of the various ways in which fallowing influences the survival of pathogens during the period between successive plantings of susceptible crops, through effects on the carry-over of inoculum, in soil-inhabiting vectors or crop/non-crop host plants. Several important vinuses survive in the debris or seed from previous crops or weeds to become foci for subsequent spread, as illustrated by examples from tropical and temperate crops including sugar-cane, sugar-beet, potato and hop. Areas being fallowed can also become refugia from which pathogens or vectors spread to adjacent crops, but this possibility has received little attention. - This emphasizes the need for further studies on all aspects of fallowing procedures. Those have a continuing role to play in both traditional and modern methods of agriculture and for diverse biological, agronomic, socio-economic and cultural reasons.

Faculté des Sciences Agronomiques de Gembloux 5030 Belgium

444

EXPERTMENTS IN WHEAT/BALION AGROECOSYSTERS AND IMPLICATIONS FOR PEST MRNAGRLENT

\author{
D. A. Ea11, R. W. Smiley, and P. 2. Rasourusen
}

Fallowing during the rowner prior to seeding winter wheat is practicad on two millien hectares in semi-arid, winterdominant precipitation regions of the northrest v.3.A. This practics anserres soil moisture far autum planting and has practice sonserres soil moistare far antam planking and dynamias of plant pathogens and veeds. The fallow period rinter, and conttnues mitil winter, wheat is seeded the following automn (october). Shallor tillage is used duxing the 14-month fallow pariod to redice evaporative molotwre loss and to control veeds. Detrimental aspects of fallow Include coil erosion from wind and watar, declining soir organic matier, reduced microbial bicmass, and joss of crop prod stabilizes wheat yields over seasons and nitigates damage from diseases and woeds. Soctetal concerns over soil erosion and declining soil quality have stimulated research on alternative practices to minimize or elinizate the fallow period.

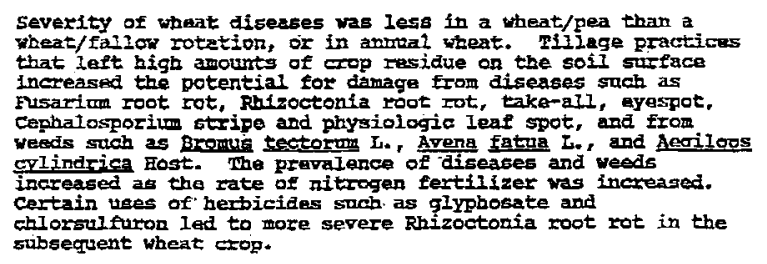
stibsequent wheat stop.

Tillage and nitrogen factilization practices used in winter wheat/fallow sotations influenced soil properties guch as organfic matter, microbial biomess, soil pH, and distribution of crop residues and weed seeds. Wach of these changres in soil environment were correlated with commenswrate efferts on severity of plant diseases and/or weed infestations. This research on the influence of fallowing practices has improved the integrated management of diseases and weeds in winter wheat/ fallow rotatious.

Oregon state Dniversity, F.O. Box 370, Pendleton, Oregon, U.S.A. 97802 . 
RESIDUAL EFFECTS OF SULPHONYL UREA DERIVATIVES IN THE CROP ROTATION AND WAYS OF THEIR OVERCOMING.

\section{T. Makhankova}

Sulphonyl urea derivatives have become very important for broad-leaf weeds control in different crops. Ear cereals, maize and rice have proved to be the most resistent crops to this group of chemicals. Side by side with positive properties of these products, such as low application rates, safety for warm-blooded and enironment, some products from this chemical group, in particular, chlorsulephuron and similar compounds, are able to damage broad-leaf crops, even applied at the lowest rates, which is manifested is residual effects on rotation crops. Resistance to chlorsulphuron and other sulphonyl urea derivatives of plants belonging to different botanical groups (Fabaceae Lindl., Brassicaceae Burnett., Cucurbitaceae Juss., Rubiaceae Juss., Apiaceae Lindl. and others) has been evaluated in vivo and in vitro. It has been stated, that plants from different families have demonstrated different reactions to herbicides. Soil and climatic characterictics of the region determine to a great extent herbicide residual effect manifestation. Ous tests have been conducted in the Urals, Western Sibeiria and North-Western region of European Russia. Experimental data on in vivo and in vitro tests, proving distinctions in resistance to sulphonyl urea derivatives of plants belonging to different botanical groups, are given in the presentation.

All-Russian Institute for Plant Protection, Shosse Podbelsky 3 , St.Petersburg-Pushkin, 189620, Russia

NO-INPUT CROP EDGE MANAGEMENT: 5 YEARS OF PRACTICE IN CEREALS w. Joente

Lowering the input of agrochemicals in crop edges and thereby enforcing wildife, as well as serving crop and environmental protection can be considered a key subject in sustainable agriculture. If no fertilizer and no herbicides are applied,
what happens to weed populations and crop yields? On-farm practice revealed that, given a choice between various management options, farmers tend to minimize risks and labour and maximize profits, including financial compensation of a specified management. Af ter several years most
choose for'grassy strips; (mown for silage) or sown choose for 'grassy strips', (mown for silage) or sown
vegetation ('green manure') along the arable crops. Only a few farmers grew cereals permanently in a $3-4 \mathrm{~m}$ wide crop edge. Here other arguments and personal interests were mentioned
(landscape, butterflies, flowering plants, pest (landscape, butterflies,
control/biodiversity).

In field experiments on sand, with crop edge strips (sumner-barley and rye) along a field with rotation of mays, barley and rye, the influence of fertilizer (F) and/or herbicide $(\mathrm{H})$ on yield and weeds was compared.

- The no input strips $(-\mathrm{H}-\mathrm{F})$ showed relatively large yield losses (up to 508 ) and a large weed biomass with many species, from the first year onwards. Species numbers increased only slightly, After 5 years Spergula arvensis, Matricaria recutita, Vicia cracca and V. hirsuta comdoninated, the latter two winding species overtopping the crop.

- The $(+H+F)$ strips reflect current practice, with low weed biomass and low number of weed species, Polygonum convolvulus locally dominating in the crop canopy.

- After five years the $(-\mathrm{H}+\mathrm{F})$ strips had a rather low weed biomass from a high number of weed species (here Matricaria recutita and Chenopodi um album of ten dominate, with Polygonum convolvulus and Vicia hirsuta dominating the crop canopy. Conclusions:

Without a financial compensation for yield losses farmers generally do not favour no-input crop edge managements. On the other hand, they do not experience an increased incidence of pests and diseases. Perennial weeds could be controlled locally.

Experiments in unfertilized, unsprayed cexeal strips show yield losses of up to ca. $50 \%$ and large weed-biomass with many species. In unsprayed, fertilized strips the cereals compete effectively with the weeds; here, the smaller weed-biomass stems from many weed species.

Permanently growing cereals tends to increase problems with climbing and/or with clonal perennial weeds.

Dept. of TPE - Weed Science Section

Agricultural University

6700 AK Wageningen NL
FALLOWING AND NON-CROP IIABTTATS: IMPLICATIONS FOR INSACT MANAGEMENT AND BIOLOCICAL CONTROL

\section{A. Landis}

Insect abundance is influenced by the array of habitats and resources in agroecosystems. By altering the temporal and spatial distribution of these landscape elements, fallowing may either increase pest pressure, or reduce it by increasing binlogical control. In most cases, a dynamic interaction of both effects occurs. The influence of agricultural landscape strueture on parasitoid movement and level of parasitism was examined in two North American systems. Parasitism of Ostrinia nubilalis by $E$. terebrans was increased by proximity to particular non-crop habitats. Parasitism of Pseudaletia unipuncta by a community of parasitoids was improved as landscape complexity increased.

Department of Entomology, 104 B Pesticide Research Center, Michigan State University, East Lansing, MI 18824-1115.
448

SOIL SANITATION BY GROWING RESISTANT GREEN MANURE TRAP CROPS AS ROTATIONAL SET ASIDE

\section{HEINBROEK, W. AND MUNNING. R.G.}

Resistant cruciferous graen manure crops, sown before mid August, can reduce the population of Heterodera schachtii and Heterodera trifolii f.sp. betae substantially, if soil temperatures are favourable. However under NW-European weather conditions nematode trapping might be poor. This can be improved by drilling resistant green manure crops in spring as a main crop in a rotational set aside. The sanitation effect combined with set aside premiums will render application of such a crop as profitable.

Better than growing a resistant cruciferous crop twice a year, the nematode trapping effect can be improved by lengthening the vegetation period. This can be accomplished by a mowing regime and/or application of special cultivars. Since white mustard did not show mowing regime and/or application of special cultivars. Since white mustard did not sho

any substantial regrowth, only fodder radish cultivars are suitable for this purpose. The effects of some resistant cultivars, particularly those selected for a long vegetation period, nearly equalled the application of cis-dichloropropene with respect to Selections, cultivars and crops resistant to the root knot nematodes Melödogyne hapla and $M$. chitwoodi were tested in the greenhouse and on severely infested fields. Since black fallow (without any weeds present during the season) will reduce infestation by root knot nematodes drastically, the expected extra effect of a resistant trap crop will be small. However root knot nematodes generally occur in sandy soil with a high population of host wews, which will weeds, which will restrict hactual under these conditions fodder radish cultivars could provide a contribution to the control of $M$. chitwoodi.

Although different crops can reduce the level of Polymyxa betae in soil, a substantial effect on the infestation and sugar-beet crop losses by Beet Necrotic Yeliow Vein Virus could not be detected. Some positive influence of green manure crops reducing the damage in sugar beets a subsequent year, might be attributed to a better soil drainage, reducing the chance of early penetration of zoöspores of P. betae into the hairy roots.

Sugar Beat Research Institure (IRS), P.O. Box 32, NL-4600 AA Bergen op Zoom. The Netherlands. 
YEASTS THAT BIOLOGICALLY CONTROL GRAY MOLD OF APPLE CAUSED BY BOTRYTIS CINEREA.

A. B. Filonow, H. Vishniac, J. Anderson, and w. Janisiewicz.

The potential of yeasts to suppress gray mold was assessed. Yeasts were grown in glucose-yeast extract-peptone liquid culture for $24-48 \mathrm{~h}$, washed twice by centrifugation in $50 \mathrm{ml}$ phosphate buffer ( $\mathrm{pH} 6.8$ ) and resuspended in buffer to give $5 \times 10^{6}-5 \times 10^{7} \mathrm{cells} / \mathrm{ml}$. Conidia of $B$, cinerea were harvested from 2-wk-old PDA, washed, and resuspended in buffer to give $10^{*}$ cells $/ \mathrm{ml}$. Wounds ( 3 min dia. $x 3$ mim deep) were made with a nail near the stems of Golden Delicious apples. Yeast cells were applied at $10^{5}$ to $10^{6}$ cells per wound. Apples with nontreated wounds or wounds treated with benomyl were the controls. After 30-60 min, each wound was inoculated with 200 to 400 conidia. Apples were incubated at 22-24 \& for 5-7 days, afterwhich they wera assessed for disease incidence and lesion dianeter. Of 27 I-Y22, Filobasidium floriforme Y7454, Rhodotorula aurantiaca $\mathrm{Y}-\mathrm{Y} 22$, Filobasidium floriforme $\mathrm{Y7454}$, Rhodotorula aurant
$\mathrm{Y} 1581$, Sporobolomyces albo-rubescens $\mathrm{Y} 1293$ and $S$. roseus strains 24257 and 28988 prevented or greatly reduced $(P=0.05)$ strains 24257 and 28988 prevented or greatly reduced ( $P=0.05$ )
the incidence of gray mold and the diameter of lesions in the incidence of gray wold and the diameter of lesions in
treated apples. Several yeasts suppressed gray mold as well or treated apples. Several yeasts suppressed gray mold as well or days incubation at 4-5 c. At 22-24 C, gray mold control days incubation at 4-5 C. At 22-24 C, gray mold control increased as the concentration of yeast cells per wound increased from $5 \times 10^{2}$ to $5 \times 10^{4}$ cells. Some yeasts, e.g. C.
laurentif BSR-I-Y22 were effective at concentrations as low as
$5 \times 10^{2}$ cells per wound. Competition for sucrose as a biocontrol wechanis: was Investigated. Nylon cloth pieces $\left(4 \mathrm{~cm}^{2} ; 5 \mathrm{um}\right.$ mesh) bearing $10^{4}-10^{\circ}$ conidia/ $\mathrm{cm}^{2}$ were aseptically incubated in contact with $10^{\circ}$ yeast cells in 100 ul of $10 \mathrm{my}$ sucrose in planchets. After $36-48 \mathrm{~h}$, germination of conidia in contact with yeast suspensions was suppressed 90-1008 compared to that of conidia in contact only with sucrose. Reducing the yeast cell concentrations in planchets by 100-fold increased germination; however, it still was suppressed $(46-80 \%)$ compared to controls. Utilization after $48 \mathrm{~h}$ of "C-labeled sucrose by isolates of $s$. roseus in planchets was 4-6 times greater than that of conidia in planchets. Suppression of ethylene production by yeasts in wounded apples as a biocontrol echaniso was studied. Wounded, wounded + yeast and nonwounded apples were enclosed in air-tight glass jars and the headspace was periodically analyzed by gas chromatography for $24 \mathrm{~h}$.

wounded apples produced more $\mathrm{C}_{2} \mathrm{H}_{4}$ than nonwounded ones, whereas $s$. roseus reduced $\mathrm{C}_{2} \mathrm{H}_{4}$ output compared to that from wounded, but, nontreated apples.

Department of Plant Pathology, Oklahoma state University, Stillwater, Oklahoma, USA. 74078.

451

\section{Biologieal control of Chenopodium album with Ascockyta coulina}

\section{Kempennar P.J.M.M. Horsten and P.C. Scheepens}

In 1991 a whdy on the use of pycnidiospores of the fungus Ascochyta caulina (P. Kast) v.d. As \& v. Kest. as a myocherticak againet the weed Chenopodium album L. was started. $C$. album is an amnual plant, and considered at troublesome weed in many arable crops. A. condina is a facultative, plant pathogenic fungur

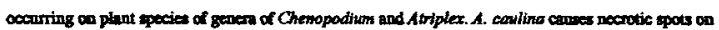
aerial parts of infocted plants.

Three control strategies of $C$. albwen were investigated: application of spores to the soil (pre emergencos), application of spores to juvenile plants (post emergence) and application of upores to flowering plants. Application of spores wo the soil was studied under controlled conditions in a greenhorse. Doxis-fiect relationships were demonstrated between spore density in the soil and mortality. LD som was circa $10^{\circ}$ epores. ${ }^{2}$ soil. Application of spores to jovenile plants was studied under field conditions. Experiments were carried out in sugar beet and majze crops. Efficacy of $A$. cantina strongly depended on evailsbility of weter on plants. When it rained after spore application $50 \%$ to $80 \%$ mortality of C. album plangte was attained. Surviving $C$. album plants were atrongly reduced in gromth and corrpetitivenesh Application of spore to flowering plants we also studied under field condtions. Wo demonstrated that flowess and reads of C. albus can be infectiod by the A. conilina. Some treatments resulted in complete prevention of seed production.

We conchuded that $A$. conlina has perspectives to control $C$. album in arable crops. Pre emergencon application of spores to the soil appeared to be a promising control strategy, but has to be tested in the field yet. Application of spores to juvenile plants was very effiotive under conditions that faveur infoction. To improve efficacy of A. cendithe under adverse exvireamental conditions, a formolation has to be developed which makes infiction less depended on emvironmental conditions. Joint application of spores and a herticide is another cption. Application of spores to flowering plants can be a method to prevent seed production of $C$. album plants that escaped other control measures.
BIOLOGICAL CONTROL OF POSTHARVEST ROTS OF APPLES

\section{M.L. GULLINO}

Several pathogens, such as Penicillium expansum, Botrytis cinerea, Monilia sp.. Alternaria sp.. can cause severe postharvest rots on apples. Their incidence varies according to disear, storage conditions and duration, and strategies of disease management followed in the field. For instance, brown rot ls becoming increasingly important on apples grown under strict IPM strategies and, even more, In organically grown fungicides applied as postharvest treatments, disease control is not always complete, due to the widespread presence of populations of some pathogens resistant to benzimidazoles. Moreover, postharvest treatments encounter increasing obstacles due to limited availability of registered compounds and to the general request by the consumers of less treated commodities. Under such conditions, the development and availability of biocontrol agents active against postharvest rots looks particularly promising.

Two strains of Candida pulcherxima, coded as 4.4 and 2.33, and one bacterial isolate, Pseudomonas sp. Me 134, were screened among hundreds of microrganisms for their good blocontrol activity against Eotrytis and Penicillium rots and brown rot. The selected biocontrol agents gave consistent results both at 2 and 22 "C in trials carried out under semi-commerclal conditions, in the presence of artificial inoculation. well against Botrytis and Fenicillium rots, while pseudomonas sp. Me 134, used at $10^{8}$ cells/ml, was particularly effective against brown rot. Their good activity was confirmed under comerclal conditions, in the presence of natural infections. However, further research is needed in order to improve the activity of biocontrol agents, also by developing effective formulations which should permit to obtain more conststent results.

The mechanisms of action of the selected blocontrol agents are discussed, also in relation with the search for the best methods of application.

DI.VA.P.R.A. - Patología Vegetale, Via Giuria 15, 10126 Torino, Italy.
452

ADVANCE IN RESEARCH ON MASS PRODUCIION THCHNTQUES OF NATURAL, ENEMIES FOR BIOLOGICAL PEST CONTROL IN CHINA

\section{Liying Ii} China is the country rich of natural enemies of pests. Great strides
forward have been made in research on mass production technigues of main species of natural enenies and their extension to practice of biological plant protection in china during the last 40 years. More than 19 species plant protection in crina during the last 40 years. More than species in parasitoids and 18 species of predators

in laboratories or biccontrol stations : Parasitoids - Anastatus japonicus, Aphidius gifuensis, Bracon greeni, Bracon nigrorufum, Diaeretiella rapae, Dibracys Cavus, Habrobracon hebe-
tor, Macrocentrus linearis, Ontsira plalliatus, Scleroderma guani, Spator, Macrocentrus linearis, Ontsira plalliatus, Scleroderma guanj, Spalangia endius, Telencmus dendrolimusi, Tetrastichus hagenowi, Irichogratha confusum, Trichograma dendrolini, Trichograma evanescens, Trichotranma japonicum

Predators - Amblyseius spp., Crrysopa spp., Coccinella septempunctata, Cryptolaemus montrouzieri, Cryptolestes turcicus, Excanthecona furcellata, Leis axyridis, orius sauteri, Phytoseiulus persinilis, podisus maculiventris, Symonycha grandis, coelophora biplagtata, Pseudosymus sp., Typhlodramus spp., xylocoris

consicerable progress was made also in research on in vitro rearing techniques of the following parasitoids : Anastatus japonicus, Bracon greeni, Dibrachys cavus, habrobracon hebetor, Ontsira plalliatus, sclerodema guani, Telencrus dendrolimusi, Tetrastichus hagenowii, Trichogramma spp. Augmentation and releases of above parasitoids and predators were implemented successfully in China as an important measure in IFM of com,
tton, rice, sugarcane, vegetables, fruit trees, forest and urban.

Prof. Liying Li, Guangdong Entcmological Institute, Xingang West Road 105, Guangzhou 510260, The People's Republic of China 


\section{BIOLOGICAL DISEASE CONTROL IN GREENHOUSE CULTIVATION}

\section{o. K. Mohammadi}

There is a growing demand for biological pesticides in greenhouse cultivation. Numerous promising biological control agents have been isolated and tested in research projects throughout the world. However, the number of available commercial products is still limited. Mycostop biofungicide is among the pioneers in the field of biological disease control. The product is based on Streptomyces bacteria and it is formulated as wettable powder. So far, it is registered for control of seed-borne and soil-borne diseases in more than 10 countries. The main application of Mycostop is in greenhouse cultivation of vegetables and omamentals, such as cucumber, gerbera, carnation and cyclamen. Besides controlling wilt and root diseases, using Mycostop has resulted in increased yield even in relatively healthy vegetation both in experimental and commercial use. Mycostop fits also to integrated pest managernent and it is thus an interesting choice in modern greenhouse cultivation.

Kemira Agro Oy, Espoo Research Centre, P.O. Box 44, FIN-02271 Espoo, Finland
INTEGRATION OF BIOLGGICAL AND OTHER WEED CONTROL IN ANNUAL PASTURE - CROP ROTATIONS

J.K. Scott, R. G. Shivas and P. B. Yeoh

In south western Australia agricultural production is largely based on a rotation of annual crops (wheat, grain legumes and canola) with annual pastures of subterranean clover (Trifolium subterrameum) and grasses. The pasture rotation is grazed by sheep, raises soil nitrogen levels and provides a disease break for the following crops. The region has a Mediterranean-type climate with hot dry summers and mild wet winters. An important annual winter weed in this system is doublegee, Emex australis (Polygonaceae), which is difficult to control because of its long-lived seed and unpalatability. Factors involved in the management of this weed isclude grazing intensity, and herbicides. Herbicide control of $E$. australis in pasture can be uneconomic and can cause damage to clover. Herbicide control of a range of weeds is essential during the crop phase. Biological control of $E$. australis is being investigated within the context of integration with existing weed management. The significant biological control agents already present are an aphid, Brachycaudus rumexicolens and a stem blight fungus, Phomopsis emecis. Minor biological control agents include a pergid sawfly, Lophythoma analis. New potential biological control agents include the weevil, Apion miniaum. The aphid arsived accidentally in Australia in the 1980's and causes significant reduction of seed set, plant and seed size in $E$. ausiralis. The seed of $E$. australis has a spiny coating (achene) which affects dormancy. One effect of the aphid is to cause a reduction in achene size which leads to a loss of seed dormancy. However, the aphid is not known to aestivate, and because the weed is absent during summer and part of autumn, it needs to find alternative hosts or re-invade from cooler regions. The aphid has a host range that includes many plants in the Polygonaceae. This indicates that augmentation of the aphid numbers on alternative hosts during autumn could increase the effectiveness of this biological control agent. Farmers could either conserve Polygonaceae plants found in native remnant vegetation or plant perennial or summer-growing Polygonaceae that are suitable hosts. An example of both cases is Muehlenbeckita adpressa. An increased aphid induced stress on the weed resulting in reduced seed dormancy could cause most seed of $E$. anstralis to germinate the following year. This would enable the weed to be readily removed by the herbicides used during the crop phase of the rotation. A second biological control agent, the sawly, is abundant is some years and would be favoured by the same alternative hosts provided for the aphid. However, the sawfly larvae feed on the leaves and appears to have little impact on seed production. The third agent already established is the fungus Phomopsis emecis. It kills about $20 \%$ of the seed. Low herbicide dosages did not encourage the fungus presence and mycoherbicide potential was investigated. Augmentation of the abundance of this fungus will rot be attempted because it lacks altemative hosts, the early growth phases of the weed escape infection by not hoving stems, and the fungus produces a powerful mammalian toxin, phomopsin A. New biological control agents are being studied to see if they are suitable to include into the pasture - crop rotation. The main insect is Apion minialtm, which being from Israel, has the potential to survive the hot dry summers characteristic of south western Australia.

CSIRO Division of Entomology, Private Bag, P. O. Wembley 6014, Australia

456

RHIZOBACTERIA-INDUCED RESISTANCE TO BACTERIAL WILT OF CUCURBTS AND CONTROL OF THE CUCUMBER BEETLE VECTOR

G.W. Zahnder, C. Yao, J.W. Kloepper, R. Shelby, G. Wei and O. Chambliss

Selected plant growth-promoting rhizobacteria (PGPR) were isolated, cultured and applied to 'Straight 8 ' cultivar cucumber seed and transplants. Cucumber plants treated with several of these PGPR strains exhibited significantly increased growth and reduced incidence of bacterial wilt disease compared with non-bacterized control plants. Subsequent fie!d experiments indicated that the severity of bacterial wilt disease and the numbers of the cucumber beetle vector were significantly lower on PGPRtreated plants compared with nontreated control plants. Cotyledon leaves from greenhouse-grown "bitter" cucumber plants treated with PGPR contained significantly lower amounts of the cucumber beetle feading stimulant "cucurbitacin $C^{n}$, compared with nontreated plants fbased on HPLC analysis!. These results suggest that, in addition to inducing resistance to bacterial wilt disease, PGPR exert a negative effect on cucumber beetle feeding by reducing plant concentrations of cucurbitacin C. This was demonstrated in greenhouse cage experiments, where cucumber beetle feeding and associated spread of bacterial wilt disease were both significantly reduced on PGPR-treated cucumber plants compared with non-bacterized control plants.

Department of Entomology

206 Extension Hall

Auburn University, Alabama 36849-5629

USA for its complementarity to Eh252. These two strains inhibit the fire blight pathogen by differe mechanisms, do not inhibit each other on Petri dishes and have different optimum of iemperature $\left(28^{\circ} \mathrm{C}\right.$ vs $\left.37^{\circ} \mathrm{C}\right)$. For two years we followed the dynamic of population of these two strains sprayed together or separately on apple and pear flowers. No difference in either the rate of growth or the level of population could be detected whether the strains were sprayed together or not; indicating that they are compatible on those flowers. When Eh252 and A506 were sprayed together on apple or Asian pear flowers the level of control of fire blight was similar to that of either strain sprayed separately. We are now looking at other mixtures including biological control agents that produce inhibitory compounds for which no cross resistance with that of Eh252 has been found. Since the genes necessary for production of the peptide antibiotic from Eh252 have been cloned and are being sequenced, we are also looking at the possibility of expressing these genes in other biological control agents than Eh252, such as $P$. fluorescens. Since the main site of infection for fire blight is the flower we also investigated the potential of honey bees, as described by $\mathbf{S}$. V. Thomson, as a delivery mechanisms for these biological control agents. In preliminary experiments we showed that bees carried effectively the nixture Eh252 and A506 onto apple flowers when these two strains were first mixed with some pollen as a carrier. All these results constitute the first building blocks of a strategy of biological control of fire blight. 


\section{IN VITRO ANTIBODY GENERATION}

\section{Hennie R. Hoogenboom}

CESAME at Depanment Pathology, University Hospital Maastricht, P.O. Box 5800,6202 AZ Maastricht, the Netherlands; tel. 31-43-874611; FAX 31-43-876613.

Phage antibody technology seeks to harness the power of Darwinian selection, by providing a diverse repertoire of structures, and selecting out a (rare) structure with binding activity (for review see ref. 1). Antibody fragments of predefined binding specificity and high affinity are isolated rapidly from such diverse repertoires of antibody $V$-genes, bypassing hybridoma technology and even immunization. Such antibodies have potential as reagents for research, tumor diagnosis and in tumor therapy.

Repertoires of antibody V-genes can be provided by PCR amplification of the rearranged $V$-genes from populations of lymphocytes, or assembied entirely in vitro from $V$ gene segments, and cloned for display of associated heavy and light chain variable domains on the surface of filamentous bacteriophage. Rare phages are selected from the repertoire by binding to antigen; soluble antibody fragments are expressed from infected bacteria. Antibodies with many different binding specificities have been isolated from the same phage repertoire, and technologies based on in vivo recombination (combinatorial infection') developed to obtain 'super' libraries. Thus human antibody fragments have been isolated from anibody repertoires up to 60 billion clones in size, and with specificities against foreign or self anvigens, including haptens, carbohydrates, secreted and cell surface proteins, viral coat proleins and intracellular antigens from the lumen of the endoplasmic reticulum and the nucleus. From those large libraries, antibodies have binding affinities up to $3 \mathrm{nM}$, which suffices for many applications. For some purposes it is necessary to improve the binding affinities; this can be achieved by mimicking the process of somatic hypermutation, by directed mutation of certain areas of the selected antibody $V$-genes, by shuffling with mutation-rich natural repertoires or by random mutagenesis and selection.

In conclusion, this in vitro immune system can be used io rapidly generate human antibodies of high affinity and, in principle, with specificity for any chosen antigen.

Ref.: Winter, Griffiths, Hawkins and Hoogenboom. Ann rev. Immunol. 12, 433-455 (1994).

A THIRD GENERATION DNA MARKER FOR THE IDENTIFICATION OF PLANT PATHOGENS: AFLP MARKERS RECOGNIZE VIRULENCE GROUPS IN POTATO CYST NEMATODES

J.N.A.M. Rouppe van der Voort, R.T. Folkertsma, P. M. van Zandvoort, K. E. de Groot, F. J. Gommers and I. Bakker.

Amplified fragment length polymorphism (AFLP) analysis is a novel PCR-based DNA fingerprinting technique (Benelux patent no. 825962 by Keygene N. V., Wageningen, The Netherlands). Prior to amplification, genomic DNA is digested and oligonucleotide adapters are ligated to the restriction fragments. These fragments are amplified for template multiplication by primers complementary to the adapters. By addition of selective nucleotides at the $3^{*}$ end of the primers only a subset of the restriction fragments will be amplified. AFLP fingerprints can be tailored to produce patterns of varying complexity by changing the extension of the primer sequence.

Per AFLP reaction, 50 to 75 fragments were amplified from template DNA of populations of potato cyst nematodes. Two types of DNA polymorphisms were resolved: (1) presence/absence polymorphisms and (2) polymorphisms in intensity representing putative alleles. Analyses of both types of polymorphisms revealed virulence groups of Globodera rosrochiensis with similarity between molecular data and the former pathotype designation. The classification of populations of $G$. pailida is less straightforward.

Department of Nernatology, Wageningen Agricultural University, P.O. Box 8123,6700 ES Wageningen, The Netherlands.
USE OF MOYYOCIONAI. ANTIBODIES TO DETECT, 'VISUALISE' AND QUANTIFY RHIZOCTONIA SOLANI and TRICHODERMA HARZLANUM IN SOILS

\section{C.R.THORNTON 1.2 F.M. DEWEY 1 and C.A. GILLIGAN 2}

Species and genus-specific monoclonal ancibodies have been raised to Rhizoctonis solani and Trichoderma harzianum respectively. These antibodies have been used to develop highly sensitive immunoassays to detect and quantify the biomass of these two fungi in complex microbial environments such as soil. The assays include enzymelinked immunosorbent assays (ELISA), immunomagnetic bead assays, immunofluorescence and a novel immunoblotting technique that allows the 'non-destructive' visualization of fungal development in situ. Recent experimencs on the biological control of $R$. solani by $T$. harzianum will be presented to illustrate how these immunological methods have been successfully applied to monitor the spatial and temporal dynamics of interacting populations of fungi in soils.

1.Deparment of Plant Sciences, University of Oxford, $S$. Parks Rd. Oxford. OX1 3RB.

2. Department of Plant Sciences, University of Cambridge, Downing Street, Cambridge, CB2 3EA
460

DIFFERENTIATION OF SEROLOGICALLY INDISTINGUISHABLE BEET NECROTIC YELLOW VIRUS ORIGINS FROM VARIOUS PARTS OF THE WORLD BY MEANS OF RFLP- AND SSCP-ANALYSES OF RT-PCR PRODUCTS

R. Koenig, P. Lüddecke and $M$. Kruse

Rhizomania-affected sugarbeet were received from more than 100 different sugarbeet fields in Europe. Asia and the USA. Various methods were tried to obtain beet necrotic yellow vein virus (BNVVV) RNAs 1 to 4 from these samples for RT-PCR (reverse transcription - polymerase chain reaction). Immunocapture (IC) of virus particles and immediate annealing with oligo-dT followed by reverse transcription proved to be the most efficient method for cDNA preparation. RT. PCR products were obtained for various regions of BNYVV RNAs 1 to 4. The genome regions which were chosen for amplification were suspected to be especially important for the pathogenic properties of the virus. RNA 3 has a pronounced influence on the strength of symptoms in leaves of test plants and in sugarbeet, RNA 4 greatly improves the transmission of the virus by polymyxa betae, the triple gene block area on RNA 2 is essential for the cell to cell spread of the virus and the coat protein gene region may be necessary for the long distant movement of the virus in infected plants. The 3-terminal region of RNA 1 was also amplified. With the RT-PCR products obtained for these regions of the viral genome restriction fragment length polymorphism (RFLP) and single strand conformation polymorphism (SSCP) analyses were carried out. Both types of analyses clearly indicated the existence of two major BNVVV strain groups which. were named type $A$ and type B. The A-type was found in Greece, former Yugoslavia, parts of Austria, Slovakia, Italy, Spain, Belgium, The Netherlands, in the one sample obtained from England, and also in the few samples obtained from Asia (Turkey, Kasachstan and Japan) and the USA. The 8-type was detected in most rhizomania-affected areas in France and Germany. Mixtures of the two types were found in borderline regions of the distribution areas of the two major strain groups in some parts of France, Austria and Germany (Kruse et al., J. Gen. Virol. 75, 1835-1842, 1994).

SSCP-analyses preceded by immuno-capture RT-PCR proved to be faster than RFL.F analyses. In addition, mutants and sLbstrains were detected more readily. Thus, some French BNYVV origins from an area where rizomania is especially severe were found to differ in their RNAS 1 and 4 and also in some other genome properties from all other A-type BNYVV origins.

Comparison of nucleotide sequences revealed that the majority of the nucleotide differences between the A- and the B-type occur in the third triplet position. The amino acid exchanges in the coat protein area are outside the four previously determined antigenic regions which are accessible on the surface of the virus particles and are involved in the formation of continuous and presumably also discontinuous epitopes. This may explain why no serological differences between various BNYVV origins have been found.

Biologische Bundesanstalt für Land- und Forstwirtschaft, Institut für Biochemie und Pflanzenvirologie, Messeweg 11, D38106 Braunschweig, Germany 
GENETIC DIVERSITY OF PSEUDOMONAS SOLANACEARUM IN JAPAN AND SOUTHEAST ASIA

\section{HORITA and K. TSUCHIYA}

Strains of $\underline{P}$. solanacearum differ in host range, pathogenicity and physiological properties, in which 5 races and 5 biovars have been described, respectively. In Japan and Southeast Asia, this pathogen was isolated from many kinds of host plants besides solanaceous crops.

The phylogenetic grouping based on RFLP analysis of the 93 selected strains of $P$. solanacearum, differing in geographical origin and isolated hosts collected from Japan and Southeast Asia resulted in three major clusters. Cluster 1 comprised the Japanes race $1 /$ biovars 1 to 4 , Chinese and most of the strains from Southeast Asia. Cluster 2 included a type strain from the USA and one strain from Southeast Asia. Cluster 3 included Japanese race 3 and BDB (Blood disease bacteria of banana), a bacterium related to $\underline{P}$ solanacearum. Cluster 1 was also subdivided into 2 to 4 subgroups. Each cluster was mostly correlated with geographical origins. Furthermore, Japanese race 1 strains were divided into three major cluster groups, in which 6 subgroups were included, and each group was basically corresponding to each biovar.

National Institute of Agrobiological Resources, 2-1-2 Kannondai, Tsukuba, 305 Japan

\section{3}

PHYSIOLOGICAL, GENETIC AND PATHOLOGICAL CHARACTERIZATION OF STRAINS OF XANTHOMONAS CAMPESTRIS PV. MANIHOTIS, INCITANT OF CASSAVA BACTERIAL BLIGHT, FROM WEST AND CENTRAL AFRICA

$\frac{\text { K. Wydra }}{\text { Fanou }}{ }^{\text {A }}$ And Fessehaie ${ }^{2}$, K. Assigbetse ${ }^{3}$, V. Verdier ${ }^{3}$, J. D. Janse ${ }^{4}$, A.

1 International Institute for Tropical Agriculture, Cotonou, Benin

2 Institute for Plant Pathology and Plant Protection, Göttingen, Germany 3 ORSTOM, Montpallier, France

4 Plant Protection Service, Dept. Bacteriology, Wageningen, Netherlands

In Ghana, Benin, Nigeria, Cameroon and Uganda 325 strains of Xanthomonas campestris pv. manihotis ( $\mathrm{Xcm}$ ) were collected from cassava (Manihot esculenta Crantz), including 13 isolates from wild Manihot species from experimental fields at the International Institute of Tropical Agriculture (IITA) in Ibadan, Nigeria. Biochemical, physiological, genetic and pathogenic features of selected strains were determined.

Metabolic fingerprinting using tests for amylase activity and for metabolization of 96 carbon sources (BIOLOG) revealed considerable variations between the strains. The program for complete linkage analysis was modified by evaluating the color intensity of the microplate wells as indicator for different levels of metabolic activity. The analysis resulted in the formation of 4 groups.

Also, the fatty acid composition of the strains varied. A hundred and fifty strains, including 30 reference strains from Latin America and Asia, showed mostly horrogeneous fatty acid profiles specific to the pathovar level. Principal component analysis revealed one big rather homogeneous group, while 7 nonAfrican strains formed a second group, different from group $I$ and heterogeneous among themselves.

Assessment of genetic diversity by restriction fragment length polymorphism (RFLP) patterns usine an r-RNA probe revealed differences only among nonAfrican stains, while the use of the plasmid probe F3Eco resulted in the classification of African strains in several groups.

When 250 strains were tested for virulence, great differences between isolates were recorded. Highly virulent strains appeared in each ocological zone- the Sudan savanna, the forest-savanna transition zone, the mid-altitude and the high rainfall zone.
USE OF POLYMERASE CHAIN REACTION (PCR) TO DETECT CYMBIDIUM MOSAIC POTEXVIRUS IN ORCHIDS

N. Abdul-Samad, M. Mat, S. Napis and K. Yusoff

Cymbidium mosaic potexvirus (CyMV) is one of the most prevalent among the viruses infecting a wide range of orchid genera causing losses in flower quality. A rapid and sensitive enzymatic amplification procedure was carried out for the detection of CyMV in undiluted crude tissue extracts as well as total nucleic acids extracted from infected tissue. The primers used was as described by Lim et al. (1993) that amplified 313 bp and 227 bp fragments. All the CyMV isolates used in this study gave the expected sized products following amplification. Nucleic acid hybridization confirmed the identity of the amplified products. The technique was found to be more sensitive than ELISA, dotELISA and IDIBA in detecting CyMV in orchids.

Department of Biochemistry and Microbiology

Universiti Pertanian Malaysia

43400 Serdang. Selangor

Maiaysia

464

CAN WE DEVELOP DURABLE RESISTANCE OF CROPPING SYSTEMS TO PEST COMPLEXES?

F. Gould

Most of the classic studies on durable host plant resistance have been developed by plant pathologists. Entomologists have often followed in the tracks of plant pathologists and have used their concepts and models in thinking about durable host plant resistance to insects. These models have been useful but differences between most pathogens and insects in population structure and mode of reproduction could population structure and mode of reproduction resistant germplasm (or deployment patterns of that germplasm) expected to result in the best durability.

I will present results of simulation models and experiments that demonstrate the importance of mate selection, as well as larval and adult movement in determining the types of germplasm and deployment strategies that are best suited for slowing the rate at which specific insect species adapt to resistant host plants. I will use examples of insect pests that differ most dramatically in their population structure and mode of reproduction to illustrate that the best deployment strategy for slowing genetic adaptation in one insect may be the worst for another insect.

Because most crops have multiple pests including insects, mites and pathogens, a major challenge facing us is to develop cropping systems that have durable resistance to key species within pest complexes. I will discuss how plant pathologists and entomologists could work together in guiding the development of such cropping systems.

Department of Entomology

North Carolina State University

Raleigh, NC 27695-7634 USA 
THE RECOGNITION, GENETIC BASIS AND EXPLOITATION OF DURABLE RESISTANCE TO DISEASES IN PLANT BREEDING.

\section{Ruy Johnson}

Resistance of a crop plant cultivar to a disease is durable if it remains effective during widespread cultivation of the cultivar for a prolonged period of years. The interpretation of this definition depends on the relative performance of other cultivars, the cultivation system and the host-pathogen interaction. Some hostpathogen systems show high specificity of interaction, whereas others do not. Experience shows that no single genetic or phenotypic model is valid for durable resistance. It ranges from single genes to more complex systems and from complete io quantitative expression. It may, is some cases, occur even where strains of the pathogen with virulence develop but do not spread. Some examples from virus diseases illustrate this point. Resistance that could legitimately be described as having demonstrated durability may fail, also illustrated by a virus disease and some specific gene combinations. Recent information will be given about durable resistance to disease in various host-pathogen sytems including the rust diseases of cereals. Brief reference will also be made to its possible exploitation in plant breeding and the potential for success and failure.

John Innes Centre, Colney, Norwich NR4 7UH, UK
MGR FINGERPRINT DIVERSITY IN HIMALAYAS AND SMALL SCALE PARASEXUAL EXCHANGE OF DNA CHALLENGF ASSUMPTION OF STRICT CLONALITY IN MAGNAPORTHE GRISFA

\section{R. S. Zeigler, J. Kumar, R. Scott, and R. J. Nelson.}

The development of durable resistance against causal agent (Magnaporthe grisea) of the blast disease of rice has been an imporant, though largely elusive, goal of breeders and pathologists for much of this century. The identification of quantitative and qualitative resistance genes, their characterization and development of strategies for their deployment can be greatly aided by understanding the structure and characteristics of target pathogen populations. Recent studies of $M$. grisea suggest that populations in the Americas and in breeding nurseries and fields of modern rice cultivars in Asia are composed of subpopulations, inferred to be lineages derived by strictly clonal descent from a common ancestor. Proposed population-targeted blast resistance breeding strategies depend upon lineages baving distinct virulence spectra and assume no recombination anong lineages. The most common tool for defining lineages is DNA fingerprinting using EcoRI digests of total genomic DNA probed using the repetitive sequence MGR 586. Pairwise comparisons of the $50-80$ bands in typical fingerprints has yielded groups with overall similarity among isolates of $80-100 \%$, and significantly less between group sirrularity. We asked if a lineage population is present under traditional Asian rice cultivation systems, and chose as a study site the blast-prone central Himalayan hills where traditional rice cultivation, together with millets (a host of $M$. grisea), still occurs. A total of 90 haplotypes (isolates with distinct band patterns) were identified from 105 monoconidial rice isolates. At the level of $80 \%$ DNA fingerprint similarity, 38 groups were identified. One field, sampled over two years, yielded 11 "lineages" out of 26 isolates analyzed the first year, and 12 "lineages" from 27 isolates the second year, with only 2 "lineages" recovered in both years--a diversity far greater than reported elsewhere. Analysis of association between locus pairs of single copy probes showed a high degree of gametic equilibrium in the same sets of isolates. Male fertile rice isolates crossed readily with female fertile isolates from millets. The high fingerprint diversity and gantetic phase equilibrium together with the presence of fertile field strains suggested that sexual recombination may be occurring in the field in the Himalayan hills Aralysis of DNA fingerprints of reisolates from pairings (Petri plate) between isolates from different lineages (selected because of their tendency to form tufts and form anastomoses upon pairing) indicated introgression of from two to $10 \mathrm{MGR}$ bands from one isolate into the MGR profile of the other. This suggested that parasexual recombinants could be erroneously classified as members of a strictly clonally derived lineage. The simple lineage structure observed in the Americas and in some circumstances in Asia may resuli from founder effects from limted introductions and/or severe bottle necks imposed on populations following widespread cultivation of modern cultivars with major resistance genes. Assumptions regaroing the strictly clonal nature of reproduction in $M$. grisea, the clear lineage structure of populations, and the strict genetic isolation of different lineages must be carefully reassessed.

International Rice Research Institute, P.O. Box933, Manila, Philippines

468

\section{APPROACHES TO STABLE RESISTANCE USING CLONES AS DISEASE RESISTANCE GENES}

467

Jones JDG, Hammond-Kosack K, Jones D, Thomas C, Harrison K, and TomesLacruz MA.

The recent isolation of several plant disease resistance genes has provoked considerable interest in the question of whether these cloned genes could be used to deliver both broad spectrum and stable disease resistance. We have isolated a tomato gene, $C f-9$, that confers resistance to races of the fungal pathogen Cladosporium fulvum that carry the corresponding Avr-9 gene. Expressing Avr-9 in a plant that carries the $C f-9$ gene is lethal. How then can a plant be produced in which the $C f-9 / A v r-9$ interaction activates defense responses, but lethality is avoided? Our objective currently is to modify the Cf-9/Avr-9 interaction so that the cure will not be worse than the disease. In principle, one could try and produce weak alleles of $C f-9$ andfor Avr-9 so that the defense response was not activated so strongly. An alternative is to use a variegation strategy. $C f-9$ alleles that carry the maize transposon $D s$ extibit somatic excision of $D s$ in the presence of transposase expressed from another locus, $s A c$. This somatic excision gives rise to small clones or sectors in which the $C f-9$ function is restored. If the plant also expresses the fungal $A v r-9$ gene, these sectors exhibit the defense response and die. The result is a plant that is variegated for a defense-related necrosis. Plants that exhibit this phenotype show significant increases in resistance to Cladosporium fulvum, and to two unrelated fungal pathogens, powdery mildew and Phytophthora infestans. It is hoped that the Cf-9/Avr-9 interaction can be further manipulated to deliver useful $P$. infestans resistance in potato.

The Sainsłury Laboratory, John Innes Centre, Norwich Research Park, Colney Lane, Norwich NR4 7UH, U.K.
AN APPROACH TOWARDS ATTAINING DURABLE RESISTANCE TO RUSTS IN WHEAT

\section{R.P. Singh, S. Rajaram}

Ruse diseases pose ar important threat to the stable wheat production in most wheat growing environments in the world. production in most wheat growing environments in the world.
The International Maize and wheat Improvement Center (CIMMYT) has a global mandate to improve germplasm suitable for has a global mandate to improve getmplasm suitable for utilization in less developed countries. Among the several possible approaches currentiy known to reduce losses due to
rust diseases; CIMMYT's strategy is to search for, understand renetic basis, and incorporate durable sources of resistance in the high-yielding germplasm. Durable resistances to rusts in wheat usually result from the additive interactions of few (between 3 to 5 ) minor genes giving partial resistance (slow (between 3 to 5) minor genes giving partial resistance (slow rusting type). When these partial genes are present alone, the level of resistance in not complete. However, resistances based on interactions of such genes are highly effective and
stable across environments. The Sr2 - complex derived from stable across environments. The Sr2 - complex derived from "Hope", lr34 -, and Yr18 - complexes from "Frontana" have the only named genes involved in the interactions and confer slow rusting when present alone. Presence of these genes can be indicated by the linked morphological markers: pseudo-black chaff for $\mathrm{Sr}$, and leaf tip necrosis for $\mathrm{Lr} 34$ and $\mathrm{Yr} 18$. The resistance conferred by the gene $L \times 34$ is of non-hypersensitive type. The steps being followed at CIMMYT to generate diverse combinations of such genes include: limiting the use of currently effective hypersensitive genes; crossing of parents that carry diverse sets of additive genes; exposing the segregating generations to high disease pressure generated by artificially created epidemics with selected pathotypes; selection of plants or lines with low terminal disease severity; multilocational testing to evaluate the effectiveness and stability of resistance across environments; and genetic analysis of resistance in selected wheats for the confirmation of the presence of additive genes.

Identification of DNA markers, linked to each of the partial genes, should aid the identification and development of diverse resistance combinations.

CIMMYT, Int., Lisboa 27, Col, Juárez, Apdo. Postal 6-64l, 06600 México, D.F., México. respectively. The genes Sr2, Lr34, and Yr18 are currently 


\section{DURABLE RESISTANCE TO THE LI:TTUCE DOWNY MILDEW}

K. Reinink, A. Leboda, R. Groenwold

Downy mildew caused by Bremia lacucae is an important disease in all lettuce (Lachuca sativa) production areas. Growers control the disease with furgicides and resistant cultivars. Breeders have relied heavily on gene-for-gene resistance conferred by dominant Dn (downy mildew) genes, of which many have been described. Until now all of these $\mathrm{Dm}$-genes have been effective for only a few years, because introductions of cultivars with new Dm-genes, or a new combination of $D m$-genes, have rapidly been followed by the appearance of new races of B. lactucae. The lack of durability of Dm-gene resistance has stimulated research into other types of resistance, that may be more durable.

The types of resistance that have been investigated are the quantitative resistance found in lettuce cultivars without effective $D \mathrm{~m}$-genes, and resistance found in wild Lactuca species. Quantitative resistance observed in cultivated lettuce did not show race-specificity, and is expected to be durable. However, the levels of quantitative resistance found in the cultivated lettuce are not high enough to provide sufficient disease control under environmental conditions favourable to disease development. In the wild species Lactuco saligna a very high level of resistance to B.lactucae was found. Altention was drawn to this species because all tested accessions of $L$. saligna were highly or completely resistant to aj] races of B. lactucoe used. Characlerization of this resistance has shown several differences with resistance conferred by $D n$-genes. Studies of the segregation of resistance in $F_{2}$ populations of crosses of L. saligna x $L$. sativa showed two distinct types of segregation. Most $\mathrm{F}_{2}$ crosses indicated an oligogenic inheritance of resistance, with only a low fraction of the $F_{2}$ progeny having the same level of resistance as the L. saligna parent. Only a few combinations of $F_{2}$ populations and $B$.lachucae races showed segregation ratios indicative of a monogenic dominant inheritance of resistance, as expected when the resistance is conferred by $D m$-genes. These tesults strongly suggested that some accessions of $L$.soligno carry race-specific Dm-genes, but that the general high level of resistance of this species to lettuce downy mildew is not based on these $D m$-genes, and must be caused by a high degree of basic incompatibility between L.saligna and lettuce downy mildew. Furthermore it was found that some lettuce cultivars carry a gene that can suppress the oligogenic resistance from L saligna, but not the resistance conferred by a putative $D m$-gene. Quantitative microscopical studies of the infection process showed that the oligogenic resistance behaves very different from Dm-gene resistance or from quantitative resistance in cultivated lettuce. The development of $B$. lactucae is halted later in the infection cycle than with hypersensitive resistance.

For lettuce breeding this means that a new resistance mechanism, providing a high level of resistance to B.lactucae is available. The resistance can be transferred to cultivated lettuce by interspecific crosses and selection. Because of its different mecharism, the resistance from L.saligna may be more durable than the Dm-gene sesistances used until now.

DLO-Centre for Plant Breeding and Reproduction Research (CPRO-DLO), P.O. box 16, 6700 AA, Wageningen, The Netherlands.

MULTILOCATION EVALUATION OF QUJANTITATIVE RESISTANCE TO RICE LEAF BLAST

\section{SW Ana C G McLaren}

The area under the disease progress curve (AUDPC) of 13 rice cultivars was evaluated at 17 sites in 10 countries, to determine the efficiency and stability of quan titative resistance abserved under field conditions. Genctype $x$ Environment (GxE) interaction models including stability regressions and additive main effects and multiplicative interaction (AMMil) models, were used to study GxE interaction for rice blast resistance. Twenty-six percent and $22 \%$ of the total sum of squares (SS) are accounted for by the genotype and environment components, respectively, whereas, $52 \%$ of the total SS is accounted for by GXE interaction. Stability regressions only acoount for $29 \%$ of the $G \times E$ SS, thile the analysis of variance for the AMMI model indicates that the first two interaction principal components (IPCA) account for $80 \%$ of the variation in the GXE interaction term. The first interaction principle component accounts for about $50 \%$ of the GXE SS and closely relates to the virutence composition of blast population. The second interaction principle component accounts for $30 \%$ of the GXE SS and relates to conduciveness of the environment for blast development as measured by overall disease progress. This confirms that the quantitative resistance, observed in multilocation evaluation, is the combined expression of partial resistance and race-specific qualitative resistance, in interaction with biotic and abiotic environmental factors. The quantitative resistance of IR64, Milyang 55 (Samgangbyeo), B3016 TB-260-3-2-1-1, IR60, and IR36 appears highly effective and stable. The greater contribution of the GXE component compared to that of the genolype component points to the important role of the efficiency of race-specific qualitative resistance in addition to partial resistance in the quantitative expression of host resistance under different field conditions.

INGER, Genetic Resources Center, The International Rice Research Institute,

P.O. Box 933, 1099 Manila, Pnilippines
EXPRESSION OF RESISTANCE BY EUCALYPTUS CAMALDULENSIS AND $E$ BLAKELYITO FEEDING BY PSYLLIDS OF THE GENUS CAROIASPINA.

F.B. Flovo and R.A. Farros:

Two species of psylid in the genus Cardiaspina feed on the parenchyria of leaves of Eucalyptus camaldulensis and $E$. blakelyi causing necrosis. During outbreaks, leat necrosis is extensive resuiting in total defoliation, dieback and even death of host trees. Some individual trees and genetic populations of these two species of eucalypt have shown resistance to psyllid attack which appears to be related to leat surtace morphology and composition of the the time of oviposition site the time of ovipositon site selection and as antibis against early instar psylid nymphs. The two expressions ol resistance are not always exhibied by the same trees. In lact, some genetic populations of trees are preferred for oviposition yet highly antibiotic to developing nymphs. The signilicance of these findings are discussed in terms of evolutionary biology and the breeding of psyltid-resistant trees for sustainable pest
management.

CStRO Division of Entomology, GPO Box 1700, Canberra ACT 2601, AUSTRALIA 
AGRICULTURAL INDIGENOUS KHOWLEDGR

IM UPPER CITARUA RTVER RASIM

WEST JAVA PROVINCE, IMDORESIA

By

Mani Diuangsih, Oekan S. Abdoellah, Budhi Gunawan, and Parikesit.

In Indonesia as well as in many parts of the worla, agricultural indigenous knowledge has successfully been agricultural indigenous knowledge has successfully been socio-cultural settings. These knowledges have been socio-cultural seteings These knowledges have been retained, adapted and implemented for survival by people ofter in environutntally sustainable mode. particular commuity to utilize and manage natural resources.

Currently, however, along with rapid devalopwent in agricultural sector and market system, many farmers are forced to adopt to more sophisticated practices. In the present time, traditional practices or knowledges in agriculture are often regarded to have some linitations in fulfilling the basic need of human beings. New technology is implemented to substitute the traditional practices. New high-yielding comodities are being introduced to replace local ones. Overall, there is a strong tendency that some traditional practices begin to fade. Many farmers are willing to leave what they have been doing for generations in order to adapt themselves to this development.

Rapid Rural Appraisal is used to explore the existence of traditional practices in Upper Citarum River Basin and to assess their persistence in the middle of current development in agricultural sector. This study also emphasizes the future prospect of traditional nractices in agricultural activities in this area.

Institute of Ecology, Research Institute, Padjadjaran University, Jalan Sekeloa, Bandung 40132, Indonesia.

475

TITLE; NIGERIAN SYSTEMS OF TRADITIONAL KNOWLEDGE AND MODERN SYSTEMS

AUTHOR: OUGHAYOMS DAVID ATTE

The Introduction of 'modern' systems of thought and life fito the area that is now known as Nigeria by the British and other European peoples over the last one hundred years has led to considerable changes in the life of the people. This is evident 1n new systems of agriculture and the overall economy, political organisations, culture, educational systems, technology and even life style. Hardly any part of Nfgexian 11fe remafns untouched to varying extent. Yet the vast majority of Nigerians, as much as $80 \%$ are supported by systems agricultural, socio-cultural, political, architectural, medical, technological, etc - which they developed themselves and which use indigenous resources and depend on indigenous or traditional knowledge and ezpertise. Many of the traditional systems have been modified in small ways by the adoption of 'modern' exogenous knowledge and technology, but still retain the original bullding struts.

This paper descrtbes the nature, processes and importance of indigenous or traditional knowledge systems in different flelds of life in N1geria though emphasis is placed on agriculture. It argues that the introduction of 'modern' systems, though colonial and neo-colonial process which neglected the tradtional knowledge, technology and resources of the people has led to dualtsation fa development with fto attendant internal probiems as weli as the condition political, economic and technological dependency in which the Nigerian nacton state and people here found themselves.

Finally the paper argues that (a) indigenous knowledge 1s st111 extensively used to meet the needs of the people, (b) analyses the extensively used to meet the needs of the people, (b) analyses
difflculties tn adopting modern systems and (c) submits that dificultes fn adopting modern systems and (c) submits that in N1geria with which godern knowledge must comoperate for the mutual benefit of both systems.

IRED (Development Innovations and Networks)

West African Sub-Regional office

19 Balogun Street, Anifowose

P 0 BOX 326
IKEJA - LAGOS

Te1. $+234-1-4938014$

Fax. $+234-1-4937723$
SCHEME FOR AN INTEGRATION OF THE TRADITIONAI KNOWLEDGE AND A NEW TECHNOLOGY FOR PLANT PROTECTION SYSTEM IN BRAZLL.

\section{Suzuki, J. ; Yoshida, T; Harnick,F.A.R}

The survival and the interactions among higher plants constitute the central line of this project, where the local traditional knowledge has the most relevant role.

Calling for a solution the small holder farmers, are disappearing from the brazilian agricultural landscape and it is giving rise to a continuous process of the nual break up. Jointly with this weightly occurrence, all the peasants experience acquired for years along are being lost too. Unless you search for a solution, it could result in a country unpredictable consequence.

Not only by recovering this folk crop knowledge from remnant areas, but also affording a proper use of their own resources in benefits of rural settlement seem to be the best way for raising these important activities to the country development. So a multidiciplinary involvement has been carried out to reach such a fragile social section aiming to an experience exchange and to assess the potential of its own natural resources.

The basis of the proposed technology is the Contact and Rhizospheric Transference System (CRTS), developed according to the recent knowledge reached on both, allelopathy and rhizospheric effects.

The plant root and shoot, growing in a very contrasted environment must be in a continuous flow-exchange of some kind of signaling agent that would be able to keep both, rool and shoot always integrated and updated. The CRTS was developed to exploit some plant to plant effects growing closely and since they are normally gently in the nature, they must be amplified by some techniques such as root densing, grown in a fine mesh compartnent, providing a roots fuld contact and the thizospheric transference between the studjed plant-root systern

Developing these studies on selected species etnobotanically assessed from working areas, could result in a low cost technique, leading to a sustannabe crop protection, based on plant biodiversity, specially plentiful in the tropics. Results of the expenments carried out with weeds and soil plant pathogen control and soil plant nutrition effects will be presented.

Faculdade de Ciencias Agranas e Veterinarias de Jaboticabal,UNESP, Departamento de Tecnologia, 14870-000, Jaboticabal SP, Brasil.

476

INDIGENOUS PEST MANAGEMENT SYSTEMS AMONG LATIN AMERICAN TRADITIONAL FARKERS

Miguel A. Altieri

Traditional farmers in Latin America have inherited and developed complex farming systews based on diversified cropping patterns. complex farming systems based on diversified. croppling packerns, and biological synergisms resulting from such complexity provide and biological aynergisus resulting fer mechanisus for such farming systews to sponsor their own soll fertilly, productivity and crop procection. about solls, plants, blo-lndicators and antmals is also the and in some cases this knowledge has been the basis for the This paper describes some traditional farming systems in Latin Amis paper describes some traditional farming systems in the effects of such practices on pest populations and damage.

Division of Biological Control, University of California, Berkeley 1050 San Pablo Ave, Albany, California 94706 and Consorclo Lat inoAmericano sobre agroecologia (CLADES), Santiago, Chile. 
THE INTERFACE BETWEEN AND THE INTEGRATION OF INDIGENOUS KNOWLEDGE SYSTEMS AND MODERN TECHNOLOGIES

D. Michael Warred

A growing anray of case studies is indicating the utility of understanding indigenous knowledge systems in facilitating sustainable approaches to development. Indigenous know ledge is community-based or ethnicallyfacilitating sustainable approaches to development. Indigenous knowledge is community-based or ethnically-
based knowledge that bas evolved at the local level. By recording such krowledge systems, development professionals gain insights into the decision-making processes within a community. Recorded knowledge professionals gain insights into the decision-making processes wiskin a comnitaity. Recorded knowledge
systems cas be compered and contrasted with the counterpan globat krowledge system. This provides mechanisons for evaluating the strengths and weaknesses of each system within the conlext of the micromechanisons for evaluating the strengths and weakoseses of each system within the conlext of the micro-
environment. In many recent case studies we discover thal probleme regarted as bigh priority within a commusity are also high priority within the scientific community. Mis discovery greatly enhances communojeations and participatory research and decision-making betwecn the clientele group and the developmest professional. This interactive flow of information between the indigenous and the counterpart cevelo

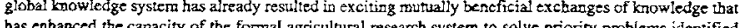

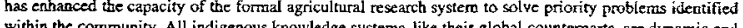
within the community. All indigenous knowledge systerns, like their global counterparts, are dynamic and encorporate aurnerous ideas from exogenous systems. This presentation will outline the process of techaology transfer within the context of indigenous knowledge systenus, focusing on case studies related to indigenous plant protection practices that, until recently, bave been largely overlooked by agricultural research and development enterprises. A survey of case studies localed at the CIKARO Docurnentation Unit and available soon on the intemet will be provided. The role of the growing global terwork of regional and national indigenous knowledge resounce centers in facilitating this collaborative approach to plant protection
research will be outlined.

CIKARD, 318 Curtiss Hall lowa State University
PLANT PROTECTION PRACIICES IN INDIA BASED ON INDIGENOUS KNOWIFDGE M.G. CHANORAKANTH

Ancient rexts of India - 'Varahamihira Brihathsamhitha' (Sth Century AD). 'Vriksha Ayirveda' of 'Lokopakakra' (9th Century AD), and 'Vriksha Ayurveda' of 'Sararigadhasa Simhitha' (13th Century $\Lambda D)$. provide recomimendations of the plant protection practices based on indigenous knowledge. They provide indications of an integrated approach to control crop pests and discases through soil, seed, plant and cnvironmental treatnent. The seed treatment with cowdeng, mikk, juice of Solanum indicum, render coconut water, Embelia ribus, and cow ghee is preseribed. As prephylactory metasure for disease cont
the incese of F.mbelia ribes. Commelina mukul, Fish meat, tumeric, mustard, flowers of Terminalia that incese of F.minelia ribes. Comimelina mukul, Fish meat, tummeric, mustard, flowers of Terminalia tomentosa $W \& A$ is mentioned. The prophylaclory pest control measure includes soil application of the mixture of Focrula narthex Loiss., Acorus calamus L., pepper, Embelia ribus. Cashew seed, Cheilanthes farinosa, mustard, cow hom powder in cow urine. These practices will be highlighted in a scparale paper. In this note we present the results of the experiments conducted for
systems of pest ind disease control highlightied in the ancient texts.

The USDA Bureau of Chemistry and Soils published the list of plants found in India possessing The USDA Burteau of Chemistry and Soils published the list of plants found in India possessing
insecticidal value in 1931 . Puttundriah \& Lakstiminarayana Bhatta (during 1935-39) tested the insecticidal value in 1934. Putturudriah \& Lakstminarayana Bhatta (during 1935-39) tested the
insecticidal properties of 45 species of plants in Mysore (southern lndia) on 4 caterpillars Plutella insecticidal properties of 45 species of plants in Mysore (southern lndia) on 4 catemillars Plutella
maculipennis Curt., Euprocis fraterna Moore., Prodenia litura Fab., Crocidolomia binotalis Zell; surface maculipennis Curt., Euproctis fraterna Moore., Prodenia litura Fab., Crocidolomia binotalis Zell; surface
grasshopper Epacromia tariulus Fab; and soft scales (green bug) Cocus viridis colernani Kkannan. They grasshopper Epacromia tariulus Fab; and soft scales (green bug) Cocus viridis colemani Kkannan. They
documented that seeds of Tephrosia candida and Anona squamosa and stem bark of Mudulea suberosa documented that seeds of Tephrosia candida and Anona squarnosa, and stem bark of Mudulea suberosa
possessed bigh degree of insecticidal potency. For getting spray emulsions, the hot industrial alcohol possessed high degree of insecticidal potency. For getting spray emulsions, the hot industrial alcohol
extracts of $M$. suberosa bark and $T$. candida seeds have to be mixed with molasses and dituted with water.

The ancient knowledge of 'Ayurveda' (Indian system of human medicine) prescribed 'Panchagavya gritha' - preparation from five products of cow viz,, urine, dung juice, milk, curd and ghee for curing epilepsy, jaundice and fever. Innovations in using 'Panchagavya' to control plant diseases were developed by a team of plant pathologists (Padmodaya and Ramachandra Reddy, 1994). They found that the fermented, diluted (10) filtrate formulation of ghee $(2 \%)$, Curds $(5 \%)$, Milk (5\%), fresh cowdung (48\%), cow urine $(40 \%)$, + common salt $(2 \%)$, yeast $(0.01 \%)$ to most effectively control the fusarium oxysporum seeding distase and wilt in comalo under pot culture and field conditions. This provided a benefit cost ratio of $21: 1$ compared with Carbendazim (50\% WP) which gave onjy $4 ; 1$. This modified 'Panchagavya' formulation effectively controlled the Panama disease of Banana caused by Fusarium oxysporum for the initial three months (Shamarao and Siddarariaiah), 1995). The altemate sprays of neem seed kemiel extract $(4 \%)$ and Triazaphos $(1.5 \mathrm{mi})$ at fornightly interval was found to effectively control murda disease of capsicum (Muniyappa, 1994). Use of neem extracts and R.D. 9 Replins controlled the leaf curl virus in tomato transmited by the whire fly Bemisia tabaci (Muniyappa 1995 pe). Similarly neem was found to be an effective nematicide (Nanjegurda, $1995 \mathrm{pc}$ ). The derails of several other studies will be provided in the main paper.

India has many innovative farmers who began experimenting with indigenous knowledge systems on their own and bcyan practicing and propayating this knowledge among their peers. In this regard the efforts of Purushothama Rao (Thirthahalli, South India) and his team of innovative fammers in developing various manurial, pesticidal and disease controlling formulations based on irdigenous knowledge are to be appreciated and tested further it the laboratories.

Department of Agricultural Economics, University of Agricultural Sciences, Ilebbal, Bangalore 560024, India. Phune: 91-080-3356328, FAX: 91-080-3330277
480

POPULATION DYNAMIC MODELLING - A NEW TOOL IN TERRESTRIAL ECO. TOXICOLOC'Y'

\section{A. Axelsen}

One of the important challenges in ecotoxicology is to extend results from laboratory tests to predictions of field effects. This is very difficult due to interactions between species in field ecosystems, animal behaviour, variation in temperatures and soil chemistry.

Mathematical modelling seems to be a promising tool to achieve an extension of laboratory results to field effects, because of the possibility of integrating information about toxicity, population dynamics, competition, predator-prey relationships, temperature sensitivity, chemical fate and exposure. The core element of a mathematic model with this capability is a population dynamical model, which can cope with interactions between species, population development and temperature effects. The mode] type is driven by temperature and the physiological demands of the organisms and the population dynamics is controlled by series of distributed delay procedures. Used as an ecotoxicological tool, the model must be able to calculate population effects, and if coupled with an exposure model and a chemical fate model, it is possible th calculate the indirect ecosystem effects of chemicals in nature, based on input from single species laboratory tests. Such models can be used to run scenarios under a wide range of climatic conditions, calculate worst case events and by aid of Monte-Carlo simulations with reasonable ranges of input parameters be used to asses the risk of certain events, such as local extinction of a species.

The model type and simulation results from a soil ecosystern model and a plant-herbivore model will be presented.

Department of Terrestrial Ecology

National Environmental Research Institute

Vejlsavej 25

P.O. Box 314

DK-s600) Silkeborg

[jenmark 
M. Klein

According to the EU Registration Directive, 91/414, concerring the placing of plant protection product the foundation for a hamonised system of registration of these producis was laid. It became clear in the development of the krmexes which followed, that mathematical modelling and Predicted Environmental Concentrations (PEC's) were going to play an important role in the decisionmaking process

A clear djstinction can be made for exposure models developed for pesticides between models dealing with inputs to ecosystems and models dealing with fate in ecosysterns.

Important input routes which have to be considered when talking about exposure models are spraydrift during application, transport via runoff water with suspended sediment, and transport through drainage systems.

Today, fate models are available simulating the behaviour of agricultural chemicals in ground water and surface water as well as in the soil. Some of these models (for example leaching models) are already in use in different member countries within the registration of pesticides. But as no exposure model includes all processes which may occur under outdoor conditions, vaidation and/or catibration of exposure models becomes important in particular when used for segistration purposes. Therefore, the question of validation will surely be in the focus of further work with the exposure models

Fraunhofer-Insizut fir Umweitchemie und Ökotoxikologie,

D-57392 Schmallenberg. Oermany
483

EXPOSURE ASSESSMENT IN FIELD DITCHES: THE TOXSWA MODEL

\section{P.I. Adriaanse and P. Groenendijk}

Application of pesticides to agricultural fields can result in the entry of residues into surface waters. Aquatic organisms can then be exposed to the pesticide. An essential element of risk assessment for these organisms is the prediction of their exposure to pesticides. Mathematical models are increasingly used for concentration estimations. They integrate existing knowledge and they can predict concentrations for more than one specific situation.

The TOXSWA model (TOXic substances in Surface WAters) describes the fate and behavicur of pesticides in a field ditch including the underlying sediment. It has been developed to assess the acute and chronic exposure of aquatic organisms and it has been mentioned in the latest changes in the registration procedure in the Netherlands.

In TOXSWA pesticides can enter the field ditch (i) by drift or atmospheric deposition, (ii) by surface runoff, or \{iil\} by drainage or leaching through the soil. Also point sources (e.g. leakage of a storage tank) can introduce pesticides into the ditch. TOXSWA considers three types of processes: transformation (first order with separate rate coefficients for ditch and sediment), sorption (Freundlich for sediment and suspended solids, linear for macrophytes) and transport (in ditch advection and dispersion, in sediment diffusion as well).

To assess which parameters are crucial for an accurate prediction of pesticide concentrations a sensitivity analysis is being executed. So far calculation results have shown to be very sensitive to the parameters describing sorption to macrophytes. As few literature data of this process exist measurements are carried out to determine the sorption coefficient of various pesticides to three different types of macrophytes.

First steps have been made to validate the TOXSWA model. Measured concen trations of the insecticide chlorpyrifos in microcosms $\{1.1 \mathrm{~m} \times 1.1 \mathrm{~m} \times 0.7 \mathrm{~m}$ \} and mesocosms $(40 \mathrm{~m} \times 3.5 \mathrm{~m} \times 0.5 \mathrm{~m})$ corresponded well to calculated concentrations of TOXSWA. Data for the herbicide linuron in both systems will become available in the near future.

Results of the sensitivity analysis and the initial phase of the yalidation will be presented and illustrated with a few appealing examples.
484

Modelling the spatial dynamics of predators in sprayed fam systems

Dr Paul Jepson

Dept Entomology, Oregon State University, Cordley Hall, Corvallis 97331, Oregon, USA

This paper will review recent field research and mathematical modeling programs which expiore the impact of pesticides on natural enemies in sprayed farming systerns. it will review the nature of the toxic effects of pesticides in the specific systerns being modelled and then explore the value of mathematical modeis whict seek to extrapolate these effects the system level, beyond that which is normally explored experimentally. Although this approach has dangers and limitations, it does generate new questions for experimentalists and highlight those combinations of agriculturat practices that are potentially the most hazardous. 
Validation of a terrestrial food-web model (CATS-1) for toxicants in meadows



PO. Box i. 3720 BA Bithisent. The Nerheriands

A common problem in the application and validation of ecotoxicologicul models is the absace of comprehensive datasers and or rime-series measurements. Generally data have to be collected from differen studies. In an ideal situation, process paramelers (such as rates for assimilation, growth, moniality etc.) are available from laboratory research and field data can be used for model calihraion and validation. In reality, a mix of data from the laboratory. the open literature and field data are available for model calibration.

A model for contaminant accumulacion in terrestrial and aquatic ecosystems (CATS) was applied to accumulation of heavy metals in meadows. Calibration of CATS models is based on random generation of parameter combinations. Output from every model simulation was compared with field and literature data to assess the validity of parameter combinations Parameter combinations leading to realistic model calculations are accepted. Rejected parameter combinations are those that lead to model output outside one or more of specitied ranges. This acceptation/rejection step is adapted from the uncertain but bounded' concept of Hornberger \& Spear.

Model validation on time-series of bioaccumulation was not possible due to lack of data. However, an extensive monitoring programme was available of bioaccumulation in meadows on different types of soil, Validation of the CATS-1 model, applied to different soil
The P. Tratas and Tom Aldenbers Jahwaton for water and arinking water research types, was performed and will be discussed.

FROM PESTICIDE USE TO PESTICIDE CONCENTRATIONS IN SURFACE WATER J.S. Buurma and J.C.M. Koopman

In this paper the emission of pesticides to surface water is constdered. Attention is paid to the fmportance of the varfous emission routes and to the resulting pesticide concentrations in surface water. Moreover the Finaliy atrention-points for research and development are put forward.

The results are based at the application of the calculation procedure for ten active ingredients. Since the investigation had che character of a judnnalssance, no absolute amouts are mentloned. For the same reason no judgennerts are made regarding the feasibility of the coramitnents and long

The use of the selected pesticides was calculated for 80 water management distrlcts in the Netherlands. Fox this purpose the average use in the target cxops of the pesticides concerned was calculated from the Farm Accountancy Data Network of LEI-DLO. Next this averages were multiplied The resulting total use for the Netherlands natched the figures of sales.

The emission loads of the various emission routes were calculated with the "Pesticide Entssie SCreening-model yoor Oppervlaktewater" (PESCO-model). This model depicts the relevance of the different emisston routes on both the natlonal level and on water management district level. Drift, run-off and equipment cleaning appear to be the most important emission routes for field-grown crops. For glasshou
limited to make conclusions. The output (- emission load) of the PESCo-model was used as input for the
calculation of the expected pesticide concentrations in surface water. For this calculation the model SOH-3 was applied. The model shows sudden concentration peaks after each pesticide application. The peaks fade away the peaks more than one hundred times higher than the pursued values.

Results of technical research on use reduction and emfssion reditetion of pesticides were collected. The use reduction options were categorized into three classes: sprayless zones, spraylng techniques and infrastructure. Dependent on the width of sprayless zones drift reductions of $60-90 \mathrm{z}$ and run-off reductions of $4.24 \%$ can be obtained. With spraying techniques deift run-off reductions of $4-24 \%$ can he obtained. With spraying techniques drift strongly depend on croptype en pesttype. The Dutch technical research on reduction of pesticide emission is strongly and equipment and equipment cleaning are also important emission routes, In order to routes run-off and equipment cleaning should get more attention.

J.S. Buurma
Agricultural Economics Research Institute (LEI-DLO)

P.O. Box 29703

NL- 2502 LS THE HAGUE
The Netherlands

488

487

\section{OVERVIEW OF THE PESTICIDE MARKET}

K. von Grebmer

The global market is expected to stagnate for a short time but then start to grow slightly. European markets after two years of sharp decline may continue to shrink further due to the new CAP reform and possible crop imports from Eastern Europe. In Eastern Europe the liberalization of the agricultural sector has been much slower and more difficult to establish than predicted. Prospects in the former Soviet Republic remain difficult. Demand for US agricultural product remains strong, fueled by population growth and economic expansion in regions such as Asia. Predictions about Latin America are difficult due to economic and political uncertainties. The situation in Africa is bardly encouraging but it is not expected that conditions will substantially improve over mid-term. The Asian/Pacific region is one of the few regions still offering potential for future growth. A substantial growth potential is seen in China. The crop protection market is underdeveloped and has an enormous volume potential. Other countries in the region may move from subsistence to commercial farming. The protected and saturated Japanese home market shows a declining trend.

Despite the increasing need for modern farming, initiatives to reduce the use of Crop Protection Products emerge in Europe and North America. The trend seems to be 'less is better' in order to take care of the environment and the limited resources. However, this approach reflects only quantitative aspects of the issue, the qualitative side is missing. According to independent experts, growing needs for food are not a theoretical justification of agrochemical industry, but rather a growing world issue by next years. Industry can play a key role by providing products, support and know-how to the developing countries and by participating in action plans.

Modern intensive farming and CPPs optimized use can ensure that production will continue to meet increasing world-wide requirements, contribute to a steady and affordable food supply, increase farm incomes and secure an economic and viable agriculture remaining compatible with the environment and with human health. Industry is working and seeking permanently to reduce possible impacts to the enviromment through innovations in different areas. CPPs have a firm place in 'Sustainable Agriculture', an objective agreed upon in many programs and projects of international organizations (WHO, FAO, Rio Summit, etc.). The behaviour of NGO's, industry and UN-organizations could gradually shift from confrontation to cooperation in order to achieve success in the greater goal; adequately feeding the additional three-thousand-million people expected by the year 2025 . 
Implementation of the FaO Code of Conduct an Induetry viewpolnt. M.R. Thomas.

1995 sees the 10 th Arniveraary of the FAO International Code of Conduct on the Distribution and Ues of Pesticides. A period in which there have been considerable changes in pesticide distribution and use, both inside and outalde the industry. Many of the moro hazardous products have or are in the process of being replaced. This, along with advances in pockaging, labelling, increased famer education and training and the uge of intrinsically more active pesticides with reduced environmental
impact are all leading to improved pest management techniques, impact are all leading to improved pest management techniques,
with1n modern Bubtainable farming Bystems. The FAo code of Conduct. within modern Bubtainable farming systers. The FAo code of Conduct has played a part in this evolutionary procese. But in mogt cases to improved global pesticide management and control has been through the influence of enforced legislative controls.

The ability to control and manage pesticides has been clearly demonstrated through Industry's Safe Ube projects in Guatema la, Kenya and Thal Iand. Here, GIFAP, the Industry Association, has shown that a concarted effort by Governments, Indugtry, Famer and other intexested parties can bring enomous benefita to those who have to use and manage pesticides. It is only through the ability to use pesticldes in well designed Intagrated crop management systeng that developing countries will be able to provide safe, sugtalnable and affordable food supplies to feed theit increasing populations.

For the forseeable future,global food production will continue to rely on the benefits which pesticides can provido. But if we want to Lmprove management and control of pesticides in a professional manner it is easential to augment regulatory controls.Voluntary Codes and Conventions can point the way but have baen shown within the Safo Use Frojects and elsewhere to be no substitute for properly anforced legislation. To this end it is time for the Intemational Community to take a positive ate towards Global pesticide management and help Developing Countries in thair search for a better tomorrow. In this respect GIFAP and ita members will continue to play a positiva role.

M.R.Thomas, GIFAP

c/o Zeneca Agrochericals. Stewardship \&afery Department,

Pernhurst,

Haslemere.

Surrey,

UR.

GU27 $3 \mathrm{JE}$

\section{OESOLETE PESTICIDES IN DEVELOPING COUNTRIES}

Developing countrles havo large slocks of opsolete pesticides. These art pesticides that cannot be used any longer because they have deteriorated as a result of prolonged storage, or because theif use has boen bannad while they were still kept in store.

Obsolete pesticides are regarded as hazerdous waste. Dus to lack of environmentally sound disposal facilitios in developing countries, the total volume of such stocks is constantly increasing. Locetions at which these stocks are stored rarely meet international standards for the sete storege of stocks are stoped paraly meet international standards for the sete atorege of pesticldes and many tocks ere in daplorabla stata. Often, drums sre even start lesking soon. These stocks pose a severe and imminent thiteat to humen health and the environment. The total of such stocks in non.OECD countilet is estimated to be in excess of 100.000 tonnes and Includes lerge quentities of or gano-chlorine compounds such as DOT. Dieldin and BHC. A significant part of the obsolete pesticide stocks are latt-overs of pesticldes that countries abtained under ald arrangements. In addition, there are large quantitles of soll that are heavily contaminated ano should bs isagrded as :oxic wate.

Various factors have contributed to the accumulation of obsolete pestlcides in developing countrles. The main foctors are: overstocking at a result of difficulties in forecasting outbreaks of migratory pests: poor assessment of requirements; inappropriate formulations or containers; excessive donatlons: absence of adequate storage facilities and poor stock manacement.

These pesticides sre to be disposed of in a safe and environmentally sound manne? Presently, the prafarrad dlsposal method is hloh temperature incineration in a dedicatod hezardous waste Incinerator. With the exception of incineration in a dedicated hezarcous waste incinerator. With the exception of fome newly ndustriellsed countries. there are no hazardous waste lncineretion
facilities in developing countries. This meane that the waste may have to be shipped to an inclnerator in an industiblised country that is witling to accapt the waste for destructlon. The cDsts of such operatlons are bevond the finBncial posslbllities of individual developing countries.

Urgent action is requirad to prevent anvironmental disasters. Laaking and deteriorated stocks are to be repacked, while funds ara being sought for safe apteriorated stocks are to be repacked, while funds are being sought for sole and of great imporance thet the necessary measures are taken, both by and of great importance thet the necessary messures are taken, both by such stocks. International assistanes is required, both techmically and financlally. With financial assistance of the Qovernment of the Netherlands. FAO le undertaking several actlvities to paisa awargness tbout this prablem in developing countries and to enhance a broad involvement of the international donor community in addressing this problem.

H.P. van der Wulp and A. Wodageneh

492

Study of the occupational Health of Indonesian Farmers who Spray peaticides Misa Kishi; Norbert Hirschhorn**; Marlina Djajadisastra*;
Latifa N. Satterlee*; Shelley Strowman**; Russell Dilts*. Latifa N. Satterlee*; Shelley Strowman**; Russell Dilts*.
* Food and Agriculture Organization Integrated Fest Management * Food and Agriculture Organizat

** Johr Snow Inc., Boston, Massachusetts * Johr. Hirschhorn is currently Visiting Professor, University of
Minnesota School of public Health, Box 97,420 Delaware Street, Minnesota School of Public Health, Box 97,420 Delaware Street, S.E., Minneapo
addressed.)

SUMMARY

A prospective study was carried out with 214 Indonesian farmers who sprayed pesticides on shallot crops. Observations of 905 spray operations were made at weekly visits to each farmer over a three-month period. Signs and symptoms of acute illness were reported by the farmers and observed by the interviewers at the time of spraying and compared to an agricultural season without spraying. Signs and symptoms typically associated with pesticide toxicity were significantly greater in frequency, by two- to nearly fifty-fold in the spray period compared to the non-spray period, while symptoms not normally associated with pesticide poisoning did not change significantly over time. Symptom rates returned to baseline in the next non-spray season. By one criterion of pesticide poisoning - three or more neurobehavioural and intestinal signs and symptoms - 21\% of spray operations resulted in poisoning. Farmers sprayed often, used multiple hazardous pesticides and their skin and clothes were often wetted with the spray solution. These exposure variables were significantly and independently associated with the number of signs and symptoms encountered. No farmer used the commonly-recommended protective garb of impermeable gloves, hats, footwear, masks and coveraIls. Such garb would be unacceptably hot in a tropical dimate and too costly for the average farmer. We recommend policies to restrict the use of thay operations by promotion of integrated pest management, already proved successful in Indonesia as elsewhere. 
THE IMPLEMENTATION OF THE CODE OF CONDUCT

A CONSUMER, ENVIRONMENTAL PERSPECTIVE Sarojeni $V$. Rengam

The International Code of Conduct on the Distribution and Use of Pesticides was unanimously adopted in 1985 at FAO's biennial Conference.

Although the code provided a yardstick to measure pestcide marketing and use practices, it did not go far enough. Since the Code's main assumption is that pesticides are necessary, it does not recognize non-chemical methods of pest control nor addresses the issue of risk or even pesticide (PIC) and only three references to integrated pest managuent (IPM).

Disappointed with the Code, citizens groups, especially the Pestivide Disappointed with the Code, cltizens groups, especially the pestivide
Action Network (PAN), went to work to monitor the Code's implementation and Actian Network (PAN), went to work to monitor the Code's implementation and
to gather evidence of pesticide problems, particularly in the South. PAN's to gather evidence of pesticide problems, particularly in the South. PAN's
monitoring reports showed inadequate labelling, misleading advertising, and monitoring reports showed inadequate labelling, misleading advertising, and
problews with use. The reports provided inpetas for PIC's adoption in 1987. problems with use. The reports provided inpetus for PIC's adoption in 1987 .
Now, cen years after the Code's adoption, the contamination of humans and Now, cen years after the Code's adoption, the contamination of humans and
the enviroment by pesticides is still evident. A soon-to-be-published PAN the environment by pesticides is still evident. A soon-to-be-published PAN Asia and the Pacific's survey in Asia of 2000 farmers and agricultural workers, rostly women, shows many cases of untrained workers spraying without protective clothing; farmers and workers suffering from pesticide poisoning with symptons ranging from chronic dermatitis to breaching difficulties: lack of information and knowledge on use and precautions and
a litany of other problems with pesticide use.

To tackle the problems with pesticides comprehensive and clearly understandable national policies on pesticde reduction in agriculture and other sectors would be needed. Such polictes should support the aims of national agriculture including the production of food and address the issues of sustainability, biodiversity, and rural development. Such a

reduction policy would reduce the dependency on chemical pest control, the risks to health and environment and the use of pesticides. It is not sufficient to regulate pesticides on a "one chemical at the time"-basis. A
transparent decision raking process is needer to separate political and scientific decisions about pesticides.

Also needed are pest management systems that fully utilize natural resources, including host plant resistance, cultural methods, and minimal or none external inputs (especially pesticides). A system based on ecological principles and utilizing che best possible mixture of traditional and modern techniques, while conserving beneficial organisms, natural enemies and biodiversity. Such an agriculture already exists in the sustainable agriculture and IPM-systems that are being practised in Asia.

Finally, only through the concerted efforts of governments, international agencies, media, citizens groups, farmers and especially women farmers, can we truly tackle the problem of feeding the world without polluting ourselves and our environment.
The International code of Conduct on the Distribution and Use of Pesticides: Results of the Second Questionnaire on the Implementation and Progress with the prior Informed consent procedure.

Van der Graaff, N.A.; Adam, A.V.

Food and Agriculture Organization of the United Nations; Rome, Italy.

SUMMARY

The International Code of Conduct on the Distribution and Use of Pesticides was adopted unanimously by the FAO Conference in 1985. Subsequently, it was amended to include the Erior in 1985. Subsequently, it was amenced to include the Frior
Informed Consent Procedure in 1989 . The Code represents a wide
consensus among Governments, Pesticide Industry and NonGovernmental Organizations on the management of pesticides.

The Code is supported by technical guidelines, which provide detailed guidance on its implementation. FAO and bilateral aid agencies, in particular GTZ, have been
instrumental in providing assistance to developing countries instrumental in providin

At the adoption of the Code in 1985, the FAo Conference requested governments to monitor its observance in collaboration with the Director-General of FAO. The Organization has undertaken two detailed surveys on the implementation, one in 1986 and one in 1993. A short overview is provided of the results of the 1993 questionnaire.

Although progress has been made since 1986, serious deficiencies in pesticice maragement remain, in particular in Africa. Although the situation in Asia and Latin America is better than in Africa, there remains considerable scope for improvements. Pesticide legislation and regulations have improved; however, distribution, trade and use practices showed only limited advances.

The implementation of the Prior Informed consent Procedure is reviewed. In general, countries find the procedure useful but substantial attention is still required to mechanisms in the country to implement the procedure. Negotiations will start soon to make the PIC procedure binding.

In conclusion, although it is evident that progress has been made, a great deal remains to be done to help developing countries ensure the safe, efficient and environmentally sound management of pesticides, within the principles of Integrated Pest Management and sustainable development.

THE USE OF ANTAGONISTIC MICROORGANISMS TO TREAT SEEDS AGAINST SOIL/SEED-BORNE PATHOGENS OF COWPEA.

A.ADEKUNLE, K.F. CARDWELL, D.A.FLORINI, AND T.IKOTUN

Five fungi and one actinomycete, Trichoderma sp., T. koningi Oudem, T. harzianum Rifai, Penicillium purpurogenum O. Stoll Cladosporium oxysporum Berk. \& Curtis and Streptomyces sp., were isolated form soil dilutions and tested in vitro for their antagonistic behaviour against three plant patbogens: Macrophomina phaseolina (Tassi) Goid., Colletotrichum truncatum (Schw.) Andrus \& Moore and Rhizoctonia solani Kuhn. Agar plugs of individual cultures of antagonists and pathogens were paired on potato dextrose agar with the antagonist in the centre and each pathogen at four peripheral points on the plates; plates were kept for observation for 2 weeks. In the presence of the three Trichoderma species the average colony diameter of the pathogens were $2.0 \mathrm{~cm}, 1.6 \mathrm{~cm}$ and $2.0 \mathrm{~cm}$ for, $\underline{M}$. phaseolina, C. truncatum and $\underline{R}$. solani, respectively. $\underline{\text { p. purpurogenum }}$ stopped growth of $M$. phaseolina at an average colony diameter of $2.7 \mathrm{~cm}$ and $\underline{C}$. truncatum at $3.2 \mathrm{~cm}$ but did not control $\underline{\mathrm{R}}$. solani. Similarly, C. truncatum gave weak control of $\mathrm{M}$. phaseolina and $\mathrm{C}$. truncatum and did not control $\mathrm{R}$. solani. These Streptomyces sp. controlled growth of all the three pathogens. In pot trials, germination percentages of seeds in soil infested with sclerotia of $\mathrm{M}$. phaseolina were calculated at 21 days after planting. When seeds were treated with benomyl (Benlate WP 50 ) at $0.5 \mathrm{~g} \mathrm{a.i.} / 50 \mathrm{~g}$ of seeds, $86 \%$ of seeds germinated. When seeds were treated in aqueous spore suspensions of $\underline{T}$. harzianum and $\underline{T}$. koningi $\underline{i}$ containing starch as an adhesive, $66 \%$ and $54 \%$ respectively of the seeds germinated. Seed germination with $\underline{T}$. harzianum was significantly better $(\mathrm{P}<0.05)$ than that of the untreated control where only $44 \%$ germinated.

ADEKUNLE, A

MAIZE PATHOLOGY LAB. I.I.T.A.

C/O L.W. LAMBOURN \& CO. LTD, CAROLYN HOUSE. 26 DINGWALL ROAD, CROYDON CR 9 3EE ENGLAND. 
Effect of Endosulfan, Neem seed kernel suspension and Bacillus thuringiensis on chickpea podborers.

H.Adldoost, S.Oroumchi ( Agricultural Research Center Urmeyeh) M.Safar abzadeh (University of Urmeyeh) and S.Izadyar (plant pest and Diseases Research Institute Tehran, Iran)

In the Northwest of Iran, chickpea is attacked by Heliothis viriplaca ( Hufangel), Helicoverpa armigera (hubner) and Heliothis peltigera (Denis \& Schiffermuller), the latter being only of ninor importance. Under rainfed conditions, major damage is caused by H.viriplaca and pod infestation usually ranges from 6 to $16 \%$.

In a field experiment conducted in a randomized complete block design in 1994, a commercial formulation of Bacillus thuringensis (Bactospeine) Neem Seed kernel suspension in water (N.S.K.S) and Endosulfan were tested against larvae of Heliothis $\underline{S p}$ in the 2 nd and $3 r d$ stages, using a knapsack sprayer.

Results revealed that on the $3 \mathrm{rd}$ and 6 th days after application, there were no significont differences in the efficiency between treatments at $\alpha=0.01 .10$ days ofter treatment, Endosulfan and N.S.KS ranked in group A and B.T. ranked in group B at $\alpha=0.01$.

499

USE OF ATTENUATED ISOLATES OF TOBACCO MOSAIC VIRUS TO CROSS-PROTECT AGAINST SEVERE INFECTIONS OF THE VIRUS IN IBARAKI, JAPAN

Atiri, G.I., Tsuda, S., and Fujisawa, I.

Mosaic disease of pepper, caused by the pepper strain of tobacco mosaic virus (TMV-P), is the most serious limitation to pepper production in ibaraki Prefecture, a major growing area of Japan. Besides long-term breeding for resistance, the use of attenuater isolates to cross-protect against more severe strains of the virus have been previously attempted. In this study, leaf samples of a field-collected isolate of TMV-P, which is serologically indistinguishable from the prevalent type strain in Japan and inducing typical severe symptoms of mosaic, crinkling, and puckering were attenuated in a growth chamber ( $80 \%$ r.h., $8000 \mathrm{~lx}$ ) at both low $(15 \circ \mathrm{C})$ and high $(37 \circ \mathrm{C})$ temperatures for periods of 14 , 28 and 42 days, respectively. After each exposure period, samples were used to mechanically inoculate 4-week-old glutinosa tobacco leaves, the assay host, in which pinprick necrotic local lesions were produced. Single lesions were excised and each was used to inoculate 3 -week-old pepper plants (Capsicum annuum $\mathrm{cv}$. New Tosahikari) at the two-leaf stage. A total of 122 and 48 single-lesion isolates were thus used after low and high temperature treatments respectively. Of these, 11 and 12 isolates produced symptomless and mild infections in pepper at low temperature, while 2 and 12 isolates produced symptomless and mild infection at high temperature. Symptomless plants produced necrotic local lesions when back-inoculated to glutinosa tobacco. When treated isolates were $e^{\mu}$ first inoculated on pepper, followed by inoculation with the severe isolate 10 days later, about $73.9 \%$ ( 17 out of 23 isolates at low temperature) and $42.8 \%$ (6 out of 14 isolates at high temperature) partially or completely prevented infection by the severe isolate.

Plant Biotechnology Institute, Ibaraki Agricultural Centre, Iwama, Nishi-lbaraki, 319-02 Japan

*First author is grateful to the IFS (Sweden) for research support and to the MIF (Japan) for the provision of a travel grant.Current address of first author- Dept. of Agricultural Biology, University of Ibadan, Ibadan, Nigeria.
BIOLOGICAL CONTROL OF FUSARIUM WILT OF BASIL (OCIMUM BASILICUM L.)

A. Garibaldi, G. MinUto, M.L. GUlLino

Fusarium wilt, incited by Fusarium oxysporum f.sp. basilici, is a major problem in the cultivation of basil (Ocimum basilicum), a cash crop grown under glasshouse (approximately 65 ha) in the Riviera Ligure, in Northern Italy. Control of $\underline{F}$. pasilici, which can be transmitted by infected seeds, is complicated by the limited availability of fungicides and by restriction in the use of some fumigants.

Saprophytic Fusarium sp., isolated from Fusarium-suppressive soils, active against several formae speciales of Fusarium oxysporum, proved effective also against Fusarium wilt of basil in several experimental trials carried out under sovere diseaso pressure. Antagonistic Fusarium spp. were used as a soil treatment ( $10 \mathrm{CFU} / \mathrm{mL})$ and/or seed dressing $\left(3 \times 10^{7}-10^{8} \mathrm{CFU} / \mathrm{g}\right)$. soil infestation with the antagonists was carried out at the same time as soll infestation with $\mathrm{F}$. basilici (applied at $10^{3}$ $5 \times 10^{3} \mathrm{CFU} / \mathrm{ml}$ of soil). An interval of $10-15$ days was left before seeding basil, in order to permit both pathogen and antagonist to colonise the soil. One soil treatment with the isolate $251 / 2$ IB of saprophytic Fusarium oxysporum effectively protects subsequent basil crops for several months. The exclusive use of such isolate for seed dressing did not control Fusarium wilt. The combination of soil treatment with seed dressing generally did not improve wilt control in comparison with soil treatment alone. The results obtained, in the presence of a high disease pressure, confirm the possibility of using antagonistic Fusarium spp. 'for effective control of Fusarium wilt of basil. In order to obtain consistent and longlasting wilt control, the ratio between antagonist and pathogen propagules must be al least 20 . The long-lasting effect shown by one soil treatment with antagonistic Fusarium spp. protects the crop for several months. The good results obtained on the variety "Fine verde", highly susceptible to Fusarium wilt, are more easily achieved with the cv "Genovese a foglie giganti" widely grown in Italy and less susceptible to Fusarium wilt.

DI.VA.P.R.A. - Patologia Vegetale, Via Giuria 15, 10126 Torino, Italy.
500

THE FAUNA OF THE WEED Cardaria draba ( L.) Desv. IN CROATIA

\section{Igrc Bař̌ić, P. Dinarina}

Cardaria draba ( L.) Desvaux (Brassicaceae) - hoary cress or white top (CADDR) is a weed species which originate from the Balcans. It is spread in many countries of Europe, especially in southern and middle Europe, without being of greater importance to agriculnural crops. In the U.S. it is spread in most states, and its presence is also registered in Canada, Australia etc. 'The importance in the U.S. is shown by the interess of the USDA to establish the fauna of this weed in Croatia in order to find out potential agents of biccontrol. Results mentioned in this paper are obtained in a research project supported by the USDA.

On C. draba 63 different species of insects were collected. In field and trough feeding tests conducted in the laboratory the feeding on this weed was proved for 43 species of them.

For some polyphagous insect species no data about their behaviour on C. draba were known. Therefore in laboratory trials data about the development of Brevicoryne brassicae ( L.) (Homoptera: Aphididae), Eurydema oleraceum (L.) (Heteroptera: Pentatomidae) and Phyllotreta nemorum L. (Coleoptera: Halticinae) feeding on C. draba wexe established. Some data on the biology of Sirocalus floralis Payk. (Coleoptera: Curculionidae) were established also. For some insect species no data about their feeding on C. draba was found in disponible literature. In laboratory trials the full development of Adelphocoris lineolatus (Gz.) (Heteroptera: Miridae) on C. draba was established. C. draba is also a suitable host for Oxycarenus lavaterrae H.S. (Heteroptera: Lygaeidae), Aphis verbasci Schrank (Homoptera: Aphididae) and a yet not identified Apion (Coleoptera: Curculionidae) species.

O. lavaterrae, S. floralis, and two leaf mining dipterous larvae identified as Phytonyza atricornis Meigen and Liriomyza strigata Meigen (both Diptera: Agromyzidae) should be submitted to further trials in order to find out their suitability as biocontrol agents.

Prof.dr. Jasminka Igrc-Bartić, Paculty of Agriculture, Institute for Agricultural Zoology, Svetosimunska 25, 41.000 Zagrcb, Croatia. 
SPORIDESMIUM SCLEROTIVORUM UECKER, AYERS ET ADAMS AS A NEW AGENT FOR BIOLOGICAL CONTROL OF PLANT WHITE ROT IN UKRAINE.

\section{V.S.Chaban}

Sclerotinia sclerotiorum (lib.) d By. - pathogen of white rot is one of most spread pathogens of many plants in Ukraine. The main source of this disease is sclerotia of the fungus in soil. Their surviving largely depends on presence and activity of antagonistic microorganisms. In 1990-1993 investigation of antagonistic fungi of sunflower white rot pathogen was done. 91 samples of soil from 15 regions of Ukraine was analyzed. Detection and isolation of antagonistic fungi was done by method of "baits". The new microparasite was found. It was identified on the basis of studying of morphological and anatomical peculiarities as Sporidesmium sclerotivorum Uecker, Ayers et Adams, which was registered in Ukraine in the first time. On inoculated sclerotia in laboratory experiments in petri dishes mycelium of S.sclerotivorum appears in 6-7 d., sporulation - after 14-15 d. 50-60\% of sclerotia were infected by mycroparasite. Infection of sclerotia, which were inoculated by spore suspention of S.sclerotivorum and placed in soil, was: in 4 $w-73,1 \%$, in $6 w-100 \%$. The infected selerotia had dark center, easy crushed. Control sclerotia, as a rule, sprouted into stroma remaining white solid center. So it was showed, that S.sclerotivorım have high antagonistic and/or parasitic potential and may be used in working out of biological control of plant white rot.

Plant Protection Institute, Vasilkovskaya st. 33,

Kiev, Ukraine 252022

503

SCREENING OF MICROBES THAT ATTACH TO BOTRYTIS CINEREA IYPHAE BEFORE TESTS FOR BIOCONTROL ACTIVITY.

\section{D.W.M. Cook, P.G.Long.}

A new approach to screening mictobial biological control agents against plant pathogens is proposed as a more efficient alternative to in vitro plate tests.

An assay was designed to detect and isolate bacteria and yeast species capable of attaching to the hyphae of Botrytis cinerea Pers. and to then separate them from the huge numbers of loosely associated microbes and other contaminants. This preliminary screening should greatly reduce the number of organisms to be screened in vivo for biocontrol activity. In preliminary studies, mixtures of bacteria and yeasts from phylloplane washings were incubated in sterile distilled water with $B$. cinerea hyphae. After a crude washing procedure microscopic observations showed microbes attaching to the hyphae.

An isolate (isolate $211 \mathrm{ex}$. Dr C. Wilson USDA) of the yeast Debaromyces hansenii (Zopf) Lodder \& Kregervan Rij. a biocontrol agent capable of attachment to $B$. cinerea, was used to develop an apparatus and protocols for the consistent removal of loosely associated and other contaminant microbes. Tests of assay performance showed that contaminant levels in the apparatus could be reduced to between 2 and 10 cells per million applied to the system. Further the assay conditions did not cause cohesive failure between the test yeast (D. hansenii) and the pathogen hyphal surface. Currently, microbes collected by this screening technique are being tested in vivo for biocontrol activity.

Department of Plant Science.

Massey University.

Private Bag 11-222 Palmerston North. NEW ZEALAND.
Biological control of potato pathogens with selected saprophytic soil bacteria

\section{Ciampi and E. Schnettler.}

The study and development of bacterial antagonists to biologically control plant pathogens as bioinoculants is based on the existence of a series of inhibitory palationships between microorganisms under natural conditions. In general the ideal inhibitory system consists in preventing the proliferation and development of pathogens populations to harmful levels for the host plant. Over the years, several selected bacteria have been studied under controlled and field conditions to control several diseases of cultivated potato (Solanum tuberosum subsp. tuberosum).

Saprophytic soil bacteria isolated from Chilean soils were initially selected by their capacity to cause intubitions of Rhizoctonia solani, Streptomyces scabies, Pseudomonas solanacearum and Erwinia (carotovora group). Many strains were studied and few of them were finally selected after establishing their taxonomic position and saprophytic capacity.

Experiments conducted in plants growing in pots under semi-controlled conditions revealed that some strains were capable to inhibit the pathogen. Field experiments confirmed previous results, and in most cases the selected antagonistic strains prevented disease establishment.

Studies conducted to control R. solani indicated that treated tubers with antagonist isolates showed less infection and less presence of sclerotia than nontreated tubers. In pot experiments to control bacterial wilt with a special formulation of encapsulated bacteria indicated less presence of wilted plants and less latent infection in daughter tubers. Field experiments to control soft rot Erwinia showed that bacteria must persist a long time in the soil to prevent infection of daughter tubers. Also, experiments to control common scab in potato indicates the possibility of using new species of Streptomyces isolated from the Chilos Island. Finally results gathered over the past 5 years clearly show that formulation and persistence in the soil for a long period of time of antagonistic bacteria is a critical condition to develop a strategy to control most soil borne potato pathogens.

Institute of Plant Protection and Production, Faculty of Agronomic Sciences, Austral University of Chile, Casilla 567, Valdivia, Chile. Research developed thanks to the contribution of SAREC, IAO, FONDECYT 52-92 and DID-UACH.
UTLIZATION OF ENTOMOPATHOGENIC FUNGI IN THE BIOLOGICAL CONTROL OF WHTEKLIES (Bemisia spp.)

\section{G. O. Costa \& R. Creamer}

Fungi collected from whiteflies (Bemisia tabaci and $B$. argentifolii) in Imperial Valley, a desert agricultural area in Southern California, U.S.A., were tested for entomopathogenicity. Only saprophytes or weak pathogens were found. Other entomopathogenic fungi were then tested for pathogenicity to whiteflies. Conidia suspended in water at a concentration of $10^{9}$ spores $/ \mathrm{ml}$ added to Tween-20 $0.05 \%$ were applied to whitefly colonies containing all life stages of the insect. The petioles of detached bean leaves infested with whiteflies were inserted in wet foam and placed inside a container covered by a small tube cage which was considered a practical bioassay; enough time was allowed for evaluation of mortality by the biological control agent. The cages were maintained at $25^{\circ} \mathrm{C}$ under $90 \%$ RH. Mortality caused by several isolates in the genuses Paecilomyces, Verticillium, Beauveria and Penicillium were determined at 5 to 7 days after spraying, which was measured as percentage of dead whiteflies compared to the watersprayed control. Fuagal sporulation was observed on some of the dead whiteflies. The dead insects were then subjected to superficial sterilization for 1 min. in $0.05 \%$ sodium hypoclorite and placed in PDA media to confirm the presence of the sprayed fungus internally. The water-sprayed control causes some mortality due to water damage of the fragile insect. Some whiteflies also showed mycelial growth due to saprophytes. Among the tested fungi, Paecilomyces amoeno-roseus appeared to be the more promising as a biological agent against the whiteflies under the tested conditions. However, the fungus lost its pathogenicity after several generations on agar media, which could be overcome by frequent passages through the insect. Paecilomyces amoeno-roseus was more agressive against adult whiteflies than nymphs or eggs.

Department of Plant Pathology, University of California, Riverside, CA, 92521-0122, U.S.A. 
BIOTIC AND ABIOTIC FACTORS INFLUENCING THE EFFECTIVENESS OF BIOENHANCED MULCHES IN THE CONTROL OF Phytophthora ROOT ROT OF AVOCADO

\section{J. L. da S. Costa, J. A. Menge, W. L. Casale, F. Guillemet}

The effect of bioenhanced mulches (artificially inoculated with biocontrol agents) on the control of Phytophthora root rot of avocados (Persea americana) was investigated. The results of 12-mo. greenhouse experiments indicated that the degree of maturation of some mulches could be correlated to their levels of toxicity to avocado clones. Sudan grass when aged for over 6 montbs in stationary piles, was toxic to avocado plants and it was not able to sustain the active populations of artificially inoculated Gliocladium virens and Trichoderma harzianum. Laboratory bioassays using sudan grass leachates demonstrated that increasing mulch maturation $(0,6,12$, and 18 mo.), correlated with increasing toxicity to $P$. indica seedlings, indicated by the visual dark necrotic symptoms on the roots. An increase on potentially toxic organic acids and nitrites, and a decrease on $\mathrm{pH}$ and $\mathrm{C} / \mathrm{N}$ ratio was observed on the older sudan grass mulches. The same levels of maturation in a yardwaste mulch (grass clippings, leaves, hardwood bark, 1:1:1) did not present any adverse effect on avocado clones. However, the ability of yardwaste to sustain high populations of the artificially inoculated biocontrol agents (BCAs) was significantly reduced on the mulches at the 12- and 18-mo. maturity levels. The bioenhanced yardwaste $(0-$ and 6 -mo. maturity levels), were able to efficiently deliver BCAs to the avocado root zone, improve soil structure and increase organic matter resulting in significantly healthier avocado plants than control plants. The BCAs were detected in the soils for up to 8 months after the pots were top dressed with the bioenhanced mulches: however, fluorescein diacetate hydrolysis tests indicated that the BCAs can significantly lose their activity after the 4th month.

Department of Plant Pathology, University of California, Riverside, CA, 92521-0122, U.S.A.

\section{7}

BIOLDGICAL CONTROL OF POWDERY MILDEW AND MILDEW OF CUCUMBER WITH ENTEROEACTER CLOACEAE IORDAN

Qlga Georgieva, Georgi Georgiev

In laboratory and greenhouse studies, the antagonistic activity of the bacterium strain Enterobacter cloaceae (Iordan) to powdery mildew caused by Erysiphe cichoracearum (D.C.) and Sphaerotheca tuliginea (Pol1) and mildew caused by Pseudoperonospora cubensis (Rostowz) have been investigated. The application of strain Enterobacter cloaceas B-I as a liquid product (titer of 2.1010 cells per millilitre) provided up to 70-60\% disease control and was as effective as Methyl Thiophanate or Propamocarb Hydrochloride in reducing powdery mildew and mildew of cucumber.

olga Georgieva

"Maritza" Vegetable Crops Research Institute

32 Erazovsko Chaussee 5 tr.

4003 Plovoiv,

B u l garia
EFEECT OF GURIZL DEPTH ON THE GERMINATION OF BromHE FIqLDUE Roth. AND Bromus rubens L. seeds. POSSIBILITY OF CULTURAL CONTROL.

\section{EENNI}

The influence of burial depth $(0 \mathrm{~cm}, 2.5 \mathrm{~cm}, 5 \mathrm{~cm}, 7.5 \mathrm{~cm}, 10$ $\mathrm{cm}, 12.5 \mathrm{~cm}, 15 \mathrm{~cm}, 20 \mathrm{~cm}$ and $22 \mathrm{~cm}$ ) in three soils ( sandy, Eandy clay loam and clay, on the germination of Bromus righdug Roth. and Bromus rubens L.seeds was investigated. The germination rate of the two weeds seeds was linearly and strongly correlated with the depth burled and the soil texture: the germination percentage of seeds buried to the depth of $10 \mathrm{~cm}$ in the sandy clay loam soll was about 0 for Bromus figldus Roth. and 33 f for Bromus rubens $\mathrm{L}$. These results show that there is an opportunity of controling these weeds by cultural means.

Laboratory of plant ecology Institute of Blology, University of Setif 19000 Algerla.

508

LONGTERM BIOCONTROL OF SCLEROTINIA SCLEROTIORLM BY CONOTHYRUM MINITANS.

M. Gerlagh, H.M. Goossen-van de Geijn and N.J. Fokkema

Sclerotinia sclerotiorum is a necrotrophic pathogenic fungus of worldwide Sclerotina sclerotiorum is a necrotrophic pathogenic fungus of worldwide
occurrence with a very wide host spectrum. Survival is by sclerotia, which occurrence with a very wide host spectrum. Survival is by sclerotia, which
produce apothecia, from which ascospores are released. Ascospores infect plants through moribond tissue. There is no secondary dissemination in the crop by vegetative spores. The mycoparasite Coniothyrium minitans destroys sclerotia and thus potentially reduces future inoculum pressure.

In a five-year field experiment on soil naturally infested with 5 . sclerotiorum, potato, bean, carrot and chicony were grown in succession. The treatments, aimed at hitting mycelium and sclerotia of S. sclerotiorum on infected plants, consisted in spraying three times during the second hast of the vegetation period with suspensions of conidia of $C$. minitans or Trichoderma spp. and a control. Treatments were randomized in ten replicate blocks. Plots were situated in a corn field to prevent spores of the antagonists to cross-contaminate other treatments. In the plots apothecia were scored as estimates of soil infestation with sclerotia. Disease incidence was assessed by visual rating

Only in bean disease incidence was considerable. Only this crop therefore contributed to appreciable addition of fresh sclerotia to the soil. In the first and second year no effect of treatments on apothecial number and disease incidence was found. In the third year the only effect was reduction of apothecia in the $C$. minitans plots with chicory, two years after a bean crop. The delayed effect is due to the turning action of ploughing. which brought sclerotia from the 1990 bean crop back to the surface after two years. With $C$ minitans, per $100 \mathrm{~m}$ row length 11 sclerotia had germinated to produce apothecia, compared to 122 and 100 for the Trichoderma treatment and control, respectively. For the same plots the figures were 26 against 148 and 95 in 1993, and 4 against 36 and 14 in 1994. No significant effect of treatment on disease incidence in bean was found except for 1994, when beans were grown again on the same plots as in 1990 . Numbers of infected plants at both sides of $100 \mathrm{~m}$ row length were 529 for $C$. minitans treatment against 1023 for Trichoderma, and 964 for the control. These figures illustrate the potential for long term control of 5 . sclerotiorum by $C$. minitans, which was about $50 \%$ under prevailing field conditions.

M. Gerlagh, DLO Research Institute for Plant Protection (IPO-DLO),

P.O. $80 \times 9060,6700 \mathrm{GW}$ Wageningen, The Netherlands. 
USE OF THE RHABDITID NEMATODE PHASMARHABDITIS HIERMAPIRODITA FOR BIOLOGICAL CONTROL OF SLUGS IN ARABLE CROPS

\section{M.J. Wilson, L. Hughes, D. Barahona and D.M. Glen}

Slugs are important pests of a wide range of arable crops, especially winter wheat and oilseed rape. They are potentially amongst the most troublesome pests in less intensive environmentally favourable arable farming systems because of the effects on slug populations of non-plough tillage, incorporation of crop residues, crop rotations and the use of cover crops The rhabditid nematode Phasmarhabditis hemaphrodita would be panticularly suitable for use in less intensive arable systems, as the nematode has no adverse effects on beneficial invertebrates. mammals and birds.

Field experiments on timing of nematode application indicate that there is little or no benefit from nematode application up to 2 weeks prior to sowing a crop of cereals or rape compared to application at the time of drilling. Application to moist soil is thought to be more important than the precise tining of application. Initial field trials indicate that mixing nematodes into soil after application protects them from dry surface soil conditions, without loss of efficacy. Laboratory studies indicate that slugs avoid areas of soil treated with the nematodes, offering the possibility that band treatments could be used, thus reducing the field dose applied, without loss of efficacy.

The effects of field application of $P$. hermaphrodita on the molluse fauna in field margins has been investigated in two field experiments where nematodes were applied to $12 \mathrm{~m} \times 12 \mathrm{~m}$ plots adjacent to field margins after drilling oilseed rape. In both experiments, several species of snails and slugs were common in the field margins adjacent to the treated areas. No adverse effects on molluscs in field margins have been detected, although laboratory tests revealed that certain species of snaits were inherently susceptible. Experiments on vertical and horizontal movement of the nematodes in medium-loam soil and sand showed that the vast majority of nematodes did not move more than $1-2 \mathrm{~cm}$ vertically or horizontally from the site of placement. These results imply that application of nematodes to arable soil is unlikely to result in significant spread of nematodes to semi-natural habitats and therefore poses no significant threat to molluscs living in semi-natural habitats adjacent to treated areas.

This research is part of a project jointly funded by the Agricultural Genetics Company Ltd and the Ministry of Agriculture, Fisheries and Food, in the LINK Technologies for Sustainable Farming Systems Programme.

IACR-Long Astiton Research Station, Department of Agricultural Sciences, University of Bristol, Long Ashton, Bristol BS18 9AF, UK

511

STUDIES OA THE SYNERGISTS FOR IN VITRO REARING TRICHOGRAMA SPP.

Shichou Han, Qiaoxian Chen, Liying Li

Solutions of seven kinds of powder (polyviny? alcohol, gelatin, white latex, agar, cassava root, rice flour, and starch) were selected as symergists to improve the oviposition of Trichograma dendrolimi, $T$. confusum and $\mathrm{T}$. evanescens with in vitro rearing method. All the seven solutions improved the trichogramatid oviposition much on the artificial "eggs". Specific differentiation of trichogramnatid ovipositional response to these synergists was observed. So, the most effective and economical synergists can be used for increasing the efficiency of rearing different species of Trichogranma in vitro.

Mr. Shichou Han, Guangdong Entomological Institute, Xingang west Road 105. Guangzhou 510260, The People's Republic of China
BIOLOGICAL CONTROL OF CUCUMBER FUSARIUM ROOT ROT CAUSAL AGENTS IN GLASSHOUSES

\section{L.D.Grishechkina}

Biological control of disease complex in the process of vegetable crops cultivation in glasshouses is becoming more and more important. This method of control of soil pathogenes, causing toot rots, hasn't found an adequate large scale use not only because of its labour-intensiveness and inconstancy of results, but because of limited assortment of biological control agents as well. The possibility of microbiological preparations Agrofil and Bactophyt application to control cucumber Fusarium root rot causal agents has been studied by the author. It has been detected that soil population of Fusarium oxysponim f.cucumerinum is considerably effected by these preparations. Thus, after soil application of Agrofil the number of the pathogene infection units has reduced nearly by 3-4 times. Bactophyt effect was manifested slower. This has been proved by results, obtained while conducting large-scale industrial application. In the variant with Agrofil number of dead plants was 2 times less as compared to the non-treated control. Besides a protective effect, a stimulating activity of the two microbiological preparations on development and productivity of cucumber plant has been observed. Bactophyt efficacy can be considerably increased by the use of biologically active substrates on the basis of a complex microbiological preparation. Microorganism association as a component of this preparation possesses an antagonistic and mycolytical activity on phytopathogenic fungi. including Fusarium gen. and increases plant resistance to phytoinfections after bacteriazation of glasshouse soil.

All-Russian Institute for Plant Ptotection, Shosse Podbelsky 3,

St.Petersburg-Pushkin, 189620, Russia

512

BIOLOGICAL CONTROL FOR SEEDLING DAMPING-OFF DISEASES.

\section{A. R. Harris, D. A. Schisler and P. G. Adkins}

Damping-off diseases due to Rhizoctonia solani and Pythium spp. cause major losses in a wide range of seedlings worldwide. Bacteria and fungi were isolated from South Australian nursery potting media that were suppressive to Rhizoctonia solani. They were assayed singly for ability to suppress damping off in bedding plants and to promote growth. Seedlings were grown in pasteurized potting medium with or without added damping-off fungi in glasshouse and growth cabinet tests. Two isolates of binucleate Rhizoctonia consistently reduced damping-off caused by Rhizoctonia solani, Pythium ultimum and $P$. irregulare. Two isolates of bacteria also reduced damping-off caused by Pythium ultimum. The effective antagonists suppressed damping-off significantly better than standard fungicide drenches in most experiments, and better than the commercial biological control bacterial isolate, Bacillus subilis A13, in all experiments. Biological control was achieved on seedlings of capsicum, celosia, petunia and brussels sprouts. In the absence of added pathogens, three isolates of bacteria and one of binucleate Rhizoctonia significantly increased shoot dry weights of capsicum seedlings in three of four experiments. Several isolates have potential for commercial development as biological controls for damping-off and growth promotion in bedding plants.

CSIRO Division of Soils, Private Bag No. 2, Glen Osmond, S.A. 5064, Australia. 
DECREASE IN INCIDENCE OF PYTHIUM FRE-EMERGENCE UAMPING-OFF OF FIELD PEA BY SEEO TREATMENT WITH BACILLIS SPP. ANU METALAXYL.

\section{S.F. Hwang}

Trends in crop diversification have resulted in the increased production of many special crops in Alberta. Field pea (Pisum sativum var. arvense (L.) is we1l-adapted to temperate climates and can withstand considerable frost. Farmers in north-central Alberta have recentiy been encouraged to include field pea as an alternative cash crop or in rotation with winter cereals. In Alberta, where environmental conditions at planting time are unpredictable, sudden drops in teriperature after planting often result in cold soil conditions adverse to rapid germination and predispose seeds and seedlings of field pea to pythium spo. infection. Pythium seed rot and seedling daming off have become major limiting factors in the production of field pea in Alberta. Studies were conducted to determine the inhibitory effect of $B$. subtilis and 8 . polymyxa on Pythium spp.; and, to examine the comparative effects of $\bar{B}$. subtilis or $\underline{B}$. polymyxa and metalaxy of 10 strains rot of field pea under controlled condition of listrains tested in paired culture, four strains of $B$. subtifis and two strains of $B$. polymyxa had an inhibitory effect subtilis - culture filtrates and bacterial suspensions of $\underline{E}$. Subtilis and $\frac{E}{\text { in vitro. }}$. polymyxa were also antagoristic to pythium sp. in vitro, Growth of $P$. irregulare and $P$. $u 1$ timum was reduced

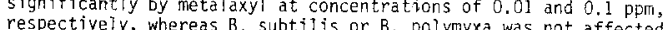
respectively, whereas $B$. subtifis or $B$. polymyxa was not affected at the same concentrations.

The biocontrol agent, B. subtilis or 3 . polymyxa, and the fungicide metalaxyl were evaluated alone and in combination for reducing seed and root rot of field pea CV. Radley caused by $P$. irregulare or P. Ultimum. Seed inoculation with B. Eubtil is or B. polymyxa did not cause significant increase in percent seedling survival, plant height, and shoot and root dry weights compared to nontreate seeds in infested soil. Greater seedling survival, plant height, and shoot and root dry weights were observed for seed treated with metalaxyl at full recomended rate than with $\underline{B}$. subtilis or $B$. polymyxa. However, the integration of $B$. Subtilis or $B$. polymyxa with metalaxyl at half recommended rate was the most effective method for reducing Pythium seed and root rat. The percent seedling survival, plant height, shoot and root dry weights did not differ significantly between seed treated with $B$. Subtilis or 8 . poimyxa plus metalaxyl alone at full rate. Nontreated seeds planted in noninfested soil had the greatest seedling survival, plant height, and shoot and root dry weights. This study suggested that a combination of Bacillus Spp, and metalaxyl at a lower rate could be used to improve the establishment and growth of field pea in Pythium-infested soils.

Alberta Environmental Centre, Vegrevilie, Alberta, Canada T9C 1T4.

515

BIOLOGICAL CONTROL OVER GYPSY MOTH (OCNERIA DISPAR L.) NUMBER IN WESTERN SIBERIA

\section{A.V.llyinykh, S.A. Bakhvalov, S.A.Mamontova}

Within 1992-1994 activities on protection against the gypsy moth have been carried out in birch and aspen forests in a number of Siberian provinces. As agents of biological control the preparation Virin - NSh based on the nuclear polyhedrosis virus and the bacterial preparation lepidocide have been used. The viral preparation was applied by manual treatment of the insects' eggs on the area comprising $10-15 \%$ of the total area infected by the gypsy moth. Expenditure of the preparation varied from $30 \mathrm{~g}$ up to $120 \mathrm{~g}$ per a hectare, the quantity of polyhedrons was $10^{9}$ per $1 \mathrm{~g}$ of the preparation. Lepidocide was used by total treatment of trees by land spraying in the period mass emergence of young larvae. The bacterial preparation was used on the areas with a high degree of a defoliation threat $(75-100 \%)$, the expenditure of the preparation was $1-2 \mathrm{~kg}$ per a hectare. A decrease of the gypsy moth number being determined by the insects' eggs was from 32 up to $82 \%$. Apart from mortality of the insects from polyhedrosis and bacteriosis resulted from applying the biological preparations, the mortality of the gypsy moth from entomophages and ecological factors have been recorded. The both preparations have not prevented defoliation of the forests in the year of usage on the areas with a high density of the gypsy moth ( 4 oviposition and over per one tree). These areas were treated for the second time on the following year; on the following season slight defoliation occurred and tree's crown was recreated in all the infected areas. On the whole, the state of the trees with the gypsy moth population can be estimated as satisfactory and economically valued losses of the trees have not occurred. The majority of existing seats of the gypsy moth have either faded or entered the stage of a crisis

Institute of Biology Siberian Branch of Russian Academy of Sciences, Frunze 11, Novosibirsk, Russia 630091
BIOLOGICAL CONTROL, OF THE SCALES, DREPANOCOCCUS CHITON IN CARAMBOLA ORCHARD

A.G. Ibrahim

The scale, Drepanococcus chiton (Green) (Homoptera: Coccidae) is a pest of carambola trees in Malaysia. The high population of the scale is obvious during the dry spell causing drying of shoots and flower stalks. A female scale is capable of producing an average of $1081 \pm 256$ eggs. The incubation period of eggs was one week and the percentage of hatching was $97.9 \pm 1.1$. The three nymphal stages of the scale can be differentiated based on the dimension of the head capsules. The development of egg to maturity of female scales was 49.9 pon days. The winged male

Field observations showed that the gravid scales are being parasitized by an egg-predator, Eunotus sp. (Hymenoptera: steromalidae). The average monthly parasitisation by Eunotus spp. was $40.6 \%$. The parasitic larvae had 5 instars and it consumed $93.2 \%$ of the total eggs of the gravid scale. The sex ratio of male to female was almost 1.1 . The potential of Eunotus spp. for the biological control of $D$.
orchard of the tropics are discussed.

Department of Plant Protection

Universiti Pertanian Malaysia

43400 UPM, Serdang, Selangor,

Malaysia.
516

BIOLOGICAL CONTROL OVER NUN MOTH (OCNERIA MONACA L.) NUMBER

\section{S.A. Bakhvalov, A.V. llynykh, G.V.Larionov}

Within three last decades there have been a number of large outbreaks of mass reproduction of the nun moth in pine tree forests of Western Siberia, the Urals, and Northern Kasakhstan. The total area of the pest's population for the indicated periodconstitutes over 100000 hectares. The viral preparation Virin - PShM and the bacterial preparation lepidocide have been used to biologically suppress the nun moth in the considerable part of the seats. The preparations were applied by aerialapplication, land spraying, and by aerosol generator as well. Aerosol treatment was carried out under thermo-mechanic mode of the generator with 10-50 micrometers dispersed composition of aerosol. Norms of the biopreparations' expenditure varied from 0.3 up to $2 \mathrm{~kg}$ of the bacterial and from $2 \times 10^{11}$ up to $5 \times 10^{12}$ polyhedrons of the viral preparation. None of the used ways of applying the biopreparations has shown stable results. Variability of efficiency indicators was very high: ranging fromthe absence of effectiveness up to $91.5 \%$. The average indicators of the efficiency for the period of the studies have varied from $26 \%$ up to $72 \%$. Aerial application has proved to be the most reliable way of applying the biopreparations in terms ofstability of the results and degree of the efficiency. Due to a long incubation period of insects' disease, even at a high degree of final indices of the efficiency, the viral preparation is not able to prevent considerable defoliation of needles incase larvae's density exceeds $3-5 \times 10^{5}$ per 1 hectare. From the viewpoint of reliability and efficiency the tested biopreparations can be really competitive with chemical insecticides only under the following expenditure norm: $1.5-2 \mathrm{~kg}$ oflepidocide per one hectare and $3-5 \times 10^{12}$ polyhedrons of the virus.

Institute of Biology Siberian Branch of Russian Academy of Sciences, Frunze 11, Novosibirsk, Russia 630091 
CULTIVATION OF NUCLEAR POLYHEDROSIS VIRUS OF GYPSY MOTH (OCNERIA DISPAR L.) IN VIVO FOR CONTROL OVER NUMBER OF INSECT

\section{A.V.llynykh, S.A. Mamontova}

Applying a technique of mathematical designing in the series of many years experiments a composition of an artificial nutrient medium for the gypsy moth larvae culture has been worked out. We have selected the components of the artificial nutrientmedium (corn flour, soy-bean flour. feed yeast, ascorbic acid, agar-agar) which do not require complicated technology in the process of work and provide normal development of the insects. As a response function we have used the growth rate of thelarvae: an indicator associated with an individual mass of the insects. Under laboratory conditions we have produced seven sequential generations of the gypsy moth, developed storage conditions of eggs passing through a diapause. For each stage of the culture process optimal conditions have been determined: i.e, density of species for a unit of volume, air temperature and humidity, time of infecting insects, a quantity of inoculant, time of collecting insects, and other parameters, which give maximal yield of the virus under minimal input. Using of preservatives and working under aseptic conditions have allowed us to produce viral material without re-location of larvae. We have used conventional equipment used for cultivation of insects and baculoviruses. Relying on gained results we have designed a system for producing nuclear polyhedrosis virus of the gypsy moth enabling $2 \times 10^{9}$ of polyhedrons per one larva to be produced. Total amount of the virus produced by $3-4$ people staff forthe season is $1.2 \times 10^{15}$ polyhedrons. This quantity is enough to treat some 30 thousand hectares of birch and aspen forests.

Institute of Biology Siberian Branch of Russian Academy of Sciences, Frunze 11, Novosibirsk, Russia 630091

519

THE RESISTANCE SCREENING OF STEINERNEMA CARPOCAPSAE STRAINS TO THE PREDATORY FUNGUS ARTHROBOTRYS DACETYLOIDES

\section{H. Jian, Y. F. Xue, X. Z. Liu}

Carposina nipponensis is a very important insect pest in apple orchard in China. The results of large scale application of entomopathogenic nematode Steinemema carpocapsae to control this insect pest in recent years show that nematode is an alternative of insectcide for soil treatment. The investigation also shows that the population of nematode released declines rapidly due to the effects of abiotic and biotic factor. The previous survey reveals that Arthrobotrys dacetyloides is the domination specie of nematode predatory fungus in Beijing area. To obtain the better control of $C$. carposina, it is necessary to screen more resistance of $S$. carpocapsae strains to the fungus.

Three nematode strains of $S$ carpocapsae BW, Mexkapow and A24 were selected to test the susceptibility to predatory fungus in this research. The fungus grows on PDA plate about 15 days, then well growth of hyphae were mixed with sterilized soil on the proportion of 3 dishes for per kilo soil. The soil of control treatment will not received the fungus. About $80 \mathrm{~g}$ soil with or without fungus (water content $16.7 \%$ ) were put into plastic jars ( 4.5 by $5.4 \mathrm{~cm}$ ), separately. And each jar was pipetted into $2 \mathrm{ml}$ nematode suspension contained $400 \mathrm{~J} 3$. The Galleria larvae bait method is used to evaluate survival nematode resistance. The more nematode number recovers from the soil with fungus, the more resists to the predatory fungus.

The results show that the number of nematode declines gradually no matter which nematode strains in soil with or without fungus; But the decreasing speed of nematode number in soil with the fungus is faster than in the soil without fungus; The averaged recovered nematode number of $\mathrm{BW}, \mathrm{A2} 4$ and Mexkapow are 64,10 and $3.4 \mathrm{~J} / 3$ at 15 th day sampling from the soil with fungus, separately. It demonstrate that BW strain is more resistance to the predatory fungus Arthroboirys daceryloides than other two nematode strains. In order to achieve better control results for $C$. nipponensis, it is suggestion that current being used A24 nematode strain should be replaced by BW strain.

Institute of Biological Control, Chinese Academy of Agricultural Sciences, Beijing 100081, P. R. China.
520

INTEGRATED USE OF TWO ANTAGONISTIC YEASTS TO POST-HARVEST TREATMENTS AGAINST DISEASES ON APPLES.

\section{M.H. Jijakli, P. Lepoivre}

In order to integrate biological control to other alternative sanitary measures, three treatments consisting of dipping the apples in water at $45^{\circ} \mathrm{C}$ for $10 \mathrm{~min}$, or in a water suspension of a mixture of $P$. anomala (strain $\mathrm{K}$ ) and Candida sake (strain O) $\left(10^{7} \mathrm{cfu} / \mathrm{ml}\right.$ each) for $2 \mathrm{~min}$ or in an emulsion of a film-forming antitranspirant (Nu-Film-P, 2\%) for $2 \mathrm{~min}$, were carried out separately or in combination on postharvest Golden Delicious. The separate or combined effects of these treatments were studied on the quality of fruits and the incidence of naturally occurring decay caused by different pathogens. The physico-chemical quality parameters (weight, size, skin coior, firmness, acidity and BRIX degree) were not affected by any of the treatments. Heat therapy alone reduced the incidence of Gloeosporium spp. infection from $54.4 \%$ (untreated apples) to $4.6 \%$, but heat treated apples were more sensitive to Penicillum spp. The application of yeasts in Nu-Film-P following heat therapy decreased the infection due to this pathogen.

These results were confirmed by a second year of trials.

Faculté Universitaire des Sciences Agronomiques, Laboratoire de Pathologie Végétale, 5030, Gembloux, Belgium. 
BIOLOGICAL, MANAGEMENT OF MACROPHOMINA PHASEOLINA BY TRICHODERMA HARZIANUM.

\section{E.Z. Khalifa and C.M. Liddell}

In tropical and subtropicl regions, including Egypt, Macrophomina phaseolina is known to cause seedling blight, charcoal rot, root and stem rots of a wide range of cultivated crops, specially under conditions of high soil temperature and low soil moisture. Safe and effective control strategies for soilborne pathogenic fungi are currently being developed using biological control agents. Such strategies help to avoid the fungicidal hazards and minimize the level of environmental pollution. Three isclates of Trichoderma harzianum, two from Egypt and one from the U.S.A, were screened in vitro and in vivo for their ability or potential against four isolates of $M$. phaseolina, one from Egypt and three from the U.S.A. The tested isolates of $T$. harzianum proved to be hyperparasites to all tested isolates of the causal organism. Cell-free metabolites of $T$. harzianum inhibited the growth and the microsclerotial germination of the pathogen. Under growth chamber condition, $T$. harzianum significantly decreased damping - off and root rot of beans. Population of propagules (colony fungal units) and recovery of microsclerotia from bean thizosphere were also significantly decreased due to the effect of the biocontrol agent. The tested isolates of T.harzianum were different in their effect against the M.phaseolina isolates.

Faculty of Agriculture, Minufiya University, Shibin el-Kom, Egypt

523

PROTECTION OP PUSARIUM WILT OF CUCUMBER BY NONPATHOGENIC STRAINS OF FUSARIUM OXYSPORUM IN GREENHOUSE AND PITLDS

Sung-Seok Yang, Choong Hoe Kim

One hundred fifty four out of 262 isolates of fusarium oxysporum obtained from healthy plant tissues of $g$ different crops and their rhtzosphere solls were found to be nonpathogenlc to cucumber plants. The nonpathogentc strains were less frequently isolated from healthy plants of cucumber and water melon, compared to other crops. When cucumber seedlings were grown in pots containing the soil-corn meal mixed media that were inoculated with each of the 154 nonpatbogenic tsolates and then transplanted into pathogenInfested natural soil, 21 1solates effectively protected cucumber plants from fusarlum wit 1nfections. A year later in 1993, 9 out of 21 13olates had sustalned their protectlve effect, and with one exception they were found to be not pathogenic to water melon, chinese meion, tomato and sesame. In the three separate greenhouse tests, where cucumber seedings were ralsed in the nonpathogentc strain-1nfested soll-corn meal mixed media, and then transplanted Into pathogen-Infested fleld soll, nine nonpathogenic strains varied in their protection efficacy from $15.5 \%$ to $90.3 \%$ on the average. In the field experiments with the strain 4-1 that showed best protection in the greenhouse tests, fusarium wilt infections In the strain 4-1 treated plots were reduced to $1.3 \%$ and $18.1 \%$, compared to $10.7 \%$ and $55.5 \%$ infections in the untreated control plots, lst and 2 nd experiment, respectively. Strain 4-1 incited minor discoloration of roots of cucumber plants at higher inoculum level as $10^{5}$ conldia/g soil. Both the pathogen and the nonpathognic strain $4-1$ were frequently re-1solated from cucurber roots. On the contrary, the nonpathogenic strain was scarcely found in the cucumber stems, unlike the pathogen.

Plant Pathology Division, Agrlcultural Sclence and Technology Institute, Suwon 441-707, Korea
_I. A., Khmel., T. A. Sorokina, N. B. Lemanova

Screening of soil-born bacteria isolated in various geographic zones of the former USSR resulted in selection of two Pseudomonas strains ( $P$. aureofaciens 135 and $P$. fluorescens 1163) with wide range of antagonistic activity against pathogenic bacteria and fungi. The strains have efficacy for biological control of crown gall on grapevine in greenhouse experinents. Dipping cuttings of grapevine in bacterial suspension decreased $2-9$ times the number of plants with tumours. The treatment of soil, seeds and seedlings with suspension of bacteria protected the plants of cucunser, tomato and carnation against pathogenic Fusarium spp. , reduced significantly the occurence of root rot of wheat and cabbage, mould of winter wheat and sunf lower.
524

ORIVS SPP. AS PREDATORS OF WESTERN FLOWER THRIPS, FRANKLINIELLA OCCIDENTALIS, IN ISRAEL

Raisa Chyzik. Mels Klien, Yaxr Ben-Dov, Ravel Putshkov

The western flower thrlps (WFT), Franklintella occidentalfs, was introduced into Israel in 1987 . Since then it has become a noxious, pest to a large variety of plants. During a search for blological enemies of the WFT, several species of bugs of the genus orius predatory to WFT were discovered in differnt areas of Israel. Most of the species found were $o$. niger, 0 . albidipensis and 0. laevigatus. Whereas in the central aree the predom ant species on field crops was 0 . niger (88\%) and on flowers (Gladiolus and Ageratum) we found 0 . nlger (Sas) and valley), 0 . Iaevigatus was the most prom hart of Israel (82z) on fteld crops; 0 . niger was 188. In the prothern ary part of Israel, we found $78 \%$ of $a$. nigex and the rest were 0 . albidipennis and 0 . Iaevigatus. A few other species. such as 0 . horvathi and so far unidentified ones were in negligible numbers. The predatory efficacy of 0 . niger in the WFT population has not yet been investigated.

Department of Entomology, ARo, The Volcani Center, Bet Dagan 50250 ISRAEL 
BREEGING OF PERENNIAL RYEGRASS AND TALL FESCUE TO RESISTANT TO BLUEGRASS WEBWORM BY ARTIFICIAL INOCULATION OF ACREMONIUM ENDOPHY'TES

Hircnoci GOGA, Yoshio HIRAI, Ken-ichi KANDA, Takao TSUKIBOSHI and Tsutomu UEMATSU

Perennial ryegrass (Lolium perenne) and tall fescue (Fegtuca arupdinacea) were imparted the resistance to bluegrags webworm

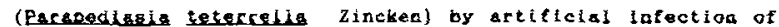
Acremeniug lelid and A. coenephlelum. rospectively. The Acremonium endophytes were isolated from perenaial ryegrass and tall fescue which had the resistance to the larvae of bluegrass webworm. The endophytes were inoculated by inserting the mycelia from the pure culture into the meristematic region at the function of the mesocotyl and coleoptile of 4-6 days-old seedings. The hyphas of the Acremenium endophytes in the inoculated plants were checked under 1 ight microscopy $1-2$ tontan after inoculation. The plants infocted with the endopbyte hed the feeding deterrent to the bluegraes wobworm and aurvival rate of the larvao on the infactod leat bladeg definitely decressed compared with the uniafected oneg. The endophyteg were transmitted to all the geedo which were produced on the artificially infected perennial ryegrass and tall fescue. The plants developed from the seeds also had the resistance to bluegrass webworm.

National Grassland Research Institute, Nishinasuno, Tochigi 329-27, Japan

\section{7}

IMPACT OF INDIGENDUS NATURAL ENEMIES ON RICE IEAFFOLDER POPULATIONS AND DAMAGE IN SOUTHEAST ASIA

\section{J. de Kraker}

Rice leaffolders Cnaphalocrocis medinalis and Marasmia spp. (Pyralidae: Lepidoptera) are considered a major pest in many Asian countries, and farmers often resort to insecticides to control them. To determine whether indigenous natural enemies have the capacity to prevent vield loss due to rice leaffolder, their impact was quantified and evaluated with experiments and model simulations.

The impoct of the natural enemy complex was studied in the field during several seasons in three Southeast Asian countries. In unsprayed fields leaffolder populations did not reach damaging levels, while their natural enemies were abundant. Leaffolder egg mortality, in particular egg predation, was an importent suppressive factor. Through direct field observetions the majol oge predators, crickets, were identified.

For various predetors the reletionships between age pradation rate. density of arey ard predator, and leaf surface area were quantified using cage experiments. In the laboratory prey preference and searching behaviour of the predators was studied. The results were summarized in a simple model for field egg predation. Model predictions were based on actual field densities of potential predators, and confirmed the major role of predatory crickets in leaffolder egg predation.

A combined pest-crop model was developed, describing leaftalder population dynamics - including the impact of natural enemies -and its interrelations with rice crop growth. The submodels of leaffolder egg predation, population dynamics, and impact on crop growth were validated in field experiments. Simulations with this combined model showed that a healthy rice crop is not very sensitive to leaffolder damege. Including mortality due to nalural enemies reducad yield loss to a negligible level.

These results fully support the importance of an IPM approach in which both proper crop menagement and natural enemy conservation are emphasized.

Deparman of Entomology

Waganingen Agriculturat University

P.O. $80 \times 8031$

$6700 \mathrm{EH}$ Wageningen, the Netherlands
ECOLOGICALLY SAFE MEANS TO CONTROL THFIPS IN GREENHOUSES IN RUSSIA

\section{E. Kozarzhevskaya}

At the present time biotechnical method to control pests is actively developing in Russia. Biological method is ecological strategy in pest management based on insect behavior, their natural reactions under influence of optical and acoustic signals.

Main Botanical Garden of Russian Academy of Sciences conducls the research of biotechnical means on the basis of pest reactions to the optical constructions such as colour traps, working out of ecological non-dangerous technologies for practical usage. Coloured traps for White flies, Aphids, Ants, Onion fly and Thrips showed high level of activity.

The most common Thrips found on protected crops are Thrips tabaci and more recently Western flower thrips, which has been widely spread in many countries. The Western flower thrips is extremely harmful pest that damages about 250 species of agriculture and horticultural plants including tomatoes, cucumbers, roses, chrysanthemums, herberas and others in greenhouses and indoor conditions.

It has penetrated Russia several years ago with plants from European countries and for the first time was noticed in such regions like St.Petersburg, Moscow and Novgorod. Now this dangerous pest is distributed with plants mostly with flowers from one greenhouse to another and continue to penetrate from foreign countries.

Western flower thrips has already become a real scourge of vegetable and ornamental production in some regions. Farms bear great expenses due to the yield losses.

Our research revealed that the blue color traps among white, yellow, green. red, brown and siver were the most active ones and 2 blue hues among 27 investigated were the most effective. The pratarance to ditferent blue nues by females and males was noted. The traps are painted with blue due-stuff of a definite colour hue and enthomological glue - "Lipofix", elaborated at the Chemistry Institute Bashkir Academy of Sciences.

Chemicals practically do not control the Western flower thrips, which has become qute resistant to many pyrethroids, phosphor- and chlor- organic preparations.

Main Botanical Garden, Russian Academy of Sciences, 4, Botanicheskaya Stre日t, 127276 Moscow, Russia

528

PHYTOMYZA ORORANCHLA KALT. [DIPTERA: AGROMYZIDAE] DN MOROCCO AND ITS POTENTAL USE FOR BIOLOGICAL CONTROL OF ORORANCHE SPP.

\section{O. Klein, LKroschel and J. Sauerbor}

The cultivation of food legumes, mainly of faba bean (Vicia faba L.), in Morosco is of importance as a protein source in human nutrition and for the preservation of the soil fertility in rotation with cereals. The cropping of legumes is hampered by the root parasitic weed Orobanche crenats. The control of $O$. crencela with chemical and other cultural measures has not been successful due to the partly negative effects on the crop and the complicated handling. The biological control of $\boldsymbol{O}$. crenata with the broomrape miner Phytomyza orobumchia seems therefore to be an interesting and promising alternative. $P$. orobanchia is indigenous in the Mediterranean region and is strictly monophageous associated with Orobanche spp. To achieve an effective biological control with $P$. arobanchia mass rearing and release of the broomrape miner are necessary to strengthen the natural population. Different rearing methods have been developed in the former Sovietunion. Since artificial rearing is not possible broemrspe spikes containing pupae of $P$. orobonchia have to be collected and stored during the winter period. The hatched flies can then be released as soon as the broomrape appears in the field. Factors limiting the population of the bromrape miner as there are hyperparasitism, destruction of pupae by tillage or feexing to death can be avoided by means of controlled reaving. The present investigations should give more informalion on the natural distribution of $P$. orobanchia as well as on its population dynamics under the geographic and climatic conditions of Moroceo for an as on its population dynamics under the geographic and climatic conditions of Morocso for an
evalustion of a potential use as biological control agent. The studies were carnied out from evalustion of a potential use as biologica' control agent. The studies wee
February to August 1994 in the provinces of Fes, Taounate and Meknes.

The field survey on the natural distribution of $P$. orobanchia showed that on every field with emerged broomrape $P$. orobonchia was present. $93 \%$ of the broomrape spikes were infested with larvae and pupre. The infestation lead to an average reduction of $96 \%$ of the seed production per capsule. With an average of $49 \%$ of infested capsules, the total reduction of the production per capsule. With an average of $49 \%$ of infested capsules, the total reduction seed production reached $47 \%$ due to the natural occurrence of $P$. orobanchia in the field.
Adults of $P$. orobamchia were caught with different trapping methods (like malaise trap, yeliow sticky trap and sweeping net) from the beginning of March until the beginning of June with the highest number of 17 adults per week in the malaise trap in the first half of May. Depending on the location and the climatic conditions 3 to 4 generations developed during this time.

$93 \%$ of the pupae of the first, $61 \%$ of the second and $1 \%$ of the third generation hatched. The remaining pupae entered into diapause. $16 \%$ of the pupae have been parssitized by 3 hymenopterous species of the family Chalcidoidea. Parasitized pupae were observed from the beginning of mid-May.

Considering the period of lowest rate of hatching, highest amount of pupae per Orobranche spike and lowest parasitization rate of the pupae the optimal time for the collection of Orobanche spikes needed for mass rearing and release of $P$. orobanchia would be the fist two weeks of May under the given circumstances of Morocco.

University of Hohenheim, Instituse of Plant Praduction in the Tropies and Subtropics (380), D-70593 Stutgart, Germany 
NEW METHOD FOR SURVIVAL COMPARISON OP FUNGAL PATHOGENS IN DIFPERENT SOIL CONDITIONS

\section{T. Lagutina, N. Vorobyov}

The method on base of membrane filter technique and the computer programs for analyzing survival of pathogenic fung1 in soll were worked out. According to this method the inoculum of pathogenic fung1 is placed on membrane fllter and introduced Into soll. After some incubation perlods the membrane 111ters are taken out from soll and the number of test-pathogens are counted by a plate dilution method. These experimental data are processed using the developed computer programs.

Mathematical model bases on the following decision of differential equations:

$$
\frac{1}{x}=\left(\frac{1}{x_{n}}-A\right) \cdot \exp (-B \cdot t)+A
$$

where $X$ - number of test-pathogen; $X_{n}$ - the initial number of test-pathogen; $t$ - incubation period; $\mathrm{A}, \mathrm{B}$ - constants.

The dynamic parameter " $B$ " permit to determine the intensity of number change of pathogentc fung1 on the membrane iliter. The static parameter " $A$ " characterizes the limit of lungal number. Using the parameters " $A, B$ " we compared the survival of pathogen1c fung1 in different soll conditions.

Th1s method was used for studying the survival of pathogenic fung1 Pusariun oxysporun in different solis with different content of organic and mineral fertilizers. The intensity of reduction of $F$.oxysporum was greater in soll with manure than in soll without one. The limit number of F.oxysporum on the membrane fliter approaches to zero.

A11 - Russian Research Institute for Agricultural mlcroblology St - Petersburg, Pushk1n, Podbelsky sh.3, 189620, Russia

\section{1}

IN VITRO REARING OF TRICHOGRAMMA SPP. WITH ARTIFICIAL MEDIA WITHOUT INSECT HEMCLYMPH

\section{Wenhui Liu, Simon A. Grenier}

Great progress has been made recently in research on in vitro rearing of Trichograma spp. in China, France etc. But, the insect hemolymph, as a main important caponent of the artificial medium, is difficult to be obtained in Western Countries. It is very urgent to solve the problem of artificial diets without insect hemolymph. In this paper, the anount of amino-acides and osmotic pressure of substitute for insect hemolymph in the artificial media were analysed. Trichograrma dendrolimi and $T$. brassicae were reared completely with a selected medium without insect hemolymph. The pupation of $\mathrm{T}$. dendrolimi and $\mathrm{T}$. brassicae was $16.48,6.878$ respectively. The adult emergence of them was $24.078,21.43 \%$ respectively. The influence of different size and thickness of the artificial chorion on trichogramatid oviposition was studied too.

Prof. Wenhui Liu, Guangdong Entomological Institute, Xingang West Road 105, Guangzhou 510260, The People's republic of China
B IOLOGICAL CONTROL OF RIIZOCTONIA SOLANI ON POTATOES IN STORAGE WITH VLRTICILLIUM BIGUTTATUM.

J.G. Lamera, R. Wustman, L.G.M. Gorris and G.J. Kriatian.

The fungus Rhyoctonia solant is pathogenic to a range of plants including potatoes. The crop growth and yield are affected and the quality of the seed tubers 1 s reduced by the presence of $R$. solant sclerotia adhering on the surface. This tuber-borne inoculum is especially harmful to the early sprout growth in spring.

Verticillium biguttatum has been found to be a hyperparasite of $R$. solant, that can Inactivate the sclerotia and hyphe tduring storage for at least six to elght weeks at elevated temperatures of $17-20^{\circ} \mathrm{C}$ (Jager and Velv1s, 1988). The present study was set up by PAGV and ATO-DLO to investigate under practical conditions to what extent the fnactivation pextod is tempcrature dependent and whether the storage temperature can be lowered after reachlng a certaln temperature degree-day sum (TDS).

Five $\mathrm{kg}$ samples of potatocs $\mathrm{cv}$. Bintje infected with $R$. solani wete put in netced bags and soaked in a solution of $1-3 * 10^{5}$ spores of $V$. biguttatum per mil (with 0,52 carboxymethylcellulose; Koppert BV). These bags were burried in $1 \mathrm{~m}^{3}$ wooden storage boxes with freshly harvested potatoes (four bags per box). Four boxes were placed in storage rooms at $16^{\circ} \mathrm{C}, 20^{\circ} \mathrm{C}$ or $24^{\circ} \mathrm{C}$ at maximum relative humidity. Each box was taken out of these rooms and the bags were placed $1 \mathrm{n}$ a room at $6^{\circ} \mathrm{C}$, as soon as a TDS of 50, 100, 150 or 200 (basal temperature $14^{\circ} \mathrm{C}$ ) was reached. The samples were intensively graded to remove dead sclerotia fron the tuber surface. Fifty sclerotia from 25 tubers per bag were placed on wet paper for one day. Hyphal outgrowth was then assessed to deternine the viability-1ndex. S1x tubers per sample of different IDS, that had a iight contamination of $R$. solant, were put in a bucket with potting so1l. After the potato stems had emerged fror. the surface, the $R$. solani atrack on the white underground stems was assessed. Experiments performed over two successive years proved that the viability of the sclerotia on the potato surface substancially decreased to a low level already when TDS was 50 . At TDS $=100$ a minimum level was reached. The viability-index of the sclerotia correlated well with the infestation of the stems taken out of the buckets. The dead sclerotia could not be removed by grading or brushing or alternative dry-wet perlods. It is concluded that a temperature sum over a certaln period is a better method than a fixed period of 6-8 weeks. When this period is too long, sllver scurf might give more problems.

Literature: Jager, G. and H. Velvis, 1988. Inactivation of sclerotia of Rhizoctonia solant on potsto tubers by Verticlllium biguttatum, a sollborne mycoparasice. Neth. J. P1. Path. 94, 225-231.

Address: PAGV, P.0.Box 430, 8200 AK Lelystad.

532

STUDY ON BAIT FORMULATION OF ENTOMOPATHOGENIC NEMATODES AGAINST GERMAN COCKROACH

\section{Xianqun Luan Huaiwen Yang}

Entomopathogenic nematode is one promised biocontrol agent, which has been used successfully to control soil-inhabiting insect pests and pests in other eavironments. Proper formulation is one of the keys to prompt the effectiveness of nematodes. In order to find suitable formulation of nematodes to control german cockroach, We compared several formulations of nematodes.

$10 \mathrm{ml}$ nematodes suspension containing 25,000 nematodes were pipetted onto and bait was placed on a sponge pad $(6.0$ by $2.0 \mathrm{~cm}$ ) that was placed in a petri dish $(6.0$ by $1.5 \mathrm{~cm})$. The combination of all of them composed a bait station, which was placed in a jar $(48 \times 36 \times 12$ $\mathrm{cm}$ ) with the inner lip greased with petroleum;mineral oil (3:2) to prevent german cockroach to escape. Food container and water trough were placed at the bottom of the jar. A pile of paper folding provided harborage for german cockroach. Thirty german cockroach (10 female, 10 male and 10 late-stage nymphs) were placed in each jar. Jars were replicated three times for each bait. They were hold for 5 days at $24-26{ }^{\circ} \mathrm{C}$ and $50 \% \mathrm{RH}$. Mortality was recorded daily for each stage and sex. PB Pheromones (periplanone-B), 11 \# pheromones (one extract from plant) and boric acid nematodes bait stations were used to be tested. Control treatment were identical without bait station.

The result demonstrated that both pheromones didn't promoted nematodes infectivity significantly $(p=0.05)$ compared with control treatment, $41.4 \%, 31.1 \%$ and $30 \%$ of german cockroach mortality respectively after 120 hours treat. At the same time, boric acid killed $83.3 \%$ german cockroach. In addition, the susceptibilities were significantly different between female, male and late-stage nyphms, the relative order of susceptibilities were female $>$ male $>$ nyphme, the LDso were 42 hours, 72 hours and 91.4 hours, respectively, as well as 68 hours for the mixed group for boric acid treatment. This test demonstrate that PB and 11 \# pheromones were not suitable for german cockroach control, that may be caused the different between cockroach spices. Boric acid had been used to control against german cockroach, but the treat time is too long. Test result is suggestion that nematodes and boric acid used together could accelerate the german cockroach death and improve the effectiveness of cockroach control.

Institute of Biological Control, China Academy of Agriculture Sciences Beijing 100081, P. R. of China 


\section{USE OF ENDOPHYTIC BAC'TERIA AGAINST POSTHARVEST DISEASES OF FRUITS AND VEGETABLES}

\section{Mari. M.Guizzardi and G.C.Pratella}

Preliminary findings clearly show the presence of a bacterial population in apparently healthy plant tissues. The endophyte population exhibited several strains capable of controlling the following pathogens of postharvest diseases: Monilinia laxa, Rhizopus stolonifer, Botrytis cinerea.

The isolated bacterial strains were tested against $M$. laxa and $R$. stolonifer (on nectarines, plums and apricots) and $B$. cinerea (on pears), $R$. stolonifer proved to be more difficult to control and it was evident the influence of different varieties on control of $M$. laxa. Some antagonistic strains significantly reduced grey mould on cy Conference pears artificially inoculated with $B$. cinerea and stored at $20^{\circ} \mathrm{C}$ for at least nine days. Their application is compatible with Iprodione-based antifungal treatment; the bacterial-antagonist mixed with the fungicide (at a rate of $50 \mathrm{ppm}$ a.i.) resulted in greater control of the pathogen as compared to the single ingredient.. The antifungal activity of these endophytic bacteria is probably due to competition for nutrients rather than to production of antibiotic substances since it did'nt find any antifungal activity in the cell-free filtrates. Our results point out a strong specificity in the host-pathogen-antagonist combination. In fact few strains proved to be effective many than hosts and the three pathogens tested.

Endophytic antagonistic strains are tested also on tomatoes against $B$. cinerea. They were mature green or red, to study a different susceptibility to grey mould and consequently a different effectiveness of biological treatment. Moreover, tomatoes, stored at $2{ }^{\circ} \mathrm{C}$ for 10 days, were treated with antagonist bacteria to evaluate their efficacy on vegetables with chilling injury. Results suggest that it is possible to control $B$. cinerea on tomatoes with endophytic bacteria aside from ripening and low-Lemperature stress. It exists also the possibility of successfully growing antagonist bacteria on nonspecific substrates, obtained wiht lower costs.

CRIOF - Università di Bologna - V.F. Re, 8 - 40121 Bologna, Italy

EXPLORATION OF THE REI ATIONSHIPS BETWEEN PREDATORS AND THEIR PREY SPESIES IN THE ALPALFA FIEDS

\section{A, Mateeva, M. Vassileva, N. Bentcheva}

The studies were carried in the recion of Plovdiv on areas where not chemical control had been applied.

The alin pests of alfalfa are Phytonomus variabilis Herbst, Phytodecta fornicata Brüg, lcyrt hosiphon pisum Harrig. Species of the family coccinellidae predominate anong the aphidofages, while the species of the frimilies syrphidae and chrysopidae are eneountered in low nubers. The parastes Anhidfus ervi Hal are predowinanted.

The ratio between predators and leaf pests in spring in favour of predators, later curing the period of mass reproduction in favour of pesta in July and August in favour of predators and in Autumn - only of leaf aphids.

A eleary expresed post tive and regression linear correlation between leaf pests on elfalfa crops and their predators, which shows that predators have a positive effect in the regulation of the pupulation numbers of leaf pesta.

As a result of the study made, the most appropriate parameters of interaction have been established with different technologies of cultivation.

Regression equations have been worked out giving the change in the insect density as depending on the climatic and agroy technical factors.

ANTAGONISM OF BACHLIIS SPP. AGAINST PSEUDOMONAS SOIANACFARUM (SMITH) SMITH AND GROWTH PROMOTION ON TOMATO PLANTS

\section{E.B.DA SILVEIRA R.R.R.MARIANQ; S J.MICHEREFF \& M MENEZES}

Thirty isolates of Bacillus from rhizosphere of several crops were tested for antagonistic activity against Pseudomonas solanacearum under greenhouse conditions. Two methods of application were compared: irrigation of the seedbed substrate five days before planting and seed bacterization sixteen hours before planting. Tomato seedlings 20 days old were transplanted into plastic pots containing natural soil previously infested with $120 \mathrm{ml}$ of $P$. solomacearum suspension containing $8 \times 10^{8} \mathrm{cfu} / \mathrm{ml}$. Seventeen days after transplanting plants were evaltated and the hacterial wilt index (BWI) was calculated. BA24, BA46 and BA3 applied to the substrate induced lower BWI giving 54.60; 46.60 and $42.60 \%$ of disease control respectively. These antagonists were evaluated in relation and $42.60 \%$ of disease control respectively. These antagonists were evaluated in relation
to five concentrations of $P$. solanaceartm $\left(1 \times 10^{7}, 4 \times 10^{7}, 8 \times 10^{7}, 2 \times 10^{8}, 6 \times 10^{8}\right)$. and five pathogen isolates from Recife (RE), Serra Talhada (ST), Vitóna de Sto. Antão (V1, V2) and Ibimirim (IB). The antagonists were also tested against the isolate RE in five soil types, alfisol (Cachoeirinha), ultisols (Moreno, Paulista, Vitória de Sto. Antão) and entisol (Recife). All cities are iocated in the state of Pernambuco, Northeastem Brazil. Antagonists were applied by substrate irrigation and BWI and proportion of Brazil. Antagonists were applied by substrate irrigation and BWI and proportion of
area under disease progression curve (PALDPC) were calculated. In general PAUDPC increased with raising of pathogen inoculum. The lowest level of BWI was observed in the treatment with BA24 (4.20) in the concentration $1 \times 10^{7} \mathrm{cfu} / \mathrm{ml}$, which corresponded to $11.20 \%$ control. The different isolates of the pathogen did not show virulence variability and the interaction BA24 x V2 presented lowest BWI. All treatments induced high numbers of PALDPC for isolates RE, IB and ST. The ultisols from Moreno and Virbria de Sto Antazo showed natural suppressivity to the pathogen. with emphasis for the interaction BAJ $x$ Moreno. Among the conductive soils the treatment BA46 evidenced the best level of disease control $(21,13 \%)$. Antibiosis "in vitto" could not be detected by using the filter paper disc technique. The ability of promoting plant growth was compared among the same three isolates applied as seed bacterization. The parameters evaluated after planting were emergency at 10 days, height and dry weight parameters evaliated after planting were emergency at 10 days, height and dry weight
at 25 days. However the emergency was not improved significantly BA24 and BA46 induced height and dry weight increases of $7.27,1030$ and $21.97 ; 7.36 \%$ respectively.

UFRPE, DEPA, Fitossanidade, Dois Irmãos, 52171-900, Recife, PE, BRAZIL

536

INTERACTION BETWEEN THE ENTOMOSTATIC PLANT AZADIRACHTA INDICA AND THE HOST / PARASITOID SYSTEM PIERIS RAPAE/ HYPOSOTER EBENINUS

\section{M.MATTER}

2. technique was schemed to simulate field exposure of pieris rapae larvae to neem seed oil - treated or untreated cabbage leaves for different periods before being subjected to the parasitoid. In a free choice test, larvae surviving neem treatment were more responded to parasitism than the corresponding untreated hosts of the same age. prolongation in larval developnent due to neem treatment was advantageous for the parasitioid as it extended the exposure period of the preferable target instars. Nevertheless. parasitization of previously neem - treated caterpillars accentuated premature host death and fewer parasitoids were obta ned. Moreover, Farasitodids surviving host treatment had significart longer duration than those developed in untreated hasts.

Prof,Dr, MAMDOUH MAHER MATTER

Pests and Plant protection Dpt.

National Research Centre

El - Tahrir St., Dokki, Cairo, EGYPT .

POSTAL CODE 12622 
COMPARISONS OF VERTICILLIUM LECANII STRAINS FOR EFFECTS ON PLANT-PARASITIC NEMATODES.

\section{$\underline{\text { S. L. F. Meyer and R. J. Meyer. }}$}

A strain of Verticillium lecanii (ATCC 58909) was found to be antagonistic to Heterodera glycines (soybean cyst nematode) eggs in petri dish assays. Four mutants with increased tolerance to the fungicide benomyl were induced with ultraviolet radiation from this wild type strain. All five strains were compared in

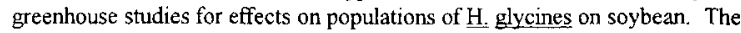
strains were incorporated into alginate prills and the prills were applied near plant roots at two rates: ca. $0.1 \%$ and $1.0 \%$ dry prill weight per weight air-dried compost/sand mixture. Each pot received 8000 nematode eggs and juveniles. At the higher prill application rate, two of the mutant strains caused significant reductions ( $46 \%$ and $57 \%$, respectively) in numbers of $\underline{\mathrm{H}}$. glycines cysts produced per pot compared to untreated controls. The wild type strain and one of the effective mutant strains were also evaluated in separate greenhouse tests at application rates of $0.003 \%, 0.007 \%, 0.01 \%, 0.02 \%, 0.1 \%$, and $0.2 \%$ dry prill weight per weight air-dried compost/sand mixture. When $300 \mathrm{H}$. glycines eggs and juveniles were added to each pot, both fungus strains caused significant reductions in cyst numbers at prill application rates as low as $0.02 \%$. Application of the wild type strain resulted in a mean reduction of $42 \%$ compared to untreated controls; application of the mutant strain resulted in a 39\% decrease. Significant mean reductions in cyst numbers of $58 \%$ (mutant strain) and $47 \%$ (wild type strain) occurred at the highest prill application rate $(0.2 \%)$ as well. When nematodes were added at 10,000 eggs and juveniles per pot, the wild type strain did not significantly decrease number of cysts produced per pot. However, the mutant strain caused a statistically significant population reduction of $34 \%$ compared to untreated control pots. This occurred at the highest prill application rate: $0.2 \% \mathrm{dry}$ prill weight per weight compost/sand mixture.

USDA, ARS, Nematology Laboratory, BIdg. 011A, Rm. 153, BARC-West, 10300 Baltimore Avenue, Beltsville, MD, 20705-2350, U.S.A.

539

THE POTENTIAL OF Talaromyces flavus AS A BIOCONTROL AGENT FOR CONTROLLING Vericillium dahtiae

\section{M.P.M. Nagtzaam}

A biocontrol agent has to colonize the root to interfere with infection of the host by Verticillium dahliae. The ability of Talaromyces flavus to colonize the growing roots from inoculated seeds was studied with potato (Solcnum tuberosum) and eggplants (Solanum melongena). The fungus was recovered from root segments $>6 \mathrm{~cm}$ below the seed. The number of colony forming units per unit root length decreased linearly with increasing distance from the inoculation site.

The potential of $T$. flavus to reduce the population density of $V$. dahliae in soil was evaluated. Microsclerotia were embedded in nylon mesh and buried in soil. It was demonstrated that the fungus grew saprophytic through soil and significantly affected the viability of microsclerotia.

In two independent field experiments $T$. flavus was evaluated as a biocontrol agent of $V$. dahliae. The antagonist reduced stem infection and stem colonization by $V$. dahliae at low, but not at high densities of the pathogen in soil. Although, the population density of $T$. flovus in soil had not decreased during one year after application, the antagonist did not suppress stem infection or colonization by $V$. dahliae in the crop of the second year.

Department of Phytopathology, Wageningen Agricultural University, Binnenhaven 9, P.O. Box 8025,6700 EE Wageningen, The Netherlands
AUGMENT ATIVE BIOLOGICAL CONTROL OF SILVERLEAF WHITEFLY IN A DESERT AND GREENHOUSE CROPPING SYSTEM.

\section{Oscar Minkenberg}

The mass-rearing of Erctmocerte sp. ex. Arizona at the University of Arizona north campus has allowed us to produce large quantities of wasps viz. millions per week, for evaluation against silverleaf whitefly in commercial plant production systems (CIBA Bunting Ltd, U.K., is sponsoring the rearing). Production of Bemisia parasitoids in high numbers is feasible and extensive research of their ecology and applicability is ongoing. The crops for which we report herein the potential use of Eretmocerus, are cotton in the Imperial Valley, California, and greenhouse poinsettias in two regions in the U.S.A.

In cotton, we demonstrated that $B$ argentifolii can be controlled by inoculative releases of Eretmocerus, at least in field cages, and that parasitism was highest in early August at $61 \%$. Density of whitefly pupae in the high release treatment was approximately one fifth of the control treatment (i.e., no parasitoid releases). Yield of seed cotton was almost three times higher in the high release cages in comparison with those of the low parasitoid release or control cages. In the 1993 season, we showed that at a release rate of 32 parasitoids per plant whitefly levels were 0.0 at the end of the season. A release rate of 4 to 32 parasitoids per plant was required to achieve control in cages. Releases of high numbers of parasitoids in relatively small field plots were less effective; parasitism and whitefly density did not differ between release and control plots.

A possible explanation for the observed ineffectiveness in field plots is the immigration of adult whiteflies during June and July in the Imperial Valley. To counteract a steep increase in adult whiteflies, we examined the application of an oil solution to the upper carropy, Adult whitefles manly reside in the top of the plants, whereas most whitefly nymphs and pupae are located in the center of the plant; parasitoids are released at the bottom. The integration of inoculative releases with oil treatments was examined on a 3.5 ha field plot in 1994. Although reasonable levels of parasitism were reached, whitefly levels were unacceptably high and yield was low (1.3 bales per acre). Given the rates of parasitoids needed and their current costs, and the whitefly pressure in desert agriculture, I conclude that augmentative biological control of $B$. argentifolit in cotton is not effective nor economically feasible in the Southwest.

For greenhouse poinsettias, life table studies by M. Hoddle, J.P. Sanderson \& R.G. van Driesche showed that Eretmocerus caused $99.1 \%$ mortality in B. argentifolii cohorts on glasshouse poinsettias (release rate was 3 females per plant per week). Cage evaluation studies and two large scale greenhouse trials in the Northeast have confirmed that Eretmocerus is a biological control candidate against whiteflies on commercial poinsettias. Studies on the use of inundative releases in poinsettias in southern California are underway.

Department of Entomology, University of Arizona, Tucson, AZ 85721 , U.S.A.

540

BIOLOGICAL CONTROL OF PLASMODIOPHORA BRASSICAE USING RHIZOPLANE FUNGI

Narisawa, $K$, and Hashiba, T.

Rhizoplane fungi showed antagonism to Plasmodiophora brassicae Wor., which causes club root disease. Antagonists were isolated from the root surfaces of chinese cabbage (Brassica campestris) using a modified Harley-Waid root-washing method (Harley and Waid, 1955). After washing, $1 \mathrm{~cm}$ length root pieces were placed on petri dishes containing $50 \%$ corn-meal agar. All fungi growing from the root pieces were cultured on $50 \%$ com-meal agar including $10 \mathrm{~g}$ of Malt extract and $\mathrm{zg}$ of Yeast extract per liter. One hundred and eighty-six strains of fungi were isolated ( 23 fungal taxa were identified) from 450 root pieces. These rhizoplane fungi were prepared for biological control studies of club root disease. The fungal cultures were incubated on potato-dextrose agar in petri dishes for several days at room temperature. The lengths of incubation depended on the fungal growth. Surface sterilized seeds of $B$. campestris were placed on a colony of each isolate growing on the medium. These seedlings were transplanted to plastic pots containing $200 \mathrm{ml}$ of sterile soil and kept in a greenhouse at a temperature of 20-25C. One month after transplanting resting spores of $P$. brassicae were inoculated into the soil at a concentration of $4 \times 10^{6}$ spores/g of dried soil. Disease symptoms in roots were assessed two months after planting. Twelve out of 186 isolates significantly reduced the incidence of club root disease \{disease index (Yoshikawa et al., 1977) was under 20\}. Ten isolates had sterile mycelia that were white, orange, or black, and two other isolates were Mortierella species. Microscopic observation showed that the hyphae of the sterile mycelia entered through root hairs of $B$. campestris and became colonized within cortical cells of the plant. Mortierella species colonized and covered the surface of roots by hyphal mats. We concluded that the colonization inside and outside of the roots prevented club root development in $P$. brassicae.

Plant Biotechnology Institute Ibaraki Agricultural Center, Ago, Iwama, Nishi-Ibaraki 319-02 Japan 
FITNESS OF TRICHODERMA SPP. AGAINST MACROPHOMINA PHASEOLINA AND SCLEROTIUM ROLFSII INFECTING SUNFLOWER AND SOYBEAN.

\section{Nazim, E.Z. Khalifa, M.Z. El-Shanawani and M.A.Awad}

Soybean and sunflower are important oil seed crops, in Egypt. They occupy the second fank after cotton in oil seed production and the cultivated area.

Under the local conditions both crops are attacked by many soil borne pathogens. Mocrophomina phoseolina Tassi (Goid) and Scleroium rolfsii Sacc. causing charcoal and stalk rots, respectively, are considered the most pathogenic fungi. They are responsible for economic loss in the number of plants and consequently the seed yield. The field observations showed that the loss in the number of plants due to both pathogens may reach 20 and $15 \%$ on sunflower and soybean, respectively.

In the course of this study, infected samples of stalks and roots of both crops were collected to isolate the causal organisms and the associated fungi. Also, samples of the rhizosphere of both crops in addition to faba bean were collected. Trichoderma harzianum, T. pseudokoningii and $T$. hamatum was isolated and comprising 44.44, 38.89 and $16.67 \%$ of the total isolates, respectively. All isolates of $T$. harziantum were found in the soybean rhizosphere, whereas, $T$. pseudokoningii were isolated from soybean and sunflower rhizosphere. No isolates of $T$. hamatum were found in the rhizosphere of sunflower, but all isolates obtained were found in soybean and faba bean thizosphere. The growth rate of the different isolates of the antagonistic fungi was tested at different temperatures $\left\{: 8,28\right.$ and $\left.38^{\circ} \mathrm{C}\right)$ to select the suitable isolates to be applied. Isolates of fast growth are more effective as bjological agents.

The mode of action of the different isolates of the biological agents was also studied against both pathogens, either for antagonism or hyperparasitism. Isolates of low and high lemperature requirments were obtained. Both antagonism and hyperparasitism were recorded with some isolates showing their effectiveness.

Faculty of Agriculture, Minufiya University, Shibin el-Kom, Egypt.

543

STRA MULCB AFD ENCAPSULATED RACILLUS THURIKGIEASIS AFRET LADTBIRD BEETLES (COLBOPTERA: COCCIMZLLIDAE) PRRDATING ON EGES ANB SWALI LARFAE OF LEPTINOTARSA DECBMIIMEATA(COLEOPTERA: CMRYSOMERIDAE).

\section{B. ODOHCO AND R. FOSTER}

Field teats were conducted in 1992 and 1993 at the $O^{\prime} N$ eall and Pinney farms in Indian, USA, to deterwine the effects of straw mulch, encapsulated Bec1llus thuringlensis var. tenebrionis (M-TRAK) and esfentalerate (Avana XI. $0.66 \mathrm{EC}$ ) on Colleomegilla maculata (L), a predator, and Coccinella septempunctata (Coleoptera: Cocclnellidae)a ladybird beetle that associates with eggs, first and second instars of Colorado potato beetle(CPB). pest and predator counts were low in both seasons and sites. Plancs under mulch and Bt treatwents had fewer aggs, larvae and predators than plants on non wulch and non insecticide control. Effects of mulch, and Bt on pests and predators were comparable to that of esfenvaletate and non mulch treatment, the standard growers" practice. C. maculata usually predate on the CPB and on the altarnate food sources found in other refugia other than potato. The alternate food source sustain the precator population when the beetie populations are low.

Namulonge Agricuitural and Animal Production Research Institute, P.0. Box 7084 Kampala, Uganda.
EFFORTS TO DEVELOP A MYCOHERBICIDE TO CONTROL PURPLE LOOSESTRIFE IN THE UNITED STATES.

\section{B. E. Nyvall and A. Hu}

Purple loosestrife (Lythrum salicaria $\mathbf{L}_{\text {. }}$ ) is a perennial introduced into the United States from Europe in the 1830s. The plant has spread throughout the United States, forming a monoculture by crowding out native vegetation in wetlands thereby reducing their value as wildlife habitat. Most biological control work has focused on insects; however, mycoherbicides either alone or in combination with insects may also be an altemative to chemical weed control in these environmentally sensitive sites. In a 3-year study, fungi were isolated from diseased purple loosestrife plants at 16 sites throughout

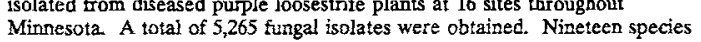
in 26 genera were identiffed of which 18 species in 24 general had not been reported from purple loosestrife in the United States. Fungal cultures were selected and 6-wk-old purple loosestrife plants misted with spore suspension at various spore concentrations depending upon spore size. Several carriers for the spore suspensions have also been studied to avoid placing inoculated the spore suspensions have aiso been studied to avoid placing inoculated
plants at $100 \%$ RH and to duplicate conditions in situ. Disease was evaluated at $7-14$ days on a scale of $0-5(0=$ no disease, $5=$ dead plant). Three fungi, Alternaria alternata (Fr.:Fr.) Keissl., Botrytis cinerea Pers.Fr. and Colletotrichum truncamm (Schwein.) Andrus \& W.D. Moore (obtained in the southem United States) consistently cause high disease. Plants were either killed, had numerous leaf spots or stunted due to necrosis of apical growing points. Results of a limited field study indicate B. cinerea may be an effective pathogen in situ.

University of Minnesota, North Central Experiment Station, 1861 Highway 169 East, Grand Rapids, MN 55744, USA.
544

STUDIES ON CONTROL OF ORACELIA ACUTA (LABDELL) WITH CRYPTOLAENUS MONIROU2IERI MUSANT

Hong Pang, Cai Tang

Oracella acuta (Labdell) was introduced illegally into China from USA. Now $Q$. acuta has been distributed to several counties of Guangdong Province of China. It danages pine trees severely. There is tendency towards in China from uSSR in 1955 and its colonized population was first found in 1979 in Guangzhou, Foshan. In April of 1993, when small population of $\underline{C}$. montrouzieri was released in pine forest of Heshan County, good efficiency of preying on $Q$. acuta and mass reproduction of c. montrouzieri were obdiet consisting of pupal hemolyneh of Antheraes pernyi, royal jelly, honey, diet consisting of pupal hemolymoh of Antheraes pernyi, royal jelly, hor
chicken yolk, etc., supplemented with some citrus mealybug, planococcass citri (Risso). Experiments showed, that the maximm of corrected mortality of 0 . acuta on screened trees was 88.78 , and 888 on unscreened trees.

Ms. Jong Pang, Guangdong Entanological Institute, Xingang west Road 105, Guangzhou 510260. The People's Republic of China 
BIOGEOGRAPHY A TOOL FOR THE BIOLOGICAL CONTROL OF

\section{Y. Papillon and B. Sicard}

Ecological, ethological and physiological approaches have shown that : (1) on a regional scale, rodent species are narrowly vs widely spread out, according to a climatic gradient (these species are spelled climatic vs ubiquist), (2) on a microlocal scale, rodent species are specialist vs generalist, when environmental occupation strategy is strict vs large, (3) feeding behavior and boring, correlate with the seasonality of trophical resources and digging. In view of these results we distinguish three types of environments : stable, fluctuating, and arid. Nevertheless, the study of the distribution of Acomys sp. in Burkina Faso indicates that in order to understand the general distribution of rodent in the soudano-sahelian region we must develop a biogeographical study. The main objective of this study is to establish a typology of coincidences in space exploitation by men and rodents, and the second objective is the optimisation of pullulation fight and prediction. Landscape typology leads to the survey of elementary units of environment with a third level of perception the local analysis of the space structure in their natural surroundings to evaluate the filters and barriers effects on species dispersion.

Dr. Papillon Yves, ORSTOM, BP. 2528, Bamako, MALI. RODENT POPULATIONS IN THE SOUDANO-SAHELIAN REGION

Bfological control of helicoverpa zea (Boddie) in field and sweet corn through Trichogramma pretiosum filey in the state of São Paulo, Brazil ${ }^{1}$

I.R.P. EARRA ; L.A.N. SA'; R.A. ZUCCHI

It is very common to observe a very high natural parasitigm of the com earworn Helicoverpa zea, by Trichogramma pretiosum under field conditions, in the State of São paulo, Brazil. The parasitism is always superior to 508 and very often reachs levels of 958 in field corn. However, this high level of parasitism is observed only in the last generation of the pest, when the damage has already occurred. The inundative release of $T$. pretiosun, reared on a factitious host (Anagasta kuehniella), may be an alternative method to control the corn earworm. In this method, releases can be done at the beginning of the corn earworm oviposition period, which occurs at the onset of the corn ailking stage. For the "Agrocica super Doce" sweet corn variety, it was observed that the oviposition peak of $H$. zea coincides with the blooming stage of the corn. The population dynamicy of the corn earworm egga was correlated with the corn phenology, and we found that the first egg Laying occurred 65 days after the germination. We observed that the $H$. zea biological control through $r$. pretiosum is possible, if we consider some aspects such as: selection of the most effective $T$. pretiosum strain and three releases of $T$. pretiosurn in a 4-day interval, with the first release when 358 of the plants is at silking stage and 758 of the male inflorescence has appeared. The parasitoid release resulted in an increase of parasitization and reduction of the $H$. zea damage up to 278 in corn ears. For field corn (dent corn variety C555) in two counties of the state of São Paulo, the release must also be done at the beginning of the $H$. zea egg laying. The dispersal capacity of $T$. pretiosum, 36 hours after release, ranged from 80 to $102 \mathrm{~m}^{2}$. The results of this study indicated that releases for controlling the corn earworm should be done in 100 points/ha. For field releases, it was observed an increase of up to 5 times in parasitism of $H$, zea eggs when $100,000 \mathrm{~T}$. pretiosum adults/ha were released three times. $H$. zea damage in corn ears in $T$. pretiosum released areas was reduced $26 \%$, showing the potential of this parasitoid for the control of the pest. The most agressive strain under laboratory conditions showed parasitism capacity higher in the first four days of the female's life.

1 Research supported by Fundacăo Banco do Brasil (FBB

'Depto. de Entomologia-ESAlQ/USP, CP.9, 13418-900, Piracicaba-SP-Brazil

3 EMBRAFA/CNPMA * Jaguariúna, SP

548

ROLE AND BIOLOGICAL CONTROL POTENTIAL OF THREE VERTICILLIUM SPECIES, RHIZOCTONIA SOLANI AND OTHER SOIL BORNE FUNGI ON THE INCIDENCE OF WILT, PREMATURE SENESCENCE AND YIELD OF POTATOES

\section{H.W. (Bud) Platt}

Verticillium wilt of potatees is generally found to be caused by albo-ctrum or $V$. dahliae, but in Ohio and elsewhere, associations with nematodes has been recognized as frequent causes of potato early dying. In the eastern coastal regions of Canada, V. albo-atrum predominates in the cool, moist conditions during the growing season. However, when warmer drier periods occur, plants often senesce prematurely without the expression of typical verticillium wilt symptoms.

Isolation of fungal and nematode species from potato fields with symptoms of premature senescence has revealed the presence of a variety of potential pathogens. These included such common fungi as three Verticillium species, Rhizoctonia solani, several Fusarium species, Colletotrichum coccodes, etc. Resident nematode specie contributions to this phenomenon were not significant. Replicated field studies involving single or multiple pathogen inoculations were conducted over several years. Several of the more common fungal pathogens and a few less common ones were found to contribute to a disease complex. The results of these investigations and potential for biological control will be presented in more detail.

Agriculture and Agri-Food Canada, Charlottetown Research Centre, Charlottetown, P.E.I., Canada C1A 7M8 FUNGI GENUS FUSARIUM. ability to secrete the chitinolityc enzymes' complex. Strains $(A, B)$ with the highest level plants against Fusarium exysponim under greenhouse conditions: the number of diseased
MYCOLITICAL ENZYMES OF BACTERUM SERRATIA MARCESCENS IN MECHANISMS OF BIOLOGICAL CONTROL OF PHYTOPATHOGENIC

\section{S.L.TJUTEREV, E.V.POPOVA, M.V.SOKOLOVA, L.K.KHATZKEVICH, A.V.KHOTJANOVICH}

Microorganisms capable of lysing other organisns play an important role in microbia equilibrium in soil and serve as a powerful tool for microbiological control. Especially it concems to several bacteria and fungi which have produced hydrolytic enzymes that degrade a cell wall of plant pathogens. Anrong such microotganisms there is a gram-negative bacterium $S$ marcescens, secreting several chitinolytic and some other hydrolytic enzymes. The bacterium S. marcescens, secreting several chituolytic and some other hydrolycic enzymes. The aim of out research is to study the role of bactenum's Serratia marcescens lytic enzymes in biocontrol mechanisms of soil-bom plant pathogens. We carried out comparative study of the antagonistic properties of three strains of $S$ marcescens with different levels of extracellular hydrolytic enzymes' activities (chitinase, glucanase, cellulase, protease) against a wide range (20) fungal pathogens (in vitro). The data showed that bacterial strains were essential different in their antifungal activities. The differences in antifungal activity of strains correlate with their of chitinase activity inhibited mycelial growth of such fungi as F.culmorum, F.oxysponm, H.sativum, V.dahliae in the range of $70-80 \%$. The strain C which doesn't F.oxysponm, H.sativum, V.dahliae in the range of $70-80 \%$. The strain $\mathbf{C}$ which doesn't
produce extracellular chitinase suppresses the growth of these fungi only by $25-45 \%$. The produce extracellular chitinase suppresses the grouth of these fungi only by $25-45 \%$. The
system bacteria S.marcescens - fungus F. Dxysporum has been used as a model for studing the dynamic of induction of bacterial lytic enxymes and their role in the degradation of vegetative and reproductive organs of this fungus. The strains of S.marcescens which are differ in their ability to attack micelium of F.oxysporum and utilize their propagules, are also differ in their capacity of secreting lytic enzymes produced by them. Strain A with the highest level of chitinase and antifungal activity was tested in the protection of cucumber plants against fusarium axysponum under greenhouse conditions: the number of diseased plants is reduced by $30-50 \%$ after bacteria. treatminent; the number of death-roll plants is
decreased in 2,5 times, yield of cucumbers increase up to $33-50 \%$. At present we are creating decreased in 2,5 times, yield of cucumbers increase up to $33-50 \%$. At present we are creating
the library of lytic enzymes' genes of the bacterial strain (A) with the highest chitinolytic enzymes' activity and which can be used for genetic transformation of microorganismsantagonists to increase their antifungal properties and to construct the vector systems useful for plant transformation. 


\section{9}

FACTORS AFFECTING THE RELEASE ANI PERCEPTION OF SEX PHEROMONES IN THE PREDATORY MITE PHYTOSEIULUS PERSIMILIS

\section{A. H. RASMY and H. E. HUSSEIN}

A laboratory test of two-choice assays, based on ferale calling, revealed that the response of males of the predatory mite Phytoseiulus persimilis A.-H. to females is adversely affected by mating and negatively correlated with age. The early age characterized by the highest release of sex pheromones by the predatory females coincides with that of male perception. Mating of both sexes reduced significantly the attraction of males to females. Results showed a1so, that the highest percentage of mating attempts was recorded in the early morning, then sharply declined in the noon time. The intensity of males to mate with females increased again at the sun set under natural day light regim. However, the present study shows that the release of sex pheromones in this predatory mite appears to be hormonally controlled by such factors i.e. day time, mating and age. These findings may contribute to a better understanding of reproduction in predacious mites.

Plant Protection Dept., National hesearch Centre, Dokki, Cairo, Egspt.

551

BIOLOGical CONTROL OF PENICILLTUM RXPANSUM IN ARGENTINa

A. Cumino, M. Ressini, S. Di Masi, A. Dobra, D. Engler.

Blue mold caused by Penicillium expansum Link., is a major postharvest disease of apples in Alto valle de R10 Negro y Neuquen. For years, fungicides were routinely used to control this disease after harvest. Pathogens resistance to fungicides and concern for public safety have combined to increase interest in alternative methods to fungicidal treatment in controlling blue mold. This has lead to rapid development of biologicai control research. The objective of this study was to determine antagonists within the epifitic microflora of the apples before harvest. Potential antagonists were obtafned from the surface of apple fruits and aerial monitoring in apple orchards. Al1 colonies (bacteria, yeast and filamentous fungi) were purified by single-colony isolations on potato-dextrose agar (PDA). In vitro screening was conducted by plating water suspensions of the potential antagonists ( $3 \times 10^{\circ}$ colony-forming units/ml) and the pathogen $\left(1 \times 10^{4}\right.$ conidia/ml) in Petri plates with PDA. In vivo screening was carried out on fully mature apples cv Red Delicious. The apples were wound-inoculated with the above mentioned concentrations of antagonists and pathogen. Inhibition of spore germination, mycelial growth and lesions diameters were evaluated. 117 isolations were tested in vitro and in vivo. OnIy 2 bacterial isolates and 3 yeasts isolates were selected for the prevention of the blue mold. The bacterial isolates were identified as Pseudononas spp.

INTA - EEA Alto valle. Casilla de Correos 782, (8332) General Roca, Rio Negro. Argentina.
CURRENT STATUS OF BIOLOGICAL CONTROL FOR THE HEMLOCK WOOLLY ADELGID IN THE U.S.A.

R, C. Reardon, M. E. Montgomery

The hewlock woolly adelgld (Hwh), Adelges tsugae Annand (Homoptera: Adelgidae) is an Important pest of eastern hemlock, Tsuga canadensis, and Carolina hemlock, I. carolingana, in the eastern U.S. A. Native natural enemies do not affectively reguiate HWA populations. No parasites of adelgids are knom but there are effective predators of HWA in Japan, including the coccinellid beetle Nephus kompirasanus and the oribatid'mtte Diapterobates humeralis. Colonies of these predators are being maincained in the laboratory. Also, two coccine 1 ind
beetles Scymus suturalis and Harmonia axyridis are being evaluated

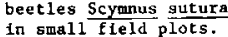

U.S. Forest Service 180 Canfield St. Morgantown, wV 26505 U.S.A.

552

BIOLOGICAL CONTROL OP SOME PATHOGENIC FUNGI BY BACILLUS SP.

A. Saniewska, L. B. Orlikowski, A. Wojdyła

In an in vitxo bioassay and greenhouse trials, isolate $\$ 13$ of Bacillus sp. isolated from rotted Hippeastrum bulb, was evaluated as biological control agent of formae spec. of Fusarium oxysporum, phytophthora cryptogea and $P$. cinnamomi. In the previous study it was found that strain $\$ 13$ inhibited mycelial growth of Phoma narcissi and development of red spot symptoms on Hippeastrum and Narcissus bulbs (Saniewska et al., 1993, 1994). Additionally, the strain applied as soil drench reduced development of phytophthora foot rot of gerbera. In the present work it was found that tested isolate inhibited in vitro mycelial growth of $F$. oxysporum $f$. sp. callistephi, f, sp. dianthi, f, sp. lilii and f, sp. tulipae ( $=$ Fot). Application of bacterial suspension as tulip bulbs soaking 24 hrs before their inoculation with Fot significantly reduced the development of rotted spots. Soaking of bulbs in bacterial suspension 24 hrs after inoculation with the pathogen was ineffective.

In the control of Sphaerotheca pannosa var. rosae, application of bacteria as spraying twicely at 7-day-intervals gave similar effect like triforine. Higher number of bacterial cells in stock culture was, in general, more effective in the disease control than its suspensions. The filtrate of strains $\$ 13$ gave similar effect in the pathogen control as suspension containing bacterial cells. Media used for antagonist growth influenced its efficacy. The strain produced on PDA was more effective than on other media.

Literature

Saniewska A., Sobiczewski P., Orlikowski L. B., 1993. Phytopathologia polonica

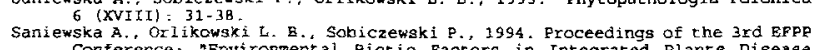
Conference : Environmental Bictic Factors in Integrated
Control $1^{t}$, Poznah. Poland, September 5-9, 1994 (in print).

Research Institute of pomology and Floriculture

pomologicana 18, 96-100 skierniewice, poland 
MICROBIAL CONTROL OF Monochamus altematus HOPE (COLEOPTERA: CERAMBYCIDAE) BY APPLICATION OF NONWOVEN

FABRIC STRIPS WITH Beauveria bassiana (DEUTEROMYCOTYNA:

\section{HYPHOMYCETES) ON INFESTED TREE TRUNKS}

\section{Shimazu, D. Tsuchiya, $\underline{H}$. Sato and T. Kushida}

The pine sawyer, Monochamus altematus transmits the pine wood nematode, Bursaphelenchus xylophy/us, which causes the pine wilt desease. Therefore, this insect has been the most important pest of pine forests in Japan Beauveria bassiana cultured on nonwoven fabric strips was applied in the field to control $M$. alternatus lanve under the bark. In the summer, the strips with the fungus were placed using various methods on the bark of pine logs infested with $M$. altematus larvae. Approximately $80 \%$ on average and a maximum of $100 \%$ of the larvae were infected and killed with the fungus using this method. Differences in mortalities between application methods of strips were not clear. This method was also effective for branches infested with $M$. altematus larvae, the utilization of nonwoven fabric strips impregnated the fungus was found to be more effective than ordinal spraying and more practical than the implantation of wheat-bran pellets containing the fungus which was developed previously by the authors.

Forestry and Forest Produdts Research Institute, P. O. Box 16. Tsukuba Norin Kenkyu Danchinai lbaraki 305, Japan.
BIOLOGICAL CONTROL OF STRIGA HERMONTHICA WITH FUSARIUM NYGAMAI IN COTE D'IVOIRE

\section{J. SAUERBORN, A.A. ABBASHER, J. KROSCHEL, D.W. CORNES, A. ZOSCHKE,} K.T. HINE

The parasitic weed Striga hermonthica is a serious problem affecting maize, sorghum and millet crops mainly in the Savannah region of Africa. Infestation by $S$. hermonthica can result in total crop loss and no practical solution to this weed problem exists. Failure of conventional methods for the control of $S$. hermonthica has led to new approaches like the use of Fuscrium nygamai as a mycoherbicide.

Following excellent results in glasshouse trials, a joint field testing programme was organised in Ivoiry Coast by Ciba-Geigy, Basel, Switzerland in collaboration with the supra-regional project "Ecology and Management of Parasitic Weeds" of the Deutsche Gesellschaft fur Technische Zusammenarbeit (GTZ) and the University of Hohenheim. Nine fieid trials were carried out over two seasons in fields with high naturally occurring infestations of Striga hermonihica.

Two field trials carried out in 1993 examined application of $F$. mygamai, at either $1 \times 10^{3}$ or $2 \times 10^{13}$ conidia (mainly microconidia) per hectare, as a conidial suspension applied as a spray or fermented on sorghum grains and applied using the sorghum as a carrier. Applications were made in maize crops both pre- and post-emergence of $S$. hermonthica. No reduction in the number of $S$. hermonthica plants emerging was observed between treatments, however after emergence $S$. hermonthica plants began to show symptoms of $F$. nygamai infection. Treatments of $F$. mygamal on sorghum grain at planting resulted in an improvement in maize vigour and the highest yield (grain or biomass) although any differences were not statistically significant. Post-emergence treatments applied over pre-emergence treatments did not improve S. hermonthica control.

As a result of these trials the second season concentrated on treatments made at planting. Treatments of $F$. myganai were formulated as either dry powders, dry granules or were again fermented on sorghum grain, which was identified as the most promising treatment in the first season. Seven trials were carried out which examined a range of treatments applied either in the maize planting hole or incorporated into the plot surface. No significant differences were the maize planting hole or incorporated into the plot surface. No significant differences were
observed with respect to $S$. hermonthica plants emerging but visual assessments recorded a $10-50 \%$ reduction of $S$. hermonthica biomass due to $F$. mygamai infection. The higher levels of $S$. hermonthica biomass reduction resulted in a corresponding improvement in maize vigour but maize yield was only significantly increased in comparison to untreated plots at one site. Variation in the results achieved between sites cannot be satisfactorily explained at the current point in time but may be related to rainfall or temperature. Modifications in the application technique may also result in more consistent activity.

The site of one of the 1993 trials was replanted with maize in 1994 to evaluate the persistence of $F$. nygamai. No further treatments were made but $S$. hermonthica plants emerging were clearly infected by $F$, nygamai and the population density proved to be lower than areas untreated in 1993. This indicates that $F$, nygamai is able to survive in the soil for a period of at least one year.

The results of these trials confirm the potential of $F$, mgamai as a mycoherbicide for the control of $S$. hermonthica, although several biological questions still remain open

University of Giesser, Tropical Crop Scjence, Schottstr. 2, 35390 Giessen, Gernany

BIOCHEMICAL AND BIOPHYSICAL TESTS FOR SELECTION OF BACILLUS THURINGIENSIS VIRULENT CLONES

\section{E.Schyolokova and V.Shevtsov}

The study of inderect biochemical and biophysical criteria could be correlated with entomocidal toxicity in different serovarieties of Bacillus thuringiensis (Bt) has been carried out.

The most virulent for Porthetrla dispar Bt subsp. dendrollmus clones have had high activity of lecitinase. The isolates of Bt subsp. gallerlae with high activity against Galleria mellonella have been characterized by significant activity of phospholipase $c$ and penicillinase but low exoprotease and chltinase production. The entomocidal toxicity of Bt subsp, galleriae have not increased if the chitinase was added to the caterpiliars fodder.

The correlation have not been found between insectlcide toxicity of Bt subsp. kurstaki clones and amylase, exoprotease, alkali phosphatase, lecitinase activities. The virulent clones have surpassed the high contents of crystal protein and the low harvest of spores. They have possessed higher level of electrophoretic cells mobility in free flow and lower level of chemotaxls to oxygen and glutamic acid than mildly active ones. The obtained results have shown what inderect biochemfcal and blophysical criteria can be used in screening of virulent Bt strains.

102, Lenin str., Serpukhov, Moscow Region 142283, Russia, $\operatorname{Fax}(095) 1168980$.

\section{were approximately $5 \times 10^{5}$ cells/wound in two days and as high as $1 \times 10^{7}$} cells/wound in five days. Colonization by antagonists expressed as cells/wound or as a percentage of the total bacterial population present in wounds, varied depending on the specific antagonist pair analyzed. The attractiveness of biologically controlling Fusarium dry rot is increased by protecting wounds with pairs of antagonists since such treatment can greatly reduce the effective dose required for disease control.

USDA-ARS, National Center for Agricultural Utilization Research, Peoria, IL, USA 61604. 
BIOLOGICAL CONTROL OF FUNGUS DISEASES OF CROPPED PLANTS IN ROMANIA

Tatiana Eugenia \$̦ESAN and T BAICU

Since 1932 when Weindling revealed that saprophytic fungus Trichodermo viride (Tlignorum) is able to inhibit some soilborne pathogenic fungi, a new era was opened to research in the domain of use of biological means to control plant pathogens, particularly through antagonistic and mycoparasilic fungi. Starting with 1955, the Intemational Organisation for Biological Control (IOBC) was founded, currently the Intemational Organisation for Biological and Integrated Control of Noxious Arimals and Plants, also including a working group entitled "Biological Control of Fungal and Bacterial Pathogens"

In Romania investigations on biological control of plant pathogens began at the Research Institute for Plant Protection, Bucharest, having here at present great share.

Romanian research on siological control of plans fungal pathogens are in line with the following directions (Baicu. 1992; Sesar \& Bajcu, 1993)

I. Basic research seeking for new sources of biological means. new antagonists. etc, referring to

I.1. Biological research on antagonistic fungi: Trichoderma viride. Trichothecium roseum, Gfocladium roseum Coniothyium minirans. Fusarium Iteritium. Fucoccum purpursicens Rusan, 1973; Sesan, 1985 1994; Dumitray, 1983; Sesan \& Crisan, 1988; \$esan \& Csep, 1991-1994; \$esan \& Baicu, 1993 etc.);

I.2. Obtaining new biotpes of biological agents, mutants resistant to fungicides, etc. (Trichoderma viride) (\$esan \& Baicu, 1989; Baicu. Șesan \& Gogoaşă, 1989);

1.3. Phyllosplere investigation as source of biological control agents and influesce of biotic and abjotic factors,

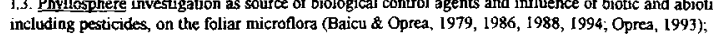
II. Applicative research, including:

II. Applicative research, including:
II.1. Ways to produce biological means. manufacturing technologies, and namely laboratory and pilot-plant I.1. Ways to produce biological means. manufacturing lechnologies, and namely laboratory and pilot-plant
technologies for mass growing of Trichoderma viride isolates biologically active against various pathogens technologies for mass growing of Trichoderma viride isolates biologically active against various pathogens
(\$esan, 1985, 1986; Sesan \& Oancea, 1993, 1994), formulation of T.viride isolates for soil (granules) and secd (Sesan, 1985, 1986; Sesan \& Oancea, 1993, 1994),
(dusts) treazments (Baim, Sesan \& Oancea, 1994);

(dusts) treatments (Baicu, \$esan \& Oancea, 1994);
II.2. Technologies for application of biological means, particularly for bioproducts based on T.viride, under II.2. Technologies for application of biological means, particularly for bioproducts based on T.viride, under
various ecological and cropping conditions (grains, industrial plants, medicinal herbs, annual pulses, various ecological and crop
strawberry. grapevine, etc.);

strawberry. grapevine, etc.);
II.3. Development of integrated control systems, encompassing biological control elements for bean, soybean, II.3. Development of integrated control systems, encompassing biological control elements for bean, soybean,
sunflowes, potato crops (Baicu, Gogoasł \& Sexan, 1986, 1988; lliescu et al., 1983, 1985, 1989), and selectivity sunflowes, potato crops (Baicu, Gogoasł \& Sesan, 1986, 1988; Iliescu et al., 1983, 1985, 1989), and selectivity
of some pesticides to T.viridg (Baicu, 1982, 1986), Coniothyrimm minitans (Sesan \& Baicu, 1993), Fusarium of some pesticides to T.viride (Baicu, 1982, 1986), Coniothyrium minitons (Sesan \& Bacr.
loterilism (Sesan, Opres \& Baicu, 1992) and other fungi beneficial to biological protection. loteritimm (\$esan, Oprea \& Baicu, 1992) and other fungi beneficial to biological protection.
III. In the funure, developmental research already starled svil] be expanded, baving as goals: III. In the future, developmental research already starled will be expanded, baving as
III.1. Facilities for production of misrobiological products to control plant pathogens, III.1. Facilities for production of misrobiological products to contro
III.2. Technologies for application of plant protection bioproducts:

III.2. Technologies for application of plant protection bioproducts:
Target-pathogens studied till now in Romania are: Fusarium spp. (wheat, maize, cotton, chickpea, vegetables), Nigraspora onzae (neaize), Pythism debaryanum, Rhizoctomia solani (sugarbet, cotion, annual pulses, vegetables), Phoma belae, Aphanomyces spp. (sugarbeet), Ascochyta rabiei (chickpea), Sclerotimia sclerofionum (surflower, soybean, lettuce, tomato, cucumber, stored carrots), Botrytis cinerea (sunflower. strawberry, grapevine), Stemphylium radicinum (stored carrots), Colletotrichum lindemuthianum (bean), Alternaria solani (potato), Uncinula necotor, Phomopsis vilicola, Eutypa lata (grapevine). Fomes annosus (conifers), Armillaria melleo (grapevine, conifers), etc.

Research Institute for Plant Protection - Bd. Ion lonescu de la Brad no.8- 71592 Bucharest 1 - Romania
THE EFFECT OF TRICHODERMA HARZIANUM ON PHYTOPHTHORA DRECHSLERI : THE CAUSAL. AGENT OF CUCUMBER DAMPING-OFF.

A. Sharifi - Tehran ${ }^{*}$, S. Nazari

One of the most important diseages of cucurbites lncluding cucumber in Iran 1s Phytophthora damping-off. Alavi (1973) and Etebarian (1978) have reported $20 \%$ and $35 \%$ damage to cucurbits due to this disease. The effect of Trichodem harzianum Rfal on the causal agent of disease ( Phytophthora drechsleri Tucker) were studied in vitro and in green -house. The results showed that the antagonist was able to reduce the damage of disease. This reduction was $35 \%$. The mechan1sms by which the antagonist effects the pathogen were ant1biosis, lysis and hyphal contact. Integrated Trichoderma and Fungicides (metalaxyl- Mancozeb- Propamocarb) applications did not show a considerable differ from using Trichoderma alone in control of disease. Using Trichoderma increased the weight of cucumber seeding compare with control treatment.

* Department of Plant Protection, Faculty of Agriculture, 31584 Karaj-IRAN
560

MANAGEMENT OF PEARL MILLET DOWNY MILDEW DISEASE USING ANTAGONISTS

Shishupala, S., Shetty, H.S.

Downy mildew disease of pearl millet caused by Sclerospora graminicola is a major blotic constraint for the crop production. Disease management strategles using host resistance has not been fully employed because of lack of durable resigtance source among the host cultivars coupled with development of new virulent strains of the pathogen. Use of metalaxyl formulations were also effective but many ingensitive strains of the pathogen are being identified against this for antagontatic fungl from rhizosphere, thizoplane for antagonistit fungl from rhizosphere, thizoplane and phylloplane. Selected microbes and their prevent asexual sporulation and sporangial germiantion of S. graminicola in in vitro
bioassays. Trichoderma harzianum and vere capable of preventing asexual sporulation of the pathogen when applied to the infected leaf. the pathogen when applied to the infected leaf. Both the species prevented sporangial germination
of s. graminicola in 1 in vitro assays. Culture
filtrates of Trichoderm spp. Asperglilus niger and filtrates of Trichoderma spp. Aspergilius niger and chaetomium globosum falled to prevent asexual germination was prevented by their culture filtrates. The organisms applied to soll in the form of wheat bran culture reduced downy mildew disease incldence significantly under greenhouse conditions. Culture filtrates used as follar spray significantly reduced the disease incidence fn susceptible pear1 millet cultivar. Fleld trial conducted under artificial epiphyototic conditions by deploying antagonists singly or in combination with metslaxyl formulations reduced downy milew incidence significantly. Among the combination treatments, solI amendment of $T$. viride along with follar spray of Ridomil MZ 72 WP $4 \mathrm{~g} / 1$ was found potential for blological control of pearl millet of downy mildew disease.

Downy Mlldew Research Laboratory, Department of Studies in Applied Botany, Manasagangotri, Unlversity of Mysore, Mysore - 570 006 , Indta. 
IPM FOR SCAPTERISCUS MOLE CRICKETS IN FLORIDA TURF

J. H. Frank, J. P. Parkman, D. E. Short

Three South American species of r.ole crickets of the genus Scapteriscus are together the most damaging irsect pests of turf grasses in Florida. An estimate in the mid-1980s blasied them for $\$ 44$ milion annually in damage and control costs when all control was by use of chemical pesticides. Control is now being exerted by a parasitoid fly and an entomopathogenic nematode from South Anerica. The fly (Ormia depleta: Tachinidae) is a classical biological control agent which, since 1988, has established a population in 32 counties. The nematode (Steinernema
scapterisci: Steinernematidae) is sold as a biopesticide, but has attriscapterisci: Steinernematidae) is sold as a bi

Entonology \& Nematology Dept, University of Flozida, Gainesvilie, FL 32611-0620, USA.
THE EFFTCACY OF NUCLEAR POL YHEDROSIS VIRUSES FOR THE CONTROL OF ARMYWORMS SPODOPTERA LITURA ON SOYBEAN AND TOBACCO IN JAVA

S. Mangoendihardjo, A. Pollet, M. Sarazic and Wagiyana

Mamestrin plus containing Nuclear Polyhedrosis Virus (NPV) of Mamestra brassicae and Cypermethrin, local strain of Spodoptera litura NPV, and chemical insecticides were tested in Java against armyworn on soybean and tobacco, during 1993-1994.

Mamestrin plus as well as local strain of NPV worked well and may replace chemical insecticides. The use of NPV is safe to the natural enemies of armyworms and of other pests on both crops.

UGM-ORSTOM, Sekip L2, 55281 Yogyakarta, Indonesia. Fax + 62 - 274 - 560267.

\section{3}

DISEASE-AND/OR PATHOGEN SUPPRESSION FOR BIOLOGICAL CONTROL OF SHEATH BLICHT OF RICE.

Sandra Taekema*, Iris Kolkman* and Kasem Soytong**

*Faculty of Environmental Science, Prof. H.C. Van Hall Institute, Hereweg 99 Groningen, The Netherlands, Faculty of Agricultutal Technology, King Mongkut is Institute of Technology Lodkrabang, Bargkok 10520, That land.

This research was done to flid a blological method to control the pathogen, Rhizoctonia solan which causes very sertously sheath Blight of rice (Oryza sativa) In thailand. Sought is for the promiaing biological control agents which can elther suppress the pathogen or the disease. In bi-culture test, two fungt were screened for their ability to inhibit the growth of $\mathrm{R}$. solani The screened antagonistic fungi, Trichoderma harzianum TH 01 and Chaetomiun cupreum KMIT 3003 both showed an antagonistic effect on the pathogen. These potentially antagonista were then tested for their pathogen-and/or disease suppression. As resulta can be reported that both antagonlata have the ability to suppress the pathogen as well as the disease of Sheath Blight in the soll. As comparison of the biological control, the chemical fungicide (PCNB) is used. The latter treatment has given a worse reault. In this teat $\underline{C}$. cupreum works a little more effective than $\underline{T}$ - harzianum. It 1 s also shown that treatments with antagonista like $\underline{\mathrm{C}}$. cupreum or $\underline{\mathrm{T}}$. harzianum with or without $\underline{\mathrm{R}}$. solan gave a better plant fresh weight than chemical trestment snd non-trested one.
564

USE OF AMBLYSEIUS WOMERSLEYT (SCHICHA) AS A PREDACIOUS MITE IN JAPAN

T. Hamamura and $\underline{\text { H. Suenaga }}$

A predacious mite, A womersleyi, which is native of Japan, have an effect on the population of Kanzawa spider mite, Tetranychus kanzawai Kishida, in tea fields (Hamamura,1986). Recently another predacious mite, Phytoseitulius persimitis Athias-Henriot is introduced into Japan from northem Europe to control spider mites in greenhouses. However, high temperatures in greenhouses and insectiside spraying for the control of other pests such as aphids and white flies may restrict the use of $P$. persimilis. We investigated the abilities of predation and fertility of $A$ womersleyi and $P$. persimilis at different temperatures. Below $30^{\circ} \mathrm{C}, A$ womersleyi was inferior to $P$. persimilis in both abilities, but above $30^{\circ} \mathrm{C}, A$ womersleyi was superior to $P$. persimilis. Some populations of $A$ womersleyi collected in tea fields in Japan were resistant to many insectisides usually sprayed in the fields (Hanamura, 1986; Mochizuki, 1990), however, newly introduced $P$. persimilis was susceptible to many insectisides (Shinkaji, 1976).

These results indicate that $A$ womersleyi is more usefull than $P$. persimilis for the control of the spider mites in greenhouses in Japan.

National Researcb Institute of Vegetables, Omamental Plants and Tea 
Usage of chemical pesticides is prohibited because of possible effect on humas health, so application of biological agents for disease control became very actual. Considerable attention was paid during last decade to use of bioagents naturally existing on surface of fruits and vegetables. The goals of our investigation were the search of useful microflora and study the possibility of its use as biocontrol mean Survey of storage houses has been undertaken during 1990-94 and 157 strains of Streptomyces were isolated. 37 strains showed low fungistatic activity or did not have it at all. In vitro investigations with Alternaria alternata, Aspergillus flavus, Botrytis cinerea, Fusarium oxysporum, Fsolani, Penicillium expansum and Verticillium dahliae as test-cultures showed that a number from the rest 20 Streptomyces possesses very high fungistatic actjvity. The most active 3 strains (Sreptomyces flavus-26, S.albosporius-9) and S.albosporius-13), that inhibited growth of all test-cultures were selected for the next studies. Study of growth
conditions showed that the highest fungistatic activity of cultural liquid was observed at growth on liquid medium SR-1 during 3 days on rotary shaker at $26-28^{\circ} \mathrm{C}$. The artificial introduction of these strains on the surface of storaged fruits and vegetables and use their cultural liquids as control mean of disease development have been made. It has been noted that artificially introduced Streptomyces were able to protect harvested products at the concentration $10^{4}-10^{5} \mathrm{cells} / \mathrm{ml}$. At this concentration S. Ravus-26. S.albosporius-9 and S.albosporius-13 increased percentage of noninfected fruits and vegetables from ca. 60 till 80,73 and $78 \%$, respectively, at the same time they did not affect the quality. In vitro investigation of fungistatic activity of cultural liquid showed that even at 1000-times dilution the antibiotic compounds of S.flavus- 26 keep their activity enough to control growth of test-cultures, at the same time 100 -time dilution was preferable to maintain antifungal ability of cultural liquid of two rest. Treatments of fruits and vegetables by cultural liquid of Streptomyces showed that the most preferable concentration was $0.02-0.04 \%$ of initial. At this concentration antifungal compounds of cultural liquid were able to keep products' losses on the low level. So, conclusion has been made that there is possibility of use of S.flavus-26, S.albosporius-9 and S.albosporius-13 as biocontrol agents of fungal diseases during storage period, though there is still necessity of further study of mechanism of their action on fungi and possible effect on quality of fruits and vegetables being stored.

\section{Institute of microbiology}

To, A.Kadiry str.

Tashkent

Uzbekistan

700128

567

BIOCONTROI. OF POST-HARVEST DISEASES ON APPLE BY ETHANOLASSIMILATING YEASTS

V.A. Todirash

The yeasts isolated from the surface of apple fruits were tested for their ability to reduce lesion development in Golden Delicious and Aidared apple after challenge with the post-harvest pathogens Penicillium expansum Link ex Thom. and Botrytis cinerea Pers ex Fr. The isolate Yo exhibited high antagonistic activity, growth in liquid mineral medium with ethanol as a nutrient in the absence of vitamins. The isolate identification shows morphological and biochemical characteristics similar to Candida sp. The deposited strains Candida utilis Y322, Candida guilliermondii Y858, Candida peiliculosa Y9 and Candida didensiae $\mathrm{Y} 10$ also inhibited lesion development.

A selective mineral salt medium was developed for isolation and growth of ethanol-assimilating yeasts. The results indicate the feasibility of application of yeast mixtures against post-harvest diseases.

Institute of Biological Plant Protection, Dacia street 58, 277050 Kishinev, Moldova
Naime Z.TEKELI Nedim UYGUN Ismail KARACA

The East Mediterranear region is the most important citrus growing area in Turkey, where about $70 \%$ of the production is concentrated. Since the production is increasing year by year, insect pests are more and more a limiting factor in production.

A most serious pest is Aonidiella aurantii (Maskell) (Homoptera: Diaspididae) causing high losses in yield and fruit quality in each year. Because insecticides showed no long lasting effect in controlling $A$. aurantit and often destroy the natural balance, biological control is the only reliable tecnique in managing this pest. Several parasitoids and predators are used to control A. aurantii.. A common predator in Turkey is the polyphagus coccinellid Chilocorus bipusiulatus (L.). Mass release into orchards, heavely infested by $A$. aurantii may prove useful in controlling, this pest succesfully. The development of efficient mass production of predatory insects depends strongly on suitable prey, which is easy to rear and does not negatively affect development and reproduction.

In this study different scale insects were used as prey for C. bipustulatus: A. aurantii, Aspidiotus nerii Bouche, and Pseudaulacaspis pentagona (Targioni). Demographic life-tables were constructed by combining the mean developmental period with the daily fertility rate of $C$. bipustulatus at constant $25^{\circ} \mathrm{C}$ for each prey. Developmental time, longevity, fecundity and sex ratio of the predator were strongly influenced by different preys. Individuals fed with $A$. nerii displayed the highest net reproduction rate $\left(\mathrm{R}_{0}\right)$ and intrinsic rate of increase $\left(\mathrm{r}_{\mathrm{m}}\right)$, and the shortest generation time compared to both other diaspidid species.

Since A. nerii was the most suitable prey and is easy to rear, this species is now used to develop a mass production of $C$. bipustulatus.

Department of Plant Protectin, Faculty of Agriculture, University of Cukurova, 01330 Adana. TURKEY
568

PRODUCTION OF ANTIBIOTIC SUBSTANCES AND SUPPRESSION OF SOILBOREE PLANT PATHOGENS BY A STRAIN OF PSEUDOMONAS FLUORESCENS AND ITS Tn5 MUTANTS

\section{K. TSUCHIYA ${ }^{3}$, Y. HOMMA ${ }^{2}$, T. NAKAYAMA ${ }^{3}$, S. TAHARA ${ }^{3}$ and M. HORITA ${ }^{1}$}

Pseudomonas fluorescens strain LRB3H1 is an effective antagonist of various plant pathogens by showing antibiosis against such as Rhizoctonia solani, Phytium ultimum and Gaeumannomyces graminis var. tritici for fungi and Clavibacter michiganensis subsp. michiganensis, Erwinia carotovora subsp. carotovora and others. The bacterium produced several metabolites, notably hydrogen cyanide (HCN), 2,4diacetyl phloroglucinol (PhI) and unidentified fluorescent substances.

Tn5 insertion mutants of LRB3W1 were screened for loss or decreased in productivity of $\mathrm{Phl}$, fluorescent substances or HCN. Antibiotic activity of LRB3W1 and Tn5 mutants against R. solani, P. ultimum and Gaeumannomyces graminis var. tritici coincided with productivity of Phl. Suppresive effect on the incidence of take-all of wheat by those strains correlated with their antibiotics productivity when the concentration of the pathogen was relatively low.

1 National Institute of Agrobiological Resources, 2-1-2, Kannondai, Tsukuba, Ibaraki 305, Japan

${ }^{2}$ Hokkaido National Agricultural Experiment Station, Hitsujigaoka-1, Toyohira, Sapporo 062, Japan

${ }^{3}$ Hokkaido University, Kita-9 Nishi-9, Kitaku, Sappora 060 , Japan 
WEEDS IN PASTURE AREAS IN THE SOUTHI- EAST MEDITERRANEAN REGION OF TURKEY AND THEIR POSSIBLE CONTROL BY NATURAL ENYMYES

$$
\begin{array}{ccc}
\text { Nedim UYGUN } & \text { N. Kemal KOC } & \text { Nezihi UYGUR } \\
\text { Ismail KARACA } & \text { Sibel UYGUR } & \text { Mustafa KÜSEK } \\
& \text { Naime Z. TEKEL! }
\end{array}
$$

The South-east Mediterranean region has a wide range of pasture area for animal breeding. The land use is characterized by extensive grazing that led to an encroachment of steppe formations. Certain plants are widespread because morphological (e.g., thoms and spines) and physiological (e.g., ethereal oils) adaptions allow them to resist the impacts of livestock.

Reducing of overgrazing would probably reduce some of these problems, however, culturel behavior and the exclusive dependence on livestock of local farmers hinder from any change. On the other hand, chemical, physical or cultural control methods of weeds are insufficient not only because of their adverse effect on nature but also because of difficulties in application. Biological control of weeds seems to be the most appropriate method in such ecosystems.

The aim of the present study was to determine the weeds in pasture lands and to find out natural antagonists associated with weeds. Promising antagonists are now being studied in taboratory experiments on their impacts on weeds.

In the iwo-year study, 39 weed species, belonging to 16 families were found. Up to now, 14 insect species, belonging to 3 order and 8 families were determined atracking weeds. Some additional species were not detemined up to now and are in progress of identification. Besides insecls. 15 fungi belonging to 2 classes were infecting weeds in pasture land. The results of the laboratory experiments on the impact of insects and fungi on weeds are forthcoming.

Department of Plan: Protection, Faculty of Agriculnure, University of Çukurova, 01330 Adana, TURKEY

571

VERTICILLIUM LECANII, A POTENTIAL BIOLOGICAL CONTROL AGENT FOR CUCUMBER POWDERY MILDEW

\section{M.A. Verhaar \& T. Hijwegen}

Cucumber powdery mildew, Sphaerotheca fuliginea, is a major disease, causing damage in all commercial cultivars of cucumbers grown in the Netherlands. Currently we are studying the prospects of the mycoparasite Verticillium lecanii to provide an alternative to the frequent application of fungicides.

A method to study the behaviour of cucumber powdery mitdew and $V$. lecanii under different conditions was developed. Second or third leaves of three weeks old cucumber plants were placed in $50 \%$ Hoagland solution. After one week most leaves had rooted and on this leaves the behaviour of potential biological control agents on mildew could be measured for about three weeks. Various $V$. lecanii isolates were tested for their parasitizing capacity, growth rate, sporulation and humidity requirements.

To test parasitizing capacity leaves were inoculated with mildew in a vacuum-operated settling tower (Reifschneider and Boiteux, 1988). Five days later these mildewed leaves were sprayed with spore suspensions of $10^{6}$ spores* $^{*} \mathrm{ml}^{-1}$. After one and two weeks the percetage affected mildew was assessed.

To measure growth of $V$. lecanii isolates on mildew, leaves were inoculated with one colony forming unit of two to three mildew conidia. Ten days later $10 \mu \mathrm{l}$ droplets containing $10^{6}$ conidia ${ }^{*} \mathrm{ml}^{-1}$ were introduced in the centre of healthy mildew colonies. The growth of the isolates was measured the following two weeks. Humidity requirements of $V$. lecanii isolates were tested by measuring the growth velocities on water agar with decreasing water potentials.

Based on the data obtained, the $V$. lecanii isolate performing best as biological control agent against cucumber powdery mildew will be selected.

Reifschneider F. J. B. and Boiteux L. S., 1988. A vacuum-operated settling tower for inoculation of powdery mildew fungi. Phytopathology Vol. 78 (11): 1463-1465.

Department of Phytopathology, Wageningen Agricultural University, P.O. Box 8025,6700 EE Wageningen, The Netherlands



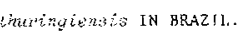

VALICENTE, F.H.'

Fall armyworm, spodoptem fragiperda, is one of the most important corn insect pests in Brazil, and its dämage can reduce production up to $34 \%$. Survey of isolates of bacolomims and strains of $B$. thumingiensio has been done in different corn producing regions in Brazil. Sine 1985 about 15000 larvae have been collected from southeastern of Brazil from which 21 isolates of Bachlovimas were harvested. Sore of these materiais have been worked and we of the isolates produced as a settable powder has been very effective against fall arnyworm, if infestation is the Eield is below $30 \%$ and larvae do not exceed $7.5 \mathrm{~cm}$. Larvae infected with Bacutovimis reduce feeding in $93 \%$ and die mostly within 6 days after infection. Also, Baoulovime produced as wettable powder has been used together with reduced dosages of chemical inseccicides and through irrigation water. About 4000 hectares has been sprayed with this Baculovirus with. good results. About 182 soil samples and spme grain dust samples have leen collected from different regions of Brazil, and about 22 strains of $B$. thipingiensis have been harvested. Some of these strains were washed and the powder coxin tested against fall arayworm in the laboratory, showitg good results, but most of the strains tested did not kill more than $50 \%$ of young larvae. Also the mixture of $B$. thuringiensis with Eaculovims in the same suspension has been a promising aim to control fall armyworm.

1. CNPMS/EMBRAPA - C. Postal 151 - 35.701-970 - Sete Lagoas, MG - Brazi1

572

BIOTIC EFFECT OF SOME SELECTED FUNGI AGAINST THREE FORMAE SPECIALES OF FUSARTUM OXYSPORUM SCHLECHT.

\section{Z. Weber, M,Werner, D. Fruzyriska-Józwak}

In laboratory experiaent the blotic effect of eight species of soll fungl against three formae speclales of Fusarium oxysporum was assessed. Blotic effect depended on an isolate of F. oxysporum a particular forma specialis, and of antagonistic fungus as well as on temperature. On average for five temperatures $\left(10,15,20,25\right.$, and $\left.30^{\circ} \mathrm{C}\right)$ the highest blotic effect was found in Trichoderma harzianum (isolate 658). T.longlbrachiatum (1solate D-645), T.hamatum (1solate C-606), T.virtide (isolates $A-129$ and $85 / 1$ ), and Zygorhynchus wolleri (isolate F-920). In the mean for ten soil 1solates, the above -mentioned blotic effect was displayed in relation to: F.oxysporum f.sp.dianthi (1solate IMI 141130) at $10^{\circ} \mathrm{C}, \mathrm{F}$. oxysporum (isolate 1 Prom Gysophlla panlculata) at $15^{\circ}$ and $20^{\circ} \mathrm{C}, \mathrm{F}$-oxysporum (isolates DII 141130 and 1 from Dianthus as well as isolate 1 from G.pantculata) at $25^{\circ} \mathrm{C}$, and F.oxysporum f.sp.asparagi (1solate IMI 141128) at $30^{\circ} \mathrm{C}$. The bighest blotic effect was found at $20^{\circ} \mathrm{C}$ in Absiòa californica, and Actinomucor elegans, at $25^{\circ} \mathrm{C}$ in Trichoderma spp., at $30^{\circ} \mathrm{C}$ in $\mathrm{Z}$.moellerl, at $25^{\circ}$ and $30^{\circ} \mathrm{C}$ in Pentcill1um funtculosum.

Agricultural UnIversity, Department of Phytopathology

u1. Dąbrowskiego 959, 60-594 Poznań, Poland 
ROOT AND STEM ROT PATHOGENS FROM CASSAVA AND THEIR ANTAGONISTS, COLLECTED IN CAMEROON, NIGERIA AND BENIN

\section{Afouda ${ }^{1}, \underline{\text { K. Wydra }}{ }^{2}$ and K. Rudolph ${ }^{1}$}

1 International Institute for Tropical Agriculture, Cotonou, Benin 2 Institute for Plant Pathology and Plant Protection, Göttingen, RFA

Root and stem rot samples were collected from cassava from different regions in Cameroon, Nigeria and Benin. The causal pathogens were identified as Botryodiplodia theobromae, Sclerotium rolfsil, Rhizoctonia solani, Sphaerostilbe sp. and Fusarium $\mathrm{sp}$.

Fungal antagonists were isolated from the rotten plant samples and from the adjacent rhizosphere soll. Strains of the species Trichoderma were tested for antagonistic potential against the root rot pathogens in in vitro tests.

The Trichoderma strains varied in inhibitory efficacy, and several promising strains were selected as possible biological control agents.

International Institute for Tropical Agriculture (ITTA), BP 08-0932, Cotonou, Republic of Benin
CROSSING BETWEEN AN INTRODUCED AND AN INDIGENOUS TORYMID PARASITOIDS IN BIOLOGICAL CONTROL OF THE CHESTNUT GALL WASP IN JAPAN

E. Yano, K, Yara, M. Shiga (National Institute of Agro-Environmental Sciences, Japan), M. Osakabe (Fruit Tree Research Station, Japan), H, Izawa(Tottori Horticultural Experiment Station, Japan) and $S$. Moriya (Okinawa Prefectural Agricultural Experiment Station, Japan\}

The chestnut gall wasp. Dryocosmus kuriphilus Yasumatsu, is one of the most serious pests of chestnut trees in Japan. It is assumed to have been introduced from China around 1940 . A torymid parasitoid, Torymus sinensis, was introduced from china into Japan and released at Tsukuba in the Kanto Plains in 1982. After establishment, the parasitoid spreaded gradually in the Kanto plains and caused a considerable reduction of the density of galls of the wasp. Before the introduction of $r$. sinensis, a closely related species. T. beneficus is indigenoug parasitoid of the wasp. Possibilities for occurrence of interspecific crossing in the field between the two species was suggested in crossing experiments. The two torymid species and the hybrid individuals produced by the crossing can be identified by the malic enzyme(ME) zymogram pattern. Galls of the chestnut gall wasp were collected at several sites of the Kanto plains and emerged adults of torymid parasitoids from the galls were kept for the isozyme discrimination for several years. The occurrence of hybrid individuals in these samples was checked by the ME zymogram. The results suggest a low rate of interspecific crossing.

Eizi Yano(National Institute of Agro-Environmental Sciences, 3-1-1, Kannondai, Tsukuba, Ibaraki, 305 Japan)
575

EFFECT OF LIGHT AND TEMPERATURE ON TRIALEURODES VAPORARIORUM WESTWOOD AND ENCARSIA FORMOSA GAHAN

\section{A. ZABUDSKAYA}

Experiments were performed under controlled conditions. The main variables were temperature, photoperiod and light intensity. Comparison of the results allows recommendations on
manipulation of the glasshouse environment to favour Each variant included $10-20$ individuals conditions were as
follows temperature $-16,22,28^{\circ} \mathrm{C}$ photoperiod $-12,15$. follows: temperature $-16,22,28^{\circ} \mathrm{C}$ photoperiod $-12,15$, $18 \mathrm{~h}$; light intensity function.

Temperature greatly influenced fecurdity in 7 . vaporariorum: 70 -72 eggs at $16^{\circ} \mathrm{C}, 131$ eggs at $22^{\circ} \mathrm{C}, 83$ eggs at $28^{\circ} \mathrm{C}$. The highest $r(0,126)$ was observed at $28^{\circ} \mathrm{C}$ at high photoperiod and light intensity values. At $22^{\circ} \mathrm{C},{ }^{r}$ for $T$.vaporariorum fell to 0101 - 1116 and, at $16 \mathrm{C}$, the value was very low 0,020 $0,044)$. Though $T$. vaporariorum had maximum biotic potential at
$28^{\circ} \mathrm{C}$, populations developed rapidly even at $22^{\circ} \mathrm{C}$, due to the $28 \mathrm{C}$, populations developed

high fecundity of the pest. It is useful to compare the development rate of pests with that of their entomophages. For T. vaporariorum and $E$. formosa, all biological indices of the parasite were lower at $16^{\circ} \mathrm{C}$ than those of the pest. This explains the low effectiveness of E.formosa in winter. At $22^{\circ} \mathrm{C}$, longevity of adult E. formosa was increased two-fold and fecundity 20 times. At this temperature, the fecundity of the pest was still higher than
of the parasite but, since the latter had a much shorter of the parasite but, since the latter had a much shorter
development period, pest control could be assured: $T$. vaporariorum populations increased 2,5 times in 10 days,
while E. formosa populations increased 3 times. At $28^{\circ} \mathrm{C}$, the respective figures were 2,7 and 6 times, which allowed $E$. formosa to control the pest very effectively.

Comparison of the relationships within this pair between $T$.vaporariorum and E.formosa of phytophage and entomophage confirms that good timing and conditions for rapid entomophage development are essential the successful biological control. Mathematical models of such relationships between $T$. vaporariorum and $E$. formosa were constructed.

Mathematical model of $T$.vaporariorum development

$\hat{f}_{m}=0,1051+0,0383 x_{1}+0,0062 x_{2}+0,0043 x_{3}-0,0034 x_{1} x_{2}+$ $+0,0024 x_{1}-0,0014 x_{x}-0,0371 x^{2}+0,0034 x_{2}^{2}+0,0039 x_{3}^{2}$ Mathematical model of E.formosa development

$+0,0048 x_{3}+0,0051 x_{1} x_{2}+$

$+0,0054 x_{1} x_{3}-0,0024 x_{2} x_{3}-0,0298 x_{1}^{2}+0,0012 x_{2}^{2}+0,0092 x_{3}^{2}$.

Where $x$ - temperature, $x_{2}$ - photoperiod, $x_{3}$ - light intensity. The resdits of mathematiclil models analyses ${ }^{3}$ are according to experimental data.

34, 54/A, L. Shevtsovoi st., Ulyanovsk, Russia, 432027

Moscow State University, Uiyanovsk Branch.
576

INCREASING RATES OF PARASITISM OF THE LARVAL PARASITOID BRACON BREYICORNIS WESM (HYMENOITERA: BRACONIDAE) B BY USING SEMIOCHEMICALS AND A SUPPLEMENTARY FOOD.

\section{F.N. ZAKI AND M. SALEH}

The kairomonal effect of hexane extract of the larvae of 0 . nubilalis and $\mathrm{S}$. cretzca on $\mathrm{B}$. brevicornis adults was evaTuated through olfactometer tests. The parasitoid males showed no definite response to any kairomone. Females were attracted to the kairomone of $S$. cretica more than to that of o.nubilalis Highly significant effect of the sex phermone of $\mathrm{o}$. nubilalig on the females of $\underline{B}$. brevicornis was recorded. Kairomones hexane extracts of larvael varied in their effect on the released $B$. brevicornis parasitolds. The kairomone of $\mathrm{S}$. cretica increased o. nubilalis and of $\underline{\mathrm{s}}$. 12ttoralis were not significantly effective.

Spraying molasses solution (108) on the corn stalks before releaspng B. brevicornis parasitoids increased the rate of parasitism from 7.79 to 28.2 .8 . The concentration of 5 gave insignificant increase in the parasitisation rate.

PROF. DT. F. N. ZAKI

PLANT PROTECTION DEPT.,

NATIONAL RESEARCH CENTRE,

EL-TAHRIR ST. CODE 12622

DOKKI, CAIRO, EGYYT. 
CONTROL EFFICIENCY ON COTTON SEEDLING DISEASES AND EFFECT ON SEEDLING GROWTH BY 5 BACILLUS STRAINS

X.J.Zhang, G.N.Zhu, W.G.Miao, et al.

5 Bacillus strains (C-22, C-37, C-54, C-57 and C-62) from 5 plant rhizosphere soils inhibited intensively several pathogens of cotton, including rhizoctonia rot (Rhizoctonia solani), anthrax (Colletotrichum gossypii), yellow dwarf (Verticillium dahliae), wilt (Fusarium vasinfectum), boll blight (Phytophthora boehamiae) and leaf spot (Cercospora gossypina). In laboratory tests, C-62 increased germination of cotton seeds, the others had no effect on seed germination. Seedling diseases were effectively controlled when seeds were treated with suspensions of 5 strains, and the height, fresh weight, leaf numbers and stem-width of cotton seedling were significantly increased when compared with control or Ling-Fu Mixture (a chemical fungicide) treatment. Over 2-3 Year's trails in Jiangsu, Henan and Shandong Provinces, similar results were obtained with C-62, C-37 and C-54 as a liquid or powder formula, with a general control efficiency of $40 \%-60 \%$ on rhizoctonia rot and anthrax when seeds were dipped or coated at a dosages of $5 \%-25 \%$ of seed weight, and with more rapid germination and higher quality of seedling.

Dept. of Plant Protection, Nanjing Agricultural University, Nanjing 210095, P.R. China

579

COLONIZATION OF B3 (BACILLUS SUBTILIS) ON ROOT AND STEM-BASE OF WHEAT

\section{X.J.Zhang, J.Zhao, and J. S. Wang}

Colonizing activities of B3 (Bacillus subtilis) on wheat root and stem-base and its effecting-factors were studied in our laboratory. It was found that in natural soils, B3 could colonize and survive over 60 and 42 days on wheat root and stem-base, respectively. B3 grew better when seeds were coated with 24 hour-old culture of B3 than those with 48 or 72 hour-old cultures. Addition of NB to B3 inoculum, mixed treatment of B3 with fungicides or soil-sterile can increase B3 population on wheat roots.

Dept. of Plant Protection, Nanjing Agricultural Uinversity, Nanjing 210095, P.R. China
SCREENING OF BACTERIAL ANTAGONISTS AGAINST SEVERAL IMPORTANT CROP PLANT PATHOGENS

\section{X.J.ZHANG, W.G MIAO, G.N ZHU and J.S WANG}

Fifty soil samples were collected from rhizosphere of 26 plant species in 12 locations of 6 provinces. After isolation and purification, 322 strains (mainly Bacillus spp.) were obtained. Their antagonistic activity was tested against 36 important crop plant pathogens in rice, wheat, cotton,tomato, Chinese cabbage and faba bean and so on. Of the 322 strains, 195 were found to be antagonistic to one or more pathogenic fungi and 178 to bacteria.

Dept. of Plant Protection, Nanjing Agricultural University, Nanjing 210095, P. R. China

580

MECHANISM OF CAIFENGNING B2 (BACILLUS SUBTILIS) ON CONTROLLING TO SCLEROTINIA BLIGHT OF RAPE

X.J Zhang, A.M Wu, and J.S. Wang

Cell-free culture filtrate (CFCF) of B2 (Bacillus subtilis) showed significant effect on germination of sclerotinia and formation of apothecium of Sclerotinia sclerotiorum. B2-CFCF at lower dosage (3.3\%) increased germination rate of sclerotinia by $20.4 \%$, but with a abnormal growth and no differentiation of germinating-apothecium. Contrastly, higher dosage of B2-CFCF inhibited germination of sclerotinia, and no sclerotinia germinated when B2-CFCF dosage was up to $16.7 \%$. Parenchyma tissues in non-germinated sclerotinia was disintegrated, with irregular cavity. Seed germination power and seedling growth of rape were promoted by $\mathrm{B} 2$ suspension at all dilution but the stock suspention, with the most significant effect to root growth at 500 times, and to bud growth at 50 times. Activities of peroxidase (PO), polyphenol oxidase (PPO), phenylalanine aminolyase (PAL) and superoxide dismutase (SOD) and content of phenolics in plants treated with B2 were variously increased, depending on growth stages of rape and soil type (natural or infested). Isozyme electrophoresis revealed 1-2 new bands ( $\mathrm{Rf} 0.048, \mathrm{Rf}$ 0.069 ) of PO and 2 new bands (Rf 0.063 , Rf 0.081 ) of SOD in plants treated with $B 2$.

Department of Plant Protection, Nanjing Agricultural University, Nanjing 210095, P.R. China. 
MICROBIAL POPULATION ON APPLE PEELS AND THEIR EFFECT ON 2 MAJOR PATHOGENS IN APPLE

\section{J. Zhang, Y. Xu, and J. Y. Wang}

Analysis on peel microflora of 8 apple varieties freshly-harvested or market-saled indicated that there existed a population of $4.87 \times 10^{3} \mathrm{CFU}-7.38 \times 10^{5} \mathrm{CFU}$ and 2.63 $\mathrm{X} 10^{2} \mathrm{CFU}-4.75 \times 10^{3} \mathrm{CFU}$ of bacteria and fungi per gram of peel, respectively. 273 bacterial strains and 75 fungal strains are obtained after isolation and purification. In plate tests, 77 bacterial and 20 fungi are antagonistic to 2 major storage pathogens, Gloeosporium fructigenum and Macrophoma kawatsokai. 9 strains showing extensive inhibition to the 2 pathogens, can effectively reduce apple rot to different degree when the pathogens are inoculated into fruits. Straints NA-W42 and K'B-X71 controled anthracnose by $96.3 \%$ and $88.9 \%$, respectively, and strain PG-W11a inhibited ring rot by $55.6 \%$.

Department of Plant Protection, Nanjing Agricultural University, Nanjing 210095, P. R. China

583

BACILLUS THURINGIENSIS SUBSPECIES KURSTAKI AS A MICROBIAL BIOCONTROL AGENT

Burtzeva L.I Kalmikova G.V.

Bacillus thuringiensis subsp. kurstaki (Btk), the most used and commercially available isolat?, is toxic to the larvae of a number of a lepidopteran pest species. The insecticidal activity of Btk products is based mainly on the so-called delta-endotoxin which is formed during the sporulation process and deposited in a crystalline form within the sporangium. The strains of Btk form parasporal crystals with embedded bodies (pathovar K-1) and without them (pathovar K-73). There are considerable variation of toxicity between different strains. There are no general rules applicable to the selection of strains and no sumple procedure for screening out the most promising. We used to plate a large number of Bik strains of different origin on a number of media. In general media consisted of a carbon and energy source like glucose, nitrogen sources such as peptone and yeast extract and mineral salt: We discovered six morphological variants of Btk strains. All morphovariants were found to exhibit a distinctive colony morphology which facilitated their isolation. These include strains which differ besides colonial characteristics metabolic capacity, in rate of growth and infectivity. Each susceptible insect species has a characteristic response which may be different from other species. Three insect species with different responses were therefore used bioassay. These were Lymantria dispar L., Loxostege striticalis $\mathrm{L}$., Aedes aegypty $\mathrm{L}$.. The $\boldsymbol{L} \boldsymbol{D}_{\mathrm{so}}$ values were determined for each morphovars by examining larval viability. Cross - antagonism berween different bacteria of each morphovars was found. This is at least indicative that bacteriocinogeny might play some ecological role.

Institute of Systematic and Animal Ecology, Frunze 11,

630039 Novosibirsk, Russia.

\section{BIOLOGICQL IHSECTICIDE FOR COLEOPTERA COHTROL}

Azizbekyan R.R., Minenkova I.B., Shagov E.H.

Insects belonging to the order Coleoptera are a the cost agronanically inportant pest. which danage potatoes. tomatnes. eggplants, cabbage etc. The losses of crop achieve to $20-30 \%$. For creation of ecological safe biological insecticide for control of Coleoptera larvae we use the original strain Bacillus thuringiensis. The active substance is protein endotoxin (erystalls). The strain synthesizes no heat-stable exotexin. The protein crystal is very active against different kind of beetle. hut nontoxic for Lepidopteran and Dipteran larvae. An insecticidal composition have formulated in a conventional canner using the nixture spores and crystals together with suitahle carriers, diluents, enulsifieres and dispersants. The Insecticide conposition have forsulated as a paste-like preparation, good soluble in water. "COLORABO" -is the Tradenane for a product. " COLORAFo" is econonically beneficially for producing and application. a field trials of "COLQRaDQ" uas conducted to prove the binlogical effect in Russia,Ukraina. Bellorussia during 5 years. The crop of potatoes, tonatoes, eggplants, cabbage were protected. The target pest is Colorado potato beetle. Lena. Phaedon , etc. Biological activity of "COLORADO" is $80-85 \%$. Depending on the population of insects harvest addition of crop achieve 25-30\%

Institute of Genetics and Selection of Industrial Microorganisus. 113545. Moscov b.1 1-st Borozhny pr.1. Russia
584

BACTERIOCINOGENY AND HYDROPHOBICITY OF BACILUUS THURINGIENSIS MORPHOVARIANTS AS PROTECIIVE FACIORS IN NATURE.

Kalmikova G.V., Burtzeva L.I.

Bacillus thuringiensis (Bt) based preparations are widely used to control the insect population. The biological activity of these insecticides is due to the combined action of parasporal crystals and spores. In addition spores introduced into environment are of ecological importance. Usually, the researches focus their attention on the preparation action and further spore existence in nature is out of the scope of their attention. One of the factors responsible for microorganism behavior in biogeocenosis is adhesion. The bacterial adhesion is mainly depend on hydrophobicity of the cell surface. The aim of this work is to compare the spore hydrophobicity of Bt ssp.kurstaki morphovariants and their antagonistic relations. The results showed the difference of cell surface hydrophobicity for the Bt ssp.kurstaki morphovariants. The composition of a sporulation medium considerably influenced the spore hydrophobicity. In the glucose-free medium strains of all but one morphovariants were hydrophilous (hydrophilous index $0 \%$ ) while in the yeast and glucose-containing medium this index has ranged within $0-65 \%$, depending on the morphovariant. We believe that different conditions of cultivation can change the synthesis of microbic metabolites which are able to influence the spore adhesion. The investigations of antagonistic relations between morphovariants have shown the strains of four morphotypes to produce bacteriocyne-like substances. Thus, when introducing Bt ssp.kurstaki populations, morphovariants with hydrophobic surface and capable at synthesis of antibacterial substances appear to have preservation advantages in nature.

Institute of Systematic and Animal Ecology, Frunze 11. 630091 Novosibirsk Russia. 
CONTROL OF EUROPEAN CORN BORER (OSTRINIA NUBILALIS HB LEPIDOPTERA PYRALIDAE) ON STRING-BEANS, BY MICROBIOLOGICAL INSECTICIDES BASED ON BACILLUS THURINGIENSIS

\section{G. M. Cuito, A. Pollini}

Larvae of European Corn Borer (O. nubilalis) damage frequently industrial string-bear crops in Northern Italy, eating the pods in the summertime (July - September). The plants are susceptibles to this insect from fruit-setting to harvest, about twenty days Notwithstanding food industries make the farmers to do a severe control programme with chemical insecticides (in general four treatments)

In 1993-1994 field trials were carried out to verify the efficacy of new insecticides based on B. thumgiensis, compared with chemicals and an unteatred control (U.T.C.). The experimental design was a randomized block with four replicates: each plot measured $30 \mathrm{sqm}$ in 1993 and $500 \mathrm{sqm}$ in 1994. Data were processed by ANOVA, and by Tukey multiple range test.

The beginning of protection programme was subordinate to the capture of adults in cone traps, baited with sex pheromone (strain "EZ") and phenylacetaldehyde (PAA). The damage was checked at the harvest as wormy pods percentage; this value in U.T.C. was about $3 \%$, however it is considered high by food industry, which rejects, in that case, all field production.

In 1993 three treatments were sprayed in the trial, and four ones in the crop. The insecticides compared were: B.th. v. kurstaki, strain SA11 (Delfin = Javelin); B.th. $v$. kurstaki, strain EG2348 (Rapax = Condor OF); B.th v. kurstaki, strain EG2371 (Lepinox $=$ Cutlass WP); B.th. v. kurstaki, strain HD1 (Biobit HPWP); B.th. v. aizawai (Centari); B.th. v. Kurstaki strain HD1 (Biobit XL); Teflubenzuron (Nomolt); Fluvalinate (Klartan); U.T.C. The best result was reached by Cutlass WP $(0.02 \%)$, followed by Klartan $(0.14 \%)$, Condor OF $(0.21 \%)$, Centari $(0.29 \%)$, Biobit XL $(0.36 \%)$ and Javelin $(0.37 \%)$ at the same statistical level, Biobit HPWP $(0.61 \%)$ and finally Nomolt $(1.87 \%)$ and UTC (2.5\%)

In 1994 two treatments were sprayed in the plots and four applications in the crop. The insecticides compared were: B.th. $v$. kurstaki, strain SA11 (Delfin = Javelin); B.th. $v$. kurstaki strain HD1 (Biobit HPWP) B th v kurstaki NN6058500 by Novo Nordisk- $B$ th v. kurstaki, strain EG2348 (Rapax = Condor OF) at $2.4 \mathrm{l} /$ ha and $1.8 \mathrm{l} / \mathrm{ha}$; Fluvalinate (Klartan); four treatments (two Acephate and two Deltamethrin) of crop defence programme; U.T.C. Four treatments got the best result $(0.11 \%)$, but also two applications with Klartan $(0.2 \%)$ were highly significant, then in decrescent order Javelin $(0.32 \%)$ and Biobit HPWP $(0.34 \%)$; Condor OF - 2.4 / $/$ ha $(0.51 \%)$ and NN6058500 (0.51\%); finaliy Condor OF $-1.8 \mathrm{l} / \mathrm{ha}(0.88 \%)$ and U.T.C. $(2.4 \%)$.

These trials confirm the possibility to carry out less treatments in string-beans crops, reaching the same outcomes, at the normal fevel of $O$. nubilalis infestation; the microbiological treatments are a very good alternative to chemicals; Fluvalinate is more effective than other chemicals in European Corn Borer control.

Giovanna Maria Curto, Aldo Pollini - Regione Emilia Romagna, Osservatorio Malattie' delle Piante (Plant Protection Service) - via Corticella n¹33, 40129 - Bologna (Italy)

587

BIOLOGICAL CONTROL OF BOTRYTIS CINEREA IN CUCUMBER AND TOMATO

\section{A.J: Dik, J. Köhl, N.J. Fokkema, Y. Elad \& D. Shtienberg}

Botrytis cinerea Pers; Fr. is an ubiquitous pathogen which causes severe losses in many fruit, vegetable and ornamental crops and which can be especially important in greentouse production. In tomato and cucumber, $B$. cinerea infects leaves, stems, flowers and fruits. In heated greenhouses, climate control can largely prevent infection of leaves, flowers and fruits, but stem infection leading to plant death is an important problem. In a collaborative project with researchers in the Volcani Center in Israel and IPO-DLO in The Netherlands, the possibilities for biological control of $B$. cinerea are the subject of research.

In Israel, bacteria and yeasts were selected on the basis of their effect on germination of $B$. cinerea conidia and germ tube elongation on leaves, disease severity, sporulation of the pathogen on leaves and stem segments and on disease of whole tomato plants. A few microorganisms were found to be at least as effective as the known biocontrol agent Trichoderma harzianum T39.

In The Netherlands, a bioassay was developed to test the effect of bacteria, yeasts and filamentous fungi on infection of $B$. cinerea on stem pieces and on sporulation of the pathogen. The most successful biocontrol agents were tested on wounds in greenhouse-grown cucumbers. All biological control agents were at least as successful as the standard fungicide treatment. Selected biocontrol agents are currently being tested under difterent climatic conditions in growth chambers and on whole cucumber plants in greenhouses with different climate settings.
COTTON PGPR AND MINOR PATHOGEN:ISOLATION,IDENTIFICATION AND THEIR INTERACTION IN THE RHIZOSPHERE

\section{Dan,H.,C.Wang and D.Wang}

The rhizosphere microorganisms can be divided into three groups according to their effects on plant growth:the beneficial(e.g.PGPR,Plant-Growth-Promoting-Rhizobacteria), the harmful(e.g.minor pathogen) and the neutral.We present here the first report of minor pathogen of cotton and the interaction between PGPR and the minor pathogen in situ.From 563 isolates from cotton rhizosphere,8 PGPR and 3 minor pathogens were selected and identified with aggressive root coloning ability. When treated as seed inocutant in pot experiment, the PGPR strains stimulated seed emergence(by 8-86\%), seedling growth(by 12 . $82 \%$ ) and reduced disease incidence of Rhizoctonia solani(by $37-88 \%$ ), and Fusarium oxysporum isp.vansinfectum(F.o.x) (by 13-71\%); 3 of 8 PGPR also caused growth promotion In wheat seedling(by 11-67), 1 of 8 PGPR caused inhibition effect on carrot seedling growth. In pot experiment the minor pathogens produced cotton seedling growth retardation along with hypycotyl twisted,root system brown-colored and root branches reduced. In the following field experiment, the PGPR stains increased the cotton fibre yield by $6-31 \%$, the disease controlling effect against R.solant and F.o.x. were $32-76 \%$ and $14-17 \%$, respectively, According to the results of 30 biochemical reaction and " $G+C$ " values, $P G P R$ were identified as Pseudomonas putida,P.fluorescens, Bacillus subtilis,B.cereus and Xanthomonas campestris; the minor pathogens were $B$. subtilis and P.fluorescens. In in vitro assays,PGPR strains inhibited or stimulated the growth of the taget pathogens and the minor pathogens. When co-inoculating PGPA and minor pathogens of cotton seeds with different combination, seedling growth could be either normal,stimulated or inhibited,suggesting that PGPR could partially or completely suppress minor pathogens in situ. The mechanism of disease suppresion by PGPR is under investigated. Preliminary results showed an antibiotic played the major role.

Department of Plant Protection,Huazhong Agricultural University, Wuhan 430070, China. Present address:Department of Plant Science, The University of Manitoba, Winnipeg R3T 2N2,Canada

The research was supported by IFS(International Foundation for Science,at Sweden) and NNSFC(National Natura! Science Foundation of China, at Beiling).

588

Burkholderia cegacia UPR 5C, a potential biofungicide for bean charcoal rot and southern blight in Puerto Rico

R. Echavez-Badel, A. Sanchez-Paniagua, R. Campo-Arana and E.C. Schroder. Department of Crop Protection, Univ. Puerto Rico, Mayaguez, Puerto Rico 00681-5000 USA.

Strain UPR 5C of Burkholderia cepacia isolated from Phaseolus vulgaris rhizosphere has been tested to protect bean against the charcoal rot (Macrophomina phaseolina) and southern blight (Sclerotium rolfsii). Bean seeds inoculated by dipping in a bacterial suspension of approximately $10^{\circ}$ cells $\mathrm{mL}^{-1}$ protected bean seedlings grown in a mixed soil infested with $M$. phaseolina and $S$. rolfsii. Significant differences $(P=0.05)$ for charcoal rot and southern blight severity infection between UPR 5C and the control (fungus alone) have and southern blight severity infection between UPR $5 \mathrm{C}$ and the control (fungus alone) have
been found. UPR $5 \mathrm{C}$ also increased plant survival and growth. This study suggests that possible mechanisms for the inhibition of fungi pathogens by UPR 5C could be production of volatile substances and/or antibiotics. 
New Microbial Preparation "Bioplant-K" Favorable for the Blological Protection of Plants.

\section{V.T. Emtsev}

We have been studying capacity of microorganisms in rnizocoenosis of veretable crops to thhibit the phy topathogenic fungi. Klebsiella planticola strain ISHA-91 got out of cucumber rhizoplane demonstrated high fungistatic act. ivity. The treatment of potatoes tubers with preparation "Bioplant-K" (made on the basis of Klebsiella planticola strain TSHA-91) under the conditions of field experiment in two times decreased desease of Rhizoctonia sp. potatoes and to a considerable extent increased effectiveness than the fungicide "Tecto-450". Studies of "Bioplant-K" treatment influence to the yields of vegetables showed
distinct positive effect: yield grew by $21-31 \%$.

Department of Microbiology, Timirjazey Agricultural Academy, Timir jazevskaja 50, 127550 Moscow, Russia

591

\section{BIOCONTROL OF ROOT ROT DISEASE OF CASUARINA EQUISETIFOLIA BY VAM FUNGI}

\author{
N. RAMAN AND S. ELUMALAI
}

Casuarina equisetifolio is a multipurpose tree having both mycorrhizal and actinorhizal association, is used in land reclamation, coastal vegetation development, saline soil improvement, mine spoils reclamation etc. In India C.equisetifolia has been introduced for several purposes. Our investigation reports the interesting disease root rot of C.equisetifolia which was controlled reports the inter

Seedlings were raised by surface sterilized seeds of C.equisetifolia, in nurseries. At different stages of growth of the seedlings occurrence of disease caused by Rhizoctonia solani was recorded in high incidence, on the diseased caused by Rhizoctonia solani was recorded in high incidence, on the diseased
seedlings. The seedlings affected with root rot showed yellowing of the foliage seedlings. The seedlings affected with root rot showed yellowing of the foliage cucumeris (Frank) Donk. was isolated consistently from all the diseased seedlings. The VAM fungi Glomus mosseae was mass multiplied on onion plants and were inoculated into the nursery seedlings of C.equisetifolia. After three months, VAM fungal inoculated seedlings showed increased amount of three months, VAM fungal inoculated seedlings showed increased amount of
chlorophyll, protein, soluble sugars, lipid, IAA, cytokinin and gibberellins. VAM fungus effectively controlled the root rot disease in C.equisetifolia.

\section{CENTRE FOR ADVANCED STUDIES IN BOTANY,} UNIVERSITY OF MADRAS, GUINDY CAMPUS MADRAS - 600 025, TAMIL NADU, INDIA
BIOCONTROL OF FUSARIUM WILT OF MUSKMELON WITH DIFFERENT FUNGAL APPLICATION METHODS

\author{
M.I. EL-Refaei, M.I. E. Barakat and O.Y. Shalaby
}

The causal organism was very resticted in pathogenicity. It was only parhogenic to muskmelon (Cucumis melo L.). whereas it was not pathogenic to other cucurbits as cucumber, squash and watermelon. Screening trials carried out for different fungal isolates to study their potential to control fusarium wilt of muskmelon. caused by Fusarium oxysporum f.sp. melonis. All fungal isolates showed different reactions with antagonistic effect in vitro and Vivo, the fungal isolates which revealed better, however, Talaromyces flavus and Trichoderma harzian um were the best to be used as antagonists in this study. Under greenhouse conditions, the two fungi were tested against Fusarium oxysporum f.sp. melonis with differnt application methods to control the disease. Soil infestation as an antagonists application revealed lower disease severity and the fungus was not found at the re-isolation process. Inoculum concentration of $5 \%(\mathrm{v} / \mathrm{v})$ for both antagonists and pathogen showed good effect in biological control study than $3 \%$ or $10 \%$. One week interval between the antagonists and causal pathogen application was doing well in minimizing wilt disease severity than other longer time. Peatmoss soil is the best for either Talaromyces flavus or Trichoderma harzianum in minimising disease severity and also seedling appear healthy with suitable plant height than other treatments. Level of $60 \%$ Waxal nutrient solution as a fertilization treatment indicate low amount of muskmelon wilt disease severity and the pathogen was not appeared in re-isolation trials.

M.I. EL-Refaei, Professor of plant pathology, college of Agriculture at Fayoum. Cairo University, Egypt

592

BIOLOGICAL WEED CONTROL USING RUST FUNGI

\section{J. Frantzenn and H. Müller-Schärer}

Rust fungi are evaluated on use for biological weed control. The evaluation is carried out by using an approach that considers various factors reducing the impact of pathogens on plant populations. These factors are, escape, avoidance, resistance, tolerance, and compensation. Compensation is considered within and between plants. The approach is applied to two weed pathosystems: Cirsium arvense (L.) Scop. - Puccinia punctiformis (Str.) Röhl, and Senecio vulgaris L. - Puccinia lagenophorae Cooke. Following this approach, escape and resistance have been demonstrated as factors reducing the impact of $\underline{P}$. punctiformis on populations of $\underline{C}$ arvense. Avoidance and tolerance could not be demonstrated as relevant factors. Compensation is suggested by the results as relevant factor. The research on

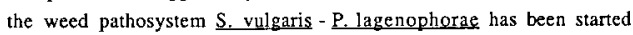
recently. First results suggest resistance as a factor that may reduce the impact of $\underline{P}$. lagenophorae on $\underline{S}$. vulgaris populations. Compensation between plants has been demonstrated as relevant factor in this pathosystem.

Research will be continued to estimate the relative meaning of each factor for reduction of the impact that a rust fungus may exert on a weed population. Subsequently, appropriate strategies might be developed to control weed populations by the rust fungi.

Botanical Institute, University of Fribourg,

3, rue Albert-Gockel, 1700-Fribourg, Switzerland 
THE DEVELOPMENT OF BIOLOGICAL CONTROL IN THE APPLE ORCHARDS OF THE LKRAINE

Victor FuRSOV

At present, environmental research, atudies of ecosyatem and systematic investigations of plant and animal taxa have interrelated closely. One of the taskg of modern agriculture is the effective protection of field crops from pesta and the elaboration of environmentally safe systems of IPM. Apple orchards are one of the moat heav1ly pesticide-treated crops in the ukraine. The optimization of peatictide use in the apple orchards has been developed for the lateat years in the ukraine on the basis of the research of complexes of entomophages. In the Ukraine the main apple pests are repregented by follar, frult-injuring and lea-mining insects. The jor pests of generative and vegetstive organs of an apple-trees belong to Lepidopters. The complex of Lepidoptera may be devided into two ecological groups: with hiddenly living (many families of Microlepidoptera) and freely living caterilliars (Geometridse, Noctuidae, Lasiocampldae and oth.). About 1000 species of parasitolds and predators are associated with thege phytophagous pests. in this complex the domi nant groups of parasitic Hymenoptera belong to Ichneumonidae, Braconidae, Eulophidae. In the apple orchards of the Ukraine the studied complex of parasitolds includes about 180 species from 67 geners of chalcidold wasps and 385 speciea from 117 genera of ichneumonold wasps. The research of specific diversity and trophic interactions of parasitoids, associated with the principal pest groups injuring generative and vegetative organs of an apple-trees in the Ukraine is in the progress now.

Institute of Zoology of Ukrainian Academy of Sciences, Kiev-30, Bogdan Khmelnitgkiy Street, 15, 252601, UKRAINE.

Fax: (044)-224-1568. E-ma11: entomoleizoology. kiev. ua

595

BIOLOGICAL CONTROL OF CROWN AND ROOT ROT OF SUGARBEETS CAUSED BY RHIZOCTORY SOEANI IN A DISEASE NURSERY

Halloin.J.M., Bugbee, W.M., and Theurer, J.C.

A disease nursery used for more than 20 years in evaluation of resistance of sugarbeets (Beta vulgaris L.) to crown and root rots caused by RHizoctonia solani (AG 2-2) in recent years has failed to produce disease suitably severe to discriminate resistant from partially resistant germplasms. The nursery employs a two year rotation between sugarbeets and alfalfa (Medicago sativa L.); and annually the sugarbeets are inoculated six weeks after planting by dispensing into their crowns, millet caryopses on which the fungus has been grown. Experiments were done to determine if biological control of the pathogen was responsible for low disease severity, Highly susceptible to highly resistant sugarbeet genotypes were planted in the established nursery and in an adjacent field with no prior history as a root rot disease nursery. In two of three years, the disease was more severe at the new sites than in the established nursery. Inoculated plants at the new sites also were more stunted. Biological control of Rhizoctonia seems a likely cause of the decreased disease severity in the established nursery. However, microbiological assays of soil from the sites revealed no consistent differences in populations of fungi, fluorescent pseudomonas spp., or actinonycetes. Isolates of actinomycetes and Trichoderma spp. inhibitory to $R$. solani have been obtained from the soil samples, but their role in the apparent biological control is unclear.

ARS, USDA, Department of Botany and Plant Pathology, Michigan State University, East Lansing, MI 48824, USA
USE AND DEVELOPMENT OF AN ENTOMOPATHOGENIC FUNGUS TO CONTROL HOPLOCHELUS MARGINAL IS (COLEOPTERA: SCARABAEIDAE), A MAJOR PEST OF SUGARCANE IN REUNION ISLAND

R. GOEBEL', 8.VERCAMBRE ${ }^{2}$

The soil pest Hoplochelus marginalis was introduced accidentaliy from Madagascar and the first damages in sugarcane crops were reported in Reunion in 1981 .

Now, this white grub is known as a major pest for the farmers and Now, this white grub is known as a major pest for the farmers and he is present on a large part of surgarcane areas, particu

Management strategies to control this pest have been focused since 1987 on the use of an entomopathogenic fungus beauveria since 1987 on the use of an entomopathogenic fungus Beauveria moronglartici, which procures a successful

The work on biological control and its involvement in farming systems and extension are discussed in this paper.

1 : CIRAD-CA, Station de la Bretagne

97487 Saint-Denis, ILE DE LA REUNION 
INTEGRATON OF BIOLOGICAL. AND CHEMICAL CONTROLS IN POTATO

G.E. Long. T.H. Ro. H.H. Tobo, T.F. Unrun. N.N. Kabalo, G XU

The green peach aphid (GPA), Myzus persicce (Sulzer) is a vector of over 50 plant virus diseases worktwide. In the northwestern USA it transmits pototo leofroll virus to commerclally grown potato, reducing yields and causing rejectlon of crops due to intemal disorders in infected tubers.

8iological control by parositoids hos not been effectiva h preventling sprecd of virus in potato fields, probably because low femperatures in pofoto growing areas hoven't allowed the rapid development of parasitism earty in the season. We hove adapted o strain of Aphelinus asychis, a parcsitold of cereal aphlds in France to attack GPA on potato in the state of Washington USA. This adapted strain is effectlve under low, early seasonal temperatures. But during midsummer, immigration rates of GPA into potato may reach 2.5M alatce per hectare per week. Unmanaged parasitoid population

Aphids flying into commercial potato fields may not require control meosures to limit the Aphids flying into commercial potato fields may not require control meosures to limit the
spread of potato leafroll virus until most of the colonzers hove landed, becausa winged 5pread of potato leafroll virus until most of the colonzers have landed, because winged
ophld colonizers may not carry virus early in their migratory fllghts. Potted potatces aphld colonizers may not carry virus early in their migratory flights. Potted poto tested as virus free by Polymerose Chain Reaction (PCR) and Enzyme-Lniked
immunasorbent Assay (ELSA) were exposed to colonzing aphids before, during, and Immunasorbent Assay (ELISA) were exposed to colonzing aphids before, during, and
just after migratory flights. Potaloes exposed prior to. or during the month-long intensive just after migratory flights. Potaloes exposed prior to. or during the month-long intensive
flight period cveraged only 3 percent infection. However, pototoes exposed after the flight period overaged only 3 percent infection. However, potatoes exposed aftert

A single application of a selectlve aphicide during the week following peck immlgration con kill most colontzing aphids, and will target those most likely to be corrylng virus. Even selective aphicides moy still kill free-living parcsitoids, and parasitoids in hosts that have not vet been mummified. Parcsitoids in mummified hasts are more protected, and

Tested on small research plots, early season inoculation with parasitoids in aphid murmmies, and non-virulferous wingless adult GPA Increased vields by $10.8 \%$ over control plots, and no increases in incidence of virus. Inis information can be used by growers and crop consultants to reduce the use of insecticides to limit potato leafroll
virus in commercial potato.

Department of Entomology. Washington State University. Pullman WA 99164-6382 USA

599

MORPHOLOGICAL, PHYSIOLOGICAL AND BIOCHEMICAL STUDY OF BACILLUS SUBTILIS - AN AGENT OF BIOLOGICAL CONTROL OF PLANTS

R.N.Mannanov, J.Safiyazov, R.K.Sattarova, D.U.Akhmedova.

In our investigation we studied the morphological, physiological and some biochemical properties of endophytic bacterium of genus Bacillus. Preliminary studies of influence of this strain on phytopathogenic pro- and eucariotic microorganisms in vitro have shown antimicrobal activity against agents of the main cotton diseases: Xanthomonas malvacearum, Rhizoctonia solani, Verticillium dahliae and Fusarium vasinfecturn. In field trials pre-sowing treatment of cotton seeds in cultural liquid of this strain has demonstrated decreasing of affection of plants by phytopathogenic microorganisms. It also substantially increased the per centage of seed germination and cotton yield.

The morphological and physiological analyses of culture have allowed to identify this strain as species Bacillus subtilis.

It is known, that many species of the genus Bacillus produce different antibiotics, which are synthesized through a ribosomal or non-ribosomal mechanisms. Some antibiotics such as tyrocidine, gramicidine, and bacitracin are synthesized nonribosomally by the multienzyme thiotemplate mechanism. Sufractin and mycobacillin are also synthesized non-ribosomally but by a mechanism that, apparently, is different from that of the multienzyme thiotemplate. Other antibiotics such as subtilin are gene encoded and ribosomally synthesized.

In our biochemical analyses the extraction and partial purification of antibjotic substanses have been made. The ninhydrin test of extracted antibiotic has given a positive result that confirms its peptide nature.

Future experiments will be attempted in hope of determining the structure of peptide antibiotic as well as for the further examination the mechanisms of antibiotic's action on cells of phytopathogens with a prospect of application in plant protection.

Institute of Microbiology,

Academy of Sciences of Republic Uzbekistan

7b. A. Kodiry str., 700128

Tashkent

Republic of Uzbekistan
SELECTION AND ACTION WECHANISM OF ANTAGONISTS AGAINST COTTON BOLL PIYYTOPHTHORA BLIGH?

Ma, Ping Li, Shezeng Chen, Xiobua Liu, Zengzhi

88 isolates of Trictoderas sp. and 103 isolates of bacteria were collected from Brodiag, Dingriog, Hengshui, Rongcheng, Beijing, Najing in 1993 sad 1994. The antagonistic aetivitg was sated in Petri disteg aganst Phytaptibora boetweriae, the pathogea caused cotton boll pot. According to their growth speeds 88 strains of Trichoderma were divided into four groups. 16 strains which belong to 18 a a 2 ad group growed very fast and encircled the colony of $P$. boeberias soonly inhibited the growth of the pathogen. Furthermore, they could grow on the colong of $P$, boebmerige and make the pathogen hypha weak. The antagosisom of Trichoderma was dominant on the competition of the space and cutrition with P. boebmeriag. Alother test showed that 4 of the 16 shraing could secset toxic oaterials which destroy the pathogen hyphe. And the inhibiting sreawere observed between Trictoderms sp. and $p$ boebmerias. The teating of 103 strains of bacteria showed that they secreted toxic substances to inbibit the gromth of P. boeboeriae ith very clearly inbibiting area. 103 strains of bacteria pere divided into groups by their inbibiting rates. The biossay of 8 strains of beteria with highly inhibitiog activity was carried out on the cotton bolls gaingt $P$. boebmerise. The results ahowed that 4 of the 8 straigs bad good relative contral effects (60-80\%). Meanhile the bioassay indicated that the relatire control effecta mete directly influenced by different application time. The control effect of sorgying the antagonitic bacteria and then innoculating?. Boebmerise 24 hours later was much higher than that of sprasing the bacteria and innoculating the pathogea imediately.

Institute of Plant Protection

Hebei Academy of Agricultural

sed Foreatry Sciences

73 East Pass Street

Baoding, Hebet 071000

P. R. China

600

BIOLOGICAL CONTROL OF EUROPEAN RED MITE PANONYCHUS ULMI (KOCH) (ACARINA, TETRANYCHIDAE) ON APPLE

T.K.VERESCHAGINA, V.A.MATSIUK As alternative to chemical control of European red mite on
apple the predatory mite Amblyseius californicus McGregor apple the predatory mite Amblyseius californicus McGregor
(Acarina, phytoseiidae) was tested in the Republic of Moldova and North Caucases region of Russia in 1991-1993. To receive large- scale detachments of predator mass reareing procedure was developed. Fredatory mite feed on two-spotted spider mite Tetranychus urticae (Koch), which in its turn is maintained on soybean. Soybean plants at the 2-leaf stage infested both prey soybean. Soybean plants at the 2-1eaf stage infested both prey and predator at the relation near 15:1. After $35-40$ days when the relation prey: predator change to $0,5: 1$ material is
for realese in the field. One could receive up to $35-40$

for realese in the field. One could receive up to $35-40$ thousand of predatory mite on $1 \mathrm{sq} m$ of unheated glasshouse in May-June. Amblyseius is released on apple trees on soybean plants, which cut off directly at the day of application. The rate changed from 25 to 35 thousand per 1 ha and the
of pests should not exceed 3 mites per leaf. There are of pests should not exceed 3 mites per leaf. There are
approximately 70 points of realising per 1 ha, i,e. $300-400$ predators on each treated tree. In all trials the effectiveness of Amblyseius was not less than $80 \%$. Especially high effect was received in 1992, when predator was released twicely in June 15 and 25, totally 25 thousand/ha in 10 year old; orchard on the area of 14 ha. At that time the density of Panonychus was $3.5 /$ leafe on the average. One month and a half ago the number of the pests on this plot has reduced to $0.6 / 1$ eaf. On the rest untreated orchard (near $100 \mathrm{ha}$ ) inspite of special spraying with omite $30 \mathrm{w}$ in the midale of July the number of red mite increased up to $61.5 / 1$ eafe. Due to serious damage the foliage untimely fold down in August. On treated plot the number of the overwintering eggs of Panonychus were less than 3 per $1 \mathrm{~m}$ of 2 year twig, while on the rest area reach to 40 one.

277060 V.Matsiuk, Cuza Voda 25/4, ap.25, Kishinev, Moldova. 
BIOLOGICAL CONTROL OF THE GRAPE MOTH LOBESIA BOTRANA DEN.

\section{V.A. MATSIUK, D.V. DERGACHOV}

Parasite Dibrachys cavus walk, (Hymenoptera, Pteromalidae) is of great importance in the grape noth number regulation. The experience of its application on vineyards in Krasnodar region of Russia and in the Republic of Moldova on the area of $5-7$ sufficiently high effectiveness against grape moth. This parasite usually is noted as secondary, but on some hosts it parasite usually is noted as secondary, but on some hosts it which pupae are protected more or less with hard covers (cocoon or chitin integument in Ely pupa). Under laboratory condit prepupae of the greated wax moth. It is possible Only 5-7\% of larvae have died during 6 months of storage. The parasite is moth is infested by parasite at the stage of the last instar application on grape consists in destruction of those pest's stages which define the density and harmfulness of subsequent generation. At least three releasing against each generation of grape moth are required. The releasing rate is 10 thousand per discovered on 100 bunch of grape. Each one additional larvae leads to increase of the rate on 1 thousand parasite

individuals. To distribute parasite evenly $20-30$ points of releasing per hectar are enough. At sumer the effectiveness of Dibrachys makes up $75-80 \%$, whereas usage in september reduces the overwintering generation on more than $80 \%$

277060 , V.Matsiuk, Cuza voda 25/4, ap. 25, Kishinev, Moldova. ET SCHIFF. (LEPIDOPTERA, TORTRICIDAE). develops as primary parasite. Female prefer to infest hosts
which pupae are protected more or less with hard covers (cocoon prepupae of the greated wax moth. It is possible to store the released at the pupa stage inside of laboratory host. Grape larva or one-day pupa. Because of this the tactic of parasite hectar when 10 or less grape moth larvae are

FUNGICIDAL PROPERTIES OF SOME TRICHODERMA SPP AGAINST MANGO STEM-END ROT PATHOGEN BOTRYODIPLODIA THEOBROMAE

Meah, H.B. and Begum, S.N.

Three Trichaderma spp. were tested for their antagonistic activities against Botryodiolodio theobromae cause of mango sten-end rot through inhibition zone technique in semi-solid media and extract inoculation with the pathogen. Clear zone of inhibition and no growth of the test pathogen in the extract was observed. The inocula, after 3 days to 1 week, were taken out of the extract and when placed in fresh culture medis failed to show any growth. The extract was evaporated to powder in water-bath. The powder dissolved in sterile water did not support the growth of $B$. theobromae. Powder was less effective than the extract. Powder, obtained through evaporation of the extract in flane when dissolved in sterile water suppressed the pathogen growth but did not kill it completely as it was indicated by growth of the pathogen on transfer to fresh aediun. This indicates a fungistatic action of the active principle due to partial loss of activity because of heating. The extract when kept at $600 \mathrm{C}$ for 5 minute killed the fungus but failed to do so when incubated at $100 \circ \mathrm{C}$. The $\mathrm{p}^{H}$ of the extract ranged from 5.0 to 7.5 which when nodified to $5.0-6.5$ did not affect the inhibitory action of the extract. The three species Trichoderms harzianun, $T$. koningii and $T$. hanatum exhibited differential inhibitory action. Preinoculation treatment of mature mangoes with powder prevented rot development while post-inoculation treatment was ineffective.

Professor, Department of Plant Pathology, Bangladesh Agricultural University, Mynensingh 2202. Bangladesh.

604

DIFFERENT APPROACHES IN BIOLOGICAL CONTROL OF PLANT DESEASES

Novikova I.I., Boikova I.V., Bikova G.A., Kalko G.V., Sergeyeva M.E.

liyertaeva S.N.

14 species of whiteflies (Homoptere, Aleyrodoldea) were revealed in Turkmenistan. Bemiaia tabaci Gennadius was noted here for the pirst time as pest of agricultural crops in open fields and in greenhouses at last years. Now chemical method ig the main method in crop protection against B.tabaci in Turkmentstan. Our investigations are devoted to natural enemies of cotton whitefly on esriculturel, ornamental and natural plants. For example, the revealing natural complex of entomophages of this whitefly in cotton fields includes 6 spectes of parasitoids and 10 species of predators. The perastolds are belonging to the families Aphelinidae ( 5 species) and Eulophldae ( 1 species) from the order Hymenoptera. The predators are belonging to the family Coccinellidae ( 5 species) from the order Coleoptera and to the families Viridae, Nabidae, Anthocoridae (5 species) from the order Hemiptere. In 1990-1991 were introduced into Turkmenistan from Uzbekistan parasitoidg Encargia formosa Gahan and E.Inaron Walker ageinst whiteflieg. But their effect in parag1tization Benisia tabacl was low. By our data, on egeplants Encarsia formosa infected 5,1-7,2\%, Eretmocerus mundus Mercet 92,8-94,9\% and Encarsia inaron infected not him, in splte of repeated production of this parasitoid. We produced E. mundus on eggplants and recelved average more than 1000 exemplares of paragit1zed pupae B.tabaci from one leaf. Artificial reservetions of this parasitoid can be created near greenhouses in sumer for following use E.mundus inside them to receive good effect by low flrst population density of pest and to reduce chemical treatments. Weasures for preservation of natural enemies in IFM will promote to rise of their effectiveness in the fields.

Institute of Zoology, Acadeny of Sciences of Turkmenistan 59 Azady Str., 744000 Ashgabat, Turkmenistan
There are two approaches in biological control of phytopathogens. They are appliing alive microorganisms for introduction to ecosystem (antagonists and mycoparasites) and using of antibiotics and other biologically active metabolites with the potential of either as fungicides or bactericides. The preparations of first type are intended for the making of specifical flavorable conditions for plants in the system host-phytopathogen-antagonist. Such preparations defend plants of different deseases using complex mechanisms of action containg influence on pathogen, plant and their microflore. The biopreparations of the second type are used for the supression of outbreaks of dangerous desease. Modern methods of screening. estimation of biological activity of antagonistic strains of microorganisms are working out at the All-Russian Institute for plant Protection. We investigate mechanisms of action of strains antagonists in the laboratory model systems and in tests fields. We have prepared some new biopreparations with the different type of action. Some of them - Alirin B. Alirin C - preparations for plant protection of soil-borne and other fungal pathogenes (Fusarium. Phytophthora, Oidium, Erysiphe etc.); Chrysomal and Globerine - metabolite biopreparations with complex effect (fungicidal and phytoregulatory): Gamair and Bioline antibacterial preparations with the high level of activity. We have made the technology of production and employment. The continuation of the work in this direction and making a wider variety of ecological harmless remedies for plant protection against deseases will make it possible to keep the structure of agrobiocenosis and their stability.

Novikova I.I. Institute for Plant Protection. Podbelsky sh.. 3. St.Petersburg - Pushkin, 189620, Russia. 
ON-STATION EVALUATION OF BACILLUS THURINGIENS IS FOR THE LNTEGRATED CONTROL GE CHILO PARTELLUS AND C. ORICHAICOCILIELLUS ON ELITE MAIZE AND SORGHLY

M.O. Odindo, A.M. Nour, S.o. Ajala, and K. Ampong-Nyarko

Bacillus thuringiensis var. kurstaki was evaluated on elite sorghum and maize gentoypes in order to deternine the prospects for its utilization in the management of insect pests in small scale farms. The pathogen was fermented in a basic medium of soybean, molasses, and dihydrogen sodium phosphate, and sprayed on 10 maize and 22 sorghum genotypes in 6 sites in the coastal region of Kenya. The sites had been selected on the basis of their variation in soll type, level and distribution of rainfall, vegetation
type, and other physical and climatic factors. The study sites were also selected as points for introduction of sorghum, a hitherto alien crop in the region.

All the plots had a natural infestation of mixed populations of Chilo partellus and C. orichalcociliellus. A $2 \%$ pathogen suspension was applied directly into the leafwhorl at the rate of $501 /$ ha, using a 51 shoulder-held sprayer. All malze and sorghum genotypes were treated at 3 weeks atter plant emergence (WAE) in one of three strips. The second strip was treated with a chemical insecticide (Furadan, carbofuran) applied as 5: granular formulation, at the rate of $9 \mathrm{~kg}$ per ha. The third strip was not treated (1nfested control plots). The level of follar damage was recorded at 5 WAE. All plots were harvested at 15 WAE and the yield of various crop genotypes and treatments assessed. Further, a random sample of 15 plants was taken from each plot, dissected, and records taken on tunnel length, infestat
entry/emergence holes.

Folfar application of $B$. thuringiensis reduced borer infestation on Foliar application of B. thuringiensis reduced borer infestation on
the sorghum and maize genotypes tested In all areas where c. partel.lus/C. orichalcociliellus were prevalent. Up to $17,4 \%$ increase in yield was recorded in sites of high borer incidence. Proportion of plants showing
foliar damage, or deadheart, was 29.4 and $1.2 \%$ in B. thuringlensis-treated plants compared to 31.5 and $2.1 \%$ in the non-sprayed control plots

respectively. Insecticide-treated plots gave higher yield in some sites, although there was no significant difference between insecticide- and pathogen-treated plots in others. B. thuringiensis was therefore shown to be a viable alternative for the control of cereal sten borers in the tropics.

The International Centre of Insect Physiology and Ecology, P.o. Box 30 MBITA, Kenya.

607

EFFECTIVENESS OF THE, ENTOMOPATHOGEN BE,AUVERIA BASSIANA IN THE CONTROL OF THE COFFEE BERRY BORER IN CHIAPAS, MEXICO.

V. Diaz-Vicente, V. M. Pinto and I. Mendez-Lopez

The coffee berry borer Hypothenemus hampei Ferr, (Coleop: Scolytidae) is present in Mexico since 1978, and has become the main insect pest of the crop. Presently, this insect is distributed in more than 115 , 000 ha. of the principal coffee growing areas of the country. Its control has been made, basically, by the use of the endosulfan insecticlde. However, reports from other countries about the coffee berry borer resistance to this compound, has forced to look for another alternatives of control. Thus, the present research work was carried out to evaluate, under fleld conditions, the effectiveness of different conidal concentrations of the entomopathogenic fungus Beauveria bassiana (Vals.) Vull1, alone or mixed with copper oxichloride which is used for the control of coffee rust, Hemileia vastatrix, also present in Mexico.

The $B$. bassiana was obtained from Ecuador and was multiplied in the laboratories of the experimental station of Rosario Izapa, Chiapas, using wheat bran as a substrate. The fungus was applied from May to June, 1992, on coffee trees of cultivar Garnica in farms of Cacahoatan county from Chlapas state in southern Mexico.

The following ten treatments were evaluated: 500, 1000, 1500 and $2000 \mathrm{ppm}$ of B. bassiana aione, and 500, 1000, 1500 and $2000 \mathrm{ppm}$ of $B$. bassiana plus $6.6 \mathrm{gr}$ of copper oxichloride; endosulfan and the control. The readings of the coffee berry borer mortality percentages were made $5,15,25,35$ and 45 days after applications, taking as a sample 100 coffee fruits which was disected to observe alive and dead borers. The results showed that the best treatment was the endosulfan insecticide w1th 88,2 mean percentage of mortality, following by $B$. bassiana $2000 \mathrm{ppm}$ alone, with a mortality of $62.4 \%$ Statistically, the fungal treatments showed no difference among them but there was difference with respect to the insecticide and control. with which the lowest percentage of mortality was obtained $(18.8 \%$.

Depto. Parasitologia-UACH. Chapingo, Mex. 56230. MExiCO
BIOCONTROL OF A DAMPING-OFF OF TOMATO BY BACILLUS SUBTILIS NB22

\section{N. Orihara, T.Ano, M.Shoda}

Bacilus subtilis NB22 which was isolated from compost as a producer of antifugal antibiotic iturin suppresses the growth of various plant pathogens not only in vitro but also in vivo and is expected to be as a potential biological control agent.

In this study, the suppressive mechanism was analyzed by the follwing experiments using a pot test of tomato and a fungal pathogen of Rhizoctonia solani which causes a damping-off of tomato.

Vegetative cells of NB22 were introduced into a soil, but the viable cell number in soil declined quickly. However, when the spores of NB22 were inoculated into a soil, the spores of NB22 is a critical factor for the biological control by NB22. When the cells of NB22 was recovered from soil samples of the roots of tomatoes after the inoculation of spores of NB22, about $30 \%$ of the recovered NB22 was found to be vegetatibe cells, indicating that the suppressive effect by NB22 is due to the produciton of iturin in soil by vegetative cells of NB22.

An iturin non-producing mutant, N $\triangle 2$ was derived from wild NB22 by genetic manipulation. Occurrence of the plant disease between $\mathrm{NB} 22$ and $\mathrm{N} \Delta 2$ is expected to be different due to the different productivity of iturin between the two. The plant test on pots is under progress.

From these experiments, the evidence that iturin is closely associated with the suppressive effect in soil will be demonstrated.

Kanagawa Horticultural Experimental Station

1217 Ninomiya, Ninomiya-machi, Naka-gun,

Kanagawa-ken, 259-01 JAPAN

FUNGAL PATHOGENS AND SUGAR ESTERS AS ALTERNATIVES TO CHEMICAL CONTROL OF PEAR PSYLLA (PSYLLIDAE: HOMOPTERA).

\section{G. J. Puterka}

The pear psylla, Cacopsylla pyricola Foerster, and related species are serious pests throughout the pear growing regions of the world. Extensive use of insecticides in the United States has caused this pest to develop resistance to most classes of insecticides. Consequently, there is an immediate need for developing alternatives to insecticides for pear psylla control. Research on two biological agents, fungal pathogens and a sugar ester extracted from Nicotiana gossej Domin, was conducted to determine if they were viable components of a pear IPM program aimed at pesticide reduction.

Two fungal pathogens, Beauveria bassiana and Paecilomyces fumosoroseus, showed the greatest potential for control of pear psylla in laboratory bioassays. Applications of conidia at rates equivalent to $2.5 \times 10^{13}$ spores/hectare were evaluated in 1993 and 1994 . In May 1993, applying fungal spores in $1 \%$ Sunoil obtained 25 and $40 \%$ nymphal mortality for $B$. bassiana and $P$. fumosoroseus respectively, 5 days after application. In September 1993, 1\% Sunoil was reduced to $0.1 \%$ because of some slight phytotoxicity problems. Treatments obtained 46 and $36 \%$ control for $B$. bassiana and $P$. fumosoroseus, respectively, 7 days after application. In the June 1994, another trial using both pathogens formulated in $0.5 \%$ Sunoil obtained 38 and $23 \%$ control for B. bassiana and $P$. fumosoroseus, respectively, 7 days after application. These tests indicated that B. bassiana was obtaining $\mathrm{ca}$. $10 \%$ higher mortalities than $\mathrm{C}$. fumosoroseus. Further research is being conducted on spore concentration, formulation, and multiple applications to determine if greater control can be achieved.

Sugar-esters extracted from the leaf trichomes of wild tobacco, N. gossej, have previously been shown to be active against various soft bodied insects. Laboratory studies indicated that this compound had insecticidal activity against pear psylla nymphs and adults at concentrations of $0.1 \%$ in aqueous solution. Field tests conducted in Washington and West Virginia orchards in 1993 and 1994 found that applications of $0.1 \%$ sugar ester obtained $70-85 \%$ control while $0.15 \%$ sugar ester obtained $80-95 \%$ control. Control with this compound exceeded those obtained by insecticidal soaps or chemical insecticides applied at recommended rates.

Both of these biological agents show great promise as alternatives to chemical control of pear psylla. These biological agents place in an IPM program for pear will greatly depend or their selectivity. Moderate levels of control between 40$70 \%$ could be acceptable if natural insect enemies are spared. Furthermore, these biological agents would be used in a multifaceted IPM approach instead of used singly. Integrating biological agents such as these, along with "soft" pesticide programs, will be a more effective biorational approach than using broad-spectrum chemical pesticides who's continued use eventually becomes ineffective against pear psylla. 
Aphids are injurious pests of vegetables and ornamental crops in greenhouses. Among the Hyzus persicae Sulz. and Aphis gossypil Glov. are the wost harmful. Blological potential of these aphids is rather nigh. It is recorded aphid resistance to insecticides. That's why the problem of biological control of these pests is very urgent. After studying of many parasites from families Aphididae and Aphllinidae for a long time wo chose two endoparasites Aphidius matricariae Hal. against Hyzus persicae and Iysiphlebus testacelpes Cres. agalngt Aphis gossypii. The former is indigenous species, the second is introduced from Mexico to Moldova.

we worked out the original method of mass rasing of these parasites on an alternative host, Schizaphis gramina Rond. The technology of mass rearing is very simple and cheap. Iife tables have been composed under different temperatures: from 15 up to $30^{\circ} \mathrm{c}$. The op timum temperatures for mass rearing of A.matricariae and L.testacipes are 25 and $20^{\circ} \mathrm{C}$ respectively. Their biotic potentials are highest possible in these condi tions. lass rearing of parasites on greenbug for a long time does not deteriorate their biological indices. These parasites are considered as the most effective ones among aphidophages tried before. They can reproduce successfuliy under greenhouse conditions and give comp lete control of aphids up to their suppression. A.mat ricarize and L.testaceipes are applied in big commercial glasshouses on tomato, cucumber, swet pepper in Moldova, Ukrain, Russia in 1986-1994.

\section{B.S. Shiyko}

Laboratory of IPU, Institute of Blological Plant Prot. P.Box.427, ML $277049 \mathrm{Kishinev,Moldova,Phone} \mathrm{(3732)} \mathrm{570-474.}$

611 NEW DATA ON THE PATHOGENICITY OF MRCROSPORIDIA NOSEMA GRYLII: IN FAVOUR OF ITS APPLICATION IN THE BIOLOGICAL CONTROI

\section{J. Sokolova , V.Dolgikh, O.Antonova \& I.Issi}

Investigations of interactions between the microsporidia Nosema grylli (Microsporidia, Nosematidae) and its host the cricket Gryllus bimaculatus (Orthoptera, Gryllidae) revealed several features of pathogenicity, that makes Nosema grylli prospective in biological control against Orthoptera. (1) The invasion with microsporidia retards the development of crickets and leads badly infected crickets to death. (2) It also prevents the maturation of female gonads. Heavily infected females are uncapable to oviposit. Microsporidia seem to influence on the female sexual system through the hormonal control or by the general reducing of the energetic resourses of the host organism. Gonads themselves do not contain parasites (fat body is the prevalent site of $\mathrm{N}$.grylli development). In cases of lower infection of crickets the oviposition is possible, but it is reduced considerably. In few cases the transovarial transmission of $\mathrm{N}$ grylli had been marked. The lowering of the fecundity together with 1ransovarial transmission makes this microsporidia especially promising as a long term microbiological control agent. (3) The infected fat body is strongly hypertrophied due to intensive spore formation in its cells. Crickets are easily bred in laboratory and thus are ideally suited for the mass production of microsporidia spores. (4) Locusta migratoria migratorioides was susceptible to the oral infection with N.grylli. Mature spores were revealed in locust fat bodies three weeks after experimental infection, though a really severe pathological effect on the locusts hadn't been observed. The infection also prevented the hatching from normally laid eggs. We suppose that the pathogenicity N grylli can be increased by application of spores in earlier stage of locust development. The works in this direction are in progress. The research is supported by Russian Foundation of Fundamental Sciences (94-04-12972).

I) .Sokolova Julia, Iaboratory of microbiological control, All-Russian Institute for Plant Protection, Shosse Podbelskogo,3, St.Petersburg-Pushkin, 189620, Russia.
The sugar-beet nematode Heterodera schachti i Schm. was firstly discovered in Ukraine even in 1936. Now it is widely spreading all over the country. The highest invasion level of this parasite is in the fields of seed-breeding farms in Vinnitsa. Kiev, Tcherkassy, Zhitomir and Tchernigov regions. It reaches approximately 3000 juveni les and eggs in $100 \mathrm{~cm} 3$ of soll, the losses of yield are 52-90 centners/ha $(20-30 \%)$; of seeds 2-10 centners/ha (20-50\%).

The inclusion of rape and oil radish which are good hostplants to sugar-beet crop rotations increased the nematode population density. However, the strong time limited "catch" sowing of these cultures (Jess than 45 days) can be used in nematode control. One and two times growing of rape (after the winter wheat and peas) reduced invas! on level to $47-64 \%$ and $53-69 \%$ respectively; of oll radtsh - to $88 \%$ and $77 \%$ respectively. The "catch" sowing of resistant to nematode German varjeties of mustard "Martigena" and ofl radish "Remonta" reduced the population density of Heterodera schachtif to 9598\%. Potato cyst nematode Globodera rostochlensis Woll. was firstjy found in Ukraine in 1963. Now it is spreading in 11 from 14 potato-seed regions on the areas approximately 6000 ha. More than $80 \%$ of these lands are private fields.

The growing of resistant and tolerant to nematode potato var 1 eties, which are giving good yield on the infested solls and cleaning them from cysts is only beginning in Ukraine. There were bred 44 hybrids with partial resistance to Globodera rostochiensis. Four Ukraintan varieties - Prolisok. Vodograd, Bereginja, Dobrotchin were effective in biological control of nematodes. They cleaned the soil from cysts to 42-95\%; German varieties Pamir, Karlena, Minerva, Likaria - to 54-99\%.

252022 UKRAINE, Kiev-22, Vasilkovskaya str. 33.

Institute of Plant Protection. Laboratory of Nematology.

612

CHAETOMIUM AS A BIOCONTROL AGENT AGAINST PLANT PATHOGENS.

Kasem Soytong Faculty of Agricultural Technology, King Mongkut's Institute of Technology Ladkrabang, Bangkok 10520, Thailand. Ifs research project No. c/1552-2.

The techniques for delivery of biocontrol agents have been employed. The specific effective strains of Chaetomium cupreum KMIT-N 4320 and KMIT 3003 and Chaetomium globosum KMIT-N 0802 were separately formulated into 2-forms as biopellets and blopowder. The biopellets were formulated with sodium alginate which formed in round shape with the size of $3 \mathrm{~mm}$. diameter after dried overnight. Tests showed that the pellets formation with Calcium salt could be survived more than 2-years as the biopowder. Inoculum strategies testing for disease and pathoger suppression in pot experiments showed that the biopeliets and blopoweder produced from Chaetomium have played a role for efther pathogen or disease suppression or both tn controlling Fusartum witt of tomato. In the fleld tralls, the pellets and powder forms of blocontrol agents were tested its effect in different environmental condition in the Northeast, East and Central Parts of Thailand to prevent tomato wilt caused by Fusarium oxysporum f. sp. 1ycopercisi and basal stem rot of corn which caused by Sclerotium rolfsil . Results showed that all tested biopellets and blopowder have significantly reduced tomato wilt and basal atem rot of corn at all tested locations as effective as chemical treated ones when compared with the controls. It was shown that Chaetomium treated-plant with any forms of bloproducts improved stands and yields over the non-treated one at all tested locations. This project has cleary shown a practical method of maintaining the biocontrol potential enhancing their applicability in the field conditions. The other seriously diseases like root and stem rot of Duriun and Citrus, anthracnose of Mango and Citrus are belng done in progress by using the another specific strains of Chaetomium produced in pellets which is being tested in the fields will be reported. 
INTERRELATIONSHIPS OF SCLEROTINIA BULBORUM ON TULIPS WITH GLIOCLADIUM CATENULATUM, O. VIRENS AND TRICHODERMA LIGNORUM

O.B.Tkschenke, J.M.Plotnikova, T.P.Alekseeva

The purpose of this work was to test our isolates of ollocladiun catenulatum and $G$. virens which were effective against some potato tuber pathogens: Fusarium sambucinum, Phoms exigus var exigua, Geotrichum candidum, Alternaris alternacs, and Fusariumspp. (Alekseeva et al., 1992) as a biocontrol agent against sclerotinia bulborum (Wakk,) Sacc, in comparison with untreated soil, commercial Trichodermin and Baitan. The antagonists propagated on wheat grains and in suspension were applied in soil around tulip base at planting. G. catenulatum str. 453 reduced tulip black slime incidence. Amount of diseased tulip plants decreased after G. catenulatum propagule application on wheat grains and in suspension in 1.5 an 2.5 times, respectively, compared with tulip cultivated on untreated soil. Trichodermin was not effective. Baitan was in 1.2 times more offective than $G$. catenulatum. Tulip planting and overwintering occured at unfavourable temperatures for antagonist preventing its development. We suggested that $G$. catenulatym had a fungistatic effect on $s$. bulborum and at summer application $\underline{G}$. satenulatumwill be more effective and might control s. bulborum development.

Dep. Plant Protection, Main Botanical Garden, Russian Academy of Sciences, Botanicheskaya, 4, Moscow 127276, Russia

615

BIOLOGICAL CONTROL OF MITES IN AUSTRALIAN VITICULTURE: IMPLEMENTATION AND SUSTAINABILITY

Jennifer Whitney and David G. James

By comparison with the rest of the world, Australian viticulture presents an agroecosystem which is essentially stable. Research conducted since 1989, has shown that biological control using indigenous predatory mites (Phytoseiidae), is a practical management option in most Australian viticultural regions. The dominant Phytoseiids (from a fauna of approximately 25 species) are Typhlodromus doreenae Schicha, Typhlodromus dossei Schicha and Amblyseius victoriensis (Womersley). These predators prey on the grapevine mite pests, Bunch mite (Brevipalpus spp), Blister mite (Colomerus vitis (Pagenstecher)) and Rust mite (Calepitrimerus vitis (Nalepa)). All three phytoseiids are generalist predators. $T$. doreenae is a particularly effective predator due to its tolerance to a variety of viticultural pesticides, its climatic habitat range and the fact that it remains on the vine year-round, overwintering on bark and canes. These features are all key factors to the success of this system. Sustainability of this natural system can be further enhanced, by developing regional control strategies, cultural and management practises and an effective mass rearing technique for future commercialisation. Research in these areas has been encouraging to date and is on schedule for completion in September, 1996.

Yanco Agricultural Institute, New South Wales Agriculnure, PMB, Yanco, New South Wales 2703 Australia
EVALUATION OF TAENTOLELLA PHIALOSPERMA AS BIOCONTROL AGENT AGAINST THE SOILBORNE PLANT DISEASES CAUSED BY PYTHIUM APHANIDERMATUM AND DEMATOFHORA NECARIX

T. Watanabe

Potential antagonists against damping-off of cucumber seedlings caused by Pythium aphanidermatum and white root disease of Japanese black pine seedlings by Dematophora necatrix were selected among six new fungal species assayed. Taeriolella phialosperma was found most effective as a biocontrol agent. These biocontrol agents were evaluated for their effects on the emergence and health of seedlings grown in the soil over the co-cultures of pathogen and antagonist in plates (soil-over-co-culture-inoculation method), or by sowing the seeds soaked in the spore suspensions of the antagonist in the artificially infested soils, Biocontrols of the soilborne plant diseases by this fungus were successful in six separate experiments in this study. Forestry and Forest Products Research Institute, P.o.Box 16. Tsukuba Science City, Ibaraki 305, Japan.

616

STUDIES ON EFFICACY OF GRANULOSIS VIRUS IN BIOLOGICAL CONTROL OF CODLING MOTH (CARPOCAPSA POMONELLA L.) IN POLAND

\section{J. Ziemnicka, T. Badowska-Czubik, W.A. Kanapackaja, I.T. Korol}

The viral insecticide based on granulosis virus Virin-Gjap (from Belorusian Institute of Plant Protection, Miñsk) was tested in laboratory of Instjtute of Plant Protection in Poznañ and in experimental orchard of institute of Pomology and Floriculture in Skierniewice. The first treatment was conducted at the stage of "black head", the second after 10 days and next after 21 days (from the first treatment) at the following concentrations: 1.8 $\times 10^{9}, 9 \times 10^{9}\left(10^{12} \mathrm{GV} / \mathrm{ha}\right)$ and $2.7 \times 10^{10} \mathrm{GV}$ per tree $\left(10^{13} \mathrm{GV} / \mathrm{ha}\right)$. Efficacy of the viral insecticide was compared with the chemical Owadofos pl 50 (fenitrotion 50 ) $0.15 \%$ and unsprayded control. The effectiveness of Virin-Gjap was estimated in both picked crop and drops. Degree of the fruit damage was determined upon the analysis of shallow entry and deep entry.

Our results confirmed previous studies. The best efficacy of Virin-Gjap bioinsecticide in codling moth control in Polish orchards was acquired by three times treatment at the virus concentration not lower than $10^{10} \mathrm{GV}$ per tree $\left(10^{13} \mathrm{GV} / \mathrm{ha}\right)$

Institute of Plant Protection, Miczurina 20, 60-318 Poznañ (Poland) 
Trichograma SPECIES (HYMENOPTERA, TRICHOGRAMMATIDRE) USED IN BIOLOGTCAL CONTROL PROGRAM IN BRAZIL.

\section{R. Z. Zucchi. J.R.F. Parra, R.C. Monteiro}

Fourteen species of Trichogramma are recorded in Brazil. However. Only two species (T. pretiosum Riley, 1879 and $T$. Galloi zucchi, 1988) are being curxenty used in biological control programs of agricultural pests in Bxazil. Both species are being studied in a long term project of the Department of Entomology (ESALQ) at University of São Páulo. T. pretiosum is the most conmon species in Brazil. It das been reported from eggs of 10 host insect species on eight crops. $T$. pretiosum natural parasitism can reach up to $98 \%$ and $89 \%$ in the last generations of Alabana argillacea (Hübner) and Heliothis virescens (Fabr.) respectively, on cotton crop, even after the application of wide spectrum insecticides. Also, its natural parasitism is high for Anticarsia gemmatalis Hủbner, and experimental results have shown the efficiency of $T$. pretiosum to control Helicoverpa zea (Boddie) on corn. However, this parasitoid is not efficient against spodoptera frugiperda (J.E. Smith), because its eggs are laid in overlapping layers and covered with scales from abdoner of ovipositing fenale. In the Nor theast region of Brazil, $T$. pretiosum is being used to control scrobipalpuloides absoluta (Meyrick) on tomato crops. As such, it is associated with okher biological agents and released on area of 1,500 ha. T. galloi only has been collected from eggs of sugarcane borer Diatraea saccharalis (Fabr,), Eor which it is the most common egs pazasitoid in Brazil. Based on experimental tests, $J$. galloi has shown to be efficient. when 200,000 individuals/ha are released in the beginning of the sugarcane borer egg population.It was originally reported from Brazil, but it occurs also in Uruguay and Bolivia. Besides these two species, other Brazilian species can be considered as potencial biological control agents, due to the high natural parasitism observed, specially on forest pests. For example, T. maxacalii voegele \& Pointel, 1980 has been collected exclusively from eggs of Euselasia sp. (Lep., Riodinidae) on Eucalyptus sp., in which the natural levels of parasitism reach 908 and parasitoid emergence is approximately $100 \%$.

Research supported by FBB, FINEP, CNPq and FAPESP

Departamento de Entomologia, ESALQ/usp, Caixa Postal 9. 13418-900 Piracicaba, SP. Btasil.

CONSTRUCTION OF A COMBINED ACTION BIOINSECTICIDE PRODUCER ACTIVE AGAINST COLORADO POTATO BEETLE

\section{A.P. Dobritsa}

Colorado potato beetle is one of the major pests of potato and other Solanaceae. For biological control of this pest, formulations on the basis of the entomopathogenic bacterium Bacillus thuringiensis (Bt) are mainly used. Commercial Bt-based bioinsecticides which are used to control Colorado potato beetle may be divided into two groups: those based on strains producing $\beta$-exotoxin ("Bitoxibacillin" and others) and those based on strains producing Coleoptera-specific $\delta$-endotoxin ("Novodor" and others). The toxins have different mechanisms of action; therefore, construction of a Bt strain able to produce both toxins might result is obtaining a bioinsecticide of combined action. The advantages of such preparations would be their higher toxic activity and minimization of the risk of arising of Bt-resistant pests. Bt entomotoxin genes are often located on conjugative plasmids and thus may be transferred between $\mathrm{Bt}$ strains by conjugation. However, it has turned out that it is difficult to combine the $\beta$-exotoxin and insecticidal CryIII-protein determinants in one genome by this way, therefore another approach has been proposed. The latter includes the following steps: construction of transposable elements harboring cry genes, integration of these elements into conjugative plasmids and transfer of the modified plasmids into Bt recipient strains. To realize the approach, a recombinant plasmid, pTV241, containing the cryIIIA gene within the transposon Tn917 was constructed and introduced by electroporation into a number of Bt strains, including Bt subsp. thuringiensis Berliner 1715, subsp. kurstaki HD1 and subsp. morrisoni DC. Transformants of the three strains were mated with a plasmidless Bacillus cereus strain and $B$. cereus clones carrying the plasmids modified as a result of Tn917-cryIILA transposition were selected. The plasmids are conjugative and a $50-\mathrm{kb}$ plasmid originated from Bt subsp. morrisoni $D C$ was transferred by conjugation to Bt subsp. thuringiensis 98 (Exo+Cry-). The plasmid is maintained in transconjugant clones with high stability, so that $98-99 \%$ of the cells harbor the plasmid after 100 generations. Insecticidal activity of the clones producing both Coleoptera-specific $\delta$-endotoxin and $\beta$-exotoxin is $3-4$ times higher than that of producers of "Bitoxibacillin" and "Novodor".
ENHANCING THE BIOCONTROL ABILITY OF TRICHODERMA 5PP, AGAINST SCLEROTIUM ROLFST

SUSEELENDRA DESAT* E. SCHL TSER In the light of increasinc TRINSCIOUSNESS To THE ENVIRONMENT" managing the soll-boxne pathoyens against which host reststance is rarely available and the pesticides recommended are required in large quantities which lead to the problems of environmental pollution and quantities which lead to the problems of environmental pollution and 60 years agr, trichoderma as a commercial biocontrol agent is still under-deployed due to lack of an integrated research approach, industrial support, and inherent problems of the biocontrol agents. The tools of modern molecular biology have given an opporturity to genetically Improve the stralns of the biocontrol agents to pool together desirable characters such as more than one mode of parasitization, resistance to seed dressing fungicides, adaptability to different agro-ecological zones, longer shelf-life, and rhizosphere competence if, they are heritable. For this purpose, protocols were developed for introducing marker genes into desirable parental strains, protoplast fusion, and carrier systems for easy and better transfer of biocontrol agent from laboratory to fleld. Isolates of Trichoderma spp. were systematically catalogued for their modes of biocontrol against Sclerotium rolfsil, stably herltable marker characters, and other desirable tralts. Out of 44 1solates. nine were effectlve in dual culture, three produced volatiles, one was reslstant to benomyl, one had rhlzosphere competence. three produced non-valatiles, 19 macerated and killed sclerotia. and 19 inhibited Botryts clnerea also. The growth of 5 .rolfsil was inhibited only in contact with the volatile substances. $\frac{\text { Scanning electron }}{4}$ microscopy reveoled that $\underline{T}$.harzianum could penetrate the sclerotial bodies and proliferated inside.

Hygromycin or phloeomych resistance genes available on plasmid constructs PAN7-1 and PANB-1, respectively, were Introduced through electroporation into the spore suspensions of parental stralns. The strength of $10 \mathrm{kV} . \mathrm{cm}$, $3 \mu \mathrm{F}$ capacitance, and 400 ohms zesistance. Protoplasts of Trichoderma spp. Were isolated by incubating ioh old Protoplasts of Trichoderma $5 \mathrm{pp}$. Were 15olated by incubating loh old mycelium in Novozym234 $16 \mathrm{mg} \cdot \mathrm{ml}$, for $4 \mathrm{~h}$, purifled and regererated. Benomyl resistant and hygromycin resistant parental protoplasts were aligned by a current of $1 \mathrm{MHz}$ frequency against the electrodes for 30
sec. Immediately after, 5 pulses each of $4.1 \mathrm{kV}, \mathrm{cm}$ field strength for sec. Irnmediately after, 5 pulses each of $4.1 \mathrm{kV}$.cm field strength for 75 Msec at 1 min intervals were given
tsolated and regenerated successfully. Pelletization in $1 \%$ alginate + $1 \%$ clay or $1 \%$ alginate $+1 \%$ clay $+1 \%$
wheat bran followed by an overnight treatment of the pellets in PEC4000 wheation retained the viability of Trichoderma for more than 90 days.

* National Research Centre for Groundnut, P.B. S, Ivragar Road. JUNAGADH - 362 O01, Gujarat, INOIA

620

BIOCONTROL. POTENTIAL OF NEW, GENETICALLY ALTERED STRAINS OF AN ENTOMOPATHOGENIC NEMATODE, Steinernema feftiae.

\section{Tomalak}

Artificial selection, strain hytridization and genetic engineering have recently become major approaches to improve biocontrol potential of entomopathogenic nernatodes in tamilias Steinemenatidae and Heterornabditidae. In the reported study spontaneous and chemically induced mutagenesis has produced a number of morphologically and behaviorally new phenotypes of infective juveniles in Steinemema feltias. The observed changes have been caused by mutations in major genes and they affected various elements of cuticular and muscular structures. as well as a nervous system of the nematodes. The functional relationship between the changed phenotype of infective juveniles and their effectiveness in finding and infecting target insects has been examined against sciarid fllos Bradysia paupera and Lycoriefla soliani, and the Wastem Fluwar Thrips, Frankliniella occidentalis. In lescoratory and greenhouse pot experiments most $\alpha$ the mitant phenotypes appeared is be disadvartageous to the nematosias' actinity. tectucing their overall effectiveness against the pests. However, a number of mutam strains with new traits, including spectic modifications of lateral tields, moderately spiral amangement of muscles, reduced sizes of infective juveniles and altered osmofic preterences showed improved movement activity, host-linding and penetration irto the hemocoel of the tested insects. The new traits are presently used in construction of more effective nematode strains for biological control of difficutt pests.

Department of Biołogical Pest Control and Quarantine, Institute of Plant Protection. Miczurina 20, 60-318 Poznan, Poland 
Umarov Fi.F.

During study of genome of alfid $f_{\text {is }}$ CXM1, the symbiosis with nodule bacteria has been studied. Molecular and genetical analysis of Fhizobium meliloti genome has showed that leut bi asynthesis gene takes part in nitrogen fixatirc symbiosis with alfalfa. Mutations in leu genes of bacteria carry to symbiosis distruction.

We have created Phizobium meliloti gene litrary and with it help cloned leut into pSUP202 vector. Transfer of this gene into Rhizobitm meliloti strain (Fix', Leu-) has shown the genotipe reconstruction and symbiosie." Also we have studied in teraction of leu* gene with different varieties of alfalfa.

The research described in this publication wes made possible in part by Grant $N$ Ru bogo from International Science Foundation

Institute of Microbiology

, Republice of Uabekistan

Te1. 7 (3712\} 41-95-52 Fax: $7\{3712\} 41-71-29$

E-majl umarove silk.glas.apc,org
T. Wai, J.R. Stomite: M.E. Tousignant, J.M. Kaper

Cucunber mosaic virus (CMV) causes disease in a wide variety of ccononically ic virus lcrvi causes disease in a wide variety of and cucurbits. Severe crop plants, including tomato, pepper, various parts of the us gast Asia and in the Mediterrancan various parts region traditional methods of controlling CMv intestations, 1.e. chemical control of the lnsect vector and breeding for CMN resistant varieties, have not been successful in combating CMV epldenics of tomato. Therefore, a novel biocontrol approach employing the concept of this tield test. Satellite RNAs axe believed to compete for the replicase enzyme encoded by the helper yirus, thereby reducing virus titers. 'uC82B', transformed with the benign om satellite S-CARNA 5 (McGarvey et al..' 1994): 'Lichun'

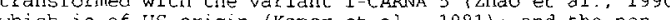
which is of us origin (Kaper et al., 1981); and the nontransformed parental lines were used to decermine the effects of this technology on virus and satellite titers and on the fruit yield. Seedlings of each variety were mechanically challengeinoculated with CARNA 5-free CMV RNA and transplanted to the field. Foliar symptoms were scored weekly and samples taken to monitor for the presence of CMV coat protein titers by ELISA and to probe for the carsa 5 satellites by dot blotting. The yield of the satellite-transformed UC82B line inoculated with CMV increased the total marketable fruit yield by 508 compaxed with the non-transformed UC82B line. Yields for the satellitetransformed Lichun line seemed higher, but were not detemined due to the indeterminate nature of the Lichun lines.

Molecular Plant Pathology Laboratory

plant Sciences Institute

Beltsville Agricultural Research Center - West

Agricultural Research Service

United States Department of Agriculture

Room 252, Building 011A

10300 Baltimore Avenue

Beltsvilie. Maryland 20705 USA
623

\section{THE USE OF PCR FOR THE CHARACTERIZATION OF BANANA BRACT MOSAIC AGENT}

\section{ANCEAU, M-L. ISKRA-CARUANA, D. COLINET, J. KUMMERT and P. LEPOIVRE}

Banana bract mosaic virus (BBMV) has recently been reported from the Philippines and India. It is transmitted mecanically, by aphids and through planting material. Symptoms can be difficult to discern, but yield losses of up to $40 \%$ can occur. Filamentous particle morphology (Munez, 1992; Caruana 1993) and its non persistent aphid transmission suggest that the virus belongs to the potyvirus group.

In order to investigate BBMV genome, a combined assay of Reverse Transcription and $P C R$ using degenerate primers specific for the potyvirus genus (Colinet ef al., 1993) was applied on total RNA extracted from BBMV infected plant originating from Philippines (Laboratoire de Virologie, CIRADFHLOR/LPRC, Montpellier, France).

This assay yielded a fragment of approximately $1.3 \mathrm{~kb}$ spanning the coding region for the $\mathrm{C}$-terminal part of the RNA polymerase and the $\mathrm{N}$-terminal part of the coat protein of the virus. This fragment was cloned and partially sequenced. Analysis of the putative amino acid sequence deduced from the nucleotide sequence revealed the presence of consensus regions which confirmed the identification of this virus as a polyvirus.

This work was financially supported by the International Network for the Improvement of Banana and Plantain (INIBAP-IPGRI) and the Belgian Administration for Development Cooperation (BADC)

Laboratoire de Pathologie Végetale, Faculté Universitaire des Sciences Agronomiques, 8-5030 Gembloux, Belgium.
624

DETECTION OF BEAN YELLOW MOSAIC VIRUS IN GLADIOLUS CORMS BY ENZYME-LINKED IMMUNOSORBENT ASSAY ON NITROCELLULOSE MEMBRANE (DOT-ELISA)

\section{G. Bellardi, C. Rubies-Autonell, V. Vicchi (*)}

Bean yellow mosaic virus (BYMV) is ubiquitous in gladiolus (Gladiolus $\mathrm{x}$ grandiflons) crops; alone or in mixed infections with other viruses, it reduces vield and flower quality. For certification of corms, virus-tested propagation stocks require reliable and rapid detection methods to be applied directly to corms or cormels. Rapid assay of BYMV by enzyme-linked immunosorbent assay (ELISA) is only possible in gladiolus leaves and flowers but very difficult in the corms due to its low concentration. Preliminary ELISA experiments showed improved BYMV detection after cutting the corms and testing the cut surface, as previously reported by other authors. We investigated whether ELISA on nitrocellulose membranes (dot-ELISA) was more sensitive for BYMV detection in gladiolus corms. The corms tested, belonging to commercial mixed cultivars, had been stored at $4{ }^{\circ} \mathrm{C}$. Dot-ELISA was applied to both dormant corm and bud tissues using as extraction buffer $0.05 \mathrm{M}$-sodium carbonate at $\mathrm{pH} 9.6$ at different dilutions: $1 / 10 ; 1 / 25$; $1 / 50 ; 1 / 100 ; 1 / 500$. Several experiments were carried out by adding to the buffer : $\mathrm{Na}_{2} \mathrm{SO} 3+$ Polyvinylpynolidone (PVP) $(10$ or $20 \mathrm{~g} /) ; \mathrm{Na}_{2} \mathrm{SO}_{3}+\mathrm{PVP}_{(20 \mathrm{~g} /)}+$ ovalbumin; PVP $(20 \mathrm{~g} /)+$ ovalbumin. Nitrocellulose membranes (NCM) $(0.45 \mu$ Millipore $\mathrm{HAHY}$ ), rnsed five times in $10 \mathrm{mM}$ Tris- $\mathrm{HCl}$ buffer, $\mathrm{pH} 7.4$, plus $0.05 \%$ Tween 80 (TBS-80), were incubated in the blocking agent, consisting of TBS-80 plus $3 \%$ bovine senum albumin (BSA), for $1.5 \mathrm{~h}$ at $37^{\circ} \mathrm{C}$. Alkaline phosphatase conjugate to virusspecific gamma globulin (1:100 dilution) of ELISA kit (Sanofi) was added and NCM stored overnight at $4^{\circ} \mathrm{C}$. The substrate was prepared mixing $(1: 1)$ Fast Blue salt and Naphthol AS-MX phosphate; the enzymatic reaction was terminated after 15-30 min. by rinsing the NMC in distilied water. Among the buffers tested the best one was added with $10 \mathrm{~g} / \mathrm{l}$ of $\mathrm{PVP}+\mathrm{Na}_{2} \mathrm{SO}_{3}$ : oxidated dots appeared with extraction buffer alone and when ovalbumin was present; best results were also observed when both corm and bud tissues were diluted $1 / 25$. Under these conditions $13 / 15$ buds tested and $7 / 18$ bulbs tested were infected. Dot-ELISA appcared more sensitive than DAS-ELISA. It is known that the polymerase chain reaction (PCR) is the best method for BYMV detection in gladiolus coms; however PCR cannot be used as a routine' method. It is not, in fact, easy to apply in certification schemes and not economic. In conclusion we can say that dot-ELISA can be used as method for a preliminary screening of gladiolus propagation stocks, followed by PCR to check BYM presence in the corms BYMV-free.

Istinto di Patologia Vegetale, Università degli Studi di Bologna, Via F. Re, 8, 40126 Bologna, Italy. (") Osservatorio Regionale per Je Malattie delle Piante, Via di Corticella 133,40129 Bologna, Italy 
MOLECULAR DETECTION AND IDENTIFICATION OF PHYTOPLASMAS INFECTING ELOWER CROPS

A. Bertaccini, M. Vibio, B. Hostachy, C. Hiruki, D. Louro

Mycoplasmalike organisms (MLOs), recently renamed phytoplasmas, are wall less prokaryotes associated with plant diseases worldwide, but knowledge about their epidemiology is very limited: their identification is hindered by the inability to isolases are known to occurr and cause severe production losses especially on ornamental and flower crops where the normal morphology is compromised by their presence inside the phloem. morphology is compromised by their presence inside the phloem. smas associated with ranunculus phyllody in French and Italian Riviera, with gladiolus virescence, aster yellows, periwinkle reen petals and Chrysanthemum yellows in Italy, with gladiolus and calendula virescence in Prance, with limonium virescence in Portugal, and with hydrangea virescence in Italy and in Canada. resh field collected samples from asymptomatic and symptomatic plants were used for nucleic acid extraction and in polymerase 作 plasmas 16s rDNA sequences, The infected samples ylelded speciFic bNA bands of 1.2 or $1.1 \mathrm{~kb}$, according with the primer pairs employed. The phytoplasma identification was performed by restriction fragment lenght polymoxphism (RRL) analyses of the amplified sequences with restriction endonucleases that cleave sites characterizing different phytoplasma groups and subgroups. All the infected samples contained phytoplasmas belonging to different subgroups of the aster yellows group (AY or $16 \mathrm{~S}$ rRNA group. I): The ranunculus samples from the Italian Riviera and the limonium plants from Portugal contained phytoplasmas belonging to subgroup $I-C$, while the ranunculus from French Riviera and the hydrangea from Canada contained phytoplasmas subgroup IB. Also gladiolus and calendula from France and chrysanthemum, hydrangea, aster, and most of the gladiola from Italy were infected with phytoplasmas of the subgroup $\mathrm{I}-\mathrm{B}$. Only in one sample of gladiolus collected in Italy, phytoplasmas belonging to subgroup I-A were detected. The periwinkle sample from Italy was infected with phytoplasmas belonging to the new subgroup I-G recently described.

It is very important to highlight that these molecular tools can enable to early detect and identify the phytoplasma population infecting flower crops to prevent outbreaks of diseases that, can be spread by vectors and plant host in a new geographical region becoming a pest difficult to control.

on the other hand the close genetic relationship among these phytoplasmas indicates their potential of spread in annual crops through maintenance in common insect vectors and/or plants hosts or their spreading with nursery materials.

Istituto di Patologia Vegetale, via Filippo Re 8, 40126, Bologna- Italy

627

IDENTIFICATION AND CHARACTERIZATION OF FOUR MEMBERS OF THE POTYVIRIDAE INFECTING SWEETEOTATO BY THE POLYMERASE CHAIN REACTION

D. Colinet, O. Duterme, J. Kummert, P. Lepoivre

Two degenerate primers, derived from conserved regions in the genome of potyviruses, were designed to amplify the variable 5'-terminal region of the coat protein cistron. Sequence analysis of the fragments amplified from mixediyinfected sweetpotato clones from china allowed to identify three distinct potyviruses, sweetpotato feathery mottle virus (SPFMV), sweetpotato latent virus and a previously unidentidied virus named sweet potato virus $G$ (SPVG). RACE (Rapid Amplification of CDNA Ends) method was used to clone the $3^{\prime}$ end of the genome of SPVG. Sequence analysis of this region indicated that SPVG should be considered as closely related to, though distinct from SPFMV.

Two other degenerate primers were designed from conserved regions in the putative RNA polymerase of potyviruses and bymoviruses. Sequence analysis of the fragment amplified from the whitefly-transmitted sweetpotato mild mottle virus (SPMMV) with these primers, brought further evidence that this virus is a member of the Potyviridae. Moreover, application of the RACE protocol followed by the sequence analysis of the region corresponding to the coat protein cistron confirmed that SPMMV should be included in a distinct genus.

Faculté Universitaire des Sciences Agronomiques, Laboratoire de Pathologie Végétale, 5030 Gembloux (Belgium)
THE USE OF NESTED PRIMERS IN PCR FOR THE DETECTION OF PHYTOPHTHORA FRAGARIAE IN STRAWBERRY

\section{P.J.M. Bonants, M. Hagenaar-de Weerdt}

Phytophthora fragariae Hickman causes red core disease in strawberry. The fungus is a quarantine organism. It is difficult to detect the fungus visually and early primary infections in propagation beds and the planting of runners from infected but symptomless resistant cultivars can easily occur without being noticed. Consequently all plant material for export must be checked for the presence of the fungus by another detection method.

$P$. cactorum is a closely related pathogen of strawberry but it is not considered to be a quarantine organism. A detection method for $P$. fragariae therefore should be highly specific.

The current detection method for $P$. fragariae, the Duncan root tip test, has several disadvantages. It is time-consuming ( $5-6$ weeks), it requires taxonomic experience and it has to be performed at about $12^{\circ} \mathrm{C}$. Serological methods (ELISA) to detect $P$. fragariae in strawberry failed because of lack of specificity and/or sensitivity.

Recently several publications appeared on fungal detection based on the PCR method It has been successfully applied for the detection of e.g. Verticilium sp.. Phoma tracheiphila, Gaumannomyces graminis and Mycosphaerella fijiensis. Sensitivity and specificity seemed no limiting factors any more.

PCR (polymerase chain reaction) primers were available that could be used to differentiate between $P$. fragariae and $P$. cactorum and that made it possible to detect $P$. fragariae in heavily infected raspbery roots (Stammler et al., 1993)

Starting from that a nested PCR was developed that can detect very small amounts of the fungus in infected strawberry roots before symptoms are visible. in such cases it was not possible to detect the fungus by a single PCR. The new inner primers are complementary to the internal transcribed spacer (IIS) sequences in the ribosomal DNA. Also zoospores of $P$. fragariae var. fragariae could be detected with this 5 at of nested primers and even a very small amount of mycelium was positive in PCR. We could detect one zoospore in a water sample.

Using this method it was feasible to detect the presence of $P$. fragariae var. fragariae in water samples taken from Duncan tests, performed by the Inspection Service.

DLO Research Institute for Plant Protection (IPO-DLO), Department of Detection, P.O. Box $9060,6700 \mathrm{GW}$ Wageningen, the Netherlands.

628

\section{THE USE OF PCR FOR THE IDENTIFICATION AND CHARACTERIZATION OF FIVE STRAINS OF YAM MOSAIC VIRUS}

\section{O. DUTERME, D. COLINET, J. KUMMERT and P. LEPOIVRE}

In order to characterize different strains of the Yam Mosaic Virus (YMV), a combined assay of Reverse Transcription and PCR utilizing degenerate primers specific for the potyvinus group was applied on total RNA extracted from YMV infected plants. This assay was performed on three YMV infected yam clones (one originated from IvoryCoast: YMV-COT, and two originated from Burkina-Faso: YMV-BU1 and YMV-BU2) and on Nicotiana benthamiama mechanically inoculated with the reference strains either YMV-12 received from Dr Dubern (Montpellier) or YMV-TOG received from Dr Adam (Braunschweig)

These assays yielded for each strain a fragment of about $1.3 \mathrm{~kb}$ spanning the coding region for the C-terminal part of the RNA polymerase and the $\mathrm{N}$-terminal part of the coat protein of the viruses. The amplified fragments have been cloned and the region corresponding to the N-terminal part of the coat protein was sequenced. Analysis of the putative amino acid sequences deduced from the nucleotide sequences of the five amplified fragments revealed the presence of various consensus regions characteristic of potyviruses. Amino acid sequence comparison of the $\mathrm{N}$-terminal part of the coat protein deduced from the five amplified fragments revealed high sequence identity ranging from $71.5 \%$ to $97.5 \%$, thus suggesting that the fragments were amplified from strains of a same virus. The most surprising result is the absence of a stretch of 12 amino acid for YMV-BU1 and YMV-BU2 compared to the other strains. Such sequence diversity in the amino terminal end of the coat protein have already been reported for other potyviruses.

Faculté Universiaire des Sciences Agronomiques, Laboratoire de Pathologie Végétale, 5030 Gembloux (Belgium) 
POLYMERASE CHATN REACTION TECHNOLOGY AS A TOOL FOR DETECTION AND IDENTIFICATION OF PLANT VIROIDS

\section{A. Hadidi}

The polymerase chain reaction (PCR) is an in vltro method in which trace amounts of DNA or transertps are ampIIfiet rapidly with very high specificity and fidelity using oligonucleotide primers and Tag DNA polymerase in a simple automated reaction. The amplified DNA after agarose or polyacrylamide gel electrophoresis, hybridization with labeled probes, or by colorimetric assay after affinity with labeled probes, or by colorimetric assay after affinity binding. The avallabillty of nucleotfde sequences of virolds has made possible the development of reverse transcription (RT).PCR assays For improving the detection and diagnosis of several viroids from their respective hosts. We have used what might be considered a variation on the inverse PCR when amplifying virolds of known nucleotide sequences. Since by definition virolds are circular RNA molecules, no circularization is required, but using primers that anneal to the central conserved region on the viroid with $3^{\prime}$ end facing away from each other, we have successfully amplified full length DNA copies of a number of viroids. These viroids include potato splnde tuber, ehrysanthemum stunt, apple scar skin, adapple apple, pear rusty skin, peach laten mosaic, plum dapple. cltrus exocorts, dild citrus, exocortis (CVIla), cilrus cachexla (CVIIb) grapevine speckle, and others. Using RT-PGR, drect detection of potato spindle tuber viroid from tubers and seeds is now possible withaut che need to germinate tubers or seeds into seedlings as required by other known detectlon methods. Only a few days Instead of several months to a few years are required for positive detection and identification of fruit crop viroids. The detection of viroids by RT-PCR requires 1 to $100 \mathrm{pg}$ of cotal nucle1c acids from infected tissue and it is 10 to 100 -fold more sensitive than viroid detection by hybridization and 2500 -fold more sensitive than return gel electrophoresis analysis. Because of its great sensitivity, the PC provides a good alternative to other diagnostic methods and can speed diagnosis, reduce the sample size required, and often eliminates the need for radioactive probes.

Bldg. 011A, Ron 106, BARC.W, National Germplasm Resources Laboratory, Agricultural Research Service, U.S. Department of Agriculture,

Beltsville, MD 20705, U.S.A.

\section{DETECTION OF CLAVIBACTER MICHIGANENSIS SSP.SEPEDONICUS AND XANTHOMONAS CAMPESTRIS PV. PELARGONII BY MEANS OF POLYMERASE CHAIN REACTION}

\section{R.-M. Leiser, D.Hasselmann, J.Schumacher}

In order to increase the sensitvity of detection for both bacterial pathogens, PCR approaches have been developed. In addition, a very useful sample preparation has been developed which utilizes Qiaquick spin columns (Qiagen GmbH, Germany). Through this method, all substances from the primary extract that are potentially PCR-inhibiting can be eliminated.

For Clavibacter michiganensis ssp. sepedonicus (Cms) the sequence of a 600 bp BamHI-fragment from its $49.5 \mathrm{kbp}$ plasmid has been utilized to design a nestedprimer PCF approach which yield's the needed sensitivity for detecting as litlle as 10 cells per reaction with a volume of $50 \mu$ l. This corresponds to a sensitivity of less than 10 cells per $5 \ldots 25 \mathrm{ml}$ pure bacterial culture or one heel end of an infected tuber among 200 noninfected ones. In control experiments this sensitivity exceeded convincingly the immunofluorescence technique.

On the basis of a new method for the differential clone screening, several pathovarspecific sequences for $X$ anthomonas campestris pv. pelargonii $\left(X_{c} p\right)$ have been recognized and cloned. After extensive hybridization studies with nine Xcp isolates and seven non-pelargonium isolates, one sequence has been selected for further experiments. Again a nested-primer PCR approach has been designed using the chosen sequence. This PCR procedure gave a sensitivity which is at least two chosen sequence. This PCR procedure gave a sensitivity which is at least two
orders of magnitude greater than ELISA- and IF-techniques - the more commonly used methods for the detection of XcP.

For both Cms and Xcp the nested-primer PCR approach has been designed in a so-called one-tube procedure in order to avoid cross contamination during the otherwise necessary pipetting steps.

R.-M. Leiser

PHYTOTEST Pflanzenservice GmbH and NewLab Diagnostic Systems GmbH, Max-Planck-Sir. 15a

D-40699 Erkrath
SENSITIVE DETECTION OF CHRYSANTHEMUM STUNT VIROID BY ENZYMATIC CDNA AMPLIFICATION

M. Hoooftman, A.M. Shamloul, M. Arts, A. van Zaaijen, A. Hadidi

Reverse transcription-polymerase chain reaction ( $R T-P C R$ ) assays were developed for the detection and identification of chrysanthemum stunt viroid (CSVd) from sap or total nucleic acid extracts of infected dried or freshly collected chrysan the mum leaves. DNA primers (20-25 nucleotides in length) specific for CSVd sequence were used for cDNA synthesis and specific amplification of CSVd. The antisense primer is complementary to CSVd nucleotides $68-87$ in the central conserved region and the sense primer is homologous to CSVd nucleotides $88-112$. The size of the major RTPCR product from CSVd-infected tissue was the same as full length CSVd (356 bp). This product was absent from uninfected tissue. Replacement of CSVd primers with those of potato spindle tuber viroid resulted in amplification of a smaller product, 325 bp, from infected tissue. A full length CSVd cDNA 356 bp product was detected from $100 \mathrm{ng}$ to as little as $0.1 \mathrm{ng}$ of total initial nucleic acids from infected tissue. The amount of amplified CSVd cDNA decreased as total initial nucleic acids decreased. Similarly, a 356 bp CSVd cDNA profuct was detected from clarified sap extracts of infected tissue diluted 100 to 1000 fold. Treatment of diluted extracts with GeneReleaser polymeric matrix to bind to RT-PCR inhibitors may increase the sensitivity of the assay. The RT-PCR assay is more sensitive than existing detection methods and provides information about viroid detection from chrysanthemum without requiring large samples or molecular hybridization.

Nederlandse Algemene Keuringsdienst voor Bloemisterij-en Boomkwekerijgewassen (NAKB) Johan de Wittiaan 12, 2517 JR Den Haag

Bldg. 011A, Rm 106, BARC-W, National Germplasm Resources Laboratory, Agricultural Research Service, U.S. Department of Agriculture, Beltsville, MD 20705, U.S.A.

632

ANALYSIS OF MOLBCULAR NECHANISMS OF THE PLANT-PATHOCWN SYSTEW

E.A. Ivanova, G.K. Vafina, I.E. Bikbulatova,

E.E. Tchernycheva

An attempt has been made at the elucideting endogenous molecular-genetic mechanisms of the forming of the plantpathogen syster. To analyse supramolecular nuclear structures in the process of unfolding of morfogenetic and onthogenatic development progreme in the pertod of Juvenile stage of heterotrophto phase has teken the common wheat (Triticun eestivum L.) of Moskoxskya-35, that ig the receptive wheat to Ustilago tritiai. Inoculation of the plant of Jatilago tritici's agent in flower phage were made by the vacuum method in the breeding nursery. Germinating wheat seeds as the non-infected and the infected by tho pathogen were carried out in the costings of the fllter papers mojatened distilled water. Cell nuclei and their supramolecular structures were isolated from seedings, that were similax on physsology, and their separate organs. Difference in the functioning of the endogenous redox and hydrolytic processes of cell nuclel as heelthy and infected seedings of the wheat is discussed.

Institute of Biology, Ufa Scientific Centre of the Russian Academy of Sciences, pr. Octybrya 69, U19 450054, Bashkirta 
NUCLEOTIDE SEQLENCE OF THE $3^{\prime}$-TERMINAL REGION OF RNA1 OF SATSUMA DWARF VIRUS AND APPLICATION TO RTPCR FOR IMPROVED DETECTION

T. Iwanami and $\mathrm{H}$. Iek 1

Satsuma dwarf virus (SDV), which is the causal agent of satsuma dwarf of satsuma mandarin (citrus unshiu Marc.)is an unclassified virus. Although particle morphology and size of coat protein of SDV are similar to those of the comovirus group, $S D V$ is seroglcally related to neither cowpea mosaic virus (CPMV) nor bean pod mottle virus (BPMV). We therefore carried out a detailed investigation of the genome structure of SDV in order to analyze the relationship between SDV and comoviruses. SDV contains two species of RNAS, RNA1 (1.9 X 10 daltons) and RNA2 (1.7 X 10 daltons). In this paper, we report the sequence of the $3^{\prime}-$ terminal 3116 nucleotides of satsuma dwarf virus (SDV) RNA1. The sequence contained a part of a single open reading frame of 2865 nucleotides and a non-coding region of 251 nucleotides upstream of the poly(A) tail. The $C$-terminal reglon of the orF was apparently homologous to the RNA-dependent polymerase of RNA viruses. The amino acid sequence of this region showed the highest homology with that of the comovirus group $(28 \%$, cowpea mosaic virus, CPMV) followed by the nepovirus group (25\%, grapevine fanleaf virus). The homology with other viruses was low. The calculated homology between SDV and CPMV (28\%) in comparison with homologies among viruses in the comovirus group (51-61\%) suggested that SDV should be classified into a distinct new plant virus group which is similar to the comovirus and nepovirus group. Furthermore, application to RT-PCR for improved detection of SDV from citrus will be discussed.

Okitsu Branch, Fruit Tree Research Station, okitsu, Shimizu, Shizuoka 424-02, JAPAN

DETECTION AND IDENTIFICATION OF PLANT PATHOCENIC XANTHCMONADS ASSOCLATED WITH PEPPER AND TOMATO SEEDS BY AMPLIFICATION OF DNA SEQUENCES RELATED TO THE HFP GENES

F.P. Leite Jr., J.B. Jones, G.C. Somodi, G.V. Minsavage, R.E. Stall

A method based on anplification and analysis of DNA sequences related to the hypersensitive reaction and pathogenicity (hrp) gene cluster was examined for detection of plant pathogenic xanthomonads associated with pepper and tomato seeds. The method of detection comprises the extraction of total DNA from seed washings to which sodium ascorbate and insoluble polyvinylpolypyrrolidone were added. Two sets of oligonucleotide primers selected from the nucleotide sequence of the hrp gene cluster of Xanthomonas campestais pv. vesicaturia were used in the reaction for DNA amplification. $x$. compestris pv. vesicatoria was detected in preparations containing cells added to pepper and tomato seeds extracts. Identification of $X$. compestris pv.vesicatoric was attained from band profiles after endonuclease digestion of the amplified DNA fragment. This endonuclease digestion step is advantageous as a control to detect nontarget plant pathogenic xanthomonads that might be on the plant material. The sensitivity of the method ranged from about $10^{2}$ to $10^{3} \mathrm{CF} / \mathrm{ml}$ of seed washings. This was 100 to 1000 times fewer than the minirsm number of celis detected with an enzyme-linked immunosorbent assay (ELISA). X. campestris pv. vesicatoria was also detected in washings obtained from several lots of naturally contaminated pepper and tomato seeds, of which one lot contained background bacterial microflora larger than $10^{7} \mathrm{cFU} / \mathrm{g}$ of seeds. The attemgts to recover plant pathogenic xanthomonads from these naturally contaminated seed lots by plating washings on both yeast-extract nutrient agar and Tween $B$ media were unsuccessful. However, the presence of viable cells of $X$. compestrus pv. vesicatoria in five of the eleven seed lots which tested positively for the bacterium in the DNA amplification was confirmed by detection of the bacterium in seedings from these seeds.

LAPAR, Caixa Postal, 1331 - 86001-970 Londrina, PR, Brazil
DIAGNOSIS OF THREE VIRUS DISEASES OF POMACEOUS TREES

1. Kummert, G. Rufflard, D. Colinet, O. Duterme, P. Lepoivre.

Apple chlorotic leaf spot virus (ACLSV), Apple stem grooving virus (ASGV) and Apple stem pitting virus (ASPV) isolates, and complexes of these viruses, multiplied on herbaceous host plants have been used for the development of detection methods based on RT-PCR and dot blot hybridization. Degenerate primers were designed from comparative analysis of nucleotide sequences published for 2 isolates of ACLSV and one isolate of each ASGV and ASPV. Different sets of primers were used for amplification of viral sequences from reversed transcribed total RNA preparations of virus-infected leaves of Chenopodium quinoa or Nicotiana occidentalis. According to the primers selected, amplifications were obtained for either one, two, or all the three viruses studied. Specific and sensitive detections were also obtained by immunocapture RT-PCR for ACLSV and ASGV. Selected purified amplification products have been cloned in pBluescript in order to develop probes for use in dot blot hybridization. Digoxigenin labelled probes thus obtained have been shown specific for the only detection of the virus isolate which originated the cloned amplification product.

The results observed during this work illustrate the difficulty to identify the viral agent(s) infecting a fruit tree on basis of the indexing on ligneous indicators by grafting ${ }_{r}$ and the prevalence of multiple infections even in some reference collected materials.

The RT-PCR, immunocapture RT-PCR and dot blot hybridization techniques thus developped will be adapted for direct use on ligneous material, and specially mother trees, with the aim of defining protocols for certification of virus-free propagating material in nuclear stocks.

Centre National de Phytovirologie IRSIA, Laboratoire de Pathologie Végétale, Faculté Universitaire des Sciences Agronorniques, 5030 Gembloux (Belgium).

636

\section{IN VITRO EXPRESSION OF GEMINIVIRUS COAT PROTEINS AND THE USE OF} EXPRESSED FUSION PROTEINS FOR PRODUCING ANTISERA

\section{S.LIU, M. PINNER, M. S. HAIDER, P. G. MARKHAM}

Geminiviruses are a group of plant pathogens with economic importance, especially in Africa, South America, South East Asia and Mediterranean. The genome of whitefly (Bemisia tabaci)-transmitted geminiviruses usually comprises two similar-sized single-stranded DNAs, denoted DNA A and B, but exceptions occur with some tomato infecting leaf curl geminivirus, which have only monopartite genome. All leafhopper-transmitted geminiviruses also have a monopartite genome. Most of the geminiviruses are very difficult to purify because the capsids disrupt easily and produce poor yields of coat protein, which makes it difficult to raise good quality antiserum for detecting these viruses. In contrast, it is comparatively easy to isolate geminivinus genomes, to subseguently clone and to In contrast, it is comparatively easy to isolate geminivinus genomes, to subseguently clone and to
sequence these genomes. Using the sequence information, it is possible to express the coat proteins sequence these genomes. Using the sequence information, it is possible to express the coat proteins
in vitro, to purify the expressed proteins and to produce antisera in the normal manner. Here we in vitro, to purify the expressed proteins and to produce antisera in the normal manner. Here we
report our results of using a fusion-protein expression system to express the coat proteins of four geminiviruses and discuss the quality of the antisera produced.

The coat proteins of African cassava mosaic virus (ACMV), Asystasia golden mosaic virus (AGMV), Beet curly top virus (BCTV) and tobacco golden mosaic virus (TGMV) were amplified from the fulllength clones of the above viruses by PCR and cloned into pMAL-C fusion-protein expression vector. The coat protein genes were inserted down stream of maltose binding protein (MPB) and expressed as a MBP fusion proteins in $E$. coli (IM83 strain). The four coat proteins were all successfully expressed in the bacteria cell and the fusion proteins were then purified by amylose resin successfully expressed in the bacteria cell and the fusion proteins were then purified by amylose resin
affinity column, and injected into rabbits to produce antiserum. The expressed coat proteins were easily cut out from the fusion proteins using $X$-a factor. The resulting antiserum against fusion proteins were tested by ELISA and Western blot.

The antiserum raised against ACMV fusion protein could be used in both ELISA and Western blot tests to detect ACMV as both purified virus and ACMV infected plant sap, and it could also be used in ISEM and immuno gold labelling (IGL). Moreover, this antiserum showed that it could cross-react with five different whitefly-transmitted geminiviruses tested in both ELISA and Western blot. The antisera against BCTV and TGMV products could be used in Western blot tests but despite cross antisera against BCTV and TGMV products could be used in Western blot tests but despite cross
absorbtion gave significant background reaction. The antiserum against AGMY product is being evaluated.

The results demonstrate that using fusion protein expression technique to express vins protein is realistic as an alternative method to produce antiserum. It can also be used to produce virus proteins for other uses such as for the study of virus protein functions. This method has a potential advantage for producing antisera and diagnosing the virus diseases with those viruses which are not easy to isolate and purify.

Address: Department of virus research, John Innes Centre, Norwich Research Park, Colney Lane, Norwich, NR4 7UH, UK. 
RAPID TOMATO SEEDLING ASSAY FOR VIRULENT ISOLATES OF VERTICILLIUM DAHLIAE, THE TOMATO WILT PATHOGEN

\section{c. Madhosingh}

A rapid 10-minute tomato seedling assay was developed for determining the relative wilt capacity of isolates and races of Verticillium dahliae, the tomato wilt pathogen. The procedures for the assay require that 8 -day old cv. Bonny Best tomato seedlings be dipped in 20-day cell-free concentrated culture filtrates of the verticillium isolates, which were grown in caapek-Dox medium with 28 Bactorcasamino acids(CDA). The seedlings in the culture filtrates were then incubated at $30 \%$ under artificial light (1200 ft/candles) at 28 relative humidity in a wind stream of $100-150$ metershin. The assay gaodlings were grown under low light cond elongated stems wich wer more susceptible to the wilt effects of the culture filtrates. Th relative pathogenicity of the isolates was determined by pattern of cell-free filtrate wilt among the isolates was the same as that for the disease caused by cultures af the isolates. The assay was used to determine the production of milt toxins by Race and Race 2 and new isolates of Verticillium dahliae and $t$ identify toxic chromatographic fractions of culture filtrates. The assay was also useful in determining the relative resistance of to cultivars to the verticillium toxins.

Pest Management Research Center, Agriculture and Agri-Food Canada, 1391 Sandford Street, London, Ontario, Canada N5V 4T3


verticillium isolates and assessing the development of disease. Th

SOME MOLECULAR PROPERTIES OF AN ENTOMOPOXVIRUS OF THE CLPREOUS CHAFER, ANOMALA CUPREA HOPE

(COLEOPTERA; SCARABAEIDAE)

\section{W. Mitsuhashi and M. Sato}

A larva of the cupreous chafer, Anomala cuprea is a major pest in many crops and forestry trees in Japan. An entomopoxvirus is pathogenic to this insect and it may be considered as a potential biocontrol agent. In the present study, some properties of major proteins and genes of the virus were investigated. The molecular sizes of the major proteins, fusolin and spheroidin, of two occlusion bodies (spindle and spheroid) were estimated as $46 \mathrm{KD}$ a and about $100 \mathrm{KDa}$ from the analysis of SDS-PAGE, respectively. The restriction patterns of the viral DNA digested with EcoRI, $H$ indIII and BamHI were analyzed, and a few bands among them seemed to be submolar ones. Several regions of spheroidin gene were amplified by polymerase chain reaction (PCR) using the primers which were synthesized from the sequence data of the spheroidin gene of an entomopoxvirus of Melolontha melolontha (Sanz et al., 1994). These PCR products were cloned into Bluescript $\left(\mathrm{ks}^{+}\right)$and sequenced by the dye deoxy terminater method.

National Institute of Sericultural and Entomological Science,

Tsukuba, Ibaraki 305. Japan

640

IDENTIFYING VERTICILLIUM WILT CAUSAL AGENTS IN SOIL AND POTATO TISSUES WITH PCR-BASED ASSAYS

\section{HW_(Bud) Platt}

Plum pox potyvirus (PPV), with another virus of the same group, prunus latent virus (PLV), is the only potyvirus infecting stone fruits. PLV reacts positively in ELISA with PPV antiserum, but negatively with PPV DNA primers specific for the $3^{\prime}$ non-coding region (NCR) of PPV in reverse transcription polymerase chain reaction ( $\mathrm{RT}-\mathrm{PCR}$ ) assay.

PPV has been found on plum, apricot, peach and, recently, on sour and sweet cherry. The tremendous economical impact of this and sweet eherry. The tremendous economical impact of this disease for the first three crops has raisad the necessity of establishing protocols for the diagnosis or the virus followed, when positive, by compulsory eradication prograns. Until now, LLISA and biological tests are the official diagnostic tor Two years ago we have started a large-scale survey to verify the sensitivity of molecular assays alternative to ELISA, especially during seasonal periods when it is difficult

It was possible to demonstrate that: i) either dot-blot hybridization or (RT-PCR) assays were consistenly more sensitive than ELISA; i i) the 3' NCR product from infected sweet cherry
indicated that the virus is an isolate of PDV.

Dipartimento di Biologia, Difesa e Biotecnologie Agro-Eorestali, Universita degli Studi delia Basilicata, via N. Sauro 85, I85100 , Potenza, Italy.

National Germplasm Resources Laboratory, Agricultural Research Service, United States Department of Agriculture, Beltsville, Maryland, 20705, USA.
Verticillium wilt occurs in most potato production areas and is primarily caused by $V$, albo-atrum and/or V. dahliae. However, $V$. tricorpus, a less pathogenic species is often isolated from diseased tissues. Disease management successes are based upon accurate assessment of pathogen preserce in soil and plant tissues. Pathogen detection has involved traditional soil and plant tissue plating methods including the use of selective media. Unfortunately, this often takes several weeks before accurate pathogen identifications can be made and these methods can be unreliable if other faster growing organisms mask the presence of the pathogen(s).

Development of DNA "probes" for these three species has enabled pathogen presence determinations within a few days. In addition, this technique is much less susceptible to "false-negatives" as minute amounts of DNA can be detected and reactions to other organisms have never been recorded despite extensive testing. These and other results on the use of this detection technique will be presented.

Agriculture and Agri-Food Canada, Charlottetown Research Centre Charlottetown, P.E.I., Canada C1A 7M8 
DEVELOPMENT OF A MULTIPLEX FCR SEFT HEALTH TEST TO DETECT AND IDENTIFY THREE SEED BORNE FUNGAI. PATHOGENS OF BARLEY.

\section{J. Alderson, E. J. A. Blakemore and J. C. Reeves}

Diseases of barley caused by Pyrenophora graminea Ito and Kuribayashi (leaf stripe) and the two forms of $P$. teres (f. teres and f. maculata Smedegaard-Peterson, causing net blotch and a spot form of net blotch respectively) are capable of producing economically significant yietd losses. P.graminea is strictly seed borne; $P$. teres can also be seed bome but is mainly transmitted locally from plant debris. For seed health testing to be effective in the management of these diseases each pathogen must be unambiguously identifiable. This is difficult using traditional agar plate or blotter tests and confirmation of fungal identity by time-consuming pathogenicity testing may be required.

The disadvantages of traditional seed health testing methods have led to much interest in the development of DNA techniques which have the potential to confer advantages of speed. accuracy of diagnosis and sensitivity of detection. Identification and detection methods employing the polymerase chain reaction (PCR) have been used to identify seed borne organisms as a means of improving seed health testing and both the potential of this new technology and its applicability are now confirmed.

Multiplex PCR uses multiple sets of primers in a single PCR reaction to amplify from more than one template DNA that may be present in the reaction mix. The PCR products can be detected either on the basis of their molecular weight or by using different labels on each primer set. In this way it is possible to have the normal advantages of simplex PCR (i.e. sensitivity and specificity) as well as the capability of detecting more than one pathogen in a single test. This would make a seed health test more cost effective and efficient in its use of resources and time.

Two strategies have been used to obtain primers for identifying and detecting Pyrenophora spp.. The first, random amplified polymorphic DNAs (RAPDs), has been successful in identifying a DNA amplification product specific for each of the Pyrenophora spp. found on barley i.e. P. graminea, $P$. teres and $P$. teres f. maculata. The specificity of these DNA amplification products has been confirmed by DNA hybridisation analysis. These DNA amplification products are being cloned and sequenced so that specific primers may be designed for use in multiplex PCR. An alternative strategy used ribosomal RNA gene sequences, which have been useful in taxonomic studies because they consist of highly conserved regions and also variable intemal transcribed spacer (ITS) regions, useful for identification at an intraspecific level. Sequence data was obtained for the ITS region from different isolates of Pyrenophora spp. Differences in the sequence of the ITS between the Pyrenophora spp. enable potentially specific primers for $P$. graminea, $P$. teres. $P$. teres f. maculata. to be designed.

Screening a large number of geographically diverse Pyrenophora spp. isolates with primers obtained from both strategies will be carried out before developing a multiplex PCR seed health test.

National Institute of Agricultural Botany, Molecular Biology and Diagnostics Section, Huntingdon Rd, Cambridge, CB3 OLE, UK.

643

DETECTION AND IDENTIFICATION OF PSEUDOMONAS SYRINGAE PV. ATROPURPUREA BY PCR AMPLIFICATION OF THE PATHOVAR SPECIFIC PLASMID

Y. Takahashi, T. Omura, H. Hibino and M. Sato

Pseudomonas syringae pv. aropurpurea harbored the indigenous plasmid pCOR 1 which correlates with pathogenicity and coronatine biosynthesis (Sato et al., 1983). In the present study, eleven cligonucleotides primers (P1 - P11) derived from the E1 fragment (17 kb) of pCOR 1 were investigated for their ability to detect pCOR 1 by PCR analysis. Each primers were constructed from the sequencing data of ends of the PstI-digested fragments of El region. PCR was performed using plasmids or total DNA obtained with heat treatment as a template DNA. A total of 127 strains, including 18 strains of pv, atropurpurea, 52 strains of 38 different pathovars of $P$. syringae, 7 strains of coronatine-minus mutant of pv. atropurpurea, and 37 strains of different bacterial species. were tested by PCR to amplify the specific fragments of pCORl. All primer sets amplified the similar PCR products indistinguishable by restriction analysis against all strains of pv. atropurpurea, suggesting that pCOR1 might be a pathovar-specific plasmid. Whereas these primers amplifjed no PCR product against most bacterial strains or those of DNA sequence resembling to parts of the E1 fragment against some strains of $P$. syringae pathovars. Especialiy the primer set PI-P2 could clearly distinguish pv. atropurpurea from the other coronatine-producing pathovars of $P$. syringae such as pv. glycinea and pv. maculicola. Moreover, even 0.1 to $1.0 \mathrm{CFU}$ of bacterial cells was detectable when amplified by P1-P2 primer. Thus, highly sensitive and specific PCR method was developed to detect and identify $P$. syringae pv. atropurpurea.

National Institute of Sericultural and Entomological Science,

Tsukuba, Ibaraki 305, Japan
DETECTION AND DIFEERENTTATION OF COCOA SWOH,LEN SHOOT VIRUS LSOLATES BY POLYMERASE CHALN REACTLON

Sammy T. Sackey, Katrin Hof fman, H. Joseph Vetten, Edgar Maisse, Daniel Adomako and Ceorge $K$. Owusu

Cocoa swollen shoot disease is caused by isolates of a bacilliform DNA virus, Classification has been based on biological properties such as symptoms induced in Amelonado cocoa, vector species, and in Chana, the location from which isolated. This classification suggests the disease is caused by strains of one virus, rather than the possible presence of different viruses. The aim of the present work was to evaluate the use of polymerase chain reaction for detection and identification of swollen shoot virus isolates. Two pairs of degenerate oligonucleotide primers derived from sequences of conserved regions of four Badnaviruses described as universal badna primers, and one pair derived from the putative coat protein region of cocoa swollen shoot virus Agou 1 from the Republic of Togo. were used in polymerase chain reactions. The DNA amplification products from several isolates tested were further analysed by southern blot and hybridisation analysis using cloned DNA frovi two dominant isolates as labelled probes. Results show that there were three main DNA products using the unversal Badna primers, and that wost of the isolates tested yielded only two products of 500 and 1000 base pairs in molecular weight. Those isolates which yelded a third product of 1700 base pairs also yielded a 1500 base pairs product with the CSSV specific primers. The latter group are also characterised by being severe in their effect on the cacao host. Hybridisation analysis showed the 500 base product is common to all isolates, but that nucleotide sequencing of the larger fragments might provide a more reliable basis for the classification of cocos swollen shoot viruses.

Cocoa Research Institute of Ghana, P.0. Box 8, New Tafo Akin, Ghana.

644

(IM)POSSIBILITIES RELATED TO THE DEVELOPMENT OF MOLECULAR TOOLS FOR THE DETECTION OF TWO APHID-BORNE STRAWBERRY VIRUSES

G. Leone, C.D. Schoen, R. Miglino, M.N.J. van Druten, W.W.G. Limpens

Routine detection of the major aphid-borne strawberry viruses in strawbern propagative material is still dependent on a time-consuming leaf-graft indexing on indicator plants. Attempts to develop alternative, rapid detection methods have been hampered up to now by the unusual properties of these viruses.

For strawberry mottle virus (SMOV), a not yet identified virus, we found and characterized infection-specific dsRNA. This dsRNA can be purified directly from strawberry and used as a diagnostic tool but the method is too labour-intensive for replacing the current indexing procedure. Therefore, we decided to make a CDNA library from purified dsRNA in order to establish virus specific probes and sequences which eventually can be used for reverse transcriptase amplification technology. However, attempts to produce cDNA failed. The main reason for this is the inefficient denaturation of dSRNA. Our experience in dealing with this probiem will be discussed For strawberry crinkle virus ( $\mathrm{SCV}$ ), a rhabdovirus, we have been able to obtain monocionals specific for the nucleocapsid proteins. Isolation of the viral RNA (about $3 \%$ of the total virus particles) in order to make a CDNA library was severely hindered by low virus concentrations in plant material. A method combining immunocapture of intact virus particles from plant material and RNA extraction is in development and results will be discussed.

DLO Research institute for Plant Protection (IPO-DLO), Binnenhaven 5, 6709 PD Wageningen, The Netherlands 
PRODUCTION, CHARACTERIZATION AND APPLICATION OF MONOCLONAL ANTIBODIES FOR DIAGNOSES OF XANTHOMONAS CAMPESTRIS PV. MANGIFERAEINDICAF. (XCM)

\section{K. TSUCHIYA, C. d' URSEL and Y. NOZU}

Eleven and 14 monoclonal antibodies (MABs) were established from the mice which immunized with sonicated cells of a strain XCH 2919 and a mixture of live and formaldehyde-fixed cells of XCM 1717, respectively. MABs thus established were classified into at least three groups based on the reactivity to various $X C M$ strains and other related bacteria by using the indirect ABC-ELISA. The 1E2El, one of the most specific MAB, reacted with only strain XCM 2919. MABs such as $1 \mathrm{~A} 7 \mathrm{H} 12 \mathrm{G} 3$ recognized mainly $\mathrm{XCM}$ strains and a few pathovars of $X$. campestris. The third group such as $21 F 1 F 9$, 2E12G12D4 and 7ClC4 also reacted with other Xanthomonas spp. The dilution limit of the first $11 \mathrm{MABs}$ and the second 14 ones comprised $100 \sim 12,500$ and $100 \sim 125,000$ in ABC-ELISA, respectively. All isotypes were included in thase MARs.

The detection limit on 4 different strains of XCM for 3 selected MäBs was comprised ca $10^{3}$ and ca $10^{4}$ cells $/ \mathrm{ml}$ and was decreased 10 times by sonication treatment of antigens. of twenty six samples of infected leaves collected from 0gasawara island, Japan, $90 \%$ of those gave a positive reaction with two selected MABs, one for its high sensitivity (1A7H12G3) and another one for its specificity (21F1F9).

National Institute of Agrobiological Resources, 2-1-2, Kannondai, Tsukuba, Ibaraki 305, Japan

647

CHARACIERIZATION OF THE LASIODIPIODIA CONIDIAL SIAGE OF BOTRYOSPHAERIA COCOGENA, IHE CAUSAL AGENT OF COCONUT LEAF BLIGHT IN BRAZIL

\section{D.R.N. WARWICK, J.L. RENARD, G. BLAHA.}

Coconut is a small holder crop, that is ecologically correct, offers a broad range of dietary income and employment opportunifies in the tropics. Leaf blight. coused by Lasiodiplodia theobromae, is associated with two ascomycetes, and the symptoms include rapid wilting, browing or reddening of individual leaves. The purpose of this work was to evaluate isolates of Lasiodiplodia for isozyme variability, comparing with the pathogenicity to coconut plants and to culture characteristics. Lasiodipiodia isolates from coconuts leaves were morphologically different from isolates from other hosts and from coconuts fruits. All isolates from cocoriuts had spores size averaging $20 \mu \mathrm{m} \times 8 \mu \mathrm{m}$ and had $\mathrm{a}$ white mycelium. The non pathogenic isolates had in the average larger conidio $(24 \mu \mathrm{m} \times 10 \mu \mathrm{m})$ and much darker mycelium and generally a good growth on oat medium. Two major patterns of isozyme polymorphism \{eletrophoretic types; were found, based on malate deshydrogenase, the Te 1 pathogenic to coconuts and Te 2 non pathogenic. Correspondence was found among pathogenicity, eletrophoretic type and morphological characters. Geografic origin was not to be associated with the differences found. It seems apropriate to give the isolates pathogenic to coconuts another denomination.

CIRAD CP, BP 5035, 34032 MONTPELLIER. FRANCE
DISTRIBUTION OF SIGATOKA LEAF SPOTS IN UGANDA AS DETERMINED BY SPECIES-SPECIFIC PCR.

W.K. Tushemereirwe, A. Johanson, E.B. Karamura and C.S. Gold.

Mycosphaerella thiensis and M. musicoia are common foliar pathogens of East African Highland bananas (EA-AAA) and they occur together. The two pathogens cannot be distinguished by symptoms. Until recently, the most reliable method of distinguishing them involved analyzing the differences between their conidia and conidiophores but in some environments these are not readily produced. This complicates mapping their distribution and estimating their relative importance.

Polymerase chain reaction (PCR) tests which are based on detection of the Mycosphaerella spp, by amplification and visualization of part of the fungal genetic information are now considered the most precise identification method for the two banana pathogens. PCR tests were carried out using species-specific oligonucleotide primers designed from ribosomal DNA ITS sequences. The tests were used to study the distribution and composition of the pathogens at different elevations and on selected cultivars. This was done by determining the incidence of each pathogen in a known number of samples. The study revealed that the exotic cultivars banana clones not endemic to East Africal supported more $M$. musicola than the highland bananas. Exotic cultivar "Kayinja" (ABB Pisang awak) had the highest incidence of $M$. musicola. The incidence of $M$. fijiensis was the same on all cultivars but the damage caused varied with cultivars. Below the altitude of 1500 metres the two pathogens were present on all the cultivars. At 1500 metres above sea level and above, all cultivars supported only $M$. musicota. It is concluded that "Kayinia" can be used as a reference cultivar for monitoring $M$. musicola in a given area.

W.K. Tushemereirwe, Kawanda Agricultural Research Institute, P.O. Box 7065. Kampala, Fax: 256567635 Phone: 567158.

648

\section{CONTROL OF POTATO VIRUS Y USING MINERAL OILS.}

\section{E.K.ALLAM, M.ABO EL-NASR, S.EL. ZEMAITY AND A.EL-SAWY}

Mineral oils have been found to reduce aphid transmission of non and semi-persistent plant viruses. Potato virus Y (PVY), a non-persistant transmitted virus, is wide spread in potato plantations in Egypt. Experoments were carried out in greenhouse and in field to study the effect of spraying potato plants with different concentrations $(1,2$ and 3\%) of paraffin oil emulsion on PVY transmitted by aphid (Myzus persica Sulz.).

Greenhouse experiments showed a relation between the concentration of the oil used and the period elapsed from acquisition and inoculation of the virus. A complete inhibition of virus transmission can be achieved with low concentrations of oil (1 and $2 \%$ ) within a period of 6 hrs., while the period can be prolonged to 24 hrs. when using high oil concentration (3\%). Feeding insects on sprayed potatoes for acquisition and inoculation of the virus gave a complete inhibtion of virus transmission.

Field experituents using 7 cvs. of potatoes, sprayed 4 times with paraffin oil emulsion with concentration of 1,2 , and $3 \%$ caused a reduction of $41.1,43.0$, and $46.5 \%$ of virus transmission respectively.

Faculty of Agriculture, Ain Shams University

Shoubra El-Khema, Cairo, Egypt

Phone no. 2223642 Fax no. sed _ ict 1461

P. Box. 68 Hadaic Shoubra 
EVALUATION OF NEW ALKYL AMMONIUM COMPOUNDS AS WOOD PRESERVATIVES

\section{g. ANOUF}

The activity in wood preservation of new compounds relative to the N-alkyl isoindolinium family has been evaluated and compared with those of alkyl ammonium compounds possessing one classic fat length.

The activity antitermitic and fongicides to Basidiomycetes lignivores, in petri-dish, then on wood samples of beech and pine according to the nature of the convenient fungi were measured by using the European standard (EN 113 \& EN 117).

The new study showed that these products present a certain interest as wood preservations.

Key-word: $\quad$-Wood preservation

-Alkyl aminonium compounds

-Fungicides

-Insecticides

11821, Alfred Laliberté

11821, Alfred Lal
MONTREAL (Quebec)

CANADA

651

THE DRY PELLETS ARE ATTRACTED TO THE WATER VOLE (ARVICOLA TERRESTRIS T.)

V.A. Bykovsky, A.A. Yakovlev

Flour or sublimate carote are known as attractive basis of rodenticide baits to the water vole,but it's not right for dry grain baits, according to the publications. The water voles recived the dry pea, oats, sunflover seed or bran pellets in our experiments. The naiv animals (29 males and fewales, or more 80 of the 36 laboratory experimental group feed more $2 \mathrm{~g}$ of pea, oats or sunflover geed pellets. Peed reaction to the bran peliets vas veaker. Average feeding of flour baits that kind like of this, was more then $2 \mathrm{~g} / \mathrm{night} /$ /repetability too ( $N=72$, field experiments in Leningrad and Novosibirsk Regions). Difference hardness of the grains, on the one hand, and the flour and the pellets, on the other hand, caused this results probably. The sucrose increased feeding of the pea flour from 88 to 33 (sucrose content was from 28 to 32 in the bait). The cooking salt increased feeding of the bait that kind like this to 98 (salt content was 18 ), and 28 content of the salt reduce this index. Thus, the pea, oats or sunflower seed pellets with several rodenticides may be us against the water vole.

ALL-RUSSIAN INSTITUTE OF PLANT PROTECTION, Podbelsky Sheste, 3 , ST.PETERBURG - PUSHRIN, RUSSIA, 189620.
NEN BIOPESTICIDES EROM ACTINOMYGGTWS PRODUCING BIOAUTIVE SECONDARY IIIIABOLI'LS

I.V. Bolkova, Ju. i..konev, 1.1. Novikova, V.A.Pavluskin

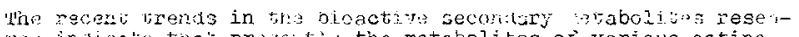

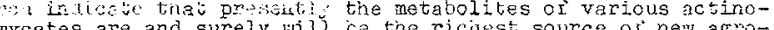
aycetes are and surely will ce the ricilest source oi new agro biologically useful areatis or leal coibounds. Up to our dass about 8900 are produedby actinomycetes. The overwhelming majority of these compounds are antibiotics (antimicrobial, anti-
fungal, antiviral compounds), but recently the number of the diPungal, antiviral compounds), but recently the number of the di-
scovered otber bioactive compounds (insecticides, herbicides) scovered other bioactive compounds (ingecticides, herbicides)
is considerably increasing. Owx work includes the screening, is considerably increasing. Our work includes the screening, isolation of the effective metabolites, the determination oz their biological activities and chemical formulae and the optimizationon their production. We have got the rich collection of Nicroorganism for Plant Protection (CNPP), which was registered in $700 P$ WDW und $\$ 760$ in 1994 . We have selected these strains from the soil of different geographic regions (Russia, india, China, Vjetnam, Italy) lihese strains form secondary metabolites with a potential either as antibiotics, or insecticides, or acaricides, or nematocides. We have prepared several new effective biopreparations: insecticides against different pests (apple, bean, peach aphid, spider mite, greenhouse whitefly. Chese biopreparation are altemative to the chemical pesicides. ruey arc nontoxic, non phytotoxic. Tle have made also several new biopreparation - plant-grow-helping stimulaters, fungicldes and antiviral preparations.

I.V.Bolkova, All-Rusaian Institute for Plant Protection, 3, Podbelskoe shosse, St-Petersburg-Pusbkin, 189620, Rugsia

652

TEE INDUCTION OF THE RESISTANCE TO VIRUSES USING BIOANTIOXIDANTS IS A NEW DIRECTION IN PLANT PROTECTION

\section{P.C.Chjntea, I.T. Lahmatova, T.D.Verderevscaia}

Viruses are known to cause disorders in plant metabolism associated with the energetic processes, namely, phytosynthetic and redox. The enzyme activation in the redox cycle leads to the shift of the competitive correlation of the enzyotic and freg radical oxidationg towards the free radical ore. The process is eccompanied by the relogse of free peroxide radicala that contribute to the 1nactivation of sulfhydril groupg of proteins, disgocletion and suppreagion of oxidative phosphorylation, deformetion, awelling and aticking, and sometimes destmaction of mitochondria, activation of autolysis processes. The biological mechanism of the bioantioxidant ection consists in the shift of the competitive correlation towards enzymes. Bloantioxidants ragulate the degree of the guppressive effect of the free radical oxidation on most metabolic processeg. The application of nontoxic antioxidants in physiological concentrations intensifies the system of natural bioantioxidants and enhences its efficiencies. In this connection, naturally-occurring compounds, possesalng antioxidative properties, nemely sterold glycosides (SG), were studied as inducers of plant resistence to viruses. It has been found that $S G$ may reduce the infectivity of tobacco mosalc virus by 30-70\%, leaf necrotic ring spot virus of atone crops by $39-56 \%$, plum pox virus by $58 \%$. It has been shown on the MU-tometo model that the decrease in the pathogen infectivity occurs due to the activation of protective systems of host plants, i.e. enhancement of rhibonuclease activity, alteration of cell protein composition etc.

P.C.Chintea, Ingtitute of Geneties, Acad. Sci. 20 Padurilor str. Chisinau 277002 Moldova 
THE INFLUENCE OF CHEMICAL IMAUNIZERS OF THE STEROID NATURE ON THE DEVELOPMENT OF TOBACCO TRANS PLANT SEEDLINGS

\section{N.S.Rigociuc, E.D.Calchei, P.C.Chinteg}

Tobacco is affected with fungal, bacterial and viral diseases during the period of transplant seeding growing. Seed presowing treatment is the most ecologically pure method to induce plent resistance to abiotic and blotic environmental factors. Chemical immuizers are capable, one way or another, of raducing pethogen activity and enhancing the trangplant seedliag resistence to pethogens. While atudying the sction of immunizers on germination energy it was established that seed trestment with these substances decreases the period of their germinetion. Subsequent observation of transplant geedlinga under conditiong of the greenhouse showed that all the developmental stages of transplant seedings produced from the treated seeds initiated earlier than in the control. The experimental transplant seedlings were not affected with seedling rots, bacterial spot and bronzetop of tometo which tella about the increase in the general resistance of plants. Seed presoking and immunizer treatment of seedlings at the stage of ear initiation reaulted in a better development of seedlings in the experiment in comparison with the control and where only seeds were trested. The output of the standard seedlings is bigher by an average of 180 planto/sq in than in the control. Seedling belght, weight of dry mags, dry roots follow the seme regularity, and the duration of the period of seedlings reduces by 6 days. The area of nursery may be cut by $10 \%-15 \%$ due to the maximua output of the standard transplant seedings. Plant reslatance to pathogens and yielding capacity increase.

P.C.Chintea, Institute of Genetics, Acad. Sc1, 20 Padurilor str. Chisinau 277002 Moldova

655

SYNTHESIS AND BIOLOGICAL ACTIVITIES OF NEW FUNGICIDAL DIFLUOROMETHOXY ARALKYLAMINOPYAMIDINES.

Katutoshi Fuji, Masaru Moritomo, Toshinobu Tanaka,

Yasuhisa Fukuda, Yoshinori Yamanaka

In our research program leading to the the discovery of agricultural chemicals by modifying aminopyrimidines, difluoromethoxyaralkylaminopyrimidines were synthesized, and thelr biological activities were examined.

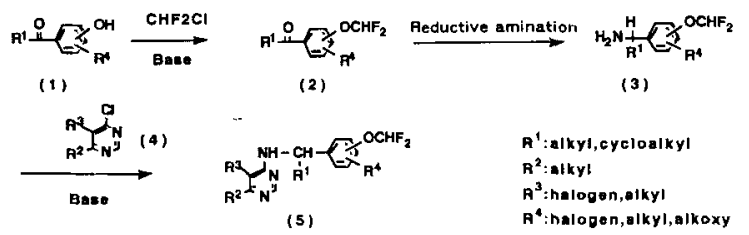

Fle Synthesle route of aralkylaminopyrimidines $(s)$

These compounds showed potent fungicidal effect (E.graminis of barley, Precondita of wheat). Modifications of these compounds were examined by changing substituents of pyrimldine ring, of benzyl position and of benzene ring. The structure-activity relationship is shown as below.

F1: $\mathrm{CH} 3, \mathrm{C} 2 \mathrm{H} 5>\mathrm{C}-\mathrm{C} 3 \mathrm{H} 5>\mathrm{i}-\mathrm{C} 3 \mathrm{H} 7$

P2: $\mathrm{C} 2 \mathrm{H} 5 \geqq \mathrm{CH} 3 \geqq n-\mathrm{C} 3 \mathrm{H} 7$

$\mathrm{R} 3: \mathrm{C}: \geq \mathrm{Br}>\mathrm{F}>>$ alkyl

R4: $\mathrm{H}>$ halogen, alkyl, alkoxy

Substituted position of OCHF2 in benzene ring : $\quad 4$ - position> 3-position

As a result, $( \pm$ )-5-chioro $\mathrm{N}$-[1-[4-(difluoromethoxy)phenyl]propyl]-6-methlpyrimidin-4 amine (UBF-002) was selected as a candidate. It is specifically effective to powdery mildew and to rust on cereals and omamental plants but there is no cross-resistance between this compound and triadimefon. Therefore, it can be presumed that the mode of action of this compound is different from DMI fungicides. UBF-002 is now being ceveloped as a novel type of fungicide.

Agrochemical Research Department, Ube Research Laboratory,

Ube Industries Ltd., 1978-5 kogushi, Ube city, Yamaguchi-ken 755, Japan
THE PROSPECTS OP PHYTOREGULATOR APPLICATION IN PLANT PROTECTION

G.L.Hatevosyan, A.I.Drizhachenko

Some ecological, phyolology-blochemlcal and pharmacological aspects of phytoregulator application in control of weeds, pests and diseaser are discussed with speolal reference to interactions of pesticides ith plent hormonal system. Tho regulatory mecbani ams which able to 1 noresse the crop plants adaptab1lity and dooreage their susceptibility to pests and diseases on the basi $B$ of application of growth regulators are examined.

The moin relations bave been revealed and the rundemantals bave beon elaborated of phytopharmacological applioation of phytoregulators - groth otimilators (aurine, gibberolins, oftokinins) and growth inhtoitors (ABA, etholono groducors, rotardante) - as meang of pest and disease managenant. 8poolal consideration is belng given to the aohievements in creation of antidotes and psevdofungs 01 des and in application of these preparations in moderm agricultural preotioe. Fermentative and phytohormonal modieloations in pertiolde influence have been analyzed by mimerous examples. Whe mechandsms of inductive adeptation have been revealed. Whioh are baged on Inorease of nonspectfio real stance capacity of plant della as a rebult of growth regulators influenoe. the regulta of an invest1 gation into new polynuctionai phytoregulators of the type of BII and BBF (tMbifos, bify phy difoset) are presented. Their high growt stimatory actlitj is acoompanied by impact on Inoxease of reglatance of potato to bllght, oucumber to powdery mildew and anthrakmopo, oabbage to blackensing and poot sirellins. The phrtoreghLatory erfect of these preparations was found to be che to stimiation of endogente phytohormones, most notably the cytokinting. The new phytoregulators deorease membrane conduetance of plaanalemma and tonoplest sugge日ting that cell re81stance to external influencies is increased. The revealed effecta probably determine the blnary action of nep plutoregilators as Doth Groxth atimulators and Induotors of plant reasitance.

The jo1nt epplication of phytoregulators and pestio1des, 1ncluding thair prolonged fortus and compositions based on biopolymer chitozone, was demonstrated to be of great practical and enviromental importance in cultivation of vegetabies.

Basseynata str. 71 ap.21.8t.Petereburg H-211, 196211, Ruegta

\section{6}

INVESTIGATION OF RELEASE KINETICS OF RADIOLABELLED HERBICIDES IMUOBILIZED IN YARIOUS POLYMER MATRICES AND APPLICATION TO WEED

M.GÜRER, A.KARTAL, M.ERCAN, M.MUTLU

Glyphosate and Propanil used in different phases of crop growth of plants were immobilized by several types of polymer matrices. The dynamic behaviour of the immobilized and free herbicides were followed after labelling with radioisotopes such as $125 I$ ( $\tau_{\frac{I}{2}}=60$ days ) and

$6 S_{Z n}\left(T_{\frac{1}{2}}=245\right.$ days $)$. The kinetics of controlled release of labelled herbicides were studied in batch and continuous systems. The optinum impobilization technique which maintains the first order release kinetics were applied to the pot experiment studies for biological effectiveness and transfer to/ftom plants.

In 80 days, after application, the inhibition rates of Lolium sp. were62\%, $86 \%$ and $45^{\%}$ for Glyphosate Ca-alginate, Glyphosate gelatinmatrix and Glyphosate a.i. respectively. This inhibition rates were $50 \%$, 68\% and 45\% for Propanil Ca-alginate, Propanil gelatin-matrix and Propanil a.i., respectively.

Phytotoxic and inhibition effects were not observed on test plants (maize and rice).

Forest Research Institute P.0.Box 24,06501 Bahçelievler, Ankara, TÜPKIYYE 
PHYTOMINERALOTHERAPY BY THE USEFUL ION OF POTASSIUM OR SODIUM BICARBONATE

\section{Yasuo Homma}

A part of organic synthesised compounds hecame social problems through their environmental pollutions, and resistant pathogens or tolerant insects and weeds for them did also became a big problem worldwide. In such circumstances, we succeeded in developing fungicides in Japan, namely potassium bicarbonate base Kaligreen $\$$, sodium bicarbonate base Harmomate and copper sulfate base G-fine.Namely 18-Crown-6 or 15-Crown-5 solutions $\left(10^{-2} \sim 10^{-1} \mathrm{M}\right)$ taken in sodium ion $\left(\mathrm{Na}^{+}\right)$or potassium ion $\left(\mathrm{K}^{+}\right)$derived from sodium or potassium bicarbonate $\left(10^{-2} \mathrm{M}\right)$ were tested for effect on the conidial germination of Pyricularia oryzae or curative effect of cucumber powdery mildew Sphaerotheca fuliginea Sodium or potassium bicarbonate $10^{-2} \mathrm{M}$ solution did show a little inhibitory effect on conidial germination of $P$.oryzae or curative effect of cucumber powdery mildew S. fuliginea. However, the solutions of 18 -Crown-6 or 15-Crown-5 complex salts had inhibitory effect 10 or more times. Therefore, we formulated sodium or potassium bicarbonate and copper sulfate with giycerides. By the results of many trials for formulating them, we could get better formulated sodium, potassium bicarbonate or copper sulfate. They were registered as fungicides. Namely (1) Potassium bicarbonate formulations $800-1000 \mathrm{ppm}$ were able to suppress strawberry, cucumber, tobacco, and rose powdery mildews.(2) Sodium bicarbonale formulation 800-1000 ppm was also able to suppress strawberry, cucumber and melon powdery mildews. And (3) Copper sulfate formulation 80-160 ppm had preventive effect on cucumber angular spots etc, also bacterial or fungel diseases. The former two play a role in nutrition and are a material for photosynthesis. The latter is an element for some copper enzymes since plants sometimes become copper dificient. Therefore, we consider these formulations as phytominerals (plant therapy minerals).

The definitions of phytominerals :

1. To play controlling role for diseases or insects

2. To act as effective (nutrition, disease resistance etc.) on host plants.

I propose such products as the above mentioned functions called

"Phytominerals" and "Phytomineralotherapy" as science.

Fungitron Laboratory, The Institute of Physical and Chemical Research, Wako-shi,Saitama,351-01,Japan

PHYSIOLOGICAL STUDIES OF QUIZALOFOP-R-TERFURIL ON SOrghum halepense $L$.Pers

\section{E.Lequizamón, M. Gattuso, S. Gattuso, J.I.Vitta, E. Purricelli, D. Tuesca.}

A set of different experiments were designed to evaluate the performance of the herbicide according to:

a) different water status of the weed.

b) different additives of the herbicide.

c) different rhizome lengths.

All experiments were set up according to appropiate statitiscal designs \& replicates. Herbicide effects were evaluated through visual ratings and regrowth countings. Averages were separated through LSD techniques at 0.05 probability level.

A complementary series of anatomical \& histological studies on the foliar surfaces and the crown of the weed were performed, in order to explore herbicide effects The herbicide showed a good performance on rhizome bud control under different water status (pots under field capacity and $50 \%$ of field capacity), additives (crop oils \& emulsificants) and thizome lengths.

Epidermic surfaces examined through SEM (Scanning Electronic Microscopy) and TEM (Transmission Electronic Microscopy) showed different wax structures when grown under different water status. Cuticle width and stomata numbers were also different.

Crown tissues showed the effects of herbicide translocation, primarily in floem and then extended to xylem and parenchyma. Crowns of piants grown under water stress showed higher sylica deposits

Cátedra de Malezas y Botánica. Dptos de Producción Vegetal y Biología y Recursos Naturales. Facultad de Ciencias Agrarias. Universidad Nacional de Rosario. C.C 14. (2123) .Zavalla.Universidad Nacional de Rosario. Argentina.

\section{CALIXIN AS PLANT PROTECTANT AND PLANT GROWTH REGULANT OF TEA PLANTE}

\section{Lalithakumari}

Calixin is developed as a fungicide but its plant growth regulating effects are well documented in Tomato plants (Rathnavathy, 1989). Similar plant growth regulating activity was observed on tea plants when used against blister blight. Calixin increased the leaf area, leaf length, chlorophyll a and b, carotenoid, nucleic acids, proteins, auxin, phenol and amino acid contents in tea leaves. Treatment with Calixin has caused a general hypertrophy of all the tissues of the leaf. However, basic organization and number of cell layers of the leaf remain unaltered with calixin treatment. The stomatal size has been increased, which naturaliy reduces the number of stomata per unit area. Increase in cell size coupled with increase in the size of the air spaces may be presumed to increase the photosynthetic efficiency of the leaves on treatment with Calixin. It is presumed that calixin inhibits ergosterol sypthesis and $G A$ synthesis through inhibition of Itc-demethylation reaction thereby juducing isopyrenoid pathway. The interference of 14 c-demethylation consequently block sterol and gibberellin biosynthesis. A shift in the balance of sterol and important plant hormones including gibberellins, abscisic acid, cytokinin and IAA might account for calixin's plant growth regulatory activity. The uitimate effect could be dependant on the dynamic equilibrium attained by these hormones at a specific stage of composition and the physical properties of tea shoot tips that renders them uniquely suitable for the manufacture of tea beverage products.

D. Lalithakumari, Centre for Advanced studies in Botany, University of Madras, Guindy Campus, Madras 600 025, India.
660

EFFECTS OF SEED COATING FORMULATION ON THE HISTOPATHOLOAY AND ULTRASTRUCTURE OF ERYSIPHE GRAMINIS F.SP. TRITICI IN WHEAT

\section{J. Y. Li, Z. S. Kang, C. Y. Shen}

Seed coating treatment has been proved to be an effective way in controlling seedborne and soilborne diseases and insects at seedling stage. Sixteen seed coating formulations (SCF) for different crops have been developed at Beijing Agricultural University and most of them have been manufactured and used in different parts of China. The seed coating treatment offers an integrated pest control in one operation before planting, improves the seed sowing quality and enhances the production yield substantially with savings of seeds, chemicals, labour and cost. The total extension acreage in China was about 8.7 million ha. for the period of 1984 to 1993 , increasing the total production yield of grain, cotton and oil crops about 4.4 million tons with estimated profit of 640 million US dollars. SCF No. 17 is an effective formulation against wheat powdery mildew at seedling stage and is widely used in several parts of China.

Seedlings from wheat seeds coated with seed coating formulation (SCF) No. 17 which contains fungicide triadimenol and other chemicals were inoculated with a virulent isolate of Erysiphe graminis f.sp. tritici. Observation by light and electron microscopy showed that SCF No. 17 increased frequence of malformed appressorium and decreased successful infection considerably. Only primary haustorium was formed in each infection site and usually became malformed. There were some electron-dense materials in extrahaustorial matrix. Mycelial growth and branching were strongly inhibited and mycelial tips were swollen obviously (see the table). Accumulation of small vesicles between mycelial and haustorial plasmalemma and their walls made their walls thicken irregularly. Necrotic host cells in infection sites were similar to hypersensitive necrosis of resistant cultivarar.

Table Effect on the haustorlum development of powdery mildew

\begin{tabular}{ccccc}
\hline \multirow{2}{*}{ TRT. } & \multicolumn{4}{c}{ length of haustoria $(\mu \mathrm{m})$ of different time after inoculation } \\
\cline { 2 - 5 } & $24 \mathrm{~h}$ & $48 \mathrm{~h}$ & $72 \mathrm{~h}$ & $96 \mathrm{~h}$ \\
\hline CK & $18.2 \mathrm{a}$ & $64.3 \mathrm{a}$ & $80.7 \mathrm{a}$ & $93.5 \mathrm{a}$ \\
1 & $14.7 \mathrm{a}$ & $33.8 \mathrm{~b}$ & $40.1 \mathrm{~b}$ & $45.6 \mathrm{~b}$ \\
$\mathrm{I}$ & $12.3 \mathrm{a}$ & $16.5 \mathrm{c}$ & $15.7 \mathrm{c}$ & $17.3 \mathrm{c}$ \\
\hline
\end{tabular}

Jinyu Li, Seed Coating Chemicals R/D Center, Beijing Agricultural University, No. 2 W. St. Yuan-Ming-Yuan, Beijing 100094, P. R. China 
MODERN SPRAYING TECHNOLOGIES FOR RATIONAL AND SAFE APPLICATION OF PLANT PROTECTION MEANS

Lysov A.K. Nikitin N.V., Makalsky L.H., Tcirin A.A.

The further improvement of spraying technology is directed on the reduction of the dose of pesticides without decrease of biological efficiency, on reduction or complete exception of blowing off of small drops during the treatment and also on elimination of large drops flowing down from the treated leaf surface.

Blowing off of small drops and large drops flowing down from the treated leaf surface leads to wasteful losses of pesticides and pollution of the environment. One of the ways of increasement of the efficiency and ecologisity of protective measures is the passage on the spraying technology with the small drops separation and also application of electrostatic spraying systems.

So, the spraying technology with the small drops separation permits to exclude the penetration into the environment of drops with the size up to $60 \mathrm{~m}$ and large drops flowing down from the treated leaf surface. Due to the small drops separation the volume of drops with the size more than $250 \mathrm{mkm}$ does not exceed $5 \%$ from the total volume of the dispersing liquid.

The tests of the spraying technology with the small drops separation bave shown its essential advantages on comparison with the conventional spraying. Ecologisity, small dose of a working liquid ( $10 \ldots 20 \mathrm{l} / \mathrm{ha}$ ), maintenance of 2 times decrease of the dose of pesticides permit manufacturers of agricultural production to reduce essentially the costs of the measures of plant protection.

Studies and tests of the spraying technology with the drops electrocharging have shown, that the given technology enables a 5 ...10 times reduction of the recommended dose of pesticides on a number of cultures without decrease of biological efficiency. Drops electrocharging solves the problem of blowing off into the environment of small-sized drops and exclude the problem of large drops flowing down from the leaf surface on the earth because only drops with the size less than $100 \mathrm{mkn}$ can be electrocharged. Creation of devices, ensuring continuous control of electrocharging process increases the perspectiveness of the wider use of this technology in practice.

ALL-RUSSIAN INSTITUTE OF PLANT PROTECTION, Podbelsky Shosse, 3, ST.PETERSBURG-PUSHKIN, RUSSLA, 189620.
EFFECTIVENESS OF TEBUCONAZOLE FORMULATIONS IN THE CONTROL OF YELLOW RUST OF BARLEY (PUCCInia strifformis f.Sp. hordeil IN MEXICO.

C. Mendoza-Zamora, E. Hernández-Mendieta \& s. RomeroCova. $\varepsilon F$. Ponce

Yellow rust of barley (Puccinia strifformis f.sp. hordei) has become the main disease of this crop in Mexico since 1988, and Folicur $250 \mathrm{C.E}$. (tebuconazole $250 \mathrm{C} . \mathrm{E} .1$ has shown to be the most efficient fungicide to control this disease. Nevertheless, in an attempt to find sorething bettex to control the pathogen, the new fungicides and formulations available in the barley growing areas of Mexico were evaluated. According to the results it may be concluded that at all dosages tested $(0.5,0.4,0.35$ and $0.31 / \mathrm{ha})$ tebuconazole 250 E.W. (Folicur 250 E.W.) protected the cultivars Esmeralda and Esperanza against yellow rust for 35 days after application, having a mean efficiency of 98.7 and 95.98 respectively during all this time and in the two areas where it was tested. The Fluzilazole (Punch 40 C.E.) and tebuconazole 360 S.C. (Elite 360 S.C.) had almost the same efficiency, 51.9 and $51.6 \%$ respectiveiy; whereas tridemorph (Calixin 750 C.E.). Hexaconazole (Anvil S S.C.) and propiconazole (Tilt 250 C.E.) showed percentages of efficiency lower than 398 . The calixin (1.0 1/ha) protected well the crop only for 10 days, and Tilt as well as Anvil gave protection for approximately 15 days but it was not satisfactory.

Universidad Autonoma Chapingo. Departamento de Parasitología Agrícola. Km. 38.5. Carr. Mexico-Texcoco. C.P. 56230. Chapingo, Edo. de Mexico. MEXICO.

\section{3}

SEED TREATMENT WITH TEBUCONAZOLE + THIRAM AND TEBUCONAZOLE + IMIDACLORRID FOR THE CONTROL OF BARLEY DISEASES AND THE RUSSIAN APHID (Diuraphis nOxia).

\section{Mendoza-zamora, E. Ponce-González \& E. Hernández} Mendieta.

The yellow rust (Puccinia strifformis west) reduces the yield of barley, in Mexico, to 50\%, and is more severe when it attacks seedlings of late sowings: the net blotch (Helminthosporium teres), considering economical importance, is the second disease of barley, and the Russian aphid the first pest of the crop during early stages of development. In order to reduce damages by these pests the effectiveness of the mixtures tebuconazole + thiram (Raxil + Thiram) and tebuconazole + imidacloprid (Raxil + Gaucho) were evaluated. The raixture tebuconazole + imidacloprid protected the barley against yellow rust, net blotch and the aphid D. noxia for a longer period of time than the other mixture. Similarly, the tebuconazole + imidacloprid mixture at the dosage of $1.0 \mathrm{l}+0.07 \mathrm{~kg} / 100$ $\mathrm{kg}$ of seed better controlied yellow rust, net blotch and Russian aphid than at the other dosages tested. Such a dosage protected barley for 56 days against heavy incidences of yellow rust; however, it did not so against H. teres. On the other hand, $D$. noxia was satisfactorily controlled if $0.07 \mathrm{~kg}$ of the insecticide imidacloprid was added to the mixture. The seed treatment with tebuconazole + thiram did not control either yellow rust or net blotch as it was expected.

Universidad Autónoma Chapingo, Departamento de Parasitología Agricola, km. 38.5 Carr. Mexico-Texcoco. C.F. 56230. Chapingo, Edo. de Mexico. MEXICO.
664

\section{DIRECT IDENTIFICATION OF SULFONYLUREA BERAICIDES AND ITS MASS SPECTRONETRY (TOF-PDMS)}

Vadym D. Chivanov, Aratoly K Mishnev, Victor K Kabanetz, Nikolai S. Kravchenko, Vera N. Sarbash, Tat'yana A. Onychko, Ludmi la 1. Grebenik, Sergey A. Aksyonov

Ihe increasing use of "soft" mass spectrometry methods [1] for pesticides and its metabolites in real samples is chiefly the result of their sultability for thermaly labile. the result of their sultability for thermally labile, non-volatile and highly polar pesticides [2], including sulfonyiurea herbicides. TOF-PDM is therefore becoming an
inportant tool for simultaneous analysis of the different inportant tool for simultaneous analysis of the different
sulfonylurea herbicides and its metabolites in biomatrixes. Sulfonylurea herbicides and its metabolites in biomatrixes. multicomponent extracts from plant tissues. In addition to selectivity, use of a TOF-PDM offers herbicides and its metabolites identification and can eliminate sample clean-up cteps, We applied the method for the aralysis of
metsulfuron-methyl DPX-T6376 (1) and chlorsujfuron DPX-W4189 metsulfuron-methyl DPX-T6376 (I) and chlorsujfuron DPX-W4189
(II) in model system. The basic components vere (I), (II) and (II) in model system The basic components vere (I), (II) and
crude unfractionated gomogenate from wheat leaves. Samples. were crude unfractionated gomogenate from wheat leaves. Samples were
generally prepared as a $1: 1: 100(\mathrm{w} / \mathrm{w})$ pixture of the generally prepared as a $1: 1: 100$ (w/w) mixture of the
(1). (II) and multicomponent biomatrix. Upon drying, the sample (1), (II) and milticomponent biomatrix. Upon drying, the sample
formed a thin amorphous Iilm on gold or silicon slides. All
analysis were done using a commercial TOF-PDMS mass spectrometer MSBX (PA"Selmi", Ukraine). The abundant ion sygnals are observed at $\mathrm{N} z 358,2 ; 396,8 ; 434,8$ and $\mathrm{m} / \mathrm{z} 382,0 ; 420,1 ; 458,2$ which correspond in mass to the molecular complexes [(II) $+\mathrm{H}]+$; I(II)+KI+; [(II) $+2 \mathrm{~K}-\mathrm{H}]+$ and $[(1)+\mathrm{H}]+; \quad[(\mathrm{I})+\mathrm{K}]+;[(\mathrm{I})+2 \mathrm{~K}-\mathrm{HI}+$; I(I I $+\mathrm{KI}+;[(\mathrm{II})+2 \mathrm{~K}-\mathrm{H}]+$ and $[(1)+\mathrm{H}]+;[(\mathrm{I})+\mathrm{K}]+;[(I)+2 \mathrm{~K}-\mathrm{HI}+$,
respect ively. The spectra contains the protonated hydrolysis respectively. The spectra contains the protonated hydrolysis
products: triazine amine at $\mathrm{m} z$ z 141 and other fragment ions at
$\mathrm{m} / \mathrm{z} 168,200$. A target detection levels rere $0,1-0,5$ $\mathrm{T} / 2$ 168, 200. A target detection levels vere $0,1-0,5$
Hg/sample. TOF-PDN has emerged as a sensitive and selective
analytical methodology for the simultaneous identification of analytical methodology for the simultaneous identification of
two different sulfonylurea herbicides in a fast and efficient yay. This methodology is especially applicable to low minimal clean-up prior to mass spectroscopy determination.

Feferences

1. L. M Shalaby, S. George //Liquid Chromatography/Mass Spectrometry, ACS Series, vol. 420, $1990,76$. 2. G. Allmaier, E. R. Schmid, P. Roepstorff //Bioned. Environ. Mass Spectrom, 1990, 19, 75.

Department Crop Protection, Agricuitural Institute, Petropavlovskaya Str., 57, Sumy-30, 244030 Ukraine. 
THE RESISTANCE REGULATION OF POTATO PLANTS TO DISEASES WITH USE OE PHYSIOLOGICAL ACTIVE AGENTS

\section{A.N.Vedeneev, V.P.Deeva}

Potato plants with different genotypes distinguishing on resistance to phytophthora infestans were treated with quartazine or fungicides (ridomil and cuprozane) and their for budding stage. The resistance regulation to fungous diseases was estimated through the monitoring of ghytoalexins (rishitin and lubimin) accumulation. The quartazine and the mixture spraying induce the de novo synthesis and accumulation of phytoalexins in the potato leaves in 5 hours. The unresistant cultivar reaction was essentialiy weaker than that in the resistant one. In result of the chemical effect, the dissociation of accumulated phytoalexins starts in s days but their levels are higher several times in comparison with control. In 7 days some plants were infected with Phytophtora infestans zoospores and isolated from the healthy ones. The peak of phytoalexins concentration accumulated de novo was concidered as a response of infected plants to the treatment. However, the highest peak levels were observed in the variants treated with quartazine. The cultivars differed essentially in peak amplitudes. The plants exhibited weak reaction to the infection after fungicides (without quartazine) spraying. In next 20 days the variations of the phytoalexins level were observed in the infected plants. Evidently, the variations were stipulated by new sporeing cycles of the fungus. Nevertheless, the tendency to phytoalexins accumulation increase in the plants treated with quartazine in comparison with non-treated plants was observed. The same tendency was shown in non-infected plants. In 30 days after treatment the cesistant cultivar plants were characterized by phytoalexins level 2-3 times higher than that in the unresistant ones. The data obtained demonstrated that the treated plants before the possible infection acquire and maintain heigher resistance to the disease over a long period of time. It was shown that quartazine does not posses the direct fungicide activity. obviously, quartazine changes the plant's metabolism and creates unfavourable conditions for fungus development by means of increasing the level of antibiotic metabolites phytoalexins accumulation.

Inst.Exp.Botany, Belarus Acad.Sci.,F.Skoryna 27., Minsk 220733 . BELARUS.

\section{S. C. Anand}

Soybean cyst nematode (SCN), Heterodera glycines Ichinohe is a serious pest of soybean (Glycine max L. Merr.). Arnong the resistant soybean germplasms, plant introduction (PI) 437654 is the only line in U.S. Soybeat World collection which is resistant to all known races of $\mathrm{SCN}$ in the United States. PI 437654 is a black seeded line in maturity group III which is agronomically very poor and shatters at maturity. This line has extensively been used in breeding programmes to evolve SCN resistant cultivars. Hartwig, a soybean cultivar in maturity group $v$ was developed from the cross PI $437654 \mathrm{x}$ Forrest ${ }^{3}$. Both PI 437654 and Hartwig appear to be almost immune to SCN. Efforts to develop an SCN population which could parasitize these soybeans have beex unsuccessful. The juveniles in the cysts which may, sometimes, develop on PI 437654 or Hartwig are incapable of producing any viable female adult in the subsequent generation. The observations of the nematode in plant roots indicated that both male and female juveniles could enter the roots of PI 437654 and Hartwig. Although, several males developed into adults, female juveniles ceasied to develop beyond $J_{3}$ or $J_{4}$ stage. Genetics of resistance to SCN indicated the presence of one dominant and two recessive genes conditioning resistance to each of the races 3 \& 14 in the crossing FI 437654 (R) $x$ Essex(S). The resistance to race 5 was controlled by two dominant and two recessive genes in the same cross. Altrough resistance to any individual race in PI 437654 did not appear to be different from those of other resistant lines, it is the only germplasm line which has an accumulation of all the genes required for complete resistance to aII SCN races. Because of the large number of resistance genes in PI 437654 providing broad spectrum of resistance, this line is likely to provide longer lasting resistance to $\mathrm{SCN}$.

University of Missouri-Columbia, P. O. Box 160, Portageville, MO 63873 , USA.
DEVELOPMENT OF SUGARCANE VARIETIES WITH RESISTANCE TO AUSTRALIAN CANEGRUES

P.G. Allsogr, T.K. MCGhie, G.R. Smith, R. Ford, M.C. Cox

Management options to minimise the impact of canegrubs on Australian sugarcane are heavily dependant on the use of synthetic insecticides. In developing a successful IPM program for canegrubs, control options must be broadened. Flant resistance offers the potential for an easily deliverable and environmentally acceptable mänagement option. Previous anecdotal reports suggest that Australian cultivars vary in their resistance to canegrubs. We have screened many sugarcane clones (from the Saccharun complex) and have shown that they do vary in their resistance to reduction in tops, roots and stubble and in their effects on canegrub development and survival. Froteinase inhibitors and lectins with activity against canegrubs have been identified and are being introduced into the sugarcane genome. Toxins from Bacillus thuringiensis have shown little potential and are not being used further. We present a rationale of using resistant plants as a control option and of its incorporation into the breeding program. Projected research will focus on screening more available sugarcane clones for resistance, identifying chemical mechanisms involved in antibiotic effects, screening further antimetabolites for possible incorporation into the genome, and proving the potential resistance of geneticaliy engineered plants

Bureau of Sugar Experiment Stations, P.O. Box 651, Bundaberg 4670, Australia

668

GENETICS OF RESISTANCE TO YELLOW (STRIPE) RUST IN CIMMYT WHEATS

Hari P. Bimb and Roy Johnson

CIMMYT (International Maize and Wheat Improvement Center) wheat cultivars are grown on more than $\mathbf{4 0}$ million hectares in many developing countries. Resistance to the three rusts is an important breeding objective and yellow rust, common in cool maritime or high altitude cultivation, has received less antention than the other two rusts. The project is to understand the genetics of resistance to yellow rust in CIMMYT cuitivars, including some from Nepal and Pakistan. Tests with many CIMMYT cuitivars, including some from Nepal and Pakistan. Tests with many the gene $Y_{r} g_{\text {often in }}$ association with $Y r 7$ and $Y_{r} A$. Until recently, $Y r 9$, carried on the chromosome 1B-1R, remained effective in Pakistan but virulence has now occurred and some cuitivars have become highly susceptible. Tests of adult plants, using a race with virulence for $Y r 7, \operatorname{Yr} 9$ and $Y r A$ show that some cultivars are susceptible, but some have good adult plant resistance. In some cases this may be derived from the durably resistant source Anza and be potentially durable. Crosses between resistant and susceptible cultivars have been developed to F3 to investigate the genetic basis of their resistance.

John Innes Centre, Colney, Norwich NR4 7UH, UK 
USING ADAPTIVE POTENTIAL OF CULTIVATED PLANTS FOR RESISTANCE TO DISEASES AND PESTS

Chesmokov Yu.V., Bomozentro I.V.

The intensiffoation of plant industry on tise basta of wide application of high-ylelding varietios and plant uybrids, fortilization, pestioldes, Irrigation and moohanization wothoda ensured the significant inorease of the yield capsoity of main agricultural aropa. The neceasity for algniflcant increasing of yleld capacity of cultivated plents on the basia of raising their total and speosic adeptivity requires the developuent of efficient methods which allow to provide the purposeful manipulation by chromosomes and gene blocka of the given specier, genus, femily. One of the atepe in solution of thio problem is the increasing of the arallable genotypic rariability and partioularly the recombination apectrum in interepecteio and intergener1o hybridization. An 1mportant place in plant adaptivo breeding takes the derelopment of varietieg reslatad to difeaser and peats. We are of opinion that plant resistance to pathogen demages (mostly in the coses of horisontal reslotance) la to be congldered taking into sooount the perculiarities of the genetio nature of adaptire reactions.

In the system of meang of plant diseaser and peste oontros a apecial attention must be paid to the integrity of "host-peraofte-environment" aystem along with adaptive breeding and the conplexity of breeding- and agratechnologiael programs. In thif connection of comparable intereat are from our point of vien the poselbilitieg of uaing a difference in the rateg of cultigen ecotype formation and pathogen sace formation procesa and also $a$ wider study of the practical application of regulator compounde (horrenes, pheromonee etc.) and sethods of cell and genetlo engineering (genetic trasformetion, oomatio hybridization) for derelopment of resiatance to dieeser and pesta in plant protection.

Institute of Genetica, Moldarian Academy of Selences, Leanaya 20 Kishiner 277018 , Holdors

671

WHEAT CYTOPLASM AS A POSSIBLE FACTOR OF BIOLOGICAL CONTROL OF THE LEAF RUST ADAPTATION

\section{E.A. Voluevich and A.A. Buloychlk}

Adaptation of 5 leaf rust clones, differed in virulence, wos analised of ter their long-term passaging on seedlings of 7 wheat introspecies cytoplasm substitution lines with nucleor genome of $\mathrm{cv}$. Leningradka and cytoplasms of Triticum aestivum cultivars developed by prof. 0.6. Davydenko. Significont effect of plant cytoplasm on reproductive ability (per $1 \mathrm{~cm}^{2}$ of leof area) of reisolates produced ofter 20 passages of the clones on seedlings of the lines was reveoled.

A most part of fungus clones increosed reproductivity ofter long-term coevolution of host-plant and pothogen. Reisolotes of the low aggressive clone $K 5$ had reduced spore formation on the line with cytoplasm of the mutant form 1003 up to the control level (cv. Leningradko) while reproductivity of initial clone exeeded it 1.5-fold. Two such cases of pathogen reproductivity reduction under long-term passaging was observed for reisolates of the 20-th passoge of the low aggressive clone $\mathrm{N} 37$ on lines with cytoplasms of 1003 and cv. Kubanka. For reisolates of high aggressive clones there were no cases of reduction of their adaptive capacities, however reproductivity of some reisolates wos lower than on the cr. Leningradko.

The decrease and preservation of reproductivity of some clones of leof rust ofter passaging are evidence of the cytoplasm influence on the pothogen adoptation process. Hence, some cytoplasms of host-plant can be a genetic barrier in pathogen adaptation.

Institute of Genetics and Cytology, Belarus Academy of Sciences, F. Skorina st., 27, Minsk 220072, Belarus. A NEW RESISTANT GENES TO PUCCINIA RECONOITA
F. SP. IRITICI IN ONE WHEAT BACKGRDUND

*Boskovie, J., Boskovit, M., Prodanovic, G. The best way to control the agent of wheat leaf rust (Puc-
Elnid recandita $f$ sp. tritici) is the development and use of resistant varieties. To successfully differentiate paraItic papuletions and to obtain usable sources of registance, it is necessary to have a sufficient number of effective resistance genes. In international analyses of Puccinis recandita f. sp. triticl population of European-Mediterranean regions isogenic Lr resistant lines used, have not shown satisfactory results. It has becone clear severa) years ood that these regions meed now, more officient differential resistance genes, as well as sources of resistance. From International Wheat Rusts Nurseries after several years of testing, eighth genetically different sources of resistance were selected and crossed with recurente parents varleties Princ and Strake. Hybrid combinations of the first backcrosse ( $F_{1} b_{1}$ ) have been tested in the seedling - tage with threp International cultures of $P$. recondita $\frac{t r i t i c l}{10}$ (Yu-13-19-1, H-13-9-1; and $\mathrm{CZ}-13-\mathrm{Ar}-3$ ). The ratio in $F_{1} b_{1}$ of elghth hybrids have shown the presence of one two or three resistance genes. Inheritance of resistance were dominant, and complementary effect of three resistant genes were only in hybrids $66 / 4 \times 5$ tarke and $32 / 2 \times$ Starke. Considerable influence of recurente parent genotype to the number of resistant genes in donors used were demonstrated. Thus, the resistant donors 26 and 94 with Princ have had one gene pair, but the same donors with Starke two resistant genes. Differences were found among the lines with a Ingle resistance gene, and that was observed in one recurrent parent crossed with the donor possessing the same resistance gene. That means again, that the recurrent parent plays an important rola in the expression of resistance. On the other side, it was established considerable influence of the pathogen culture to the number af resistance genes in the donors used. The same crossing combinations tested with ane pathogen culture resulted in presence of two resistance gen
one resistance gene.

* Boskovic Jelena, Faculty of Agriculture, Institute for Plant Protection. Tra D. Obradovica G, 21000 Novi Sad, YugosIavia.

672

RESISTANCE OF SOLANACEOUS PLANTS TO COLORADO POTATO BEETLE, LEPTINOTARSA DECEMLINEATA SAY (COLEOPTERA: CHRYSOMELIDAE) IN CONNECTION WITH THE INTRA-SPECIFIC VARIABILITY OF THE PEST.

\section{S.R.Fasulati}

The problem of potato breeding for resistance to the Colorado potato beetle (CPB) is complicated by the fact that $C P B$ is a very variable species. According to our data (Fasulati, I988,1993,1994; Fasulati, Vilkova, Ivashchenko, 1994), CPB has a lot of genotypical forms (isoreagents and biotypes) in each its population. These forms have different feeding adaptations for species and varieties of Solanaceous plants. The adaptations of CPB forms are interconnected with the morphological features of insects, such as 9 morphs of pronotum patterns of imagoes and 4-5 colour variations of eggs. Many of such CPB forms have a different ability to develop on the same species and varieties, including the varieties which are known as resistant ones, of potato (Solanum tuberosum, varieties which are known as resistant ones, of potato (Solanum tuberosum,
S.andigenum, S.demissum, S. chaccense, S.commersoni, etc.), egg-plant (S.melongena), tomato (Lycopersicon esculentum). For example, the insect survival varies from 0 to $80-100 \%$ for the larvae of different morph of $C P B$ which fed on the same resistant potato varieties (Zov, Arina, some hybrids). We have also revealed one CPB form which is able to survive on Mexican groundcherry, Physalis ixocarpa.

The genetical variability of CPB provided a basis for its wide adaptability The geographical distribution of $\mathrm{CPB}$ is accompanying by intensive microevolutionary processes. The feeding conditions play a decisive role in $C P B$ microevolution. As a result, the geographical populations of the pest and the complexes of them are differing in genetical and phenotypical structure in different climatic zones. Thus, the differences between them in frequences of 9 pronotum morphs are significant ( $P<0.01 ; F$-test). Accordingly, such populations are differing in feeding and other ecological adaptations (Fasulati, 1988,1993). So the resistance of some plant varieties to CPB have a zonal specificity in field conditions. It has been shown for potato cultivars Arsy, Lugovskoy, Malakhit, Ogonyok, Omega, Zarafshan,etc., and for tomatoes. For example, the tomatoes are heavily injured by CPB nowadays in USA (Cantelo, Cantwell,1983) and in some places of Northern Caucausis (Hushtov,1994). We also observed the different survival of larvae (from 0 to $35.6 \%$ ) of the different european CPB populations which fed by tomato leaves in laboratory tests populations which fed by
(Fasulati,Vilkova, Ivashchenko, 1994).

In connection with the intra-specific peculiarities of the pest, it is desirable to investigate the Solanaceous plant resources and to evaluate new varieties for resistance to $\mathrm{CPB}$ in different climatic zones and in laboratory conditions. It will be useful for revealing the plant varieties with resistance to the most forms of CPB.

All-Russian Institute for Plant Protection, Shosse Podbelsky 3, St.PetersburgPushkin, 189620, Russia 
DESIGN AND USE OF A COMPUTER SIMULATION MODEL TO EVALUATE GERMPLASM FOR ANTIBIOTIC RESISTANCE TO ALEYRODIDAE.

\section{Wilant van Giessen, Chris Mollema \& Kent Elsey}

SARAH is a deterministic simulation model of whitefly population growth based on whitefy life-history components determined on individual plants (SARAH $=$ Software for the assessment of Antibiotic $\underline{\text { Resistance to }}$ Aleyrodidae in $\underline{H}$ ost plants). The model serves as a tool to combine these components and to obtain a single criterion for (antibiotic) resistance. This criterion is the decrease in simulated intrinsic population growth rate, $r_{s}$, relative to the $r_{s}$ value determined on a susceptible control genotype. This simulation-model based evaluation method offers a standardized way to quantify levels of antibiotic whitefly resistance and enhances efficiency in breeding programs. The evaluation method was tested using the greenhouse whitefly, Trialeurodes vaporariorum Westwood (Homoptera: Aleyrodidae), on tomato and the sweetpotato whitefly, Bemisia tabaci Gennadius (Homoptera: Aleyrodidae), on tomato, eggplant, collard, and pepper. A high correlation was found between results from a sensitivity analysis of SARAH and results from a similar sensitivity analysis of a validated whitefly population growth simulation model that was developed by Yano et al. (1989). A significant positive correlation was observed between simulated intrinsic population growth rate $\left(r_{s}\right)$ and sex-ratio (\# females/\# males) for $T$. vaporariorum on tomato.

USDA-ARS, U.S. Vegetable Laboratory, 2875 Savannah Highway, Charleston, South Carolina 29414, USA.

\section{CHARACTERIZATION AND INHERITANCE OF DURABLB LEAF RUST RESISTANCE IN} TWO INDIAN WHEATS (Triticum aestivum)

\section{R. G. Saini 1, A.K. Gupta 2}

Wheat cultivars HD2009 and "Kundan" have shown durable resistance to leaf rust (Puccinia recondita tritici) in India under a variety of environmental conditions. variants $77-1$ and $77-2$ of race 77 are predominant constituents of the Indian leaf rust race nora. Their presence in field tests eliminates the effect of all known $L r$ genes so far identified from Triticum aestivum. Therefore, wheats showing field resistance in an epiphytotic of these variants must carry new and as yet undescribed leaf rust resistance genes. Disease severity on both these cultivars does not exceed $30 S$ in field tesis against variants $77-1$ and $77+2$. In glasshouse tests the seedlings of these cultivars developed susceptible infection types against varlant $77-1$ but the adult plants were resistant (infection type $=$;), indicating that these two wheats carry hypersensitive adult plant resistance against variant $77-1$. The seedlings and adult plants of both these wheats, however, developed only susceptible infection types $(33+)$ against variant (77-2). Since varlant 77-2 showing virulence on adult plants of HD2009 and "Kundan" was also used for field tests, the hypersensitive adult plant resistance of these two wheats against variant $77-1$ is not involved in their field resistance. Although the seedlings and adult plants of RL6058 (Lr34), a source of durable leaf rust resistance developed susceptible infection types $(33+)$ against variants 77-1 and 77-2, the disease severity on RL6058 did not exceed $5 S$ in field tests. In contrast to this the disease severity on Agra Local and WL711 (Lr13) also showing susceptible infection types on seedlings and adult plants was 70S and 90S, respectively. The field resistance of HD2009, "Kundan" and RL6058 against a mixture of $77-1$ and $77-2$, was therefore, due to factor(s) which do not evoke hypersensitive response against these two variants. This non- hypersensitive response was evident in juvenile plants of HD2009, "Kundan" and RL6058. This also expressed as longer gap between uredial appearance and eruption on seedlings of
HD2009, as longer latent period on seedlings of "Kundan", and as longer latent period, smaller uredia and longer gap between uredial appearance and eruption on seedlings of RL6058 (Lr34). The field resistance of HD2009, "Kundan" and RL6058 appears to be due to the cumulative effect of one or more of such components of non- hypersensitive resistance over many rust cycles. Genetic studies on the fleld non- hypersensitive resistance over many rust cycles. Genetic studies on the feld
grown adult plants in $F_{1}$ and $F_{2}$ generations from crosses of cultuvars tiD2009 and grown adult plants in $F_{1}$ and $F_{2}$ generations from crosses of cultuars HO$^{2009}$ and
"Kund an" with Agra Local revealed presence of a partially dominant gene conferring low disease severity to each of these wheats in field tests against a mixture of variants $77-1$ and $77-2$. This gene in HD2009 and "Kundan" was identified as Lr34 (RL6058) through allelic tests.

1. Deptt. of Genetics, PAU, Ludhiana-141004 (Pb.), INDIA 2. Director, CSIR Complex, Palampur-176 061 (IIP), INDIA

\section{5}

TRANSGRESSION OF LATENCY PERIOD IN SSD F\& SPRING WHEAT LINES INFECTED WITH WHEAT LEAF FUST

Th. Jacobs and D.J. van der Gaag

Latency period (LP5O) was studied in Single Seed Derived F8 lines of crosses between spring wheat genotypes with high levels of partial resistance. Fiag leaves of plants from SSD F8 lines were inoculated with a monospore culture of wheat leaf rust, (Puccinia recondita f.sp. tritici).

In the crosses Westphal12A $\times \mathrm{BH} 1146$ (WE $\times \mathrm{BH}$ ) and Akabozy $\times \mathrm{BH} 1146(\mathrm{AK} \times \mathrm{BH}\}$ the relative latency period (LP5O) showed transgression towards susceptibility, no plants were found which showed a LP5O longer than the most resistant parent (table 1). In the F2 generation of the crosses with BH1146 transgression towards both shorter and longer LP5O was reported (2). but no transgression has been observed in the F3 generation of the part selected for longer LP5O in the F2 (1). It can be concluded that $\mathrm{BH} 1146$ contains a specific combination of resistance genes which is difficult to retrieve in $\mathrm{F} 8$ lines.

In the cross Westphal12A $\times$ AKabozu (WE $\times$ AK) transgression towards both longer and shorter LP5D was observed. In the cross between Akabozu and Westphat12A no shorter LP50 was observed. In the cross between Akabozu and Westpha112A no transgression was observed in the $F 2$ generation $\{2\}$, transgression towards both sides
was observed in the $F 5$ generation (1). These results indicate that accumulation of resistance genes is possible using these parents.

Table 1. Number of SSD F8 lines per LP50 class of three crosses.

Cross class mean of relative LP5O

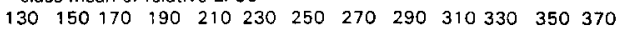

\begin{tabular}{lrrrrrrrrrrrrr}
\hline WE $\times \mathrm{BH}$ & & $4^{\mathrm{t}}$ & 11 & 11 & 21 & $* 13$ & 19 & 6 & 3 & 4 & $*$ & & \\
$\mathrm{AK} \times \mathrm{BH}$ & $3^{1}$ & $6^{\mathrm{t}}$ & $13^{\mathrm{t}}$ & 12 & 9 & 7 & $* 7$ & 6 & 4 & 4 & $*$ & 3 & 1 \\
WE $\times \mathrm{AK}$ & $3^{\mathrm{t}}$ & $8^{\mathrm{t}}$ & $3^{\mathrm{t}}$ & 7 & 3 & $* 5$ & $* 5$ & 4 & 9 & 1 & $4^{\mathrm{t}}$ & $2^{1}$ &
\end{tabular}

* = LP50 of parents: WE $227.1+1-35.8 ;$ BH 329.0 +1-24.2; AK 253.7+/-35.7

$\mathrm{t}=\mathrm{transgressive} \mathrm{lines}$

1. L.H.M. Broers, Th. Jacobs, 1989. The inheritance of host plant effect on latency period of wheat leaf rust in spring wheat. II: Number of segregating factors and
evidence for vansgressive segregations in F3 and F5 generations. Euphytica 44i31,207-214.

2. Jacobs, Th.. L.H.M. Broers, 1989. I: Estimation of gene action and number of effective factors in F1,F2 and backcross generations. Euphytica 44(3), 197-206.
676

POSSIBILITIES FOR POLEN SELECTION FOR USTILAGO MAYDIS (BECKM.) UNGER RESISTANCE IN MAIZE

M.N.Lazu, O.P.Gutsulyak

Microgametophytic selection as a method of breeding plants for resistance to abiotic environmental factors has been proposed fairly recently. Pollen afford possibilities for extensive manipulation of sporophytic genome by artificially selecting and evaluating the gametophyte. Fresh mature pollen was placed on a nutrient medium supplemented with Ustilago maydis extract in various concentrations $(0.0-1.0 \mathrm{mg} / \mathrm{ml})$, the control variant containing no toxin. The pollen was germinated at $28^{\circ} \mathrm{C}$ for 40 min. When the nutrien medium was supplemented with the U.maydis extract in a concetration of $0.5 \mathrm{mg} / \mathrm{ml}$, the genotypes examined markedly differed from one another with respect to relative proportions (z) of the germinated pollen grains and pollen tube length. The resistant Iine 092 showed a 28.38 reduction in the percentage of germinated pollen grains relative to the control. the reduction in pollen tube length being 25.18. In the sensitive 1 ines zemundo-2454 and MAN-123, the relative percent of pollen tube length is 50 lower than in the control $(62.2 \%$ and 58.68, respectively). Therefore, pollen tubes and pollen grains of the more resistant genotypes are less prone to inhibition by metabolities of the pathogen. At a concetration of $1.0 \mathrm{mg} / \mathrm{ml}$, the pollen grain germination rate and pollen tube length varied according to the genotype. All the lines examined showed a significant reduction in percentage of the germinated pollen grains. Thus, the extract concentration of $0.5 \mathrm{mg} / \mathrm{ml}$, appeares to be an optimal in differentiating maize genotypes with respect to their resistance to $U$, maydis and in breding for resistance to corn smut.

Lazu M.N. - Leading Scientific Researcher, Doctor in Biology, Institute of Genetics, Academy of Sciences, 20 pădurilor str., Chisină 277002 R.Moldova 


\section{A STRATEGY FOR CONSTRAINING ERGOT DISEASE EPIDEMICS IN F,} HYBRID SORGHUM PRODUCTION

\section{H.A.G. Hassan, P.G. Mantie, N.W. McLaren}

The ergot fungus Claviceps africana is an important factor in the economics of producing $F_{1}$ hybrids of grain sorghum in Africa. The effect on male-sterile lines, deletion of ovaries and encouragement of saprophytic fungal spoilage of developing grains, is qualitatively the same as the effect in self fertile sorghums. However, the effect nay be quantitatively much more significant on account of the greater temporal opportunity for infection of unfertilised ovaries of male-steriles and the tendency of the pathogen to express secondary conidiation on exuded honeydew under relatively cool and humid climatic conditions. Secondary conidia are produced by iterative germination of macrospores immediately below the surface of exuded honeydew so that the new spore is formed above the surface and readily becomes airbone (Mycological Research 93, 497, 1989). Local epidemics of ergot disease are caused by this phenomenon (Plant Pathology 42, 368, 1993) and wider epidemics may also be initiated. Germination of macrospores in situ may be physiologically allowed by the rather low sugar content of the honeydew exuded via the large sphacelial biomass of this ergot fungus when it parasitises sorghum ovaries (Mycological Research 95, 1101, 1991). Consequently, a strategy that contrives a higher sugar content of exuded honeydew might suppress secondary conidiation. Such a strategy might be achieved by incorporation of a sweet sorghum character into the genome of the male-sterile line, if some sweet sorghums could be shown to support an altered parasitic behaviour of the pathogen.

The first phase of experimental field comparison of a range of 30 sweet sorghums and 10 grain sorghums in South Africa was completed under irrigated conditions in northern Natal in 1994 Whereas all grain sorghums supported typical whitened honeydew symptoms of profuse secondary conidiation associated with low sugar content of honeydew, secondary conidiation was not observed on the honeydew exuded from several sweet sorghum lines even after rain, and the consequent conditions of high humidity. Sugar analysis of the honeydew from these sweet sorghums revealed rather high residual concentrations of oligosaccharides. The study is being extended to be more comprehensive amongst available lines of sweet sorghums and to evaluate the interaction between the pathogen's metabolism of the pian's sucrose and the expression of secondary conidiation.

Biochemistry Department, Imperial College of Science, Technology and Medicine, London SW7 $2 \mathrm{AY}$, UK and Grain Crops Institute, Potchefstroom, Republic of South Africa.
A STRATEGY FOR DEVELOPING DURABLE RESISTANCE TO BACTERIAL WLT (Pseudomonas solanacearum, race 1) IN TOMATO

P. Prior, S. Bart, A. Darrasse, M. Béramis, S. Leclercq and G. Anaïs

The worldwide tropical and subtropical devasting bacterial wilt (BW) on tomato remains a persistent disease. Plant resistance through integrated management resulted in a site specific control strategy. External wilt symptoms associated with oozing index are a major criterion used to reject susceptible materials in a breeding program for BW resistance. This procedure appears only effective during the hot season favourable to wilt syndrom, but does not allow to select material among all other symptomless tomatoes.

From the Freach West Indies, a recent review reported that all symptomless plants were latently infected by Pseudomonas solanacearum regardless of the genetic background for resistance to BW. Restriction of bacterial invasiveness in the stem was associated with field resistance: the higher the resistance, the lower the stem colonization.

Thirteen tomato cultivars, ranging from susceptible to resistant, were field evaluated both for wilt incidence and bacterial colonization at collar and midstem sites of symptomless plants. Experiments were conducted during colder and warmer months, unfavourable $(0-30 \% \mathrm{BW})$ and favourable $(0-80 \% \mathrm{BW})$ to the disease, respectively. From the cold season, susceptibility of tomato cultivars could not be discerned using wilt incidence criterion. In contrast, tomato was accurately classified as susceptible, moderately resistant and resistant to $\mathrm{BW}$ on the basis of $P$. solanacearum colonization frequencies, concerning infections which evolved into wilt symptoms and asymptomatic plants carrying latent infections. This predictive model was consistent with the final BW ranging established from field results obtained during the favourable, hot season. Bacterial colonization frequencies at collar is reported as the most adequate indicator for quantifying tolerance to $P$. solonacearum and resistance to wilt, regardless of wilt observation which is highly influenced by environmental variations.

Institut National de la Recherche Agronomique, Unité de Recherches en Productions Végétales; Phytobactériologie \& Amélioration des Plantes, BP 1232, 97185, Pointe à Pitre, Guadeloupe (FWn).

UNDERSTANDING PEST RESISTANCE MECHANISMS AND ITS CONTRIBUTION TO CROP IMPROVEMENT

\section{P C Stevenson F M Kimmins}

Plant resistance to pests and diseases is widely used in IPM progranmes but the biological basis of resistance and the nature of the interaction between the plant and pest usually remains unknown. Empirical screening trials have been used effectively as a means of identifying resistance and provide a relatively straightforward route to the rapid deployment of new cultivars. Examples are presented here that demonstrate the advantages to be gained from understanding resistance mechanisms. This work complements both conventional breeding and biotechnological approaches to crop improvement and can also contribute to the development of durable pest resistance.

We have shown that the defence mechanism of chickpeas (Cicer arietinum) to fungal wilt (Fusarium oxysporum f.sp. ciceri) is not effective against all geographical variants of the pathogen thus the deployment of this resistarce showfd be managed according to pathotype distribution. In another example we have found no effective resistance in cultivated groundnuts (Arachis hypogaea) to its major lepidopteran pests in India. We have identified resistance factors effective against a range of Lepidoptera in wild species of Arachis and shown that the components can be transferred by conventional breeding.

Resistance mechanisms to homopteran pests have also been studied. These insects are important because they not only cause damage directly but also indirectly as virus vectors. Thus resistance to the insect vector may also affect vious spread. For example a mechanism of resistance identified in groundouts reduced development of Aphis craccivora as well as the incidence of the persistent virus disease, groundnut rosette. We know that the mechanism disrupts phloem feeding but it also promotes probing so it will not disrupt the transmission of non-persistent vinuses and may even increase their spread. Finally, feeding deterrents to the brown planthopper, Nilaparvata lugens, located in the phloem of rice are common to both cultivars and land races of rice (Oryza sativa). These compounds both reduce feeding and inhibit development. In wild species of rice, however, the mechanisms identified prevent phloem location by $\mathrm{BPH}$ and thus may provide a more potent defence which in the advent of gene transfer in rice could be incorporated into the cultivated germplasm. 
THE WAYS TO MAKS PROVISION FOR RESISTANCE OF THS WINTER WHEAT TO PEST INSBCTS IN THE FOEEST-STEPPE ZONE IN UKRAINE

\section{G.I. Vasechko}

The density of actually all the pest insects would be kept on the level below a threshold of darage for host-plants when using some agronomic and breeding measures. The former consiats in changing of terms of the sowing depending on forecast of weather for October. When the forecast of air temperatures is close to jearly average or below it, the sowing should be practiaed in the second ten-days period of September. At the forecast showing warm October, the sowing should be done in the third ten-days period of September. In so doing in autumn, the plants grow in the conditions admissible for the wheat but unfovourable for nearly all the species of the pest insects. The breeding measures consist in the activity directed to supply wheat varieties with the traits that aerve as factors of resistance to the pest insects. These traits are the following: 1. The developed bility to regenerate lost sprouts, 2. The intensive growth in spring ( up to flowering ), 3. The bigh resistance to lodging. The first trait is valuable at outbreaks of sprout-damaging flies in autum and spring - the Hessian fly, the frit fly and others. The second trait protects from the green-bug and other sucking insects ( if the flowering is finished before end of May, the se peats appearing in June do not make aignificant damage). The third trait protects from sumer generations of the flios and stem saw-wasp. The traits suppress patbogenes and are valuable as components of the total system of vitalitg and productivity of the weat.

Plant Protection Institute, Vasilkovskaya str. 33, Kiev-22, 252022, Ukraine.

THE ECOLOGICAL ROLE OF PLANT IMMUNITY IN AGROECOSYSTEMS

\section{N.A.Vilkova}

The breeding and extensive use of crop cultivars which are resistant to pests, diseases and abiotic environmental factors is a large ecological and social problem in up-to-date plant industry. The cultivar, as a biological means of production, has a dominant role in agroecosystem. It constitutes an exogenic environment-forming factor for all the autotrophic and heterotrophic organisms within the agrocenosis. On the other hand, the crop cultivar plays the role of endogenic factor as a food resource for consuments. Thus, the plant resistance to all damaging agents and environmental factors is the main mechanism of the ecological stability of the agroecosystem as a whole due to optimizing of the system of trophic interrelations and of homeostatical mechanisms of agroecosystem Crop resistant cultivars, in distinction to application of pesticides, provide long-standing decrease of yield losses and depression of pest poptslation and epiphytoties It is obvious that the growing of resistant cultivars is best suited to the requirements of economical effectiveness of plant industry and environmental security.

Hence, the improvement of plant breeding to resistance is a principal way for increasing of a roecosystems' stability. With that in view, we carry out the ecological studies aimed to evaluate the possibilities of single pest genotype to cambine acceptable level of productivity and the high resistance to pests and diseases. Some plant genotypes which are resistant to pest-pathogens complexes have been revealed The logical models (idiotypes) of pest-resistant cultivars have been developed for wheat, potato, cabbage, cotton, carrot and russian turnip on a basis of the revealed resistance mechanisms of these crops to pests

The plants, as producents determine the directionality of tranfsfer of substances, energy and create complicated informative background for all the consumers. It is responsible for vitality and all types of communications as well as character of divergent and convergent evolution of all the members of ecosystem. The plants, as a result of convergent evolution, appear to be heteromorphy structures at different levels of symbiogenesis with heterotrophic organisms, including the parasitism. The directionality of pest microevolutionary processes was demonstrated to be determined by interastion between its special and populational specificity as well as presence of different resistance mechanisms in plants. An increase of resistant cultivars plantations allows to control also pest microevolutinary processes.

The results of studying of ecology of plant immunity, forming and acting of such consortiums as "plant -phytophagan", "plant - pathogen" and "plant phytophagan -entomophagan" and microevolution of phyto- and entomophagans as well, can forme the basis of optimally strategy of the use of resistant plant cultivars in agroecosystems.

All-Russian Institute for Plant Protection, Sh.Podbelsky 3, St.Petersburg-Pushkin, 189620, Russia.
The sources of triticale resistance to diseases in Moldova

Yeverita E. C. , Euiucli P. I. , Coteinicova L. C.

in 1991-1993 a study was performed to assess the resistance of the hexaploid triticale collection ( 240 stooks and cultivars) to powdery mildey (Erysiphe Eraminis Dch) at the natural bacground to show that the crop is resistant to the above disease. In 1991 wheat was strongly affected with powdery mildew due to high humidity. All the stocks and cultivars of triticale exhibited a high resistance to the disease under those conditions. Brown rust (Pucoinia triticine Eryks) affects triticale to a various extent. According to the degree of tolerance the triticale collection has been divided into several groups of rezistance based on a ranking scale as follows: 9 (very strong), 7 (strong), 5 (average), 3 (weak), 1 (very weak). The conditions of 1991-1993 were favorable for the affection of triticale with the disease. of 240 genotypes more than a half were resistant to the agent, no signs of the disease were found on leaves (9). 40 genotypes or $16 \%$ of plants were very weakly affected (7). 48 samples or $20 \%$ of plants were infected to on average extent (5). 32 genotypes or $14 \%$ were affected to a lamge extent ( 3 and 1 ), leaves were covered with large pustules of spores. The samples of (Donskaya semidwarf $x$ Chulpan) $x$ triticale and LT 363 from Polond belonged to those Eroups. The primary and secondary triticale of our breeding such as Gordeiforme 640 x Chulpan, Gordeiforme $640 \mathrm{x}$ Short-Stemmel 69, Gorde iforme $640 \times$ Voshod 2, PRAG 45/2 x KAD 2, 6 IA $502 \times$ KAD 2, Plovdiv $1 \mathrm{x}$ KAD 2 , PRAG $39 / 1 \mathrm{x}$ barley, KAD 2/917, KAD 5 , sultivars from Roumania TF 5 , TF 6 , and from Poland $S T$ 7r5/8 represent the best genotypes for the resistanse to brown rust. They were employed in further orossings as sources of resistance and new triticale cultivars such as INGEN 12, INGEN 93 and INGEN e/94 yere developed based on them The cultivars KAD $2 / 91 \%$, recognized in Moldova, and a promising KAD 5 possess a complex resistance to the above diseases.

institute of Genetics, Acad. Sci. 20 Padurilor str. Chisinau, 277002, Noldova

684

SPIDER MITE TETRANYCHUS URTICAE $K$. AND THRIPS ONION (THRIPS TABACI L) ON THE CUCUMBER PLANT IN GLASSHOUSE: INTERASTIONS BETWEEN PESTS

\section{V.A.Razdoburdin}

The larvae of thrips can be often found whithin local nidus of spider mite on cucumber leaf plates. In such cases the nidus of spider mite can occupy the square 10-100 times less than the leaf square, uncrowded with plant pests at all. As this fact takes place in conditions, which are rather close to ecological vacuum, we can propose, that thrips desire for rather close to ecological vacuum, we can propose, that thrips desire for
feeding optimization is the reason to form the aggregations between the feeding optimization is the reason to form the aggregations between the
various species of pests (mite + thrips). The behaviur of thrips larvae in presence of the spider mite was investigated on the leaf disks in Petri dishes in laboratory. We have shown that thrips can feed on eggs and larvae of mite. Attacks of thrips larvae aganist adult mites were met with reverse. Mites body is covered with hair. If thrips touch the hair mite disturbed by it, he runs away. The same result was given in mite disturbed by it, he runs away. The same result was given in laboratory experiments with thrips Frankliniella occidentalis $P$. Thrips
was more aggressive towards the mite, than the Thrips tabaci was. In laboratory conditions the larvae of F.occidentalis fed 0.9-1.1 eggs of mite a day. The larvae of T.tabaci fed 2-3 times eggs less. Cannibalism is also more typical for F.occidentalis. The larvae of F.occidentalis have fed 2 times less number of mite larvae, than it fed eggs. The genotype of feeding plant influenced on predatory activity of both species of thrips on mite. Tomato plant is less preferable feeding plant for thrips and mite mite. Tomato plant is less preferable feeding plant for thrips and mite
both, than the cucumber plant is. On tomato plant (variety: Krasnaya strela) the larvae of both thrips species fed $1.3-2$ times as more eggs, as it was on cucumber (Variety: Tumir). On tomato plant there were fed 1.5-1.8 times as more numbers of mite larvae which were fed by thrips Foccidentalis as on cucumber was. The predatory mites phytoseiidae (Amblyseius burkeri $H$ and Acucumeris $O$ ) are usually used for (Amblyseius burkeri $H$. and A.cucumeris $O$.) are usually used for
biological control towards thrips in glasshouses. We suppose that thrips biological control towards thrips in glasshouses. We
can feed a several number of these predatory eggs.

All-Russian Institute for Plant Protection, Shosse Podbelskyi 3, St.Petersburg-Pushkin, 189620, Russia 

DUVIRA, A 100\% LEAFROLL VIRUS (PLRV) INFECTED POTATO (SOLAnUm
tubEROSUm L.) CULTIVAR MAINTAINS SATISFACTORY MARKETABLE YIELD

J.A.C de SOUZA-DIAS ${ }^{(*)}$; A.S. COSTA; P.B. GALlo; G. GONZALES

Duvira is a parental unknown potato genotype which was found 5 years ago in a potato growing region in the State of Sá Paulo. Brazil. It rose interests on mild PLRV strain selection and cross-protection studies due to: (A) a history of more than 30 years of sucessive plantings with home saved seed; (B) 100\% PLRV years of sucessive, plantings with home saved seed; (B)

Experimental screenhouse and field evaluations under high PLRV inoculum pressure, showed satisfactory yield performance of tDuvira when maintained as home saved seed potato and without aphicide treatments (Souza-Dias, et al., 1993. An. Potato J. 70 (11):843). During the last season (August-Decetuter, 1994), 'Duvi ra' was yield evaluated within a 'Bintje" comercial table potato crop. The results showed the following means and respective standard deriations for 20 random hills/clone, on weight (kg) / number of tubers ranging in diameter from $<20 ;>20<40 ;>40<60$
$>60<80 ;>80<100 ;>100 \mathrm{mg}$ :

Duv1ra-1 (original clone, not thermatherapy treated) $=0.93$ $(.490) / 0.15(.4), 3.3(3), 4.7(2), 3.4(2), 1.8(3), 0.0(0)$ Duvira-2 (5th field multiplication after PLRV-freed by thermo(2),0.0(0)

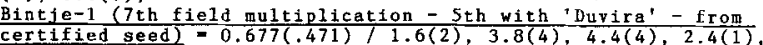
certified seed) Bintje-2 (2th field multiplication - 2th with 'Duvira' - from $\frac{\text { imported seed) }}{1.0(1) .0(1) \text {. }}$

Preliminary assay for mild PLRV stratn in 'Durira' was done by ransferring (top graft) to 'Bintje' and 'Aracy', but progenies secondary PLRV syaptoms were not different from their controls, inoculated with a normal PLRV strain. These results indicated no mild strain involvement. However, the marketable yield maintenance of Duvira-1 and 2 , with a gain of 37.5 and $45.5 \%$, respectively, over Bintje-1, indicates mechanism of PLRV tolerance in 'Duvira', which is under investigation. Since 'Duvira' has a 'Bintje'-like tuber phenotype, with apparently good cooking qualities, it is possible that 'Duvira' will be an option for potato growers, where the 'Bintje' seed-potato fast PLRV degeneration problem is recurrent. It will also be a self sustainable cultivar because of low seed costs and of treatments.

(*) Instituto Agronomico de Campinas. Seçăo de Virologia Fitot, C.P. 28, CEP 13020 314943. CNPq Fellow. Work patially supported by FUNDACÃO IAC/
POTATO GROWERS.

AIR-ASSISTED SPRA YING IN ARABLE FARMING: DEPOSITION AND BIOLOGICAL EFFICACY

\section{L.C. van de Zande, H.A.J. Porskamp, J.M.G.P. Michielsen, R. Meier, M.T. van} Uzendoorn

In a series of experiments the deposition of spray liquid and the biological effects were determined in arable crops using a sprayer equipped with an air-assisted system to convey the spray droplets to and in the crop. In three growing seasons the use of air assistance was compared with conventional spraying on three experimental sites for potatoes and winter wheat. The biological effects were determined in randomized field tests, with spray concentrations of the active ingredient varying from 0 to $100 \%$ of the dose recommended, and volume rates of $150 \mathrm{l} \mathrm{ha}^{-1}, 225 \mathrm{l} \mathrm{ha}^{-1}$ and $300 \mathrm{l} \mathrm{ha}^{-1}$ for potatoes and volume rates of 100 $1 \mathrm{ha}^{-1}$ and $2001 \mathrm{ha}^{-1}$ for winter wheat.

The deposition of the spray liquid was measured at different times of spraying during the growing season of the crops. The total deposition and deposition pattern on the crop was established by washing paper strips which had been suspended at different heights in the crop, using the dye Brilliant Sulfo Flavine (BSF). In addition to absolute deposition (quantity of chemical), the coverage and the droplet spectrum on the target area were determined by video recordings of the spray deposition on water-sensitive paper that was suspended in the crop. The video recordings were analysed by means of vision technology.

Biological effects of the sprayings were investigated by quantifying the number of plants infected and the percentage of the leaf area covered with Phytophthora infestans in potatoes. In winter wheat the percentages of the leaf area covered with brown rust (Puccinia recondita) and leaf spot (Septoria tritici) on the three top level leaves were quantified. In each growing season the number of infected leaves or level of infected area of the plants was measured on each plot at weekly intervals from the time of first infection. The number of infected potato tubers was determined at harvest and after circa two months of storage

The use of air assistance resulted in a better penetration of the spray in the crop, and a more evenly spread distribution over the crop. At lower spray volumes the total deposition on the plants was significantly higher for air-assisted spraying. Significant differences were found in the biological efficacy between volume rates and dosage.

In potatoes, at the time of first infection and on individual recording dates, the infection of the air-assisted plots was significantly lower. Weekly full-dose fields sprayed with air assistance resulted in a significantly better leaf late blight control. During the whole spray season the effect of the dose of active ingredient was significant for the infected leaves and for the number of tubers infected. However, the relation between leaf and tuber infestation and dosage was not clear.

In winter wheat a sprayed volume of $2001 \mathrm{ha}^{-1}$ sometimes resulted in a disease control better than $1001 \mathrm{ha}^{-1}$. Air assistance was significantly better at a $1001 \mathrm{ha}^{-1}$ volume rate. Full dosage and $75 \%$ dosage of active ingredient resulted in better disease control than $50 \%$ dosage.

IMAG-DLO, [nstitute of Agricultural and Environmental Engineering, Mansholtlaan 10-12, P. O.Box 43, 6700 AA Wageningen, The Netheriands

PAGV, Research Station for Arable Farming and Field Production of Vegetables,

Edelhertweg 1, P.O.Box 430, 8200 AK Lelystad, The Netherlands

COMPUTER MODEL SIMULATION AND FIELD EXPERIMENTAL. MEASUREMENT OF PRIMARY AND SECONDARY AIR DRIFTS OF PESTICIDES IN DIFFERENT METHODS OF PESTICIDE APPLICATION

\section{Y.Samsonov, V. Makarov, K. Koutsenogii, 1 V. Raputa, 'O. Vinkenstern}

The ingress of pesticides to air takes place during pesticide treatments of crops. Besides, there is due to contact of air with objects, polluted by pesticides. By the global winds air polltution extend over the whole space. The local primary and secondary air drits of pesticides beyond the boundaries of treated fields bring the largest direct and potential dangers for people. The primary drift means that a portion of pesticide substances (as aerosols or small droplets ) is carried away by wind beyond the boundaries of the treated field just during the treatment. The secondary drift is caused by the appearance of pesticide residues on vegetation and soll carred there during the treatment in the course of subsequent vaporization of residues the pesticide vapouts can also be carried away by wind. Both the primary and secondary drifts have potential ecological and medical dangers, since they can get into human organism and poliute biosphere components. The quantities of these dritts depend on the methods of pesticide application, on specific application rates, on meteophysical and landscape conditions, on physico-chemical properties of pesticides, etc. The quantitative and qualitative estimates of the pesticide dritts in various methods of their applications have been presented. The special aerosol generators with adjustable sizes of aerosols (the mass-median diameters can be varied within $1-30 \mathrm{mcm}$ ) was used in the so-called Optimum Aerosol Technology (OAT) for field treatments. The airplane and ground airblast sprayers were used in field experiments also. The experimental data were obtained during the tests with OAT for wheat protection in steppe areas of North Kazakhstan (1980-1982, 100,000 hectares) and Novosibirsk region (1985 - $1989,50,000$ hectares). Both the experimental and calculated data coupled with the mathematical computer models of OAT have been used. The models involve numerical computations of the equations for turbulent diffusion of zerosol or vapour impurities in the atmosphere surface layer allowing for aerosol size distribution, efficiencies of aerosol sedimentation on pest insects, on vegetation and soil. The timedependent concentrations of pesticide in air $\mathrm{C}(\mathrm{x}, t)$ and the potential specific inhalation intakes in human organism $D(x)$ have been found. The distances $x$ (up to $3 \mathrm{~km}$ ) from the aerosol generator path or from the field boundary were varied, as well as pesticide vaporization rates. The meteophysical parameters of atmospheric surface layer were varied to correspond to night ( stable atmosphere stratification ) and day conditions ( unstable stratification). The specific inhalation intakes $D(x)$ have been compared with the corresponding acceptable daily intakes ( $A D I$ ) for some insecticides organophosphorous, pyrethroids ). It was established that the potential pesticide intakes after the OAT do not exceed ADI, while after spraying the secondary drifts often exceed the ADI $(2-8$ times $)$.

Institute of Chemical Kinetics \& Combustion, Siberian Branch of the Russian Academy of Science, 630090 . Novosibirsk-90, Russia

- Computing Centre, Siberian Branch of Russian Academy of Sciences

688

EFFECT OF CROP, NOZZLE TYPE AND WIND SPEED ON SPRAY DRIFT: A MODEL APPROACH

\section{H.J. Holterman, J.C. van de Zande, J.F.M. Huijsmans}

The application of crop protection chemicals still brings about considerable emission into the environment. The application of pesticides to field crops usually takes place using a boom sprayer provided with hydraulic spray nozzles. The spray cloud produced contains a wide variety of drop sizes. The loss of spray droplets due to drift should be minimized but can never be avoided altogether as minimizing drift (large droplets) conflicts with enlarging the biological efficacy (small droplets). Especially small droplets are very susceptible to windinduced drift.

In the Multi-Year Crop Protection Plan the Dutch govemment plans to restrict the use and reduce the emission of crop protection chemicals. Especially drift to the surface water must be minimized. Assessment of potential drift-reducing techniques demands a quantification of drift. Quantifying spray drift by field trials is difficult because of the large number of parameters involved, some of which cannot even be controlled.

To investigate spray drift a computer model has been worked out to simulate drift as it occurs in field operations with a conventional boom sprayer. The model covers meteorological aspects (e.g. wind speed, relative humidity, turbulence intensity, air temperature) and factors related to the spraying technique (e.g. nozzle type, droplet size distribution, liquid pressure, boom height, forward speed). Spray drift from a whole boom sprayer is estimated by summing the drift as computed for each nozzle separately. The model is particularly suited to predict the nearby $(0-10 \mathrm{~m})$ distribution of chemicals as a function of downwind distance from the crop edge. The model is also suited to assess drift-reducing methods e.g. involving a no-spray zone and low-drift nozzle types. Experimental field trials to validate the model show that downwind droplet deposition is well predicted.

The effect of a no-spray zone at the border of the crop field was quantified. Increasing the no-spray zone lowers the emission to the soil surface next to the field border $(0-10 \mathrm{~m})$, the decrease is higher if a crop is grown on the no-spray zone instead of allowing the zone to lie fallow. Model calculations predict that the major factors affecting spray drift are average wind speed and boom height above crop canopy. Using flat fan nozzles an increasing wind speed of $2 \mathrm{~m} \mathrm{~s}^{-1}$ to $5 \mathrm{~m} \mathrm{~s}^{-1}$ increased the spray drift by about $50 \%$. With increasing spray boom height above the crop surface from $0.5 \mathrm{~m}$ to $1.0 \mathrm{~m}$, spray drift had about doubled. The effect of nozzles with fine, middle or coarse spray classes on spray drift is quantified. The coarser the nozzle type, the lower the drift amount appeared to be. The use of a low-drift nozzle to spray a coarser spray class at the same volume rate, results in a lower spray drift.

IMAG-DLO, Institute of Agricultural and Environmental Engineering, Mansholtuaan 10-12, P.O.Box 43, 6700 AA Wageningen, The Netherlands 
EMISSION-REDUCING APPLICATION TECHNIQUES: FIELD MEASUREMENTS OF SPRAY DRIFT IN ARABLE FARMING

L.F.M. Huijsmans, J.C. van de Zande, H.A.J. Porskamp, J.M.G.P. Michielsen

Reducing the use of chemicals now having top priority, more attention needs to be paid to achieving a better leaf coverage with less chemical. Furthermore, emission of crop protection chemicals is a major problem in chemical crop protection. New spray application techniques might improve the deposition and reduction of drift.

Research has been set up to study the effects of improved application techniques on the reduction of the emission to surface water, soil and air. A measuring programme has been set up to deternine the emissions for various techniques. New methods have been developed to be implemented in field surveys to measure the emission in the field, the deposition on the soil surface undemeath the crop cover and the spray drift outside the field.

In a series of field trials air-assisted spraying was compared with conventional spraying in a potato crop in the growing season. The effect of low-drift nozzles on spray drift was also quantified, as well as the effect of a no-spray zone. Measurements were done on a bare soil surface and in a ditch, downwind of the crop.

Emission measurements were carried out by adding the fluorescent dye Brilliant Sulfo Flavine (BSF) to the spray agent and placing collectors in and outside the field. The emission to the soil was measured by placing collectors between and on top of the potato ridges. Emission of spray liquid next to the field on the soil surface was measured with collectors placed up to $15 \mathrm{~m}$ downwind from the field edge. After the spraying, the dye was extracted from the collectors. The rate was measured by fluorimetry and expressed per surface area of the collector. The deposits measured were expressed as percentages of the application rate of the sprayer (spray dose).

The effect of air assistance on soil emission underneath a potato crop was significant. Deposition on the soil surface on top of the potato ridges and between the ridges was significantly higher for air-assisted spraying at both volumes of $150 \mathrm{l} \mathrm{ha}{ }^{-1}$ and $300 \mathrm{l} \mathrm{ha}$; with no difference in emission between the two volumes. Deposition on top of the potato ridges is higher than between ridges.

Air assistance clearly affected the reduction of drift; compared to a conventional sprayer without air assistance, a $50 \%$ emission reduction was found. The effect of a comparable lowdrift nozzle on drift was lower than that of using air assistance

A clear picture of the performance of the present new developments compared with a conventional sprayer is given.

IMAG-DLO, Institute of Agricultural and Environmental Engineering, Mansholtlaan 10-12, P.O.Box 43, 6700 AA Wageningen, The Netherlands

691

\section{DATABASES ON PESTICIDES AND THE ENVIRONMENT AS AN INFORMATION KEY TOOL FOR THE LMPLEMENTATION OF IPM}

B.C. Schifiers, J.F. Cooper, J. Deuse, D. Jourdain, G. Keck, J. Savelkoul

The Lome Convention (Lome IV) contains a number of provisions directly relevant to pesticides, IPM, and the environment and development. Article 40 specifies that the Community shall provide available technical information on pesticides in a view to belp the ACP states to develop a suitable and safe use of these products. Suitable pest control methods should be used in an integrated manner (IPM) and pesticides should be used on an as needed basis only. In such an IPM strategy, the effects of pesticides on human and animal health, on the environment and sustainability of the agricultural system should be carefully considered. The most immediate problem is the lack of information. Rational control requires decisions to be made in the field and the provision of alternative choices is essential. Action must not be blind but the appropriate result of an informed decision. It is intended, therefore, to develop the databases to the point where they can fully enable the rational use of pesticides as a first step in the implememation of IPM and thence sustainable agriculture. The "Database on Pesticides and the Environment" programmes, funded by the Commission of the European Union (DG VIII \& DG I), contain a comprehensive information on various aspects of pesticides used in Africa (29 countries) and in the ASEAN region (Brunei Danussalam, Indonesia, Malaysia, Philippines, Singapore and Thailand) inchuding use recommendations, properties (physical, biological, chenical, etc ), commercial products, agronomic uses, registration information, behaviour (mode of action, ecotoxicity,...) and effects on human and animal health, and the environment. They allow quick and easy retrieval of information using an ORACLE system (for Africa) or an IBM compatible personal computer (for Asig) Approximately 6,000 products are described in the databases. Data were collected from various sources and in cooperation with different national and international organizations. The data, provided by five european institutions, were reviewed and verified by the participating countries. The countries themselves contributed their national pesticide use recommendations on various applications such as crop protection, post harvest and seed treatment, invasions control, public health, and plant growth regulators. In addition, active ingredient data sheets from various sources such as EPA WHO, FAO, RSC and available PIC data were also included in the databases. This information is expected to benefit pesticide regulatory authorities, poison control centres for humans and animals, environmental protection agencies, and other organizations concerned with the safe use and management of pesticides.

IPHYTROP Network on Pesticides

Faculte des Sciences Agronomiques

UER Chimic analytique et Phytopharmacie

Passage des Deportés, 2

B-5030 GEMBLOUX (Belgjum)
CONTRIBUTION OF FOLK MEDICINE IN THE EVOLUTION OF NATURAL PLANT PROTECTION IN SOUTH AFRICA

Poswal, M.A.T.

In South Africa, numerous plant species are known to be utilized in traditional medicine for the treatment of diseases of fungal, bacterla insecticldal properties, while others are used as anthelmintics. Preliminary observations of aromatic plants, used in the production of essential oils, suggest that these could oerve as ingect repellents and attractants. The woalth of information on folk medicine, coupled with
the diversity of south Af 1 ican flora, has led to the study of medicinal plants as a starting point for pharmaceutical research. Results of ethnopharmacological gtudies initiated in 1974 indicates that plant extracts of certain plant species exhibited antimicrobial/antifungal activity. Similarly, research baged on the knowledge of folk medicine should contributa to the evolut ion of natural plant protection in south Afred, such that the potential of natural peaticides are astablished resource-poor farmers lo discused. Department of Agronomy, Faculty of Agriculture, University of Fort
Hare, Private sag $\mathrm{x} 1314$, Alice 5700, South Africa
692

PESTICIDE AWARENESS IN THE TROPICS A CASE STUDY OF JAMAICA'S FARMING SECTOR.

\section{P. Whylie, J. Myers, J, von Dueszein}

Jamaica is one of the larger islands in the Caribbean. The annual use of pesticides in this country amounts to 1.3 million $\mathrm{Kg}$ which represents approximately $1.5 \mathrm{Kg}$ per capita of its rural population. In order to devise an effective awareness programme on pesticides, the Pesticides Control Authority in Jamaica commissioned a baseline, national survey targeting the farming sector. One thousand predominantly small farmers from all parishes of the country were interviewed in June/July 1994 by a firm of professional pollsters according to a stratified sampling method. Twenty six questions were presented including request for information on purchase, handling, storage, use, poisonings and disposal of pesticides.

The results show that $44 \%$ of pesticides are not purchased in the original containers. The farm store was the main source of pesticides $(85 \%)$ and also provided most information on use. Regarding protective gear, $55 \%$ of farmers never or rarely wear any form of protective gear. This may be partly due to the fact that $27 \%$ of famers do not read the labels on the pesticide containers. Most farmers $\{61 \%)$ store pesticides locked away. Of the $4 \%$ that said pesticides were stored in their homes, $3 \%$ reported that pesticides were stored in the kitchen. To improve efficacy, $33 \%$ of farmers modify or increase the concentration recommended. Most farmers apply pesticides as soon as pests appear $(49 \%)$, or an a scheduled basis $(36 \%)$. Some $4 \%$ of the respondents admitted cleaning equipment in the river. For disposal empty pesticide containers or bags are usually thrown away scattered or placed in a garbage heap rather than being buried or burned. It is estimated that every fitth farmer had at some time experienced poisoning effects of pesticides, although over $70 \%$ knew not to eat, drink or smoke while mixing or using pesticides.

Public awareness programmes have to be undertaken to convey the message of the dangers of pesticides. The importance of the Farm Store Manager as a facilitator cannot be ignored. Innovative methods must be found to provide protective gear that is comfortable and will be willingly worn by farmers in the tropics. A play comprising ten skits on pesticide use is being performed in rura communities and may represent a socio-culturally sound vehicle for information transfer on safe use of pesticides in Jamaica.

Pesticides Control Authority, 10 Caiedonia Ave, Kingston 5, Jamaica W. tellfax: +1 -1809/960-0775 


\section{INDIGENOUS KNOWLEDGE SYSTEMS IN CASSAVA PLANT PROTECTION, CAMEROON.}

\section{I.T. Ambe.}

Cassava production in Cameroon is plagued by many pests and diseases. These pests include rodents, mammals, birds, arthropods mollusks and weeds. Diseases are fungal, bacterial and viral in nature. Some physiological disorders have been of great concern. A brain storming workshop was organised in Cameroon and many indigenous knowledge systems in cassava plant protection were identifled. Farmers in Cameroon use indigenous control methods of pests and diseases on cassava which have not been documented. These Indigenous systems were grouped into four categories: cultural (mechanlical and farming practices), chemical, biological and plant-host resistance. Some of the control methods were grouped under more than one category. There is need for research to further address some of the identified methods. Realising that there exist work needs to be carried out in areas of research, through surveys to really document the existing indigenous systems of plant protection for other crops in Cameroon.

Institute of Agronomic Research (IRA), Ekona, PMB 25. BUEA, Cameroon. rich indigenous methods of controlling plant pests and diseases, further

AN OVERVIEW OF INDIGENOLS KNOWLEDGE SYSTEMS FOR FIELD AND STORAGE PEST CONTROL IN NIGERIA

I.D. Erinle

Pests (sensu lato) constitute a serious constraint to crop production in both traditional and modern cropping systems. Subsistence farmers are often conditioned to rationalize losses where no control measures are available. In several cases, however, there are proven, low cost indigenous technologies, particularly for insect, avian and weed pests. The most important of these in Nigeria .......................................... pests of seedlings and foliage of vegetables, storage pests, annual peeds and vertebrate pests. Mixed cropping as a traditional system of crop production has several proved advantages over monocropping, pest control being one of them. Despite the availability of indigenous pest control options, crop losses in traditional cropping systems are often still too high and militate against the attainment of self-sufficiency in the production of food, feed and fibre. Research to fortify and improve on sustainable low input technologies so as to offer the farmer a wider menu of options is very essential. It is increasingly apparent to most researchers that this is best attained through participatory approaches, with the farmer playing as vital a role as the researcher. For this to flourish, however, requires the support of appropriate institutions and services.

Department of Crop Protection, Institute for Agricultural Research, Ahroadu Bello University, P.M.B. 1044, Zaria, Nigeria

696

AN INVESTIGATION INTO THE INDIGENOUS CROP PROTECTION PRACTICES OF THE PEOPLE IN THE EASTERN EXTENSION ZONE OF LAGOS STATE, NIGERIA

Y.O.K. Osikanlu, N.A. Amusa and D.M. Warren

Indian sub-continent is diverse in physiography and rich in both ethnic and plant diversity. There are more than 500 ethnic groups (ca 60 million) inhabiting different parts of India. The areas inhabited by the tribals are rich in plant resources. Some of the major ethnic groups are Gond, bhil, Mina and Santall (Central India); Boro, Garo, Khasi, and Lepcha (North-East India); Amchi, Gaddi, Jaunsari, Ladald, and Nepall (Himalayan regions); Irula, Kodaku and Toda (South India); and Andamani, Jarwa, Onge (Andaman \& Nicobar). Even today most of them are practicing primitive agriculture, based on indigenous knowledge. They conserve numerous land races and wild relatives by growing traditional varieties and through taboos, myths, and facts about forests, trees, and plants. Indigenous methods are used by different tribal communities for protecting crops and planting materials from insect-pests. Tribais have two types of agro- systems ie. arable farming. and shifting cultivation (known as (known as Bewar, hum, and Parda), Although such type of agricultural practice is banned by the government, nevertheless, it is being practiced in some tribes. Most of the communities cultivate primitive cultivars like pseudo-cereals, millets, and pulses. Seeds are sun-dried and preserved in the air-tight containers along with leaves of some insect repelling plant (Azadirachts indics, Murrayz koenigil, Acorus calamus, etc.). These containers are made from bamboo strlps plastered with a mixture of mud and straw. Rhizomes and tuberous are mostly preserved elther in the pits after giving some specific treatment or dried in shade and kept for future use. Seeds and truits of spices and condiments crops are preserved in dried bottlegourds used as a container. Pulses are mixed with millets after giving some treatment with milk and/or leaf extracts. Primitive agro-systems are the store house of knowledge and drought hardy, stress tolerant, disease resistant land races. Tests done in laboratory and small scale fleld trtals on Azadirachta indica and Acorus calamus have shown the efficacy of these for protecting stored grains from pest attacks. Traditional practice in some tribes of South India to tip their hands in milk before transplanting tobacco seedlings is known to protect crops from many viruses. Recent observation (Swami, Swarup and Dash, 1993) that bovine milk carries an iron binding glycoproteln, lactoferrin showing powerful rlbonuclease activity which prohibits microbial growth, provides a scientific explanation to this practice. Practices to conserve and manage thelr crops are intimately known to the tribal people for millennia. These require special attention and documentation for developing eco-friendly crop protection methods.

CSIR Complex, Post Box No. 6, Palampur-176 061 (IIP), INDIA
A study was undertaken to investigate the indigenous crop protection practices for the various crops grown in the Eastern Extension Zone of Lagos State, Nigeria. A structured questionaire was used to collect information relevant to the study.

The findings showed that $81.4 \%$ of the farmers were 40 years old and above. $59.3 \%$ of the farmers carried out their farming activities in Epe Local Government Area of the zone. $64.7 \%$ of the respondings engaged in farming as their primary occupation and $89.4 \%$ had farm holdings of not more than 5 ha. It was also found that many of the farmers had a reasonably long farming experience, $74 \%$ with over 10 years of experience.

Disease contral measures were applied at planting, pre and post planting stages of the crops. Ash treatment was used by some farmers in treating tomato, pepper and cassava but a negligible few used it on maize. $36.6 \%$ harvested fruits of infected tomatoes green. Use of certified seed was practiced by some farmers in controlling diseases of the crops at preplanting stage.

Control of diseases at the planting stage was very minimal and about $56.7 \%$ of the respondents did not apply any control measure to tomato, pepper and cassava, respectively.

Institute of Agricultural Research and Training, Obafemi Awolowo University, P.M.B. 5029, Ibadan, Nigeria; Department of Agricultural Biology, University of Ibadan, Ibadan, Nigeria; Centre for Indigenous Knowledge for Agriculture \& Rural Development, 318 Curtiss Hall, Iowa State University, Ames, Iowa 50011, USA. 
CONTRIBUTION OF FOLK MEDICINE IN THE EVOLUTION OF NATURAL PLANT PROTECTION IN SOUTH AFRICA

M.A.T. Poswal

In South Africa, numerous plant species are known to be utilized in traditional medicine for the treatment of diseases of fungal, bacterial or viral origin. Some of the plant species are said to possess insecticidal properties, while others are used as anthelmintics. Preliminary observations of aromatic plants, used in the production of essential oils, suggest that these could serve as insect repellents and attractants. The wealth of information on folk medicine, coupled with the diversity of South African flora, has led to the study of medicinal plants as a starting point for pharmaceutical research. Results of ethopharmalogical studies initiated in 1974 indicate that plant extracts of certain plant species exhibited antimicrobial/antifungal activity. Similarly, research based on the knowledge of folk medicine should contribute to the evolution of natural plant protection in South Africa, such that the potential of natural pesticides are established and exploited. Their role in integrated pest management systems for resource-poor farmers is discussed.

Department of Agronomy, Faculty of Agriculture, University of Fort Hare, Private Bag X1314, Alice 5700, South Africa

699

\section{CURING CITRUS FRUITS TO CONTROL POSTHARVEST DISEASE}

J.W. Eckert, M. Ratnayake, J.R. Sievert, R.R. Stange

Curing oranges and lemons at $32^{\circ} \mathrm{C}$ and $90 \% \mathrm{RH}$ for $2-3 \mathrm{~d}$ controlled Penicillium digitatum, the principle wound pathogen, but not $P$. italicum or Geotrichum candidum. The curing treatment was lethal to germinating $\boldsymbol{P}$. digitatum spores and favorable for the biosynthesis of fungitoxic aromatic aldehydes and the deposition of ligninlike polymers at wound sites in the fruit exocarp. The curing process was enhanced by several chemical and biological agents.

Department of Plant Pathology, University of California, Riverside, CA 92521, USA.

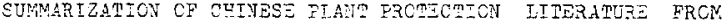
ANCIENT TIME TO 1993.

Jang Huafu

This article was written on the basis of the worke entitied

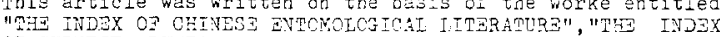

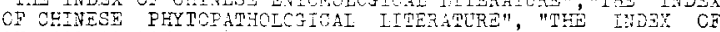

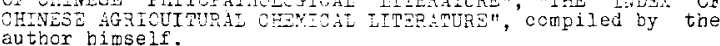

1. Entomological Iiterature: There are 108,474 pieces of records on insects liteature. There are 10,315 pieces of records on insects iltercture before the foundation of $P$.R china, after that there are 98,159 pieces of recoeds on insecti literature. It is 9 times than the tine before the foundation of P.R.China. There are 293 regularly and irregularly pubiisheó journals anj newspaters in china irregularly published journals an newspafers in china.
mhere are 32 sorts before foundation of china, after that
there are 211 sorts.

there are 211 sorts.

. Fhy topathological iitarature: There are 81,031 pieces cf records on phytopathological iiterature. There are 6,352 pieces of recoris on phytopatiological literature before the fcundation of P.R.China, after that there are 74,679 pleces of records on phytopathological literature. It is 10 times than the time before the founcation of F.R. China. There are 98 regularly and irreguiarly published journals in China. There are 9 sorts before foundation of ohina, after that there are 89 sorts.

3. Agricultural chemical Literature: There are 109,513 pieces cf records on agnicultural chemical litereture, of which, 34,311 are pubiicly fublished papers from the chinese journals, newspapers and books;19,902 are literature from restricted publications, 15,722 are translated papere. 39,408 are translated abstracts from foreign sources, 170 are literature on agricultural chemicals in China. written inforeign languages eitiber by chinese scholars or by foreign scholars. Among the total of 109,513 literature10,125 items were published before the founding of $F$. China while the rest 99,388 items were published after the founding of $p . R$. Chioa in 1949 , with the latter outnumbering the former by Dore than 9 times. There are 37 regularly and irregularly published journals and newspapers in china. Before the establishment of the F. china there was only 1 while after the establishment of the agricultural chemical journals and newspapers.

Wang Huafu, No. 10 Guang Ming Hu Tong, Jianusi City, Teilong jiang Province 154002 F. . China

Tiamusi Agricultural coliege

\section{ChARACTEAIZING INDIGENOUS KNOWLEDGE SYSTEMS WITH EXAMPLES FROM ETHIOPIA}

by Tewolde B. G. Egziabher"

Discussing indigenous knowledge in an audience of scientists calls for contrasting it with scientific knowledge, taking the fatter as the standard.

"Science" is taken to be an endeavour to understand the causation of natural phenomena. But so is indigenous knowledge. Science is also contrasted with religion in searching for possible explanations while the former uses revelation as the immutable explanation of natural phenomena Depending on while the former uses revelalion as the cmnulable explanation of netantfic and the religious. For this eligious beliets, an indiwdul's kns reason, the distinction of scientific knowledge as exclusive of religious knowledge is a mere menta construct, which can help the individual organize his/her knowledge but is. otherwise of little value in its application. Indigenous knowledge does not make this reductionist distinctlon. However, indwidual items of knowiedge are classified as immutable. which includes those that are of religious origin. and changeable with new information.

Science is often described as being characterized by observation, hypothesis generation, experimentation and/or new observation, induction and deduction. All these characteristics apply to that part of indigenous knowledge which is not based on religlous beliefs. These are illustrated with examples from Ethiopia.

However, the historians of science, so far almost all from, or coopted into, industrialized societies, ify to make it look that these universal traits of human knowedge apply only to "science". This is partly because their knowledge base is only "science", their "indigenous knowledge" having largely been subsumed into, or replaced by, "scientffic knowledge". The fact that the method of transmission of knowledge in the industrialized countries is through the written word whilst in most other countries writing either does not exist or is more or less restricted to religion, makes the orally iransmitted knowledge alien.

A usual consequence of the alienation of an advantaged group is the creation of an aristocracy which creates artificial barriers to keep the lower classes out. Two of the barriers aristocratic "science" has created are the virtual disregard as non-existent of:

- what has not been written

- what has always been and has thus not been "dlscovered".

Using the first barrier, we have created the dichotomy between "science" and "Indigenous knowledge"

Using the second barrier, we have considered indigenous knowledge as structurless. After the failure of the "scientific" requirement tor absolute proof, "science" now looks for the most probable explanation of the "scientific" requirement tor absolute proof, "science" now looks tor the most probable explanation as its basis. This is precisely what indigenous knowedge has always done including, however, also religious and other forms of knowledge in the determination of probabilttes. In one aspect, this brings reductionist science up to the level of holism of indigenous knowedga. However, reductionism has still knowledge is more holistic.

A new synthesis is required. "Science" should get rid of its aristocratic trappings. With the holistic focus of indigenous knowtedge, and with the new tods developed by "science", a rewarding expedition into the human knowledge base is possible.

National Herbarium, P.O.Box 3434, Addis Ababa, Ethiopia. 
GENETICALLY ENGINEERED MICROBIAL BIOPESTICIDES: IS THERE LIFE AFTER TRANSGENIC PLANTS?

\section{W.D. Gelernter}

In 1996, transgenic crops that express Bacillus thuringiensis ( $B$ ) toxins are scheduled for market introduction. In light of the improved levels of insect control observed for transgenic plants, a reassessment of the role of microbia blopesticides in crop protection is necessary. Do microbial biopesticides have positive features that set them apan from transgenic crops? For which crops and markets will biopesticides have the greatest potential? Will commercialization of biopesticides continue once transgenic crops are introduced?

These questions will be addressed by providing an overview of microbial based geneticatly engineered products that are currently available, and those that are likely to become available in the near future. The advantages and disadvantages of these biopesticides will be contrasted with those of transgenic crops, and the most appropriate niches for each type of product will be discussed. A proposed direction for future biopesticide research and development efforts will also be presented.

\section{PACE Consulting, 1267 Dlamond Street} San Diego, CA 92109 USA
BIOFUMUOATION - USINO BRASSICA SPECIES TO CONTROL PESTS AND DISEASES IN AUSTRALIAN AGRICULTURE AND HORTICULTURE.

\section{L.A. Kirkegaard, I.N. Mathiessen and J.M. Desmarchelier}

Brassica plants meicase bincidd compounds, principally isothiocyanstes (ITCs) produced during the breakdown of glutusinolates in crop residues. These compounds are known to suppiess a range of insects, nematustes and fungi. Brassica species and varieties differ in the rype concentration and insects, nerrutsetes and fungi. Brassica specties and varietes differ in the type, concentration and
distribution of glucosinolates in their tissues which provides an toppontunity to select or breed varieties with enhanced pest control properties. An understanding of the distibution in plants, the release pattern imes seril and the effecery of the chemicals is necessary to fully exploit the potertial of "biofumigation" as a part of an integrated pent and disegse managemeat program. We discuss the results of current reseurch in Austratia which airns to determine the potential of biofumigation by brassicas in the cerenl and potalo industries.

Recent field experiments have shown that wheat grown after brassicas outyielded wheat grown after other break crops such as oats or linseed (Angus ef al. 1991, Kirkegand et al. 1994). In addition early growth and yield of wheat following Indian mustard was significandly better than wheat following canola. Angus et al. (1991) speculated that improved proweth may have resulted from suppression of soil-borne wheut pathogens by TTCs released from Brassica residues and that the higher levels of different rypes present in Indian mustard were responsible for its superior break erop efferts. This hypothesis was supported by the resuits of experinents invescigacing the suppresivion of the take-all fungus (Gaeumunnomyces graminir var, tritio) by Brassica root piects in viro (Angus et a/. 1904). Further in vitro studies have indicaled the effectiveness of ITCs released fron: Brassica reviduev in a range of soil-bome fungal patbogens and differences in the sensitivity of fungal species to the $\mathrm{TrC}$ (Kirkegaard ard Wong unpub.).

Biofumigation using Brassica crops is being investigaved as a pan of an integnased pest manugemen strutezy for the control of soil incer pests of potatoes. Potan growers currenly use synthetic ITCs at
a cost of $\$ 600 \mathrm{ha}$ for conorol of white frimged weevil (Graphognathus leucoloma (Bohemani)) and a cost of $\$ 600$ ha for control of white fringed weevil (Gruphognuthus leucoloma (Bnhemani)) and African black beetle (Hereronyehus arator (Fabricius). The odults of both species attack tubers and
cause significant economic damage in low aburdunce. The small larvac of the whitefringed weevil hatch

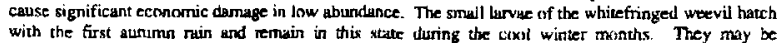

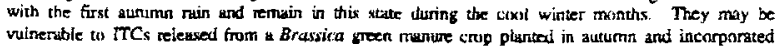
before potatoes are planted in spring. The African black beetle spends winter in the adjult stuge, however brassitas producing high levels of glucosinolates could drive them away or interfere with their breeding in spring. Several zoil hrone diveases uf potatues (e R Rizoctonia) may also cendidates for contonl or suppression using biofumigation.

CSIRO Division of Plant Industry, GFO Box 1600, Canberra, ACT 2601 Australia. 


\section{G. Shaner and G. Buechley}

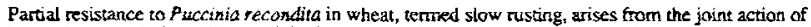
longer larent period, smaller uredinia that produce fewer spores, and a radured densiny of uredinta conger larent penod, smaller uredinia that produce fewer spores, and a reduced density of uredinta differen manife stavions of a common underlying mechanism of resistance that relards the difeerent manife stanons of a common underlying mechanism of resstance that retards the
development of the fungus in the host. The frequency of penetration of the fungus is the same on development of the fungus in the host. The frequency of penetration of the fingus is the same
slow- and fasi-nsting cultrvars. Once the fungus begins ramifying through the mesophyll, slow- and fast-nsting cultrvars. Once the fungus begins ramifying through bhe mesophyll,
however, it grows at a slower rate and takes longer to reach the mass necessary to produce however, it grows at a slower rate and takes longer to reach the mass necessary to produce
urediniospores in a slow-rusting cultivar. Once a uredinium has erupted on a slow-rusting cultivar, urediniospores in a slow-rusting cultivar. Once a uredinium
its rate of expansion is slower than in a fast-rusting cultivar.

Latent period has been the most reliable component for studying inheritance of slow rusting. We inoculate plants in the boot stage and estimate the percentage of uredinia that have erupted each day. Uredinia erupt from about 6 to 10 days after infection on a fast-rusting cultivar, and from about 7 to 20 days on the most slow-rusting cultivars. For genetic studies, a mean latent period is calculated from the frequency distribution for time of erupcion of uredinia on each plant. Early work with F2 plants, F3 families, and back cross progeny suggested that long latent period was condirioned by the additive action of 2 or 3 partially recessive genes. Progeny of crosses between slow-rusting
culnivars showed transgressive segregation for latent period.

More recently we have sndied F6-derived F7 families, each tracing to a different F2 plant, from a cross between slow rusting cultivar CI 13227 and fast-rusting cultivas Suwon 92 . Use of these nearly homozygous families eliminated the confounding effects of domiriance, and replication of lines allowed betuer separation of genetic and environmental variance. There was a wide range in latent period among F2 piants of this cross, and highly significant differences in latens period among families. The distributions of lacent period for $F 2$ plants and 53 and 57 family neans were skewed, with population means significanly below mudparental values. Daza from $F 7$ families suggest that 3 loci with epistabic effects control latent period Approximately $3 / 4$ of the $F$ families had larent penods below the parental midpoint. Possibly one of the three loci has a major effect on latent period.

These same families were grown in the field in 1994, in the F8 generation. There were highly significant differences among families in rust development. Because of the considerable difference in date of spike emergence among families, the area under the disease progress curve divided by the duration of the epidemic on each family was a useful statistic to compare families. The distribution
of AUDPC/day was approximately normal. There was a significant negative correlation between latent period and disease development in the field.

Slow rusting does not appear to be a geneticaily complex trait, and it should be possible to work with it in a wheat breeding program. That few wheat breeding programs include intentional crossing and selection for slow rusting may result from the lack of a simple and rapid means of assessnent rather than from the genetic complexity of the trait.

Department of Botany and Plant Pathology Purdue University

1155 Lilly Hall

West Lafayerte, lndiana 47907-1155, USA
L.T Colon, D.J. Budding, I Hoogendoorn

The oomycete Phytophthora infestans causes a destructive disease of potato worldwide. known as potato late blight. Symptorns of the disease are grevish brown necrotic lesions, expanding at a rate of up to about

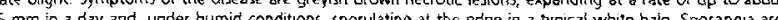

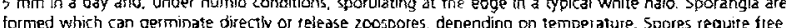
wer on he len for water on the leaves for germinalion. They insth by direct pepell An incer the host cell. Whin 48 hours. spapsionther sporangia. Under favourable conditions the disease can kili he follage of a susceptible crop in a few weeks ame Tuber infectin, especialy in heavy soll, is the tradional means to bidge the periad beeween wo growing seasons. Infected tubers in pctato waste ples or planted as seed may produce the first infected plants of the season, which supply the initial inoculum that starts the late bilght epidemic. Since around 1980 both the A1 and the a2 compatibility type of the pathogen occur in Europe. In mixed infections of both types in one leaf, sexuai reproduction can occur giving sise to cospores. This type of spore can probably survive in the soil for several years and also sene as initial inoculum the next growing season. Phytophthora infestans has shown a great capacity for adaptation, to fungicides but also to host resistance The late blight problem seemed solved when at the begirining of this century major resistance genes from Sotanum demissum were found and transfersed to potato. However, before the first resistant vatiety was
actually introduced, virulent races had already appeared. With the introduction of the sexual grcle in Europe actually introduced, virulent races had already appeared. With the introduction of the sexual cycte in Europe, durability of this and of ather resistances may be even lower than before. In contrast, the partial resistance present in 5 . tuberosum appears durable. Unfortunately, its level is not high enough to sufficiently prot
potato crops, and the combinztion with early maturity has not yet been achieved. A number of other potato crops, and the combination with early maturity has not yet been achieved. A number of other be estimated lacking this information, the approach which seems most feasible is accumulating as much genes for parcial resistance as possible. Perhaps in the future this will be easier, if marker aided selection for individual genes or QTS (quantative trait loci) becomes feasible.

8reeding for partial resistance to $p$ infestans has to take into acrount all aspects of the disease cycle Resistant varieties should be resistant both in the ioliage and in the tuber. Partiat resistance in the foliage can be thought to consist of several resistance components, influencing disease cycle parameters such as infection efficiency, lesion growth rate, latent period (generation time) and sporulation capaciny. Resistance to stem infection, which can kill an othemise uninfected plant by causing the stem to break ott easily, is arother factor to take into account. It has been shown in simulation studies by Van Oijen (1992) that infection efficiency and lesion growth rate probably have the greatest impact on the epidemic development of potato late blight, whereas latent period and sporulation capacity appear less important. in a field of one partially resistant variety, the resistance effects of these four components can simply be added to estimate the effect of combined components. However, traditional selection for partial resistance in field trials makes use of small plots highly subject to interplot interference. Host genotypes with low sporulation capacity and use of small plots highly subject to interplot interference. Host genotypes with low sporulation capacity and long latent periods will not necessarily be selected, while they can still contribute significantly to resist
Our analyses of components of resistance to $P$. infestans in the potato (Solanum tuberosum) arid in a Our analyses of components of resistance to $P$. infestans in the potato (Solanum tuberosum) and in a number of wild Solanum species, show that the importance of specific components varies among species. In S. tuberosum for example, sporulation capacity (the number of spores produced per unit infected leat area) showed higher genetic variation than infection efficiency and lesion growth rate. In S. berthayirii, a South American species, infection efficiency appeared the most important compontent. In the selection of crossing parents it may therefore be usetul to consider components of resistance in addition to overall resistance. On costly to be carried out routinely

Oijen, M. van, 1972. Selection and use of a mathematical model to evaiuate components of resistance to Phytopthora infestans in potato. Netherlands Journal of Plant Pathology 98: 192.202

DLO.Centre for Plant Breeding and Reproduction Research (CPRO-DLO), PO BOX 15, 5700 24 Wageningen. the Netherlands

\section{DURABLE DISEASE RESISTANCE IN PEANUT}

\section{B.B. Shew, M. K. Beute, H. T. Stalker, and T. G. Isleib.}

Arachis hypogaea is grown in warm climates throughout the world and several pathogens cause extensive losses. Because high yields require intensive management, there is a great need to increase production and quality in low-input cropping systems. In North Carolina (NC), the root, peg and pod rot (CBR) caused by Cylindrocladium parasiticum is an important disense of peanus. Chemical control of CBR is expensive and incomplete. Initial screening of $A$. hypogaed revealed partial resistance to CBR and the germplasm line NC 3033 was released in 1976. Expreasion of resistance to CBR varies with the level of inoculum present in soil; susceptible genotypes become severely diseased at inoculum densities as low as 0.5 microsclerotia (ms)/g soil whereas NC 303 ] expresses moderate resistance at $50 \mathrm{~ms} / \mathrm{g}$. Although the association between CBR resistance. small seed size, and low yield first found in NC 3033 hampered efforts to produce resistant cultivars, NC 8C was released in 1983. The cuttivar NC 10 C replaced NC $8 \mathrm{C}$ in 1991 Optimum performance of NC $10 \mathrm{C}$ requires proper rotations, delayed planting, neralade control, and fumigation. Advanced generation breeding lines currently are being evaluated that will give ise to cultivars with greater CBR resistance. Early and late leaf spots occur world wide on pesnut and cause nearly complete defoliation and dramatic yield losses in the absence of frequent fungicide sprays. Immunity is lacking in $A$. hypogaea, but moderate to high partial resistance has been found. Resistance breeding in NC has focused on earty leaf spot, which predominates there. Several components of resistance identified in greenhouse tests were correlated with Gieid performance. Resistance generally is thought to be under additive genetic control, but occasionally dominance or epistatic effects have been reported. Breeding lines with high yield and quality, and parital resistance to early leafspot have been selected. Highly resistant genotypes also have been found in some exotic plant introductions, but this germplasm often has smal seeds, low yields, and low combining ability for resistance. Very high resistance to leaf spots bas been found in wild species of Arachis. Resistant germplasm lines at the same ploidy leyel as cultivated peanut were derived from $A$. cordenasii and released. Resistance again sppeared to be negatively correlated with large sed size and high yield, but sufficient variation among lines exists to allow selection for these traits. These lines also may be of great use in areas where large seod size is not of primary importance. Resistance to leaf spots is inherited independently and planting of cultivars with resistance to only one leaf spot may result in exchange of one disease for the other. Resistance to early lesf spot is somewhst unstable across locations, pertups because temperature influences resistance expression differentialiy in some genotypes. Therefore. deployment of leaf spot resistance will require careful monitoring of pathogen populations. Recognition of the importance of pariel resistance has led to remarkable progess in cultivas and germpiasin development is $A$. hppogaea since 1970 . In addition, very high levels of resistance or immunity to major pathogens have been found in wild Arachis spp. Recent progress in lowering barriers to interspecific crossing, mapping of quantitative trait loci, and developing transformation systems will make these resources more accessible to peanut breeders.

North Carolina State University, Box 7616, Raleigh, NC 27695-7616 
H.H. Geiger ${ }^{1}$, T. Miedaner ${ }^{2}$, H.G. Welz ${ }^{1}$

Classical genetic analyses of quantitative resistance (QR) have substantially contributed to our understanding of host-parasite interaction in many plant pathosystems. In most experiments, variation in $Q R$ was shown to be controlled by genes located on several chromosomes. Differences between generation means or progenies within generations were mainly attributable to additive gene action while dominance and epistasis played an important role in particular cases only. Heritability estinates were generally high and recurrent selection studies demonstrated that QR can be improved considerably by breeding. Experimental evidence of pathotype specificity was obtained in only few studies, i.e. durability of $Q R$ seems to prevail.

Molecular marker techniques recently offered new approaches for studying the genetics of QR. Linkage analysis in segregating generations allows to identify, localize and manipulate QR loci individually. Molecular markers can therefore be used to (1) directly determine the number of genes involved in $Q R$ and its components, (2) assess gene action and interaction, (3) discriminate between pleiotropism and linkage disequilibrium as causes of genetic correlations between $Q R$ components, (4) analyze whether pathotype-specific and non-specific resistance genes may be allelic, (5) study interactions with pathogen genotypes, plant organs, developmental stages, and abiotic factors at the indjidual $Q R$ gene level, (6) transfer $Q R$ to susceptible, but otherwise elite materials by marker-assisted backcrossing, (7) investigate homologies of $Q R$ mechanisms in different host species, and (8) isolate $Q R$ genes by map-based techniques. However, the marker approach can only detect genes or chromosome segments with rather large effects on $Q R$. Variation due to minor genes still remains a domain of classical quantitative genetics. Experimentai data from marker studies in various plant pathosystems is used to iliustrate of the foregoing issues.

\section{University of Hohenheim}

' 350 Institute of Plant Breeding, Seed Science, and Population Genetics

${ }^{2} 720$ State Plant Breeding Institute

D-70593 Stuttgart, Germany

807

TKE ROLE OF PLANT GROFTH DEVELOAMINT REGULATORS (PHYTOHORMONES) IN THE RESISTANCE OF PLANTS TO THE HARMFUL PESTS.

\section{Eugene Kislin}

The role of plant growth development regulators in the resistance of some cultivated plants against harmful pests has been studied. Experiments were carried out at All-Russian Institute for Plant Protection (St Petersburg) and Institute of Viticulture (Kishinev) up 1985 to 1994. Observations were made for fields and laboratories of maize, wheat, barley, horse bean, cucumber and grapevine. The effects of the pre-treatment of seeds of some agricultural plants (wheat, pea, maize, cucumber) with exogenous regulators (indole-3acetic (IAA), gibberellic (GA), abscisic (ABA) acids, and 6benzylaminopurine (6-BAP)) on the resistance of adult plants to harmful pests (Aphis fabae L., Shizaphis graminium Rond, Oscinella frit L., and Tetranichus urticue Koch.) were investigated. The extent of biological damage of plants has been also investigated. Resistant and susceptible plants were used. Varieties, species and
hybrids of grapevine lVitis vinifera I., V. Vinifera L. \& V.


labrusca $L ., V$, riparia Michx. $x V$. rupestris Scheele. 101-14, V. monticola Buchl., degrees of resistance against the Phylloxera vastatrix flanch. have been studied with regard to ABA in leaves of adult plants damaged by the pest. The phytohormones were assayed by means of TLC, GLC and HPLC methods. It was found that IAA $(100 \mathrm{mg} / \mathrm{L})$, and GA $(25 \mathrm{mg} / \mathrm{L})$ considerably decreased the numbers of cereal aphids which were being fed on wheat and maize, while 6-BAP $(25,100 \mathrm{mg} / \mathrm{L})$, IAA $(25 \mathrm{mg} / \mathrm{L})$ and $\mathrm{GA}(100 \mathrm{mg} / \mathrm{L})$ increased the extent of damage of plants grown under the field conditions. IAA caused the increase of maize damage by frit fly while GA decreased it. However 6-BAP $\{100 \mathrm{mg} / \mathrm{L}\}$ considerably decreased the damage by Tetranichus urticue Koch. and inhibited the stem growth of cucumber plants in the greenhouse. The increase of pea resistance to Aphis fabae L. Was observed after changed the growth and development All the studied substances have insects leaf galls. The ABA levels in undamaged leaves was depended from species. Maximum of ABA content has been observed in the uninfested leaves of leaves of Vitis vinifera I.. Changes in ABA content in the uninfested leaves were correlated with the resistance of grapevine to leaf form of the insect pests. Obviously induction of ABA and development of microorganisms (bacterium and fungus) inside gall. It has been also assumed that the resistance of plants to gall. It has been also assumed that the resistance of plants to harmful pests maght be caused by the changes in the assimilate flow phytohomones.

All-Russian Institute for Plant Protection, Shosse Podbelskyi,3, st Petersburg-Pushkin, 189620, RUSSIA E-mail: vizreriam.spb.su
Th. Jacobs and D.J. van der Gaag

Components of partial resistance were studied in 54 Single Seed Derived F8 lines of a cross between two spring wheat genotypes with a high level of partial resistance. Four flag leaves each of five plants per F8 line were inoculated with a monospore culture of wheat leaf rust. (Puccinia recondita f.sp. tritic). Fluorescent stain was used for histoiogical observations.

Longer latency period (LP50) was correlated witb lower Infection Type (IT), lower Infection Frequency (IF), higher percentages of aborted infection structures, and higher percentages of non sporulating colonies.

Hypersensitive cell collapse was visiblo as autofluorescence. Nearly all aborted infection structures was associated with cell collapse (Fig.1). Lower infection Type was associated with more autofluorescence in established colonies.

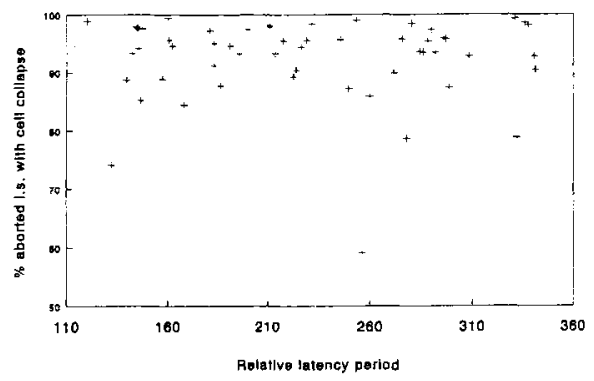

The correlations between LP5O and the components IF, \% aborted infection structures and $\%$ non sporutating colonies are conform expectations. A relation between cell wall appositions and aborted infections structures has been observed (1). No mechanism has been postulated for the post-haustorial growth retardation of established colonies. Encapsulation of haustoria has been excluded (2). Results reported here suggest that Encapsulation of haustoria has been excluded (2). Results reported here suggest that
hypersensitive cell collapse is the main causal factor of growth retardation. Contact between the host ceil and the haustorium of the fungus could lead to both the death of the infection structures (lower (F) and a reduced growth of established colonies (longer LP50).

1. Jacobs, Th., 1989. Journat of Phytopathology 127, 239-249.

2. Jacobs, Th., F.P. van Hee and F.M. Engels, 1993. Mededelingen van de taculteit van Landbouwwetenschappen 58 (1):1.9 Rijksuniversiteit van Gent, Beigium.

Department of Plant Breeding, Wageningen Agricultural University, P.O. Box 386, 6700 AJ Wageningen, the Netherlands

\section{8}

ANTIXENOSIS RESISTANCE IN SUBTERRANEAN CLOVER TO REDLEGGED EARTH MTE

\section{T.J. Ridsdill-Swith, Y. Jiaug, E.L. Ghisalberti}

Redlegged earth mite, Halotydeus destructor (Acarina: Penthaleidae) is a major pest of annual pasture legumes in southem Australia and in particular of subterranean clover, Trifolium subiemoneum. Varieties of subclover with seedling resistance to redlegged earth mite have been identified through screening for feeding damage. Protection of seedlings of these annual plants from mite attack is a major aim of this project. Mites tend to aggregate when feeding, leaving silvery patches of damage. In choice experimeats the mites distinguish within $3 \mathrm{~h}$ between detached cotyledons of resistant and suscentible subclover varieties. As a result of this behaviour feeding mites tend to aggregate on the preferred host. Counts of mites on the cotyledons gave a quicker and easier measure of feeding than other methods of measuring feeding. These results provide strong evidence for antixenosis (antifeeding) resistance in the cotyledons.

A bioassay was developed to investigate any antifeeding effects of non-volatile plant extracts. However, in spite of extensive testing no plant extracts associated with resistant varieties showed antifeeding properties. A physical factor, cotyledon toughness, was identified as a factor in the resistance. There are no obvious differences in the visible stricture of coryledions of resistant and susceptible varieties, and the mechanisms are still being investigared. Cotyledor roughness has now been tested in over 30 varieties of three subfamilies of subclover and in Medicago sp. varieties, and is negatively correlated with feeding damage in all but one of these varieties.

A bioassay has also been developed to test for plant volatiles, and volatiles extracted from cotyledions have beer shown to repel the mites at close range. Some volatiles are present in higher concentrations in resistant than susceptible varieties. It is suggested that the mites detect them when they feed on the resistant cotyledons. Work is proceeding to identify the compounds involved

This project is part of a subprogram in the Co-operative Research Centre on Legunes for Mediterranean Agriculture (CLIMA), involving plant breeders, agronomists, entomologists and chemists. Resistant varieties are being selected on the basis of screening and of mite responses to the different varieties. In addition plant breeders are crossing the best resistant varietses with vaneties having superior agronomic qualities. The antixeaosis resistance factors reported bere affect mite feeding behaviour and are more likely to provide stable long term resistance than highly toxic antimetabolic resistance factors. We are optimistic that commercial subclover varieties with seedling resistance to redlegged earth mite will be released in the next few years which will reduce the considerable losses to farm production curreatly oceuring from this pest.

Co-operative Research Centre for Legumes in Mediterranean Agriculture, and CSTRO Division of Entomology, University of Westem Australia, Nedlands, WA 6009, Australia. 
COMMUNICATION TECHNOLOGIES FOR INFORMATION PROVISION AND ADVISORY COMMUNICATION IN DUTCH AGRICULTURE A STATE-OF-THEART IN CONCEPTUAL AND PRACTICAL TERMS

\section{R. Ausher}

Since microcomputers are already there in the developing countries, the issue is not whether to use them, but how to appropriately apply available microcomputer they have proved themselves. Agricultural production they have proved themselves. Agricultural production systems in the developed and the developing world have much in common. The basic difference lies in the levels of input and output, crop intensity and productivity. Farmer's decision-making processes and needs for updated information are becoming more complex and demanding. In order to sustain and improve environment-friendly productivity there is a parallel need for integrated data, information, know-how and technologies. The conflict between precision within a narrow range of operating conditions (high data: parameter ratio) and generality (low data:parameter ratio) is the main problem affecting the transportability of decision-support systems utilizing biological parameters. Following this conceptual framework, a survey of actual computer-based utilizations in support of various agricultural extension functions in the developing world has been initiated. The survey focuses on the real benefits of computer use, and the problems of this area requiring intervention and remedies. Its analysis is helpful in crystallizing more efficient ways of matching the opportunities offered by computer technologies with the specific needs of various agricultural extension systems and extension crop protection more specifically.

Ministry of Agriculture, Extension Service, P.O.Box 7054 , Tel-Aviv 61070, Israel.

811

\section{IPM TRAINING APPROACH USED IN VIETNAM}

\section{Marion Fredrix and Pham Thi Nhat}

Vietnam started a training programme in Integrated Pest Management (IPM) in Rice in 1992. Farmers are the main target group of the IPM training. The main sources of information for farmers are the study fields, and their own experiences in rice growing. Farmers learn methods how to approach problems, and how to find solutions by conducting experiments.

Farmers attend Farmer Field Schools (FFS) in their village. A FFS consists of a group of 25-30 farmers that meet one morning every week for the duration of a rice crop, about 3.5 months. Each FFS sets up a study field, divided into an IPM and Farmer Practice (FP) plot. Weekly farmers make observations in both treatements, looking at all factors in the rice ecosystem: rice plants, weeds, pests, natural enemies, diseases, weeds, water, and weather conditions. After observation each group analyzes the field situation by making an ecosystem drawing. and discusses the findings and makes a management decision for the field. A group discussion follows. Besides weekly ecosystem analysis farmers do special exercises, like insect zoo (to study predation capacity, parasitism, life cycles), effects of pesticides on natural enemies, on health. Also additional field studies are set up by the farmers, like defolation (to simulate leaf damage), detillering (to simulate stemborer damage) and exclusion cages for Brown Planthopper.

During the FFS regularly team building exercises are done, to create strong farmer groups. The FFS is facilitated by Plant Protection extension workers, who attended a full time season long Training of Trainers Course, in which they learn about IPM training methods and planning. Also farmers who attended a FFS and that are interested in becoming farmer-trainers conduct FFSs in their area. IPM farmers maintain their yields, reduce pesticide use and get higher benefits. More important is that in many villages IPM farmers continue to play an active role in spreading IPM in their area. IPM clubs are established in many places. These clubs do a range of activities: organize and conduct FFSs, conduct studies like rice fish culnure, soybean IPM. Some also cooperate with researchers (international and national) to get a better knowledge of blast disease, and to improve gene deployment strategies for a longer term control.

At present over 950 extension workers have participated in TOTs, and over 100,000 farmers in FFSs. Local govermment provides funds for about $30-40 \%$ of FFSs, showing a strong commitment to the XPM programme. The governments of Australia and the Netherlands provide funding for IPM in Vietnam through the FAO.

FAO-IPM Programme, FAOR, 3 Nguyen Gia Thieu, Hanoi, Vietnam

\section{Leeuwis}

In recent years a considerable amount of (state-subsidized) projects were launched in order to stimulate the use and development of computer-based decision aids for farmers and extension workers. Not unlike automation projects in other societal realms, many 'agro-informatics' projects have failed to meet their original objectives. Both the quantitative and qualitative use of the software and hardware packages which were produced remains unsatisfactory (Klink 1991: NRLO 1991; Geuze 1991).

Amongst those involved with 'agro-informatics' there seems to be a fair amount of consensus on how this situation can be alleviated. The most umportant recommendation is that supervision and extension activities need to be launched in order to improve the use of software products by farmers. The idea is that existing packages are (even if they can still be improved) quite adequate (Klink, 1991:i) and that large segments of farmers -because of their assumably limited analytical capacities- need to be helped to adequately interpret and analyze the available information (Geuze 1991:137; Klink 1991:i; NRLO 1991:10; Ziggers 1991:12). In essence, this mode of thinking implies that agro-informaticians tend to fall back on rather outdated models of technology transfer and extension. More recent theoretical insights, however suggest that the technologies on offer themselves may be problematic, and that this is somehow related to the complex interactions and active negotiations that occur among the multiple actors involved (i.e. researchers, extersion workers, policy makers, project staff, farmers, software designers, commercial softwate industries etc.)

In my paper, I will first point to various shoncomings in the way adoption or nonadoption of computer-based decision aids (which I will label communication technologies') is currently explained and conceptualised. Second, I will argue that -in line with my theoretical critique- we must move away from technologically based descriptions and analyses of communication technology use. In relation to this I will provide a functional classification of such technologies. Third, I will identify a number of ways in which communication technologies may fail to anticipate the environment in which they are supposed to be used. In doing so, I will argue that the concept of 'information-needs' is highly problematical, and of limired value if it comes to directing communication technology development. Finally, I will reflect on currently proposed solutions for the 'adoption-crisis' (see above), and indicate how social scientists may contribute to the development of more appropriate communication technologies.

Department of Communication and Innovation Studies, Wageningen Agricultural University, Hollandseweg $1,6706 \mathrm{KN}$ Wageningen, The Netherlands

812

INFORMATION TECHNOLOGY IN SUPPORT OF NGO BASED EXTENSION AND EDUCATION IN GHANA: A CASE STUDY FROM NORTHERN GHANA

\section{D.M. Millar}

I start this paper by making an un-professional division between computer-based information technology and non computer-based information technology. I make this lame dichotomy because all the literature I have come in contact with see IT in terms of modem technology; as if IT never existed before the computer era and as if we those in this part of the World never have operational ITs of our own.

In their article, Robling and Engel (1991) wrote that "IT refers to computer-based systems used for collecting, exchange, processing and/or producing information".

In the same publication, Leeuwis (1991), introduces a social dimension to computerbased TTs by asking who the users of ITs were. He argues convincingly that researchers and extensionists were also ultimate users of $\mathrm{IT}$ to pronote their scientific models. The 'people aspect' of computer-based ITs was now beginning to become an issue.

Nitsch (1991) took the debate further by his discourse on the formal rationalities of science versus adaptive rationalities of farmers. He postujates that even in the advanced countries where computers have their home, the complexities of farming and the number of variables that a farmer has to consider in one decision making process makes computers incomplete in their services.

I wish, in this write-up, to discuss IT in its broadest sense: beyond computer-based ITs, by drawing on a NGO's experiences with the Participatory Technology Development Model (PTD) of Northem Ghana. The PTD Model is based largely on Rural Peoples's Information Technology (RPIT) which is deduced form their different forms of information generation and use as reflected in their modes of experimentations namely; Curiosity experiments, Problem solving experiments, Adaptive experiments and Socialyeer pressure experiments.

In discussing the aspects of RPIT that influence education in the PTD model, I high-light some aspects of NGO training that have evolved as a result of the need to build up NGO capacities to enable them deal to effectively with the realities on the ground; the inperfection of NGO actions notwithstanding.

In concluding. I take the stand that there is the need for the integration of non computerbased ITs with computer-based ITs; the formal/informal with the indigenous. I favour the development of models of ITs within tural peoples' environments (Jaboratories) narrowing the professional distances and capturing adapuve rationalitiesfrealities. For recommendation, I look at participation and sustainability from a new paradigm by advocating dialogical development and enhancement, 'Farmer-Led' modelling in ITs, reorganising to strengthen the User sub-system and re-orienting extension and research. 


\section{H.F.M. ten Berge, W.A.H. Rossing, A. Elings}

A systems research network for rice was established in aight Asian countries with 15 national agricultural research centres (NARCs) via the SARP project. The goats were to build human capacity in systems research (short term) and to improve rice based cropping systems through higher research efficiency (long term).

NARCs are participating by interdisciplinary teams. A 'team establishment phase' covered six years, and included three international training programs. It was followed by an 'application phase', currently addressing six 'application programs'. Mechanisms used in capacity building after the formal training phase are case studies, team visits by project staff, joint research planning and output analysis, shor: ( 3 months) and long (1 year) fellowships for exchange visits of team members, annual thematic workshops, PhD fellowships, support to local (national) training courses, a proceedings series for project publications, and the development and distribution of user-friendly shells to facilitate the use of simulation models

One of the joint research programs addresses crop protection. This program aims at quantitatively explaining effects of selected pests and diseases on growth and production of rice, based on insights into the effects on crop physiology. Attention is focused on stemborers, bacterial leaf blight (Xanthomonas campestris pv. oryzae) and blast (Pyricularia oryzae). In the paper, application of the approach to the rice-bacterial leaf blight pathosystem is used to illustrate the role of information technology. Limitations experienced by the NARCs, and in particular by crop protection researchers, with this methodology are discussed. In part, these stem from a lack of crop awareness among scientists in crop protection. Traditionally, training curricula focus on pests and their control methods and largely ignore the important role of the crop and its responses to pest attack. This situation appears to be wide-spread and calls for further deveiopment of interactive dynamic training materials.

DLO Research Institute for Agrobiology and Soil Fertility

P.O. Box 14

6700 AA Wageningen, The Netherlands
T.R. Fasulo

Developing worthwhile computer knowledgebases or training software for any area where agricultural extension provides service can be beneficial for all concerned. Well designed software will save extension personnel time and money, and can result in a large number of clients continually receiving instruction in the latest pest control techniques. Developing such software, however, can be expensive in time and money in that it initially requires extensive resources and thoughtful design. On the other hand, developing poorly designed software not only wastes resources, but alienates both users and granting agencies to any future projects. Unlike software with mass market appeal, agriculiurally related software has a much smaller market. In addition, targeted agricultural users of such software are generally not as technologically sophisticated as those in other areas of business. It is important to remember that no matter how important the information is, if it is not easy to access or presented in an attractive format then the program will not be used. By recognizing the above principles, this department has successfully developed a number of software packages for the urban pest, agricultural and omamental industries that are being distributed internationally. These packages are distributed by the University of Florida, the United States Department of Agriculture, industry associations, and private corporations.

Department of Entomology \& Nematology

University of Florida

Gainesville, Florida 32611-0649

USA

fasulo@gnv.ifas.gnv.edu (Internet)
The Australian Cooperative Research Centre for Tropical Pest Management (CTPM) produces computer programs that provide high quality, technical support for pest management R \& D, training and decision support. End-users include policy makers, research and extension scientists, students, crop consultants and farmers.

Two distinctive features characterise CTPM software

- user-friendly - simple but powerful tools to undertake well specified tasks

- generic - users, trainers or decision support developers can use or easily adapt the software for their own specific, pesi management probiems.

Four software products illustrate how this generic approach is being implemented

DIAGNOSIS for Crop Protection - a training tool designed to "simulate" real problems that the user (students, extension agents, crop consultants) has to solve. The program allows the user to investigate the problem using draw-down menus and uses text, allows the user to investigate the problem using draw-down menus and uses text, to users on how to improve their diagnostic skills. Diagnosis comes complete with a to users on how to improve their diagnostic skills. Diagnosis comes complete with a
builder that allows trainers to develop their own scenarios without requining any further builder that all
programming.

BUGMATCH for cotton - Developed for Rhone-Poulenc Australia, this program is designed to help with the identification of pest and beneficial insects in cotton and to educate users (growers and consultants) on the benefits of IPM. Colour images and video tion and concepts. A broad reforence section provides users with easy access to information on importance, damage, distribution and monitoring of the main species in Australia.

CLIMEX - A research and decision support tool for predicting the potential distribution and relative abundance of species in relation to ctimate. CLIMEX is used in over 20 countries (by research scientists and policy makers) to provide information on insect, weed, pathogen and vertebrate distribution for quarantine and biological control purposes. A new Windows version, recently released, includes many improved features.

GENSECT - The ultimate, use-friendly population model, provides users (research scientists and students) with a means of building a population model of any species simply by clicking on menu options and answering questions. GENSECT has a modular structure, with each module dealing with a specific population process, such as development, reproduction, mortality and diapause, for each set of user-defined lifecycle stages, such as eggs, seeds, flowering stages or adult insects. These modules can also link to other modules in other programs, such as the met-manager in CLIMEX for Windows). A core version of GENSECT will be released in early 1996. Subsequent versions will incorporate additional teatures as they are developed.

Cooperative Research Centre for Tropical Pest Management, Gehrman Laboratories, The University of Queensiand, St Lucia, Brisbane, Qld 4072, Australia
816

\section{A DECISION-SUPPORT SYSTEM FOR INTEGRATED CROP MANAGE- MENT OF GREENHOUSE CROPS}

\author{
J.L. Shipp, N.D. Clarke, W.R. Jarvis, A.P. Papadopoulos and T.J. Jewett
}

The use of decision-support systems in crop protection is a rapidly growing area. In greenhouse crop management, the industry is technologically advanced and computer literate. Computerized environmental control and fertigation systems are in wide commercial use and offer opportunities for precise and versatile crop management. Environmental regulations, fewer effective registered pesticides, worker health concerns and cousumer demand for safer food products are forcing the greenhouse industry to reduce its use of chemicals for control of pests and its use of fertilizers and water. These demands can be met in greenhouse production because the crops are grown in an enclosed environment, biological and cultural control strategies are available, and soilless media and closed-feeding systems are used.

At the Harrow Research Centre, a decision-support system consisting of an expert system and a database management system has been developed to integrate pest protection and production strategies for greenhouse crops. A key component of the decision-support system is the identification and resolution of conflicting recommendations. The system has initially been developed for greenhouse cucumber and tomato, but can also be easily adapted to other greenhouse crops. The system contains expert knowledge in six areas: crop production, conflict resolution and the identification, control, forecasting and prevention of crop disorders. The database component manages information for accounting, specifications of the greenhouse operation, job planning, fruit yield and quality, pest incidence, preparing reports and many other aspects of the greenhouse operation.

The decision-support system requires a 386 personal computer and Microsoft Windows $^{\mathrm{TM}}$ to operate it. Greenhouse growers have had input into the design and evaluation of the system throughout the developmental phase. The system will be field tested in 1995/96. Although growers are the intended main user, other users would include agricultural advisors, agribusiness, researchers and educational institutions.

Agriculture and Agri-Food Canada, Research Centre, Harrow, Ontarion, Canada NOR 1G0 
NOVEL INSECTICIDES WITH SELECTIVE PROPERTIES: MECHANISM AND IMPORTANCE IN IPM PROGRAMS

\section{Ishaaya and A.R. Horowitz}

Novel insecticides with selective properties have been introduced in recent years as components in IPM programs for controlling agricultural pests in Israel. Buprofezin, a chitin synthesis inhlbitor affects embryogenesis and larval stages of plant hoppers, whiteflies and scale insects. It is not ovicidal but hoppers, whiteflies and scale insects. It is not ovicidal but suppresses embryogenesis upon exposure of females to treated plants. The concentration for 50t larval mortality of the Whiter fy Bemisia tabaci is $6 \mathrm{mg}$ (AI)/liter in the spray solution. Under field conditions the compound has vapor phase toxicity, affecting bidden larvae present at the lawer surface of the leaves. The juvenoid compound pyrifroxyfen suppresses embryogenesis and adubt formation upon treatment of $B$. tabacr females and larvae. It affects $0-2-d a y-o l d$ eggs and exhlbits translaminar activity affecting hidden eggs and larvae present on the lower surface of the leaves. Diafenthiuron, a thiourea derivative affects mitochondrian oxidation, acting specifically on mites, aphids and whiteflies. The compound affects all stages of $\mathbf{B}$. tabaci in the order, larvae $a d u l t s>$ pupaeseggs. Pymetrozine, a novel triazinone derivative acts specifically on the sucking muscles of aphids and whiteflies, resulting in starvation an mortality, The chloropyridyl derivatives, imidacloprid and acetamipxid, which are in progress development and marketing, are systemic and foliar insecticides acting specifically on sucking insects such as aphids and whiteflies; if lised as systemic insecticides through sol selective insecticides.

Ald the above compounds have different biochemical modes of action and can be alternated to prevent development of resiscance, for controlling whiteflies and aphids.

The search for potent acylureas has led to the development of new compounds such as chlorfluazuron, teflubenzuron, nexaflumuron and triflumuron. These compounds are today important components for controlling lepidopterous pests such as spodoptera littoralts in vegetables and field crops and the grapevine moths lobesia botrana and cryptoblables gnidiella in vineyards. In addition to the benzoyphenyl ureas, several ecdysone agonists are under development and marketing such, as FH-5992 (tebuferozide) and $\mathrm{RH}-248 \mathrm{~S}$. Both of them are powerful compounds against various lepidopteran pests.

The above compounds have relatively low toxicity to man and natural enemies and are considered important components in IPM programs in fiela crops, ornamentals and orchards. For preventing development of resistance, an insecticide resistance management strategy should be developed aiming at alternating compounds with different modes of action and restricting the use of each compound to one treatment per season.

Department of Entomology, Agricultural Research Drganization, The volcani Center, Bet Dagan 50250, Israel

819

\section{REDUCTION OF VIRUS TRANSMISSION AFTER APPLICATION OF PYMETROZNE}

Daniele Fugg, J. Scott Fergusson and Claude Flückiger

Pymetrozine is the first compound from a new class of insecticidally active ingredients with a novel mode of action. Treated insects do not die irnmediately, but quickly stop feeding. New trials carried out searching to clarify the practical significance of this mode of action indicate that the transmission of persistent viruses is efficiently controlled, which was confirmed under field conditions on potatoes. Pymetrozine has both contact and systemic activity. Both foliar and soil application lead to effective control. The compound also has translaminar activity Pymetrozine was discovered by Ciba Crop Protection and is being developed worldwide for the control of aphids and whiteflies in vegetables, ornamentals, field crops. hop, decidxous fruit and citrus, as well as brown planthopper in rice. The control of aphids is achieved with 10-20 g/hl, whilst the control of whiteflies requires 20-30 g/hl. Pymetrazine has been introduced in Switzerland in 1994, and in Ecuador in 1995. The compound is safe to most nontarget arthropods, and of low hazard to birds and fish.

Ciba-Geigy Limited, Insect Control, PP 7.314, CH-4002 Basle, Switzerland
ECDYSONE AGONISTS: BIOCHEMICAL AND BIOLOGICAL ASPECTS

\section{G. Smagghe and D. Degheele}

The substituted diberzoyl hydrazines, RH-5849 and tebufenozide, are the first potent nonsteroidal agonists of ecdysteroids against insect larvae of different orders, especially Lepidoptera. The discovery of such compounds generates great interest as they provide important tools in research on ecdysteroid hormone activity, and the opportunity to be used in pest control. Tebufenozide is more active and caterpillar selective than RH-5849, suggesting that this compound may be an excellent insect growth regulator in the control of lepidopterous pests, even in integrated pest management programs. This overvizw considers the specific acdysieroid-mimieking activity of these compounds as well as their atvantages for research and agricuiture

Laboratory of Agrozoology, Faculty of Agricultural and Applied Biological Sciences. University of Gent, Coupure Links 653 , B-9000 Gent, Belgium.

820

IMIDACLOPRID, A NOVEL CHLORONICOTINYL NNSECTICIDE, BIOLOGICAL ACTIVITY AND AGRICULTURAL IMPORTANCE

\section{W. Leicht}

In 1984, our partmers at Nihon Bayer Agrochem synthesised a series of compounds which we later named chloronicotinyl insecticides. From this series, imidacloprid was selected and developed as an insecticide in a joint action by our Japanese and German researchers. The relationship of this novel insecticide to nicotine, to the nitromethylenes developed by the Shell company in the late seventies and to newly described pharmaceutical painkillers found in the skin of frogs are reviewed. Besides structural similarities, all these compounds share the nicotinic acetylcholine receptor as the molecular site of action. Evidence is presented that the low mammalian toxicity of the compound may be due to a selective agonistic effect at the insect receptor. Resistance of insects towards commercial insecticides is very often due to changes at the molecular target siles. Since most of the cornmercially applied insecricides are directed at molecular target sites other than the nicotinic acetylcholine

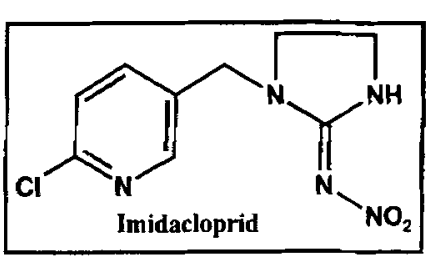
receptor, imidacloprid is especially effective against insects resistant to conventional insecticides. A moderate water solubility confers root-systemic properties to imidacloprid allowing to protect plants against sucking and mining insects after soil or seed application. This is demonstrated by the excellent control of aphids in sugar beet and cereals which is achieved by imidacloprid applied as seed dressing. Also the use of granules in rice culture using seedling boxes is a further example of soil application. However, imidacloprid can also be used as foliar spray and was shown to be effective against white flies and scale insects in citnus groves. A promising new paint application was developed for aphid control in hops.

Bayes AG, Crop Proteution, Development, Centre Monheirn. D-51368 Leverkusen, Germany 


\section{A. R. Horowitz, and I. Ishaaya}

Since the late 1980s, various novel insecticides, mostly insect growth regulators (IGRs) were introduced in Israel against cotton, vegetable and ornamental pests. The insecticides included: vuproferin, pyriproxyfen, diafenthiuron and imidacloprid for controlling the whitefly Bemisia tabaci (Gennadius), and berzoylphenyl ureas such as teflubenzuron, chlorfluazuron, hexaflumuron and lufenuron against lepidopteran pests, especially the Egyptian cotton leafworm, Spodoptera littoralis (Boisduval). pests to the above insecticides, various countermeasures were pests to the above insecticides, various countermeasures were initially employed as a insecticide resistance management (IRM) strategy in Israeli cotton since 2987 . The main principles were: restricting the use of the insecticides to a one-pest-generation period (approximately one month) alternating compounds with different modes of action, and minimizing the number of treatments according to provisional action threshold (thus preserving natural
enemies populations). enemies populations).

The implementation of the strategy was accompanied by an extensive resistance monitoring program conducted during the summer in cotton fields and throughout the year in various greenhouses. Base-1ine bioassays for susceptibility of key pests to the most important novel insecticides were carried out prior the resistancemonitoring in field populations.

The resistance monitoring results showed that the susceptibility of $B$. tabaci to buprofezin and pyriproxyfen in cotcon fields (with an IRM strategy) was maintained, although some decrease in susceptibility to insecticides was found late in the cotton growing season following applications. Due to the restricted use of the novel compounds, and consequently a reduction of selection pressure, those insecticides could be applied in the following season when the pest populations were again susceptible to both compounds. on the other hand, moderate to high resistance levels to buprofezin (ca 20 -fold) and pyriproxyfen (ca 500-fold). respectively, were observed in greenhouse (without an IRM strategy) after successive use of these compounds. Recent assays have shown that in pyriproxyfen- of buprofezin-resistant strains of $B$. tabaci, no appreciable cross-resistance was observed among pyrigroxyfen. buprofezin, diafenthiuron and imidacloprid. Thus. these compounds can be use as alternative insecticides in an IRM strategy.

The monitoring of resistance in $S$. 1ittoralis to the IGRs, chlorfluazuron, teflubenzuron and hexaflumuron started late in the 1992 season with the collection of eggs and neonates from fields after applications of these compounds. The results indicated that the susceptibility of the larvae to chlorfluazuron was not altered after five years use. Only a slight increase in tolerance to teflubenzuron and hexaflumuron was observed.

The rational use of insecticides has resulted in maintaining the susceptibility of pests to the various groupe of insecticides and substantially reduced insecticide applications.

Department of Entomology, Agricultural Research Organization, The Volcani Center, Bet Dagan 50250, Israel.

\section{3}

BIOACTIVITY OF LJGNANS AND MECHANISM OF ACTION ON HERBIVOROUS INSECTS

B.J.R. Philogane

Lignans are phenylpropanoid secondary metabolites often containing a methylene dioxyphenyl group which can inhibit the cytochrome $\mathrm{P}-450$ dependant oxidation enzymes utilized by insects for the detoxification of insecticides. These compounds are widely distributed in plants and new occurrences are continually being reported. Lignans are most abundant in the Pinacede, Podophyllaceae, Rutaceae and Lautaceae.

A wide range of biological activities have been attributed to lignans: antibacterial, antimihotic, antioxidant, antifeedant and growth inhibition. Lignass alone have limited activity on insects particularly at the concentrations naturally occurring in plants. But in association with other allelochemicals or with synthetic pesticides they become guite toxic through their synergistic action.

We have investiguted the biouctivity of several lignans vis-j-vik a polyphagous insect. which is also a well established pest, the European corn borer, Ostinia nubilalis. Our research bas focussed on the activityof dillapiol, disesantamin. episansambin, cubebin, sesamolin,pinerlonguminurie and a neolignan, conocapan,

Resuits indicate that lignians have both synergistic and insecticidal action and that they play a major role in the protection of several plant species againse phytophagous insects.

University of Ottawa

550 Cumberland Street

P.O. Box 450, Station $F$

KIN 6N 5
AND BIOLOGICAL ACTEVITY

M. Matsuda, Odawara Research Center, Ninoon Soda Co., Ltd.

Acetamiprid, NI-25, $\quad N^{2}-((6-$ chtoro-3-pyridyl $)$ methyl $)-N^{2}-$ cyano-N ${ }^{1}$-metnylacetamidine, is a novel broad spectrum insectiride invented by Nippon Soda Co.luta. It is under world-wide scale develoment for the insect control in many croos. Aceramiorid has excellent activity agaust Hemiptera, Levidoptera, Coleoptera Thysanoptera and others on fruit trees, vegetables and tea. It shows also high activity aginst resistant insects to conventimnal inserticides. The compound shows high insecticidal actiritv by soil or seed ereatment with its systemic action as well as by foliar application aqainst anhids, thrios and leoidonterous insects on vegetahles. It is also effective against leafminers with its translaminar action and agajnst fruit moths with its ovicidal and larvicidal activities on fruit trees and tea. The pest insects treated with acetamintid show rabid boisoning symotoms such as excitement and paralysis, and they die. From our electrophysiological and biochemical studies of mode of action. acetamiorid is considered to act as an agonist to nicotinic acetylcholine receotor of insect rentral nervous system.

345 Takada, Odawara, Kanagawa, 250-02, Japan

824

Efficacy of some neu insecticides against the colorado Potato Beetle Lept inotarsa decerlineats say in poland Maria Pawinska

Institiute Potato Research, 76-009 Bonin, Poland

In Poland, the arrival of the colorado Potato Beetle in the 1950 cast a shadow over the country's most important crop. because after cereals, potatoes are biggest crop. The CPB infests about 907 of the growing area and accounts for a 57 loss of production even after chenical treatment. In an untreated field, loss of yield can be as hight as about 70\%. The cultivation of potatoes trends completely upon insecticides in controlling this pest and nost farmers are obliged to control crops using insecticides, with one to three treatwents annually. The control of CPB using the phosphoroorganic issecticides dominated In the 1983-1987 vegetation seasons, the pyretroids in seasons 1988-1992 and bensultap in 1993-1994. Rational control of CPE are introduced into the agricultural practice using insecticides with the ner chenical groups: fipronil, acetaniprid, inidacloprid, "ecologically accepted - the selectivity activated in time"- flucykloksuron, hexaflumuron, lufenuron, teflubenzuron (Insect Growth Regulators) and Biological Insecticides based on Bacl/lus thuringienis (B.t.t.), ained at nininising the occurrence of resistance in the colorado potato Beetle. The bioactivity of the chitin synthesis inhibitors and B.t.t. used in the comercial formution against larvae of $C P B$ in field experiments vas evaluated during 1985-94, ecetamiprid and fipronil in 1993-94 and imiadcloprid (insecticide seed dressing treatment) - 1991-94. All compounds showed hight potential in the control of the pest. 
PROCESSES AND PRODUCTS IN GLOBAL CHANGES OF ATMOSPHERE, LAND USE AND CLIMATE, AND THEIR IMPLICATIONS FOR PEST IMPACT RESEARCH

\section{S.V. Krupa}

Our knowledge of global climate change has many uncertainties. Whether global air temperature will increase, by how much, and when, are subject to debate, but there is little doubt that tropospheric concentrations of several trace gases are increasing. While possible increases in the average air temperature is a product of these changes, the increases in the trace gases alone will have an effect on agriculture. Increases in the ambient concentration of carbon djoxde are expected to have a positive net effect on crop production. In contrast, any increases in the penetration of surface level ultraviolet- $B(280-320 \mathrm{~nm})$ radiation, and known increases in surface ozone concentrations, are considered to have adverse effects on certain crops. Our present knowledge of the joint effects on crops of elevated levels of carbon dioxide, ultraviolet-B radiation and ozone, and possible alterations in air temperature and precipitation patterns, is virtually zero. Therefore, any predictions of the effects of global climate change on agriculture are subject to significant uncertainties. In contrast, coupling of climate change (only temperature and precipitation) models to crop production have led to a number of predictions of future scenarios. In spite of their present limitations, results from these efforts can be useful in planning for future agriculture.

Department of Plant Pathology, University of Minnesota, St, Paul, MN 55108, USA

MODELING AND PREDICTING THE IMPACT OF CLIMATIC CHANGE ON DISEASES AND WEEDS AND THEIR INTERACTIONS WTTH THE HOST PLANTS

\footnotetext{
M.J. Kropff 1.2 , P.S. Teng ${ }^{3}$

${ }_{1}$ Department of Theoretical Production Ecology, Agricultural University. Bomsesteeg 47, 6708 PD Wageringen, The Nerheriands

2 DLO-Research Instituze for Agrobiology and Soil Fertility (AB-DLO), P.O. Box 14,6700 AA Wageringen, The Netherlands

${ }^{3}$ The Intemational Rice Research Institute, P.O. Box 933, 1099 Manila, Philippines
}

Changes in global climate are expected to alter the distribution, abundance and dynamics of pests insects, weeds and diseases) and pest-plant interactions. Because of the complexity of these interactions, models that are based on the mechanisms of crop pest interactions are needed to estimate such changes. Several approaches have been developed to link pest models with cro models by identifying coupling points for pest and crop models for different pest-crop combinations.

For the estmation of the effect of global change pests, crops and their interactions, the component need to be moteled with the relevant climatic factors as driving variables. For diseases and weeds, such models have been developed and linked to crop models. The combination models were used to evaluate the consequences of climate change.

Specific exaraples will be given for the effect of global change on the disease leaf blast in rice and the effect of weeds on rice and sugarbeet. Studies on the eftect of temperature and rainfall changes in Asia on blast epidemics and effects on rice, showed that rainfall changes had no significant effect on blast epidemics, whereas temperature strongly affected the dynamics in several agro-ecological zones. For weeds it was found that the effect of temperature changes depended on the specific competition situation, including the date of weed emergence, the physiology of the crop and weed species and the environment. The use of models to predict the impact of elimatic change on pest and crop-pest interactions is discussed.
828

\section{PEST RISK ANALYSIS AND CLIMATE CHANGE}

R.W. Sutherst, T. Yonow, N. White and S. Skilton

The status of arthropod pests, weeds and diseases will alter in response to climate change in different environments. Clients seeking information on likely impacts have different needs for information depending on their particular concerns. A process is described and illıstrated of pest risk analysis in relation to impacts on agriculture and natural ecosystems resulting from elimate change. It consists of a hierarchical approach which depends on the needs of the customer and the quality of data that is available. A suite of tools ranging from expert systems, climate-matching models, population simulation models and GIS are appropriate in different situations. The future needs for research and the opportunities for synergism from international collaboration through the IGBP-GCTE network are discussed.

Cooperative Research Centre for Tropical Pest Management, The University of Queensland, Brisbane, Qld, Australia 4072 
NEW PEST PROBLEMS N EUROPE, AND THE ROLE OF THE GLOBAL WARMING

F. Kozár

Significant northward invasion of several southern insect pests (for example Pseudaulacaspis pentagona) was observed in the last years in different parts of Europe. In a lesser extent some species showed south-east extension of their distribution, as the Pulvinaria regalis, or the Phyllonorychter leucographella. Although this later species is also considered to be of Mediterranean origin. There is a more complex situation with Bemisia tabaci. This species, together with some other whitefly species, shows a northward spreading in Southern Europe in field conditions. B. tabaci has also spread very quickly in greenhouses of Northem and Central Europe. If this species appears in field conditions in Central Europe, it would not be easy to establish, whether it is an infestation originating from local greenhouses, or if there is a northward movement of this species in the nature.

New outbreaks of termophilous insects were observed in different countries, such as Ceratitis capitata in Southern Europe, Ceroplastes japonicus in North Italy, or the recent outbreak of the Dociostaurus maroccanus in Hungary, etc. New pests were also introduced into different countries such as Diabrotica virgifera in Yugoslavia, or the appearance of two Ragolethis species in Italy and Switzerland (all are north-americanian origin). There are also every year a lot of pest introductions in European greenhouses from tropical countries, especially of different scale insect species on flowers and ornamental plants. There are new problems with migrant southern species, such as the recent outbreaks of the Helicoverpa armigera in 1993-1994 in Hungary. The appearance of Ceratitis capitata in traps in Hungary during the past years could also be a result of immigration of flies from southem Europe, but might also have been introduced with infested fruits. According to literature this species can not overwinter in. Central Europe, not even in winters milder than the average.

The northward expansion and the new outbreaks of several termophilous insect pests could be explained with long periods of years with mild winters, unusual, long-lasting droughts, and with subsequent hot summers in Europe. The high number of introductions of new pests is the result of the increasing intensity of world trade. It makes almost impossible to stop the appearance of new pests by mean of quarantine. The question remains open, whether changing climate helps the establishment and outbreak of these new pest species.

Plant Protection Institute, Hungarian Academy of Sciences. dr. F. Kozár. H1525, Budapest, P.O.Box 102, HUNGARY

\section{1}

\section{POLICIES OF MULTI- AND BILATERAL DONORS - DO THEY REALLY MELP IPM?}

\section{G. Fleischer}

International donors of development aid have contributed a lot to raising the intensity of agricultural production in deveioping countries. In reaction to the growing problems with the spread of indiscriminate pesticide use, multi- and bilateral donors have set up guidelines on pesticide management and pesticide use in projects. A survey among ten international donors was carried out aiming at the evaluation of the status quo of donor policies and guidelines.

Since the mid of the eighties, financing institutions (World Bank, Asian Development Bank, European Union, IFAD, and German Kreditanstalt für Wiederaufbau) started to implement administrative provisions on pesticide supply and assistance to developing countries. The supply of the most toxic substances (WHO classes la and (b) is discouraged but not ruled out. The consequences of supplying and promoting pesticides to farmers shail be evaluated in an environmental impact analysis. Some donors started with advice on national pesticide price policy and with nationwide pest management programs, including the support of IPM training.

Technical cooperation agencies (FAO, USAID, Overseas Development Administration, German Technical Cooperation Agency GTZ) have already a tradition in conducting research and development as well as extension in IPM. However, the acproach has too long been too specific to single pesis and crops. Donor support for establishing institutions on pesticide legislation and regulatory affairs is comparatively new. Among the organisations with coordination objectives, FAO established global conventions (e.g. International Code of Conduct) and, together with UNEP, compliance provisions (Prior Infomed Consent). The activities strve for setting a minimum standard on information exchange, trade and pesticide management. OECD established advisory guidelines for member countries. However, the role of the coordinating institutions is limited since compliance is generally voluntary.

Major shortcomings of the cument approach of pesticide policies of international donors are the following:

- Procurement guidelines in general lack an assessment of the need for the proposed pesticide aid. As a first step the ban of the most dangerous pesticides in donor aid programs has not yet been implemented.

- A conclusive strategy of how to combine agricultural development and natural resource management is still missing. Up to now, there is a reliance on mitigative measures regarding negative side effects.

- Intemational donor policies lack the coordination in pesticide policies between donor agencies. IPM too often is implemented in isolated projects whereas at the national level pesticide policy formulation is neglected. In Sub-Saharan Africa, donors still supply a considerable amount of pesticides to developing countries.

The commiment to IPM as a major principle in plant protection policies has been dectared. But a common understanding is still lacking about which components can take part in an IPM program and - even more important - which measures must not be included.
CLIMATE VARIATIONS AND POPULATION OUTBREAKS IN RONDENTS OF THE SAHELIAN REGION.

B_sicard

The sahelian region contains numerous rodent species whose episodic population outbreaks lead to considerable damage to farm produce with disastrous effects on the economy. These outbreaks are generally related to the selective effect of some environmental factors which act slowly (at the multi annual level) on the population dyramic of rodents. Neverthless, a such relation fails to explain how a climatical disturbance (at the seasonal level) may induce a population outbreak appearing suddenly in some species only. To obtain the information needed to forecast the effect of this or that climatical disturbance on the population dynamic of this or thas species, we sudied the regulation of the poputation dynamic in the thain rodent species from 1984-1992 in Burkina Faso, and since 1992 in Mali. These eco-etho-physiological studies lead us to the conclusion that the annual variation of some environmental factors (daylength. temperature, air humidity, food, and some chemical cues) are speciesspecifically perceived by the central nervous system of rodents, and speciesspecifically used for the synchronisation of the main biological functions specifically used for the synchronisation of the main biological functions
(reproduction, mobility and aestivation) involved in the regulation of the population dynamic. Consequently, a disturbance of the annual variations of the synchronizers may induce a sudden demographic outbreak, or a sudden demographic collapse, depending on the species. In order to establish a typology of the effect of the main climatical disturbances of the soudanosahelian climate on rodent demography, we actually investigate the mechanism of the synchronuzing effect. The first question is : does this effect result (1) from the perception of a combination of synchronizers at a given time, or (2) from the perception of the specific chronological order of the variations of the

synchronizers in the standard climatical year. Some results obtained in Arvicanthis niloticus and Gerbillus nigeriae, two species known for their population outbreaks, are presented.

Dr. Sicard Bnuno, ORSTOM, BP. 2528, Bamako, MALI

832

Plant Protection Services as constrains to I.P.M.?

\section{R.F. Raske}

Integrated Pest Management (IPM) projects are since the early Seventies an important part of the Technical Cooperation Programes ( $T . c_{\text {. }}$ ) of the Federal Republic of Germany-lpm-projects became very early one of the priority areas within agricultural projects.

Perennial (coconuts, fruits,coffee) as well annul crops (vegetables, cotton, cereals, legumes, root-and tuber crops) are dealt with all over the world in at present about 25 different projects.Planning, implementing and securing sustainability faced and still faces considerable difficulties and constrains within the project and its implementing agencies as well as concerning the external frame conditions.

some major obstacles,aside the financial limitations, are:

-A strongly technical oriented split-up within plant protection services (e.g.entomology, plant pathology, virology etc.) hampers holistic appraaches. -Inconsistencies within the tasks and organisation of plant protection agencies render

-responsibility for IPM strategy development,

-status of distributors of (subsidized) chemical pesticides,

-coordination between research stations, technical institutions and extension services

rather difficult.

-Limited confidence of farmers into government programes.

-Inadequate extension systems to transfer the IPM messages to our target groups.

-Time frames available aze too short for the proper implementation of a sound IPM strategy.

Possible ways and means to overcome constrains are discussed,

e.g.:-intensified baseline surveys,

-systematic involvement of farmers and other target groups,

-cooperation with Non-Government-Organzations ( $\mathrm{NGO}^{\circ} \mathrm{s}$ ),

-appropriate phasing of the overall IPM programe,

just to mention a few. 
INSTITUTIONAL CONSTRAINTS TO IPM IMPLEMENTATION FOR FOOD CROPS IN WEST AFRICA

A. Youdeowei ${ }^{1}$, A. Adesina ${ }^{1}$ and S. Mboob ${ }^{2}$

When IPM was first introduced in 1978, the Philippine program for rice prodiction was based on a pesticide-oriented national crop protection policy that complemented a well-established trade in pesticides. Beginning from large institutional changes in 1986 the Philippines came to recognize IFM as the most viable and sustainable aliernative to tarmers' dependence on harmful, chemical pesticides. In 1993 IPM moved from policy declarafion to full implementation as a Presidential program called Kasaganaan ng Sakahan at Kalikasan (KASAKALIKASAN), meaning: "bounty in agriculture and nature."

With the enactment of the Local Government Code in 1992, the legal mandate was provided for devolving authority, responsibilities for decision-making, and internal revenue allotments to the local government units. The devolution has been uniquely seen by KASAKALIKASAN as an opportunity to encourage communities to build up their capabilities for implementing and thence owning IPM. IPM has covered 27 provinces in 13 regions for almost 20,000 larmers who are now promoting IPM in their own farms and neighboring areas. The Philippine Fertilizer and Pesticide Authority reports that in the Cordilleras of Luzon, IPM-trared vegetable farmers have reduced pest cide applications from over twenty to only two per season, with at least as much yield, and with improved health and income. In Davao del Norte, Mindanao the volume of chemical products sold, primarily for rice, in 1994 dropped by $38.25 \%$ and the value, by $25.9 \%$ while production increased.

The farmer-oriented approach of IPM is is not a commodity-oriented approach. Nor is it premised on the "transier of technologies" to farmers, but on farmers' initiatives, experiential learning, and discovery by farmers and technicians in the field. The National Research and Extension Agenda 2000, however, still promotes a commodity orientation, together with the "rransier of location-specific technologies to farmers. ." and remains an institutional constraint.

The acriculture bureaucracy's approach to research and extension would be strengthened it IPM's farmer-orientation is further specified to include ... as a vital imperative -. the woman-farmer orientation. KASAKALIKASAN calls for the "Incorporation of a mechanism to facilitate women farmers' access to IPM trainirg... [0 reach a] $30 \%$ female participation rate by the end of the program period, both as trainers and recipients of IPM." This maans revisiting the IPM tramework for community participation and inserting the gender framework in its overall dasign and implementation to further accelerate the ongoing process of IPM success in Philipones fields. Strategies should:

- incorporate gender sensitivity and awareness in the education, training, and delivery of IPM based on recognition of the multiple roles of women beyond their participation in the IPM program itself:

- promote through IPM training processes equal access to knowledge, resources, opportunities, and benefits:

- include in IPM technology design and adaptation concepts and practices to increase women's production and income while rectucing their work burden:

As communities are fast adhering to the IPM philosophy of local initiative and ownership, government cannot simply sit back. How soon it can reoriert practice to fit principle remains to be seen. But IPM is recognized for showing the way to the nation's envisioned environment of farmer empowerment and sustainable progress in the countryside.

Assistant Secretary for Policy and Planning, Department of Agriculture, Elliptical Road. Diliman, Quezon City, PHILIFPINES

835

CONSTRAINTS AND OPPORTUNITIES OF IMPLEMENTING BIOLOGICAL CONTROL IN BRAZIL

\section{E.MOSCARDI}

Significant programes of biological control have been developed in Brazit, such as: 1. production and release of imported parasitoids against the sugarcane borer, Diatraea sacccharalis; 2. use of the fungus Metarhizium anisopliae against sugarcane spitt le bugs (Hom. Cercopidae) in the Northeast; 3. production and release of imported parasitoids agatnst wheat aph ids in the South; and 4. use of a nuclear polyhedros is virus (NPV) aga inst the soybeanSouth; and 4. use of a nuclear polyhedros is virus (NPV) aga inst the soybean-
defol iating caterpitlar Anticarsia gemmatalis. Other in it iat ives include: the KPV of Spodotera frugiperda in corn; a granulosis virus of the cassava caterpillar. Erinnyis ello, the fungus Beauveria bassiana against banana weevils and termites; and egg parasitoids against soybean stink bugs. The reasons and strategies behind each programe varied with the characteristics of each cropping syster and institutions involved. In sugarcane chenical control is usually not feasible, leading to a rapid adoption of technologies control is usually not feasible, leading to a rapid adoption of technologies by farwer's associations, which established thenseives product ion units of
biological agents. In the case of soybeans and other crops where chemicals biological agents. In the case of soybeans and other crops where chemicals
provide "efficient control", the use of biological agents was not a farmers' demand, but rather a demand by the soctety in general for lower environmental contanination and lower levels of chemical restdues in foods. Therefore, the long lasting culture among farmers of applying "effective and cheap" chenicals had to be changed gradually through proper technology transfer chemicals had to be changed gradually through proper technology transfer strategies. These activities included pilot programs at farmer level, for at
least tim seasons, before effect ive inplementation of the programes, such as least tim seasons, before effective implementation of the programes, such as
the cases of the use of a NPV aga inst $A_{x}$. genatalis and egg parasitotds aga inst stink bugs in soybean. Despite the cases of successess, there are several opportunities for developing other programes in cropping systems, forests, and pastures in Brazil. However, there are some constraints to the develop, of these programes or the expansion of the existing ones, which include; 1. lack of government policies tomards implementation of biological include; L. lack of government policies touards implementat ion of biological
contral programes; 2. lack of legislation and protocols for registrat ion of
b lological products; 3. lack of suff icient funds to support research needs biological products; 3 . lack of sufficient funds to support research needs
and to qualify human resources in the area; 4. lack of highly qualified researchers and structure of extension to explore the full potential of available biocontrol agents, at regional or local conditions; 5 . low awareness of faroers to environental problems and their reststance to changes; 5 . narketing agressiveness of chemical comanies; 7. low prices changes; 5 . marketing agressiveness of chemical comanies; 7. low prices
of chenicals; 8 . low interest of private companies to develop biological of chemicals; 8. low interest of private companies to deve lop biological
control products; and 9. technical difficulties of implementing large scale production and quality control of biological products.

EMBRAPA-CNPSO, C.P.1061, 86001-970, LONDRINA, PR, BRAZIL
Food crops in much of sub-humid and semi-arid West Africa face the dual problems of poor rainfall and high losses from pests. To reduce the level of losses, most countries have historically encouraged the use of pesticides. Current estimates of pesticide imports into Africa average about UDS 500 million, imports into West Africa account for about $20 \%$ of this value. Continued reliance on pesticides for pest control has serious implications for sustainable farm productivity and farmers' health. This paper critically reviews major factors that have historically constrained the implementation of IPM approach for food crops in West Africa. These factors are: the structure and systems of national agricuitural extension and plant protection services which are often 'pesticide-use' based, inappropriate approaches in crop protection research, poor linkages and communication between government agencies which service the farming community, and isappropriate government and donor policies which encourage continued provision of development assistance in the form of pesticides. Lastly, IPM implementation is seriously constrained by the general unwillingness of the governments and pesticides companies to fully adopt and implement the FAO International Code of Conduct on the Distribution and Use of Pesticides.

'West Africa Rice Development Association (WARDA), Bouaké, Ivory Coast, and ${ }^{2}$ FAO Regional Office, Accra, Ghana
836

REDUCTION OF HAZARDOUS PESTICIDE USE: STICK OR CARROT?

J.A.W.A. Reus

There is an increasing pressure on the farming community to reduce the use of pesticides and to adopt untegrated crop protection methods. In some countries policy plans have even been adopt untegrated crop protection methods. In some counte

introduced with specific pestucide use reduction targets.
An important question is how farmers can be motivated to reduce the use of hazardous An important question is how farmers can be motivated to reduce the use of hazardous
pesticides and to adopt methods of crop protection which have a lower environnental impace. in pesticides and to adopt methods of crop protection which have a lower environmental impact
general, direct regulation like imposing rules and laws does not really motivate agrarians to general, direct regulation like imposing rules and laws does not really motvate agrarians to
change their farming practice. There is a risk that farmers put more energy into an artempt to get change their farming practice. There is a risk that farmers put more energy into an attempt to ge
round a regulation instead of adopting new crop protection techniques. Regulation is therefore only effective in situations where control is easy to carry out. lit crop protection and pesticide Only effective in situat ons where con
use these situations are rather scarce.

use these situations are rather scarce.
There is therefore a clear need for additional policy instruments to motivate agrarians and to There is therefore a clear need for additional policy instruments to motivate agrarians and to
implemert integrated crop protection methods. In many countries, active measures to implement implemert integrated crop protection methods. In many countries, active measures to implemen
IPM are still lacking, which results in serious irstitutional constraints. In the Netherlands, the IPM are still lacking, which results in serious institutional constraints. In the Netherlands, the
Centre for Ariculture and Environment (CLM) has gained experience with motivating policy Cente for Ariculture and Environment (CLM) has gained experience with mativating policy
instruments for farmers. In short, the strategy which is followed consists of three steps: (1) instruments for farmers. In short, the strategy which is followed consists of three steps: (1)
making the environmental burden due to pesticides quantifiable, so that a fammer becomes aware making the environmental burden due to pesticides quantifiable, so that a famer becomes aw
of the problems and can see the results of his efforts, (2) tanslating results of research on of the problems and can see the results of his efforts. (2) tanslating results of research on
integrated crop protection to practical advices on farm level. (3) giving excra financial incentives
to forenunners. to foremuners. for pesticides was developed. This yardstick assigns 'environmental impact points' to each pesticide with regard to contamination of groundwater, effects on water organisms and effects on soil organisms. It enables farmers to choose a pesticide with the least hamful effect on the environment and to measure the progress they make towards more environmentally sound crop protection.

Translating research results to practical advices on farm level is done in close co-operation with groups of farmers. For example. in the main flower bulb-growing areas of the Netherlands studygroups of bulb growers are set up. The group members discuss opportunities and studygroups of bulb growers are set up. The group members discuss oppor
constraines of several crop protection methods and evaluate there results. of hazardous pesticides in some ground water protection areas, farmers were rewarded when they substantially reduced the use of pesticides with a high leaching potential. As a result the number of environment impact points for groundwater contamination declined with more than $95 \%$. Market organisations may also have a large role in the implementation of integrated crop protecaon methods. A large store chain in the Netherlands stared to place demands on its suppliers with regard to the quantity and quality of the pesticides used. At the moment a more general environmental certification (geen label) for agncultural products is being introduced in the Netherlands. This green label has been developed in close co-operation with producers, retailers, government and consumers and enviromental organisations.

Centre for Agriculture and Environment

P.O. Box 10015

3505 AA Utrecht

The Netheriands
In order to make the environmental burden of pesticides quantifiable an environmental yardstick

Financial or market incentives have proved to be a major stimulus to farmers to reduce the use 


\section{DRDent}

Scientific training equips scientists for specialist research, while existing qualification and reward systems seek to promote specialist endeavour in preference to more generalist approaches. IPM is dependent for it success on multidisciplinary research inputs, but pest management research is essentially divided along strict disciplinary lines. Scientists from entomology, plant pathology and weed science specialise, for instance, in subjects such as chemical control, hostplant resistance or cultural control. Within this framework even further levels of specialisation occur as scientists address particular pest problems. The consequence of this emphasis on research specialisation is the development of specific terminologies, jargon and concepts, as well as different modes of enquiry and standards of proof. While such specialism promotes communication and understanding within a discipline the same process causes compartmentalisation, reduces communication and inhibits collaboration between disciplines. Such factors limit the likelihood of successful interdisciplinary collaborative IPM programmes and hence, the development of integrated solutions to pest management problems. There is a need to address some of the institutional constraints which maintain this situation and introduce new structures. mechanisms and approaches which promote interdisciplinary enquiry in pest management.

The key institutional requirements for improving opportunities for interdisciplinary research in IPM may be classified under three headings (i) appraisal and reward systems (ii) organisational structures and (iii) training in interdisciplinary research management.

The limitations and means by which scientific achievement is measured and rewarded in terms of specialists, generalists and interdisciplinary research is considered and suggestions for changes to existing arrangements are put forward.

Fixed organisational structures are inappropriate for scientists involved in interdisciplinary collaboration. Alternative, more open and flexible structures which promote interdisciplinary enquiry are outlined.

The management of interdisciplinary research represents one of the most challenging of human resource management activities. The techniques for integration of inputs from disparate specialist groups are described, in particular those required for the establishment of a shared paradigm, appropriate communication networks and programme planning and monitoring procedures. The need for training scientists in the use of these techniques of research management are discussed.

School of Pure \& Applied Biology, University of Wales, Cardiff, PO Box 915 , Cardiff, CF1 3TL, U.K.

\section{IN INDONESIA}

\section{K. Lntung} Since 1989 Indonesia has developed national IPM farmers training program in rice,
soybean, and vegetables. Approximately 300,000 rice farmers and 10,000 vegetable soybean, and vegetables. Approximately 300,000 rice farmers and 10,000 vegetable farmers have undergone training through IPM Field School system which urilize participatory approach in making IPM implementation decisions by farmers. During one planting season training period farmers are learning and practising in making proper pest management decision, based on analysis of information which they gathered through their weekly monitoring activities.

Most of IPM farmers have changed their attitude and behaviour in managing their own field and its pest problems, they are able to reduce pest control cost and increase their net profit. However since vast amount of rice farmers are untouched by IPM training program, most Indonesian farmers are still practising conventional approach of pest control.

Some constraints challenged the IPM implementation in the form of different opinions and perceptions on the concept and technology of IPM between decision makers, researchers, and field workers. Strong bureaucratic and egosectorial system in the Government are not too conducive for the development of participatory and partnership approaches in IPM field implementation. The ongoing national intensification programs in attaining annual food crops production target in the decision makers level has little concern in reducing the use of agriculture inputs such as fertilizers and pesticides.

The transdisciplinary research programs are not in the priority list of research managers and policy makers. Most Indonesian researchers rarely expose, train, and support themselves to work in an integrated and interdiscipinary environment; which

Farmers as an individual and a group should have a chance and acquire ability to make their own decision in managing their own field based on IPM principles. The current agriculture extension practices which treat farmers more as a massive and passive objects, should step forward into treating farmers as dynamic subjects and working partners.

State Ministry for the Environment,

Jln. Merdeka Barat 15

Jakarta 10110

Indonesia.
840

\section{THE SPS AGREEMENT AND ITS LIKELY EFEECTS ON TRADE IN PLANT} PRODUCTS AND UPON QUARANTINE
J. Magalluăes

With the entry inte force of the World Trade Organization on 1 January 1999 and with the progressive implementation of the commitments negotiated in the Uruguay Round getting under way, the international fremework within which domestic and trade policies are conducted in the plant and plant products sector will urdergo important changes. Not only measures such as quantitative restrictions, variable levies. minimum import prices and voluntary export restraints are now prohibited, but most export subsidies are banned and the Agreement on the Application of Sanitary and Phytosanitary Measures (SPS Agrecment) will prevent the unjustified use of sanitary requirements as a means to restrict plant and plant products trade.

To meet their obligations under the SPS Agreement in the plant and plant products sector, governments essentially have two alternatives: to undertake an actual assesament of risks involved in each cast, or to base their requirements on international standarda, guidelises and recommendations as developed by the FAO Intermational Plant Protection Convention. If governments act in one of these two ways, they will be in compliance with their obligations under the WTO. More stringent standards are allowed by the Arrangement, but governments who apply such standards can be challenged to provide a scientific justification for their use. Dispute setlement procedures are unified and strengthened (including an Appeals Body) under the WTO. In a dispute under the SPS Agreement involving scientific or technical issues, a panel should seek advice from experts.

The Agreement encompasses a number of important concepts or principles, running from harmonization and equivalence to the recognition of free-areas, consistency in risk assessment, transparency, monitoring of harmonization, technical assistance, etc. which will constinte regular agenda items for the meetings of the SPS Comraitree, the specin) WTO body established to oversee the implementation of the Agreement. In its first meeting, held in March 1995, the Committee immediately initiated the discussion of some of these substantive items.
Joāo Magalihtes

World Trade Organization

Agriculture and Commodities Division

154. Rue de Lausanne

CH-1211 GENEVE 21 
FORMATION AND IMPLICATION OF INTERNATIONAL STANDARDS FOR PHYTOSANTTARY MEASURES

\section{Hedley}

The Food and Agriculture Organization (FAO) of the United Nations is, in cooperation with regional plant protection organizations, responsible for the development of international standards for phytosanitary measures. Standards are being developed in response to the requirements of the WTO Sanitary and Phytosanitary Agreement.

An interim grechanism for the production of standards is being followed which involves the preparation of draft standards by expert groups, the distribution of the drafts to all FAO Memabers for comment, and the endorsement of the standards by the FAO Governing Bodies, the Connitiee on Agriesture, the FAC Council and the FAO Conference A group of phytosanitary experts nominated by regional plant protection organizations, with the IPPC Secretariat, oversee the standards through their various stages. This interim mechanism is under review. The standard Principles of Plan Quarantine as Related to International Trade" was endorsed by the Twenty-sixth Session of FAO Conference, 1993 and two further standards, "Guidelines for Pest Risk Analysis" and Code of Conduct for the Import and release of Exotic Biological Control Agents", are expected to be endorsed by the Twenty-seventh Session of the FAO Conference, 1995.

The implications of the development and implementation of standards under the Sanitary and Phytosanitary Agreement for national plant protection organization (NPPOs) are considerable. NPPOs will veed additional resources to be able to deal with their responsibilities in commenting on draft international standards the undertaking of pest risk analyses associated with the raising of new import regulations and the justification of present regulations, the notification of regulations and any requirements associated with dispute resolution procedures.

Secretariai of the International Plant Protection Convention

Food and Agricalture Ojganization of the United Navions

Viale celle Terwe if Caracalla, 00100 Rome. lialy
844

BPFECTIVARSS OF PEST SPRKAD IIAITATION BY REGULATIOA AND

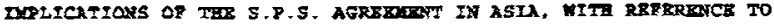
MRTAYSIA

M.A. JAMIL

Malaysia remains to be one of the world's leading exporter of oil palm, natural rubber, tropical timber, cocoa beans and pepper. However, Malaysia is lagging behind in its export of eresh agricultural produce. The Plant Quarantine the laws relating to control, prevention and eradication of plant pests. The Plant Quarantine Regulations 1981, regulate the entry of agricultural produce, products, soil and beneficial organisms. The Regulations emphasise on minimizing the transfer of pests of major economic crops such as oilpalm, rubber, cocoa and coconut. It is generally felt that measures such as these that heip Malaysia sustain its position as the major exporter of such crops. Numerous pests are known to be asociated with these crops, but Malaysia has been fortunate, so far, in not having any serious exotic peet established within its borders. However, over the years, there have beer. cases of entry of plant quarantine pests for other less important crops. These pests, unless eradicated could become significant in the future. The strength and weaknesses of the plant Quarantine Act and Regulations to minimise istroduction of plant pests through imporcation of agricultural combodities, and the partial success in the implementation of regulatory measures are bighighted Additionally, this paper identifies the reasons for pest establishment and proposes some solutions that could be undertaken. The plant Quarantine Act and Regulations are not meant to be technical trade barrier to discriminate trading partners. Malaysia strongly supports the SPS Agreement, and will continue to solicit expertise in phytosanitary measures from member countries.

Department of Agriculture Malaysia

Wisma Tani

Jalan Sultan Sallehuddin

50632 KUALA LUMPUR

MALAYSIA. 


\section{R Black}

Germplasm importation for development projects may introduce pests that are as great a risk as those associated with trade imports or smuggling. Major projects often fail adequately to anticipate these risks. Experience shows that full safeguards to obtain pestand disease-free material may not be implemented. Projects may even be halted or abandoned when the host government refuses to allow importation of germplasm. These considerations have so fas applied mainly to publicly-funded development and diversification projects. As commercially-oriented agriculture increases in lessdeveloped countries, demand for germplasm importation creates additional pressures on local plant health authorities. In the future the latter will generally lack adequate resources for plant quarantine. A planned approach to germplasm procurement would help relieve this pressure and avoid many plant quarantine problems.

Germplasm requirements should be considered during project conception, planning and throughout the project cycle. National plant health authorities should be consulted and account taken of the local capacity to monitor bealth of imported germplasm. Increasing general awareness of plant quarantine issues among non-specialist project and donor agency staff by providing guidelines that will enable them to assess whether germplasm can be imported safely.

To meet this need the NRI has prepared briefings on plant quarantine for use by development personnel and for inclusion in ODA's Manual of Environmental Appraisal which is used as part of the assessment process for all new projects. This material could serve as a model for the adoption of a more proactive approach to plant quarantine by other developmental organisations.

Natural Resources Institute, Central Avenue, Chatham Maritime, Kent, ME4 4TB, United Kingdom.

Since its foundation in 1907 American Cyanamid's investment in agrochemical research has produced a stream of innovative new produets which have placed the company at the forefront of crop protection technology.

Rapidly escalating research and development costs, together with political policies influencing agriculture have created a period of unprecedented change in the agrochemical industry as we approach the year 2000 .

The world population is forecast to double in the next forty years, reaching 8.5 billion by the year 2025. This tremendous increase creates a major challenge in the development of sustainable food production systems to meet the needs of future generations. To meet these needs will require close collaboration between the agrochemical and plant breeding industries, as well as those involved in agricultural food policies. Without high-yield agricultural systems the extensification of land cultivations will increase, without inevitable damage to the environment.

New and increasingly effective crop protection products and crop varieties and hybrids will be needed to support the high-yield agriculture of the future. Those challenges can only be met if industry can expect reasonable return on the very significant research and development investment required. Critical issues will be the patent protection of biotechnology inventions and patent term restoration. Regulatory systems must respect the protection of proprietary data, and operate under strictly scientific criteria rather than less objective, and sometimes politically motivated criteria, seen recently in certain European countries.

Against this background, Cyanamid is committed to continued investment in its world class research and development activity, producing high quality new agricuttural products to heip meet the world's food requirement into the next Millennium.

Cyanamid International, Agricultural Products Division, chaussée de Tirlemont 105, B-5030 Gembloux, Belgium.

\section{M.Los}

The imidazolinone herbicides were the result of some fourteen years of research. During this time a variety of chemical structures with interesting but not commercial activity were discovered. The progression to the extremely active pyridine derivatives will be described together with the determination of their mode of action. This knowledge allowed the differentiation between proherbicides and herbicides as well as their intrinsic activity. The utility of these herbicides has been extended by the development of imidazolinone-resistant crops. The impact of this new series of herbicides on agriculture and the implications for herbicide research will be discussed.

American Cyanamid Company, PO Box 400, Princeton, NJ 08543 


\section{IMIDAZOL.INONE-RESISTANT CROPS}

\section{W. Clayton, R. Krueger}

The imidazolinone herbicides have many advantageous features that make them near-ideal weed control agents including a broad spectrum of weed control activity, low product use rates, Jow environmental impact, low toxicity to mammals and non-target animals, and application timing flexibility. This profile, coupled with the fact that these herbicides are already registered in many countries for a variety of uses, serves as a strong incentive for development of imidazolinone-resistant crops.

Imidazolinone-resistant crops offer a favourable alternative to the traditional approach of identifying and developing new crop herbicides. Producing a specific crop that is resistant to currently registered imidazolinone herbicides supplements Cyanamid's search for novel, selective herbicides for the crop while reducing herbicide registration costs and increasing grower herbicide options for the crop.

Imidazolinone-resistant crop plants can provide a multitude of new weed control opportunities. The decision to introduce novel imidazolinone-resistance into a crop depends upon an assessment of the opportunities for both the crop and herbicide for which novel tolerance can be introduced. Cyanamid's guiding principles in assessing

imidazolinone-resistant crop opportunities and efforts in development of imidazolinone-resistant crops will be summarised.

American Cyanamid Company, Agriculnural Research Division, P.O. Box 400, Princeton, NI 08543-0400, U.S.A.

851

THE CINNAMIC ACID AMIDE FUNGICIDE DIMETHOMORPH

\section{R. Mengel}

Control of diseases in key economic crops such as vines and potatoes is becoming increasingly demanding in the face of rising resistance to fungicides and the legitimate requirements that use of crop protection agents be carried out in an environmentally responsible way. The Cyanamid cinnamic acid amide fungicide dimethomorph has properties which enable it to make a significant contribution to the resolution of both of these problems. Dimethomorph is a fungicide with selective activity against Oomycete fungi including vine downy mildew, potato late blight and related diseases caused by the genus Phytophthora. Dimethomorph has a biological mode of action which involves interference in the formation of the fungal cell wall and which is different from that of fungicides already on the market. It shows no cross-resistance to such products. It can this be used to control fungal strains which have developed resistance to products such as pbenylamides. A comprehensive anti-resistance strategy has accompanied the development of dimethomorph for the maket right from the start.

Comprehensive studies have also shown that dimethomorph responis well to current demands for products with low environmental impact. It has little or no effect on mammals, birds, fish and other aquatic organisms. It does not accumulate or leach and thus is most unlikely to appear in the ground water. Furthermore, it is not harmful to earthworms, bees or beneficial arthropods. These properties make dimethomorph an excellent candidate for use in integrated crop protection programmes. Such programmes, developed supported by both the farming communities, state authorities and the producers of crop protection agencies seek to utilise all potential methods of increasing production from a crop in a simultaneously applied, integrated, manner. These may include tilling methods, crop rotation, use of suitable varieties, biological and biotechnological methods and the appropriate use of chemical crop protection agents. An object of such programmes is to minimise negative effects on the environment whilst at the same time ensuring sufficient crop yields to be economical and make foodstuffs available at reasonable costs. This aim must be considered to be one of the comerstones of a future sustainable agriculture. It is in the nature of integrated crop protection that only chemical agents with minimal environmental impact can sensibly help achieve its goals. Cyanamid is convinced that its dimethomorph fungicide possesses the properties necessary to make it an appropriate partner in such programmes and thus a significant contributor to a sustainable agriculture.
PYRROLE INSECTICIDES :

THE DISCOVERY AND DEVELOPMENT OF A NEW INSECTICIDE

\section{B. Bemstein and D.Hunt}

Modified fermentation isolates have provided stgnificant products over the years, especially in the pharmaceutical arema. Several products, such as abamectin, have also benefited the agricultural crop protection area. Screening of fermentation broths was initiated by the Agricultural Research Division of American Cyanamid several years ago. This led to the discovery of an insecticidally active natural product dioxapyrrolomycin. Chemical enhancement of this lead led to a large effort that ultimately provided several development candidates, including $\mathrm{AC} 303,630$.

Much of the activity surrounding the synthesis program centred on the preparation of an array of halogenated and trifluoromethyl substituted 2-arylpyrroles. The chemical modification of dioxapyrrolomycin to AC 303,630 was optimised through the use of QSAR.

AC 303,630 has demonstrated efficacy against a wide variety of economic posts including the orders Acari, Coleoptera, Diptera, Heteroptera, Homoptera and Lepidoptera, in a wide array of cropping situations. Insecticidal activity has been demonstrated via ingestion and cuticular contact of the material. However, greater toxicity has been observed in larvae via ingestion vs. contact.

The pyrroles have demonstrated excellent activity on susceptible species, even where resistance to current insecticides such as pyrethroids, carbamate and organophosphates is significant. Multiple cross resistance has not been observed in extersive testing, thus $A C$ 303,360 shows significant opportunity for use in controlling pests in crops such as cotton, vegetables and top fruit where insecticide resistance has become a problem.

American Cyanamid Company, P.O. Box 400, Princeton, NJ 08543-0400, U.S.A.

852

SUSTAINABLE PERFORMANCE MANAGEMENT - THE CYANAMID APPROACH

P.K. Leonard

Resistance problems do not occur by chance. It is human behaviour that creates thern. The way we use crop protection products has a direct impact on the rate at which resistance may develop.

The need to manage resistance has never been greater as it becomes more challenging to replace established chemistries and modes of action with new ones. The need to protect long term performance of our best products is more important than ever. Resistance development must be minimised if sustainable world food production is to continue to benefit from modem environmentally triendly crop protection products.

Historically, research has focused on understanding resistance mechanisms and processes. Too much attention has been paid to understanding the problem, not enough to solving it. Ultimately it is our behaviour that must be changed if we are to succeed in managing the threat of resistance. A greater emphasis needs to be placed on changing the way we use crop protection products to reduce the threat of resistance.

Cyanamid is committed to maintaining the performance of its products by ensuring that they are used in ways that minimise the risk of resistance. This proactive strategy, aimed at changing behaviours, will be illustrated with specific reference to the acaricide tebufenpyrad, the fingicide dimethomorph and to the imidazolinone herbicides.

Cyanamid International, Agricultural Products Division, European Regional Office, chaussée de Tirlemont 105, B-5030 Gembloux, Belgium. 


\section{W. Atwood}

Today, although we have agricultural surpluses in many countries, there are still a significant number of people who are hungry or malnourished. Furthermore, in the next 30 years, the global population will grow from over five billion to more than seven billion, increasing the demand for food and the need for increased agricultural production.

There are several definitions for sustainable agriculture. In its most basic form, agriculture must be sustainable if we are to continue to feed the growing world population. The question to be discussed is how to minimise environmental impact while producing adequate food and fibre. Excessive sediment in surface waters represents one of the major environmental costs of agriculture. Key to reducing this impact, while protecting groundwater and wildlife habitat, is to intensively manage the world's most productive land, use conservation tillage and low-dose herbicides for effective and economic weed control and maximum yields.

American Cyanamid Company, P.O. Box 400, Princeton, NJ 08543-0400, U.S.A.

855

THE EFFECT OF CROP COVER AND MICROCLIMATE ON ACTIVITY OF GROUND SURFACE PREDATORS

\section{A. Honęk, J. Štolcová, Z. Martinková}

The activity of insects on ground surface is affected by several factors including density of the plant stand and its correlate - microclimate. In 1992 - 1994, we investigated, the activity of surface predators in an experimental fallow field, by means of pitfall traps. The $25 \times 25 \mathrm{~m}$ field was divided into $5 \times 5 \mathrm{~m}$ plots grown by a naturally established weed stand or mowed and superficially ploughed in midJune and late July. A series of pitfall traps placed on an interface between the plots was operated throughout the year, other traps were placed on the bare ground of the ploughed plots and in the centre of the weed grown plots (controls). The bare ground and control traps was operated in June - August, as long as the ploughed surface remained free of newly established plants. The traps were emptied daily and the effect of temperature and plant cover on catch size was investigated.

With increasing ambient temperature, catch size of selected predator species (carabids Pseudoophonus rufipes, Harpalus affinis, H. tardus, Poecilus cupreus, Anchomenus dorsalis, Amara convexiuscula, Bembidion lampros) increased by $6 \%$ per $1{ }^{\circ} \mathrm{C}$ increase of average temperature. Plant cover substantially affected the arthropod surface activity. The size and diversity of catches of predators in control and bare ground plots were correlated. The variation was determined by weather and seasonal variation of species abundance. Some species preferred the inside of plant stands, others preferred bare ground, or showed little preference. The preference for dense stand was positively correlated with species body size (with several notable exceptions), and may indicate a thermoregulation or cryptic function of the sheltering in plant stands. The magnitude of site preferences was influenced by ambient temperature. Under low temperature the catches on weedy and bare ground surface were similar and the difference increased with temperature. Phytophagans were less affected and their catches were generally greater on bare ground. This may be caused by an increased walking activity due to failure of finding the host plant as well as less intense predation on bare ground.

A. Honæk, Research Institute of Crop Production, 16106 Praha-Ruzyne, Czech Republic
Authors: $\quad$ Peter A.C. Ooi \& Peter E. Kenmore

Integrated Pest Management (IPM) researchers and scientists in Indonesia, Malaysia, Philippines and Vietnam have known for decades that natural enemies provide fundamenta control of herviborous insects in rice and cabbage. This knowledge has not reached the vast majority of farmers. Top-down systems concentrate on delivering inputs like resistant varieties and pesticides, or simple messages like uni-dimensional thresholds, but fail to help farmers understand the concepts and fieid indicators of biological control. Unless farmers understanc biological control they cannot derive maximum benefit from other inputs. They often apply insecticides to rice fields planted with insect-resistant varieties, which accelerates the evolution of virulence in insect populations. When farmers cannot recognize parasitized pest larvae on cabbages, they continue to treat with insecticides and prevent the establishment of parasitoids. Farmers, however, who understand the role of natural enemies through discovery-based education often become resource persons for other farmers. They inspire others to develop expertise in IPM experimentation and practice.

In over 25,000 Asian villages farmers practice and develop IPM. In Farmers Fielo Schools (FFSs) people leam about the role of natural enemies within an agro-ecosystem framework. Rice farmers observe herbivores, detritovores, and natural enemies during weekly field visits. To investigate and understand the function of arthropods, farmers collect them in clear plastic bottles or bags and observe their feeding habits. Observation and discussion lead to discovery by farmers themselves of the key concepts involved. Farmers also compare populations of herbivores and predators in insecticide-treated and untreated fields. This helps farmers to discover the population dyamics of arthropods, the regulation of herbivores in an agro-ecosystem, and the disruptive effects of insecticides. This knowledge enables farmers to decide on the best way to conserve natural enemies in rice fields. Field exclusion cages further strengthen farmers' comprehension of natural enemies. They compare populations of a common herbivore (e.g. the brown planthopper) in the presence and absence of predators. Farmers come to understand the roles of different trophic levels in creating functional biodiversity which motivates them to conserve these essential ecological processes. Similarly, cabbage farmers are abie to discover the role of the parasitoid, Diadegma semiclausem Hellen (Ichneumonidae), in controlling the diamondback moth. They collect larvae from field sites without spraying and determine the level of parasitism. In addition, the level of parasitism can be compared between sprayed and unsprayed fieids. This understanding of the role of parasitoids helps farmers to conserve $D$. semiclausem by avoiding the use of chemical insecticides. Without an understanding of the role of ratural enemies, farmers are not able to appreciate the need to conserve these beneficials. Farmers who comprehend the role of natural enemies are more judicious and less profligate in the use of chemical insecticides. Experimentation and discovery are the key to understanding, and are indispensible to the development of farmer expertise in IPM.

FAO Inter-country Programme for IPM in Rice in South and Southeast Asia, P.Q. Box 3700 MCPO, 1277 Makati, M. Manila, PHILIPPINES

856

EFFECTS OF COLLEMBOLA ON SOME SOIL-BORNE CEREAL PATHOGEN FUNGI

\section{G. Innocenti, M.A. Sabatini}

We studied under laboratory conditions the interactions between some species of Collembola and four of the most important pathogen fungi of the foot and root of cereals.

Specimens of Onychiturus pseudogranulosus, $O$ armatus and $O$. tuberculatus introduced in Petri dishes containing agar medium inoculated with Gaeumannomyces graminis var. tritici, Fusarium culmorum and Rhizoctonia sp. actively fed on the hyphae of these fungi, and in the case of $G$. graminis tritici, the Collembola dug into the agar to reach the hyphae growing inside. The animals did not have a highly specific feeding preference even when the fungi were present in the same dish contemporaneously. These animals thus may have a role in biological control of the tested fungi.

Experiments carried out to study the interactions between the Collembola and another important fungal pathogen, Bipolaris sorokiniana demonstrated that the fungus had a repellent and lethal effect on the animals. Our studies pointed out that the hyphae produced one or more metabolites that are lethal for several species of Collembola ( $O$. pseudogranulosus, $O$. armatus, $O$. tuberculatus, Mesaphorura krausbaueri, Megalothorax minimus, Isotomodes productus, Isotoma notabilis, Cryptopygus thermophilus). Additional studies indicated that $B$. sorokiniana do not produce the toxic substance or substances throughout their life cycle; this or these metabolites are released when only hyphae are present, whereas we observed that the conidia were appetized and disseminated with the feces of Collembola. These conidia still maintained the capacity to germinate even after having passed through the animal alimentary tract.

Dipartimento Protezione Valorizzazione Agroalimentare, Università degli Studi, via Filippo Re 8, 40126 Bologna, Italy 
Steinernema riobravis $n . s p$. (Rhabditida : Steinernematidae) an alteraative for supressing insect pest populations.

LLeera G., J. Raulston, S.D. Pair, H.E Cabanillas and G O Poinar Ir

Actual demand for bropesticides as an alternative to chemical pesticider is ixcreasing due to concern about pesticide resides in crops and hazards to the environament

Nenatode species of the fanily Steinernematidae inctude some of the nost promising agents for biological control of agriothura' insect pess.

Sieinemema riobravis $\mathrm{n}$. $8 \mathrm{p}$. Was originally derected around laritude $26^{\circ} \mathrm{N}$, losgatude $98^{\circ} \mathrm{W}$, and altituite

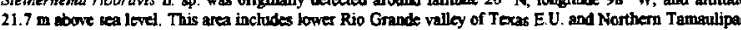

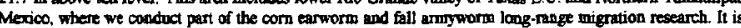

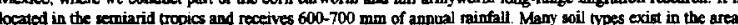
they generalty consise of $25.70 \%$ clasy, $15-65 \%$ sand, $15 \%$ sill, and s pH of $>8$. Aboul 200,000 ha of irrigmed com are plamed annually in this region in February and carly March. Fruting normally begins in irrigmed com are plamed annually in this region in Februasy and carly March. Fruiting normally begins in carty to mid-May, and mature corn earworm and fall anwyousm larvate exit the corn to pupate in late May infest this crop.

infest this crop.
Corn earnofir and fall armyworm excavated from soil in fruting cornfields in these areas to provide Corn earnofin and fall armyworm excavated from soil in fruiting cornfields in these arcas to provide that aumetous propupac and pupee were parasitized by a Steinernemo op. nematode.

Over a 5-yr period of this investigation, parasitized corn earworm and fall armyworm were observed in 34 and $24 \%$ of all fields samplod respectively. Altbough fower corn earworm prepupecen were excavated than appae and exuria combined, a significantly higher perpentage of the prepupae were parasitized. Similarty. a significanty higher percentage of fall armyworm prepupae were parasitized compared wish pupee. Averaged ver all years, 11.6 and $9.3 \%$ of all com earworm and fall armyworm excenated were parasitized by Streinernema riobravis $\mathrm{n}$ sp., respection

Moat of the fields observed in this ntwdy are cropped onox each year. Atthought these fields recerve irrigation Moat of the fields observed in this thrdy are cropped onos each year. Athonght these fields recerve irrigation Suring the oxm-erowing season, they are exposed to extendod dry periods for the rectainder of


The iowe Rio Crande valley Texas and Nortbers Tamadipas Mexico cora crop produces billions of cort

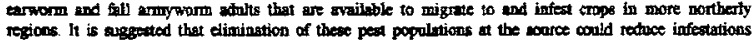



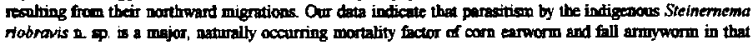
nobravis o ap. is a major, naturally cocarning mortality frctor of cam earworm and fall armyorm in that area. This nematode magy be a frim

and fill arimyworm popalntions. intermedia as indicated by the segative results in the crossbrocting lests. Albo, mosphoilogical characters

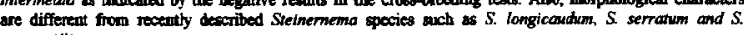
neocurrilis

Steinernema riobrovis a. op. third instar infoctive juveniles cmer the hemoocel of insects, deliver their ansociated bacteria, complete usually two generation, and then emerge from the insoct cadaver as infoctive joveriiles.

Each Steinernemetid species bas its own particalar symbiotic "strain" or subspocies. Further studies on ymbiotic becterium, Xenorhabchus ap., associated with S. nabrowis nt $\$$. will provide detailed idemtification and its role as a bioinescticide.

SesLs Loena-Gallardo INSTITUTO NACIONAL DE INTESTIGACIONES FORESTALES AGRICOLAS Y PECUARLS. APDO. 172 RIO BRAVO TAM. MEXICO

THE ROLE OF THE COVER CROP IN BIOLOGICAL CONTROL OF MITES USING PHYTOSEIULUS PERSIMILIS IN APPLE ORCHARDS IN SOUTH AFRICA

\section{K.L. Pringle}

The study was conducted in two blocks of apple orchards over a four year period. Phytoseiulus persimilis persisted from year to year in one of the blocks (block $A$ ) and in the other it had to be introduced each year (block B). In block A I. urticae was recorded on 29 plant species in the cover crop and $P$. persimilis on 21 of these, while in block B I. urticae was recorded on 22 plants of which 15 also hosted $P$. persimilis. In both blocks most of the records of $\underline{T}$. urticae and $\underline{P}$. persimilis in the cover crop were from January to April when their population levels were high in the apple trees. This indicated that both mite species colonise the cover crop from the trees as a result of population pressure. The preferred host plants for both mite species in block A were Geranium molle. Medicago Dolymorpha and two species of Sonchus. During April and May when mites were establishing in the cover crop these plants accounted for $23 \%$ and $18 \%$ respectively of the total cover. In block $B$ these plants accounted for $7.5 \%$ and $10.5 \%$ respectively in April and May. Oxalis pes-caprae and Plantago lanceolata were the dominant plants in block $\mathbf{B}$ during April and May, accounting for $35 \%$ of the cover during both months. $Q$. pes caprae occurred under the drip line of the trees and both mite species were recorded on this plant for a short period when the mites started leaving the trees. Few records of $T$

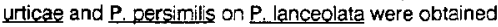

Department of Entomology and Nematology,

University of Stellenbosch

STELLENBOSCH 7600 ,

Republic of South Africa
THE IMPACT OF IPM IMPLEMENTATION ON COTTON, WHEAT AND VEGETABLES IN THE CENTRAL SUDAN

Z. T. Debrowski, A. A. Abdelrahman, A. Alsaffar and C. M. Beije

The FAO/Government of Sudan cooperative project on Integrated Pest Managewent (IPM) on Cotton financed since 1979 by the Government of the Netherlands has done an impressive contribution during its I - III Phases in detherlands has done an impressive contribution during its I - III Phases in demonstrating that the pesticide spraying cannot provide sustainable protection
of crops. It was proven that natural enemies occurring in the agro-ecosystems of crops. It was proven that natural enemies occurring in the agro-ecosystems
should be an integral part of the control measures, reason for which pesticide should be an integral part of the control measures, reason for which pesticide of pests are present and that biological control does not play an important role.

Raised ETLs for the chemical control of four key insect pests in cotton and one key pest in wheat are now implemented in the Gezira and Rahad Scheme. As a result, the number of sprays in the Gezira Scheme has dropped from $5-6$ in 80ties to 4.9 in 1993 to 3 in 1994 in cotton and from 1.7 to 0.9 in wheat. This means a saving of approximately $2.6 \times 10^{6}$ US $\$$ in cotton and $1.3 \times 10^{6}$ US\$ in wheat (calculation using the 1994 cotton and wheat areas and current exchange rate). In the Rahad Scheme, the number of sprays in cotton was reduced from 4.6 in 1993 to 3.1 in 1994 . This means a saving of $0.7 \times 10^{6}$ US\$. The total donor contribution to the FAO/ARC project amounts to $8.3 \times 10^{6}$ US $\$$ for the four respective project phases from 1979 to 1995 . From these data it can be concluded that the donor contribution to the project is less than the savings in pesticides of two years of IPM implementation in cotton and wheat in the Gezira and Rahad Scheme ( Report of the Tripartite Review Mission, 21 - 30th July 1994).

In addition to the measurable economical return, the project staff and the ARC counterparts noticed the positive effect of reducing aerial spraying on the occurrence of natural enemies on other crops in the cotton based agroecosystem in the Rahad and Gezira Scheme, indicating an improvement of environmental quality in the both agricultural schemes.

Since January 1993, the IPM Project has shifted its major activities from cotton to vegetables where pesticide misuse and risks for heslth and environment were large. First IPM options ("menus", "tool boxes") for tomato and onion, the major vegetable crops grown in the central Sudan were prepared based on vegetable crops grown in the central Sudan were prepared based on
recommendations of two national workshop (with participation of more than 300 recommendations of two national workshop (with participation of more than 300 researchers, extensionists, plant protection and horticulture specialists and 220
farmers). Regular pest surveys and critical evaluation of presently used production systems of vegetable crops and their effect on pest damage on 300 production systems of vegetable crops and
vegetable farms were carried out in 1993.

In developing a new implementation strategy for vegetable IPM in the Sudan, the project has drawn on the experiences of ather FAO IPM projects, particularly project has drawn on the experiences of ather FAO IPM projects, particularly in Southeast Asia, and has adopted the IPM Farmer Field School (FFS) approach. In this approach, on-farm research is integrated with extension: farmers are The results of FFS are analyzed to provide feed-back to the process of IPM The results of FFS are analyzed to provide feed-back to the process of IPM technology development. Significant changes in cultural practices and use of insecticides
17 in 1994 .

FAO/ARC IPM Froject, ARC, Wad Medani

860

EPIZOOTICS CAUSED BY ENTOMOPHTHORALEAN FUNGI IN PEA APHID POPULATION AND EVALUATION OF THE CONIDIOBOLUS OBSCURUS (RESTING SPORES) AS BIOCONTROL AGENT

\section{E.G.Voronina, G.R.Lednev, E.V.Roma}

Studies for epizootics induced by entomophthoralean fungi (zygomycetes: Entomophthorales) in pea aphid population (Acyrthosiphon pisum) within about two decades have revealed significant impact of this pathogens on control of host population density. In central region of Russia the epizootics occured each year, although usually too late to protect the crops from aphid damage. If crop is on flowering stage and disease level of aphids exceed $25 \%$ effectively suppression of aphid popula$t$ ion below economic injury levels will be observed.

Within growing season and on different stages of epizootic the specific fungus species prevalence was observed, namely, conidiobolus obscurus infection appeared early but within all season infection levels never exceeded $10 \%$; Erynia neaphidis infection dominated within expanded epizootic period. E.neoaphidis seems to be a main epizootic inducing agent. Essential differences was revealed in disease nature on diverse type of legumes, level of infection was 2-4 $t$ imes more of pea aphid population infesting vetch was $2-4$ times more than on clover. Data obtained in voronezh region were used to identify the predictive regression model for disease development in pea aphid population. The predictors are the host density and infec$t$ ion level of aphid population on date preceeding 3 week to prediction, sum of effective temperatures and cumulative precipitation and number of rainy days in preceeding 1.5 month. verification of model was made using data obtained in orel region and confirmation was good.

Results obtained from field experiments for release of C.obscurus resting spores (produced in vitro) suggest that disease development may be induced 1-2 week earlief than naturally on levels $2-3$ times exceding natural infection. However, low horizontal transmission of introduced pathogen was found that may be explained by loss of clue epizootological properties of fungus under cultivation. Some ecological conditions of resting spore germination are studied for prediction of their behaviour in situ.

Voronina E.G., All-Russian Plent Protection Institute, 3, Podbelskogo Road, St.-Petersburg - Puschkin. 189620, Russia. 
THE MTKKER-KILLER DILEMMA IN ARTHROPOD PREDATOR-PREY INTERACTIONS

\section{Maurice Sabelis, Minus van Baalen \& Bas Pels}

Some arthropod predator-prey systems on plants exhibit a metapopulation structure with violent, multi-generation population fluctuations at a local spatial scale, suggesting that the predators first drive their phytophagous prey to extinction and then disperse in search for other prey populations. Other predator-prey systems do not normally show such extreme dynamic behaviour; they may show large fluctuations but no prey extinction or they are characterized by a high frequency of host plant overexploitation by the phytophagous prey escaping suppression by the predators or atherwise. While such differences in dymamical behaviour certainly exist between species, it may well be that they also show up at the intraspecific level, once given proper attention. Clearly for selecting biological control agents it is useful to identify the underlying biological properties that lead to either type of local population and to assess how much genetic variation exists with respect to these properties.

Using a caricatural model of local predator-prey dynamics based on the assumption of exponential growth and constant rate of predation it is shown which life history features, predation and dispersal traits are likely to give rise to prey extermination. Moreover, it is shown by ESS-analysis that maximization of the intrinsic rate of population increase and suppression of dispersal until after prey extermination (Killer-strategy) is often superior to alternative exploitation strategies, such as behaviour reducing predation pressure which in turn leads to a larger prey supply in the future (Miller-strategy). However, there is still room for Milker strategies to evolve when dispersal between local populations is such that the frequency of co-invasion involving both Millkers and Killers is low; bence, the Milker-Killer Dilemma.

The experimental evidence for Killer-like exploitation strategies is reviewed and a research program for assessing the occurrence of Milker-like strategies is discussed.

Department of Pure and Applied Ecology, Section Population Biology, University of Amsterdam, Kruislaan 320, 1098 SM Amsterdam, The Netberlands

USE AND LIMTTATIONS OF THE HYPERMEDIA TECHNIQUE IN THE PLANT PROTECTION FIELD

\section{J. Carrascal, L. F. Pau and L. Reiner}

The use of hypermedia techniques in plant protection is analysed in view of its possibilities to make access to knowledge and information more comprehensible, and to accelerate and disseminate their transfer. Different hypermedia applications in plant protection are surveyed and the characteristics of such systems are reviewed in terms of information structure, contents, system architecture, user information browsing and support. Requirements, future possibilities, and limitations are finally discussed.

Technische Universität München

Institut fûr Ackerbau und informatik im Planzenbau

Lange Polnt 51

85350 Freislng-Weihenstephan

Germany

\section{ELEMENTS OF SUCCESSFUL IPM PROGRAMS USED IN CALIFORNIA}

\section{F. G. Zalom}

A comprehensive and successful Integrated Pest Management (IPM) program for an agricultural system requires willing and cooperative growers, support of organizations who market or purchase their products, dedicated research and extension personnel who can produce a body of relevant information concerning farming practices, pest biology and pest management tactics, and a supportive regulatory structure. IPM is a challenging educational subject because of its complexity and intensive emphasis on management which requires individuals who wish to apply it to understand broader ecological relationships in addition to crop production practices.

In the early 1970 's, a program for state licensing of "Pest Control Advisers" (PCA's) was initiated in California. In order for farmers to use a pesticide, they must have a written recommendation from a licensed PCA and file a notice of intent to make a pesticide application with the local regulatory official representing the state who must determine within $\mathbf{2 4}$ hours if the application should be permitted. Individuals licensed as PCA's must have a college degree in agriculture or biological sciences, a specified amount of experience, pass a written examination in laws and regulations as weil as in various pest control disciplines, an once licensed must receive 20 hours of continuing education credits each year. Over 3900 individuals in California are licensed. Use of IPM practices by PCA's is a good indicator of overall adoption or potential adoption because of their key role in grower's pest control decisions.

PCA's have been the target audience for research and extension efforts of the University of California's Statewide IPM Project which was funded by the state legislature in 1979 to foster more rapid implementation of IPM practices. A survey of California PCA's showed levels of use of twenty selected IPM monitoring techniques by the advisors ranged from 10.7 to $93.6 \%$. The most widely adopted of the IPM techniques tended to be those that had been available longest, those which had been actively demonstrated by extension IPM staff and those which involved the researcher at the field as well as the laboratory level. Over $86 \%$ of the active PCA's have at least one of the University of California's IPM manuals which are written for specific crops, and the average adviser had 3 or 4 manuals. Almost 500 have accounts to access the University's IMPACT computer system which contains databases of weather, phenology models, pest management guidelines, and pesticide use data. More recently, organizations have been developing to promote adoption of IPM which include diverse teams of stakeholders such as growers, educators, researchers, environmental groups, government agencies, and various elements of the agricultural industry.

UC Statewide Integrated Pest Management Project University of California

Davis, CA 95616 U.S.A.

864

LINKING DISEASE AND WEATHER FORECAST SYSTEMS: EVOLUTION AND RESOLUTION.

R. C. Seen arid J. M. Russo

Most plant disease forecast systems need accurate and timely weather information. Many different mechanisms have been developed to acquire and process weather information for disease forecasts However inherent limitations to these methods include the small area served by the weather station and the cost of providing multiple stations across farms or regions. New approaches using disease models, mesoscale weather forecasts, and geographic information system (GIS) techniques can now overcome these limitations. Output from mesoscale weather models can be combined with local geophysical conditions to estimate detailed weather parameters for specific areas as small as $1 \mathrm{~km}^{2}$, up to $48 \mathrm{~h}$ in advance Composite weather estimates can be used to generate high-resolution disease forecast- or risk-maps for entire regions. These techriques can be applied to most regions of the world where reliable digital elevation models exist. Examoles of disease models (infection and phenology forecasts) that have beer experimentally linked to high-resolution weather information. include apple scab (Venturia inaequalis) and grapevine downy mildew (Plasmopara viticola). Both models need temperature, relative humidity, and surface wetness as inputs. Temperature and humidity can be reliably estimated by the high-resolution methods: surface wetness estimates require additional refinement. However, utility of the information need not be based on absolute accuracy of the weather information, but whether the models advised correct disease management decisions. In a similar approach, high-resolution climate data can be usea to estimate biological risk of disease development by new or exotic plant pathogens.

Department of plant Pathology, Cornel1 University, New York State Agricultural Experiment Station, Geneva, NY, USA 14456. 
SUCCESSES OF INTEGRATED PLANT PROTECTION SYSTEMS - THE RELATION BETKEEN DETECTION OF PLANT DISEASES AND ACTION THRESHOLDS

verreet, J.-A.

Control of fungal diseases has a high priority for assuring yield i.e., for avoiding yield losses. Current routine aqricultural practice is to time treatments to control diseases of wheat, barley. sugar is to tine treatrents to control diseases of wheat, barley. sugar beet an other crops only in relation to plant development. Such treatments, based on principles or insurance as well as on ignoin the infection procal benaviour rarely intervene speciflcally in the infection process and ignore changes in disease progress to year depending year. to year. depending on the biological requirements of the pathogen and meteorological conditions, treatments related to the growth is a of the plant is present at the tiwe of application or if the sprays interfere accidentally with the epidemic. From an enidemiological point of vi ew these growth stage-oriented treatments are randomly timed and only randomly successful. Decreasing yield prices demand the lowest oossible production input, and this can be schieved only if the epidemiological behaviour of the pathogens is predicted accurately on a regional scale.

Integrated plant protection demands that pesticides are apolied only when required by the state of the infection and that the pathogen be clearly identified. Treatment are necessary at the most vulnerable stages of the fungal population. Application of fungicides at this time should have great economical and ecological benefit. To follow the princioles of integrated plant protection management and to realise this objective, certain preconditions must be met: 1 . Qualitative as well as quantitative diagnosis of the pathogen present is required. A crucial factor in ensuring the targeted use of fungicides is a reliable diagnosis, especial$1 y$ of pathogens, which induce nonspecific necrosis and of which fungal structures are not recognizable macroscopically. In many cases misinterpretations of symptoms are subject of non-justified treatments. 2. Scientifically developed threshold values for disease control, hased on the population dynamics of the pathogens are necessary. The attainment of the specific threshold value in the population signals the optimised pesticide treatment. Such integrated threshold values must be able to react flexibly to any plant cultivation and environmental change, such as fertilzer use, sowing date crop rotation, continous cereals variety to use. sowing cor variety totly the use of threshold values means considerable improvement of olant protection measures in every case, because they use the specific and current population dynamics of the pathogens.

3. Modern, pathogen-specific fungicides must be available. The successes of several integrated plant protection systems in different crops will be demonstrated

Prof. Dr. J.-A. Verreet, Christian-Albrechts-lniversität Kiel, Institut für Phytopathologie, Hermann-Rodewald-Str . 9; 24118 Kiel, FRG

867

\section{SITRAMA: INTERACTIVE SYSTEM FOR WEED MANAGEMENT}

\section{E. Lequizamón, A. Casali, R. Corti, E. D'Agostino, L. Dip, D. Faccini \& J. Pluss.}

The major task that have to be met in the agricultural systems in the next decades is the achievement of equal productivity levels with lower agrochemicais inputs. The development of techniques to support those "sustainable" systems is consequently highly relevant.

This is the purpose of SITRAMA, in which the integration of expert systems with conventional programming techniques allows a robust and efficient development in a complex domain.

SITRAMA is a computer system that deals with weed management in soybeans, a highly relevant crop of Argentina. It uses the Knowledge Engineering Techniques, and it is designed under four modules integrated in the same environment of programming. The system as a whole is structured using object oriented programming

Module ! allows the recognition of a weed seedling, from a knowledge base containing 40 specis, the most widespread and common in the soybean crop. Strategies followed for the identification simplifies the conventional dicotomic clasification and concentrates the recognition procedure on relevant characters. In such way, the information requirements to the user are minimal and reasoning procedure of the expert is emulated.

Module II calculates crop yield reduction taking into account weed density and distribution.

Module III recommends a control estrategy for the weeds, taking into account the climatic conditions, land preparation and previous infestations.

Module IV makes economic analisis under conventional (Cost/Benefit, Margins maximisation) or advanced techniques ( Risk, Uncertainty)

SITRAMA Knowledge Base is being structurated under production rules and object classes, which allows to work with inherited properties. The inference engine uses this estructure to reach conclusion tasks of each module.

System prototype is ceveloped using a pool of Artificial Intelligence tools, built on LeLisp, a Lisp dialect. The software have a group of programs (SmeciD) for the generation of the expert system and also a tool for the design of the graphic interphase (Aida-Masai(B)) which is very important to achieve an optimum interactivity with final user. SITRAMA is being designed to run in an IBM-PCs $386 / 486$ computer with a SVGA 24 bit graphic card and a doublespeed CD Rom lectot.

Cátedra de Malezas Facultad de Ciencias Agrarias Dpto. de Producción Vegetal. C.C. 14 (2123). Zavalla / Dpto de Sistemas e Informática. Facultad de Ingenieria. Pellegrini 250.(2000).Rosario. Universidad Nacional de Rosario. Argentina. Fax : $54-41-257164$
COMMERCIALIZATION OF DIAGNOSTIC KITS FOR ROUTINE USE - SUCCESSES AND FAILURES

\section{S. A. Miller}

Since the early $1980^{\prime}$ s, technologies for detection of plant pathogens have been developed for the agricultural marketplace. Nearly all tests have been based on the enzyme-linked immunosorbent assay, although recently products and services based on the polymerase chain reaction assay have also been introduced. While many kits are technologically innovative, marketing them has proven to be difficult, and many of the companies attempting to commercialize kits in the 1980's are no longer involved. Specific examples of technological and marketing successes and failures will be presented.

Dept. of Plant Pathology, The Ohio State University/OARDC, 1680 Madison Ave., Wooster, OH U.S.A. 44691

868

UNCERTAINTY AND TACTICAL DECISION SUPPORT IN WINTER WHEAT PEST MANAGEMENT

\section{W.A.H. Rossing, R.A. Daamen, E.M.T. Hendrix \& M.J.W. Jansen}

Calculation of damage thresholds is an important element of systems which support tactical pest management decisions. Dynamic models are used to relate observed pest and disease levels to financial loss. Usually, uncertainty about inputs and parameters in the models is ignored. Recommended actions are adjusted to be on the safe side, i.e. biased to chemical control, to account for risk aversion. Such prescriptive approach to decision support does not give insight into the riskiness of altemative decisions and may promote overuse of pesticides.

An alternative, consultative approsch is proposed in which the consequences of decision alternatives are analyzed in terms of the generally applicable, but potentially conflicting objectives 'retum on expenditure' and 'insurance'. These objectives are made operational by defining the profitability of stralegy $A$ compared to strategy $B$ as the probability that $A$ results in lower costs than $B$. Risk associated with a strategy is defined as the 0.90 quantile of the probability distribution of costs associated with that strategy. A selection criterion must be applied to identify the optimal strategy. Since selection criteria depend on the risk attitude of an individual farmer and may change with time, it is argued that in a decision support approach emphasis should be on presentation of consequences of alternative decisions rather than on optimization.

To illustrate the approach, uncertainty about the costs of different decisions on chemical control of cereal aphids (especially Sitobion avenae) and brown rust (Puccinia recondita) in winter wheat is calculated with an upgraded version of a well validated deterministic model based on the decision support system EPIPRE. Sources of uncertainty, which comprise estimates of initial state and parameters, future weather, and white noise, are modelled as random inputs. It is shown that ignoring uncertainty leads to wrong recommendations and potential overuse of pesticides. The consultative framework is implemented in nomograms.

A Monte Carlo procedure was developed to rank the various sources of uncertainty with respect to their contribution to output uncertainty. Uncertainty about population relative growth rates was found to contribute most to uncertainty about model predictions. The procedure provides an objective framework for prioritizing future research efforts aimed at improving the system's predictive capabilities.

Reference

Rossing, W.A.H., Daamen, R.A. \& Hendrix, E.M.T., 1994. A framework to support decisions on chemical pest control under uncertainty, applied to supervised control of aphids and brown rust in winter wheat. Crop Protection, 13:25-34. 
MECHANICAL DISTRIBUTION OF PREDACIOUS INSECTS AND ARTHROPODS UNIFORMTTY OF RELEASE AND PHYSICAL DAMAGE TO ORGANISMS.

\section{K. Giles I. Gardner, H.E. Studer}

Augmentative and imundative releases of predacious insects and arthropods are commonly made in field crops such as strawberries and grapes by manual distribution of predatorcarrier mixes. A mechanical system for field distribution of predacious mites was developed for use with ground vehicles. The systern released commercial mixtures of vermiculite carrier and Phytosejulus persimilis mites. Release rate of the mites was controlled through rotational spoed of a metering plate and the size of cells on the plate; calibration was analogous to that of a pesticide sprayer. Laboratory and field testing found the system to uniformly release mobile mites with an average sccuracy of $98 \%$ of the decired release rates from 32,000 to 92,000 predatorsha. Concentration of organisms in released mix was consistent, indicating uniform release. Mecharical release required significantly less time and labor than mamual release. Uniformity and accuracy of release were significantly improved by use of the mechanical distributor

Initial mechanization efforts determined that mites could be easily damaged by agitation of the vermiculite - mite mixture. The results revealed the necessity for biological evaluation of mechanical handling effects on biocontrol organisms. Many biological control organisms are mixed into carrier materials prior to shipment and release. Excessive vibration and agitation may significantly reduce vibbility of the organisms. The mechanical distributor developed for mite distribution was used to distribute lacewing (Chrysoperla) eggs mixed in vermiculite and rice hull carriers. Eggs were reclaimed and incubated Hatch rates were not affected by use of the mechanical device.

Department of Biological \& Agricultural Engineering University of California - Davis

Davis, CA USA $95616-5294$
872

Problems arising and practical solution found in the Netherlands in 1mpleaenting the new European regulatory regime for plant protection products.

\section{H. de Heer} The process of 1 mplementing environmental criteria in the Dutch Pesticide
Act has been complicated with a fairly long history. The implementation of Act has been complicated with a fairly long history. The implementation of
the first set of environmental criteria (persistence, leaching and aquatio the first set of environmental criteria (persistence, leaching and aquation Principles of the Directive $91 / 414 / E E C$.

Principles of the Directive The further elaboration of the other criteria mentioned in the Uniform protection and efficacy) is on-going now.

protection and efficacy) is on-going now. will be done where and as far possible simul caneous1y in September 1995

The complete implementation process including all the Annexes of the Directive $91 / 414$, will take about one year.

Thus, the transition process from a national registration process towards a harmonized European policy will by gradual.

Problems arising and practical solutions found in the Netherlands in implementing the new regulatoriy regime for plant protection products will be discussed.

Ministry of Agriculture, Hature Management and Fisheries Department Agriculture

73 Bezuldenhoutseveg

P.0. Box 20401

2500 EK The Hague, The Netherlands. 
THE POTENTIAL FOR MINIMIZING RESIDUE DATA REQUIREMENTS FOR MINOR CROPS - A NATIONAL AND EUROPEAN PERSPECTIVE

\section{$\underline{\text { U. Banasiak, K. Hohgardt, H. G. Nolting }}$}

The question of comparability of residue test results and possible extrapolations is not new, but is now being discussed as much as never before on both the national and intemational level. The reasons for that are different. The probiem is particularly evident in the authorization of plant protection products for minor crops, where it is unprofitable for manufacturers to bring about residue data because of the poor prospects of tumover. As a consequence, supervised trials are hardly ever carried out to produce relevant residue data.

It is now the task to define, on the basis of present knowledge and experience and taking account of the worst case, the conditions under which the residue behaviour of plant protection products in different crops of one group is comparable. In such cases, present data may be transferred from one situation to another, which allows to decisively reduce the extent of, or even dispense with, separate trials. We propose an official testing scheme which tests the possibilities of extrapolation by answering key questions on the substance's mode of action (by contact or systemic) and mode of application (before flowering, to the soil, to the plant).

Since 1993, the Federal Biological Research Centre for Agriculture and Forestry (BBA) has built up the Residue Data Information System (RUEDIS), which stores residue data from supervised trials together with relevant secondary information on the crop, treatment, weather, analytical methods etc. It may be expected that a unique fund of computer data will be available for evaluations within the next few years. The RUEDIS data bank is meant to be an instrument to work out models for extrapolation of the residue behaviour of plant protection products within selected crop grouping systems on the basis of standard methods to establish comparability (for instance, the ratio between the crops' surface and mass, different increase in mass etc.). It is also to help establish rules for comparability by defining criteria for acceptable deviations from use pattems and possibilities of data bridging between similar compounds.

There is no foreseeable solution to this complex problem yet. So, besides scientifically justified extrapolations, purely pragmatic regulations, based on convention and plausibility, will continue to be needed in the future.

Dr. Ursula Banasiak, Biologische Bundesanstalt für Land- und Forstwirtschaft, Fachgruppe Chemische Mittelpruffung, Messeweg 11-12, D-38104 Braunschweig
STRATEGIES AND CRITERIA FOR THE MUTUAL RECOGNITION OF AUTHORIZATIONS IN THE CONTEXT OF THE EUROPEAN REGULATORY SYSTEM FOR PLANT PROTECTION PRODUCTS

\section{Mark R. Lynch}

The new European regime for the authorization of plont protection products involves opproval of active substances at Community level and authorization of preparations at national level in accordance with Community rules. The mutual recognition of tests and analyses conducted in other Member States and of suthorizations granted by the competent outhorities of other Membes States, on the bosis of substontiated claims as to the comparability of agriculturol, plant heolth and
environmental (including climatic) conditions, constitute a central teature of the regime.

When an active substance has been included in Annex I, a decision will have been taken, in principle and subject to compliance with the conditions end restrictions associated with the inclusion in Annex l, that preporations containing it comply with the requirements of the regime relating to the safeguarding of human and animal health and the protection of the environment. Consequently, the outhorization process for individual preparations, serves to confirm that each individual preparation sotisfies thase requirements, taking account of local conditions and the particulat uses and manner of use proposed for it.

Where the provisions relating to the mutual recognition of on authorization is invoked, the examination of documented claims os to comparability, canducted to establish the extent to which such a cleim has been substantiated, serves to confirm the relevence of an authorization decision made in one Member State to conditions in onother Membar State.

In justifying claims that the conclusions reached by ane Member State are relevant to conditions in another Member State and therefore that conditions are comparable, it is necessary that a comparison be compiled, submitted and avaluated with respect to all ralevent foctors and conditions which could have on impect on the performence of the plent protection product and on its effects on humen and animal health and the environment. Relevant supporting documentetion must be provided to substantiate the comparisons made.

Since in individual cases some, but not all, of the factors and conditions concerned, will be reievant and furthermore since some of those are likely to be of more importance than others to the decision making process, siered approach for each relevant sector, involying the identification of the critical maxing process, a tier dapprasch for each relovant sector, involving the identication of the critical factors involved. is proposed. That approach is intended to ensure that only relevant been given due weight. Finally, a tiered decision making scheme for the integration of the decisions made for each individual
sector is proposed. That scheme is intended to facilitate the making of an overall decision with sector is proposed. That scheme is intended to tacilitate the making of an overall decision with
respect to the applicestion made. while ensuring thas key decision making criteria ore given due respect

Pesticide Control Service, Depertment of Agriculture, Food and Forestry, Abbotstown Labor story Complex, Abbotstown, Costletenock, Dublin 15, trelend

THE EXPERIENCES OF THE NETHERLANDS IN PROGRESSING THE AUTHORIZATION OF MINOR USES IN THE NETHERLANDS

Kuijpers, Laetitia A.M. and Straathof, Harry.

The Netherlands has to deal with relatively many minor uses of pesticides. For various reasons it is becoming harder to introduce pesticides for minor uses into the market and keep them there. In a workshop in 1994 al1 parties involved in the Netherlands were willing to attack the problem. After this workshop a project Minor Uses was initiated. This project - a cooperation of government, pesticide manufacturers and agribusiness - aims to explore the possible solutions and to arrive at a differentiated authorization system regarding minor uses. Four sub-projects were set up to concentrate on the following items: pilots, bottlenecks, finances and authorization policy. The project started in august 1994. The first stage of the project will be evaluated after one year. This paper illustrates the project and gives the results obtained so far in this project.

Ministry of Agriculture, Nature Management and Fisheries Plant Health Department

P.0. Bax 20401

$2500 \mathrm{EK}$ The Hague 
Changes in land use and agriculture are of all ages. Shifts in demand due to increased population numbers and change in diet and the change in supply due to agricultural productivity have caused considerable changes in Jand use and agricultural methods. Long term analyses show fluctuations in land use, considerable contractions and expansion of cultivated land, but only modest changes in agricultural productivity per unit of acreage. Only during the last decennia tremendous changes in agricultural productivity per unit of acreage (ha) were made possible by better agricultural methods and use of external inputs. That has changed the nature of agriculture considerably. Soil and labour productivity have increased in the industrialized world with factors between $7+10$ and $100-300$ respectively. In the developing world that shift is taking place at this moment. What may be expected in the future may be studied with scenarios that combine the explicitized objectives of policy makers and the technical possibilities derived from scientific analysis and studies. Explorative studies on agricultrual productivity may take place at various aggregation levels World food studies show what may be achieved at various places with different types of agrotechnology. The number of people that may be fed on a world level varies between $11.10^{9}-44.10^{9}$ depending on agricultural methods and diet. At a desaggregated level, studies consider the political priorities such as socioeconomic, environmental and agricultural objectives. The conflicts and trade offs between various objectives may be shown by the scenario studies at that level. An illustration is given by the study on land use in the European union. The amount of land use may vary between $40-70.10^{6}$ ha of land (at present $128.10^{6} \mathrm{ha}$ ) and the amount of pesticides in use between $20-80.10^{6} \mathrm{~kg}$ (at present $400.10^{6} \mathrm{~kg}$ ). There are ample opportunities for a dramatic decrease in pesticide use without a negative impact on land productivity and total agricultural produce. The technologies that enable such a shift are not yet adopted and the absence of that change is partly due to political, partly due to cultural inertia.

The role of scenario studies to explore options, to show perspectives and utopia, to demonstrate trade offs and to structure thinking for future developments will be illustrated in this symposium at various aggregation levels and for various time horzons.

Prof.dr.is. R. Rabbinge

Department of Theoretical Production Ecology

Bornsesteeg 47

P.O.B. 430

6700 AK Wageningen

the Netherlands

\section{ChARACTERIZING PEST CONSTRAINTS TO RICE PRODUCTION IN ASIA: APPROACHES TO IDENTIFY RELATIONSHIPS BETWEEN PEST PROFILES AND PRODUCTION SITUATIONS, AND TO DELINEATE IPM RECOMMENDATION DOMAINS}

\section{S.Savary, P.S.Teng, H.M.Singh, R.K.Srlvastava, and F.A. Elazegul}

Rice is a good model crop to address the characterization of pest constraints to production, and options to alleviate them: in Asia, rice is grown in an extremely wide range of production situations, and the diversity of its pests is large. In this presentation, the term 'pest' encompasses any harmful agent (i.e.. pathogens, insects, and weeds), and we concentrate on irrigated and rainfed lowland rice crops. Methodologies have been developed to simultaneously consider the association between production situations (including patterns of cropping practices) and pest profiles (the combination of pests that affect a crop in a given season, and may result in a yield reduction). Surveys have been conducted in the Philippines and in India that demonstrate a strong linkage between pattems of cropping practices and pest profiles. Both types of crop attributes are strongly associated with variation in yield in actual farmer' $s$ fields. The approach provides scope for further research, especially for the identification of key pests in a given production situation, and the delineation of recommendation domains for IPM. While quantitative surveys provide measurement of the risk probability attached to a particular pest / pest profile (i.e. the probability of its occurrence in a given production situation). they do not allow to quantify directly risk magnitude (the quantitative consequence of its occurrence in terms of yield reduction). During the past years, IRRI has developed an experimental crop loss database where pest profiles and patterns of cropping practices are manipulated in a series of factorial experiments. These experiments provide a measure of the attainable yield corresponding to a given production situation, and therefore allow a direct measurement of yield losses. The combination of risk probability and risk magnitude data will allow an analysis of the risk attached to rice pests in a range of production situations. The need for such analysis is particularly acute in a fast-changing agriculture, such as the ricebased agriculture in Asia.

IRRI-ORSTOM Joint Project on rice pest constraints characterization, P.O. Box 933, 1099 Manila, Philippines. 
BETWEEN MARKET AND ENVIRONMENT: EXPLOR|NG OPTIONS FOR ENVIRONMENTALLL FRIENDLIER FLOWER BULB PRODUCTION SYSTEMS

W.A.H. Rossing, J.E. Jansma, F.J. de Ruijter \& J. Schans

Flower bult production systems in the Netherlands rely et present on inputs of large amounts of pesticides and nutrients. Farmers are under political and public pressure to design production systems which meat both eccnomical and environmental objectives. Experimental research at the cropping system level is expensive and time consuming, and may consider only few technical alternatives. Atternatively, explorative modelling studies can be used to identify potentially successful production systems, thus directing empincal research. Aim of this study is to explore flower bulb production systems which meet economical and environmental objectives at the cropping system level.

A multi-criteria decision problem approach is used. One economic and two environmental objectives are formulated in consultation with a group of flower bulb growers and environmentalists who intend to use the results as a starting point for discussion about sustainability issues. The economic objective is maximization of gross farm margin, i.e. financia yield minus costs of non-factor inputs, casual (not skilled) labour and machine use. The environmental objectives are minimization of pesticide input (in $\mathrm{kg}$ active ingredient per hectare) and minimization of nitrogen surplus (in $\mathrm{kg} \mathrm{N}$ per hectare). Important constraints comprise farm size, the possibility to rent additional land free of soil-bome pests and diseases, and the variety of crops that may be grown. Production techniques were defined according to four characteristics: 'soil type and health', 'cropping frequency', 'crop protection regime' and 'nutrien regime '. Their inputs and outputs were quantified as technical coefficients which were used in a linear programming model. By optimizing farm gross margin with increasingly tight constraints on the environmental objectives, the trade-off between market and environment was explored. The analysis was carried out for two tarm sizes ( $15 \mathrm{ha}, 3$ permanent labour. and $25 \mathrm{ha}, 4$ permanent labouri, with and without rent of disease-free land, and for the crops tulip, daflodib, illy, narcisus and winter wheat as a tytosanitary intermediate crop.

Results show the trade-off between pesticide immission and nitrogen emission on the one hand, and farm gross margin on the other. Using maximum tolerable levels of pesticide immisston ( $48 \mathrm{~kg}$ a.i. per ha) and nitrogen emission (pending final government decision, $140 \mathrm{~kg} \mathrm{~N}$ per ha) as starting-points, restricting pesticide use has a smalier effect on gross margin than restricting nitrogen surplus. Farm gross margin increases strongly when a 'break crop', such as winter wheat, is used in the cropping system. Sensitivity analyses showed increases of $50 \%$ in farm gross margin due to improved soil health, notwithstanding the low gross margin of winter wheat. Land rent is shown to be another major factor in maintaining relatively high farm gross margins while satisfying environmental restrictions.

The results show the trade-oft between economic and environmental goals and the potential of various production techniques to reach these goals. They are used currently as a basis for discussion on sustainability issues by a group of advanced bulb growers and environmentalists. Moreover, the results direct experiments at commercial tarms and on research stations aimed at implementation of ecologically and economically sound production techniques.

Walter A.H. Rossing, Deparment of Theoretical Production Ecology. Wageningen Agricuitura University, P.O. Box 430, 6700 AK Wageningen, the Netherlands; e-mail: rossing rcl,wau.nl

883

\section{CROP PROTECTION BETWEEN DREAMS AND REALITY}

Günther Voss

Without crop protection, there would be less untouched nature, less food for an ever-increasing world population, higher food prices for all and a reduced and fluctuating income for farmers. Despite this, large sections of our society take a negative view of crop protection. The cause is clear; inadequate match of perceived benefits and risks. Four points will be emphasised. (a) The frequent stance of "pro natural, contra synthetic chemicals" is unscientific. (b) Crop production at required rates is not and will never be possible without synthetic crop protectants. (c) By copying strategies as designed by nature, competent and responsible research and registration have made significant progress in providing society with safer, more effective, and ecologically acceptable plant protection agents and methods. (4) A successful implementation of integrated pest management relies on a continuing and rational trialogue between society, agriculture and industry.

Crop Protection Division, Ciba-Geigy Limited, $\mathrm{CH}-4002$ Basef

\section{S. Jannai} Following crop diversification and intensification in India in the last species are becoming prominent. Increased use of pesticides has led to several enviromenta] problens. We owe a healthy environwent and good food for our future generation. The seocnd Green Revolution has to be ushered in with a difference. The future pesticides would ned to heve greater specificity of action, biodegradability and safety to non-target organises and lesser overall enviromental pollution. Pesticide apray achedules by calendar have given way to their need-based application taking into consideration the economic threshold level, cost/benefit and benefit/risk ratios, pest resistance to pesticides, pest resurgence, adverse effects on reneficial organisms, and hazards of pestictde residues. Integrated pest maragement [IPM] is gaining momentum in any crop ocosystems in India, and the recently introduced Farmers' Field Schools on IPM have created much the recently introduced Form

India is the second largest annufacturer of pesticides in Asia today, and is the third largest consumer of pesticides in the world. Total installed capacity of pesticides is 124,000 MT. Nearly $68 x$ to $75 x$ of total consumption has been for crop protection and the romaining is used in public health. Currently, pesticides are being used in about 25x of our total cultivated ares. Insect icides occupy 78.00x of the total pesticides, fungicides 10.50\%, herbicides 10.00\%, and others 1.50\%. Dotton crop consumes 41.30x of pesticides, rice 13.30\%, vegetables 13.00x, plantation crops 7.10\%, and others including large areas of coarse cereals, pulses, oil leeds, etc., 25.30\%. The consumption of technical grade pesticides in Indis has increased from 434 MT in 1954 to 85,030 MT in 1994-95 and the usage per hectare from $2.2 \mathrm{~g}$ in 1950 to $650 \mathrm{~g}$ in 1994-95. In June 1993 , the Government has banned 12 pesticides and another 17 in
1994 , besides restricting the use of 13 pesticides. Introduct ion of systemic fungioides and sterol inhibiting fungicides of curative action is encouraged. The share of biological ('Boft' pesticides] is expected to increase at a very fast rate in the coming 5 years to reach 3-5x of the total market. The estimated denand of 600,000 litres of neem products by 1996-97 has been on the pessimistic side. The gap botween deand and supply is expected to be filled in by now botanical pesticides of which neew has the maximum potential. Use of B.t., NPVs, parasitoids and predators, fungal antagonists, insect sex phercmones, etc., will increase considerably. Developing atabie basic formulations of entomopathogens for maintaining pathogen viability and virulence would receive greater attention. Biotechrology and genetic ens ineering mould enable development of more efficient microbial pesticides. Higher yielding crop cultivers with durable resistance to pests and pethogens would be available. The concepts and practices of IFy would ga in much monentum in order to stabilise crop yields, increase farmers' profits, and restore ecological balance.

Centre for Research on Sustainable Agricultural and Rural Development, M.S. Sware inathan Research Foundation, Hadras 600 L13, India.

884

\section{TOWARDS SUSTAINABLE CROP PROTECTION IN AUSTRALIA}

\section{G. Evans and P.C. Rowland}

Despite Australia's large size, it does not have an abundance of arable land and less than $4 \%$ is used annually for cropping. Conservation tillage systems, based on herbicides, have protected fragile and erodible soils while enabling cropping intensity to double over the past two decades. However sustainable limits may have been reached, or even exceeded. Other concerns include the emergence of herbicide-resistant weeds and unknown environmental consequences of herbicide use.

Australia relies heavily on rural exports and is sensitive to the demands of international markets on matters of food safety including pesticide residues. Governments and industry actively seek ways to improve the efficiency of pesticide use, recognising also the contribution this makes to sustainability of our agricultural systems. Initiatives include the National Strategy for Ecologically Sustainable Development, support for research into IPM and biological control, new education and training programs and the elimination of hazardous pesticides through the Existing Chemical Feview Program. This paper will review the progress made to date in improving crop protection in Australia.

Bureau of Resource Sciences, PO Box E11, Queen Victoria Terrace, Parkes, ACT 2600, Australia. 
CROF LOSSES AND THE EFFICACY OF CROP PROTECTION - A CRITICAL ASSESSMENT

\section{E.C. Oerke}

Actual crop losses; i.e. despite of the present crop protection practices, and potential crop losses - i.e. a 'no control scenario' - of rice, wheat, barley, maize, white potatoes, soybean, cotton and coffee were estimated by evaluating data from current literature and field experiments. Total losses were calculated from yield reductions due to pathogens, animal pests and weeds on a regional, continental and global level. Actual yield and production data as well as crop loss estimates were compared with data for 1965 (Cramer 1967). Since 1965, world-wide production of most crops has been increased considerably. Simultaneously, crop losses in wheat, white potatoes, barley and rice also increased by $4 \%$ to $10 \%$. In other crops, losses remained largely unchanged or slightly decreased (coffee, $5 \%$. The different developments in various crops and regions indicate a complex interdependence between crop protection intensity and attainable yield

The efficacy of mechanical, chemical and biological crop protection practices actually used was calculated as percentage of potential crop losses prevented by these actual control measures. In 1988-90, the efficacy was highest in cotton (55\%), whereas it reached only $34 \%$ to $38 \%$ in the most imporant food crops rice, wheat and maize. The variability among cropping areas was aiso high: In the eight crops, in Western Europe an average of $61 \%$ of potential crop losses was prevented, in North America and Ocearia the percentage was $44 \%$, in all other regions only $38 \%$. Due to the small share of Westem Europe in world-wide production of $8 \%$, the efficacy of actual crop protection is only $40 \%$ on a global level.

The assessment of actual crop protection efficacy indicated that (1) the importance of crop protection is mainly determined by the magnitude of potential crop losses due to pathogens, animat pests and weeds and by the economic threshold levels, respectively; (if) the differences in crop protection efficacy among regions are due to differences in the susceptibility of pests to control measures, in the availability of efficient control practices, and in the know-how of the farmers. However, the magnitude of actual crop losses and crop protection efficacy cannot be used as an indicator of the economy and/or the necessity of actual crop protection measures as the socio-economic background of crop production highly varies among cropping areas and crops.

E.-C. Oerke. Institut für Pflanzenkrankheiten, Universităt Bonn, D-53115 Bonn, Germany

887

BEHAVIOUR OF ARTHROPODS IN RRLATION TO BIOAVAILABILITY OF PYRETHROIDS

G. Jagers op Akkerhuis

G. Jagers op Akkerhuis
vrije Universiteit Amsterdam

Effects of nerve-poisons on arthropod behaviour will be discussed in relation to peripheral sensory targets and pesticide bioavailability. Experimental results will concern effects of the pyrethroid insecticide deltamethrin on the behaviour of the linyphiid spider oedothorax apicatus

It will be shown that with respect to effects on spider behaviour, the bioavailability concept has to be used in two different ways. 1 . In the case of internal concentrations, bioavailability refers to the dynamic concentration balance of the toxicant at relevant neuronal and neurosecretory target sites. 2. In the case of peripheral sensory targets bioavailability is mediated by behaviour dependent contact with contaminated substrate and the resulting partitioning balance at the contact site.

The use of the "interface" concept in ecological risk assessment of pesticides will be advocated. The interface refers to all channels via which an organism interacts with its environment. This can be separated in a "perception interface" relating to all sensory information about the environment, and an "activity interface" with respect to environment oriented behaviour.

Vrije Universiteit Amsterdam

Faculty of Biology

De Boelelaan 1087

I081 HV Amsterdam
TOWARDS A SYSTEMATIC EVALUATION OF PESTICIDE RESISTANCE RISKS

A.J.W. Rotteveel. J.W.F.M. de Goelj and J.F. van Gemerden

Pesticide resistance problems are increasing wotldwide and pesticide availability is in many countries exoding for various reasons. In order to keep useful products on the maxket as long as possible, it is becoming imperative to devise and use resistance management strategies. One factor in any such system is the evaluation of the specific resistance risks of the pesticide uses involved. This paper describes a general qualitative evaluation system for resistance risks of pesticide applications being currently developed. This risk evaluation system uses a total of six risk categories. The system is proposed as a tool in the (re)registration proces, but is not envisaged as resulting in the refusal of registration of active ingredients.

Plant Protection Service, P.0. Box 9102, NL-6700 HC Wageningen, The Netherlands

888

NON-TARGET EFFECTS OF INSECT GROWTH REGULATORS ON COCCINELLIO BIOCONTROL AGENTS AND THE IPM COMPATIBILTTY OF SUCH PRODUCTS

\section{Hattingh and B.A. Tate}

Insect growth regulators (GRs) have widely been claimed to fulfill the need for effective plant protection products which are compatible with IPM However, field experience in the southern African citrus industrv has indicated that their use may result in extensive pest repercussions. The advent, in this region, of the commercial use of the juvenile hormone analogue pyriproxyfen, resulted in a resurgence in the economic pest status of cottony cushion scale after a century of complete biological control. This was due to the disruptive effect of the product on the coccinellid biocontrol agents of the genus Rodolia. Sirrilariy, the increased use of IGRs in the southern African citrus industry has resulted in the near elimination of another economically valuable coccinellid biocontrol agent Chilocorus nigritus. Field experience indicated that the detrimental effects of spraying IGRs were highly persistent. Bioassays were conducted to determine the duration of residue toxicity of a number of IGRs (juvenile hormone analogues and chitin synthesis inhibitors) under conditions of field weathering. The economically important coccinellids $C$. nigritus and Cryptolaemus montrouzieri were used as indicators. The bioassays confirmed that the residues were highly persistent under field conditions with residues from a single application of pyriproxyfen still capable of inducing complete sterility in C. nigritus after 19 weeks of field weathering. It was concluded that the IGRs tested, could not be considered to be compatible with IPM in the southern African citrus industry, where coccinellids are important biocontrol agents.

Outspan Citrus Centre, P.O. Box 28, Nelspruit 1200, South Africa 


\section{S.Dorn}

Crop yields of cassava, the staple food of around 500 million of the poorest people in Africa, Latin America and Asia, are reduced by up to $75 \%$ by the cassava mealybug (Phenacoccus sp.). Biological control by mass release of antagonists started in Latin America in 1994 and in Africa in the early 80 's.

Our studies in Atrica and Latin America put emphasis on diversified cassava agroecosystems.

Reliable and sustainable pest control for an extended region with its diverse successfully been established, two strains originating from ecologically differen zones in South America were studied as possible candidates for mass releases to complement the established parasite. The spectrum of potential preys for $\mathrm{H}$. notata was found to be narrow, but still large enough to ensure the survival of the populations even in times of limited mealybug availability. The differences between the two strains were found to be minor in their life tables, but marked in their functional and numerical response as well as in selected behavioral characteristics. Experiments in the field showed that mealybug infested cassava plants situated between heathy plants were located by $H$. notata within a short period of time. Studies in Latin America are aimed at analyzing the mechanisms involved in the attraction of antagonists to the attacked host plant. Special attention is being paid to chemical stimuli released from infested plants. Furthermore, tests will also be carried out to analyze the searching behavior of the antagonists in cassava intercropped with leguminous plants.

Institute of Plant Sciences, Applied Entomology, Swiss Federal Institute of Technology (ETH), Clausiusstr. 25, CH-8092 Zürich. Switzerland. ocological conditions usually requires different antagonist species and ecotypes. In Africa, where the parasitic encyrtid wasp Epidinocarsis lopezi (DeSantis) has

Author: Peter Kenmore and Jonathan Pincus

WHAT WE LEARYED BY THE 1990s -

- g.obui tectinologles misy work in highly modified. itrigated agroecosystems. but dor I generaliy work in highly variable raitfed agroceosystems where local solutions must be found

- Farmers' tield schools (extension) \& participatory technology development (research) can link with farmers" R\&D: - host plant resistance and pesticides are unsusta inable if farmers lack knowiedge of how they act on agroecosystems. - pesticides counter yteld-reducing. not yicld limiting faciors and need not be subsidized to increase food predustivn.

- hosi plant resistance - however genetically engineered - is as vulnerable to pest evolution as are pesticides. SHOLLD PUT US ON SCHEDULE FOR LNCED's AGENDA 21 BY THE YEAR 2000 with. Valional IPM policies in over 25 Non-OECD countries with acceptance by govermment policy makers and front
line exiension staff that farmers can and should be in charge of local- farm- - tield- . crop- level decision making. more farmers field schools and and participatory lechrology development connected to courageous researchers enabling IPNI-nurtured partnerships to apply: 1) nutrient analyses and management: 2) genetic conservation and deployment: 3) crop physiology; 4) diagnostics to ident ify and analyze disease progerss curves:

- nerworks of farmers' groups facilitated by government and NGO extension systems:

- farm.station and policy understanding of the roles of yield determining, yield limiting. and yield reducing faciors: - general acceptance of the lack of relationship between insecticide use and food production

- informed criticism by NGOS, scientists. and genera] public of stalte-supported or -facilitated pesticide marketing. - stricter examination, and limitation of pesticides in development assistuniv in lire with OECD-DAC guidelines - IPM-promoting policies and pracices in grant- and inrestement-doner insilutions;

WHAT DOES THIS SCENARIO MEAN FOR IPM SCIENTISTS?

- colleagial but accountable partnerships among and with farmers' groups. comununity organizers, and extcnsionists (Borifacio. Goodell. Jiggins):

- more DPM teams with broader and deeper local experience solving local problems (Benties. Roling).

- more hypotheses generated from an order-of-magnitude more people involved in problem-solving:

- beter hspothesis (esting: 1) more people doing the testing, and 2) new (ectruques emerging (Richards):

facing and overcoming obstacles like: 1) privale bureaucracies: agro-cherrical. textile manufacturers. food marketers. 2 ) public bureatcracies: crop profection (eg. long range forecasting). extension (eg. social marketingh. conzrodity hoards: and 3) historical but dysfunctional policies (public and private) that persist under shon time horizons and simplifiving assumptions.

- better questions: not only those answerabie within experinent station methods but confront nalure and culture even when uneomfortable. (eg. Sperling on varietal selection. Loevinsots on cropping intensification. Ashby on fertilizer management).

- oppomunity to create beuer tools to make and analyze more detailed local observations (Barfield);

WHICH FR UVEWORKS MIGHT HELP US TRAVERSE A CHANGE OF ATTITLDE?

Seizing new opportunitics to solve big problems facing us at local levels will mean changing our attitudes. There are biological. political. and social theories that provide stimulating models of how to disuribule problems among many small units each with the capability to propose and test local solutions that will later self-assemble: - deyelopmental/embryological- undifferentialed cells as units with simple instructions that assemble an organism through simple communication with neighbor cells (Wolpert):

- neural networks - brain cells with rules for communication asking for assistance from other cells (Minsky): - paraltel processing - in massive computers (Hilles):

- neuronal selection - memory and personality as selective reconstruction of brain cell configurations (Edclman): - natural selection of themata - in scientific thiniking over generations of human thought (Toulmin):

- civic community - building local social capital to make democrary work (Punnarn):

- communicative action - creating local public spheres of dialogue (rather than burcaucracies) that define, debale communicative action - creating

FAO PM. Programme - P.O. Box 186+ Manila. Philippines

THE DEVELOPMENT OF AN IPM PROGRAMME TO CONTROL THE EUCALYPT DEFOLIATING BEETLE CHRYSOPHTHARTA BIMACULATA OLIVIER (COLEOPTERA:CHRYSOMELIDAE)

\section{LLMadden, A R Clarke and H J Elliott}

The Cooperative Research Centre for Temperate Hardwood Forestry was established in 1991 to integrate an applied approach to production forestry in southern Australia through programmes on Soil and Stand Management, Genetics and Tree Breeding and Resource Protection.

A major project, within the Resource Protection programme, aims to develop a better understanding of environmentally compatible control options for Chrysophtharta bimaculata, the major pest of plantation eucalypts in Tasmania. These strategies will involve the replacement of a dependence on synthetic insecticides with commercial formulations of Bacillus thurigiensis var. tenebrionis and the potential or more effective use of

- lure trees of other, more susceptible species

- biological control including parasitoids and pathogens

- insecticides at sub-toxic levels, and

- host tree resistance/tolerance through breeding and silvicultural management

The advantages and disadvantages of these strategies will be discussed as they apply to the development of a sustainable forest industry, while complying to standards determined by government agencies and the concerns of the general public.

CRC for Temperate Hardwood Forestry

Locked Bag No. 2, Post Office

Sandy Bay TASMANIA 7005

Australia

\section{2 \\ BIOLOGICAL CONTROL OF MITES IN AUSTRALIAN VITICULTURE: STATE OF THE ART}

\section{D.G. James and J. Whitney}

The development of biological control of mites in Australian viticulture is an excellent example of cost-effective research which is providing a practical and sustainable alternative to chemical control. Based on the use of indigenous predatory mites (Phytoseiidae), biological mite control in vineyards is being rapidly adopted throughout Australian viticulture. Detection of miticide residues in dried grapes in 1988 stimulated research into this previously unstudied acarine system and led to identification of a sigrificant phytoseiid fauna which preys upon the grapevine mite pests (Brevipalpus spp., Colomerus vitis (Pagenstecher), Calepitrimerus vitis (Nalepa)). The dominant phytoseids (from a fauna of approximately 25 species) are Typhlodromus donenae Schicha, Typhlodromus dossei Schicha and Amblyseius victoriensis (Womersley). T. doneenae, alone, or in combination with either of the other two species, maintains small, non-damaging populations of the three pest mite species. All three phytoseids are generalist predators which maintain large populations on vines during summer, even in the absence of mite pests. T. doreenae occurs on vines throughout the year and is well adapted to a range of climatic conditions. It also possesses tolerance to a range of viticultural pesticides allowing it to be integrated in commercial vineyard management. $T$. doreenae is currently being mass-produced in a pollen based rearing system, for introduction to viticultural regions in Australia which currently lack this predator. It is expected that biological control will be the industry standard for mite management in Australian viticulture by 1997 , saving an estimated $\mathbf{A} \$ 3.5 \mathrm{M}$ per annum in chemical control costs.

Yanco Agricultural Institute, New South Wales Agriculture, PMB, Yanco, New South Wales 2703 Australia 
The role of husk cover and maize cultivar in the population density of Prostephanus truncatus Horn (Coleoptera; Bostrichidae) in rural grain stores in West Africa

\section{W.G. Meikle, P. Degbey, C. Borgemeister, C. Adda, B. Djomamou, and} R.H. Markham

The effects of maize cultivar on $P$. truncatus density in the field, and on larval life table parameters in the laboratory, were evaluated at the IITA research station near Calavi, Atlantic Province, Benin. The field experiment, carried out at Ex Soprova. Mono Province, was designed to evaluate the effects maize variety, using cobs with and without husk, on grain losses and insect pest density in rural stores Eight maize varieties were evaluated, including two local varieties, two improved IITA varieties and four lines from the germplasm collection of CIMMYT Mexico, that had shown varying degrees of susceptibility to $P$. iruncatus attack in laboratory conditions. The maize. grown under uniform conditions at Ex Soprova, was stored in $75 \mathrm{~kg}$ lots in granaries consisting of a split-bamboo cylinder on a bamboo platiorm, with three replicates per variety. After three months seven of the varieties stored with the husk had significantly lower grain loss scores than the same varieties stored without the husk. The varieties from the Mexican collection fared worse on average than the Atrican varieties, both with and without the husk Both P. truncatus and Sitophilus zeamais density were significantly correlated with grain losses. After six months pest density and grain losses were high among all treatments. The effects of varietal grain characteristics on $P$. truncatus larval growth and survivorship were evaluated on the single grain level. $P$. truncatus eggs were placed in holes drilled in kernels of tour of the varieties used in the field experment and the kernels were placed in CTH chambers at $30 \mathrm{C}$ and $75 \%$ r.h. (ca. 13-14\% grain moisture content). The developmental state and weight in dry matter of the larvae from ten kernels were determined every two days. No significant differences were observed among the varieties in terms of larval developmental time, growth or survivorship. These results suggest that good husk cover plays an important role in the resistance to insect pests of maize stored in traditional granaries, and this role may be more important than grain characteristics. Also, the effect of environmental factors on the expression of varietal characteristics was unclear and may intluence the efficacy of any varietal resistance. Laboratory experiments should be closely linked with field experiments to evaluate varieties selected for use by smallholder farmers.

International Institute of Tropical Agriculture, IITA-Benin B.P. 08-0932, Cotonou, Benin

895

UTIIIZNG Amblyseius scutalis (A.H.) TO CONTROL Eutetranychus orientalis (KLEIN) ON CITRUS TREES

M.E. El-Halawany, M.A. Abdel-Samed, H.M. Ebrahim and K.M. El-Saied

Experiments were carried out under field conditions to estimate the effect of the predatory mite Amblyseius sctutalis (A.H.) against citrus brown mite Eutetranychus orientalis (Klein) at El-Ismailia Govemorate on citrus trees. The predatory mite $A$. scutalis was released on January 9, 1992 at 40-70 adult females per tree (according to the size of the tree) when infestation level of the prey citrus brown mite $E$. orientalis reached 202 per leaf of citrus. The percentage reduction on the population density was 82.88 after 12 months of release, and then decreased to $52.46 \%$ reduction in E. orientalis after 22 months of the retease. So, it was felt necessary to carry out the second release of $A$. scutalis on November 25,1993 , when the infestation with moving stages of $E$ orientalts reached 4.9 individuals per leaf. The reduction percentages of E. orientalis after one year from the second release of the predator mite was $\mathbf{7 0 . 1 9}$, To serve the biological control programme the side effect of three Organophosphorus compounds (Dimethoate, Malathion and Sumithion) six fungicides (Saprol, Nimrod, Tilit, Afugan, Robigan and Calixin) and Neem WP were tested against adult females of $A$. scutalis under laboratory conditions. Also the side effect of 13 acaricides and some Minerol oils were tested against $A$. scutalis under field conditions. All fungicides tested: Neem WP, three acaricides (Vertimec, Cascade and N.N.I.) and Mineral oils were harmless to the predatory mite $A$. scustalis. Thus we can use those compounds in the programme of biological control.

Plant Protection Research Institute, Dokki, Giza, Egypt
NGO-GO COLLABORATION IN IMPLEMEENTING IPM IN THAILAND: WILL ING BUT UNEASY PARTNERS

\section{M.J. Ishii-Eiteman and N. Kaophong}

Despite a government policy of Integrated Pest Management, pesticide use in Thailand has soared in recent years. Farmers express frustration with their increasing reliance on chemical means of pest control, but for complex reasons they are often inhibited from exploring ecological alternatives. Local people's organizations and grassroots non-goveramental organizations (NGOs) have responded by working at the village level to find ecologically and economically sustainable solutions. While excelling in the adaptation of local knowledge to site-specific conditions and in the mobilization of community participation, the degree to which these organizations contribute to or influence uational pest mamagement strategies is woefully limited. Larger development institutions, on the other hand, such as government agencies, academic and research institutes, enjoy comparatively greater financial, technical and extension resources, but are typically less effective in communicating with local villagers and in re-directing research priorities to meet the needs of the poorest. In fact, the skills and resources of faruers, NGOs, government agencies and research institutes are in many ways complementary and inter-agency collaboration can go far in overcoming the institutional constraints which individual organizations face.

To facilitate collaboration between NGOs, government agencies and researchers, Save the Children/US (SC) initiated a three-year project on institutional partnering focussing on integrated pest management strategies in Thailand. In March 1993, SC convened a planning workshop for farmers, governmental and nongovernmental organizations interested in working together to develop IPM strategies for rice-based cropping systems in the Upper Central Plain of Thailand. As a result, participants agreed to form an "IPM Working Group" and to proceed actively with the organization of a season-long field-based training for farmers and NGOs. The training, held during the rice-growing season from July to November, emphasized group participation in developing an appropniate neld-based curriculum, which ensures relevance of training content to farmers' needs and, most importantly, actively engaged participants in the process of their own education. The content quickly expanded beyond the more traditional "insect pest-oriented" approach of many Rice IPM Trainings, and embraced wider concerns of soil fertility, rice growth and development, natural farming, cultural methods in weed management, agroforestry and rotational cropping, which ultinately lead to more sustainable crop production with fewer pest disturbances. The dynamic exchange between farmers, NGO and government extensionists led the trainiag on to the social and economic dimensions of farm management and the critical importance of community organizing as a tool to escape from indebtness and poverty.

The 5-month exercise in inter-agency collaboration yielded a rich set of experiences, from the technical to social to institutional aspects of integrated pest management. It also demonstrated both the enormous benefits of working together and sharing diverse skills as well as the challenges and difficulties in communicating creatively across traditional institutional barriers.

Save the Children, P.o. Box 49, Amphur Mutang, Nakhon Sawan, 60000, Thailand 
PARASITISM OF HETERODERA SCHACHTII BY PAECILOMYCES FUMOSOROSEUS

FATEMY, S.

Iran has more than 150,000 hectares under cultivation of sugare beet. Among many other nematodes $\underline{H}$. Schachtii is the most important parasite of sugare beet which in some areas causes severe yield lass. Khorasan the main sugare beet growing province with nearly $80 \%$ of its fields infested with this nematode, in some parts has a population as high as $140 \mathrm{eggs} / \mathrm{g}$ soil. the overall yield loss of upto 57 due to this nematode has been reported from this province. The rotation is not.a common practice and nematicides are hardly being used by farmers. Therefore biological control may remain one of the alternative methods of con troling this nematode. In order to exploit endewic natural agents, survags are being carried out during which $\underline{P}$. funosoroseus was isolated from $\underline{H}$. schachtii cysts. Its pathogenicity against eggs, females and cysts of beet cyst nematode was therefore examined in vitro. Infection of separated eggs measured on water agar at $15,20 \& 25^{\circ} \mathrm{C}$ was $64.5,78.3 \&$ 87.37 respectively. At $25^{\circ} \mathrm{C}$ all cysts and females were infected by fungus with percent infe ction of egrs vithin cysts and live females being 22.3 \& 75.4 respectively. Apparently $\underline{p}$. fumosoroseus did not infect mature eggs or juveniles in these tests.

Plant Pests and Diseases Research Inst. POBox 19395-1454, Tehran, Iran.

899

THE APPLICATION OF TRICHOGRANA (HMM, TRICHOGRANATIDAE) FOR THE BIOLOGICAL CONIROL PROCRAMMDS IN THE UARAINE

\section{victor FUREOV}

The ece-parasitolds, Trichograma species, are the important biological control apents in the likraine. Now the commercial cultures of Trichocramm are produced in about 15 main and 45 repional biological factor 108 in 15 different districts of the ukraine. The bett regional blo-laboratories are working in the Cherkasey Dletrict. Now three apecles of Trichoprame are reared in the laboratories: T.pinto1, T. dendrolimi, T. telengat. At the current time the proposed T. evanescens is not reared in the commercial cultures. Wany records or provious application of T.evaneBcens have to belong to T.pintol. Now T.pintol is the doninant apecies recorded from all studied laboratories.

Now T.pintol is applied by seasonal colonization arainst aome noxious Noctuidae, Ostrinia nubilalis, Lozcstepe sticticalis on aupar bet, cabbage, vegetablea, prain crops, lepuminoug crope, perennial prasses. T.dendrollmi and T. telengal are recomended to be ubed apainst Carpocapese pownolia and othor Tortricidae in apple orcharde. Nine indigenous apecies of Trichorama are found in the natural and apricultural ecolopical cominities of the Ukraine: T. dendrol imi, T.pintol, T.evareacens, T.telengal, T. embryophaqum, T.eleqantum, T. principium, T.eemblidis, T. piooum Indipenous apeoles aro used for the creation of commercial cultures or Trichocrama. It 18 recommended to change the culture of T.pintol on more productive culture of T. evanescens in the western diatricts of the lkraine. It was proposed to keep the elite maternal culture of T.evanescens and other speciea in one oentral blo-laboratory.

Institute of Zoolocy of Lkrainian Acadeny of Sciences, Kiev-30, Bogdan Khmelnitakiy Street, 15, 252601, Ukraine.

Fax: (044)-224-1569. E-ma11: entomoleizoolocy. k1ev.us
HOLCOTHORAX TESTACEIPES RATZ. (HYM, ENCYRTIDAE) EGT-LARVAL PARASITOID OF LEAF-MINING LEPIDOPTERA IN URRAINE

\section{Victor FURSOV, Serpej SVIRIDOV}

Holcothorax testacelpes Ratz. is widely distributed polyembryonic ex-larval parasitoid of leaf-mining mothe (Graciliarildae). H. testaceipes has became a very effective parasitoid of Phyllonorycter pyrifolliella Grem and Ph. blancardella F. in the fruit orchards of the ukraine. The level of paragitiastion may be about $90 x$. Holcothorax attacks the equ of its host, and parasitold develope polyembryonically within five larval ingtars. The adulta of H. teatacelpes emerge from a mumifled cuticle of its hat. In Ukraine 2-3 ceneration of Holcothorax are observed. Pupae and larvae of Holcothorax are overwintering inaide the miners in falled leaves. The emergence of Holcothorax 18 occured in May, and about 7 days later than the emeronce of leaf-mining mothe. In the Ukraine Holcothorax is involved in a complex of 39 epecies of parasitoids of leaf-minars on epple trees. Holcothorax has 5 apecies of hyperparasitolds.

The tendency of increasing of a number of Holcothorax was Indicated durin last 10 yearg of observations in the apple orchards of the Uikraine. The abundancy of Holcothorax may be explained by 1 ts more high fertility as well as phenomenon or polyembryony and its hicher resiatance to chemical treatments. Small larva of holcothorax are leas suffered frow auch hyperparasitolds as Symiesis (Eulophidae) than lare larvae of Apanteles bicolor Nees (Braconidae). Laually 8-14 larvas of Holcothorax are developing in one larva of leaf-minine mothe. One larva of most inportant hyperparasitoid, S. Berlcelcomis Nees, damaqes 2 larves or pupee of H. testaceipes.

Institute of Zoolony of Ukrainien Masdeny of Scienoes, Klev-30, Bogtan Khmolnitgkiy Street, 15, 252601, UKRAINE.

Fax: (044)-224-1569. E-mail: entomole1zoolowy. klev. ua

900

NATURALIY-OCCURRING BIOLOGICAL CONTROL - A NEGLECTED COMPONENT IN DEVELOPING INTEGRATED CONTROL PROGRAMS

\section{GONZAIEZ}

Naturally-occurring biological control is a neglected but essential cornerstone in developing area-wide integrated control programs. Without effective understanding of the impact from naturally-occurring arthropod enemies, long-term implementation of integrated control programs is not environmentally or economically feasible. Attempts to implement introduction and/or augmentation biological control programs also requires an understanding of the naturally-occurring biological control factors. Long-term effective biological control programs will only persist when additional IPM mortality factors complement rather than compete, over-lap, duplicate, or detract from natuallyoccurring arthropod enemies. Examples are given from California cotton pest control programs. Insecticide check and economic threshold sudies were used to document the effectiveness of naturally-occuring biological control species.

Department of Biological Control, University of California, Riverside, Califomia 92521 


\section{INFLUENCE OF PASSAGING ON THE PATHOGENICITY OF VERTICILLIUM LECANII ZIMM.}

\section{E. Loginova, J. Kuzmanova, B. Boev, K. Sapundzieva}

The pathogenicity of isolates from the Bulgarian strain $\mathrm{N} 3$ of $V$. lecanii, isolated from dead larvae of whitefly (Trialeurodes vaporariorum), has been studied. They have been obtained by passaging several times through the whitefly (B $3 / 30$ and $B 3 / 50$ ) and the cotton leaf aphid ( $A$ $3 / 30$ and $A 3 / 70$ ). The diata of their action against whitefly larvae, Aphis gossypil and Frankliniella occidentalis, were reponted. The results had shown that to the specific host the pathogenicity of the passages improved in comparison with the initial strain.

To A. gossypii B $3 / 50$ has a greater and a faster effect even than $A$ $3 / 70$, passaged through the same host. Analogous data of accelerating the pathogenic process and increasing the pathogenicity was obtained for $F$. accidentalis in $B 3 / 50$ compared with the initial strain $N 3$ as well as $A 3 / 30$ and $A 3 / 70$. Therefore, despite their common origin, the passages display different aggressiveness to the tested insects, as a result of which the pathogenicity is changed towards the nonspecific hosts.

The changes in the strain toxicity correlated with its their morphological and biochemical difference.

Bulgaria, Plovdiv $-4000,12$ Mendeleev Str., Agricultural University

903

THE LARVAL PARASITOID WASP OF THE ORIENTAL, FRITT FLY N TAIWAN

LEE, WEN.YUNG

The oriental fruit fly, Bactrocera dorsalis is one of the economic intoxtant insect pests in Taiwan island. The Government and the Agricultural Organization employ the control method of use of the Anaihilation Technique with the attractants of the poisoned methyl eugenol. Taiwan entomologists have been seeking integrated control methods with the food attractants, attractants for oviposition and others. The Braconid wasp, Diachasmimorpha longicaudatus which is an efficient larval parasitoid of the oriental fruit fly were introduced from Hawaii and have been successfully raised in the laboratory, recently established in the guava orchards as well. The present report was made to have an understanding to the biological characteristic of this wasp and to know how to use this wasp in the imegrated control program of the oriental fruit fly in Taiwan.

Instinte of Zoology, Academia Sinica, Nankong, Taipei 115, Taiwan, R.O.C.

\section{THE EFFECT OF DIFFERENT STRAINS OF VERTICILLIUM LECANII ZIMM. \\ AGAINST FRANKLINIELLA OCCIDENTALIS PERG.}

\section{B. Boev, H. Serman, E. Loginova, J. Kuzmanova}

At the Humboldt University - Berlin /Germany/, the Research Institute for Vegetable Crops "Maritsa" and the Agricultural University, the last two Plovdiv-Bulgaria, some experiments were carried out to establish effect of Verticilium lecanii Zimm against $F$. occidentalis. Bulgarian strains $\mathrm{N} 3$ (isolated from whitelly larvae) and $N 3\left(50^{\text {th }}\right.$ passage through whitefly). and a German strain $V 24$ were used. Different spore concentrations $(T$ $10^{6}, 10^{7}, 10^{8}$ ) were tested both at $20^{\circ} \mathrm{C}$ and $25^{\circ} \mathrm{C}$. The results had shown that the virulence of strains $\mathrm{N} 3$ and $\mathrm{V} 24$ was similar, while that from N 3/50 was with a higher initial and a stronger effect. The best results were obtained from $T 10^{8}$ at $25^{\circ} \mathrm{C}$. The difference between the spore concentrations of the strains at $20^{\circ} \mathrm{C}$ was about the same but the speed of mortality was lower. The sporulation under these conditions was in weaker extent. It was established that the pathogenous process was accelerated depending on the increassing of spore concentration. It was found that the difference between the effects obtained from $T 10^{\circ}$ and $\mathrm{T} 10^{\prime}$ was insignificant. Therefore we consider that for practical point of view $T 10^{7}$ was the best.

Bulgaria, Plovdiv - 4000, 12 Mendeleev Str., Agricultural University

904

EFFICIENCY OF NATURAL ENEMIES OF OULEMA MELANOPUS (L.)

\section{A.A.Marnedov}

The crop damage is the function positively pest density dependent and negatively density of beneficials dependent. Therefore natural enemies must be a part of the threshold concept. The more beneficials present in crop the more pest density is permitted without yield losses. The emergent larvae of 0 . melanopus on cereals damage leaf surface. Consecutive population entering the next instar (or stage: egg larva) is accompanied by population mortality. Eggs and larvae are attacked by a lot of predators. Mortality of each life stage is effected by removing a percentage of insect in each life stage. To know the percentage is the possibility to predict the damage level (having the information about initial density of the pest - egg, and damage per unit of each instar larvae). For this study (1990-1992) eggs and larvae was exposed in various sites on a winter wheat field and the percentage of their disappearance was compared with predator densities in the sites. The egg mortality was about $50 \%$, 1 -st instar larvae - 60, 2-nd - 40, 3-rd - 20, 4-th - 20. So total mortality (eggstlarvae) was over $90 \%$. Correlation analysis shows there are positive correlations between the daily percentage of egg disappearance and the density of Tachyporus hipnonum $(r=0.41)$, Nabis sp. $(r=0.52)$ and Coccinella 7 -gunctata $(s=0.34)$. So there is a basis for egression model development

Podbelsky shosse, 3, St -Petersburg-Pushkin, 189620 Russia

All-Russian instinute for plant protection (VIZR) 
STUDIES CN THE PREYING EFFICIENCY CF PREDATORY CROCK BEETLE, AUT OCKATES AENEUS PARRY (JRICTFNOTCMIDAE:
COLEOPTERA) ON THE WHITE GRUB, HOLCTRICHIA J.ONGJPENNIS BLANCH. IN LABORATORY CONDITIONS.

\section{P.N. MISHRA, M.P. SINGH}

In India, wite gruks (Scarabaeidae : Coleoptera) popolarly known as root grube have pored an alarming situation in the country as root grube have pored an alarming situation in the country twenty two srecies of white grube recorded in the Gerbwal region twenty two srecies of white grube recorded in the Gerbwal region
of U.F. hille, Holotrichia Jongipennis Elanch. (CCleoptera: Melolonthidae) is a predominant species prevalent in U.F. hills. The beetles emerge 1 n the end of May after the first monsom rains and defoliate the varioug fruit plum, walnt snd eheztnut. The beetles are the potent source of root grube which are sublerranean and ereed various field crops grown under rainfed conditions during rainy season. The Fredatory crock beetle, Autocrates aeneus Parry is a voracious feeder of beetles and grubs hoth of Holotrichia longipennis during the active season i.e., June-July. laboratory studies have shown that the average lengevity of female predator was 27.50 days while in case of male the average
longevity was 27.0 days. longevity was 27.0 cays. Male predator consumed 3.70 beetles/night and 4.70 grubs/day of $\underline{H}$. longipernis while femele AutocrateE consumed 3.24 beetles/right and 4.45 grubs/day caged in glasf acquaria in lab. conditions. The emerpence of predator Eynchronize with the emergence of root grubs beetles, $H$. longifennis during rainy season. The precator was obeerved to be attracted towards the light source for preying ufon the root grub beetles trapped on light source and thus it might be playing an important role in the natural contral of root grub beetles in U.P. hills. The data on its longevity, nocturnal habit and preyinp potential euggest that the predator must be conerved in nature.

Senior Scientist Entomslogy

Hill Campus, G.B. Pant Univ, of Agri. Tech., Ranichauri.



PEST MANAGEMENT SYSTEMS FOR EGGPLANT ARTHROPODS: A PLAN TO COUNTER PEST RESURGENCE RESULTING FROM THE DESTRUCTION OF NATURAL ENEMIES.

\section{H. Nemoto}

Egaplant in an experimental plot were continuously sprayed with the insecticide permethrin, milbemectin, phenthoste, and imidacloprid to evaluate their respective side-effects. Effect of populations of pests and of their natural enemies were assessed. The results revealed the importance of natural enemies such as anthocorid bugs, Orius spp. Application fo pesticid may cause resurgence of pest population because of (1) the development of resiatance by the pest and (2) nonselective killing of predators. The autbor there for attempted to develop methods of control of pests that would no affect substantially natural enemies. Imidacloprid which is highly effective against Aphis gassypit and Thrips palmi caused resurgence of the spider mite Telramychus kanzawai. Milbemectin which exerts minimal adverse effect of Orius spp. when used in combination with imidacloprid maximized the latter's advantages while minimizing it disadvantages.

Saitama Horticultural Experiment Station, Rokumanbu, Kuki, Saitama 346 Japan.
908

ANALYSIS OF SOYE VARIATION SOURCES IN RELEASING PHYTOSEIULUS PERSIMILIS IN FIORIDA STRAWBERRY FIELDS

\section{J. F, price and $K$. A. Sorenson}

Florida produces over 2000 ha. of strawberries for the winter fresh market; about 300 ha. are produced with phytoseiulus persimilis Athias-Henriot predatory mites as the control agent for the primary pest, the twospotted spider mite (Tetranychus urticae koch). Predators are mixed with vermicuite and are shipped to farmers in ca. $500 \mathrm{ml}$ bottles then released into the fields by hand. The releasing process is costly and may be imprecise because quantities of the predator may remain in the bottle after all of the vermiculite has been dispensed. Three important sources of variation in the numbers of predators that remained in the bottle were studied: 1 ) Release of the
predators by manually shaking the contents from the shipping predators by manually shaking the contents from the shipping bottle or release of the contents by manualiy operating a
device that dropped ca. I ml of the contents of the bottle device that dropped ca. l ml of the contents of the bottle
per dose (dispensing method), 2) dispensing the contents at per dose (dispensing method), 2) dispensing the

(temperature), and 3 ) remixing the contents (rolling on its (temperature), and 3) remixing the contents (rolling on 1ts axis and end over end) every three minutes or not at ail during the 15 minute dispensing process (remixing practice)
The interaction of dispensing method $X$ temperature and of The interaction of dispensing method $x$ tempezature and of temperature $X$ remixing practice were significant. Fewest
predators remained in the bottles when the mechanical device predators remained in the bottles when the mechanical
was employed and the contents were held at $4^{\circ} \mathrm{C}$; most was employed and the contents were held at $4^{\circ} \mathrm{C}$; most remained in the bottles when the mechanical device was
employed, but contents were dispensed at $22^{\circ} \mathrm{C}$. Fewest remained in the bottles when contents were dispensed at $4^{\circ} \mathrm{C}$ regardless of remixing practice; most remained in the bottles when contents were dispensed at $22^{\circ} \mathrm{C}$ and contents ware not remixed during the dispensing operation. Results of these studios are useful to release predators efficiently and to design efficient, automatic devices for release into gtrawberry fields.

Univeratty of Florida, Gulf Coast Research and Education Center, 5007 60th Street East, Bradenton, Florida, U.S.A. 34203 . 
NATURAL PREDATORY BUG ARMA CUSTOS AS POSSIBLE AGENT AGAINST LEPTINOTARSA DECEMLINEATA

\section{A.H.Saulich}

In biological control of adventive pests the imported entomophages usually are utilized. For example, positive results from the use of the north american hemipterous bugs Podisus maculiventris and Perillus bioculatus (Pentatomidae: Asopinae) against the Colorado potato beetle are expected. However, as it was discovered in laboratory and field experiments, the seasonal cycles of these bugs don't correspond to the environmental climatic conditions in new ranges and beneficial species die out when unfavourable seasons become. In this connection the natural enemies are of interest for use in biological control, among them species of the same subfamily Asopinae, all members of which are predators. Unfortunately according to literature data the majority of Asopinae have univoltine seasonal cycles. It makes a lot of problems in insects cultivation.

The predatory bug Arma custos was investigated in details. By special experiments it was established that univoltine population from the forest-steppe zone of Russia has facultative diapause controlled by qualitative photoperiodic reaction of the long day type. Thanks to this it is possible to maintain the active laboratory oviture of A.custos independently of seasonal time. Further investigations are necessary to confirm truth univoltinism in other Asopinae bugs which traditionally considered as univoltine.

Biological Research Institute of St.Petersburg State University

Stary Peterhof, Oranienbaumskoye sh. 2

198904 St.Petersburg

Russia
911

THE REACTION OF AMBLYSEIUS CUCUMERIS ON SPATIAL DICTRIBUTION OF PREY THRIPS TABACI LIND. AS INDEX OF ITS SUPRESSION V.B. Shevchenko

It is established that degree and terms of entomophage ability for control (especially for A.cucumeris, with its low functional response to T.tabaci larvae density, low speed of spread and searching ability) depend to great extend on place and density of its release. One meets difficulties appreciating predator's role in pest number control taking into consideration environmental heterogeneity, variability of density and prey spatial distribution. This is why only quantitative characters of predator - prey system in glasshouse (such as frequency of meeting, infection coefficient, average density in sample) are not sufficient, especially if purpose of investigation is background for control measures

The effect of entomophage release tactics on pest control has been evaluated in present investigation. Variants differed both in rate and number of releases and measure of spatial distribution variability of samples taking or not into account corresponding characters of $T$.tabaci population in glasshouse. Mutual variability of density spatial distribution of predator and prey in glasshouse was considered as indirect estimation of entomophage control effect.

management of pest population was attained by purposeful and regular beneficial agent releases.

we have made following conclusions while assessing the results: -the most important characters of entomophage are: functional reaction to pest density, ability to concentrate in places of phytophage aggregation, its searching activity, conjugation with pest in time and space; -degree and term of realization of ability for control pest releases;

-increase of entomophage individuals aggregation results in increase of total control effect;

-effective control of thrips population is accompanied by transformation of regulatory aggregative distribution into randomly dispersed ore.

Mutual variablity of density spatial distribution of predator and prey in glasshouse was considered as indirect estimation of and prey in glasshouse was

Management of pest population was attained by purposeful and Management of pest population was
regular beneficial agent releases.

Dr.valery B. Shevchenko

Laboratory of IPM, Institute of Biological Plant Protection

P.Box. 427

ML 277049 Kishinev, Moldova

Phone (3732) 570-474; Fax (3732) 579-641
912

LARVAE AND PUPAE OF THE ANGOUMOUS GRAIN MOTH, SITOTROGA CEREALELLA OLIV., AS FOOD FOR MASS REARING OF PREDACEOUS AND PARASITOID INSECTS

V.A.Shlyakhtich, E.I.Negru

Sitotroga cerealella eggs are widely used for a rather long time for rearing of Trichograma sp., Chrysopa carnea steph.. podisus maculiventris Say, Cryptolaemus montrozieri Mulsant and some other entomophages. The angomois grain moth is produced on barley, wheat or corn Yielding not more than $1,2 \mathrm{~kg} / 100 \mathrm{~kg}$ of grain. The use of late instar larvae and pupae of this insect for mass rearing of entomophages allows to increase the yield of food biomass up to $30-40 \mathrm{~kg} / 100 \mathrm{~kg}$ of grain, $1 . e$. about 30 times. However, development of Sitotroga larvae and formation of
pupae takes place inside the grain. Hence, it does not allow to pupae takes place inside the grain. Hence, it does not allow to
get them in sufficient quantities using the existing get them in sufficient quantities using the existing solve this problem.

Experiments have proved possibility normal development on such food for the lacewing, Ch, carnea (which is applied against aphids and other sucking insects on different crops in greenhouses and in the field), the bug $P$. maculiventris (used against the colorado potato beetle on solonaceous crops), ectoparasite Bracon hebetor Say (against noctuids on cotton),
pupal parastte Chouioia cunea Jang (promising entomophage of the
fall webworm, Hyphantria cunea Drury).

fall webworm, Hyphantria cunea Drury). sitotroga larvae and pupae allows to cut over 20 times expenses necessary to rear this predator as compared to the existing technologies. Analogous tendency has been revealed for other above mentioned entomophages.

Dr.Vladimir A.Shlyakhtich

Head of Departament of Entomology, Institute of Biological Plant Protection

P.Box 427

ML 277049 Kishinev, Moldova

Phone (3732) 22-49-20, Fax (3732) 57-96-41 

STUDIBS OH THE PREDATORY MITES ASSOCIATED WITH SPIDER MITES ON
YEGETABEB CROPS IN BASTRR IHDIA.

\section{Singh. R. R.}

In recent years there has been increased interest in the study of predatory nites associated Hith crop nite pests in India because of the harnful effects of pesticides and partly because of the intensiveness of research in Integrated Mite Hanagenent. A survoy carried out to record the predatory nites rith phytophagous nites on vegetable erops revealed that twenty four species of predatory nites were most comon on vegetable crops. Twenty four spacies of nite predators, which belongs to fanily - Phytoseiidae, Tydeidae, Stignaeidae. Cheyletidae and Anystidae. Due to indiscrininate use of pesticides on vegetable crops, predatory nites have become very scarce and collectod irregularly on vegetable crops. During rocent years this has rosulted in froguent out break of nites on vogetable crops in this region. of change in pest status is the red spider nite. Tetranychus Iudeni 2acher. Regular out breaks of this nite on vegetables have made this nite a distinctive and alarning pest. An axporiment pas conducted to see the effect of seven pesticides against this mito and its predators. Some insecticides have been obzerved to be detrinental to predatory fauna.

Department of Entonology \& Agril.zoology, Institute of Agricultural Scienco, Banaras Hindu University, Varanasi221005 , INDIA.
SEASONAL ABUNDANCE OF CARABIDS IN CABBAGE FIELD AND THEIR POTENTIAL AS PREDATORS OF DIAMONDBACK MOTH, PLUTELLA XYLOSTELLA, LARVAE.

\section{H. Suenaga and T. Hamamura}

Seasonal abundance of carabid beetles in a cabbage field, adjacent old fields and the field boundaries was investigated over the period May - December in 1994 in Mie prefecture in central Japan using pitfall traps. The most abundant species was Amara congrua Morawitz, followed by Harpajus griseus (Panzer), H. sinicus Hope, Chlaenius micans (Fabricius), and $H$ tinctulus Bates. These five species formed $65 \%$ of the total capture. A congrua and $H$. tinctulus, $C$. micans, and $H$. griseus and $H$. sinicus showed peaks of capture in May-Jul, Aug.-Sept, and Sept- Nov., respectively. C. micans occurred in higher numbers in the cabbage field, while the other species occurred in higher numbers in the adjacent old fields and the field boundaries.

The potential of adult carabids (H. grisers, $H$. sinicus, and $C$. micans) as predators of diamondback moth larvae were estimated in our laboratory. Adult carabids of each species ( 8 or 16 individuals) were introduced into an experimental enclosure of $48 \times 33 \mathrm{~cm}$, in which 8 young cabbage plants had been planted. About 40 diamondback moth larvae were released on the cabbages. The carabids were allowed to prey upon the diamondback moth larve for 4 days at $25^{\circ} \mathrm{C}$ under the photoperiod of 14L:10D. C. micans consumed $89 \%$ of the total larvae. On the contrary, $H$. griseus and $H$. sinicus consumed less than $10 \%$ of the total larvae. We observed $C$. micans crawling on the cabbage leaves even during the day.

National Research Institute of vegetables, Omamental Plants and Tea, Ano, Mie 514-23, JAPAN

\section{6}

EFFECT OF FLOODING ON THE SURVIVAL AND PATHOGENICITY OF FUSARIUY OXYSPORIM F. SP. CEPAE IN PADDY SOIL.

\section{$\underline{\text { Kinji Tanaka, Eiji Kubota and Hideki Terao }}$}

Onion bulb rot caused by Fusariun axysparun $f$. sp. cepae an sumer stored bulbs has recently become prevalent throughout the onion growing areas in Saga Prefecture. The investigation in the present study demonstrated that onion plants grown in bulb rot infested soil previously flooded with water developed less severe bulb rot than in infested non-flooded paddy soil. Flooding during June to August prior to sov onion seeds on seedling bed was successful controlled Fusarium oxysporum f. sp. cepae and severerity of bulb rot was observed to decrease during storage. The incidence of onion bulb rot in the non-flooded and flooded soil $x a s \quad 42 x$ and $22 \%$. respectively. At high temperature $(30 \mathrm{C})$, the number of viable Fusarium gropagules in flooded soil decreased more rapidly than in non-flooded soil after two weeks. It is concluded that bulb rot of onion can be successfully controlled by flooding.

Saga University, Honjou-machi 1 Saga 840 Japan
V. Táborsíy, Czech Agricultural University in Prague Department of Plant Protection Kamýcká 129 16521 Prague 6 - Suchdo1 Czech Republic E-mail: TABORSKY_Y @ AGRO.VSZ.CZ 
EFFECTS OF FOUR INSECTICIDES ON THE PREDATORY STINK BUG POOISUS MACULIVENTRIS

\section{A. De Cock, P. De Clercq, L. Tirry \& D. Degheele}

The asopine bug Podisus maculventris (Say) is a potential biocontrol agent of several lepidopteran and coleopteran crop pests. The susceptibility of this predatory bug to the insecticides diflubenzuron, pyriproxyfen, diafenthiuron and imidacloprid was investigated in the laboratory. Toxicity of these compounds by topical application, exposure to treated glass plates, and ingestion via treated drinking water to fifth-instar nymphs and female adults of $P$. maculiventris was assessed. The insect growth regulators diflubenzuron and pyriproxyen did not affect the viability of adult predators. The benzoylphenylurea compound diflubenzuron was harmless to nymphs when applied topically or by residual contact, but was highly toxic when ingested. The juvenile hormone mimic pyriproxyfen caused high mortality to nymphs by all ways of exposure tested. Diafenthiuron, a novel thiourea compound, was toxic to both nymphs and adults of $P$. maculiventris by ingestion and residual contact, but not by topical application. The nitroguanidine insecticide imidacloprid had adverse effects on the survival of nymphs and adults of the predator whatever the method of exposure. The results suggest that integrated pest management programmes involving these insecticides should regard possible side-effects to this beneficial insect.

Laboratory of Agrozoology, Faculty of Agricultural and Applied Biological Sciences, University of Gent, Coupure Links 653, B-9000 Gent, Belgium
DIAPAUSE OF PREDATORY BUG PODISUS MACULIVENTRIS IMPORTANT PART OF TECHNOLOGICAL CYCLE IN THE MASS REARING

AS

\section{T.A. Volkovich}

The seasonal cycles of natural insects populations consist of two alternating periods the active development and diapause. But in artificial conditions the continuous rearing (one generation after another) is widespread while it brings to degeneration of bioculture. Just this way is used in the mass rearing of the bug Podisus maculiventris usually. This species was introduced from USA (Missury) as the perspective predator of Leptinotarsa decemlineata. In Russia it may be used by method "of the flooding outputs" only. That is wby the improvement of the mass rearing technology is actual. As it was discovered in expenments P.maculiventris has short and nondeep imaginal diapause. Photoperiod $11 \mathrm{~L}: 13 \mathrm{D}$ and temperature $20^{\circ} \mathrm{C}$ are the most favourable conditions for diapause induction. These conditions must to affect on larval and imaginal stages during 18-20 days. The species of prey has important significance too. Diapause ends in the 1,5 months but bugs can be keep without mortality in the course of 3 months under $8^{\circ} \mathrm{C}$. The incorporation to the cycle two-step storage elongates this period. The productivity of the bugs culture is rised when diapause is included to the cycle of mass rearing.

St.Petersburg State University

Biological Research Institute

Oranienbaumskoye sh.2, Stary Peterhof

198904 St.Petersburg, Russia
920

\author{
A REVIEW OF THE PALAEARCTIC CHALCIDOID WASPS \\ (HYM., CHALCIDOIDEA) DEVELOPING IN THE PLANT SEEDS
}

ZEROVA M.D.

Some speoies and genera of chalcidoid wasps are knom as phytophagous. In the Palaearctics the largest ecologioal groups of phytophagous ohalcidoid wasps are represented by the gall-formers and seed-eaters. The complex of geod-eaters includes 122 speoies from three families: Eurytomidae (85 species), Torymidae (36 species), Pteromalidae (1 species). In the Palaearctics the range of host plants is formed by more than 200 species of 16 families of Vasculares plants. The seeds of various cultivated and wild plants are infested by the phytophagous chalcidoid wasps: Rosaceae (apricot, plum, apple), Fabaceae (Trifolium, Vicia), Unbelliferae (carrot, coriander, fennel), Anacordiaceae (Pistacea) and many others. Some species, like Eurytoma amigdali, belong to the karantine subjects. Some species (Eurytoma gibbus, $E . r o d d i)$ danage the seeds of arricultural grasses (Trifolium, Vicia). Seed-eaters can play an economically important role in agriculture, forestry and nature in general.

Nost of chalcidoid seed-eaters are characterized by the narrow trophio speoialization. Many trophic interactions are neakly studied. The researoh on the taxonomy, species diversity, trophical relations, peculiarities of distribution, economical importance is in the propress now.

The research of phytophagous chalcidoid wasps has also a theoretical interest. The origin and evolution of some groups of phytophagous ohalcidoid wasps inside a highly specialized superfamily of parasitic hymenopterans may be conducted as a special biological phenomenon.

Institute of Zoology of Ukrainian Academy of Soiences, Kiev-30, Bogdan Khmelnitskiy street, 15, 252601, UKRAINE. Fax: (044)-224-1569. E-mail: entomoleizoology. kiev.ua 
INTEGRATED Heliothis armigera MANAGEMENT WITH GIVING PRIORITY TO BIOLOGICAL CONTROL IN VERY EARLY-MATURING COTTON PRODUCTION AREA OF CHINA

Y. Zhou, X. Zhang, T. Li, M. Lian

Middle of the province shanxi is one of the very early-maturing cotton production areas of china. The fluctuation of cotton bollworm (Heliothis armigera) has caused serious losses in cotton production since 1992. Aimed at utilizing the natural control effects, strengthening the research of biological control technique, and keeping the ecological balance, we experimented single technique of non-chemical-pesticide control of Heliothis armigera in 1993. In 1994 we integrated the cotton bollworm management with giving priority to biological control.

1. Sexual hormone trap was used in the bollworm development prognoses to guide the control of eggs and larva. This measure supplied companied with other prognoses methods precise schedule of spraying and releasing of natural enemy.

2. In time inset of parasitic insect and entomopathogenic bacteria played a important roll. Shanxi is a semiarid area. To gain an effective control, we have introduced a field protection and reproduction device to avoid dryness for Trichogramma spp. Later in 1994 we have imported Trichogramma embedded in artificial eggs that was manufactured by Guangdong Academy of Agxicultural sciences and suited for large area application of pest control. In order to reduce the chemical pesticide usage, agent of Bacillus thuringiensis was sprayed in the hatching phase of bollworm eggs. 3. Cultural methods took part in the integrated boliworm management Through i.)ploughing and irrigation in autumn; ii.) planting trapplant-belt to reduce the amount of imago; iii.) deteriorating the living environment of Heliothis armigera through biological and ecological measure.

Shanxi Academy of Agricultural Sciences, 2 Chang Feng street, Taiyuan 030006 , FR China

\section{3}

SUPPRESSION OF RHIZOCTONIA SOLANI ANASTOMOSIS GROUP 8 IN AUSTRALIA AND ITS BIOLOGICAL NATURE

\section{B. M. Wiseman, S. M. Neate, K. Ophel-Keller and S. E. Smith}

The nature of suppression of a field soil showing a decline in the Rhizoctonia barepatch disease of wheat (causal agent $R$.solan AG-8), in a minimurn tillage system in southern Australia was investigated. The suppressive characteristics of the soil could be transfered to an autoclaved or pasteurised soil by adding $10 \%(\mathrm{w} / \mathrm{w})$ of the unsterilized soil. This resulted in lower disease levels following inoculation with R.solani AG-8. No transfer of suppression was observed when non-suppressive soil was added to the autoclaved or pasteurised soil. Gamma iradiaton or pasteurisation at $80^{\circ} \mathrm{C}$ for 30 minutes eliminated the ability of the soil to suppress disease. This is the first report of biologically based suppression of this root rotting disease of wheat caused by $R$.solani AG-8.

Co-operative Research Centre for Soil and Land Management PMB 1 Glen Osmond 5064

AUSTRALLA

\section{A NEW PARASTTOID OF THE MAIZE STEMBORER, BUSSEOLA FUSCA ( LEPIDOPTERA: NOCTUIDAE)}

\section{K. YITAFERU}

The new Braconid parasitoid was reared from Busseola fusca larvae in eastern Ethiopia and it has recently been identified as Dilichogenidea fuscivora Walker at the CAB-International Institute of Entomology. This species has only been collected so far from Ethiopia. It is by far the major larval parasitoid of $B$. fusca around Alemaya area (eastem Ethiopia). A Ceraphronid hyperparasitoid, Aphanogmus sp. was found to parasitize $D$. fuscivora in the field. The biology of this new species is described based on field and laboratory studies. How the parasitoid, $D$. fuscivora survives during the dry season is explained. Its potential as a possible future biological control agent and as a promising component of IPM is emphasised

\section{Alemaya University \\ P. O. Box 138 \\ Dire Dawa \\ Ethiopia}

924

PREDICTING DISEASE OUTBREAKS TO REDUCE FUNGICIDE APPLICATIONS IN PROCESSING-TOMATOES, CARROTS AND CELERY

Bolkan. H. A., Reinert, W. R., Curtis, R. K., Larsen, D. J., and Bidersthadt, D. L.

Anthracnose fruit rot (caused by Colletotrichum coccodes (Wallr.) S.J. Hughes), black mold (caused Altemaria allemara (Fr.:Fr.) Keissl.), late blight (caused by Phytophthora infestans (Mont.) de Bary), and Septoria late blight (caused by Septoria apiicola Speg.) can inflict large economic losses in processing-tomatoes (Lycopersicon esculentum Mill.) and celery (Apilon graveolens L. var. dulce (Mill.) Pers.) respectively. Similarly, Alternaria dauci (Kuhn) Groves \& Skolko), the cause of Alternaria leaf blight of carrots (Daucus carota L. var. sativa Hoffm.), is widespread throughout the processing-carrot growing areas of eastern North America. Traditionally, these diseases are controlled with protectant fungicide applied on caiendar base, and depending on the disease the number of sprays can range from 2 to 10 per season per crop. The preventive strategy is successful in protecting yields and quality. Public concerns, however, about pesticide residues is forcing processors and growers to fine alternative control strategies to reduce the use of synthetic pesticides. Disease forecasting systems have shown to be successful in reducing fungicide applications. To help growers make the switch from calendar base fungicide applications to only when needed, Campbell Soup Company has adopted the TOMCAST forecasting system (R.E. Pitblado, 1992) for Anthracnose fruit rot, black mold, Altemaria leaf blight, and Septoria blight, and the PCM W.R. Steverson, 1993)/TOM-CAST system for late blight. In 1994 growing season, using these systems, fungicide applications to control Anthracnose fruit rot on processingtomatoes in Ohio was reduced from 9.0 applications/hectare to 5.5 applications/hectare (i.e., about $38.9 \%$ reduction). During the same period, celery growers in California who used the TOM-CAST system reduced their fungicide applications from 7.0 applications to zero (i.e., $100 \%$ reduction) for Septoria blight control. Similarly, processing-tomato growers used 1.2 fewer sprays per field to control black mold (about $36 \%$ reduction). Growers at Campbell's Sinalopasta, Mexico, operations who used the PCM/TOM-CAST system were able to reduce their fungicide applications to control late blight from 8.0 applications/hectare to 3.7 applications/hectare (i.e., 53.7\% reduction). Growers cost savings (application and fungicide costs) on 890 hectares of processing-tomatoes in Ohio was $\$ 258.8 /$ hectare, for Septoria blight control on 24 hectares total cost savings were $\$ 50,000$, and for late blight disease in Mexico on 2000 hectares the cost savings were $\$ 65,000$.

Bolkan, H.A., Campbell Research and Development, 28605 County Road 104, Davis, California, USA, 95616 
THE OSE OF WEATHER PARAIETERS TO REDUCE FUNGICIDE APPLICATIONS IN POTATO, ONIONS AND BRUSSEIS SPROUTS.

\section{E. Bouma}

Three weather based decision support systems in the Netherlands are based on weather data measured in the crop. Prophy for control of late blight (Phytophthora infestans) in potatoes, MYcos for control of Mycospherelle brassicicola in Brussels sprouts and BOTCAST/SIV for the control of Botrytis squamosa In onions. Prophy. MYCOS and BOTCAST/SIV all use a small automatical weather atation which is measuring weather data in the crop.

The development of almost all fung1 depends on the environmental circumstances in the crop. Especlally temperature, relative humidity (rh) and stances in the crop. Especlally temperature, relative humidity (rh) and influence of the temperature, th and the duration of the leafwetness on the development of the funat, is and the duration of the leafwetness on the development of the fungl, lt is possible to estimate the disease severity. The weather parameters have to be measured in the location where the fung develop, because most of the time these meteorological parameters diffe
from the meteorological parameters measured at official helght (1.50$2.00 \mathrm{~m})$. Especially when the canopy is closed, the differences are large. The temperature in the crop can differ $3-4^{\circ} \mathrm{C}$ compared to the temperatur at offclal helght. The relative humidity can differ up to 158 from the $r$ messured at official helght. Also the leafwetness period can differ a lot.

Prophy, calculates the advice for the next application date. Weather para meters (temperature, rh and precipitation) are used together with some other parameters (e.g. cv resistance, fungicide), in order to recommend a spraying interval. For a good advice Prophy also needs the three day weather forecast. This information comes from one of the two offictal weather services in the Netherlands. In field trials a reduction of the numbers of applications has been reached of $10 z$, compared with a seven-day sprayin scheme without having a greater risk of a late blight tuberinfection. About 150 farmers in the Netherlands use this system to control late blight. Prophy has been developed by Prolion and PAGV.

The applications to control M. brassicicola with the help of Mros, are based on temperature, rh and precipitation. The system takes into account periods with a high rh, calculates the dangerous periods and gives the farmer an advice when to spray. The system has been tested succesfully by a group of farmers in 1994 and will be commercially introduced in 1995. Whith the help of the systen a reduction of $50 \mathrm{z}$ of the fungicide applications has been realised. MYCOS has been developed by Prolion and PAGY.

BOTCAST/SIV. The Botcast-module can be used to supervise the timing of the first spray, while the SIV-module is suitable to time the successive sprays. The models were developed in Canada and the us and validated for Dutch circumstances. With the use of the models a 57 in reduction of the fungicide applications was realised compared to weekly spraying progran and did not lead to a yield depression. The system is used by s5 farmers who are organised in user-groups.

Research Station for Arable Farming and Pield Production of Vegetables (PAGV), P.O. Box 430, B200 AX Lelystad, the Netherlands

analysis OF PREVAlent trend of Plant DISEASE By genetic PROGRAMMING

Y. Cai

Prevalent trend of dwarf virus of late rice was analyzed by genetic programming. The success rate reaches rather high. The results show that the genetic programming method is good, and therefore it might be referred as an effective assistant technique for the forecast of plant disease.

Keywords: dwarf virus, Genetic Programming

Shanghai Research Center of Technology, Chinese Academy of Sciences, Shanghai, 200233, Chin
THE INFLUENCE OF TTETHERCONDITIONS ON CHOICE AND TIMING OF PESTICIDES

E. Botung

The efficacy of agrochemicals against pests, diseases and weeds depends very much on weather parameters. Knowledge of the reaction of pesticides to very inefficient control. Formutiont Inefficient contror. layer at the upperside of the leaf are important for the speed and way of uptake of the active ingredient by the leaves. The thickness of these waxlayers is influenced by meteorological parameters. It is important to know how weather parameters measured in the crop are related with the measure-
ments on official height $(1.5-2 \mathrm{~m})$. Pesticide formulations can be divided in two groups:-apolar (water-repellent) - polar (fat-repellent). The spolar group of the products diffuses easily through the waxlayer because they are able to solve the fatty and waxy matter quite weIl. The degree of dissolution depends on the temperature. Apolar substances are rainfast very quick and are less dependent on weather conditions.

The diffusion of the polar products is much more difficult. The absorption takes place through small pores in the waxlayer and the cutin structure in the cuticle. It is a process of diffusion and can only take place under sufficiently moist conditions. The absorption of polar products is slower compared with the apolar and often a longer period of humid circumstances is necessary for absorption.

Another classification of crop protection agents with regard to weather parameters is according to their biological activity: Funglcides, most contact fungicides need dry, sunny weather after application, the product can dry quickly on the leaf. Rain after application shortens the period of efficacy, showers with a high intensity have wore effect than rain periods with a low intensity. In case of systeric fungicides, the temperature and relative humldity are more important for effective action. Temperatures between $15^{\circ}$ and $22^{\circ} \mathrm{C}$ and a relative humidity $>/ 80$ ensure rapid results. With herbicides it is important to discriminate between those weather con. ditions that have an effect on absorption fter application and those that ditions that have an effect on absorption after application and those that have an effect on the mode of action in the plants. With regard to appli. cation, the herbicldes may be divided into soil and contact herbicides. Soll herbicides have to be applied on motst soil and should be followed by a short perlod of dry weather. After that, rain is necessary for the product to thoroughly penetrate the soil. In the case of contact herbicides, the nature of the formulation has an important effect on absorption. The Emulsified Concentrates (BC) are not very sensitive to the weather, while the others (polar) are. Optimum weather conditions after application can follow from the mode of action of the herbicide and/or any selective action.

Insecticides are very sensitive to weather conditions, particularly to rain and radiation. Rain within one hour of application washes off approximately and radiation. Rain within one hour of application washes off approximately 50 of the active ingredient. The apolar formulations are less vulnerable in this respect than other cypes of formulations. There is a large diffe-
rence in temperature sensitivity between the different types of insecti. rence

Research Station for Arable Parming and Field Production of Vegetables (PAGV), P.O. Box 430, 8200 AR Lelystad, the Netherlands.

928

APPLICATION OF ARTIFICIAL NEURAL NETWORK ON ANALYSIS OF THE PREVAILING SYSTEM OF Botrytis fabae

Y. Cai

The analysis of the prevailing level of Botrytis fabae by artificial neural network was presented in this paper. And the prevailing system of $B$. fabae of Sheyang County from 1973-1984 were collected as an object of study. The successful rate reached $100 \%$.

The results showed that the performance of the neural network approach is good, and therefore it might be referred to as an effective assistant technique for the prevailing level of plant disease.

Keywords: Botrytis fabae, Prevailing Level, Self-Organization Artificial Neural Network, T. Kohonen Model of 3-D

Shanghai Research Center of Technology, Chinese Academy of Sciences, Shanghai, 200233, China 
THE APPLICATION OF ARTIFICIAL NEURAL NETWORK IN EPIDEMIC TREND OF WHEAT POWDERY MUDEW

Y. Cai

In this paper, the back-propagation model, which is one of the typical artificial neural network models, is used to establish a predicting model for the medium-short term (60 - 90 days) prediction of wheat powdery mildew, the success rate of which reaches $100 \%$. The results showed that the neural network approach performs well and it might be an effective assistant tool in the prediction of epidemic trend for plants.

Keywords: Wheat Powdery Mildew, Predicting Model, Artificial Neural Network, Back-propagation Model

Shanghai Research Center of Technology, Chinese Academy of Sciences, Shanghai, 200233, China

931

THE APPLICATION OF GENETIC ALGORITHM IN DISCRIMINATING DYNAMTC MODELS OF WHEAT POWDERY MILEW DEVELOPMENT

Y. Cai

Genetic algorithm is used to discriminate the parameters of dynamic model of wheat powdery mildew development (S-curve; $y=k /\left(1+a^{*} \exp \left(-b^{*} t\right)\right)$ ). By comparison with the calculating result in document 1 , the result shows that it operates wel and is expected to be widely used in analyzing various dynamic models of plant protection and plant diseases.

Keywords: Wheat Powdery Mildew, Dynamic Model, Genetic Algorithm

Shanghai Research Center of Technology, Chinese Academy of Sciences, Shanghai, 200233, Ching
THE GENETIC PROGRAMMING ON FORECASTING Gibberella zeae of WHEAT

Y. Cai

The genetic programming to forecast the wheat disease caused by Gibberella zeae was presented in this paper, and a group of samples was collected to study. The success reached was rather high. The results show that the performance of the approach is good, and therefore it might be referred as an effective assistant technique for forecasting plant diseases.

Keywords: Genetic Programming, Gibberella zeae on wheat

Shanghai Research Center of Technology, Chinese Academy of Seiences, Shanghai, 200233, Ching

932

ECOLOGICAL AND MATHEMATICAL GROUNDING TO OPTIMIZE APPLICATION OF BIOLOGICAL CONTROL MEANS (ON THE EXAMPLE OF PHEROMONES AND TRICHOGRAMMA SPP.)

v. Catana Principal possibility to increase effectiveness of biological
control means have been proved by mathematical modeling. Developed ecological and mathematical models have been used to optimize existing components of application technologies for biological control means. They include the following sequence in biological control means. They include the following sequence in brassicae L.: monitoring and forecast of pest number dynamics by means of pheromone traps and its suppression (of necessary) with the egg parasite Trichogramma spp.

Generalized conceptual model has been developed for relationship with pheromone traps with natural pest population. The model allows to optimize strategy for trap application depending on values of studied parameters characterizing certain population. Conceptual analytical model has been proposed for relationship between two insect populations according to the type "parasite host" the effect of lateness is the main element. It gives possibility to develop optimal tactics for preparation and number of releases of Trichograma spp. (of different age) in the field.

Conceptual models and experimental data on application of pheromones traps and Trichogramma spp. against Mamestra brassicae in cabbage agrocenoses served as the base for developing regression models for short term forecast of pest egg number and expected effectiveness of Trichogramma spp. effectiveness depending on phytosanitary situation and quality of applied biomaterlal. Regression models have been built which allow to develop optimal schemes for mass rearing of Trichograma spp. with different age. It provides reliable effectiveness of Trichograma spp. and release number reduction.

Dr.Vasile D. Catana

Head of Laboratory of Forecasting, Institute of Biological Plant Protection

P. Box 427

MI 277049 Kishinev, Moldova

Phone (3732) 57-04-29; Fax (3732) 57-96-41 
DEVELOPMENT OF A COMPUTER BASED IPM PROGRAM FOR PESTS OF CULTIVATED MUSHROOMS

\section{A.D. Clift and M.A. Terras}

A program shell, INFORMER*, has been developed that has an inference engine and support for text, graphics and external simulation models. We have used this shell to package information on pest identification, problem solving and pest management in cultivated mushrooms in Australia.

Initially there are a series of text/graphics to scroll through to determine the general problem. Once this has been selected, the procedure is repeated to better define the information required. At this second level, the selection leads to the detailed information required, featuring text, graphics and the output from a simulation model.

The program is Windows ${ }^{*}$ based and is available as an EXE file run through Windows. The basic material included is easily updated and new modules added.

This version includes information on sciarids, phorids and the impact of farm management including chemical use, physical exciusion and presence of weed moulds.

Biological and Chemical Research institute, PMB 10, Rydalmere, NSW 2116, Australia.

935

THE DECISION-SUPPORT SYSTEM PRO_PLANT FOR CEREAL DISEASE CONTROL: SCIENTIFIC BACKGROUND AND PRACTICAL EXPERIENCES

J.Frahm, Th. Volk and U. Streit

The task of the knowledge-based advisory system PRO_PLANT is to help farmers to reduce the input of pesticides to a minimum while giving them economic returns as good or aven better as with high-input routine sprays with their potential environmental hazards (e.g. ground and surface water contamination). At first, the expert system was developed for cereal diseases and was extended to pests in rape. weeds in maize, diseases in sugarbeet and growth regulators in cereals in 1994. It can be used by censultants and farmers as a stend-alone varsion.

The scientific basis of the system for cereal disease control is a control strategy that is relatad to the amount of disease and to the intection probabilities. The probabilities of infection are calculated from topical meteorological data. Besides, available fungicides are described in detail (e.g. their curative efficiency). Additionally, PRO_PLANT considers information about cultivar resistance, growth stage of the crop and nitrogen manuring. Thus, the right ingredient should be applied in the right quantity and at the right time. The knowledge has been derived from experts and from field trials over more than 10 years. Necessary weather data come from the "Deutscher Wetterdienst" (the official meteorological service in Germany) via videotex system or from a small home-run meteorological station.

Official advices for plant protection in North Rhine Westphalia are given with the aid of the PRO PLANT-system from 1992 on. Since 1993 the program is distributed commercially all over Germany, at first in a version for MS-DOS, now running under MS-Windows. It is used by official and private advisory offices as well as by farmers. Some licenses have been installed in other eurcpean countries. The experiences made in the last years show, thet farmers can educe fungicideinput elmost by $30 \%$, either by reduction of treatments or dosage reduction. Even with little training, tarmers can use the system for managing cereal disease control. The department of agricultural informatics at the University of Münster has realized PRO_PLANT in cooperation with the Institute for Plant Protection. The project is financially supported by the Ministry for Environment, Regional Planning and Agriculture of North Rhine Westphalia.

Institute for Plant Protection, Seed Testing and Apicultural Research, Nevinghoff 40, 48147 Münster, Germany
DEW FORMATION, DEW DRYING, LEAF WETNESS DURATION AND THE EFFECT OF THE FLOW REGIMES ON THESE PROCESSES.

\section{R.M.M.El-kilani, A.F.G Jacooss and A. Van Pul.}

ABSTRACT. The processes of dew formation and dew drying control the duration of leaf wetness af different heights within plant canopies. The leaf wetness duration period and the climatic conditions (temperature, humidity) during the night and the early morning hours within the canopy control fungal disease development. The correet definition of these environmental conditions, combined with the use of fungal distase spores environmental requirements, could be used to predict the probability of fungal disease breakout.

The coupling of the air flow regime within plant canopies to the flow regime above controls all the above mertioned processes. A detailed canopy climate model which considers these processes is used to predict the effect of the coupling of the flow regirne within plant canopies to the flow above, during night time and early morning hours, on the leaf wetness duration.

The model is compared against a set of collected measurements and seems promising. The model is used to study the effect of the above mentioned processes and their parameterizations on the probability of disease occurrence. We try to simplify the prediction procedures.

Department of Meteorology, Agricultural University P. O. Box 9101 , NL 6700 HB Wageningen, The Netherlands.

936

\section{NEURAL NETWORK MODELS OF WHEAT LEAF WETNESS}

\section{J. Francl, S. Panigrahi, and T. Padhi.}

Most bacterial and fungal pathogens require specific minimal periods of free moisture on plant surfaces for infection and reproduction. Also, disease severity is frequently worsened when wetness duration is extended past the minimum length. Thus, disease forecasting models should measure directly or be able to predict free moisture events and their duration. Neural network modeling is a method of pattern analysis based on a parallel distributed information processing structure containing highly interconnected processing elements or nodes Neural network models have been used to optimize patten matching for classification, process control, and prediction. Flag leaf wetness of wheat sown at three planting densities (45, 90, and 135 $\mathrm{kg} / \mathrm{ha}$ ) was etrulated by replicated flat plate resistance sensors placed in the crop at flag leaf height. Three neural network models of free moisture status were developed by learing the pattem of sensor output for 1,728 half-hourty readings of environmental variables. A full-input set model (FIS) had as input typical automated weather station data, as well as wetness data from a sensor placed above the crop, maximum wind gust, and temperature and relative humidity within the crop. A reduced-input set model (RIS) used the weather station data and the above-crop wetness sensor data. A minimum-input set model (MIS) was based solely on information commonly available from automatic weather stations. For each model, a backpropagation architecture produced optimal models by trial-and-error selection of parameters. Neural network models were validated on 298 half-hourly environmental readings from six arbitrarily chosen time periods not included in the training. Linear regression showed the models explained from 42 to $79 \%$ of the variation in wetness readings. Much of the model error was related to underprediction of high resistance values (dryness) and error was lowest between midnight and $0700 \mathrm{hr}$. The accuracy of model prediction for wetness duration depended on the threshold value between wet and dry states. Averaged across three thresholds (200,600, and 1000 kohms resistance) and three planting densities, the FIS, RIS, and MIS models had mean absolute errors of $1.2,1.3$, and $1.7 \mathrm{hr}$, respectively, and were within $2 \mathrm{hr}$ of the actual wetness duration $79 \%, 79 \%$, and $72 \%$ of the time. Similarly, the FIS, RIS, and MIS models accurately predicted the free moisture status in $89 \%, 88 \%$, and $83 \%$ of the 298 cases, respectively. Results compare favorably with previously published deterministic models of leaf wetness duration. The anount of error in the MIS model is probably tolerable for forecasting fobar diseases of wheat bot ite higher error level of MIS ys. FiS and RIS models indicates the importance of hoving wetness dals as input. Further improvement in model accuracy will depend on adding more observations and locations for model training, testing neural network architectures other than backpropagation, and using time series analysis.

Department of Plant Pathology. North Dakota State University, Fargo, ND 58105-5012 USA 
TURBO TOM-CAST: USER-FRIENDLY SOFTWARE TO RUN THE TOM-CAST DISEASE WARNING SYSTEM FOR TOMATOES

\section{R. A. Erickson, M.L. Gleason, R.E. Pitblado}

Turbo TOM-CAST, a computerized software program to operate the TOM-CAST disease warning system for field grown tomatoes (developed by R.E. Pitblado, Ridgetown College, Ridgetown, Ontario, Canada), was devised to help make TOM-CAST readily accessible to users possessing any level of computer expertise. TOM-CAST uses weather data (duration of wetness periods and average air temperature during these periods) to assess the risk of crop loss caused by early blight (pathogen: Alternaria solani), Septoria leaf spot (Septonia lycopersici), and anthracnose fruit rot (Colletotrichum coccodes) and to recommend timing of fungicide sprays. TOM-CASI assigns daily numerical ratings of disease risk called Disease Severity Values (DSV's) and recommends applying a fungicide spray when the sum of DSV's since the previous spray equals or exceeds a predetermined threshold. The primary functions of the Turbo TOMCAST software are management of collected weather data and computation of DSV's based on that data. Weather data can be input manually or from datalogger files. The software enables the user to access both daily and cumulative summaries of collected weather data and DSV's computed from this data. The summaries are provided in the form of a report that includes the total number of wet periods, the duration of each wet period, the average temperature during a wet period, DSV's computed for each wet period, daily DSV total, cumulative DSV's since the previous fungicide spray, and cumulative DSV's for the entire monitoring period. Turbo TOMCAST can also be used to notify the user automatically when preset thresholds are reached, signaling that a fungicide spray is recommended. The program is designed to operate on IBMcompatible microcomputers which use the MS-DOS operating system. Copies of the software and written documentation are available for \$25 U.S. from M.L. Gleason at the address below.

Department of Plant Pathology, 351 Bessey Hall, Iowa State University, Ames, Iowa 50011 , U.S.A (electronic mail: mgleason@iastate.edu)
OPTIMAL BIOLOGICAL CONTROL OF TWO-SPOTTED SPIDER MITE THROUGH STOCHASTIC SIMULATION

\section{J. Horn, D. K. Pearl, and R. Bartoszynski}

We have developed a detailed stochastic predator-prey model that dynamically mimics the complexities of environmental realities. The model follows eggs, nymphs and adults of both predator and prey populations along with their level of chemical resistance, the growth of the plants under attack, and the temperature of the surrounding greenhouse environment. We have developed this model using the interaction between fetranychus uricae, (the two-spotted spider mite of 2SSM) and its predator Phyloseiulus persimilis (PPE) in a greenhouse cucumber system in order to develop, and apply to cucumbers grown in a state-of-the-art greenhouse, the general methodology of finding the optimal strategy for biological control of 2SSM by the release of PPE. The stochastic model has been programmed to produce a fast, extremely realistic simulation model of the development and interaction of the populations of prey and predators, including the damage to the crop done by $2 S S M$. We are conducting experiments in which a suggested strategy is compared with other selected strategies, in order to iltustrate the correspondence between simulation and the reality. An essential component of this is refinement of the stochastic model to incorporate the results of our experiments moving from single plant to multiple plant systems and from Lima beans to cucumbers. The technology developed is being transferred to a practical production situation involving cucumbers in a commercial sized greenhouse.

Department of Entomology, The Ohio State University, 1735 Neil Ave., Columbus, OH 43210, USA
939

NEW WAYS IN CONTROLLING OIL SEED RAPE PESTS WTTH THE AID OF THE DECISION SUPPORT SYSTEM PRO_PLANT

A. Johnen, J. Frahm and M. Gruenewald

The computer- aided decision support system Pro Plant, originally developed for controlling cereal diseases, has been extended for managing pests in winter rape seeds. Considering not only the amount of adult beetles as monitored by yeilow traps it also uses weather data for prognosis of flight conditions, egg laying periods and larval development. The decision support system has been validated for several years in the different growing regions (rotations, sowing dates and height above sea level) in Westfalen-Lippe. It has been introduced to farmers and consultants in 1994.

In autumn mainly the cabbage stem flea bettle (Psylliodes chrysocephata) is considered. The amount of the beetles colonising the oil seed rape crop must by controlled after first emergence with the aid of yellow traps. Since the larvae are most damaging, the system calculates the egg laying period of the beetles and the appearance of the first larvae $\left(200^{\circ} \mathrm{C}\right.$ degree-days-sum of daily mean temperature above $4{ }^{\circ} \mathrm{C}$ starting from the beginning of the egg laying period). Depending on site and season the spraying of pyrethroid insecticides can be delayed in autumn or under special conditions in early spring. Spring treatments can control the first arriving cabbage stem weevils (Ceutorhynchus quadridens) too.

In early spring cabbage stem weevils ( $C$. quadridens) are the first arrivers followed by blossom beetles (Meligethes aeneus), seed weevils (Ceutorhynchus assimilis) and pod midges (Dasineura brassicae) with temperatures above $15^{\circ} \mathrm{C}$ in late april. Rape stem weevils (Ceutorhynchus napi) are not present in the growing area.

Egg laying of the cabbage stem weevils ( $C$. quadridens) mainly depends on temperature and hours of daily sunshine. Therefore it often starts several days or even weeks after arriving of the adult weevils in the fields. In calculating the possible beginning of egg laying the system gives advice in delaying spring applications of pyrethroids. The consideration of the conditions for egg production makes it possible to control cabbage stem weevils ( $C$. quadridens) together with the main migration of blossom beetles (M. aeneus) and the begioning of pod weevil ( $C$. assimilis) migration. In many years the main pests can be controlled with one spray at all and a good efficacy.

The main part of the program is the phenological model using weather data for calculating several biological processes (flight conditions for migration of beetles, egg laying periods, hatch of larvae). The data are presented in a graphic. Several other useful data (growing conditions of the crop. data about pesticide application and performance, field expectations) are processed too. The 1995 -Version runs under MS-Windows.

Institut for Agricultural Informatics, University of Muenster, Kleimannstr. 5, 48149 Muenster
940

NEW COMPUTERIZED FORECASTING SYSTEM (BLIGHTAS) AND ESTIMATION OF YIELD LOSS FOR RICE SHEATH BLIGHT DISEASE

\section{T. Kobayashi, T. Ijiri and T. Hashiba}

New computerized system(BLIGHTAS) for forecasting the development of rice sheath blight disease was based on the relative height of the uppermost lesions to the plant height(X, vertical development of the disease) and the percentage of the diseased hills(A, horizontal development of the disease). The $X$ value was calculated from the daily average temperature, relative humidity and susceptibility of the leaf sheath. On the other hand, the A value was calculated the daily average temperature, relative humidity and the number of the sclerotia per 10 ares in paddy field. In this system, the yield loss of fully ripened kernels in $\mathrm{kg}$ per 10 ares $(\mathrm{L})$ is expressed as follows: $L=(41.3 \mathrm{X}-826.2) \mathrm{A} / 1,000$. BLIGHTAS is consisted of three major program, analytical program of diurnal variation of temperature and relative humidity, analytical program of annual variation of temperature and relative humidity and forecasting program for sheath blight disease. Each program was constructed with about 30 subroutines. The total number of steps of the program amounts to about 5,000. BLIGHTAS is developed for estimating the expected yield loss of two months later. It has been confirmed that the yield loss predicted by BLIGHTAS was very close to the actual yield loss.

Faculty of Agriculture, Tohoku University, Sendai 981, Japan. 


\section{K. P. Koutsenogi}

The report gives a new conception of the estimation of the efficiency of pesticide application. The conception is based on the investigation of the processes of wind turbulent diffusion of aerosols in the atmospheric surface layer, of the hitting of aerosols at the targets and on the physical and mathematical computer simulations of the above processes

Experimental and theoretical data are presented on the influence of aerosol sizes on the efficiency of aerosol hitting on pest insect bodies and on plant leaves as well as on influence of aerosol size distribution. of meteophysical parameters of the atmospheric surface layer of the kinds of plants and the landscape, etc, on the aerosol diffusion along the treated field. It is proved that for each concrete situation there are such aerosol sizes when the amount of aerosol pesticide substance hitting just on insect body will be maximum. These aerosol sizes are called the optimum

The field and laboratory studies have been performed to investigate the dependencies of the optimal sizes on the kinds of agricultural and forest plants and pest insects, on the meteorological and landscape conditions, on the width of treated fields, etc.

A few mathematical computer models were proposed to calculate the optimal aerosol diameters, the minimum of the application rates of pesticides. etc. The special aerosol technics for pesticide aerosol treatments have been designed, produced and tested on the area of a few hundred thousands of hectares in the 80-5 and 90-5 in the different regions of the former USSR.

The given technics and so-called the Optimum Aerosol Technology have some advantageous over the aerial and ground sprayings:

1. A 2 - 10-times reduction of specific application rate of pesticides

2. A $10-30$-times decrease of the pesticide residues in vegetation and soil.

3. A 10 - 100 -times increase of the working rates of field treatments.

Institute of Chemical Kinetics and Combustion, Siberian Branch of the Russian Academy of Sciences, 630090, Novosibirsk-90, Russia

943

INTEGRAIED SYSTENS FOR DECISION SUPPORT NN PLANT PRODUCTION IN NORWAY

\section{i. A. Magans}

The developreat of new information technology has paved the way for dramatic changes in information delivery in plant production. Since 1988 a national project conapsised of several plant protection theme thas been in operation in Norway. The main objective is to tume the use of pesticide prot to a che

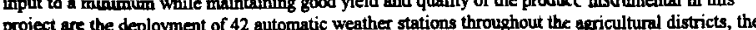
project are the deployment of 42 automatic weather stations throughout the agricultural districts, the
co-operation of some 700 farmers scouting their own crops and some 100 farmer-owned research rings

belping rescarch-based models operate at a high level of automatisation.

Weather data also include two and five-day prognoses updated four times a day. Information on weather atd specific warnings in plant protection are disseminated by means of an electronic bulletin board (BBS). A voice bcard system linking the telephone with huge expert systems provides decision support to any user day and night. In 1994, 14000 calls were registered on the BBS and some 7000 calls were registered on the voice board Both systerns are drawing on information in shared databases updated by several specific modules, plant protection in cereals, potato, fruit and vegetables. Irrigation scheduling in severol crops is included in the system.

Nonwegian Crop Research Institute, Plant Protection, N-1432 As, Norway
GROWN ROSE SEEDLINGS.

\section{A.J. van Kuik}

Powdery mildew on roses caused by Sphaerotheca pannosa var. rosae is a major problem in raising outdoor rose seedlings. Control by weekly applications of fungicides is the conventional method. Present ecological concerns about frequent sprays of fungicides are serious. Powdery mildew development is strongly correlated with weather conditions.

To reduce the number of applications a computerized forecasting model has been generated to identify periods when environmental conditions are favorable for powdery mildew development. The model was based on the following environmental parameters: temperature, leaf-wetness, relative air humidity. Data were registrated houxly by an automated weather station. The forecasting model used the data to calculate the length of the latency period at that time. The strategy of the model was to spray with a fungicide just before a latency penod was passed. A new latency period started after each spray In field trials from 1991 to 1994 the model strategy was tested in the cultivation of rose seedlings. Disease severity data from plots subjected to model strategy were compared with nonsprayed checks and with weekly sprays that started 4 to 6 weeks after sowing. There were no differences between the model strategy and the weekly schedule, with regard to disease severity during the season and the final growth of the rose plant as measured by the stem diameter. The computerized forecast model reduced fungicide sprays by 30 to 50 percent and achieved the same level of control as the weekly schedule.

Research Station for Nursery Stock, P.O. Box 118, 2770 AC Boskoop, the Netherlands.

944

OPTIMUM AEROSOL TECHNOLOGIES OF INSECTICIDE APPLICATION IN PLANT PROTECTION

E.I.Kirov, VI.Makarov, Y.N.Samsonov, A.A.Alekseev, O.V.Chankina, N.N.Gorbunov, I.B. Knorr

The experimental results on the efficiency of aerosol technologies of the application of insecticides against Loxostege sticticalis $L$. and for the protection of the coreal crops from some pest insects are given. The experiments were carried out in North Kazaknstan and West Siberia from 1980 to 1987 . More than 40 experiments were performed over the area West Siberia from 1980 to 1987 . More than 40 experiments were performed over the area
of about 150,000 ha. The crop area were treated by a special generator with controllable of about 150,000 ha. The crop area were treated by a special generator with controllable
dispersion of aerosols (GCD). The generator can be used to obtain insecticide zerosols with optimum droplet sizes varying from 1-2 to $25-30 \mu$ (optimum for each concrete situation) The main unit of the generator is a aerial gas-turbine compressor put on a truck. Aerosol technologies can be used against the following species of pest insects: Sitobion avenae F Schizaphis graminum Rond Macrosteles laevis Rib. Psammotettix stritus Haplothrips tritici Haplothips thitici $\mathrm{K}$ anceps Schiff. Oscinella pusilla Mg. Special aerosol technologies have been worked out against Loxostege sticticalis $\mathrm{L}$. both the larva and the imago. The following insecticide commercial formulations were used: methyl parathion $50 \%$ and $40 \%$ emulsiable concentrates (e.c.); fenitrothion (metathion), $50 \%$ e.c.; phoxim, $50 \%$ e.c. (volaton) and $90 \%$ solution; phosalon, $35 \%$ c.e. cypermethrin ("Cymbush", $25 \%$ and "Ripcord", $40 \%$ c.e.) fenvaierate ("Sumicidin"), $20 \%$ c.e.; permethrin ("Rovicurt"), 25\% ce. All experiments were carried out at nights wien the so-called stable stratification of surface atmospheric layer (air temperature inversion) appears and aerosol cloud is near the ground. Finally. we (air temperature inversion) appears and aerosol cloud is near the ground. Finally, we 1).

Table 1. Main technological characteristics of the different methods of insecticide application.

\begin{tabular}{|c|c|c|c|}
\hline $\begin{array}{c}\text { Technological } \\
\text { characteristics }\end{array}$ & Aerosol VCD & Aerial spraying & Ground spraying \\
\hline Particle size, $\mu$ & $1-15$ & $200-300$ & $200-300$ \\
\hline Efficient range, $\mathrm{km}$ & $0.5-3.0$ & 0.040 & 0.015 \\
\hline Treatment rate, ha/day & $600-2000$ & below 550 & Delow 100 \\
\hline Dose of OP, $\mathrm{kg} / \mathrm{ha}$ & $0.07-0.15$ & $0.4-0.7$ & $0.4-0.7$ \\
\hline Dose of fuel, itha & $0.15-0.32$ & $1.0-1.5$ & $0.5 \cdot 1.0$ \\
\hline
\end{tabular}

The results are clear enough and need no additional remarks. In aerosol technologies with optimum dispersion compared with spraying, the main technological parameters of insecticide application were improved 34 times and more.

Institute of Chemical Kinetics and Combustion, Siberian Branch of Russian Academy of Sciences, Institutskaya 3 Novosibirst, 630090, Russia. 
C. J Meir

Lack of or inadequate training is often cited as one of the most important constraints to the implementation of integrated pest management (IPM) by smaliholder farmers. Non-formal, participatory education techniques have been shown to be more effective than traditional teaching in helping adults learn, both with regard to the design and the execution of training. But how can you judg whether or not a training course really was participatory? This paper discusses various analyticai techniques which we have used to evaluate how participatory IPM training courses are in reality and to indicate how farmer participation might be maximised. I developed these techniques as part of a study of training courses in insect and disease management for Smanduras and other Central American institutions. I use a combination of objective and subjective analyses. The objective
analyses involve the use of different graphs. One graph provides an instant analyses involve the use of different graphs. One graph provides an instant
visual overview of the variety and participative nature of the different activities which took olace during the course. Others show to what extent the activities which took olace during the course. Others show to what extent the farmers participated individually and as a group, and what sort of effort the
trainer was making to encourage their participation. Time analyses show us on what themes we spent most of the training time. The subjective analyses on what themes we spent most of the training time. The subjective analyses
deal qualitatively with the objectives and planning of the training, the deal qualitatively with the objectives and planning of the training, the presentation of the concepts necessary to understand the IPM technologis
discussed, the style of training, the vocabulary and the materials used.

The use of these analytical techniques has aroused considerable interest both from trainers and from the farmers they train. They have helped us think from trainers and from the farmers they train. They have helped us think about participation in practical terms and have encouraged us to structure our
courses to involve the farmers we train more, both as a group and courses to involve the farmers we train more, both as a group and
individually. In this way we feel we are maximising our overall impact as well as the impact in each community to which the individua) farmers (change as the inpact in each community to which the individua) farmers (change agents) will return with their IPM messages. Our analyses have also helped us to focus our activities more closely on meeting farmers' noeds and to balance the time allocated to the different themes we wish to discuss. Experience has shown us that the attitude and skills of the trainer are probably the factors which most influence the impact of our courses on the farmers we train. The analytical techniques we use have holped individua? weaknesses and have encouraged them to share thei strengths with others.

We try to analyze our training on a daily basis to allow us to incorporate changes quickly and respond to the needs of the farmers we train. We feel these techniques have made us more aware of what farmer participation in IPM training really means and of how to achieve it. And from our experience, more farmer participation in IPM training means more farmers putting IPM into practice in the field.

Hillsides IPM Programme, Department of Crop Protection, Zamorano, (Panamerican School of Agriculture), PO Box 93, Tegucigalpa, Honduras, Central America, and Imperial College Centre for Environmental Technology, 48 Prince's Gardens. South Kensington, London, SW7 2PE, United Kingdom.

947

VIRTUAL PLANTS: SIMULATING 3-D GROWTH AND OTHER RESPONSES OF PLANTS TO DAMAGE

Peter M. Room and Jim S. Hanan

Computers are becoming powerful enough to allow biologists to investigate the complex, three-dimensional (3-D), dynamics of growth by individual plants.

Plants are modular organisms adapted for gathering diffuse resources from 3-D space and plant architecture (3-D structure) plays a key role in determining yield and competitive ability. The architecture observed is the result of earlier addition and loss of modular parts in particular positions. The value of a part to a plant, the likelihood that it will be damaged and the plant's response to damage are strongly affected by the geometrical and topological relationships of the part to the rest of plant. Despite such significance, the spatial dynamics of plant parts remain largely unexplored due to the difficulty of collecting and manipulating data. Now, severa! types of 3-D digitisers are available and there is software which uses growth rules derived from digitiser measurements to create "vinual plants." Virtual plants are computer abstractions containing sufficient 3-D information on growth for realistic images of successive growth stages to be generated.

The value of virtual plants lies in the information they contain on the numbers, shapes, sizes, positions and orientations of parts and in the way they make explicit the relationship between a set of growth rules and the architectures which emerge from them. Virtual plants are tools for gaining understanding at a level intermediate berween the cellular level of genes and physiology and the stand level of biomass. Virtual experiments can be performed by changing growt th rules to explore numerous scenarios prior to designing real experiments. As well as numerical output, the simulations can produce static pictures or animatjons of plant architecture which are either schematic or realistic. These images will be very useful in agricultural education and in decision-making by farmers, agronotnists and pest managers.

In management of pests and pathogens, it is anticipated that virtual plants will be used to improve the definition of action thresholds through simulation of interactions between plant architecture, pesticide deposition, canopy microclimate, insect and pathogen movement and damage, and plant compensatory growth. In biological control of weeds, rules may emerge for which weed architectures and types of herbivore or pathogen damage lead to effective control when brought together. Virtual experiments will also indicate new ideotypes for plant breeders, improved agronomic and pruning strategies for field and horticultural crops and plantation trees, and information on the $3-D$ distribution of biomass useful in fire and remote-sensing research.

The use of sonic digitisers to measure the 3-D structure of plants and the expresssion of growth niles as computer comands are described. The results of manipulating virtual plants in virtual experiments will be illustrated.

CSIRO Division of Entomology and Cooperative Research Centre for Tropical Pest Management, P.B.No. 3, Indooroopilly, 4068, Australia.Email: room@ctpm.uq.oz.au

\section{8}

PROPHY: A COAPUTERISED EXPERTISYSTEM FOR CONTROL OF LATE BLIGET IN POTATOES IN THE RETEIRRLANDS.

\section{E.T.A.M. Schepera, W. Hugreren}

In order to effectively control Phytophthore Infestans in potatoes wh a minimum input of fungicides, decisions have to be based on information of a number of sopects. A computerised expertaystem (Prophy), developed by Prolion and PAGV, can support dec181ons of farmers by integrating all avallable knowledge on relevant aspects for control of $P$. Infestans. In the Dutch veraion of Prophy the first treatment is malniy triggered by the development of the crop and the varietal resiatance. This 18 when a seagitive variety has reached a height of $15 \mathrm{~cm}$. In a wore resiatent variety spraylag starta 10 days latex. When late blight is observed in che region it is recomended to spray earlier.

The follow-up treatments are scheduled by combining the protection status of a crop with the weatber conditions. It 18 agawed that a sensitive potato cy 18 protected for 8 days after opraying with a protectant fungicide. In more realotent cr's this period to extended witb 1,2 or 3 days, depending on the degree of resiatance. Several parametera cas influence the duration of this period. Pirat of all the applicarion of a reduced doane can ghorten the protection perlod. The degree with which agw lesves heve developed since the Inst trentmeat can lengthen or shorten the protection period whth one day. The wash-off effect of fungicides caused by rainshowers is integrated by shortening the period with 3 daya at most. Relative humidity (rb) and temperature have a large influence on the aporulation, germination and infection of $P$. Infestans. These parametera are measured in the crop. A day 1a considered favourable for the development of $P$. Infestans when in a periad from $8 \mathrm{pm}$ on the previous dey till 12 am on the day 1tgelf, a period of at least 6 hours with a high rh occurs, followed by at least 2 hours of lesfwetness or rain. The temperature range $188-25^{\circ} \mathrm{C}$. The number of favourable days in a perfod of 3 to 10 days ago shortens or extende the durstion of protection with 2 days at woot. Pavourable days of 1 and 2 days ago are not included because infections that mighe have occured can not yet contribute to the disesse pressure. Obaervations of viable Iegtons close to the site, overrule the effect of the calculaced favourable days. The "assimed" protection period for a cercain cv can only be shortened with 3 dage at wost. Prophy formulates a recovmendation on the basio of the calculated protection period and the wenther forecast. When for certals reasoss procection 18 no longer present fand the weacberconditiono are favourable for infection, a cyorandi-contalning fungleide is recomended. Irial results in 1993 and 1994 showed that Prophy effectively controlled late blight with leas oprayo when coxpared to 7 -day apraying scheme.

Research Station for Arable Farming and Field Production of Vegetable (PAGV) P.0.Bor 430, 8200 AR Lelyatad, The Netherlands Prolion Development, Rrome Spleringweg 289 B, 2141 BS $\nabla 1$ f fhuizen (KL) 
The use of PC-Plant Protection - a decision support system for crop PROTECTION - IN EDUCATION AND EXTENSION IN DENMARK.

Secher, Bo J.M.

The decision, in 1986, on an action plan to reduce pesticide use in Denmark by $50 \%$ did led to increased research in the potentials of reduced dosages. A decision support system developed by The Danish Institute of Plant and Soil Science and The Danish Agricultural Advisory Center (PC-Plant Protection) implements this research combined with a detailed use of threshold values to support decisions on treatment need, choice of pesticides and the appropriate dosage for the actual problem. The pest and di-sease model of the system has been commercially available since 1993. In 1994, 1100 licenses were in use at agricultural schools and with advisers and farmers. The system was evaluated by agricultural advisers in 1990, 1991 and 1993. Usability and use-fulness of the recommendation mode was judged to be user friendly and the model to be reliable and meeting the advisors requirements. The recommendation model for pest and disease control has been field validated since 1990. The validation in winter wheat (54 trials), spring barley ( 54 trials) and winter barley ( 36 trials) has shown that the model is able to handle the control of pests and diseases to a satisfactory level, without affecting the farmers' gross margins. The model was able to adjust pesticide use to large yearly variations and on average consumption of pesticides in the plots treated according to the model was well below the level in the reference plots and the commonly used strategies by danish farmers.

Danish institute of Plant and Soil Science

Lottenborgvei 2 , OK 2800 Lyngby. Fax: +4545872210

DIAGNOSIS - A NOVEI, MULTIMEDIA, COMPUTER-BASED APPROACH TO TRAINING CROP PROTECTION PRACTITIONERS

\section{T.M. Stewart, B. P. Blackshaw, S. Duncan, M. L. Dale,} M. P. Zalucki and G.A. Nonton

The muitimedia computer package DIAGNOSIS provides a training aid to crop protection students for pest and disease diagnosis. The program simulates field and laboratory scenarios, in which students must actively seek clues and interpret observations on the cause of plant problems. Output may consist of text, graphics and video. The software allows the simple construction of local scenarios by individual tutors. Once students have recorded their diagnosis, justification and recommendations for action, they receive an automatic de-briefing on their problemsolving approach. Student input is recorded to disk for later assessment by the tutor (Crop Protection: In Press)

T.M. Stewart G.A. Norton

Dept. of Plant Science Co-operative Research Centre for Tropical Pest Management Massey University Private Bag Palmerston North Geturnann Laboratories University of Queensland

New Zealand
Brisbane QLD 4072

Australia
CROP GROWTH BASED MODEL OF FOLIAR UPTAKE AND REDISTRIBUTION OF PESTICIDES

K.V.Novozhilov, T.M.Petrova, N.N.Semenova

Assessment of the potential effectiveness and safety of plant protection products requires in the quantitative analysis of their interactions with the enviromment and particularly with the treated crop. With this aim a simulation crop growth based model describing the behavior of foliar applied pesticides in treated leaves has been developed. The model assumes two pathways of pesticides uptake (through cuticle and through stomata), penetration in according with hypothesis of passive transport and first order transformation on/into leaves. The designing model is a compartmental research-oriented one. Consideration of the cuticle as separating compartment permits to estimate the accumulation of the pesticides into cuticle in dependence on its thickness and density as well as on the partition and diffusion coefficients. Within this framework the model is reduced to analytical expression permitting to estimate the influence of pesticides decompositions rates and water content in leaf tissue on degree of its penetration. The sensitivity of the pesticides redistribution in leaves was analyzed in regard to the factors affecting their penetration in two situations: (i) while treatment a cabbage long and short duration cultives (c.v. Nomer Pervy and Slava) with organophosphorous (malathion, diazinon, phoxim) and pyrethroid (zeta-cypermethrin) insecticides; (ii) while treatment the winter wheat (c.v. Bezostaya 1) growing under different water supply (boghara and irrigation) with organophosphorous (parathion - methyl, dimethoate) and pyrethroid (cypermethrin, fenvalerate) insecticides. In the case (i) it was found that increasing of the insecticides content in the leaves of a long duration cultive is caused primarily by decreasing the rate of the compound decomposition into a plant and in less extent by changing in the leaf morphology (e.g. cuticle thicikness, stomata frequency). In the case (ii) the changes in a leaf structure and permeability of the epidermal cells of the plants due to the different growing conditions have a major effect on the differences in the preparations redistribution. Thus developed simulation model integrates the effecta of the pesticides physico-chemical and treated crop morphological properties as well as growth conditions and could be used as foundation for evaluating toxicity some group of insecticides taking into account the specific peculiarities of phytophages and entomophages and their behavior during ontogenes.

All-Russian Institute for Plant Protection, (VIZR), 189620, shosse Podbelsky 3, st-Petersburg, Pushkin, Russia

DISSEMINATING IPM AMONG SMALL COTTON FARMERS IN INDIA

B.D. Adiman 1. N.M. Murkumbi

INTRODUCTION: With 11.6 million Hectares under cotton. India has the largest cotton-growing area in the world. Unfortunately there for plant protection leading to large-scale pesticide resistance and resurgence of pests. Since 1992 the first author has organised IPM farmers from sowing to the picking stage. Cultural. mechanical. farmers from sowing to the picking stage. Cultural, mect

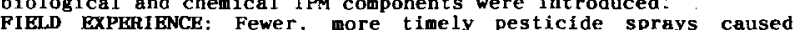
FIElD LxPEAENCL: Fewer, more timely pesticide sprays caused increase in population of predators (adult, grubs as well as egg masses). Movement of honey-bees in the adopted cotton fields was observed after an absence of many years. $21 \%$ of hellcoverpa armigera eggs were found parasitized after release of the egg parasite Trichogramma chilonis. Pheromone traps were used to monitor the above pest as well as the pink bollworm. The farmers were taught to use such traps as a "bio-clock" for choosing between different IPM tools. Sprays of NPV and the non-toxic repellant RAKSHAK were found to control bollworms effectively in the early stages without harming the natural enemies. These agents used in combination with pyrethrolds gave better and longer-lasting results than pyrethroid alone. Ladyfinger and marigold were planted along the barders as trap crops. Nipping of the terminal bud at 110 DAS reduced egg-laying of Helicoverpa armigera and gave easier access for spraying the lower section of the plants. Early sowing. weeding-out of alternate hosts of pests and judicious use of irrigation and fertilizers were other cultural components of IPM. RRSULTS: We have succeeded in cutting the usage of chemical pesticides by $50 x$ and also Increased the net yield, Results compared to non-IPM fields in same village are summarlsed belcw:

Increase in Yield \begin{tabular}{lrrr} 
Decrease in plant protection cost & 7 vs 12 & 6 vs 10 \\
\hline
\end{tabular} FRUSTRATIONS: Farmers were unable to identify pests. Ignorance of the biology of pests was an hindrance in monitoring and decisionmaking. This led to untimely and/or unnecessary spraying of pesticides. Some of the IPM tools were slow-acting so we also had to check that chemicals had not been sprayed in our absence. Polyphagous pests found year-round sustenance because of the different crops being cultivated in adjacent fields. Small size of holdings (average $2 \mathrm{Ha}$ ) hindered co-ordination and organization of large-scale efforts. The supply of various IPM tools such as predators, parasftes and pathogens was inadequate. Also some of these had a short shelf-life and stringent storage conditions which could not always be adhered to. Finally, the lack of personne! trained in IPM methods meant that only one team is available to disseminate IPM over a cotton-growing area exceeding $50.000 \mathrm{Hg}$

B.D. Adimani

Assistant Director of Agriculture (Plant Protection)

office of the Principal Agricultural officer

Shivali Nagar. Belgaum. 590016 INDIA 
INTEGATED COMTROL OF ORYCTES MONOCEROS ON DATE PALNS IN NIGERIA.

(1) trapping studies.

By C.I. AISAGEONHI

ASTACT

Four plastic bucket trops containing $2 \mathrm{ml}$. Ethyl chrysanthemumate and a control were set up on 4 Date palm genepools at Dutse. They were renewed at four various time intervals. Oryctes recoveries were monitored for 4 nonths and compared with numbers of oryctes physically encountered within same period. 17 oryctes monoceros were encountered on palns on the whole, in the 4 genepools, within the period, but nork was recovered from the athyl chrysantinemuate traps. The differences In the monthly mean numbers of $\underline{0}$. monoceros physically encountered in the various genepools was not cignificant $(P=\$ .05)$. Though $Q$. monoceron was not recovered in the traps other insects - Piesoctorus ap. Macrotarmes op, sebaethe torrida, pracile helvale, Mylebrlo 6p. Juloidis coillaudt

- Sternocere Xunil and Apis mellifera were however recovered. DNTOMOLOGX DNISION, NIGSNIAN IMSTITURE FOR OLL PAIH IISENACA, P.M.E. 1030, MENIN CITY.

955

CONTROL OF FUSARIUM OXYSFORUM F.SP. ASPARAGI IN FIELD SOIL AMENDED WITH CROP RESIDUES AND TARPED WITH PLASTIC SHEETING

Win J. Blok, Jan G. Lanoers and Gerrit J. Bollen

Fusarium oxysponum f.sp. asparagi (Foa) incites a rot of stem bases, rhizomes and roots of asparagus. The pathogen is the cause of asparagus replant-bound early decline, which is one of the main constraines to asparagus production in the Netherlands. The fungus is present in the whole rootable layer and survives asparagus-free periods for many years. Currently, effective ways of control are not available.

Soil flooding has proven to be an effective practice to control a number of soilborne pathogens. It was hypothesized that destruction of pathogen propagules is due to the combination of low oxygen levels and toxic products generated during decomposition of organic material. For flooding a watertight layer needs to be present in soil. However, such a layer normally does not occur in asparagus fielos because the crop roots deeply and soils with a watertight layer are not used for asparagus production. Therefore, we tried to realize anaerobiosis in soil by a combination of amendment with crop residues and tarping with polyethylene sheeting with a low diffusion rate for oxygen. In laboratory experiments it was found that low oxygen levels $(<0.5$ vol \%) were obtained within one or a few days in soil amended with various crop residues at a rate of $0.5 \%$ (dw/dw) and incubated in gastight glass viats. After an incubation period of 7 weeks a 100-3000 fold reduccion in pathogen population density was found. A strong reduction was also obtained under field conditions. Plots $(4 \times 4.5 \mathrm{~m})$ without or with crop residues of fresh cabbage or fresh grass $\left(0.5\right.$ and $0.8 \mathrm{~kg}$ (dw) ha ${ }^{-1}$, respectively) were tarped with plastic sheeting or left untarped. Each of the six treatments was replicated three times. In each plot two nylon bags with chlamydospores in taic powder at a rate of $2.1 \times 10^{3} \mathrm{cfu}$ Foa $\mathrm{g}^{-1}$ were buried at a depth of $15 \mathrm{~cm}$. During the experimental period oxygen concentration and redox potential of the soil were measured in four additional plots. After 15 weeks samples were recovered and plated onto fusariumselective Komada medium. Irrespective of amendment with crop residues, the infestation level of the samples from the nontarped plots was slightly decreased, viz from the original $2.1 \times 10^{7} \mathrm{cfu}^{-5}$ to $0.7-1.3 \times 10^{6} \mathrm{cfu} \mathrm{g}^{-1}$. However, in the amended soil tarped with plastic sheeting the inoculum was reduced to below the lower limit of detection (15 cfu $\mathrm{g}^{-1}$ ). In the nonamended, tarped soil, inoculum level of the samples was $1.2 \times 10^{6} \mathrm{cfu}^{-1}$.
SEED SOAKING IN ACETYLSALICYLIC ACID FOR ELICITATION OF INDUCED SYSTEMUC RESISTANCE TO POWDERY MILDEW OF CUCURBITS

M.M. Aly, M.A. Attwa and M.K. Ali

Cucurbits are attacked by many important foliar diseases. Powdery mildew caused by Sphaerotheca fuliginea is one of them. Lack of resistant varieties makes the control of this disease dependent on fungicide application which causes environmental hazards in addition to increasing production costs. Many reports indicated that foliar application with acetylsalicylic acid (Asprin) in the seedling stage is effective in inducing a systemic resistance. Our work aimed at inducing a systemic resistance in an earlier stage of growth, in addition to be easily applicable and less expensive.

Three host plants were used in this study, curcumber (cucumis sativus $\mathbf{L}$.), squash (cucumis pepo L.) and melon (Cucumis melo L.). Seeds were soaked in different concentrations of asprin i.e. $2.5,5.0,7.5$ or $10 \mathrm{mM}$ for $24 \mathrm{~h}$ before sowing, as compared to 2.5 and $5 \mathrm{mM}$ foliar application in seedling stage. Results showed that seed application was more effective than foliar application. Treatments of 2.5 and $5.0 \mathrm{mM}$ were the most effective with both cucumber and squash. Reduction in disease severity, in different trials, ranged from 50 to $64 \%$ for both crops. As for melon, the best treatments were 7.5 and 10.0 $\mathrm{mM}$, reduction in disease severity ranged from 62 to $80 \%$. Treatment by 10.0 $\mathrm{mM}$ had te best effect on growth characters, as it doubled the length of runners, increased number of leaves by $23 \%$, fresh and dry weight/plant by 34 and $43 \%$. Results were promising for field trials which are under investigation.

Faculty of Agriculture, Shams University, P.O. Box 68 Hadayek Shoubra, 11242 Cairo, Egypt.

956

THE STAGE OF INTEGRATED CONTROL OF WHEAT DISEASE IN ROMANIA

I. Bobeş and M. Tomşa

Wheat is grown in Ronania annually on a suface of 3 million ha. Most favourable soil and climatic conditions for its cultivation are to be found with counties in the south-west of the country. Yields are between $2000-6000 \mathrm{~kg} / \mathrm{ha}$, i.e. an average of $3000 \mathrm{~kg} / \mathrm{ha}$. Of the registered varieties cultivated on large areas we mention: Aniversar, Apulun, Ariesan, Dacia, Esters, Favorit, Flamura, Fundules, Gabriela, Iulia, Libelula, Lovrin, Moldova, Potaisa, Silvana, Suceava, Timisan, Transilvania, Turda. More than 20 phytopathogenic agents attack wheat with varying occurrence and intensity; degree of attack varies between 10 and 50 per cent. Most important of these are the following: Fusarium nivale, F. graminearum, Septoria tritici, S. nodorum, Helminthosporium sativum, H. gramineum, Cercosporella herpotrichoides, Ophiobolus graminis, Tiphula incarnata, Erysiphe graminis, Cladosponium herbarum, Puccinia graminis, P. rriticina, P. striformis, Tilletia caries, $T$. foetida and $T$. controversa. Wheat disease control is based on prognosis and warning within integrated protectiou carried out by both classic and modern methods: physical, in stand, biological, chemical. Chemotherapy is applied both to seeds and plants. Frequently used methods are the following: dusting, wet dusting slur, spraying and immersion. Of the phytopharmaceutical products utilized there are to be mentioned Bayton, Benit, Beret, Criptodin, Quinolate, Prelude, Raxil, Tirametox, Vincit, Vitavax, etc. There has been proved - by scientific investigations that the highest economic efficacy is that of $1: 6$ lei $-1: 80$ lei in the control of wheat diseases, this one has the highest expectation.

Univ. Agric., str. Mănăştur 3, Cluj-Napoca, 3400 Romania. Phone 064-196384, Fax 064-193792. 
INFLUENCE OF IRRIGATION METHODS ON SPREAD OF PYTHIUM APHANIOGRMATUM AND PHYTOPHTHORA PARASITICA DURING PRODUCTION OF TWO GREENHOUSE CROPS

\section{S. L. von Broembsen, J.M. Dole, A. Fallon}

The effects of four irrigation methods (handwatering, microtube, capillary mat, and ebb and flow) on spread of two zoosporic fungi, Pythium aphanidermatum and Phytophthora parasitica, and on development of root disease caused by these pathogens was studied during production of poinsettias and vincas, respectively. Sixteen pot blocks of 'Gutbier V-14' poinsettias arranged four by four were established by transplanting rooted cuttings into pots of media infested with $P$. aphanidematim or no pathogen. For studies of disease spread, only the center four pots of each block were treated, but for development studies al pots were treated. Disease development caused by $P$. aphanidermatum was greatest for capillary mat irrigation with $75 \%$ plant deaths, compared with 56,38 and $25 \%$ for handwatering, microtube and ebb and flow, respectively. Disease spread occurred only with the two subirrigation methods. Spread to outer plants was $33 \%$ for $\boldsymbol{\theta b b}$ and flow and $22 \%$ for capillary mat. Spread of $P$. parasitica from a central infestation to surrounding plants during greemhouse production was studied for vinca (Catharanthus roseus) seedlings grown in flats or $6.4 \mathrm{~cm}$ pots and irrigated by capillary mat, ebb and flow or handwatering. Spread to surrounding plants in flats was 62,88 and $100 \%$ for capillary mat, ebb and flow and handwatering, respectively. For pots, pathogen spread was less and was dependent on initial concentration of inoculum in the soilless medium. Spread to piants in surrounding pots with ebb and flow was 55,14 and $7 \%$ for 10,1 and $0.1 \%$ inoculum, respectively. Spread occurred only at the $10 \%$ indeculum level from capillary mat ( $4.7 \%$ spread) and handwatering $(2.4 \%$ spread) irrigation. $P$. parasitica was recovered from ebb and flow reservoirs, capillary mats, and trays under handwatered plants during this trial. Clearly, irrigation method was shown to influence both spread of zoosporic fungal pathogens and diseases caused by these pathogens, and thus is an important tool for managing such diseases of greenhouse crops.

Department of Plant Pathology, Oklahoma State University, Stillwater OK 74078 USA

959

DEVELOPHENT OF DISEASE MANAGEMENT PRACTICES FOR SUSTAINABLE PRODUCTION OF CON PEA (vigna Unquiculata L Walps) IN SEMI ARID AREA OF RAJASTHAN, INDIA.

\section{A.S. CGEEA}

A project to develop disease management practices for cow pea Vigna Unquuiculata - L Walps production was undertaken in semi arid area of Rajasthan. A survey to understand the current Cow pea farming practices and farmers perceptions of diseases as a constraint to production revealed that cow pea (Vigna Unguiculata- L Walps) a grown is a secondary crop requiring low inputs for control of diseases. Diseases were identified as a major limiting factor in cow pea grain production

To achieve the low inputs reguirements for control of the diseases, the combined effects of different croping systems, resistant cultivars and reduced fugicides use were examined, This was in order to develop cheaper, profitable and efficient component technologies for increased cow pea grain production.

Different profitable fugicidal spray level were obtained with resistant cow pea cultivars. Three fungicidal spray intervals at bud initiation, flowering and padding stages of plant growth was identified as optimal with substantial increase in grain yield and profit margin.

H.S.CHEEMA, Assistant Profegsor, Department of Plant Pathology Agricultural Research Station, RAU, Durgapura, Jaipur-302017.

\section{RELATING FOLIAR DISEASE TO LOSS OF LIGHT} INTERCEPTING GREEN LEAF AREA.

R. J. Bryson, N. D. Paveley, R. K. Scott and R. Sylvester-Bradley.

Crop growth and yield relates to both the crops green area index (the number of units of planar area of green stem, leaves and ears per unit area of ground that they accupy) and the incident solar radiation. Loss of green area, and hence a reduction in light capture, due to foliar pathogens can have a significant effect on crop growth and yield. Traditionally, estimates of canopy loss dine to folar diseases have been made by measuring the percentage of leaf area exhibiting disease symptoms either at a particular growth stage or expressed as the integrated area under a disease progress curve (AUDPC). However, measurements of disease based on a percentage do not take into account canopy size and therefore cannot be reliably related to light interception over a season. Consequently, the relationship of disease to yield loss varies from site-to-site and season-toseason. Assessments of percentage disease sytnptoms present are therefore inadequate determinants for yield loss estimates. Considerable uncertainty still underlies yield loss predictions and hence economic decisions on disease management. Studies at ADAS Terrington in the UK have been carried out to investigate actual green area loss due to yellow nust (Puccinia striiformis) in whole crop canopies and on individual leaf layers. Yellow rust was found to significantily decrease light intercepting green leaf area, particularly in large canopies, and caused leaves to 'shrink'. The eventual aim of this work is to develop a model to relate loss of green area within a canopy to changes in light interception and to therefore precict disease induced yield losses. More ascurate determination of yield loss due to foliar disease will aid the rationalisation of pesticide use

Dr. R. J. Bryson. (University of Nottingham) c/o. ADAS Anstey Hall, Maris Lane, Trumpington. Cambridge. UK. CB2 2LF

960

TNTRODUCTION OF PHEROMONES FOR MATING DISRIUPTION OF CROP PESTS IN DEVELOPING COINTRIES

D Chamberlain, A Cork, L McVeigh, G Moawad', Z Ahmad ${ }^{2}, \mathrm{~K}$ Krishnaiah ${ }^{3}$

Mating disruption with fomulations of synthetic sex pheromones is increasingly accepted in the developed world as a technique that is compatible with a wide range of other components in IPM strategies for control of lepidopterous pests. This approach is also applicable in developing countries which are becoming dependant upon chemical insecticides and face the problems of insecticide resistance in key crop pests

After over ten years of development, pheromones are now widely used for the control of pink bollworm on cotton in Egypt, and duning 1994 nearly half the collon crop was treated with pheromone formulations. In Egypt cotton pest control is implemented by Government agencies and pink bollworm is the single main pest whereas in Pakistan pest control in cotton is the responsibility of individual farmers and a variety of bollworms are important; experience gained from using pheromones in these two systems will be compared and discussed.

Different crops require different application techniques and the experiences on cotton will be compared with those gained during work to introduce mating disruption with pheromone for control of yellow stemborer on rice in India where success is even more dependant upon coordinated activities of many small farmers.

Natural Resources Institute, Central Avenue, Chatham Maritime Kent, ME4 4TB, United Kingdom

'Ministry of Agriculture, Cajro, Egypt ${ }^{2}$ Central Cotion Research Institute, Multan, Pakistan ${ }^{3}$ ICAR Direciorate of Rice Research, Rajendranagar, Hyderabad, India 
EFFECT OF LATE WEEDING ON YIELD OF SPRING ROW CROPS

$$
\text { D.D... Orazic D., G.0., Glusac D., M.G., Malidza G. }
$$

Under modern conditions of spring row crops breeding, herbicides and their promptly application play a very important role in weeding.

Since spring row crops possess a week competitive ability because of the wide space between the rows at the early stage of growth, which permits strong development of weeds according to data of Kojic and sinzar (1985), the aim weeds according to da ta of kojlc and sinzar (1985) the aim of weeds influence of weeds on corn, soybean. Sugar beet and sunflower crops when the asplication of herbicides was postponed 10 to 20 the according to the optimal date. We also investigated practical usage depending on mechanical harvesting.

an the basis of the results obtained during a threeyear investigation period we can conclude that late weeding of spring row crops significantly decreased the yield of crops. The later is the weeding, the greater is the percentage of decreased yield. Sugar beet crop is the most susceptinle to the presence of weeds at the early stage of the vegetation, while soybean and corn are more competitive with weeds.

A ten-day delay of mechanical weeding of sugar beet croo decreased the root yield by $24 \%$, and if the delay was twenty days it decreased the yield by $46:$

$I^{*}$ the weeding of soybean crop was deleyed for ten days the yield was decreased by $23 \%$, and a delay of twenty days decreased the yield by $30 \%$

When the weeding of corn crop was delayed for ten days the yiald was decreased by 10:, while a twenty-day delay of weeding decreased the yield by $20 \%$.

Institute of Field and Vegetable crops, Maksima Gorkog 30 , 21000 Novi Sad, Yugoslavia
CONTROL OF APHIOS IN ICEBERG LETTUCE BY SYSTEMIC INSECTICIDE (IMIDACLOPRID) APPLIED TO PELLETED SEEOS AND EFFECT ON GERMINATION.

\section{A. Ester and N.B.M. Brantjes*}

Arer head formation by closure of the leaves, aphids inside the heads are out of reach for contact insecticides. Such aphids build up colonies, and make iceberg lettuce heads unmarkotable. Prevention against aphids has to protect the entire plant only till about one week aner head formation. After that moment the aphids on the outside of the heads can be removed wnh contact insecticides.

We aimed at complete protection during 6 to 7 weeks by a systemic insecticide, on the seeds. We also studied field sprays for the completion of the aphid control till harvest.

Field experiments have been made in 1993 and 1994 at four locations scattered over The Netherlands, with seeds of the varieties Abba in 1993 and Strada in 1994 provided, by Leen do Mos. Seeds were sown in peat blocks, those peat blocks were based on a potting compost and had a volume of $64 \mathrm{~cm}^{3}$ which were conform the standard foutine with plantraisers in The Netherlands. Two weeks atter sowing the peat blocks were planted out in open field and received the kocal standard cultural practise, except for the omission of standard insecticide protections. Plants were spaced at $40 \times 35 \mathrm{~cm}$, which resulted in 65,000 plants per hectare. Imidacloprid was administered to the seeds in combination with the standard 3-3.5 mm pellet. A neutral polymer prevented dusting of the insecticide. Dosages expressed as grams active ingredient per $\mathrm{kg}$ of raw sods were: $150,250,400,800,950$ and 1200 gram.

Intansity of phytotoxic etfects during germination tests ( 6 cays, top of poning soil $17^{\circ} \mathrm{C}$ ) related to dosage. Dosages over 950 gram resutted in unacceptable seedling detormation. Segdlings on peatblocks with minor detormations recovered, and resulted in normally sized heathy plants. in 1993 and 1994 the lovej of aphid protection related to the dosage. $400 \mathrm{gram}$ was insufficient and 800 gram gave good protection for more than 7 weeks atter drilling. After 8 weoks the protection became insufficient in all dosages. Syrphid lavae, as aphid predators, on treated plants showed no negative effects.

Seven weeks ather drilling, till one week betore harvest, the crop was sprayed with Pinmicart $(0.25$ liter a a./ha) each week. Four days betore harvest a final application of Heptenophos $(0.275$ liter a.i.ha) was given. This combination of seed and crop treatment gave complete aphid control till havest.

Under normal summer conditions in field production of iceberg lettuce the seed application of 62.5 gram a.i. insecticide per hectare can replace at least the first 6 crop treatments, each of $221 \mathrm{ml}$ a. per hoctare. The advantages of application on seeds consist of; firstly a substantial reduction in the quantity of utilised insecticides; secondly a reduction in the costs for spraying 6 times; and thirdly for the grower a reduction of the heath risks from exposures during spraying. The combination of sed dressing with a systemic insecticide and final crop spraying with a difterert type of insecticide is considered to torm a precaution against umwanted selection for a specitic insecticide tolerance in aphids.

Research Station for Arable Farming and Field Production of Vegetables. (PAGV) P.O. Box 430 . 8200 AK Lelystad, The Netherlands

"Leen de MOs BV, Seeds. PO. Box 54, 2590 AB 's-Gravenzande, The Nethertands.
963

A PILOT VLLAGE APPROACH: INTEGRATING RESEARCH AND EXTENSION IN A COMMUNITY-IMPLEMENTED CITRUS IPM PROGRAMME IN BHUTAN

\section{E.H. L. yan Schoubroeck, H. R. Feijen}

In the lower hills of Bhutan, mandarin (Citrus reticulota) forms the main cash crop for farmers. The Chinese Citrus Fly Bactrocera minco (Enderlein) (Diptera, Tephritidae) has been identified as the main pest. The larvae induce premature fruit dropping in a late stage. Drop percentages are almost equivalent to yield loss. Orchards with drop percentages ranging from 50 to $75 \%$ are common, though archards with much lower percentages are also found. Between 1987 and 1993 preliminary economic data on crop loss and ecological data of the fly were collected. Feasible control methods, like dropped-fruit destruction and beit-splashing were identified. From 1993 onward, a "pibt village" approach was used to implement a participany IPM programme for control of $B$. minax and other citrus pests. The programme has been implemented in six villages. Field demonstrations of all phases of the life cycle of the fly have been organized. The link with "late fruit drop" was demonstrated. Control methods have been discussed and demonstrated and input reguirewents handed to the village committees. Day-to-day supervicion was carried out by extension agents and key farmers, while plant protection suff participated during key periods of pest development and control exercises. The village approach led to progress on four levels. 1) Farmers gained knowledge on fly bioiogy and IPM aspects life pest monitoring, selective use of pesticides and natural control. 2) Essential ecological data of the fly (such as attraction periods to bait, larval and pupal densities, emergence rates and fruit drop rates in treated and non-treated villages) were collected by a detwork of farmers, extension agents and plant protection staff. 3) Ideas on feasibility of control measures at village level were developed, as were ideas on communal aspects of control and its organization. 4) Stills and motivation of local extension staff increased. Outreach activities have started in neighbouring villages. In the near future, fly control will be implemented all over the citrus growing areas in Bhutan based on results otonined in the pilot viliages. The same pilox village approsech is being used in Bhutun to implement IPM methods in apple, porato and vegetables in onder to control various insect pests, diseases and yestebrate pests.

IPMD Project, P.O. Box 639, Thimphu, Bhutan, Fax +975-2-23940
964

\section{WAVELENGTH SELECTIVE MULCHES PROVIDE A NOVEL APPROACH TO THE MANAGEMENT OF ROOT-KNOT NEMATODES ON} SOLANACEOUS CROPS.

\section{B.A. Fortnum, D. Decoteau, and M. J. Kasperbauer}

Root-knot nematodes (Meloidogyne spp.) commonly infest vegetable and field crops in temperate regions of the world and are a limiting factor in crop production. Tomato and tobacco producers in the USA routinely apply nematicides to suppress root-knot nematodes and enhance yields. The widespread use of nematicides increases the risk of air and groundwater contamination. Plastic mulches, in concert with nematicides, are commonly used in the producion of vegetables to enbanced water and fertilizer management, and to control weeds. Upwardly reflected light from colored mulches has been shown to have a phytoregulatory role on plant growth, hastening or retarding maturity, and affecting crop yield. Photomorphogenesis in plants is regulated by light in the red (R) and far-red (FR) portion of the spectrum. Tomato plants infected with $M$. incognita and irradiated with light containing a high FR/ $R$ ratio at the end of the day produce fewer eggs and egg masses than plants receiving a low FRR ratio. Therefore, it is apparent that the reproductivity of the nematode in roots is influenced by the light environment of the shoots. In our studies the effects of different colored mulches on the quantity and spectra of reflected light, earliness of fnit set, fruit yield, and root-knot disease were examined in field grown tomatoes and tobacco over a nine year period. The following data is a summary of these trials, portions of early studies have been published. Plant growth and fruit yield were recorded in tomato plants inoculated with $M$. incognite at initial populations ( $\mathrm{Pi}$ ) of $0,10,50,100$, or $200\left(\mathrm{X} \mathrm{10} \mathbf{1 0}^{3}\right.$ ) eggs per plant, and grown over white, black or red plastic mulch. Tobacco was grown in a field containing a natural infestation of $M$. arenario. Tomatces grown over white mulch had greater yields $(P=0.05)$ in the spring $(42 \%)$ and fall $(36 \%)$ than similar plants grown over black mulch. Tomato growth and fruit yields declined as $\mathrm{Pi}$ increased. Tomatoes grown over red mulch in the spring matured earlier. Hastening crop maturity reduced the impact of late season nematode damage through avoidance. Tomato plants inoculated with 200 (X $103)$ eggs and grown over white mulch or red mulch yielded $(102$, and $111 \%$, respectively) greater than similar plants grown over black mulch (7.4 kg and $7.7 \mathrm{~kg}$ vs. $3.6 \mathrm{~kg} /$ plot, respectively). Tobacco grown over red and white mulch yielded ( $24 \%$ and $21 \%$, respectively) greater than similar plants grown over black mulch. Colored mulches provide a novel approach to modifying plant growth and root-knot disease.

Bruce A. Fortnum, Clemson University, Pee Dee Research and Education Center, Rt 1 Box 531, Florence, South Carolina, 29501-9603, USA. 
A FARM BASED DOT ELISA KIT FOR PREDICTING ASCOSPORE DOSE OF VENTURIA NAEOUALIS

A. R. Gotlieb, L.P. Berkett, W.E. MacHardy, J. L. Bergdahl, and D.K. Sutton

Current management of apple scab is based on effective control of primary infections. This is accomplished with repeated applications of fungicides during the period when ascospores are being released from overwintered leaves on the orchard floor. of the primary stage of disease devel leaves on the orchard floor. Successful management of the primary stage of disease development reduces the need for further control measures
during the remainder of the growing season. This reduction in fungicide use translates into during the remainder of the growing season. This reduction in fungicide use tran
savings in production costs to the apple producers and mitigates concern about savings in production costs to the apple producers and mitigates concern about
environmental contamination from overuse of fungicides and the development of fungicide resistance. To efficiently schedule fungicide applications for apple scab, growers need to know when ascospores are mature, when a level of spores is high enough for economic damage, and when environmental conditions fulfill infection requirements. Presently, apple growers can easily identify infection periods but they have no way to evaluate the presence of mature ascospores or the potential for ascospore release or the threshold of spores needed for infection during a wetting period. The development of a system that would enable the apple producer to accurately and rapidly measure ascospore maturity and potential level of ascospores in their own orchard would lead to more informed decisions on the necessity of fungicide applications. We have developed an 'on-farm' antibody based kit to monitor mature ascospores of $V$. inaequalis. When combined with a model which estimates ascospore maturity and a threshold for significant first infection, an orchard system can be implemented with the safe delay of as many as three sprays. This system combines five features: 1) maturation of ascospores under natural orchard conditions, 2) release of ascospores and capture on an assay medium, 3) detection of ascospores with an immunoassay, 4) Relation of the assay of first spores trapped to a model which predicts the level of spores present in relation to degree days, 5) safety of delay of sprays determined experimentally as the model progresses. Thus far we have been able to detect a single spore being released from a leaf. We have been able to delay up to three fungicide sprays in commercial orchards with a low potential ascospore dose (PAD) without crop loss. Work in progress to perfect a model to predict potential ascospore dose so that it can be effectively used by the farmer just prior to the onset of the growing season will be discussed.

Alan A. Gotlieb, Plant and Soil Science Department, University of Vermont, Burlington, VT, 05405, U.S.A.

967

IPM FOR SMALLHOLDER FARMERS: TRAINING FOR CHANGE

C J Meir

This paper examines the major components which need to be considered in the development of an integrated pest management (IPM) training programme for small farmers, drawing on the experience of the Hillsides IPM Programme of Zamorano in Honduras. The dynamism inherent in the development of a training programme is illustrated by examples of the changes that the Hillsides IPM Prog
train.

Lack of training has been identified as one of the most important constraints to the implementation of IPM by small farmers. Developing an IPM training programme for small farmers is a dynamic process which must centre on the
farmer if it is to achieve results in the field. Training should encourage farmers to change by helping them learn new ideas, put some of these into practice, and evaluate the results. Our objective as IPM trainers is to support IPMers. In order to support the learning prose 1PMers. In order to support the learning process we need not only to offer follow-up afterwards. These follow-up afterwards. These activities enable us to focus the course content

Course design can be split into three elements: technical content, activities and materials, and teaching style. The technical content of Zamorano's courses aims materials, and teaching style. The technical content of Zamorano's courses aims IPM. Over time this has developed to include application of these concepts to specific pest problems experienced by the farmers being trained. Courses have specific pest problems experienced by the farmers being trained. Courses have a hish practical component and include a variety of activities ranging from discussions through song and drama to energisers. Over time these have expanded to include problem-solving in small groups. The style of teaching is the most important of the three elements and the one that has changed least. It is based on respect for farmers and their knowledge. This grew out of the work of the Department's anthropologist, Dr Jeff Bentley, who initiated the Hillsides IPM training programme. Trainers treat farmers as colleagues, encouraging them to participate fully by using the farmers' own vocabulary and drawing out their knowledge to build new concepts on existing ones. This teaching style has an enormous impact on farmers: they remember the courses years after they received the training. A recent development in our teaching the trainers.

The main conclusions are that for training to change we need to work closely with farmers and focus the content of courses to respond to their needs. We need to evaluate our work on a daily basis to enable us to adapt and improve our training. Finally, the quality of the training and hence its impact depends almost entirely on the trainers. We need to search out quality trainers and give them the space and resources to grow so that our training programme can also grow to meet the changing needs of today's smallholder farmers.

Hillsides IPM Programme, Department of Crop Protection, Zamorano, (Panamerican School of Agriculture), Apdo 93, Tegucigalpa, Honduras, Central America, and Imperial College Centre for Environmental Technology, 48 Prince's Gardens, South Kensington, London, SW7 2PE, United Kingdom.
TECHNICAL AND ECONOMIC FEASIBILITY OF MECHANICAL WEED CONTROL IN ARABLE FARMING IN THE NETHERLANDS

\section{A.T. Krikke, R.Y. van der Weide and F.G. Wijnands}

The reappraisal of mechanical weed control is not longer based on environmental considerations like minimising input and independence on herbicides. Economic and agronomic considerations, like the costs of chemical inputs and problems with resistant weed species are at least as important. The last five years practice oriented research in the Netherlands has looked intensively into the technical and economic feasibility of mechanical weed control and its integration with other (chemical) methods in more sustainable weed control strategies for a range of crops.

By using combinations of harrowing, hoeing, ridging and row spraying substantial reductions in the amount of pesticide used could be achieved. Expressed as reduction in active ingredient use: about $40 \%$ in chicory, $55 \%$ in sugar beet and winter wheat, $90 \%$ in silage maize and even $100 \%$ in potato, summer barley and legumes, without affecting yield.

What agronomically is feasible is not necessarily always technically or economically teasible. These aspects play an important role in the attitude of farmers toward application of the new strategies. In economic studies these new strategies were integrated in a whole farm context, enabling a full evaluation and appraisal of the shitts in inputs, labour and required machinery. In many situations the financial savings on herbicides are sutficient to compensate for the extra manpower and machinery. At farm level the workload increases and the pattern of labour demand changes, with May and June being particularly busy months. On some types of farms in the Netherlands this shift in labour peaks can only be accommodated if the working width of the row sprayers and hoeing implements is adjusted correspondingly. The maximal financial reward for the extra labour required for mechanical weed control varies between NLG 30 and NLG 100 per hour. For the relatively small areas of silage maize on livestock farms, the benefit however is marginal or may even be negative.

On 38 innovative tarms practising integrated arable farming systems, where sustainable weed control strategies were introduced, the reduction of herbicide inpu: amounted to some $60 \%$ compared to the farm specific reference of $1987-1989$, saving approximately 110 NLG per ha direct costs.

However, farmers in general still show some reluctance to introduce new strategies of weed control, mainly because of associated feelings of risks, extra hours required and the feasibility under varying weather and soil conditions. Succesful introduction requires, alongside this type of studies, skilled and committed farmers

Research Station for Arable Farming and Field Production of Vegetables P.O.Box 430 8200 AK Lelystad

The Netherlands

\section{8}

\section{TEACHNOLOGICAL APPROACHES TO THE IMPLEMENTATION OF THE STRATEGIES OF INTEGRATED PEST MANAGEMENT}

\author{
LUU XIANJIN" TU CHENGWEN" CAO CHEYANG *
}

The implementation and extension of the tacties and strategies is now quite difficult in China. Some teachnological approaches to transfer the knowledge - based extension to the material-based were made in our laboratory in reoent years. Based on the cological study focusing on the three - way relationship among crop, pest and natural enemy, and the investigation on the control practioe in the rice fields, a series of integrated pest management agents (of dynamic nature) on the rice pests management were developed, and the products were widely acoepted by the extension workers and the farmers. To obtain a sustainable management of the cotton bollmorm (Hetiocuerpa armigen ), a novel insecticide formulation were designed. Field tests have proved that the new chemical application planning centred on this mixture would protuce accumulative high residual effects on the cotton bollworm. So the combinations of knowledges and teachnicians are essential for these approaches.

* : Institute of Plant Protection Jiangsu Academy of Agricultural Sciences Nanjing 210014 P. R. China 


\section{THRESHOLDS: DETRIMENTAL TO WEED MANAGEMENT?}

\section{R. F. Norris}

Weed management decision making using principles based on the population biology of insects is not ecologically sound. Plants are producers in an ecosystem trophic dynamics sense. Their population biology is different from that of insects, which are consumers. Reproductive potential of most weed species is much higher on an individual basis than that of insects. Many annual weeds produce in excess of 10,000 new individuals in a single generation, and several have over a 1:1000000 fecundity ratio. Recent work shows that seed production is much higher for many weed species than is currently recognized. This high reproductive capacity leads to explosive population dynamics that cannot be achieved by most pest arthropods except over several generations. Sceds of most weed species exhibit dormancy. This means that population decline is relatively slow, and often takes place over several years. Dormancy and slow loss of viability leads to the development of a seedbank in the soil. Most pest insects have no equivalent to this. Allowing understory weeds, although not causing yield loss in competitive crops like com (Zea mays L.), to produce seed and resupply the seedbank is ensuring that weed management will be required in less competitive crops in rotation. The adoption of single-season economic thresholds for weed management implies that the seedbank cannot be manipulated. This premise should be challenged. Utilizing economic thresholds for weed management mandates that high levels of weed control input will be required for each crop. For thresholds to be useful as an aid to decision making there must be a technique for measuring the size of the population. This is difficult for weeds due to the presence of the sedbank. Counting growing plants is only a partial estimate of the total population, and may give misleading information about implications of short-term control strategies. Population dynamics models predict that the threshold for weeds with high seed output and short seedbank life, such as bamyardgrass (Echinochloa crus-galli (L.) Beauv.), should be such that no seeds are produced. For example, a single harnyardgrass plant in a hectare of sugarbets (Beto vulgaris L.) in Califonia produces sufficient seds to reinfest, with dispersal by human activity, the entire hectare at a density that will mandate weed control in following crops like tomatces (Lycopersicon esculentum $\mathrm{L}$.) or dry heans (Phaseolus vulgaris L.). For weeds with long-lived seeds in the soil, such as velvetleaf (Abutilon theophrasti Medicus), the concept of single-season economic thresholds is devastating when applied to spread of the weed into areas not previously occupied. For invading woed species with a persistent seedbank the threshold should also be zero. The use of thresholds in both of these situations is detrimental to management of these weeds. Several farms in California have adopted zero seed production thresholds for management of weeds. Their weed management programs have changed from a concept of control to achieve a successful crop, to a strategy aimed at stopping weed reinfestation. These farmers claim that the fatter strategy is economically superior to that using thresholds. In some cases adoption of zero seed production for weed management has resulted in decreased reliance on herbicides as weed control can be attained by use of non-chemical means.

Weed Science Program, Vegetable Crops Department, University of California, Davis, CA 25616, USA.

971

EXPLAINING PLANT DISEASE TO RURAL FARMERS: EMPOWERING THE PRODUCER.

Sherwood, S.G.

Due to the invisibility of pathogens and limited access to information, rural farmers of Central America do not understand the cause of biotic plant diseases. Without such knowledge, their management practices are supposititious, and efforts may be useful, useless, and even harmful. Cornell University and the Panamerican School of Agriculture (El Zamorano) developed and tested a course on plant disease management for small production farmers. They conducted 15 courses in Honduras and Nicaragua during 1994 with over 200 farmers and extensionists participating. The course built on local knowledge and relied on creativity, motivation, and leadership to empower the producer to manage plant disease problems. Farmers were cager to understand plant disease, and they mastered abstract ideas related to plant-pathogen interactions. Participants learned to identify common disease problems by pathogen type and explain disease life cycles. With their new knowledge and ability, farmers improved management strategies. As a result, they decreased wasteful pesticide use and adopted practices that should stabilize and improve their production systems.

IPM for Small Producers in Nicaragua, MIP-Zamorano, P-126, Managua, Nicaragua. telefax: (505) $265-7114$.
DETERMINATION OF THE SPILOCAEA OLEAGINA INFECTIONS SEVERITY IN OLIVE TREE (OLEA EUROPEA) THROUGH OUT ARTIFICIAL VISION.

\section{M.L. Soriano, J. Cabrera, A. Porras, I. Marcilla.}

The Olive tree (Olea europea) occupies in Spain 2.000 .000 hectares, being the first country in the world on cultivated surface, but its rentability is being affected by several diseases caused by fungus, specially the disease caused by Spilocaea oleagina, wich affects the $100 \%$ of the cultivated surface in Castilla-La Mancha (center of Spain). The severity of the ocasionated symptoms are relationed with the rentability of the crops which is diminished in a $20 \%$ by this disease due to the fall of the leaves provocated and the death of the productive buds. The illness manifests itself with necrotic marks affecting in bigger or less percentage to the foliar surface. The control of the disease is made by copper fungicides in preventive aplications which requires the stoppage of the fungus in the fabrics of the plant before the visible presence of the symptoms through the technique described by LOPRIENO-TENERINI. The evaluation of these symptoms, and so those visible, has been made in the traditional way of visu through the scale severity application depending on the percentage of the affected foliar surface. This work presents a computer program for the application of the artificial vision to the study of the foliar diseases, that permits from image capted by scanner and digitalized by personal computer -basing on the color differences of the bealthy foliar surface and in the ill one- quantify the severity of the visible infections and in those which are being incubated with a high level of resolution and in a rapid way, which facilitates the taking of decisions in the control strategies.

E.U.I.T.A. University of Castilla-La Mancha.

Ronda de Calatraya s/a. 13004 Ciudad Real. Spain

972

WATER STRESS AND SEED TREATYENT EFFECTIVENESS IN SIBERIA

Toropova E.U. Novosibirsk Agricultural University

Continental climate of the Western Siberia restricts application of seed treatments for the control of connon root rot (Holninthosporiun sativun) on spring wheat. Laboratory and field experiments showed, that aptimal soil hunidity (about $50 z$ of nax moisture) during seed sprouting is a linit factor for fungicides activity (Baytan-universal, Vitavax 200 FF, Suni-8, THTD). Water stress in the beginnig of the growing season resulted in the negative effect of seed treatment and increased canmon root rot incidence on $15-40 x$.

water stress in the niddle of the season, tipical for siberia, provided the best conditions for root rot development (root rot incidence increased 7 times in the season), so any seed treatment was able to control the disease during only first 30 days after sowing. In rosult, there was no difference in root rot incidence and yield between treated and cheching variants by harvesting. Under optimal alinatic conditions (about $60 x$ of nax noisture) seod troatment epfectiveness depended on infection levels in seeds and soil and could increase yield on $40 x$.

633126 Russia, Novosibirsh region, Rrasnoobsh, a/y 18 


\section{J.R. Velastegui and M.G. Sánchez}

Swiss Technical Cooperation funded a research work at the University of Ambato Ecuador (2,868 m elevation) between 1992-1993. A 7HP portable steam generator was locally constructed ( $1.95 \mathrm{~m}$ long, $1.05 \mathrm{~m}$ wide and $1.65 \mathrm{~m}$ high) equiped with: boiler tubular type; diesel burner; a presuretrol and a McDonnell to control automatically pressure and buming functioning; tap water supply ( 52 liters/cart); and a water pump. The steam goes through a hose to a four wheeled metallic cart $(1.8 \times 0.95 \times 0.45 \mathrm{~m})$ carrying $0.75 \mathrm{~m} 3$ of dry and crumbled soil covered by an impermeable tarp. The stean is distributed through three perforated metallic pipes placed at the bottom of the cart. When the boiler is cold, water takes $\mathbf{4 5}$ min. to generate steam. Application of steam is at $82^{\circ} \mathrm{C}$, on average, at least during $30 \mathrm{~min}$. Thus, $0.75 \mathrm{m3}$ of soil takes between 35 and $\mathbf{4 5} \mathrm{min}$ to be disinfected, depending on soil texture. The usual pressure to do it is 1.0-1.5 Mpa. The steam generated is $117.6 \mathrm{Jb} / \mathrm{h}$, the heat output is $285,947 \mathrm{BTU} / \mathrm{h}$ and the heat losses 2,211 BTU/h. Diesel consumption is about $2 \mathrm{gal} / \mathrm{h}$, so 5 gal for $5-6$ carts a day,that is 2.95 US dol. At 1994 the cost of the generator and the cart is 3,400 US dol.

$100 \%$ mortality was obtained when applying steam treatment on main soil pests: Sclerotinia sclerotionum fungus scierotia; Phytlophaga insect larvae; and Dature stramonium weed seeds.

Once the research finished, at the beginning of 1994, the equipment was transported to one of the Fruit Experimental Station from INIAP (National Agricultural Research Institute) to be used in routine soil disinfections. At the end of 1994 the results are completely satisfactory because apple rootstocks for farmers growing in soil disinfected by steam are three times bigger and vigorous than those growing in soils under other treatments and none basal or root disease is present. Moreover, almost all the seeds for those rootstocks had germinated, in contrast with those under the other treatments.

Therefore, the technique is reliable, easy to do, safe, ecologycally "clean", and cheaper, because the equipment would last 10-15 years and the disinfected soil can be stored within closed rooms without recontamination risks. Soil disinfection with steam is not yet in use in Ecuador and it would match succesfully with other pest control measures in IPM programs, both in greenhouses and at open air, and would also be useful for disinfecting farm buildings, water/milk containers, pots, farm tools, etc.

UNIVERSITY OF AMBATO, P.O. BOX 596, AMBATO-ECUADOR

975

MULTIPLE TACIICS FOR MANAGING TUFTED APPLE BUD MOTH (LEPIDOPTERA : TORTRICIDAE) IN NORTH CAROLINA APPLE SYSTEMS

J, F. Walgenbach and D. M. Borchert

The tufted apple bud moth (TABM), Platynota idaeusalis (Walker), is the most important direct insect pest of apples in North' Carolina, USA. The development of multiple in North carolina, USA. The development of multiple
chlorpyrifos and phosmet) has led to the use of carbamates chlorpyrifos and phosmet) has led to the use of carbamates these insecticides have reduced populations of the predatory beetle stethorus punctum (Le conte), which is a key predator of European red mite. The use of Bacillus thuringiensis products and pheromone-mediated mating disruption, which are compatible with the ERM mite biological control program, have been evaluated as alternative strategies for managing TABM. Laboratory bioassays with purified Bt endotoxin proteins showed that cry IA (a). IB and IC were most active, While IA(b) and IA(C) were least active, and ID and IF were intermediate in activity against TABM larvae. In the field the use of Bt products with the highest IB and IC content provided comparable levels of control as the most effective organophosphate (encapsulated methyl-parathion). Pheromonebased mating disruption (1,000 dispensers/ha loaded with 50:50 E11-14:AC and E11-14:OH) has been an effective alternative under low-density TABM populations. In orchards with high density TABM populations, mating disruption has been ineffective or not consistent. In an effort to reduce the intensity of TABM populations in high density orchards and to improve the efficacy of mating disruption, spring applications of the pyrethroid insecticide esfenvalerate were applied to the ground cover to kill overwintering TABM larvae. This tactic enabled mating disruption to effectively control the first TABM generation, but damage increased during the second generation. Although the cost of the Bt and mating disruption approaches are higher than synthetic insecticides, increasing levels of TABM insecticide resistance and consumer concerns about pesticide residues is expected to increase the use of these alternative management tactics.

MHCREC, N. C. State University, 2016 Fanning Bridge Rd., Fletcher, NC 28732
BUD") CONTROL

\section{Y.I.Vlasov}

The mycoplasma disease-tomato stolbur is spread at the south regions of Russia. Different species of leafhoppers are vectors of the stolbur mycoplasma just as of similar disease, known abroad as "big bud". At present the possibility of tomatoes protection from stolbur by ecologically safe methols exists in Russia. Use of the early-season varieties of tomatoes and application of the early spring outdoor planting are recommended at the zones of epiphytoty, because in that case tomatoes form basic yield before mass leafhoppers' colonization and, accordingly, before noticeable stolbur development. At the North Caucasus this recommendation was successfully checked up with the towato varieties Utro, Volgogradsky rannji (Bogoutdinov, 1992, Vlasov, 1994). At the same time under conditions of Krasnodar Territory the large tometo collection was estimated on field resistance to stolbur by the methods, devised at the institute (Samsonova, Vlasov, Besedina, et al., 1991, Sakalieva, 1992). As a result tomato varieties with high resistance to the disease were revealed, those are Rogusansky, Capitan, Rannyi Uzbekistan, Fakel, Narvik, Titan, Maja.

All-Russian Research Institute for Plant Protection, s.Podbelskogo,3, St.Petersbourg-Pushkin, 189620 Russia Phone: $476-84-89$
976

\section{GENERATIONAL SUCCESSION OF CHEMICALLY INDUCED RESISTANCE TO BLAST DISEASE IN RICE PLANTS}

\section{ARIMOTO, Y., HOMMA ,Y., YOSHINO, R. AND SAITO, S.}

We found that resistance to rice blast disease caused by Pyricularia oryzae can be induced in rice plants by soaking rice seeds in DL-alanine dodecylester hydrochloride solution $(500 \mathrm{mg} / \mathrm{l})$.

Rice seeds were soaked in the chemical solution (treatment : T) or distilled water (control : $\mathrm{C}$ ), and incubated at $30 \mathrm{C}$ for 3 days. Both seeds were sown and cultivated in a greenhouse or paddy field until harvest to obtain 2 nd generation seeds. The 2nd generation seeds harvested from the T-seedlings were soaked again either in the chemical solution or distilled water to prepare T-T-seedlings and T-C-seedlings. These procedures were repeated for preparing $3 \mathrm{rd}$ and 4 th generation seedlings.

The outbreak of leaf and panicle blast disease on rice plants $(\mathrm{T})$ decreased to $25 \%$ (in a greenhouse). The chemically induced resistance is persistent without decrease up to 3 rd generation seedlings. and blast severity on the plants was reduced to $39 \%$ (T-C and $28 \%$ (T-C-C) in greenhouse tests, and $48 \%$ ( $\mathrm{T}-\mathrm{C}$ ) in field tests.

When rice seeds in alternate generations were treated with the chemical, protective effect remained without decrease in all generation from the original to 4 th. These seedlings showed $75 \%$ (T-T), $61 \%$ (T-C), $87 \%$ (T-C-T) and $97 \%$ (T-C-T-C) reduction in disease incidences, respectively.

On the rice plant $(T)$ in which blast resistance was chemically induced, conidia of $P$. orizae germinated and formed appressoria, and then its hyphae penetrated into the host tissues, however, the invading hyphae were restricted in epidermal and adjoining cells without subsequent penetration. 
EFFECTIVE CONTROL OF THE TOBACCO WHITEFLY, BEMISIA TABACI, USING THE INSECT GROWTH REGULATOR "APPLAUD".

\section{D.Bedford, G.I.Harwood, R.P.Tucker and P.G.Markham}

Over the past decade, the tobacco whitefly, Bemisia tabaci, has increasingly become more serious as a pest to agriculture worldwide. It has caused extreme crop losses by feeding honeydew contamination and plant virus transmission. This increase has predominantly been associated with the appearance of a new biotype, the " $\mathrm{B}$ " biotype, which is more fecund than most other established biotypes, colonises a far greater range of host piant species and induces phytotoxic damage in many crop species. The "B" biotype which exhibits a very high reproduction rate has proved to be extremely difficult to control with insecticides. Finding effective control methods for this whitefly (along with the many plant viruses it can acquire and transmit) is now paramount for global crop protection.

Integrated pest management (IPM) systems are currently being developed and tested, aimed at overcoming or preventing the resistance and tolerance problems that might occur through the use of mono-treatment control methods. In this case specific predators, parasites and/or fungal pathogens are used alongside selective chemical treatments. APPLAUD, a buprofezin based treatment that disrupts chitin production in Homopteran, sucking-insects has been available for whitefly control within IPM in parts of Europe since the late 1980s and offers a high level of selectiveness.

The dosage approved in the United Kingdom for control of the glasshouse whitefly, Trialeurodes vaporanorum, is set at $0.3 \mathrm{ml}$ of APPLAUD per litre of water $(75 \mathrm{ppm}$ buprofezin). This application concentration has also been used elsewhere in Europe on infestations of $B$, tabaci as they begin to appear on glasshouse and field crops. However concern over the effectiveness of APPLAUD at controlling $B$. tabaci at this rate, have arisen.

APPLAUD was tested against early instar "B" and non "B" biotype larvae of B. tabaci at a dose rate of $0.3,0.6$ and $1.2 \mathrm{ml}$ per litre of water. The $0.3 \mathrm{ml} / \mathrm{l}$ treatment al lowed a significant amount of larvae to survive and develop. However the $0.6 \mathrm{ml} / 1$ treatment allowed only a few of the " $\mathrm{B}$ " biotypes to survive, while the $1.2 \mathrm{ml} / 1$ treatment achieved total control within these tests. Progeny from the survivors of the $0.3 \mathrm{ml} / 1$ and $0.6 \mathrm{ml} / 1$ treatments were also totally controlled by APPLAUD at $1.2 \mathrm{ml} / 1$

This higher dose of APPLAUD is extremely effective at controlling all tested biotypes of $B$. tabaci by killing the early instars. It was also shown to reduce egg viability.

John Innes Centre, Colney Lane, Norwich, Norfolk, NR4 7UH. United Kingdom.
EVALUATION OF FIPRONIL FOR USE AGAINST MUSHROOM PESTS

\section{A.D. Clift and M.A. Terras}

Fipronil (Rhône Poulenc MB46030), a new phenyl pyrazole insecticide that acts by blocking the GABA regulated chloride channel, provides excellent control af many soil insects. We determined the activity of fipronil against several mushroom pests (sciarids, Lycoriella mali; mushroom phorids, Megaselia halterata and mushroom white cecids, Heteropeza pygmaea) in the laboratory. In a laboratory system, over $90 \%$ control was achieved at $1 \mathrm{mg} / \mathrm{kg}$ medium for sciarids, but $10 \mathrm{mg} / \mathrm{kg}$ was required for phorids and cecids.

Further work in an experimental mushroom growing unit, when fipronil was incorporated into the casing layer at $10 \mathrm{mg}$ active/ $\mathrm{kg}$ final casing, indicated higher rates would be required for sciarids. Doubling the rate to $20 \mathrm{mg} / \mathrm{kg}$ provided excellent protection against sciarids and cecids for the eight weeks from application of the casing layer to termination of harvest. This higher rate provided over $90 \%$ suppression of mushroom phorids, the only insecticide tested in the unit over the last 15 years to do so.

Fipronil is effective against the dipterous pests tested and is compatible with commercial mushroom production. There is no single insecticide available to the mushroom industry that will control sciarids, phorids and cecids. It is proposed that this insecticide be used as a casing treatment incorporated at $20 \mathrm{mg} \mathrm{ai} \mathrm{kg}^{-1}$, equivalent to $16 \mathrm{~g}$ product in $300 \mathrm{~L}$ bale peatmoss. At this rate there were negligible survivors in the treated casing from infestation experiments. Larvae in the compost were not exposed to the chemical. Good physical exclusion is therefore required to minimise compost infestation. Late in the growing cycle large larvae could move from the compost into the treated casing, which could lead to development of fipronil resistance. Fipronil should only be used as part of an IPM program, rather than to be relied on as the sole control measure.

It is possible to have very even incorporation of insecticides in the casing layer by adding the chemical to the water used in preparing the casing layer. Watering on is not as efficient. Similarly compost treatments are not recommended because of difficulty of uniform treatment. Further, the casing treatment was providing good protection under very heavy pest pressure. Fipronil has been found to be active against insects resistant to pyrethroid, cyclodiene and organophosphate insecticides, so it would be expected to be effective against the insecticide resistant sciarids and phorids in Europe and North America.

Biological and Chemical Research Institute, PMB 10, Rydalmere, NSW 2116, Australia

980

A NEW TETRAZINE ACARICIDE SZI-121, LEAD OPTIMALISATION

J. Hajimichael, Gy. Horváth, L. Pap, S. Botár, I. Székely

(L) AND EPIPHYAS POSTVITTANA (WALKER) (LEPIDOPTERA: TORTRICIDAE) AND THEIR ROLE IN EVOLUTION OF APPLE PEST MANAGEMENT.

\section{G. M. Gurr, W. G. Thwaite, B. J. Valentine.}

Results are presented from a field evaluation of insect grown regulators (IGRs) tebufenozide and fenoxycarb over three production seasons in an apple orchard in New South Wales, Australia. In the first two production seasons of the trial considerable pest pressure was indicated by sex pheromone trap catches of both codling moth Cydia pomonella (L) and lightorown apple moth Epiphyas postrittana (Walker) yet IGR treatments maintained damage levels to fruit at harvest (including winctalls) below $1 \%$ for both pests which was equivalent to control afforded by azinphos-methyl. Tebufenozide gave better control than fenoxycarb of E. postvittana. Numbers of secondary pests were higher in plots under azinphos-methyl treatment than under either IGR treatment and this was attributed to the lower numbers of natural enemies observed. Limb tapping in the second production season showed spiders, Stethorus $\mathrm{sp}$. adults and larvae, and apple dimpling bug. Campylomma liebknechti (Girault) nymphs were all significantly more mumerous in IGR treated trees. In the third (current) production season, vacuum sampling of trees just prior to the first insecticide applications, indicated that lower densities of spiders had persisted in plots last treated with azinphos-mettyl in the previous summer than in IGR treated plots. Findings are interpreted in relation to the contribution which such target specific compounds may make to the evolution of pest management in apples as a strategy which is relatively acceptable to growers and more compatible with biological control and so help meet the growing demand for pesticide reduction.

Orange Agriculturat College, the University of Sydney, Orange, NSW, 2800 . Because of the growing economic importance of phytophagous mites new potent and selective
acaricides are continuously needed for mite manngement. The tetrazine type acaricides having acaricides are continuously needed for mite manngement. The tetrazine type acaricides havin specific ovicide action seems to meet hise requirements.

Lead-molecule and our synthesis plan

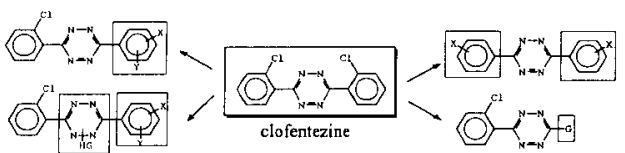

Excerpts of the chemical structure-hiological activity relationship

According to the results, asymmetric compounds have higher activity than the symmetric ones with the exemption of chloro substituted molecules. Our team recognised that the introduction of fluorine atom into ortho position of the phenyl group resulted in the development of translaminar, fluorine atom into ortho position or the phenyl group resulted in the development of translaminar, transa the phenl group is the best. More or fewer fluorine atoms on the phenyl at position 2 and 6



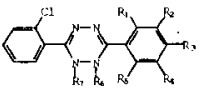

Feat of halogen sutstintion of phenyl ning on inherent and

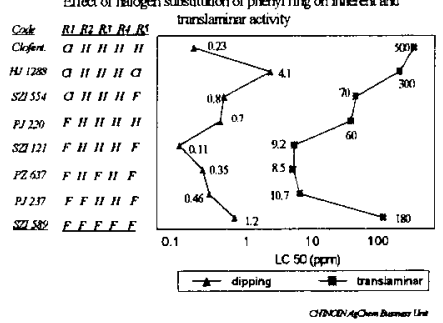

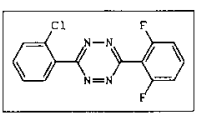

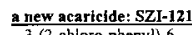

3-(2-chloro-phenyl) 6 -

$1,2,4,5$-tetrazine

The advantages of SZI-121: Selective to phytophagous and chrysalis stages It has excellent translaminar and transovarion activity o very effective via vapou phase and has long lasting
pate It is highly effective to 
AEROSOLS OF BIOINSECTICIDES AND PYRETHROIDS AGAINST CABBAGE MOTH Mamestra brassicae L.

\section{V.P. Khodyrev, E.I. Kirov, V.V. Gaevoy}

Treatment was performed using an aerosol generator with controlied dispersion, operating in a pneumomechanical regime. Working solutions were based on a $20 \%$ water-glycerin emulsion. Generator moved at night with downward air currents. Treatment productivity was $500 \mathrm{ha} \mathrm{hr}^{-1}$. The variants of treatment with high biological effectiveness obtained in 1985-1994 are presented. A bacterial preparation Lepidocide (based on Bacillus turingiensis), stabilized powder in a dose of $100 \mathrm{~g} / \mathrm{ha}_{1}$ viral preparation Virin-X, 1011 polyhedrae per 1 ha and their mixtures caused the mortality of caterpillars by $44 \%, 41 \%$ and $79 \%$ at the width $400 \mathrm{~m}$ from the generator path. Lepidocide in a dose of $300 \mathrm{~g} / \mathrm{ha}+$ Virin- $X .3 \times 10^{10}$ polyhedrae per 1 ha with repeated treatment in 11 days reduced the number of insects by $88 \%$ at $900 \mathrm{~m}$ from the generator path. The usage of bioinsecticides and their mixtures is more effective for the peak number of cabbage moth (as it was in 1985-1987) when insects are more sensitive. During the last years the increase in the number of pests has been observed.

Sumicidine ( $20 \%$ e.c., 0.15 / / ha) and Sumi-alfa ( $5 \%$ e.c. 0.15 I / ha) were also employed. In this case a biological efficiency amounts to $97 \%$ at $900 \mathrm{~m}$ from the generator path. The cabbage moth is controlled together with different species of leaf betles.

Institute of Biology Siberian Branch. Russian Academy of Sciences Frunze str. 11. Novosibirsk 630091, Russia.
NOTES AEOUT TESTING OF THE STERILANT ACTIVITY OF OVICIDES

$$
\text { L. Pap. E. Bleicher and J. Hajimichael }
$$

The integrated mite control needs selective miticides compatible with biological control methods. From this respect the group of ovicides acting solely on the eggs of phytophagous mites have been in the centre of interest. After first representatives of physiological selective ovicides, clofentezine and hexithyazox, a new improved compound (SZI-121) was presented recently. Working with this compounds we found that the standard biological methods proved to be often unsuitable to recognise practically important features of the compounds

For example to assess the overall effect of ovicides on mite populations the sterilant activity is generally considered. In routine test the exposed female mites are transferred to untreated leaves and are allowed to lay eggs for 24 or 48 hours. The eggs are counted and incubated for hatching. The number of unhatched eggs refers the sterilant activity.

We performed a comparative laboratory test to compare the sterilant activity of SZI$121200 \mathrm{SC}$, Apollo $500 \mathrm{SC}$ and Nissuron $10 \mathrm{WP}$. if the time resalution was enhanced and the egg production also monitored, we found large difference between the tested ovicides. They acted distinctly on egg production and egg fertility depending on the period of laying. In the $0-12$ hour period the number of laid eggs did not differ significantly. However contrary with Apollo and Nissuron, \$Zl-121 significantly suppressed the egg production in the $0-24$ and $24-48$ hour periods. Apollo failed to show sterilant activity. The sterilant activity of SZl-121 and Nissuron were decreased by chronological order. The freshly laid eggs were more sensible than the older ones. In the first period (0-12 hours) the efficacy of $5 \mathrm{Zl}-121$ was excellent $(97.0 \pm 3.0 \%)$ and significantly higher comparing to Nissuron $(74.3 \pm 6.1$ $\%$ ). There was no significant difference between them as for the 0-24 hour period. After 24 hours the eggs become as viable as control probably due to depletion. Finally we concluded that in the case of SZI-121 the suppressed egg production. amplifies the overall efficacy in the $0-24$ period. Moreover the suppressing effect on egg production remained high after depletion of the compound in 24-48 hour period. Probably this effect contributes to elevated field efficacy of SZI-12 1 .
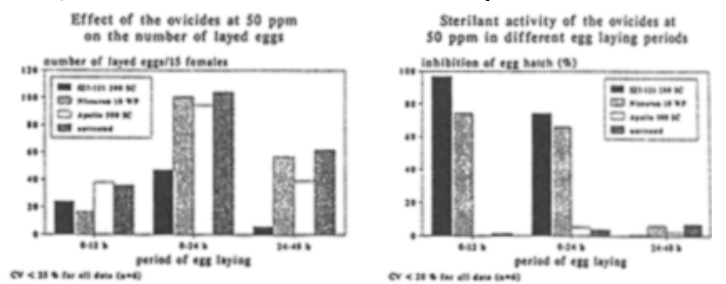

CHINOIN Pharmaceutical and Chemical Works Co. Ltd

AgChem Business Unit, H-1780 Budapest P.O.B. 49. Hungary

984

EFFECTS OF HYDROXAMIC ACID DERIVATES ON DEVELOPMENT OF CEREAL APHIDS.

\section{A.B.Vereschagina}

Benzoxazolin-2-one (BOA) and its 6-methoxyderivative (MBOA) fall into decomposition products of hydroxamic acid which are of importance in plant immunity to pests and diseases. There is no agreement among authors regarding toxic effect of these compounds on aphids. From our point of view, the significance of this resistance barrier heve to be decreased in the case of intercellular penetration of aphid stylets in a consequence of the specificity of its "working" only in response to destruction of cell walls or cell contents. It has to be effective, however, when aphids stylets penetrate phloem tubes in intracellular way. In this connection we have tried to evaluate the influence of these hydroxamic acid derivates on aphid species which differ in their phytotoxity and type of stylet penetration into plant tissues. For this purpose aphid species Rhopalosiphum padi L. and Shizaphis graminum Rond. were fed through parafilm membrane by BOA $(0.004 \%$ - BOA $4 ; 0.02 \%-\mathrm{BOA} 20)$ and MBOA $(0.005 \%-$ MBOA $5 ; 0.024 \%-$ MBOA 24) water solutions and by AFD which contained derivates in the same concentrations. Control aphids fed on maize line A-619, water and AFD without derivates.

The comparison studies of the mortality of Rh.padi and Schgraminum females revealed that Sch.graminum was influenced by toxic derivates to a lesser degree than Rh.padi. The mortality of Sch.graminum females after 3 days of feeding on AFD with hydroxamic acid derivates constitutes 40\% (BOA 4), 46.6\% (BOA 20), 40\% (MBOA 5), 50\% (MBOA 24) as compared with $63.3,70,70$ and $76.6 \% \%$ respectively for Rh padi mortality. Probably Sch.graminium posseses some system of neutralization of these derivates, since penetration of its stylets destroy plant tissues and "turn on" plant resistance reaction. Thus, the different reaction of the studied aphid species to the derivates of hydroxamc acid can be responsible for different varietal resistance of cereals to them.

It is notable that fecundity of aphids sufficiently increased in all variants of the trial as result of their transfering from arificial diet to maize, although as a whole it was less than one of the control specimens which feed on maize only.

All-Russian Institute for Plant Protection, Podbelsky sh. 3 , St.Petersburg-Pushkin, 189620, Russia $\|$
Its efficacy, toxicological and environmental features make the SZI-121 an ideal tool of integrated mite management. 
INSECTICIDE SYNERGISTIC, INSECT GROWTH DISRUPTION AND INSECTICIDAL ACTIVITY OF SOME NOVEL OXIME ETHERS AND ESTERS

S.Walia, Y.S.Saxena, O.Koul and H.Chowdhury

The widespread anxiety about the adverse effects of conventional highly toxic and persistent insecticidess have led to the increasing tendency to ban or restrict their use in crop protection. Insecticide synergists and insect growth regulators, being environmentally benign, are considered as chemicals of choice and are incorporated increasingly in integrated pest and resistance management programmes. A series of oxime ethers/esters derived from 3,4-dimethoxybenzaldoxime, 3-methoxy-4-ethoxy benzaldoxime, piperonal oxime, m-phenoxybenzaldoxime, dillaldehyde oxime and acetyl dihydrodillapiole oxime have been synthesized and evaluated for their insecticide synergistic, insect growth disruption and insecticidal activity. Among the potent synergistic compounds, pentoxyiminomethyl benzo-1,3-dioxole imparted the highest activity with natural pyrethrins against the test insect Tribolium castaneum. It was found superior to comercially available insecticide synergist piperonylbutoxide (PBO). Among the compounds exhibiting insect growth disruption activity, n-propyl and cyanoethyl ether of m-phenoxybenzaldoxime gave outstanding performance. When injected into the haemolymph or topically applied to Schistocerca gregaria fifth instar nymphs o $0.5-1.0$ ug/nymph, these compounds recorded 85-90\% $\mathrm{N}, \mathrm{O}-\mathrm{methoxy}$ and $\mathrm{N}, \mathrm{O}-\mathrm{i}$ sopropoxyiminomethyl benzo-1,3-dioxole which at 5 ug dose level (injection) showed $60-758$ inhibition in insect growth. At 3 ug level, the latter compound was still capable of inflicting 508 deformities. Similarly m-phenoxy benzaldoxime esters of cyclopropane carboxylic acid, m-phenoxy benzoic acid and 3 -methyl-2-(4-chlorophenyl) butyric acid exhibited both, insecticidal and insect growth discuption properties. Some of these compounds were active against Achoea janata larvae. Structure-activity relationships in these series revealed that for maximum activity, the optimum chain length in oxime ether moiety should not exceed 3-4 carbon atoms. to decrewaing around the aromatic nucleus also contributed to decrease in activity. Details of these investigations will

Division of Agricultural Chemicals Indian Agricultural Research Institute New Delhi - 110012 , India

987

REGISTRATION OF THE MYCOSTOP® IN THE UNITED STATES - A CASE HISTORY

K. Kuparinen, P. Ronkainen, J. Uoti, and E. Butts

Kemira Agro Oy registered MYCOSTOP in the United States under the "Subdivision M of the Pesticide Testing Guidelines - Microbial and Biochemical Pest Control Agents.' Kemira submitted product chemistry, including taxonomic information, acute toxicity and pathogenicity, and ecological effects data. MYCOSTOP is a biofungicide used to control witt and root diseases primarily caused by fusarium in vegetable and omamental crops. The product contains Straptomyces grisoovindis Strain K61. MYCOSTOP is also The product contains Straptomyces griseovindis Strain K61. MYCOSTOP is also
registered in Finland, Bulgaria, Hungary, Switzerland, Chile, Denmark, Estonia, Sweden, registered in Finland, Bulgaria, Hungary, Switzerland, Chile, Denmark, Estonia, Sweden,
and Russia. This presentation will consider the regulatory process that Kemira dealt with to test and register MYCOSTOP in the United States

Kemira Agro Oy

Porkkalankatu 3

P.O. Box 330

00101 Helsinki, FINLAND
FUNGICIDE TREATMISTT OF CEREAL SEEDS IN NORWAY ACCORDING TO NEED

Brodal, G.

Norway has a strategy to reduce the use of pesticides, including seed treatment fungicides, and to ensure their use only according to need. From the year 1990 , seed lots of spring cereals have been tested routinely for seed-borne pathogens and assessed for the need of treatment. In barley and oats, the osmotic blotter method are used to detect infections of Drechslera spp. and the method are used to detect infections of Drechslera spp. and the Bipolaris sorokiniana. In wheat, the freezing bloteer method are Bipolaris sorokiniana. In wheat, the freezing blotter method are lots are recommended for treatment when infections, as percentage infected seeds, reach the following thresholds:

-2 D. graminea $/ D$. teres in 6 -rowed barley cvs.

barley crs.

25 Fusarium spp. in barley

$\geq 10$ B. Soroxiniana in barley

$\geq 25$ D. avenae in oats:

$\geq 15$ Fusarium spp. in oats;

$\geq 15$ Fusarium spp. in wheat

$\geq 5$ i nodorum in wheat.

Fungicide seed treatment is also recommended if germination is considerably improved by a rout ine test treatment. The reed for treatment, differs between species, cultivars, growing areas and years. In average over the five years 1990 to 1994 , the treatment have been reduced with 35 compared to the extent of treatment before seed health testing became routine. Because of unusual healthy cereal seed harvested 1994, only about 20 of the seed lots used 1995 have been recommended for treatment. This corresponds to about $2.1 \mathrm{mi} / \mathrm{l}$ dollars $(12.75 \mathrm{mill}$ Nkr) in saved fungicide costs and 1300 kilogram less chemicals (active ingredients\} added to the environment.

The treatment of cereal seeds only according to need is voluntary and based on advisory analysis. However, the experience so far. says that both the seed companies and the farmers, for economic reasons and envirpnmental concern, only treat their seed when $1 \mathrm{t}$ is recommended. Further, with knowledge of which pathogens that occur in a seed lot, it is possible to choose the most appropriate fungicide(s).

Norwegian Agricultural Inspection Service, Seed Testing Station, Fellesbygget, N-1432 Às, Norway

988

REGISTRATION OF THE BIOCHEMICAL AZADIRACHTIN IN THE UNITED STATES A CASE HISTORY

\section{E.R. Butts and J. Martineal}

The United States Environmental Protection Agency has established regulations and guidelines that provide a mechanism for registration of biochemicals requiring significantly less data than are required to register conventional chemical pesticides. These registration requirements are detailed in the "Pesticide Assessment Subdivision M: Biorational Pesticides" and in Title $\mathbf{4 0}$ of the U.S. Code of Federal Regulations Part 158. A registrant may request EPA to classify as a biochemical, a compound that has a nontoxic mode of action and that occurs in nature for is structurally similar and functionally identical to one that occurs in nature). Once that classification is granted functionaily identical to one that occurs in nature). Once that classification is granted, the registrant must conduct product chemistry, acute and subchronic toxicology, and
ecological effects studies to support the registration. AgriDyne Technologies, Inc. has registered azadirachtin under the tradenames of AZATIN ${ }^{\bullet}$, ALIGN*, and TURPLEX This presentation will consider the regulatory process that AgriDyne dealt with to obtain EPA registration of azadirachtin as a biochemical.

E.R. Butts intemational, inc.

26 Sherman Court

P.O. Box 764

Fairfield, CT 06430 U.S.A. 
THE EFFECTIVITY OF MODERN PESTICIDES IN THE CONDITION CIF ANTROPOGENOUS CONTAMINATION FOR AGROCENOSES

Ellipas A.S., Oulianenko L.N., Krokhlov G.N., Yuorevich I.N.

By growing crops on radioactive contaminated areas with application of measures to ontain clear products (satisfyira the standards) in many ceses the ohyto-sanitary situation is significantiy changed. That is why the crop protection from post und deseases is growing in role and responsibility for chosce of used means and methods is increasina. The esseritial features reqired of preparation choice are their high effectiveness and ecological socurity what is conditioned.In order to ecologizate protective measuras the microbiological preparations such as boveriri, bitoxibacililin and others find currently wide use for treatment of potatoes from colorado beetle. In our investigations "Novodor" was used $<a$ fluid concentrate containing $2 \%$ of active albumen of bacteria Bacilicus thuringiensis subsp. tenebrionis produced by the firm "Novo-Nordisk"). This preparation was tested under conditions of tield experiment CBryansk reaion, Novozybkov district, contanination density on radiocausium $-0,56 \mathrm{mBg} / \mathrm{s} \cdot \mathrm{m}$ ) on potatos of divided into districts medium variety "Novekiy". The treatment of potatoe plantations was carried out in amount, of 41 of preparation per ha. During the treatment the mumber of Colorado betie amounted to $604 \mathrm{sn.1} 10$ plant. In this case the larvae of youncer aces were ciresent for the post part $(91,4 x)$ in the population what provided high offectivenese of the preparetion. Alrody 4 davs later. the typical igns of entomopathogenic iffect of the biopreparation were noted. Reduction of pest number on the 7 th day after the treatment was $92 x$ and that of plant population 92,5x. During the 10-14th days after the treatment the larvae of the 1st and those of the 2nd age appeared in potatoe plentations but the pest mumber did not reach economic threshold of harmflness till drying the leafy tops of root vegetables. It should be pointed out that the biopreparation did not increas casium transport into potato tubers. Thus. the conducted investigations lead one to recommend application of "Novodor" a a highly efficient and ecologically safe biopreparation for potatoe protection on radioactive cantaminated territories.

Obninsk, Kaluga Region, 249020 Rusala

Ruseien Institute of Agricultural Radiology and Agroecology

991

IN GEARCH OF THE WAY FOR MINIMIZATION THE LBAGE OF PEBTICIDES ON THE RADIOACTIUE CONTAMINATED LANDS

\section{Qullianenke L.N., Filipas A.s.}

Radioactive contamination of large territories of agricultural lands as a result of the Chernobyl NPP accident brings attention of workers of agroindustrial complex to serise of problems. The main of them is development of crop growing technologies on decreasing radionuclide transfer into the yield and reducing anthropogemic loed on agroecosvitems. raking it into account the ways and methods for plant protection which reduce pesticide use are to be foind. It mav be achiaved at the cost of develooment and iritroduction of ecologically clean practices on increace of olant residance to naratud organisas. One of this ptactices is aresowing cultivation of eeede by complex preparatione providing a greater growth intensity of eedinge, Increasing growth proceseses of plants, high compatitiveness of crops as compared to the weed vegetation as well as icreasing resistance of plants to widely - distributed phytopatogen cespecially during the critical growth stages). The investigations conducted by us show that use of compounds with biological activity combined with baitanmuniversal and trace lemerit additives for presowing incrustation of soeds of barley growing on agricultural lands of the Non-chernozem Zarie of Russia (Novosyboov region) with radioactive contamination deneity of $0,56 \mathrm{MBq} / \mathrm{s}$.m provided a favourable ohyto-yaritary Ituation in sowings: damage by Drechslere teres, relative to the control reduced from 3 to 5 times and that ore by root rot: - by a factor of 7-10. By none of decese arents did cconente threshold of harafulness exceed. Thus, the necesnity of entifungel use for treatment of Dlants during vegetation (1-3 troatments) was excluded. The results obtained by us Llow to suggest that a stable phyto-sanctary ituation in crop cowing: on redioactive contaminated areas may be achieved at the cost of ueing complex preparations with biologicd ectivity for incrustation of sesd what is of great importence for minimization of pesticide use and reducing tectrogenic load on agrocenoses.
EFFICACY OF YEAST AUTOLYSATE BATT SPRAYS IN CONTROLLING FRUIT FLIES INFESTING CAPSICUM IN TONGA.

Nemeve P.S., Langi T.F.

Poisoned bait sprays containing an attractant (protein hydrolysate), water (carrier) and an insecticide (Malathion), have been used worldwide, over the years, for controlling fruit Hies. The efficacy of yeast autolysate ( an improved trit fly bait devetoped in Australia), was investigated, in pre - harvest controf of fruit flies intesting capsicum(Capsicum annum L.), in Tonga. Two concentrations: $80 \mathrm{~m} / \mathrm{s}$ and $50 \mathrm{~m} / \mathrm{s}$ of yeast autolysate / litre of ban mixure. were tested during 1993 and 1994 respectively. Good trut fly control was achieved at both concentratons of the batt, applied weekty, as a course spray to each ptant in every third row. Weekly damage levels during the baiting period, of more than 12 weeks, in both trials . varied between $0.7 \%$ in the treated plots, compared to levels as hign as $97.100 \%$ in the untreated plots. The data obtained, demonstrated further, the bait effectiveness during the warm summer days, when fruit fly pressure was betieved to be high. The fruits found infested during the damage assessments were processed in the laboratory for adutt fly emergence, to determine the fruit fly species breeding in the capsicum. Only Bactrocera tacialis (Diptera:Tephritidae), an endemic species, was reared from the capsicum during the course of the trials. The species showed preference of mature green fruts to young immature fruits, however the latter becarne equally vuinerable to infestation if the former went out of season. The economic and ecological implications of bait spray technique, as well as methods of improving the efficacy of bait spray treatments are discussed.

Fruit Fly Project

Ministry of Agriculture and Forestry

Vaini Research Station

P.O. Box 14, Nuku'alota, Tonga, South Pacific.

Phane: (676) $32125 / 23571$

Fax: (676) $32132 / 24271$

992

ON POTENTIALITY OF APPLICATION OF PHOTOCHEMICALLY UNSTABLE INSECTICIDES IN OPTIMUM AEROSOL TECHNOLOGY OF PLANT PROTECTION

\section{Y. Samsonoy, V. Makarov, K. Koutsenogii}

The negative ecological consequences of the insecticide applications are often related to their persistence in various biosphere components. Due to long residence

times of insecticide poilution in plant, soil, water and atmosphere they are dangerous for people and environment. Possible it would be desirable to use the photo unstable insecticides having shot residence times in air and on open surfaces of leaves or soil. However, it is difficult to use such insecticides in conventional methods of aerial and ground spraying. Here the dispersed insecticide first precipitates on a plant surface thus forming a pesticide deposit. Only then pest insects come into contact with the deposit, accumulate a lethai cose of insecticide and die. For a number of reasons, the period of accumulation cover many hours or a few days. It is evident that photo unstable insecticides which quickly decompose under sunlight do not give such a time reserve.

It is possible to use photo unstable insecticides in the so-called Optimum Aerosol Technology (OAT) of insecticides application. in this technology the lethal cose is accumulated not at contact with deposit but upon the hit of aerosois directly on insect bodies just during passage of aerosol cloud across the treated field. The cloud moves due to wind Aerosols hit on insects via the inertial mechanism of aerosol precipitation. Its efficiency is strongly dependent on aerosol sizes. The main peculiarity of OAT is that each concrete situation needs aerosols of certain, so-called optimum sizes, which provide relatively selective hit of particles on pest insects, but not on plants and soil. In this case the application rates of insecticides would be minimum but would provide the required mortality of pest insects within the planned width of treated area. For the reasons connected with the processes of wind diffusion of aerosols in the so-called stable stratified atmospheric surface layer, the optimum aerosol treatments are performed at night only.

Since in OAT the lethal dose hits on insects just during the night treatment, it is assumed that the photo unstable insecticide could effectively be used as aerosols. In this case, the night treatments must provide the required mortality of pest insects, but insecticide that have been precipitated on plants, soit or diffused in air must fast be phololyzed next morning (day) under sunlight to harmless substances. A fast photo destruction of insecticide pollution could decrease potential danger of pesticide application for people and environment. A few special areas of effective aerosol application just of photo unstable insectici-

des in plant protection could be proposed.

Institute of Chemical Kinetics and Combustion, Siberian Branch of the Russian Academy of Sciences, 630090 , Novosibirsk-90, Russia 
CONSTRAINTS TO CHEMICAL CONTROL OF MAIZE STEMBORER (Chilo partellus Swinhoe) IN MOZAMBIQUE

P.A.Segeren, w. Slobbe, E. Rafael

Although cultural practices like early sowing, field cleaning and the practice of a closed season reduce stemborers to some extent, farmers depend on the use of pesticides for satisfactory control. However, pesticides and spraying equipment, if available at ali, are out of reach of most smallfarmers. Results of experiments on the effect of application of locally made granular formulations and of some botanical pesticides, both designed for use by this target group, are presented.

Address: Departamento Sanidade Vegetal, C.P.4588, Maputo, MOZAMBIOUE

EFFECT OF INTERCELLULAR FLUIDS ON THE INTERACTION OF PEA WITH Pseudomonas syringae PV. pisi

\author{
A.D. Akpa, S.A. Archer
}

The effect of simultaneous treatment with intercellular fluids (IFs) and live bacteria on the responses of pea cultivars Early Onward, Hurst Green Shaft, Fortune and Kelvedon Wonder to inoculation with races 2 and 4 of Pseudomonas syringae pv. pisi was investigated. The IFs were obtained from compatible interactions of $P$. syringae pv. pisi ( $P$ sp) and pea using a modified vacuum infiltration technique. Treatment with IFs alone or in mixture with bacteria resulted in a compatible-type reaction (CTR) on the universal suscept, Kelvedon Wonder. On cultivar Fortune, resistant to both races, sole treatments with the IFs or in mixture with bacteria resulted in an incompatible-type reaction; however, IF4 showed no symptoms and a mixture of

resulted in CTR. Simultaneous treatment with an IF and the bacterial race that produced it results in an incompatible-type reaction in cultivars resistant or susceptible to the race involved, whereas mixed treatment with an IF and the bacterial race different from the one that produced it results in a CTR in cultivars susceptible to the race involved. It is concluded that in incompatibletype reaction, the IFs appear to be specifically recognized by the host, whereas in CTR, they are not.

Department of Crop Protection, Institute for Agricultural Research, Ahmadu Bello University, P.M.B. 1044, Zaria, Nigeria
VARIETAL BASIS OF FUNGICIDAL EFFICACY AGAINST STAGONOSPORA NODORLM BERK IN WHEAT

\section{Tvarủžek, K. Klem}

The field trials were established under artificial inoculation. The inoculum was applied at $D C 51$, and some fungicides with a different type of action were sprayed 7 days later. Visual symptoms of infection on spikes and leaves and fingl yield effects were determined in a set of nine winter wheat genotypes.

AL'DPC values indicated the significance of the factor of variety for the success of fungicidal application. The varieties with genetically based resistance to Stagonospora nodorum Berk. mostly showed the insignificant yield increase after fungicidal spraying.

On the other hand, susceptible varieties usually showed high yield increase. These results suggest that the chemical control of Stagonospora nodorum Berk will not be necessary for all varieties, some fungicide treatments will be uselness.

Agricultural Research Institute Kroměřì, Co. Ltd.

Havlíkkova 2787

76701 Kroměřiz

Czech Republic

996

VIRULENCE STUDY OF STAGONOSPORA NODORUM ISOLATES ON SELECTED CULTIVARS OF TRITICALE AND BREAD WHEAT IN SINGLE AND MIXED INOCULATIONS

\section{E. Arseniuk, P. Czembor \& A.L. Scharen}

Relative virulence of 15 Stagonospora (Septoria) nodorum (SNB) monopycnidiospore isolates and their mixture was studied under field conditions on a differential set of triticale and bread wheat cultivars. The isolates originated from diseased triticale and wheat plants sampled in diverse geographical regions of Pland.

Significant effects of isolates, cultivars and cultivar $x$ isolate interactions were detected. Since the interaction of isolates $x$ cultivars was significant the term virulence is used. The mixture of isolates was classified in the largest group (cluster) $(n=6)$ of isolates showing intermediate vinulence level on the cultivars tested. In comparison to single isolates the differential capacity of the isolate mixture was also reduced. Relationships between mean isolate virulences and cultivar variances of SNB reactions were not significant for leaves (moderate SNB level) and significant for heads (low SNB level) A single pathogenic isolate used for screening of breeding materials may already provide a reliable information on their SNB resistance.

Plant Breeding and Acclimatization Institute, Radzików, 05-870 Błonie, Poland 
POSSIBLE RELATION OF ACTIVE OXYGEN TO PARTIAL RESISTANCE OF RICE TO BLAST

\section{Pasechnik T.D., Aver'yanov A.A., Lapikova V.P., Kovalenko E.D.}

The early stages of the blast fungus (Magnaporthe grisea) development in relation to rice varietal resistance were studied. Drop diffusates of both healthy and infected leaves of the susceptible cultivar (Sha-tiao-tsao) inhibited the fungus spore germination weakly. This action of healthy leaves of the vertically (Zenith and Tadukan) and partially resistant (Shimokita, Norin 18, China 1039 and CNAB kweichow) cultivars varied in strength being dependent on a fungal isolate used. In the infected counterparts the toxicity was always high. The appressorial formation was suppressed stronger on leaves of resistant (Tadukan and Shimokita) than of susceptible cultivars. The fungitoxicity of any diffusate was diminished after addition of catalase, hydroxyl radical scavengers, or iron chelator desferrioxamine. In contrast to the vertically resistant cultivars, the toxic action of the partially resistant ones did not increase under light and was not (or weakly) inhibited by superoxide dismutase, boiling, or long storage. It seems that the suppression of an ectophytic development of a pathogen by host's excretions contributes to the disease resistance. The mechanism of the suppression is mediated by active oxygen species $\mathrm{H}_{2} \mathrm{O}_{2}, . \mathrm{OH}$ and, in some cases, $\mathrm{O}_{2}^{-}$) and transition metals but is different in vertically and partially resistant cultivars.

Research Institute of Phytopathology, p/o B.Vyazemy, Moscow region 143050, Russia

\section{9}

RESULTS ON THE MAIZE RESISTANCE TO THE EUROPEAN CORN BORER (OSTRINIA NUBILALIS)

\section{Al. Băroulescu, O. Cosmin}

In Romania, the most important yield losses caused by Ostrinia nubilalis are due to its stalk attack. Because of this, a maize breeding program for resistance to stalk tunnelling is in progress. For detecting resistance sources, annually, over 700 maize forms were tested under artificial infestation conditions. As a result of these investigations a series of data were obtained. Repeated testing of the resistant inbred lines obtained by inbreeding and selection of different maize forms crossed with sources of resistance resulted in the development of inbred lines with a constant level of resistance. By crossing these inbred lines with maize forms with acceptable levels of agronomic performance, new hybrid combinations were achieved. From these, only inbred lines with low level of attack were selected and tested in the subsequent three years. After that, a new cycle of crossing and screening for insect resistance was carried out. After the $3^{\text {rd }}$ experimental cycle, maize inbred lines with an appreciable degree of resistance to the European Corn Borer have been obtained, also having substantially increased agronomic characters. Taking into account the data of the last year of the $3^{\text {rd }}$ experimental cycle it is noticed that the attack by the borer was 8-fold lower, as judged by the length of tunnels, and 6-fold lower, as expressed by the number of larvae in the maize resistant inbred fines as compared to that of highly susceptible lines. As to the behaviour of maize hybrids existing in practice or only under test, it is worth to stress that all of them were affected by the borer, however two hybrids, not originating from resistance sources, showed a lower attack but insufficient to ensure a satisfactory protection against the attack of this pest.

Al. Bărbulescu, Research Institute for Cereals and Industrial Crops Fundulea, 8264 Fundulea, District Căararași, Romania
In the past 10 years in a Moscow region greenhouse tomatoes have been hardly damaged by phytophthora rots of roots, stens, leaves and fruits. Some years of the epidemics $100 \%$ of damaged plants were noticed. Under prevalling climatic conditions the Phytophthora diseases in the case of the hard ones result in mass death of both seedlings and adult plants. The diseases characterised by high diversity of symptons, which has never been observed formely, for example, root rots of fruiting plants or rots of stens right up to the upex. For the first time the following 4 species of the genus Phytophthora have been determined in the same area: P.parasitica $(38 \%)$, P.cactorum (37\%), P.capsici (19\%), P.cryptogea (6\% of 72 anallysed isolates). Computerzed method was used for for species identification. The isolates have pathogenic specialization to infect host - plant organs. Collection of wild and cultivated tomatoes samples (120 genotypes) were evaluated on resistance to phytophthora species. For this aim the new original method allowed to test both detached organs and growing plants was used. The base of this method is the inoculation of sterile sand with a suspension of Phytophthora zoospores ( 6000 spores $/ \mathrm{ml}$ ) in knop mediua. Plants have been grown or transplanted on accrdance of needed age in a trays $(20 / 12 / 3 \mathrm{~cm}$ - for seedlings, $40 / 40 / 15$ - for adult), which were full of sand evenly saturated with the inoculum (Knop medium in a control). Detached organs were placed on surface of sand saturated in the same way. Tomato resistance to the pathogen has been registered on 7 and 14 days after inoculation. In result of the evaluation tested tomato samples were divided into 4 groups depending upon the level of their resistance: high and middle resistant, also high and middle susceptible. Resistant genotypes make up $8 \%$ of the tested ones. Some of the evaluated samples were characterised by nigh resistance not only to soilborn species, but as previously shown to P.infestans too, for instance L. pimpinellifolium (3990), selective lines 372, 349, 342 NISTIO. Frequently distinctions in resistance for different organs of the same samples were noticed. However L.pimpinellifolium 3990 had high resistance of all organs. obtained data have been confirmed by good resistance of the samples under natural infestation.

Dep. Mycol. \& Algol., Moscow University, Moscow 119899, Russia.

\section{0}

INTROGRESSIVE RECOMBINATION AND ADAPTATION TO PESTS AND DISEASES.

Bastrygine A.S., Senyuk V.I.

Mutation and recombinations are considered as the maln sources of plant genotypic variability conditioning their adaptive potential.

The comparative study of the peculiarities of plant adaptive potential clearly indicates that wild species and primitive forms have - in comparison with cultigens - consicerably wider spectrum of adaptation to abiotic fectors (pests etc.) and to the unfavourable environmental conditions (pollution etc.) as well. Therefore the combination of the high potential of productivity and resistance to the unfevourable enviromental factors and diseases in cultivated plants has inevitale bearing on the developing of and using tile methods of coverning the recombination process.

of freat importance is the further improvement of methods for accounting the natural and induced recombinogenesis for developuent of durable resistance to diseases and pests. Here a special role may belong to electrophoretical analysis due to its high resolution power and productivity. Considering possible negative consequences of the exogenic factors influonce (particularly the environment pollutints such as pests) on the recombination frequency and spectrum the efficient test systems must be developed for higher plants with the aim of monitoring these changes.

Lab of srotein Chemistry, Moldavian state Jniversity,

irateevich str. 60 , Kishinev 277094 , ivoldova 
RESISTANCE TO CERCOSPORA LEAF SPOT IN SUGARBEET: A SIMULATED APPROACH.

V. Rossi, P. Bactilani, P. Racca, S. Giosuè.

It is known that Cercospora leaf spot resistance in sugarbeet reduces the progress rate of epidemics, according to the Vanderplank's concept of horizontal resistance; therefore, level of horizontal resistance of several genotypes may be powerfully quantified by changes in the apparent infection rate. However, in sugarbeet, as well as in other pathogens, the infection rate calculated for epidemics progressing under different environmental conditions do not reflect accurately the level of resistance; in fact, the reduction of infection rate in the resistant genotypes changes significantly in time and space. It forces breeders to repeat trials in several environmental conditions, increasing selection time and cost.

According to other host-pathogen systems, a better evaluation of host resistance level could be made by measuring each component of horizontal resistance. In the case of sugarbeet, several mechanisms of host resistance are well studied, but sufficient information about components of resistance are not yet available. Using four sugarbeet genotypes which show different disease levels under field conditions, we carried out several experiments under controlled and natural conditions. We observed that the following components of horizontal resistance significantly influence the progress of epidemics: infection efficiency of conidia, lenght of incubation period, size of necrotic spots, amount of conidia produced on necrotic spots. We used a modified version of the simulation model CERCODEP to simulate the effect of each component of horizontal resistance on the progress of Cercospora leaf spot epidemics in several environmental conditions.

We think that 1) the measurement of resistance components of selecting genotypes in simple monocyclic experiments under controlled conditions and 2) the use of CERCODEP model to simulate their effect on disease progress in field under a wide range of environmental conditions may lead to a) an increase of the accuracy of genotype resistance level assessment, and b) a significant reduction of time and cost required for genotype evaluation.

Istituto di Entomologia e Patologia vegetale, U.C.S.C., Via E. Parmense 84 - 29100 Piacenza-Italy. Tel. ++39523599254 E-Mail PATOLO@PC.UNICATT.IT

1003

HISTOLOGICAL OBSERVATIONS ON THE EFFECT OF QUANTITATIVE RESISTANCE ON THE DEVELOPMENT OF PUCCINIA STRIIFORMIS F.SP. TRITICI.

\section{H. M. Broers, A. M. Lopéz Atilano}

Flag leaves of one highly susceptible and three quantitatively resistant (QR) spring bread wheat genotypes were brush-inoculated in two independent experiments with urediospores of yellow rust to study the effect of resistance on development of the fungus during the first six days of its infection cycle. Survival ratios indicated that the most important bottleneck implemented by $\mathrm{QR}$ was the formation of appressorium-like structures (ALS). On QR-genotypes, twice as many germ tubes cvergrew stomata than on the susceptible Morocco resulting in a lower infection frequency. Most of the infection structures (IS) which terminated on a stoma were able to torm an substomatal vesicle (SSV). However, the percentage of infection structures with ALS which also formed añ SSV decreased in time in the three QR-genotypes but not in Morocco suggesting that some SSV were disintegrated due to resistance. On Morocco, between 81 and $91 \%$ of the infection structures with an SSV were able to form infection hyphae with haustorium mother cells (HMC) within six days after inoculation. On resistant genotypes, the formation of the first hyphae was retarded as only between 48 and $72 \%$ of infection structures with SSV formed first infection hyphae. Forty-eight hrs after inoculation, no genotype had infection structures with more than five HMC. Six days after inoculation all infection structures with infection hyphae were considered established (more than five HMC) on Morocco. On the resistant genotypes, 71 to $96 \%$ of those infection structures were considered established. This result shows that the development of the fungus is slower on resistant genotypes.

This temporal study of the yellow rust infection cycle explained the differences between susceptibility on one hand and quantitative resistance on the other. However, the differences in level of OR (measured as latency period and infection frequency) are only partially explained by the results of this study. Therefore, it is argued that after the first six days of fungus development, events happen that differentiate among the levels of resistance.

Department of Plant Breeding, Wageningen Agricultura| University, P. O. Box 386, 6700 Aw Wageningen, The Netherlands / International Maize and Wheat Improvement Center (CIMMYT), Lisboa 27, Apdo Postal 6-641, 06600 Mexico D.F., Mexico
ENHANCEMENT OF RESISTANCE TO PUCCINIA RECONDITA TRITICI OF WHEAT BY INCORPORATION THE STRONG GENES IN SOURCES OF RESISTANCE

*Boskovie, J.. Boskovie, M., Prodanovie, G.

It is well know that the best way to control wheat leaf It is woll know that the best way to control wheat leaf rust caused by Puccinie recendita tritici is the developtests of International Leaf Rust Nurseries in the past, ixteen resistant donors have been selected and crossed with a recurent parents wheat varieties Princ and Starke, as a background- In other to enhancement the resistance of these hybrids, eight selected the most interesting lines have been crossed with only effective isogenic lines con taining the strong gones Lrg, Lr19 and Lra4. The genetic analysis of twanty two crossing combinations have buen re alized by testing with thres pathotypes of Puceinie resendita tritici (BO-F. 12/89/ 15.w. 8/89 and Ch1.w. 14/89) in $F_{2}$ gereration. The different segregation ratios between rewistant and cusceptiblo plants within the progenies of all crossing combinations proved that no ane of the resistan donors did not cantained the strong resistent genes Lra, Lr19 and Lre4. It means, that our hybrid lines contained resistant genes from the donors and in addition three strong resistant genes. Some segregation ratios demonstrat ed dominance of resistance and the other complementary dominant geres. Important influence to segregation ratios hove had the cultures of $P$. recondita tritici used. Thus, segregation ratios and the number of resistant venes of 21 cross comblnations were different using first two cultures but where the wawe with the donor 94. Selected hybrid wheat lines from this crosses hove been tusted in ELRWN nurberjes on the large European-Mediterramean regions and showed very good resistance.

"Bokkovic Jelena, Faculty of Aoriculture, Institute for Plant Protection, Trg D. Obradovita B, 21000 Novi Sad, Yugoslavia.

1004

STABILITY OF THE ALIEN CYTOPLASM EFFECTS ON PARTIAL RESISTANCE OF WHEAT ALLOPLASMIC LINES TO POWDERY MILDEW POPULATIONS

\section{A.A. Bulovenik and E.A. Voluevich}

The objects under investigation were 31 wheat alloplasmic lines with nuclear genome of Penjamo 62 (P62) cultivor and cytoplasms of Triticum, Aegilops, Agropyron species developed by prof. I. Panayotov. Cytoplasmic effects on the components of partial resistance, percentage of disease development and reproductive abilitity of powdery mildew population (per $1 \mathrm{~cm}^{2}$ of leaf area), on the two antogeresis stages, 3ro and flag leaves, during three years were analized. For most of alloplosmic lines these porameters were shown to depend on the environment and, perhaps, changes in pathogen population. However, stability of cytoplasmic effect manifestotion on resistance was revealed for several lines. The line with cytoplasm of $A e$. sharonensis was detected to keep lower percentage of disease development on the third leaf in comporison with P62. Plants of the alloplasmic line with cytoplasm of $T$. zhukovskyi at the same growth stage had both analized components of partial resistance on the level of P62 for three years of experiment. At the late ontogenesis stoges alloplasmic lines with cytoplasms of $A e$. aucheri, Ae. mutica and Ae. biuncialis steady stimulated reproductive ability of the pathogen in comporison with euplasmic control. Line with the cytoplasm of $\mathrm{Ae}$, shoronensis had decreased level of both parameters of resistance on the flog leave in comparison with control independently of the year of experiment. The anolysis of alien cytoplasm effects on the percentage of disease development showed preservation of this paraneter at the control level in spite of the ontogenesis stage for olloplasmic line with the Ae. longissima cytoplasm and its significont increase for Ae. variabilis one. Fungus reproductive ability on the line (monococcum)-P62 during plant ontogenesis did not differ from spore-formation on the euplasmic control.

Institute of Genetics and Cytology, Belarus Academy of Sciences, F. Skorina st., 27, Minsk 220072, Belarus. 
The Effect of Russlan Wheat Aphid Feeding On Chlorophyll a Fluorescence Induction KInetics of Resistant and Susceptible Cereals

\section{JOHN D.BURR AND NORM C. ELLIOTT}

Flant entries that had tested previously as resistant or susceptible to Russian wheat aphid. Diuraphis noxis, were used to evaluate the effect of aphid feeding on leaf chlorophyll content and in vivo chlorophyll fuorescence induction kinetics. $D$. noxia feeding for $96 \mathrm{~h}$ caused significant reductions in Chlorophyll $a_{1}$ Chlorophyll $b_{1}$ and total chlorophyll content in susceptible wheat ('PAVON', 'TAM $W$-101') and barley ("MNTERMALT). Total ehlorophyll content and constituent chlorophyli $a$ and $b$ levels were not significantly affected by $D$. noxia in resistant wheat ( $P|366616, P| 372129$ ) or barley ( $P \mid 366450)$ and in neither resistant (PI 386156) nor susceptible ('BEAGLE 82') triticale. $D$ noxia infestation resulted in significant alterations of the primary fluorescence induction transients. When compared with noninfested control plants, infested susceptible wheat and barley showed significantly increased non-variable fiuorescence $\left(F_{0}\right)$ and decreased maximal fiuorescence $\left(F_{m}\right)$ and variable fluorescence $\left(F_{v}\right)$. In contrast. values for these parameters did not differ significantly among the infested and noninfested controls of resistant plants. When compared with control plants. the photochemical efficiency of photosystem II $\left(F_{v} / F_{m}\right)$ and the half rise time from $F_{0}-F_{m}\left(t_{1 / 2}\right)$ were reduced significantly in the infested susceptible entries but remained relatively unchanged for the infested resistant cultivars. No significant differences between infested and noninfested plants were observed in the measured chlorophyll fluorescence parameters for either the resistant or the susceptible triticale which implied a much different resistance mechanism(s) to 0 . noxia. The results of this investigation suggest that $D$. noxia damage goes beyond the simple removal of photosynthates from the plant. The substantial decrease in $F_{v} / F_{m}$ following aphid infestation for the susceptible wheat and barley indicated a significant decrease in the capacity and efficiency of the primary photochemistry of photosystem II.

\section{USDA-Agricultural Research Service}

Plant Science \& Water Conservation Laboratory

1301 N. Western Street

Stillwater, Oklahoma 74075 USA
THE INFLUENCE OF MUTANT LOCI ON TOMATO RESISTANCE TO FUSARIOSE

Chernov A. A. , Maliarchuc G. G.

Correlations are known plant resistance to phytopathogen affection and some morphological or biochemical characteristics (Robinson et al. . 1970; Medina and Stevens, 1980). The present study aims at studying the relation between the morphological markers $\theta$. ful (chromosome 4) and hl, a (chromosome 11) and tomato resistance to Fusarium oxysporum $f$. $\mathrm{sp}$. lyoopersici. The studies were carried out at a common senetic backeround in the Fa population (ko $628 \times$ Moldavian eariy). In all, 2000 plants were studied. The size of sumpling is sufficient to exclude effects related to segregating for other traits. The inoculated plants were surveyed for 20 days after inoculation and were visually classified into two categories: healthy - no external or internal symptoms, and diseased - completely dry, witing or sturited with vascular browning. The genetic analysis of F2 segregation in the experiment and control showed that the markers e, ful and a decrease the plant resistance to fusariose significantly $(P<0.001)$. The share in the segregation made, as compared with the control, $40.5 \%$ for the e marker, $10.7 \%$ for the ful marker, $47.6 \%$ for the a marker. The hl marker did not reduce the resistance to the pathogen statistically significantly (P<D. 01). The effect of gene interaction increasin the resistance is possibie for the a and e markers, i.e. the share of plants having the aase senotype in the experiment made $55.6 \%$ as compared with the control, which exceeds the similar rat 10 for the a and e markers considered individualiy. Thus, the hl gene is most promising to be employed as a genetic marker in the study of the resistance to fusariose, as it has no negative pleiotropic effect on the trait and is located in the same group of lincage with the genes responsible for resistance to fusariose.

Institute of Genetics, Acad.Sci., 20 Padurilor str. Kishinev 27roo2 Moldova

1008

CHARACTERIZATION OF MNTHOHOARS CAMESTRTS PV VESICATORIA IN THE FRENCR AEST IRDIES AND EVMLUATIOA OF RESISTANCE IN TOMATO.

A. Darragse, A. Col6, s. Leclercq, S. Bart, G. Ano and P. Prior

Bacterial spot disease caused by Xanthomonas campestris pv vesicatoria is recognized as a serious disease of tomato plants in most of the tropical countries, including the French west Indies. Field and quality of fresh-market production are severily affected, especially in arable lands with an irrigation program supporting the development of intensive vegetable production.

As for other bacterial diseases, growing resistant tomato plants remains the major control strategy, with site specific crop managenent. Breeding program for resistance imply local evaluation of available sources of resistance and evaluation of the pathogen population variability.

Two hundred Btrains vere collected in Guadeloupe and Martinique (FWI). Results from biochemical and physiological features and pathogenicity tests allowed a preliminary selection of 30 straing (including reference strains) to search for molecular markers of polymorphism. RAPD and RFLP (HrP and 16g+23s regions) studies are in progress. Markers which are consistent with biological properties of strains will be used to characterize the entire collection.

In addition, ten tomato lines were evaluated both for leaves and fruit resistance during summer 1994 in Guadeloupe. Diseese severity under natural infections was compared with artificial spray infections using indigenous strain G12-1. Hawai 7998 , Ohio 4013 and sugar were confirmed to have leaf resistance to $x \in v$. Additional resibtance to bacterial spot vere observed on the fruits of Ohio 4013 and Sugar. Artificial infection is reparted to be more efficient in screening for resistant material when compared to natural contaminations. Experimental data on the polymorphism of straine of Xev from the FWI are presented. This variability will be further considered in the breeding program by using a more representative inoculum in the selection procecture.

Institut National de la Recherche Agronomique, Unité de Recherches en Productions Végtales, Phytobactériologie Amelioration des Plantes, BP 1232, 97185, Pointe a Pitre Cedex, Guadeloupe (FWI). 
HEAD BLIGHT REACTIONS OF WHEAT CULTIVARS TO FUSARIUM

GRAMINEARUM.

\section{H.R. Etebarian and M. Torab1}

Head blight reactions of 27 Irantan, three Chinese and two United States midwestern wheat cultivars were determined in field trials against a mixture of 11 1solates of Fusarium graminearum that had been collected from different locations In Gorgan and Mazendaran of Iran.A randomized complete block design with 3 replicates was used. Plants were inoculated when the main spikes of the earliest cultivars began emerging from the boot, and inoculation were cont inued every other day until all cultivars had been inoculated 10 times. The methods of determination of Percentage of necrotic spike (incidence) and visual rating scales were used for evaluation of wheat cultivars. The results indicated That chinese cultivars Su Mat 3, Shanght 5 and Shanghi 3, Minnesota cultfvar Marshal, Iranian cultivars, Omid and Ghafghaz vere most resistant but Iranian cultivars Bolani, Darab, Ghods, Falat, Sorkh Tokhm, Azadi, Arvand Mutant, Golestan and Minnesota cultivar Wheaton were among the most severly infected.

Abourayhan Institute, Tehran University P.0. Box 11365/7117, Tehran, IRAK.

1011

HYPERSENSITIVITY AS A MAJOR PLANT INDUCED DEFENSE AGAINST GALLING INSECTS

G.W. Fernandes, A.C.F. Lara, L.M. Araújo, L.R. Allain

We report on the mortality factors affecting the survivorship of an undescribed cecidomyid (Diptera: Cecidomyidae) leaf galle on Eauhinia sp (Leguminosae) in a cerrado vegetation in Minas Gerais, Brazil during a two year period. Our goal was to unrave the importance of plant driven mortality on the galling population, a much relevant but neglected aspect in insect herbivore population dynamics. Hypersensitivity is an important host defensive response to infection by gall-forming insects. This plant induced defense encompasses morphological and histological changes terminating in the death and/or necrosis of the infected tissue. It leads to a disruption of nutrient supplies to the larva and the production of many toxic metabolites resulting in the cessation of gall growth. We also studied the relationship between plant vigor and hypersensitive reaction. A recent hypothesis proposes that insect herbivores attack the most vigorous plants or plant modules where their larvae achieve an increased performance. We verified whether gallers suffered aifferential mortality in the plant module (shoots) population. We randomly sampled 50 shoots on 20 plants in both 1993 and 1994 and recorded shoot length, number of leaves per shoot, number of gallec leaves, and number of galls to correlate with galler success (predation, parasitism, pathogens, and hypersensitivity). The most relevant gall mortality factor was caused by plant hypersensitive reactions. In both years; mortality caused by this plant resistance mechanism was higher than 908 . Plants were able to locate the gall inducing site very early in the gall development and elicit a response. plants also varied significatively in their response to insect attack. Thus, hypersensitive reaction was very effective as a defense mechanism whereby plants escaped herbivory. We found no significant relationship between galling success and plant module vigor (shoot length, number
leaves per shoot). Hypersensitive reaction may be under the leaves per shoot). Hypersensitive reaction may be under influence of other plant traits, such as plant hormones, secondary chemistry, and/or under the influence galler ability to manipulate the host tissue. The inclusion of plant resistance as a phenomenon in life table analysis is needed to unde preference and larval performance in insect herbivores.

Ecologia Evolutiva de Herbivoros Tropicais/DBG, CF 486, ICB Universidade Federal de Minas Gerais. 30.161-970 Belo Horizonte
(MG) BRA2IL.



Fandeeve E.

5 genotypes of triticele culivared in joldove vere studied in the experiments. Immature erbryo vere cultiveted on the nutrient meitum 55 a cul ture? fil trete of the Fusertum ortsporum ent medium 5. A culture filte of the Fuseriur. oxisporum. and fusaric asid isoleted from the garne filtrate vere used as selective cgants in the selection of resiatant lines. The con certretion of theoulturel filtrste and fusaric asid vas from 0 to $35 \%$ and from 0 to $150 \mathrm{me} / 3$, respectively. The traits of
the freauency of callus pormation, cellus weight and gize, fre-
cuency of embryo germinetion and root formation were seored. cuency of embryo germinetion and root fometion were scored. The results obtained show thet the frequency of callus formation on media conteining the cultural filtrete is relatively lorer then thet on medie supnlemented with fusaric esid for turel filtrete as compared with fusaric esid. Furthermore, the incese of thecultural filtrete concentretion in the nutrient medium conposition ebove 35 leads to reduction of the frecuency of ceilus formation to $O$, , but to incrsase in the freau ency of embryo germination to gor: depending on the culturei filtrete concentration and senotype. This proves the presencof phytohomones in the cul tural filtrete that contribute to renid germination of embryos, which, in its turn, mekes it impossibie to select resistart Iines. The employment of fussric csid in the composition of the nutrient medium leeds to the decresse in the frecuency of cellus formation, weght arc size of calluses in comperison wit the control. The concentration atint cenotypes for tie restotance to fuseric esid. whis, it cin be concluded thet fuseric esid and the cultural filtrate ere selective cgerts ir. the composition of nutrient media for aeloctine triticale ines resistent to fusarioge in the In tro culture, but fusaric asid is most efficient ir. this ca. : a males it posgible to rork it the level ci csilus, it is cresent in netabcites of $\varepsilon 11$ the fungl of the Fusertus, genus; 1 the prineipal pathotorin of the fuscriom genus end

277002, Institute of Genetics, str. Fedurft 20, Kishinev,
loldove.

\section{2}

IDENTIFICATION OF SOME ASPARAGUS VARIETIES RESISTANT OR TOLERANT TO PUCCINIA ASPARAG I D.C.

F. Fiune

Fesearch has been carried out to evaluate the behaviour of some varieties and hybrids of asparagus (Asparagus officinalis $\mathrm{L}$.) against asparagus rust caused by Puccinja asparadi D.C. The Puccinlaceae fungus 15 responsible for serlous danage, in Ital. particularly where environiental conditions are very favourable. spring, decoloured, yellowish and oblong spots are produced on epinean parts of young plants. Pycnia and aecia appeared on the epigean parts of young plants. Pycnia and aecia appeared on the
spots. The pycnia are the means by which new races of rust arise spots. The pycnia are the means by which new races of rust arsse $t i n g$ new races of the fungus. Aeciospores infection gives rise ting hew races of the fungus. Aeciospores infection gives risering the summer and autum, the ureciospores then serve as the repeating stage and are wind-borne to plants where new generations of urediospores are formed. Blackist telia, at first sub-epiderinc and then dehiscent, thus completing the life cycle. The plants can parch, the below spears are suffering and pathogens. The control of asparagus rust is possible by using chemicals such as zineb, mancozeb, maneb, bitertanole, alone or in combination with captan and EBI cyproconazole and tebucanazograms on the resistant variety study, 18 asparagus varieties and hybrids have been compared, replicated four tames in a randomized complete block experiment design, in order to search that ones showing the best benaviour to escape the disease. The phytopathological observations have been carried out on november when the control plots have showed $100 x$ of infected plants. For each plots, the number of diseased plants has been registered; al] the plants have been grouped into infection classes, calculating the frequences. Therefore the disease severity have been rated according to the following scale: $0=$ no symptoms: more $10 x$ of snoot lenght: 2 = less $20 x$ of infected shoots with up to 20 pustoles for no more $20 x$ of shoot length; $3=1$ ess $40 x$ of infected shoots wath up to 80 pustoles for no more $50 x$ of shoot length; 4 = less $80 x$ of infected shoots with up to 200 pustoles and over, confluent too, for no nore Box of shoot lengnt; 3 = completely infected or dead plants. For plots, the severity and diffusion of the infection have been obtained by resorting gnificantly, the best hehaviour. They have exhibited $37 x$ (plants only belonging to class 1 ) and $42 x$ of diseased plants, $9 x$ and $16 x$ of Mckinney index, respectively. The most susceptible plots have showed $100 x$ of infected plants and $100 x$ of MEKinney index. The resistant plants have stiowed a good tolerance to Fusarium oxysporun f.sp. asparagi Cohen and Rhizoctonia violacea Tul. too Sezione Blologia, Fisiologia e Difesa, Istituto Sperimentale per
l'Drticoltura, Via Cavalleggeri 25,84098 Pontecagnano-Sa, Italy 


\section{VARIETAL RESISTANCE AND CHEMICAL CONTROL OF WHEAT}

(TRITICUM AESTIUUM L.) BLAST (PYRICULARIA GRISEA)

Pest problems can cause severe crop damage to groundnut (Arachis hypogaea L.). with diseases frequently among the most yieldhypogae L.). With diseases frequently among the most yieldinto breeding for disease resistance in groundnut. Diseases that have been evaluated include late leaf spot (Cercosporidium personatum Berk. \& Curt. Deighton), early leaf spot (Cercospora arachidicola Hori), southern stem rot (Sclerotium rolfsi Sacc.), rust (Puccinia arachidis speg.), web blotch (Phoma arachidicola Marasas, Pauer, \& Boerema), cylindrocladium black rot (Cylindrocladium crotolaria (Loos, Bell sobers), spotted wilt virus (TSWV). Using field, greenhouse, and laboratory procedures significant progress has been made in identifying peanut genotypes with multiple disease resistance. identifying peanut genotypes with multiple disease resistance.
Emphasis has been placed on resistance to late leaf spot (CP). Emphasis has been placed on resistance to late leaf spot (CP).
stem rot (SR), and TSWV, which are primary disease problems' in stem rot (SR), and TSWV, which are primary disease problems in
the southeastern USA production area. The Southern Runner the southeastern USA production area. The Southern Runner variety (1986) was released as a result of this effort, with
resistance to CP, SR, rust, web blotch and TSWV. Breeding resistance to $C P$, $S R$, rust, web blotch and TSWV. Breeding
material with even better resistance to $C P$, $S R$ and TSWV have material with even better resistance to $C P$, SR, and TSWV have been developed and identified in recent years. Field studies have shown that some of these lines produce pod $y$ ields over 4000 $\mathrm{kg} \mathrm{ha}$ with no fungicide sprays for leaf spot control, compared to $3200 \mathrm{~kg} \mathrm{ha}$ for Southern Runner and less than $2000 \mathrm{~kg} \mathrm{ha}{ }^{-1}$ for Florunner. Some of these partially resistant genotypes produce pod yields over $5000 \mathrm{~kg} \mathrm{ha}^{-1}$ with four or less foliar fungicide applications. PI203396 is a primary source of resistance to late leaf spot. The main mechanisms of resistance are longer latent periods, reduced lesion size, and reduced sporulation. Groundnut genotypes that have shown good resistance to $C P, S R$, and TSWV include F79X4-6-2, UF81206-2-24-B, and UF91108. All of these genotypes have shown significant yield and disease resistance advantages over Southern Runner in field tests in Florida. In s. rolfsii inoculated, paired-plot yield tests, using a 1-5 disease severity scale, $79 \times 4-6-2$ rated a 2.2 , compared to 4.1 for the susceptible Florunner. Pod yield loss was 23.48 for $79 \times 4-6$ 2 , compared to 63.68 for Florunner. Several genotypes with good resistance to $C P$ and $S R$ have recently been identified as having good resistance to TSWV. Prospects appear to be very good for the release of a commercially acceptable peanut cultivar with excellent production and resistance to $C P, S R$, and TSWV.

North Florida Research and Education Center, University of Florida, 3925 Highway 71 , Marianna, Florida 32446.

1015

RELATIVE SUSCEPTIBILITY AND RESISTANCE OF SOME SORGHUM GENOTYPES TO SPIDER MITES WTTH INFLUENCE OF FERTILIZER SOURCES

A. Taha Hassan, M.S. Abd-El-Fattab

Twenty sorghum cultivars were evaluated for their relative susceptibility to the red spider mite Tetranychus urticae Koch infestation during the season 1992 with influence of fertilizer sources on population abundance of mites.

Obtained results indicated that the level of mite infestation were devided into four categories: Highly resistant, resistant, intermediate and susceptible, whereas sorghum cultivars local 29 and Sel 113 expressed high resistance while Giza 15, Sel.G.pp III, local $1 / 89$, local $56 / 82$, local $53 / 82$, local $36 / 82$, local $33 / 82$ and local $31 / 82$ were resistant. The opposite was found with family 334 , local $6 / 77$, local $218 / 77$ and local $217 / 77$ which expressed high level infestation while the rest of cultivars were intermediate in their level infestation.

Data idicated that population abundance of spider mites was affected by fertilizer sources, whereas adding nitrogen above increasing population density of mites than mixed with potassium or potassium alone.

Plant Protection Research Institute, Nadi El-Said Street Dokki, Cairo, 12611 Egypt; Field Crops Research Institute, Nadi El-Said Street, Dokki, Cairo, 12611 Egypt.

\section{A.C. GOULART; F. de A. PAIVA; P.J M ANDRADE}

The wheat blast disease, caused by Pyricularia grisea, was reported by the first time in 1985, at the Northern region of the Parana State. Since then, the disease spread to other states and nowadays wheat blast is present at all wheat growing regions. Pynicularia species were not reported as causing economical damage to wheat in other parts of the world, but in Brail the seriousness of the situation was demonstrated in Mato Grosso do Sul State, where the yield loss was estimated to be $29 \%(862 \mathrm{~kg} / \mathrm{ha})$. The objective of this work was to evaluale the behaviour of different wheat cultivars in relation to the blast under field conditions as well as to select fungicides to control $P$. grisea. The experiments were carried out under field conditions, during 1990 and 1991, at Itapora county, Mato Grosso do Sul State, Brazil. For the varietal resistance experiments, 25 wheat cultivars were evaluated and for the chemical control the used cultivar was Anahuac in both years. The following fungicides were tested: mancozeb $(2,000 \mathrm{~g} \mathrm{a.i} / \mathrm{ha})$, methyl thiophanate + mancozeb $(490+$ $2,240)$, trifenil tin acetate + mancozeb $(88+1,248)$, tricyclazole $(225)$, tebuconazole $(250)$, prochloraz (450), fusilazole (125) and untreated control. The fungicides were sprayed omto the plants three times. The first at Zadoks growth stage 54, with two more with a twelve days inierval. The evaluations for both experiments were made at the laboratory and only the spikes that showed the characteristic blast symptons (black infection point in the rachis) were considered as infected. The least infection index was observed on the BH 1146 cultivar, which showed no more than $5 \%$ of infected spikes. Incidence of up to $25 \%$ was observed on the BR 18-Terens, BR 21-Nhandeva and BR 40-Tuiúca cultivars. On the other tested aultivars, the incidence varied from $35 \%$ to $99.5 \%$ of infected spiles. As for the fungiciles, tricyclazole and tebuconazole, followed by methyl thiophanate + mancozeb and mancozeb were the best for controlling wheat blast. Poor control of this disease was observed (maximum of $39 \%$ ), showing the low efficiency of the tested fungicides. All fungicide treatments increased yield above the unsprayed control, with distinction to tricyclazole and tebuconazole. A correlation analysis showed in both years, astinction to highly significative correlation coefficient between spikes infected by $P$. grised and yield.

EMBR 4PA-CPAO Caixa Postal 661, 79804-970 - Dourados, MS. Brazil.

1016

ULTRASTRUCTURAL LOCALIZATION OF BACTERIAL POLYSACCHARIDE IN XYLEM VESSELS OF RICE LEAVES INFECTED WITH Xanthomonas oryzae pv. oryzae

O. Horino, M. Watahe, Y. Kubo and S. Tsuge

Infection of bacterial leaf blight of rice with Xanthomonas oryzae pv. oryzae (XOO) is accompanied by the formation of two types of fibrillar materials in xylem vessels where the causal bacteria have invaded. Staining ultrathin section with alkaline bismuth, electron dense and lucent fibrillar materials (DFM, LFM) were observed in our previous study. DFM was major material in infected xylem vessels and often filled the xylem vessel. LFM was localized in the area close to bacterial cells. The main purpose of this investigation is to elucidate properties of DFM and the relationship between DFM and bacterial extracellular polysaccharide (EPS) by immuno-electron microscopy. The antibody could detect EPS at concentrations above $0.1 \mu \mathrm{g} / \mathrm{ml}$ using ELISA. Immuno-electron microscopic observation of xylem vessels of rice leaves infected with XOO showed that the colloidal gold labeling was over the outer layer and cell surface of the bacteria. No gold particles were observed for the other parts, including xylem ressel walls and DFM that filled the infected xylem vessels. Xylem ressel walls often degenerated and DFM was likely to be derived from degenerated cell walls. XOO was markedly enveloped by DFM in the xylem vessels. This strongly suggests that the DFM was not the EPS. The results also showed that localization of EPS coincided with that of bacterial cells and was restricted to the area close to bacterial cells. The DFM was not observed in uninoculated rice leaves. The amount of EPS may not be enough to fill and plug xylem vessels and prevent water flow. Therefore, we conclude that EPS is not directly involved in the virulence of XOO. However, it is not clear whether EPS may play some role other than facilitating the movement of bacteria, which, in turn, may affect the induction of symptoms by XOO. 
1017

\section{BIOLGGICALLY ACTIVE PLANT COMPOUNDS AS REGULATORS} OF PLANT-PHYTOPHAGAN TROPHIC INTERACTIONS.

\section{L.S.Ivashchenko}

The studies of biochemical mechanisms of host plant - insect interactions have been conducted over period of years. The dominance of food connections was revealed as a consequence of the investigation of the phytophagan - host plant system. The physiological barrier has been demonstrated to be among the main wechanisms of plant resistance to pests. This barrier is conditioned by the action of physiologically active plant compounds. The growing and reproductive functions of insects were found to be depressed in a consequence of the feeding on resistant plant genotypes. The resistance of some crops icereals, solanaceous, cotton has been demonstrated to be concerned with structure and concentrations of plant secondary metabolites, such as poliphenols (DIMBOA, gossipol, tannin) and alcaloids ( - tomatine, demissine). An increase in concetrations of these compounds determines the enchancement of oxidase (cytochromoxidase, succinoxidase monoaminoxidase) astivity. At the same time the activity of such oxidases as polyphenoloxidase and peroxidase is reduced as a result of changes in energetical metabolism. The plant secondary metabolites include in metabolism of phytophpgans. As a result, toxic compounds may arise, and the additional consumption of energy became necessary for its neutralization. At the same time the fraction composition of proteins, ratio of aminoacids, lipids and fatty acids are changed. This fact substantiates the proposal that plant secondary metabolites play a large part in regulation of phytophagan metabolism.(The investigations were partly supported by Russian Fund for Fundamental Investigations, grant N $93-04-21920$ )

A1-Russian Instituto for Plant Protection, Sh.Podbolscy 3, St.Petersburg-Pushkin, 189620, Russta

1019

PATHOGENTC YARIABILITY OF PERONOSCLEROSPORA SORGHI (WESTON AND UPPAL) C.G. SHAW ON SOREHIJY IN AFRICA

C.H. Bock ${ }^{2}$, M.J. Jeger, L.K. Mughogho, K.F. Cardwell, V. Adenle, G. Kaula, E. Mtisi, and D. Mukansabimana

Sorghum downy wildew (SDM), caused by $P$. sorghi infects both sorghum and maize crops and can result in severe loss of yield as systemically infected plants are usually sterile and thus produce no grain. The most desirable stracegy to contzol this pathogen is through host plant resistance However, pathogenic variability within $F$. sorghi has greviously resulted in the breakdown of resistance in the USA. In Africa, as improved.

genetically-uniform varieties replace the traditional. locally-adapted land races, the pathogen population will come under increasing selection for pathotypes to infect these new varieties. The widespread and potentially damaging nature of this disease makes it desirable to obtain information on the pathogenic variability of $P$. sorghi in Africa, which will give an indication of the durability of resistance.

Nine isolates of $P$. sorghi were sampled from different geographic locations and hosts in Africa: from sorghum (Sorghum bicolor). two from Zimbabwe, one each from Zambia, Kenya and Rwanda; from maize (Zea mais), two from Zimbabwe, one from Mozambique; and from wild sorghum ( $S$. arundinecium), one from 2 ambia. Conidia suspensions ( $1 \times 10^{\prime}$ conidia/ml) of these 1solates were prepared Erom systemically infected leaf material and individualiy spray inoculated, unili run-off, onto 2-day old seedlings of fourteen varieties of container grown sorghum. The varieties inoculated were QL-3, TX 412, RTX 7078, DMS 6S2, 82BDM499, 85E0N495, SC414-12E, RTX430, SC170-617. CSj541, SClSSC, SV-1, SV-2 and TAM428. The 1noculacion was undertaken in a controlled environment ( $22 \circ \mathrm{C}$. $>95 \mathrm{RH})$ using a hand held sprayer. The different varieties were assessed for the incidence of systemic SDM infection at one week intervals up to three weeks after inoculation. Cluster anslysis of the disease incidence data with respect to isolate indicated that the pathogenicity of isolates from geographically-close locations was similar, regardless of host. Those from Zimbabwe were least pathogenic, causing a susceptible reaction in fewest varieties - the incidence of systemic infection exceeded $80 \%$ in only three of the varieties tested (DMW 652, RTX 7079 and IX 412). The isolate sampled from sorghum in Ruanda was the most pachogeric, the Incidence of systemic infection exceeded $80 z$ on nine of the varieties tested. Only the varieties QL-3 and SC155C renained resistant to all nine isolates. The variety sv-1, which is commercially available in Zimbabwe was resistant to the isolates of $P$. sorghi from 2 id abwe, but was susceptible to the isolates from 2 ambis, Mozambique, Rwanda and Kenya.

These results demonstrate significant variation in pachogenicity among populations of $p$. sorghi in Africa. This has implications for resistance breeding. quaranci the deployment of presenty avallable resistant varieties, and disease between different regions.

1 Natural Resources Institute, Central Avenue, Chatham Maratide, Chatham, Kent KEA $4 \mathrm{IB}$, England. UK.
1018

In vicro gelection of callus resistant to Cercosporidiug

personatue and regeneration of plants in groundnut

(Arachis bypogaea [.)

PBROMAL VELRATACHALAM AND MARAYARASAMY P. JAYABALAM

Callus cultures were established from hypocotyl explants of arachis hypogaea L. cultivar TMV-7 (Tikka disease gugceptible) on MS medium supplemented with NAA $(4.0 \mathrm{mg} / 1)$ and $\mathrm{KIN}(0.5 \mathrm{mg} / 1)$. Growth of callus was monitored over a 30-day-period in the presence of varying concentrations of culture filtrates of the pathogen. Calluses resistant to the crude filtrate of Cercosporidiu personatum were selected by plating them onto a layer of callus growth medium. The medium was containing various concentrations of the fungal culture filtrate. Toxin resistant calluses were recovered from selective media. The resistant calluses were used for plant regeneration. Regenerated plants were transferted to greenhouse condition. Plant Tissue Culture Unit, Department of Plant Science
Bharathidasan University, Tiruchirapali-620 024, India.

1020

ROLL OF PUBESCENCE IN RESISTANCE OF SOYBEAN TO THE FALSE MELON BEETLE, ATRACHYA MENETRIESI FALDERMANN.

Hiroo Kanno

Resistance of soybean to the false melon beetle (FMB) was investigated in the field and labora tory using randomly selected genotypes of soybean with varying levels of pubescence on leaves. There was a negative with varying levels of pubescence on leaves. There was a negative correlation between trichome density on leaves and level of leaf damage
caused by feeding of FMB. Most of the genotypes which exhibited caused by feeding of FMB. Most of the genotypes which exhibited high level of resistance to FMB were highly pubescent. The differences
of damage level of leaves bet ween most pubescent variety, T-207, and of damage level of leaves between most pubescent variety, T-207, and
glabrous variety. Mumoh hadaka, did not change even after soaking glabrous variety, Mumoh hadaka, did not change even after soaking
leaves into methanol to remove chemical substances on leaf surface. leaves into methanol to remove chemical substances on leaf surface.
it means that this phenomenon does not concern with any chemicals It means that this phenomenon does not concern with any chemicals.
The normal type of trichome only confirmed on soybean leaf by SEM The normal type of trichome only confirmed on soybean leaf by SEM
observation. There was no typical type of glandular trichome which produce some kinds of substance. Trichomes might act as mechanica barrier to feeding behavior of FMB. The pubescence therefore indicates to be an important factor associated with resistance of soybean to
FMB. Several other aspects of the resistance are also discussed.

Tohoku National Agricultural Experiment Station, Yotsuya, Omagari Akita 014-01. JAPAN. 
ROLE OF PEROXIDASE AND POLYPHENOL OXIDASE IN WILD RYE RESISTANCE TO RUST

\section{E.P.Komarova, I.A.Davidovich}

Investigations were carried out in Derzhavin rye (hybrid form) with polygenic resistance to all rust types inierited from Kupriyanov wild rye. Cultivated rye susceptible to pathorgen was used as an alternative variant. For ascertaining a true importance of peroxidase (PO) and polyphenol axidase (PFO) in developing immune responses of rye (against a background of contradictory literature evidence) we have studied substrato specificity of these enzymes and effect of $\mathrm{pH}$ on their activity. It was revealed that depending on $\mathrm{pH}$ of reaction medium (phosphate buffer pH 5.5-8.5) not only PO- and PPO activity of rye varied sharply (from maximum to absolute inactivation) but their substrate specificity too. On the basis of the resuits obtained a new methodological approach to studying PO and PPO that makes experiment conditions realistic in terms of natural surrounding medium and functioning of enzymes was developed by us (according to medium $\mathrm{pH}$ and substrates). Intraceliular pH in rye leaves in pathogenesis dynamics was also determined (Doklady Akademil lauk Belarusi, 1989-1995). Thus, for analysins the role of substrate- and pH-dependent poiysubstrate enzyines of PO and PFO in piant immunity, hydrogen donors (? substrates for $P O$ and 4 - for PPO), with which the $y$ have high enzyou-substrate affinity in weak-alkaline reaction medium ( $\mathrm{pH} 7.5$ ) corresponding to $\mathrm{pH}$ of rye cell cytosol, were used for the first time. As a result, rye Po was revealed to be of great importance in developing rust resistance factors, since its activity level in infected leaves of Derzhavin rye is 2-3-fold (depending on substrate) as much as in tissues of susceptible rye. FPO is not involved in defense reaction of rye plants against rust pathogen.

Institute of Experimental Botany, Belarus Academy of Sciences, F.Skorina st.27, Minsk 220072, Republic of Belarus.

\section{IDRITIPICATION OF SLOW ROST RXPRRSSION TO BRONN RUST} (PUCCINIA RECONDITA TRITICI) IN INDIAN WERATS

\section{J. Kuear, L.B. Goel and S. Nagarajan}

Using a computer software (developed at CIMMYT, Mexico), the AUDPC values were calculated by processing the data of rust severities recorded at 78,88 and 98 th days of sowing. The test entries' number 255 included important released cultivars and lines advanced to be identified for release. Cultivars/lines were categorised into subgroups depending upon the extent of AUDPC value. AUDPC values between $0-10$ for 93 lines shows that they possess strong vertical resistance (VR) which may eventually get matched by the evolution of new pathogenic forms. Lines grouped into the AUDPC range of $11-100$ and totaling 83 showed initial brown rust infection with pustules of moderately resistant (MR-Type) type leading to slower brown rust development compared to the susceptible checks (with AUDPC value $>2000)$. Such genotypes probably possess vertical resistance genes apart from the adult plant resistance (APR). These genotypes may prove to be durable in field since they possess just vertical resistance only. In another 43 lines, epidemic initiated with susceptible pustules similar to that of the check, however, subsequently the rate of disease development remained slow resulting in poor terminal value (AUDPC $>101-<200$ ). Such a resistance is visualised to stay longer under field and remain durable. As many as 43 lines possessing higher value(AUDPC $>200$ but (1500) may not do satisfy even the requirements for usage as resistance stocks. The AuDPC based selection of material is being advocated in the Indian wheat programme for developing wheat genotypes for cultivation over the Indo-Gengetic plain.

Directorate of wheat Research

Post Box No. 158,

Karnal - 132 001.

INDIA
THE POPULATION STRUCTURE OF FUNGI OF THE PUSARTM LK.EX FR. GENUS ON SOYA UNDER CONDITIONS OF MOLDOVA

\section{KORETSKY LYUBA}

I4 species and 9 varietles of Pusarium (2400 isoletes) were isolated from infeoted soedlings and wilting plants in various ocological zones of Moldove. Qualitative differencer nere revosiod between straina according to their pethogenialty, and oultivara were established which differentiate anong them most distinctly. Under conditions of Moldova F.oxyeporum forms 5 types differing in morphological/cultural properties. Differences were found botween local populations and those of remote goographical regions (Krasnoder Territory, Par kast, Middle Agla). The study on the role of the host plant in the variability of populations showed that many direction selection of olones for the trait of pathogenfeity takes place in cultivers differing in the genotype. It hag been eateblished that cultivars possessing various levals of resiatance may sorve as a medium in selecting parasite olonos selection of the most pathogento olones occurring on reaistent variotias. The introduction of a new oultivar K-003 realatant to abiotic environmental faotore and furarioge in the plantings of Moldova in I994 influencod the structure of Pusarium populations. Thus, this yoar highly pethogenlo clones have appesrat which were not eerlier encountered in Holdove. They aro not sble of suppressing genes responsible for the resistanoe in this cultivar, but atrongly afect susceptible ones. Laboretory passagea of F.oxysporum (I0 generations) through reaigtant soya stocks induced the increased pathogenicity, through susceptible decreased one. This phenomenon ta espeoially aigniflcant at the critical lavels of tempereture and humidity.

Ingtitute of Genetica, Acod. Sc1., 20 Padurilor atr. Chisinau 277002 Moldova

\section{4}

\section{RESISTANCE OF TROPICAL CROPS TO THE PARASITIC ANGIOSPERM STRIGA}

\section{J.A. Lane, D.V. Child, T.H.M. Moore \& J.A. Bailey}

The obligate pathogenic angiosperm Striga is one of the major constraints to cowpea and cereal production in the semi-arid tropics of Africa. Yield losses can be significant (20-50\%). Siriga species are root parasites, and each Striga plant produces up to 25,000 seeds which can remain viable in the soil for up to 20 years. These factors make control of Striga extremely difficult. Culnural control methods can reduce low Striga infestations, but are rarely successful once parasite numbers increase. Crop resistance offers one of the most potentially feasible options for control. Sources of complete resistance to $S$. gesnerioides have been identified in cowpea (Vigna unguiculata). The resistant cowpea variety B301, which IITA has shown is due to dominant genes is being widely used in West African breeding programmes. There are two mechanisms of resistance in cowpea, and both are highly effective. Parasite death follows the hypersensitive response of host tissue around invading Striga radicles or parasite tubercles form on cowpea roots, but the shoots fail to elongate and further parasite development is halted.

Resistant cowpeas are currently being prepared for release across West Africa. However, the potential durability of the resistance is unknown as there are different parasite races which vary in virulence. To assess the extent of the variation, 45 samples of $S$. gesnerioides were collected from seven countries in West Africa in collaboration with IITA and NARS. The variation in virulence of these samples was determined using a differential series of cowpea varieties grown using an in vitro technique. The geographic distribution of the three known races of the parasite was mapped. Two new parasite races were identified. One race came from Cameroon and was mapped. Two new parasite races were identified. One race came from Cameroon and
eastern Nigeria, whilst the other was only found in southern Benin. $S$. gesnerioides from southern Benin was unique in that it was virulent on cowpea variety B301, which was previously thought to be resistant across West Africa. Accurate knowledge of race distribution is essential for planning the deployment of resistance.

Complementary research focuses on cereals. $S$. hermonthica and $S$. asiatica which are distributed widely across Africa parasitise maize, millets and sorghum. Unlike cowpea, there is no complete resistances to Strig $a$ in cereals. Therefore, wild progenitors and relatives of maize and sorghum have been evaluated for novel resistance to Striga. Two sources of resistance have been identified in accessions of Zea diploperennis and Sorghtum versicolor, wild relatives of maize and sorghum respectively. Resistance was expressed after penetration of host roots by Striga and resulted in parasite dean or very retarded development. All $S$. versicolor plants tested were resistant to Striga, but only $15 \%$ of $Z$. diploperennis plants were resistant to $S$. hermonthica, whilst $70 \%$ were resistant to $S$. asiatica. This suggests that wild relatives of cereals may be good sources of resistance genes. These results provide the first characterisation of the nanure of resistance to Striga in Zea germplasm. Variation of virulence of $S$. asiatica and $S$. hermonthica is also evident. Thus, resistant material will have to be tested against Striga species and strains from across Africa to assess the likely stability of resistance. Research was funded by the UK Overseas Development Administration.

IACR-Long Ashton Research Station, Department of Agricultural Sciences, University of Bristol, Long Ashton, Bristol BS18 9AF, UK. 
THE LINES OF COMMON WHEAT DURABLE RESISTANCE TO SOME DISEASES

\section{I.F. Lapochkina, D.A.Solomatin, V.A.PukhaIskiy.}

The spring common wheat $(2 n=42)$ variety Rodina and samples of Ae. speltoides $(2 n=14)$ with resistance genes to powdery mildew, brown rust and stimulating homoeologous chromosome pairing upon crossing with wheat were used. Two approaches were used in the research: common hybridization and hybridization combined with gamma-irradiation of Ae. speltoides podlen with co-60 at dozes $1-10 \mathrm{kR}$. Lines of wheat with durable resistance to puccinia recondita and Erysiphe graminis tritici were created. Resistance of lines is explained by as substitution so addition of alien chromosames and translocation of Ae. speltoides genetic material to the common wheat genome. Some of lines carry morphologycal marker characters of $\mathrm{Ae}$. speltoides and cytologicaly constant.

Agricultural Research Institute of Non-Chernozem Zone, 143013 , Nemchinovka, Moscow region, Russia.

\section{7}

\section{INDDCED RBSISTANCE TO ROOT ROTS AND SEED MOLD IN CORN}

M.N.La2u, A.I.Iurcu, P.C.Chintea

During the bast decade the induction of plant resistance using biologically active natural substances has received a special interest. Corn root rot susceptible stocks $\mathbb{R i n} 016, \mathbf{7 7}$ and three steroid glycosides were studied. Glycoside treatment of seeds under stress conditions resulted in an inducing effect. Ocher glycosides were not active at low temperatures. However, 10 days later at the temperature of $14-17^{\circ} \mathrm{C}$ all the glycosides stimulated growth of seedlings by $2-2.5$ times as compared with the control. Glycoside immunized seeds were affected by mold at less extent, except for the seeds treated with $\alpha$-tomatine at the concentration of $0.005 \%$, and the $\mathrm{TF}_{1}$ treated $10.001 \%$ and $0.08 t)$ seeds were not affected at all. Giycoside action was studied as regards seed gemmination and seedling growt on the background of artificial infection with fusarium during sowing under ope imum growing conditions of climatic chamber. Glycoside seed treatment contributed to high laboratory emergence in the presence of the pathogen. The glycosides caused a decrease in the rate of corn seedling root infection with fusariose by 2.5 times. Field date confirm that seed treatment with glycosides increased field emergence and decreased the rate of plant affection by corn smut, ear fusariose, cork moth, which resulted in producing capacity increase. Thus, the results received prove the efficiencies of glycoside application to increase corn producing capacity and resistance to a number of biotic and abiotic environmental factors. Seed pre-sowing treatmenc stimulates seed emergence, corn growth and development, thereby inducjing plant protective responses to parnogens.

Lazu M.N. - Leading Scientific Researcher, Doctor in Biology, Institute of Genetics, Academy of sciences, 20 pădurilor str., Chiłină 277002 R.Moldova
MESHOD OF TESTIHG TUEER RESISTAYCI TO POTATO BLACITEC IAZAREV A.

It is improved variant of lcboratery method of schiciele J.(1972) to estimate the susceptibility of potato tubers to vlackied pathogen. Using speciaily made hollow core-borers or augersyringe for taking samples (15 mun in diamiter) the cilinders are removed from tubers. For this purpose special device is used from stainless steel of the following construction. Sevon blades of $0.5 \mathrm{~mm}$ in tbicksess,20 mm in length and $20 \mathrm{~mm}$ in widtb are wountec in consecutive criler at intervals of $10 \mathrm{fir}$ on the base frame. sfter pressiag this device on potato cylinders be bave got discs of presuretive thickness. The density of the bacterial suspension (mixture of $3-4$ strains of pathogen) is at $5 \times 10^{8}$ cells/ml.40-50 discs from every cultivar are taken for inoculating. The inoculation of pieces is: cirried out by pricking then with dissecting needie previously dipped at $5 \mathrm{~mm}$ into the bacterial suspension. The temperature and the period of inoculation during experiments are $25^{\circ} \mathrm{C}$ and $24 \mathrm{~h}$. 'ine level of infection of potato cultivers is proposed to estimate measuring roting area on surface of every disc and average area of damage according to sample. The potato cultivars according to tuber susceptibily are variously grouped as follows:0-fully resistant (no danage on infected pieces): I-quite resistant (rotting area up to $10 \mathrm{~mm}^{2}$ ); II-relative resistant $\left(10.1-15.0 \mathrm{~mm}^{2}\right)$; III-middle resistant $\left(15.1-25.0 \mathrm{~mm}^{2}\right.$ ): IV-aon-resistant (fully susceptible)(more 25 $\mathrm{min}^{2}$ ). The cultivar is considered comparatively resistant at scaled estimation zanging from $O$ to II. It is necessery to uso middle resistant cultivars. In comnection with this the proposed analysis of potato sbould be supplemented.by estimating stem susceptibility to the pathogen, for example the laboratoryvegetative method by Schneider J.(1972).

All-Russian Institute for Plant Protection Podbelsky sh. 3, Pasbkin-St.Petersburg-8, 189620, RUSSTA

1028

GENETIC ANALISIS OP RESISTANCE TO POWDERY MILDEW (ERYSTPHE GRAMINIS Y.SP. TRITICT) IN TRITIOUR HONOCOCCUN $\mathrm{L}$.

\section{T. V. Lebedeva}

Resistance of the VIR T monococcum collection was studied in relation to powdery mildew. Two monopustulosus isolates and population of the patogen were ueed for inoculation 102 accessions at the eeedling stage. Phytopathological anelisis revealed a conglderable yariability of disease resistance within the diploid wheat. In some samples on increase in susceptibility was noted at higher temperature $\left(t^{\circ}=25-28^{\circ} \mathrm{C}\right)$. Inberitance of reaiatance was tested in 65 samples of cultivated eincorn. They were found noninfected when inoculated itb population of the fungus and two ioolates. The 7 and 72 eredings were tegted againat both races. Disease resiotance in this diplofd wheat was controlled both the recessive and domfnent factora. Yost of the accessions had one dominant gene; in the other amples a digenic control of the resiatance was observed. It was found that samples from Turkey possesed one and the awe dominant gene; 12 occesions of the different origin had the other common domlnant gene for resiatance at the aeedling stage. Genes for resistance to powdery mildew in T. monococcum were high effective and differed from maxy known alleles of the genes of the cownon wheat.

Department of Genetica, N.I.Vavilov All-Rugalan Institute of Plant Induatry (VIR), Hoskovakoje shosee 11, St.Petersourg, Pughkin, 189620, Russia 
THE INHERITANCE OF THE RESISTANCE TO BASAI STEM TUSARIOSE IN: TRITICALE

Celine A.Iunascu, Petru I.Buiucli, Efiria C.Veveriţä.

Under conditions of the Moldove Republic the ranifestation of fuseriose in triticale is diverse, namely, ear fusariose, ear bIindness, seed infection, embryo death, necrosis of the besal stem (NBS). The latter syrptom occurs practically in all plants, however, 1 ts developrent depends on the degree of the sample resistance. We consider resistence to NBS as a component of triticale resistance to fuserioge. Sirce this is the least investigated symptom from the zeretic viewpint, we almed at reveeling the inheritarce petterr of the resistance to it. Inheritence was show to cepend both on the level of parental stock resistance and on the gene combination in $F_{I}$ hybrida in each particuIer case. Inheritance was established to occur according to the dorinant positive type, however, the dominance degree $\left(F_{F}\right)$ is expressed in rether a high renge from -0.52 to -3.47, i.e. from the intermediate form to superdominance (minus reans the reduction in the infection rete). Fositive transriessions have been rainly revesled, at which the resistance of individual $F_{I}$ hybrids exceeds thet of the resistant perent itself by hearly two tires. The negative transpregsion hes also been pound $\left(\mathrm{H}_{\mathrm{p}}=+9.67\right)$ in the reciprocal crogsins with the rost sensitive materral stock involvec, which proves the perticieation of both nuclear and olasta rares in the senetic determination of triticale resisterce to root rot.

Gelire L.Iupascu, 20 Eadurilor str., Institute of Genetics, Chisinau, 277002, The Moldova Republic

1031

EFFECT OF ALLOPLASMON ON THE COMPLEX OF PHENOLIC CONJUGATES OF HEALTHY AND SEPTORIA NODORUM INFECTED WHEAT SEEDLINGS

\section{E.A. Voluevich, A.P. Volynets, L.A. Pshenichnaya,} A.Y. Lupey and G.V. Morozik

The objects under investigation were 5 wheot alloplasmic lines of $\mathrm{cr}$. Chinese Spring with cytoplasms of Aegilops comoso (C 05). Triticum dicoccoides (C 21), Ae. ventricosa (C 36), Ae, crosso (C 55), Ae. vovilovii (C 56), developed by prof. K. Tsunewaki, and the euplasmic cultivar (C 52). C 55, $C 56$ and $C$ 21 were significantiy more resistant to high agressive isolate of Septoria nodorum than C 05, C 36 and C 52. The composition and quantitative content of flavonoid glycosides and ethers of phenolcarboxilic acids in heal thy and $S$. nodorum infected wheat seedlings 1,3 and 6 days ofter inoculation were examined. The highest content of flavonoid glycosides in uninjured plants wos detected in $C 21$ and $C 55$, the lowest - in $C 05$ ond $C 52 . C 21$ and $C 55$ also had increased level of phenolcarboxilic acid ethers whereas $C 36$ and $C$ o5 had decreased one. Content of phenolic conjugates fell under influence of severe infection. The capacity to compensate the flavonoid glucoside content upto the level of heal thy plants was expressed better in the more resistant lines. 55, 56 and 21 had significantly more post-inoculation decrease of total quantity of ethers and, especially, ethers of nydroxycinnamic acids than other lines. The possible recson of the intensive decrease of phenolic ethers in the more resistont lines may be their act1ve inclusion in biosynthesis of defence biopolymers (lignin, cutin, suberin). Under influence of infection the formation of high fluorescent phenolic compounds which were absent in healthy plants wos established. During the disease development quontity of these substances increosed. Alloplosmic lines considerably differed in their composition and content, 6 days ofter inoculation $C 55, C 21$ and $C 52$ had the moximum number of compounds induced by infection.

Institute of Genetics and Cytology, Belorus Acadeny of Sciences, F. Skorina st., 27, Minsk 220072, Belarus.
METHODS FOR DIAGNOSIS AND IMHAKCMMBNT OF TRITICALE AND WHEAT RESISTAICE TO ROOT ROTS

Gelina A.Ludescu, Pavel C.ChIntea, Anetol Gh.Jacotá

During recent years the diegnosis and enhancegent of plant resistance to root rots as one of the ways to reduce the fungicide pressing or reject their utilisation have accuired a special attention in view of protection of triticale and wheat, as well as other cereal crops.. The long terw studies have show that the presence of anthocyan coloring on the coleoptile of plants grown on an overturned agar disc containing nvcelfurn of the pathogenic strain the degree of Tusarium spo. infection of seeds from ireshly harvested plants ray be effectively used as indices in laboratory tests for triticale resistance to fusariose. Under fleld conditions indices as follows may be employed:anther lenght (this index reflects the dissenination of mycelium up the vessels and associated water supply of the plant upper portion);injury of the stem basal portion which shows resistance to the symptom development; grain content and weight of IOCO geeds. Trees Indices should be scored on plents grom on the erticical infectlve background. The research carried out shoved that at the infection of wheat seeds with s. sramineemim schrobe at the level of 2.0-2.5 (on a 3 rankins scile basis) at the temperatures of $20-22 / I 6-I 8^{\circ}$ and $T C-I 2 /$ $6-8^{\circ} \mathrm{C}$ (day/night) the combined application of Baitan $(7.5 \mathrm{~g} / \mathrm{I}$ ) and steroid giycoside Moldstim $(0.1 \mathrm{~g} / 1)$ turned out to be rost effectivo of all the combinations used. The treatrent resulted in reduction of the rate of the basal stem infect1on to $. . I-0.2$ and the increase in total biomase by $67.3 \%$ and $42.2 \%$, respectively, at the above terperature regires as compcred with the infected planta. Thus, employing Moldstim may result in the fourfold reduction of the Baitan concentrotion $(30.0 \mathrm{z} / 1)$ proposed for prosowing treatrent of wheat seeds.

Galina 1.rupaşcu, Institute of Genetics, Acadery of Sciences, 20 Päduricor str., hisginău, 277002, The Noldova Rerublec

1032

INHERITANCE OP LEAP RUST RESISTANCE IN MRXTCLN SOFT MHEAT SAMPLZS

\section{I.A.Makarova, I.G.Od1ntsova}

Inheritance of seadling resiatance to Puccinis recondita in Mexican gpring soft wheat scessions k-58848, k-58849, $k-58850, k-58835, k-58836, k-58837, k-58838, k-58839$ has been studied. Reactions of samples to Infeatation with natural population of leaf rugt pathogen and clones virulent to Ir23 gene are deacribed. Segregation of F2 hybrids from crosses of reaistant specimens with susceptible variety Saratovikaya 29, Thatcher lines - testers of known effective genes for resiatance Lr9, Lr19, Lr23, Ir24 and inter Be Is presented. In cases with non-clear borders of phenotyp1c classes, the presence of gegregationg in hybrid combinationg was checked in the F3. The presence of hytherto anknown genes for seeding regiatance is supposed in samples $k-58835, k-58836$ (Lrk2), k-58839 (Irk3), k-58837 (IrM4), $k-58838$ ( Lr $¥ 5$ ). The accessions $k-58848, k-58849$, and $k-58850$ have per two identical genes for registance, of which one is $\operatorname{Ir} 23$.

Acknowledgment. This research was gupported in part by the grant 93-04-21033 Irom RPFI.

Department of Genetics, N.I.Vavilov All-Rusgian Institute of Plant Industry (YIR), Moskovakoje shosse 11, St.Petersburg, Pushkin, 189620, Russia 
RESISTANCE OF SOME PEAR GENOTYPES TO PEAR PSYLLA PSYLA PYRI L.

S. Milenković, S. Stamenkovíć

Pear psylla (Psylla pyri L.) is a major pest in the plantation orchards in Yugoslavia. Insecticides have been mostly used in the control of this pest so far. The paper presents the first obtained results of the study on resistance of some pear cultivars to pear psylla.

The investigations were conducted in varietal plantings of the Fruit and Grape Research Centre, Cacak over the 1991-1994 period. The resistance was determined in the conditions of natural infestation and high population pressure of Psylla pyri. L. The criteria for resistance evaluation were grouped as follows: parameters of population dynamics of pear psylla leggs, larvae and imagos) and parameters of damage degree (defoliation, honeydew and sooty mould).

The tests included 20 pear cultivars. The majority of cultivars were found to be ei.ther susceptible or highly susceptible. Moderate resistance was shown by three cultivars (Magness, Karamanka and Vidovača).

Besides being resistant to pear psylla, local cultivars Karaman$\mathrm{ka}$ and vidovaca are resistant to Venturia prina Ad. These cultivars shoula be used for breeding other resistant cultivars due to their good pomological properties.

Slobodan Mi.lenković

ARI "Serbia", Fruit and Grape Research Centre, Cacak

ARI "Serbia", Fruija

Kralja Petra
32000 Cacak

Yugosiavia
COMPARISON OF SUSCEPTIBILITY OF CUCURBITA

SPP.TO FUSARIUM SOLANI F.SP. CUCURBITAE

RACE 1 .

Mochizuki,M.11, Nagao,H.11, Sato,K.21, and Ogiwara, S.2)

Fusarium wilt of cucumber has been controlled grafting onto Cucurbita ficifolia 'Kurodane'. However, grafting does not control the foot rot caused by Fusarium solani $E$. sp. cucurbitae race 1. We examined the reactions of Cucurbita spp. to Fusarium root rot by evaluating disease severity to find resistant breeding materials. Susceptibilities of Cucurbit plants varied. The severity of seedling invasion was determined after 40 days of planting by the disease severity which was defined according to the degree of discoloration of hypocotyl and root. Cultivars 'Suzunari-nishiki' and 'Miyako' of Cucurbita maxima were more susceptible than C.pepo 'Zam Squash'and C.moschata 'Yashiro-yon-go'.C.pepo 'Zam Squash'and C. moschata 'Yashiro-yon-go'were severely affected only by 3 isolates. The disease indices for these cultivars showed a discontinuity for infection. Cucumber 'Ootone-ichi-go'also was less susceptible to these isolates except a isolate (P1-2). Aggressiveness among the isolates to cucurbits also varied.C.pepo and

C.moschata have a potential gene sources

for Fusarium root rot resistance.

1) Faculty of Horticulture, Chiba University, Matsudo 271 Japan; 2) Nihon Horticultural Production Institute, Matsudo 271 Japan.

1036

HEW GRISS FOR IEAP RUST RBSISTAFCB IN WHEAT

\section{I.G.Odintsova, N.A.Makarova}

Expressed genotype for dlesese resistence depends on parasite population structure which in Europe is other than in Amer1ca and Australia, where most of known investigations were done. Fe used Buropean populetions and clones of Puccinia rocondita virulent for the Thatcher lines th all known genes for leaf mat resistance except of highly effective Lrg, Lr19, Lr23, Lr24. Uaual methods of hybridological analysis mere used including teats with universally susceptible rarioty, teata for alleligm with Ir9, Ir19, Ir23, Ir24 and wth each other. For chromosome localisation monosomics of Kazakhstanskaja 126 mere used. Pive new genes mere 1dentifled: IrH in accession $k-44748$, LrM1 $(k-51070)$, LrG $(k-48198)$, Lril6 $(k-58840)$, and Irsp. The genes $I r H$ and IrWl are located on chromosomes 31 and 54 reapectively, where no genes for leaf rust resiatance are known. The gene lrM6 is located on chromosome $3 \mathrm{D}$ as the known gene $\mathrm{Lr} 24$ is. But it is independent of Ir24 and unlike of Ir24 is not linked w1th Sr24. The gene LrG is located on chromosome $I B$ as Ir25 is, but it is not linked with glfadines triplet characteriatic for Ir26. The Irsp gene was transferred from Legliopa speltoldes into soft wheat. It is closely linked with effective genes for stem (Srsp) and yellow (Yrsp) ruete resistance and with gametoc1dal (Ge) gene which killa in heterosygous aporofits the gametes without the same Ge allel. Jaknown effective genes for registance againgt Furopesa populations of F.recondta are not seldom. Such genes may be useful for plant breeding, phytopathology and genetics. Acknowled ment. This research was supported in part by the Brent 93-04-21033 erom RPFI.

Department of Genetics, N.I.Vavilov Ll-Rugeian Institute of Plant Industry (VIR), Moskovskoje shosse 11, St.Petersburg, Pushkin, 189620, Kugsia
S.K.Nayar, Head, Directorate of wheat Research Regional Station, Flowerdale, Shimla 171002 (INDIA). 
VIRUS - HOST INTERACTIONS IN CONBINATION TOMATO - TMV

T.I. Odinteova, M.S. Turishchevs, E.N. Andreeva, V.A. Pukhalsky

Development of effective tactic against viral infection includes an analyais of plant natural resistance and acquired immunty by virus strains with low pathogenicity (plant vaccine). The mode of plant - virus interaction depende both on plant and virue genotype. We report protein study in tomatoes inoculated Fith different TMN strains.

Isogenic tomato lines with various im resistance genes and aifferent IhV otrains were used in this study.

HPLC anelysig of the component composition of leaf acid-soluble proteing in susceptible tomatoes showed that infection is accompanied by the appeerance of several proteina, One of them, P36, appeared homologous to the Iur coat protein starting from the 7.4 th position of the polypeptide chain. Simllax protein was detected in tomatoes carrying In-2 $2^{2}$ infected with $2^{2}$ otrain. An analysis of soluble and membrane chloroplast protelns showed that, in plants with pronounced diseage symptome, a viral coat protein accumulates, and its content correlatea with symptom severity. At the same time, the content of photosystem II polypeptides is decreased. The inhibition of photosystem II activity in plants whth yellow-green mosalc was shown by low-temperature fluoracence spectra. Transeriptional setivity of chloroplast was inhibited upon infection with severe miv strains. Infection with attensted $\nabla_{69} \mathrm{WWN}$ strain did not reault in the synthesis of new plant proteins and eignificent decreese in transeriptional activity of chloroplasts.

Vavilov Ingtitute of General Genetics, Rusgian Academy of Sclences, Gubkin str. 3, II7809 Moscow B-333, Rusais

1039

BROWN RUST OF WHEAT IN AUSTRALASIA - RECENT EXPERIENCES AND MMPLICATIONS FOR RESISTANCE BREEDING

\section{R.F. Park, R. A. McIntosh and G. N. Brown.}

Wheat brown rust (caused by Puccimia recondita f. sp. tritici, Prt) occurs in all wheat growing regions of Australia and New Zealand. In the past, yield losses due to the disease were generaily regions of Australia and New Zealand. In the past, yield losses due to the disease were generaily
low, although a severe epidemic in Westem Australia during 1992 resulted in individual crop losses estimated at up to $37 \%$

Pathogenicity surveys of $\mathrm{Pr}$ in Australasia have been particularly informative because of the geographic isolation of Australasia from other cereal growing regions of the world. Annual surveys during 1980 - 1993 detected 21 distinct pathotypes for the first time. Most (16) of these surveys durng $1980-1993$ detected 21 distinct pathotypes for the first time. Most (16) of these pathogenicity. Comparative pathogenicity and isozyme studies have provided strong evidence that the remaining five pathotypes originated from outside Australasia.

An important component of breeding for resistance to cereal rusts is anticipating future pathogenic changes in the pathogen population. For example, virulence for the gene $2+24$ has not been detected in Australasia, and in recent years a very large proportion of the wheat area sown in Queensland (45.6\% in 1993) and New South Wales (35.1\% in 1993) was protected by this gene. Virulence for $L r 24$ occurs in other cereal growing regions such as South Africa and the USA, and it is considered likely that in the future, this gene will be overcome in Australasis

Anticipatory breeding is more difficult when new pathotypes are introduced from other regions. Such exotic pathotypes may possess new vinulences, virulence combinations, and / or different fitness attributes. The detection of the $L r / 3$ vinilent pathotype $53-1,(6),(7), 10,11$ in 198 resulted in the susceptibility of several cultivars. The pathotype reached epidemic leveis in central Queensland in 1988. Pathotype 64-11, first detected in 1990, increased in frequency in northern New South Wales, and was commondy isolated from hybrid wheats heterozygous for $L r / 3$. This pathotype is now known to combine virulence for $L r /$ with partial virulence for $L r / 3$. Of greater significance was the introduction of pathotype 104-2,3,(6),(7),11, first detected in 1984. After just 5 years, this pathotype, or the derivative $104-1,2,3,(6),(7), 11$, was the most common pathotype of $P r t$ isolated from all wheat growing regions in Australasia In the apparent absence of any beneficial virulence attributes, it appears that this pathotype may possess other attributes which confer a competitive advantage under Australasian conditions.

Various sources of adult plant resistance (APR) are now being considered as a means of diversifying resistance to $P r t$ Greenhouse tests have confirmed virulence in Australasian Prt isolates for the APR genes $L r / 2, L r / 3$, and $L r 22 b$. Whereas virulence for $L r 22 b$ has been very common since at least the early 1960 s, frequencies of virulence for $L r / 2$ and $L r / 3$ deciined during 1989-1992. Both $L r I 2$ and $L r / 3$ are still effective against all current pathotypes if used in combination with certain seedling resistance genes. APR genes with the greatest potential are $L r 22 a, L r 3-1, L r 35$, and $L r 37$. The gene $L r 34$ confers slow rusting, and one approach being considezed is to incorporate this gene into all wheats which are very susceptible to Prt. Gene $2 r 37$ has been deployed in the cultivars Trident, Sunbri, Sunvale and Sunstate

University of Sydney, Plant Breeding Institute Cobbitty. Private Mail Bag 11, Camden, NSW 2570. Australia
DYNAMIC SYSTEMS OF HOST METABOLISM ASSOCIATED WITH HAUSTORIUM FORMATION OF Peronospora parasitica DV JAPANESE RADISH

K. Yoshida and T. Olgguchi

Peronospora parasitica Pers. ex Fr., the causal agent of Japanese radish downy mildew, formed haustoria in the root tissues $\left(5 \times 5 \times 3 \mathrm{~mm}_{\mathbf{3}}\right.$ ) of Japanese radish (cv. Shirokubimiyashige) in the same manner as the leaf tissues. But in the root tissues treated with hest $\left(50{ }^{\circ} \mathrm{C}\right.$-more than $\left.20 \mathrm{sec}\right)$ prior to inoculation, haustorium formation (HF) was suppressed and the fungus iovaded the cell by hyphae-like structures, similar to germ-tubes, lacking the specific Auorescence of haustoria by membrane bound calcium ions. The inhibition disappeared after $24 \mathrm{~h}$ in the tissues treated at $50^{\circ} \mathrm{C}$-30 sec (Ht tissues). Resistant reactions were suppressed in the beat-treated tissues and the tissues were infected with Colletotrichum lagenarium, a non-pathogen. HF also was inhibited by metabolic inhibitors, such as chlorotetracycline (CTC), EGTA, K-252a, W-7, okadaic acid, $\mathrm{Na}_{3} \mathrm{VO}_{4}$ and cytochalastine $\mathrm{B}$ (CB). By the CTC and CB-treatment above, invasion of the cell by hypha-like structures, inhibition of plasma streaming, and induction of infection by a non-pathogenic fungus appeared in much the same way as in the Ht tissue. The inhibition of HF by CTC or heat treatment disappeared with the addition of protein kinase activator or calcium ionophore A23187. Patterns of the protein phosphorylated in vitro in the membrane fraction isolated from root tissue of Japanese radish changed after infection and the activity of protein kirrase depending on calciun ions and calnodulin increased. The protein kinase in the fraction was associated with the F-actin fraction. These results suggest that dynamic systems of host metabolisms containing protein phosphorylation and actin polymerization may be associated with $\mathrm{HF}$ of downy mildew fungus.

College of Agriculture, Ehime University, Tarumi, Matsuyama, 790 Japan

1040

EVALUation of resistance of WILO SPECIES OF the potato TU THE COLORADO BEETLE.

Pedko V.R., Smelyanets V.P., Korol T.S.

With the aim of the analysis of potato resistance to the Colorado beetle, it has been developed the principle of selection of resistant forms among wild species of the potato according to four vectors of adaptivity and five scales. The category of vectors includes:duration of development from egg to adult stages (this is time-vector - $t$ ), mortality during the period of the studies (this is mortality-vector - d), weight (vector of mass $-m$ ), emerging of beetles of the first generation (vector of aftereffect $-\mathbf{k}$ ). The first scale embraces the most resistant species. When feeding by them, the Colorado beetle characterizes by prolonged period of the development $(t=30-40$ days), high mortality $(d=50-76,7 \%)$, low mass $(m=15,6 \mathrm{mg})$ and low emergene $(k=0-6.7 \%)$. This scale can be expressed by the formula: $t^{5} \wedge d^{5} \wedge m^{1} \wedge k^{1}$. The second scale includes rather resistant species which render the following effect on the Colorado beetle when feeding by them: $t-27-29$ days, $d-30-40 \%, m-23,9-29,9$ $\mathrm{mg}, k-12,5-20 \%$. Its formula is: $t^{4} \wedge d^{4} \wedge m^{2} \wedge k^{2}$.

The third scale means the following: $t-24-26$ days, $d-16,7-27,7 \%, m-44,9-48,0 \mathrm{mg}$, $k-34,2-42,9 \%$. Its formula is: $t^{3} \wedge d^{3} \wedge m^{3} \wedge_{k} 3$.

The fourth scale: $t-21-25$ days, $d-7-13 \%, m-50,8-60,0 \mathrm{mg}, \mathrm{k}-54,5-60 \%$. Its formutia is: $t^{2} \wedge \delta^{2} \wedge \mathrm{m}^{4} \wedge \mathrm{k}^{4}$.

The fifth scale (the susceptibie species): $t-17$ days or less, $d-0 \%, m-81,0-87,0$ $m g, k-73,5-87,5 \%$. its formula is: $11 \wedge d^{1} \wedge m^{5} \wedge k^{5}$

Ukraine 252022 Kiev-22, Vasilkovskaya, 33, Plant Protection Institute 
THE ROLE OF ELISA DEMECTTON TI BREEDTIG OP PEACFES FOR RISTSTAITE TO FIUM POX VIRUS

J.Polekx, I. Oukropec

The detection of plum pox virug (PFV) by EIISA was carried out in the flowers and leaves of infected peach trees during regetation period. Prv was easily and reliably detected in lowers fron the beglnnlng to the end of fowering, and in leaves durlng the lay in leaves during the hay wes half or the concentration in flomers. It is proposed to determine the relative concentration of PPV in lowers (April) and in leaves (mid-lay) for evaluation of resistance of peach trees to PFV. Lower values of relative concentration of pry were determined in infected peach trees of Favorta wore.thi. Ill and Bnvoy varieties. Peach varieties characterized by Iow relative concentration of PPV are reconmended for breeding of peacher for resistance to PFV.

Dr.jaroslav Polák, Division of Phytomedicine, Research Institute of Crop Production, Drmovská 507, 161 06 Praha 6, Czecin Republic

\section{V. Pop}

BEHAVIOUR OF SOME TOMATO VARIETIES TO TOMATO SPOTTED WILT VIRUS

Since 1990 , in Romania, a new virus disease has broken out, especially, in tomato crops grown in our very big commercial glasshouses. My experimental results showed that the disease is produced by tomato spotted wilt vinus (TSWV) and that the vector of vinus is the trips species Frankliniella occidentalis, recently introduced in our country as well as in other European countries.

The disease is very dangerous, making, practically, impossibie to grow tomato plants in greenhouses in the 2-nd (summer) cycle. Therefore, a complex research programme concerning, especially, the epidemiology and control of the disease and its vector was initiated. As part of this programme, experiments and other investigations are carried out in five glasshouse complexes, located in different parts of the country, in which a variety of plants species are grown.

The control of TSWV is very difficult, no method applied alone being enough effective. Therefore, our purpose is to elaborate a complex of integrated control measures, efficiently applicable in glasshouses with different crops.

Since the use of resistant varieties is a very important control measure, we have investigated the behaviour of numerous tomato varieties, recently created by breeders for glasshouse growing, to the natural infection and artificial inoculation with TSWV.

Data concerning the reaction of the verieties to the natural infection were obtained either by observations made direct in usualy crops or in special experimental plots established in commercial glasshouses, whereas the artificial juice inoculations were done in experimental glasshouse

Among the investigated material, no variety with resistance of vertical type was found But our results show that the studied vanieties differ greatly from each others concerning the proportion of infected plants, some of them having a resistance of orizontal type. Under low infection pressure, the varieties with a high resistance of this type were practically not infected by TSWV. Comprehensive data concerning the bihaviour of investigated tomato varieties to TSWV will be presented in the extended paper.

Research Institute for Plant Protection, Bd.Ion lonescu de la Brad 8, 71592 - Bucharest - Romania

1044

\section{VETHOD OF CABBAGE EVALUATION}

FOR ITS RESISTANCE TO SLIMY BACTERIOSIS

A. N. Samokhvalov, U. B. Rogachev.

Erwinia carotovora pv. carotovora population is charasterized by a high degree hoterogenecity in Moscow region. Maximum virulence (4 to 5 values of magnitude) hes been found in ten isolates separated from cabbage seed plants of Amager 511 and Podarok varieties. A method of varietal cabbage evaluation for resistance to sliny bacteriosis is elaborated based on pathogen inoculation of the midrib middle of a leaf with a dose of $4 \times 10$ cel3s/ml and subsequent calculating the extent of maceration of the leaf blade according to our 7 point evaluation scale. The method allowed to differentiate variet ies of cuitivated white cabbage according to their resistance to the pathogen into three groups: moderately resistant; medium susceptable and highly susceptable plants. 
CONTROL OF POTATO CYST NEMATODES WTTH PARTIALLY RESISTANT POTATO VARIETIES.

\section{C.H. Schomaker \& T.H. Been}

A large number of potato varieties have partial resistance to some pathotypes of potato cyst nematodes, for which they are susceptible according to the Descriptive List of Potato Varieties. This means that these varieties multiply the nematodes less strongly than susceptible ones and therefore, less control is needed by means of soil fumigation or crop rotation. Although relative susceptibilities are reasonable constant, the multiplication of potato cyst nematodes at one initial population density can vary strongly between fields and years, because of large variations of the maximal multiplication number $a$ and the maximal population density $M$ in the (simplified) stochastic model for population dynamics: $P f=M(1-\exp (-a P i / M)(1)$ (Seinhorst, 1993)

Therefore it is impossible to predict population densities in individual fields using only mean values of $a$ and $M$, let alone from only the mean value of $a$, as is common practice in temporary advisory systems. A better approach would be to calculate, with the aid of equation (1) and the frequency distributions of $a$ en $M$, the probabilities of a range of the population densities after several potato crops, given a certain initial population density, the relative susceptibility of the potato varieties to be used, the effect of crop rotation and the effect of control measures. By combining equation (1) with the relation between initial population density and relative yield:

$y=m+(1-m) 0.95^{p / \cdot \cdot 1}$ (2) (Seinhorst, 1986) probabilities at certain relatieve yield reductions (= probabilities at certain population densities) can be calculated. With the results of these calculations farmers can evaluate the risks of cropping several potato cultivars with a known relative susceptibility to the nematode population involved and choose combinations with the largest probability at a maximal financial retum.

DLO Research Institute for Plant Protection (IPO-DLO), PO Box 9060, NL-6700 GW Wageningen, The Netherlands. For E-mail use in \%"th.been@IPO.AGRO.NL".

1047

VIRULENCE MONITORING AND YELLOW RUST RESISTANCE IN WHEAT

$$
\text { S. Sharma and C.B. Karki }
$$

\section{Abstract}

Yellow rust (puccinia strifformis west. f. sp. tritici) is a major problen of wheat in hilly region of Nepal. Wheat grain yield loss up to $20 \%$ was recorded when there was moderate infection of the rust. Agronomically superior and other promising wheat cultivars are susceptible to the rust. This is assumed to be due to the change in virulence of the rust parhogen.

With a view to monitor the rust pathotypes in Nepal several rust samples from the country were analysed at the Research Institute for Plant Protection (IPO). Wageningen. The Netherlands. Virulence monitoring was also carried out in Nepal. by planting wheat differentials at several locations in the field. Yellow rust pathotypes so far recorded are $4 \mathrm{E} 0,4 \mathrm{E} 16,70 \mathrm{E} 16,68 \mathrm{E} 16,7 \mathrm{E} 150$, OE16, 2E0, 6E0, 6E16, 7E150 I5E150, $15 \mathrm{E} 158,70 \mathrm{~B} 0$ and 66E18. The pathotype $7 \mathrm{E} 150$ is the most prevalent and it attacked several promising wheat most prevalent and it attacked several promising wheat varieties/lines including a popular and widely grown variet
RR 21 (Sonalika). Wheat cultivars Annapurna-1, Annapurna2. Annapurna-3 and other promising lines NI 539, NL645. $W \dot{Z} 685$ and $W \mathbb{R} 689$ have shown resistance to many prevalent WR 685 and WK 689 have shown resistance to many prevalent pathotypes of the rust both under field and grouth chamber conditions. Recently yellow rust resistance gene postulation on some advanced as well as local wheat varieties were done. Wheat cultivars of Nepal were postulated to contain Yr 2, lines grown in Nepal were postulated contain Yr 9 in addition to other resistant genes. Ffforts are being made to utilize wild emmer derivatives in our breeding programme to develope high yielding, superior, rust resistant cultivar.

Lumle Agricultural Research Centre

P.O. Box No. 1, Pokhara

Gandaki Zone (Nepal)

Phone : 061-20385/21399
PECULIARITIES OF DIFFERENTIATION OF PRIMARY INFECTION STRUCTURES OF ERYSIPHE GRAMINIS TRITICI AS AN INDICATOR OF RESISTANCE OF WHEAT VARIETIES TO PENETRATION OF POWDERY MILDEW INFECTION

\section{G. V. Serezhkina}

The results of investigations of pathosystems at obligate parasitism show that the formation of infection structures of the parasite is genetically programmed and infection structures with common morphology are formed on the susceptible host plant at the optimal invironmental conditions. Our study of differentiation of infection structures of Erysiphe graminis tritici was held on the wheat-agropyron varieties of the new wheat species Triticum agropyrotriticum Cicin, carrying new adaptive valuable characteristics, and among them, resistance to diseases owing to intergeneric hybridization of wheat and agropyron species. At optimal conditions conidium of E. graminis tritici on susceptible variety forms very compact primary infection structures with short germ tubes and oval appressorium. Various kinds of deviations in morphology of infection structures of the pathogen from normal, genetically conditioned pathway of development could be observed on the resistant varieties. The deviations, evidently, are determined by some factors characteristic for the resistant host plant. Our experiments have shown that if less than 30 per cent of normal infection structures were formed in a variety, then the variety could be considered as resistant one to pathogen penetration. Scanning electron microscopy observations have clearly shown breakages in adhesion between the parasite and the host plant in a zone of penetration, which led to sliding of infection structures from the surface of resistant plants, making penetration impossible. So on the basis of statistic analyses of the data of light and scanning electron microscopy research of relationships of powdery mildew fungi with wheat varieties it allows to suggest at ncw method, based on a possibility to determine plant resistance to parasite penetration by morphology of primary infection structures of the parasite.

Matn Botanica! Garden RAS

Botanitcheskaya st. 4

127276 Moscow Russia
1048

INVESTIGATING RESISTANT CULTIVARS OF TOMATOES TO BROOMRAPE (OROBANCHE AEGYPTIACA PERS.)

\section{P. SHIMI}

Dodder damage to tomatoes in Iran is quite extensive and an effective control of the parasite is not yet known. Many resistant and/or tolerant tomato cultivars have been reported from different parts of the world, but almost all of them have been proven to be not resistant or tolerant by othe researchers. This paper is the result of three years of research involving 300 cultivars of tomatoes at phase 1 , and 82 cultivars at phase 2. The experiments were conducted in the fjeld in the framework of an augmented design using ten plants per plot. The tomatoes were inoculated with 2 mgs of broomrape seed at the time of tramsplanting. Data collected for evaluation included percent of tomato plants infected per cultivar. and number of broomrape flower heads per tomato plant. Results showed the cultivars Primo Front (Italy), DE 3075 (Denmark), $C_{2}$ (Iran), CL-11040-0-29-1-0 (Taiwan), and Castle Peel II (Italy) resistant to Orobanche aegyptiaca. Many other cultivars with less than $50 \%$ infection were tolerant. Presence of resistance genes in above tomato cultivars is evident. This calls for genetic engineers to transform the genes and produce resistant cultivar(s) of tomatoes to this very important parasite

Plant Pests and Diseases Research Institute, P.0.80x 1454. Postal code 19395. Tehran-Iran 
FEEDING SELECTIVITY OF INSECT PESTS AND EVALUATION OF CROP VARIETAL RESISTANCE

(BY THE EXAMPLE OF SPRING WHEAT - SUNN PEST SYSTEM).

\section{E. A. Sinelnikov, N. A. Vilkova, N. V. Burinskaya, S M. Tombol, A. N. Guida}

The plant immunity to insects is due to complex of mechanisms of plant resistance which influence phytophagans in a variety of fashions' in many cases it is difficult to reveal some key resistance factor. By this reason the plant characters which are in direct responsibility for cepressing of pest development and multiplication are rather rarely used as a criteria in evaluations of pest resistance. The sources of plant resistance to pests are usually revealed from small-plo1 germplasm collections by the use of indices of plant-pest interactions, such as pest numbers and level of plant injury by the pest. However, insect pesis encounter great envronmental andhutitional diversity, akin to intercropping situation (van Emden, 1987), in smalt-plot trials. So, it seems to be worthwhile to study how and in which extent insect preferences can infuence resistance evaiuations in different plant-pest systems under the conditions of germplasm collections and other small-plot trials

Spacial-temporal interactions of the natural sunn pest (Eurygaster integriceps Put. Hem. Scutelleridae) population with spring wheat cultivars were studied through the period of wheat ontogenesis under the conditions of germplasm collection in Krasnodar (South Russia). It has been found that the relative attractivity of wheat cultivats to the overwintered and summer sunn pest generations is quite different. The growth and developmental rate at early stages of plant ontogenesis seems to be the main varietal character determining cultivar attractivity for the overwintered sunn pest adults, which is in agreement with the previous data (Shapiro, Vilkova, 1973) The bugs prefered more developed plants of early ripening cultrvars. The food preferences of overwintered bugs determined the distribution of eggs and significantly influenced the distribution of larvae (including older stages) among the test cultivars. The relative attractivity of wheat cultivars for bugs changes at later stages of wheat ontogenesis. The significant re-distribution of the pest numbers among the cultivar plots was revealed when the adults of summer generation became prevailing in the population. Late nipening common wheat cultivars were mainly prefered to early npening ones, while within the mid-ripening group the vulnerability of endosperms to hydrolitic decomposition by bug enzymes ( by Ekman, et al, 1973) played a leading role in varietal preference by the pest. The pest preferences seem to be clirscted by relative nutritional and energetic value of wheat cuttivars which changes during crop ontogenesis. The endosperm resistance to hydralitic decomposition by bug enzymes is a character of antibiotlc significance (Vilkova, 1980: Ekman. Vilkova, 1973). Hence, the attractivity of spring wheat cultrvars for the summer generation of $E$ integriceps is related to their antibiotic properties on the other hand the influence of food preferences of the overwhintered generation on larval numbers and consequent grain injury by the pest can introduce some difficulties in estimation of wheat varietal resistance to $E$. integriceps under the conditions of small plot trials.

All-Russian institute for Plant Protection,

Podbelsky av.3. St. -Petersburg-Pushkin, 189620, Russia

1051

IDENTIFCATION OF RUCE BLAST RESISTANCE FOR IMPROVED AROMATC RICE VARIETIES IN THARAND

\section{P. Sirichunya, A. Surin, A. Vannavichit, S. Tragoonnung}

Rice blast disease has caused serious yield losses in the North and Northeast of Thailand in recent years. Because of this there is considerable interest in developing varieties with durable resistance to the causal agent, Pyricularia grisea. Resistance to blast is a complex trait. Loci like blast resistance will allow the breeder to efficiently transfer the trait into a more favourable genetic background.

The KDML105, a low yielding landrace, is a well known rice both in the local and export markets for its superb cooking quality, most of all, its aroma. Thai aromatic rice is susceptible to blast at every stage of growth. The CT9993 lime, an upland rice from the Center of International Tropical Agriculture (CIAT) is high yielding with excellent durable blast resistance tested in the Northeast of Thailand. The progenies from these two parents are expected to be an ideal segregating population for mapping genes controlling durable blast resistance and aroma. Segregating material of KDML $305 \times C T 9993$ was tested for level of blast resistance following the standard Evaluation System by IRRI at Ubonrajtani Rice Research Center (URRC) and the Rice disease branch in the Department of Agriculture (DOA). The result showed that 42 from 81 lines were resistant while RD23 and KDML305 were very susceptible.

The major genes for blast resistance will be mapped using KDML105 resistance mutants. Sixty eight KDML mutants, selected from gamma-ray treated seeds, will be provided by Rice Research Institute. The mutant lines were selected for improved agronomic traits, blast resistance and aroma. All mutant bines derived from the family KDML'86 G204 were showing complete resistance to blast at URRC. One hundred and sixty RAPD primers from Operron have been screened on genomic DNA of a set of KDMI mutant lines. Three primers produce products specific for the resistant or susceptible lines. The patterns from primer OPMJJ, OPOis and OPE17 were clearly specific to resistance scores. The specific PCR products, isolated from agarose gel electrophoresis, will be used to develop RFLP markers for further mapping.

RFLP Laboratory, Central Laboratory, KU, Campus Kampangsaen, Nakormphatorn, Thailand, tel 034-351399-457, fax 034-351908

$$
\text { Dr. Gajendra singh }
$$

In the present studies more than 100 varieties of mango have been screened against hoppers both under natura? infestation and artificial released conditions. Nylon screen bags and polystyrine bags sleeved at both ends were used for releasing the hoppers on panicles. Observations were recorded on the hopper population inside the bags to find out their survival/development and multiplication and fruit set. Observations were also recorded on hoppex population and fruil set outside the bags on panicles as well as on trunk.

Result of the trials conducted during $1902-93$ and Result of the trials conducted during $1902-93$ and
$1993-1994$ indicated that very few varieties could tolerate hopper infestation. Varieties Pulhora, Amin, Kakaria, Amin, Kala Hapus, Ramkela, Pau, Annanas, Kalank Goa, Keshar Basti were resistant types to hoppers. Toletant varieties included Dudha peda, Goff of Navasari, Padiri, Azwain, NX Humayudin, Tamancha, Eruvadi Banglora, Malihabadi, Neelum, Mallika, Banglora, panja Pasand, Langra Rampur, etc. While the varieties $N$ Chausa, Hapus, Himsagar, Rahiri, Panchadrakalsa, Eruvadi Romani, Kodurchinnarson, Gulab khas, Green, Vanraj, Kalapaddy, Mithua Malda, Sonakuliu, Nilyora, Hathizhool, Kazalio, N x Fanchadrakelsa, Cecil, Asadio, Baramalda, Ladavio, Rajapuri, Pathar, Naspati, Bijoragarh, Maharaja of Mysore, Popatpari, Fakira, Keshar, Chinnaswaranarekha, Vellakachi Cherumani, Malgoē, Hazoor Pasand, etc. were susceptible.

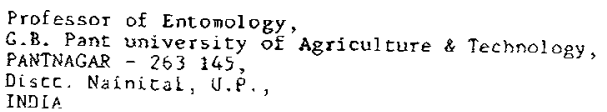

1052

REACTION OF THE PROTECTIVE SYSTEM OF THE PINE (PINUS SILVESTRIS) TO DAMAGE BY RHYACIONIA BUOLIANA SCHIFF, (LEPID.: TORTRICIDAE).

\section{Smelyanets V.P.}

The reaction of protective system of the pine has been studyed during 9 years. In so doing it has been recorded the changing of concentration of essential oils of definite groups of trees in dependance on attacks by the pine shoot moth. In part of trees $(54,3 \%)$ the attack leads to increasing of the concentration. In others $(25,7 \%)$ the concentration is decreased. The rest of trees $(20.0 \%)$ does not react on the attacks. The colonies density of the pest during this period is in the ranges 17,8-0,1 pest per tree. The activity of protective system of the ecosystem reaches up to $80 \%$. The highest activity of the protective system is marked in the group of pines with concentration of essential oils $0,2+0,05 \%$, that represents the main trophical niche of the pest in the stand, where there is the directioned selection of the pest population on tree's viability. The lageffect of the protective system is $2-3$ years. It is suggested the group of pines that does not react to the changes of density of attacks is a trophic niche of the pest in an ecosystem. In the mechanism of protective reaction of the pine it has been traced the positive and negative feedback.

Ukraine 252022 Kiev-22, Vasikorskaya, 33, Plant Protection Institute 


\section{Q.v. Solodukhina}

Brown rust (Fuccinia dispersa Erikss. et Henn) is a widely distributed disease of winter rye in Rusia. Considerable harm is being done to short atem varieties resulting in a oignificantly lower grain yield. It has been experimentally proved that every $1 \%$ of the increase in susceptibility caued the decrease of 1000 grein weight for $0,12-0,418$. The high harmfulness of brown ruat indicatea to the necegatty of increasing the efforta on the deveiopment of resiatant varieties. Success in this field may be achieved if proper initial material and knowledge on the regularities of reaistance inheritance are available. The perennial wild opecier (Secale montanum) and some rare varletien of cereal rye (s.cereale) in whose populations some resistant genotypes occur may serve as sources of the race-specific reolatance to brown ruet. Fure wild formb of rye are of littic help being directly used in breeding. Plant resistance to the digease were revealed in 38 cultivars. In each case, registance to brown rust was shown to be controlled by a dominant allele of a aingle gene. In order to identify genes with monofactor control of resiatance we have auggested a method based on test crossings of diheterozygotes by identified genes. Resistance to a population of brown rust was, for the first time demongtrated, to be controlled by the genes Ir4 (in cultivars Sanim, I - 125/79, Baltiskaya RPd, and Chernigovekaya FPd) and Lr5 (Immunnaya 1). Brown rust reaistance in the cultivar Chulpan RPd eppeared to be determined by a gene that was nonallelic to Lr4 (temporarily deaignated Lr6)

Department of Genetics, N.I. Vavilov All-Ruagian Institute of Plant Industry (VIR), Moskovskoje shosse 11 , St. Petersburg, Pushkin, 189620, Russia

ON THE SEARTH OF DISEASE RESISTANT BIOTYPES WITHIN THE POPIRATION OF RLSSIAH AHD DUTCH REPRODUCT ION OF EUROPEAN HINTER WHEAT VARIETIES

S. K. Temirbekova, E. V. Poruva, E. Beys, Jan D. Van. Kansvelt

The intensification of the asricultural production combining with unfavourable ablotic factors led to the mass enzyme - mycotic seed exhaustion affection of cereals (EMSE). This disease is so called "disease of propress". As a result of the EMSE non-infectlous stage development the excellent nutritious medium for fusariosis, septorlosis and alternariosis of spikes is creat.ed. The yield losses reach 30-50\%, simultaneously the quality of grain gets worse. Resistant varieties have not been available.

After t.wo years of parallel joint. investigations of Russian and Dit.ch reproduction winter wheat varieties Okapi, Herzos. Recktor, Obelisk, Mirinovskaya 808, 7arib it has been indicated the following:

1. Electrophoretic analysis of elladine spectra of Dutch and Russian reproduction variet.les has proved the absence of genetic variations for both reproductions independentiy of crowing conditions and protein content. But in spite of genetic ldentity, varieties reproductions (Dutch and Russien) are different. according to winter and frost resistance, ripening, resistance for non infectlous and infectious EUSE stages (biological seed shock and Fusarium spp. . Septoria nodorum), Erysipte graminis, Puccinia recondita.

2. We were ploneers in finding out an 1mprint of adaptiviness property for the varieties from electrophoremrames.

3. Blotypes resistant to fusarlosis, alternariosis, spike septoriosis and stresses have been picked out within the population of varieties Okapi, Recktor. The disease resistant blotypes have been sowed for reproduction.

Moscow department of VIR

Timiryezev gt $r$. 3, Mikhrevo,

Noscow region 142810 . Risssia

FAX: $007-(095)-222-65-11$ BOX H 1194 MOY18
A. Surin

Rice blast disease has caused serious yield losses in the area of growing rice in Thailand. Because of this there is considerable interest in developing varieties with resistance to the causal agent Pyricularia grisea. Any new cultivars must have slender, translucent grains with intermediate amylose content to sustain rice production in Thailand and to ensure World export market for Thaj rice. Therefore isolates of the causal fungus named $P$. grisea from various locations should be identified in group of virulent or non-virulent. The result will be useful for breeders. The rice leaves of 7-10 days old of $\mathrm{RD} 23$ were laid on selective media (benximidazole agar 95\% WP.) in a petri dish. We use 3 leaves/isolate with 3 replications. Then spore suspensions from Chiengrai, Nakorpathum, Chachogsao were inoculated directly to the rice leaves. Then kept at room temperature 5 days, the symptoms of leaf blast will appear. Then the lesion size on each of the leaves was measured, and gelatin slides were stuck to the lesion for counting the numbers of spores. The lesion size, no of $P$. grisea/lesion were analyzed. The results indicated that there were highly significant differences of lesion size $(\mathrm{cv}=19.5 \%)$ and numbers of spores/lesion (cv $=37.8 \%)$

The spores of $\boldsymbol{P}$. grisea from Chachoeugsao and Chiengrai province can cause large lesions and high numbers of spores. Thus, before recommendation the new cultivars should be tested as to the effectiveness of resistance to $P$. grisea from two locations.

The pure cultures of $P$. grisea from Ubonrajchathanae and Sorattaneae can infect the partial resistance SPR 90. Differences in lesion size were highly significant $(\mathrm{cv}=\mathbf{2 0 . 9 \%})$

We can conclude that the causal agent $P$. grisea from the North, Northeast, the South and the Central of Thailand did not belong to the same group. The $P$. grisea from the North, Northeast and Central part of Thailand are virulent.

Rice Disease Branch, Plant Pathology \& Microbiology Division, Department of Agriculture, Bangkhan, Bangkok 10900, Thailand

1056

ADULT PLANT RESISTANCE OF WHEAT TO POWDERY MILDEW (ERYSIPHE GRAMINIS DC $F$. SP. TRITICI MARCFAL) - A POSSIBLE WAY OF PATHOGEN CONTROL

\section{Trarüžek, K. Klem}

A set of winter whegt varieties registered for growing in the Czech Republic have been assessed for their reaction to powdery mildew in laboratory tests and field trials. The $\mathrm{Pm} \mathrm{2,6}$ and $\mathrm{Pm} 4 \mathrm{~b}$ resistance genes are still effective, only. These findings obtained in the laboratory tests with a large collection of pathogen isolates are in good correlation with field test results from early growing stages.

However, the reaction type can be significantly changed during the vegetation period. Genotypes with effective genes of resistance in their genomes can show a susceptible type of reaction and, on the contrary, varieties missing resistance genes can change this reaction to resistant one due to field resistance. The importance of these reaction types is discussed.

Agricultural Research Institute Kroměřǐ, Co. Ltd.

Havlickova 2787

76701 Kroměřín

Czech Republic 
GENETICS OF WHEAT RESISTANCE TO DARK BROWN LEAF SPOT BLOTCH

\section{G. myryentin}

Leat spot blotch of wheat caused by Bipolaria sorokiniana is wide epread and economically important disease, eepecialIy under warm hurid conditions. The most common used chemical method for protection is not efficient when epiphythoty occurs. Whe at genotypes with some level of resistance were identified in India, USA, Germany, but all of them are very auscetible in eedling teat after inoculation with several pathogen atrains from Ruseia. We have evaluated the reactions of over 500 samples of Triticum aestivum, 300 samples of another Triticum epecies and 100 of hegilops ones. Three semples of common wheat have a good level of registance: 181-5 (Canada), selection line from Mexico 120, and IWWR 208/85 (Bulgaria); the mogt of Aegilops tauschil amplea possess high level of resiatance; other cenotypes are susceptikle. FM, $\mathrm{P} 2$, and $\mathrm{F} 3$ from croses of resiatant amples with ausceptible ones (Lusiteno, Vega) and between them were evaluated for disease reactions after inoculation with strain $T$ of pathogen; reciprccals were included. From the analyais of segregations we concluded that each of 3 samples has 1 gene for resistance and the geneg are independent. Gene of IWWR 208/85 is dominant at middle inoculation pressure and recessive at high one, that of 181-5 is dominant at all pressures and gene of Mexico 120 is recegsive at middle and noneffective at high level of inoculation. Analysis of amphiploids T. aestivum $x$ Ae.taugchif revealed the ouppregaion of Ae.tauschii reaistance by comon wheat genome.

Department of Genetics, N.I.Vavilov All-Russian

Inetitute of Plant Industry (VIR), Moskovskoje shosee 11, St.Peteraburg, Pushkin, 189620, Russia

1059

RELATIONSHIP BETWEEN RESISTANCE TOMI GENEVIRULENT MELOIDOGYNEINCOGNITA AND HEAT-STABLE NEMATODE RESISTANCE IN LYCOPERCICON PERUVIANUM.

L.C. Veremis and P.A. Roberts

The inheritance of heat-stable resistance to the root-knot nematode, Meloidogyne incognita (Kofoid and White) Chitwood and its relationship to resistance to gene $M i$-virulent nematode isolates. Was investigated on resistant clones of Lycopersicon peruvianum L. Resistance gene expression in parental L.peruvianum clones P.I. 270435-2R2, 270435-3MH, 126443. $1 \mathrm{MH}$ and $126440-9 \mathrm{MH}$ and their $\mathrm{F}_{1}, \mathrm{~F}_{2}$, and $\mathrm{TC}_{1}$ generations was evaluated using an index of resistance based on egg masses and eggs per gram of root. The segregation ratios were determined in experiments carried out at $25 \mathrm{C}$ ( $M i$ expressed) and $32 \mathrm{C}$ ( $M i$ not expressed) constant temperatures $w$ ith $M i$-avirulent and $M i$-virulent nematode isolates. Parental clones P.I. $126443-1 \mathrm{MH}, 270435-2 \mathrm{R} 2,270435-3 \mathrm{MH}$, and their $\mathrm{Fi}_{1}$ progenies were resistant at both temperatures to an $M i$-avirulent isolate. Also parental clones P.I. 270435-3MH, 126443-1MH and their F l progenies were resistant to an Mi-virulent isolate of $M$. incognita. To generate test-cross populations, $F_{1}$ progenies of $270435-3 \mathrm{MH} \times 270435-2 \mathrm{R} 2,270435-3 \mathrm{MH} \mathrm{X}$

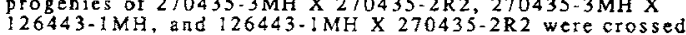
with L.peruvianum P.I. 126440 clone $9 \mathrm{MH}$, susceptible at high temperature. At $32 \mathrm{C}$ all TC1 generations gave temperature. At $32 C$ all $T C 1$ generations gave
resistantisusceptible (R:S) ratios of $3: 1$. These results indicated that heat-stable resistance is conferred by differe (independent) single dominant genes in each of the clones
L.peruvianum P.I. $126443-1 \mathrm{MH}, 270435-2 \mathrm{R} 2$ and $270435-3 \mathrm{MH}$.
The heat-stable resistance genes are expressed when heatThe heat-stable resistance genes are expressed when heatsensitjve gene $M i$ is not expressed. One TCl population evaluated with the Mi-virulent nematode isolate segregated with an R:S ratio of $3: 1$. These results indicated that resistance to the Mi-virulent isolate is conferred by different independent dominant genes in the resistant clones L.peruvianum P. $126443-1 \mathrm{MH}$ and $270435-3 \mathrm{MH}$. Additional screening with the same individual plant clones of the $\mathrm{TC}_{1}$ segregating population suggested weak linkage of heat-stable and $M i$-virulent resistance genes. That some individual TC 1 clones were susceptible at $32 \mathrm{C}$ but resistant to the $M i$-virulent nematode isolate and vice verse, suggested that heat-stable and Mi-virulent incompatibility resistance are not conferred by the same genes in the tomato genome.

Deparment of Nematology, University of Califomia, Riverside, CA 92521.
ABOUT THE EVALUATION OF FORAGE GRASSES AIMING RESISTANCE TO THE SPITTLEBUGS (HOMOPTERA: CERCOPIDAE)

\section{J. R. VALÉRIO}

In spite of some progress, the screening methodology of forage grasses resistant to spittlebugs needs adjustments as well as further development of new evaluation procedures. Herein are a few useful considerations as regard to this line of research: 1) data obtained with a particular spittlebug species should not be generalized, it is of paramount importance to repeat the same assays with the different important spittlebug species. While the nymphal survival of Aeneolamia varia was of only $1 \%$ on the Brachiaria introduction BRA 005215 , it was $88 \%$ for Zulia entreriana. In addition, the spittlebug Mahanarva fimbriolata was found to be more toxicogenic than the species $Z$. entreriana, causing $20 \%$ more damage in $\boldsymbol{B}$. decumbens; 2) for feeding preference trials using spittlebug adults it is suggested to standardize the number of tillers per plant. Positive correlation was observed between numbers of $\boldsymbol{Z}$. entreriana adults on the plant and the number of tillers of B. decumbens plants; 3 ) when comparing different forage grass introductions for damage caused by adult spittlebug, more differences were evident with younger plants infested for shorter periods of time. When plants of different ages $(20,35,65$ and 80 days old) of the susceptible $B$. decumbens and the resistant $B$. brizantha $c v$. Marandu were infested with five adults per pot, during $2,4,6,8$ and 10 days, more distinctive damage between the susceptible and resistant plants were observed in 20 days old plants infested for only two days; and 4) as regard to damage by adults, it is important to remember that spittlebug females, possibly due to different nutritional requirements, cause greater damage than males. Therefore, is necessary to standardize adult damage evaluation trials either for males or females.

\section{EMBRAPA - CNPGC. C. Postal 154 - CEP 79002-970 - Campo} Grande, MS, BRAZIL

FAX (067) 7632245 - Email: jraul@cnpgc1.embrapa.anms.br

\section{0}

THE BIOLOGICAI CHARACTERS OF THE FUNGUS PSEUDOPERONOSPORA CUBENSIS ROSTOVS IN VIEW OF THE PROBLEM OF CUCJMERR RESISTANCE TO FAISE UILEW

\section{E.A.Vlasova}

Cucumber resistance to false mildew was studied at three regions of Russia, in Moldavia and Tadgjikistan. The pathogene possesses high aggressivity and ia able to invade all overground organs of plants and infects all new growing tissues. The early disease development on cotyledons favour the accumulation of infection, followed by infestation of the rest of the overground organs. The teadency to expansion of the spectrum of susceptible cultivars and varieties among cucurbitaceae was shown up and the largest compatibility with the cucumber genotypes was noted. Infection of melon and pumpkin was achieved by inoculation; vegetable marrou became weakly infected, water-melon and custard squash were unsusceptible. As a whole, the agent of the cucumber false mildew has the exceptionaliy effective mechanisms for overcoming the resistance of the wide genotype diversity of the host-plant. Never-the-less, the works, accomplished by us and our colleagues (Madjldova, Vlasova, 1990, Straystar, 1991, Vlasova, 1992) show the possibility of selection of the sources of resistance and relatively resistant and tolerant cucumber varieties. It was shown that the resistance level to false mildew coincides with the resistance level to true mildew in many of cucumber specimens. The varieties, resistant to peronosporosis were revealed: C.hardwikii, Rishu, Sanazu, Mid, Chipper, Score, MSU 9410, Centurion, Mig, Beregovoy, Decan, Contact, Cascade, Vernisaz, Epilog, etc.

St.Petersbourg State Agricultural University, Chier of phytopathology, Leningradskoye s.2,k.9 St.Petersbourg-Pushkin, 189620 Russia Phone $476-04-36$ 
THE ROLS OF WHEAT GRIONOS IN CHANG ING THE GERETIC SARUCTURS OT T.CARIBS POPUIATIONS

A.Y. Itamaleev, R.F . Isaev, R.R.Thasanov, A.A.Iamaleevo (Departeent of Biochenistry and Cytochemiatry, Ufa Scientific Centre, Russian Academy of Sciences)

Regarding the problems of genomic selection it is of great interest to investigate the effect of wheat genomes on the genetic structure of T.caries populations. Practically all the ditic structure of .caries populations. Practicalyy al the diploid wheat species and egylopses - donors of
genomes - have been taren into consideration.

The pathogenic passage from soft wheat through T.monococcum The pathogenic passage from sort weat through T.monococcum and r.beoticum plants (donors of the c genome, 2nit) results In changling the genetic structure or the basic ror. No virulonce to the Bt 4 resistence gene occura when analjsing with a monogenic difforentiating set. The back pasage of this pathogone on the audeptible species also results in a certain the aggression to the Bt-1 and $\mathrm{Bt}-7$ resistence genes lowered the aggression to the Bt-1 and Bt-7 resistence genes lovered abruptly while any virulence to the Bt 4 resistence gene does
not occur. So, it may be concluded that the transition of the pathogenic basic race under ney conditions changes its genetic structure to an essential degree and, accordingly, the T.caries specialization.

The complication of the virulence spectrum in wheat genomes is a consequence of the erolutionary process. Hutations and introgressions in the genomic individual zones may appear as the direct causes. Gene engineering methods suggest to solve many problems such as interpreting initial products of resistence and virulence genes.

Department of Biochemistry and Cytochemistry, of Seientific Centre, Russian lcaderay of Sciences, Prospelt Ortyabrya 69, Ura 450054, Russia

1063

RESISTANCE DIFFERENTIATION AND MATURE-PLANT RESISTANCE OF BANANA PLANTS AGAINST BANANA BUNCHY TOP VIRUS

Z.J. Zhou*, L.H. Xie, Q.Y. Lin, G.K. Liu

Banana bunchy top disease (BBTD) is the most economically important disease of bananas in China. It has been reconded in the provinces of Fujian, Guangdong, Guangxi, Hainan, Yunnan and Taiwan. There has been some research on the variety resistance against the disease, however, resistant varieties are less acceptable to growers and consumers, thus limiting their use. Our studies have shown differences of disease incidence in sitz and in vitro among banana genotypes. Following inoculation tests, AAA genome bananas were generally shown to be higtly susceptible, AAB bananas were susceptible and ABB bananas were resistant.

It was also found that many plants with an AAA genome bananas had survived for decades in the habitually epidemic areas in the plantations. These plants were shown to have a variation of resistance against the virus. The most resistant plant strain, designed as Xinjiao No.1, was chosen and confirmed to be free of all banana viruses found in China. Its suckers were then used for culturing explants and micropropagating plantlets by a micropropagation factory. Further resistance test of the micropropagated plantlets showed that they were as highly susceptible as $\mathrm{cv}$. Taiwanjiao belonging w AAA genome type, but as the plantlets grew higher and older, they became more resistant. The resistance of the older plants was therefore referred as mature-plant resistance. With the use of these micropropagated plantlets in a proper location and season, the plantations can be kept free of BBTD. These result opened an altemative way to use the genotype resistance as a measure to control the disease. It also showed that good resistance could give full play to banana burchy top virus orly when it is adequately managed by planting the micropropagated AAA plantlets early in the season to produce "mature-plant " by the time of high aphid vector infestation. The prospects for using resistance as a control measure for the disease will be funher discussed.

Inst of Plant Virology.Fujian Agricultural University.Fuzhou 350002, P.R. China; Present address for comespondence: Dept. Vinus Res., John Innes Insinite,Norwich NR 47 UH, UK.
1064

INTEGRATED MANAGEMENT OF MALATHION DEGRADATION BY WILD LEGUME RHI ZOBI UM

L.V.Gangawane and fomain P. Francts Soll Microbiology and Pesticides Laboratory,

Department of Botany.

Dr. Babasaheb Ambedikar Marathwada University,

Aurangabad 431004, MS, Indla

Degradation of a pesticide compound in the environment and in agricultural solls is desirable as soon as the pest or disease is controlled. Microbial processes play an important role in the biological degradetion or transformation of pesticides. In the present investion a the ability of Rhizobium isolated from the nodules of wild legumes such as Indigofera echinata I olandulosa I duthei I tinctoria Crotolaria orixensi echinata 1 1.8landulosa, ${ }_{1}$ duthei, 1. 1inctoria, crotolaria orixensi and Thephrotia purpurea. Al the 22 isolates were efficient for nodulation and $N$ ina maxlmum degradathon than other lsolates. Enzymes like phosphatase and carboxymuse malathon. Rhizoblum. TLC and HPLC study lndicated the melatnion dicarboxylic acid, 0-dimethylos plasmid protle could not locate gene on smaller plasmids. Use of root exudates (Vitariln B-12), Fusartum oxysporum. Mg, Mn, Mb, Bo, $P$ (Superhosphate), $X$ (murlate or potash) alongwith Rhizoblum 1solate RId-12 or mutant RId-12 UV-B increased melathion degradation
significantly within 10 days.

Prof. L.V. Gangawane, soll Microbiology and Pesticides Laboratory, Department of Botany, Dr. Babasaheb Ambedkar Marathwada University, Aurangabad 431004, MS, India. 
EFFECTS OF OOMGINAT IONS OF PEST IOIDES AND PHYSICAL, OHEMIOAL
AND ENVIRONMENTAL STRESSES ON FREE RADICAL OXIDATIVE PROCESSES IN PLANT TISSUE

\section{N. Loginova, L. M Karpova, G. L. Egorova} The effects of combinations of pesticides and physical,
chemical and environmental stresses on tree radical oxidation in treaed plants (wheat, potato, carrot) were studied. This was accomplished by the monitoring superoxide radiaal [0; was accomplished by the monitoring superoxide radiad [0; catalase, peroxidase [antiradical enzymes] and hydroxilating activities in plants treated by different types of activities in plants treated by different types of estimation of bound peroxidase solubilization. Among the stress-factor there were: iv-irradiation; extreme temperature-chilling there: and $+35-45^{\circ} \mathrm{C}$; ${ }^{2}$ chemical stress-non-physiological concentration of $\mathrm{Cu}^{2+}$ ions high non-baianced level of the fertilizers. It was shown that active oxygen species are most potent destructive agents in plants exposed to combinations of pesticides and other stresses. The strong synergetic effect of pesticides and chemical stress on $\mathrm{O}_{2}$-generation and of lipid peroxidation products in plant tissue were revealed. These processes were accelarated by superoxide dismatase inhibitors. The combinations of pesticides and chemical stress caused decrease of the antioxidant activity of fungicides and increase of the herbicide activity. Lipid peroxidation activation in exposed to pesticides, chemical and the temperature stresses was accompanied by the weakening of lipid-prote in interactions in biomembranes and following solubilization of bound peroxidase and the changes of chemiluminescence parameters. A combination of pesticides and temperature stress (chiliting) caused $\mathrm{H}_{2} \mathrm{O}_{2}$ accumulation as a esult of catalase and peroxidase activities inhibition. In model experiments this combination was used with active oxvgen species generating system (UV-irradiation+ $\mathrm{H}_{2} \mathrm{O}_{2}$, . In that case the strong suppression of the frotective ant iradical systens was discovered. It is important, that hydroxylating system responsible for xenobiotics detoxification was severely inhibited by this combination too, especially when $\mathrm{Cu}^{2}$ was added. It seems that there is threshold of restriction on antiradical protection. It may be imoosed if oxygen radicals formation and peroxidation of membrame lipids combination with environmental and physico-chemical st resses - temperature, UV-irradiation, non-balanced and over-dosed fert itare, of the free radical oxidat ive metabolism in plant cells.

All Russian Institute of Plant Protection, Podbelskogo shauss. 3, 182620 Pushkin, St.-Petersburg Region. Russia
THE INFLUENCE OF STORAGE CONDITIONS IN THE QUALITY CONTROL ANALYSIS OF PESTICIDE SAMPLES

Fernandez, C., Neves. Fl, Seltzer, H., Gaese, $\mathbf{H}$

The degradation of active ingredients is not only important for the evaluation of ecotoxicological behavior of pesticide but can also influence their efficacy in the application. Quality control as conducted by the Ministry of Agriculture of the Säo Pauio State/Brazil is therefor of great importance.

The ethylenebisdithiocarbamates are inherently unstable and have been found to degrade to different products including ethylenethiourea, ethyleneurea, 2 . imidazoline, ethylenethiuram monosulfide and ethylenebisisothiocyanate. The extent of decomposition of the formulation depends upon storage temperature, time, light exposure and relative humidity.

The purpose of this study was to determine the influence of samplig and storage conditions which the formulation may be subjected under the inspection process. The model atrempts to simulate this conditions.

Results are presented to show the degradation of MANCOZEB depending on

- container materials

- storage temperature

- moisture content

- sun radiation exposure

- storage time.

CATI - Serviço de Análises e Diagnósticos Av. Brasil, $n^{\circ} 2340,13073-001$ Campinas - SP, Brazil

POSSIBLE EFFECT OF GLOBAL WARMING ON RICE LEAFFOLDER

R. Zhang, K.L. Heong, I.T. Domingo

The effect of global warming on rice leaffolder(RLF), Cnaphalocrocis medinalis was evaluated under the


The temperature range of 25 and $300^{\circ}$ is favourable to the growth of RLF population, in which the survival rate from egg to adult ranges between 23.1 and 36.68 and the fecundity is higher. However, the lethal influence of temperature is significantly enhanced as temperature reaches to $35^{\circ} \mathrm{C}$ at which the eggs were unable to hatch and the adults also failed to lay eggs. Larval parasitization is low at $35^{\circ} \mathrm{C}$ and none of larvae were parasitized at $40^{\circ} \mathrm{C}$ as the parasitoids were unable to survive. The hibernation area would move northward 1-20N latitude and the occurrence would increase $1-2$ generations if global warming occurs.

Research Institute of Entomology, Zhongshan University Guangzhou 510275, P.R. China 
NITROGEN APPLICATION TIMING FOR INTEGRATED MANAGEMENT OF BROMUS TECTORUM IN WINTER WHEAT

D. A. Ball, D. J. Wysocki, and T. G. Chastain

Management of Bromus tectorum L. in winter wheat presents a major constraint to adoption of conservation tillage system in the Pacific Northwest region of the U.S.A. This region produces wheat under annual precipitation ranging from 250 to $700 \mathrm{~mm}$, and is subject to high rates of soil erosion due to steeply rolling topography. Conservation tillage systems are being implemented to mitigate soil erosion. Changes in nitrogen management practices have been proposed as a method to protect water resources from excess nitrate nitrogen. Changing nitrogen management practices produce changes in whed populations and their competitive relationship wheat, thereby offering potential for improving the integrated management of Bromus tectorum. To integrate weed
control and fertilization practices a study was undertaken to control and fertilization practices a study was undertaken nitrogen management practices in this environment.

Field experiments were conducted over two seasons at two locations in areas with low annual precipitation $(300-460$ $\mathrm{min})$. Main treatments consisted of $\mathrm{N}$-fertilizer rates and application timings using a new point-injection fertilizer technology in a winter wheat/summer fallow rotation. Treatments consisted of an untreated control, $N$ conventionally applied in fallow prior to planting, $N$ applied at planting, $N$ applied in growing crop using point injection split applications of $\mathrm{N}$ applied in fallow plus $\mathrm{N}$ applied in growing crop using point injection, and split applications of $N$ at planting plus $\mathbf{N}$ applied in growing crop. Main treatments were split into two subplots, one of which was seeded with Bromus tectorum prior to wheat planting and the other not seeded. Measurements were made of wheet growth and grain yield, and Bromus tectorum percent cover and dry matter production.

Results are from plots that were subjected to the same fertilizer treatments for two consecutive cropping cycles. In one location when cropping season precipitation was $95 \mathrm{~mm}$ above average split application of precipitation was 95 m above average, split application of $N$ and later application full $N$ application rates applied in fallow and to the full $N$ application rates applied in fallow and to the untreated control in the absence of Bromus tectorum: When Bromus tectorum was present at levels interfering with whea growth, weed dry matter production was increased by split applications and later application timings. The increased interference from Bromus tectorum from these fertilizer split application timings.

Columbia Basin Agricultural Research Center, oregon state University, $P$. O. Box 370 , Pendleton, OR 97801. USA

1071

PESTS, YIELDS, AND YiELD GAPS AS RELATED TO PRODUCTION SITUATIONS IN FARMERS' FIELDS IN RAINFED LOWLAND RICE AREAS OF SOUTHEAST ASIA.

H. O. Pinnschmigh, F. dela Peña, N. Cabulisan, V. Chamarerk, N. D. Long, and P. S. Teng.

Crop protection and cropping intensification treatments were integrated with holistic field surveys conducted at RL sites in the Mekong River Delta (S Vietnam), NE Thailand, and NW Luzon (Philippines) in 1992 and 1993. Data on crop development, yield, dynamics of disease- and other pest severity levels, weather-, water-, and soil conditions, and crop- and pest management practices were collected. Simulation and empirical modelling approaches were employed to estimate potential yields limited by weather, $N$, and water plus their corresponding yieid gaps. Correlation and factor analyses were done to characterize patterns of and interrelations among cropping- and pest control practices, factors determining productivity levels, and severity levels and yield effects of pests. High yieids were associated with parameters of high soil productivity, such as soil carbon content, high fertilizer and pest control inputs, little drought stress, and high pest severities. The total yield gap was estimated to be around $30.50 \%$ and be mainly due to $\mathrm{N}$ limitation. The estimated yield gap due to factors other than $\mathrm{N}$ and water limitation was around $10-30 \%$. Intensive chemical crop protection increased yields by less than $10 \%$ but did not consistently affect overall disease-and other pest severity leveis in comparison with the control. Yield gaps were positively correlated with pest severity levels after yield effects due to $\mathrm{N}$ limitation were accounted for. Sucking insects such as brown plant hopper and rice bugs, the dirty panicle complex, stem rot, weeds, stem borers and other tiller damaging pests, blast, sheath blight, brown spot, and bacterial leaf blight were among the key pests with respect to incidence and severity levels. Leaf yellowing and root rot were severe at some Vietnamese sites. Thai sites were characterized by less intensive cropping practices and less favorable soil conditions, compared to Philippine and Vietnamese sites. The data indicate that raised production levels will increase pest intestation levels while the actual economic importance of these remains unclear.

Entomology and Plant Pathology Division, International Rice Research Institute, P. O. Box 933, 1099 Manila, Philippines.

\section{THE PERSPECTIVE OF HEAVIER DAMAGES AND THE NEED FOR NEW CONTROL METHODS OF HETERODERA SCHACHTII SCHM.RELATED TO CLIMATE, ECONOMICAL, AND TECHNICAL CHANGES IN POLISH AGRICULTURE.}

\section{H.Banaszak}

For several years there has been a change in climate in Poland. It is warmer, the winters come later and therefore shorter; they are also without substantial snowfall which brings moisture. These changes are the cause of moisture deficit soil during the Spring and Summer months. The damages from Heterodera schachtii Schm. is heavier in moisture deficit soil than in optimally moist soil. The highest influence on the increase of Heterodera schachtii Schm. damage may be due to the specialised short rotations of sugarbeet and the cultivation of rape in sugarbeet rotation which are connected with economical changes in Polish agriculture. These factors change the high number of pests in the field. Another change in increased pest damage may be the technical dissemination of new sugarbeet seed and changes in the assortment and technical application of herbicides in sugarbeet cultivation. The sugarbeet plants from technologically new seeds are less resistant to disease connected with Heterodera schachtii Schm. The new assortment and the application of herbicides has not restrained the incubation of Heterodera schachtii Schm. larvae as effectively as the old assortment and application of herbicides.

We must look for new biological control methods of Heterodera schachtii Schm. because chemical control methods of this pest aren't effective and ecological. These related factores will been shown in this poster.

Dr. Heliodor Banaszak, manager

Plant Protection Institute in Poznan

Field Experimental Station in Torun

Zwirki i Wigury 73 str.

87-100 Torun

Tel/Fax:202-23

Poland

\section{2}

DEVELOPMENT TRENDS AND ECONOMICS OF THE CHEMICAL CONTROL IN THE IPM IN RUSSIA

\section{ZAKHARENKO V. A.}

In accordance with the areas occupied by agricultural crops, the distribution of harmful organisms and indices of their harmfulness to the crops losses in the Russia are estimated an average about $28 \%$ all crops every year in 1985-1990, more then $30 \%$ in 1991-1994. In the system of protection measures for 30 years preferential development has the chemical method. The saved yield crops due the pesticides application are reflected $17.4 \%$ all crops, 62.1 $\%$ of the annual crop losses in 1985-1990. But the usage of chemicals for the pest control leads to such problems as environmental pollution, development of resistance to pesticides, harmful effects on non-target organism and threat to the health of man. The economic losses in the Russia connected with the negative effect of pesticides is estimated as $14.6 \%$ of the saved yield crop due to the pesticides. In condition of modern agriculture chemical of control of harmful organisms should correspond to progressive integration of plant protection. Predictive calculation indicates that the integrated protection, according to economic indices, is excessive if introduced on all sowing areas. It can be effeciently introduced in zones with intensive agriculture, where high dosages of pesticides per hectar are used and the danger of their side effect is great (area 30\% of arable land). In other regions it is advisable to maintain the traditionally established system of plant protection with moderate or without use of pesticides. 
Molecular basis of pathogenisity in Corchorus capsularis Var. snow white.

Hosne-ara-Begum and A.I.Khandakar,

Bangladesh Jute Research Institute, Dhaka.

Corchorus capsularis and C.olitorius are two cultivated species of jute and are major fibre crops in South East Asia. In Banglades it alone earns thirty percent of the country's export earning. Very recently Bangladesh Jute Research Institute has developed a variety of white jute (Corchorus capsularig) which produced fibre snow-white in colour and is called snow white jute. It attracted buyers and fetched higher price than the fibre produced from other cultivars of jute. But unfortunatelg enongh it was observed that the snow-white jute was auch more susceptable to sted-rot diseases than other existing varieties.

Scientific investigations have been undertaken to unmask the reason of such pathogenisity in the variety under report through both microbial and cbemical studies. The stem-rot disease was coused by Macrophomia phaseolina. The growth of the fungal patbogen was more or less double on dry weight basis in the water soluble extract of the stem of the variaty snow-white at 60 days than that of some other cultivars in production. Chemical studies reverled that the water soluble extract of the young stem of the variety snow-white contained three times more sugar but lesa protein content than those in other cultivars ( $\mathrm{CV}-1$ and Lalshachi).

$A$ bioassay was established with the stimulant water soluble extract. On purification of the extract through organic solvant aystem, Sephax column, ion-exchange chromatograpby and paper chromatography identified the stimulant material as mannose, a monosacharide sugax. Mas-spectral studies will be conducted to confirm the chemical identity of the stimulant material.However, the pure sample of mannoge proved to be really stimulating more than other monosacharides in the growth of Macrophomina phaseolina that produced stem-rot in snow-white jute.

\section{CHARATERZATION OF' THE GARLIC WHITE SPOT} Almaguel Léridas J. Ramirez, Guadalupe Gómez, S. Jiménez, E. Pérez, Magałys Suárez, M.
Elena Harty and J. Cortinas.

The gartic white spot is a non-infectious pathology, which was known in Cuba since 1981. The highest damages are present during Janury-February, where the mos! severe affectations reach up to $30-40 \%$ of the planted areas (most problematic years) and high harvest losses occur. The "Criollo"variety is the most sensible when reaches 50-60 days of it cycle. In 1991 this pathology caused strong losses to garlic crop. Several experiments were conducted under laboratory conditions simulating acid rains $(\mathrm{pH} 3.3-4.5)$ and a regiter ozone device wass installed at the closer Meterological Station in Batabanó during the 1992-1993 season. Crop areas belonging to the private and state propieties were evaluated in order to charaterize the appearence and development of the white spot. In this work it was determinated that the patology is produced by ozone, polhsion, which come to Cuba as a result of the meterological fenomena. Three spot types were observed: apical spot or "streamer type", "alive white spol" and "white freckels", depending on the variety, leaf consistency, plant structure, elc. In any case the first symptom is an invisible clorosis that appears 24-48 hrs later when critical ozone concentration occurs and after 3-4 dzys evotves to a typical milly white spot (most known symptom) that laots 7-10 days and starts the leaves dying (known damage) making them hang from the false stem during the rest of the plant cycle. An amount of 45-55\% losses was observed due to plant cycle shortening, plant fading and bulb development interruption.

Instituto de Investigaciones de Sanidad Vegetal. Calle 110 \# $514 \% 5$ ta B y 5ta F, Playa.C Habana. Cuba.
EFFECTS OF SOIL AMENDMENTS ON VESICULAR-ARBUSCULAR MYCORRHIZAL FUNG

\section{S. Nemex}

Three sites were prepared for citrus groves in Florida in 1985 - 86. Seven soil amendments were preplant deep-tilled $1.2-15 \mathrm{~m}$. deep and $1.5 \mathrm{~m}$ wide in the row. Treatments were limestone $\left(56,000 \mathrm{~kg} \cdot \mathrm{hg}^{-1}\right)$, commercial gypsum $(1120$ and $2240 \mathrm{~kg}$. hat), phosphogypsum $\left(11,200 \mathrm{~kg} \cdot \mathrm{ha}^{-1}\right)$, phosphoclay $(89,000 \mathrm{~kg}$. ha-1), humate $(86,912$ kg. ha $\left.\mathrm{a}^{-1}\right)$, shrimp waste $(20,451$ and $81,805 \mathrm{~kg}$. ha- $)$ and peat $\left(280,000 \mathrm{~kg} \cdot \mathrm{ha} \mathrm{a}^{-1}\right)$.

Deep-tilled controls and a no-till control were established at sites $A$ and $B$ and a deeptilled control at site C. Mycornizal fumgus infection was determined in roots in 1987 at sites $A$ and $B$ and in 1989 in site C. Fungus infection ranged from 6 (no-till control) to $64 \%$ in site $A ; 64$ to $81 \%$ in site $B$; and 15 to $47 \%$ in site C. In all sites, amendments did not improve percent infection, and vesicle, and hyphae ratings significantly over the deep-tilled control. In site A, percent infection in the limestone treatment was the highest (64) and was significantly better than infection in phosphogypsum, peat, and no-fill control treatments. No treatment had a suppressive effect on infection. Phosphoclay, phosphogypsum and peat applied to soil in pots amended with Glomus mossece or $G$. imtroradices and planted with citrus had no depressive effect on infection. Humate added to soil in pots, however, at $314 \mathrm{~kg}^{-h^{-1}}$ reduced $G$ mossea infection. In general, these amendments when deep-tilled in field soils do no after the infection levels of VAM fungi.

USDA, ARS

2120 Camden Rd.

Orlando, FL 32803
1076

POPULATION TREND OF THE CHRISOMELID BEETLE COPa OCcidentalis ON LATE SEASON FLUTED PUMPKIN (Telfaria occidentalis)

Author: UKO EJIGHIONWU.E.

Ielfaira occidentalis known as fluted pumpkin is a leafy Vegetable valued highty during the late season because of its scarcity due to no rainfall. Within these period (october - March) under research the leaves are ravaged by a particular insect pest known as Copa Occidentalis and never to come back again until the same time next season. These research was carried out to determine the highest month of Occurance using three Local Cultivers, Ubong afaria I, II, III, having difference in Morphology in relation to size colour, and texture of the leaf and seed, and to find out preference if any. A feeding test was carried out to determin the preference of Beetle to the different cultivers and a statistical analysis was run using the ANOVA Table at ( $P \quad 0.5$ ) and using LSD to check if there is preference in feeding habit of the Beetle to the leaves of the
pumpkin. The population of the insect was determined by "Scoop net" Method of counting and a graph drawn on the population against each month to determine the population trand of the insect. The highest population occurence was during the month of December and there was no significant preference between UBONG afaira I. \& II, but ubong afaira IJI has preference which could be attributed due to its hard texture and coarsness.

NO. 5 OMOTOLA STREET IWAYA, YABA, LAGOS. NIGERIA. FACULTY OF AGRICULTURE DEPARTMENT OF CROP SCIENCE INIVERSITY OF CALABAR

P.M.B. IIIS CALABAR, CROSS RIVER STATE, NIGERLA. 
ROLE OF SYNTHETIC CHEMISTRY AND BIOTRANSFORMATIONS FOR AGROCHEMISTRY

\section{Kenji MORI}

Natural product chemistry has continuously supplied lead compounds useful in agrochemicals research. For example, the discovery of pyrethrins was the beginning of the pyrethroid industries today. It qujte often happens, however, that a bioactive natural product is obcainable onily in a limited amount. Synthetic chemistry is the most powerful helper in that case to provide the natural product itself or even its more potent mimics. Examples will be given among the bioactive natural products which were studied by my own group in Tokyo. They include serricornin (1, the cigarette beetle pheromone), japonilure (2, the Lapanese beetle pheromone), disparlure ( 3 , the gypsy moth pheromone), brassinolide ( 4 , a phytohormone), and glycinoeclepin A (5, a harching stimulus against soybean cyst nematode).

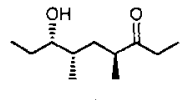

1

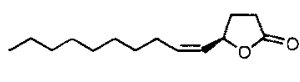

2

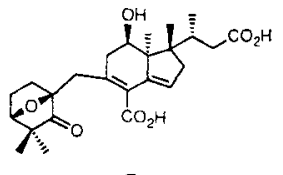

5

Department of Chemistry, Science University of Tokyo, Kagurazaka 1-3, Shinjuku-ku, Tokyo 162, Japan

1203

\section{IHE FUNGICIDAL ACTIVITY OF SORAPHEN A AND ITS DERIVATIVES}

Gemard Hötle." Anthony C. O'Sulivan, Mans Reichentoach. and Marius Sutter"

"Ciba-Geigy AG, CH-4002 Basel, Switzerland.

+Gesellschaft für Biotechnologische Forchung D-38124 Braunschweig, Germany

Soraphen A 1 was isolated recendy from the myxobacterium Sorangium cellulosum by Höfle et al. at the Gesellschaft für Biotechnologische Forschung at Braunschweig. It was screened as a crop protection agent in greenhouse trials. and was found to possess excellent activity against fungal pathogens on plants. ${ }^{2}$ The fungicidal activity of 1 proved to be so interesting that the producing strain was reexamined. More than 20 congeneric metabolites were then isolated, building a family of naturally occurring soraphen derivatives. ${ }^{3} \mathrm{At}$ the same time a collaborative derivatisation program was initiated starting from the major metabolite soraphen $A$. In this presentation the fungicidal activity of Soraphen $A$ will be compared with that of its derivatives.

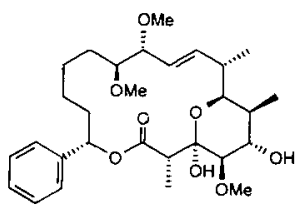

Soraphen A

1. Bedor, N.; Schomberg, D.; Gerth, H.; Reichenbact, H.; Höle, G. Liebigs Antr. Chem. 1993, 1017. Gerth. K.; Bedorf, N.; Issckik. B.: Höne. G.; Reichenback. H. J. Ámibioties 1994, 47, 23-31.

2. Gesellschaft fur Biotechnologische Forschung and Ciba-Geigy AG EP 282455 A2.

3. Bedorf. N.; Böhlendorf, B;; Forche, E.; Gerth, K.; Höne, G.; Irschik, H.; Jansen. R.; Kunze. B.; Reichenbach, H.; Sasse, F.; Sreinmea, H.; Trowitzch-Kienast, W.; Pachlatko, P. GBF and Cibz-Geigy Ef 358606 (9 Seps. (988) (Chem. Abs. 1990.113. 4l2l3s).
SCREENING OF BASIDIOMYCETES FOR NEW ANTIFUNGAL COMPOUNDS : THE STROBILURIN STORY

Timm Anke, Wolfgang steglich

The production of antimicrobial compounds is observed in cultures of many basidiomycetes. However, most of the compounds isolated so far exhibit a broad and unselective toxicity towards pro- and eucaryotes. Many of these compounds are either sesquiterpenoids or acetylenes. Among the very first selective antifungal antibiotics to be detected in our screening were the strobilurins $A$ and $B$ produced by strobilurus tenacelius, a small edible agaric. Subsequently strobilurins and related oudemansins were detected in cultures of many other basidiomycetes and one ascomycete from different locations both in tropical and temperate climates. The strobilurins exhibit high antifungal activities against ascomycetes, basidionycetes, zygomycetes, and oomycetes by interfering with electron transport mediated by the mitochondrial bcy complex. The new target and the lack of taxicity towards mammals provided a strong incentive to synthesize both the natural products and a great number of analogues and derivatives. Synthetic efforts in industry finally resulted in compounds with considerably improved activity and, important for an application in the field, light stabiiity.
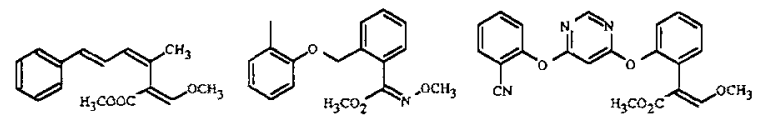
Strobilunin A
Strobilunus ssp. BAS $490 \mathrm{~F}$
BASF AG

ICIAS504

ICI

Prof. Dr. T. Anke, LB Biotechnologie der Universität, Paul-Ehrlich-Str. 23, D-67663 Kaiserslautern, FRG
1204

A SERIES OF DICYCLIC INSECT JUVENILE HORMONE ANALOGS OF THF CZECH ORIGINR: TWENTY YEARS OF DEVELOPMAENT

\section{Z. Wirnmer, M. Rejzek, M. Zarevücka, J. Kuldová, I. Hrdý, V. Nèmec and M. Roınañuk}

Several milestones may he seen in the research of insect juvenile hormones during its 75 year long history. Let us mention the Kupec's discuvery of the "brain" hormone in 1920's, followed by the Wigglesworth's finding of an "inhibitory" hormone in 1936, for which he suggested the name juvenile hormone $(\mathrm{JH})$ in 1940 , and by the Slama's and Williams's discovery of the "paper tactor". later identified as juvabion. This compound became one of the first naturally occurring insect juvenile hormone analogs (JHAs), and started a new era of the insect $\mathrm{JH}$ research, resulting in the isolation and identitication of five compounds, being considered to repiesent the insect $\mathrm{JHs}$, and in the synthesis of hundreds of compounds displaying the same sort of biological activity as the natural JHs show. Since the mid-1970's, scientists, having dealt with the research in this area, have increased their effort to develop more stable, but not persistent, and environmentally safe structures. In 1975. Swiss (Ciba-(ieigy) and (zech scientists published new series of dicyclit SHAs.

This contribution summarizes the twenty year history of development of dicyclic JHAS, based un 2-(4-hydroxybenzyl)-1-cyclialkanones, which have been developed in the Institute of Organic Chemistry and Biochemistry in Prague. The initial research started in 1975 with the synthesis and structure-activity relationship studies in several 2*(4-hydroxybenzyl)-1-cyclohexanone terivatives. After having prived promising biokgical activity with two or three series of the IHAs, the attenturn has heen utmed to the main goal namely is explain, why the derivarives of 2-(4-hydroxybenzy)-1-cyclwhexanone have heen chosen for this investigation. The ketonic function of the saturated ning enables easy derivatizing, which may finally result in preparation of several related compounds, differing in their physiconemical properties. This research has been motivated by the preceding discovery of juvenogens (Sláma and Romañuk, 1976). The term juvenugen has been used w indicate complex substances capable of liberating a biologically active mulecule ( $=$ a JHA) under the influence of hintic or abiotic factors. In this context, simple reduction of uriginally present ketonic function in the molecule to an alcoholic we results in: (a) a synthesis of separable cis and trans isomers of the appropriate JlAAs, (b) an introduction of an easily transformable function, which lurther derivatizing may enable preparation uf a broad range of different compourds and (c) a porential synthesis of chiral JHAs. Synthesis of juvenogenic glycosides, glycerides, fanty acid eslers and wther derivatives has extended remarkably both the way of research in inseci endacrinology, and of application of JHAs in insect pest control. Polar iuvenogens enable systemx application against sucking insect species \{aphids, heteropterans etc.), apolar juvenugens enable ural applization by mixing with the appripriate food fior tested insect species, and all of them enable application by spraying piant surface or an area tos the protected against insect pesis. Positive results of the structure-activity relationship studies provided with about twenty series of compounds derived from 2-(4-hydroxybenzyl)-1-cyelohex anone have initiated the research consisting in modification of the size of the saturated ring and in its further substitution in inder on investigate the strutture-activity relathinships in even mure details. Successfut syathesis of steremisumers of several fHAs noens ither apportunity to augment the understanding of the insect $\mathrm{JH}$ (and $\mathrm{JHA}$ ) mode of action. The investigation on chiral JHAs has now heen the basic target of the present research program in this area. 
DISCOVERY AND BIOCHEMICAL MODE OF ACTION OF THE PHENYLPYRROLE FUNGICIDE FENPICLONIL

Ad Jespers', Maarten de Waard ${ }^{2}$

CGA 142705 (fenpiclonil) and CGA 173506 (fludioxonil) are the first phenylpyrrole fungicides commercially developed by CIBA-GEIGY AG, Basel. Fungi among the Ascomycetes, Basidiomycetes and Deuteromycetes are sensitive. Benzimidazoleand dicarboximide-resistant field isolates are not cross resistan with phenylpyrroles.

Fungicidal activity of phenylpytroles was first recognized in pyrrolnitrin, which is produced by several Pseudomonas species The use of these bacteria in biological control programs has been investigated, but never gained large scale application. Pyrrolnitrin was not commercialised as a fungicide in agriculture because of its low stability in the light and its difficult and inefficient full synthesis. One-step-synthesis of phenylpyrroles became feasible when a strong electron-withdrawing cyanide group was introduced in the 3-position of the pyrrole ring. Since stability was improved as well, the cyanopyroles were the basis of the now commercialised phenylpyrroles fenpiclonil and fludioxonil.
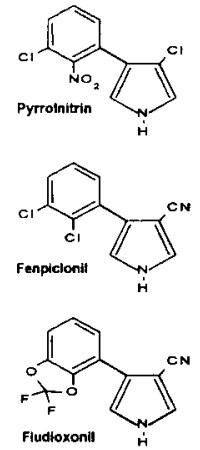

The biochemical mode of action of fenpiclonil has been studied in the fungus Fusarium sulphureum (Schiecht), one of the causal organisms of damping off in cereals, of which control by fenpiclonil is recommended. By monitoring the interference of fenpiclonil with fungal metabolism, the accumulation and incorporation of glucose into fungal glycans appeared to be most strongly inhibited. However, when elimination of carbon dioxide from glucose and the behaviour of various glucose analogues was studied, it was concluded that the observed effects are best explained when fenpiclonil would inhibit trans-membrane transport associated glucose phosphorylation, the most plausible mode of action of fenpiclonil

Ad B.K Jespers, H\&PC Research, P.O.Box 16299, 2500 AE The Hague, The Netherlands. Maarten A de Waard, Wageningen Agricultural University, P.O. Box 8025, 6700 EE Wageningen, The Netherlands

We acknowledge CIBA-GEIGY AG, Basel for fully financing this study

1207

CYSTEINE-RICH ANTIMICROBIAL PEPTIDES FROM PLANTS AS FACTORS FOR INCREASING DISEASE RESISTANCE IN CROPS BY MOLECULAR BREEDING

Broekaer W.F ${ }^{1}$, Terras F.R.G.', De Bolle M.F.C. ${ }^{1}$, Cammue B.P.A. ${ }^{1}$, Osborn R.W. ${ }^{2}$, Rees S.B. ${ }^{2}$

We have purified a series of cysteine-rich antimicrobial peptides from various plant seeds. Based on their amino acid sequences, these peptides can be classified in at least six different classes. Each of these classes has its own characteristic biological activity properties. In the case of $5 \mathrm{kDa}$ antimicrobial peptides from Raphanus sativus we have studied the expression pattern of these proteins by immunocytochemistry and RNA blot experiments. In mature seeds, the antimicrobial peptides are most abundant in the outer cell wall of peripheral cell layers of seed organs, and in leaves the expression of the peptides is strongly induced upon fungal infection. To see if constitutive expression of such antimicrobial peptides in a heterologous plant may confer enhanced disease resistance, we have made several constructs based on cDNA sequences of the different antimicrobial peptides and introduced these constructs into tobacco. Transgenic tobacco plants expressing the antimicrobial peptides at about $0.1 \%$ of total protein content have been regenerated and bred to the homozygous stage. Some of these transgenic lines show enhanced resistance against foliar fungal pathogens.

${ }^{\prime}$ F.A. Janssens Laboratorium voor Genetica, Katholieke Universiteit Leuven, Willem de Croylaan 42, B-3001 Heverlee (Belgium); ${ }^{2}$ ZENECA Agrochemicals, Jealott's Hill Research Station, Bracknell, Berks RGI 2 6EY (UK)

\section{STEREOSELECTIVE; ENZYME CATALYZED PRODUCIION OF} L-PHOSPHINOTHRICIN

K. Bartsch, A. Schulz and U. Kragl

L-phosphinothricin (L-homoalanine-4-yl-(methyl)phosphinic acid) I (L-PPT) is the active ingredient of the herbicide Basta. A coupled, enzymatic process for the synthesis of the active L-enantiomer from its non chiral 2-ketoacid precursor (2-oxo-4-[(hydroxy)(methyl) phosphinoyl]butyric acid] (PPO) was developed by application of two bacterial transaminases.

In the first step of the reaction PPO is converted to L-PPT with 4-aminobutyrate:2-ketogluta-rate transaminase (EC 2.6.1.19) from $E$. coli using glutamate as the amino donor.

In the second step glutamate is regenerated from aspartate by aspartate:2-ketoglutarate transaminase (EC 2.6.1.1) from Bacillus stearothermophilus. The simultaneous action of both enzymes allows quantitative product yields by using only moderate molar excess of the amino donors glutamate and aspartate, since the reaction by-product oxalacetate decarboxylates spontaneously to pyruvate in aqueous solution.

The genes gabT from E. coli and aspC from Bacillus stearothermophilus encoding the two transaminases have been cloned and sequenced and were used for the construction of procaryotic expression plasmids.

The two proteins were partially purified from recombinant $E$. coli overexpression strains and - after immobilization - applied to an enzyme reactor for the intermittent (repetitive batch) and continous production of L-PPT. The reacior was operated at $50^{\circ} \mathrm{C}$ and $\mathrm{pH}=8.0$ in the presence of $10 \mu \mathrm{M}$ pyridoxal phosphate and with a molar ratio of the substrates PPO : glutamate : aspartate $=1$ : $0.2: 1.2$. Conversion rates of up to $90 \%$ and product yields of up to $85 \mathrm{~g} /$ were obtained in the enzyme process. The optical purity of the produced $\mathrm{L}$ phosphinothricin was $>\mathbf{9 9 . 9} \%$.

Klaus Bartsch

Hoechst Schering AgrEvo GmbH

Biochemical Research, $\mathrm{H} 872 \mathrm{~N}$

D-65926 Frankfurt

Germany

1208

The importance of scouting techniques in cotton IPM.

\section{G.A.Matthews,}

Abstract.

Yields of seed cotton have been increased very significantly since modern chemical techniques have been used. IIowever, simplistic approaches to the use of insecticides on a calendar pest populations with subsequent crop failures in many areas.

Crop monitoring or "scouting" is not new, but has seldom been operated on an extensive regular basis in a format that allows informed decision making. Some countries have monitoring systems that allow retrospective analysis of pest infestations but the farmer requires simple techniques of judging the severity of a pest population in relation to plant development that will enable minimal intervention with insecticides as part of an integrated pest management progranime.

This paper discusses experiences with scouting techniques, including crop inspection and pheromone trapping, and how data is utilised in certain countries. 
The Wellesbourne pest forecasting models are based on rates of insect development and use a Monte Carlo simulation method to allow for variation within the insect population. The method is feasible because it uses a fixed number of individuals from one generation to the next and simulates the timing of pest activity rather than changes in pest numbers. The forecasts use standard meteorological data (air and soil temperatures) and forecasts can be produced for any locality provided appropriate weather data are available. Forecasts have pollen beetles (Mcligcthes, sp) and the large narcissus fly (Morodon cquesiris). The forecasts predict periods of pesi activity and can be used to indicate when insecticide treatinents should be applied to have maximum impact on the pest population and minimum impact on predators and parasitoids As part of the validation process, HRI Wellesbourne, funded by the Horticulture Development Council, has sent weekly forecasts to about 400 growers.

Horticulture Research International, Wellesbourne, Warwick CV35 9EF.UK.

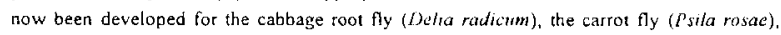

Commercial banana production is vitally important to rany developing country and small island economies of the tropics. Smallholder cultivation of Musa as a staple food is equally important in local production and consumption in many parts of Latín America, Sub-Saharan Africa and S.E. Asia. The history of the banana industry has intimately been linked with the pests and diseases that have afflicted and continue to afflict this crop. The major pest constraints are: the fungal diseases, black Sigatoka (Mycosphaerella fijiensis), Panama disease (Fusarium oxysporum f.sp. cubense). and post-harvest rots usullly involving Collecotrichum musae: the bacterial disease Moko (Pseudomonas solanacearum); the virus disease bunchy top (vectored by the banana aphid Pentalonia nigronervo-
sa); and the invertebrate pests, burrowing nematode (Radopholus similis) sa): and the invertebrate pests, burrowing

and weevil borer (Cosmopolites sorditus). For each of these pest constraints different control strategies have dominated pest management practices, including highly intensive chemical control for black Sigatoka, national exadication programes fot Moko and bunchy top, screening germplasm for virus diseases, and international breeding and germplasm improvement programes. Monitoring and early warning systems for sone aspect of pest status have been practised for most of these constraints. Molecular probes have been developed and use to distinguish among Mycosphaerella spp. and to monitor the spread of black Sigatoka in E. Africa and the Caribbean, and to improve virus detection methods for use in quarantine. Biochemical assays have been developed as diagnostic tools to detect strains of $F$. oxysporum $f . s p$ cubense in E. Africa, where Panama disease symptoms are prevalent in Uganda and the Kenya highlands, and for Race 4 of the pathogen (to which comercial cavendish cultivars are susceptible) in the sub-tropics. Fungicide insensitivity in Mycosphaerella and post-harvest pathogens is routinely assessed in the windward Islands. The monitoring of fruit quality on arrival in importing countries provides rapid information on post-harvest diseases and possible problems in plantation pest management. Monitoring and legal controls for Moko in Grenada have largely prevented the spread of this disease to other Caribbean islands. Inspec. tion and roguing of bunchy top diseased plants in New South wales has contained but not eradicated the problen and similar approaches are being adopted in some tropical areas. Contamination of newly planted areas with soil invertebrate pests, especially nematodes, will pose a considerable threat given limitations on future use of nematicides such as non-volatile organophosphates/carbomates, and will require careful monitoring. Greater use should be made of micropropagated planting material . material certified free of invertebrate pests and pathogens, although this can lead to new problems if susceptibility to virus infection is
enhanced. Despite the many achievements, much can still be done to make better use of monitoring and early warning systems in banana pest better use of monitoring and early warning systems in banana pest
management. There is a particular danger of currently minor pathogens management. There is a particular danger
becoming significant on new cultivars.

${ }^{1}$ Department of Phytopathology, Wageningen Agricultural University, P.O. Box $8025,6700 \mathrm{EE}$ Wageningen, The Netherlands.

\section{RISK ASSESSMENT FOR RICE PLANTHOPPER AND TUNGRO DISEASE OUTBREAKS}

\section{J Holt, T C B Chancellor, D R Reynolds}

Because of economic and environmental reasons all pesticide treatments carried out today should be based on thresholds or risk evaluations. In Dermark the decision support system PC-Plant Protection is used as a tool which combines information on thresholds with recommendations for treatments using adjusted dosages. In order to carry out disease risk assessments in individual fields farmers are provided with simple and clear methods which enables them to make decisions on whether a fungicide treatment is needed. Incidence is used for assessment of mildew (Erysiphe graminis) and rust (Puccinia striffomis, Puccinia hordei), counting percent plants with attack at growth stages between GS 26-31 and percent plants with attack on 3 upper leaves from GS 32-71. Risk of eyespot (Pseudocercosporella herpotrichoides) can be carried out in spring either using field information (previous crop, pre-previous crop, date of drilling and weather data) or using inciderice. Development of eyespot in May and June is, however, very weather dependent, and in many cases makes the risk assessment unreliable. The risk evaluation of leaf diseases like Septoria nodorwm and Septoria tritici, Rhynchosporium secalis and Drechslera teres are based on days with precipitation of more than $1 \mathrm{~mm}$ pr day and susceptibility of varieties. On a national level maps are generated giving accumulated values for precipitation an other climate parameters. In addition weekly reports are made from a monitoring system run by The Danish Agricultural Advisory Center. Winter wheat is assessed at 55 localities, winter bariey at 30 localities and spring barley at 40 localities. From GS $26-31$ percent plants with attack is assessed in untreated plots of different varieties. From GS 32 plants with attack on the upper three leaves are assessed, using the same assessments methods as in PC-Plant-Protection. This information gives a picture of the general disease situation throughout the country. From 1985 to 1993 The Danish Institute of Plant and Soil Science has been monitoring pests and diseases in farmers fields by operating a field monitoring system. In 1992 and 1993 this system did use the farmers own touch tone telephone as the interface to the system including the recommendation model in PC-Plant Protection. This field monitoring system did provide actual information on the level of pests and diseases in Denmark, as well as good data on yearly variations in the need for fungicide treatments. ELISA lab-kits have been tested as a tool to provide more precise threshold decisions on eyespot and $S$. nodorum $/ S$. tritici. The methods have proven to give reliable diagnosis of the present diseases, but so far the methods have to be quantified more precisely. The presymptomatic reactions measured by ELISA can be a useful 'window' when optimizing disease control using adjusted dosages. Advantages of the ELISA method compared to using weather data alone have to be justified in economic terms. The aim in the future is to make better use of climatic information, including information from local weather stations in order to give better prediction of the actual disease risk situation.

We illustrate the role of risk assessment in rice pest management by considering two important problems: rice tungro vinus disease (RTVD) and migatory planthoppers, particularly the Brown Planthopper (BPH). Both occur sporadically, but require different approaches to risk assessment. Rice varieties incorporating field resistance to RTVD (based on vector resistance) are widely grown despite the fact that RTVD risk is generally low. Recent work on the epidemiology of RTVD was able to specify the risk of infection in new plantings according to proximity to existing infection sources; epidemiological models confirmed these findings. Potential may exist, therefore, to reduce selection pressure on the vectors by restricting the use of resistant varieties to areas where chance of infection was high.

In the tropics, BPH population growth is constrained by natural regulatory mechanisms and the main risk of outbreaks is that posed by insecticide-induced resurgence. In the temperate rice growing regions, long-distance migration is critical and immigration rate and temperature are the principal determinants of population size in the crop. In Zhejiang Province, China, simulation models have been used to interpret monitoring information in order to achieve more effective control. Planthopper early warning systems utilise weather and source area pest information; there may be potential to improve prediction using insect-monitoring radar.

Natural Resources Institute, Central Avenue, Chatham Maritime, Kent, ME4 4TB, United Kingdom 


\section{Van den Ende, J.E.}

Existing advisory systems on integrated pest management in apple in Europe are focused on optimal timing of monitoring and control of single pests without using local observations in the orchard. Although general advices may guide plant protection on farms, advices based on local observations on pests improves decision making and makes early pest warnings of value to individual growers. In the Netherlands a computerized advisory system (Gaby) for apple IPM has been developed to support decision making of individual fruit growers. Gaby's comparative originality is its block (or orchard) specificity and its integrated approach towards pest control. The system provides clear monitoring recomendations only when this is appropriate for a particular pest in the orchard by using information concerning orchard

characteristics, phenology of pests, previous field observations and insecticide applications. It recommends treatments, only if control thresholds are exceeded, while indicating the most appropriate type and concentration of insecticide and the optimal moment for control. It is the growers's responsibility to provide good input data, that will result in the best recommendation. Proper use of Gaby prevents redundant insecticide use and unnecessary monitoring. Future developments are focused on incorporating Gaby and existing disease management systems in an on-line plant protection advisory system for individual fruit growers.

Research Station for Fruit Growing, Brugstraat 51, 4475 AN, Wilhelmiradorp, The Netherlands.

\section{5}

A SAMPLING METHOD FOR THE DETECTION OF POTATO CYST NEMATODES (GLOBODERA SPP)

\section{T.H. Been \& C.H.Schomaker}

To reduce the use of nematicides in The Netherlands a new sampling method for the detection of potato cyst nematodes has been developed. The method should detect small infestations with a predefined probability so that farmers would stop applying soil fumigation as a precaution. Also, if the foci could be localized, the method could minimize the area a control measure must be applied to. As many infestations are still small when they are detected, other control measures than soil fumigation, which has a poor cost to benefit ratio, can be applied.

Patchy infestations in all growing areas in The Netherlands with different history and cropping frequency were sampled. More than 40 foci were analyzed and an exponential model describing the size and shape of an infestation focus could be developed. From the resuits it can be concluded that the data from all growing areas fit well to the model of expected cyst densities within a focus. Differences in parameter values of the model between growing areas appeared to be highly improbable So, one detection method for small infestation foci suffices for all growing areas.

A pilor version of the method is already in broad use in The Netherlands in the seed and consumption potato areas. In comparison with the reference years used by the Multi Year Crop Protection Plan, soil fumigation decreased by $90 \%$ in the Fievopolders, where the sampling system was introduced first, and by an average of $60 \%$ in all growing areas in The Netherlands. Since 1992 the sampling system is used as a basis for an advisory system. Research is now directed to improvements on the estimation of the size of the infestation focus from the sampling data.

DLO Research Institute for Plant Protection (IPO-DLO), PO BOX 9060, NL-6700 GW Wageningen, The Netherlands. E-mail in\%"t.t.been@IPO.AGRO.NL"

\section{Srivastava, C.P.}

The widespread and indiscriminate use of chemical pesticides have led to the development of resistance in about 600 pests (insects, weeds, pathogens) on global basis. As a result the worldwide shift towards integrated pest management have worldwide shift towards integrated pest management have
brought the behaviour modifying chemicals (BMC),
particularly pheromones to the forefront of pest control. particularly pheromones to the forefront of pest control. Fheromones have been identified for about 1068 insect species. The most exploited classes of pheromones in pest control are the sex pheromones and the aggreyation pheromones. Presently sex pheromones are being used widely for monitoring and also as an early warning indicator. In India about 25 pheronones of agrlcultural: horticultural and storage pests are commercially available. Hany of them are being used for detecting loth the presence and the density of the pest species. various trap designs have been developed to monitor the specific insect pests. For developing a good trap design the knowledge of insect behaviour is at pre-requisite. The environmental factors such as wind velocity and temperature influence the trap catches considerably. The pheronone catch the insects even at very low population and thus can be used as an early warning indicator of pest incidence. Pheromone traps are also used to estimate the optimum time to apply insecticides and to ensure the judicious use of insecticides. For having a good monitoring system the relationship between the trap catches and the corresponding field infestation estinate should be consistent across time and space. The pheronone traps can also be used to determine the maximum threat period of a varticular insect pest in a region. This trap data can also be used for designing the cultural pest control for the crops in a particular region. crop maturities and the sowing dates of the cultivated host plants can be manipulated to ensure that the most pest plants can be manipulated to ensure that the most pest vulnerable stage of the crops does not coincide with the peak activity of the concerned pest. In Inda the most litura are being monitored with pheromone traps from last 15 and 10 years respectively, across country. The present Department of Entomology \& Agril. 20ology, Institute of 221005 . INDIA.

1216

SUSTAINABLE CROP PROTECTION: RESIDUE ASPECTS AND CONSUMER SAFETY

\section{J.W. Dornseiffen and W.H. van Eck}

Chemical crop protection inevitably nay result in residues in agricultural produce, to which consumers may become exposed via the route of dietary intake.

The setting of maximum residue limits (MRLs) is generally part of the registration procedure of pesticides and is subject to harmonization both on a world-wide scale in the codex Alimentarius commission (CAC) as well as in the European Union and other economic groupings. In the (near) future, MRLs endorsed by the CAC within the framework of the so-called SPS Agreement of the World Trade Organization (WTO) will serve as reference points in case of international trade disputes.

MRLs are not derived from toxicological end points (Acceptable Daily Intakes) but are entirely based on Good Agricultural Practices (GAPs). The GAP of a pesticide includes parameters like dosage, time and frequency of application and preharvest interval, and is therefore determined by crop cultivation practices, characteristics of pests and diseases, including epidemiological phenomena and intrinsic characteristics of the pesticide (i.e. systemic versus non-systemic). Thus, an MRL is basically a technical limit.

However, the ultimate acceptability of MRLs is based on a risk assessment procedure taking into account relevant toxicological end points, derived from an extensive data base, and dietary intake patterns varying not only from one region to another, but also showing great variability anongst different population groupings.

It is obvious that sustainable crop protection practices may significantly affect the GAP of a particular pesticide and as a result also its mRLs.

It can be assumed that consumers will most likely not benefit significantly from changing crop protection practices, and in some cases even negative aspects are to be expected, as is illustrated in this paper.

Ministry of Health, Welfare and sports,

Directorate for Food and product Safety.

Section of Chemical Substances,

Sir winston Churchilllaan 368

P.O. Box 3008

$2280 \mathrm{MK}$ Rijswijk,

The Netherlands. 
The main objective of this study is to compile and compare pesticide-residue data from recent Total Diet Studies performed in different countries of Europe and North America. However, the lack of consistency among methods used for sampling, analysis, processing and reporting impedes comparability of residue data. Because of such differences, comparisons between countries are often difficult to make, and only trends within a country may at times be established.

A first point of difference may be the choice of the foods and contaminants that are monitored. Residue data can be collected in different ways: random sampling and surveillance or compliance Residue data can be collected in different ways: random sampling and surveillance or compliance
sampling. Random sampling reflects overall estimates of dietary and pesticide intake. Surveillance or sampling. Random sampling reflects overałl estimates of dietary and pesticide intake. Survell
compliance samples are directed to problem areas suspected of violating tolerance levels.

Obviously negative observations are more frequent in a random sampling program. On the other hand, surveillance or compliance sampling may provide primarily positive values, because these types of sampling are designed to intercept violations.

Identification, separation and calculation of the discrete observation categories are essential. Yet many residue report compilations do not discriminate between the categories of sampling, so that the results of many residue data sets are skewed due to inadequate sampling plans or a heavy emphasis on of many residue data sets are skewed due to inadequate sampling
pesticides that have a high potential for violation or health risk.

pesticides that have a high potential for violation or health risk.
Also sampling may be biased to cover only a part of the population (e.g. avg. person, adults [male/female], teenagers [maie/female], intants....).

To summarize one can say that in each total diet study the composition of the diet, the number of samples taken and the consumer to whom the study is geared is to be well defined.

A second item, the lack of commonality among the performed analytical methods (Multi-Residue Method and/or Single-Residue Method), the number of chemicals included (e.g. metabolites and degradation products) and the limits of detection associated with it, is limiting also the utility of the data for use in exposure assessment and is hampering the comparability.

The proportion of the samples exceeding MRL's (Maximum Residue Leveisi can be totally different depending on the number of methods taken under consideration. Also MRL's differ from country to country so that this can be another cause of difference in \% of samples with residues above the MRL.

Also, the processing and reporting of data on residue levels can result in important differences. Residues that exceed MRL's are subjected to confirmation and are included in the residue report. Residues below the tolerance levels are usually reported but not always confirmed. Further, when a given method includes chemicals that are not present above LOD (limit of detection), these absences are not always specified in the final residue report.

In calculating averages, some use only positive data (above the LOD), some include the LOD for nondetectables, and others enter a zero for non-detectables.

It is thus difficult to judge the quality associated with individual residue results or averages.

At last it is also important to know which part of the crop is analysed and the kind of performed preparation.

Conclusion: guidelines for organizing and conducting dietary intake studies must be set up to make comparison between various studies on a global basis possible.

Department of Crop Protection Chemistry, Faculty of Agricultural and Applied Biological Sciences University of Gent, Coupure Links 653, B-9000 Gent

THE OPINION OF TOWN POPULATION IN SLOVENIA ABOUT CON. TAMINATION OF AGRICULTURAL PRODUCTS WITH PESTICIDE RESIDUES

\section{J. Maček}

In Slovenia regionally representative monitoring of contamination of agricultural products is being performed for twenty years (1973-1992). Generally, the contamination is very low if not negligeable. Accordingly, we wanted to investigate the opinion of town population in Slovenia about the contamination of these products. An extensive questionary including more than 2000 respondents was applied. The results show that $70 \%$ of all the respondents consider pesticide residues the most important food contaminants when speaking of plant products, this number decreases to $61 \%$ when speaking of food of animal origin. It is obvious that this general opinion has nothing to do with the actual state of affairs. About $70-80 \%$ of consumers are willing to by alternatively grown products in the case that they were only up to 10 $\%$ more expensive.

Biotechnical Faculty, SLO-61111 Ljubljana, Jamnikarjeva 10t, P.O. Box 95, Slovenia 
CURRENT STATUS AND FUTURE PERSPECTIVES OF IGRS FOR THE CONTROL OF STOREO PRODUCT INSECTS

H. Oberiander D. L. Silhacek. E Shaaya, and I. Ishaaya

The control of stored product insects with insect growth regulators (IGRs) was heralded in the 1970 s as a potentially strong afternative to the use of traditional insecticides. iritially, the IGRs included compounds that mimicked the insect's own jurenle hormone Subsequently, chitin. included compounds that mimicked he insects own juvenile hormone. Subsequently, chitin synthesis inmibitors and ecdysteroid agonists have been added to the list of available IGRs. Extensive laboratory studies have been conducted on the physiojogical and biochemical effects of IGRs on stored product insects. However, there has teen less emphasis on developing practical applications of these materials for stored product insects in the past decade. There is a new imperative to examine IGRs for the protection of stored protutis which results from no longer having some eariter control measures available, and particularty by the loss of the use of methyl bromide by the end of the century. Therefore. it is clear that a fresh evaluation of the We need to bok at the new generations of IGRs and at new approaches to the application and usage of these materials. We have investigated the action and efficacy of various IGRs, including juvenile hormone-like compounds, ecdysteroid agonists and chitin synthesis inhibitors on the RH 5849, inhibited larval growth at low doses, and was toxic at concentrations greater that 15 ppm. Simuttaneous treatment with a juvenile hormone mimic methoprene, allowed continued growth, but metamorphosis was prevented. In other tests the juvenoid agonists, fenoxycarb and pyriproxyfen, were effective in preventing egg hatch when treatment was begun during the first 18 hours of embryogenesis. Tests with chitin synthesis intibitors showed that chlorfluazuron and teflubenzuron were particularty effective against first instar larvae. Thus, our research provides some new perspectives on the potential utilization of IGRs for the protection of post harvest commodities.

Herbert Oberiander. Insect Attractants, Behavior and Basic Biology Research Labcratory, United States Department of Agriculture, Agricultural Research Service, P. O. Box $\$ 4565$, Gainesville, Flonda, 14565, US.A potential role of IGRs for the protection of stored products from insect infestation must be made Indian meal moth both in vivo and in vitro. For example, administration of an ecdysteroid agonist.

PLANT OILS AS FUMIGANTS AND CONTACT INSECTICIDES FOR THE CONTROL OF STORED-PRODUCT INSECTS

E. Shaaya, M. Kostjukovsky

The funigant toxicity of a large number of essential oils extracted from various spices and herb plants and some of their major constituents was assessed against a number of major stored-product insects. Three groups of active materiais were distinguished according to their activity against the various insects. Recently we have isolated a compound which was found most active of all the essential oils and their constituents which were tested against stored-product insects. A concentration of $1.8 \mathrm{~g} / \mathrm{m}^{3}$ air caused 100 mortality of all adult insects tested after $24 \mathrm{~h}$ exposure. Tribolium castaneum was found most resistant of all insects to essential oils and their monoterpenes. The activities of these compounds are insect and stage specific. The possible involvement of the enzyme acetylcholinesterase in the susceptibility of the insects to these compounds was examined.

The biological activity of a large number of edible oils and fatty acids was also assessed. It was possible to show a close correlation between the chain length of the fatty acids and their biological activity. This study showed that edible oils are potential control agents against stored-product insects. This technology can be very useful on the farm level in developing countries, where grain is stored in small quantities for household use.

plant oils, being natural products, can play an important role in the the strategy of stored-grain protection and eliminate the need for, and risks associated with, the use of insecticides.

Dept, of stored Product

The Volcani Center

50250 Bet Dagan, ISRAEL

\section{4}

\section{PEST MANAGEMENT OF STORED PRODUCTS USING LOW TEMPERATURE}

\section{H. Nakakita and $\mathrm{H}$. Ikenaga}

\section{T.W. Philfips}

Development of semiochemical-based pest management systems in storedproducts is being necessitated by the loss of traditional residual and fumigant pesticides. Pheromones are known from over 30 species of stored-product insects. The most successful applications of these compounds are as attractants in traps for detecting and monitoring pests in storage facilities. attractants in traps for detecting and monitoring pests in storage facilities. Pecent research has focused on improving trapping technology by discovery
of additional pheromones, incorporation of food odor smergists, developing of additional pheromones, incorporation of food odor symergists, developing
better slow-release tormulations, and improving trap design. New better slow-release formulations, and improving trap design. New
developments include identification of sexpheromones for the cowpea weevil, Callosobruchus maculatus, and synthesis of effective phercmone analogues for the sawtoothed grain beetle, Orzaephilus surnamensis. Pheromonebaited traps for storage moths (Pyralidae: Phycitinae) are very effective and are used extensively in biorational pest manegement schemes. Tuaps ipr beetle pests are being dith novel pitfall-typo designs lit utilize foodpests are being doveloped with novel pittall-type designs that utilize foodderived attractants and pheromones. Direct control of storage pests with pheromones can be approached with the attracticide and mating disruption methods. Both techniques utilize synthetic female sex pheromones to prevent males from reproducing. Recent research is investigating attractants for female moths. Volatiles from food sources attract female Indianmeal moths, Plodia interpunctella, and secretions from conspecific larvae contain oviposition stimulants for females. Semiochemicals to manipulate female behavior will provide new tools for many different pest management approaches. Natural products derived from plants or other sources show potential as repellents or teeding deterrents against storage pests. Su potential as repants or they could be strategically incorporated into papers or glues for protection of packaged foods. Semiochemicals may soon be available to manipulate the behavior of beneficial natural enemies of storage pests. Bracon hebetor, a parasitic wasp of pyralid moth pests. displays directed orientation to host-related stimuli after it has had contact experience with host kairomone. Thus the host-finding efficiency ot natural enemies in biological control programs could be improved with the use of kairomones in mass-rearing or release protocols. The role of semiochemicals in stored-product pest management is increasing as more biorational methods are emptoyed.

USDA ARS, Tropical Fruit and Vegetable Research Laboratory, P.O. Box 4459 , Hilo, HI 96720 USA
Low temperature storage can be considered an ideal method for grain preservation, since it can regulate insect infestation and maintain the stored grain in good quality for long periods of time. The insect's body temperature decreases according to this of the surrounding, because of it's poikilothermic character. Thus, low temperature will be able to control insects selectively. This method for insect control is especially useful for stored product insects, the majority of which are known to be of tropical of subtropical origin, adapted to high (around $30^{\circ} \mathrm{C}$.) in which their mulciplication is optimal. Lowered temperature inhibits the metabolic rate of insects, which cause many physiological phenomena such as motioness, inhibiting the chance of mating, reducing ealing activity, delaying development and growth rate. Although the intensity of the above symptoms is influenced by the degree of low temperature used, $10-15^{\circ} \mathrm{C}$ are commonly recommended for the prevention of damage done by stored product insects. The idea of using low temperature storage has a history longer than 60 years and many pioneers recommended this method as useful for grain storage. However, it's practical application throughout the world was somewhat limited except in Japan, where brown rice has been stored in large scale (about 4 million tons) in warehouses at low temperature $\left(15^{\circ} \mathrm{C}\right)$. Presently, the use of most of the conventional chemicals, either grain protectants or fumigants have been restricted because of problems like toxic residues in foodgrains, the development of insect resistance and the damage done to the environment.

Thus, it is time to reconsider the benefits of this method, and to extend its use more widely in the pest management of stored products.

National Food Research Institute, Ministry of Agriculture, Forestry and Fisheries, Tsukuba, Ibaraki, 305 Japan 
CHLLED AERATION FOR PEST MANAGEMENT IN STORED FOOD GRAINS

\section{E. Maier, L. J. Mason and R. A. Rulon}

Pesticides are used to treat bulk stored grains at several points in the food processing chain. A grain chiller urilizes a portable refrigeration system to control the temperature and relative humidity of the air entering the grain storage structure independent of ambient conditions. As a result, grain chilling provides a non - chemical conditioning and preservation lechnology for cereal grains and oilseeds. The process of chilled aeration allows for conditioning of large grain masses to desired temperature and moisture levels at any time during the storage season. This low temperature preservation method is an alternative to treating food grains with pesticides for product quality control.

During the summer of 1994, four bins were evaluated for the chemical-free pes: management and conditioning of popcorn. Two of the bins were managed with intensive aeration for moisture conditioning and several fumigations for pest control. The other two bins were managed with the Purdue prototype grain chiller. These two bins were monitored and chilled only as needed throughout the summer. The grain ternperature in the chilled bins remained below the critical $15^{\circ} \mathrm{C}$ limit for insect development. Grain temperatures in the bins conditioned with ambient aeration were $7-20^{\circ} \mathrm{C}$ higher during the same period. By Mid-September, the challed bins had not required a single funigation and had insect levels significantly below those found in the furnigated bins. Additionally, the chilled popcorn was conditioned to precisely the moisture content desired for processing. Furthermore, an economic analysis of chilled storage versus traditional insect control and conditioning methods has shown grain chilling to be competitive. Grain chilling appears to be both effective means of pest control and quality management for storing popcom and other food grains and oilseeds in an ecologically safe manner. collod wersus Amblem Aartion

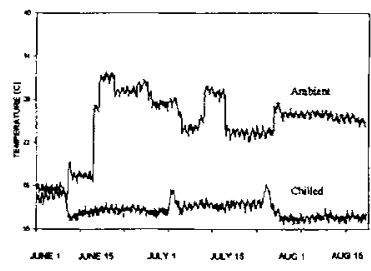

Dirk E. Maier

Agricultural Engineering Dept.

Purdue University

W. Lafayette, Indiana 47907-1146

U.S.A.

\section{CURRENT STATUS AND FUTURE PERSPECTIVES OF INERT DUSTS FOR THE CONTROL OF STORED PRODUCT INSECTS}

\section{Peter Golob}

For many years, inen dusts particularly silica derivatives, have been krown to be effective insecticides. However, cost constraints and fears regarding the effects of inhalation of finally-divided silica particles prevented their use in storage. In recent years, the growing problem of insect resistance to conventional orgacophosphate and pyrethroid insecticides has necessitated the search for alternative methods of protecting grain during storage. Inert dusts, new formulations, are beginning to be used to this end.

In Australia, 'Dryacide', a diatomaceous earth coated with amorphous silica gel has been found to provide excellent protection against insect attack to wheat stored in flat bulks when sprayed as a thin coating over the grain. Applied as a slunry to concrete store walls the dried powder remains active for more than a year, substantially longer than corresponding conventional chemicals applied as wettable powders. Dryacide has also been shown to enhance the fumigation with phosphine in silos with less than optimal conditions of gas-retention.

Inert dusts aiso have potential for use in developing countries as alternatives to dilute dust insecticides for use on small scale farms. Work in Zimbsbwe and in Ghans has demonstrated that both pulses and maize can be protected against storage insect pests by treatment with activated silicates.

Clearly, amorphous silica and silica aerogels have the potential to replace organophosphate and pyrethroid insecticides as grain storage protectants. Much more fieid testing needs to be undertaken before these materials become widely accepted but their use does point to a solution to some of the problems being experienced with conventional insecticides.
THE PESTICIDAL EFFECT OF TEN BOTANICALS AND FOUR VEGETABLE OILS IN THE MANAGEMENT OF THREE CALLOSOBRUCHUS SPECIES

\section{R.H.S. Rajapakse , H.F. Van Emden}

The possible use of ten botanicals and four vegetable oils in managing the bruchid beetles of legumes, Callosobruchug chinensis, C. maculatus and $\mathrm{C}$. rhodesianus was investigated. All four oils tested (corn, groundnut, sunflower and sesame) significantly reduced the oviposition of all three bruchid species at 10 $\mathrm{m} 1 / \mathrm{kg}$ and also significantly reduced the longevity of adults of $C$. maculatus and $C$. chinensis at this dose. Only corn and sunflower oil caused a significant reduction of longevity of $c$. Thadesianus at $10 \mathrm{ml} / \mathrm{kg}$. The number of eggs laid by all three bruchid species was significantly reduced in treatments to wich powders of Cymbopogon citratus, Cinnamomum camphora and Derris inudata had been added.

Department of Agricultural Biology.

Faculty of Agriculture.

University of Ruhuna, Mapalana,

Kamburupitiya,

Sri Lanka.

\section{8}

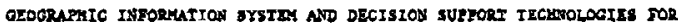
GRASHOPYg WANAGETET

*. P. Kema, J. S. Bazry The duel objectfves of protecting Amertca's apriculture and protectiog

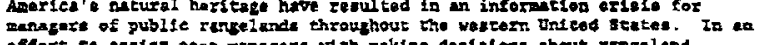


Erakthopper contral, tho United Statox Dapartapat of AfrteulturoAorfeuitural lestazch Sazvice (USDh-Als) and USDA-Amian and Plene


Wich APhIS porsonnol throughout tho westera Unitod stato: to dovelop a corputer decleion support package callod lopper, Hopper, developed undor the 2987-1994 cooperative Grasshopper Intogratod Pant Managemont Projoct, providas ansigtence to ranchers and decision mikers evalusting inmovative strategfor Lor zangeland grasthopper mangemont. Usins recont advancos computer tochnoloky. Hoppos bas boen dosigned to be very uenc fiflondly

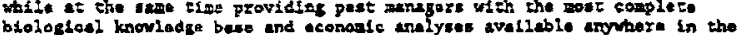

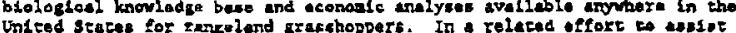
Inteed states for renfolend Frafahoppers. In relaced offort to ansin proper ecosystam nangement of thited States rengelends, the Rangelind

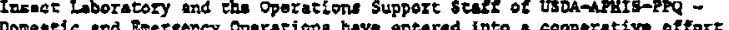
Domentic and Eangency Oporazions have ontored lato a cooperative offort information syatem, to poet manapment professtonsls in the 17 ventern

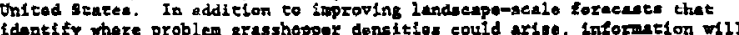
fdentify where problem grassheppex densitios could arise. Laformation will also bo avallable on insese and plant cominity composition that cab be uned to help deflan the potential inpacte of humen incerventions. This cooparative effort is expeted to sliow for the incegration of obg ictives cooceming the protection of the egriculture as vell as the netive ecospstame of Aserico's rangelands.

United Scetes Deparement of Acriculture, Agriculeural Researeh Sezvice, Rangolesd inece Laboratory. Bozenn, Yontant, U.S.A., 59717 
GAASSHOPPER CONTROL IN CANADA WITH SPECIAL REFERENCE TO ALBERTA GRASSLANDS

\section{Dan L. Johnson}

Grasshoppers are native grassland pests. The multi-species nature of the pest aspect of grasshoppers means that they are found throughout the year, attack a wice range of plants, and vary in impact from serious pests to beneficial species. The most significant losses are to cereal crops and crops and and 1991 grasshoppers caused losses of over $\$ 300$ million in the Canadian Prairie provinces (all other insect crop pest accounted for total losses of ca. $\$ 73$ million). Grasshopper control is conducted by private land owners rarely by government. During the period 1985-1991, farmers and pasture managers in Alberta sprayed $1,820,000$ ha for grasshopper control, using 745,000 L of grasshopper insecticides in 1985-86 alone. Concerns regarding indirect impacts of extensive spraying include damage to bees and grassland birds that rely on these insects for food. Cooperative research on improving grasshopper control in the grassiand region of Canada has focused in three areas:

Improved forecasting. An annual forecast is based (since 1988) on a simple GIS-assisted analysis of results of an annual adult survey, in Alberta typically every $10 \mathrm{~km}$ in an area of 200,000 ha. The resulting long-term population database assists validation of biophysical and ecological process models. It is also being used to model responses to climate change, to characterize ecoregions, and to forecast insecticide requirements.

Alternative insect control agents. Insecticides used for grasshopper control in Canada (e.g. carbofuran) are under review. Research in Alberta on alternatives includes assessment of impacts of insecticides on non-target vertebrates and arthropods; dose-response testing, formulation and lethal and sublethal impacts of fungal and protozoan entomopathogens, nove insecticides, such as natural plant toxins (e.g., glucosides and alkaloids 1995). dye-sensitized photooxidation (1995), phenylpyrazole (fipronil, 1989-90:; parasitic flies; and improved toxic baits.

Ecological studies. Grasshoppers are important consumers on 7 million ha of grazing land in Alberta. Studies on the population dynamics and trophic ecology, as consumers and as an avian food resource, are in progress in natural areas and in also intensively managed grazing reserves.

Agriculture and Agri-food Canada Research Centre, P.O. Box 3000, Lethbridge, Alberta, Canada, T1J 481.
LOCUST AND GRASSHOPPER CONTROL IN AUSTRALIA

GHS Hooper

Five endemic acridid species can cause economic damage to crops and pastures in Australia, but four of these are only important at irregular intervals. However, in the eastern part of the continent populations of the Australian plague locust, Chortoicetes terminifera (Walker), can increase to levels that warrant control in most years.

In 1974 the four eastern mainland States joined with the Commonwealth Government to form the Australian Plague Locust Commission (APLC). This organisation is responsible for control of $C$. terminifera in about two million square kilometres of inland eastern Australia, and in certain circumstances it is also responsible for control of the migratory locust, Locusta migrotoria migratorioides (R. \& F.), and the spur-throated locust, Nomadacris guttulosa (Walker).

Depending on the species involved, and where in each the State the outbreak occurs, responsibility for control can devolve to landholders, State departments of agriculture, and/or the APLC. The objective of control can vary from protection of crops and pastures to limiting the potential for damage by reducing the total size of the locust population.

To meet its charter the APLC routinely monitors the distribution and development of locust populations by ground survey. To compensate for having only seventeen permanent staff it has embraced modern technology. It has a remote sensing capability which enables the distribution of rainfall (the most remote sensing capability which enables the distribution of rainfall (the embarked on the development of a decision support system to enhance its forecasting capability.

In addition to undertaking control of all significant outbreaks of C. terminifera the APLC has adopted the philosophy of controlling all nymphal and adult populations that it believes could develop into significant outbreaks. Hence it will initiate a control campaign if at least ten square kilometres of band or swarm targets are detected.

A rapid response capability is essential to achieve effective locust control. The APLC ensures it meets this requirement by having (i) a stockpile of insecticide, (ii) spray aircraft on contract, (iii) good HF and UHF radio communications, and (iv) a short command strueture.

Australian Plague Locust Commission

GPO Box 858

Canberra ACT 2601

Australia

THE ROLE OF SEMIO-CHEMICALS IN DESERT LOCUST GREGARISATION

Ahmed Hassanali

Gregarisation is largely a density driven process initially associated with increased encounters between the insects. The process involves a physiological transformation of individual insects and associated behavioural changes as well as developmental synchronisation of the individuals across the whole population. The process begins with increased encounters between solitarious individuals in the initial stages of crowding, followed by those between solitarious insects and gregarious/gregarising counterparts, and finally by those between gregarious insects.

Our research to-date has focused on the role of semiochemicals in modulating such important characteristics of the gregarious-phase desert locusts as cohesion, synchronous maturation and mass egg-laying at common sites. A complex of 5 sets of pheromone systems has been implicated and largely characterised. An overview of the status of the research will be presented. The probable part played by these pheromones in the earlier stages of gregarisation will also be described.

The International Centre of Insect Physiology and Ecology, P.O. Box 30772, Nairobi, Kenya. 
PHEROMONAL MODULATION OF AGGREGATION BEHAVIOUR IN THE DESERT LOCUST, SCHISTOCERCA GREGARIA (FORSKAL)

\section{Obeng-Ofori, D. Torto, B, Njagi, P.G.N. and Hassanali, A.}

The most striking feature of the deser locust is its ability to reversibly transform between two morphologically, physiologically and behaviourlly distinct phases: solitaria and gregaria. The solitary phase prevails in the recession areas as dispersed individuals in very low densities in scattered populations, feeding on a limited range of desert plants and reproducing only when environmental conditions are sufficiently favourable. The gregarious phase is characterised by highly cohesive behaviour, longdistance migratory aptitude, polyphagy, synchronous and accelerated maturation and communal egglaying, making the deser locust such a devastating pest. Pheromones are implicated in the perpetuation of these gregarious characteristics. The aggregation pheromone system of gregarious Schistocerca gregania is a complex derived from the insects themselves and their faeces. This has been demonstrated in GC-EAD. GC-MS and behavioural assays. Behavioural assays showed that two distinct sets of releaser pheromones modulate the aggregation behaviour of the desert locust: a juvenile aggregation pheromone specific to the nymps and an adult aggregation pheromone specific to the adult stages. There was no sexual differentiation in the production or response to nymphal pheromone system. Young adults (immature adults) of either sex did not produce a stimulus with significant activity. Of the older adults (sexually mature adults), only the males produced the aggregation stimulus to which both sexes were equally responsive. The adult aggregation pheromone system consists of phenylacetonitrile guaiacol, phenol and benzaldehyde. Mature solitary desert locust adults were also responsive behaviourally and electrophysiologically to the components of the adult pheromone system. The identification of the nymphal pheromone system is not yet complete. Five components: phenol, guaiacol, benzaldehyde, acetophenone and phenylethanol have been identified in the nymphal volatiles. The behavioural activity of phenol, guaiacol and benzaldehyde have been established so far.

Faecal volatiles are part of the aggregation pheromone complex. The nymphs

aggregate in response to their faecal volatiles and to those of young adults but were indifferent to volatiles emitted from older adult faeces. The young and older adults even showed less specificity, they were cross-responsive to each other's faecal volatiles and to those of the nymphs. Phenol and guaiacol were the most predominant electrophysiologically active compounds of nymphal and young adult faeces. Faecal volatiles of older adults contained phenylacetonitrile in addition to phenol and guaiacol.

A better understanding of the gregarisation-solitarisation process could open up ways of interfering with the gregarisation behaviour of the desert locust and possibly help develop new intervention strategies for biorational management of the insect.

ICIPE Research Centre P.O. Box 30772 Nairobi, Kenya.
MORPHOMETRICAL CHARACTERISTICS AS THE INDICATORS OF TRENDS IN POPUIATION DYNAMICS OF THE MOROCCAN LOCUST, DOCTOSTAURUS MAROCCANUS (THUNBERG， 1815) (ORTHOPTERA: ACRIDIDAE)

\section{A. V.Latchininsky}

Moroccan locust Dociostaurus maroccanus (Thunberg, 1815) is the pest of high economical importance in at least 30 countries from the Canaries to Afghanistan (Latchininsky \& Launois-Luong, 1992). In 1993 and 1994 , more than 1.000000 ha were treated against this very mobile and extremely detriment species in Central Asia and kazakhstan (Latchininsky, 1994). To diminish the chemical load on kazakhstan (Latchininsky, 1994). To diminish the chemical load on the environat in the ecotonal zones inhabited by the pest, it is necessary to evaluate correctly the current state of pest population dynamics and to predict its future tendencies. For this purpose, a new comprehensive scale of morphometrical phase length (F) to hind femur length (F) ratios are utillzed: elyeron length (E) to hind fexur length (F), pronotum length (P) to head width (C) and hind femur length (F) to head width (C). As we have shown earlier (Latchininsky, 1990), the combination of these characters complemented by pigmentation scale of scaf (1972), would be sufficient to estabilsh the current phase state of population. Moreover, in some cases, the adequate characterization of phase could be achieved by use of a single morphometrical trait, e.g. hind femur length. Having the relevant data for 3-4 or more consequent years, it is possible to predict the direction of future changes in population dynamics, as it was effectuated in our case studies in Tadjikistan (1986-1990) and Uzbekistan (1990-1994). Distribution area of the species being discontinuous, the average $E / F$ ratio examined in the individuals of populations from 19 different zones appeared to be subject to geographical variation. For example, the $E / F$ ratio of the solitarious males varies from 1.32 in Moldova to 1.52 in Hungary. E/F of gregarious males varies from 1.62 in Uzbekistan to 1.85 in Montenegro. In solitarious females, the $E / F$ ratio is the lowest (1.38) in Azerbaijan to be the highest $(1.60)$ in N.Caucasus. Relevant values for $E / F$ of gregarious females are 1.58 (Spain) and 1.81 (Algeria). Sex differences are well pronounced in $\mathrm{E} / \mathrm{F}$ values of solitarious phase to be less expressed in gregarious. The proposed alscriminative approach to different geographical populations in the study of the trends of Moroccan locust population dynamics is shown to produce the best results in pest forecasting. Advantages and limitations of the method are discussed.

VIZR; 3, Podbelsky street, 189620 st.-Petersburg - Pushkin, Russia.

A RADAR METHOD FOR MONITORING THE MIGRATION OF DESERT LOCUSTS

\section{J R Riley, D R Reynolds, A D Smith}

Recent developments in radar entomology have led to an inexpensive method of using vertical-looking radar (VLR) to automatically monitor the migration of insect pests. This paper describes an evaluation of VLR as a monitor of solitarious desert locust migration in West Africa. The radar has been operated at three sites in Mauritania, and its measurements of the speed, direction and orientation (heading) of nocturnal overflying insects were found to agree satisfactorily with independent, simultaneous measurements made with a conventional scanning radar. Unlike previously used entomological radars, VLR has the capacity to estimate the body-mass of individual overflying insects, and it was thus able to distinguish locusts from other high-flying noctumal insect migrants. The radar was found to cope satisfactorily with the density and complexity of the different aerial fauna at the three experimental sites. It is concluded that a network of VLRs could routinely provide information about the nocturnal, high-altitude migration of desert locusts in their normal habitat, and that this information would provide a valuable supplement to the data acquired for forecasting operations by ground survey teams.

Natural Resources Institute, Radar Unit, North Site, Leigh Sinton Road, Malvern, Worcestershire, WR 14 1LL, United Kingdom 


\section{BIOLOGICAL CONTROL IN COTTON-WHEAT CROPPING SYSTEMS IN} NORTHERN CHNA

\section{J. Xia \& W. van der Werf}

Cotton is a major crop in China. It is intensively treated with pesticides against insects. One of the earliest pests in the cotton growing season is cotton aphid, Aphis gossypii. Chemical control of this species destroys natural enemy complexes. This sets off a pesticide treadmill during the rest of the growing season.

Seven spot ladybird, Coccinella septempunctata, is a major predator of cotton aphid. Its occurrence in cotton can be promoted by intercropping with wheat or oil seed rape. These crops mature when cotton is planted (May). During maturation, aphid populations on wheat and oil seed rape decline, causing natural enemies to move to other food sources, i.c. cotton aphid.

The effectiveness of biocontrol by ladybirds depends on the timing and extent of coccinellid arrival in the aphid populations. If they arrive too late, the effect is insufficient. Timely immigration of coccinellids can be promoted by intercropping. Intercropping at a small scale, e.g. sequences of one row of cotton and a few rows of wheat, is probably more effective than intercropping at larger scales, i.e. multiple rows of cotton interspersed with multiple rows of wheat.

We conduct experimental and modelling research to answer three questions: 1. Which ratios or absolute densities of cotton aphids and lady birds yield biological control, depending upon temperature, crop development stage, presence of other natural enemies, and stage composition of the populations of the two species?

2. How are the timing and extent of lady bird migration from wheat into adjacent cotton affected by the scale of intercropping, development stage of wheat, wheat aphid density, and by weather factors?

3. What are effective intercropping strategies promoting biocontrol?

The modelling serves to relate observations on dispersal behaviour, life history and spatial distribution to population dynamics and biocontrol. At the conference, we will present results obtained during the 1994 and 1995 growing seasons.

Cotton Research Institute; Chinese Academy of Agricultural Sciences; Anyang; Henan 455112; CHINA; fax 86372224952 \& Wageningen Agricultural University; Department of Theoretical Production Ecology; P.O. Box 430; 6700 AK Wageningen; Netherlands; Email: Wopke.vanderWerf@staff.tpe.wau.nl

THE FOLK FARMER AND THE GLOBAL MONOCROP ECONOMY: RESPONSES TO DEGRADING ENVIRONMENTS IN GHANA

\section{K.S. Amanor}

This paper examines farmers' strategies in the forest and transitional zones This paper examines farmers' strategies in the forest and transitional zones land degradation and the attendant problems of weeds, pests and soil fertility. Four different farming systems are examined including cocot in the forest. high input maize monocropping under permanent agriculture in the transitional zone, maize intercrops under natural fallowing systems in the forest, and yam farming in the transitional forest area. Cocoa and maize monocropping form the most important farming systems in which formal sector research is carried out. Maize intercropping and yam farming are sectors which depend on technology largely developed and adapted by farsners and are cultuated in the context of bush fallowing. Maizs monocropping and cocoa farming are systems of permanent cultivation using high hiputs and a narrow range of modern varletes. Their cultivation has resulted in the excess removal of the forest vegetation. impoverished solls. build up of pest populations and diminishing returns to farmers as input prices increase. As a consequence, in the maize monocrop system farmers are increasingly turning away from permanent maize cultivation with fertilizers to crop rotations with legumes. In the cocoa system, plagued by new strains of black pod disease, farmers are abandoning cocos and turning to a variety of food crops. In the yam and maize-cassava system farmers rely on natural fallows because root crops are reputed to yield poor quality tubers when grown with inorganic fertilizers. In recent years the maize-cassava intercrop has suffered from serious problems resulting from the domination of the forest fallow by Chromolaena odorata, a weed often introduced by government services as a cover crop. This plant has increased weeding requirements and destroyed the synergic interactions within the agroecosystem. It also harbours the variegated grasshopper a major pest of cassava. Yam farmers maintain a wide selection of genetic materials. Including unpalatable wild yams, which enables them to continually select characters to changing conditions and to find substitute varieties for plants varieties which have become susceptible to disease. The main problems for yam farmers are pressures on the land from population increase and the expansion of commercial monocrop maize farming. Many of the perspectives of small-scale farmers mirror debates among researchers about the environment and sustainable agriculture. Strategies developed by small-scale farmers are seen as containing valid principles which can form the basls for developing more habitat-management oriented research for IPM. The potential of this dependsupon political economic factors concerned with the role of rural agricultural sector in international and national economies, and agricultural policy directions.
Cost-benefit analysis and farmer's perception of crop egde management in the Netherlands.

G.R, de \$noo

Centre of Environmental Science, Leiden University

P.O. Box 9518

2300 RA Leiden

The Netherlands

There is a growing interest in establishing unsprayed crop edges for the purpose of nature conservation, integrated pest management or reducing pesticide drift to surrounding areas. In the Dutch Field Margin Project, nor only the ecological and environmental implications of crop edge management were studied but also the costs of the measures taken and farmer's perception.

In a field experiment in the Haarlemmermeerpolder, in winter wheat, sugar bet and potatoes unsprayed crop edges were created, on which no herbicides or insecticides were used. Yield quantity and quality from the unsprayed edges was compared with that from sprayed edges. Relative to the sprayed crop edges yield reduction in the unsprayed edges was about $30 \%$ in sugar beet, $2 \%$ in potatoes and $13 \%$ in winter wheat. In most cases the quality of the yield was not changed. Balancing the yield losses against the benefits, viz. reduced pesticide costs, it can be concluded that with winter wheat and potatoes, unsprayed crop edges can be well adopted in agricultural practice. In sugar beet the cost is too high.

For the acceptance of the crop edge management in farming practice, a survey among 31 arable famers with experience of crop edge management all over the Netherlands demonstrates the relevance of farmers' perception. Statistical analysis of survey data show that of several types, such as unsprayed crop edges in cereals, potatoes, unsprayed cereal edges along other crops, grass strips or set-aside strips, farmers preferred unsprayed edges in cereals or grass strips for agronomical, farming equipment related and sociopsychological reasons. It is above all the width of the margin that is important for acceptance crop edge management in farming practice. With regard to the payment system, farmers prefer a guaranteed reward instead of a payment for 'nature result'.

1240

\section{POLYCULTURE FOR THE MANAGEAENT OF CHILI APHIDS AND THER NATURAL}

M.Y. Hussein A.S. Norani.

The effects of plant diversity (polyculture) on the abundance of Aphis gossypil Clover (the main vector of chilli viruses) and of Menochllus sexmaculatus Fabr. (the main predator) were studied in replicated fleld plots representing monoculture, diculture and triculture a groecosystems. The population trends for A. gosyoil and $M$. sexmaculatus were ecompared among the three types of agroecosvstem. The M. sexmaculatus were ecompared among the three types of agroecosvstem. The
incldence of chiffi veinal mottle virus i c aMN and vield of fresh chifies were also compared. The results showed that the activities of vectors and the predators were affected by the presence of companion crops which were planted in between chillies. The vector members were consistentiy higher on chillies when planted as a monoculture. Similarly, the population of $M$. sexmaculatus was significantly higher in the dicuiture and triculture as compared to monoculture. In the diculture system, maize planted in between rows of chillies was effectiveiy employed as a barrier crop and caused a significant reduction in the numbers of vectors trapped and poor aphid colony establishment on chillies. This has resulted in lesser activity of vectors and subsequent lower spread or cVMv. Lower vector numbers is especlally desirable in preventing initial transmission and spread of the virus on chillies. Better results were obtained in the case of chilli-brinal diculture but not with chilli-long bean combination. In the triculture system, a perennial legume crop, Leucaena, was planted together with chill and brinjal. The resultant increase in the population of $M$. Sexmaculatus proved the Natural Enemles Hypothesis which predicts a positive correlation between ricnness in plant species and natural enemy abundance. The successful regulation and suppression of $A$. gossypl/ populations through habitat management sisgnifies that the management of vectors and their natural enemies in chilli agroecosystem is in consonent with the ideals of integrated pest management in the tropics.

Plant Protection Department Universiti Pertanian Malavsia 43400 upM, Serdang, Selangor Malaysia 
EFFECTS OF FLOODING, SOIL FUMIGATION, AND COMPOSTED ORGANIC HOUSEHOLD WASTE ON PYTHIUM ROOT ROT IN BULBOUS IRIS

\section{Z. 1. Némethy}

During the survey of virus diseases of gladioli (Gladiolus L. hybrides ) cucumber mosaic cucumovirus (CMV), bean yellow mosaic potyvirus (BYMV), tobacco rattle tobravirus (TRV), and tobacco necrosis necrovirus (TNV) were frequently found in plants. While these viruses are polyphage and their vectors exist on the growing spots, we supposed that weeds growing among gladioli may also be infected by those ones. To identify virus infections of weeds we collected leaves and roots of 146 samples, with green, yellow mosaic or mottling or without symptoms, from 4 locations last two years. The 30 examined weed species belonged to families: Amaranthaceae, Boraginaceac, Caryophyllaceae, Chenopodiaceae, Compositae, Convolvulaceae, Cruciferae, Fabaceae Labiatae, Malvaceae, Plantaginaceae, Polygonaceae, Portulacaceae, Resedaceae, Scrophulariaceae, Solanaceae.

Infection rates of weeds, measured by ELISA tests, were the following: BYMV $81 \%$, TRV $49 \%$, TNV $34 \%$, CMV $25 \%$, healthy $8 \% .36 \%$ of plants were infected by only one virus, while $56 \%$ of samples showed complex virus infection.

All samples (31) of Chenopodium spp. were infected by BYMV, and in the majority $2-4$ viruses were identified. High rate of complex virus infection were detected in the samples of Cirsium arvense, Cirsium brachycephalum, Convolvulus arvensis, Datura stramonium, Reseda lutea, Sinapis arvensis, Solanum nigrum, Stachys annua, too. These species are susceptible to viruses infecting gladioli and may be sources of virus infection and spread.

BYMV were common in species: Amaranthus, Ambrosia, Cirsium, Datura, Heliotropium, Galinsoga, Reseda, Solanum, Stachys, while TRV in Cirsium, Convolvulus, Datura, Poligonum, Reseda, and TRV in Reseda, Sinapis.

To avoid virus epidemy and to keep gladiolus plants virusfree, it is advisible to kill weeds, as virusreservoirs in the gladioli.

University of Horticulture and Food Industry Department of Plant Pathology

H-1502 BUDAPEST 112 POBOX 53

\section{THE BASIC CHARACTERISTICS OF ALFALFA PLAN BUG POPULATIONS}

IN THE SEASONAL DEVELOPMENT PROCESSES

\section{N.N.Gorbunov}

Alfalfa plan bugs have been accounted regularly (twice a week) during vegetative season. In retrospect 5 stages of populations formations have been distinguished in the frames of each fluctuations; 1 ) single appearance; 2) size growing of population; 3) size maximum;4) size decrease; and 5) stop of bug's development. Twelve seasonal observation have been carried out. Besides in each population age composition, sex ratio, the average species mass, the community mass and reproductive potential have been determined. At the first stage 10 percent of number and 2 percent of mass have been accumulated for larva and 10 percent of the both indexes for adults. The males were dominated. The females started to form eggs. The second stage was characterized by almost double increasing of bug's number 25 percent of total number and 9 percent of biomass were accumulated for adults. The number of males and females became equal. The reproductive potential was realized to 20 percent. The next stage was characierized by most specimen's number which increased in 3.3 times for lana and in 2.2 times for adults comparing with previous stage. During that stage 50 percent of number and 38 percent of biomass were accumulated for larva and 50 percent of number and 62 percent of biomass for adults. The females started to be dominated in populations. The reproductive potential was realized to 56 percent. The size decrease stage was charactenzed by decreasing of species number in 1.3-1.5 times comparatively with previous stage. During that period 75 percent of number and 74 percent of biomass for larva and 75 percent of number and 86 percent of biomass for adults were accumulated. The female share was increased to 65 percent. The reproductive potential was realized to 82 percent. The last stage was characterized by subsequent decreasing of species number and biomass These results have shown that optimal period for insect account and crop protection measures should be the second stage of population formation. In this case theoretical calculations discovered minimal crop loss after insecticidal treatments. It could result 75 percent in number control for larva and 75 percent for number control and 68 percent in biomass control for imago.

\section{G.J. van Os and W.J.M. van Gulik.}

Pythium is a soil-borne fungus which causes root rot in several bulb crops. To reduce the use and dependence on fungicides, non-chemical control methods have to be developed. Pythium is a fast growing fungus which is susceptible to competition by other microorganisms. A light intestation in non-sterilized soil causes moderate root rot, wheress the same infestation in sterilized soil leads to severe disease development. Pot experiments were performed to study the impact of several cultural practices on Pythium root rot in bulbous iris in relation to the condition of the soil microflora. Flooding and soil fumigation are generally applied in ornamental bulb culture to control some diseases and weeds. Flooding, however, does not kill Pythium. Flooding of infested field soil resulted in enhanced root rot compared to the non-flooded treatment. A similar effect was found when Pythium was introduced after soil fumigation with methylisothiocyanate or dichloropropene. Infestation of fumigated field soil resulted in enhanced root rot compared to infestation of non-fumigated soil. In the absence of other microorganisms (heat-sterilized soil) flooding and fumigation treatments had no effect on the disease development, indicating the crucial role of the microflora in the adverse effects of flooding and fumigation.

Apparently Pythium benefits from the elimination or disturbance of the soil microflora by sterilization, flooding, or fumigation. To restore the natural disease suppression in treated soils the effect of adding composted organic household weste was investigated. The introduction of microorganisms by adding $1 \%$ matured compost (equivalent to max. allowed dose for field application) to heat-sterilized soit one week prior to infestation with Pythium, resulted in a reduction of the pathogen population and the disease development compared to treatments without compost or with sterilized compost. Further experiments are performed to determine the effects of compost application after flooding or fumigation of fiald soil.

Bulb Research Centre, PO Box 85, 2160 AB Lisse, The Netherlands
1244

\section{IETEGRATED MARAGEAIEST OP SOYBEAR IHSECT PESTS}

RUMAR, N.G., VENKATARAVANAPPA, B. AND RAJAGOPAL, D.

An experiment was carried out to manage the insect pests of soybean cxop during the rainy season of 1992 and 1993 under rainfed conditions. The experiment included three main treatments viz., soybean seed treatment with chlorpyriphos 20 EC (4 $\mathrm{ml} / \mathrm{kg}$ seed) followed by foliar application of endosulfan $35 \mathrm{EC}$ $(0.058)(\mathrm{ST}+\mathrm{FA})$, foliar application of endosulfan (0.058) alone need based (FA) and untreated check. Each main treatment included five subtreatments i.e., soybean monocrop, soybean as intercrop with pigeonpea, maize, fingermtllet and sorghum. The results indicated significant reauction in stem tunnelling due to stemfly, Melanacromyza sojae $2 \mathrm{hnt}$. In soybean ST+FA treatment (15.878). It was on par with FA treatment (17.418). Among the subtreatments soybean (+maize) recorded least atemfly tunnelling (13.798) and was on par with soybean when grown as intercrop with finger millet (15.178), Borghum (15.47\%), pigeonpea (16.52\%) and soybean monocrop (18.398). Significantly less leaflet damage by the leaf miner, Aproaerema modicella (Deventer) was observed in the ST+FA treatment (18.898). However, the incidence of the American serpentine leaf miner, Liriomyza trifolii (Burgess) on soybean wae more in the insecticide trested plots than unprotected plots. No significant difference in Helicoverpa armigera (Hubner) damage to pods was noticed among the main treatments. Higher soybean seed yield was obtained in the ST+FA treatment followed by FA and untreated check.

Department of Entomology, University of Agricultural Sciences, G.K.V.K., Bangalore 560 065, INDIA. 
MAMAGEMENT OF SORGHUM INSECT PESTS

B. Narasimha Rao $i, T$. Ramesh Babu 2

Insects infesting sorghum can be categorised into soil pests, foliage, stem and earnead feeders and storage pests, the major ones being shoot fly, stem borer, armyworm, orain midge and a complex of earhead pest which take a major toll of sorghum yields $(12 \%)$. Shoot fly. Atherigon soccata Rond. damage varies with sowing time. Two peak activity periods of shoot fly (August and November) can ae avoided by adonting sowing soon after onset of monsoon in rainy season and October sowing in postrainy season. Application of nitrogen demonstrated that in post rimy mitrogen enthances the incidence. A minimum of $40 \mathrm{~kg}$ N per hectare is required. Seed treatrient with carbofuran $40 F \quad F \quad 125 \mathrm{~g} / \mathrm{kg}$ seed signifiCantly reduced the shoot fly incidence. Soil application

Stem borer, Chilo partellus (Swinhoe) incidence, in general, will be more in summer and post-rainy season crops compared to rainy season crop. Similar to shoot fly, stem borer incidence also was very high with zero level nitrogen. Application of insecticidal dusts, viz., carbaryl 5\%, phosalone $4 \%$, endosulfan $4 \%$, quinalphos $1.5 \%$, etc., a $12 \mathrm{~kg} / \mathrm{ha}$ in whorls was reparted to be highly effective in reducing the steri borer incidence. hese dusts were also proved to be equally effective against armywrm, Mythimna separata (Waiker).

Early-sown crops generally escape midge, Contarinia sorghicola (Coquiliett) damage, while late-flowering ones suffer serious damage when climatic conditions are favourable. Carbaryl, carbofuran, chlor pyrifos, dichlorvos, endosulfan, monocrotephos and phosalone have been tested and recormended under different situations. Similarly, earhead bug, Calocoris angustatus (Leth.) aiso can effectively be checked with severat insecticidal dust formulations.

Professor of Entomology, Department of Entomology, College of Rgriculture, Rajendranagar, Hyderabad-500 030, India.
REDUCING THE INCIDENCE OF MAIZE STREAK VIRUS BY

EXPLOITING THE BEHAVIOUR OF ITS LEAFHOPPER (CICADULINA SPP.) VECTORS

\section{W W Page}

Studies in Uganda on the epidemiology of maize streak virus disease indicate that males and females of the vectors (Cicadulina spp.) differ in their behavoiur within a maize crop and this may have a significant effect on disease incidence. Up to $90 \%$ of the lealhoppers caught in the upper canopy of maize are female whereas males constitute up to $90 \%$ of the population caught close to the ground. Cicadulina mbila have been shown to communicate during mate seeking behaviour by vibrating the leaf on which they are standing. It is hypothesised that males move from stem to stem through the maize stand close to the ground in search of females which tend to sit higher up in the whorls of the plant. It is suggested that the opportunities for males to locate females are enhanced in the maize over the denser vegetation presented by most of the wild grasses that act as preferred hosts for feeding and oviposition. By intercropping with various crop plants which produce thicker foliage near the ground, it should be possible to manipulate the insects behaviour to make the maize crop less attractive for mating activity. Evidence is presented to show that intercropping does reduce the incidence of maize streak disease in a maize crop and that spacing and plant height may also affect behaviour and the incidence of maze streak

Natural Resources Institute, Central Avenue, Chatham Maritime, Kent, ME4 4TB, United Kingdom

\section{7}

A PRELIMINARY STUDY ON THE INHIBITORY EFFECTS OF COMPOST EXTRACTS TO CUCUMBER DOWNY MILDEW AND ITS MECHANISMS

L. Ma, F. Gao, Y. Wu, X. Qiąo

Compost extracts can effectively reduce the damage by Phytophthor infestans on potatoes and tomato, Erysiphe graminis on barly and wheat, Erysiphae betae on sugar beets, Sphaerotheca fuliginea on cucumbers (Weltzien 1989, 1990). The authors studied the inhibitory effects of 4 compost extracts, made from dungs of horse, cow, sheep, and pig, on cucumber downy mildew (Pseudoperonospora cubensis (Berk. et curt.) Rost) under greenhouse condition and its possible mechanisms preliminarily.

The compost extracts of horse and cow dung shown effective control of cucumber downy mildew. The relative efficacies were correspondingly $67.33^{\circ}$ and $66.1 \%$ as compared with control. The compost extracts of sheep and pig dung acted less effective $\left(46.5^{\circ}\right.$ and $57.3 \%$ correspondingly) and unstable.

Liu et al.(1993)reported that contents of chlorophyll and peroxidase activity were correlated with the resistance of cucumber to downy mildew. The compost extracts could significantly increase the contents of chlorophyll and peroxidase activity. All the cucumber plants treated with 4 compost extracts contained more chlorophyll (24.2\%, 23.1\%, 20.5\%, $19.1 \%$ correspondingly) than that in control cucumber (18.19\%). In compost extract treated cucumber plants the peroxidase activity was also higher than that in control cucumber. In cucumber plant treated with compost extract of sheep dung the peroxidase activity was $194.48 \mathrm{mg}-\mathrm{H}_{2} \mathrm{O}_{2} \mathrm{~g}^{-3} \mathrm{~min}^{-3}$, while in that treated with compost extracts of cow, horse, pig dung the peroxidase activity were 180.2, $176.12,176.12 \mathrm{mg}-\mathrm{H}_{2} \mathrm{O}_{2} \mathrm{~g}^{-1} \mathrm{~min}^{-1}$ correspondingly. This value was by control cucumber $149.6 \mathrm{mg}-\mathrm{H}_{2} \mathrm{O}_{2}$ $\mathrm{g}^{-1} \mathrm{~min}^{-1}$.

The Effects of compost extracts can be a complex of biotic and abiotic factors. These factors may act in various degrees under different conditions. After sterilizing of the compost extracts the relative efficacy increased in $21.7 \%$ by horse dung, $47.65 \frac{}{6}$ by sheep dung, but decreased in $18.5 \%$ by cow dung and $45.3 \%$ by pig dung.

Institute of Plant Protection, Shanxi Academy of Agricultural Sciences, 64 North Nongke Road, Taiyuan 030031, PR China

\section{8}

PROPHYLACTIC EFFECTS OF COMPOST EXTRACTS ON WHEAT LEAF RUST (Puccinia recondita var.)

L. Ma, F, Gao, Y. Wu, X. Q.iaO

Compost has been used as a organic fertilizer worldwide and for a long time. But studies on the effect of compost extracts on various plant diseases were still in the very beginning. Weltzien(1989) reported that the aqueous extracts of good fermented compost could effectively reduce the incidence and intensity of the disease after it was prophylactic applied on the leaf surface. In order to confirming the effect of compost extracts on wheat leaf rust (Puccinia recondita var.) the authorg conducted a repeated experiment using aqueous extracts of 3 -months composted horse dung, cow dung, sheep dung, and pig dung in wheat varieties Mingxief69 (highly sensitive to $F$, recondita) and Ringyang 181 (middle resistant to $P$. recondita).

1. The relative efficacies of 4 compost extracts, that made from 3 -months composts of fresh horse dung, cow dung, sheep dung, and pig dung mixed with vegetable orchard soil and water, were by middle resistant variety all $>85 \%$ as compared with control, in witch the compost extracts were applied to the surface of wheat leaf 48 hours before inoculation with $P$. recondita spores. 2. The compost extracts shown inhibition effects in both highly sensitive and middie resistant varieties of wheat to $p$. recondita. But the effect was stronger by middle resistant variety than by highly sensitive variety.

3. By both wheat varieties the compost extract of pig dung shown best effectiveness. The relative effecacy was 99.98 by Pingyang 181 (middle resistant variety) and $62.6 \%$ by Mingxie169 (highly sensitive variety).

4. Relatively larger deviations between the repeats were observed. This must be related to the source of the dung, composting time, amendments etc. Further studies on this aspects are in progress.

Institute of Plant Protection, Shanxi Academy of Agricultural Sciences, 64 North Nongke Road, Taiyuan 030031, PR China 
1249

EFFECT OF GAMMA IRRADIATION ON AFLATOXIN PRODUCTION BY ASPERGIILUS PARASTTICUS

Zeinab H. Kheiralla*, Nagwa L. Hassanin** and Amra H.***

\section{Abstract}

Exposure of aflatoxins produced by $\underline{A}$. parasiticus grown on sterile raw peanuts to specific gamma imadiation doses induced reduction in aflatoxins with increasing gamma irradiation dose than did aflatoxin from non-irradiated gterile raw peanuts (contro)\}. The reduction of allatoxins ranged from 9.3 to $29.7 \% ; 13.9$ to $37,3 \%$; 0.96 to $16.8 \%$ and 0.72 to $60.3 \%$ for $A F B_{1}, A F B_{2}, A F G_{1}$ and $A F O_{2}$, when the culture was irradiated at 0.5 to $\$ \mathrm{kGy}$ respectively. On the other hand, non-sterile raw peanuts that was inoculated with conidia of the fungus and then incubated for two weeks at $30^{\circ} \mathrm{C}$ following irradiation resulted in reduction in aflatoxins ranged from 18.2 to $89 \%$; 26 to $96 \% ; 35.4$ to $85.2 \%$ and 17.3 to $76.4 \%$ for $A F B_{1}, A F B_{2}, A_{1}$ and $A F G_{2}$ respectively.

Irradiated raw peanuts culture following inoculation with conidia of the fungus and then incubated for two weeks at $30^{\circ} \mathrm{C}$ resulted in reduction of toxin(s) production ranged from 4.9 to $28.4 \% ; 16.8$ to $54.7 \% ; 1.71$ to $18.6 \%$ and from 9.13 to $30.4 \%$ in $A F B_{1}, A F B_{2}, A F G_{1}$ and $A F G_{2}$ when cultures irradiated at 0.5 to $5 \mathrm{kGy}$ respectively. Inoculation of either sterile or non-sterile raw peanuts cultures following irradiation resulted in completely degradation of aflatoxin(s) at bigher doses between 2.5 to $5 \mathrm{kGy}$ for sterile substrates and at $2.5104 \mathrm{kGy}$ for non-sterile raw peanuts.

* Botany Dept., Woman's College, Ain Shams Univ., Cairo, Egypt.

Biochemistry and Nutrition Dept., Woman's College, Ain Shams Univ., Cairo, Egypt.

*** Mycotoxins Central Lab., National Research Center, Dokki, Cairo, Egypt.
BIOLOGICAL CONTROL OF POBTHARVEST ROTS OF TABLE GRAPE, KIWIFRUIT AWD GTRAWBERRY BY YEASTS

\section{G. Lima, A. Ippolito, P. Nigro and M. Balerno}

Due to the restriction on the use of synthetic fungicides, safe and effective alternative methods of controlling postharvest diseases of fruits and vegetables need to be developed. Biological control by means of microorganisms recovered from fruit surfaces has proven promising. Among the putative antagonists, yeasts appear particularly suitable due to their ability to grow rapidiy, to colonize the wound site and to compete with the pathogens for space and nutrient. Moreover, they seem capable of interacting dire

More than 200 yeasts (inciuding yeast-1ixe fungi), isolated on nutrient yeast-dextrose agar from fresh or stored fruits and vegetables, were preliminary tested for their in vivo activity against Botrytis sinerea Pers.

The most effective isolates were tested in biological control trials on table grape, kiwifruit and strawberry, using grape berries wounded by removing the pedicel, kiwifruits and strawberries wounded in the equatorial zone. Thirty $\mu 1$ of yeast suspension containing $4-6 \times 10^{8}$ cfu/ml were placed in each wound and after $2 \mathrm{~h}$ the wounds were inoculated with $10 \mu \mathrm{l}$ of a conidial suspension $\left(1.5 \times 10^{4}\right.$ conidia/ml) of B. cinerea. The fruits were stored for 5 days at $20^{\circ} \mathrm{C}$ and $95-98^{\circ} \mathrm{RH}$. The isolates L47, 160 and 466 , among those tested, were the most effective against the pathogen reducing grey mold infection more than 858 by comparison to the control. On table grape these isolates were also effective in controliing Rhizopus stolonifer Erhenb. and aspergillus niger van Tiegh. The isolates, identified as Aureobasidium pullulans (L47), Candida vanderwaltil (L60) and $c$. oleophila (266) enhanced their activity when applied in 28 calcium chloride.

The isolates 447 and $L 66$ were also assayed in field trials against . c. cinerea on table grape. L47 was effective in controlling the pathoges on stored $\left(0^{\circ} \mathrm{C}\right)$ bunches and in the field, in anner comparable to that exerted by dicarboxidide treatrents. The population of 497 lasted significantiy higher in comparison with that of 566 , both in stored bunches and in the field.

When yeasts application was combined with curing in trials on stored $\left(a^{\circ} \mathrm{C}\right)$ kiwifruits, the control of Botrytis infection was significantiy improved.

The isolates L47, L60 and $L 66$ were able to grow in presence of relatively high concentration of some of the main fungicides of relatively high concentration of some of the main fungicides observations on same of the possible modes of action of the most observations on some of the possib

Hork supported by a Grant of the Kationat Research Council of Italy, Special project RAISA, Subproject 4

Dipartimento di Protezione delle Piante dalle Malattie University of Bari, via Amendola 165/A - 70126, Bari, Italy.

\section{2}

EFPICACY EVALUATION OF BACILLUS THURINGTENSIS AND NEEW OIL, AZADTRACTA INOICA ON POTATO TUBER-MOTH IN THE FIELO ANO DURING STORAGE.

S.A. Salem and M.S. Foda

A new approaches were used for evaluation of the efficacy of. Bacillus thuringiensis and pure neem seed oil on the potato tuber-moth, Phthorimaea operculell.a in the field and store. Both the two materials were found equally effective on the potato tuber-moth infestation. The infestation percentage could be significantly reduced by frequent application of both in the field up to harvest and also during the storage. No clear synergistic effect could be observed upon application of the two preparations simultaneously. The results obtained suggest their possible use as substitutes for hazardeous chemical insecticides in combating the pest both in the field and during storage.

* Dept. of Plant Protection and Microbial Chemistry, National Research Center, Dokki, Cairo, Egypt. 
ON THE WAY TO AN INTEGRATED STORED PRODUCT PEST

MANAGEMENT: BIOLOGICAL CONTROL WITH ENTOMOPHAGOUS INSECTS

\section{Al-Kirshi,A.-G., Prozell, S., Reichmuth, Ch.,Schöller, M.}

Natural enemies are known for all major stored product pest insects. Biological control has been developed to be competitive in some areas of plant protection like fruit- and vegetable protection. Feasibility of rearing and application of natural enemies have been proven. Warehouses with stored product commodities are a closed system, like greenhouses, where the application of biological control agents is most effective. The use of insect predators and parasitoids for stored product protection comprises advantages compared with chemical methods. The use of antagonistic insects is not known to be poisonous to humans.

The use of biological control strategies against stored product pests is increasing mainly due to the growing concem towards chemical pesticides. Other than biological control measures the use of pesticides includes problems of resistant insect species, workers safety and pollution. The introduction of biological control methods is enhanced by the decreasing number of available pesticides. In developing countries, biological pest control measures offer alternatives to expensive insecticides.

Meanwhile, in stored product protection results from practice and large-scale experiments are available and some parasitic wasp species are officially accepted for use. Investigations about egg- and larval parasitoids are presented and the potential of biological control methods as part of an integrated pest management is discussed.

Federal Biological Research Centre for Agriculture and Forestry, Institute for Stored Product Protection, Königin-Luise-Str. 19, 14195 Berlin, Germany.

\section{EFFECTSOFLOWTEMPERATUREONGROWTH,GERMINATION AND PATHOGENICITY OF PHOMA BETAE IN STORAGE}

\section{Shiping Tlan, Paolo Bertolini, Marta Mari}

The effects of low temperature on mycelial growth, spore germination and pathogenicity of Phoma betae were studied in culture and on young root of sugar beet at temperatures of 20,10, $4,2,0,-2$ and $-4^{\circ} \mathrm{C}$. P. betae was able to grow and germinate on potato dextrose agar at temperature as low as -4 , but the radial growth of mycelium was significantly reduced and spore germination was delayed at temperatures below $0^{\circ} \mathrm{C}$. Disease incidence and lesion development of sugar beet roots by $P$. betae were also inhibited with decreasing of temperature from 20 to $-2^{\circ} \mathrm{C}$. The infection of $P$, betae on wound-inoculated roots occurred more early with a higher disease incidence as compared to that on unwound-inoculation roots. $P$. betae was capable of penetrating directly unwounded roots. The infection by contact with decayed ones is also an important way for spread of the disease in storage. Sugar beet roots stored at $-2^{\circ} \mathrm{C}$ showed slightly symptoms of freezing injury.

CRIOF, Department of Protection and Improvement of Agricultural Food Products, University of Bologna, Via Gandolfi 19, 40057 Cadriano Bologna, Italy
ALTERNATIVE PROTECTION OF STOKE U CONPEA AGAINST Callosobruchus maculatus F. (COLEOPTERA : BKUCHIDAE) USING BOScia senegalensis (PERS.) LAM. EX POIR. (CAPYAKACEAE) ALONE OK IN COMBINATION WITH HERMETICAL STORAGE.

D. SECKK

Biological activity of the sahelian shrub Boscia senegalensis has been evaluated on callosobrucnus maculatus which is the major insect pest of storea cowpea in west Africa. when added to cowpeas at $2-4 \times(w / w)$, fresh ground fruits and leaves caused $80-100 \times$ mortality in $C$. maculatus adults and reduced or completely inhibited the oviposition of the females. Chemical investigations conducted on the plant, using GC MS techniques, demonstrated that the insecticidal effect of $B$. senegalensis is due to the liberation of Methylisothiocyanate from a glucosinolate precursor (glucocapparin) contained in Bosia fruits and leaves.

The combination of $B$. senegalensis use with hermetical storage permitted to obtain $100 \times$ protection in infested cowpea seeds. because of a complete inhibition of a new generation of the Cowpea Beetle.

These results have prospects in the developinent of safe and indigenous botanicals against stored-product insects in developing countries.

Laboratoire d'entomologie des denrées stockées Institut Sénégalais de kechercies Agricoles/DKCSI boite Postale 240 Saint Louis, SENEGAL.

Tel. 0022161 1751 Fax : 61'18 91 .
1256

\section{TRAPPING AND ESTIMATION OF LOSSES AND DAMAGE CAUSED BY} OLIVE FLY Bactrocera oleae (GMEL) IN THE SAIS. MOROCCO.

AFELLAH M., BEN HAMADI I., CHEMSEDDINE M., BOUGHDAD A., HIALL A.

Trapping and estimation of losses caused by the olive fly were conducted in five orchards of olive which include 2 varieties for olive oil (Moroccan Picholine and Languedoc Picholine), and 2 varieties for caned olive (Gordal and Meslalla). Four orchards were located at $550 \mathrm{~m}$ altitude and one was at $300 \mathrm{~m}$. Trapping was based on McPhail, on sexual traps and yellow glued plates. The catching evolution using McPhail showed two main periods of high activity of this devastator in the automn and Spring. The captures were very low or nul in the Summer in high altitude areas, but in the plains they remain until June. This insect showed high preference for Gordale variety indicating a strong preference of adulte flies for the big dimensions among the caned ofives. For other trapping devices, the pheromone were less attractive than the McPhail trap, but were highly selective, and the yellow plates were ineficient towards the Bactrocera oleae. The losses reached about $3,8 \mathrm{~kg}$ and $2 \mathrm{~kg}$ per tree for Gordale and Meslalla respectively. These losses consisted of fruits presenting damage by the fly on the harvesting day besides the high number of infested shed fruits. For the varieties for olive oil, the effects of the flies increased the oif acid content which reached $4 \%$ when the damage was about $41 \%$ and the production was not stored before trituration. This oil is therefore classed as non suitable for consommation because it is in the category of "refined oil". Also, the trituration of a sample of slightly infested olives (11\%) gave an "Extra virgin oil" (rate of acidity $0,8 \%$ ) without stockpiling before trituration. The rate increased to $3,5 \%$ twenty days after storage with the same level of infestation.

AFFELLAH M. INRA/Laboratoire de Zoologie BP 578 Meknès Maroc. 

Rhopalosiphum padi (L.) and Schizaphius graminum (Rond.). Adults and nymp of both species commonly intermixed or resided and nym to together at the same location on the plants, but later in the season (beginning of March), $R$. padi usually become more abundant on stems and ears. The development of aphids population continue through January until April. Both species began to appear on the plants nearly during the first weak of January. Then, thei populations increased gradually to reach $10 \%$ of the maximal leve in about 6 weeks later. Aphids population increased to peak numbers nearly in the middle of March (milky ripenning stage of the plant). The population then declined, so that by late March and beginning of April both species were found in few numbers The decline may have been practicaliy in response to the host plant maturity and the invasion or the natural enemies. The time passed Erom the first migracion of aphids on the wheat field until their disappearance was about 12 weeks. The highest level of population density was recorded after about 10 weeks from initial aphid infestation. This means that the establishment of cereal aphids population in the fleld can be expected in the beginning of February, whereas the peak number should be expected in middle of March. The lower limit of the initial infestation which resulted an economic damage was 0.25 individual/tiller, whereas an initial infestation rate of 0.5 individual/tiller or more incuced a significant losses in the yield $>34 \%$. The reduction in grain yield due to natural aphid infestation reached an average of $18 \%$.

Prof. Abdel-Wahab Moh. Ali

Dept. Plant Protection, College of Agric.

University of Assiut, 71516 Assiut,

Egypt Leaf eating caterpiliars are serious pests of oil
palm in Malaysia. These include the bagworms (Psychidae), nettle caterpillars (Limacodidae) and hairy caterpiliars (Lymantridae). Normally, these pests are well controlled by natural enemies. However, when natural control breaks down, these pests can build up rapidly and cause severe defoliation of the palm canopy. An average crop loss of $30 \%$ over a period
following 508 defoliation.

A Monitoring and Surveillance System (MSS) has been developed to counter these pests. It relies on developed to counter these pests. It relies on
regular bi-weekly census following detection of pest regular bi-weekly census following detection of pest
infestation. This continues for several months after successful control. When thresholds of 5 to 10 caterpillars, depending on species, are exceeded, caterpillars, depending on species, are exceeded, control is effected by judicious foliar application of selective insecticides or by trunk injection of systemic insecticides. Additional criteria for absence of natural control, early caterptilar stage with no or few live cocoons.

Proper training of plantation personnel is crucial for successful implementation of the MSS. They are trained to recognise early symptoms of caterpillar damage on fronds, identify caterpillar species and stages in life-cycle select census palms and fronds, carry out actual caterpiliar counts and record ali relevant data.

The data is sent to Ebor Research, a central research station, for processing. This involves data entry using Turbo Basic Programing Language, creation of an interface file and generation of colour coded maps to reflect 5 levels of caterpiliar and live cocoon infestations using Harvard Graphics Version 3 software. Recommendations are rapidly issued to the respective plantations based on the summary produced. Integrated pest management of leaf eating MSS system. Sime Darby Plantations, Ebor Research, Locked Bag No.
7202 , Batu Tiga, 40706 Shah Alam, Selangor, MAIAYSIA.

EFFECT OF TIMING OF CONTROL OF FOLIAR DISEASES ON MULTIPLE PEST EPIDEMICS

\section{G. Eversmeyer and C. L. Kramer} Leaf rust caused by Puccinia recondita Rob. ex Desm. f.sp. tritici,
Septoria leaf blotch caused by Mycosphaerella graminicola (Fuckei) Septoria leaf blotch caused by Mycosphaerella graminicola (Fuckei) Schroeter, and tan spot caused by Pyrenophora tritici-repentis diseases of wheat (Triticum aestivum L.) in the hard red winter diseases of wheat (Triticum aestinum L.) in the hard red winter wheat region of the rust have averaged 7.5 . 11.4, 10.1 and $1.0 \%$ over the last four years in Kansas alone. Under epidemic conditions, yield losses caused by Septoria leaf blotch range from 30 t $50 \%$ on susceptible cultivars in Southern Kansas. Losses caused by tan spot have ranged from 20 to $50 \%$ in the same fields. Yield increases resulting from control of individual foliar pathogens of wheat have been extensively documented. Most of these studies however, are directed at determining the effect of a single disease rather than
the interactive effects of two or more pests. Rarely is only one disease observed to occur in a wheat crop under field conditions.

Application of foliar sprays of triadimefon at several growt stages on wheat cultivars Newton, TAM107, Karl, and Arkan were used to control foliar pathogens. Single applications of triadimefor after the milk growth stage on susceptible cultivars resulted in from 24 to 68 increase in yield. Increases in yield resulting from control of pests on resistant cultivars ranged from 4 to $12 \%$. Area under tissue destruction curves (AUTDC) $(r=-0.71)$ and percent severity $(r=-0.77)$ of leaf rust were negatively correlated with percent yield increase. AUTDC $(r=0.61)$ and percent severity $(x=0.53)$ of leaf rust were positively correlated with percent yield increase. AUTDC of Septoria leaf blotch $(r=-0.65)$ and tan spot $(r=-0.88)$ were also negatively correlated with yields.

Merle G. Eversmeyer, Research Leader Rm. 4008 Throckmorton Hall Kansas State University
DEVELOPMENT OF A SEMI-SELECTIVE MEDIUM FOR QUICK AND EASY DETECTION OF XANTHOMONAS CAMPESTRIS PV. MANIHOTIS, INCITANT OF CASSAVA BACTERIAL BLIGHT

\section{A. Fessehaie ${ }^{1}, K$. Wydra ${ }^{2}$ and K. Rudolph 1}

1 Institute for Plant Pathology and Plant Protection, Göttingen, RFA

2 International Institute for Tropical Agriculture, Cotonou, Benin

A semi-selective medium for isolation of Xanthomonas campestris py manihotis $(\mathrm{X} \mathrm{cm})$, incitant of cassava bacterial blight, was developed. Several carbon and nitrogen sources were tested for selectivity towards $\mathrm{Xcm}$, and antibiotics were screened for inhibitory effect on the epiphytic bacteria of cassava.

Trehalose and ammoniumchloride were identified as optimal carbon and nitrogen source, respectively. From eighteen antibiotics screened, three were chosen, which did not inhibit the pathogen but totally prevented growth of epiphytic bacteria.

Different basic media were tested using the selected carbon and nitrogen source and screened for their effect on growth and plating efficiency of $\mathrm{Xcm}$. Within the finally developed medium the selectivity of the antibiotics cefazolin, lincomycin and fosfomycin was tested and their concentration in mixture optimized. These experiments resulted in the following composition of the $\mathrm{Xcm}-\mathrm{semi}$ selective medium:

$1 \mathrm{~g}$ yeast extract, $1 \mathrm{~g}$ glucose, $9 \mathrm{~g}$ trehalose, $3 \mathrm{~g}$ dipotassiumhydrogenphosphate, $1 \mathrm{~g}$ sodiumdihydrogenphosphate, $0.3 \mathrm{~g}$ magnesiumsulfate, $1 \mathrm{~g}$ ammoniumchloride, $25 \mathrm{mg}$ cefazolin, 1000 microgram lincomycin, $2.5 \mathrm{mg}$ fosfomycin, $200 \mathrm{mg}$ cycloheximide and $14 \mathrm{~g}$ agar, the $\mathrm{pH}$ was adjusted at 7.0 .

The semi-selective medium was tested under field conditions and was shown to facilitate isolation of $\mathrm{Xcm}$ from diseased and symptomless leaves. The medium will be used for epidemiological studies on the survival of $\mathrm{Xcm}$ on weeds. in plant debris and in the soil.

Institute for Plant Pathology and Plant Protection, University of Göttingen, Grisebachstr. 6, 37077 Göttingen, Germany 


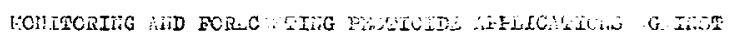

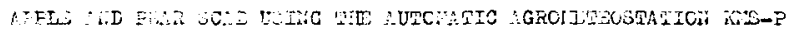

\section{a.... ipodorova}

buring the opcration oi the …- (inton MAn, iustria) in Fort-

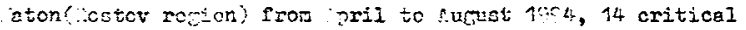
periods for scab infection in aple and pear trees vere rccerded. eovy infection ws signilec 3 tines, sediun tines and lifilt in the rest of the cascs.

In ccordince with those sinncle and on the basis of the studies on the timine of primery (xpril $3,1,4$; and second:ry (iay $: 1, \cdots$ infection and jant ghenolosy, control trentients with Mor and $\lambda$ to were aplied in the orchard. in sone crses for tecinical ressons there we a tire lne in surings but this las was nevor seyend the tiwe durinc vilich the products

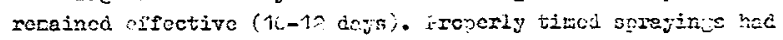
a good erfect in controlling scab on imits and lerves. the zercent of liscaced lants veriod irois 6 to 55 dopondint on ayple and ron cultiver, mile the discase index varied from

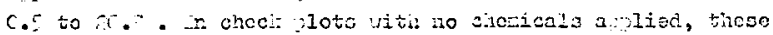

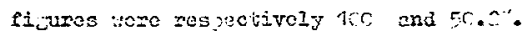

For tive vurposes oi co-muter--seisted estination of scab infection lovels is a busis $z e r$ disease forecrst, it is desiroblo that tic levice siouli includo a sensor for moneurin: scaj infection lovels on tile surface of a lanf on fruit accordins to a scole of $i$ to 5 with the demajo threshold of $5(10)$.

hon used in the "hrtu" prograw, the s-i stetion operated fairly vell and its readings were in accorderco uth tho real situation ia tise orchari, coyering on wather conditions and diseese develonsent.

\section{1-iiliesian Tent :rotection Enstitute}

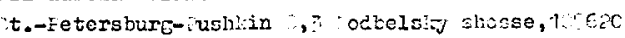

Russia

Telephone: (912) 4705170 ; Tolefax: (212) 47043.03

\section{3}

COMPUTER-ASSISTED, TEATHBR-LFI UST FEKOHONE VONITORINO OF ORCHARD FTSTS

I.Ya.Srichanov and L.P. Ferger

All-Rusgian Plant Protection Inati tute, St.Petersburg Rusgia

is syatem of nest nonitoring, using the agrometeorological stetion KNS-P (Anton PAAR, Austria) and a network of ohersnone traps, hes been onerated in orchards in the North Caucagus (Azov district in the Rostov region). Pased on the correlation analygiz of phenological and weather data, it hes been mossible to distinguish Io or I2 critical neriods of effective heet gume during the seeson when the whole negt onmolex is nost likely to be controlled by insecticides. Thus, for examble, at the effective heat sum of $220^{\circ}$ only one insecticide or sorey nixture appication is sufficient to reduce the numbers of aonle, olum and oriental fruit noths, apple ernine and lear nining moths, fall webworns, and scales and mites. The effective heat sum of $420^{\circ}$ is ontimal for control of the secono generetion of codling moths, some leaf miners, fall wetworns, and scales and nites. The neec of pesticide anolications for the rain nesta is determined from the results of aheromone tranoing. To sianlify the nonitoring system, it is sugzested that for key pest snecies samples ghould not be taken (using pheromone trans for adults or visual surveys of olante for larvae) until after fruit setting when the effective heat sums of $I 30$, $\hat{2} 20,310,420 \ldots$ degrees have been reached. For exemnle. the critical effective heat sums for the coditing moth are $220,420,660$ and $7^{\circ} 0$ degrees. So, if this nest soecies is the key one in the farm and if its damage threshold deternined from moth centures into the treps is exceeded, then there is no need in monitoring and control of other lenidopteran vests.
NOCTURNAL SKY COVER PERSISTENCE IN DES MOINES, IOWA, U.S.A.

\section{V.L. Boulaevskaia, S.E. Taylor, K.J. Koehler, M.L.Gleason}

Atmospheric cloud cover (sky cover) is an important determinant of dew formation and duration because the presence of clouds influences terrestrial radiation balance. If sky cover during the night could be estimated from variables routinely measured at most weather stations in the U.S., these estimates could be used to improve the accuracy of dew estimation models without requiring visual observations of sky cover, which are made routinely at only a few sites. The relationship of sky cover observed during the night to sky cover observed during the previous evering and subsequent morning was investigated using July 1990, 1991, and 1992 data for Des Moines, Iowa. The most frequent patterns were entirely clear and enirely cloudy nights. Values of sky cover observations in the evening and the following morning were strong indicators of the sky cover values during the intervening night. Averaged observations of overnight sky cover were regressed on the overnight change in air temperature and on average relative humidity during the night. Average relative humidity during the night was found to be a strong predictor $\left(r^{2}=0.82\right)$ of completely clear or completely cloudy overnight sky cover patterns, but not of intermediate patterns. Overnight change in air temperature was a weaker predictor $\left(r^{2}=0.61\right)$ of completely clear or completely cloudy nights. We have shown that for certain subsets of nights in Des Moines, sky cover values in the preceding evening and subsequent moming, as well as average relative humidity during the night, are strong predictors of sicy cover at night.

Department of Plant Pathology, 351 Bessey Hall, Iowa State University, Ames, IA 50011, U.S.A. (electronic mail of 4th author: mgleason@iastate.edu)

OCCURRENCE, DEVELOPMENT, AND CONTROL BY FUNGICIDES OF SEPTORIA NODORUM, S TRITICI, AND PSEUDOCERCOSPORELLA SEPTORIA NODORUM, S TRITICI, AND PSEUDOCERCOSPORELLA
HERPOTRICHOIDES ON WHEAT IN POLAND AS DETERMINED BY DIAGNOSTIC IMMUNOASSAYS

C.M. Smith, C. Janczak, M. Korbas, Z. Pokacka, M. Glazek, Z. Weber, J. Kus, A. Mroz, T. Zawadzki

The occurrence, development, and control by fungicides of Septoria nodorum, S. tritici, and Psestocercosporella herpotrichoides on winter wheat in Poland in 1993 were investigated using DuPont diagnostic immunoassays. Main shoots of 1993 were investigated using DuPont diagnostic immunoassays. Main shoots of
thisty wheat plants per field were collected at several times between tillering thirty wheat plants per field were collected at several times between tillering
(April) and grain ripening (July). The shoots were bulked by stem bases, leai (April) and grain ripening (July). The shoots were bulked by stem bases, leaf
layers, and heads. Samples were then tested in the 'Advisor' enzyme-linked layers, and heads. Samples were then tested in the 'Advisor' enzyme-linked
immunosorbent assays (ELISA) for $S$. nodorum and S. tritici (leaves and heads) and $P$. herpotrichoides (stem bases). To investigate disease occurrence and development, samples were collocted from 25 wheat fields, located throughout Poland, which had not been treated with fungicides. ELISA results showed that at all locations, the three pathogens were present at tillering and attained high levels by milk development stage. S. nodorum and $S$. tritici initially occurred in lower foliage and spread to upper leaf levels and heads by anthesis to milk
development. $P$. herpotrichoides intection increased signiticantly between development. $P$. herpotrichoides intection increased signilicantly between effect of fungicides, a field trial was established in southeastern Poland. Treatments consisted of either an untreated control or a foliar fungicide spray program of DuPont's 'Alert' 375 SC (flusilazole $125 \mathrm{~g} / \mathrm{/}+$ carbendazim $250 \mathrm{~g} / \mathrm{l}$ ) applied at 1 l/ha at first node, followed by 'Cerelux' $510 \mathrm{EC}$ (flusilazole $160 \mathrm{~g} / \mathrm{l}+$ tridemorph $350 \mathrm{~g} / 1)$ applied at $0.8 \mathrm{l} / \mathrm{ha}$ at booting. Flusilazole fungicide combinations were highly effective in controlling $S$. nodorum, $S$. tritici, and combinations were highly effective in controlling $S$. nodorum, $S$. tritici, and
$P$. herpotrichoides and exhibited excellent residual activity. These results $P$. herpotrichoides and exhibited excellent residual activity. These results
demonstrate the importance of the three cereal pathogens in Poland as well as demonstrate the importance of the three cereal pathogens in Poland as well as
the utility of diagnostic immunoassays and flusilazole fungicide in the integrated control of these diseases in Eastern Central Europe.

E. I. DuPont de Nemours and Company, Agricultural Products, Stine-Haskell Research Center, Newark, Delaware 19714-0030 U.S.A. and Plant Protection Institute, ul. Miczurina 20,60-318 Poznan 1, Poland 
PRODUCTION OF CONIDIA OF PERONOSCLEROSPORA SORGHI (GESTON AND UPPAL) C.G. SHAW ON SORGHUM IN AFRICA

C.H. Bock ${ }^{1}$, M.J. Jeger, L.K. Mughogho, and K.F. Carduell

The sources of inoculur for epidemics of sorghum downy mildew (Peronoscleyospora sorghi) on sorghum or maize are soilborne, sexually. produced oospores or asexually produced conidia. Information on the potential for asexual spore production and the conditions that influence it
during the period of early crop growth when plants are susceptible to infection, will indicate the role of conidia in epidemic development in particular region.

The daily production of conidia of $p$. sorghi was investigated using a 7 -day The dally production of conidia of p. sorghi was favestigated using a 7 -day Apri2) and $1993 / 94$ (Novenber 1993 april 1994) at Yatopos in 7 imbabwe. Apri2) and log3/94 (Novaber The spore samplers were situated adfacent to crops of sorghum that we Thected wich downy alldew during both seasons. Primary weather data. including 4hour maximum, minimum and mean cenperatures, 4 -hour mean windspeed and daily rainfall, were recorded at were sampled intermittently throughout the sampling period. Hovever, during the sampling period in 1993/gh very few conidla were sampled after February conditions under which conidia where sampled in the vicinity of infected crops was sinilar during both seasons. The conidia were sampled between 2330 and 0830 hours only, but most frequently between 0330 and 0530 hours. production of confida was profilic, but irregulat, being closely associated with rainfall. Up to 6000 conidia were sampled per $\mathbf{m}^{3}$ per hour. On 736 of the nights on which conidia were sampled it had rained up to three days earlier, the rain presumably providing adequate humidity for sporulation. The few nights when conidia were sampled but without rain for the previous five days may be associated with still conditions and the presence of a humid air mass, although $r, h$. was not recorded. Temperatures on nights when conidia where sampled ranged from 10-21 ${ }^{\circ} \mathrm{C}$, conidia being sampled most frequently on nights when the temperature was $15-19{ }^{\circ} \mathrm{C}$. Windspeeds were less than $2 \mathrm{~ms}^{-1}$ on over $60 z$ of nights when conidia were sampled. Low windspeeds would favour high r.h., which would support spore production. The irregular production of conidia of $P$. sorghi appeared to have a close dependency on the distribution of the rainfall in this area, which is typical of the semi-arid tropics. The rain would provide transient conditions of high $r, h$, which would be sufficient to support sporulation for a few days only, provided the temperature was between 10 and $26{ }^{\circ} \mathrm{C}$. Low windspeeds would be less likely to reduce $r . h$. as the humid air within the crop boundary layer is less likely to be mixed, so conidia would be produced under comparatively still conditions. This suggests that in the semi-arid areas of southem Africa crops will be infected only if the date of planting and the period of early crop growth and susceptibility coincides with a period of high moisture and temperatures suicable for conidia production.

Natural Resources Institute, Gentral Avenue, Chatham Maritime, Chatham, Kent ME4 4TB, England, UK.

1267

BIOECOLOGICAL OUTLINES OF COLORADO BEETE LEPTINOTARSA DECEMLINEATA (SAY) (COLEOPTERA: CHRYSOMELIDAE) A NEW ESTABLISHED PEST IN IRAN

\section{H. KAZEMI and J. ARDABILI}

The Colorado beetle was one of the quarantine pests in Iran. Kazemi first reported the pest in the Ardabil region in 1984. The bioecological studies were carried out by the authors from the same year. The hibernating insects are tolerant to winter cold. In spring (late April and May) as soon as the young leaves of potato plants appear, the overwintering adult beetles emerge from the ground and begin to feed on the leaves. The eggs are laid in batches underside of the leaves and other weeds and plants of the potato field. The maximum nurber of eggs were 82 in a batch. Our study showed that one overwintering female insect had laid up to 920 eggs. After 7 to 15 days the eggs hatch into larvae and start to feed. The larval stage lasts about 16-28 days in the Ardabil region depending on the temperature. The fully grown larvae burrow a few centimeter (usually 3 ) into the soil to pupate. The pupal stage lasts about 15-25 days. This insect has 2 generations per year in the Ardabil region. The Colorado beetle apart from the potato family (Solanaceae) also feeds on other plants such as Hyoscyamus sp. and Senecio spp. The insect has an obligatory diapuse.

The insect is now an established pest and can be found in East and West Azarbaidjan. Ardabil and Gillan provinces of Iran. The percentage of infestation in the field was between 5 and $50 \%$.

Thiodan $35 \% \mathrm{EC}$. Zolon $35 \% \mathrm{EC}$ and Larvin $80 \%$ W.P. are being used as a chemical control against this insect ac the moment. The larvae of Chrysopa sp. is one of the pretators that feeds on the eggs of the Colorado beetles. Attempts are being made for the use of microbial control measures against the pest.

Faculty of Agriculture, University of Tabriz, Tabriz/Iran.
THE COMMON VOLE (MICROTUS ARVALIS Pall.) AS A MODEL OBJECT FOR REMOTE SENSING IN AGROCENOSES

\section{F.A.Karlik, G.Ye.Sergheyev}

Life activity signs of the common vole as indices for remote sensing in agrocenoses were studied in steppe areas of Ciscaucasia, the zone of severe damage by the pest. On sampling plots of $10 \times 10,25 \times 25$ and $100 \times 100 \mathrm{~m}$ located within perennial grasses and winter grain crops inhabited by rodents at different population levels, all vole colonies were mapped on a scale of $1: 100$ and 1:200. As elements of mapping. signs of burrowing and grazing activities were used, i.e. burrow openings, burrow openings with earth hillocks and groups of hillocks, and spots of fully destroyed vegetation. Here also the complete removal trapping of rodents was carried out on a multi-day basis. The stage of population dynamics was determined from demographic structure of captured individuals and other indices used in ecological research. On the basis of statistical treatment of the data, a non-linear regression model was constructed permitting population densities of the common vole to be tentatively estimated by signs of its burrowing activity. The resulting objective relation was used to develop methods of aerovisual surveyes of agricultural lands for vole infestations. Fields are surveyed from board a light helicopter or plane flying at the height of $100-200 \mathrm{~m}$ by a surveyor who gives an expert estimation of infestations. Expert estimations are expressed as numerical scores of 1 to 5. Each score corresponds to a certain gradation of density ranging from the minimum seen from the air to the maximum established for a given zone. An experienced surveyor can only make an error within one class interval of the scoring system suggested. But errors of this kind are of no much significance in decision making on adequate tactics of pest control.

3, Podbelsky shosse, St.Petersburg-Pushkin, 189620 RUSSIA All-Russian Institute of Plant Protection (VIZR)

\section{8}

EMPIRICAL MODEL FOR PREDICTION OF RICE DAMAGE BY RHIZOCTONIA SOLANI

C. R. KIM, C. H. RIM.

Emplrical models to predict degree of damage by rice sheath blight at maturing stage were constructed based on 17 -year data obtained during 1976-1994 crop seasons. The following data were collected 10 times by 10-day interval from June 21 to september 21: plant height (PHE), number of tiller (NOT), number of diseased tiller (NDT), top lesion height (TLH: highest position of lesion in each hill), percent diseased h1ll (PDH), percent diseased tiller (PDT), percent top lesion height vs plant height (PLH). Degree of damage (DD) was checked only one time at maturing stage. DD could be predicted by the regression equation; $\mathrm{DD}=-5.91+2.11 \mathrm{PHE}-4.02 \mathrm{NDT}-0.50 \mathrm{PDH}+2.17 \mathrm{PDT}-$ $7.55 \mathrm{TLH}$ with $\mathrm{R}$-square value of 0.7180 on July 11 (just after maximum tillering stage) under standard nitrogen fertilizer level. The DD derived during vegetative stage can be used to estimate the yield loss by sheath bilght using the following equation: $Y=0.55 \mathrm{X}$ $1.70\left(R^{2}=0.7962\right)$, where $Y$ is yield loss and $X$ is $D D$.

Plant Pathology Division, Agricultural Sclence and Technology Institute (ASTI), 249 Seodun-dong, Suwon 441-707, Rorea 
IDENTIFICATION OF GEOGRAPHICAL POPULATIONS OF THE GRASS MOTH Loxostege sticticalis L. BY THE SR-XRF TECHNIQUE

\section{I.B.Knorr, E.N.Naumova, V.A.Trounova, I.P. Dolbnya. K.V.Zolotarev}

Central problem in the protection of crops against the grass moth is a question devoted to the mass migrations of the imaginal phase of this pest. Unfortunately, a hypothesis on the mass moth migrations is not accepted by all specialists. This can be explained by absence of the direct evidences of such the migrations supported by available facts and experimental data. The currently available data reported by Russian specialists are usually supported by visual observations and logical assumptions only. Study of grass moth migrations presents methodical difficulties, since it is necessary to register a nuge number of the moths moving over hundreds and thousands kilometers by the system of cyclonic winds formed in the lower troposphere. Our long-standing study on the grass moth carried out in the Asiatic regions of Russia and neighboring territories of Kazakhstan has shown that the study of grass moth migrations is connected with the need to distinguish between separate geographical populations. To our opinion, an idea to analyze the element composition of moth body using the SR-XRF technique seems to be successful and perspective. This technique has been shown to offer a lot of advantages over the emission spectro- scopy approach previously used by the authors, including its higher accuracy and simplicity. The SRXRF technique ailows to determine more than 30 chemical elements simultaneously. Besides, examined samples can be stored for long time to be repeatedly analyzed, if it is necessary. Hereafter, probably, it will be possible to determine "element-markers" or groups of elements being more specific for each a separate geographical population of the grass moth that could became a reliable basis for its identification.

Institute of Biology Siberian Branch of Russian Academy of Sciences, Frunze 11, Novosibirsk, Russia 630091

1271

\section{DETECTION OF PLANT VIRUSES IN THE HUNGARIAN RIVERS AND LAKES}

\section{E. Pocsai, J. Horváth, N. Juretiê, G.K. Szabó and A. Vida}

Recensly a considerabie attention has been drawn to the researches on the occurrence of plant viruses in natural waters. This matter raises many questions that are of imporance from the points of view of booth epidemiology and preservation of nature. It is already proved that plant viruses can occur in tivers and lakes and accordingly it means a new way of vinus spreading. It is very likely that plant viruses are adsorbed to soil colloids that protects them from inactivation for a longer period.

In Hungary, 25 water samples were collected from the main rivers and lakes in July of 1994. After concentration by ultracentrifugation, water samples were tested by ELISA for the presence of 24 plant viruses. Results of serological test were confimed by electron maicroscopical investigations and in some cases by biological tests.

According to the results, approached from serology, electron microscopy and biological invesugations, eight plant viruses were detected from the 24 tested samples. The most frequent viruses were potato $Y$ poryvinus and plum pox potyvinus. Besides the above mentioned two viruses, the presence of potato $S$ carlavirus, alfalfa mosaic virus, the following strains of barley yellow dwarf luteovinus: PAV , RPV , RMV and MAV, tobacco etch potyvinus, tobacco mosaic tobamovirus and maize dwarf mosaic potyvirus were also detected.

As far as the authors are aware, and according to the available literature, among the eight detected viruses, seven /alfalfa mosaic virus, barley yeliow dwarf mosaic potyvinus, phum pox potyvirus, potato S carlavirus, potato $\mathrm{Y}$ potyvinus and tobacco etch potyvirus / are new records for natural waters.

Plant Health and Soil Conservation Station of County Fejér,

2481 Velence, Ország street 230.

HUNGARY
KNOW THE ENEMY: WHAT HAPPENS WHEN FARMERS OISCOVER THE REAL HEROES (AND MORE ABOUT THE MONSTERS) IN THEIR FIELDS?

\section{3 Meir}

Most farmer training programmes in integrated pest management (IPM) aim to transfer technology from the trainer's head to the farmer's field. Zamorano's Hillsides IPM Programme, based in Honduras, is different. We aim to share our knowledge of the basic concepts on which IPM is built with farmers, and thus to complement their of ten considerable knowledge of the farmers, anvironment. This paper examines the impact of the participatory training in insect management which Zamorano offers to Central
makes recommendations for future work.

Our three day courses teach farmers five basic concepts which they generally know little or nothing about: insect reproduction, predation, parasitism, entomopathogens and the manipulation of natural enemies. Although some of the details are often forgotten, most of the farmers we train still remember the basic principles of all five of these concepts several years after receiving the training. The knowledge they gain opens up a whole new world of natural nearly always has some impact on their crop protection practices.

Almost all the farmers we train stop killing wasps and ants (two of the most important groups of predators) and many actively protect them and/or create habitats for them in their fields. Many farmers reduce their pesticide use in some way: some may stop spraying an insect because they now realise it is not a pest, some have fewer pest problems due to attractici: and/or conservation of natural enemies, and others prefer to use botanical pesticides. Some farmers construct traps and others simply search out and kill the insect pest at the egg stage now that they know what these turn into. Mixed cropping systems or crop rotations in order to reduce pest damage are less popular, but some farmers have tried this option. A few farmers stop using pesticides altogether, convinced that the damage to themselves, the environment and their new-found friends is too great a cost.

The results of our training demonstrate clearly that smallholder farmers are capable of assimilating new concepts and applying them to their own situations to produce original IPM strategies and improve productivity. However, we stil have a lot of ground to cover. We find that farmers often forget details which can be important and that the considerable enthusiasm the course generates is not always put to full use. We would also like to see less use of botanicals and more integrated cropping systems as well as more farmers passing on more of their knowledge. We are working to improve farmers' ability to apply the concepts we teach to their own pest problems. We are collaborating with NGOs who encourage farmers to use soil conservation techniques and green manures, and to grow a greater variety of crops. We are trying to devolve some of responsibility for using their new ideas to the farming communities we train, whilst providing them with support and access to more information. Our hope that with these measures, the future will see increasing numbers of IPM heroes emerging from farmers' fields.

Hillsides IPM Programme, Department of Crop Protection, Zamorano, (Panamerican School of Agriculture), PO Box 93, Tegucigalpa, Honduras, Central America, and Imperial College Centre for Environmental Technology, 48 Prince's Gardens, South Kensington, London, SW7 2PE, United Kingdom.

MONITORING TECHNIQUES POR DETECTING RESISTANCE IN HELICOVERPA ARMIGERA (Hubner).

\section{S. GOUTHAMAN and A.REGUPATHY}

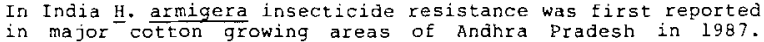
in major cotton growing areas of Andhra Pradesh in 1987 . Subsequently the resistance to insectcides like cypermethrin,
fenvalerate, endosulfan and quinalphos has been reported from fenvalerate, endosulfan and quinalphos has been reported from Punjab, Haryana, Maharashtra and Tamil Nadu states. The effective resistance management depends on the successful
monitoring programme. The Natural fesources Institute (NRI), monitoring programme. The Natural Resources Institute (NRI), UK in collaboration with the International Crops Research
Institute for the Semi - Arid Tropics (ICRISAT), Hyderabad, Institute for the Semi - Arid Tropics (ICRISAT), Hyderabad,
India and the Indian Council of Agricultural Research (ICAR), New Delhi, India initiated a collaborative programme involving New Delhi, India initiated a collaborative programme involving seven centres. In this monitoring programme the discriminating doses for fenvalerate $(0.2 \mathrm{ug})$, cypermethrin $(0.1 \mathrm{ug}$ ) and endosulfan (10 ug) have been fixed using the susceptible (third instar) cultures available at NRI, UK and Australia for topical application method. The high tech nature of topical application method defers many field level workers and marginal farmers with low literacy rate for adoption for decision making on the choice of insecticide to be applied.
Hence a choice of detection methods should be made available Hence a choice of detection methods should be made available determined by the level of technical support available to any monitoring programme. As on date no susceptible line of $\underline{H}$. armigera is maintained or available in India. Indirect metho $\bar{d}$ bioassay viz., vial, boquet, spray tower and larval dip for commoniy used insecticides, endosulfan, guinalphos, chlorpyriphos, fenvalerate and cypermethrin. This involves :

1. Working out $\log$-dose-probit-mortality lines for the five insecticides by five bioassay methods including topical method with third instar TNAU culture.

2. Multiplying discriminating doses (DD) available for susceptible cultures of NRI and Australia by topical application by the factor of ratio of $\mathrm{LD}_{99}$ of topical and other methods.

3. Validation of extrapolated DDs by testing on different $\underline{H}$. armigera populations.

The studies indicated that DDs arrived for Eive different methods inflicted mortality with standard error (SE) varying from 3.9 to 7.1 when batches of 50 insects were used. The variation could be reduced when more number of insects per test are used. 
REMOTE SENSING MONITORING OF CEREAL SYST NEMATODE (HETERODERA AVENAE WALLENWEBER 1924) IN AGROECOSYSTEMS OF RUSSLA NORTH-WEST

\section{M.I.Saulich., F.A.Karlik, L.A.Guskova, T.G.Terentyeva} populations and in an effort to improvement of Plant Protection Service information security investigations were conducted with the use of air-visual observations and a large-scale air-photography on normal films. Diagnostic signs are focuses of damages in oat and barley crops at a period from begining of its booting to full maturation stage

The basic information are collected during observations from helicopters in routes. On the basis of a complex of diagnostic signs, highly reliable estimations of causative agents of the damages are made. Then using an expert scale of $0-5$, the degree to which nematodes are spread in grain crops by area damaged is estimated. Thus a general description of crop infestation is gave and first determined a location of nematode focuses.

Within specific fields an exact mapping of focuses are fulfiled from large-scale airphotographs (1:2000). To ease photographs interpretation a computer program, permiting to identify images of nematode focuses by its shape, sizes, color variations, and peculiarities of mutual placing.

Over three years using remote sensing diagnostics grain crops were observated from helicopters in Leningrad region. A good agreement between results of air-visual detection of focuses and its real terrestrial placing was proved.

In perspective an application of motor deltaplanes and light aircrafts "AVIATICA$890^{n}$ will permit sharply to rise an efficiency of aviation machinery emploument for purposes of remote sensing determination of nematode focuses placing in grain crops.

All-Russian Institute of Plant Prolection, ch. Podbelscogo 3 ,

St.Peterturg, Pushikin, 189620, RUSSIA
For scientific substantiation of monitoring tecnology of cereal cyst nematode

CONTROL OF DISEASES IN WINTER WHEAT HITH APPROPRIATE DOSE RATES AND WEATHER-BASED DECISION SUPPORT SYSTEMS

\section{H.T.A.M. Schepera, E. Bouma}

In three crials over 1993/94 full and appropriate dose fung1c1de progranmes were evaluated on winter wheat. Two cva, Ritmo and Florids with disesse

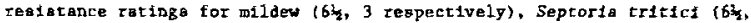
62) and brosin rust $(6, \delta)$ were ereated with the fungteide programes.

In 1993 cv Florida was treated at GS 65 with $1,251 /$ ha(1002) Sportakbelta and 1,0 1/ha(100z) Matador. Also splitted applications from GS 31 to GS 75 were carried out with $5 \times 20 x, 2 \times 50 x, 5 \times 10 z$ and $2 \times 25 x$. In the untreated plota disease developed to low-fnternediate levela: milew (3x), s. trittel (7t) and brom ruse (132). All fungiclde programes reduced $S$. erfitci and brown rust compared to the untreated. Amang the programpes were no differeaces. Programes increased pield with $7-127$ over the untreated $(9780 \mathrm{~kg} / \mathrm{hr})$.

In 1994 co Florids was treated with $1 \times 1000,2 \times 25 \%$ and $1 \times 507$ Sportik Delca. Also programee were carried out in which $0,251 /$ ha $(-252)$ Corbel or 0,12 2/ha $(-257)$ Bayfidan was applied at GS 31 followed by a treatment at GS 39 43 with 252 Sportak Delta or $25 z$ Matador or $0,21 /$ ha $(=25 z)$ Alto or 0,37 $1 /$ ha $(-25 x)$ Opus Tesm. Also four decision support systems were tested namely: Epipre, Integrated Disease RIsk, Pro_Plant and PC Plant Protection. Moderate levels of $S$. tritfet (7z) and brown rust (6z) developed after GS 65 in the untreated plote. Brown tust was reduced by all programes. There was a tendency that programmes with a marginal effect on $S$. tritici resulted in lower ylelds. Programes increased yleld with $4-10$ over the untreated $(7054 \mathrm{~kg} / \mathrm{ha}$ ). Half-rate progeamines with $2 \times 25 \%$ Sportak Deita, $2 \times 25 \pi$ Opus Team (IDR) and $25 \mathrm{zay} f 1 \mathrm{dan}+25 \mathrm{x}$ Opus Team resulted in effective disease control and the blgbeat argins over costs.

In 1994 cV Ritmo was treated at GS 30 with 50 or 2002 Corbel and at GS 57 59 sith 50 or 1002 Matador. Also splitted applications at GS 30 and GS 37 39 were carried out witb $25 z$ Corbei $+25 *$ Alto, 253 Corbel+ $25 z$ Sportak DeIta, 25z Corbe1+ 25x Matador aad 25x Bayfidan+ 25x Opus Team. Alao the above mentioned systems were tested. At GS 30 the incidence of mildew was 437 . At as 73-77 the severity in the untreated plots was as follows; mildew (12z), s. tritfci $(5,5 \pi)$ and brown rust $(17 \pi)$. Brown rust and $s$. trfticl were reduced by all programes. Programmes increased field with $15-192$ over the untreated $(8878 \mathrm{~kg} / \mathrm{ha})$. Programes which were less effective on m11dew resulted tn lower gields. All half-rate programmes and decision support systems, except Epipre ( $2 \times 1002$ Opus Team at CS 57-59), resulted in higher margins over costs than the $2 x 100 z$ programme (1x Corbel + 1x Matador).

Research Station for Arable Farming and Field Production of Vegetables p.0. Box 430, 8200 AK Lelystad, The Netherlands, Telephone (0)3200-9111]

DISEASE FORECASTING FOR MANAGEMENT OF LEAF SPOT DISEASES ON A SUSCEPTIBLE AND A PARTIALLY-RESISTANT GROUNDNUT CULTIVAR

\section{F. M. Shokes and D. W. Gorbet} Leaf spot diseases of groundnut can be a major cause of pod yield
loss. Serious loss of photosynthetic area can occur due to loss. Serious loss of photosynthetic area can occur due to defoliation if these diseases are not controlled. Early leaf spot, caused by Cercospora arachidicola Hori (CA), and late leaf spot, caused by Cercosporidium personatum Berk. \& Curt.jén dore currently managed by application of fungicides during the growing season in the USA. In the southeastern growing area fungicides are often applied beginning 30-40 days after planting until 2-weeks before harvest for a total of 7-8 applications. In the Virginia-Carolina area where $\mathrm{CA}$ predominates, leaf spot forecasting models have been in use for the past decade. In the southeast $C P$ was predominant until the past two years and models have only recently been developed to manage both early and late leaf spot in this area. About 8 years ago the cultivar southern Runner, which has partial resistance to $C P$, was released. Testing of late leaf spot models began in north Florida five years ago. One model that has been tested, AU-PNUTS, was developed at Auburn University (Plant Disease 77:722-725). This model uses only a rain gauge and spray determinations are based on the number of rain events that have occurred since the last fungicide application. A second model developed at the University of Georgia (Phytopathology 80:989) uses a computercontrolled weather station (ENVIROCASTER, Neogen Corp., E. lansing. MI). This model uses temperature and hours of leaf wetness to predict when infection periods have occurred and protection is needed. Both models have bees successful in saving spray applications in a given year, although they predict differently at times, when sprays are needed. From 1-3 sprays have been saved when compared to spray applications based on a 10-14-day calendar schedule. The models typically work best for leaf spot control when a systemic fungicide, such as tebuconazole, is used in the program. The predictive models are adequate when the protectant fungicide chlorothalonil is used. However, yielas in a given year may be increased as much as 1128 $\mathrm{kg} / \mathrm{ha}$ with Southern Runner and $1793 \mathrm{~kg} / \mathrm{ha}$ with Florunner when tebuconazole is used. The eradicant activity of tebuconazole is beneficial in decreasing leaf spot severity and there is added benefit from soilborne disease control. Both models work weI when the partially resistant cultivar is used. Disease levels are generaliy lower, yields are similar and yield differences between the treated and untreated sround and yield differences partially resistant cultivar.

North Florida Research and Education Center, University of Florida, Route 3 Box 4370 , Quincy, Florida 32351 .

\section{PREDICTION OF RICE BLAST DISEASE BY USING INDICATOR PLANTS}

\section{A. Surin, P. Arunyanart, W. Rodjanahusdin and P.S. Teng}

Rice blast disease caused by Pyricularia grisea is the major disease of rice in Thailand. Many methods are used to overcome yield loss caused by this disease. We tried to use indicator plant to predict disease occurrence in order to control blast disease.

RD 23 seedlings were used as the indicator plant to monitor rice blast disease in the field. The experiment was conducted in an irrigated area of the farmers' field at Pathumtani province during the dry season (Nov, '93 - Febr. '94). Three bulks of size $50 \times 50 \mathrm{~cm}$ containing RD 23 seedlings of 7 - 10 days old were laid in the field where RD 23 variety was planted. The seedling bulks were changed weekly. In the experimental field, a hygrothermograph and a simple spore trap were set up in order to collect climatic data and count the number of $P$. grisea spores.

Blast disease on indicator plants and on rice plants in the field were investigated weekly. The symptom was first observed on the indicators one week after laying the bulks in the field with $5 \%$ disease severity (\% infected leaf area). A week later, leaf blast occurred on $\mathrm{RD} 23$ in the field at about 5\% disease severity. It might due to temperature, relative humidity, number of spores and disease severity on indicator plant throughout the crop were recorded and

We can conclude that temperature influences disease severity and incidence of blast disease. Disease severity on indicated plant was correlated to disease incidence in the field. $\left(r^{2}=0.84^{* *}\right)$. Because disease incidence in the field occurred 2 weeks after disease appearance on indicator plant, indicator plants can be used as an index to predict the occurrence of blast disease in the crop.

Rice Pathology Research Group. Plant Pathology and Microbiology Division, Department of Agriculture, Bangkok 10900, Thailand statistically analysis was performed. 
DETECTION OF SYSTEMIC INFECTIONS OF DYER'S WOAD (ISATIS TINCTORLA) BY THE BIOCONTROL RUST, PUCCINIA THLASPEOS, USING THE POLYMERASE CHAIN REACTION

\section{S.V Thomson, B.R. Kropp, and K.M. Flint}

Dyer's woad (Isatis tinctoria) is a member of the cruciferae family that was introduced into the U.S. from Europe and historically used as a dye plant. It has become naturalized with few enemies and poses a threat to rangeland in the western U.S. Discovery of a systemic rust, tentatively identified as Puccinia thlaspeos, offers promise as a biological control without environmental disruption. The rust was discovered in Idaho in 1978 on woad plants, but other reported hosts growing in the same area were not infected. The natural ranges of Puccinia thlespeos and dyer's woad overlap in Europe, but the rust has not been reported as a pathogen of dyer's woad outside of Utah and Idaho. Rust specific polymerase chain reaction (PCR) primers enabled the detection of the fungus in both symptomatic and asymptomatic but infected plants. The PCR test confirmed the presence of the fungus in the rosette stage of plants six to nine months prior to symptom development at bolting. The PCR test has been used to detect the fungus in asymptomatic parts of diseased plants and in some apparently healthy plants. It is also being used to determine the success of inoculation attempts and the location of infections in plants.

Dept. of Biology, Utah State Univ., Logan, UT., USA, 84322-5305
PORECASTING POR STRATEGIC CONTROL OF THE APRICAN ARHYYWOR - TTRE USE OF SATELIITE IMAGERY TO LOCATR RAINSTORYS ASSOCIATED WITH THE FORYATION OF OUTBREAKS.

\section{Y R Tucker} African armyworm, spodoptera exempta (Walker) is a
serious pest of cereals in Africa. The moths are known to migrate long distances and evidence from radar migrate long distances and evidence from radar that high density armyworm outbreaks are most likely to occur when flying moths are concentrated by rainstorms.

The Meteosat satelifte provides a means of monitoring rainstorms at half-hourly intervals throughout the day and night. The presence of night-time rainstorms, especially following dry periods, has been used for several years to help identify areas where armyworm moths
may be concentrated. Empirically, Meteosat data are considered to help strategic control by reducing the area needed to be surveyed to locate and control initial outbreaks. However, no quantitative assessment of the usefulness of the method has been published.

The present study seeks to remady this amission with an aralysis of African armyworm outbreaks and the location of rainstorms in eastern Africa in October - November 1992. It is intended to update these analyses with data for 1993 and 1994 .

The 1992 data showed a significant association between armyworm outbreaks and rainstorms although trap data are needed to zeduce the number of false positives

(rainstorms not followed by outbreaks). Individual

arnyworm outbreaks were found to be particularly

associated with the edge of rainstorms, as would be

expected if the concentrating mechanism was the low-level

outdraught from such storms.

Natural Resources Institute

Central Avenue

Chatham Maritime

Chatham

Kent

ME4 $4 \mathrm{~TB}$

PESTICIDE USE, ENVIRONMENTAL BURDEN AND THE ECONOMIC PERFORMANCE OF ARABLE FARMS IN THE NETHERLANDS

H. Janssen, A.J. Oskam and R.A.N. Viiftigschild

The Dutch government aims at reducing pesticide input in agriculture, both in volume and in environmental effect. Monitoring studies, based on the LEI-DLO Farm Accountancy Data Network, show a reduction of volume over the past few years, but aiso a considerable dispersion in use between farms. This paper investigates whether arable farmers show a consistent behaviour of pesticide application in the course of the time. Moreover the relation between environmental burden - measured by means of an environmental yardstick developed by CLM - and pesticide use per hectare has been determined. A model that explains the relation between the level of pesticide application, intensity and quality production and the economic performance of arable tarms has been developed.

Department of Agricultural Economics and Policy, Wageningen Agricultural University, P.O. Box 8130, NL-6700 EW Wageningen, The Netherlands 
MONITORING PECULIARITIES OF PLANT DISEASES CONNECTED WITH PATHOGENS' COMPLEX.

Vlasov D. Yu., N1kolaev P.M.

Many diseases of apricultural plants are caused by complex of pathogenic fungi. The most important conditions determining the development of such complex are the state of host-plants. agroecological factors and infectious potential. As an example of mixed infections, root rot of wheat in Rostov region and clover leaf spots in S.-Petersburg region have been investigated. 19 species of fungi from different ecological and taxonomic groups were found in diseased roots of wheat. Among them there were obligate and elective parasites, mycorrhizal fungi and saprophytes. This complex was sufficiently stable. Scme specles of fungi (usually 3-5) were predominant for all perfod of vegetation of wheat. Hence, succession of attendant species took place on the different stages of development of the host-plants. The application of some soil herbicides broke the balance between species of fungi and root rot increased. The infection and colonization of above ground plant parts of clover by fungi was very similar with the same process in the roots of wheat. It was shown that the display of clover leaf spots was connected with suffictently stable complex of fung1. O1seased plants belonging to 4 species of Irifolium had specific composition of predominant and attendant species of fungi. As a result, stable and specific cormunities of fungt arise in the conditions of arrocenosis and they have an ability to determine the state of the cenosis in the whole. The conception may be valuable for development of the biological control strategy.

Saint-Petersburg State University, Department of Botany. 199034, S.-Petersburg, Universitetskaya emb. 7/9, Russia.

1283

FIELD MONITORING OF PHYTOSANITAR CONDITIONS:
CENOCONSORTIAL APPROACH

\section{A.F.Zubkov}

The aim of an investigator in the field monitoring is to determine and to estimate interrelations in the biocenosis, but very often this problem is almost insoluble. The method of quantitative estimation of biocenotic interactions - harmfulness phytophages end the role of entomophages - hasn't been elaborated well enough because the whole population is considered as the elementary unit of biocenosis. This misunderstanding leads to poor results from researehes of every population, one at a time, but not of interspecific relations. The author has developed the method of cenoconsortial analysis of biocenotic intenrelations as the most comprehensive characteristics in the field ecological monitoring, where under the specially organized observations are elementary biocenosis formations "cenoconsortiya", i.e. the whole complex of directly interacting organisms of all the throphic levels (Zubkov,C.-x.биология,N3,1989 end N3,1992). To deternine the relations between organisms of different species within a cenoconsortium the original algorithm has been elaborated which with the help of some contrivances pennits statistical analysis of observed data collected from marked cenoconsortiagrounds (Zubkov et al, Зоолог.журнал,61,N2, 1982; Энтомол.обозрение, 66, N4, 1987; Вестник c.-х.бнологии, N12, 1989). These methods are constituent parts of the methodology of the new branch "Agrobiocenological phythosanitary diagnostics" (Zubkov, 1993), based on the conception of cenoconsortial structure of agrobiogeocenosis (stable agroecosystem such as bjogeocenosis), which is formed upon arable lands within balanced field crop rotation (Zubkov,Экология, 2, 1992).

All-Russian institute for plant protection (VIZR)

189620, h.Podbeiskogo, 3, St.-Petersburg, Pushkin, Russia

\section{MONITORING OF SUNFLOWER DISEASES IN RUSSIA}

\section{V.I.Yakutkin}

Protection of sunflower crops against diseases is one of the major research problems in Russia. During the recent 20 years the most common and harmful diseases of sunflower have been white and grey rots and downy mildew. More and more damage is now being caused by Phomopsis. At present a scientific strategy has been developed in Russia of sunflower control on the basis of crop health monitoring. It involves methods and procedures of diseases recording and analysis of the resuits using multi-year, long-term and short-term predictions.

It has been found that the spatial occurence of sunflower diseases in agrocenoses obeys the law negative binomial distribution. The mean $(\bar{X})$ and the variance $\left(S^{2}\right)$ for crop infestation can be described by formula $S^{2}=2.493 \bar{X}^{0.634}$ which permits better diseases recordings with predetermined accuracy. The biological damage threshold is $5 \%$ of totally rots-affected heads or downy mildew or Phomopsis-affected stems. Multi-year prediction, on the basis statistical classification of tenitory of Russia with respect to disease incidence, permits disease dynamics to be predicted 5 years in advance for large areas of the country. Long-term prediction defines more exactly the seasonal dynamics of diseases on a territory, region or district scale, using linear approximation. Short-term prediction serves to model the disease dynamics for near furure in a separate administrative district, farm or field, using hydrothermal ratio (GTK) or polynomial.

Thus, the disease monitoring, involving continuous surveying of fields for diseases and step-by-step analysis of the results using the system of predictions, permits better protection of sunflower at different levels such as territory, region, district, farm an field.

189620 St-Petersburg, Pushkin-6, Podbelsky 3, Russia

All-Russian Plant Protection Institute (VIZR)

\section{4}

\section{THE EFFECTS OF NEEM EXTRACTS ON THE NUTRITION INDICES AND DEVELOPMENT OF HELOTHIS ARIIGERA (LEPIDOPTERA: NOCTUIDAE) LARVAE}

\section{Ahmad and $M$. Kisdarjono}

The extracts from the neem tree, Azadiracht Indice (Meliaceas) wore administered olther in artificial dief or by injection to the final (fffth) instar Holiothis amigera larvae.

In general, the relative consumption and growth rates, as well as the efficiency of food use, were significantly lower on all treatment. Interestingly, the value of E.C.I. (efiiciency of conversion of ingested food to blomass) and E.C.D. (efficiency of conversion of digested food to biomass) were significantly higher on larvae fod on diet with 75 PPM neem extract than that of the larvae fed on diet with 50 PPH neem extract. Compared with the controls in both artificial diet and injection, the development time was significantly longer for all treatments. At all treatments, the noem extracts significantly disupted the larval-pupal development On higher dosages, substantlal development anomalios results upon pupation.

Inter University Center for Lfe Sciences and Department of Blology, Institut Teknologi Bandung, Jalan Ganesh: 10 Bandung 40132, INDONESIA 
EFFICACY OF NEEM (Azadircha indica) AGAINST CODLING MOTH, Cydia Pomonella (Linnaeus) (Lepidoptera: Tortricidae)

\section{AMIN A. AL HEMYARI AND ALINTAZEE}

Studies were conduted at Oregon State University, USA Under laboratory conditions to determine the effect of Neem Seed Exterct (NSE) against Codling moth, Cydia Pomonella
(Linnaeus). The extract was diluted by distilled water to reach $0,01,0.5$ and 1 \% Concentrations. NSE Provided one hundred percent larval mortality at a rate of $1 \%$ within $48 \mathrm{hrs}$ post-treatment. At low Conc. 0.5 rates, low mortality was noticed within 48 hrs but nearly one hundred percent mortality was seen 48 days after treatment. Popation did not occur with $0.5 \%$ Conc. ,however at 0.1 and $0.01 \%$ Larvae were pupated but non of them were emerged to normal aduct. At lower rate, NSE caused extreme prolongation, antifeedant re-
sponse, repellency and growth reglatory activity.

P.O. BOX 13020 Sana'a, Yemen
SOME ASPECTS OF THE BIOLOGY AND CONTROL OF THE RICE MOTH CORCYRA CEPHALONICA ON SOME PULSES IN GHANA

Allotey, J. and Azalekor, W.

The life history and control of the rice moth Corcyca cephalonica were studied on groundnut, bambara groundnut and cowpea under amblent laboratory conditions. The mean developmental periods of c. cephalonica ranged from $33.28 \pm 0,23$ to $45.25 \pm 1.84$ days on whole, broken and powdered forms of the food media. Egs hatchability was observed to be 837 , longevities of the adults were found to be from $1.5 \pm 0.5$ to $16.45 \pm 1.17$ days for males and from 1.5 to $11.93 \pm 1.27$ days for females. Sex-ratios of emerged $\underline{C}$. cephalonica on the food media ranged from $1: 1$ to $1: 1.7$ ( $;$; ) while fecundities of $\mathrm{C}$. cephalonica on the food media ranged from $128 \pm$ 4.94 to $157 \pm 7.87$.

Citrus sinensis was the wost efficacious of the three plant materials (ie.) C. sinensis, Chromolaena, Eichormia) used as seed protectants against c. cephalonica on groundnut. At $2.5 \mathrm{~g}$ C. sinensis per $40 \mathrm{~g}$ groundnut only $40 \%$ of the introduced $C$. cephalonica larvae emerged as aduits as compared to $81.11 \%$ emergence in the control jars.

DR. JOSEPH ALLOTEY DEPARTMENT OF ZOOLOGY UNIVERSITY OF GHANA P.0. BOX 67 LEGON, GHANA.

1288

POTENTIALITY OF SOME HIGHER PLANT PRODUCTS AS BOTANICAL FUNGITOXICANTS

N.K.DUBEY, A.K. MISHRA, N.KISHORE During Screening of different parts of 80 angiospermic
species, the leaves of Cymbopogon citratus, Ocimum canum, species, the leaves of Cymbopogon citratus, Ocimum canum,
citrus medica, prunus persica and Trichyspermum ammi Citrus medica, Prunus persica and $\frac{\text { Trichyspermum }}{\text { exhibited ammi }}$ exhibited absolute fungitoxicity against Aspergillus flavus:
the storaye fungus of stored food commodities while the the storaye fungus of stored food commodities while the
leaves of Chenopodium ambrosioides, Lippia alba and rhizomes leaves of Chenopodium ambrosioides, Lippia alba and rhizomes of Zingiber Cassumunar showed strong antifungal activity aphanidermatum causing damping-off तiseases. The fungitoxic Factors of the effective plants were isolated and standardized by different phytochemical methods, The fungitoxic properties viz lethal dose, Nature of toxicity, spectrum, shelf-life, thermostability of the isolated products were determined. These have shown higher efficacy than some prevalent synthetic fungicides. The essential oils of C. citratus and 0 . canum were tested for their fumigant activity for control of hiodeterioration of some fruits and stored food commodities and these have shown their efficacy as post harvest fumigants by protecting the treated commodities for a longer duration. The animaltoxic investigations with the Cymbopoyon and citrus oils vere carried out and the oils were found to be nonanimal toxic in nature. The oil of c. ambrosioides exhibited its fruitfulness in significant control of damping-off disease of tomato when used in seed treatment. The products thus constitute a novel source of plant disease controlling ayents and may be exploited as biodegradable natural pesticides.

Department of Botany, Banaras Hindu University, Varanasi-221 005, India.
Jepartient of Plant Frotection, vasir's collebe of isriculture Univeraity of Aden, nhormaksar D.j.sox 6172 aden, heyuolic of Yemen 

EFFECT OF TRYPSIN INHIBITORS FROM BUCKWHEAT SEEDS ON GUT
PROTEASE ACTIVITIES OF KERATINOLYTIC, PHYTOPHAGOUS AND STORED-PRODUCT INSECT LARVAE

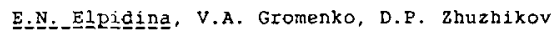

In recent years a role of proteinase inhibitors in plant protection against pests has been intensively investigated. Their toxic effect on insects was established in feeding trials on artificial diets (1) and in genetic engineering experiments which resulted in enchanced resistance of transgenic plants to insects $(2,3)$. Insect gut proteases are the most likely targets of protease inhibitors, and there have been several examples of the latter being active in in vitro assays against gut proteases $(4,5)$, but the iaformation about their interaction is 1 imited, sometimes contradictory and does not allow one to get a systemic view on this problem.

This abstract presents the results of a comparative study of gut proteases from the larvae of three groups of insects, having different food specialization: keratinolytic (Ephestia cautella Wlk.) phytophagous (Pieris brassicae I.) and stored-product insects (Tenebrio molitor L.). The effect of $\mathrm{pH}$ on partially purified digestive protease activities in the three species was investigated, as well as the action of specific inhibitors and activators of serine cysteine and metalloproteinases on the major enzyme activities. proteolytic activities towards variety of syathetic substrates were also studied. The variety of syathetic substrates were also studied. The inhibitory effects of pure trypsin inhibitors isolated from buckwheat seeds on

thoroughly examined. The data obtained will belp in the identification of the most "useful" candidates among plant inhibitors of proteases for transfer to ather plant species via genetic engineering to
produce insect-resistant cultivars of different plants. References

1. Steffens R., Fox F.R., Kassel B., 1978. J. Agric. Food Chem, $26: 170-174$

2. Hilder V.A., Gatehouse A.M.R., Boulter D., 4987, Nature $330: 160-163$

3. Johnson R., Narvaez J., An G., kyan C.A.. 1989. Proc. Natl Acad. Sci. USA $86: 9871-9875$

4. Birk Y., Gertler A., Khalef S., 1963. Biochim, Biophys. Acta $67: 326-328$ 5. Christeller J.T. Markwick N.P. Burgess P.J., 1994. Arch,
Insect Biochem. Physiol. 25:159-173

A.N, Belozersky Institute of Physico-Chemical Biology, Moscow State University, Moscow 119899 , Rusia

1291

USE OF AN EXTRACT OF GLIRICIDIA SEPIUM, A COMMON LEGUMINOUS TREE IN CENTRAL AMERICA, IO CONTROL WHIIE FLIES IN GREEN BEANS

LGrossman, L.Bradshaw, K.Golden, and G.Valladares

White flies (Bemesia tabaci) have been threatening a variety of vegetable crops in Costa Rica for the last several years through transmission of viral diseases, in particular the Geminivirus. This study attempted to find solutions to the problem of white fly infestation of green bean crops using a participatory approach in the Central Valley county of Atenas, Costa Rica. With the input of local producers, it was decided to test an extract from the locally available tree of Gliticidit sepium. Popular knowledge commonly holds G. sepium has insecticidal properties. Extract was prepared by soaking leaves for $24-48$ hours in water, then filtering the liquid. When experimental plots reached a threshold of 10 or more adults per 50 leaves the extract was applied. Experimental plots were on farm and contrasted to adjacent control plots which received no treatment against white fly. We found that number of flies present decreased significantly after application of the extract and that the appearance of viral symptoms was delayed in the experimental plots. However, in the experimental plots bean plants did show symptoms of toxicity. At this time, it is not clear whether the toxicity was due to the concentration of the G.sepium, or to the adherent which was used with the extract. It can be concluded that G sepikm is an effective control agent against $B$, tabaci , but the appropriate dosage and adherent needs to be determined. Also, to reduce problems of resistance, the G. sepium extract should not be used as the only control, but rather in conjunction with other management options a part of an integrated technology package

Centro de Estudios sobre Desarrollo Sostenible, School for Field Studies, Apdo. 1504013, Atenas, Alajuela, Costa Rica
GLUCOSINOLATES AND THEIR BREAKDOWN PRODUCTS AS IN VITRO GROWTH INHIBITORS OF PHYTOPATHOGENIC FUNGI

\section{P. Kachlicki, M. Jędryczka, E. Lewartowska, L. Frencel}

Glucosinolates are characteristic compounds of cruciferous plants. Their breakdown products, obtained in reaction catalyzed by endogenous enzyme - myrosinase, are known to have toxic properties. The active products are in most cases isothiocyanates structurally related to original glucosinolates. Effects of these compounds on growth of 5 Fusarium oxysporum special forms (callistephi, dianthi, lini, lupini and pisi) and an oilseed rape pathogen Alternaria brassicicola were studied. In some combinations strong inhibition of fungi growth on solid Czapek-Dox medium was observed. The most effective were hydrolysis products of glucoalyssin (5-methylsulphinyl pentyl glucosinolate) and mixture of rape (Brassica napus) glucosinolates. Native glucosinolates influenced fungal growth to much lower extent.

Institute of Plant Genetics, Strzeszyńska 34, 60-479 Poznań, Poland

1292

ISOLATION OF INHIBITORY SUBSTANCE IN CITRUS STEM BUTTON AGAINST DIAPORTHE CITRI

\section{M.HOSODA,Y.HOMMA and D.HOSOKAWA}

Japan has much rain during the period of fruit development ( June to September). The diseases of citus fruit are caused by the rainbome pathogens Diaporthe citi , Xanthomonas campestris py cirri and so on. These pathogens infect fruits or young leaves and may cause considerable injury to truit rinds, thereby resulting in serious economic losses.

$D$. ciri has penetrated into the pedicel of the fruits, it can remain latent until near the end of storage. Citrus should have a preinhibitin within the stem-end of the fruits, so $D$. citri cannot penetrate the preinhibitin barrier.

This study indicates that one of inhibitory substances against $D$. citri is isolated from citrus stem button.

Stem butons ( 1000 samples) were extracted with methanol. The methanol fraction was evaporated. The ininibitory fraction from the methanol extract was purified by chromatography ( ethyl acetate - water ). The (active) fraction was melt in the ethyl acetate phase. The active fraction was purified by thin layer chromatography (silica gel), developed by $n$-hexane : ethyl acetate $=1: 1$ ( the active fraction $R f 0.7$ ), then toluene (the active fraction Rf 0.04 ). The active fraction was detected by the blue strong fluorescence through UV $366 \mathrm{~nm}$. Next the active fraction was purified by high perfomance liquid chromatography ( SENSYU SCIENTIFIC CO.,LTD HPLC SYSTEM , column : ODS-H $\mathrm{UV}: 365 \mathrm{~nm}$ flow $: 1.0 \mathrm{~mm} / \mathrm{min}$ ). The active fraction was the strong peak and retention time about $7.5 \mathrm{~min}$.

The substance punfied by above process was rested for antifungal activity ,being revealed that inhibitory activity against $D$. citri pycnospore germination was the concentration of 200 ppm. For the chemical characteristic of the substance, the mass spectrum indicated that the molecule weight was 368 . I will identify the chernical structure of the substance by various instrumental aralyses.

Phytopathology Laboratory, Tokyo University of Agriculture and Thedhology, Fuchu-sti , Tokyo, 183, Japan 
INSECTICIDAL PROTEINS WITH POTENTIAL FOR USE IN THE CONTROL OF COWPEA PESTS. I. Clavigralla tomentosicollis.

\section{Jackai.L.E. N. R. E. Shade and L. L. Murdock}

Different amounts of three compounds, wheat germ agglutinin (WGA), para. aminophenylalanine (PAPA) and a cysteine proteinase inhibitor (E-64), were incorporated into artificial cowpea seeds and fed to the brown cowpea coreid bug, Clavigralla tementosicollis, to determine the effects on some demographic statistics of this insect. $\mathrm{C}$. Iomentosicollis is an important pod and seed pest of cowpea, yigna unguiculata, in Africa. All three compounds caused high nymphal motalities at the higher doses tested $70 \%$ for 0.01 WGA: $100 \%$ for nymphal mortalities at the higher doses tested $70 \%$ tor 0.01 WGA: $100 \%$ tor
0.006 PAPA; $75 \%$ for 0.001 E-64 w/w). E-64 had a lower LD 50 than WEA and 0.006 PAPA; $75 \%$ tor 0.001 E. $64 \mathrm{w} / \mathrm{w}$ ). E-64 had a lower $\mathrm{LD}_{50}$ than $\mathrm{W}$
PAPA. At doses between 0.6 and $1 \%$ ( $w / w$ ) PAPA caused mortalities comparable to those of Viana vexillate (accession TVnu 72) in which this compound occurs naturally. 12 days atter infestation. Neither of the other compounds was comparable to TVnu 72 after the same duration of development. Presumably, comparable doses of E-64 would have resulted in higher mortality than what was recorded on WGA and PAPA. Also. insects took longer 10 develoo on E-64 although survivors were about the same size in all treatments. These results suggest that useful genes exist among edible plant sources that can be used in the genetic transformation of cowpea for pest these proteins, that would open the way for the transtormation of cowpea for improved resistance to pest damage as a component of IPM on this important crop. The genes can be obtained commercially. Since these proteins are from food plants. the risks to human health should be minimal, if any.

International Institute of Tropical Agriculture, Oyo Road. PMB 5320, Ibadan. Nigeria. WA resistance. If further testing contirms the insecticidal value and human safety of

\section{STUDIES ON THE CHEMICAL CONTROL OF PLANT VIRUS DISEASE BY A2}

Shan Jiang, Yu-Xia Hou, Xue-Liu Guo, Rong Zhang and Sheng-Hai Bai

The A-2 is made of soybean lecithin and alkane monosulfonate (E-30). It has been certified that it could suppress the infection of tobacco mosaic virus (TMV) to tobacco in glass-house. The field tests about A-2 were conducted by spraying it one time every is days on tomato, pepper and sugar beet. The total application of A-2 is three times from seedling stage. The test of the A-2 against Rhizomania of sugarbeet was performed in two fieids. The $20 \%$ granule DHT (2,4-dioxohexahydro-1,3,5-triazine) and 10\% NS-83 water form, a vincide for plant in China, were used as reference chemicals in these lest DHT was applied only once following the first spray application of A-2. The treatment way of NS-83 was same with A-2's. The results, as shown in the following tables, demonstrated that the A-2 possessed higher antiphytoviral activity broad-spectrumly. The ELISA analysis indicated that A-2 and the reference vinucides could decrease the accumulation of beet necrotic yellow vein virus (BNYVV) in the root of sugar beet (Beta valgris $\mathrm{L}$. suken 83 (2) at similar activity level. It also remarkably reduce the symptom of virus disease of tomato and sweet pepper caused by TMV and cucumber mosaic virus (CMV) more efficient than the NS-83 and DHT. These would support the A-2 as a new plant virucide apply to the chemical control of plant virus disease in practice.

\begin{tabular}{|c|c|c|c|c|c|c|}
\hline Disease & Treastment & $\begin{array}{l}\text { Concentration } \\
\text { (mig kg }{ }^{-1} \text { ) }\end{array}$ & $\begin{array}{c}\text { Mean } \\
\text { Checked } \\
\text { Piants }\end{array}$ & \multicolumn{2}{|c|}{$\begin{array}{l}\text { Mean Infection } \\
\text { Index }(\%)\end{array}$} & $\begin{array}{l}\text { Reduction of } \\
\text { Symmptom (\%) }\end{array}$ \\
\hline \multirow{3}{*}{$\begin{array}{l}\text { Pepper } \\
\text { mosaic } \\
\text { virus } \\
\end{array}$} & Control & 0.0 & 67.0 & \multicolumn{2}{|c|}{30.5} & 0.0 \\
\hline & $A-2$ & 500.0 & 543 & \multicolumn{2}{|c|}{$\frac{30.5}{5.9}$} & 80.7 \\
\hline & NS- 83 & 1000.0 & 53.7 & \multicolumn{2}{|c|}{141} & 53.8 \\
\hline \multirow{6}{*}{$\begin{array}{l}\text { Tomato } \\
\text { mosaic } \\
\text { virus }\end{array}$} & Control & 0.0 & 1110 & & 28.5 & 0.0 \\
\hline & \multirow{3}{*}{$A-2$} & 2500 & 76.3 & \multicolumn{2}{|r|}{9.9} & 653 \\
\hline & & 500.0 & 797 & \multicolumn{2}{|c|}{9.5} & 66.7 \\
\hline & & 1000.0 & 81.0 & \multirow{2}{*}{\multicolumn{2}{|c|}{$\begin{array}{r}4.6 \\
202\end{array}$}} & 83.9 \\
\hline & $\sqrt{\mathrm{NS}-83}$ & 1000.0 & 790 & & & 29.1 \\
\hline & DHT & $30 \mathrm{mg}$ & 78.3 & \multicolumn{2}{|c|}{17.4} & 38.9 \\
\hline & Treatment & $\begin{array}{c}\text { Concentration } \\
\left.\text { (mg kg } \text { kg }^{-1}\right)\end{array}$ & $\begin{array}{l}\text { Clecked } \\
\text { Plants }\end{array}$ & \multicolumn{2}{|c|}{$\begin{array}{c}\text { Mean Optical } \\
\text { Density in ELISA }\end{array}$} & $\begin{array}{l}\text { Mean Reduction } \\
\text { of BNrVW } \\
\text { Amount }(\%)\end{array}$ \\
\hline & Control & 0.0 & 30 & 0.103 & 0.190 & 0.0 \\
\hline \multirow{6}{*}{$\begin{array}{l}\text { Rhizomania } \\
\text { of sugar } \\
\text { beet }\end{array}$} & \multirow{3}{*}{$A-2$} & 2500 & 30 & 0.067 & 0.147 & 270 \\
\hline & & 500.0 & 30 & 0.050 & 0.133 & 37.5 \\
\hline & & 10000 & 30 & 0.060 & 0.093 & 47.8 \\
\hline & NS-83 & 10000 & 30 & 0.030 & 0.127 & 46.4 \\
\hline & \multirow[t]{2}{*}{ DHT } & $30.0 \mathrm{mg}$ & 30 & 0.127 & 0.137 & 9.9 \\
\hline & & $600 \mathrm{mg}$ & 30 & 0.033 & 0.113 & 50.2 \\
\hline
\end{tabular}

Depariment of Applied Agricultural Chemistry, Beijing Agriculurat Uniwersity, Beijing i06094, China

\section{5}

\section{THE HOST RESISTANCE TO PATHOGENS CAUSED BY A-2}

Shan Jiang, Xue-Liu Guo, Yu-Xia Hou and Shu-Yun Mu

The A-2 consists of soybean lecithin and alkane monosulfonate (E-30). Previous studies have shown that it possesses higher antiphytoviral activity to hinder the infection of plant virus. Otherwise, as shown in the following tables, the results of field trials demonstrated that A-2 reduced remarkably the infected plants by the Fusarium oxysporum Schlecht. $\mathrm{f}$. sp. vasinfectum Snyd. et Hans. which leads to the sweet pepper fusarium wit on sweet pepper, and the symptom caused by the Alternaria solami (Ellis et Martin) Jones et Grout. which brings about the tomato early blight on tomato beside its antiphytoviral activity. The NS-83 was used as a reference chemical in these tests because all of them are the membrane lipid substance. The chemicals sprayed three times from the seedling stage (once every 15 days). As a phytovinucide. NS-83 has been reported that its one of major antiphytovial mechanisms is to induce the high level resistance of host to plant virus and some fungus pathogens. Therefore, these results could suggest that A-2 could contribute to inducing the resistance of host plant as NS-83 performed, but more efficient than it did.

\begin{tabular}{|c|c|c|c|c|c|c|c|}
\hline Disease & Treatment & $\begin{array}{c}\text { Concentration } \\
\left(m g . \mathrm{kg}^{-1}\right)\end{array}$ & \multicolumn{2}{|c|}{$\begin{array}{c}\text { Mean Checked } \\
\text { plants }\end{array}$} & \multicolumn{2}{|c|}{$\begin{array}{l}\text { Mean Infection } \\
\text { Index }(\%)\end{array}$} & $\begin{array}{l}\text { Reduction of } \\
\text { Symptom (\%) }\end{array}$ \\
\hline \multirow{3}{*}{$\begin{array}{l}\text { Tomato } \\
\text { early } \\
\text { olight }\end{array}$} & Control & 0.0 & \multicolumn{2}{|l|}{1010} & & 23.5 & 0.0 \\
\hline & A-2 & 500.0 & \multicolumn{2}{|l|}{79.0} & & 9.5 & 59.6 \\
\hline & NS-83 & 1000.0 & \multicolumn{2}{|l|}{79.0} & & 18.7 & 20.6 \\
\hline Disease & Treatment & $\begin{array}{c}\text { Concentration } \\
\left.\text { (mg. } \mathrm{kg}^{-1}\right)\end{array}$ & $\begin{array}{l}\text { Mean } \\
\text { Checked } \\
\text { Plants }\end{array}$ & \multicolumn{2}{|c|}{$\begin{array}{c}\text { Mean } \\
\text { Infected } \\
\text { Plants }\end{array}$} & $\begin{array}{c}\text { Incidence } \\
(\%)\end{array}$ & $\begin{array}{l}\text { Reduction } \\
\text { of Infected } \\
\text { Plants (\%) }\end{array}$ \\
\hline \multirow{3}{*}{$\begin{array}{l}\text { Sweet pepper } \\
\text { fusarium } \\
\text { wilt }\end{array}$} & Control & 0.0 & 171.3 & \multicolumn{2}{|c|}{69.0} & 40.3 & 0.0 \\
\hline & NS.83 & 1000.0 & 173.3 & \multirow{2}{*}{\multicolumn{2}{|c|}{$\begin{array}{l}50.7 \\
28.7\end{array}$}} & 29.3 & 27.3 \\
\hline & $A-2$ & 5000 & 177.0 & & & $16 \overline{2}$ & 598 \\
\hline
\end{tabular}

On the other way, the studies showed that there were two novel proteins in the intercellular fluid of the leaves of tobacco (Nicotiana tabacum cv. "Samsun" NN) and sugar beet (Beia valgris L. tianyan 301 ) treated by soybean lecithin and $E-30$ other than in the control plants, which is called as the pathogenesis-related proteins (PRs). Seven PRs in tobacco and sugar beet were accompany with high level resistance of host plant at the same time after NS-83 treated. That would be in favour of the hypothesis that links the resistance induced with the PRs. Therefore, although the PRs induced by the E-30 and soybean lecithin was limited, this would still suggest that should contribute. partly or as a sign at least, to the host resistance as well as the NS-83 did The details about the factor of resistance of host plant by the A-2 treatment should be studied in future. As the soybean lecithin and E-30 are easy degraded and no problem of toxicity to the environment and animal, These would be one of useful ways for the plant disease chemical control.

\section{NEW APPROACH TO RESEARCH OF ENVIRONMENTALLY SAFE MICROBLAL INSECTICDEE PRODUCERS}

\section{T.G. Judina and L.I. Ljutikova}

The discovery of insects species susceptible to Bacillus thuringiensis (Bt) deltaendotoxims is difficult as it is based on empirical methods. We propose a scheme of purposeful search of target insects from different orders, which bave a susceptibility to Bt delta-endotoxins and also to protein inclusions of other microorganisms. Assessment and comparison of antibacterial activity levels of protein inclusions by patent have principle significance of proposed scheme. Inclusion proteins of B. sphaericus, B. laterosporus, B. thuringiensis subspp. thuringiensis, kurstaki, dendrolimus, tenebrionis, galleriae and so on are distinguished by different indexes of antibacterial activity. There is positive correlation between antibacterial and insecticidal actions of delta-endotoxins. It was proposed explanation of correlation reasons. Although delta-endotoxins are characterized by different antibacterial activity levels measured by specific activity units, the latter do not depend on specificity of insecticidal effect and characterize membranotropic activity potentials of these polypeptides. If the indexes of antibacterial activity differ from one another more than 2-fold, there is high probability to find insect species from new orders or other organisms susceptible to delta endotoxins with unusually high index. So, antibacterial activity indexes of delta-endotoxins specific for Lepidoptera and Coleoptera and also delta-endotoxins of $B$. thuringienses subsp. neoleonensis were approximately 7,23 and $70 \mathrm{SU} / \mathrm{mg}$, accordingly. We did not register insecticidal effects against termite Anacanthotermes ahngerianus Jacobs (Isoptera) among 24 serotypes of $B$. thuringiensis examined earlier as mixtures of spores and crystals or crystal proteins. It was assumed on the base of antibacterial activity index and information about isolation region (Mexico), that termites have susceptibility to subsp. neoleonensis delta-endotoxins. Our assumption was confirmed by biotests. LC-50 and LT-50 were calculated. So, the use of the elaborated scheme permitted us to discover the representative of a fourth order of insects (Isopterea) susceptible to action of Bt delts-endotoxins.

M.V. Lomonosov Moscow State University, 119899, Russia 
N.V.Kandybin O.V.Smernov G.V.Samoukina

Working out and 2troduction of new microbial preparations 15 extremely necessary for expansion of alternative methods sphere of plant protection. All these measures settle the ecological, yleld keeping problems. the problems of food and agriculture raw materials quality increase. To achieve these altis the search of microorganisms, possesing man's liseful features is belng carried out. For the last years in All-Russian Research Institute for Agricultural Microbiology (St.- Petersburg, ecologically safe media for pest control on the basis of the new strains were producted. BACICOL is a new preparation of Bacillus thuringiensis, highly eflective for coleoptera insects - phytopnages. there were Bot data on its useful application in different geographical zones to protect potato, cabbage, berry plantationsicereal crops. AcTikik a preparation on the basis of a streptomycete - 1 s applied to control Colorado potato beetle and spider mites in greenhouses especially. It is produced bi several formulations. It has uniquelly low application dose - $30 \mathrm{~g} / \mathrm{ha}$. Both preparations and their producers have bern successfully treated for safety for a man, non-target
species and phytotoxicity.

189620 St.- Petersburg. Pushkin -6. Podvelsky -3. Russia, All-Russian Restearch Institute for Agricultural Mitrobiology

Effect of validoxylamine A (VAA), a trehalase inhibitor, on the flight and and reproduction in Periplaneta americana

\section{Y. Kono, M. Takahashi, K. Matsushita, M. Nishina and Y. Kameda}

VAA, an amino sugat, whose glucoside, validamycin $A$, bas been used for a long time as a fungicide against Rhyzoctonia solani disease, is a potent and specific inhibitor of trehalases from various organisms (Kameda et al.,1987). I-50 values of VAA against trehalases of thoracic muscle and ovaries of the cockroach were determined to be $1.4 \times 10^{-3} \mathrm{M}$ and $5 \times 10^{-9}$ $M$, respectively. When VAA ( 5 or $50 \mathrm{ug} /$ insect) was injected into the adult cockroaches, trehalase activity of both tissues was mostly inhibited for more than 2 weeks. One day after the injection, the males were unable to continue flying for $2.5 \mathrm{~min}$ though untreated adults flew for about $15 \mathrm{~min}$. The haemolymph trehalose concentration increased to about 3 times the normal level, and trehalose was not metabolized in the tissues. One week after the injection, degeneration of oocyte was observed in the females. The sclerotization of ootheca deposited by the female was incomplete, and no larvae hatched from the ootheca. Taking the specificity of inhibitory activity of VAA into account, the abnormal phenomena observed were thought to be solely caused by the suppression of trehalose metabolism.

National Institute of Health, Department of Medical Entomology, Toyama 1-23-1, Shinjuku-ku, Tokyo 162, Japan
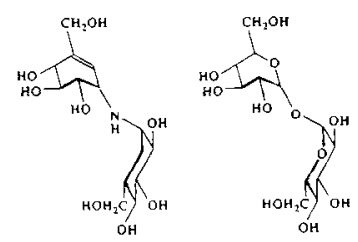

juurianov I.F., Karpuls i.r., banzelesova li.e.'.

It's iound that steroid slycostide $\alpha$-tomatin, broush into liquid nutrient medium for growing or fungts lielminthosporium tures Sacc., caused net blotch of barley, in share j00, 150, 50, 5 and I meg/ma, in the fomer four concentrations suppressed of cevelopment of the fungus and lowered of mycelial mass on $2 I$ day of culturing accordingly to $45 \%$, $3 \%, 43 \%$ and $53 \%$ as compared with control (the ane medium without of $x$-tonatin) and slightig (to II4\%) incresed of fungus development in the latter concentration. By electron-nicroscopic investigation it's revealed that the cell wall anci also plasnatic anc otner membranes are evicently the sites of action of $\alpha$-tomatin on fungous cel1. so wi bo oberved enlightement and lose of lyor structure ox the call walls and lover contrast or lack of the visible external and intemal membranes in mycelial cultures affected with $0.01 \%$ oi $\alpha$-tomatin. When barley leaves, infectec with if veres, are cut oiti and incubated on $0,004 \%$ water solutions or beazinidazole with addition of $0,01 \%, 0,001 \%$ and $0,000 \%$ or $\alpha$-tomatin (in experimental variants) or without ô it (in control), we revealed after 5 and 7 days of incubation the inhibition of enizrgement of necrotizing brown spots on the leaves at the all concentfitions of the preparation, although these differences to IO-I2 days were expressed less marked. It's also found distinct inibition of development of stem rust on rye, when leaves or jeedings in a day arter inoculation are cut off and put on the same solutions of $\alpha$-tomatin as above. ihemical iduunization of berley and rye seeds nith $0,01 \%$ of this preparation and aduitional treatment oi these plants at the phase tubulation with $0,005 \%$ solution in the rield conditions are promoted to raising of stability or these crop plants against influences of aiseases and unfavourable factors of enviroments and are improved oi structure of harvest.

220733. Institute of Experimental Botany of the scagemy of Sciences, Minsk, Skorina str., 27, Belarus. 
UTHLIZATION OF SOME NATURAL PRODUCTS AS BIOCHEMICAL CONTROL AGENTS AGANST THE COTTON LEAFWORM Spodoptera littoralis (Biosd.)

\section{M.I. Mogahed* and A.H. Mohanna**}

Trials were conducted under laboratory conditions to investigate the insecticidal activities of Ammi majus and Lupinus termis extracts on some biological features of the cotton leafworm, Spodoptera littoralis.

The negative correlation was noticed towards the hatchability percentages of the treated eggs and the extract concentration of both plants. Concentrations of 3 and $4 \%$ ( $w / v)$ plant extract caused the highest mortality percentages for the 2nd and the 3rd larval instars of $S$. littoralis. The 3rd larval instar was more susceptible to $A$, majus extract than the 1st and 2 nd instars.

The duration period of feeding larvae and produced pupae were affected with the increase of the extract's concentration. The duration of treated 1st and 2nd larval instars and produced pupae were significantly shorter than untreated ones. The concentrations of 1, 2 and $3 \%$ plant extract of Lupinus termis enhanced the development of 1st and 3rd instar larvae. Moreover, concentrations of 4 and $5 \%(w / v)$ extract of $L$. termis caused the prolongation of larval duration, and also caused growth retardation of non-feeding stages (prepupae and pupae) resulting from treated 1st and 3rd larval instars of S. littoralis.

* Dept. of Pests and Plant Protection, National Research Center, Dokki, Cairo, Egypt

** Efficient Productivity Institute, Zagazig University, Zagaxig, Egypt

1303

EFFECTIVENESS OF GARLIC HOMOGENATE IN THE CONTROL OP PATHOGEMIC FUIGI

L. B., Orlikowski, A. Saniewska, Cz. Skrzypczak, A. Wojdyla

Extract from garlic (AIlium sativum) cloves or leaves has been used against several human diseases, insects, viruses and recently against plant pathogenic fungi. Tansey and Appleton (1975) found antifungal properties in vitro of garlic against 42 genera and species caused mainly human diseases. Since the last 20 years more studies have been concerned the effectiveness of garlic in the inhibition of plant pathogens, among them species of Fusarium, Phytophthora and powdery mildew fungi. It is already known that aqueous extracts of garlic or its derivatives inhibit in vitro the growth of many but not all species of fungi. A few in vivo experiments indicated that garlic cloves homogenate or ajoene control red spot of Hippeastrum and Narcissus incited by phoma narcissi, phytophthora foot rot of gerbera (Saniewska 1992, Orlikowski and Saniewska 1994) and strongly reduced powdery mildew and leaf spot development on cucumber, tomato and roses (Reimers et al. 1993)

In our studies effectiveness of cloves homogenate was determined against Phytophthora cryptogea on gerbera, Fusarium oxysporum f. sp. tulipae $(=F O t)$ on tulip bulbs and Sphaerotheca pannosa var. rosae and Diplocarpon rosae on roses. Soaking of gerbera from in vitro multiplication in 2 aqueous garlic extract before planting did not inhibit Fhytophthora fot rot development. Application of such extract immediately after planting into artificialy infected peat decreased disease severity about $50 \%$. One hour dipping of tulip bulbs, artificialy inoculated with Fot $24 \mathrm{hrs}$ before treatment in 2.5\% garlic extract gave similar effect as methyl tiophanate or benomyl. The percentage of diseased bulbs decreased about 3 times in comparison to nontreated material. Both, in greenhouse and field trials garlic aqueous extracts applied as weekly spraying significantly decreased development applied as weekly spraying significantly decreased development and spread of powdery mildew and black lear spot. In greenhouse trial, the concentration 0.125 was already effective in the disease control. On one cultivar of rose spraying of plants at dose $20 \mathrm{~g}$ of garlic/1, development of mycelium
of $S$. pannosa var. rosae was not observed.

Literature Orlikowski L.B., Saniewska A., 1994. Proceedings of the 3rd EFPP Conference: "Environtental Biotic Factors in Integrated Plant Disease Control", Poznan' Poland, September $5-9,1994$, (in print).

Reimers F.. Smolka S. E., Werres S., Elank-Schumacher K., wagner G., 1993.

Z. Pflkrankh. Pelschutz 100:622-633.

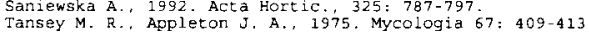

Research Institute of Pomology and Floriculture

Pomologiczna 18,96-100 Skierniewice, Poland

THE POSSIBLE CONTROL OF EURYGASTER INTEGRICEPS PUT.BY TREATING EGGS WITH PYRIPROXYFEN.

HASSAN DAROOGEH NOKHODCHERY ${ }^{1}$ - ALI ZARNEGAR ${ }^{2}$

During a laboratory study carried out in IRAN, showed that treatment of eggs of Eurygaster integriceps put. with juvenile hormone mimic( pyriproxyfen / either directly or via the maternal inhibit embryogenesis. The eggs treated with pyriproxyfen remained green with no differentiation visible by external examination. The sensitivity of eggs to the pyriproxyfen depended on the progress of embryogenesis; the freshly deposited eggs were most sensitive; the sensitivity decreased in older eggs and those eggs supplied withe pyriproxyfen day 3 or later developed normally.

Plant pests and Diseases Research Institute, P.0.Box 1454, Code19395 Tehran - IRAN 
Henryk Pospieszny

Soluble chitin oligomers, chitosan, microcrystalline chitosan, cartoxymethyl chitosan and chitosan sulfate were examinated for their inhibitory effect against viruses infected bean. Chitin derivatives applied by spraying or inoculation, protected bean plants against local and systemic infection caused by Alfalfa mosaic virus (AIMV), Bean yellow mosaic virus (BYMV), Bean common mosaic virus (BCMV), Peanut stunt virus (PSV), Bean golden mosaic virus (BGMV), Tobacco mosaic virus (TMV) and Tobacco necrosis virus (TNV). Spraying of bean plants with $0.1 \%$ or $0.01 \%$ solutions of chitin oligomers and chitosan completely reduced the number of local lesion production by AIMV, TMV and TNV. Preinfectional treatment of bean plants with chitin derivatives protected $70 \%$ $100 \%$ of them from systemic infection of BCMV, BYMV, PSV, AIMV and BGMV. Microcrystalline chitosan, cartoxymethyl chitosan and chitosan sulfate are a little weaker inhibitors of virus infection then chitin oligomers and chitosan. Pretreated parts of bean plants were protected from infection of mechanically transmitted viruses for several days because of the hard washable film formed by the chitin derivativers. Chitin derivatives induced resistance against vinses also in untreated parts of the treated bean plant. When the lower leaves were treated with chitin derivatives and upper one's were inoculated with AlMV or TMV, the number of local tesions produced by viruses were reduced by about $60 \%-70 \%$. Preinfectional root treatment with chitosan induced systemic resistance of bean to AIMV. These facts indicated that chitin derivatives had influence on bean susceptibility rather than had direct effect on virus. The fact that inhibitory activity is transiocatable within plant indicates that either inhibitors are mobile or that they induce the production of compounds of the antivirus activity. The mechanism (s) of inducation of resistance seems to be temperature dependent. Chitin oligomers and chitosan protected bean plants also against BCMV transmitted by aphids.

\section{Henryk Pospieszny}

ul. Miczurina 20

60-318 Poznań

Poland
S. M. Khod jibaeva, L. T. Pulatova, G. A. Kasymova

It has been isoleted from grey-raerth soils of Uzbekistan the Streptomices herochimiense h.34 th strain, inhibiting growth and development of gormoz, root decaying and vilt stimuluses of cotton-plant. The conditions of pigment complex have been optimized, which cause its growth-stimulating and fungicide properties. on nutritive medium, containing glucose, peptone, seccndary wastes (fruit squeezings out, rice brans) the yeild of pigment complex was $2 \mathrm{~g} / 1$. The temperature of melting is about $125-130^{\circ} \mathrm{C}$.

According to mass-spectrum data its molecular weight (mass) is equal to 600. Ik-spectrum has revealed the availabitity of olefines $(\mathrm{C}=0)$, metoxyl- $\left(\mathrm{C}-\mathrm{O}-\mathrm{CH}_{3}\right)$, methyl- $\left(\mathrm{CH}_{3}, \mathrm{NH}_{2}\right)$ and carbonyl groups. The maximums of adsorption in range of $\mathrm{UV}$ and visible light are in the field of $245 \mathrm{~nm}, 290 \mathrm{~nm}$ and 530 nm. Its empiric formula it $\mathrm{C}_{2} 6 \mathrm{H}_{35} \mathrm{~N}_{3} \mathrm{O}_{2}$.

Thus, basing on spectral analysis date, mass-spectrum, elementary composition and emperic formula, the pigment isolated from Streptomices herochimiense h. 34 th strain may attribute to prodigiozin-like compounds. In laboratory and field conditions it has been revealed its inhibiting action on growth and devolopment of some phitopathogenic fungi Fusarium oxysporum, Virticillium dahliae, Rhizoctonia solani and ogommoz stumulus of cotton-piant Xantomonas mal vacearum.

Institute of Uicroblology Academy of Science Republic of Uzbekistan, 7b.str. A.Kad1ry Teghkent 700128
1307

GUMATE FROTECT ACIICN AGAINCE NET BLCCH IN BARLEY

G.N. Shanbanovich, Z.J.Serova, I.K.Ges, S.S. Ling

The present study of gumate treatment of barley geeds showed a decrease of net bloch incidences in growne plants curing all vegetation period. It was shown that gumate preparations (oxydate, hydro- and oxygumete) have a remote action on leaves infection. Extent of infection wag lower in plants with seeds treated by gumates. This inhibitory effect was shown both at the early stages of plant development and at the milk-wax period. rbove effect stopped only at the last stages of plant vegetation. It was suegest that physiological-biochemicel basis of gunate frotect effect connects with more effective nucleicprotein netabolism. Irucleic acids and protein synthesis was stimulated by gunate treatnent simultaneously barley firmess to deseases. Cbviously, Eunate treatment of seeds activates plant genome functions, synthesis of proteins wich regulated barley metaboligm, connected with reatrict spreading of infection. It was detected any different reactions on gumate treatment among studied barley varieties.

Institut of Experinental Botany icademy of Sciences, T. Jkorina st. 27 , Minsk.22ccic Belarus

"Institut of agriculture and feeds of Republic Belarus.

Timiryazev: street, 1; zhodino. $22<160$, Belarus
1308

STUDIES OF EXPLORING CROP PROTECTION AGENTS FROM LABIATAE PLANTS

Shang Zhizhen, Xu Jianhua, Zhang Yieguang, Qiu Yutong, Liu Zhun, Chen Huanming

With an attempt to explore biorational crop protection agents, we have investigated antifeedant and growth regulatory activities of extracts and/or chemicals related to three Labiatae plants, Ajuga nipponensis, A. decumbens, and Teucrium quadriforum which are widely distributed in Southern China. The target pests in test were such economically important Lepidopteran larvae as Pieris rapae, Plutelia xylostella, Ostrinia furnacalis, Mythimno separata, and Prodenta litura.

Anong all the extracts of aerial part of $A$. nipponensis, the chloroform extract $C$ was found to possess the strongest antifeedant activity against the 5th instar larvae of Pieris rapae; its antifeeding ratio(AR) was $78.9 \%$ at the concentration of $0.5 \%$, and its $\mathrm{AFC}_{50}$ was $0.12 \%$ in no-choice test In leaf-disk method for toxicity test, extract $\mathrm{A}$ and $\mathrm{C}$ exhibited growth regulatory effects, leading the treated larvae to malformation and death. Their ED 50 were $1.36 \mathrm{mg} / \mathrm{g}$ and $0.34 \mathrm{mg} / \mathrm{g}$, respectively. When tested with the $4 \mathrm{th}$ instar larvae of Plutella xylosfella, extract $C$ showed certain stomach and contact actions as well as its antifeeding and growth interfering efficacies. Its $\mathrm{LD}_{50}$ was $0.22 \%$ for topical application and $0.29 \%$ for leaf-disk test while its $\mathrm{AFC}_{50}$ was $023 \%$ in the latter. Both the methanol and chloroform extracts demonstrated potent antifeeding effects for Prodenia litura, and Mythimna separata. At $0.5 \%$, extract $C$ had ARs above $90 \%$ against both species in choice test; in no-choice test, the AR was $60.5 \%$ for the 5 th instar larvae of $P$. litura and $46.7 \%$ for the 4 th larvae of $M$. separata, respectively.

From Ajuga decumbens and Teucrium quadriforum, Ajugacumbin A with known antifeedant activity and Teucvidin (1) with unkown activity were isolated and identified. Through chemical modification(s) and/or further extraction from the plants, 44 other clerodane diterpenoids were obtained. Out of them, 12 compounds have been tested for antifeedant activity and exhibited different levels of activities. Teucvidin (3), a derivative of Teucvidin (1), was found to be the most potent antifeedant against the 4th instar tarvae of M. separata. Its AR was $100 \%$ at the concentration of $0.1 \%$ in no-choice test, and $64.4 \%$ at $0.0125 \%$ in choice test. They were also effective in inhibiting the feeding of the 5 th instar larvae of Ostrimia furnacalis and of the 3th instar larvae of Plutella xylostella. To elucidate the mechanism of their antifeedant activities, preliminary eletrophysiological investigations have been done with $O$. furnaclis tarvae and showed that these clerodane diterpenoids could stimulate a certain sensory neuron in the medial maxillary sensillum to produce action potentials other than those induced by sodium chloride and sugar

Elemento-Organic Chemistry Laboratory, Nankai University, Tianjin 300071, P.R.China 


\section{N.I.Shtemenko, L.F. Zamorueva, Dukachova O.V.}

Surface waxes of leaves and seeds makes hydrophobic layer wich protects plant from pests and penetration of environmental pollutants. Only with development of chromatographical methods some progress in their study was possible due to significant heterogeniety of these substances. Plants of different types were investigated on composition of the protective layer. The main substances were hydrocarbons and ethers of long chain alkohols with fatty acids. Hydrocarbon. were investigated by GLC method, high molecular weight ethers - by HPLC, after hydrolysis and derivatization tatty acids and long chain alkogols were analyzed by GEC again. Most of steps of investigation were accomanied by TLC and spectral methods. It was shown, that not only types of plants, but even wild tipes and adult plants of maize differ on content of free hydrocarbons, fatty acids and alkohols. For example, surface lipids of seeds of ordinary forms of maize had more heterogenical spectrum of hydrocarbons, fatty acids with more long chain and letter unsauration than mutant forms. These forms differ on their stability to diceases. The most differences in leave waxes were tound in the fraction of fatty acids. It seems so that such plants as Zea mais L., Picea Caradensis and Hoya carnosa R.Br. has different schemes of biosynthesis of this classes of compounds. especially changes concern elongation-decarboxylation enzyme systems, wich are active in plants.

Institute of Biology of Dniepropetrovsk State University, Gagarin ave 72 , Dniepropetrovsk 320625 , Ukrain

\section{A. P. Volynets, L. A. Pshenichnaya and V.P. Shuiranov}

Bioiofical activity of steroid glycosides, their interaction with phytohormones. infiuence on the growth and development of bariey piants and phytopathogenic fungus. Hetminthosporium teres Succ., vausing agent of barley net biotch ulsease, were invesizigated. Unaracter of changes of barley hormonal complex aiter treatment with sterolu glyoosides was examined. Steroid siycosides possessed hormone-ilke activity of auxin and cytoininin iyge: They stimulated plant growth and rooting, formation and preservation of plements in experiaents in vitro. At the jane tine they strenthered auxin and cytokinin activity in concentrations lower 5 mg/i. Refuiatory activity of steroid Hiycosides also was leveaied for their influence on barley plants and phytopathogenic lungus $\ddot{k}$. teres. At the concentrauiuns oi 5-50 mg'l they depressed fungus growth but stimulated or did iot efiect an the plant growth. A treatment of plants with jierois glycoisides lecreased percentage of diseased piants and degree of bar"iey infection by leaf diseases and root lots. Simul taneously a glain productivity of bariey was raised. In piants ireateu with steroid glycosides the content of auxin and cytokinin was raised and stabllized at the high jevel. In all experiments activity of spirostanol glycosides was hipher than furostanoi ones. Trus, ratural steroid elycosides Mày be attributed to antiblotic substances with regulatory acilivity. Their protective effect is connecteu with their owr 'Equlatory ability as weli with activation aind stablication of hormonal inetabolism of infected piants.

institube of Experimental Botany, belalus kitavemy of Sciences, F. Shorina st, 2\%, Minsk 2zoot2, Belarus.

\section{AFTER APPIICATION OF THE CFREALS, POTATO AND VTNEYARDS}

L.F. Vassyuk, A.P.Kozhemyakov, T.A.Popova, V.K.Tchebotar,

Y.D.Shenin*, L.F.Kruglikova*

Flavobacterin is a biopreparation for increasing the productivity of cereal,fodder crops and vegetables. Seed inoculation by flavobacteria increased the yield of cereals by $0.3-0.5 \mathrm{tha}$, fodjer grasses by 1.4-1.8 tha, fodder sorghum by $2.4-4 \mathrm{tha}$, vegetables by 1.7-6 tha.Bacterization has improved the plant quality by increasing the content of protein, phosphons, lyzine and ascorbic acid.

Biopreparation is created on the base of associative nitrogen fixing bacteria L-30 belonging to genus Flavobacserium.

Mode of positive action of biopreparation is connected with active nitrogen fixation on the plant roots as well as with the improving of mineral and water uptake, phytohormones production and fungistatic activity.

Numerous greenhouse and field tests data showed that the flavobacteria produce substances suppressing phytopathogens, causing Rhizoctonia, Fusariwm and Uncimula affection. The rhizoctoina and wet rot on the potato tubers decreased by $2-4$ times after inoculation of potato with Flavobacterium sp.,strain L-30. The bacterization of sugar beet seeds decreased the number of piants damaged by Rhizoctonia and Fusarium by 3 4 times under field tests. Application of flavobacteria metabolites for spraing on the leaves of vine decreased the true mildew (Uncimla) spreading from $40 \%$ to $22 \%$

The active substances was extracted from culture medium with orgaric solvents The organic layer was evaporated to dryness under reduced pressure and the residue was applied to a plate of silica gel and developed first with n-buthanol-acetic acid-water (3:1:1) followed with benzene-methanol-acetic acid (45:8:4). The antibiotic is a a complex from two components with $R_{r}=0.43$ and 0.89 in first component.

The UV spectrum of the component with $\mathrm{R}_{r}=0.43$ showed the shoulder absorption band at $250-270 \mathrm{~nm}$. The IR spectrum of this component showed the characteristic absorption band of hydroxygroups, carbonyl-group and amido-group. All data showed that this antibiotic belong to the polypeptide. The both components of antibiotic showed high activity against phytopathogenic bacteria and fungi.

Resedrch Institute for Agricultural Microbiology,

Shosse Podbelskogo 3.St Petersburg-Pushkin-8, 189620, RUSSIA

Research Technological Institute of Antibiotics and Medical Enzymes,

Joint-Stock Company*, St.Petersburg, Ogorodnicov prospect 23

1312 BTOSYNTHESIS OF THE SECONDARY METABOLITES IAASIS OF
APHIDICIDE BIOPESTISIDES BY ENTOMOPHTHORA THAXTERIANA PETCH (ZYGOMYCETES: ENTOMOPHTHORALES )

Voronina, E.G.. Mukamolova T.G.

Since 1970 year the conception of the use of entomophthosalean fungi in IPM concluded in development of biopesticides with epizootic type of action (mycosidin). It has been determined the peculiarities process of development of Entomophthora thaxteriana fungus $f$ rom vegetative to reproductive stage and specific requirements of this fungus in nitrogen. carbon. phosphorus and mineral nutrition resoutses. It has been shown that introduction of the resting spores produced in vitro into pea aphid population have resulted in the enhancement of epizootics in host population only under specific conditions. that were favorauble for natural epizootic.

The charges of pathogenesis have occured under adaptation of investigeted isolates to in vitro development. The fungus has began to produce the toxic metabolites. and to colonize the body of host preferably after toxic effect (post mortem) or, namely. it has passed to saprotrofic status. Which followed by changes in virulence. conidiezenesis and type of transmission in host populstion. Heace for successful introduction of resting spores produced in vitro it is necessary to investigate the changes in host-pathogen system influenced by saprotrofic status of pathogen.

The capacity of entomophthoralean fungj to produce the toxic metabolites was the basis for works on development of preparation with fast and contact action against aphids. The dynamic of toxin production have been strdied and effective solvents and methods for receiveng of preparations have been determined. Toxic compounds extracting with lipid fraction are the base of mycoaphidin $T$. but untoxic lioids presented in large amount make worse its preparation form. So, the hydrolysis was applied for production of preparation called entox. Yield of the entox is 2 times more than mycoaphidin and the entox has better capacity to emulgate in water. No significant differences in biological effectivness of this preparations under investigated concentration were detected.The preparations are harmless for bees. entomophagoes and warm-blooded animal.

Voronina E.G., AlI-Russisn Plant Protection Instilute. J, Podbelskogo Road. St.-Petersburg - Puschitin, 189620, RUSS1A. 


\section{EVALUATION OF CINNAMIC ACD DERIVATIVES AS REPELLENTS FOR CROP PROTECTION}

\section{R.W. Watkins, E.L. Gill and D.P. Cowan}

Non-lethal chemicals, that deter berbivores from feeding on crops, have considerable potential as relatively benign methods of reducing damage caused by pest species. Secondary plant substances have a profound effect on food selection by herbivores and therefore are a potential source of feeding deterrents. The presence of such chemicals in certain species myy serve as a warning that the plant is unpalatable, and encourages herbivores to feed on altermative food sources. Aversion (immediate or learned) to these substances appears to be common to both vertebrates and invertebrates.

The feeding preferences of bullininches, Pyrrhyla pyrrhyla for fruit-bud extracts have been correlated with biochemical differences between these extracts. Those that were aversive to birds were found to contain high concentrations of secondary metabolites, in particular a family of aromatic acids known as cinnamic acids. No-choice feeding trials were carried out to quantify the doseresponse relationships for a range of cinnamic acid derivatives against feral pigeons (Columbia livia). The most potent feeding deterrent identified by this screen, cinnamamide, can significantly reduce food consumption at concentrations as low as $0.09 \% \mathrm{w} / \mathrm{w}$; this effect increases with concentration and food consumption can be completely abolished at applications of $0.6 \% w / w$. Subsequent trials showed this compound to be an effective, benign repellent with activity against a broad spectrum of pests including birds, rats, mice and slugs.

This paper describes the work undertaken to determine the relationships between the molecular structure of these cinnamic acid derivatives, their repellent activity and their potential to prevent vertebrate and invertebrate damage to crops.

Vertebrate Pest Management Group, Central Science Laboratory, Ministry of Agriculture, Fisheries \& Food, London Road, Slough, UK, SL1 7HJ

$$
1315
$$

ASCEMDING AND DESCENDING TRAKSLOCATION OF

14 C-MALATHIOH IN PLAhT-SOIL ECO-SYSTEM

M.A.Abo-El-Seoud ${ }^{1}$ and A.M.Shans-EI-Din ${ }^{2}$

A glass house experiment was achleved to measure the movement, fate and translocation of C-mslathion applied to cabbage plants. Cabbage seediinga were transplanted in cabbage plants. Cabbage seedlings were transplanted in
plastic pots contain $1 \mathrm{Kg}$ of alluvial or sandy soil. After
growth period of 2 months, the radioactive pesticide was applied to leaves $(300,000 \mathrm{cpm})$ or soils (500,000 cpm) of
the grown plants. Plant and soil semples were harvested at interval of 2 days and up to 14 days after application. Radioactivity was transported and detected in stems and rootg after 4 and 10 days respectively, of foliar application, however, no ${ }^{4} \mathrm{C}-$ residues could be transported it recovered in either of the used soils. On the other hand, Pic-malathion was penetrated and detected in cabbage roots after 2 days of soil application, then it was translocated up to stems after 6 and 4 days and to leaves after 10 and 6 days witb respect to the alluvial and sandy soil-grown plants, respectiveiy. Amounts of $\mathrm{c}$-residues remained in plants, respectiveiY. Amounts of $C$-residues remained in alluvial soil were higher than in sandy soil. Inturn, cabbage grown in sandy treated soils contained more radiocarbon than those grown in alluvial treated soil. In general, ascending translocation of the radioactive material from roots and up to leaves was comparatively faster rather than the descending transport from leaves and down to roots.

1-Agric. Dept. of Soils and Water Res., Atomic Energy Authority, Inshas, P.O. Box 13759, Egypt

2- Plant Protection Dept., Fac. of Agriculture, Moshtohor, Egypt.
USE OF SYNTHETIC SEY PHBRONONE FOR CONIROL GRAPHOLI THA FUNEBRANA F.BY DISORTENTATION METHOD

I.Zared1 shco, T. Nastas, G. Roshca, N. Fedoseev

The use of sex pheromones for disorientation is one from ecologicaliy pure methods of plant protection. In the elaboration of pheromone application technology for these objects the important role $1 \mathrm{~s}$ played by right choice of pheromone composition, rates of 1 is expense and dispensers, which have to assure defintte speed and duration of substance release in atmosphere and to protect it from environment factors. In the tests on Grapholitha funebrana F.disorientation we were using 65\% cis-3-dodecenylecetate, $2 \%$ trans-8-dodeceny lacetate and $33 \%$ dodecylacetate mixture as pheromone and pellicle coupositions on the base of polyvinglchlorld,elaborated by us as dispensers and containing 10 to $50 \mathrm{mg}$ of pheromone in different versions. The method effectivity was belng estimated by suppression of male catching in control traps and bJ decreasing of frutt injuring,and quality of dispensers was being done that by duration of their work and by values of pheromon secretion speeds.All tested versions (pheromone expense rates 25 to $5 \mathrm{~g} / \mathrm{ha}$ ) provided high level of male disorientation (more $99 \%$ ) and decreasing of fruit injuring to the level of chemical gtandard (1-2\%). The greatest interest is version with minimum pheromone expense $(5 \mathrm{~B} / \mathrm{ha})$, with that the speed of secretion of base pheromone component was changing in limits 1,14 to $0,02 \mathrm{mg} /$ day from one dispenser during one month. Proposed method of using of pelliclo dispensers allowed with noticeable decreasing of pheromone expense rates (to $5 \mathrm{mg} / \mathrm{ha}$ ) to realize pest disorientation wh thigh effectivity and to avold the application of pesticides for plum protection against Graphoit tha runebrana $F$.

Dr.Irina A.Zavelishco

Institute of Biological Plant Protection, Dacia 50,ap. 17 ML 277062 Kishinev, Moldova,Phone (3732)539-233, Fax (3732) 579-641

\section{6}

RESIDUES AND PERSISTENCE OF SOHE ORGAHOPHOSPHORUS INSECTICIDES APPLIED TO CABEAGE PLANTS

M.A. Abo-El-Seoud ${ }^{2}$, A.H. Shams-EI-D1n ${ }^{2}$, L.N.Danial ${ }^{1}$ and $S, M$, Alumed $^{2}$

Cabbage plants grown in a field experiment were sprayed with E.C. formulations of malathion, pirimiphos methyl and prothiofos at the rates of 570,600 and $750 \mathrm{gm}$ a.i. per feddan, residues of the three insecticides were analysed by GLC. Results revealed that initial deposits was varied; the highest deposit was that of malathion followed by pirimiphos methyl, and the lowest initial deposit was that of prothiofos. Initial deposits on inner leaves were less than the outer leaves. Prothiofos showed more persistence than malathion, the least persistent toxicant was pirimiphosmethyl. Persistence of the toxicants was lower on inner leaves, probably owing to lower initial deposits. Deposits were within the permissible limits after 12,12 and 15 days for malathion, pirimiphos-methyl and prothiofos respectively, while on inner leaves, these intervals were 6,8 and 12 days for the same insecticides respectively.

1- Agric. Dept. of Soils and Water Res., Atomic Energy Authority, Inshas, Egypt

2- Plant Protection Dept., Fac. of Agriculture Moshtohor, Egypt. 
CHLORPYRIFOS AND FENTTROTHION RESIDUES IN CUCUMBER RIND AND PULP DETERMINED BY GAS CHROMATOGRAPHY.

\section{J.C.FERST and G.S. de Bactista}

chlorpyrifos and fenitrothion regidues in cucunber rind and pulp wer determined by gas chromatography in the state of são Paulo, Brazil, to study their behavior after applications for pickleworms (Diaphania spp.) and leafminers (Liriomyza spp.) control. The insecticides were sprayed twice (until run-off) in a 10-day interval, during the frutification period, at the following dosages: chloxpyrifos 72 and 144 a. 1.12001 water; fenitrothion 150 and $300 \mathrm{~g}$ a $i .100 \mathrm{l}$; and control The treatments were replicated three times. The applications were done with a nanual back-pack sprayer, and a volume of application of 500 $1 /$ ha, approximately. Samples $(n-180)$ of all treatments, each one consisting of ca. $5 \mathrm{~kg}$ of non-washed fruits, were collected at zero, 3 and 8 days after the first application; and zero, 8 and 14 days after the second one. The rind and pulp were analysed separately. Sub-samples (1 $\mathrm{kg}$ ) wexe homogenized with a blendor and the amount of $100 \mathrm{~g}$ of the material was kept in a deep freezer (for up to 5 days) until the tests were performed. Residues were extracted with acetone, followed by partition with methylene chloride. Quantitative determination was carried out in a gas chromatograph equipped with an alkaline flame ionization detector (AFID). The quantitation $1 \mathrm{imit}$ was $0.02 \mathrm{mg} / \mathrm{kg}$ (ppm) for both insecticides in both substrates, and recoveries ranged from 75 to 1158 foz chlorbyzifos and from 88 to 125 for fenitrochion. Residues of both insecticides in the rind showed fast dissipation, with the degradation half-lives of $3-4$ days $f O I$ chlorpyrifos and 1 day for fenitrothion. In the pulp, residues of the former wexe not detected in any sampling date; and for the latter, residues were detected only in some samples collected after its first application at levels up to 0.0 $\mathrm{mg} / \mathrm{kg}$. Because of maximum residue leveis (MRLs) for pesticides in cucumber axe established in whole fruit basis, fenityothion residues were below its MRL $(0.5 \mathrm{mg} / \mathrm{kg}) 14$ days after last application (safety intervall.

Department of Entomology, ESALQ, University of São Pauto 13418-900 Piracicaba-SP, BRAZIL

1319

PESTICIDE RESIDUES IN PLANT PRODUCTS IN SLOVENIA IN THE PERIOD 1973-1992

\section{J. Maček}

The paper discusses the contamination of plant products with pesticide residues based on a monitoring performed in the past twenty years (19731992). The following plant products were included in the study: potato tubers, maize grain, hope cones, grapes, fruits (mainly apples and pears) and various vegetables. Samples were taken at random irrespective either of previous or eventual previous use of pesticides. They are regionaily representative. As it can be seen from tables the contamination of plant products in the period discussed was very moderate according to EEC normatives, it means it is surprisingly low.

Biotechnical Faculty SLO-61111-Ljubljana, Jamnikarjeva 101, P.O. Box 95 Slovenia

\section{MONITORING OF PESTICIDE RESIDUES IN INDIAN ENVIRONMENT}

S.K. Handa

The use of pesticides is very important for meeting the increasing needs of a growing population and for controlling vector-borne diseases, particularly in developing countries. According to an estimate, almost one half of the potential food production of less developed countries is lost due to insects, plant pathogens, weeds, rodents and birds. However, the use of pesticides is accompanied by a variety of undesirable environmental effects. The effects include excessive mortality, and reduce reproduction potentials in organisms such as birds and fish, changes in abundance of species and diversity of ecosystems, and production of pesticide resistance in targets and non targets organisms. Residues of pesticides also contribute significantly towards contamination of air, water, soil and food. Many western countries have established intensive monitoring programmes to estimate the levels of pesticide residues in both abiotic and biotic components of the environment. In order to co-ordinate the research work on pesticide residues, the Indian Council of Agricultural Research Work sponsored the All India Co-ordinated Research Work on Pesticide Residues with the objective to co-ordinate the research work on pesticide residues in agricultural produce and other components at all matural levels, to monitor the pesticide residues in abjotic and biotic environments, and to study the dissipation of pesticide residues in crops from supervised trials with recommended pesticides and to work out the safe time limits between pesticide application and consumption of the product. The All India Co-ordinated Research Project has seventeen research centres all over India. Monitoring studies carried out by the research centres of the project revealed the presence of DDT and $\mathrm{HCH}$ in cereals, millets, oilseeds, vegetables, spices, fruits, animal and human tissues, feed concentrate and straw. Thus, there is a need to replace these pesticides with easily biodegradable pesticides. The salient research findings on monitoring of pesticide residues in the Indian environment will be discussed in the seminar.

Division of Agricultural Chemicals and Project co-ordinator, All India Co-ordinated Research, Project on Pesticide Residues, Division of Agricultural Chemicals, Indian Agricultural Research Institute, New Delhi 110012, India.

1320

UPTAKE OF FUNGICDE, THIABENDAZOLE BY OYSTER MUSHROOM (PLEUROTUS OSTREATUS) CULTIVATED ON A SAW-DUST MEDIUM

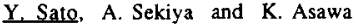

A fungicide, thiabendazole (TBZ) has been used for control of Trichoderma which is injurious to oyster mushroom (Pleurotus ostreatus) in Japan. In the present study, the uptake of $\mathrm{TBZ}$ treated to a saw-dust medium by fruit bodies of oyster mushroom was investigated. The fungicide was treated to the medium at the concentration of a practical use (Exp. 1), or concentrations of 10 times (Exp. 2) and 50 times (Exp. 3) as much as that of Exp. 1. The fruit bodies, mycelia and media were sampled at intervals after cultivation on these media. The residual analysis of TBZ was conducted by high-performance liquid chromatograph (HPLC) with fluorimetric detector. The fungicide was detectable in all fruit bodies of oyster mushroom collected from all TBZ-treated samples, showing that it transferred to fruit bodies from media. The amount of TBZ in fruit bodies from Exp. 1 was lower than the tolerance lever for $\mathrm{TBZ}$ residue in foods noticed by the Environmental Agency, Japan. Moreover, no difference of TBZ concentration in fruit bodies was observed among samples collected at the first and second flush, although TBZ concentration in the pileus was 2 to 4 times as much as that of the stem. The yield and quality of fruit bodies of the oyster mushroom lowered slightly in Exp. 2 or markedly in Exp. 3 when cultivated on media added with excessive amount of TBZ.

Forestry and Forest Products Research Institute, 1 Matsunosato, Tsukuba, Ibaraki 305, Japan 
TRANSLOCATION AND PERSISTENCE OF SOIL AND FOLIAR INSFCTICIDES IN PEA.

Surendra Singh 1, D.P.S. Bhati 2

Build up of trichlorphon, phosphamidon and dirnethoate residues in pea. Pisum sativum L. due to the control of Agromyzid pest, opliomyia phaseoli (Tryon), has been studied. Trichloxphon crystalline powder 10.5 and 1.0 kg per hectare) and foliar spray of phosphamidon and dimethoate $(0.1$ and $0.2 \%)$ were applied on 45 days old crop. The pea leaves and yreen pods were evaluated to find out the persistence of toxic residues after translocation through roots and deposits fron foliar spray. Trichlorphon reduces deyraded to safe level in leaves after 45 days of treatment while the green yods contained toxic level of trichlorphon residues after 72 days of harvest. Phosphamidon degraded to safe level ( 8 ppr) by fifth day, however, dimethoate was found to persist to toxic level even after two weeks of treatment.

Postal Address : DR, SURENDRA SINGH, 29/138, CHHIPETI, RAJAMANDI, AGRA - 282002 , INDIA.

\section{BIOTIC AND ABIOTIC FACTORS INFLUENCING THE POPULATION DYNAMICS OF THE SENEGALESE GRASSHOPPER, OEDALEUS SENEGALENSIS}

\section{J T Colvin, J Hoht. R J Cooter}

The Senegalese grasshopper, Oedaleus senegalensis (Krauss), is a sporadic pest of graminaceous crops such as millet and sorghum in much of West Africa. One hypothesis to explain the sporadic nature of outbreaks involves the build up of an egg-pod bank over several consecutive dry years. After such a dry period, mass hatching may occur with the first good rains. Predation pressure may be reduced at this time allowing the grasshopper population to reach outbreak proportions in the 2-3 generations of the rainy season. The ability of eggs to survive in the soil for several years is therefore considered to be a major factor contributing to the pest status of this species. The results of a study to investigate the above hypothesis are presented. A simulation model of the grasshopper population was also developed to enable an assessment to be made of the relative inportance of contributing factors. Results will be discussed in relation to management of this species

Natural Resources Institute, Central Avenue, Chatharn Maritime, Kent, ME4 4TB, United Kingdom
NATURAL OCCURRENCE OF MYCOTOXINS IN FUSARIUM INFECTED BEANS

T.C. Tseng, J. C. Tu

Navy bean (Phaseolus vulgaris L.) is also known as pea bean and white bean. It is destined exclusively for human consumprion. Thus, product quality and safety are important considerations. Mycotoxins in foods and food products pose serious health hazard to consumers. Like grains and cereals, navy beans are often infected by species of Fusarium in the field during the post maturation period before harvesting. The infected beans shows whitish grey to pink discolouration. Unlike grains and cereals, in which mycotoxins associated with fusarium infection have been doccumented, little investigation is made on mycotoxins of fusarium seed mould of beans. Recently, we conducted a series of investigations on the subject. This is first report on the discovery of several mycotoxins in the nanually infected mouldy navy beans with distinct whitish grey to pink discolouration. Species of Fusarium isolated form the mouldy beans include $f$ : avenaceum, $F$, culmorum, $F$, graminearum, $F$ moniliforme, $f$ axysporum and $F$ solani. Three types of bean samples were analysed for mycotoxins. These samples were $(A)$ healthy beans without any apparent discolouration,(B) beans with pink discolouration and (C) a mixture of beans with whitish grey and pink discolouration. The sample extracts were ran on TLC plates against the known standards for qualitative analysis. The results indicated that diacetoxyscripenol (DAS), deoxynivalenol (DON), T-2 toxin (T-2) and fumonisin $B t(F B t)$ were present in Type $B$ and $C$ samples but not in healthy one (Type A). Quantification of DAS,DON, and T-2 and of $F B_{1}$ were achieved by gas chromatography and high performance liquid chromatography, respectively. The results of quantification showed that (i) DAS, DON, T-2 and FB1 were not detected in the healthy sarnple, but in Type $B$ and Type $C$ samples, DAS, DON, T-2 and $\mathrm{FB}_{1}$ in the amounts of $3.3,3.1,13.5$ and $1.1 \mu \mathrm{g} / \mathrm{g}$ and of $9.2,6.5,5.5$, and $0.5 \mu \mathrm{g} / \mathrm{g}$ were detected, respectively. Further research is underway to determine which species of the isolated fiusarium is responsible for the production of a specific toxin.

The Insititute of Botany, Academia Sinica, Nankang, Taipei, Taiwan 11529, R.O.C.

1324

\section{CONTROL OF AUSTRALIAN PLAGUE LOCUST IN SOUTH AUSTRALIA}

\section{D.C. Hopkins, P.J. Warren and K.R. Henry}

The Australian plague locust, Chonoicetes terminfera, occurs naturally in the arid regions of central Australia. Population levels are largely regulated by localised rainfalt events that occur throughour this region. When population levels are high long distance migrations can lead to locust outbreaks in South Australia that threaten agricultutal and horticultural crops. These outbreaks are initiated by large flights of locust from central Australia in Marctu/April to pastoral country which ajoins the agricultural and horticultural areas of South Australia. These locusts lay eggs on arrival in these pastoral areas and these eggs undergo a diapause through the winter and hatch the following spring. If not controlled, these locusts complete their development and migrate south and damage crops and pastures. In the past (1955 \& 1976), major invasions of locust have moved to areas as far south as Adelaide causing millions of doliars worth of damage to the state's rural industries.

In South Australia, Primary Industries South Australia (PISA) joins forces with the Australian Plague Locust Commission to control locust outbreaks. Each organisation has a clearly defined area in which to work and this paper reports the control activites (methodology, logistics, etc) carried out by PISA to control a major outbreak of locusts in 1992.

Primary Industries South Australia

Box $1671 \mathrm{GPO}$

Adelaide South Australia

Australia 5001 
APPLICATION OF INSECTICIDE AEROSOLS OF OPTIMAL SIZES FOR LOCUST AND GRASSHOPPER MANAGEMENT

E.Kirov, V.Makarov, Y.Samsonov, K.Koutsenogii, A.Alekseev, V.Gaevoi, M. Sergeev, A.Lachininskii, N.Evdokimov, I.Ivanov, V.Chakhov

The effective aerosol treatments against a few kinds of locust and grasshopper have been performed in the 80-s and 90-s in the different regions of the former USSR The main peculiarity of these treatments was that insecticide aerosols of adjustable sizes $(1-30 \mathrm{mcm})$ have been used. The treatments were part of the so-called Optimum Aerosol Technology (OAT) of insecticide application. The technology is based on physical processes of wind turbulent diffusion of aerosol cloud in atmospheric layer near the surface of a treated area. The spatial diffusion is accompanied by inertial and gravitational precipitation of aerosols on insects, on plant leaves, on soil. The efficiencies of the above processes are strongly dependent on aerosol sizes, meteophysical parameters of atmosphere, kinds of plants and pest insects, etc. It is possible to provide relatively selective hit of aerosols on insect bodies, but not on leaves or soil. The direct hit of aerosols of optimal sizes just on insects characterizes the fundamental distinction of OAT against aerial and ground spraying.

Special powerful aerosol generators are designed. The generators are installed on trucks and hence are travelling. The generators move on roads along the up-wind edges of the treated areas. The effective widths of treatments vary from 200 to $2000 \mathrm{~m}$, the working rates vary from 200 to 3000 hectares per night. Aerosol treatments are performed at stable or neutral stratification of atmosphere which exist usually at night.

The treatments have been performed against Calliptamus italicus ( $L$.), Chorthipous albomarginatus (Deg.). Euchorthippus pulvinatus (Deg.). Pararcyptera microptera (F.d.W) in Northern Kazakhstan and in West Siberia ( 60,000 hectares, 1980-82 ,89-92), in Southern Tadjikistan (Aiolopus thalassinus (F.). Acrotyfus insubricus (Scop), Sphingonotus rubescens (Walk), 3,000 hectares, 1983-84), in Yakutia

(Chorthippus albomarginatus (Deg.), Euchorthippus pulvinatus (Deg.). Pararcyptera microptera $(F d W$.), 30,000 hectares, 1992-93). The organophosphorous and pyrethroids (methylparathion, fenvalerate, fluovalinate, etc) have been used. It is obvious that OAT may be used for locust management in the different parts of the world. However it would be desirable to investigate the potentiality of its application against locust before and during their migration. The locusts usually settle on land at night. Taking into account the mobility of aerosol generators and the high working rates of aerosol treatments just at night, one may suppose theif effective usage.

Institute of Chemical Kinetics \& Combustion, Siberian Branch of the Russian Academy of Sciences, 630090 , Novosibirsk -90 , Russia
INTEGRATED MANAGEMENT OF GRASSHOPPER AND LOCUST IN CHINA

LI OINGGUANG

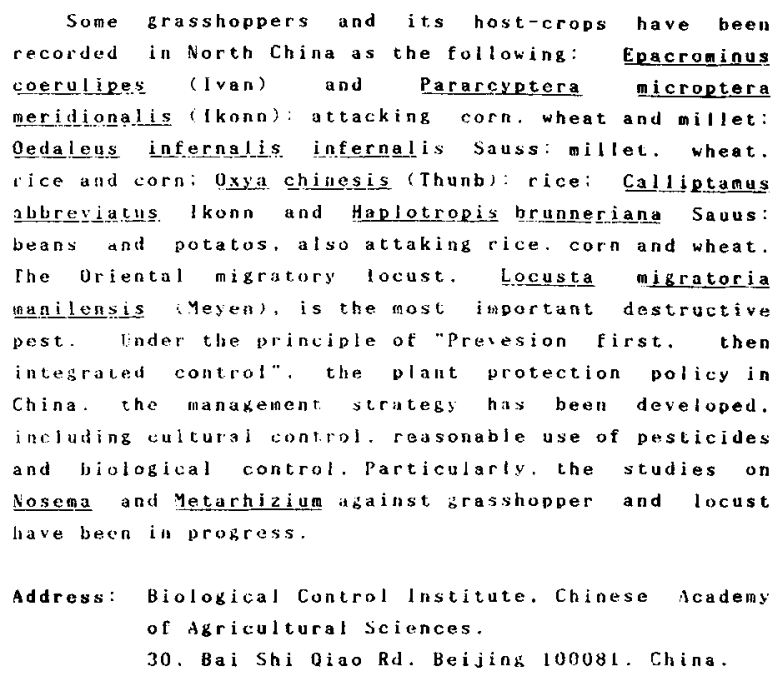

MONITORING THE MOVEMENT OF MIGRATORY INSECT PESTS - A NEW RADAR METHOD

\section{J R Riley, A D Smith, D R Reynolds}

A novel vertical-looking radar (VLR) technique has been developed which makes routine, long-term monitoring of insect migratory flight a practical and economic option. The radar transmits a plane polarised, circularly symmetric, nutating beam, in which the plane of polarisation rotates. Individual overflying insects return a time-varying signal which provides information on their size, body shape, orientation, altitude, and displacement speed and direction. All these parameters can be automatically extracted from the amplitude of the radar signal, using a specifically developed algorithm on a personal computer. Plots of the density and of the directional and altitudinal distribution of migratory flight are available, together with estimates of the body-mass distribution of the migrants. The radar provides coverage from an altitude of about $100 \mathrm{~m}$ up to $1,500 \mathrm{~m}$ above ground level for moths and grasshoppers, but the maximum altitude limit decreases with insect size. The equipment is based on the mature technology of marine radar and personal computers, and so should be inexpensive and reliable. The technique is intended for use by scientists and pest control technologists with an interest in insect migration, and it does not require specialist knowledge of radar.

Natural Resources Institute, Radar Unit, North Site, Leigh Sinton Road, Malvern, Worcestershire, WR14 ILL, United Kingdom 
BEHAVIOUR AND FOOD CONSUMPTION OF Schistocerca gregaria IN THE DESERT OF MAURETANIA. AN IMPUT TO LOCUST MANAGEMENT

H. Wilps

In the literature Schistocerco gregaria is described as an insect that daily consumes an amount of vegetation roughly equal to its own body weight. Since this insect is also migratory, in the case of mass migrations one can calculate that there will be enormous losses of vegetation. Given the polyphagy of $S$. gregaria, these losses can occur on cultivated fruit orchards or plantations, as well as on pastures. In the first case, the population of the countries involved will be directly affected, in the second case, indirect consequences can be expected, since $S$. gregaria is a competitor for the food supply of livestock. In particular in the Sahel countries, raising livestock represents the most important form of agricultural production. Both possibilities have led in the past to extensive and cost costly measures to control this pest. In the present investigation the food uptake of $S$. gregaria larvae was quantified under field trial conditions. Thereby the most important wild plants and the most common cultivated plants were offered in binary choice experiments. In all cases it was found that the amounts of food consumed were substantially less than the values given in the literature. In addition, questioning of Mauretanian stock herdsmen revealed that the food preferences of grazing livestock and $S$. gregaria larvae are different. By quantifying the number of individuals in the hopper bauds as well as the biomass of cultivated plants and grazing pasture consumable by $S$. gregaria, it has been possible to make the first estimates of the vegetation losses depending on the factors of the number of individuals, their age distribution and the resting time of the larvae in the biotopes. Based on these results and behaviour observations, new strategies for controlling this pest have been derived. These have now been successfully tried out by prelininary field trials in 1994-1995 in the breeding areas in Inchiri (Mauretania).

GTZ-Project Integrated Biological Control of Grasshoppers and Locusts, P.B. 5180, 65726 Eschborn, Germany

EFFECT OF DRY HEAT SEED TREATMENT ON SOME SEED AND SEEDLING PARAMETERS IN TOMATO

\section{A.D. Akpg and J.J. James}

Five levels of dry heat treatment and exposure periods, 60, 70, 80, 90 and 100 ${ }^{\circ} \mathrm{C}$ for $96,72,48,24$ and $0.33 \mathrm{~h}$, respectively, were evaluated in the laboratory and glasshouse for their effectiveness in improving seed germination, seedling emergence, seedling vigour, stem height, stem diameter, root length, root and shoot dry weights in tomato cultivars TI 10 and TI 160. A batch of unheated seeds served as a check.

In the laboratory experiment, cultivar TI 10 seeds heat-treated at $80{ }^{\circ} \mathrm{C}$ for 48 h showed a significantly $(P=0.05)$ improved seed germinationn and seedling vigour as compared with TI 160; furthermore, cultivar TI 10 appears more suitable for cultivation as it has lower fungal incidence. Storing heat-treated seeds for eight weeks reduced their germinability. The results of the glasshouse experiment indicate that heat-treating seeds of cultivar TI 10 significantly improved seedling vigour and stem height, while in TI 160 , root length, root and shoot dry weights were significantly improved with heat treatment at 70 and $80{ }^{\circ} \mathrm{C}$ for 72 ands $48 \mathrm{~h}$, respectively. Dry heat seed treatment at $100{ }^{\circ} \mathrm{C}$ for $0.33 \mathrm{~h}$ and to some extent $90^{\circ} \mathrm{C}$ for $24 \mathrm{~h}$ adversely affected all the seed and seedling parameters assessed.

Department of Crop Protection, Institute for Agricultural Research, Ahmadu Bello University, P.M.B. 1044, Zaria, Nigeria
1332

CHEMICAL CONTROL OF RHIZOCTONIA DISEASES OF POTATO

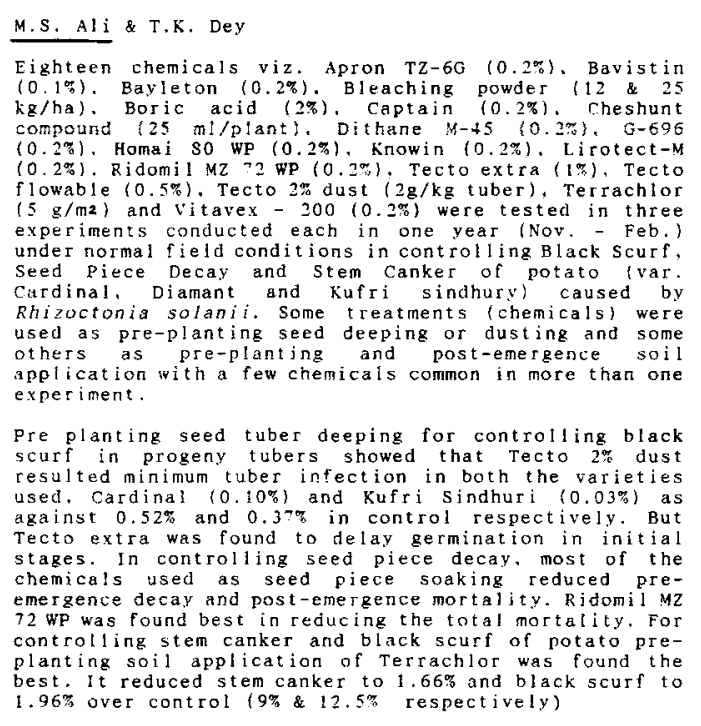

Overall yield was found to be positively influenced by the treatments. Inconsistence findings of studies may be attributed to the role of associate - organisms in controlling Rhizoctonia.

Tuber Crops Research Centre. Bangladesh Agricultural Research Institute, Joydebpur, Gazipur - 1701. Bangladesh 


\section{FUSARIUM WILT DISEASE OF TOBACCO IN MALAYSIA}

\section{A. R. Azm!}

Fusarium wilt is among the most destructive disease of tobacco in Malaysia, causing severe loss to the farmers. Based on symptoms of infected plants and parts of the plant infected, Fusarium wilt disease had been identified to consist of two different diseases. Vascular wilt disease caused by Fusarium oxysporum while root rot disease caused by $F$. solani. Usually the witt symptoms of plant infected by vascular wilt only appeared on one side of the plant, and the plant died. Observation on internal part of the plant showed that vascular tissue (usually on the wilted side) furned brown or darkened. However, plant infected by root rot showed more damage on the root system which turned black and rot. The leaves turned yeliow, the plant become stunted and died. Analysis of soil from the rhizospere of the infected plant showed that soil $\mathrm{pH}$ seemed to play an important role in determining the development of the disease. Both diseases were severe when tobacco was planted on sandy soil and low soil $\mathrm{pH}$ which ranged between 4.00 to 5.49. Planting beds treatment with soil amendment $S H$ Mixture at $1 \mathrm{mt}$ tha fourteen days before field planting showed good potential in controlling the diseases.

Tobacco Research Center, MARDI Telong, 16300 Bachok, Kelantan, MALAYSIA.
BOURCES OP SURVIVAL OF PHYTOPHTHORA INFESTANB CAUBING LATE BLIGHT DIBEASBS OF TOMATO IN COLD AREAB.

Bagirova S.F., An Jian Li, Dyakov Y.T. Survival of late blight on tomatoes in cold areas is not
undestood. Potato crops are considered to provide inoculum for infection of tomatoes. We have got evidence of distinct populations of Phytophthora infestans on potato and tomato in the same areas: collection of strains from tomatoes (TO and Ti races) and potatoes (To race) in Moscow region are differentiated in portions resistant to metalaxyl strains; $A 1$ and $A 2$ mating types and isolates of VC1 and vc2 - vegetative compatibility groups. overwintering on potato can be excluded here.since isolates of $P$. infestans with A2 mating type have been found in Europe, a source of survival may be oospores. We have investigated the formation of oospores in tomato tissue and the survival of $\mathrm{P}$. infestans in diseased tissue overwinter in cropping situations. Tomato fruits, leaves and stem pieces were inoculated with zoospores suspension of two P. infestans strains: T1A2VC2 and TOA1VC1, either individually or in combination. After inoculation, the material was placed either $5-10 \mathrm{~cm}$ deep in the soil, or on surface of the soil, either in the field, or in the glasshouse. Experiments were set up in October - November and samples were removed and microscopically. examined the following April.For the analisis of the material the 'baits' method was used - the plant tissues were placed in Petri dishes, moistened with sterile deionized water and covered with 'baits' (that is fresh fruits, leaves, seeds, or seedlings of tomato, or slices of potato tubers). After incubation this material was examined microscopically. Oospores were discovered in sampies infected with strains T1A2vc2 and TOA1Vc1 but not in samples infected with TOA1VCl individually, Formation of oospores therefor may have resulted from hybridization or from chemical induction either by the second strain, or by soil microorganisms. Late blight symptoms, oogonia and young oospores not sporangiophores or sporangia were found in the baits after contact with samples infected with T1A2VC2 and T1A2VC2 + TOA1VC1. Oospores in overwintring tomato debris, late blight symptoms and oospores in fresh tomato tissue contacted with debris, were found only in samples of the tomato race 1 in tomato tissue. aggressivenes to tomato can surviveans that race $T 1$ having high aggressivenes to tomato can survive on the crops in cold areas. However, hybrids of races $T 0$ and $T 1$ will lose complex genes provided the adaptation to infect tomatoes. Our experiments that almed at definig the intensity of oospores formation between races have shown that the number of oospores formed In T1A1XT1A2 is 3-4 times higher, than crosses with To race, wich can overwinter in potato tuber. There is portion genetic isolation between To and fi. The results or our expert festans can overwinter on tomatoes by means of oospores in cold regions. This work was supported by grant of Fund for Fundamental Science.

Dep.Mycol \& Algol., Moscow University, Moscow 119899, Russia.

1336

A NEW PLANT PROTECTION STRATEGY AGAINST GRASS MOTH Loxostege Sticticalis L. AT EURASIAN CONTINENT

\section{I.B. Knorr and A.N. Bashev}

Grass moth is well known as one of the dangerous agricultural pests. It is characterized as a polyvoltine pest with significant individual's reproductive potential, active migration and complex dynamics. In the outbreak period there appears a serious problem of the pest management on a huge area. The history of grass moth study is more than hundred years, but the efficient plant protection strategy against this pest is absent so far. In this paper, a new strategy of the pest management is described. It rests upon the life-system approach 1 and involves a study of endogenous adaptive transformation variety. Here we describe two types of the transformations which are the most important. A study of photo-thermic adaptive transformations allow us to estimate the boundaries of the pest population system and its spatial structure. These data are valuable for the analysis of a migration role and spatial management. Analysis of the adaptive transformations which result from a density change reveal the pest density threshold value when migrations begin in the system.

1Sharov, A.A. 1992. The life-system approach; a system paradigm in population ecology. - Oicos 63: 485-494

Computer Center Siberien Branch of Russian Academy of Science, Lavrentyev ave 6, Novosibirsk 630090 , Russia 
A STUDY OF ADAPTIVE TRANSFORMATIONS FOR GRASS MOTH Loxostege Sticticalis L. POPULATION MANAGEMENT

\section{A.N.Bashev}

In this paper an application of the life-system approach 1 to the problem of $L$ sticticalis management on the Irtish-Ob population system territory is discussed in connection with photo-thermic and density-dependent endogenous adaptive transtormations. The management is described by a set of $m$ - control measures: $\left(A, S_{i}, i=1,2 \ldots, m\right.$, where $A$ is target, $S$ is intensity of control which can be expressed by the negattve $\log$ - transtormed pest survival. In the case of a spacedistributed ecosystem for the target $A_{i}$ it is necessary to show the compartment coordinate $A_{i}$ and the stage of development $\Phi_{i}$ The study of the photo-thermic adaptation mechanism allows one to estimate the boundaries of the pest population system and its spatial structure. The areal is presented as a system of interacting compartments with different local dynamics which are in accordance with two warrantable types of age-structures: $L C C_{1}$ and $L C_{2}$ The compartment boundaries can be found from the condition

$$
k_{L C j} \rightarrow \max ,
$$

where $j=1,2$ and $k_{L} C_{j}$ is the mean intrinsic rate of increase. The analysis of the life-system structure shows that management must suppress pest migrations in the system. Consequently the control measures $(A, \Phi, S)_{m}$ are efficient only in the case when the pest density is above the threshold $N^{*}$ in one of the compartments and when migrations begin. The threshold value is defined on the basis of investigation of the density dependent endogenous adaptive transformations. Thus, to provide the guaranteed yield in a compartment with a probability $P$ it is necessary that pest reservation density should belong to interval $[L C L, U C L]$ with the probability $P$, where $L C L$ and $U C L$ are the lower and the upper confidence levels of the pooulation density, respectively. To fulfill the requirement of the pest migrations suppression in the system, the upper confidence level must satisfy the following inequatity

$$
U C L \leq \ln N^{*}
$$

Sharov. A A 1992. The life-system approach: a system paradigm in population ecology- Oicos 63: 485-494

Computer Center Sibenen Branch of Russian Acaderny of Science,

Lavrentyev ave 6, Novosibirsk 530090, Russia
DENDROCHROHOLOGY OP PInus hartwegIi IN PORESTED REGIONS REAR YRXICO CITT

M. A. ALARCON, L. I. DE BAUER, G. SEGURA, J. JASSO and M. ZEPEDA

Mexico C1ty, known as a highly polluted city due mainiy to heavy vehicular traffic and pooralr cliculation, is located in a broad basin at the South of the central Mexican Plateau at an elevation of $2240 \mathrm{~m}$ (7349 $\mathrm{ft}$ ). The urban vegetation, ornamental and native plants, shows poor growth and ocasionsily symptoms resembling those caused by photochemical oxidants.

Forests of the southern mountainous portion of the Mexico City area, mainly pine and fir stands, are currently being threathened by very severe symptoms consisting of stippling, chlorotic mottling and necrosis of the leaves, and general decline leading to tree death.

The patterns of ring growth of Pinus hartweg11, conf1rwed as a very sensitive spectes to oxidants, were analysed using standard dendrochronological rechniques. The results shos a period of growth decrease in the 1930 's apparently related to a below-average precipitation. However, there is a second perfiod of more drastic growth decline, beginning in the early 1970's, with no slgns of recovery up to the present. Th1s growth decline cannot be ascribed to extreme climatic conditions but it can be related to a dramatic increase in air pollutants, mainly ozone transported from the urban area. The decrease of the ring growth of P1nus hartwegit and the increase on ozone levels apparently, began simultaneously and both phenomena show an acelerated trend in recent years.

Colegio de Postgraduados, 56230 Montect11o, Mêx. Mextco

1340

ETHIOLOGY OF CACAO SOFT ROT IN BAHIA, BRAZIL

J. L. Bezerra, E.D.H.N. Luz and G. Niella

A soft rot of cacao pods has been reported in a restricted area of Bahia, Brazil causing limited losses on the production. The causal agent of the disease was identified as Macrophoma sp.. a fungus not previously reported on cacao. Examining materials collected during recent nutbreaks of the disease it vas found that the fungus prociuces a small percentage of septate conidia which make of the genus stagonospora a better choice to aceomodate the pathogen. The same fungus was later found on twigs of corindiba (Trema micrantha Blume) causing dieback. Neither cacao or Corindiba have been reported before as hosts of Stagnospora. Differences in conidial morphology, size and host range in relation to known species of the genus justify to describe the fungus as a new species, stagnospora deceptiva spec. nov.

CEPLAC/CEPEC/Fitopatologia, Kn 22 rodovia Ilheus-Itabuna, Cx. Postal 07, 45600-000 Itabuna-Bahia, Brazil. isolated from pea seeds are easily recognized on the basis certified and uncertified pea seeds lots were infected by the three species. However, the infection was higher in uncertified seeds. The seed infection by $P$. medicaginis var. pinodella was the highest followed by $M$.pinodes and $A$.pisi. The seed infection by the three pathogens was low and did not exceed 5\%. An effect of the pea pod disease severity on the Ascochyta spp. seed rate infection has been shown. The rate of infection increased with the disease severity on the pods. Even pods without any symptoms could produce infected seeds. Seeds with symptoms and without symptoms were extracted from infected pods and the three pathogens were isolated from both categories of seeds. The result shows that it is important to avoid selecting apparently healthy looking pods and healthy seeds for the pea seed production. A rapid technique for screening of pea cultivars for resistance to Mycosphaerella pinodes and Phoma medicaginis var, pinodella at seedling stage is described. Seeds are dipped in a conidial suspension and sown in pots kept at $20-24^{\circ} \mathrm{C}$ for 10 days. The disease severity is evaluated on 10-day-old seedlings by measuring the length of the necrotic areas on the hypocotyl at the point of seed attachment. 
DEVELOPMENT AND SPREAD OF PEANUT BUD NECROSIS VIRUS IN GROUNDNUT AFTER SAP INOCULATION

A.A.M. Buiel (1), D. Peters, D.V.R. Reddy and J.E. Parlevliet

Peanut bud necrosis virus (PBNV) is a serious problem to groundnut production in South Asia. Breeding for combined resistance to the virus and to the vector is the most promising solution to prevent the occurrence of this virus.

We identified several PBNV resistant groundnut genotypes using mechanical sap inoculation. Resistant genotypes showed a reduction in disease incidence (percentage of systemically infected plants) compared to the susceptible check. In this study we investigated the mechanism that causes the reduced incidence. In particular, we were interested whether the reduced incidence results from resistance to infection or from inhibition of systemic translocation. We monitored the development of virus concentration in the mechanically sap inoculated leaf and the spread to systemically infected leaves. In resistant genotypes virus multiplication was inhibited in the inoculated leaf. Nevertheless, when systemic infection occurred in resistant genotypes, virus concentration in these systemically infected leaves was comparable to that in susceptible genotypes. These results indicate that resistance of groundnut to PBNV is in fact resistance to infection.

(1) Crop Protection Division, International Crops Research Institute for the Semi-Arid Tropics, Asia Center, Patancheru P.O., Andhra Pradesh 502 324, India.

1343

Biogeneration of modified atmosphere in small grain bins for the control of storage insects.

M. Calderon, N. Paster and Miguel Mora

ABSIRACT

The principle of this method is to use a deterioration of waste plant material in small containers (biogenerators) for the proctuction of high $\mathrm{OO}_{2}$ concentrations and the consumption of $O_{2}$ (resulting in low $O_{2}$ contents). The produced, lethal to the insocts, modified atmosphere in the biogenerator, is transferred to the grain bin in which insect infestation may take place.

The different parameters of the system: biogenerator-waste materialgrain bin, were investigated and the following findings can be noted: The biogenerator, may be of any volume depending on the quantity and the type of the waste plant material (the substratum) and the size of the grain bin.

The substratum: it was concluded that wet substrata (orange peels, bananas etc) under anaerobic conditions, proctuced markedly more $\mathrm{CO}_{2}$ than dry materials (like wheat bran). The $\infty_{2}$ production was notably large in the case of suggar cane.

The grain bins, were of capacity of $200-1000 \mathrm{~kg}$ of grain.

It should be noted that this method was successfully demonstrated in costa Rica (Central America.)
ECOLOGICAL PROTECTION OF PLANT CROPS BY GROWTH REGULATORS.

Ḅulantseva E.A., Salkova E.G.

Regulation of ripening with the help of growth regulators is a very important problem, the decision of which will allow to have fresh frults during the long time. Important aspect of this problem is determination of growth regulators residue in plant material. It is necessary to establish the contents of residue matter in plant material treated by growth regulators and conformity of these contents to medical recommendetions. Named problems are regarded in examples of green house grown tomatoes and citrus fruits.

A.N.Bach Institute of Biochemistry of Russian Academy of Sciences. 33., Leninsky pr., 117074., Moscow., Russia.

1344

STEROID GLYCOSIDE AND PESTICIDE APPLICATION USED TO CONTROL TOBACCO VIRAL DISEASES

E.D.Calchei, F.G.Jitcu, P.C.Chintea, S.A.Sve

The integrated protection of tobacco allows the provision of the ecological gafety of the method in case regulations and technologies are rationally employed and strictly observed. The plant protection system including presowing treatment of tobacco seeds with steroid glycosides, pharmidofos, soil applied 7 days before sowing, and 1-2 sprayings of plants during vegetation with the insecticides decis ond ridochomecine turned out to be most rational. We consider that this is an optimum treatment as during the whole vegetation period it provides a sefe protection of planta from insect carriers of viral diseeses. Thus, the biological efficiencies in the control of tobscco trips, carriers of the virus of bronzetop of tomsto, mede $78.7 \%$ at the level of injury of 1.2 (control 3.7). High results were obtained in the control of peach moth, carrier of the virus of cucumber mosaic and Y-virus of potato. The injury of plants with pesta did not exceed $2.3 \%$ (control 10.4\%) at the degree of injury of 1.3 (control 3.2). The yielding capacity and quality of tobacco are directiy related with the treatmenta. The yield increase in the experiment made $5.9 \mathrm{c} / \mathrm{he}$, and in total, the yielding capacity mede $30.1 \mathrm{c} / \mathrm{he}$, the output of the top-quality production according to the actuel standard mode $94.5 \%$. The date received show that the presowing treatment of tobacco seeds with glycosides in the optimum combination with the minimum utilization of pesticides is a promising agronomical technique in tobacco growing which allows the exclusion of 2-3 chemical treatments of plentations with insecticides against ingect carriers of vimuses from the existing technology.

P.C.Chintea, Institute of Genetics, Acad. Sc1, 20 Padurilor str. Chisinau 277002 Moldove 
PENICILLIUN SPECIES OF POSTHARVEST DISEASES OF POME FRUITS IN ARGENTINA .

A. Dobra, S. Di Masi, M. Rossini, D. Engler.

Post-harvest diseases are responsible for considerable losses during storage of apples and pears in Alto Valle de Rio Negro $y$ Neuquen. The most important postharvest disease is associated with Penicillium. The objective of this study was to identify the species of Penicilliu. The isolates were obtained from apples and pear rots, flotations tanks in apple packinghouses, storage chambers and from epifitic microflora of the orchards. 71 of the 82 isolates collected, caused blue mold in inoculated apples. 6 species were identified, to say: P. expansum, P. crustosum, P. echinulatum, P. raistrickii, P. brevicompactur and P. raksanii of of the isolates were P. expansun, $10 \%$ P. Crustosum and the 40 remainder corresponded to the others species mentioned and 10 isolates non identified yet. Isolates of each specie were submited to growth test in vitro and in vivo at different temperatures: $22,3,0$ and $-10^{\circ} \mathrm{C}$. The grow was normal for all
isolates at $22^{\circ} \mathrm{C}$. P. raistrickii didn't growth at $0^{\circ} \mathrm{C}$ and $-1^{\circ} \mathrm{C}$ in vitro and in vivo.

Facultad de Ciencias Agrarias, Univ. Nacional del Comahue. (8303) Cinco Saltos, Rio Negro. Argentina.

1347

ROOT AND CROWN ROT OF WALNUT IN SOUTH WEST OF IRAN

\section{A. Fassihiani}

In a survey conducted in Kohkiluieh and Boirahmad province, South West of Iran, Walnut trees showed root and crown rot associated with cracks in lower crown with black gum exudate. From the diseased tissues of the root and crown plated on PDA supplemented with pimaracin ( $10 \mathrm{ppm}$ ), Ampicillin (500ppn) PCNB ( 25ppm), Benomyl ( $10 \mathrm{ppm}$ ) and Rifampicin ( $500 \mathrm{ppm}$ ), a Phytophthora species was isolated. Sporangia were produced abundart $1 y$ on hempseed suspended in non-strile soil extract 24-48 hour after incubation under flourecent 1ight. Sporangial shape varied from pear shape to obpyriform, papillate, caducous, and the L/B ratio was 1.3- 1.4. Oospores formed in singel culture within $3-4$ days both on CMA and in non-strile soil extract. Antridia and oogonia were monoclinous and the average dimention was $16 \times 14$ and $23 \times 19 \mathrm{U}$, respectively. The optimum temperature for growth was $27 \mathrm{C}$ and the fungus had condiderable growth at $5 \mathrm{C}$. Wound inoculation of the fungus to the apple fruit caused extensive brown rot and discoloration at 20C. The fungus was reisolated from the diseased tissues. The foregoing characteristics corresponds to those given for

Phytophthora cactorum. (Newhoek \& Waterhouse, 1978 ).

Fars Agricultural Research Center, P.O.Box 73415-161

Fars, Zarghan, IRAN

STUDY OF SPECIES COMPOSITION OF WEED POPULATIONS IN SOUTH RUSSIA

Eugene KISLIN and Natalia ERMOLOVA

In recent years, much interest has been given to the problem of the overgrowing of sown areas, because considerable crop loss has been observed due to weeds. Investigation is being made of the population dynamics, distribution and harmfulness of weeds. This study Considers the species composition of weeds in some regions of South Russia (Rostov Research Station of the All-Russian Institute for wheat and sunflower. The effect of different sown of maize, winter wheat and sunflower. The effect of different sown precursors on weed populations has been studied. Besides the influence of herbicides which are being used in wheat, sunflower, barley on the South Russia have been tested for their efficiency for the control. For estimating the species composition of weed population ten areas of $2500 \mathrm{~cm}^{2}$ on the sown surface were taken from 15 different places. Total areas of the wheat sowing were included about 120 ha. Density, Erequency and cover of the species in different fallow were measured using $1 \mathrm{~m}^{2}$ quadrates in the case of herbaceous species. The major species were: Amaranthus retroflexus L., Amaranthus blitoides S.
Wats, Ambrosia artemisilfolia L., Capsella bursa-pastoris (L.) Wats, Ambrosia artemisilfolia L., Capsella bursa-pastoris (L.)
Medik., Cheropodium album L., Cirsium arvense (L.) Scop., Medik., Chenopodium album L., Cirsium arvense (L.) Scop.,
Convolvulus arvensis L., Descurainia Sophia (L.) Webb. ex Prant,, Convolvulus arvensis L., Descurainia sophia (L.) Webb. ex Prantl,
Fumaria Schleicheri Soy-Will., Lactuca serriola L., Lamium Fumaria Schleicheri Soy-will., Lactuca serriola L., Lamium Setaria glauca (L.) Beauv., Sonchus arvensis L., Xanthium strumarium $L$. The major species in the winter wheat fields were Cirsium arvense (L.) Scop. and Chenopodium album L. The average population sizes were about 10 - 40 plants per square meter for Chenopodium album I. and $1.5-2.5$ for Cirsium arvense (L.) Scop. In the last two years the increasing of the seedling population of Ambrosia artemisiffolia L. has been observed. For maize the main weed species were Amaranthus retroflexus Lor malze the main weed species were Anaranthus retroflexus L., Convolvulus arvensis L. and Cirsium arvense ( $L$.) Scop. The abundance of Amaranthus retroflexus L. could be as high as 90 - 110 plants per square meter. Convolvulus arvensis L. and Cirsium arvense (L.) Scop. were the most common weeds in
sunflower. The present of maize and barley such as precursor in the wheat sown has been decreased the densities of Cirsium arvense (L.) wheat sown has been decreased the densities of Cirsium arvense (L.) Scop. Sonchus arvensis L., Fumaria Schleicheri Soy-will. and album $\mathrm{L}$. and Thlaspi arvense L. have been observed after application of bare fallow. However, the best yield was obtained after bare fallow application. In the last few years herbicide application has greatly declined due to economic difficulties. Certain weed species are becoming increasingly dominant in the area, particularly Amaranthus retroflexus L., Ambrosia artemisiifolia L., Cirsium arvense (L.) Scop., Convolvulus arvensis L, and Sonchus arvensis L.

All-Russian Institute for Plant Protection, Shosse Podbelskyi, 3, st Petersburg-Pushkin, 189620, Russia Email:vizreriam.spb.su

1348

A NEW APPROACH TO PROTECTION OF NAIZE AGAINST THE SUROPEAN CORN BORER, OSTRINIA NUBILAIIS (HBN.)

A.N. Frolov, D. S.Trishkin, K.D.Dyatlova, M.A. Ohumakov, A. G. Makhotkin

The European corn borer, Ostrinia nubilalis (Hbn.), is one of the most serious pests of maize in Russia. For the Krasnodar Territory and Rostor Region where the pest has two generations it has been shown that the moths of the overwintered generation aggregate on small areas ("action sites"), which are usually 1) located near the maize crons, 2) protected by woodland from prevalent winds, and 3) covered oy not high ( $30-70 \mathrm{sm})$ wild or cultivated vegetation. Action sites can be known beforehand from the field map. Moth densities in action sites are largely determined by the size of a field and the species comosition of plants in the area of moth aggregation and show a high correlation ( $r=$ $0.93-0.99$ ) with the percent of plants infested, number of eggs laid and larval population. Chemical control using synthetic pyrethroid sprays in action sites of overwintered moths permits a considerable reduction in ist-generation larval populations. In addition to high biological efficiency $(80-95 \%)$, less chemical is required as compared to overall treatments because only one-tenth to one-hundredth of the area under crop is treated in this case. Thus, for example, as shown for the Azov district (Rostov Region), for pyrethroid Arrivo (25\% e.c.) applied at the rate of 150 g/ha to moth action sites of $0.33 \mathrm{ha}$ in size during the $50 \%$ moth emergence reduced the damage within the field under crop of

All-Russian Institute for Plant Protection, 3, Podbelsky av, St.-Petersburg, Pushkin, 189620, Russia 


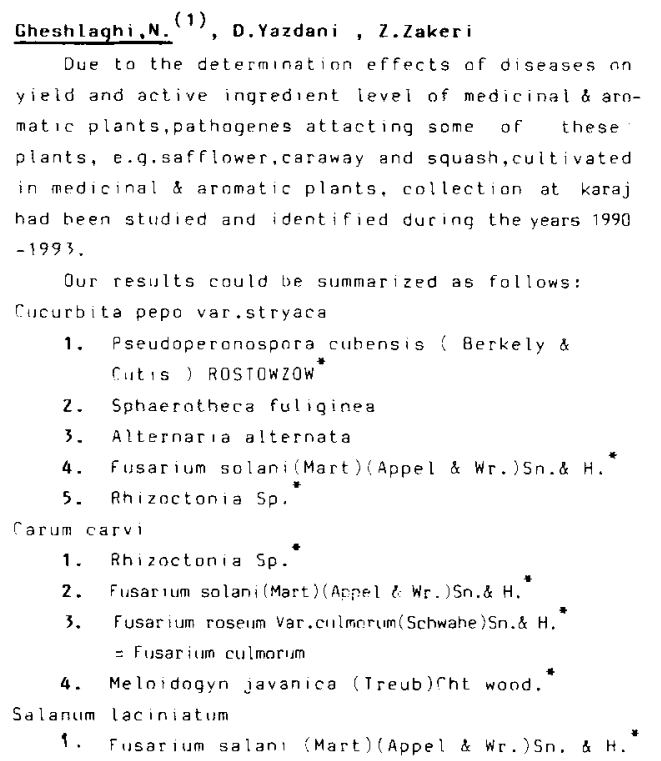

(1): Medicinal and Aromat ic Research Fenter/Jahad Daneshgahi P.0.80X.14155-4364. Tehran- Iran

* : Indicates that the identicated species is regestered for the first time in Iran.
In Vietnam nine hectares of land are used for agricultural of which $7 \mathrm{milli}$ ion ha are used for food crops. Rice is grown on $6.3 \mathrm{milli}$ ion ha $88.5 \%$ of the fieil total area.

During the last few years in the course of the country economic renovation,agricultural production particularly rice production has recorded very important progresses, and has been able to meet wich food demands of domestic consumption and for export.

Rice production in 1993 was 21,9 million, increased by $38 \%$ more than that in 1985 . The average yield per hectare was 3,43 tons, exceeded $26 \%$ in comparison with 1985 .

one of the important factors enhencing these progresses in rice production is the larger use of intensive farming syster and larger introduction in practice in Practice of Integrated Pest

Management of rice.For example.areas infected by major insect pests and diseases of rice in 1993 was inceased by 1,6 times more than that of 1985. However thank to the implementation of IPM in large scale the yield loss was not exceeded $5^{\circ}$ while pesticide use was at the same level of

that of 1985 (about 24 millions USD). Pesticide use was reduced over $50 \%$ at demonstration pilots in Mekong Delta and River Delta.

The mentioned-above data showed that the increase of rice production has became less depended upon pesticide use and more beneficial for farmers, while the reduction of pesticide use made great contribution to improving public health and environment.

FAO intercountry Programne has occupied an important place in IPM implemntation in Vietnam. We join this Programme since 1990. Thank to the technical and financial support of FAO IPM Programe following training courses has been conducted during 1992-1994.

- 15 Traing courses (TOT) for 514 Trainers.

- 916 Farmer field schools (FFS) for 22.900 farmers in 14 provinces.

The participation in IPM Programme has also strengthened the research works on rice pests (BPH, RLF) and created a new relationship between research Institutions and farmers.

The current IPM training is mainly concentrated on rice insect pests, while rice diseases (blast, bacterial rot of grain, bacterial yellow stripe) are also a big problem of rice production, especially in North and Central of Vietnam. Therefore the future IPM Programme should pay more attention to disease control technology in TOT and FFS Training. The cooperation between IPM programme with nationat rice research systen, as well as international Research Institutions should be closer and more effective. NATIONAL INSTITUTE OF PLANT PROTECTION

1352

A SURVEY OF CROPPING PRACTICES AND FOLIAR DISEASES OF PHASEOLUS BEANS IN ETHIOPIA

A. Habtu, I. Sache and J.C. Zadoks

Field surveys were conducted in three major bean growing areas of Ethiopia. Data collected included cropping systems and severities of bean diseases. We used correspondence analysis to characterize differences in disease severity between regions and seasons, and to determine associations between geographic areas and cropping systems, areas and diseases, and cropping systems and diseases. The chi-square analyses suggested a high probability of high plant density. high weediness, high bacterial blight and high anthracnose being associated with area 1 (Rift Valley). In area 2 (Sidamo) there was a high probability of high rust intensity, low plant density and low weediness. Area 3 (Keffa) was characterized by a high probability of angular and floury leaf spots. In area 1 (Rift Valley) low rust intensity was closely associated with year 1 (1990) and high rust intensity with year 4 (1993). Anthracnose and bacterial blight showed no clear association with years. Some linkages between cropping systems and disease severities were indicated. In areas 1 and 2 , there was a high probability of low rust at early sowing and a high probability of bacterial blight at high weediness and high plant density situations. The probability of observing high rust severity at high weediness was low. This study suggests that specific needs of areas, with their own production situations, must be considered in the process of developing strategies for the improvement of production and crop protection in beans.
BIOLOGICAL EFFECTS OF BACILLUS THURINGIENSIS ON THE PARASITES AND PREDATORS OF THE GREASY CUTWORM AGROTIS YPSILON

ㄴ. Hafez, H.S. Salama, R. Aboul-Ela, F.N. Zaki and M. Ragaei

Investigations have been carried out to explore the relation between the pathogen, Bacillus thuringiensis and two parasites namely, Apanteles ruficrus and Meteorug leeviventris and the predator Oriug albidipennis, associated with s. vpsilon. The biology of the parasites showed to be affected in some aspects when its host lervae vere fed on a diet containing B. thuringiensio. A gignificant reduction in reproductive potential and longevity occurred together with retardation in development. The predator 0 . albidipennis was affected in terms of nymphal duration, rate of food consumption and egg production when the larvae were fed on the treated host. The effect of the exotoxin (thuringiensin) on both parasites and predator has been also demonstrated.

Prof. Mahmoud Hafez

Department of Entomology, Faculty of Science, Cairo University, Giza, Egypt (Postal code I26I3) and National Research Centre, Department of Pests \& Plant Protection, Dokki, Cairo. 
STUDIES ON THE RESPONSE OF THE EGGS OF THREE CORCULIONIDS TO CONTRAILED ATHOSPHERPS

\section{Hohamed Youssri HASHEM}

Dept. of Econ. Entomology and Pesticides, Fac. of Agric., Cairo University, Giza, Egypt)

The response of egg stages of the three curculionids sitophilus granarius, Sitophilus zeamais and sitophilus oryzae to three atmospheres varying in their components of $\mathrm{CO}_{2}, \mathrm{O}_{2}$ and $\mathrm{N}_{2}$ at $30^{\circ} \mathrm{C}$ and $70 \% \mathrm{R} . \mathrm{H}$. were studied. These mixtures were $91.6 \% \mathrm{CD}_{2}+1.7 \% \mathrm{O}_{2}+6.78 \mathrm{~N}_{2}: 62.7 \% \mathrm{CO}_{2}+7.5 \% \mathrm{O}_{2}+29.8 \% \mathrm{~N}_{2}$ and $32.73 \mathrm{CO}_{2}+13.58 \mathrm{O}_{2}+53.84 \mathrm{~N}$. The eqgs of $\mathrm{s}$. oryzas were found to be more tolerant to the three atmosphezes than the eggs of $S$. zeamais and $S$. granarius over exposure periods

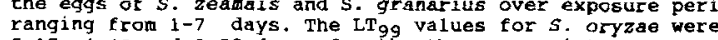
$5.17,4.40$ and 3.89 days for the three gas mixtures, respectively. The most sensitive eggs were the eggs of s. granarius, showing $\mathrm{LT}_{99}$ values of $3.88,3.22$ and 3.02 days,
respectively.
STUDIES ON ONE OF THE MAN PESTS INFESTENG SOME OR CROPS IN EGYPT

A. Taha Hassan, Sadik A. E1-Rais, Soliman M. Soliman

Oil crop (peanut Arachis hypogaesa L. and sesame Sesamum indicum L.) varieties were evaluated for their resistance to the red spider mite Tetranychus urtical Koch infestation, with influence of sowing date and fertilizer sources on population abundance of mites on plants.

A. Peanut crop: obtained results revesled that Balady variety expressed fighly susceptible, while Giza 4 was resistant, but Giza 5 was intermediate to spider mite infestation.

B. Sesame crop: Data showed that Giza 25 variety was more susceptible to spider mite infestation than Giza 32.

In both of oil crops peanut and sesame the rate of infestation is markedly influenced by planting data and fertilizer sources whereas experiments indicated that later seeded crops (15th of May) in both species suffered beavier infestation than that earlier seeded ones (15th of April). Adding nitrogen fertilizer alone had considerable role in the level of infestation of spider mites, followed by mixture of $\mathrm{N}$ and $\mathrm{K}$, while the apposite took place with adding potassium alone.

Plant Protection Research Institute (ARC), Nadi El-Said street, Dokhi, Cairo, 12611, Egyps; Soils \& Water Research Insittute (SWRT), Agricultural Rescarcb Center, Criza, Egypt.
1355

\section{INTRGRATBD DISRASE MANAGEMBNT OF BOTRYTIS GRAY MOLD OP CHICKPEA}

Haware, M.P., McDonald, D., and cenne, J.M.

Botrytis gray mold (BGM) is a major disease affecting chickpea in South Asia. Screening of chickpea germplasm has not revealed any accession with high levels of resistance to BGM. The field trials conducted at Pantnagar, India in the 1991/92 and $1992 / 93$ postrainy seasons indicated that a tall, erect genotype, ICCL 87322 sown at wider row spacing had lower disease levels and higher grain yields than at closer spacings in both fungicide (Ronilan(A))-sprayed and nonsprayed plots. It appears possible to manage BGM in chickpea by sowing tall and erect genotypes (moderately resistant) at wider spacing with judicious use of fungicides. These components are presently being developed into a management strategy for transfer to farmers.

Senior Scientist (Pathology), International Crops Research Institute for the Semi-Arid Tropics (ICRISAT) Asia Center, Patancheru 502324 , Andhra Pradesh, India
1356

Aflatoxin $B_{1}$ contamination of traditional maize stores in four agroecological zones of benin

K. Hell, K.F. Carowell, F.A. Schutz, M.Setamou

Samples were taken from 300 traditional maize stores to determine the distribution and the level of aftatoxins in different agroecological zones in Benin. The impact of different farmers practices on the resultant aflatoxin contamination was evaluated by means of a questionnaire on maize production, harvest practices, storage practices and storage problems with the same 300 farmers. The first survey conducted from September 1993 until January 1994, coinciding with the first month storage in each of the different regions, revealed the presence of allatoxin $B_{1}$ in Benin. The percentage of contaminated stores varied from $5 \%$ in the Sudan Savanna (SS) to $26.3 \%$ in the Southern Guinea Savanna (SGS), with levels of aflatoxin $B_{1}$ varying from $1.7 \mathrm{ppb}$ to $562.5 \mathrm{ppb}$.

A second survey was carried out between March and April 1994, coinciding with six months of storage, in 150 stotes of the 300 initial farmers. The percentage of stores infected with aflatoxins rose overall, particularly in the northern part of the country. In the SS $56.7 \%$ of the stores showed the presence of aflatoxin $\mathbf{B}_{1}$. in the SGS the percentage of stores infected rose minimally, with $27.5 \%$ of the stores contaminated by aflatoxin $B_{1}$ after six months of storage. The level of aflatoxin $B_{1}$ ranged from $3.1 \mathrm{ppb}$ to $500 \mathrm{ppb}$ in the second survey.

Farmer's practices that have an influence on the raised aflatoxin levels are: drying before storage, quality of the husk cover of the cobs. selection of damaged grains before storage and use of indigeneous plants or application of pesticides to protect the store during the storage period. Attack of insects in the field and in storage increased the likelihood of contamination with aflatoxins in traditional storage structures.

Kerstin Hell

International Institute of Tropical Agriculture

Biological Control Centre for Africa

BP 08-0932

Cotonou .

Republic of Benin 
POPULATION DYNAMICS OF Pseudomonas cichorii ON LETTUCE PLANTS AND INFECTION ROUTE OF $P$. cichorii

\section{Y. Hikichi, A. Saito and K. Suziki}

Populations of Pseudomonas cichorii, a causal agent of bacterial rot of lettuce, were monitored on lettuce plants cv. Success in a field at Iwate, Japan. During head formation, the bacterial populations on leaves were lognormally distributed, and those on outer leaves and head leaves increased progressively. And leaves, on which the bacterial populations were above $10^{5} \mathrm{efu} / \mathrm{g}$ fresh weight, had symptoms. The bacterial populations on the outermost head leaves positively correlated with those on the outer leaves. The percentages of lettuce plants with rotten head leaves positively correlated with that with diseased outer leaves. These results suggested that the bacterial population levels were directly related with disease incidence and that the bacterial populations on head leaves were related with the bacterial populations on outer leaves. When sections of head leaves were stained with fluorescein isothiocyanate-conjugated antibody against $\boldsymbol{P}$. cichorii and were observed under a fluorescence microscope, specific fluorescent specks derived from $P$. cichorii were observed in stomata of non-diseased leaves and intercellular spaces of epidermic and mesophyll of diseased leaves.

Iwate Biotechnology Research Center, 22-174-4 Narita, Kitakami, Iwate 024, Japan

FORMATION OF DEW AND THE DRYING PROCESS WITHIN CROP CANOPIES

\section{A.F.G. Jacobs and J. Nieveen}

Abstract. Experimentally outdoor, dew profiles within a plant canopy has been measured after dewy nights in a maize canopy and in a bariey canopy. In our canopies it appeared that dew profiles can show similar shape for various dew events. Clear unique dew profiles occurred when the plants are stiff, the stems as well as the leaves. This result suggests that it is likely to assume here that these similarity profiles are a consequent of the more or less constant foliage area distribution of a plant canopy during a growing season. Flexible plant canopies, however, behave more complex depending on the structure of the canopy and depending on the wind regime during the early hours.

The course of the drying process within the plant canopy has been monitored until the collected night-time free liquid water within the canopy was evaporated. Initially, the drying process was faster in the upper layers of the canopy. Later, when the moisture distribution has become more or less even through the whole canopy, the drying rate became approximately uniform.

The amount of dew and the drying rate during the moming hours have also been estimated by applying simple model simulations. The calculated total amount of dew agreed with the measured amount to within $6 \%$. The measured drying process, however, lagged about 1 hour behind the calculated one. Possible causes of this difference are the dripping effect and drainage down the stems which were not included in the calculations.

Department of Meteorology, Agricultural University PO Box 9101 ,

NL 6700 HB Wageningen, The Netherlands.
FACTORS AFFECTING THE DEVELOPMENT OF DARK LEAF AND POD SPOT (ALTERNARIA BRASSICAE) ON WINTER OILSEED RAPE (BRASSICA NAPUS) IN THE UK

C. $x$. HONG, B. D. L. FITT

Studies in controlled environment cabinets and glasshouse conditions demonstrated that Alternaria brassicae can produce symptoms on leaves of potted oilseed rape (Brassica napus) plants at temperatures between 6 and $25^{\circ} \mathrm{C}$ with a minimum wetness duration of $6 \mathrm{~h}$ at the extremes of this temperature range. On these leaves and on pods on detached racemes the minimum wetness duration required for symptom development was $2 \mathrm{~h}$ at temperatures between 15 and $20^{\circ} \mathrm{C}$. The incidence and severity of symptoms increased and the length of the incubation period decreased very significantly $(\mathrm{p}<0.001)$ with increasing temperature from 6 to $20^{\circ} \mathrm{C}$ and increasing wetness duration from 2 to $24 \mathrm{~h}$. At $25^{\circ} \mathrm{C}$ the incubation period was longer and the severity of symptoms less than at $20^{\circ} \mathrm{C}$.

The crucial weather factors affecting epidemic development on winter oilseed rape are low temperatures in winter and wetness duration on pods in summer. Throughout the autumn and winter wetness is not generally limiting and the number of dark leaf spot cycles and survival and efficiency of inoculur are determined by temperature. Therefore the amount of inoculum available in spring during the stem extension of winter oilseed rape plants is greatly influenced by the temperatures during autumn and winter.

Alternaria brassicae is only damaging to winter oilseed rape if severe epidemics develop on pods in the summer. At that time temperature is not limiting. The limiting factors are the availability of inoculum and the occurrence of sufficient wetness duration for infection to occur. If the UK climate changes so that the temperatures in autumn and winter increase, it is possible that more dark leaf spot will develop and more inoculum will be available in the spring. Therefore dark pod spot could become a more important problem in winter oilseed rape. An integrated strategy for control of the disease needs to be developed.

IACR-ROTHAMSTED

HARPENDEN

HERTS. AL5 2JQ

UNITED KINGDOM

\section{0}

IWPROVED WICK AVALUALION GF STHR RUST UREDOSPORBSS VIABILITY AFINAR STORAGE

Karouk V.V., Plotaikova J.M."

The means of determination of spore viability after storage relating to phytopathogenic fungi are the same tiat are usualiy applied to otiner fungi, and first of als that is definition of per cent of germinated spores, i.e. spores formed frowth tubes. But ior many pathogens that is not quite true, because growth tubes are not infectious, and therefore they are not eyt sertifica of the spore ability to reproduce themselves on the host plants. In particular, rust Iungi uredospores infect of plants by weans of infection structures namely: appresioria, intection pegs, substomatal vesicles ara iniection inprae. The intection stmuctures iormation from uredospores in vitro can evoke not at all rust jpocies, wile at least two forma speciales of ruccinia gratuinis - I.sp. uritici and f.sp.secalis, as it is shown in our experifrents, both in difierent degree possess of such abili$t_{y}$, and they can easy ditilerentiate applessoria on water agar caused. by heat sncck treatment $\left(30^{\circ}, 6\right.$, 3 ) in an one hour after the urecospores begin sroving. ie found that uredospores or $P$. rrauinis $\ddot{t}$.sp.tritici in an one nonth after storage in liquid nitrocen or in reirigerator jerminated on 66\% and on 29\% accorcingl, and dirierentiated of infection structures on $42 \%$ and 14\%; تresh spores (control) save $75 \%$ or cerminatior and $56 \%$ of infection structures ilferentiation. Ihus me see, that infectious ability cí uredospores brings down more fast, tian their ability for serminatine. lherefore, the cefinition of P.graminis uredospores viability based on their capability of iriection structures aifiereotiation it seets to be nore precise, than one baseci on ability for fermination. We propose to use or this simple and quick rethod Ior evaluaticn cf stet rust uredospores viability after storage.

20735 . Institute of Beperimental Botany of the dcadeny of sciences, Minsk, it.jkorina str., 67, Belarus.

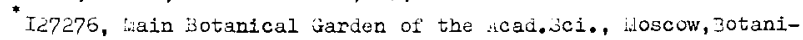
cul str.,4, iussia. 
BARLEY SCALD IN UZBEKISTAN - ISOLATION AND OILTIVATION OF PHYNCHOSPORTUM SECALIS CULTURES

Guz L.N., Khagarov B.A., Postrikova E.N.

Barley scald caused by coelomycetous funzus Rhynchosporium secains (Oud.) Dayis is the second important disease (after powdery mildew) of the culture in Uzbekistan. Its epidemics have occured on about $10 \%$ or total barley 11elds during the last 5 years (Khasanov, 1992). Thus investigations were begun to study pathogen biology and methods of barley protection from scald. Twelve specimens of infected leaves were coliected in Uzbekistan in 1993 and 1994. Flve of them produced 1golates of scald fungus. To select favorable medium ilfteen media were tested which contalned the following components in varlous quant1ties: extracts of ma1ze, yeast and potato, peptone, asparagine, casein hydrolyzate, home-made V-8 juice, solution of microelements, modifled Czapek's medium, lactose, sucrose, agar. Characters of flve fi.secalts isolates (colony morphology, color, growth rate)varied on these media considerably. The most ravorabie media contained (grams per liter):(medium 1) - maize extract - 5, peptone - 5, $\mathrm{NaCl}-5, \mathrm{CaCO}_{3}-0.5$, sucrose -20 , agar -10 , water -11 ; (medius 5) - yeast extract - 7,5, casein hydrolyzate - 3, lactose 37. $\mathrm{KH}_{2} \mathrm{PO}_{4}-1, \mathrm{MgSO}_{4}-0,5$, solution of microelements $-1 \mathrm{mI}$, agar - 10, water - 11 . Growth of lungus 1solates started on 7 8-th day of incubation and on 30 day colony size reached 35-4tm. Corresponding values on untavorable media were 15-20-th day and $3-5 \mathrm{~mm}$. Fungus colonies as microscopy showed, consisted of a number of typical conidia. They were used as inoculum in tests of 12 barley cultivars for resistance. Investigations continue to improve confdiation of fungus and to construct an synthetic pathogen population.

Institute of Genetics, Academy of Sclences.

700000 Tashkent, Glavpochtamt, P.0.B.97, Uzbek1stan

\section{CORTON PHYTOPHTHOROSIS CAUSATIVE AGENP IN UZBEKISTAN}

Shaporatova R.A., Thasanov B.A., Bazhanova R.Y., Aboutharimov A.A.

It 1s known from literature that the different fungus species of Phytophthora genus can be cotton disease causative agents. It is P. nicotianae var. parastitica in Australla, P. capsict in the USA, P.boehmertae - in China. The study of cotton phytophthorosis causative agent in Uzbekistan has been conducted to determine 1ts taxonomic status. Several 1solates of the fungus of diflerent geographic origin have been obtained for this purpose. The study of cultural, morphological and blological characters connected with species differentiating criterla has been carried out. The1T variability depending on an isolate and growth cond1tions has been detected. The Pungis culture grows rather quickly, 7-9 mm per day. The colony growth type is variable: radiate, fairly flupfy or rosette pattern ith striations, sometimes stellate"chrysanthemu" pattem. Myceltum is brarched with short projections, sometimes with swellings or gnarls. Chiamidospores are not formed in all isolates. Sporanglophores usually do not dilfer lrom other hyphae and sometimes are branched. Sporangia are oval, broadly ellipsoldal, orblcular, ifmoniform, obpyriform or of an 1rregular shape, $15-40 \times 13-20 \mathrm{~mm}$, papilia falriy protuberant, sometimes inconspicuous, without pedicel. Sporangia are non-caducous, they shed w1th a length of hyphae. Sporangla germinate only by the growth of a germ tube w1thout zoospore formation. Sexual organs (oogonia, anthertdia, oospores) are formed extreme rarely. Cardinal temperatures: $m \ln 5 \mathrm{C}$, for some 1solates $8 \mathrm{C}$; opt. $22-25^{\circ} \mathrm{O}$; max $31-32^{\circ} \mathrm{G}$. All the 1solates are low seng1tive to malach1te green. The absolute coincidence or characters of the 1solates studied with the degcription of some species in the known keys to species of Fhytophthora de Bary has not been detected. The study of cultural, morphological and physiological characters variability by means of other modern methods of invest1gations and by usage of much more 18olates w1ll allow to elucidate pathogen species.
CHANGES IN COMTON FIEDIS' PHYTOSANITARY STATE OF UZBEKISTAN

Agarkova I.V., Whokhlacheva V.E., Shapovalova R.A., thrasanov B.A.

Surveys of diseases were made in all 13 cotton-growing regions of Uzbekistan in 1992-1994. In total 110 samples of diseased plant parts were collected, includtng 72 ones of leaves, 27 - bolls and 11 - roots and crowns. The samples were analysed using m1croscope, wet chambers, pure culture technique etc. In laboratory. Results obtalned have showed the expansion of harmful diseases. Besides prevalling earlier diseases (Yerticltztum and fusartum wilts, Xanthomonas leaf spot and stem and boll rot, root rots: an increase of lesf spots, caused by Alternaria, Stemphyitum and some seplparasitic fungus spectes was observed. Changes were also seen in species composition of cotton seedling blight pathogens complex. In addition to common seedling parasites as Rhizoctonia, Fusarium and Thtelaviopsts species young infected plants yielded on agar media also Acremonium (1solation frequenty 36\%), Pythium $(9,1 \%)$, Phytophtora $(15,8 \%)$ spec1es. Common cotton (Gossypium hirsutum) cult1vars became more infected with Fusorium wilt and number of diseased plants was about $60 \mathrm{~s}$ in some flelds. Development of Verticinlum wilt was registered on ine-1ibre cotton (G.barbadense) cuitivars (maximu $27 \%$ diseased plants). A new, earlier unknown disease of cotton, caused by Phytophthora sp. was found in mary regions of Uzbekistan. Its main symptoms were boll blackening, underdeveloping, dying off and mumification. Number of fine-fibre cotton plants w1th such symptoms reached 90-100\% on some flelds. According to distribution and importance in Uzbekistan cotton diseases can approximately be arranged as following: Phytophthora blight - Fuacrtum w11 Vertictlitum wilt - leaf blotches and spots of var1ous etiolozy - Xanthomonas disorders - seedling blights and root rots.

Ingt1tute of Genet1cs, Academy of Sclences.

700000 Tashkent, Glavpochtamt, P.0.B.97, Uzbekistan.

1364

\section{PHYPOPHTHOROSIS - NEW DISEASE OF COTTON IN UZBEKISTAN}

Shapovatova R.A., Enasanov B.A., Bazhanova R.Y., Abchkartmov A.A. The most w1despead cotton diseases in Uzbek1stan are Vertictlltun and Pusaritum wilts, Xanthomonas leaf spot and stem and boll rot, root rot, Alternario leafspot. Recently the disease wh1ch differs in 1 ts symptoms from the abovementioned ones has been detected there.The speciffc character of plant infection were expressed in boll blackening and murmification, fiber rot and brown, with 1rregular borders, leaf spot, often with destruction and rall damaged t1ssues. The goal of our investigations was to determine the disease etiology, 1ts area and cotton damage degree. The infectlous nature of the disease has been proped by performing Koch's triad (1solation of the causative agent into a pure cultu$r e$, plant inoculation, pathogen reisolation). The causal organ1sm was the representative of the lower fung1 of Phytophthora genus. It was revealed by the survey of cotton fields that the territory of Uzbekistan could be divided into three zones according to the phytophthoros1s damage degree. The northern part of the Republic (Karakalpakstan and Bukhara and Navol regions) 1s less subjected to the pathogen inpluence. Cotton damage was 5-10\% there. The cultivated varletles are C-4727, Bukhara-6 (Gossyplum hirautum). The most part of the whole cotton growing zone of Uzbekistan can be considered as the moderately damaged (10-25\%): Ferghana valey, Tashkent, Syrdar'ya, Dzhizak, Samarkand, Kashkadar'ya regions. Ferghana 3, Namangan 77, K1rg1zskiy 3, C-6530, C-9070 varieties (G. nirsutum) and some others are been growing there. The cotton flelds of the southern part (Surkhandar'ya reglon) were most damaged. The yleld losses on the particular field reached $30-50 \%$ as a result of the boll rot and mumisication. The main curtivated varleties in this region is represented by the most raluable pine flbrous ones: Termes-16, Termez-24 (G. barbadense). Prytophthorosis problem in Uzbek1stan is not restr-1cted by cotton, lately the disease has had the epidemic character on tomato, melon, water-melon, pumpkin.

Institute of Genetics, Acadeny of Sciences.

700000 Tashkent, Glavpochtant, P.0.B. 97, Uzbek1stan 
Mostovoy V.A., Postnikova E.N., Khasanou B.A.

Areas under wheat have roughly been increaged in Uzbekistan since 1992 to provide people with own bread grain fully. Intens1fication of wheat production takes place and this process causes changes in pathogen species constitution and plant mycobiota. The following diseases were revealed on wheat lields in varlous regions of Uzbekistan in 1993-1994: loose smit (Ust1lago tritici (Pers.)Rostr.), common bunt (Ttlletta caries (DC.) Tul., T.foe$t i d a$ (Wallr.). Liro a.o.), powdery mildew (Erysiphe graninis DC.f.sp.tritict Marchal), yellow rust (Fuccinta atriformis West.), brown rust (Pucctnia recondita Rob.: Desm.), tan spot (Fyrenophora tritici-repentis (Died.) Drechs.), Septoria leaf blotch (uycoephaerella grominicola (Fuck.) Schroet.) and spot blotch (Cochliobolus aativus (Ito et Kur1b.) Drechs.: Dastur). In previous years prevalling diseases of wheat included yellow and brown rusts and powdery mildew; moderate to haevy yellow rust epldemics repeated once every 5-7 years. During the last years intensive development of tan spot, Septoria leaf blotch and powdery mildew on wheat is being obsemed in aome regions. Intensity of these diseaseg on wheat leaves reached 50-70\% and more in some fields at the heading-anthesis stages. It is possible that further developing of the diseases can occur because of repeated growings of wheat on the same nonirrigated areas and expanding imigated ones.

Institute of Genetics, Academy of Sciences.

700000 Tashkent, Glavpochtamt, P.B.0.97, Uzbekistan
PPEVICUR 607 SL

Subkhanculov A.A., Shapovalova R.A., Yakubov B.A., Khasarov B.A.

Frytophtoro bligth is one of the most harmpul diseases of cotton in Uzbekistan. Protection measures against this diseese here are not worked up. The goal of our investigations in 1993-1994 was the testing of Previcur 607 SL for protection of cotton plants against this disease. Apron 35 WP was used as a standard. The experiments was conducted in Surikhandar'ya reglon on the state farm flelds of Termez 16 fine-flbre cotton variety (Gossyplum barbadense). The experiment includes folloving varlants: seed treatment with Previcur - $21 / t$, seed treatment with Previcur $21 / t+$ plant spraying with Previcur $-1.51 /$ ha, plant spraying with Previcur - 1,5 l/ha, standard - seed treatment with Apron - $1 \mathrm{~kg} / \mathrm{t}$ and nontreated control. The size of a plot was $100 \mathrm{~m}^{2}$ at four replications. Seeds were treated with funglcides 10 days belore sowing. The cotton plants were sprayed with Previcur at the beginning of a buading phase. In the result of the testing it was ascertained that one spraying of plants with Previcur at a budding phase w1thout seed treetment did not protect crop against Phytophtora blight. The combination of the seed treatment with plant spraying and seed treatment only are more effective. The significant reduction in number of infected bolis was obtained in both variants and, as a result of this, increasing in the yleld of raw cotton in comparison with nontreated control and standard took place. The reduction in number of infected bolls was from 31,2 to $43,6 \%$ and yield increasing - irom 19,6 to $58,8 \%$.

Institute of Genetics, Academy of Sclences.

7C0000 Tashkent, Glappochtamt, P.0.B. 97, Uzbek1stan
1367

BIOCHFMICAL TNVESTTAATION OF FUNGUS - CALISATIVE AGENT OF GOTTON PHTTOPHT:HOROS TS

TAhohenko G.S., Yunuskhanov Sh.Yu., Shapounzova R.A.

Tr ievelop effoctive nasasures for sotton phytophthorosia control the Investrgating have been conducted to study the causative agent billogy of this diatase and meshaniam of host-pathogen interaction. The differences in electropinoretio protein spectrum of elve fhytophthory stratns have been detectel. The fungus strifias yudfel isan alter lepending on nutrient medtum. The stralre atudied were establioled to have the different tonenzyne composition (erlatase, proterse, o-diphenoloxtdase). The double diffusion in agar gel of fungus-pathoen and cotton-host prote-

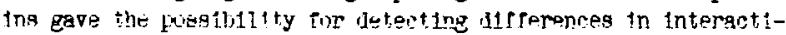
al of water-yolubie pittein rungus traction with protelns of seris of resistanl aril susceptible cotton vartetles.

Inetitute of Genetios, Acadiny of Sclences.

7no000 Taklkent, Glavpochtamt, P.O.B.97, Uzbek1stan
1368

\section{EFFECT OF THE INFECTION OF DIAPORTHE CTTRI ON THE QUALLITY OF CITRUS UNSHU FRUIT}

\section{T. Konno, Y. Homma and S. Wakimoto.}

Satuma mandarin Citrus unshu is very important fruits during winter season in Japan. The quality of fruits consists of sugars and acids content. In order to clarify the effect of citrus melanose on the quality of fruit juice, we tried to compare the acid and sugar content of inoculated fruits with them of nomal fruits. Infected twigs as inoculum was the twigs artificially inoculated with the causal fungus (Diaporthe citri). The infected twigs were hung for several days near above fruits on free. Then after rainfall, these twigs were taken off the fruits. We picked inoculated fruits and normal fruits at intervals of two days (from September 10, to October 18, 1991). We pressed juice from 12 fruits separately at twelve oclock every time, then, filtrated fruits juice and analyzed total sugars and acids of fruits juice. The total acid content of normal fruits was $1.4-1.7 \%$, and total sugar was $7.8-8.2 \%$. The result of analysis, total acid content of inoculated fruits was always $0.3-0.5 \%$ high and total sugar was also $0.3-0.8 \%$ low. Similarly, we picked inoculated fruits and normal fruits at intervals of two hours (from September 10 to 12, 1991). And we analyzed the organic acids (citric, malic acid) and sugar (glucose, fructose and saccharose) content in Citrus unshu fruit by HPLC (senshu HPLC system). The result of analysis, both of the citric acid $(0.2-0.5 \%)$ and the malic acid $(0.02-0.07 \%)$ from inoculated fruits was higher than them from normal fruits. On the other hand, sugar (glucose $0.2-0.4 \%$, fructose $0.2-0.4 \%$. saccharose $0.1-0.2 \%$ ) content of inoculated fruits was lower than them of nomal fruits. Therefore, organic acid and sugar content in Citrus unshu fruit was easily affected by the infection of melanose fungus, $D$. citri. Therefore, in order to get the fruits of high quality, it is necessary to control not only citrus melanose but other diseases with fungicides or any other way. We will report our trial for control by sodium bicarbonate formulation (Harmomate) or potassium bicarbonate formulation (Kaligreer $\left.{ }^{(}\right)$).

Tokyo University of Agriculture, 1-1-1, Sakuragaoka, Setagaya-ku, Tokyo, 156 , Japan 
THE THREAT TO SUGAR BEET PLANTATIONS OF PIESMA QUADRATLM FIEB. IN POLAND

\section{A. Korcz, L. Zielinska, A. Wojtowicz}

In Poland beet leaf bug (Piesma quadratum Fieb) usually appears on sugar beet plantations in first decade of May when apple trees blossom and leaved forests become green. Beet leaf bug always exists in higher or lower intensity in certain regions in Poland. In these regions sugar beets are infected by bet leaf curt virus transmited by its vector beet leaf bug. Infection of beets results in lower content of sugar and lower yield

\section{Institute of Plant Protection}

Miczurina 20

60-318 Poznań

Poiand
SNVIROMMEYTAL EFFBCTS ON THE DBVELOPNENT OF STEM CANKFR AND ROOT ROT IN SOYBEAN IN MOLDOVA

Koretaky Lruba, Lupashky Gellna, Sokh1rka Angela

It is difficult to carry out the selootion for goya stocks resistant to rusariose root rot and atem canker, most harmful diseases under conditions of Moldova, due to the strong influence of onvifonmental ocological factors on the diseage ooures. Barlier we have found that poeltive low temperatures and high lovels of humidty contribute to the orhancement of soya oultivar suzceptibility to fuariose. In I993-I994 wo studied the influence of abiotic factors (total alnimam and maximum tomperatures, total preoipitation) on the rate of Infection with Fuarium root rote (FRR), cotylodon fusarioge (CP) and atem canker (SC) of a complement of cultivarg differing in the duration of the regetatiwe period and the time of sowlng. A strong interrelationship was artabliahid betweon FRR and $C F$ development both at the firet period of sowing $(r=0.97)$ and at the second one ( $r=0.82)$. It was marked that there was no aigalifcant difference betwoen early - and late-ripening oultivara for PRR infection, owever early-riponing cultivars wore more affected with CF. It was also established that late-ripening cultivarg wore more sugceptiblo to SC at both poriods of sowing. Berly-ripening cultivare wero supposed to escape the digease due to the fact that the meximum development of the pethogen did not coincide with the eusoptible developmental etage of planta or because they were more infeoted with SC. It ahoud be mentioned that the weak development of fusariose contributed to the enhanced infeotion with SC. The coefficient of correlation between these indices made $-0.9 \mathrm{I}$. This tolls about the presenos of antagonisw between the agonte of the diseases wich was confirmed by the in vitro oulture.

The Institute of Genetics of the Acadeng of Sclences of the Republic Moldova, 20 Padurilor str., 277002


Bogdan Rorić

Attack of some wheat diseases can greatly affect yield. Therefore, it is not surprising, that scientists want to reduce them to a minimum. In Croatia not surprising, that scientists want to reduce them to a minimum. In Croatia the beginning of work on breeding wheat for resistance to diseases. The first targeted breeding for resistance of wheat to certain diseases was performed by dr. Mirko Koric in Osijek (1929-1946) and be subsequently the well known wheat variety as $U-1$. For une of its perents he used the Marquis which in resistant to stem rust. After the World War II, the work has recomenced in Zagreb and since 1947 the work in this fleld has continued for today. At that time stem rust was the wost serious disease and in the years of high severity, like in 1928,1932,1954 and 1956, ylelds were reduced up to 80\%. For this reason it was irportant to know which races and effectiveness of $\mathrm{Sr}$ resistance genes to the fungus Puccinia graminis f.sp.tritici were prevalent in Croatia. Itwas also Important to test different sources of resistance to find those that prove effective to stem rust. The work was so suecessfuI that today almost all wheat varieties and wheat lines in Croatia possess resistance to this disease.

By developing high-yjelding wheat varieties, and by applying suitable euitural practices, another disease known as powdery mildew became increasingly evident. Work on breeding for resistance with phytopathological investigations to this casual organism i.e. the fungus Erysiphe graminis f.sp.triticic wes initiated it 1964. The above breedins program yielded rearkable results, tharks to the investigations of pathotypes and Pm resistance genes through which effective sources of resistance were found. During many years of investigations the best effectiveness was exhibited by the Poulb and Pm2+MId resistance genes.

Problem of wheat diseases did not stop there. New diseases appeared as septoria nodorum bloteh and fusarium head blight. That infeluenced the expansion of phytopathological activities with new investigations started in 1978 and 7980 . This time, investigations of fungi Leptosphaeria nodorum and Fusarium gramineamum were started. Program on the studies of this fungi prises som main directions. Laboratory 1nvestigations and producing the most viruient isolates of Leptosphaeria nodorum and Fusartum graminearum in pure culture to be used for artificial infection at the adult stage in the fleld and at the stage of seedling in the greenhouse. In those stages sources of and at the stage of seedling in the greenhouse. In those stages sources of resistance to this diseases were found and determined. All phytopathological investigations, so far, have dealt with the study of fungi as causal organisms of various diseases, but a step forward was made when the program of breedin sources of resistance was started. The idea was to incorporate through breeding as many resistance genes as possible into certain sources of reststance both to one or several diseases. Thus improved sources of resistance developed in so called "preselection" are given to breeders to include them in their programs of developing new wheat varieties either resistant or tolerant to 
SPATIAL PATTERNS OF SPEAR ROT AND 'MARCHITEZ SORPRESIVA', FATAL DISEASES IN OIL PALM IN SURINAME

H.I. van de Lande, L. Jubithana and S.C. Bram

Abstract

Spear rot (fatal yellowing) and 'marchitez sorpresiva' (sudden wither disease, 'hartrot' or marchitez) are (sudden wither disease, 'hartrot or marchitez) are sorious diseases in oil palm. The causal agent of spear rot is yet unknown. Marchitez is associated with years Victoria, a 1,700 hectares oil palm plantation, was devastated by spear rot. Phedra, a 875 hectares plantation, is affected by both, spear rot and marchitez. A geostatistical technique (semivariogram anslysis) was used to describe and analyze initial spreading patterns of respectively, spear rot and marchitez affected trees in the field. The diffuse spreading pattern of spear rot illustrated by the random and linear models makes disease control difficult if not impossible to realize. Focal development of spear rot is typically ellipsoid or funnel-shaped with a preferential heading to the west (the dominant wind direction). The increase of the range in the spherical model gives indications of the extent of focal expansion over time. Isolated cases of marchitez can be controlled in the initial epidemic stage by destroying the diseased tree and by treating the tree circle of the affected tree including the immediately neighbouring supposedly healthy trees with an appropriate insecticide. Succesful control was attained in an area which was under high disease pressure from an adjacent marchiter affected planting. Spatial analysis of tbis marchiter aree revealed spreading patterns according to the spherical revealed spreading paterns according to marchiter focl opidemic stage of marchiter, focal formation and growth, resulting from short distance spread, is expressed by the short rapge and the low sill ia the spherical model. Later in the epidemic, diffuse spreading patterns resulted from ivation whor not disectuated timely or was not effectuated at all. Poo disease management has led to a situation where both, pil

University of Suriname, Faculty of Technological Sciences, P.0.Box 9212, Paramaribo, Suriname, South America.

MODELS OF MICROBIOLOGICAL PROCESSES FOR THE CONTROL OF DISEASES IN POTATOES UNDER THE HERBICIDES IN SIBERIA.

Myronenko L. B., Naplecova N.N.

Herbicides and other pest1cides have a long period of decomposition in siberia because of 1 ts nature conditions. The influense of zellek and zenkor herbicides on biological activity of the grey forests and leached chernozem of Western siberia on potatoes has been studied. Potatoe waz grown by Holland technology the herbisides are used in $0,4 \mathrm{~kg} / \mathrm{ha}$ and $0,6 \mathrm{~kg} / \mathrm{ha}$ respectively. That pecticides has long action on the microblota under Siberian conditions. our fleld experiments showed that the herbicide concentration inibits the respiration and nitrogen-fixing both in grey forests and leached chernozem. The decomposition of cellulose in nature conditions and the microorganism biomass changed from $2 \%$ to $10 \%$ and from 0.42 to 0,55 correspondingly. The influence of herbicides on nitrogen-fixing and respiration in the different kinds of soll studied during the potatoes vegetation period was in the range of 0,36 to 0.66 from 3.7 to 8.3 correspondingly. The respiration and nitrogen-flxing both in the grey forests and leached chernozem are the ecological targets. These changes caused by zellek and zenkor affected the biological circulation of carbon and nitrogen. The changes in the solls bioactivity under herbicides combined with the potatoes resistance to reduce phyopatogenes; Rhyzoctonia solan1. Phytophtora infestans. The comparison of potato diseases and ecological targets showed that the sickness rate decreased where bloactivity was larger, 1.e. the targets were more resistant. This means that the soll in this case is less toxic. According to our results, two model microblological processes are the best for blological control of potato diseases: respiration and nitrogen-fixing.

630039 Russia Novosibirsk Agrarian Univers1ty Dobrolubova 160

1375

Spec1es and Strain Composition of Viruses Infecting Oat and Barley in Central and North - Hestern Regions of Russia.

V.S. Obruchkov, E.E. Radchenko, Yu.A.

Var1tsev, T.N. Erokh1na

Phytopathological evaluation of small grains conducted during 1993 - 1994 in Central and North - Western regions of Russia permitted the following diseases to be recorded as most common: dware barley plants with yellow and mosa1c leaves and oat with red and mosa1c leaves. As a rule, these plants were invaded by the aph1d species : Rhopalosiphum padi and (or) sitobion avenae. Testing of 11eld samples of these plants by DAS - ELISA using peroxidase - labeled monoclonal antibodies to Barley Yellow Dwarf Virus (BYDV) permitted the identification of this v1rus. Blological tests with different aphid species (R.padi, R.maidis, S.avenae, Schizaphls graminum) demonstrated that oat and barley crops in central and North - Western regions of our country were infected by two strains of the V1rus: RPV and PAV. Besides BYDV, an unknown virus was 1solated from soll samples and oat plants with red and mosalc leaves collected near Tyer (central region of Russia). Examination of the purifled v1rus under an electron microscope (H1tach1 $H$ - 300) showed that rod - shaped particles are of about $350-700$ nm long and $21-24 \mathrm{~nm}$ w1de. As the rod - shaped v1rus is transm1tted through soll by the fungus Polymyxa graminis 1t can be tentatively attributed to furoviruses.

A11 - Russian Plant Protection Institute (VIZR), 3, Podbelsky shosse, St. - Petersburg . 189620, Russ1a.
1376

EVIDENCE FOR POLLEN AND SEED IRANSMISSION OF IHE COCONUT CADANG-CADANG VIROID IN COCOS NUCIFERA

E.P.Pacumbaba, B. Zelazny, J.C. Orense and E.P. Rillo

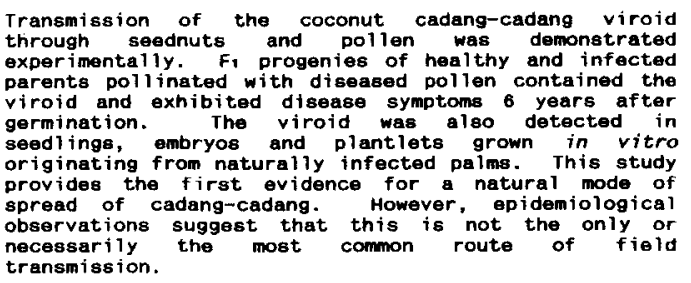

Philippine Coconut Authority, Albay Research Center, Guinobatan 4503, Albay, Philippines. 
EFFECTS OF BIRD CHERRY-OAT APHID AND CEREAL, LEAF BEETLE ON YIELD OF WINTER WHEAT VARIETIES

\section{Papp}

Resistance zests based on infestation by bird cherry-oat aphid (Rhopalosipfium padi L.) and estimation of leaf-feeding damage by cereal leaf beetic (Oulens melanopus L.) were carried out on 18 and 26 winter wheat cultivars, respectively, in 1987-1994.

Grain yield and thousand-kernel mass were measured in infested and non infested control plots in ages covered by insect nets. Highly significan: differences were found between genotypes in infestation by bird cherry-oat aphid and feeding damage by cereal leaf beetle as well as in losses to grain yield and shousand-kernel mass. Infestation by bird cherry-oar aphid varied between 20\% ('GK 7ombor') and 63\% ('GK Lili'). The most resistant cultivar, 'Downy' had $26 \%$ leaf-feeding damage by cereal leaf beetle and the most susceptible, 'GK Kincsô' $77 \%$.

Combined effect of cereal leaf beetle and bird cherry-oat aphid was observed in 1987-1990. Grain yield was reduced by $47 \%$, while the reduction of thousand-kernel mass was $40 \%$ on average. The yield of the most tolerant genotype, 'GK Korány' was reduced by $33 \%$, while that of the most susceptible one, 'Mv 13' decreased by $67 \%$. A close correlation was found berween infestation severity by bird cherry-oat aphid and yield reduction $(r=0.78, P<0.001)$. Principal component analysis and multiple regression analyses were performed to determine the relationships among tested characteristics and to quantify the effect of some of them.

In 1991-1994, when cereat leaf beetle attacked alone, the most sensitive genorype, 'GK Lili' suffered $40 \%$ loss in yield, while the most resistant one, 'Downy' 13\%. The correlation between feeding damage by cereal leaf beete and yield reduction was medium $(r=0.63, P<0.001)$.

Trichome length of the flag leaf exhibited a significant correlation with feeding damage by cereal leaf beetle $(r=-0.80, P<0.001)$

Cereal Research Institute, P.O.B. 391, Szeged, H-6701 Hungary

1379

THE EFFECT OP MINIMUM INBECTICIDE BPRAYB ON YIBLD PERTORMANCE OF COMPEA IN FOUMBOT, CMMEROON

AOTROR: I A, PRRH

The effect of minimum deltamethrin insecticide spray at $12.5 \mathrm{~g}$. a. $i /$ ha, in yield performance of 5 cowpea cultivars was studied at Foumbot. Cameroon, in 1988, 1989 and 1990. For each year, the seed yields from plants sprayed once at either 75-100\% flower bud, or 75-100t flowering (= 1 flowering spray) and once at 75-100 podaing $l=1$ post - flowering spray), were not significantly (p > 0.05) different from yields of plants sprayed fortnightly from 21 days after planting to 10 days before harvest. For each year also, the percentage losses in potential seed yield of plants receiving one flowering and one post-flowering insecticide treatments, were significantly ( $<<0.05$ ) lower than those from unsprayed plants. These percentage losses were not however significantly ( $p>0.05$ ) higher than those obtained from plants sprayed fortnighthy.

Department of Plant Protection.

Faculty of Agriculture,

University of Dschang

P.O. Box 308

Dschang, CAMEROON.
BEED YIELD REBPONBE OF 13 COWPEA COITIVARB TO DELTAMETRRIH INBECTICIDE TREATMENTS AT FOUMBOT, CAMEROON

AUTHOR: I.A. PARH and J.N. ABANE

A comparison of the seed yield potentials of two improved IITA and II Cameroonian local cowpea cultivars was studied at Foumbot, Cameroon, in 1988, 1989, 1990 and 1991. Throughout the study period, the seed yield potentials of insecticide untreated plants of Njombe local, Tengeng local, Foumbot local, Mouda local and Diongo-Ikiliwindi local, were relatively high, However, these yields were lower than those from treated plants of the different cultivars. The local cowpea cultivars showed also, a high seed yield response to fortnightly deltamethrin insecticide treatments.

Department of plant protection, Faculty of Agriculture. University of Dschang, P.O. BOX 308 Dschang, CAMEROON.

1380

INTRODUCING TEMPORAL AND SPATIAL COMPONENTS OF PLAMT DISEASF EPIDEMICS USTMG A SIMPLE GRAPHYCAL COAPUTER SIMULATION PROGRAM

\section{P.L.R. Partner \& NeRoberte}

principles of plant tiseage epidemiology are a bagic component of most courges in crop protection and are an integral pazt of the design and courses th crop protection and are an integral part of the design and implementation of modern integrated crop procection procedurea. An rnderstanding of ene prineiples of epidemiology lo thexerore reçuireme both tor students and for advisory and extension seaff. Experience of teaching epldeniology has shown that its mathematical basis can be demotivating to those whose interest is primarily the biology of the system under investigation. Some of the inertia involved in learning the principles or epldemiology can be reduced ie the gubject is program, without explicit reference to mathematics.

The computer program, SATSUMA (Spatial and Temporal Simulation inder Mechanistio Assumptiongl can be used to illustrate epidemiological principles. The same objective could be achieved using a spreadeheet package but this places restrictions on the use of the program agoociated with availability of the appropriate spreadsheet software. A simple purpose-buile program offers a mare accessible alternative.

Students work with the program at their own pace through a serieg of exercises. This presents the opportunity to explore the effects of various environmental and biological variables on the spatial pattern and temporal development of disease. Stochastic elements within the simulation confer added benefit as an educational tool by allowing the examination of the natural variation observed in epidemics defined by identical parumecer valuea. Once the students have gained a strong impression of the appearance of epidemics and an awareness of the factors which determine their development, the mathematics which are used to quantify disease incrgase can more readily be appreciated.

The mechanistic, gxid-based nature of SATSUMA and other simulators of this type has advantage relative to empixically-derived growth curve simulations, in that the consequences of different degrees of disease aggregation on the accuracy of sampling protocols can be demonatrated. In addition, changes in the accuracy of a given sampling protocol, as disease incidence changes with time, can also be illustrated. These additional attributes may be valuable in the training of farmers. advisors and extension workers who use threshold-based pesticide applications.

Institute of Ecology s Resource Management, University of Edinburgh, Wegt Mains Road, Edinburgh EH9 $35 \mathrm{G}$. UK. 
HARIFULNESS OF FUSARILM ROOT ROT ON SPRING WHEAT IN THE FOREST STEPPE AREA OF WESTERN SIBERIA

Pavlova 0. 1. Novosibirsk Agricultural University.

Fusarium root rot is one of the most serious and widespread diseases of the spring wheat in Western Siberia Harmfulness of Fusarium root rot. was studied on different levels of infection in gray forestry soil of greenhous. In the beginning of the vegetation (20 phase on the international scale) the disease development and biomass synthesis of plants had the correlation. with the number of the pathogen propagules in the soil con selective media). On the 20-th phase the primary roots were the most injured, by the harvesting ( 91 phase) epicotyls and stem bases. The infection level caused a statistically reliable loss crop yield of spring wheat. The empirical threshold of Fusarium root rot harmfullness was by 300 propagules / g soil (yield loss $10 \%$ ).

633128 Russia, Novosibirsk reg., Krasnoobsk, $16-22$

1383

SOME DANGEROUS PESTS OF LEPIDOPTERE - NOCTUIDE IN THE CABBAGE CROPS AND THE INELUENCE OF ENVTRONMENTAL FACTORS ON THE DYNAMICS OE THEIR POPULATIONS

Roman Tr., Neqru Georceta (*

*) - Research Institute for Vegetable and Flower Growing 8268 - Vidra, S.A.I., Romania

\section{Abstract}

The paper underlines that during the six years of studies the dynamics of adult populations of Lepidoptera Noctuidae species in cabbage and cauliflower crops realised a curve characterized by a numerical density maximum 1989. When the population of the year 1988 was taken as a year of reference, it represented only 54.2 by comparison with that of year 1989 .

Till 1993, this curve reqistered permanently a pronounced regression and it attained some values below the economic level for damages of 4.3 8. The authors have established the systematic classification of the biological material caught by the aid of pheromona and light traps and they have emphasized that among the constitutive species adapted to this biotope took place a permanent fight in order to occupy and extend their vital space. Population of Discestra (Mamestra) trifolii - $R \circ t \mathrm{t}$. that registered, during the 1988 - 1989 period a level of $94-978$ decreased till 1993 at a share of 19 and it was replaced by the species Mamestra suasa - D. et C., Mamestra oleracea - L., and Autographa aamma - L. The authors have underlined that the real causes that have had a negative effect on the local populations of lepidopterous noctuide species had been early autumns, frosty winters characterized by high thermic amplitudes, associated with the absence of a protecting layer of snow as well as late, cool rainy springs. Another factor that has caused the decreasing of the existing populations was the permanent presence in the fields of the parasite Trichogramma evanescens $-w e s t w$. whose annual weight in reduction of the attacks ranged between $18-348$.
Effect of early planting on the yield of sugarbeet infested with Heterodera schachtio in west Azarbaijan

\section{R.Parvizi, H . Pashazadeh}

During 1994 , effect of planting date on the yield of sugarbet was investigated in two field trials infested with sugarbeet cyst nematode ( Heterodera schachit schmidt) using a randomized complete block design. The field studies revealed that early planted plots which had develpped sufficiently at the time of nematode attack resisted infestation by the nematode whereas in the middle and late planted plots plants were severelly injured and their development inhibited . statistical evalution revealed that differences in yield were significant and the mean yield of early, middle and late planted plots in Oromieh and miandoab were $44.91,24.56,14.93$ and $45.56,28.41,23.04$ tons/ha respectively

\section{R. parvizi}

agricultural rasearch centre of west Azarbaijan

P.0.Box 365 , Oromieh, IR AN

\section{4}

SEED TRANSMISSION OF HIBISCUS LATENT RINGSPOT VIRUS (HLRV) IN HIBISCUS CANNABINUS (KENAF) AND CHENOPODIUM QUINOA

\section{Rubies-Autonell, G. Anacario}

Hibiscus latent ringspot virus (HLRV), identified in Hibiscus rosa-sinensis from Nigeria and recently in $H$. cannabinus (kenaf) in Italy, has been classified as a nepovins, but up until now no virus vector has been identified. In the three-year period 1992-94, HLRV was identified after emergence in randomly distributed siightly stunted piants with leaf chlorosis. For this reason, an investigation was made of HLRV seed transmission in some seed lots (from various origins) using experimental fields where HIRV had been identified. Seeds were sown in sterilised soil in a glasshouse at $21^{\circ} \mathrm{C} \pm 1{ }^{\circ} \mathrm{C}$ and a $16 \mathrm{~h}$ photoperiod. Kenaf seedlings were assayed after 5-6 weeks using the standard PASELISA method. The antiserum prepared was diluted in PBS buffer $1 / 500$ for both steps. Extracted sap was diluted in 1:10 PBS-Tween containing 2\% polyvinylpyrrolidone PVP MW 24,000), sodium sulfite $0.13 \%$ and $0.2 \%$ powdered chicken albumin. HLRV was detected in $17 / 76(22.3 \%)$ of kenaf plantlets cv. G4, grown from commercial seeds from Australia Chorotic leaf mottling was observed on two plantlets while the infection was symptomless in the other 15 . Seeds from the same lot were disinfected for $30 \mathrm{~min}$ with tri-natriumfosfat $10 \%$, washed 3 times in $\mathrm{H}_{2} \mathrm{O}$ and ground individually in a mortar after imbibition in $\mathrm{H}_{2} \mathrm{O}$ for $5-6$ hours; $92 / 157(58.6 \%)$ were positive to the test. This increase in the percentage of infection in whole seeds, as compared with the transmission in plantlets, induced us to test the different seed parts. After disinfection and imbibition as above, the seeds were carefully dissected with sterile forceps, separating the embryo, external coat and internal coattendosperm. Each individual part was treated for 15 minutes with tri-natriumfosfat, washed and ground. HLRV was identified in $9 / 35(25.7 \%)$ embryos, a percentage very close to that of the seedlings, demonstrating that seed infection certainly occurs through the embryo. Assays are being continued to check other parts of the seed for HLRV. The virus was not found in 50 seedlings cv. Everglades 41 , in 40 seedlings cv. BG 5238 , or in 100 seedlings cv. G4 grown from seed of other origins Seed transmission in artificial hosts such as $C$. qunoa was also studied. The seeds tested were collected from 8 infected plants inoculated when young and kept in a glasshouse. Seedlings tested about 7-8 weeks after germination showed no symptoms, but $5 / 675$ $(0.74 \%)$ plants were positive with PAS-ELISA. The results suggest that HLRV may be spread mainly by seed. International exchange of seeds leads to spreading of the virus to different geographic areas. To prevent HLRV diffusion rapid, sensitive, economic diagnostic techniques such as ELISA should be applied to check commercial seed lots.

Istituto di Patologia Vegetale, Università degli Studi di Bologna, Via F. Re, 8, 40126 Bologna. Italy. 


\section{A.R. SAGHR' ${ }^{1}$, R.K. UPADHYAY'2, G. GODAT ${ }^{1}$ AND A . MOHAMAD'}

Dodder (Cuscuta spp.) is a stem and leaf parasite which twines on several vegetable crops in the United Arab Emirates (U.A.E.). It is especially detrimeatal to Jew's mallow, mint, onions, tomato and turnip. The sandy light soils and hot climate of U.A.E. favour the germination of cuscuta seed, and the spread and twining of its shoots on the foliage of host plants. Under heavy infestation of dodder, vegetable crops may be completely destroyed. The objective of this study was to evaluate the efficacy of certain herbicides for the selective control of attached dodder on some vegetable crops in the U.A.E. Two pot experiments were conducted using five local cultivars of chard, spinach, turnip, radish and red beet grown in the greenhouse. When the seedlings were about $10 \mathrm{~cm}$ high, they were infested with $C$. campestris tondrils by placing them around the stems and leaves of these regetables. Two rates each of glutosinate- $\mathrm{NH}_{4}$ at 25 and $50 \mathrm{ppm}$ (a.i.) and giyphosate at 75 and $100 \mathrm{ppm}$ (a.i.) were sprayed on the foliage of the infested vegetable crops. The spray volume used was $100 \mathrm{ml} / \mathrm{m}^{2}$. The treatments and the controi pois, sprayed with water. were replicated $5 \mathrm{X}$ in a randornized complete block design. The results showed that post-attachment of glufosinate- $\mathrm{NH}_{4}$ at $25-50 \mathrm{ppm}$ caused $50 \%$ phytotokicity to turnip and $44 \%$ dodder control. In beets injury was minimal but parasitism did not hold. Olyphosate at $100 \mathrm{ppm}$ caused $56 \%$ phytotoxictly and $44 \%$ dodder control in turnip, and was not phytotoxic to bects, and gave $96 \%$ dodder control and excellent yield of chard leaves.

1 Faculty of Agricultural Sciences, U.A.E. University, P.O.Box 17555 , Al-Ain, U.A.E.

2 Plant protection Lab., Al-Ain Municipality, P.O.Box 1003, AI-Ain, UAE
DENSITY OF P. PENTAGONA.

Diaa A. F. Sheble

On the basis of climatological studies it is generally accepted that the increase of the amount of greenhouse gases in the atmosphere can lead to a global warming. In Hungary during the period of 1881 - 1990 when the winter temperature increased significantly a northward migration of some termophilous pest insects was observed. As a mater of fact some new insect pests have appeared in Hungary. The problems related to new insect pests in Hungary were analysed earlier by Kozár and David (1986). Kozar and Stollar (1991 a, 1991 b). In Hungary we examined the winter mortality of $P$. pentagona in 21 places at the end of the winter of 1993. We compared the mortality with the absolute minimum temperature in winter of 1992 - 1993. In Budapest minus 10 was the lowest temperature, the coldest place was Paks and Baja with minus 20 degree. The mortality was varied among $36.5-100 \%$. The lowest mortality was found in Budapest, Dumaujváros and Szeged it was among 36.5 - 66.6 $\%$. The lighest (100\%) was in Baja (South Hungary). The relationship between winter mortality and minimum degrees was not significant We found that minus 20 can not stop totally the spread of the pest. The distribution and the density of the P. pentagona were followed by pheromone traps among 1991 - 1993 in Hungary and adjacent countries in different places. The highest number of majes of $P$. pentagona was (11460) in one trap in 1991, while in 1992 it was only 1033 and in 19931629 . In central part of the country the number of collected males increased sevenfold for three years. In the eastern part of the country the number of maies in the traps decreased to zero. There were new places infested like Balatonakarattya. Gyöngyös, Szántód. Szentes, Tokod, etc. Also we put some traps near the border in Austria and Romunta but there was no infestation. In Slovakia we found in 19931679 (in Hikovce) and 557 (in Komarno) males, It seems that if the winters in the next years will be milder than the average, this pest will continue its spread northward in different parts of Europe.

Plant Protection institute. Hungarian Academy of Sciences, H-1525, P. O. Box 102, Budapest Hungary.
1387

DISPERSAL MOVEMENT OF THE DIAMONIBACK MOTI ON CKUCIFEROUS VEGETABLE FIELDS

Y. SHIRAI

The diamondback moth, Plite/la xulostella, a serious insect pest of cruciferous vegetables, has already developed a high resistance to many types of insecticide. Rotational applicalion of several insecticides with different mechanisms of insecticidal action is one of most effective control measures against this specles. P. xylostella has been reported to be a iong-distarit migratory species, however, the dispersal movement over relatively short distances on vegetable fields was influenced largely by the environmental factors which were different from the case of lorit-distalit migration. The larger-sized moths emerged in a low temperature season have inigher potential ability to undertake lung-distant flight and longer longevity in comparison with smalier-sized moths emerged in high temperature season. Undoubtecly, the larger moths from autumn to early spring are tuugh and suitavie for long-distant flight. Nevertheless, the short distant movement on vegetahie fieids was not active in the autumn or early spring, because the lower temperature below $15^{\circ}(C$ considerably the flight activity. In the mid spring to early summer, $D$. xylostella flew more actively beyond the fields and the mean flight distance indicated ca. $600 \mathrm{~m}$ from the mark-recapture experimeni. Therefore, rotational application scheduled for warmer seasons should be conducted on an appropriate arfa scale with the cooperation of regional farmers, taking into account the wide flight range of P. xylostelia.

National Institute of Agro-Environinental Sciences, Kannondai, Tsukuba, Ibaraki 305, JAPAN.
1388

MASS PRODDCTION AND APPLICATION OF VAM INOCDLA IN TROPICAL FARMING SYSTEM

\author{
(S.N.SINGH, V.P.Rao, S.E. Pawar)
}

Mass inocula of Glomug aggregatum, G.fasciculatum, G.mosseae and Gigaspora margarita are being prepared at Mycorrhiza production centre and at BAIF, Pune on Bahia grass and Guinea grass as host plants and Solrite + perlite $(1 ; 1)$ as substrate. Growth chambers are provided with light intensity at 10-15 k lux, photoperiod at 14 hours/day, temperature 25$28^{\circ} \mathrm{C}$, temperature of substrate $28-30^{\circ} \mathrm{C}$, pH of substrate 6.5-7.0 and humidity 70-80 per cent. 0.3 micron Microfilters are used for providing bacteria-free air. The plants are given fertilizers in the form of urea, rock phosphate/bonemeal and muriate of potash, and Long Ashton,s nutrient solution for ficronutrients. Plants harvested 75 days after inoculation. Inoculum containing a mixture of vam fungi hyphae, spares and VAM infected roots is dried to 5-10 percent moisture level. Nearly 1500 packets of inocula, each containing $250 \mathrm{~g}$ of inoculum, of selected VAM fungal strains have been supplied to about 100 research organizations in the country.

Vesicular Arbuscular Mycorrhiza (VAM) also applied as Biocontrol agent in protection of plant diseases in tropical farming in rural India. It has been tried for different crops and horticulture at nursery level including multipurpose tree species. It has given visible advantages in the growth, rooting and over-all growth of the plant. This will certainly help in developing resistance with different root nematodes prevalent in the tropical soil. Rhizobium and VAM have together been applied in the field and results are documented and approved in IPM as well as in sustainable Agriculture.

BAIF Laboratorieg Limited, BRIAHNAGAR, Off Pune-Nagar Road, Wagholi PUNE 412207 (MAHARASHTRA) INDIA.

Tel : $0091.212 .670408 / 670409 / 677438$ FAX: 009/212670859 
IMSTAR CONPOSIT ION OF SAFFLOHER APHID, UZQLEUCCN COMPOSTAAE (THE NB.AL) SIMGH O.V.K., GOUD T.R., AND REDDY D.D.R.

Safflower, Carthanus tinctorius inn. is infested by Safflower aphic, Uroleucon compositae (Theobald), a key pest of the crop. It was abserved that young ones constituted the major pert of colonies and the aphid population was found to depend on their rulative composean.

In order to study the pattern of incresse and inctar conposition of the aphid population infesting safflower var. Kenjira. invesigations were carried out during five rabi seesons from 1989 to 1993. The percent plant infestation was more on stern, lower surface of leaves and lower plant parts in the earlier weeks of infestation. They were seen to move upwards and reach the inflorescence and were also found on upper sunface of leaves.

Hapoing of the infestetion was done Fron colonization to peck spread of their population on the glants. The infestation patterr wäs also studied.

The trends of instar composition for five seasons were similar. The number of the first three instars had a single peal in all seasons, at peak aphid population. The ratio of paded to non-padded nymphs exceeded unity during study per $1 \times d$.

The growth rate of aphid population expressed as intrinsic rate of increase per fenale per day (rm) and potontial rate of incrcase per instar period $(\lambda)$ were estimated.

The identifying characters of $\underline{\mathrm{U}}$. comositae nymphs of norphs were studied. Using morphometric data, their diagramatic flgures were prepared.

Vice-grincipal and Jeneral Entanologist Regional Agricultural Research Station,

inchra iradesh state (India).

1391

PASSION FRUIT RESISTANCE TO COLLAR ROT: RESISTANCE OF Passiflora edulis edulis TO Fusarium solani ISOLATES WITH VARYING ALTITUDE AND SOIL PH

\section{Ssekyema, T.R. Swinburne and L.W. Burgess}

The passion fruit growing area, Mbale, Uganda, has lowland and highland ecological zones. Fusarium solani, passion fruit collar rot causal organism is found in the lowland while highlands are disease free. Soil pH was found to vary from $7.38,6.70,7.05,7.47,6.02,5.65$ to 5.22 with increasing altitude. Passiflora edulis edulis cultivar from the apparently uninfested highlands was found to be susceptible to $F$. solani isolate from the highlands with change to pot experimental environment. The paper discusses why such variations in resistance/pathogen virulence and proposes need for further investigation.

Kawanda Agricultural Research Institute, Horticulture Research Programme, P.O. Box 7065, Kampala, Uganda

INFLUEACE OF CALCIOM, IRON AND ZINC ON DEVELOPMENT OF PHYTOPETHORA FOOT ROT OF GERBERA AND FUSARIUN WILT OF CHINA ASTER

\section{Cz., Skrzypczak, L. B., Orlikowski}

Ornamental plants are grown under intensive menagement and usually at high levels of fertility. Under such conditions the effect of fertilizers and fertility levels on soil-borne pathogens may be enhanced causing greater losses or might be inhibitive. phytophthora foot rot incited by F. cryptogea have been reported on several ornamental plants growing under glass, plastic or in field and is considered to be the most serious disease of gerbera. Fusarium wilt of china aster caused by $F$. oxysporum $f . s p$. calistephi is the most dangerous threat of china aster. In some nurseries the losses caused by the fungus varied from a few to even $90 \%$. Interest in the control of pathogen by some fertilizers has been developed at the Institute (Orlikowski and Kowalczyk, 1992, Orlikowski and Skrzypczak, 1994). In our studies the effectiveness of calcium iron, and zinc chelates on in vitro growth of Phytophthora cryptogea and Fusarium oxysporum f. sp. callistephi $(=F(C)$, development of disease symptoms and population dynamics of both pathogen was determined. On water agar, the growth of $P$. cryptogea was reduced by calcium nitrate and Fe-EDDHA and stimulated by ferrous sulphate whereas the development of Foc was inhibited by zinc and ferrous sulphate applied into medium at dose $100 \mu \mathrm{g} / \mathrm{ml}$. In greenhouse trials, fertilization of plants twice a week as top-dressed with calcium nitrate, $z$ n-EDTA, zinc sulphate and Fe-EDDHA signifacantly decreased Phytophthora foot rot severity on gerbera. The development of fusarium wilt of china china aster was strongly inhibited by calcium nitrate. Fertilization of gerbera with tested compounds increased, in general, population densities of $P$. cryptogea. Calcium nitrate did not influence the pathogen development in the substrate.

Literature

Orlikowski I. B., Kowalczyk W. 1992: Prace Instytutu Sadownictwa i Kwiaciarstwa, S. B, 17, $91-98$.

Orlikowski L. B., Skrzypczak Cz., 1994: Phytopathologia Polonica $7(X I X), 49-55$.

Research Institute of Pomology and floriculture, Pomologiczna 18, 96-100 Skierniewice, Poland

1392

POPULATION DYNAMICS OF SUMMER FRUIT TORTRIX MOTH, ADOXOPHYES ORANA F.V.R. (LEPIDOPTERA, TORTRICIDAE) IN WESTERN SERBIA

\section{S.Stamenković, S., Milenković, T.Stamenković}

Summer frutt tortix moth, Adoxophyes orana F.v.R. occurs regularly in almost all apple and pear commercial orchards in Serbia. It has caused constiderable damage for many years. It was first observed in the vicinity of Belgrade in i959. Summer fruit tortrix moth has been an economically important pest in fruit production since 1970 when considerable damage of apples and pears was recorded in the region of Western Serbia. Taking into consideration the presence and the increased population density of this pest in the Cačak area over the 1976-1994 period, the study was conducted on the population dynamics and the duration of embryo development in order to determine optimal terms for applying control treatments.

The following fruit species were registered as hosts of summer fruit tortrix moth in serbia: apple, pear, quince, peach, apricot, plum, cherry and sour cherry. Apple and pear were the most cot, plum, cherry and sour cherry. Apple and pear were the most cultivars, the greatest damage was recorded with cv. Williams cultivax
$(58.0 \%)$.

In the climatic conditions of western serbia, A. orana moths deIn the climatic conditions of Western Serbia, A. orana moths develop two generations in a year with the flight period in June and August. Sumer fruit tortrix overwinters in the third larvae i.nstar. The third instar larvae leave winter shelters in ear Apriti.

The emergence of the first generation moth in the area under

observation occurs in the second half of May and lasts till early July. The emergence of the second generation moths begins most often at the end of July and in early August and the flight lasts till mid September.

The duration of embryo development in the field conditions is 8-15 days.

Svetomir Stamenković

ARI "Serbia", Fruit and Grape Research Centre, Cacak

Kralja Petra I/9

32000 cacak

Yugos lavia 
COLLETOTRICHUM GRAMINICOLA CN SORGHUM IN WEST AFRICA: DISEASE DEVELOPNENT, YIELD LOSS, CONIDI KI PRODUCPION, AND GENETIC VAR IABI I ITY

H.D.Thomas, I.Sissoko, R, A. Frederiksen, and J.Rosewich

Ieaf anthracnose of sorghum (Sorghum bicolor) is caused by the fungus colletotrichum graminicola. The development of the disease and its efrect on grainweight were studied under field conditions for three seasons (1990-1992) on 15 randomly selected fungicide-treated and unsprayed plants per subplot. Two inored sorghum cultivars were exposed to natural inoculum of the fungus in lali, west Africa. Infection on the susceptible medium maturing $(70-90$ days to $50 \%$ flowering) caudatum cultivar Is 19696 increased rapidly after anthesis and most leaves were killed by the time of Fhyslological maturity. Progress of the disease on the noderately resistant early maturing (less than 70 days to $5 \%$ lowering) guineense cultivar IS 25105 was slower after anthesis compared with 19696 , and leaves were not killed by maturity. Areas under the disease progress curve for the three years were 61,127 , and 121 for IS 18696 and 47 , 112 , and 122 for is 25105 . Yield losses in 1990, 1991, and 1992, reepectively, for Is 13696. The isease had no effect on yields for Is 25105 in log and igy 1:1 urily $4 \%$ loss in 1992. These results suggest that leaf anthracnose is serious in rest Africa on susceptible cultivars such as Is 18696 and that eerly maturing cultivars like Is 25105 are exposed to lesser inoculum during their early growth stages.

Eix sorghum isolates of $\mathrm{C}$. graminicola from Mail and Jurkina Faso produced in seven d ays 210,000 and 250,000 falcate conidia/square mm medium or potato dextrose and oatmeal agars, respectively. No oval conicia vere observed in both of these solid inedia. In two days $1 . Z$ and 2.9 milion oval conidia/ml were detected in modified Fries liquid medium (FLM) and in potato dextrose croth (PDB), respectively. As detection of ova? conidia declined in PDB at four to six days, falcate conidium production was initiated ano at 10 days between 1.9 and 4.6 million falcate conidia/ml were detected. Conidial dimorphism was not observed in FIM. Sutstrate conditions may influence production of both types of conidia. Restriction fragment length polynorphism separated six isolates of 0 . Eraminicol

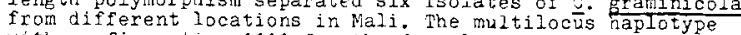
with configuration 1111 for the four locj examined was conmon to seven other isolates from either the same or different locations in iali and five ssolates from the same location in Jurkina Faso. Thus, in iest Africa genetic variability may not be linked to eeographic location.

Department of Crop Protection, Njala University College, University of Sierra Leone, PMB. Freetown, Sierra Leone

IINFLUENCE OF COWPEA AND MELON POPULATTONS ON WEED INFESTATION, CROP YIELDS AND FARMEFS" ATTITUDE TO NEW WEED CONTROL PRACTICE.

A. J. UDOH

ABSTRACT:

Small farmers in the humid regions of tropical Africa spend $30-42 \%$ of their total farm labour input in controlling veeds. Chemical weed control is normally recommended. But high cost of herbicides and environmental pollution ace specific problems with chemical veed control. A three year bio-weed control system with three populations of cowpea $\left(20,33\right.$ and $50 \times 10^{3}$ plants ha-1) and melon (5, 10 and $15 \times 10^{3}$ plants ha-1) in a cassava/matze intercrop was conducted to check weeds at 3,5 and 8 weeks after planting (WAP) in the southeastern humid rainforest zone of Nigeria. Cowpea and melon populations at $50 \times 10^{3}$ and $15 \times 10^{3}$ plants ha-1 significantly reduced weed infestation, gave the highest food energy and net income in the cropping system. Since rural women constitute $50-60 \%$ of the farming population and are a slgnificant source of labour in weeding operations then a weed control strategy with melon crop which provides ofl and protein to farm families may be highly innovating to them. Two hundred women farmers were invited to visit the research-extension plot at 5 and BWAP before weeding. At 5WAP, $75 \%$ of the farmers and at 8 WAP, $100 \%$ of them preferred to adopt $50 \times 10^{3}$ cowpea with $15 \times 10^{3}$ melon plants ha-1 since this combination gave the most effective weed control.

\section{TOMATO VIRUS DISEASES IN RUSSIA}

\section{A.E.Tsyplenkov}

Tobacco (TMV) and tomato mosaic (ToMV) viruses are wide spread on tomatoes, causing different diseases: mosaics, streak and internal browning of fruits. Cucumber mosaic virus (CMV) is more common on tomatoes at the region of the North Caucausus, at the Lower Volga region and at the Far East. The virus induces leaf filiformity, stem and fruit streak. The most CMV strains, isolated from tomatoes in Russia, belong to $\mathrm{DL}$ group, which is symptomatically close to the DTL, U,C,D groups of CMV strains known abroad (Tsyplenkov et al,1992). Tomato spotted wilt virus has the limited area and was noted at the Black Sea coast region of the Caucasus (Vlasov,1976). Tomato aspermy and potato $\mathrm{X}$-vinuses are insignificant in the protected ground. The new virus, which belongs to the Potex-group, was revealed on the foreign tomato varieties. The use of resistant tomato varieties and control of vectors are the most perspective ways of tomato protection from viruses. Home tomato hybrids Dozent, Barynia, Gloria, Romeo possess $100 \%$ resistance aganist ToMV and $30-40 \%$ one aganist CMV

All-Russian Institute for Plant Protection, Podbelsky av.3, St.Petersburg-Pushkin, 189620, Russia

1396

THE MAIT THEAT DISEASTS IY ALBATIA, ECOVOSICAL

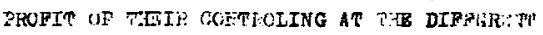

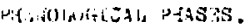

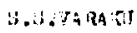


ha is planted nostly irth joesd vleat last yesra. Yarious soil and climatical conditions influence on spreading of many pathogenes in whest, but fungus diseases are more $1 \mathrm{~m}-$ -portant. $\mathrm{F}$,graminis, Septoria sp., as a Logliar diseases and Fusarium sp. (F, cummorum, T.equisetum, F. avenaceum.) as foot rot of whest are 3 lmost $z$ nnualy in our country. Chemical method for albanian farmerg remains the main method for wheat disegses controline. Treatment with frumidor is ca-rried out at different phenological phases of whest(GS??; CS51-59;G532,51-59; GS32,51-59,61-69). Based on the expenses /profit rate, for our conditions, a treatment at GS $51-57$ was more economical than those applied I time at GS32, 2 time at GS32,51-59;3 time at GS32,51-59,61-69.Different fungicldes are applied to control wheat disesses, but the best regults are achived by using of Tilt, iforizon etc.Tre-atments with frumidor affected also ag an arti-senescence having citokinin like properties(R.J.Cook,1931).

Skender S. Varaku

Instituti i wbrojtjes se Bimeve

Te1.3555222132 Durres, Albania.

Telef3x.35552a2182 
The effect of urea-fertilizer and maize-variety on the incidence of Striga hermonthica in the field

\section{T. Goverse' ${ }^{1}$, M.A. Pruiksma' ${ }^{1}$, G. Odhiambo' ${ }^{2}$, J.A.C. Verkleij ${ }^{1}$, A.H. Pieterse ${ }^{3}$ and} J.K. Ransom ${ }^{4}$

Striga hermonthica is a very noxious parasitic weed, which occurs mainly in subSaharian Africa. It parasitizes important staple crops like maize, sorghum and millet and causes serious damage to its hosts. Striga is probably, after drought, the main cause of severe yield losses in some countries. Research on Striga has been canried out for decades, but still no suitable solution for the Striga problem in Africa is found. Many experiments show beneficial effects of the use of nitrogen fertilizers to control Striga. However, the mechanisms behind this are still unclear. In vitro studies showed that urea nhibiss Striga germination and nomal development of the Striga radicle. Unfortunately, in field experiments nitrogen application was less succesful and contradictionary results were often shown.

This experiment was conducted in Kibos, Kenya, to evaluate the effect of different leveis of urea-application and maize-variety on the incidence of Striga

The results showed that he double cross hybrid 4622 supporned significant more Striga compared to the varietal hybrid H511 and the local variety Katumani. Based on the results of plantheight and stover production it appeared that vatiety $\mathrm{H622}$ suffered most from the parasite. No differences in grain yield were found between the hybrids and the considerably higher compared to Kanumani.

A significant effect of urea-application on the number of emerged Striga plants was found 12 and 14 weeks after planting. Less Striga was found in plots where $84 \mathrm{~kg}$ N/ha was applied compared to plots without any urea-treatment. No differences in Striga numbers were found between plots where urea was applied at once and plots where the amount was splitted applicated (at planting and 3 weeks after planting). Plant height and stover production remained unaffected
for all plot recieving urea-fertilizer.

1. Vrije Universileit, Faculty of Biology, Department of Ecology and Ecoloxicology, De Boelelaan 1087. 1081 HV Amsterdan, The Netheriands.

2. The Kenya Agricultural Research Institute (KARI, N.S.R.C. Kibos, P. O. Box 1221, Kisumu, Kenya 3. Royai Tropical lnstitute, Depart Kenya. hermonthica in the field. Striga come results showed that the double cross hybrid H622 supported significant more local variety, although the yield potential of the two hybrids in Striga free plots are

SIDE-EFFECTS OF LIQUID FERTILIZERS ON THE PESTS

K. Veverka, S. Pekár

On the contrary to the nutrients from the soil which influence pests via plant metabolism droplets of liquid fertilizers can hit the pests directly. Their solutions are phyrotoxic and question was if they are toxic to pests too.

All the liquid fertilizers produced in Czech republic were tested for the insecticidal activity against Tribolium confusum used as a model object. High efficiency was found in the UAN only (urea+amonium nitrate). The solutions of both compounds applied separately had no activity, highest possessed their mixture in equimolar ratio. Insecticidal activity of UAN was not substantially affected by $\mathrm{pH}$. Residual activity of the spray on inert surface was very long. The mortality of the beetles placed on glass Petri dishes 24-96 hours after spraying was $100 \%$, decreasing to $93 \%$ after one week and to 89 \% after two weeks. Surfactants with high HLB value decreased and those with low HLB value increased the insecticidal activity of UAN.

UAN was toxic for Leptinotarsa decemlineata Say adults in the laboratory tests. Vhen sprayd on eggs the hatching was only delayed, not decreased. The growth of larváe born from treated eggs was prevented for the fortnight and survival decreased to $0.0-27.0 \%$. Only first two instars were sensitive to the direct UAN spray. The older ones were not affected.

Toxicity of UAN to Meligethes aeneus. Tetranychus urticae. Eriosoma lanigerum and to the overwintering eggs of Psyla ma$1 i$ was revealed. UAN can affect the predators like Coccineidae and spiders linyphitidae. Pardosa agrestis is insensitive. UAN spraying of fields with flowering weeds is banned due to its activity as a contact and a stomach poison for honey bees. UAN exert synergistic activity with mevinphos. dimethoat, pirimiphos-methyl. methidathion.

Karel Veverka, VURV Prague-Ruzyne, Czech Republic

1400

1399

CHLOROTIC LFAF CURL OF TOMATOES-THE NEN VIRUS DISEASE IN TTRRIENISTAN

Y.I.Vlasov, T.N.Teploukhova, V.K.Lebsky, B.Sejidov

The tomato disease of the chlorotic leaf curl type, transmitted by the glasshouse whitefly (Trialeurodes vaporariorum) was noted in the protected ground of Turkmenistan in 1991 (Vlasov, Teploukhova, Orasov, Goshaev, 1994). In experiment in addition to tomatoes infection was transmitted to Nicotiana glutinosa and Datura stramonium by whiteflies and by grafting. Next years the disease became widespread in the open ground too. Whiteflies-borne diseases have been previously unknown at CIS countries. At different times various pathogenes, including geminiviruses, were isolated from infected plants. Their avallability was shown by electron microscopy. Agronomical methods and, to begin with sowing time, play an important role in the disease harmfulness reduction. Less harmful disease manifestation is ensured by late autum sowing of greenhouse tomatoes as compared with their early autumn sowing. Under field conditions, on the contrary, the early sowing time and, accordingly, the early open-ground planting provide less tomato susceptibility to the disease. Y.I.Vlasov, AllRussia Research Institute for Plant Protection, s.Podbelskogo,3, St.Fetersburs, 189620, Russia Phone: 476-84-89

\section{SUGARCANE PATHOLOGY IN NIGERLA.}

\section{PAST, PRESENT AND FUTURE}

\section{A.C. WADA}

Sugarcane Pathology is still in its infancy in Nigeria. Studies conducted on 104 cane farms in 4 states and Abuja in the Middle-blet zone of the country thow that smut (Ustilago scitaminea Syd.) and red rot (Glomerella tucumanensis(Speg) v. Arx \& E Muller) diseases with field incidence of $37.17,30.42,24.62,30.7$ and 27.399 and 13.75, 28.0, 18.0, 16 and $20 \%$ in Niger, Benue, Kwara, Plateau and Federal Capital Territory, Abuja respectively were the provalent diseases. Sugarcane leaf blast Paraphaeosphaerio michotii 0 . Erikss as low in all the cane fields visited (9-17\%). SCMV had the least distribution and incidence (5 and $6 \%$ respectively) and was observed in one location only. U. scitaminea had $100 \%$ distribution in Niger, Benue, Plateat and FCT. While in Kwara it was observed in $60 \%$ of the total fields surveyed. G. tucumanensis was recorded in all fields visited in Benue, Plateau and $F \mathrm{CT}$, while it occured in 37.5 and $80 \%$ of the surveyed cane fields in Niger and Kwara States respectively. Ratoon crops were the most severely affected by $U$. scitaminea, and G. tucumanensis was more severe on the purple type canes. The distribution, incidence and severity of these four diseases are discussed and the work plan for current and future studies outlined.

Sugarcane Research Programme National Cereals Research institute Badeggi, P.M.B. 8, Bida, Niger State, Nigeria. 
DISPERSAL OF RUST UREDINIOSPORES FROM GROUNDNUT BY WINO AND RAIN K.D.R. HADIA, D.R. BUTLER, H.A. MCCARTNEY

Rust of groundnut (Arachis hypogaea) is caused by the fungus Puccinia arachidis and the pathogen is spread ma inly by urediniospores which are predominant ly produced on lower leaf surfaces. The removal of urediniospores from inoculated plants was studied in the wind tunne1/rain tower at Rothamsted and the results used to help explain field observations of a irborne spore concentrations measured at the ICRISAT Asia Center. In the wind tunnel, infected plants containing sporulating pustules were exposed to simulated "gusty" wind with various mean wind speeds and the concentration of a irborne spores was measured down wind of the plants. Where the same plants were exposed to increasing wind speeds, few spores were caught unt il the mean wind speed reached $0.5 \mathrm{~ms}$ and the highest spore concentrations were found with winds of about 2.5 ms $^{-1}$. When plants were exposed to cont inuous wind, most of the released spores were dispersed during the first few minutes, spore concentration then declined exponent ially with $t$ ime. The concentration of a irborne spores was linearly related to the number of lesions on the source plants. In the field, urediniospore concentrations were monitored over three growing seasons. Hourly average spore concentrations were signif icantly greater when hourly
average wind speeds exceeded $2 \mathrm{~ms}^{-1}$ than at slower wind speeds. In the average wind speeds exceeded $2 \mathrm{~ms}^{-1}$ than at slower wind speeds. In the
field, spore concentrations of ten increased sharply at the onset of field, spore concentrations often increased sharply at the onset of
rain or during short showers suggesting that rain may play a part in rain or during short showers suggesting that ra in may play a part studied in the rain tower where infected plants were exposed to artificial rain with a steady wind speed of $2.0 \mathrm{~ms}^{-1}$ and spore concentrations were measured down wind. The experiments showed that very few spores were dispersed in splash droplets but impacts from rain drops dis lodged spores into the air. In cont inuous rain the majority of spores were removed during the first few minutes of exposure and there was no further spore depletion from lesions after about 10 mins. When plants were exposed to a series of rain fall events each last ing 2 min, about $75 \%$ of the trapped spores were caught
during the first event. Ra in removed between 25 and $90 \%$ of the available spores depending on the raindrop size and the rainfall intensity, large rain drops removed more spores than smal? drops. Ra in removed additiona? spores from plants even after they had been exposed to relatively high winds. For example about $30 \%$ of the available spares were removed after a $5 \mathrm{~m}$ in exposure to a mean $w$ ind speed of $3 \mathrm{~ms}^{-1}$, while twice as many were removed from the same plants durtng a subsequent 2 min exposure to rain. These results correspond with patterns of spore concentration found in the field and suggest that rainfalt removes rust urediniospores effect ively before their subsequent dispersal by wind.

IACR-Rothansted, Harpenden, Herts AL5 2JQ, UK
ICRISAT Asia Center, Patancheru,A.P. 502324, India.
THE VIRUSES ACCOMPANIED THE SUGAR BEET RHIZOMANIA DISEASE IN MIXED INEECTIONS

\section{T.A. Yakutkina}

Beet necrotic gellow vein virus (BNYVV), transmitted by the funges Polynyxa betae Keskin, is known to be the pathogene of sugar beet rhizomania. Except BNYVV the other viruses are often liagnosed in the plants, infected by rhizomania, however (Jankulova, 1984, Henry, Jones at al., 1986). Tobacco mosaic virus (TMV), tobacco necrosis virus (TNV), tobacco rattle virus (TRV) and beet mosate virus (BMV) were revealed when testing the diseased plants from the rhizomania nidi in kazakhstan (Yakutkina, Vlasov, Akperlinov, 1991). TMN and TNV prejominatei over those ones. The role of these pathogenes for rhisomania levelopment seems to be unsimple and now isn't quite clear, but their soil-borne nature is obvious. At the alif of rhizomania the fungy of the genus Olpilium, the vector of TNV, have been found out equally with the fungus P.betae. Mixed virus infections can alter the rhizomania symptoms displaying. Besides that, they make difficulties for qualitative antigene production in order to receive BNYV diagnosticums. We have proposed the method for BNYV and TMV separation on the indicator plants (Tetragonia expansa L., Chenopodium quinoa ilild, Nicotiana glutinosa) and using the specific antisera to TMV and BNYVV.

All-Russian Research Institute Ior Plant Protection s.Podbelskogo 3, St.Petersbourg-Pushkin 169620 Russia

Phone 476-84-89

\section{4}

ENHANCEMENT OF RICE PERFORMANCE AND INCIDENCE OF NATURAL FUNGAL AND INSECT DAMAGE WITH CYANOBACTERIZATION, MICROEL
NITROGEN AND CERTAIN PESTICIDES

\section{Y.G. Yanni, M.R. Sehly and F.E. Abdallah}

Inoculation of Egypt rice fields with cyanobacteria increased yield by some $30 \%$ with saving of $1 / 3$ to $1 / 2$ of the recommended rates of fertilizer-N. Asymbiotic dinitrogenfixation in the rice ecosystem is not the only contribution of cyanobacteria. Suppression of growth of aquatic macrophytes, increasing P-availability, decreasing sulphide injury, aiding soil particle aggregation and incidence of natural infection with the blast fungus Pyricularia oryzae and infestation with the stem borer Chilo
agamemnon (Bles.) and the leaf-miner Hydrellia prosternales (Deeming) were also reported.

reported. Two set of field experiments were conducted in the northern Nile Delta where fungal
and insect pests severely damage rice crop. In the first set, the rice variety Giza-176 and insect pests severely damage rice crop. In the first set, the rice variety Giza- 176 was inoculated with a soll-based inoculum containing a balanced colony-forming units
(CFU) of the dinitrogen-fixing cyanobacteria : Anabaena cylindrica, Anabaena oryzae, Aulosira fertilissima, Nostoc muscorum and Tolypothrix tenuis applied at $100 \mathrm{~kg}$ Aulosira fertilissima, Nostoc muscorum and Tolypothrix tenuis applied at $100 \mathrm{~kg}$
fresh material ( $90 \%$ moisture) / ha 5 days after transplanting along with urea at 72 or fresh material ( $90 \%$ moisture) / ha 5 days after transplanting along with urea at 72 or
$144 \mathrm{~kg} \mathrm{~N} / \mathrm{ha}$ in two equal doses: 25 days after transplanting and at the midtillering $144 \mathrm{~kg} \mathrm{~N} / \mathrm{ha}$ in two equal doses: 25 days after transplanting and at the midtillering $\mathrm{Mn}, \mathrm{Zn}, \mathrm{Cu}, \mathrm{B}, \mathrm{Mo}, \mathrm{Co}$, Vilamin B, meta-indole-acetic acid, amino acids and growth promotion substances), the fungicide Sportake 45 and a combination of $50 / 50$ of the two compounds were applied as soon as the detection of fungal infection symptoms (during the late panicle initiation stage). The second set comprised the rice variety IR-28 with the same cyanobacterization and combined- $N$ treatments along with application of the stem borer insecticides: Cyfen, Dursban or Fipronil $(28.8,12$ and 24 $\mathrm{kg} / \mathrm{ha}$, with active ingredients of 5,5 and $0.2 \%$, respectively).

Performance of rice, as indexed by plant height, productive tillering, grain and straw yields and $\mathrm{N}$-contents, the harvest index (\% of grain yield / grain $+s t r a w$ yields), grain size and the agronomic fertilizer $\mathrm{N}$-use efficiency ( $\mathrm{kg}$ grain yield $/ \mathrm{kg}$ fertilizer- $\mathrm{N}$ ) were maximized by cyanobacterization along with $72 \mathrm{~kg} \mathrm{~N} / \mathrm{ha}$ and $50 / 50$ of Vitafort plus Sportake 45. Natural infection with Pyricularia oryzae. Helminthosporium oryzae, Alternaria sp. and Sclerotium oryzae, the causative agents of the rice blast, brown spot, leaf spot and stem rot diseases, respectively, was less in the inoculated sub-subplots comparing to their non-inoculated counterparts and in case of application of 72 rather than $144 \mathrm{~kg} \mathrm{~N} / \mathrm{h}$. Inoculation of the rice variety IR-28 along with urea at 72 rather than $144 \mathrm{~kg} \mathrm{~N} / \mathrm{ha}$ and Cyfen at $28.8 \mathrm{~kg} / \mathrm{ha}$ registered the maximum grain yield and $\mathrm{N}$-content and the highest agronomic fertilizer $\mathrm{N}$-use efficiency. Effect of insecticides differed according to the amount of combined- $\mathrm{N}$ and inoculation or not with the cyanobacteria. Natural infestation with the stem borer, as indexed by numbers of dead hearts and white heads $/ \mathrm{m}^{2}$, increased with increasing urea fertilizer and was significantly less in the inoculated sub-subplots than their noninoculated counterpirts

A mechanism involving a sort of regulation of avalable- $\mathrm{N}$ in the rice ecosystem by the dinitrogen-fixing cyanobacteria is suggested to diminish excessive or sub-minimal $N$ absorption and, consequently, decrease plant susceptibility to fungal and insect pests.

Sakha Agricultural Research Station, Kafr El-Sheikh 33717, Egypt. 
THE RISE AND FALL OF CARBOFURAN IN MELON PRODUCTION IN THE MIDWESTERN UNITED STATES

\section{A. C. York}

In the early 1970's, researchers in the United States began investigating the use of carbofuran as a planting time treatment for melon insects. It was particularly effective in controlling striped cucumber beetle (Acalymma vittata F.) and spotted cucumber beetle (Diabrotica undecimpunctata howardi Barber) and thus the bacterial wilt disease transmitted by these insects. Adult beetles were controlled for a period of six to ten weeks by the systemic insecticide as they fed on the stems and leaves of melon plants, and the larvae of the betles were killed in the soil by the contact action of the insecticide. By using a single application at planting time, growers could eliminate 10 to 13 foliar applications of other products. Plan growth was increased, early harvests were increased, insecticide costs were reduced, applicator hazard was reduced, and pollinator kill reduced. In the mid1980 's in Indiana, over $90 \%$ of melon growers surveyed were using carbofuran on more than $90 \%$ of their acreage each year. At about the same time, maize growers began suffering insect control failure on maize crops when carbofuran was applied at planting time for corn rootworm larval control. While researchers first blamed resistance for the lack of control, it soon became clear that accelerated degradation of carbofuran and other carbamate insecticides was the reason for lack of insect control. On some fields where carbofuran had previously been effective for 50 to 60 days, half life was reduced to as little as 10 days. Melon growers began experiencing, on some soils, complete loss of effective control of cucumber beetles requiring a return to foliar applications of insecticide to prevent insect feeding and subsequent plant death. In the early 1990's scouting for cucumber beetles and treatment decision levels were introduced to melon growers with good success. It became obvious that growers would accept traditional integrated pest management activities on a crop previously thought to be exempt because of the disease/insect interaction. Pesticide use has been reduced with substantial savings in pesticide costs.

1158 Entomology Hall, Purdue University, West Lafayette, Indiana, U.S.A. 479071158

USE OP PHEHOLOGICAL FORECASTINJ MGHHODS IN PLANT PROTECTION

E. D.Abseshidze

The wide use of pesticides in plant protection have led to entronment pollution, removinx nontaret benericial ineects, appearing or the resistant population. The effective plant pro tection is based on rellable forecasting of pest and disease development, on the analysis of information of certain life cycle of noxious oranizas and host olunt. It is luportant or esti atiar uf the ecological situation and establisning of optizal tiufine of control. Two phenoloxical forecastinz pethons were use for cotinfzation of plant protection in seorgla-iethor of forecastinz of oranizms development by the nest rontent in ifr and the nethod of tomperaturo-phenologlcal nomogram. The rate of the developient of organizms is not direct function of the e1r teaperature. It is a certain complex runction of many varigble quantities cormine thermal complex of atmosphere. The content of heat in air was celculater by Ramzin nomoxram.

The temperature-phenological nomoram nethod is based on watchinc of temperature net of region with phenoloficel curves of species. The carried tests established the hish econosic and ecolozical efflcacy of the use of phenolorical forecastinz in lant protection.

3COPI IEILISI, ave. Jamsakhurdia I4, apt. 9

Republic of seorgla
A FIELD TRIAL OF USING Trichogramma chilonis ISHU REARED IN ARTIFICIAL EGGS TO CONTROL Heliothis armigera (HUBNER)

\section{Zhili Zhang, Juwen Wu and Suqing Wang}

A field trial was carried out using Trichogamma chilonis reared in artificial eggs to control Heliothis armigera in the cotton field, Hebei province in 1994. Three releases were made at the second generation egg stage, 351,000 wasps per hectare. The result showed that the parasitising rate was $\mathbf{4 6 , 4 \%}$, the natural parasitising rate 0 . There were five releases at the third generation egg stage, 1,530,000 wasps per hectare, parasitising rate $58,4 \%$, the natural rate also $0 . T$. chilonis reared in artificial eggs could fly far away at valid distance of 10 meters.

T. chilonis reared in Antheraea pernyi G.-M. was as a control. There were four releases at the egg stage of the third generation, $1,125,000$ wasps per hectare. The parasitising rate was $74.6 \%$. When $T$. chilonis reared in $A$. pernyi was released at egg stage of the second generation, the parasitising rate was $67.8 \%$ at normal years. The main reason of lower parasitising rate this year was abnormal climate in which temperature was much higher and humidity was much lower than usual. There were 27 days in which temperatures was above $30{ }^{\circ} \mathrm{C}$. The upper limit of development temperature of $T$. chilonis was $33{ }^{\circ} \mathrm{C}$ at laboratory. When the temperature was $35.5^{\circ} \mathrm{C}$, the parasitising rate was only $38,5 \%$, only $4.4 \%$ wasps emerged, and 67\% individuals were abnormal. $T$. chilonis released in the field at 18:00-12:00 could only survive $16 \mathrm{~h}$. Under higher temperature, accelerated embryo development decreased parasitic probability. This trial was arranged at the area in which $H$. armigera has higher resistance to agricultural chemicals. The control efficiency in the wasp-releasing areas (Trichogramma of artificial egg and natural egg) were higher than that in chemical control area (eight spraying, 24 bollworms on a hundred plants). The mortalities of $\boldsymbol{H}$. armigera were $58.3 \%$ and $83.3 \%$ in the wasp-releasing areas, the percentages of horehole cotton buds and bolls were decreased to $80.8 \%$ and $84.6 \%$, respectively, the number of natural enemies (ladybirds and lacewings) was doubled.

Institute of Plant and Environmental Protection, Beijing Academy of Agriculture and Forestry Sciences, P.O. Box 2449, Beijing 10081, China

1408

RECURRENT SELECTION FOR RESISTANCE TO NORTHERN LEAF BLIGHT OF MAIZE IN UGANDA

Adipala, E. and 0julong, H.F.

Most of the malze grown in Uganda are open-pollinated cultivars derived from low to mid-altitude tropical germplass. They express varying levels of partical resistance to northern leaf blight (NLB), a destructive disease of malze in Uganda. Of the cultivars so far tested in Uganda, Babungo 3 and EV8342-SR have shown consistently high levels of partial resistance to E. turcicum and are being used to develop new populations with acceptable levels of resistance. The programmes generally employ half-zib recurrent selections and genetic gains as high as $51.6 \%$ have been achleved within the first cycle of selection. Resistant progenies have been advanced to second cycle of selection. Progress achieved so far suggests that 1 t should be possible to develop maize populations with high levels of resistance to Bxserohilum turctcum.

Makerere University, Department of Crop Science, P.0. Box 7062, KAMPALA, UGANDA 


\section{DURABLE RESISTANCE TO LEAF RUST IN WHEAT: A CASE STUDY.}

\section{Mohammad Aslam ${ }^{1}$, M. Hussain ${ }^{1}$, N.I. Hashmi and S.2. Mustafa ${ }^{2}$.}

Resistance to leaf rust, incited by Puccinia recondita in bread wheat, Triticum aestivum, cultivars Pavon 76 , and Blue Silver was studied in the field for a period of more than 15 years at different locations in Pakistan. Both cultivars carry the gene Lr13 which conditions resistance to leaf rust. High pathogenicity corresponding to this locus in the parasite population was recorded in very high frequencies through out the period of study. Whereas reaction of the $\mathrm{cv}$ Blue Silver changed from moderately resistant to susceptible rather rapidly, viz, in a couple of years; that of the cr Pavon 76 remained almost the same during the whole period. Compared with Blue Silver, Pavon 76, therefore, exhibited a higher level of resistance which could be classified as durable. A study of the tatent period revealed that in variety Pavon 76 the incubation period was comparatively longer than in Blue Silver. In comparison with $c v$ Blue Silver the appearance of flecks as well as sporulating pustules in Pavon 76 was apparently delayed by at least one day. This relatively longer latent period in the cr Pavon 76, therefore, appears be one of the factors contributing towards durability of resistance in this cultivar.

1. Crop Diseases Research institute, and 2. Coordinated Research Programme for Wheat, Barley, and Trilicale, Pakistan Agricultural Research Council, P.O. Box 1031, Islamabad.
1411

\section{BIOLOGICAL CONTROL OF CORN STALK ROT}

\section{J. Chen, Z. H. Song ,Z. J. L iang}

Corn stalk rot caused by $P$ y thism aphonid ermatum and Fusarium gramineartum was controlled by biocontrol agents such as $T$ richod erma virid $e$ and socne bacteria. Results of corn seedling periad experiment in pots auggested that the disease was ontrotled better in the treatments of sted dressing with Titrichod erma viride or bacterha, and bole appitcation of $T$. virid $e$ in combination with seed dressing with bacteria. In the sterilized soil inoculated artificialty with these bioprotectants, the control result was as high $58.15 \%$ to $P$. aphasidermatum and $56.25 \%$ to $F$. $g$ raminearum, however, in the natural soil infected by pathogens, control result of $100 \%$ was obrained. In the field experiment, there was a better control of the disease in the treatments of seed dressing with either beteria or Trichoderma virid e. All treatod plants grew mote highly than non-treated plants did. and the former produced bjgber yield than the latter did.

Department of Plant Protection, Shenyang Agricuttural University, Shenyang 110161, Liaoning, P. R. China
BIOLOGICAL CONTROL OF RESEDA LUTEA E. (RESEDACEAE). A WEED OF CEREAL CROPS IN SOUTH AUSTRALIA

\section{P.T. Bailey and T.J. Wicks}

Reseda lutea was accidentally introduced to South Australia, probably from the Mediterranean area where it is common, but not abundant on disturbed calcareous soils. In South Australia, the plant has increased in abundance in the past 20 years so that it is now a weed of over 200,000 ha with a potential range of 1 million ha. In croplands, it competes with cereal plants, causing up to $10 \%$ yield loss. The seeds also contaminate grain at harvest, which means that the seed must later be separated from the grain. In pastures. is competes with other plants.

$R$. Iurea is a deep-rooted perennial which reproduces by seeds and also vegetatively from root segments. Plants of $\boldsymbol{R}$. lutea are fragmenred by cultivation in autumn and in spring these regenerate.
The plant remains green throughout the surmmer amongst the stubble of the previous crop and is well The plant remains green throughout the surmmer amongst the stubble of the previous crop and is well
established when the pasture rotation starts the following autumn. Planis may live for many years and develop stems $2-3 \mathrm{~cm}$ diameter. It is a prolific seeder but the seedlings suffer from competition in established communities.

R.lutea is difficult to control with herbicides and it has no effective natural enemies in Australia. This, and the fact that the Resedaceae thas tho native members in Australia or has any econortically beneficial species suggest that $R$. Iutea is a good candidate for biological control by importing ratural enemies from where it naturally occurs.

A survey of arthropods and pathogens on Reseda spp growing in the Iberian Peninsula, North Africa and southern UK made during spring, 1994, indicated a number of narual enemies which may be useful in South Australia. A weevil, Baris picicornis (Curculionidae) was found associated with $\boldsymbol{R}$. lutea over most of the area; the larvae channel into the roots. Larvae of the seed-eating beetle Bruchela rufipes (Urodontidae) were found in the seed pods of a number of plants. The flea beetle, Phyllotreta nodicornis (Chrysomelidae) adults were commonly found on the leaves.

of the four fungal pathogens detected, a leaf spot caused by Cercospora resedae and stem lesions caused by Phoma resedicola were found at some sites

These natural enemies appear to be specific to the Resedaceae and will be further tested for their suitability for importation into Australia.

South Australian Research and Development Institute, Box 1671 GPO Adelaide 5001. Australia

\section{2}

POSSIBILITIES TO WARR AND CONTROL THE LEPIDOPTERA OLIGIA STRIGILIS L. USING THE LIGHT TRAP

\section{Manuela Dănulescu}

The capture of 0ligia strigilis L. lepidoptera using the light trap has in view the emission of warnings for estabilishment the best period for the pest control and elaboration of prognasis concerning the attack. The observation carried out using this method offer informations about the biological cycle and the curve of flight of this pest with hight frecvenquency and intensity which could represent a permanent danger under favorable enviromental conditions. It is possible to provide the protection of Dactylis glomerata crops by the aplication of chemical trataments at the best period.

Grassland Research Institute Braşov ROMANIA

Cucului str. $5 \operatorname{cod} 2200$

tel/fax: 40(0) 68142119 
TAXONOMY ON THE IMPORTANT GENERA AND SPECIES OF PRATYLENCHDAE IN CHINA

\section{Y.X. Dugn, W. Z. Liu,Y. Liu}

The faxonomy history of the Pratylonchidae nomatodes was summerizod in this papes. The important genora and species (Hirschmanniella, Pratyleachoides, Pratylopchus) of Pratylench idae in China were systematically atudied 43 spocies of the family from 3 genera were described in detail, including 7 new species: Hirschmanaiclla brassicae n. sp. ,H. jiningensis n. sp. ,H. sinenzis n. sp. ,Pratylenchus angularis n. sp. ,P. dangshanensis n. sp. ,P. tobacum n. sp. and P. saccherum n. sp. ; and 16 new record specios for China. Each spocies was illustrated and discussed. All synonyms and litteratures related to tho identification of oach species were listed. Under light microscopes and Scanning Eloctron Microscopes, the key characters for identification of several species werv abserved. The relationship botween the vitrastructure and evolution in the family was also discussed.

Laboratory of Nomatology, Department of Plent Protection, Shenyang Agriculcural Uaivensity, Dongting 110161 ,Sheyang, Ching.
ROLE OF AIRBLAST SPRAYERS IN REDUCING RISKS OF PESTICIDE USE IN IPM CITRUS ORCHARDS IN THAILAND

Ellis, W; King, W; Kosaisaevee, S; Sirisingh. S; Tongsakul, S.

As part of an inter-agency cooperative programme between the Thai Department of Agnculture, Thai-German IPM Project, Natural Resources Institue (UK) and the GIFAP Safe Use Project, a model farm concept was developed and tested in Thai citrus orchards.

The overall aim was to minimize pesticide usage / exposure through a combination of farmer education, IPM training, improved application technolagy and protective clothing.

Farmer innovations to traditional boat-sprayers resulted in considerable improvements in spraying efficiency, droplet size distribution, and operator safety. Comparisons between highpressure hand spraying, a locally-produced aibalst sprayer, and a new airbiast design are presented, and pollution aspects and economic implications of such improvements within IPM systems are discussed.

Deparment of Agricultufe, Bangkhen, Bangkok 10900 THAlLAND

1416

STUDIRS ON ANTHFACNOSE OF CARAWAY (CAROM CARVT L.)

A. Evenhuis (PAGV), B. Verdam (IPO-DLO)

Caraway is a minor arable crop grown for its seeds and its etheric oil. Anthracnose, caused by Aycocentrospora acerina (Hart.) Deighton is a major disease of caraway in the Netherlands Yield loss of 758 has been recorded. Disease management is essential in order to increase the crop's yield stability. Various studies were done, in the period from 1990 until 1994, to obcain information on the fungal pathogen and to generate a set of crop protection methods based on this information.

Contaminated soil and infested caraway seeds were shown the principal inocukum sources of $M$. acerina. Disease severicy can be reduced by crop management. The effect of reducing the nitrogen level and sowing rate on anthracnose development is demonstrated other crop management methods are discussed.

Research Station for Arable Farming and Field Production of Vegerables, 80 BOX 430,8200 AK Lelystad, The Netherlands.
A critical appraisal of out whole approach to biological control concluded that our knowledge of the ecology and epidemiology of Botrytis in kiwifnat was inadequate. Epidemiological studies in 1992-1994 indicated that a range of tissues were potenual sources of inoculum. At the end the end of the flowering period, senescing male and ferrale flower tissues were the principle sources of Botrytis inoculum. In contrast, necrotic leaf tissue was the principle source of inoculum in kiwifruit vines prior to harvest. There was a significant relationship between Total potential spore production (TPSP) in experimental vines and the level of Botrytis inoculum on the fruit surface at harvest. Artificial inoculation of fruit at harvest confirmed that the greater the level of Botrytis contamination of the finit surface at harvest, the greater the level of picking scar contamination and postharvest storage ro.

A new biological control strategy has been initiated in collaboration with DLO Research Institute for Plant Protection, Wageningen, The Netherlands. This strategy is aimed at reducing the sparulation potential of Botrytis on necrotic kiwifruit tissues using antagonistic saprophytes. Laboratory and field tests indicated that selected isolares of Aliemaria, Epicoscum and Ulocladium were tolerant of intemupted wet periods. The suppression of Batrytis sponulation by some saprophytes was as high as $100 \%$. These findings are discussed in relation to fuzure biological control strategies in kiwifruit.

HortResearch, Crop Production and Protection Division, P.O. Box 51, Lincoin. Canterbury, New Zealand.

DLO Research Institute for Plant Protection, P.O. Box 9060 . 6700 GW Wageningen, The Netherlands. 
THE POSSIBLE MEANING OF FOOD CONSUMPTION RATE IN THE EVALUATION OF THE PREDATORS OF SPIDER MITE

$$
\text { C. C. Ho }
$$

Amblyseius womersleyi Shicha is commonly found in Taiwan and has a short develpmental period and fecundity comparable to $A$. fallacis Garmen. The female mite consums 3 and 32 eggs of Tetranychus kanzawai Kishida daily during develpmental period and adult stage, respecitvely. Reared on a soybean leaf approximately the size of four $\mathrm{cm}^{2}$, the adult females ceased to escape when daily food supply reached 80 spider mite eggs, and her daily fecundity attained 3 , the fecundity in life history study, after 20 spider mite eggs were offered daily. In eggplant this predator are found on those leaves which contained 100 or less spider mites. These may offer informations for developing biological control programs.

Taiwan Agricultural Reseach Institute 189 Chungcheng Rd. Wufeng, Taichung, Taiwan R. O.C
ADJUANTS FOR THE ENVIRONMENTAL SAFE TECHNOLOGY OF BEANS AND SOYA GROWING

\section{J. Kovacs, M. Koppanyi and I. Petroczi}

Hungary provides good natural conditions for growing beans and soya in the south part of the country. Lately the shortage of the water, the pests and diseases caused cutting of quality and quantity of the yield. Some regions of the country become drought-affected as the supposed consequence of the 'Greenhouse' effect. That is why the research work on drought became more important in Hungary. We investigated the effect of the Adjuvants + Pesticides combinations on the safety of the environment, the possibility of the cutting of the pesticides dose, the physiological effect of the adjuvants, the quantity and quality changes of the yield, the possibility of making more effective the use of water.

The use of adjuvants may lead to cut of the evaporation coefficient of the plants and it can reduce the evaporation of the infected plants and it can also make the use of pesticides more effective.

Hungarian Academy of Sciences, H-1051 dapest, Nadur u.7, Hungary

\section{A Taxonomic Study of Chinese Phorid Flies} (Diptera: Phoridae)

Liu Guangchun, Fang Hongping

The phorid flies or Phoridae are one of the largest families of Diptera. About 3000 species in 250 genera are known in the world fauna. China is whete the Palaearctic fauna and Oriental fauna meet and intermingle, and is tich in Phoridae resources. However, our knowledge of Chinese Phoridae is very poor. For long time the Chinese phorid fauna has been neglected

The present paper deals systematicaliy with the taxonomy of the Chinese phorid flies for the first time in China. The research history on the phorid flies both in the world. and in China is reviewed. These flies are stressed as pests of economic and hygienic importance. All important morphological terms are discussed and made consistent for use in keys and descriptions. Special attention is paid to structure of male and female terainalia and their homology. Based on the examination of about 6000 speciwens, 23 genera and 126 species of phorids are recognized. Among them, 3 genera, 1 subgenus, and 65 species are described as new to science, 9 genera and 30 species as new to China. Keys are presented to facilitate identification of all taxa, including geneta and species. All the genera and species are described or redescribed.

Dr. Liv Guangebun

Department of Plant Protection

Shenyang Agricultural University

Shenyang, Liaoning 110161

P. R. China
1420

A NEW SPECIES IN GENUS MELOIDOGYNE ON ROOTS OF GINSENG, MELOIDOGYNE CHANGBAIENSIS N. SP. (NEMATA, MELODOGYNDAE)

LIU ,W. Z. ,X.WU, Y.X.DUAN, AND Y.LIU

Root -knot nematode of american ginseng was collected trom Jian, by Changbai Mountains, Jilin province in China in September, 1990. Morphological characters of identity for this new species of namatode were observed. It is different from other species in the Meloidogyne with some important diagnotic shorter spear (7. 5-10um) in females, set off head region and constrictive at base, loger spear $(15-20 \mathrm{um})$, tail sharply pointed and gubernaculum longer $(12.5-15 \mathrm{um})$ in males, and pointed tail end in second-stage juveniles.

Department of Plant Protection, Shenyang Agricultural U niversity, Shenyang, 110161 , China. 
A NEW SPECIES OF GENUS APHELENCHOIDES, LEAF NEMATODE OFGINSENG, APHELENCHOIDES PANAXOFOLIA N.SP.

\section{(NEMATA; APHELENCHOIDAE)}

\section{LIU,W.Z. ,X.WU,Y.X.DUAN ,AND Y.LIU}

Brown spot of leaf a new disease of ginseng was found in Zuojia, Jilin Province, China during $1990-1991$. It was caused by a species of nematode in genus A phelenchoides. Morphological characters of identify for this new species of nenatode were observed. It is different from other species in the genus with some important diagnotic ; short spear $(7.5-10 \mathrm{um})$, lip region base constrictive, excretory pore between nerve ting and valve, tail ending in star mucrons, spicula in males with sharp apex and rostrum.

Department of Plant Protection, Shenyang Agricultural University, Shenyang, 110161, China

\section{INDIGENOUS KNOM.EDGE SYSTEMS}

CHARLES MUGAMBWA CM (Author)

PRESENTER E.J. VAN DE FLIERT

Subsistence farmers regulate pests without recourse to expensive and dangerous chemicals or insecticides which is not yet known to modern scientists. Increasing the species and or genetic diversity of cropping system so that several sources of resistance are used simultaneously is a key strategy to minimize losses from $p l a n t$ diseases and nematodes. Mixing different crops species or varieties can delay the onset of diseases, feduce the spread of disease carrying spores and modify environmental conditions such as humidity light, temperature and air movements so that they are less favarable to the spread of certain diseases. The leaf juice of certain plants or weeds $\mathrm{e} . \mathrm{g}$. Datura stramonium) are commonly used as pesticides. Non biological methods irclude spraying dirt, cow urine and using smoke or heat are used as a control mechanism to regulate pests and diseas. In many peasant farming system in Uganda, crops often inter-cross with their wild relatives, growing in the same fielo or in nearby rields wich results in new characteristies of plante The diversity of genetic resources is associated with rich culturesof plant tional knowledge. Maintenance of species and genetic diversity in the fields is one of the most effective strategies to create stable systems of conservation by resource poor farmers practicing low input aggriculture. Farmers have well established systems of ensuring sustainability of seeds supply and often operate in networks, thus playing a key role in the creation, maintenance and promotion of crop genetic diversity through a series of activities to sustain plant production

Programme for Cultural Reasearch and Development (PCRD) P.O.Box 93, Fort Portal

Uganda

Tel. $256-(0493) 2200$

Fax. $256-(0493) 2636$
1424

INTERACTION BETWEEN OZONE, SULFUYROTOXIDE, ALPHA AND DELTA RACES oF colle totrichum 11ndemuthianum Bri AND Cav. ON BEANS (Phaseolus vulgaris L.)

O.S. Achwanya, and L.D. Moore. A number of bean cultivars were evaluated for their response to
the alr pollutants, $\mathrm{O}_{3}$ and $\mathrm{SO}_{2}$, singly and, 1 n combination, as well as for their reaction to the alpha and delta races of Colle totrichum
lindemuthianua. Varfation in response to both the pollutants and the fungus was noted among the cultivars. Anthracnose caused up to $50 \%$ reduction in biomass in some cultivars. A negative correlation $(\mathrm{r}=-0.72, \mathrm{P}<0.0001)$ was found between disease severity and total $(\mathrm{r}=-0.72, \mathrm{P}<0.0001)$ was found between disease severity and total
plant biomass. Greater than additive effects of $\mathrm{O}_{3}+\mathrm{SO}_{2}$ mixtures were demonstrated. Chlorophyll content and biomass were found to be reliable varlables for assessing treatment effects. Ozone and $\mathrm{SO}_{2}$ appeared to stimulate disease development. Greater pollutant infury was also noted in the prezence of anthracnose.

There was an interaction between the pollutants as well as between each pollutant and the fungal disease. Implications for evaluating bean cultivars for reststance to $\underline{C}$. 1indemuthianum under polluted atmosphere are suggested.

Egerton University,

P.0. Box 536 ,

NJORO, KENYA.

but the sprouts shrivelled and died.

From apparently healthy looking stored ware tubers, all the

fungi listed for rotted minisetts were isolated except

Phoma Leveillei, Verticillium sp., Trichoderma harzianum

and Botryodiplodia theobroma. From freshly harvested seed yams from minisetts, a few fungi were isolated namety, Sclerotium roltsii, fusarium oxysporum, Aspergillus niger, Rhizopus stolonifer and Curvularia sp.

The relationship between some of these fungi and disease

development in yam is discussed. 
BIOLOGICAL CONTROL OF PYTHIUM THROUGH SEED COATING AND SEED PRIMING WITH TRICHODERMA.

Legro, b., Satter, $\mathrm{H}$. To prevent or control plantdiseases the use of
biologicals can be an alternative for chemicals which biologicals can be an alternative A Trichoderma harzianum strain was selected, based on A Trichoderma harzianum strain was selected, based on its antifungal characteristics, to study the possibilities for the compatibility in the coating and priming technology. The coating and priming technologies are among the most suitable delivery-systems available control several economically important soil-bor diseases in the seedling stage (e.g. Fusarium,
Rhizoctonia and Pythium)!

Studies with pelleted tomatoseed including this Trichoderma strain prove that it is possible to control Pythium ultimum in a bioassay. Compared with the standard treatments with chemicals still some progress has to be made. It is evident that a nutrient source must be available for the Trichoderma to optimize its antagonistic abilities. For coated tomatoseed therefore it is inevitable to provide these nutrients with the coat.

Application of Trichoderma during biopriming of tomato and cucumberseed proved to be very effective. Data obtained from the bioassay with $P$. ultimum are very stimulating. Objects with Trichoderma appeared to be superior to those without Trichoderma and better than seeds treated with chemicals. The stand of the seedlings (with rrichoderma) was comparable with those grown in clean soil. Biopriming also provided a faster and more uniform germination and thus both factors contribute to overcome the first critical phase for a seedling.

Integration of the two disciplines seedcoating and priming with Trichoderma is in study and is expected to be a very effective combination for biological control purposes.

Incotec International B.V., Westeinde 107, 1601 BL Enkhuizen, The Netherlands. (tel:02280-10782 fax:02280-20074);
IHE USE OF MR-FORMULATION AS A NOVEL AND ENVIRONMENTALIY SAFE PHOTODYNAMIC FUNGICIDE FOR THE CONIROL OF POWDERY MILDEWS

Tzeng, D. D.-S., Tzeng, H.-L. C., Chen, R.-S., Hsu, H.-K., Cheng, A.-H., Tsai, C.-T., Chen,C.-W., Hwang, T.C., Yeh, Y., and DeVay, J. E.

The control of powdery mildews on melon, cantaloupe, squash, pea, tobacco, strawberry, and grapevine were atternpted by the application of a formulation developed from the methionine-riboflavin mixture (MR), a chemical concoction reported to be photodynamically biocidal (Tzeng, 1988). The applied formulation consisted riboflavin $(26.6 \mu \mathrm{M}), \mathrm{D}, \mathrm{L}$-methionine $(1 \mathrm{mM})$, copper sulfate $(1 \mathrm{mM})$ and sodium dodecyl sulfate (1000 ppm). In greenhouse as well as field trials performed, the disease control efficacy of the test formulation was equivalent to or even superior than the compared commercially available chemicals. Upon the formulation application, mildew lawns on the infected plant parts were generally cleaned. A weekly application was found sufficient in controlling the disease as that by most conventional fungicides. An adjunct scanning electron microscopy revealed that after the chemical application, the mildew propagules residing on a diseased leaf quickly became collapsed. dismantled, and tended to lose their cellular contents and disintegrate gradually. The observed morphological destructive effects suggested strongly a contact eradicating activity by the applied test formulation. The overall performance of disease contro efficacy among the test crop species clearly demonstrated the usefulness of test formulation as an ideal alternative fungicide for the control of powdery mildews on various greenhouse as well as field crops. The formulation is unique in that it contains mainly food constituents and household use biodegradable ingredient. It can be home made; and the potential risks of unwanted residual environmental effects appears unlikely.

Department of Plant Pathology, National Chung Hsing University, Taichung 402 , Taiwan; R.O.C.
1428

\section{ULTRASTRUCTURAL STUDY OF VERTICILLIUM DAHLIAE AS A POTENTIAL ADDITIONAL METHOD OF POPULATION MONITORING IN RELATION TO PATHOGENICITY}

T.A. Vlassova

Characteristic features of Verticillium dahliae Kleb., the causal agent of Verticillium wilt, are intraspecific variability and permanent evolution. It makes necessary the regular monitoring of fungal population in the soil. In this respect mycelium ultrastructure of two $V$. dahliae strains differing in virulency to cotton plants was examined by method of transmission electron microscopy. The mycelium ultrastructure of both highly virulent and nonvirulent strains was in general typical for fungal cell. However, there were some visual differences in the details of hypha organization, especially in the mitochondrial apparatus development and lipid accumulation. The mean quantity of mitochondria in the cells of nonvirulent fungus was about twice more than in the cells of virulent one. The quantity and sizes of lipid inclusions were contrawise significantly more in the hyphae of virulent strain. The vacuolar system was some more extensive and lomasomes were more frequently observed in the latter. These peculiarities may not be interpreted as direct criteria for pathogenicity. So the mitochondrial apparatus development may be related to the trophic specificity of strain. However, the higher lipid content and vacuole capacity could be of certain importance for neutralization of plant fungicide influence. Vacuole volume may to some degree determine hypha mechanical pressure, and lomasome amount may be connected with fungal exometabolite excretion intensity; the both may be involved in plant penetration by fungus. Further investigations are needed to confirm use of present method for fungal population analyse for pathogenicity.

Department of Plant Physiology, Faculty of Biology, Moscow State University, 119899, Moscow, Russia 
Neyra, M. and P. Póner

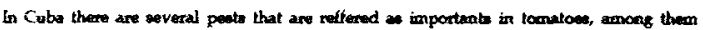

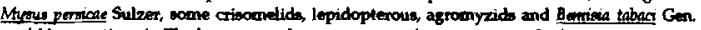


vector of the tometioe Yellow Leat Couriling Vinu (ToYLCV).

During 1993-1994, 4 experimente were conducted with Gaucho 70 WS and Contidor 35 SC (active ingredient tridedoprid) in order to control tomatoe coedling and plentation peete in (active ingredient lmidecloprid) in order

Gauchio was applied manually at a rate of $\% \mathrm{gaai} / \mathrm{kg}$ of seedh and Confidor at $1.05 \mathrm{gai} / \mathrm{m}^{2}$ of the modling using $2 L$ of water as final solution. 6 deye befose trooplanting. This application was divected to the base of the plant stalk ueing a Birchueier oprayer without nozele at a rate of $25 \mathrm{~m} /$ plant. Stenderd insatments with tradtional products were eatabliahed

Gaucho conducted to low foliar damage levela (11x), inainly protuced by Diabrotica balteata

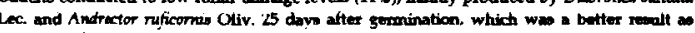

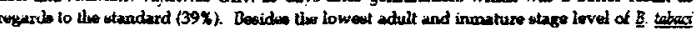

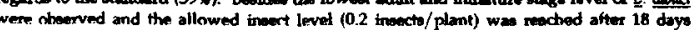
while in the stmenderd it was oberved after 9 dyys despils $4-5$ application of other azmethemixal product. On the nther hand an infortant population reduction of the

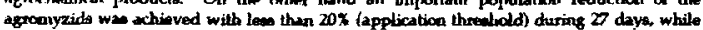
to the entandard this period lastud only 9 days.

Confidm applind mee hefire and twine after trasplanting rechosed the incidence of aculto

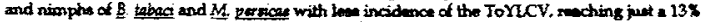
of the plants while in the standard a $49 x$ was obeerved.

Gaucho did not affect the germination potential of the tomatoe seeda. An efimulant effect

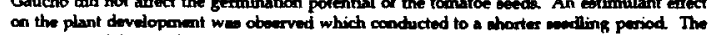
On the plant developonent wio oboerved which conchuctod to a chorter moviling period. The Grucho Cos

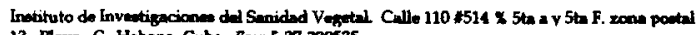
13. Playa C. Haberse, Cuba Pax; 5-37-330635
The effect of wheat varieties on longevity and reproduction

potential of Russian wheat aphid

Gh.Reza Rassoulian and L.Doulati

Plant protection Department. Agricultral College of Karaj.

University of Tehran. Iran

Russian wheat aphid has been known as one of the most important pest on wheat and barley in the recent years. This pest is spread in several state of U.S.A and canada and cause a bigh damage on wheat farms. There are many researches project in the world in order to decrease the damage of this pest in relation to find some resistance varieties of wheat to russian aphid.

In order to investigate the longevity and reperoduction of Russian wheat aphid on wheat varities, there has been sellected 13 varities of wheat as; Argentine Dastjerdi,zarand,Rashid,Shahi, Azar, Adleghadim, Adlejadia, Khalig, Naz, Sefideh, Darab and Ghods.

The plant were grown in flastic poas filled with vermculite soll, in greerhouse condition $(20-25$ and $75 *$ R.H $)$. Bfter 30 days first instar nymphs were placed on a single leaf of each varieties.Each experiment was replicated four times.Over the diuration of study ( 6 weets), longevity and reproductive data were recorded weekly.

The results showed that the longevity of aphids on all varieties were the same, but reproductive ferformance were significantly on some varieties as shabi and Sefideh. 


\section{A. A Abdernbman, Z. T. Dabrowski and A. Akettiar.}

The catastrophic consequesces of over-relinese on intensive chemikal control was demonstrated in the cotton agro-exosystem doring the 70-ties in the Gezira Scheme and was the subject of delibentions of a Crop Pest Mamegement Symposinas hedd in Khartoum, 6 - 8 Febresry, 1978 (S. El Besther, K. B. BI Tigani; Y. M. El Tayeb and H Khalifs). The symposium roconmended the initiatios of adoptive research leadieg to integrated pest control approach.

In response to an official requent from the Governmeat of the Sudma, the project entitled "Devedopereat and Application of lategrated Pest Control in Cotton and Rotational Food Crops in the Sodan" was initiated 28 sti March, 1979 for a first phase of three years and later fortber extended for consecative four pheres. The FAO was designated at the exenting ageacy, and the Miniztry of Agrieatture throagh the Agricaiteral Research Corporation (ARC) a the inplementing agescy. Since the beginning, the project has heen sponsored by the Governiment of the Netheriandx

In the lst pteser, most of the sctivities were devoted to the stady of the seasosal

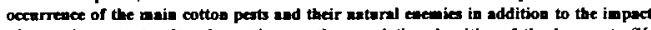
of some important cultanal practicas on the popalation densities of the key pests ( $K$. Eveleens; A. A Abdelrahman; O. S. Bindra; van Gent and D. W. Sippent. The disturbanoc of cotton eco-systen by the "packige deal" system ma identified as the major factor of the drawatic increase of spraying in the 70-ties to 8 - 9 sprays of insecticide mixteres. The increaved lint stickiness sttributed to the whitefly ont-break reduced the prices of the Sadnese cotton in the international narkets. If the 2nd pbsese (1985-89) Large scale trials condected in the fermer's fiedd of the Gerirn

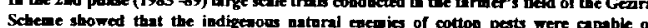

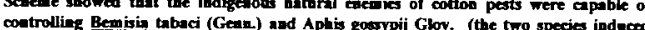
controlling bemisin habed (Geath) nod Aphis gossypii Glov. (the two species indecto more thas $50 \%$ of the anzall insecticide application). The cex parzsitoid Trichogramede pretiosang Rehy was introdaced from USA to control Helicoverpan armizera (Hb). The parasitoid bernme cotablished in all important oot Herrera, A. A. Abdernhman, A. E. M. Hesian)
lo the 3rd phase (1989-92), a critical re evaluation of the ceonomic threabold levets (ETL) for the main cottos pests showed that the ETL $s$ contd be considerably raised (2-3 folde) without sigrificant effect on the yiedd (P. A. Stan, R. Manir, A. A. Abdelnatman).

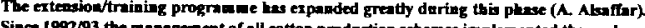
Since 1992/93 the management of all cotton production sctemes ieptemented the package recomeneded by the projert for the control of cotton pests (particalarty the mised ETLS). As a resalt, the nomber of sprays in the Geaira has dropped from 4.9 in 1992/93 to 3 in 1993/94 scason and from 4.55 to 3.1 in the Rabad Seteme; oqual to an anmual seving of $2.6 \times 10^{6}$ nod $0.7 \times 10^{6}$ USS in 1993-94, respectively.

Additional on-farme research on jessid ETL on long and sbort stable cotton varieties is now condweted in the phase four (1993-90) in addition to monitoring the effect of implemestition of the new IPM packnges on the occurrenct of the major gropss of astaral enenies is four large experimeats (in cooperntion with the Plunt Protection

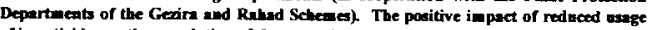
of inecticides on the popalation of the naturnl enenses on other crops in the cotton-based


involved it cotton prodection. This is in uddition to the improved envirosmental quality which is reflected is the red weed bealth besards and the wellare of the comanonity at large

FAO/ARC IPM Projeet, GCP/SUD/02SNET; ARC Gerin Station; do FAO Representative, P. O. Box 1117, KHARTOUM, Sudan.

CLASSICAL BIOLOGICAL CONTROL OF CEREAL STEMBORERS: A COLLABORATIVE PROJECT BETWEEN THE INTERNATIONAL CENTRE OF INSECT PHYSIOLOGY AND ECOLOGY AND WAGENINGEN AGRICULTURAL UNIVERSITY.

W. Overholt, S. Kimani, A. Ngi-Song, J. Mbapila, C. Onwega, P. Lammers and $\mathrm{K}$. Ogedah."

Lepidopteran stemborers are a major constraint to increase in the production of cereals in the tropics. In Africa, several species of stemborers, the majority of which are native, attack maize and sorghum. However, one of the most injurious species in eastern and southern Africa is Chilo partellus, a stemborer that was accidentally introduced from Asia sometime before 1930. The International Centre of Insect Physiology and Ecology and the Wageningen Agricultural University are collaborating in a project of classical biological control of $c$. partellus and other stemborers. A common parasitoid of $C$. Partellus in Asia, Cotesia flavipes, was imported and released in the coastal area of Kenya in 1993. Recoveries two years after releases provide strong evidence that the parasitoid is firmly established. The population of $C$. flavipes is increasing, and recoveries are being made as far as 20 kilometres from the release sites. The impact of $C$. flavipes on stemborer populations in Africa is currently under study.

"ICIPE/WAU, International Centre of Insect Physiology and Ecology, P.O. Box 30772, Nairobi, Kenya.

\section{B. Hassane}

The national of crop protection service of Chad (DPVC) was established in 1986 following severe outbreaks of locusts. It became responsible for all aspects of crop protection, but adequate functioning was hampered from the beginning by a very weak infrastructure. A project to strengthen the DPVC, was formulated and carried out by FAO between June 1991 and June 1995 with funding of the Dutch Govemment and the UNDP.

Amongst the outputs of the project was the realisation of a satisfactory infrastructure. This included the construction of laboratory space, offices and stores at headquarters in N'Djamena and at 9 regional bases. Staff received their training in crop protection, on the job and abroad Relevant information was brought together in a specialised library, a computerised data-base was developed. and basic laboratory equipment was purchased. Besides these efforts to provide the DPVC with the basic means to carry out its task, the project was also concerned with the elaboration of a national crop protection legislation. After implementation, this will provide the necessary legal context in which the DPVC can execute its duties.

In the past, the free distribution of chemical pesticides and application equipment during large scale control operations directed against outbreaks of locusts resulted in an abuse of chemical products. At present, pesticides are hardly available in Chad, but even if they were, most farmers would not have the means to purchase them. It is clear that free distribution of products will not provide an ecologically sound and durable solution. Therefore, the major objective of the project was to make a start with deveioping and introducing safe and sustainable crop protection methods which can be included in a strategy of integrated pest management (IPM).

It is essential that these methods are efficient, practical and within economic reach of the subsistence farmer. They should include a variety of preventive methods like the use of crop varieties adapted to the local conditions (drought resistance, resistance to pests and diseases) selection of good quality seed, crop rotation and chemical seed treatment. But aiso curative pest control methods are needed, as well as techniques to protect stored products. Methods like the use of wood ashes and botanical products might prove useful, but on-farm validation trials and a wider extension are still necessary.

During the project period a start was made implementing on-farm demonstrations of preventive and of non-chemical crop protection methods. Farmers are encouraged to observe and discuss differences between treated and non-treated plots. Although this approach of participatory technology development seems a promising method of extending IPM methods, it is obvious that the DPVC by itself is not capable of introducing these techniques at a large scale. To really reach large number of farmers, other channels will have to be found

The most apparent way would be to use national development organisations and NGO's who are in direct contact with large numbers of farmers. The DPVC will have to play a role of supplying the technical knowledge. Their subject matter specialists could train and guide the rural extension workers and provide specialised technical assistance like pest identification.

Direction de la Protection des Végétaux et du Conditionnement (DPVC) B.P. 441, NDjaména, Chad, Tel. 235-513692, Fax 235-515119

1603

\section{DEVELOPMENT OF IPM SYSTEMS IN ZANZIBAR}

\section{Mherik R. Said and Gerard C.A. Bruin.}

Zanzibar, like many other African countries, depends largely on agriculture for its survival. With the exception of a few largescale monocrop farmers, agriculture is characterized by subsistence, mixed cropping and short cycie bush fallow systems. Crops include food crops like cassava, banana, rice, sweet potatoes, grain legumes, sorghum, fruits and vegerzbles, and cash crops like cloves, coconuts, fruits and spices. Pests and diseases form a major constraint, 2s well as declining soil fertility.

The Plant Protection Division (PPD)

The PPD is the sole responsible agency in Zanzibar that deals with all aspects of pest control in the Isles. The approach of the PPD changed from fire fighting (1950-80) via problem analysis (1980-93), when more advanced steps were taken in determining causes, losses and threshold levels, to IPM. Starting in 1994 the trend is towards a more holistic approach, where pest and diseases are looked at in the entire cropping systems contert. As most farmers are just too poor to use external inputs, pesticide use is insignificant, and IPM is nor developed as an alternative to pesticide use, but to generate cheap and effective crop protection packages that fit in the social-economic context.

IPM development in Zanzibar ideally is characterized by:

- Farmers participation: farmers play a central role in defining priorities, experimental design, and execution of on-farm trials

- Local, on-farm development of technology

- Inventory and exploitation of existing traditional knowledge of sustainable crop protection practices

- Interdisciplinary cooperation: various researchers and extensionists work together with farmers and farmers groups.

The IPM development process

In order to be successful in implementing an PM strategy, institutional as well as technical adaptations are necessary. Consequently, the PPD went through a number of steps to redirect its vision from a conventional approach (extensionists that try to 'sell' research results to farmers) to a participatory development of local technology. This process started with a series of internal workshops to redefine the policy, mandate and focus of the PPD, and to analyze the present working methods and shortcomings. The Southeast Asian rice IPM example was studied, and useful elements indicated. Participatory tools were reviewed. Subsequently, external workshops on both islands were heid to communicate the concept of IPM and the strategy of the PPD to government officials and to other agricultural projects, and to ask their cooperation. Next a session was held to develop prionities in crops and crop protection problems. Suitable criteria were developed first, and using these, the most important crops and problems were defined. For Rice, Cassava, Banana and Vegetables interdisciplinary. 'IPM Crop Groups' were formed with the assignment to develop action plans for participatory activities that will result in local on-farm development of cheap and adequate crop protection technologies. IPM coordinators have been appointed. As IPM is very knowledge-intensive appropriate methods of both farmer training and upgrading of facilitating staff have to be found. In irrigated rice, effectivity of botanical extracts of neem and utupa (Tephrosia vogelit) to control Trichispa beetles are studied, while a comprehensive package of cultural measures to avoid Hispa damage is developed.

Plant Protection Division of Zanzibar, P.O. Box 1062, Zanzibar. Phone/Fax; +255-54-32110. 
INVESIGATLON OH TÄE DISTRIBUTION OF THE CODLIGN WOTH IN CHINA

Ruihua Iin Menguo Yao Kexun iluanq

The codling goth Laspeyresia panare/la (t.). is one of the nost important

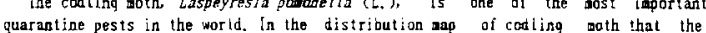

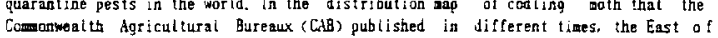
Comonmeat th Agricultural Bureaux (Cid) pubtished in different times, the East of
China especially the Bohai Bay were placed on the list of coding anth infested areas. And it caused serious influence an the export of Chinese apples and pears. Mearwhile some countries that codling moth existed requested chine to rolax the restriction of the import of apples. thus a threaten to the production of the apples and pears of China was constituted.

In order to clarify the raal distribution situation of this past in China in Shangdnag Lianning, Hebei, Beijing, Henar Gansy and Xinjiang altagether seyen

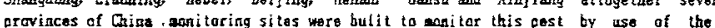



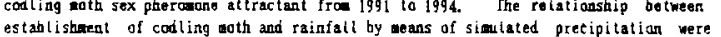

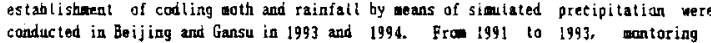
experiasents were canducted in different typos and applo orchards during Aprit to

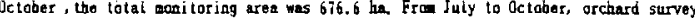
fruit chack by dissertion was carriad out. There wore altogather 49 tieas for the orchard survey and fruit dissection. It covered 82 countioss 189 different types of

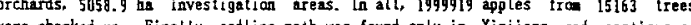

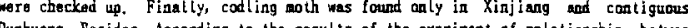
Durhuang. Basides. Accarding to the results of the exprinent of relationsthip betwees codling moth and simulated rainfall indoor. It was proisisinary demostrated that the devel lopent of codling moth was greatly inhibit tert by precipi tation. Siace the Babai Bay belongs to the teaperats zone ant it has mansoon climate. the antual rainfall usual ly cascentrates on June and August. espeerially an Juity and Augest. The average procipitation any at tain 900 per month. That eliento is unfavourable for codling

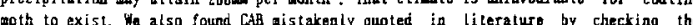
acth to chist ho also

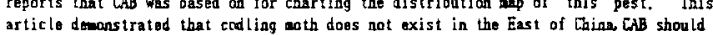
article demanstrated that cording
correct the ersor in the app.

ADDRESS OF RUTHUA JIN:

Department of Plant Frotection

Beijing agricultural University

Beijing 100094

China

\section{2}

CURRENT RESEAROH ON LEGANE VIRUSES WITH SPECIAL DMPHASIS ON GROND NUT VIRUSES IN SRI LANKA

JANAKIE C. RRJAPAKSE.

Several virus diseases were found to affect the major legume crops grown ex. Compea, unghean, black gram and groundnut in Sri Lanka. Mungbean Yellow Mosaic Virus (MMV) and Bud necrosis disease (BND) in groundinut were found to be predominant. The END was caused by tanato spotted wilt virus (TSWV) and distorted groundnut leaves with mosaic mottling positively identified as ISWV by transmission and serology. The crusal virus and transmission and serology of MMN in Sri Lanka has already been described. The TSWV was transnitted by the trrip Frankliniella schultzei. The incidence and managenent of $F_{-}$shultzei were studied during 1991-92 period. The maximum monthly trap catches of thrip species were reconded in Septenber and lowest in Jamuary and February. Intercropping with other cereal crop resulted in low incidence of thrips. The insecticide Thiodicarb were fourd to be effective in controlling aymphs of F. schultzei. The trap counts of thrip which were used an indicators of the insecticidal efficiency were insignificant for the vectors.

(Division OF PLANT PATHOLOGY, regional RESEAROH STATION, DEPARTMENT OF AGRICULTURE, ANGUNAKOLAFELESSA, SRI LANKA.)
EFFECTS OF PLANT GROWTH REGULATORS ON SCLEROTINIA SCLEROTIORUM OF SUNFLOWER

\section{G.R.Komitova, D.T.Todorov}

Sclerotinia sclerotiorum (Lib.) de Bary attacks stems and capitula of sunflower. This fungus may become a limiting factor of sunflower production.

The controt of the disease is a very important task, because fungicidal treatments are not effective enough.

The plant growth regulators (PGRs) - monomethyl ester of itaconic acid (MEIA) and monoethyl ester of succinic acid (MESA) were screened for toxicity to the colony of $\mathrm{S}$. sclerotiorum on agar. In these tests the PGRs were not toxic to sclerotia and also stimulated the production of the pathogen. However, the PGRs with concentracion 10-2 $\mathrm{M}$ inhibited the germination of sclerotia and the production of apothecia in vitro. The use MEIA and MESA may be perspectivic way to decrease ascospore production and the damase of $S$. sclerotiorum in sunflower.

Plant Protection Institute,

2230 Kostinbrod

Bulgaria
1653

PESTICIDE RESIDUES IN BREAD AND BREAKFAST CEREALS -

$$
\text { THE COMPLETE PICTURE }
$$

Matthews, W.A. and Panton, S.W.

It has been common practice for the last thirty yesrs to apply admixtures of pesticides to whest before storage, In order to prevent insect infestation. If the grain is to be stored for any length of time the applied pesticide must remaln on the grain at high enough levels to ensure it remains insect free. As a result, when the wheat is taken out of storage it will still contain residues. In this work we looked at what happens to residues of organophosphorus pesticides when wheat is processed to make bread and a high bran extruded breakfast cereal, using $14 \mathrm{C}$ labelled pesticides.

The result show that cooklng decreases the overall level of residues, the scale of reduction being dependant on the nature of the process.

However, the proportion of nonextractable residues (as defined by XUPAC)

Increased significantly. This has important 1aplications for the monitoring of pesticides residues in bread and other processed grain products, since the methods used for monitoring do not take nonextractable residues into account. Such residues could be bioavailable, so information obtaln so far using non labelled methods may not give a complete picture of the fate of residues in processed foods. 